\begin{tabular}{|c|c|}
\hline $\begin{array}{l}\text { 2. To: (Receiving Organization) } \\
\text { Distribution }\end{array}$ & $\begin{array}{l}\text { 3. From: (originating organization) } \\
\text { R. A. Watrous }\end{array}$ \\
\hline $\begin{array}{l}\text { 5. Proj./Prog./Dept./Div.: } \\
\text { TWRS }\end{array}$ & $\begin{array}{l}\text { 6. Design Authority/ Design Agent/Cog. } \\
\text { Engr.: } \\
\text { R. A. Watrous } 74640\end{array}$ \\
\hline
\end{tabular}

8. Originator Remarks:

For Approval $]_{2}$,

11. Receiver Remarks:
[] Yes [x] No
4. Related EDT No.:

NA

7. Purchase Order No.: NA

9. Equip./Component No.: NA

10. System/Btdg./Facjlity: NA

12. Major Assm. Dwg. No.: NA

13. Permit/Permit Application No.: NA

14. Required Response Date: NA

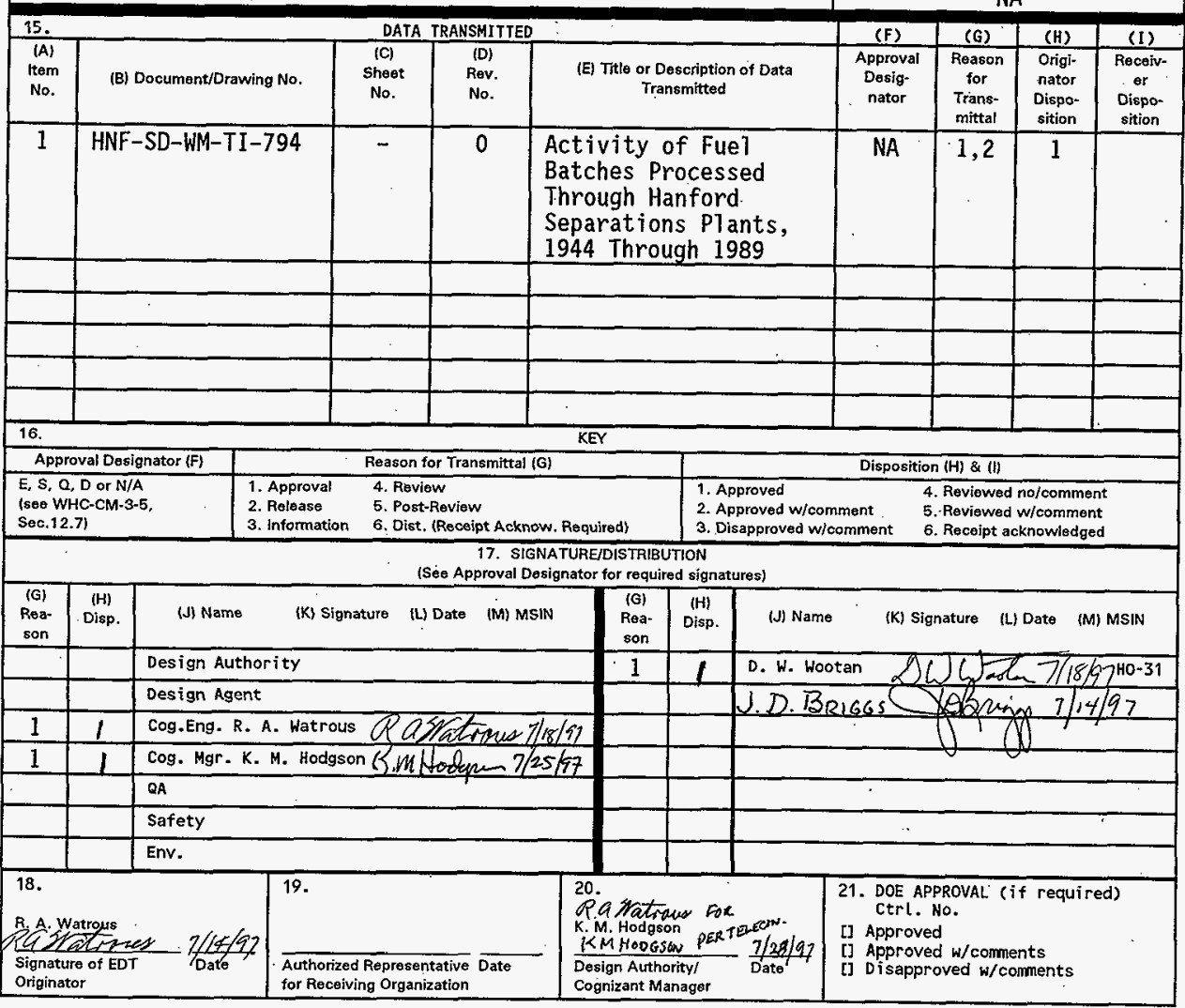




\title{
Activity of Fuel Batches Processed Through Hanford Separations Plants, 1944 Through 1989
}

\author{
R. A. Watrous and D. W. Wootan (FDNW) \\ Lockheed Martin Hanford Corporation, RichTand, WA 99352 \\ U.S. Department of Energy Contract DE-AC06-96RL13200
}

\author{
EDT/ECN: $619948 \quad \cdot$ UC: 721 \\ Org Code: 74640 Charge Code: N4G3A \\ B\&R Code: EW3120074 Total Pages: 202
}

Key Words: fuel, radionuclides

Abstract: This document provides a printout of the "Fuel Activity Database $^{18}$ (version U6) generated by the Hanford DKPRO code and transmitted to the LoS Al amos National Laboratory for input to their "Hanford Defined Waste" model of waste tank inventories. This fuel activity file consists of 1,276 records--each record representing the activity associated with a batch of spent reactor fuel processed by month (or shorter period) through individual Hanford separations plants between 1944 and 1989. Each record gives the curies for 46 key radionuclides, decayed to a common reference date of January $1,1994$.

TRADEMARK DISCLAIMER. Reference herein to any specific commercial product, process, or service by trade name, trademark, manufacturer, or otherwise, does not necessarily constitute or imply its endorsement, recommendation, or favoring by the United States Government or any agency thereof or its contractors or subcontractors.

Printed in the United States of America. To obtain copies of this document, contact: Document Control Services, P.0. Box 950, Mailstop H6-08, Richland WA 99352, Phone (509) 372-2420; Fax (509) 376-4989.
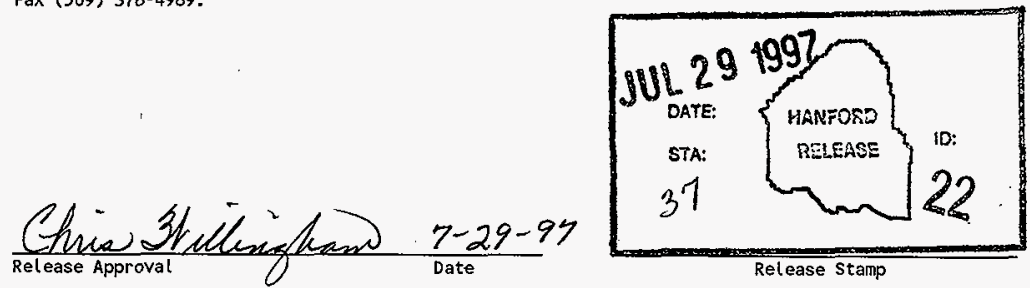


\title{
ACTIVITY OF FUEL BATCHES PROCESSED THROUGH HANFORD SEPARATIONS PLANTS, 1944 THROUGH 1989
}

July 1997

\author{
R. A. Watrous \\ Lockheed Martin Hanford Company \\ Richland, Washington \\ D. W. Wootan \\ Fluor Daniel Northwest \\ Richland, Washington
}

Prepared for

U.S. Department of Energy

Richland, Washington

DOES NOT CONTAIN CLASSIFIED OR

UNCLASSIFIED CONTROLLED

NUCLEAR INFORMATION

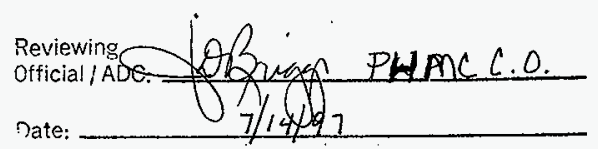


HNF-SD-WM-TI-794

Revision 0

This page intentionally left blank. 


\section{HNF-SD-WM-TI-794}

Revision 0

\section{CONTENTS}

1.0 INTRODUCTION $\ldots \ldots \ldots \ldots \ldots \ldots \ldots \ldots \ldots \ldots \ldots \ldots$

2.0 FUEL ACTIVITY FILE $\ldots \ldots \ldots \ldots \ldots \ldots \ldots \ldots \ldots \ldots$

3.0 ORIGEN2 AND DKPRO CODES $\ldots \ldots \ldots \ldots \ldots \ldots \ldots \ldots$

4.0 SIMPLIFYING ASSUMPTIONS $\ldots \ldots \ldots \ldots \ldots \ldots \ldots \ldots \ldots$

5.0 DKPRO OUTPUT SUMMATION $\ldots \ldots \ldots \ldots \ldots \ldots \ldots \ldots$

6.0 ACCURACY OF THE "UNIFIED SEPARATIONS PRODUCTION FILE" . . . . 11

7.0 REFERENCES . . . . . . . . . . . . . . . . . . . . . . . . 19

\section{APPENDIXES}

A FUEL ACTIVITY FILE $\ldots \ldots \ldots \ldots \ldots \ldots \ldots \ldots$ A-1

B DKPRO/ORIGEN2 ASSUMPTIONS AND FILES $\ldots \ldots \ldots \ldots \ldots$. . . 
HNF-SD-WM-TI-794

Revision 0

\section{LIST OF FIGURES}

1. Comparision of Metric Ton Uranium $\ldots \ldots \ldots \ldots \ldots \ldots \ldots$

2. Comparision of Megawatt Days $\ldots \ldots \ldots \ldots \ldots \ldots \ldots \ldots$

\section{LIST OF TABLES}

1. Definition of Fuel Types and Fuel Codes for Radionuclide Inventory Modelling. . . . 3

2. Summation of Fuel Activity Records. . . . . . . . . . . . 7

3. Comparison of Fuel Tonnage and Exposure From Separations Plant and

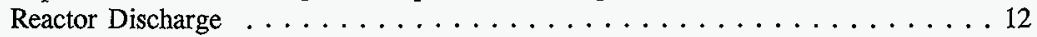

4. Comparison of Total Fuel Tonnage Between Separations Plant Records and Reactor Discharge Records . . . . . . . . . . . . . . . . . . . 15

5. Comparison of Total Fuel Exposure Between Separations Plant Records and Reactor Discharge Records . . . . . . . . . . . . . . . . . 16

6. Inventory by Fuel Type $\ldots \ldots \ldots \ldots \ldots \ldots \ldots \ldots \ldots \ldots \ldots \ldots \ldots \ldots \ldots \ldots \ldots$

7. N Reactor Fuel Shipped Offsite $\ldots \ldots \ldots \ldots \ldots \ldots \ldots \ldots$

8. Comparison of Fuel in $\mathrm{K}$ Basins $\ldots \ldots \ldots \ldots \ldots \ldots \ldots$ 
HNF-SD-WM-TI-794

Revision 0

\section{ACTIVITY OF FUEL BATCHES PROCESSED THROUGH HANFORD SEPARATIONS PLANTS, 1944 THROUGH 1989}

\subsection{INTRODUCTION}

One objective of the Standard Tank Inventory task under development at the Hanford Site is to estimate total ("global") inventories and tank-by-tank inventories for 46 key radionuclides contained in 177 high-level waste tanks.

The current strategy for generating these estimates is to modify and expand the Los Alamos National Laboratory (LANL) developed Hanford Defined Waste (HDW) model (Agnew et al. 1996) to carry data fields for these radionuclides. This existing model provides the calculational framework to identify receiver tanks for the various waste streams exiting the separations plants and to model subsequent tank-to-tank and tank-to-crib waste transfers and solubility effects.

Data on radionuclides contained in batches of discharged reactor fuel are needed as input to the HDW model. These data are generated by the ORIGEN (Croff 1980, Wittekind 1994a) and DKPRO (Schmittroth and Wootan 1997) code developed and maintained at the Hanford Site by Fluor Daniel Northwest Environmental and Nuclear Initiative group. Output from the DKPRO code is an electronic file of "pre-decayed" Fuel Activity Records that is then transmitted to Los Alamos (the HDW model). This report provides hard copy documentation of this data file.

Sections 2.0 and 3.0, below, describe the Fuel Activity Record data file and the codes by which it is generated. A discussion of simplifying assumptions is presented in Section 4.0 to serve as a basis for future corrections to the estimate of activation products. Section $\mathbf{5 . 0}$ presents the Total Fuel Activity inventory table. The detailed (batch-by-batch) fuel activity data file is presented in Appendix A. Finally, Section 6.0 presents data comparisons to help validate the accuracy of the Separations Production data file, upon which the ORIGEN/DKPRO calculations are based.

\subsection{FUEL ACTIVITY FILE}

Output from the DKPRO code is 1,276 Fuel Activity Records of pre-decayed radionuclide values, each record representing the curies associated with a batch of fuel processed by month (or shorter period) through individual separation plants, but modelled as though the fuel was held (unseparated) until the reference decay date of January 1, 1994. 


\section{Revision 0}

A hard copy of the Fuel Activity File is attached as Appendix A. . Each record is continued over eight pages and is actually made up of two groups of data: (1) Separations Production information that is contained on the first two of every eight pages, and (2) Fuel Activity Data, contained on the following six pages of every eight pages. The 1,276 fuel batch records are first grouped by the four separation plants ( $T, B$, Reduction and Oxidation [REDOX], Plutonium-Uranium Extraction [PUREX]) through which the fuel was processed; within each group the records are listed chronologically by separations date.

The Separations Production data fields provide a copy of data describing each fuel batch as follows:

- Listing Number (only appearing on this hard copy printout)

- Fuel Type code

- MTU per batch

- Estimated percent ${ }^{240} \mathrm{Pu}$ in total $\mathrm{Pu}$ (used on certain records as an index from which fuel exposure is derived)

- Fuel Exposure (MWd/MTU)

- Separations Plant

- Separations Date

- Separations Key (the "HAPO Day" corresponding to the separations date)

- Cooling Days

- A reference for the preceding data

- Total Waste Loss Fractions for Th, U, Np, and Pu

- A reference for this waste loss data.

With the exception of the Waste Loss data, these separations production data were used as control parameters to the DKPRO code; they are being passed on to the HDW model only for information/reference purposes.

The fuel activity data fields list curies (decayed to January 1, 1994) for 46 key radionuclides contained in each batch of fuel. 
The fuel activity listing given in Appendix A represents a minor rearrangement of the actual DKPRO output file. The order of listing certain radionuclides has been changed and cladding waste activity values are combined with separations waste activity values so as to simplify data input to the HDW model.

\subsection{ORIGEN2 AND DKPRO CODES}

The DKPRO code solves the general problem of modeling complex nuclear waste streams using ORIGEN2 (Croff 1980, Wittekind 1994a) radionuclide production files. ORIGEN2 is used to calculate initial radionuclide inventories with subsequent decay and. processing computed by the DKPRO code. Input to the DKPRO code includes an archive file of separations processing records by fuel batch, a file of processing directives, a file of summary directives, and a set of ORIGEN2 inventory files representing unprocessed radionuclide inventories. For each separations processing record, DKPRO calculates the radionuclide activities in that batch of fuel and then applies whatever processing instructions are provided in the processing directives file.

For the purpose of this radionuclide modeling task, Hanford Site fuels have been classified in six fuel types, as defined in Table 1. For each fuel type, ORIGEN2 was used to calculate radionuclide inventories corresponding to particular fuel exposures. As described in Appendix B, a set of fifteen ORIGEN2 output files were used to represent the six fuel types at various exposures. DKPRO interpolates with respect to burnup (using a Lagrange interpolating polynomial) for values that do not match the specific burnup on the ORIGEN2 source files.

Table 1. Definition of Fuel Types and Fuel Codes for Radionuclide Inventory Modelling.

\begin{tabular}{|c|l|}
\hline Code & \multicolumn{1}{|c|}{ Fuel definition } \\
\hline Al-0.71U & $\begin{array}{l}\text { Aluminum clad, natural enrichment uranium (0.71 percent U-235) -- either } \\
\text { solid core or I\&E. Single-pass reactor fuel. }\end{array}$ \\
\hline Al-0.94U & $\begin{array}{l}\text { Aluminum clad, "Enriched" uranium (0.94 percent U-235). Single-pass } \\
\text { reactor fuel. }\end{array}$ \\
\hline Zr-0.94U & Zircaloy clad, "Enriched" uranium (0.94 percent U-235). N Reactor fuel. \\
\hline $\mathrm{Zr-1.1U}$ & $\begin{array}{l}\text { Zircaloy clad, "Spike" uranium (0.94 percent U-235 inner fuel tube and } \\
1.25 \text { percent U-235 outer fuel tube, together averaging 1.1 percent U-235). } \\
\text { N Reactor fuel. }\end{array}$ \\
\hline $\mathrm{Zr}-2.1 \mathrm{U}$ & $\begin{array}{l}\text { Zircaloy clad, "Co-Product" driver uranium (2.1 percent U-235). N Reactor } \\
\text { fuel. }\end{array}$ \\
\hline Al-0.0Th & Aluminum clad, Thorium oxide target elements. Single-pass reactors. \\
\hline
\end{tabular}


The processing directives input to DKPRO are used to control the removal or splitting of waste streams. This flexible capability allows the DKPRO code to be used to model the chemical separation of routinely extracted elements such as uranium, plutonium, neptunium, thorium, and technetium, and the removal (to atmosphere, silver reactors, or cribs) of volatile elements such as hydrogen $\left({ }^{3} \mathrm{H}\right)$, carbon $\left({ }^{44} \mathrm{C}\right)$, and iodine $\left({ }^{129} \mathrm{I}\right)$. However, for generating the input needed for the HDW model, no processing of the fuel or removal of constituents was done with DKPRO.

The processing directives input also provides DKPRO the ability to output two sets of nuclides representing primary and secondary waste streams. Splitting factors for specific elements, as well as a default splitting factor for all other elements can be specified. Splitting factors between the primary separations waste stream and the secondary cladding waste stream for uranium, plutonium, thorium, and neptunium, and a general splitting factor for all other elements, were included in the DKPRO calculation of nuclide inventories that was provided to LANL. However, since this splitting was also incorporated into the HDW model, the splitting done by DKPRO was not used.

Approximately 1,300 fuel batch records are contained in the "Unified Separations Production File". Each record contains data on fuel type, fuel tonnage, exposure, separations date, and cooling time. These data are used by DKPRO in determining the nuclide inventory associated with each batch of fuel. To do so, DKPRO interpolates the ORIGEN2 inventory files as a function of fuel type and fuel exposure (MWd/MTU), applying separations or splitting factors, and performing decay calculations.

In the current version of the DKPRO/HDW model interface, the DKPRO code calculates radionuclide decay from the date of fuel discharge to the "standard inventory reference decay date," January 1, 1994, accounting for the unique timing of each fuel batch. This "pre-decay" feature avoids the complexity of adding decay functions for all radionuclides in the LANL portion of the overall model. However, a special correction is required in the case of ${ }^{241} \mathrm{Am}$ to account for the removal of its parent, ${ }^{241} \mathrm{Pu}$, at the fuel separation date. Note that this correction for ${ }^{241} \mathrm{Am}$ remaining in tank waste at January 1,1994 , is very sensitive to the fuel cooling time, the fraction of ${ }^{241} \mathrm{Pu}$ remaining in the waste, and the waste aging period (separation date to January 1, 1994).

Output from DKPRO is controlled by the list of output radionuclides included in the DKPRO input. The output consists of a file of "fuel activity records, " corresponding to the fuel batch records in the "Unified Separations Production File". Each fuel activity record contains the activity in curies of 46 key radionuclides.

The input and output files for the ORIGEN2 and DKPRO calculations are listed in Appendix B. 
HNF-SD-WM-TI-794

Revision 0

\subsection{SIMPLIFYING ASSUMPTIONS}

The following simplifying assumptions have been made in the current version of the DKPRO code, affecting the accuracy of radionuclide estimates:

- ORIGEN2 code values for ${ }^{14} \mathrm{C}(\mathrm{Ci} / \mathrm{MTU})$ are based on an assumed constant level of nitrogen impurity in uranium fuel cores of $50 \mathrm{p} / \mathrm{M}$ for aluminum clad fuel and $75 \mathrm{p} / \mathrm{M}$ for zirconium clad fuel. In reality, the nitrogen level varied with time, ranging from highs of $90 \mathrm{p} / \mathrm{M}$ in the late 1940 's to lows of about $8 \mathrm{p} / \mathrm{M}$ during the 1970's.

- Activation products, ${ }^{59} \mathrm{Ni}$ and ${ }^{63} \mathrm{Ni}$, are based on an assumed constant $7,800 \mathrm{p} / \mathrm{M}$ of $\mathrm{Ni}$ in aluminum cladding. (In actuality, pre-1959 aluminum alloys contained only $100 \mathrm{p} / \mathrm{M}$, while post-1959 aluminum contained Ni levels of 9,000 to $13,000 \mathrm{p} / \mathrm{M}$.)

- The ORIGEN2 estimates for activation product, ${ }^{60} \mathrm{Co}$, assume a cobalt impurity level of $10 \mathrm{p} / \mathrm{M}$ in cladding and $0 \mathrm{p} / \mathrm{M}$ in core uranium. Since actual levels of cobalt in uranium are unknown, but probably greater than zero, it is predictable that the DKPRO output results for ${ }^{60} \mathrm{Co}$ are low.

- The ORIGEN2 code's prediction of ${ }^{237} \mathrm{~Np}$ is based on an assumed constant level of $400 \mathrm{p} / \mathrm{M}^{236} \mathrm{U}$ in initial 0.947 percent enriched uranium input to the Hanford Site reactors. In actuality, the ${ }^{236} \mathrm{U}$ level varied from about $35 \mathrm{p} / \mathrm{M}$ in 1959 to over $700 \mathrm{p} / \mathrm{M}$ in enriched uranium irradiated during the 1980 's. Neptunium-237 production can be expected to vary somewhat with the ${ }^{236} \mathrm{U}$ level, but a direct proportionality is not expected (due to the multiple neutron capture paths involved in ${ }^{237} \mathrm{~Np}$ production).

Certain inaccuracies in the ORIGEN2 code are now known that also affect the accuracy of values listed here for ${ }^{79} \mathrm{Se}$ :

- Curie values for ${ }^{79} \mathrm{Se}$ calculated by ORIGEN2 are suspected of being high by a factor of about 8 due to the use of an erroneous half-life value in the ORIGEN2 data library. 
HNF-SD-WM-TI-794

Revision 0

This page intentionally left blank. 
HNF-SD-WM-TI-794

Revision 0

\subsection{DKPRO OUTPUT SUMMATION}

A summation of the 1276 waste activity records generated by the DKPRO code is presented in Table 2 for key radionuclides. These values represent the total curies of radionuclides (decayed to the common date of January 1, 1994) contained in 1276 batches of unseparated fuel. As described in Section 2.0, above, values do not account for the separation of ${ }^{3} \mathrm{H},{ }^{14} \mathrm{C}$, Tc (partial separation), ${ }^{129} \mathrm{I}$, Th, $\mathrm{U}, \mathrm{Np}$, and Pu. Also, ${ }^{241} \mathrm{Am}$ is overestimated because of it's dependance on the decay of parent, ${ }^{241} \mathrm{Pu}$.

Table 2 is presented, here, for its value in support of internal model checking. It should not be considered the "global tank inventory," although for radionuclides other than ${ }^{3} \mathrm{H},{ }^{129} \mathrm{I},{ }^{90} \mathrm{Sr},{ }^{137} \mathrm{Cs}, \mathrm{Pu}, \mathrm{U}$, and ${ }^{241} \mathrm{Am}$, it may be fairly close.

Table 2. Summation of Fuel Activity Records.

(Activity in Fuels Processed Through T, B, REDOX, and PUREX Plants)

Units: Accumulated Curies, decayed to January 1, 1994. (3 Sheets)

\begin{tabular}{|c|c|c|c|c|c|}
\hline Nuclide & $\mathrm{T}$ & $\mathrm{B}$ & REDOX & PUREX & Total fuel \\
\hline${ }^{3} \mathrm{H}$ & $2.4586 \mathrm{E}+03$ & $1.1977 \mathrm{E}+03$ & $3.3332 \mathrm{E}+04$ & $2.0056 \mathrm{E}+05$ & $2.3755 \mathrm{E}+05^{\mathrm{b}}$ \\
\hline${ }^{14} \mathrm{C}$ & $1.0587 \mathrm{E}+02$ & $5.9093 \mathrm{E}+01$ & $8.2533 \mathrm{E}+02$ & $3.9131 \mathrm{E}+03$ & $4.9034 \mathrm{E}+03^{\mathrm{a}}$ \\
\hline${ }^{59} \mathrm{Ni}$ & $3.0105 \mathrm{E}+01$ & $1.6809 \mathrm{E}+01$ & $2.2280 \mathrm{E}+02$ & $9.1338 \mathrm{E}+02$ & $1.1831 \mathrm{E}+03^{\mathrm{c}}$ \\
\hline${ }^{60} \mathrm{Co}$ & $2.6997 \mathrm{E}+01$ & $1.0746 \mathrm{E}+01$ & $6.3880 \mathrm{E}+02$ & $1.2617 \mathrm{E}+04$ & $1.3294 \mathrm{E}+04^{\mathrm{b}}$ \\
\hline${ }^{63} \mathrm{Ni}$ & $2.7314 \mathrm{E}+03$ & $1.4982 \mathrm{E}+03$ & $2.1422 \mathrm{E}+04$ & $9.1162 \mathrm{E}+04$ & $1.1681 \mathrm{E}+05^{\mathrm{c}}$ \\
\hline${ }^{79} \mathrm{Se}$ & $2.2329 \mathrm{E}+01$ & $1.2466 \mathrm{E}+01$ & $1.8944 \mathrm{E}+02$ & $7.7628 \mathrm{E}+02$ & $1.0005 \mathrm{E}+03$ \\
\hline${ }^{90} \mathrm{Sr}$ & $1.7556 \mathrm{E}+06$ & $9.2590 \mathrm{E}+05$ & $1.7987 \mathrm{E}+07$ & $8.3254 \mathrm{E}+07$ & $1.0392 \mathrm{E}+08$ \\
\hline${ }^{90} \mathrm{Y}$ & $1.7561 \mathrm{E}+06$ & $9.2617 \mathrm{E}+05$ & $1.7991 \mathrm{E}+07$ & $8.3275 \mathrm{E}+07$ & $1.0395 \mathrm{E}+08$ \\
\hline${ }^{93} \mathrm{Zr}$ & $1.0606 \mathrm{E}+02$ & $5.9214 \mathrm{E}+01$ & $9.0207 \mathrm{E}+02$ & $3.6695 \mathrm{E}+03$ & $4.7369 \mathrm{E}+03$ \\
\hline${ }^{93 m} \mathrm{Nb}$ & $8.9008 \mathrm{E}+01$ & $5.0460 \mathrm{E}+01$ & $7.0552 \mathrm{E}+02$ & $2.6318 \mathrm{E}+03$ & $3.4768 \mathrm{E}+03$ \\
\hline${ }^{99} \mathrm{Tc}$ & $7.3475 \mathrm{E}+02$ & $4.1020 \mathrm{E}+02$ & $6.2798 \mathrm{E}+03$ & $2.5727 \mathrm{E}+04$ & $3.3151 \mathrm{E}+04^{\mathrm{d}}$ \\
\hline${ }^{106} \mathrm{Ru}$ & $1.4622 \mathrm{E}-05$ & $1.0825 \mathrm{E}-06$ & $1.3195 \mathrm{E}-01$ & $1.1415 \mathrm{E}+05$ & $1.1415 \mathrm{E}+05$ \\
\hline${ }^{113 \mathrm{~m}} \mathrm{Cd}$ & $2.6964 \mathrm{E}+02$ & $1.3426 \mathrm{E}+02$ & $3.1995 \mathrm{E}+03$ & $1.8813 \mathrm{E}+04$ & $2.2416 \mathrm{E}+04$ \\
\hline${ }^{125} \mathrm{Sb}$ & $2.7220 \mathrm{E}+01$ & $7.7304 \mathrm{E}+00$ & $2.0995 \mathrm{E}+03$ & $2.1734 \mathrm{E}+05$ & $2.1948 \mathrm{E}+05$ \\
\hline${ }^{126} \mathrm{Sn}$ & $3.3597 \mathrm{E}+01$ & $1.8752 \mathrm{E}+01$ & $2.8083 \mathrm{E}+02$ & $1.1992 \mathrm{E}+03$ & $1.5324 \mathrm{E}+03$ \\
\hline${ }^{129} \mathrm{I}$ & $1.3845 \mathrm{E}+00$ & $7.7272 \mathrm{E}-01$ & $1.1942 \mathrm{E}+01$ & $5.0014 \mathrm{E}+01$ & $6.4114 \mathrm{E}+01^{\mathrm{a}}$ \\
\hline
\end{tabular}


Table 2. Summation of Fuel Activity Records.

(Activity in Fuels Processed Through T, B, REDOX, and PUREX Plants)

Units: Accumulated Curies, decayed to January 1, 1994. (3 Sheets)

\begin{tabular}{|c|c|c|c|c|c|}
\hline Nuclide & $\mathbf{T}$ & B & REDOX & PUREX & Total fuel \\
\hline${ }^{134} \mathrm{Cs}$ & $1.8980 \mathrm{E}-01$ & $5.8620 \mathrm{E}-02$ & $1.1945 \mathrm{E}+02$ & $8.8749 \mathrm{E}+04$ & $8.8868 \mathrm{E}+04$ \\
\hline${ }^{137} \mathrm{Cs}$ & $1.9919 \mathrm{E}+06$ & $1.0526 \mathrm{E}+06$ & $2.0569 \mathrm{E}+07$ & $9.6533 \mathrm{E}+07$ & $1.2015 \mathrm{E}+08$ \\
\hline${ }^{137} \mathrm{Ba}$ & $1.8843 \mathrm{~B}+06$ & $9.9573 \mathrm{E}+05$ & $1.9459 \mathrm{E}+07$ & $9.1320 \mathrm{E}+07$ & $1.1366 \mathrm{E}+08$ \\
\hline${ }^{151} \mathrm{Sm}$ & $8.3648 \mathrm{E}+04$ & $4.5873 \mathrm{E}+04$ & $6.7110 \mathrm{E}+05$ & $2.7398 \mathrm{E}+06$ & $3.5404 \mathrm{E}+06$ \\
\hline${ }^{152} \mathrm{Eu}$ & $6.3161 \mathrm{E}+00$ & $3.2721 \mathrm{~B}+00$ & $2.2865 \mathrm{E}+02$ & $1.6472 \mathrm{E}+03$ & $1.8854 \mathrm{E}+03$ \\
\hline${ }^{154} \mathrm{Eu}$ & $4.5854 \mathrm{E}+02$ & $2.2141 \mathrm{E}+02$ & $1.5532 \mathrm{E}+04$ & $1.5771 \mathrm{E}+05$ & $1.7392 \mathrm{E}+05$ \\
\hline${ }^{155} \mathrm{Eu}$ & $5.2277 \mathrm{E}+02$ & $1.9976 \mathrm{E}+02$ & $1.1460 \mathrm{E}+04$ & $1.4830 \mathrm{E}+05$ & $1.6049 \mathrm{E}+05$ \\
\hline${ }^{226} \mathrm{Ra}$ & $5.8189 \mathrm{E}-03$ & $3.6713 \mathrm{E}-03$ & $1.8391 \mathrm{E}-02$ & $4.9354 \mathrm{E}-02$ & $7.7235 \mathrm{E}-02$ \\
\hline${ }^{227} \mathrm{Ac}$ & $2.9980 \mathrm{E}-02$ & $1.8493 \mathrm{E}-02$ & $9.2668 \mathrm{E}-02$ & $1.0828 \mathrm{E}+02$ & $1.0842 \mathrm{~B}+02$ \\
\hline${ }^{228} \mathrm{Ra}$ & $2.2923 \mathrm{E}-08$ & $1.3713 \mathrm{E}-08$ & $3.7605 \mathrm{E}-07$ & $6.4400 \mathrm{E}+01$ & $6.4400 \mathrm{~B}+01$ \\
\hline${ }^{229} \mathrm{Th}$ & $4.4428 \mathrm{E}-06$ & $2.6675 \mathrm{E}-06$ & $3.3146 \mathrm{E}-05$ & $2.9159 \mathrm{E}+01$ & $2.9159 \mathrm{E}+01$ \\
\hline${ }^{231} \mathrm{~Pa}$ & $6.6192 \mathrm{E}-02$ & $3.9119 \mathrm{E}-02$ & $2.3182 \mathrm{E}-01$ & $1.9051 \mathrm{E}+02$ & $1.9085 \mathrm{E}+02$ \\
\hline${ }^{232} \mathrm{Th}$ & $2.9589 \mathrm{E}-08$ & $1.7447 \mathrm{E}-08$ & $5.2688 \mathrm{E}-07$ & $6.8849 \mathrm{E}+01$ & $6.8849 \mathrm{~B}+01^{f}$ \\
\hline${ }^{232} \mathrm{U}$ & $3.1323 \mathrm{E}-02$ & $1.7088 \mathrm{E}-02$ & $4.6124 \mathrm{E}-01$ & $3.0175 \mathrm{E}+03$ & $3.0180 \mathrm{E}+03^{\mathrm{f}}$ \\
\hline${ }^{233} \mathrm{U}$ & $1.5232 \mathrm{E}-03$ & $8.7560 \mathrm{E}-04$ & $1.4190 \mathrm{E}-02$ & $1.1719 \mathrm{E}+04$ & $1.1719 \mathrm{E}+04^{\mathrm{f}}$ \\
\hline 234 U & $1.6549 \mathrm{E}+03$ & $9.1207 \mathrm{E}+02$ & $7.7552 \mathrm{E}+03$ & $2.5284 \mathrm{E}+04$ & $3.5607 \mathrm{E}+04^{\mathrm{f}}$ \\
\hline $235 \bigcup$ & $7.3675 \mathrm{E}+01$ & $4.0584 \mathrm{E}+01$ & $3.1304 \mathrm{E}+02$ & $1.0406 \mathrm{E}+03$ & $1.4679 \mathrm{E}+03^{\mathrm{f}}$ \\
\hline${ }^{236} \mathrm{U}$ & $1.4164 \mathrm{E}+01$ & $7.9107 \mathrm{E}+00$ & $3.2831 \mathrm{E}+02$ & $8.2331 \mathrm{E}+02$ & $1.1737 \mathrm{E}+03^{\mathrm{f}}$ \\
\hline${ }^{237} \mathrm{~Np}$ & $4.5353 \mathrm{E}+00$ & $2.5313 \mathrm{~B}+00$ & $5.5477 \mathrm{E}+01$ & $2.1315 \mathrm{E}+02$ & $2.7569 \mathrm{E}+02^{f_{\mathrm{i}}}$ \\
\hline${ }^{238} \mathrm{Pu}$ & $6.3383 \mathrm{E}+02$ & $3.4613 \mathrm{~B}+02$ & $1.9217 \mathrm{E}+04$ & $1.0946 \mathrm{E}+05$ & $1.2966 \mathrm{E}+05^{\mathrm{f}}$ \\
\hline${ }^{238} \mathrm{U}$ & $1.6795 \mathrm{E}+03$ & $9.2580 \mathrm{E}+02$ & $6.5635 \mathrm{E}+03$ & $2.3757 \mathrm{E}+04$ & $3.2925 \mathrm{E}+04^{f}$ \\
\hline${ }^{239} \mathrm{Pu}$ & $9.3980 \mathrm{E}+04$ & $5.2495 \mathrm{E}+04$ & $6.7420 \mathrm{E}+05$ & $2.9005 \mathrm{E}+06$ & $3.7212 \mathrm{E}+06^{f}$ \\
\hline${ }^{240} \mathrm{Pu}$ & $8.1803 E+03$ & $4.5369 \mathrm{E}+03$ & $1.0684 \mathrm{E}+05$ & $5.5897 \mathrm{E}+05$ & $6.7853 \mathrm{E}+05^{f}$ \\
\hline${ }^{241} \mathrm{Am}$ & $5.3375 \mathrm{E}+03$ & $2.9448 \mathrm{E}+03$ & $1.1810 \mathrm{~B}+05$ & $6.7318 \mathrm{E}+05$ & $7.9956 \mathrm{E}+05$ \\
\hline${ }^{241} \mathrm{Pu}$ & $2.5460 \mathrm{E}+04$ & $1.3436 \mathrm{E}+04$ & $9.4004 \mathrm{E}+05$ & $8.9967 \mathrm{E}+06$ & $9.9757 \mathrm{E}+06^{\mathrm{f}}$ \\
\hline${ }^{242} \mathrm{Pu}$ & $1.1453 \mathrm{E}-01$ & $6.0803 \mathrm{E}-02$ & $4.4066 \mathrm{~B}+00$ & $3.7910 \mathrm{E}+01$ & $4.2492 \mathrm{E}+01^{\mathrm{f}}$ \\
\hline${ }^{242} \mathrm{Cm}$ & 1.1653E-01 & $5.9033 \mathrm{E}-02$ & $9.4060 \mathrm{~B}+00$ & $1.0034 \mathrm{E}+02$ & $1.0993 \mathrm{E}+02$ \\
\hline
\end{tabular}




\section{Revision 0}

Table 2. Summation of Fuel Activity Records.

(Activity in Fuels Processed Through T, B, REDOX, and PUREX Plants)

Units: Accumulated Curies, decayed to January 1, 1994. (3 Sheets)

\begin{tabular}{|c|c|c|c|c|c|}
\hline Nuclide & T & B & REDOX & PUREX & Total fuel \\
\hline${ }^{243} \mathrm{Am}$ & $3.8843 \mathrm{E}-02$ & $1.9711 \mathrm{E}-02$ & $3.4749 \mathrm{E}+00$ & $3.8406 \mathrm{E}+01$ & $4.1940 \mathrm{E}+01$ \\
\hline${ }^{243} \mathrm{Cm}$ & $2.4202 \mathrm{E}-03$ & $1.1748 \mathrm{E}-03$ & $8.4762 \mathrm{E}-01$ & $1.2897 \mathrm{E}+01$ & $1.3748 \mathrm{E}+01$ \\
\hline${ }^{244} \mathrm{Cm}$ & $3.5367 \mathrm{E}-02$ & $1.6981 \mathrm{E}-02$ & $1.6940 \mathrm{E}+01$ & $2.9687 \mathrm{E}+02$ & $3.1386 \mathrm{E}+02$ \\
\hline
\end{tabular}

PUREX $=$ Plutonium-Uranium Extraction

REDOX $=$ Reduction and Oxidation (Hexone) solvent extraction plant.

${ }^{2}$ Values for ${ }^{3} \mathrm{H},{ }^{14} \mathrm{C}$, and ${ }^{129} \mathrm{I}$ represent total activity in fuel, and as such do not account for significant portions of these nuclides that were routed to dissolver offgas streams or plant condensate streams.

${ }^{b}$ Values for ${ }^{60} \mathrm{Co}$ needs to be revised upward slightly to account for presence of $\mathrm{Co}$ in uranium fuel core as well as in cladding.

${ }^{6}$ Values for ${ }^{59} \mathrm{Ni}$ and ${ }^{63} \mathrm{Ni}$ do not account for increase of $\mathrm{Ni}$ in aluminum cladding during the late 1950 's.

${ }^{\mathrm{a}}$ Values for ${ }^{99} \mathrm{Tc}$ represent total activity in fuel, and as such do not account for a small fraction of Tc that may have been extracted during fuel separation.

${ }^{\text {e Values for }}{ }^{237} \mathrm{~Np}$ reflect a constant level of $400 \mathrm{p} / \mathrm{M}{ }^{236} \mathrm{U}$ in enriched uranium and as such do not account for the ramp up variation in ${ }^{236} U$ level over time.

${ }^{f}$ Values for $\mathrm{Th}, \mathrm{U}, \mathrm{Np}$, and $\mathrm{Pu}$ isotopes represent total activity in fuel, and as such do not account for the relatively large fraction of these elements that were extracted during fuel separation operations. 
HNF-SD-WM-TI-794

Revision 0

This page intentionally left blank. 
HNF-SD-WM-TI-794

Revision 0

\subsection{ACCURACY OF THE "UNIFIED SEPARATIONS PRODUCTION FUL"}

The "Unified Separations Production File" (UNIFIED6.PRN) contains 1,276 records of fuel batches processed by month (or shorter period) through individual separation plants. Fuel processing records from the separations plants (B, T, REDOX, PUREX) were used to construct this file. All of the fuel was categorized into six fuel types.

One way of assessing the validity of this file is to compare with records of the fuel discharged from the Hanford Site production reactors. All of the fuel discharged from the reactors should have been either processed through the separations plants, stored in the K Basins, or shipped offsite.

A previous study of historical plutonium production at Hanford Site (Roblyer 1994) included a comprehensive examination of reactor power production and fuel discharges. The file RCHIVE.ALL (Roblyer 1994) provides monthly mass and burnup values for fuel discharges from 1944 through 1987 from the single-pass reactors (B, C, D, DR, F, H, KE, $\mathrm{KW}$ ) and N Reactor. The discharged fuel is categorized in 25 different fuel types, as compared to the six fuel types in the separations plant file. The reactor fuel discharge data were derived from TRAC data files (Jungfleisch 1984 and 1992) with modifications and extensions based on monthly reactor reports and other data.

The detailed data in the separations production file and the reactor discharge file were summarized to provide yearly cumulative totals of fuel mass in metric tons uranium (MTU) and fuel burnup in Megawatt days (MWd). All of the aluminum clad fuel was categorized as single-pass reactor (SPR) fuel and all of the zirconium clad fuel was categorized as $\mathrm{N}$ Reactor (NR) fuel. The aluminum clad thoria fuel was included with the SPR fuel. Table 3 lists the cumulative MWd and MTU values by year for the separations plant file (UNIFIED6.PRN) and for the reactor discharge file (RCHIVE.ALL). These values are plotted in Figures 1 and 2.

Table 4 and Figure 1 compare the cumulative fuel masses in MTU for single-pass reactor and $\mathrm{N}$ Reactor fuel for the separations plant file and the reactor discharge file. In Table 4 the fuel currently in storage or processed offsite (described later) has been added to the separations plant totals to provide a more direct comparison with the amounts discharged from the reactors. The $\mathrm{N}$ Reactor totals agree very well. The single-pass reactor difference is larger in magnitude than the $\mathrm{N}$ Reactor fuel difference, but represents only a small percentage of the total. The single-pass reactor difference is most likely representative of the overall uncertainty in fuel records over more than forty years of fuel discharges and processing. 
HNF-SD-WM-TI-794

Revision 0

Table 3. Comparison of Fuel Tonnage and Exposure From Separations Plant and Reactor Discharge Records. (2 Sheets)

\begin{tabular}{|c|c|c|c|c|c|c|c|c|}
\hline \multicolumn{9}{|c|}{ Cumulative Metric Ton Uranium and Megawatt day by Year } \\
\hline & \multicolumn{4}{|c|}{ Separations Plant Data (UNIFIED6.PRN) } & \multicolumn{4}{|c|}{ Reactor Plant Data (RCHIVE.ALL) } \\
\hline Year & MTU-SPR & MTU-NR & MWd-SPR & MWd-NR & MTU-SPR & MTU-NR & MWd-SPR & MWd-NR \\
\hline 1944 & $3.27 \mathrm{E}+00$ & 0.00 & $7.92 \mathrm{E}+01$ & 0.00 & $1.28 \mathrm{E}+01$ & 0.00 & $3.10 \mathrm{E}+02$ & 0.00 \\
\hline 1945 & $5.64 \mathrm{E}+02$ & 0.00 & $1.21 \mathrm{E}+05$ & 0.00 & $6.91 \mathrm{E}+02$ & 0.00 & $1.62 \mathrm{E}+05$ & 0.00 \\
\hline 1946 & $1.34 \mathrm{E}+03$ & 0.00 & $3.07 \mathrm{E}+05$ & 0.00 & $1.44 \mathrm{E}+03$ & 0.00 & $3.32 \mathrm{E}+05$ & 0.00 \\
\hline 1947 & $1.92 \mathrm{E}+03$ & 0.00 & $4.37 \mathrm{E}+05$ & 0.00 & $2.10 \mathrm{E}+03$ & 0.00 & $4.81 \mathrm{E}+05$ & 0.00 \\
\hline 1948 & $2.64 \mathrm{E}+03$ & 0.00 & $5.99 \mathrm{E}+05$ & 0.00 & $2.91 \mathrm{E}+03$ & 0.00 & $6.67 \mathrm{E}+05$ & 0.00 \\
\hline 1949 & $3.31 \mathrm{E}+03$ & 0.00 & $8.05 \mathrm{E}+05$ & 0.00 & $3.51 \mathrm{E}+03$ & 0.00 & $8.84 \mathrm{E}+05$ & 0.00 \\
\hline 1950 & $4.12 E+03$ & 0.00 & $1.16 \mathrm{E}+06$ & 0.00 & $4.34 \mathrm{E}+03$ & 0.00 & $1.25 \mathrm{E}+06$ & 0.00 \\
\hline 1951 & $5.09 \mathrm{E}+03$ & 0.00 & $1.67 \mathrm{E}+06$ & 0.00 & $5.28 \mathrm{E}+03$ & 0.00 & $1.79 \mathrm{E}+06$ & 0.00 \\
\hline 1952 & $6.21 \mathrm{E}+03$ & 0.00 & $2.41 \mathrm{E}+06$ & 0.00 & $6.58 \mathrm{E}+03$ & 0.00 & $2.63 \mathrm{E}+06$ & 0.00 \\
\hline 1953 & $7.73 \mathrm{E}+03$ & 0.00 & $3.36 \mathrm{E}+06$ & 0.00 & $8.22 \mathrm{E}+03$ & 0.00 & $3.69 \mathrm{E}+06$ & 0.00 \\
\hline 1954 & $1.01 \mathrm{E}+04$ & 0.00 & $4.59 \mathrm{E}+06$ & 0.00 & $1.12 \mathrm{E}+04$ & 0.00 & $5.17 \mathrm{E}+06$ & 0.00 \\
\hline 1955 & $1.34 \mathrm{E}+04$ & 0.00 & $6.04 \mathrm{E}+06$ & 0.00 & $1.50 \mathrm{E}+04$ & 0.00 & $6.85 \mathrm{E}+06$ & 0.00 \\
\hline 1956 & $1.79 \mathrm{E}+04$ & 0.00 & $8.32 \mathrm{E}+06$ & 0.00 & $1.98 \mathrm{E}+04$ & 0.00 & $9.80 \mathrm{E}+06$ & 0.00 \\
\hline 1957 & $2.35 \mathrm{E}+04$ & 0.00 & $1.18 \mathrm{E}+07$ & 0.00 & $2.57 \mathrm{E}+04$ & 0.00 & $1.32 \mathrm{E}+07$ & 0.00 \\
\hline 1958 & $2.95 \mathrm{E}+04$ & 0.00 & $1.54 \mathrm{E}+07$ & 0.00 & $3.15 \mathrm{E}+04$ & 0.00 & $1.69 \mathrm{E}+07$ & 0.00 \\
\hline 1959 & $3.50 \mathrm{~B}+04$ & 0.00 & $1.97 \mathrm{E}+07$ & 0.00 & $3.74 \mathrm{E}+04$ & 0.00 & $2.16 \mathrm{E}+07$ & 0.00 \\
\hline 1960 & $4.13 E+04$ & 0.00 & $2.44 \mathrm{E}+07$ & 0.00 & $4.40 \mathrm{E}+04$ & 0.00 & $2.66 \mathrm{E}+07$ & 0.00 \\
\hline 1961 & $4.79 \mathrm{E}+04$ & 0.00 & $2.96 \mathrm{~B}+07$ & 0.00 & $5.06 \mathrm{E}+04$ & 0.00 & $3.18 \mathrm{E}+07$ & 0.00 \\
\hline 1962 & $5.43 \mathrm{E}+04$ & 0.00 & $3.43 \mathrm{E}+07$ & 0.00 & $5.73 \mathrm{E}+04$ & 0.00 & $3.68 \mathrm{E}+07$ & 0.00 \\
\hline 1963 & $6.04 \mathrm{E}+04$ & $1.54 \mathrm{E}+00$ & $3.89 \mathrm{E}+07$ & $1.50 \mathrm{E}+03$ & $6.35 E+04$ & 0.00 & $4.20 \mathrm{E}+07$ & 0.00 \\
\hline 1964 & $6.72 \mathrm{E}+04$ & $2.99 \mathrm{E}+00$ & $4.41 \mathrm{~B}+07$ & $2.17 \mathrm{~B}+03$ & $7.16 \mathrm{E}+04$ & $5.02 \mathrm{E}+01$ & $4.80 \mathrm{E}+07$ & $1.60 \mathrm{E}+04$ \\
\hline 1965 & $7.40 \mathrm{E}+04$ & $1.15 \mathrm{~B}+01$ & $4.90 \mathrm{E}+07$ & $1.72 \mathrm{~B}+04$ & $7.74 \mathrm{E}+04$ & $3.23 \mathrm{E}+02$ & $5.28 \mathrm{E}+07$ & $5.19 \mathrm{E}+05$ \\
\hline 1966 & $7.90 \mathrm{E}+04$ & $2.31 \mathrm{~B}+02$ & $5.29 \mathrm{E}+07$ & $4.61 \mathrm{E}+05$ & $8.20 \mathrm{E}+04$ & $5.09 \mathrm{E}+02$ & $5.64 \mathrm{E}+07$ & $9.47 \mathrm{E}+05$ \\
\hline 1967 & $8.33 \mathrm{E}+04$ & $3.53 \mathrm{E}+02$ & $5.66 \mathrm{E}+07$ & $7.44 \mathrm{E}+05$ & $8.61 \mathrm{E}+04$ & $8.94 \mathrm{~B}+02$ & $6.04 \mathrm{E}+07$ & $1.84 \mathrm{E}+06$ \\
\hline 1968 & $8.67 \mathrm{E}+04$ & $7.80 \mathrm{E}+02$ & $5.99 \mathrm{E}+07$ & $1.69 \mathrm{E}+06$ & $8.94 \mathrm{E}+04$ & $1.19 \mathrm{E}+03$ & $6.39 \mathrm{E}+07$ & $2.47 \mathrm{E}+06$ \\
\hline 1969 & $8.93 \mathrm{E}+04$ & $1.02 \mathrm{E}+03$ & $6.29 \mathrm{~B}+07$ & $2.15 \mathrm{~B}+06$ & $9.19 \mathrm{E}+04$ & $1.51 \mathrm{E}+03$ & $6.66 \mathrm{E}+07$ & $3.26 \mathrm{E}+06$ \\
\hline 1970 & $9.03 \mathrm{E}+04$ & $1.11 \mathrm{~B}+03$ & $6.41 E+07$ & $2.37 \mathrm{E}+06$ & $9.38 \mathrm{E}+04$ & $1.83 \mathrm{E}+03$ & $6.77 \mathrm{E}+07$ & $3.97 \mathrm{E}+06$ \\
\hline 1971 & $9.32 \mathrm{E}+04$ & $1.43 \mathrm{E}+03$ & $6.62 \mathrm{E}+07$ & $3.05 \mathrm{E}+06$ & $9.45 \mathrm{E}+04$ & $2.08 \mathrm{E}+03$ & $6.80 \mathrm{E}+07$ & $4.44 \mathrm{E}+06$ \\
\hline 1972 & $9.38 \mathrm{E}+04$ & $1.77 \mathrm{E}+03$ & $6.64 \mathrm{E}+07$ & $3.81 \mathrm{E}+06$ & $9.45 \mathrm{E}+04$ & $2.35 \mathrm{E}+03$ & $6.80 \mathrm{E}+07$ & $4.91 \mathrm{E}+06$ \\
\hline 1973 & $9.38 \mathrm{E}+04$ & $1.77 \mathrm{E}+03$ & $6.64 \mathrm{E}+07$ & $3.81 \mathrm{E}+06$ & $9.45 \mathrm{E}+04$ & $2.69 \mathrm{~B}+03$ & $6.80 \mathrm{E}+07$ & $5.67 \mathrm{E}+06$ \\
\hline 1974 & $9.38 \mathrm{E}+04$ & $1.77 \mathrm{E}+03$ & $6.64 \mathrm{E}+07$ & $3.81 \mathrm{E}+06$ & $9.45 \mathrm{E}+04$ & $2.97 \mathrm{E}+03$ & $6.80 \mathrm{E}+07$ & $6.41 \mathrm{E}+06$ \\
\hline
\end{tabular}




\section{HNF-SD-WM-TI-794}

\section{Revision 0}

Table 3. Comparison of Fuel Tonnage and Exposure From Separations Plant and Reactor Discharge Records. (2 Sheets)

\begin{tabular}{|c|c|c|c|c|c|c|c|c|}
\hline \multicolumn{8}{|c|}{ Cumulative Metric Ton Uranium and Megawatt day by Year . } \\
\hline & \multicolumn{3}{|c|}{ Separations Plant Data (UNIFIED6.PRN) } & \multicolumn{3}{|c|}{ Reactor Plant Data (RCHIVE.ALI) } \\
\hline Year & MTU-SPR & MTU-NR & MWd-SPR & MWd-NR & MTU-SPR & MTU-NR & MWd-SPR & MWd-NR \\
\hline 1975 & $9.38 \mathrm{E}+04$ & $1.77 \mathrm{E}+03$ & $6.64 \mathrm{E}+07$ & $3.81 \mathrm{E}+06$ & $9.45 \mathrm{E}+04$ & $3.20 \mathrm{E}+03$ & $6.80 \mathrm{E}+07$ & $7.04 \mathrm{E}+06$ \\
\hline 1976 & $9.38 \mathrm{E}+04$ & $1.77 \mathrm{E}+03$ & $6.64 \mathrm{E}+07$ & $3.81 \mathrm{E}+06$ & $9.45 \mathrm{E}+04$ & $3.36 \mathrm{E}+03$ & $6.80 \mathrm{E}+07$ & $7.47 \mathrm{~B}+06$ \\
\hline 1977 & $9.38 \mathrm{E}+04$ & $1.77 \mathrm{E}+03$ & $6.64 \mathrm{E}+07$ & $3.81 \mathrm{E}+06$ & $9.45 \mathrm{E}+04$ & $3.69 \mathrm{E}+03$ & $6.80 \mathrm{E}+07$ & $8.42 \mathrm{E}+06$ \\
\hline 1978 & $9.38 \mathrm{E}+04$ & $1.77 \mathrm{E}+03$ & $6.64 \mathrm{E}+07$ & $3.81 \mathrm{E}+06$ & $9.45 \mathrm{E}+04$ & $3.97 \mathrm{E}+03$ & $6.80 \mathrm{E}+07$ & $9.19 \mathrm{E}+06$ \\
\hline 1979 & $9.38 \mathrm{E}+04$ & $1.77 \mathrm{E}+03$ & $6.64 \mathrm{E}+07$ & $3.81 \mathrm{E}+06$ & $9.45 \mathrm{E}+04$ & $4.25 \mathrm{E}+03$ & $6.80 \mathrm{E}+07$ & $9.95 \mathrm{E}+06$ \\
\hline 1980 & $9.38 \mathrm{E}+04$ & $1.77 \mathrm{E}+03$ & $6.64 \mathrm{E}+07$ & $3.81 \mathrm{E}+06$ & $9.45 \mathrm{E}+04$ & $4.47 \mathrm{E}+03$ & $6.80 \mathrm{E}+07$ & $1.04 \mathrm{E}+07$ \\
\hline 1981 & $9.38 \mathrm{E}+04$ & $1.77 \mathrm{E}+03$ & $6.64 \mathrm{E}+07$ & $3.81 \mathrm{E}+06$ & $9.45 \mathrm{E}+04$ & $4.56 \mathrm{E}+03$ & $6.80 \mathrm{E}+07$ & $1.07 \mathrm{E}+07$ \\
\hline 1982 & $9.38 \mathrm{E}+04$ & $1.77 \mathrm{E}+03$ & $6.64 \mathrm{E}+07$ & $3.81 \mathrm{E}+06$ & $9.45 \mathrm{E}+04$ & $5.23 \mathrm{E}+03$ & $6.80 \mathrm{E}+07$ & $1.17 \mathrm{E}+07$ \\
\hline 1983 & $9.38 \mathrm{E}+04$ & $1.86 \mathrm{E}+03$ & $6.64 \mathrm{E}+07$ & $3.91 \mathrm{E}+06$ & $9.45 \mathrm{E}+04$ & $5.83 \mathrm{E}+03$ & $6.80 \mathrm{E}+07$ & $1.23 \mathrm{E}+07$ \\
\hline 1984 & $9.38 \mathrm{E}+04$ & $2.95 \mathrm{E}+03$ & $6.64 \mathrm{E}+07$ & $5.12 \mathrm{E}+06$ & $9.45 \mathrm{E}+04$ & $6.50 \mathrm{E}+03$ & $6.80 \mathrm{E}+07$ & $1.30 \mathrm{E}+07$ \\
\hline 1985 & $9.38 \mathrm{E}+04$ & $4.10 \mathrm{E}+03$ & $6.64 \mathrm{E}+07$ & $6.81 \mathrm{E}+06$ & $9.45 \mathrm{E}+04$ & $7.23 \mathrm{E}+03$ & $6.80 \mathrm{E}+07$ & $1.37 \mathrm{E}+07$ \\
\hline 1986 & $9.38 \mathrm{E}+04$ & $4.95 \mathrm{E}+03$ & $6.64 \mathrm{E}+07$ & $7.82 \mathrm{E}+06$ & $9.45 \mathrm{E}+04$ & $7.78 \mathrm{E}+03$ & $6.80 \mathrm{E}+07$ & $1.41 \mathrm{E}+07$ \\
\hline 1987 & $9.38 \mathrm{E}+04$ & $5.08 \mathrm{E}+03$ & $6.64 \mathrm{E}+07$ & $8.14 \mathrm{E}+06$ & $9.45 \mathrm{E}+04$ & $8.15 \mathrm{E}+03$ & $6.80 \mathrm{E}+07$ & $1.44 \mathrm{E}+07$ \\
\hline 1988 & $9.38 \mathrm{E}+04$ & $5.66 \mathrm{E}+03$ & $6.64 \mathrm{E}+07$ & $8.74 \mathrm{E}+06$ & & & & \\
\hline 1989 & $9.38 \mathrm{E}+04$ & $5.66 \mathrm{E}+03$ & $6.64 \mathrm{E}+07$ & $8.74 \mathrm{E}+06$ & & & & \\
\hline
\end{tabular}


HNF-SD-WM-TI-794

Revision 0

Figure 1. Comparision of Metric Ton Uranium.

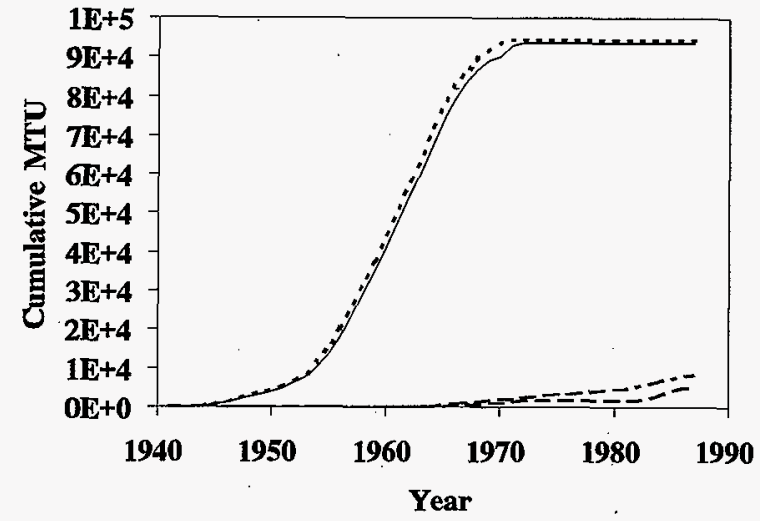

- Separations Plant-SPR - - Separations Plant-NR

-... Reactor Discharge-SPR - - Reactor Discharge-NR

Figure 2. Comparision of Megawatt Days.

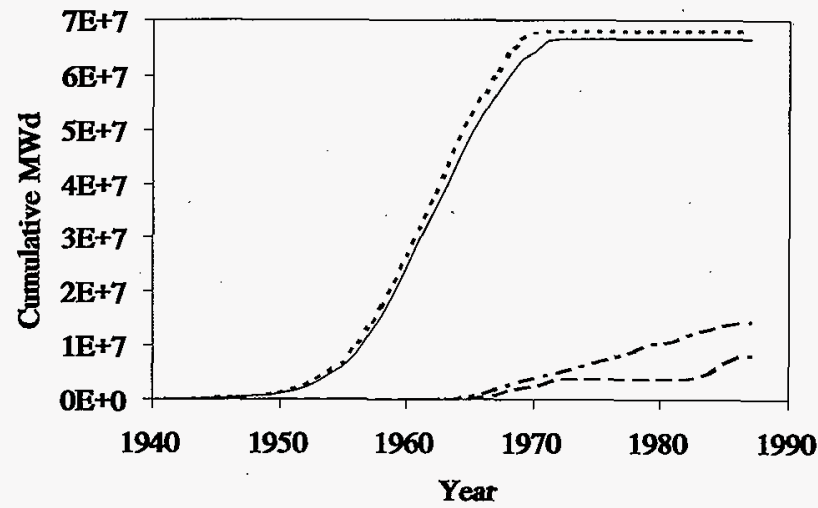

Separations Plant-SPR - - - Separations Plant-NR

- . - - Reactor Discharge-SPR - - Reactor Discharge-NR 
HNF-SD-WM-TI-794

Revision 0

Table 4. Comparison of Total Fuel Tonnage Between Separations Plant Records and Reactor Discharge Records.

\begin{tabular}{|c|c|c|c|}
\hline & $\begin{array}{l}\text { Single-pass } \\
\text { reactor MTU }\end{array}$ & $\begin{array}{l}\text { N Reactor } \\
\text { MTU }\end{array}$ & $\begin{array}{l}\text { Total } \\
\text { MTU }\end{array}$ \\
\hline $\begin{array}{l}\text { Processing Plant Records } \\
\text { Fuel Processed Onsite } \\
\text { (UNIFIED6.PRN) } \\
\text { Fuel Processed Offsite } \\
\text { Fuel Remaining in Basin Storage } \\
\text { Total: Processed and Stored }\end{array}$ & 93,748 & $\begin{array}{c}5,663 \\
379 \\
2,090 \\
8,132\end{array}$ & $\begin{array}{c}99,411 \\
379 \\
2,090 \\
101,880\end{array}$ \\
\hline $\begin{array}{l}\text { Reactor Records } \\
\text { Fuel Discharged (RCHIVE.ALL) }\end{array}$ & 94,448 & 8,147 & 102,595 \\
\hline $\begin{array}{l}\text { Fuel Discharged minus } \\
\text { Processed and Stored }\end{array}$ & 700 & 15 & 715 \\
\hline Percent difference & +0.74 & +0.18 & +0.67 \\
\hline
\end{tabular}

MTU $=$ Metric Ton Uranium.

Table 5 and Figure 2 compare the cumulative fuel exposure in MWd for single-pass reactor and $\mathrm{N}$ Reactor fuel for the separations plant file and reactor discharge file. In Table 5 the MWd for the fuel currently in storage or processed offsite (described later) has been added to the separations plant totals to provide a more direct comparison with the MWd discharged from the reactors. The differences are larger than for the MTU values, but still relatively small. There is an inherent uncertainty in the fuel exposures due to the uncertainty in overall reactor power level and individual tube powers. 
HNF-SD-WM-TI-794

Revision 0

Table 5. Comparison of Total Fuel Exposure Between Separations Plant Records and Reactor Discharge Records.

\begin{tabular}{|l|c|c|c|}
\hline & $\begin{array}{c}\text { Single-pass } \\
\text { reactor MWd }\end{array}$ & $\begin{array}{c}\text { N Reactor } \\
\mathrm{MWd}\end{array}$ & $\begin{array}{c}\text { Total } \\
\mathrm{MWd}\end{array}$ \\
\hline $\begin{array}{c}\text { Processing Plant Records } \\
\text { Fuel Processed Onsite } \\
\quad \text { (UNIFIED6.PRN) }\end{array}$ & $6.64 \mathrm{E}+07$ & $8.74 \mathrm{E}+06$ & $7.51 \mathrm{E}+07$ \\
$\begin{array}{c}\text { Fuel Processed Offsite } \\
\text { Fuel Remaining in Basin Storage } \\
\text { Total: Processed and Stored }\end{array}$ & $6.64 \mathrm{E}+07$ & $\frac{7.28 \mathrm{E}+05}{5.37 \mathrm{E}+06}$ & $\begin{array}{c}7.28 \mathrm{E}+05 \\
\frac{5.37 \mathrm{E}+06}{8.48 \mathrm{E}+07}\end{array}$ \\
\hline $\begin{array}{l}\text { Reactor Records } \\
\text { Fuel Discharged (RCHIVE.ALL) }\end{array}$ & $6.79 \mathrm{E}+07$ & $1.44 \mathrm{E}+07$ & $8.23 \mathrm{E}+07$ \\
\hline $\begin{array}{l}\text { Fuel Discharged minus } \\
\text { Processed and Stored }\end{array}$ & $1.50 \mathrm{E}+06$ & $-4.40 \mathrm{E}+05$ & $1.06 \mathrm{E}+06$ \\
\hline Percent difference & +2.2 & -3.1 & +1.3 \\
\hline
\end{tabular}

MWd = Megawatt day.

Table 6 provides a summary of the total fuel inventory by fuel type for the separations plant file and the reactor discharge file. The six fuel types are those used for the separations plant file. The largest differences were found for the enriched aluminum clad SPR fuel (Al-0.94) and enriched zirconium-clad $\mathrm{N}$ Reactor fuel ( $\mathrm{Zr}-1.1)$. Some of the differences could be due to differences in the sorting of twenty five different fuel types into six categories.

Table 6. Inventory by Fuel Type.

\begin{tabular}{|c|c|c|c|c|}
\hline Fuel type & $\begin{array}{c}\text { MTU } \\
\text { UNIFIED6.PRN }\end{array}$ & $\begin{array}{c}\text { MTU } \\
\text { RCHIVE.ALL }\end{array}$ & $\begin{array}{c}\text { MWd } \\
\text { UNIFIED6.PRN }\end{array}$ & $\begin{array}{c}\text { MWd } \\
\text { RCHIVE.ALL }\end{array}$ \\
\hline $\mathrm{Al}-0.71$ & $7.81 \mathrm{E}+04$ & $7.83 \mathrm{E}+04$ & $5.19 \mathrm{E}+07$ & $5.22 \mathrm{E}+07$ \\
\hline $\mathrm{Al}-0.94$ & $1.50 \mathrm{E}+04$ & $1.56 \mathrm{E}+04$ & $1.38 \mathrm{E}+07$ & $1.49 \mathrm{E}+07$ \\
\hline $\mathrm{Al}-0.0 \mathrm{Th}$ & $6.28 \mathrm{E}+02$ & $5.48 \mathrm{E}+02$ & $6.36 \mathrm{E}+05$ & $8.35 \mathrm{E}+05$ \\
\hline $\mathrm{Zr}-0.94$ & $5.80 \mathrm{E}+03$ & $5.76 \mathrm{E}+03$ & $9.84 \mathrm{E}+06$ & $9.82 \mathrm{E}+06$ \\
\hline $\mathrm{Zr}-1.1$ & $1.59 \mathrm{E}+03$ & $2.04 \mathrm{E}+03$ & $3.51 \mathrm{E}+06$ & $3.82 \mathrm{E}+06$ \\
\hline $\mathrm{Zr}-2.1$ & $3.59 \mathrm{E}+02$ & $3.50 \mathrm{E}+02$ & $7.67 \mathrm{E}+05$ & $7.54 \mathrm{E}+05$ \\
\hline
\end{tabular}

MTU $=$ Metric Ton Uranium

$\mathrm{MWd}=$ Megawatt day. 
The Nuclear Fuel Services (NFS) facility, at West Valley, New York, reprocessed N Reactor fuel from Hanford Site during the period of 1966 through 1971. Table 7 provides a summary of different references on the amount of $\mathrm{N}$ Reactor fuel shipped offsite. The approximately 400 MTU of N Reactor fuel shipped offsite roughly matches the difference in reactor discharge (RCHIVE.ALL file) and separations plant (UNIFIED6.PRN file) inventories shown in Table 6 for the combination of $\mathrm{Zr}-0.94$ and $\mathrm{Zr}-1.1$ fuel types. No records were found of substantial amounts of SPR fuel shipped offsite.

Table 7. N Reactor Fuel Shipped Offsite

\begin{tabular}{|c|c|c|}
\hline & MTU & MWd \\
\hline $\begin{array}{c}\text { TRAC file PCHIVE.BIG } \\
\text { (Jungfleisch 1992) }\end{array}$ & 294. & $6.24 \mathrm{E}+05$ \\
\hline Myers (1995) & 379. & $7.28 \mathrm{E}+05^{*}$ \\
\hline
\end{tabular}

MTU $=$ Metric Ton Uranium

MWd = Megawatt day

*Estimated based on average burnup of $1920 \mathrm{MWd} /$ ton.

The fuel storage basins at the Hanford Site K Reactor contain unreprocessed fuel discharged from Hanford Site reactors. The UNIFIED6.PRN separations plant file includes a separate category for all the fuel stored in the K Basins. The standard database for spent nuclear fuel in the K Basins is the Safeguards Control and Accountability Transaction System (SCATS) (Dasinger 1993). A related database is the reconstructed SCATS database (R-SCATS) (Wittekind 1994b and Schlosser 1992). Table 8 compares the total mass and burnup of basin fuel in the separations plant file with the SCATS and R-SCATS databases. The MTU values agree within 0.5 percent. The SCATS and R-SCATS databases reflect improved burnup estimates that address the effect of a segregation campaign whereby lowburnup fuel elements were selectively removed for reprocessing. This could explain the lower MWd values in the UNIFIED6.PRN file compared to the standard databases.

In general, the reactor discharge values and separations plants values for fuel mass and exposure agree fairly well. As expected, there is a time delay between the discharge from the reactor and the processing of the fuel in the separations plants. This is particularly evident for the long PUREX shutdown. Most of the differences for $\mathrm{N}$ Reactor fuel can be explained by the shipment of $\mathbf{N}$ Reactor fuel to be processed offsite. Differences for the single-pass reactor fuel can be attributed to the uncertainty in the records. 
HNF-SD-WM-TI-794

Revision 0

Table 8. Comparison of Fuel in $\mathrm{K}$ Basins.

\begin{tabular}{|c|l|c|}
\hline & MTU & MWd \\
\hline UNIFIED6.PRN & 2090. & $5.374 \mathrm{E}+06$ \\
\hline SCATS & 2099. & $5.848 \mathrm{E}+06$ \\
\hline R-SCATS & 2098. & $5.557 \mathrm{E}+06$ \\
\hline
\end{tabular}

MTU $=$ Metric Ton Uranium

MWd $=$ Megawatt day. 


\subsection{REFERENCES}

Agnew, S. F., J. Boyer, R. A. Corbin, T. B. Duran, J. R. FitzPatrick, K. A. Jurgensen, T. P. Ortiz, and B. L. Young, 1996, Hanford Tank Chemical and Radionuclide Inventories: HDW Model Rev. 3, LA-UR-96-858, Los Alamos National Laboratory, Los Alamos, New Mexico.

Croft, A. G., 1980, ORIGEN2 - A Revised and Updated Version of the Oak Ridge Isotope Generation and Depletion Code, ORNL-5621, Oak Ridge National Laboratory, Oak Ridge, Tennessee.

Dasinger, J. R., 1993, Nuclear Material Safeguards Systems User's Manual, WHC-IP-0382, Rev. 1, Westinghouse Hanford Company, Richland, Washington.

Jungfleisch, F. M., 1984, Preliminary Estimation of the Waste Inventories in Hanford Tanks Through 1980, SD-WM-TI-057, Rockwell Hanford Operations, Richland, Washington.

Jungfleisch, F. M., 1992, TRAC Data Files (U), WHC 92-0001 Disk (2A), Westinghouse Hanford Company, Richland, Washington.

Myers, L., 1995, Plutonium Recovery from Spent Fuel Processing At West Valley, New York, DP-22, West Valley, New York.

Roblyer, S. P., 1994, Plutonium and Tritium Produced in the Hanford Site Production Reactors, WHC-SD-CP-RPT-014, Westinghouse Hanford Company, Richland, Washington.

Schlosser, J. E., 1992, The FUELSEG Program, The Estimation of Isotopics of N Reactor Segregated Irradiated Fuels, WHC-SD-NR-CSWD-049, Westinghouse Hanford Company, Richland, Washington.

Schmittroth, F., 1996, Comparison of Spent Fuel Databases for $K$ Basin, WHC-SD-SNF-ANAL-011, Westinghouse Hanford Company, Richland, Washington.

Schmittroth, F., and D. Wootan, 1997, DKPRO: A Radionuclide Decay and Reprocessing Code, HNF-SD-WM-CSWD-082, Fluor Daniel Northwest, Richland, Washington.

Wittekind, W. D., 1994a, Software Certification Package for the ORIGEN2 Code, WHC-SD-NR-SWD-006, Westinghouse Hanford Company, Richland, Washington.

Wittekind, W. D., 1994b, Consolidated Fuel Decay Heat Calculations, WHC-SD-NR-ANAL-014, Westinghouse Hanford Company, Richland, Washington. 
HNF-SD-WM-TI-794

Revision 0

This page intentionally left blank. 
HNF-SD-WM-TI-794

Revision 0

\section{APPENDIX A}

\section{FUEL ACTIVITY FILE}

NOTE: Because of its size, the Fuel Activity file has been omitted from all but the arachieval copies (U.S. Department of Energy Reading Room, Central Files, TCSRC, historical file) of this distribution. 
HNF-SD-WM-TI-794

Revision 0

This page intentionally left blank. 
HNF-SD-WN-TI-794, ReV. 0

UNIFIEO SEPARATIONS PRODUCTION DATA- SEPN DATE

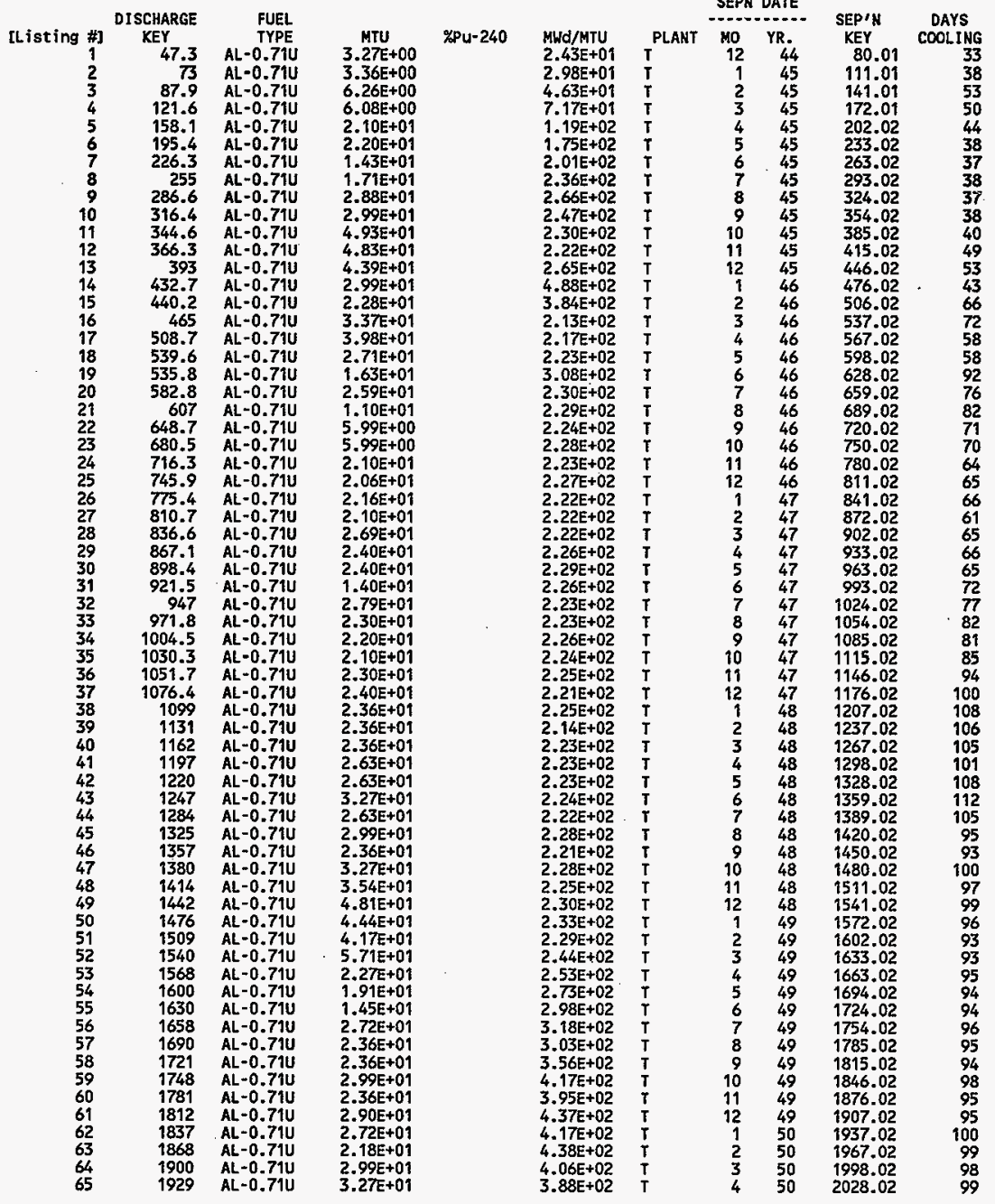


HNF-SD-WM-TJ-794, ReV. 0

UNIFIED SEPARATIONS PRODUCTION DATA- TOTAL WASTE LOSS FRACTION

[Listing \#] Fuel Data Reference

1 SPLANT.DAT 19348 9/30/93 1:19P

2 SPLANT.DAT 19348 9/30/93 1:19P

3 SPLANT.DAT 19348 9/30/93 1:19P

4 SPLANT.DAT 19348 9/30/93 1:19P

5 SPLANT.DAT 19348 9/30/93 1:19P

6 SPLANT.DAT 19348 9/30/93 1:198

7 SPLANT.DAT 19348 9/30/93 $1: 19 P$

8 SPLANT.DAT 19348 9/30/93 1:19P

9 SPLANT.DAT 19348 9/30/93 1:19P

10 SPLANT.DAT 19348 9/30/93 1:19P

11 SPLANT.DAT 19348 9/30/93 $1: 19 \mathrm{P}$

12 SPLANT.DAT 19348 9/30/93 1:19p

13 SPLANT.DAT 19348 9/30/93 1:198

14 SPLANT.DAT 19348 9/30/93 $1: 19 P$

15 SPLANT.DAT 19348 9/30/93 1:19P 16 SPLANT.DAT 19348 9/30/93 1:19P 17 SPLANT.DAT 19348 9/30/93 1:19P 18 SPLANT.DAT 19348 9/30/93 1:19P 19 SPLANT.DAT 19348 9/30/93 $1: 198$ 20 SPLANT.DAT 19348 9/30/93 1:19P 21 SPLANT.DAT 19348 9/30/93 $1: 19 \mathrm{P}$ 22 SPLANT.DAT 19348 9/30/93 1:19P 23 SPLANT.DAT 19348 9/30/93 1:19P 24 SPLANT.DAT 19348 9/30/93 1:19P 25 SPLANT.DAT 19348 9/30/93 1:19P 26 SPLANT.DAT 19348 9/30/93 1:19P 27 SPLANT.DAT 19348 9/30/93 1:19P 28 SPLANT.DAT 19348 9/30/93 $1: 198$ 29 SPLANT.DAT 19348 9/30/93 1:19P 30 SPLANT.DAT 19348 9/30/93 1:19P 31 SPLANT.DAT 19348 9/30/93 $1: 19 \mathrm{P}$ 32 SPLANT.DAT 19348 9/30/93 1:19P 33 SPLANT.DAT 19348 9/30/93 $1: 19 \mathrm{P}$ 34 SPLANT.DAT 19348 9/30/93 1:19P 35 SPLANT.DAT 19348 9/30/93 1:19P 36 SPLANT.DAT 19348 9/30/93 1:19P 37 SPLANT.DAT 19348 9/30/93 1:19P 38 SPLANT,DAT $193489 / 30 / 93 \quad 1=19 P$ 39 SPLANT.DAT 19348 9/30/93 1:19P 40 SPLANT.DAT 19348 9/30/93 $1=19 \mathrm{P}$ 41 SPLANT.DAT 19348 9/30/93 1:19P 42 SPLANT.DAT 19348 9/30/93 1:19P 43 SPLANT.OAT 19348 9/30/93 $1: 19 \mathrm{P}$ 44 SPLANT.DAT 19348 9/30/93 1:19P 45 SPLANT.DAT 19348 9/30/93 1:19P 46 SPLANT.DAT 19348 9/30/93 $i: 19 \mathrm{p}$ 47 SPLANT.DAT 19348 9/30/93 1:19P 48 SPL.ANT.DAT 19348 9/30/93 1:19P 49 SPLANT.DAT 19348 9/30/93 $1: 19 \mathrm{P}$ 50 SPLANT DAT 19348 9/30/93 $1: 19 \mathrm{P}$ 51 SPLANT.DAT 19348 9/30/93 1:19P 52 SPLANT.DAT 19348 9/30/93 1:19P 53 SPLANT.DAT 19348 9/30/93 $1: 19 \mathrm{P}$ 54 SPLANT.DAT 19348 9/30/93 1:19P 55 SPLANT.DAT 19348 9/30/93 1:19P 56 SPLANT.DAT 19348 9/30/93 $1: 19 \mathrm{P}$ 57 SPLANT.DAT 19348 9/30/93 1:19P 58 SPLANT.DAT 19348 9/30/93 1:19P 59 SPLANT.DAT 19348 9/30/93 1:19P 60 SPLANT.OAT 19348 9/30/93 1:19P 61 SPLANT,DAT 19348 9/30/93 1:19P 62 SPLANT.DAT 19348 9/30/93 $1=19 \mathrm{P}$ 63 SPLANT.DAT 19348 9/30/93 $1: 19 \mathrm{P}$ 64 SPLANT.DAT 19348 9/30/93 1:19P 65 SPLANT.DAT 19348 9/30/93 1:19P

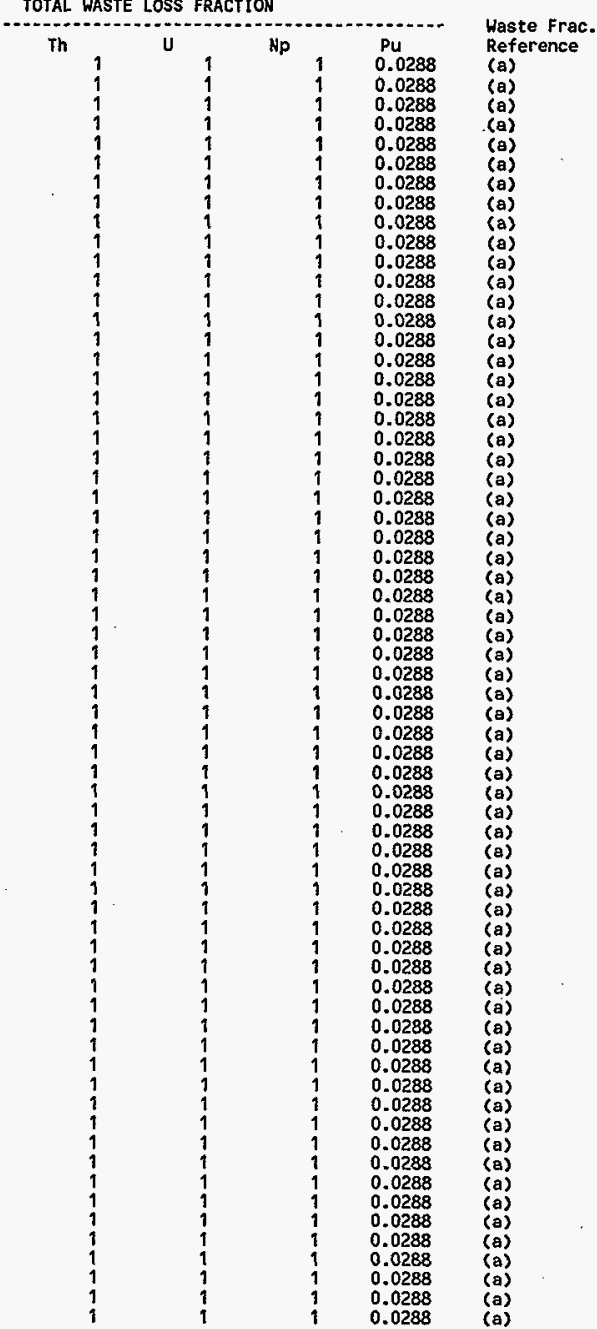


HNF-SD-WH-T1-794, REV. D

ACTIVITY IN UNSEPARATED FUEL (Curies decayed to $1 / 1 / 94$ )

\begin{tabular}{|c|c|c|c|c|c|c|c|c|c|}
\hline 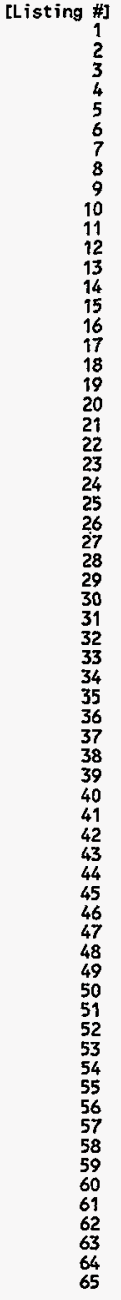 & $\begin{array}{l}H 03 \\
7.78 \mathrm{E}-02 \\
9.85 \mathrm{E}-02 \\
2.87 \mathrm{E}-01 \\
4.33 \mathrm{E}-01 \\
2.51 \mathrm{E}+00 \\
3.90 \mathrm{E}+00 \\
2.92 \mathrm{E}+00 \\
4.11 \mathrm{E}+00 \\
7.88 \mathrm{E}+00 \\
7.64 \mathrm{E}+00 \\
1.18 \mathrm{E}+01 \\
1.11 \mathrm{E}+01 \\
1.22 \mathrm{0}+01 \\
1.55 \mathrm{E}+01 \\
9.25 \mathrm{E}+00 \\
7.57 \mathrm{E}+00 \\
9.19 \mathrm{E}+00 \\
6.45 \mathrm{E}+00 \\
5.38 \mathrm{E}+00 \\
6.40 \mathrm{E}+00 \\
2.72 \mathrm{E}+00 \\
1.45 \mathrm{E}+00 \\
1.49 \mathrm{E}+00 \\
5.12 \mathrm{E}+00 \\
5.15 \mathrm{E}+00 \\
5.30 \mathrm{E}+00 \\
5.17 \mathrm{E}+00 \\
6.67 \mathrm{E}+00 \\
6.08 \mathrm{E}+00 \\
6.20 \mathrm{E}+00 \\
3.58 \mathrm{E}+00 \\
7.07 \mathrm{E}+00 \\
5.83 \mathrm{E}+00 \\
5.69 \mathrm{E}+00 \\
5.40 \mathrm{E}+00 \\
5.96 \mathrm{E}+00 \\
6.12 \mathrm{E}+00 \\
6.17 \mathrm{E}+00 \\
5.90 \mathrm{E}+00 \\
6.17 \mathrm{E}+00 \\
6.92 \mathrm{E}+00 \\
6.94 \mathrm{E}+00 \\
8.70 \mathrm{E}+00 \\
6.98 \mathrm{E}+00 \\
8.23 \mathrm{E}+00 \\
6.29 \mathrm{E}+00 \\
9.06 \mathrm{E}+00 \\
9.72 \mathrm{E}+00 \\
1.36 \mathrm{E}+01 \\
1.28 \mathrm{E}+01 \\
1.19 \mathrm{E}+01 \\
1.74 \mathrm{E}+01 \\
7.17 \mathrm{E}+00 \\
6.56 \mathrm{E}+00 \\
5.47 \mathrm{E}+00 \\
1.10 \mathrm{E}+01 \\
9.14 \mathrm{E}+00 \\
1.08 \mathrm{E}+01 \\
1.62 \mathrm{E}+01 \\
1.21 \mathrm{E}+01 \\
1.66 \mathrm{E}+01 \\
1.49 \mathrm{E}+01 \\
1.26 \mathrm{E}+01 \\
1.61 \mathrm{E}+01 \\
1.69 \mathrm{E}+01\end{array}$ & $\begin{array}{l}\text { C14 } \\
5.11 E-03 \\
6.44 \mathrm{E}-03 \\
1.87 \mathrm{E}-02 \\
2.81 \mathrm{E}-02 \\
1.61 \mathrm{E}-01 \\
2.48 \mathrm{E}-01 \\
1.85 \mathrm{E}-01 \\
2.58 \mathrm{E}-01 \\
4.91 \mathrm{E}-01 \\
4.74 \mathrm{E}-01 \\
7.28 \mathrm{E}-01 \\
6.86 \mathrm{E}-01 \\
7.45 \mathrm{E}-01 \\
9.32 \mathrm{E}-01 \\
5.59 \mathrm{E}-01 \\
4.61 \mathrm{E}-01 \\
5.55 \mathrm{E}-01 \\
3.88 \mathrm{E}-01 \\
3.22 \mathrm{E}-01 \\
3.82 \mathrm{E}-01 \\
1.62 \mathrm{E}-01 \\
8.60 \mathrm{E}-02 \\
8.77 \mathrm{E}-02 \\
3.00 \mathrm{E}-01 \\
3.00 \mathrm{E}-01 \\
3.07 \mathrm{E}-01 \\
2.98 \mathrm{E}-01 \\
3.83 \mathrm{E}-01 \\
3.48 \mathrm{E}-01 \\
3.53 \mathrm{E}-01 \\
2.03 \mathrm{E}-01 \\
4.00 \mathrm{E}-01 \\
3.28 \mathrm{E}-01 \\
3.19 \mathrm{E}-01 \\
3.01 \mathrm{E}-01 \\
3.31 \mathrm{E}-01 \\
3.39 \mathrm{E}-01 \\
3.41 \mathrm{E}-01 \\
3.24 \mathrm{E}-01 \\
3.37 \mathrm{E}-01 \\
3.76 \mathrm{E}-01 \\
3.76 \mathrm{E}-01 \\
4.69 \mathrm{E}-01 \\
3.74 \mathrm{E}-01 \\
4.39 \mathrm{E}-01 \\
3.34 \mathrm{E}-01 \\
4.78 \mathrm{E}-01 \\
5.11 \mathrm{E}-01 \\
7.11 \mathrm{E}-01 \\
6.64 \mathrm{E}-01 \\
6.14 \mathrm{E}-01 \\
8.94 \mathrm{E}-01 \\
3.68 \mathrm{E}-01 \\
3.34 \mathrm{E}-01 \\
2.77 \mathrm{E}-01 \\
5.54 \mathrm{E}-01 \\
4.58 \mathrm{E}-01 \\
5.38 \mathrm{E}-01 \\
7.97 \mathrm{E}-01 \\
5.95 \mathrm{E}-01 \\
8.09 \mathrm{E}-01 \\
7.25 \mathrm{E}-01 \\
6.09 \mathrm{E}-01 \\
7.76 \mathrm{E}-01 \\
8.10 \mathrm{E}-01\end{array}$ & $\begin{array}{l}\text { NIS9 } \\
1.46 \mathrm{E}-03 \\
1.84 \mathrm{E}-03 \\
5.34 \mathrm{E}-03 \\
8.01 \mathrm{E}-03 \\
4.60 \mathrm{E}-02 \\
7.08 \mathrm{E}-02 \\
5.27 \mathrm{E}-02 \\
7.36 \mathrm{E}-02 \\
1.40 \mathrm{E}-01 \\
1.35 \mathrm{E}-01 \\
2.08 \mathrm{E}-01 \\
1.96 \mathrm{E}-01 \\
2.12 \mathrm{E}-01 \\
2.65 \mathrm{E}-01 \\
1.59 \mathrm{E}-01 \\
1.31 \mathrm{E}-01 \\
1.58 \mathrm{E}-01 \\
1.11 \mathrm{E}-01 \\
9.16 \mathrm{E}-02 \\
1.09 \mathrm{E}-01 \\
4.61 \mathrm{E}-02 \\
2.45 \mathrm{E}-02 \\
2.50 \mathrm{E}-02 \\
8.54 \mathrm{E}-02 \\
8.55 \mathrm{E}-02 \\
8.76 \mathrm{E}-02 \\
8.50 \mathrm{E}-02 \\
1.09 \mathrm{E}-01 \\
9.90 \mathrm{E}-02 \\
1.01 \mathrm{E}-01 \\
5.78 \mathrm{E}-02 \\
1.14 \mathrm{E}-01 \\
9.35 \mathrm{E}-02 \\
9.08 \mathrm{E}-02 \\
8.58 \mathrm{E}-02 \\
9.45 \mathrm{E}-02 \\
9.66 \mathrm{E}-02 \\
9.70 \mathrm{E}-02 \\
9.23 \mathrm{E}-02 \\
9.61 \mathrm{E}-02 \\
1.07 \mathrm{E}-01 \\
1.07 \mathrm{E}-01 \\
1.34 \mathrm{E}-01 \\
1.07 \mathrm{E}-01 \\
1.25 \mathrm{E}-01 \\
9.51 \mathrm{E}-02 \\
1.36 \mathrm{E}-01 \\
1.46 \mathrm{E}-01 \\
2.03 \mathrm{E}-01 \\
1.89 \mathrm{E}-01 \\
1.75 \mathrm{E}-01 \\
2.55 \mathrm{E}-01 \\
1.05 \mathrm{E}-01 \\
9.51 \mathrm{E}-02 \\
7.88 \mathrm{E}-02 \\
1.58 \mathrm{E}-01 \\
1.30 \mathrm{E}-01 \\
1.53 \mathrm{E}-01 \\
2.27 \mathrm{E}-01 \\
1.69 \mathrm{E}-01 \\
2.30 \mathrm{E}-01 \\
2.06 \mathrm{E}-01 \\
1.73 \mathrm{E}-01 \\
2.21 \mathrm{E}-01 \\
2.31 \mathrm{E}-01\end{array}$ & $\begin{array}{l}\text { NI } 63 \\
1.25 E-01 \\
1.58 E-01 \\
4.59 E-01 \\
6.90 E-01 \\
3.97 E+00 \\
6.11 E+00 \\
4.55 E+00 \\
6.36 E+00 \\
1.21 E+01 \\
1.17 E+01 \\
1.80 E+01 \\
1.70 E+01 \\
1.84 E+01 \\
2.30 E+01 \\
1.38 E+01 \\
1.14 E+01 \\
1.38 E+01 \\
9.61 E+00 \\
7.97 E+00 \\
9.48 E+00 \\
4.01 E+00 \\
2.14 E+00 \\
2.18 E+00 \\
7.45 E+00 \\
7.47 E+00 \\
7.65 E+00 \\
7.43 E+00 \\
9.55 E+00 \\
8.67 E+00 \\
8.80 E+00 \\
5.06 E+00 \\
9.98 E+00 \\
8.20 E+00 \\
7.96 E+00 \\
7.53 E+00 \\
8.30 E+00 \\
8.49 E+00 \\
8.53 E+00 \\
8.12 E+00 \\
8.46 E+00 \\
9.44 E+00 \\
9.45 E+00 \\
1.18 E+01 \\
9.41 E+00 \\
1.10 E+01 \\
8.41 E+00 \\
1.21 E+01 \\
1.29 E+01 \\
1.79 E+01 \\
1.68 E+01 \\
1.55 E+01 \\
2.26 E+01 \\
9.30 E+00 \\
8.45 E+00 \\
7.01 E+00 \\
1.40 E+01 \\
1.16 E+01 \\
1.36 E+01 \\
2.02 E+01 \\
1.51 E+01 \\
2.06 E+01 \\
1.84 E+01 \\
1.55 E+01 \\
1.98 E+01 \\
2.06 E+01\end{array}$ & $\begin{array}{l}\text { CO60 } \\
4.99 \mathrm{E}-04 \\
6.34 \mathrm{E}-04 \\
1.85 \mathrm{E}-03 \\
2.81 \mathrm{E}-03 \\
1.63 \mathrm{E}-02 \\
2.55 \mathrm{E}-02 \\
1.91 \mathrm{E}-02 \\
2.70 \mathrm{E}-02 \\
5.20 \mathrm{E}-02 \\
5.07 \mathrm{E}-02 \\
7.87 \mathrm{E}-02 \\
7.48 \mathrm{E}-02 \\
8.19 \mathrm{E}-02 \\
1.04 \mathrm{E}-01 \\
6.23 \mathrm{E}-02 \\
5.20 \mathrm{E}-02 \\
6.37 \mathrm{E}-02 \\
4.50 \mathrm{E}-02 \\
3.72 \mathrm{E}-02 \\
4.50 \mathrm{E}-02 \\
1.92 \mathrm{E}-02 \\
1.04 \mathrm{E}-02 \\
1.07 \mathrm{E}-02 \\
3.70 \mathrm{E}-02 \\
3.75 \mathrm{E}-02 \\
3.88 \mathrm{E}-02 \\
3.81 \mathrm{E}-02 \\
4.95 \mathrm{E}-02 \\
4.53 \mathrm{E}-02 \\
4.65 \mathrm{E}-02 \\
2.70 \mathrm{E}-02 \\
5.36 \mathrm{E}-02 \\
4.45 \mathrm{E}-02 \\
4.36 \mathrm{E}-02 \\
4.17 \mathrm{E}-02 \\
4.62 \mathrm{E}-02 \\
4.77 \mathrm{E}-02 \\
4.83 \mathrm{E}-02 \\
4.65 \mathrm{E}-02 \\
4.89 \mathrm{E}-02 \\
5.53 \mathrm{E}-02 \\
5.57 \mathrm{E}-02 \\
7.02 \mathrm{E}-02 \\
5.67 \mathrm{E}-02 \\
6.75 \mathrm{E}-02 \\
5.20 \mathrm{E}-02 \\
7.51 \mathrm{E}-02 \\
8.12 \mathrm{E}-02 \\
1.14 \mathrm{E}-01 \\
1.08 \mathrm{E}-01 \\
1.01 \mathrm{E}-01 \\
1.48 \mathrm{E}-01 \\
6.17 \mathrm{E}-02 \\
5.67 \mathrm{E}-02 \\
4.75 \mathrm{E}-02 \\
9.58 \mathrm{E}-02 \\
8.02 \mathrm{E}-02 \\
9.51 \mathrm{E}-02 \\
1.42 \mathrm{E}-01 \\
1.08 \mathrm{E}-01 \\
1.48 \mathrm{E}-01 \\
1.34 \mathrm{E}-01 \\
1.13 \mathrm{E}-01 \\
1.46 \mathrm{E}-01 \\
1.54 \mathrm{E}-01\end{array}$ & $\begin{array}{l}\text { SE79 } \\
1.07 \mathrm{E}-03 \\
1.35 \mathrm{E}-03 \\
3.92 \mathrm{E}-03 \\
5.89 \mathrm{E}-03 \\
3.39 \mathrm{E}-02 \\
5.22 \mathrm{E}-02 \\
3.89 \mathrm{E}-02 \\
5.43 \mathrm{E}-02 \\
1.04 \mathrm{E}-01 \\
9.98 \mathrm{E}-02 \\
1.53 \mathrm{E}-01 \\
1.44 \mathrm{E}-01 \\
1.57 \mathrm{E}-01 \\
1.97 \mathrm{E}-01 \\
1.18 \mathrm{E}-01 \\
9.70 \mathrm{E}-02 \\
1.17 \mathrm{E}-01 \\
8.16 \mathrm{E}-02 \\
6.78 \mathrm{E}-02 \\
8.04 \mathrm{E}-02 \\
3.40 \mathrm{E}-02 \\
1.81 \mathrm{E}-02 \\
1.85 \mathrm{E}-02 \\
6.30 \mathrm{E}-02 \\
6.32 \mathrm{E}-02 \\
6.46 \mathrm{E}-02 \\
6.27 \mathrm{E}-02 \\
8.06 \mathrm{E}-02 \\
7.31 \mathrm{E}-02 \\
7.42 \mathrm{E}-02 \\
4.26 \mathrm{E}-02 \\
8.40 \mathrm{E}-02 \\
6.90 \mathrm{E}-02 \\
6.70 \mathrm{E}-02 \\
6.33 \mathrm{E}-02 \\
6.97 \mathrm{E}-02 \\
7.13 \mathrm{E}-02 \\
7.16 \mathrm{E}-02 \\
6.81 \mathrm{E}-02 \\
7.09 \mathrm{E}-02 \\
7.91 \mathrm{E}-02 \\
7.91 \mathrm{E}-02 \\
9.87 \mathrm{E}-02 \\
7.87 \mathrm{E}-02 \\
9.22 \mathrm{E}-02 \\
7.02 \mathrm{E}-02 \\
1.01 \mathrm{E}-01 \\
1.07 \mathrm{E}-01 \\
1.50 \mathrm{E}-01 \\
1.40 \mathrm{E}-01 \\
1.29 \mathrm{E}-01 \\
1.88 \mathrm{E}-01 \\
7.73 \mathrm{E}-02 \\
7.03 \mathrm{E}-02 \\
5.83 \mathrm{E}-02 \\
1.17 \mathrm{E}-01 \\
9.65 \mathrm{E}-02 \\
1.13 \mathrm{E}-01 \\
1.68 \mathrm{E}-01 \\
1.26 \mathrm{E}-01 \\
1.71 \mathrm{E}-01 \\
1.53 \mathrm{E}-01 \\
1.29 \mathrm{E}-01 \\
1.64 \mathrm{E}-01 \\
1.71 \mathrm{E}-01\end{array}$ & $\begin{array}{l}\text { SR90 } \\
7.27 E+01 \\
9.18 E+01 \\
2.67 E+02 \\
4.01 E+02 \\
2.30 E+03 \\
3.55 E+03 \\
2.64 E+03 \\
3.70 E+03 \\
7.05 E+03 \\
6.82 E+03 \\
1.05 E+04 \\
9.91 E+03 \\
1.08 E+04 \\
1.34 E+04 \\
8.06 E+03 \\
6.70 E+03 \\
8.09 E+03 \\
5.66 E+03 \\
4.68 E+03 \\
5.60 E+03 \\
2.37 E+03 \\
1.27 E+03 \\
1.29 E+03 \\
4.42 E+03 \\
4.44 E+03 \\
4.55 E+03 \\
4.43 E+03 \\
5.71 E+03 \\
5.18 E+03 \\
5.27 E+03 \\
3.03 E+03 \\
5.99 E+03 \\
4.93 E+03 \\
4.79 E+03 \\
4.54 E+03 \\
5.00 E+03 \\
5.13 E+03 \\
5.16 E+03 \\
4.92 E+03 \\
5.13 E+03 \\
5.73 E+03 \\
5.74 E+03 \\
7.17 E+03 \\
5.73 E+03 \\
6.73 E+03 \\
5.14 E+03 \\
7.37 E+03 \\
7.89 E+03 \\
1.10 E+04 \\
1.03 E+04 \\
9.55 E+03 \\
1.39 E+04 \\
5.73 E+03 \\
5.22 E+03 \\
4.33 E+03 \\
8.66 E+03 \\
7.19 E+03 \\
8.44 E+03 \\
1.25 E+04 \\
9.37 E+03 \\
1.27 E+04 \\
1.14 E+04 \\
9.62 E+03 \\
1.23 E+04 \\
1.29 E+04\end{array}$ & $\begin{array}{l}\text { Y90 } \\
7.27 E+01 \\
9.19 E+01 \\
2.67 E+02 \\
4.01 E+02 \\
2.31 E+03 \\
3.55 E+03 \\
2.65 E+03 \\
3.70 E+03 \\
7.05 E+03 \\
6.82 E+03 \\
1.05 E+04 \\
9.91 E+03 \\
1.08 E+04 \\
1.34 E+04 \\
8.06 E+03 \\
6.70 E+03 \\
8.09 E+03 \\
5.66 E+03 \\
4.69 E+03 \\
5.60 E+03 \\
2.37 E+03 \\
1.27 E+03 \\
1.29 E+03 \\
4.43 E+03 \\
4.44 E+03 \\
4.56 E+03 \\
4.43 E+03 \\
5.71 E+03 \\
5.18 E+03 \\
5.27 E+03 \\
3.03 E+03 \\
5.99 E+03 \\
4.93 E+03 \\
4.79 E+03 \\
4.54 E+03 \\
5.00 E+03 \\
5.13 E+03 \\
5.16 E+03 \\
4.92 E+03 \\
5.13 E+03 \\
5.73 E+03 \\
5.74 E+03 \\
7.17 E+03 \\
5.74 E+03 \\
6.74 E+03 \\
5.14 E+03 \\
7.38 E+03 \\
7.90 E+03 \\
1.10 E+04 \\
1.03 E+04 \\
9.55 E+03 \\
1.39 E+04 \\
5.73 E+03 \\
5.22 E+03 \\
4.33 E+03 \\
8.66 E+03 \\
7.19 E+03 \\
8.44 E+03 \\
1.25 E+04 \\
9.37 E+03 \\
1.28 E+04 \\
1.14 E+04 \\
9.62 E+03 \\
1.23 E+04 \\
1.29 E+04\end{array}$ & 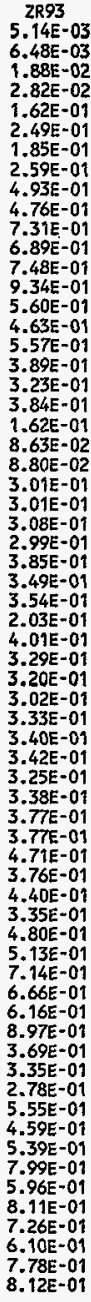 \\
\hline
\end{tabular}


HNF-SD-WM-TI-794, ReV. 0

ACTIVITY IN UNSEPARATED FUEL (Curies decayed to $1 / 1 / 94$ )

\begin{tabular}{|c|c|c|c|c|c|c|c|c|c|}
\hline 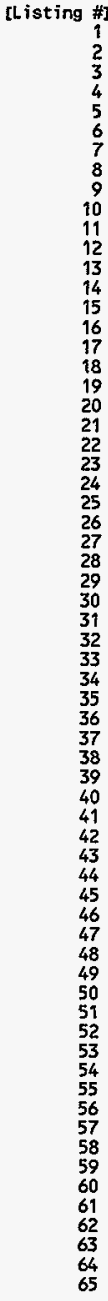 & $\begin{array}{l}\text { NB93* } \\
4.48 \mathrm{E}-03 \\
5.65 \mathrm{E}-03 \\
1.64 \mathrm{E}-02 \\
2.46 \mathrm{E}-02 \\
1.41 \mathrm{E}-01 \\
2.17 \mathrm{E}-01 \\
1.62 \mathrm{E}-01 \\
2.26 \mathrm{E}-01 \\
4.29 \mathrm{E}-01 \\
4.14 \mathrm{E}-01 \\
6.36 \mathrm{E}-01 \\
5.99 \mathrm{E}-01 \\
6.49 \mathrm{E}-01 \\
8.11 \mathrm{E}-01 \\
4.86 \mathrm{E}-01 \\
4.02 \mathrm{E}-01 \\
4.84 \mathrm{E}-01 \\
3.38 \mathrm{E}-01 \\
2.80 \mathrm{E}-01 \\
3.33 \mathrm{E}-01 \\
1.41 \mathrm{E}-01 \\
7.48 \mathrm{E}-02 \\
7.62 \mathrm{E}-02 \\
2.60 \mathrm{E}-01 \\
2.61 \mathrm{E}-01 \\
2.67 \mathrm{E}-01 \\
2.59 \mathrm{E}-01 \\
3.32 \mathrm{E}-01 \\
3.01 \mathrm{E}-01 \\
3.05 \mathrm{E}-01 \\
1.75 \mathrm{E}-01 \\
3.46 \mathrm{E}-01 \\
2.84 \mathrm{E}-01 \\
2.75 \mathrm{E}-01 \\
2.60 \mathrm{E}-01 \\
2.86 \mathrm{E}-01 \\
2.93 \mathrm{E}-01 \\
2.94 \mathrm{E}-01 \\
2.80 \mathrm{E}-01 \\
2.91 \mathrm{E}-01 \\
3.24 \mathrm{E}-01 \\
3.24 \mathrm{E}-01 \\
4.04 \mathrm{E}-01 \\
3.22 \mathrm{E}-01 \\
3.77 \mathrm{E}-01 \\
2.87 \mathrm{E}-01 \\
4.11 \mathrm{E}-01 \\
4.39 \mathrm{E}-01 \\
6.11 \mathrm{E}-01 \\
5.70 \mathrm{E}-01 \\
5.27 \mathrm{E}-01 \\
7.66 \mathrm{E}-01 \\
3.15 \mathrm{E}-01 \\
2.86 \mathrm{E}-01 \\
2.37 \mathrm{E}-01 \\
4.74 \mathrm{E}-01 \\
3.92 \mathrm{E}-01 \\
4.59 \mathrm{E}-01 \\
6.81 \mathrm{E}-01 \\
5.08 \mathrm{E}-01 \\
6.90 \mathrm{E}-01 \\
6.18 \mathrm{E}-01 \\
5.19 \mathrm{E}-01 \\
6.61 \mathrm{E}-01 \\
6.90 \mathrm{E}-01\end{array}$ & $\begin{array}{l}1 C 99 \\
3.52 E-02 \\
4.43 E=02 \\
1.29 E-01 \\
1.93 E-01 \\
1.11 E+00 \\
1.72 E+00 \\
1.28 E+00 \\
1.79 E+00 \\
3.40 E+00 \\
3.28 E+00 \\
5.04 E+00 \\
4.75 E+00 \\
5.16 E+00 \\
6.49 E+00 \\
3.88 E+00 \\
3.19 E+00 \\
3.84 E+00 \\
2.68 E+00 \\
2.23 E+00 \\
2.64 E+00 \\
1.12 E+00 \\
5.95 E-01 \\
6.06 E-01 \\
2.07 E+00 \\
2.08 E+00 \\
2.12 E+00 \\
2.06 E+00 \\
2.65 E+00 \\
2.40 E+00 \\
2.44 E+00 \\
1.40 E+00 \\
2.76 E+00 \\
2.27 E+00 \\
2.20 E+00 \\
2.08 E+00 \\
2.29 E+00 \\
2.34 E+00 \\
2.35 E+00 \\
2.24 E+00 \\
2.33 E+00 \\
2.60 E+00 \\
2.60 E+00 \\
3.24 E+00 \\
2.59 E+00 \\
3.03 E+00 \\
2.31 E+00 \\
3.31 E+00 \\
3.53 E+00 \\
4.92 E+00 \\
4.59 E+00 \\
4.25 E+00 \\
6.18 E+00 \\
2.54 E+00 \\
2.31 E+00 \\
1.92 E+00 \\
3.84 E+00 \\
3.17 E+00 \\
3.73 E+00 \\
5.54 E+00 \\
4.13 E+00 \\
5.62 E+00 \\
5.03 E+00 \\
4.23 E+00 \\
5.39 E+00 \\
5.63 E+00\end{array}$ & $\begin{array}{c}\text { RU106 } \\
1.63 \mathrm{E}-12 \\
2.17 \mathrm{E}-12 \\
6.52 \mathrm{E}-12 \\
1.05 \mathrm{E}-11 \\
6.60 \mathrm{E}-11 \\
1.11 \mathrm{E}-10 \\
8.90 \mathrm{E}-11 \\
1.33 \mathrm{E}-10 \\
2.73 \mathrm{E}-10 \\
2.76 \mathrm{E}-10 \\
4.44 \mathrm{E}-10 \\
4.35 \mathrm{E}-10 \\
5.05 \mathrm{E}-10 \\
7.44 \mathrm{E}-10 \\
4.35 \mathrm{E}-10 \\
3.49 \mathrm{E}-10 \\
4.59 \mathrm{E}-10 \\
3.40 \mathrm{E}-10 \\
2.90 \mathrm{E}-10 \\
3.64 \mathrm{E}-10 \\
1.61 \mathrm{E}-10 \\
9.26 \mathrm{E}-11 \\
1.01 \mathrm{E}-10 \\
3.67 \mathrm{E}-10 \\
3.89 \mathrm{E}-10 \\
4.20 \mathrm{E}-10 \\
4.36 \mathrm{E}-10 \\
5.87 \mathrm{E}-10 \\
5.65 \mathrm{E}-10 \\
6.08 \mathrm{E}-10 \\
3.66 \mathrm{E}-10 \\
7.53 \mathrm{E}-10 \\
6.49 \mathrm{E}-10 \\
6.72 \mathrm{E}-10 \\
6.64 \mathrm{E}-10 \\
7.62 \mathrm{E}-10 \\
8.16 \mathrm{E}-10 \\
8.56 \mathrm{E}-10 \\
8.63 \mathrm{E}-10 \\
9.54 \mathrm{E}-10 \\
1.14 \mathrm{E}-09 \\
1.19 \mathrm{E}-09 \\
1.56 \mathrm{E}-09 \\
1.33 \mathrm{E}-09 \\
1.69 \mathrm{E}-09 \\
1.37 \mathrm{E}-09 \\
2.05 \mathrm{E}-09 \\
2.33 \mathrm{E}-09 \\
3.43 \mathrm{E}-09 \\
3.41 \mathrm{E}-09 \\
3.36 \mathrm{E}-09 \\
5.20 \mathrm{E}-09 \\
2.26 \mathrm{E}-09 \\
2.20 \mathrm{E}-09 \\
1.95 \mathrm{E}-09 \\
4.14 \mathrm{E}-09 \\
3.63 \mathrm{E}-09 \\
4.61 \mathrm{E}-09 \\
7.35 \mathrm{E}-09 \\
5.80 \mathrm{E}-09 \\
8.48 \mathrm{E}-09 \\
7.91 \mathrm{E}-09 \\
7.11 \mathrm{E}-09 \\
9.47 \mathrm{E}-09 \\
1.04 \mathrm{E}-08\end{array}$ & $\begin{array}{l}\text { CD113* } \\
8.96 \mathrm{E}-03 \\
1.14 \mathrm{E}-02 \\
3.30 \mathrm{E}-02 \\
4.99 \mathrm{E}-02 \\
2.90 \mathrm{E}-01 \\
4.51 \mathrm{E}-01 \\
3.38 \mathrm{E}-01 \\
4.75 \mathrm{E}-01 \\
9.11 \mathrm{E}-01 \\
8.81 \mathrm{E}-01 \\
1.36 \mathrm{E}+00 \\
1.28 \mathrm{E}+00 \\
1.40 \mathrm{E}+00 \\
1.80 \mathrm{E}+00 \\
1.07 \mathrm{E}+00 \\
8.70 \mathrm{E}-01 \\
1.06 \mathrm{E}+00 \\
7.40 \mathrm{E}-01 \\
6.19 \mathrm{E}-01 \\
7.34 \mathrm{E}-01 \\
3.11 \mathrm{E}-01 \\
1.66 \mathrm{E}-01 \\
1.71 \mathrm{E}-01 \\
5.85 \mathrm{E}-01 \\
5.89 \mathrm{E}-01 \\
6.04 \mathrm{E}-01 \\
5.89 \mathrm{E}-01 \\
7.60 \mathrm{E}-01 \\
6.92 \mathrm{E}-01 \\
7.05 \mathrm{E}-01 \\
4.07 \mathrm{E}-01 \\
8.03 \mathrm{E}-01 \\
6.62 \mathrm{E}-01 \\
6.46 \mathrm{E}-01 \\
6.12 \mathrm{E}-01 \\
6.76 \mathrm{E}-01 \\
6.94 \mathrm{E}-01 \\
6.99 \mathrm{E}-01 \\
6.67 \mathrm{E}-01 \\
6.97 \mathrm{E}-01 \\
7.81 \mathrm{E}-01 \\
7.84 \mathrm{E}-01 \\
9.81 \mathrm{E}-01 \\
7.86 \mathrm{E}-01 \\
9.27 \mathrm{E}-01 \\
7.08 \mathrm{E}-01 \\
1.02 \mathrm{E}+00 \\
1.09 \mathrm{E}+00 \\
1.53 \mathrm{E}+00 \\
1.43 \mathrm{E}+00 \\
1.33 \mathrm{E}+00 \\
1.95 \mathrm{E}+00 \\
8.04 \mathrm{E}-01 \\
7.35 \mathrm{E}-01 \\
6.13 \mathrm{E}-01 \\
1.23 \mathrm{E}+00 \\
1.02 \mathrm{E}+00 \\
1.21 \mathrm{E}+00 \\
1.81 \mathrm{E}+00 \\
1.36 \mathrm{E}+00 \\
1.86 \mathrm{E}+00 \\
1.67 \mathrm{E}+00 \\
1.41 \mathrm{E}+00 \\
1.80 \mathrm{E}+00 \\
1.88 \mathrm{E}+00\end{array}$ & $\begin{array}{l}\text { SB125 } \\
1.85 \mathrm{E}-04 \\
2.37 \mathrm{E}-04 \\
6.96 \mathrm{E}-04 \\
1.07 \mathrm{E}-03 \\
6.33 \mathrm{E}-03 \\
1.00 \mathrm{E}-02 \\
7.64 \mathrm{E}-03 \\
1.09 \mathrm{E}-02 \\
2.13 \mathrm{E}-02 \\
2.09 \mathrm{E}-02 \\
3.27 \mathrm{E}-02 \\
3.13 \mathrm{E}-02 \\
3.47 \mathrm{E}-02 \\
4.53 \mathrm{E}-02 \\
2.71 \mathrm{E}-02 \\
2.25 \mathrm{E}-02 \\
2.79 \mathrm{E}-02 \\
1.99 \mathrm{E}-02 \\
1.66 \mathrm{E}-02 \\
2.02 \mathrm{E}-02 \\
8.69 \mathrm{E}-03 \\
4.76 \mathrm{E}-03 \\
4.96 \mathrm{E}-03 \\
1.74 \mathrm{E}-02 \\
1.78 \mathrm{E}-02 \\
1.85 \mathrm{E}-02 \\
1.84 \mathrm{E}-02 \\
2.41 \mathrm{E}-02 \\
2.23 \mathrm{E}-02 \\
2.31 \mathrm{E}-02 \\
1.35 \mathrm{E}-02 \\
2.71 \mathrm{E}-02 \\
2.27 \mathrm{E}-02 \\
2.25 \mathrm{E}-02 \\
2.16 \mathrm{E}-02 \\
2.42 \mathrm{E}-02 \\
2.51 \mathrm{E}-02 \\
2.56 \mathrm{E}-02 \\
2.49 \mathrm{E}-02 \\
2.65 \mathrm{E}-02 \\
3.03 \mathrm{E}-02 \\
3.08 \mathrm{E}-02 \\
3.91 \mathrm{E}-02 \\
3.20 \mathrm{E}-02 \\
3.86 \mathrm{E}-02 \\
3.00 \mathrm{E}-02 \\
4.37 \mathrm{E}-02 \\
4.78 \mathrm{E}-02 \\
6.78 \mathrm{E}-02 \\
6.48 \mathrm{E}-02 \\
6.14 \mathrm{E}-02 \\
9.11 \mathrm{E}-02 \\
3.82 \mathrm{E}-02 \\
3.55 \mathrm{E}-02 \\
3.02 \mathrm{E}-02 \\
6.15 \mathrm{E}-02 \\
5.20 \mathrm{E}-02 \\
6.26 \mathrm{E}-02 \\
9.49 \mathrm{E}-02 \\
7.24 \mathrm{E}-02 \\
1.01 \mathrm{E}-01 \\
9.17 \mathrm{E}-02 \\
7.89 \mathrm{E}-02 \\
1.02 \mathrm{E}-01 \\
.09 \mathrm{E}-01\end{array}$ & $\begin{array}{c}\text { SN126 } \\
1.56 \mathrm{E}-03 \\
1.97 \mathrm{E}-03 \\
5.71 \mathrm{E}-03 \\
8.60 \mathrm{E}-03 \\
4.97 \mathrm{E}-02 \\
7.69 \mathrm{E}-02 \\
5.74 \mathrm{E}-02 \\
8.05 \mathrm{E}-02 \\
1.54 \mathrm{E}-01 \\
1.48 \mathrm{E}-01 \\
2.27 \mathrm{E}-01 \\
2.14 \mathrm{E}-01 \\
2.33 \mathrm{E}-01 \\
2.99 \mathrm{E}-01 \\
1.77 \mathrm{E}-01 \\
1.43 \mathrm{E}-01 \\
1.73 \mathrm{E}-01 \\
1.21 \mathrm{E}-01 \\
1.01 \mathrm{E}-01 \\
1.19 \mathrm{E}-01 \\
5.04 \mathrm{E}-02 \\
2.68 \mathrm{E}-02 \\
2.73 \mathrm{E}-02 \\
9.33 \mathrm{E}-02 \\
9.36 \mathrm{E}-02 \\
9.57 \mathrm{E}-02 \\
9.28 \mathrm{E}-02 \\
1.19 \mathrm{E}-01 \\
1.08 \mathrm{E}-01 \\
1.10 \mathrm{E}-01 \\
6.32 \mathrm{E}-02 \\
1.24 \mathrm{E}-01 \\
1.02 \mathrm{E}-01 \\
9.93 \mathrm{E}-02 \\
9.37 \mathrm{E}-02 \\
1.03 \mathrm{E}-01 \\
1.06 \mathrm{E}-01 \\
1.06 \mathrm{E}-01 \\
1.01 \mathrm{E}-01 \\
1.05 \mathrm{E}-01 \\
1.17 \mathrm{E}-01 \\
1.17 \mathrm{E}-01 \\
1.46 \mathrm{E}-01 \\
1.17 \mathrm{E}-01 \\
1.37 \mathrm{E}-01 \\
1.04 \mathrm{E}-01 \\
1.49 \mathrm{E}-01 \\
1.59 \mathrm{E}-01 \\
2.22 \mathrm{E}-01 \\
2.07 \mathrm{E}-01 \\
1.91 \mathrm{E}-01 \\
2.79 \mathrm{E}-01 \\
1.15 \mathrm{E}-01 \\
1.05 \mathrm{E}-01 \\
8.69 \mathrm{E}-02 \\
1.74 \mathrm{E}-01 \\
1.44 \mathrm{E}-01 \\
1.70 \mathrm{E}-01 \\
2.54 \mathrm{E}-01 \\
1.89 \mathrm{E}-01 \\
2.58 \mathrm{E}-01 \\
2.31 \mathrm{E}-01 \\
1.94 \mathrm{E}-01 \\
2.47 \mathrm{E}-01 \\
2.57 \mathrm{E}-01\end{array}$ & $\begin{array}{l}1129 \\
6.42 \mathrm{E}-05 \\
8.10 \mathrm{E}-05 \\
2.35 \mathrm{E}-04 \\
3.54 \mathrm{E}-04 \\
2.05 \mathrm{E}-03 \\
3.17 \mathrm{E}-03 \\
2.37 \mathrm{E}-03 \\
3.32 \mathrm{E}-03 \\
6.34 \mathrm{E}-03 \\
6.11 \mathrm{E}-03 \\
9.36 \mathrm{E}-03 \\
8.81 \mathrm{E}-03 \\
9.61 \mathrm{E}-03 \\
1.23 \mathrm{E}-02 \\
7.30 \mathrm{E}-03 \\
5.91 \mathrm{E}-03 \\
7.13 \mathrm{E}-03 \\
4.98 \mathrm{E}-03 \\
4.17 \mathrm{E}-03 \\
4.91 \mathrm{E}-03 \\
2.08 \mathrm{E}-03 \\
1.10 \mathrm{E}-03 \\
1.13 \mathrm{E}-03 \\
3.84 \mathrm{E}-03 \\
3.86 \mathrm{E}-03 \\
3.94 \mathrm{E}-03 \\
3.83 \mathrm{E}-03 \\
4.92 \mathrm{E}-03 \\
4.46 \mathrm{E}-03 \\
4.53 \mathrm{E}-03 \\
2.60 \mathrm{E}-03 \\
5.13 \mathrm{E}-03 \\
4.21 \mathrm{E}-03 \\
4.09 \mathrm{E}-03 \\
3.86 \mathrm{E}-03 \\
4.25 \mathrm{E}-03 \\
4.35 \mathrm{E}-03 \\
4.37 \mathrm{E}-03 \\
4.15 \mathrm{E}-03 \\
4.33 \mathrm{E}-03 \\
4.83 \mathrm{E}-03 \\
4.83 \mathrm{E}-03 \\
6.02 \mathrm{E}-03 \\
4.80 \mathrm{E}-03 \\
5.63 \mathrm{E}-03 \\
4.28 \mathrm{E}-03 \\
6.15 \mathrm{E}-03 \\
6.56 \mathrm{E}-03 \\
9.14 \mathrm{E}-03 \\
8.53 \mathrm{E}-03 \\
7.89 \mathrm{E}-03 \\
1.15 \mathrm{E}-02 \\
4.733 \mathrm{E}-03 \\
4.33 \mathrm{E}-03 \\
3.58 \mathrm{E}-03 \\
7.18 \mathrm{E}-03 \\
5.94 \mathrm{E}-03 \\
7.00 \mathrm{E}-03 \\
1.05 \mathrm{E}-02 \\
7.78 \mathrm{E}-03 \\
1.06 \mathrm{E}-02 \\
9.50 \mathrm{E}-03 \\
7.99 \mathrm{E}-03 \\
1.02 \mathrm{E}-02 \\
1.06 \mathrm{E}-02\end{array}$ & $\begin{array}{c}\text { CS134 } \\
1.90 \mathrm{E}-12 \\
2.45 \mathrm{E}-12 \\
7.27 \mathrm{E}-12 \\
1.13 \mathrm{E}-11 \\
9.35 \mathrm{E}-07 \\
8.88 \mathrm{E}-06 \\
9.36 \mathrm{E}-06 \\
1.85 \mathrm{E}-05 \\
4.48 \mathrm{E}-05 \\
3.92 \mathrm{E}-05 \\
5.44 \mathrm{E}-05 \\
4.85 \mathrm{E}-05 \\
7.49 \mathrm{E}-05 \\
2.36 \mathrm{E}-04 \\
1.03 \mathrm{E}-04 \\
3.29 \mathrm{E}-05 \\
4.30 \mathrm{E}-05 \\
3.21 \mathrm{E}-05 \\
4.68 \mathrm{E}-05 \\
3.55 \mathrm{E}-05 \\
1.54 \mathrm{E}-05 \\
7.88 \mathrm{E}-06 \\
8.91 \mathrm{E}-06 \\
2.92 \mathrm{E}-05 \\
3.24 \mathrm{E}-05 \\
3.16 \mathrm{E}-05 \\
3.17 \mathrm{E}-05 \\
4.17 \mathrm{E}-05 \\
4.19 \mathrm{E}-05 \\
4.37 \mathrm{E}-05 \\
2.57 \mathrm{E}-05 \\
4.81 \mathrm{E}-05 \\
4.05 \mathrm{E}-05 \\
4.36 \mathrm{E}-05 \\
3.92 \mathrm{E}-05 \\
4.40 \mathrm{E}-05 \\
4.60 \mathrm{E}-05 \\
4.72 \mathrm{E}-05 \\
4.27 \mathrm{E}-05 \\
4.95 \mathrm{E}-05 \\
5.71 \mathrm{E}-05 \\
5.83 \mathrm{E}-05 \\
7.46 \mathrm{E}-05 \\
6.15 \mathrm{E}-05 \\
8.07 \mathrm{E}-05 \\
5.88 \mathrm{E}-05 \\
9.27 \mathrm{E}-05 \\
9.47 \mathrm{E}-05 \\
1.46 \mathrm{E}-04 \\
1.40 \mathrm{E}-04 \\
1.34 \mathrm{E}-04 \\
2.27 \mathrm{E}-04 \\
9.98 \mathrm{E}-05 \\
1.07 \mathrm{E}-04 \\
1.05 \mathrm{E}-04 \\
2.37 \mathrm{E}-04 \\
1.92 \mathrm{E}-04 \\
2.89 \mathrm{E}-04 \\
5.48 \mathrm{E}-04 \\
3.92 \mathrm{E}-04 \\
5.28 \mathrm{E}-04 \\
4.98 \mathrm{E}-04 \\
5.91 \mathrm{E}-04\end{array}$ & 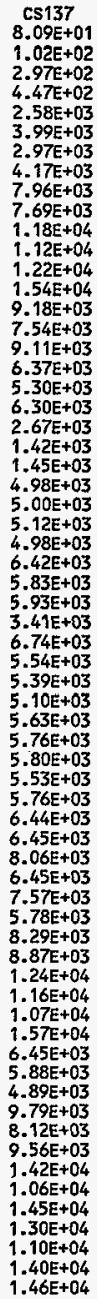 \\
\hline
\end{tabular}


HNF-SD-WM-I1-794, ReV. O

ACTIVITY IN UNSEPARATED FUEL (Curies decayed to 1/1/94)

\begin{tabular}{|c|c|c|c|c|c|c|c|c|c|}
\hline $\begin{array}{r}\text { isting } \\
1 \\
2 \\
2 \\
3 \\
4 \\
5 \\
6 \\
7 \\
7 \\
8 \\
9 \\
10 \\
11 \\
12 \\
13 \\
14 \\
15 \\
16 \\
17 \\
18 \\
19 \\
20 \\
21 \\
22 \\
23 \\
24 \\
25 \\
26 \\
27 \\
28 \\
29 \\
30 \\
31 \\
32 \\
33 \\
34 \\
35 \\
36 \\
37 \\
38 \\
39 \\
40 \\
41 \\
42 \\
43 \\
44 \\
45 \\
62 \\
46 \\
63 \\
64 \\
65\end{array}$ & $\begin{array}{l}B A 137 \\
7.65 \mathrm{E}+01 \\
9.67 \mathrm{E}+01 \\
2.81 \mathrm{E}+02 \\
4.23 \mathrm{E}+02 \\
2.44 \mathrm{E}+03 \\
3.77 \mathrm{E}+03 \\
2.81 \mathrm{E}+03 \\
3.94 \mathrm{E}+03 \\
7.53 \mathrm{E}+03 \\
7.28 \mathrm{E}+03 \\
1.12 \mathrm{E}+04 \\
1.06 \mathrm{E}+04 \\
1.15 \mathrm{E}+04 \\
1.45 \mathrm{E}+04 \\
8.68 \mathrm{E}+03 \\
7.13 \mathrm{E}+03 \\
8.62 \mathrm{E}+03 \\
6.03 \mathrm{E}+03 \\
5.02 \mathrm{E}+03 \\
5.96 \mathrm{E}+03 \\
2.52 \mathrm{E}+03 \\
1.35 \mathrm{E}+03 \\
1.38 \mathrm{E}+03 \\
4.71 \mathrm{E}+03 \\
4.73 \mathrm{E}+03 \\
4.85 \mathrm{E}+03 \\
4.71 \mathrm{E}+03 \\
6.07 \mathrm{E}+03 \\
5.52 \mathrm{E}+03 \\
5.61 \mathrm{E}+03 \\
3.23 \mathrm{E}+03 \\
6.37 \mathrm{E}+03 \\
5.24 \mathrm{E}+03 \\
5.10 \mathrm{E}+03 \\
4.83 \mathrm{E}+03 \\
5.32 \mathrm{E}+03 \\
5.45 \mathrm{E}+03 \\
5.48 \mathrm{E}+03 \\
5.23 \mathrm{E}+03 \\
5.45 \mathrm{E}+03 \\
6.09 \mathrm{E}+03 \\
6.10 \mathrm{E}+03 \\
7.63 \mathrm{E}+03 \\
6.10 \mathrm{E}+03 \\
7.17 \mathrm{E}+03 \\
5.47 \mathrm{E}+03 \\
7.84 \mathrm{E}+03 \\
8.39 \mathrm{E}+03 \\
1.17 \mathrm{E}+04 \\
1.10 \mathrm{E}+04 \\
1.02 \mathrm{E}+04 \\
1.48 \mathrm{E}+04 \\
6.10 \mathrm{E}+03 \\
5.56 \mathrm{E}+03 \\
4.62 \mathrm{E}+03 \\
9.26 \mathrm{E}+03 \\
7.68 \mathrm{E}+03 \\
9.04 \mathrm{E}+03 \\
1.35 \mathrm{E}+04 \\
1.01 \mathrm{E}+04 \\
1.37 \mathrm{E}+04 \\
1.23 \mathrm{E}+04 \\
1.04 \mathrm{E}+04 \\
1.32 \mathrm{E}+04 \\
1.38 \mathrm{E}+04\end{array}$ & $\begin{array}{c}\text { SM151 } \\
4.23 \mathrm{E}+00 \\
5.33 \mathrm{E}+00 \\
1.54 \mathrm{E}+01 \\
2.30 \mathrm{0}+01 \\
1.31 \mathrm{E}+02 \\
1.98 \mathrm{E}+02 \\
1.47 \mathrm{E}+02 \\
2.03 \mathrm{E}+02 \\
3.84 \mathrm{E}+02 \\
3.73 \mathrm{E}+02 \\
5.73 \mathrm{E}+02 \\
5.44 \mathrm{E}+02 \\
5.84 \mathrm{E}+02 \\
6.87 \mathrm{E}+02 \\
4.24 \mathrm{E}+02 \\
3.67 \mathrm{E}+02 \\
4.42 \mathrm{E}+02 \\
3.08 \mathrm{E}+02 \\
2.50 \mathrm{E}+02 \\
3.04 \mathrm{E}+02 \\
1.28 \mathrm{E}+02 \\
6.85 \mathrm{E}+01 \\
6.98 \mathrm{E}+01 \\
2.39 \mathrm{E}+02 \\
2.39 \mathrm{E}+02 \\
2.45 \mathrm{E}+02 \\
2.38 \mathrm{E}+02 \\
3.07 \mathrm{E}+02 \\
2.78 \mathrm{E}+02 \\
2.82 \mathrm{E}+02 \\
1.62 \mathrm{E}+02 \\
3.20 \mathrm{E}+02 \\
2.63 \mathrm{E}+02 \\
2.55 \mathrm{E}+02 \\
2.42 \mathrm{E}+02 \\
2.66 \mathrm{E}+02 \\
2.73 \mathrm{E}+02 \\
2.74 \mathrm{E}+02 \\
2.61 \mathrm{E}+02 \\
2.72 \mathrm{E}+02 \\
3.03 \mathrm{E}+02 \\
3.03 \mathrm{E}+02 \\
3.79 \mathrm{E}+02 \\
3.02 \mathrm{E}+02 \\
3.54 \mathrm{E}+02 \\
2.70 \mathrm{E}+02 \\
3.86 \mathrm{E}+02 \\
4.14 \mathrm{E}+02 \\
5.75 \mathrm{E}+02 \\
5.37 \mathrm{E}+02 \\
4.97 \mathrm{E}+02 \\
7.21 \mathrm{E}+02 \\
2.96 \mathrm{E}+02 \\
2.68 \mathrm{E}+02 \\
2.21 \mathrm{E}+02 \\
4.39 \mathrm{E}+02 \\
3.65 \mathrm{E}+02 \\
4.22 \mathrm{E}+02 \\
6.16 \mathrm{E}+02 \\
4.63 \mathrm{E}+02 \\
6.23 \mathrm{E}+02 \\
5.69 \mathrm{E}+02 \\
6.02 \mathrm{E}+02 \\
\end{array}$ & $\begin{array}{l}\text { EU152 } \\
0.00 \mathrm{E}+00 \\
0.00 \mathrm{E}+00 \\
0.00 \mathrm{E}+00 \\
0.00 \mathrm{E}+00 \\
0.00 \mathrm{E}+00 \\
2.05 \mathrm{E}-04 \\
1.02 \mathrm{E}-03 \\
3.13 \mathrm{E}-03 \\
8.72 \mathrm{E}-03 \\
6.79 \mathrm{E}-03 \\
8.18 \mathrm{E}-03 \\
6.59 \mathrm{E}-03 \\
1.34 \mathrm{E}-02 \\
5.69 \mathrm{E}-02 \\
2.28 \mathrm{E}-02 \\
3.71 \mathrm{E}-03 \\
4.96 \mathrm{E}-03 \\
3.81 \mathrm{E}-03 \\
8.53 \mathrm{E}-03 \\
4.44 \mathrm{E}-03 \\
1.88 \mathrm{E}-03 \\
8.58 \mathrm{E}-04 \\
1.03 \mathrm{E}-03 \\
3.02 \mathrm{E}-03 \\
3.56 \mathrm{E}-03 \\
3.12 \mathrm{E}-03 \\
3.04 \mathrm{E}-03 \\
3.93 \mathrm{E}-03 \\
4.19 \mathrm{E}-03 \\
4.28 \mathrm{E}-03 \\
2.47 \mathrm{E}-03 \\
4.16 \mathrm{E}-03 \\
3.43 \mathrm{E}-03 \\
3.92 \mathrm{E}-03 \\
3.17 \mathrm{E}-03 \\
3.50 \mathrm{E}-03 \\
3.59 \mathrm{E}-03 \\
3.62 \mathrm{E}-03 \\
2.86 \mathrm{E}-03 \\
3.62 \mathrm{E}-03 \\
4.05 \mathrm{E}-03 \\
4.07 \mathrm{E}-03 \\
5.09 \mathrm{E}-03 \\
4.08 \mathrm{E}-03 \\
5.64 \mathrm{E}-03 \\
3.68 \mathrm{E}-03 \\
6.20 \mathrm{E}-03 \\
5.67 \mathrm{E}-03 \\
9.30 \mathrm{E}-03 \\
8.72 \mathrm{E}-03 \\
8.11 \mathrm{E}-03 \\
1.52 \mathrm{E}-02 \\
6.71 \mathrm{E}-03 \\
7.70 \mathrm{E}-03 \\
7.93 \mathrm{E}-03 \\
1.84 \mathrm{E}-02 \\
1.43 \mathrm{E}-02 \\
2.28 \mathrm{E}-02 \\
4.51 \mathrm{E}-02 \\
3.08 \mathrm{E}-02 \\
5.00 \mathrm{E}-02 \\
4.15 \mathrm{E}-02 \\
3.79 \mathrm{E}-02 \\
4.29 \mathrm{E}-02 \\
4.28 \mathrm{E}-02\end{array}$ & $\begin{array}{l}\text { EU154 } \\
0.00 \mathrm{E}+00 \\
8.20 \mathrm{E}-05 \\
2.26 \mathrm{E}-03 \\
8.11 \mathrm{E}-03 \\
9.79 \mathrm{E}-02 \\
2.46 \mathrm{E}-01 \\
2.16 \mathrm{E}-01 \\
3.66 \mathrm{E}-01 \\
8.03 \mathrm{E}-01 \\
7.18 \mathrm{E}-01 \\
1.03 \mathrm{E}+00 \\
9.29 \mathrm{E}-01 \\
1.25 \mathrm{E}+00 \\
3.06 \mathrm{E}+00 \\
1.42 \mathrm{E}+00 \\
6.08 \mathrm{E}-01 \\
7.58 \mathrm{E}-01 \\
5.45 \mathrm{E}-01 \\
6.54 \mathrm{E}-01 \\
5.67 \mathrm{E}-01 \\
2.41 \mathrm{E}-01 \\
1.24 \mathrm{E}-01 \\
1.33 \mathrm{E}-01 \\
4.38 \mathrm{E}-01 \\
4.62 \mathrm{E}-01 \\
4.55 \mathrm{E}-01 \\
4.45 \mathrm{E}-01 \\
5.75 \mathrm{E}-01 \\
5.49 \mathrm{E}-01 \\
5.61 \mathrm{E}-01 \\
3.24 \mathrm{E}-01 \\
6.14 \mathrm{E}-01 \\
5.07 \mathrm{E}-01 \\
5.19 \mathrm{E}-01 \\
4.72 \mathrm{E}-01 \\
5.22 \mathrm{E}-01 \\
5.36 \mathrm{E}-01 \\
5.41 \mathrm{E}-01 \\
4.95 \mathrm{E}-01 \\
5.44 \mathrm{E}-01 \\
6.11 \mathrm{E}-01 \\
6.14 \mathrm{E}-01 \\
7.71 \mathrm{E}-01 \\
6.20 \mathrm{E}-01 \\
7.66 \mathrm{E}-01 \\
5.62 \mathrm{E}-01 \\
8.46 \mathrm{E}-01 \\
8.71 \mathrm{E}-01 \\
1.28 \mathrm{E}+00 \\
1.20 \mathrm{E}+00 \\
1.12 \mathrm{E}+00 \\
1.77 \mathrm{E}+00 \\
7.52 \mathrm{E}-01 \\
7.53 \mathrm{E}-01 \\
6.91 \mathrm{E}-01 \\
1.49 \mathrm{E}+00 \\
1.20 \mathrm{E}+00 \\
1.67 \mathrm{E}+00 \\
2.95 \mathrm{E}+00 \\
2.09 \mathrm{E}+00 \\
3.19 \mathrm{E}+00 \\
2.74 \mathrm{E}+00 \\
2.43 \mathrm{E}+00 \\
2.88 \mathrm{E}+00 \\
2.94 \mathrm{E}+00\end{array}$ & $\begin{array}{l}E U 155 \\
1.05 E-02 \\
1.33 E-02 \\
3.87 E-02 \\
5.82 E-02 \\
3.33 E-01 \\
5.08 E-01 \\
3.78 E-01 \\
5.27 E-01 \\
1.00 E+00 \\
9.86 E-01 \\
1.54 E+00 \\
1.47 E+00 \\
1.58 E+00 \\
1.82 E+00 \\
1.15 E+00 \\
1.03 E+00 \\
1.26 E+00 \\
8.87 E-01 \\
7.09 E-01 \\
8.86 E-01 \\
3.78 E-01 \\
2.05 E-01 \\
2.11 E-01 \\
7.34 E-01 \\
7.41 E-01 \\
7.70 E-01 \\
7.57 E-01 \\
9.83 E-01 \\
8.98 E-01 \\
9.22 E-01 \\
5.35 E-01 \\
1.07 E+00 \\
8.86 E-01 \\
8.67 E-01 \\
8.31 E-01 \\
9.23 E-01 \\
9.53 E-01 \\
9.65 E-01 \\
9.33 E-01 \\
9.79 E-01 \\
1.11 E+00 \\
1.12 E+00 \\
1.41 E+00 \\
1.14 E+00 \\
1.35 E+00 \\
1.05 E+00 \\
1.51 E+00 \\
1.63 E+00 \\
2.29 E+00 \\
2.17 E+00 \\
2.03 E+00 \\
2.97 E+00 \\
1.23 E+00 \\
1.12 E+00 \\
9.31 E-01 \\
1.87 E+00 \\
1.57 E+00 \\
1.82 E+00 \\
2.66 E+00 \\
2.03 E+00 \\
2.74 E+00 \\
2.50 E+00 \\
2.11 E+00 \\
2.76 E+00 \\
2.93 E+00\end{array}$ & $\begin{array}{l}\text { RA226 } \\
5.07 \mathrm{E}-06 \\
5.20 \mathrm{E}-06 \\
9.67 \mathrm{E}-06 \\
9.35 \mathrm{E}-06 \\
3.22 \mathrm{E}-05 \\
3.36 \mathrm{E}-05 \\
2.18 \mathrm{E}-05 \\
2.58 \mathrm{E}-05 \\
4.34 \mathrm{E}-05 \\
4.49 \mathrm{E}-05 \\
7.37 \mathrm{E}-05 \\
7.20 \mathrm{E}-05 \\
6.50 \mathrm{E}-05 \\
4.42 \mathrm{E}-05 \\
3.36 \mathrm{E}-05 \\
4.98 \mathrm{E}-05 \\
5.85 \mathrm{E}-05 \\
3.99 \mathrm{E}-05 \\
2.39 \mathrm{E}-05 \\
3.77 \mathrm{E}-05 \\
1.59 \mathrm{E}-05 \\
8.74 \mathrm{E}-06 \\
8.54 \mathrm{E}-06 \\
3.02 \mathrm{E}-05 \\
2.90 \mathrm{E}-05 \\
3.08 \mathrm{E}-05 \\
2.97 \mathrm{E}-05 \\
3.81 \mathrm{E}-05 \\
3.31 \mathrm{E}-05 \\
3.35 \mathrm{E}-05 \\
1.92 \mathrm{E}-05 \\
3.92 \mathrm{E}-05 \\
3.21 \mathrm{E}-05 \\
2.99 \mathrm{E}-05 \\
2.93 \mathrm{E}-05 \\
3.21 \mathrm{E}-05 \\
3.28 \mathrm{E}-05 \\
3.28 \mathrm{E}-05 \\
3.24 \mathrm{E}-05 \\
3.23 \mathrm{E}-05 \\
3.59 \mathrm{E}-05 \\
3.58 \mathrm{E}-05 \\
4.45 \mathrm{E}-05 \\
3.53 \mathrm{E}-05 \\
3.96 \mathrm{E}-05 \\
3.12 \mathrm{E}-05 \\
4.29 \mathrm{E}-05 \\
4.75 \mathrm{E}-05 \\
6.33 \mathrm{E}-05 \\
5.89 \mathrm{E}-05 \\
5.42 \mathrm{E}-05 \\
7.34 \mathrm{E}-05 \\
2.94 \mathrm{E}-05 \\
2.46 \mathrm{E}-05 \\
1.87 \mathrm{E}-05 \\
3.49 \mathrm{E}-05 \\
2.97 \mathrm{E}-05 \\
3.00 \mathrm{E}-05 \\
3.79 \mathrm{E}-05 \\
2.97 \mathrm{E}-05 \\
3.64 \mathrm{E}-05 \\
3.41 \mathrm{E}-05 \\
2.72 \mathrm{E}-05 \\
3.72 \mathrm{E}-05 \\
3.97 \mathrm{E}-05\end{array}$ & $\begin{array}{l}\text { RA228 } \\
1.37 \mathrm{E}-12 \\
1.73 \mathrm{E}-12 \\
5.00 \mathrm{E}-12 \\
7.48 \mathrm{E}-12 \\
4.27 \mathrm{E}-11 \\
6.53 \mathrm{E}-11 \\
4.84 \mathrm{E}-11 \\
6.74 \mathrm{E}-11 \\
1.28 \mathrm{E}-10 \\
1.23 \mathrm{E}-10 \\
1.89 \mathrm{E}-10 \\
1.78 \mathrm{E}-10 \\
1.92 \mathrm{E}-10 \\
2.36 \mathrm{E}-10 \\
1.42 \mathrm{E}-10 \\
1.19 \mathrm{E}-10 \\
1.42 \mathrm{E}-10 \\
9.93 \mathrm{E}-11 \\
8.19 \mathrm{E}-11 \\
9.75 \mathrm{E}-11 \\
4.12 \mathrm{E}-11 \\
2.19 \mathrm{E}-11 \\
2.22 \mathrm{E}-11 \\
7.57 \mathrm{E}-11 \\
7.57 \mathrm{E}-11 \\
7.73 \mathrm{E}-11 \\
7.48 \mathrm{E}-11 \\
9.60 \mathrm{E}-11 \\
8.68 \mathrm{E}-11 \\
8.79 \mathrm{E}-11 \\
5.04 \mathrm{E}-11 \\
9.93 \mathrm{E}-11 \\
8.14 \mathrm{E}-11 \\
7.88 \mathrm{E}-11 \\
7.44 \mathrm{E}-11 \\
8.17 \mathrm{E}-11 \\
8.35 \mathrm{E}-11 \\
8.37 \mathrm{E}-11 \\
7.95 \mathrm{E}-11 \\
8.25 \mathrm{E}-11 \\
9.18 \mathrm{E}-11 \\
9.16 \mathrm{E}-11 \\
1.14 \mathrm{E}-10 \\
9.07 \mathrm{E}-11 \\
1.06 \mathrm{E}-10 \\
8.05 \mathrm{E}-11 \\
1.15 \mathrm{E}-10 \\
1.23 \mathrm{E}-10 \\
1.70 \mathrm{E}-10 \\
1.58 \mathrm{E}-10 \\
1.46 \mathrm{E}-10 \\
2.12 \mathrm{E}-10 \\
8.70 \mathrm{E}-11 \\
7.88 \mathrm{E}-11 \\
6.50 \mathrm{E}-11 \\
1.29 \mathrm{E}-10 \\
1.07 \mathrm{E}-10 \\
1.25 \mathrm{E}-10 \\
1.84 \mathrm{E}-10 \\
1.37 \mathrm{E}-10 \\
1.86 \mathrm{E}-10 \\
1.66 \mathrm{E}-10 \\
1.39 \mathrm{E}-10 \\
1.77 \mathrm{E}-10 \\
1.85 \mathrm{E}-10\end{array}$ & $\begin{array}{c}\text { AC227 } \\
2.57 E-05 \\
2.64 \mathrm{E}-05 \\
4.90 \mathrm{E}-05 \\
4.73 \mathrm{E}-05 \\
1.62 \mathrm{E}-04 \\
1.68 \mathrm{E}-04 \\
1.08 \mathrm{E}-04 \\
1.28 \mathrm{E}-04 \\
2.15 \mathrm{E}-04 \\
2.23 \mathrm{E}-04 \\
3.67 \mathrm{E}-04 \\
3.59 \mathrm{E}-04 \\
3.23 \mathrm{E}-04 \\
2.14 \mathrm{E}-04 \\
1.65 \mathrm{E}-04 \\
2.49 \mathrm{E}-04 \\
2.93 \mathrm{E}-04 \\
2.00 \mathrm{E}-04 \\
1.18 \mathrm{E}-04 \\
1.89 \mathrm{E}-04 \\
7.95 \mathrm{E}-05 \\
4.39 \mathrm{E}-05 \\
4.28 \mathrm{E}-05 \\
1.52 \mathrm{E}-04 \\
1.46 \mathrm{E}-04 \\
1.55 \mathrm{E}-04 \\
1.50 \mathrm{E}-04 \\
1.92 \mathrm{E}-04 \\
1.67 \mathrm{E}-04 \\
1.69 \mathrm{E}-04 \\
9.68 \mathrm{E}-05 \\
1.98 \mathrm{E}-04 \\
1.62 \mathrm{E}-04 \\
1.51 \mathrm{E}-04 \\
1.48 \mathrm{E}-04 \\
1.63 \mathrm{E}-04 \\
1.66 \mathrm{E}-04 \\
1.67 \mathrm{E}-04 \\
1.65 \mathrm{E}-04 \\
1.64 \mathrm{E}-04 \\
1.82 \mathrm{E}-04 \\
1.82 \mathrm{E}-04 \\
2.26 \mathrm{E}-04 \\
1.80 \mathrm{E}-04 \\
2.02 \mathrm{E}-04 \\
1.59 \mathrm{E}-04 \\
2.19 \mathrm{E}-04 \\
2.42 \mathrm{E}-04 \\
3.23 \mathrm{E}-04 \\
3.01 \mathrm{E}-04 \\
2.77 \mathrm{E}-04 \\
3.74 \mathrm{E}-04 \\
1.50 \mathrm{E}-04 \\
1.25 \mathrm{E}-04 \\
9.49 \mathrm{E}-05 \\
1.77 \mathrm{E}-04 \\
1.51 \mathrm{E}-04 \\
1.52 \mathrm{E}-04 \\
1.90 \mathrm{E}-04 \\
1.50 \mathrm{E}-04 \\
1.83 \mathrm{E}-04 \\
1.71 \mathrm{E}-04 \\
1.37 \mathrm{E}-04 \\
1.88 \mathrm{E}-04 \\
2.01 \mathrm{E}-04\end{array}$ & $\begin{array}{c}\text { TH229 } \\
2.61 \mathrm{E}-10 \\
3.28 \mathrm{E}-10 \\
9.51 \mathrm{E}-10 \\
1.42 \mathrm{E}-09 \\
8.18 \mathrm{E}-09 \\
1.26 \mathrm{E}-08 \\
9.34 \mathrm{E}-09 \\
1.30 \mathrm{E}-08 \\
2.48 \mathrm{E}-08 \\
2.38 \mathrm{E}-08 \\
3.65 \mathrm{E}-08 \\
3.43 \mathrm{E}-08 \\
3.73 \mathrm{E}-08 \\
4.69 \mathrm{E}-08 \\
2.80 \mathrm{E}-08 \\
2.29 \mathrm{E}-08 \\
2.75 \mathrm{E}-08 \\
1.91 \mathrm{E}-08 \\
1.59 \mathrm{E}-08 \\
1.88 \mathrm{E}-08 \\
7.94 \mathrm{E}-09\end{array}$ \\
\hline
\end{tabular}


HNF-SD-WM-TI-794, ReV. 0

ACTIVITY IN UNSEPARATED FUEL (Curies decayed to 1/1/94)

\begin{tabular}{|c|c|c|c|c|c|c|c|c|c|}
\hline 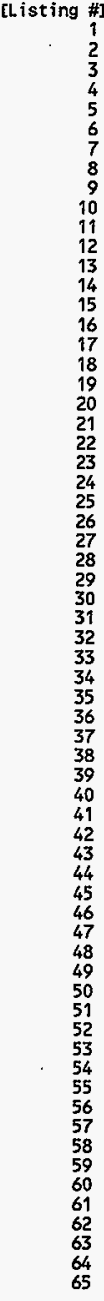 & $\begin{array}{l}\text { TH232 } \\
1.71 \mathrm{E}-12 \\
2.15 \mathrm{E}-12 \\
6.21 \mathrm{E}-12 \\
9.30 \mathrm{E}-12 \\
5.32 \mathrm{E}-11 \\
8.14 \mathrm{E}-11 \\
6.03 \mathrm{E}-11 \\
8.39 \mathrm{E}-11 \\
1.59 \mathrm{E}-10 \\
1.54 \mathrm{E}-10 \\
2.36 \mathrm{E}-10 \\
2.22 \mathrm{E}-10 \\
2.40 \mathrm{E}-10 \\
2.95 \mathrm{E}-10 \\
1.78 \mathrm{E}-10 \\
1.48 \mathrm{E}-10 \\
1.78 \mathrm{E}-10 \\
1.24 \mathrm{E}-10 \\
1.02 \mathrm{E}-10 \\
1.22 \mathrm{E}-10 \\
5.15 \mathrm{E}-11 \\
2.74 \mathrm{E}-11 \\
2.78 \mathrm{E}-11 \\
9.49 \mathrm{E}-11 \\
9.49 \mathrm{E}-11 \\
9.70 \mathrm{E}-11 \\
9.40 \mathrm{E}-11 \\
1.21 \mathrm{E}-10 \\
1.09 \mathrm{E}-10 \\
1.11 \mathrm{E}-10 \\
6.34 \mathrm{E}-11 \\
1.25 \mathrm{E}-10 \\
1.02 \mathrm{E}-10 \\
9.92 \mathrm{E}-11 \\
9.37 \mathrm{E}-11 \\
1.03 \mathrm{E}-10 \\
1.05 \mathrm{E}-10 \\
1.05 \mathrm{E}-10 \\
1.00 \mathrm{E}-10 \\
1.04 \mathrm{E}-10 \\
1.16 \mathrm{E}-10 \\
1.16 \mathrm{E}-10 \\
1.44 \mathrm{E}-10 \\
1.15 \mathrm{E}-10 \\
1.34 \mathrm{E}-10 \\
1.02 \mathrm{E}-10 \\
1.46 \mathrm{E}-10 \\
1.55 \mathrm{E}-10 \\
2.16 \mathrm{E}-10 \\
2.01 \mathrm{E}-10 \\
1.86 \mathrm{E}-10 \\
2.69 \mathrm{E}-10 \\
1.11 \mathrm{E}-10 \\
1.00 \mathrm{E}-10 \\
8.27 \mathrm{E}-11 \\
1.65 \mathrm{E}-10 \\
1.36 \mathrm{E}-10 \\
1.59 \mathrm{E}-10 \\
2.34 \mathrm{E}-10 \\
1.75 \mathrm{E}-10 \\
2.37 \mathrm{E}-10 \\
2.12 \mathrm{E}-10 \\
1.77 \mathrm{E}-10 \\
2.26 \mathrm{E}-10 \\
2.36 \mathrm{E}-10\end{array}$ & $\begin{array}{c}\text { PA231 } \\
5.20 \mathrm{E}-05 \\
5.33 \mathrm{E}-05 \\
9.91 \mathrm{E}-05 \\
9.57 \mathrm{E}-05 \\
3.28 \mathrm{E}-04 \\
3.41 \mathrm{E}-04 \\
2.20 \mathrm{E}-04 \\
2.60 \mathrm{E}-04 \\
4.38 \mathrm{E}-04 \\
4.55 \mathrm{E}-04 \\
7.49 \mathrm{E}-04 \\
7.34 \mathrm{E}-04 \\
6.60 \mathrm{E}-04 \\
4.37 \mathrm{E}-04 \\
3.37 \mathrm{E}-04 \\
5.11 \mathrm{E}-04 \\
6.01 \mathrm{E}-04 \\
4.10 \mathrm{E}-04 \\
2.43 \mathrm{E}-04 \\
3.88 \mathrm{E}-04 \\
1.64 \mathrm{E}-04 \\
9.04 \mathrm{E}-05 \\
8.84 \mathrm{E}-05 \\
3.14 \mathrm{E}-04 \\
3.02 \mathrm{E}-04 \\
3.21 \mathrm{E}-04 \\
3.11 \mathrm{E}-04 \\
3.99 \mathrm{E}-04 \\
3.47 \mathrm{E}-04 \\
3.51 \mathrm{E}-04 \\
2.01 \mathrm{E}-04 \\
4.13 \mathrm{E}-04 \\
3.39 \mathrm{E}-04 \\
3.15 \mathrm{E}-04 \\
3.10 \mathrm{E}-04 \\
3.40 \mathrm{E}-04 \\
3.48 \mathrm{E}-04 \\
3.49 \mathrm{E}-04 \\
3.45 \mathrm{E}-04 \\
3.44 \mathrm{E}-04 \\
3.83 \mathrm{E}-04 \\
3.82 \mathrm{E}-04 \\
4.76 \mathrm{E}-04 \\
3.79 \mathrm{E}-04 \\
4.26 \mathrm{E}-04 \\
3.37 \mathrm{E}-04 \\
4.63 \mathrm{E}-04 \\
5.13 \mathrm{E}-04 \\
6.86 \mathrm{E}-04 \\
6.38 \mathrm{E}-04 \\
5.90 \mathrm{E}-04 \\
7.97 \mathrm{E}-04 \\
3.20 \mathrm{E}-04 \\
2.67 \mathrm{E}-04 \\
2.03 \mathrm{E}-04 \\
3.78 \mathrm{E}-04 \\
3.23 \mathrm{E}-04 \\
3.25 \mathrm{E}-04 \\
4.08 \mathrm{E}-04 \\
3.22 \mathrm{E}-04 \\
3.93 \mathrm{E}-04 \\
3.69 \mathrm{E}-04 \\
2.95 \mathrm{E}-04 \\
4.05 \mathrm{E}-04 \\
\end{array}$ & 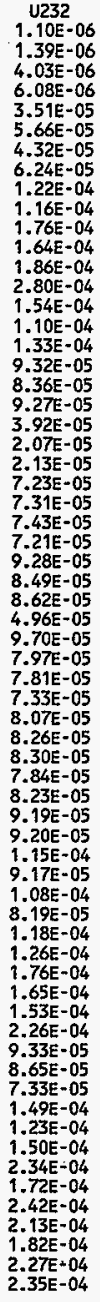 & 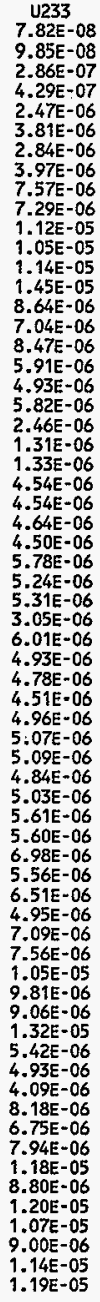 & $\begin{array}{l}\text { U234 } \\
1.08 \mathrm{E}+00 \\
1.11 \mathrm{E}+00 \\
2.07 \mathrm{E}+00 \\
2.01 \mathrm{E}+00 \\
6.96 \mathrm{E}+00 \\
7.28 \mathrm{0}+00 \\
4.73 \mathrm{E}+00 \\
5.62 \mathrm{E}+00 \\
9.50 \mathrm{E}+00 \\
9.86 \mathrm{E}+00 \\
1.62 \mathrm{E}+01 \\
1.59 \mathrm{E}+01 \\
1.44 \mathrm{E}+01 \\
9.80 \mathrm{E}+00 \\
7.47 \mathrm{E}+00 \\
1.11 \mathrm{E}+01 \\
1.31 \mathrm{E}+01 \\
8.99 \mathrm{E}+00 \\
5.37 \mathrm{E}+00 \\
8.52 \mathrm{E}+00 \\
3.60 \mathrm{E}+00 \\
1.99 \mathrm{E}+00 \\
1.95 \mathrm{E}+00 \\
6.94 \mathrm{E}+00 \\
6.69 \mathrm{E}+00 \\
7.12 \mathrm{E}+00 \\
6.91 \mathrm{E}+00 \\
8.88 \mathrm{E}+00 \\
7.74 \mathrm{E}+00 \\
7.86 \mathrm{E}+00 \\
4.52 \mathrm{E}+00 \\
9.26 \mathrm{E}+00 \\
7.61 \mathrm{E}+00 \\
7.10 \mathrm{E}+00 \\
6.98 \mathrm{E}+00 \\
7.68 \mathrm{E}+00 \\
7.86 \mathrm{E}+00 \\
7.89 \mathrm{E}+00 \\
7.82 \mathrm{E}+00 \\
7.81 \mathrm{E}+00 \\
8.72 \mathrm{E}+00 \\
8.72 \mathrm{E}+00 \\
1.09 \mathrm{E}+01 \\
8.67 \mathrm{E}+00 \\
9.77 \mathrm{E}+00 \\
7.74 \mathrm{E}+00 \\
1.07 \mathrm{E}+01 \\
1.18 \mathrm{E}+01 \\
1.59 \mathrm{E}+01 \\
1.48 \mathrm{E}+01 \\
1.37 \mathrm{E}+01 \\
1.86 \mathrm{E}+01 \\
7.47 \mathrm{E}+00 \\
6.27 \mathrm{E}+00 \\
4.77 \mathrm{E}+00 \\
8.95 \mathrm{E}+00 \\
7.65 \mathrm{E}+00 \\
7.75 \mathrm{E}+00 \\
9.82 \mathrm{E}+00 \\
7.74 \mathrm{E}+00 \\
9.51 \mathrm{E}+00 \\
8.92 \mathrm{E}+00 \\
9.82 \mathrm{E}+00+00 \\
1.05 \mathrm{E}+01\end{array}$ & $\begin{array}{c}\text { U235 } \\
5.00 \mathrm{E}-02 \\
5.14 \mathrm{E}-02 \\
9.55 \mathrm{E}-02 \\
9.24 \mathrm{E}-02 \\
3.18 \mathrm{E}-01 \\
3.30 \mathrm{E}-01 \\
2.14 \mathrm{E}-01 \\
2.53 \mathrm{E}-01 \\
4.26 \mathrm{E}-01 \\
4.43 \mathrm{E}-01 \\
7.31 \mathrm{E}-01 \\
7.17 \mathrm{E}-01 \\
6.46 \mathrm{E}-01 \\
4.28 \mathrm{E}-01 \\
3.31 \mathrm{E}-01 \\
5.02 \mathrm{E}-01 \\
5.92 \mathrm{E}-01 \\
4.05 \mathrm{E}-01 \\
2.40 \mathrm{E}-01 \\
3.84 \mathrm{E}-01 \\
1.62 \mathrm{E}-01 \\
8.99 \mathrm{E}-02 \\
8.80 \mathrm{E}-02 \\
3.13 \mathrm{E}-01 \\
3.01 \mathrm{E}-01 \\
3.21 \mathrm{E}-01 \\
3.12 \mathrm{E}-01 \\
4.01 \mathrm{E}-01 \\
3.49 \mathrm{E}-01 \\
3.54 \mathrm{E}-01 \\
2.04 \mathrm{E}-01 \\
4.17 \mathrm{E}-01 \\
3.43 \mathrm{E}-01 \\
3.20 \mathrm{E}-01 \\
3.15 \mathrm{E}-01 \\
3.46 \mathrm{E}-01 \\
3.54 \mathrm{E}-01 \\
3.56 \mathrm{E}-01 \\
3.53 \mathrm{E}-01 \\
3.52 \mathrm{E}-01 \\
3.93 \mathrm{E}-01 \\
3.93 \mathrm{E}-01 \\
4.90 \mathrm{E}-01 \\
3.91 \mathrm{E}-01 \\
4.40 \mathrm{E}-01 \\
3.49 \mathrm{E}-01 \\
4.80 \mathrm{E}-01 \\
5.34 \mathrm{E}-01 \\
7.14 \mathrm{E}-01 \\
6.66 \mathrm{E}-01 \\
6.17 \mathrm{E}-01 \\
8.35 \mathrm{E}-01 \\
3.36 \mathrm{E}-01 \\
2.81 \mathrm{E}-01 \\
2.13 \mathrm{E}-01 \\
3.99 \mathrm{E}-01 \\
3.41 \mathrm{E}-01 \\
3.44 \mathrm{E}-01 \\
4.32 \mathrm{E}-01 \\
3.42 \mathrm{E}-01 \\
4.18 \mathrm{E}-01 \\
3.93 \mathrm{E}-01 \\
3.14 \mathrm{E}-01 \\
4.33 \mathrm{E}-01 \\
4.65 \mathrm{E}-01\end{array}$ & $\begin{array}{l}\text { U236 } \\
7.03 \mathrm{E}-04 \\
8.86 \mathrm{E}-04 \\
2.57 \mathrm{E}-03 \\
3.85 \mathrm{E}-03 \\
2.20 \mathrm{E}-02 \\
3.38 \mathrm{E}-02 \\
2.51 \mathrm{E}-02 \\
3.50 \mathrm{E}-02 \\
6.65 \mathrm{E}-02 \\
6.42 \mathrm{E}-02 \\
9.87 \mathrm{E}-02 \\
9.31 \mathrm{E}-02 \\
1.01 \mathrm{E}-01 \\
1.24 \mathrm{E}-01 \\
7.49 \mathrm{E}-02 \\
6.26 \mathrm{E}-02 \\
7.54 \mathrm{E}-02 \\
5.26 \mathrm{E}-02 \\
4.34 \mathrm{E}-02 \\
5.18 \mathrm{E}-02 \\
2.19 \mathrm{E}-02 \\
1.17 \mathrm{E}-02 \\
1.19 \mathrm{E}-02 \\
4.06 \mathrm{E}-02 \\
4.07 \mathrm{E}-02 \\
4.17 \mathrm{E}-02 \\
4.04 \mathrm{E}-02 \\
5.20 \mathrm{E}-02 \\
4.71 \mathrm{E}-02 \\
4.78 \mathrm{E}-02 \\
2.75 \mathrm{E}-02 \\
5.42 \mathrm{E}-02 \\
4.45 \mathrm{E}-02 \\
4.32 \mathrm{E}-02 \\
4.08 \mathrm{E}-02 \\
4.50 \mathrm{E}-02 \\
4.60 \mathrm{E}-02 \\
4.62 \mathrm{E}-02 \\
4.40 \mathrm{E}-02 \\
4.57 \mathrm{E}-02 \\
5.10 \mathrm{E}-02 \\
5.10 \mathrm{E}-02 \\
6.36 \mathrm{E}-02 \\
5.08 \mathrm{E}-02 \\
5.94 \mathrm{E}-02 \\
4.53 \mathrm{E}-02 \\
6.48 \mathrm{E}-02 \\
6.93 \mathrm{E}-02 \\
9.64 \mathrm{E}-02 \\
8.99 \mathrm{E}-02 \\
8.32 \mathrm{E}-02 \\
1.21 \mathrm{E}-01 \\
4.97 \mathrm{E}-02 \\
4.51 \mathrm{E}-02 \\
3.73 \mathrm{E}-02 \\
7.45 \mathrm{E}-02 \\
6.17 \mathrm{E}-02 \\
7.22 \mathrm{E}-02 \\
1.07 \mathrm{E}-01 \\
7.97 \mathrm{E}-02 \\
1.08 \mathrm{E}-01 \\
9.69 \mathrm{E}-02 \\
8.12 \mathrm{E}-02 \\
1.04 \mathrm{E}-01 \\
1.09 \mathrm{E}-01\end{array}$ & $\begin{array}{c}\text { U238 } \\
1.09 E+00 \\
1.12 E+00 \\
2.09 E+00 \\
2.03 E+00 \\
7.02 E+00 \\
7.36 E+00 \\
4.78 E+00 \\
5.69 E+00 \\
9.62 E+00 \\
9.99 E+00 \\
1.64 E+01 \\
1.61 E+01 \\
1.46 E+01 \\
9.99 E+00 \\
7.60 E+00 \\
1.13 E+01 \\
1.33 E+01 \\
9.10 E+00 \\
5.45 E+00 \\
8.63 E+00 \\
3.65 E+00 \\
2.02 E+00 \\
1.98 E+00 \\
7.03 E+00 \\
6.77 E+00 \\
7.20 E+00 \\
6.99 E+00 \\
8.99 E+00 \\
7.84 E+00 \\
7.95 E+00 \\
4.57 E+00 \\
9.37 E+00 \\
7.70 E+00 \\
7.19 E+00 \\
7.06 E+00 \\
7.77 E+00 \\
7.95 E+00 \\
7.99 E+00 \\
7.91 E+00 \\
7.91 E+00 \\
8.82 E+00 \\
8.82 E+00 \\
1.10 E+01 \\
8.78 E+00 \\
9.89 E+00 \\
7.83 E+00 \\
1.08 E+01 \\
1.20 E+01 \\
1.60 E+01 \\
1.50 E+01 \\
1.39 E+01 \\
1.88 E+01 \\
7.57 E+00 \\
6.36 E+00 \\
4.84 E+00 \\
9.08 E+00 \\
7.76 E+00 \\
7.87 E+00 \\
9.99 E+00 \\
7.87 E+00 \\
9.68 E+00 \\
9.08 E+00 \\
7.28 E+00 \\
9.99 E+00 \\
1.07 E+01\end{array}$ & $\begin{array}{l}\text { NP237 } \\
2.04 \mathrm{E}-04 \\
2.58 \mathrm{E}-04 \\
7.50 \mathrm{E}-04 \\
1.13 \mathrm{E}-03 \\
6.55 \mathrm{E}-03 \\
1.02 \mathrm{E}-02 \\
7.60 \mathrm{E}-03 \\
1.07 \mathrm{E}-02 \\
2.05 \mathrm{E}-02 \\
1.97 \mathrm{E}-02 \\
3.01 \mathrm{E}-02 \\
2.83 \mathrm{E}-02 \\
3.10 \mathrm{E}-02 \\
4.09 \mathrm{E}-02 \\
2.39 \mathrm{E}-02 \\
1.90 \mathrm{E}-02 \\
2.29 \mathrm{E}-02 \\
1.60 \mathrm{E}-02 \\
1.35 \mathrm{E}-02 \\
1.58 \mathrm{E}-02 \\
6.68 \mathrm{E}-03 \\
3.55 \mathrm{E}-03 \\
3.62 \mathrm{E}-03 \\
1.24 \mathrm{E}-02 \\
1.24 \mathrm{E}-02 \\
1.27 \mathrm{E}-02 \\
1.23 \mathrm{E}-02 \\
1.58 \mathrm{E}-02 \\
1.44 \mathrm{E}-02 \\
1.46 \mathrm{E}-02 \\
8.37 \mathrm{E}-03 \\
1.65 \mathrm{E}-02 \\
1.35 \mathrm{E}-02 \\
1.32 \mathrm{E}-02 \\
1.24 \mathrm{E}-02 \\
1.37 \mathrm{E}-02 \\
1.40 \mathrm{E}-02 \\
1.40 \mathrm{E}-02 \\
1.33 \mathrm{E}-02 \\
1.39 \mathrm{E}-02 \\
1.55 \mathrm{E}-02 \\
1.55 \mathrm{E}-02 \\
1.94 \mathrm{E}-02 \\
1.54 \mathrm{E}-02 \\
1.81 \mathrm{E}-02 \\
1.38 \mathrm{E}-02 \\
1.98 \mathrm{E}-02 \\
2.11 \mathrm{E}-02 \\
2.94 \mathrm{E}-02 \\
2.74 \mathrm{E}-02 \\
2.54 \mathrm{E}-02 \\
3.70 \mathrm{E}-02 \\
1.52 \mathrm{E}-02 \\
1.39 \mathrm{E}-02 \\
1.16 \mathrm{E}-02 \\
2.33 \mathrm{E}-02 \\
1.92 \mathrm{E}-02 \\
2.28 \mathrm{E}-02 \\
3.43 \mathrm{E}-02 \\
2.55 \mathrm{E}-02 \\
3.50 \mathrm{E}-02 \\
3.12 \mathrm{E}-02 \\
3.33 \mathrm{E}-02 \\
3.47 \mathrm{E}-02\end{array}$ \\
\hline
\end{tabular}


ACTIVITY IN UNSEPARATED FUEL (Curies decayed to $1 / 1 / 94$ )

\begin{tabular}{|c|c|c|c|c|c|c|c|c|c|}
\hline 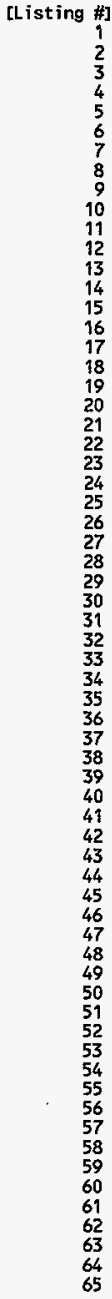 & $\begin{array}{l}\text { PU238 } \\
2.85 \mathrm{E}-03 \\
3.61 \mathrm{E}-03 \\
1.06 \mathrm{E}-02 \\
1.61 \mathrm{E}-02 \\
9.54 \mathrm{E}-02 \\
3.13 \mathrm{E}-01 \\
3.25 \mathrm{E}-01 \\
6.33 \mathrm{E}-01 \\
1.49 \mathrm{E}+00 \\
1.27 \mathrm{E}+00 \\
1.71 \mathrm{E}+00 \\
1.49 \mathrm{E}+00 \\
2.27 \mathrm{E}+00 \\
6.99 \mathrm{E}+00 \\
3.01 \mathrm{E}+00 \\
9.25 \mathrm{E}-01 \\
1.16 \mathrm{E}+00 \\
8.47 \mathrm{E}-01 \\
1.25 \mathrm{E}+00 \\
9.02 \mathrm{E}-01 \\
3.82 \mathrm{E}-01 \\
1.88 \mathrm{E}-01 \\
2.07 \mathrm{E}-01 \\
6.57 \mathrm{E}-01 \\
7.11 \mathrm{E}-01 \\
6.74 \mathrm{E}-01 \\
6.55 \mathrm{E}-01 \\
8.43 \mathrm{E}-01 \\
8.25 \mathrm{E}-01 \\
8.38 \mathrm{E}-01 \\
4.82 \mathrm{E}-01 \\
8.80 \mathrm{E}-01 \\
7.23 \mathrm{E}-01 \\
7.59 \mathrm{E}-01 \\
6.65 \mathrm{E}-01 \\
7.32 \mathrm{E}-01 \\
7.49 \mathrm{E}-01 \\
7.53 \mathrm{E}-01 \\
6.60 \mathrm{E}-01 \\
7.46 \mathrm{E}-01 \\
8.33 \mathrm{E}-01 \\
8.34 \mathrm{E}-01 \\
1.04 \mathrm{E}+00 \\
8.31 \mathrm{E}-01 \\
1.05 \mathrm{E}+00 \\
7.42 \mathrm{E}-01 \\
1.15 \mathrm{E}+00 \\
1.14 \mathrm{E}+00 \\
1.71 \mathrm{E}+00 \\
1.60 \mathrm{E}+00 \\
1.48 \mathrm{E}+00 \\
2.45 \mathrm{E}+00 \\
1.05 \mathrm{E}+00 \\
1.10 \mathrm{E}+00 \\
1.05 \mathrm{E}+00 \\
2.32 \mathrm{E}+00 \\
1.83 \mathrm{E}+00 \\
2.68 \mathrm{E}+00 \\
4.96 \mathrm{E}+00 \\
3.44 \mathrm{E}+00 \\
5.38 \mathrm{E}+00 \\
4.52 \mathrm{E}+00 \\
4.05 \mathrm{E}+00 \\
4.67 \mathrm{E}+00 \\
4.70 \mathrm{E}+00\end{array}$ & $\begin{array}{l}\text { PU239 } \\
4.81 E+00 \\
6.07 E+00 \\
1.76 E+01 \\
2.62 E+01 \\
1.50 E+02 \\
2.29 E+02 \\
1.69 E+02 \\
2.35 E+02 \\
4.46 E+02 \\
4.32 E+02 \\
6.65 E+02 \\
6.27 E+02 \\
6.76 E+02 \\
8.16 E+02 \\
4.97 E+02 \\
4.22 E+02 \\
5.08 E+02 \\
3.54 E+02 \\
2.90 E+02 \\
3.49 E+02 \\
1.48 E+02 \\
7.86 E+01 \\
8.00 E+01 \\
2.74 E+02 \\
2.74 E+02 \\
2.81 E+02 \\
2.72 E+02 \\
3.50 E+02 \\
3.17 E+02 \\
3.22 E+02 \\
1.85 E+02 \\
3.65 E+02 \\
3.00 E+02 \\
2.91 E+02 \\
2.75 E+02 \\
3.03 E+02 \\
3.10 E+02 \\
3.11 E+02 \\
2.96 E+02 \\
3.08 E+02 \\
3.44 E+02 \\
3.44 E+02 \\
4.29 E+02 \\
3.42 E+02 \\
4.00 E+02 \\
3.05 E+02 \\
4.36 E+02 \\
4.67 E+02 \\
6.49 E+02 \\
6.06 E+02 \\
5.60 E+02 \\
8.13 E+02 \\
3.34 E+02 \\
3.03 E+02 \\
2.50 E+02 \\
4.98 E+02 \\
4.13 E+02 \\
4.81 E+02 \\
7.06 E+02 \\
5.29 E+02 \\
7.14 E+02 \\
6.42 E+02 \\
5.37 E+02 \\
6.89 E+02 \\
7.20 E+02\end{array}$ & $\begin{array}{l}\text { PU240 } \\
7.98 \mathrm{E}-02 \\
1.06 \mathrm{E}-01 \\
3.59 \mathrm{E}-01 \\
6.55 \mathrm{E}-01 \\
5.02 \mathrm{E}+00 \\
1.00 \mathrm{E}+01 \\
8.22 \mathrm{E}+00 \\
1.30 \mathrm{E}+01 \\
2.71 \mathrm{E}+01 \\
2.47 \mathrm{E}+01 \\
3.60 \mathrm{E}+01 \\
3.29 \mathrm{E}+01 \\
4.11 \mathrm{E}+01 \\
8.59 \mathrm{E}+01 \\
4.17 \mathrm{E}+01 \\
2.14 \mathrm{E}+01 \\
2.62 \mathrm{E}+01 \\
1.86 \mathrm{E}+01 \\
2.00 \mathrm{E}+01 \\
1.89 \mathrm{E}+01 \\
7.98 \mathrm{E}+00 \\
4.12 \mathrm{E}+00 \\
4.33 \mathrm{E}+00 \\
1.44 \mathrm{E}+01 \\
1.48 \mathrm{E}+01 \\
1.47 \mathrm{E}+01 \\
1.43 \mathrm{E}+01 \\
1.84 \mathrm{E}+01 \\
1.72 \mathrm{E}+01 \\
1.74 \mathrm{E}+01 \\
1.00 \mathrm{E}+01 \\
1.91 \mathrm{E}+01 \\
1.57 \mathrm{E}+01 \\
1.57 \mathrm{E}+01 \\
1.44 \mathrm{E}+01 \\
1.59 \mathrm{E}+01 \\
1.63 \mathrm{E}+01 \\
1.63 \mathrm{E}+01 \\
1.51 \mathrm{E}+01 \\
1.62 \mathrm{E}+01 \\
1.80 \mathrm{E}+01 \\
1.80 \mathrm{E}+01 \\
2.25 \mathrm{E}+01 \\
1.79 \mathrm{E}+01 \\
2.17 \mathrm{E}+01 \\
1.60 \mathrm{E}+01 \\
2.36 \mathrm{E}+01 \\
2.45 \mathrm{E}+01 \\
3.51 \mathrm{E}+01 \\
3.28 \mathrm{E}+01 \\
3.03 \mathrm{E}+01 \\
4.66 \mathrm{E}+01 \\
1.95 \mathrm{E}+01 \\
1.89 \mathrm{E}+01 \\
1.67 \mathrm{E}+01 \\
3.53 \mathrm{E}+01 \\
2.85 \mathrm{~B}+01 \\
3.77 \mathrm{E}+01 \\
6.39 \mathrm{E}+01 \\
4.55 \mathrm{E}+01 \\
6.76 \mathrm{E}+01 \\
5.81 \mathrm{E}+01 \\
5.08 \mathrm{E}+01 \\
6.08 \mathrm{E}+01 \\
6.20 \mathrm{E}+01\end{array}$ & 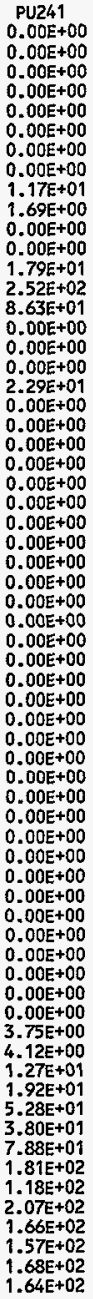 & $\begin{array}{l}\text { PU242 } \\
0.00 E+00 \\
0.00 E+00 \\
0.00 E+00 \\
0.00 E+00 \\
0.00 E+00 \\
0.00 E+00 \\
0.00 E+00 \\
0.00 E+00 \\
0.00 E+00 \\
0.00 E+00 \\
0.00 E+00 \\
0.00 E+00 \\
0.00 E+00 \\
1.40 E-03 \\
2.71 E E-04 \\
0.00 E+00 \\
0.00 E+00 \\
0.00 E+00 \\
0.00 E+00 \\
0.00 E+00 \\
0.00 E+00 \\
0.00 E+00 \\
0.00 E+00 \\
0.00 E+00 \\
0.00 E+00 \\
0.00 E+00 \\
0.00 E+00 \\
0.00 E+00 \\
0.00 E+00 \\
0.00 E+00 \\
0.00 E+00 \\
0.00 E+00 \\
0.00 E+00 \\
0.00 E+00 \\
0.00 E+00 \\
0.00 E+00 \\
0.00 E+00 \\
0.00 E+00 \\
0.00 E+00 \\
0.00 E+00 \\
0.00 E+00 \\
0.00 E+00 \\
0.00 E+00 \\
0.00 E+00 \\
0.00 E+00 \\
0.00 E+00 \\
0.00 E+00 \\
0.00 E+00 \\
0.00 E+00 \\
0.00 E+00 \\
0.00 E+00 \\
0.00 E+00 \\
0.00 E+00 \\
0.00 E+00 \\
0.00 E+00 \\
0.00 E+00 \\
0.00 E+00 \\
1.16 E-04 \\
6.42 E-04 \\
3.52 E-04 \\
8.09 E-04 \\
5.84 E-04 \\
6.08 E-04 \\
5.42 E E-04 \\
4.79 E-04\end{array}$ & $\begin{array}{l}\text { AM241 } \\
0.00 E+00 \\
0.00 E+00 \\
0.00 E+00 \\
0.00 E+00 \\
0.00 E+00 \\
0.00 E+00 \\
0.00 E+00 \\
0.00 E+00 \\
3.44 E+00 \\
4.98 E-01 \\
0.00 E+00 \\
0.00 E+00 \\
5.21 E+00 \\
7.30 E+01 \\
2.49 E+01 \\
0.00 E+00 \\
0.00 E+00 \\
0.00 E+00 \\
6.53 E+00 \\
0.00 E+00 \\
0.00 E+00 \\
0.00 E+00 \\
0.00 E+00 \\
0.00 E+00 \\
0.00 E+00 \\
0.00 E+00 \\
0.00 E+00 \\
0.00 E+00 \\
0.00 E+00 \\
0.00 E+00 \\
0.00 E+00 \\
0.00 E+00 \\
0.00 E+00 \\
0.00 E+00 \\
0.00 E+00 \\
0.00 E+00 \\
0.00 E+00 \\
0.00 E+00 \\
0.00 E+00 \\
0.00 E+00 \\
0.00 E+00 \\
0.00 E+00 \\
0.00 E+00 \\
0.00 E+00 \\
0.00 E+00 \\
0.00 E+00 \\
0.00 E+00 \\
0.00 E+00 \\
0.00 E+00 \\
0.00 E+00 \\
0.00 E+00 \\
9.24 E=01 \\
1.01 E+00 \\
3.11 E+00 \\
4.68 E+00 \\
1.28 E+01 \\
9.16 E+00 \\
1.89 E+01 \\
4.33 E+01 \\
2.82 E+01 \\
4.90 E+01 \\
3.93 E+01 \\
3.68 E+01 \\
3.94 E+01 \\
3.83 E+01\end{array}$ & 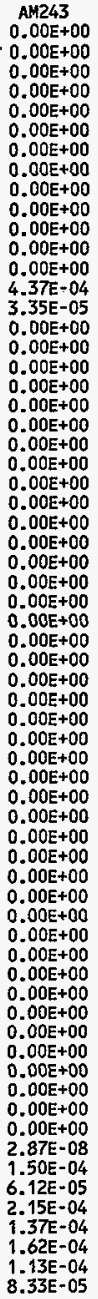 & $\begin{array}{l}\text { CN24Z } \\
0.00 E+00 \\
0.00 E+00 \\
0.00 E+00 \\
0.00 E+00 \\
0.00 E+00 \\
0.00 E+00 \\
0.00 E+00 \\
0.00 E+00 \\
0.00 E+00 \\
0.00 E+00 \\
0.00 E+00 \\
0.00 E+00 \\
0.00 E+00 \\
1.28 E-03 \\
1.04 E-04 \\
0.00 E+00 \\
0.00 E+00 \\
0.00 E+00 \\
0.00 E+00 \\
0.00 E+00 \\
0.00 E+00 \\
0.00 E+00 \\
0.00 E+00 \\
0.00 E+00 \\
0.00 E+00 \\
0.00 E+00 \\
0.00 E+00 \\
0.00 E+00 \\
0.00 E+00 \\
0.00 E+00 \\
0.00 E+00 \\
0.00 E+00 \\
0.00 E+00 \\
0.00 E+00 \\
0.00 E+00 \\
0.00 E+00 \\
0.00 E+00 \\
0.00 E+00 \\
0.00 E+00 \\
0.00 E+00 \\
0.00 E+00 \\
0.00 E+00 \\
0.00 E+00 \\
0.00 E+00 \\
0.00 E+00 \\
0.00 E+00 \\
0.00 E+00 \\
0.00 E+00 \\
0.00 E+00 \\
0.00 E+00 \\
0.00 E+00 \\
0.00 E+00 \\
0.00 E+00 \\
0.00 E+00 \\
0.00 E+00 \\
0.00 E+00 \\
0.00 E+00 \\
0.00 E+00 \\
4.53 E-04 \\
1.88 E-04 \\
6.46 E-04 \\
4.12 E=04 \\
4.86 E-04 \\
3.43 E-04 \\
2.56 E-04 \\
0\end{array}$ & $\begin{array}{l}\text { CM243 } \\
0.00 \mathrm{E}+00 \\
0.00 \mathrm{E}+00 \\
0.00 \mathrm{E}+00 \\
0.00 \mathrm{E}+00 \\
0.00 \mathrm{E}+00 \\
0.00 \mathrm{E}+00 \\
0.00 \mathrm{E}+00 \\
0.00 \mathrm{E}+00 \\
0.00 \mathrm{E}+00 \\
0.00 \mathrm{E}+00 \\
0.00 \mathrm{E}+00 \\
0.00 \mathrm{E}+00 \\
0.00 \mathrm{E}+00 \\
2.21 \mathrm{E}-05 \\
0.00 \mathrm{E}+00 \\
0.00 \mathrm{E}+00 \\
0.00 \mathrm{E}+00 \\
0.00 \mathrm{E}+00 \\
0.00 \mathrm{E}+00 \\
0.00 \mathrm{E}+00 \\
0.00 \mathrm{E}+00 \\
0.00 \mathrm{E}+00 \\
0.00 \mathrm{E}+00 \\
0.00 \mathrm{E}+00 \\
0.00 \mathrm{E}+00 \\
0.00 \mathrm{E}+00 \\
0.00 \mathrm{E}+00 \\
0.00 \mathrm{E}+00 \\
0.00 \mathrm{E}+00 \\
0.00 \mathrm{E}+00 \\
0.00 \mathrm{E}+00 \\
0.00 \mathrm{E}+00 \\
0.00 \mathrm{E}+00 \\
0.00 \mathrm{E}+00 \\
0.00 \mathrm{E}+00 \\
0.00 \mathrm{E}+00 \\
0.00 \mathrm{E}+00 \\
0.00 \mathrm{E}+00 \\
0.00 \mathrm{E}+00 \\
0.00 \mathrm{E}+00 \\
0.00 \mathrm{E}+00 \\
0.00 \mathrm{E}+00 \\
0.00 \mathrm{E}+00 \\
0.00 \mathrm{E}+00 \\
0.00 \mathrm{E}+00 \\
0.00 \mathrm{E}+00 \\
0.00 \mathrm{E}+00 \\
0.00 \mathrm{E}+00 \\
0.00 \mathrm{E}+00 \\
0.00 \mathrm{E}+00 \\
0.00 \mathrm{E}+00 \\
0.00 \mathrm{E}+00 \\
0.00 \mathrm{E}+00 \\
0.00 \mathrm{E}+00 \\
0.00 \mathrm{E}+00 \\
0.00 \mathrm{E}+00 \\
0.00 \mathrm{E}+00 \\
0.00 \mathrm{E}+00 \\
6.37 \mathrm{E}-06 \\
1.50 \mathrm{E}-06 \\
1.05 \mathrm{E}-05 \\
5.82 \mathrm{E}-06 \\
7.94 \mathrm{E}-06 \\
4.12 \mathrm{E}-06 \\
2.06 \mathrm{E}-06\end{array}$ \\
\hline
\end{tabular}


ACTIVITY IN UNSEPARATED FUEL (Curies decayed to 1/1/94)

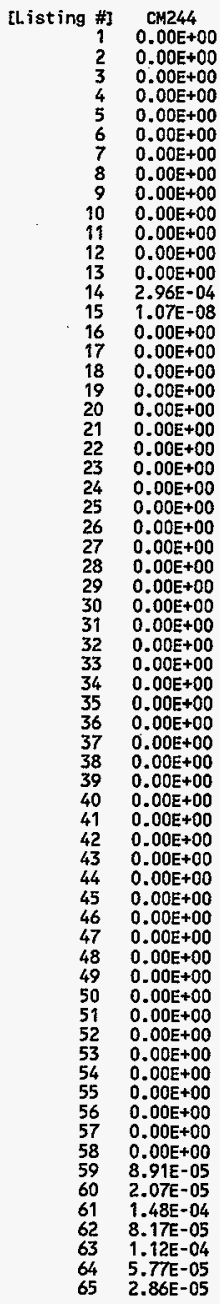


HNF-SD-WM-TI-794, ReV. 0

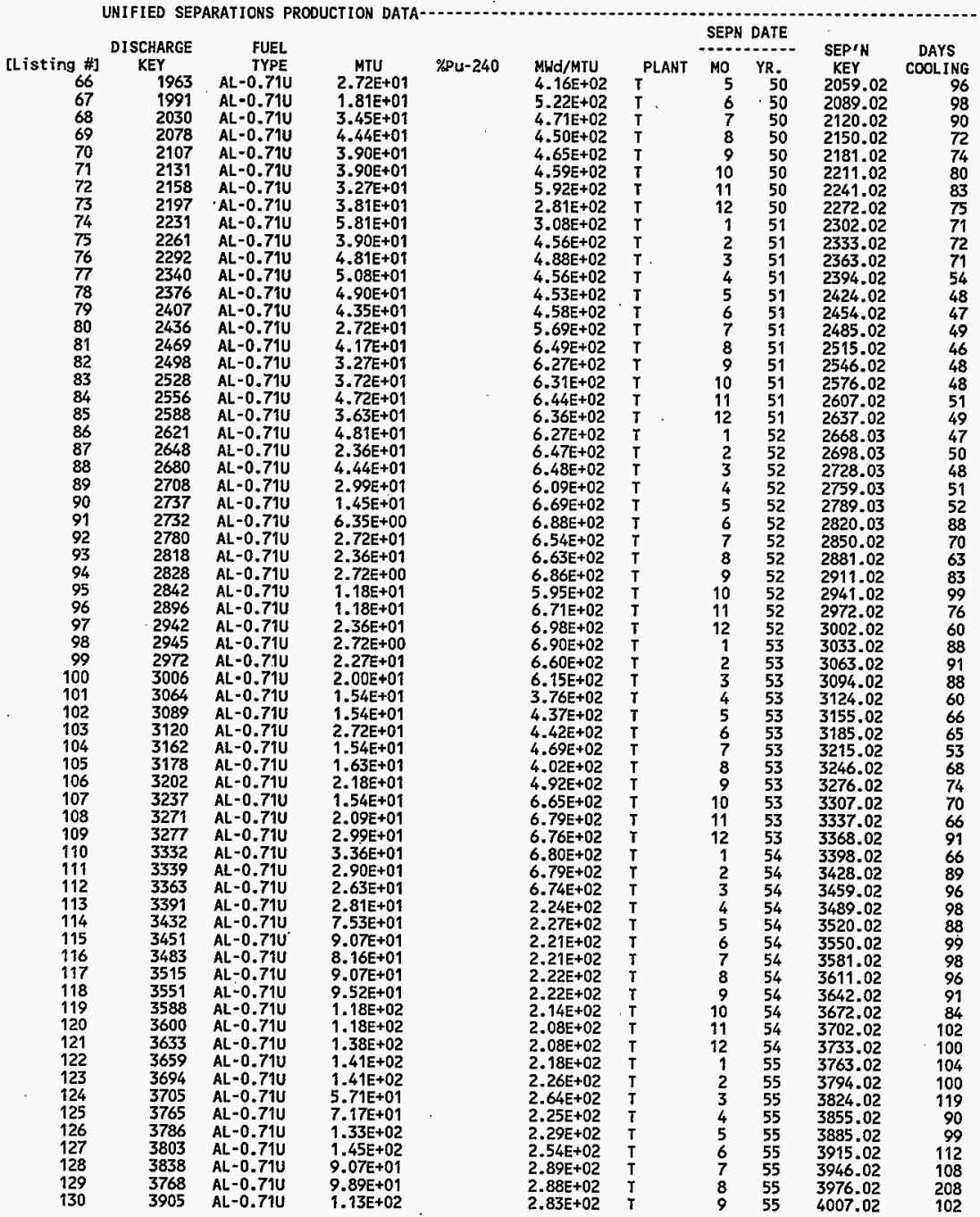


HNF-SD-WM-T1-794, ReV. 0

UNIFIED SEPARATIONS PRODUCTION DATA

TOTAL WASTE LOSS FRACTION

[Listing \#] Fuel Data Reference

66 SPLANT.DAT 19348 9/30/93 1:19P 67 SPLANT.DAT $193489 / 30 / 931: 19 P$

68 SPLANT.DAT 19348 9/30/93 1:19P 69 SPLANT.DAT 19348 9/30/93 1:19P

70 SPLANT.DAT 19348 9/30/93 $1: 19 \mathrm{P}$

71 SPLANT.DAT 19348 9/30/93 1:19P

72 SPLANT DAT $193489 / 30 / 931=19 \mathrm{P}$

73 SPLANT.DAT 19348 9/30/93 $1: 19 \mathrm{P}$

74 SPLANT.DAT 19348 9/30/93 1:19P

75 SPLANT.DAT $193489 / 30 / 93 \quad 1: 19 \mathrm{P}$

76 SPLANT.DAT 19348 9/30/93 1:19P

77 SPLANT.DAT $19348 \quad 9 / 30 / 93 \quad 1: 19 \mathrm{P}$

78 SPLANT.DAT $193489 / 30 / 931: 19 \mathrm{P}$

79 SPLANT.DAT 19348 9/30/93 1:19P

80 SPLANT.DAT 19348 9/30/93 1:19P

81 SPLANT.DAT 19348 9/30/93 1:19P

82 SPLANT.DAT 19348 9/30/93 1:19P

83 SPLANT_DAT 19348 9/30/93 1:19P

84 SPLANT.DAT 19348 9/30/93 1:19P

85 SPLANT.DAT 19348 9/30/93 1:19P

86 SPLANT.DAT 19348 9/30/93 1:19P

87 SPLANT.DAT 19348 9/30/93 1:19P

88 SPLANT.DAT $19348 \quad 9 / 30 / 93 \quad 1: 19 \mathrm{P}$

89 SPLANT.DAT 19348 9/30/93 1:19P

90 SPLANT.DAT 19348 9/30/93 1:19P

91 SPLANT DAT 19348 9/30/93 1:19P

92 SPLANT.DAT 19348 9/30/93 1:19P

93 SPLANT.DAT $19348 \% / 30 / 93$ 1:19P

94 SPLANT.DAT 19348 9/30/93 1:19P

95 SPLANT.DAT 19348 9/30/93 1:19P

96 SPLANT.DAT 19348 9/30/93 1:19P

97 SPLANT.DAT 19348 \%/30/93 $1: 19 \mathrm{P}$

98 SPLANT.DAT 19348 9/30/93 1:19P

99 SPLANT.DAT 19348 9/30/93 1:19P

100 SPLANT.DAT 19348 9/30/93 1:19P

101 SPLANT.DAT 19348 9/30/93 1:19P

102 SPLANT.DAT 19348 9/30/93 1:19P

103 SPLANT.DAT 19348 9/30/93 $1: 19 P$

104 SPLANT.DAT $193489 / 30 / 93 \% 1=19 \mathrm{P}$

105 SPLANT.DAT 19348 9/30/93 $1: 19 P$ 106 SPLANT.DAT $193489 / 30 / 931: 19 \mathrm{P}$ 107 SPLANT.DAT $193489 / 30 / 93$ 1:19P 108 SPLANT.DAT 19348 9/30/93 1:19P 109 SPLANT.DAT $193489 / 30 / 93$ 1:19P 110 SPLANT.DAT 19348 9/30/93 1:19P 111 SPLANT.DAT 19348 9/30/93 1:19P 112 SPLANT.DAT 19348 9/30/93 1:19P 113 SPLANT.DAT 19348 9/30/93 $1: 19 \mathrm{P}$ 114 SPLANT.DAT 19348 9/30/93 $1: 19 \mathrm{P}$ 115 SPLANT.DAT 19348 9/30/93 1 :19P 116 SPLANT.DAT 19348 9/30/93 1:19P 117 SPLANT.DAT 19348 9/30/93 1:19P 118 SPLANT.OAT 19348 9/30/93 1:19P 119 SPLANT.OAT 19348 9/30/93 1:19p 120 SPLANT.DAT 19348 9/30/93 1:19P 121 SPLANT.DAT $19348 \quad 9 / 30 / 93 \quad 1: 19 P$ 122 SPLANT.DAT 19348 9/30/93 $1: 19 \mathrm{P}$ 123 SPLANT_DAT $193489 / 30 / 93 \quad 1: 19 \mathrm{P}$ 124 SPLANT.DAT 19348 9/30/93 $1: 19 \mathrm{P}$ 125 SPLANT.DAT 19348 9/30/93 $1: 19 \mathrm{p}$ 126 SPLANT.DAT 19348 9/30/93 $1=19 \mathrm{P}$ 127 SPLANT.DAT 19348 9/30/93 1:19P 128 SPLANT.DAT 19348 9/30/93 $1: 19 \mathrm{P}$ 129 SPLANT.DAT 19348 9/30/93 1:19P 130 SPLANT.DAT 19348 9/30/93 1:19p

U

$1 \quad 0, \quad$ Np

1

$\begin{array}{ll}1 & 1 \\ 1 & 1\end{array}$

Pu

0.0288

0.0288

0.0288

0.0288

0.0288

0.0288

0.0288

0.0288

0.0288

0.0288

0.0288

0.0288

0.0288

0.0288

0.0288

0.0288

0.0288

0.0288

0.0288

0.0288

0.0288

0.0288

0.0288

0.0288

0.0288

0.0288

0.0288

0.0288

0.0288

0.0288

0.0288

0.0288

0.029

0.026

0.021

0.017

0.021

0.022

0.023

0.024

0.024

0.023

0.024

0.023

0.025

0.025

0.022

0.061

0.023

0.023

0.026

0.027

0.024

0.031

0.036

0.04

0.037

0.04

0.043

0.032

0.039

0.036

0.036

0.03
Waste Frac. Reference

(a)

(a)

(a)

(a)

(a)

(a)

(a)

(a)

(a)

(a)

(a)

(a)

(a)

(a)

(a)

(a)

(a)

(a)

(a)

(a)

(a)

(a)

(a)

(b)

(b)

(b)

(b)

(b)

(b)

(b)

(b)

(b)

(b)

(b)

(b)

(b)

(b)

(b)

(b)

(b)

(b)

(b)

(b)

(b)

(b)

(b)

(b)

(b) 
HNF-SD-WM-TI-794, Rev. 0

ACTIVITY IN UNSEPARATED FUEL (Curies decayed to 1/1/94)

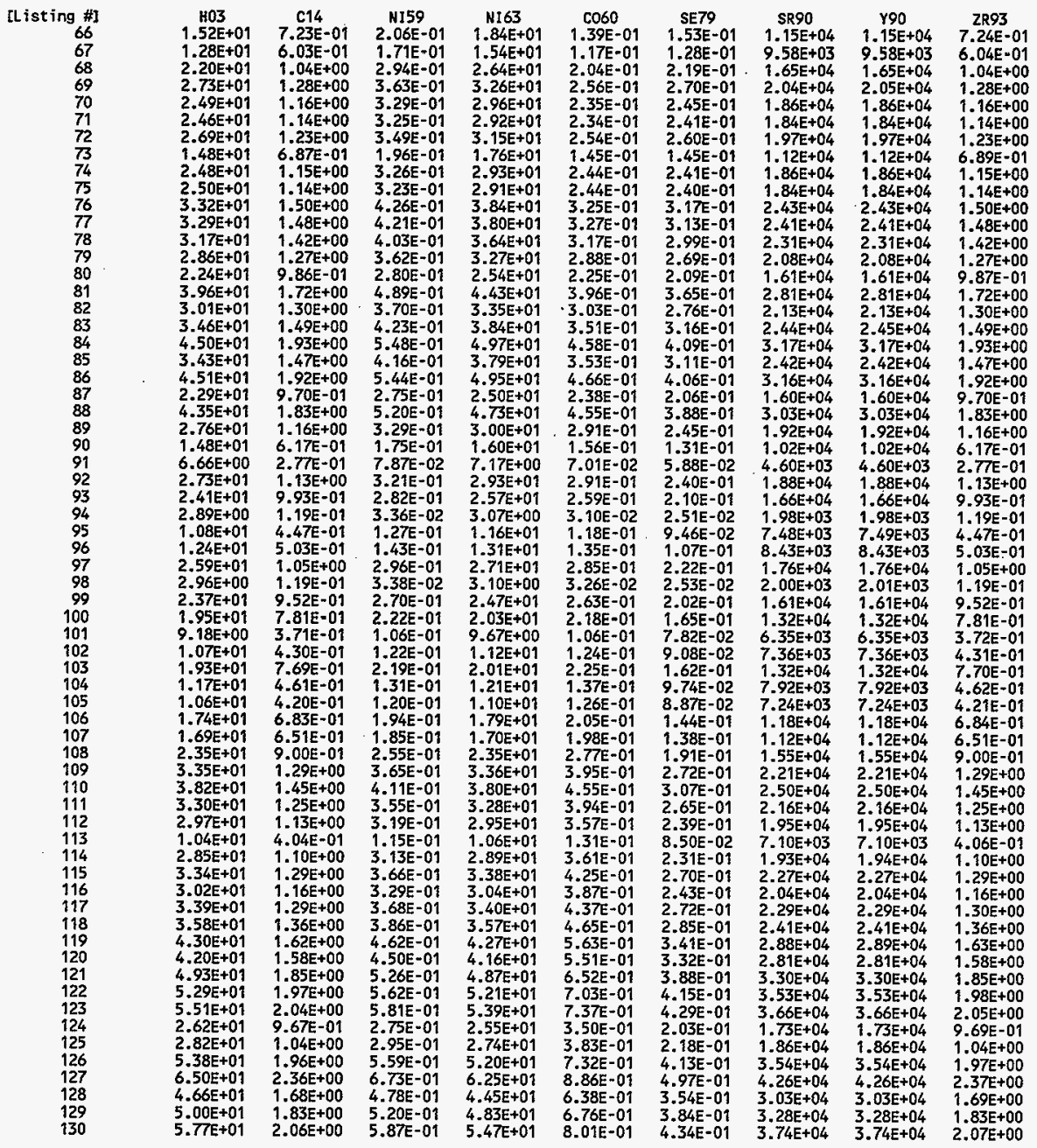


HNF-SD-WM-TI-794, Rev. 0

ACTIVITY IN UNSEPARATED FUEL (Curies decayed to 1/1/94)

\begin{tabular}{|c|c|c|c|c|c|c|c|c|c|}
\hline 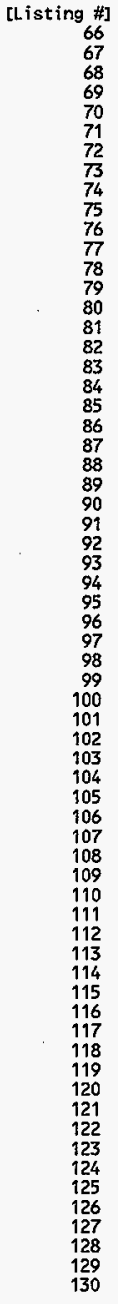 & $\begin{array}{l}\text { NB93* } \\
6.15 E-01 \\
5.13 E-01 \\
8.80 E-01 \\
1.08 E+00 \\
9.83 E-01 \\
9.69 E-01 \\
1.04 E+00 \\
5.83 E-01 \\
9.70 E-01 \\
9.62 E-01 \\
1.27 E+00 \\
1.25 E+00 \\
1.20 E+00 \\
1.07 E+00 \\
8.31 E-01 \\
1.45 E+00 \\
1.10 E+00 \\
1.26 E+00 \\
1.62 E+00 \\
1.23 E+00 \\
1.61 E+00 \\
8.14 E-01 \\
1.54 E+00 \\
9.72 E-01 \\
5.17 E-01 \\
2.32 E-01 \\
9.47 E-01 \\
8.31 E-01 \\
9.91 E-02 \\
3.74 E-01 \\
4.20 E-01 \\
8.72 E-01 \\
9.95 E-02 \\
7.94 E-01 \\
6.51 E-01 \\
3.09 E-01 \\
3.59 E-01 \\
6.40 E-01 \\
3.84 E-01 \\
3.50 E-01 \\
5.68 E-01 \\
5.40 E-01 \\
7.46 E-01 \\
1.07 E+00 \\
1.20 E+00 \\
1.04 E+00 \\
9.31 E-01 \\
3.35 E-01 \\
9.10 E-01 \\
1.06 E+00 \\
9.57 E-01 \\
1.07 E+00 \\
1.12 E+00 \\
1.34 E+00 \\
1.30 E+00 \\
1.52 E+00 \\
1.63 E+00 \\
1.68 E+00 \\
7.96 E-01 \\
8.52 E-01 \\
1.61 E+00 \\
1.94 E+00 \\
1.38 E+00 \\
1.50 E+00 \\
1.69 E+00\end{array}$ & $\begin{array}{l}1 C 99 \\
5.02 E+00 \\
4.20 E+00 \\
7.20 E+00 \\
8.87 E+00 \\
8.06 E+00 \\
7.94 E+00 \\
8.58 E+00 \\
4.75 E+00 \\
7.93 E+00 \\
7.90 E+00 \\
1.04 E+01 \\
1.03 E+01 \\
9.85 E+00 \\
8.84 E+00 \\
6.87 E+00 \\
1.20 E+01 \\
9.09 E+00 \\
1.04 E+01 \\
1.35 E+01 \\
1.03 E+01 \\
1.34 E+01 \\
6.78 E+00 \\
1.28 E+01 \\
8.09 E+00 \\
4.31 E+00 \\
1.94 E+00 \\
7.90 E+00 \\
6.94 E+00 \\
8.29 E-01 \\
3.12 E+00 \\
3.52 E+00 \\
7.31 E+00 \\
8.34 E-01 \\
6.65 E+00 \\
5.45 E+00 \\
2.57 E+00 \\
2.99 E+00 \\
5.34 E+00 \\
3.21 E+00 \\
2.92 E+00 \\
4.75 E+00 \\
4.55 E+00 \\
6.29 E+00 \\
8.98 E+00 \\
1.01 E+01 \\
8.75 E+00 \\
7.87 E+00 \\
2.79 E+00 \\
7.59 E+00 \\
8.88 E+00 \\
7.99 E+00 \\
8.92 E+00 \\
9.37 E+00 \\
1.12 E+01 \\
1.09 E+01 \\
1.28 E+01 \\
1.36 E+01 \\
1.41 E+01 \\
6.68 E+00 \\
7.15 E+00 \\
1.36 E+01 \\
1.63 E+01 \\
1.16 E+01 \\
1.26 E+01 \\
1.43 E+01\end{array}$ & $\begin{array}{l}\text { RU106 } \\
9.97 E-09 \\
9.14 \mathrm{E}-09 \\
1.65 \mathrm{E}-08 \\
2.22 \mathrm{E}-08 \\
2.14 \mathrm{E}-08 \\
2.19 \mathrm{E}-08 \\
2.63 \mathrm{E}-08 \\
1.40 \mathrm{E}-08 \\
2.51 \mathrm{E}-08 \\
2.79 \mathrm{E}-08 \\
3.94 \mathrm{E}-08 \\
4.20 \mathrm{E}-08 \\
4.31 \mathrm{E}-08 \\
4.11 \mathrm{E}-08 \\
3.51 \mathrm{E}-08 \\
6.72 \mathrm{E}-08 \\
5.33 \mathrm{E}-08 \\
6.46 \mathrm{E}-08 \\
8.88 \mathrm{E}-08 \\
7.11 \mathrm{E}-08 \\
9.89 \mathrm{E}-08 \\
5.32 \mathrm{E}-08 \\
1.06 \mathrm{E}-07 \\
6.99 \mathrm{E}-08 \\
4.02 \mathrm{E}-08 \\
1.80 \mathrm{E}-08 \\
7.93 \mathrm{E}-08 \\
7.48 \mathrm{E}-08 \\
9.24 \mathrm{E}-09 \\
3.46 \mathrm{E}-08 \\
4.43 \mathrm{E}-08 \\
1.01 \mathrm{E}-07 \\
1.16 \mathrm{E}-08 \\
9.62 \mathrm{E}-08 \\
8.29 \mathrm{E}-08 \\
4.01 \mathrm{E}-08 \\
4.98 \mathrm{E}-08 \\
9.45 \mathrm{E}-08 \\
6.21 \mathrm{E}-08 \\
5.69 \mathrm{E}-08 \\
1.000 \mathrm{E}-07 \\
1.09 \mathrm{E}-07 \\
1.60 \mathrm{E}-07 \\
2.31 \mathrm{E}-07 \\
2.90 \mathrm{E}-07 \\
2.54 \mathrm{E}-07 \\
2.38 \mathrm{E}-07 \\
7.60 \mathrm{E}-08 \\
2.23 \mathrm{E}-07 \\
2.70 \mathrm{E}-07 \\
2.58 \mathrm{E}-07 \\
3.07 \mathrm{E}-07 \\
3.45 \mathrm{E}-07 \\
4.40 \mathrm{E}-07 \\
4.38 \mathrm{E}-07 \\
5.44 \mathrm{E}-07 \\
6.14 \mathrm{E}-07 \\
6.80 \mathrm{E}-07 \\
3.33 \mathrm{E}-07 \\
3.93 \mathrm{E}-07 \\
7.77 \mathrm{E}-07 \\
9.77 \mathrm{E}-07 \\
7.52 \mathrm{E}-07 \\
1.17 \mathrm{E}-07 \\
\end{array}$ & 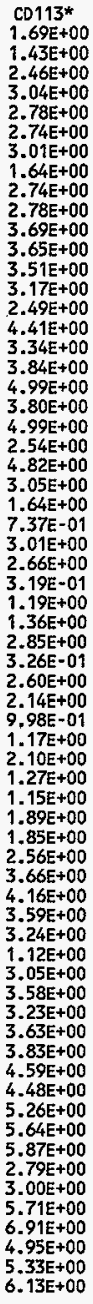 & $\begin{array}{l}\text { SB125 } \\
9.96 \mathrm{E}-02 \\
8.54 \mathrm{E}=02 \\
1.50 \mathrm{E}-01 \\
1.91 \mathrm{E}-01 \\
1.77 \mathrm{E}-01 \\
1.77 \mathrm{E}-01 \\
1.96 \mathrm{E}-01 \\
1.10 \mathrm{E}-01 \\
1.88 \mathrm{E}-01 \\
1.93 \mathrm{E}-01 \\
2.60 \mathrm{E}-01 \\
2.65 \mathrm{E}-01 \\
2.60 \mathrm{E}-01 \\
2.38 \mathrm{E}-01 \\
1.90 \mathrm{E}-01 \\
3.41 \mathrm{E}-01 \\
2.63 \mathrm{E}-01 \\
3.07 \mathrm{E}-01 \\
4.06 \mathrm{E}-01 \\
3.15 \mathrm{E}-01 \\
4.21 \mathrm{E}-01 \\
2.18 \mathrm{E}-01 \\
4.19 \mathrm{E}-01 \\
2.70 \mathrm{E}-01 \\
1.47 \mathrm{E}-01 \\
6.60 \mathrm{E}-02 \\
2.77 \mathrm{E}-01 \\
2.50 \mathrm{E}-01 \\
3.01 \mathrm{E}-02 \\
1.14 \mathrm{E}-01 \\
1.34 \mathrm{E}-01 \\
2.87 \mathrm{E}-01 \\
3.29 \mathrm{E}-02 \\
2.67 \mathrm{E}-01 \\
2.23 \mathrm{E}-01 \\
1.09 \mathrm{E}-01 \\
1.28 \mathrm{E}-01 \\
2.35 \mathrm{E}-01 \\
1.45 \mathrm{E}-01 \\
1.33 \mathrm{E}-01 \\
2.21 \mathrm{E}-01 \\
2.19 \mathrm{E}-01 \\
3.10 \mathrm{E}-01 \\
4.44 \mathrm{E}-01 \\
5.20 \mathrm{E}-01 \\
4.52 \mathrm{E}-01 \\
4.12 \mathrm{E}-01 \\
1.46 \mathrm{E}-01 \\
4.08 \mathrm{E}-01 \\
4.84 \mathrm{E}-01 \\
4.45 \mathrm{E}-01 \\
5.09 \mathrm{E}-01 \\
5.47 \mathrm{E}-01 \\
6.71 \mathrm{E}-01 \\
6.59 \mathrm{E}-01 \\
7.88 \mathrm{E}-01 \\
8.58 \mathrm{E}-01 \\
9.10 \mathrm{E}-01 \\
4.35 \mathrm{E}-01 \\
4.84 \mathrm{E}-01 \\
9.31 \mathrm{E}-01 \\
1.14 \mathrm{E}+00 \\
8.29 \mathrm{E}-01 \\
8.59 \mathrm{E}-01 \\
1.07 \mathrm{E}+00\end{array}$ & $\begin{array}{l}\text { SN126 } \\
2.30 \mathrm{E}-01 \\
1.94 \mathrm{E}-01 \\
3.31 \mathrm{E}-01 \\
4.08 \mathrm{E}-01 \\
3.71 \mathrm{E}-01 \\
3.65 \mathrm{E}-01 \\
3.99 \mathrm{E}-01 \\
2.15 \mathrm{E}-01 \\
3.60 \mathrm{E}-01 \\
3.63 \mathrm{E}-01 \\
4.80 \mathrm{E}-01 \\
4.73 \mathrm{E}-01 \\
4.52 \mathrm{E}-01 \\
4.06 \mathrm{E}-01 \\
3.19 \mathrm{E}-01 \\
5.62 \mathrm{E}-01 \\
4.24 \mathrm{E}-01 \\
4.85 \mathrm{E}-01 \\
6.30 \mathrm{E}-01 \\
4.77 \mathrm{E}-01 \\
6.24 \mathrm{E}-01 \\
3.16 \mathrm{E}-01 \\
5.97 \mathrm{E}-01 \\
3.76 \mathrm{E}-01 \\
2.02 \mathrm{E}-01 \\
9.08 \mathrm{E}-02 \\
3.69 \mathrm{E}-01 \\
3.24 \mathrm{E}-01 \\
3.88 \mathrm{E}-02 \\
1.45 \mathrm{E}-01 \\
1.64 \mathrm{E}-01 \\
3.42 \mathrm{E}-01 \\
3.91 \mathrm{E}-02 \\
3.10 \mathrm{E}-01 \\
2.54 \mathrm{E}-01 \\
1.18 \mathrm{E}-01 \\
1.37 \mathrm{E}-01 \\
2.45 \mathrm{E}-01 \\
1.48 \mathrm{E}-01 \\
1.33 \mathrm{E}-01 \\
2.19 \mathrm{E}-01 \\
2.13 \mathrm{E}-01 \\
2.94 \mathrm{E}-01 \\
4.20 \mathrm{E}-01 \\
4.74 \mathrm{E}-01 \\
4.09 \mathrm{E}-01 \\
3.68 \mathrm{E}-01 \\
1.26 \mathrm{E}-01 \\
3.42 \mathrm{E}-01 \\
4.00 \mathrm{E}-01 \\
3.60 \mathrm{E}-01 \\
4.02 \mathrm{E}-01 \\
4.22 \mathrm{E}-01 \\
5.04 \mathrm{E}-01 \\
4.91 \mathrm{E}-01 \\
5.74 \mathrm{E}-01 \\
6.14 \mathrm{E}-01 \\
6.35 \mathrm{E}-01 \\
3.02 \mathrm{E}-01 \\
3.22 \mathrm{E}-01 \\
6.12 \mathrm{E}-01 \\
7.38 \mathrm{E}-01 \\
5.72 \mathrm{E}-01 \\
6.46 \mathrm{E}-01 \\
\end{array}$ & $\begin{array}{c}\text { I129 } \\
9.47 \mathrm{E}-03 \\
7.99 \mathrm{E}-03 \\
1.37 \mathrm{E}-02 \\
1.68 \mathrm{E}-02 \\
1.53 \mathrm{E}-02 \\
1.50 \mathrm{E}-02 \\
1.64 \mathrm{E}-02 \\
8.87 \mathrm{E}-03 \\
1.48 \mathrm{E}-02 \\
1.50 \mathrm{E}-02 \\
1.98 \mathrm{E}-02 \\
1.95 \mathrm{E}-02 \\
1.86 \mathrm{E}-02 \\
1.67 \mathrm{E}-02 \\
1.31 \mathrm{E}-02 \\
2.31 \mathrm{E}-02 \\
1.75 \mathrm{E}-02 \\
2.00 \mathrm{E}-02 \\
2.59 \mathrm{E}-02 \\
1.97 \mathrm{E}-02 \\
2.57 \mathrm{E}-02 \\
1.30 \mathrm{E}-02 \\
2.46 \mathrm{E}-02 \\
1.55 \mathrm{E}-02 \\
8.30 \mathrm{E}-03 \\
3.74 \mathrm{E}-03 \\
1.52 \mathrm{E}-02 \\
1.33 \mathrm{E}-02 \\
1.60 \mathrm{E}-03 \\
5.96 \mathrm{E}-03 \\
6.77 \mathrm{E}-03 \\
1.41 \mathrm{E}-02 \\
1.61 \mathrm{E}-03 \\
1.28 \mathrm{E}-02 \\
1.05 \mathrm{E}-02 \\
4.84 \mathrm{E}-03 \\
5.65 \mathrm{E}-03 \\
1.01 \mathrm{E}-02 \\
6.08 \mathrm{E}-03 \\
5.50 \mathrm{E}-03 \\
9.02 \mathrm{E}-03 \\
8.76 \mathrm{E}-03 \\
1.21 \mathrm{E}-02 \\
1.73 \mathrm{E}-02 \\
1.95 \mathrm{E}-02 \\
1.69 \mathrm{E}-02 \\
1.52 \mathrm{E}-02 \\
5.19 \mathrm{E}-03 \\
1.41 \mathrm{E}-02 \\
1.65 \mathrm{E}-02 \\
1.48 \mathrm{E}-02 \\
1.66 \mathrm{E}-02 \\
1.774 \mathrm{E}-02 \\
2.08 \mathrm{E}-02 \\
2.02 \mathrm{E}-02 \\
2.37 \mathrm{E}-02 \\
2.53 \mathrm{E}-02 \\
2.62 \mathrm{E}-02 \\
1.25 \mathrm{E}-02 \\
1.33 \mathrm{E}-02 \\
2.52 \mathrm{E}-02 \\
3.04 \mathrm{E}-02 \\
2.36 \mathrm{E}-02 \\
2.66 \mathrm{E}-02 \\
\end{array}$ & $\begin{array}{l}\text { CS134 } \\
6.05 E-04 \\
6.96 E-04 \\
1.09 E-03 \\
1.32 E-03 \\
1.30 E-03 \\
1.24 E-03 \\
1.94 E-03 \\
3.99 E-04 \\
7.92 E-04 \\
1.39 E-03 \\
2.09 E-03 \\
1.94 E-03 \\
1.92 E-03 \\
1.78 E-03 \\
1.91 E-03 \\
4.05 E-03 \\
3.02 E-03 \\
3.55 E-03 \\
4.92 E-03 \\
3.69 E-03 \\
4.97 E-03 \\
2.69 E-03 \\
5.23 E-03 \\
3.14 E-03 \\
1.93 E-03 \\
8.91 E-04 \\
3.54 E-03 \\
3.22 E-03 \\
4.16 E-04 \\
1.32 E-03 \\
1.82 E-03 \\
4.08 E-03 \\
4.67 E-04 \\
3.56 E-03 \\
2.88 E-03 \\
7.42 E-04 \\
1.08 E-03 \\
1.99 E-03 \\
1.36 E-03 \\
1.03 E-03 \\
2.23 E-03 \\
3.22 E-03 \\
4.60 E-03 \\
6.59 E-03 \\
7.83 E-03 \\
6.81 E-03 \\
6.24 E-03 \\
4.71 E-04 \\
1.33 E-03 \\
1.58 E-03 \\
1.46 E-03 \\
1.69 E-03 \\
1.83 E-03 \\
2.07 E-03 \\
1.94 E-03 \\
2.33 E-03 \\
2.80 E-03 \\
3.14 E-03 \\
2.02 E-03 \\
1.70 E-03 \\
3.44 E-03 \\
5.06 E-03 \\
4.61 E-03 \\
4.70 E-03 \\
6.02 E-03\end{array}$ & $\begin{array}{l}\text { CS137 } \\
1.31 \mathrm{E}+04 \\
1.10 \mathrm{E}+04 \\
1.89 \mathrm{E}+04 \\
2.33 \mathrm{E}+04 \\
2.12 \mathrm{E}+04 \\
2.09 \mathrm{E}+04 \\
2.27 \mathrm{E}+04 \\
1.26 \mathrm{E}+04 \\
2.10 \mathrm{E}+04 \\
2.10 \mathrm{E}+04 \\
2.77 \mathrm{E}+04 \\
2.75 \mathrm{E}+04 \\
2.64 \mathrm{E}+04 \\
2.37 \mathrm{E}+04 \\
1.85 \mathrm{E}+04 \\
3.24 \mathrm{E}+04 \\
2.46 \mathrm{E}+04 \\
2.82 \mathrm{E}+04 \\
3.65 \mathrm{E}+04 \\
2.78 \mathrm{E}+04 \\
3.64 \mathrm{E}+04 \\
1.85 \mathrm{E}+04 \\
3.49 \mathrm{E}+04 \\
2.21 \mathrm{E}+04 \\
1.18 \mathrm{E}+04 \\
5.32 \mathrm{E}+03 \\
2.17 \mathrm{E}+04 \\
1.91 \mathrm{E}+04 \\
2.29 \mathrm{E}+03 \\
8.60 \mathrm{E}+03 \\
9.73 \mathrm{E}+03 \\
2.03 \mathrm{E}+04 \\
2.32 \mathrm{E}+03 \\
1.85 \mathrm{E}+04 \\
1.52 \mathrm{E}+04 \\
7.19 \mathrm{E}+03 \\
8.36 \mathrm{E}+03 \\
1.50 \mathrm{E}+04 \\
9.02 \mathrm{E}+03 \\
8.21 \mathrm{E}+03 \\
1.34 \mathrm{E}+04 \\
1.29 \mathrm{E}+04 \\
1.78 \mathrm{E}+04 \\
2.55 \mathrm{E}+04 \\
2.89 \mathrm{E}+04 \\
2.49 \mathrm{E}+04 \\
2.24 \mathrm{E}+04 \\
7.95 \mathrm{E}+03 \\
2.17 \mathrm{E}+04 \\
2.54 \mathrm{E}+04 \\
2.56 \mathrm{E}+04 \\
2.69 \mathrm{E}+04 \\
3.23 \mathrm{E}+04 \\
3.15 \mathrm{E}+04 \\
3.99 \mathrm{E}+04 \\
3.96 \mathrm{E}+04 \\
4.77 \mathrm{E}+04 \\
3\end{array}$ \\
\hline
\end{tabular}


HNF-SD-WM-TI -794, Rev. 0

ACTIVITY IN UNSEPARATED FUEL (Curies decayed to 1/1/94)

\begin{tabular}{|c|c|c|c|c|c|c|c|c|c|}
\hline $\begin{array}{r}\text { Listing } \# \\
66 \\
67 \\
68 \\
69 \\
70 \\
71 \\
72 \\
73 \\
74 \\
75 \\
76 \\
77 \\
78 \\
79 \\
80 \\
81 \\
82 \\
83 \\
84 \\
85 \\
86 \\
87 \\
88 \\
89 \\
90 \\
91 \\
92 \\
93 \\
94 \\
95 \\
96 \\
97 \\
98 \\
99 \\
100 \\
101 \\
102 \\
103 \\
104 \\
105 \\
106 \\
107 \\
108 \\
109 \\
110 \\
111 \\
112 \\
113 \\
114 \\
115 \\
116 \\
117 \\
138 \\
119 \\
120 \\
121 \\
122 \\
123 \\
124 \\
125 \\
126 \\
129 \\
130\end{array}$ & $\begin{array}{l}B A 137 \\
1.24 E+04 \\
1.04 E+04 \\
1.78 E+04 \\
2.20 E+04 \\
2.00 E+04 \\
1.98 E+04 \\
2.14 E+04 \\
1.19 E+04 \\
1.99 E+04 \\
1.99 E+04 \\
2.62 E+04 \\
2.60 E+04 \\
2.49 E+04 \\
2.24 E+04 \\
1.75 E+04 \\
3.07 E+04 \\
2.32 E+04 \\
2.66 E+04 \\
3.46 E+04 \\
2.63 E+04 \\
3.45 E+04 \\
1.75 E+04 \\
3.31 E+04 \\
2.09 E+04 \\
1.12 E+04 \\
5.03 E+03 \\
2.05 E+04 \\
1.81 E+04 \\
2.16 E+03 \\
8.13 E+03 \\
9.21 E+03 \\
1.92 E+04 \\
2.19 E+03 \\
1.75 E+04 \\
1.44 E+04 \\
6.80 E+03 \\
7.91 E+03 \\
1.42 E+04 \\
8.53 E+03 \\
7.76 E+03 \\
1.27 E+04 \\
1.22 E+04 \\
1.69 E+04 \\
2.41 E+04 \\
2.73 E+04 \\
2.36 E+04 \\
2.12 E+04 \\
7.52 E+03 \\
2.05 E+04 \\
2.40 E+04 \\
2.16 E+04 \\
2.42 E+04 \\
2.55 E+04 \\
3.05 E+04 \\
2.98 E+04 \\
3.49 E+04 \\
3.73 E+04 \\
3.87 E+04 \\
1.84 E+04 \\
1.97 E+04 \\
3.75 E+04 \\
4.52 E+04 \\
3.22 E+04 \\
3.49 E+04 \\
3.97 E+04\end{array}$ & $\begin{array}{c}\text { SH151 } \\
5.61 \mathrm{E}+02 \\
4.55 \mathrm{E}+02 \\
7.93 \mathrm{E}+02 \\
9.84 \mathrm{E}+02 \\
8.88 \mathrm{E}+02 \\
8.82 \mathrm{E}+02 \\
9.12 \mathrm{E}+02 \\
5.56 \mathrm{E}+02 \\
9.21 \mathrm{E}+02 \\
8.80 \mathrm{E}+02 \\
1.15 \mathrm{E}+03 \\
1.15 \mathrm{E}+03 \\
1.10 \mathrm{E}+03 \\
9.88 \mathrm{E}+02 \\
7.40 \mathrm{E}+02 \\
1.26 \mathrm{E}+03 \\
9.62 \mathrm{E}+02 \\
1.10 \mathrm{E}+03 \\
1.42 \mathrm{E}+03 \\
1.09 \mathrm{E}+03 \\
1.42 \mathrm{E}+03 \\
7.14 \mathrm{E}+02 \\
1.35 \mathrm{E}+03 \\
8.64 \mathrm{E}+02 \\
4.52 \mathrm{E}+02 \\
2.02 \mathrm{E}+02 \\
8.34 \mathrm{E}+02 \\
7.33 \mathrm{E}+02 \\
8.65 \mathrm{E}+01 \\
3.36 \mathrm{E}+02 \\
3.70 \mathrm{E}+02 \\
7.65 \mathrm{E}+02 \\
8.73 \mathrm{E}+01 \\
7.05 \mathrm{E}+02 \\
5.83 \mathrm{E}+02 \\
2.98 \mathrm{E}+02 \\
3.40 \mathrm{E}+02 \\
6.08 \mathrm{E}+02 \\
3.62 \mathrm{E}+02 \\
3.36 \mathrm{E}+02 \\
5.33 \mathrm{E}+02 \\
4.82 \mathrm{E}+02 \\
6.67 \mathrm{E}+02 \\
9.52 \mathrm{E}+02 \\
1.08 \mathrm{E}+03 \\
9.29 \mathrm{E}+02 \\
8.36 \mathrm{E}+02 \\
3.41 \mathrm{E}+02 \\
9.27 \mathrm{E}+02 \\
1.09 \mathrm{E}+03 \\
9.77 \mathrm{E}+02 \\
1.09 \mathrm{E}+03 \\
1.15 \mathrm{E}+03 \\
1.38 \mathrm{E}+03 \\
1.34 \mathrm{E}+03 \\
1.57 \mathrm{E}+03 \\
1.68 \mathrm{E}+03 \\
1.73 \mathrm{E}+03 \\
8.12 \mathrm{E}+02 \\
8.80 \mathrm{E}+02 \\
1.67 \mathrm{E}+03 \\
1.99 \mathrm{E}+03 \\
1.41 \mathrm{E}+03 \\
1.52 \mathrm{E}+03 \\
1.73 \mathrm{E}+03\end{array}$ & $\begin{array}{l}\text { EU152 } \\
4.22 \mathrm{E}-02 \\
5.05 \mathrm{E}-02 \\
7.45 \mathrm{E}-02 \\
8.59 \mathrm{E}-02 \\
8.42 \mathrm{E}-02 \\
7.74 \mathrm{E}-02 \\
1.27 \mathrm{E}-01 \\
1.85 \mathrm{E}-02 \\
3.84 \mathrm{E}-02 \\
7.85 \mathrm{E}-02 \\
1.18 \mathrm{E}-01 \\
1.03 \mathrm{E}-01 \\
9.94 \mathrm{E}-02 \\
8.96 \mathrm{E}-02 \\
9.98 \mathrm{E}-02 \\
2.11 \mathrm{E}-01 \\
1.53 \mathrm{E}-01 \\
1.76 \mathrm{E}-01 \\
2.39 \mathrm{E}-01 \\
1.74 \mathrm{E}-01 \\
2.29 \mathrm{E}-01 \\
1.22 \mathrm{E}-01 \\
2.31 \mathrm{E}-01 \\
1.34 \mathrm{E}-01 \\
8.17 \mathrm{E}-02 \\
3.81 \mathrm{E}-02 \\
1.45 \mathrm{E}-01 \\
1.28 \mathrm{E}-01 \\
1.65 \mathrm{E}-02 \\
5.07 \mathrm{E}-02 \\
6.81 \mathrm{E}-02 \\
1.48 \mathrm{E}-01 \\
1.69 \mathrm{E}-02 \\
1.25 \mathrm{E}-01 \\
9.82 \mathrm{E}-02 \\
2.10 \mathrm{E}-02 \\
3.17 \mathrm{E}-02 \\
5.70 \mathrm{E}-02 \\
3.86 \mathrm{E}-02 \\
2.73 \mathrm{E}-02 \\
6.19 \mathrm{E}-02 \\
9.25 \mathrm{E}-02 \\
1.29 \mathrm{E}-01 \\
1.84 \mathrm{E}-01 \\
2.09 \mathrm{E}-01 \\
1.81 \mathrm{E}-01 \\
1.63 \mathrm{E}-01 \\
6.17 \mathrm{E}-03 \\
1.69 \mathrm{E}-02 \\
1.98 \mathrm{E}-02 \\
1.79 \mathrm{E}-02 \\
2.01 \mathrm{E}-02 \\
2.12 \mathrm{E}-02 \\
2.07 \mathrm{E}-02 \\
1.76 \mathrm{E}-02 \\
2.07 \mathrm{E}-02 \\
2.80 \mathrm{E}-02 \\
3.25 \mathrm{E}-02 \\
2.770 \mathrm{E}-02 \\
1.66 \mathrm{E}-02 \\
3.49 \mathrm{E}-02 \\
5.97 \mathrm{E}-02 \\
6.09 \mathrm{E}-02 \\
6.55 \mathrm{E}-02 \\
7.54 \mathrm{E}-02\end{array}$ & $\begin{array}{l}E U 154 \\
2.80 E+00 \\
3.00 E+00 \\
4.65 E+00 \\
5.52 E+00 \\
5.29 E+00 \\
4.99 E+00 \\
7.26 E+00 \\
1.82 E+00 \\
3.38 E+00 \\
5.12 E+00 \\
7.41 E+00 \\
6.78 E+00 \\
6.54 E+00 \\
5.91 E+00 \\
5.93 E+00 \\
1.20 E+01 \\
8.81 E+00 \\
1.02 E+01 \\
1.37 E+01 \\
1.01 E+01 \\
1.33 E+01 \\
7.04 E+00 \\
1.34 E+01 \\
7.95 E+00 \\
4.79 E+00 \\
2.38 E+00 \\
8.44 E+00 \\
7.48 E+00 \\
9.51 E-01 \\
3.07 E+00 \\
3.98 E+00 \\
8.60 E+00 \\
9.82 E-01 \\
7.42 E+00 \\
5.90 E+00 \\
1.64 E+00 \\
2.25 E+00 \\
4.05 E+00 \\
2.65 E+00 \\
2.05 E+00 \\
4.16 E+00 \\
5.55 E+00 \\
7.73 E+00 \\
1.11 E+01 \\
1.26 E+01 \\
1.09 E+01 \\
9.86 E+00 \\
1.08 E+00 \\
2.95 E+00 \\
3.47 E+00 \\
3.14 E+00 \\
3.54 E+00 \\
3.74 E+00 \\
4.28 E+00 \\
4.06 E+00 \\
4.78 E+00 \\
5.42 E+00 \\
5.82 E+00 \\
3.32 E+00 \\
3.00 E+00 \\
5.87 E+00 \\
7.95 E+00 \\
6.60 E+00 \\
7.05 E+00 \\
8.21 E+00\end{array}$ & $\begin{array}{l}E U 155 \\
2.62 \mathrm{E}+00 \\
2.10 \mathrm{E}+00 \\
3.75 \mathrm{E}+00 \\
4.76 \mathrm{E}+00 \\
4.32 \mathrm{E}+00 \\
4.34 \mathrm{E}+00 \\
4.41 \mathrm{E}+00 \\
2.89 \mathrm{E}+00 \\
4.82 \mathrm{E}+00 \\
4.54 \mathrm{E}+00 \\
5.95 \mathrm{E}+00 \\
6.10 \mathrm{E}+00 \\
5.92 \mathrm{E}+00 \\
5.38 \mathrm{E}+00 \\
3.97 \mathrm{E}+00 \\
6.75 \mathrm{E}+00 \\
5.22 \mathrm{E}+00 \\
6.05 \mathrm{E}+00 \\
7.82 \mathrm{E}+00 \\
6.09 \mathrm{E}+00 \\
8.06 \mathrm{E}+00 \\
4.07 \mathrm{E}+00 \\
7.78 \mathrm{E}+00 \\
5.08 \mathrm{E}+00 \\
2.65 \mathrm{E}+00 \\
1.18 \mathrm{E}+00 \\
4.99 \mathrm{E}+00 \\
4.45 \mathrm{E}+00 \\
5.22 \mathrm{E}-01 \\
2.08 \mathrm{E}+00 \\
2.30 \mathrm{E}+00 \\
4.81 \mathrm{E}+00 \\
5.50 \mathrm{E}=01 \\
4.53 \mathrm{E}+00 \\
3.80 \mathrm{E}+00 \\
2.09 \mathrm{E}+00 \\
2.37 \mathrm{E}+00 \\
4.29 \mathrm{E}+00 \\
2.58 \mathrm{E}+00 \\
2.44 \mathrm{E}+00 \\
3.84 \mathrm{E}+00 \\
3.39 \mathrm{E}+00 \\
4.75 \mathrm{E}+00 \\
6.79 \mathrm{E}+00 \\
7.83 \mathrm{E}+00 \\
6.78 \mathrm{E}+00 \\
6.14 \mathrm{E}+00 \\
2.75 \mathrm{E}+00 \\
7.59 \mathrm{E}+00 \\
8.95 \mathrm{E}+00 \\
8.15 \mathrm{E}+00 \\
9.21 \mathrm{E}+00 \\
9.81 \mathrm{E}+00 \\
1.19 \mathrm{E}+01 \\
1.17 \mathrm{E}+01 \\
1.39 \mathrm{E}+01 \\
1.49 \mathrm{E}+01 \\
1.56 \mathrm{E}+01 \\
7.29 \mathrm{E}+00 \\
8.13 \mathrm{E}+00 \\
1.55 \mathrm{E}+01 \\
1.86 \mathrm{E}+01 \\
1.32 \mathrm{E}+01 \\
1.40 \mathrm{E}+01 \\
1.66 \mathrm{E}+01\end{array}$ & 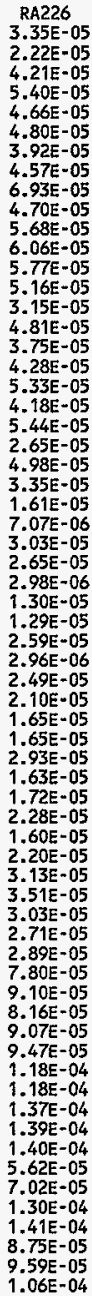 & 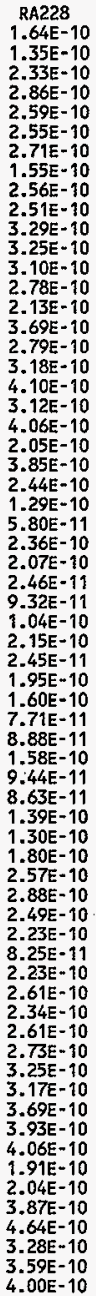 & 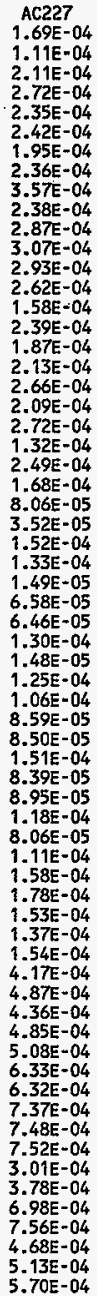 & $\begin{array}{l}\text { TH229 } \\
3.19 \mathrm{E}-08 \\
2.67 \mathrm{E}-08 \\
4.56 \mathrm{E}-08 \\
5.59 \mathrm{E}-08 \\
5.06 \mathrm{E}-08 \\
4.98 \mathrm{E}-08 \\
5.37 \mathrm{E}-08 \\
2.96 \mathrm{E}-08 \\
4.92 \mathrm{E}-08 \\
4.90 \mathrm{E}-08 \\
6.45 \mathrm{E}-08 \\
6.34 \mathrm{E}-08 \\
6.05 \mathrm{E}-08 \\
5.41 \mathrm{E}-08 \\
4.20 \mathrm{E}-08 \\
7.33 \mathrm{E}-08 \\
5.53 \mathrm{E}-08 \\
6.31 \mathrm{E}-08 \\
8.16 \mathrm{E}-08 \\
6.18 \mathrm{E}-08 \\
8.05 \mathrm{E}-08 \\
4.06 \mathrm{E}-08 \\
7.65 \mathrm{E}-08 \\
4.82 \mathrm{E}-08 \\
2.57 \mathrm{E}-08 \\
1.15 \mathrm{E}-08 \\
4.68 \mathrm{E}-08 \\
4.10 \mathrm{E}-08 \\
4.89 \mathrm{E}-09 \\
1.84 \mathrm{E}-08 \\
2.06 \mathrm{E}-08 \\
4.27 \mathrm{E}-08\end{array}$ \\
\hline
\end{tabular}


HNF-SD-WN-TI-794, ReV, 0

ACTIVITY IN UNSEPARATED FUEL (Curies decayed to 1/1/94)

\begin{tabular}{|c|c|c|c|c|c|c|c|c|c|}
\hline 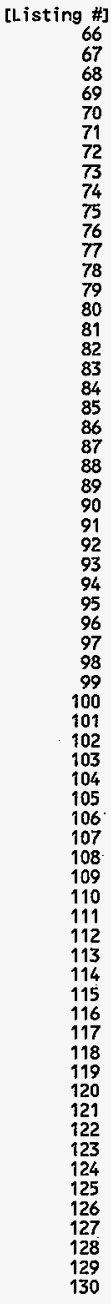 & $\begin{array}{l}\text { TH232 } \\
2.10 \mathrm{E}-10 \\
1.73 \mathrm{E}-10 \\
2.98 \mathrm{E}-10 \\
3.67 \mathrm{E}-10 \\
3.31 \mathrm{E}-10 \\
3.27 \mathrm{E}-10 \\
3.48 \mathrm{E}-10 \\
1.98 \mathrm{E}-10 \\
3.29 \mathrm{E}-10 \\
3.23 \mathrm{E}-10 \\
4.23 \mathrm{E}-10 \\
4.18 \mathrm{E}-10 \\
3.99 \mathrm{E}-10 \\
3.58 \mathrm{E}-10 \\
2.74 \mathrm{E}-10 \\
4.75 \mathrm{E}-10 \\
3.59 \mathrm{E}-10 \\
4.11 \mathrm{E}-10 \\
5.30 \mathrm{E}-10 \\
4.03 \mathrm{E}-10 \\
5.25 \mathrm{E}-10 \\
2.65 \mathrm{E}-10 \\
4.99 \mathrm{E}-10 \\
3.16 \mathrm{E}-10 \\
1.67 \mathrm{E}-10 \\
7.50 \mathrm{E}-11 \\
3.06 \mathrm{E}-10 \\
2.68 \mathrm{E}-10 \\
3.19 \mathrm{E}-11 \\
1.21 \mathrm{E}-10 \\
1.35 \mathrm{E}-10 \\
2.79 \mathrm{E}-10 \\
3.18 \mathrm{E}-11 \\
2.54 \mathrm{E}-10 \\
2.08 \mathrm{E}-10 \\
1.01 \mathrm{E}-10 \\
1.16 \mathrm{E}-10 \\
2.07 \mathrm{E}-10 \\
1.23 \mathrm{E}-10 \\
1.13 \mathrm{E}-10 \\
1.82 \mathrm{E}-10 \\
1.71 \mathrm{E}-10 \\
2.35 \mathrm{E}-10 \\
3.36 \mathrm{E}-10 \\
3.77 \mathrm{E}-10 \\
3.26 \mathrm{E}-10 \\
2.92 \mathrm{E}-10 \\
1.08 \mathrm{E}-10 \\
2.93 \mathrm{E}-10 \\
3.43 \mathrm{E}-10 \\
3.08 \mathrm{E}-10 \\
3.43 \mathrm{E}-10 \\
3.59 \mathrm{E}-10 \\
4.28 \mathrm{E}-10 \\
4.17 \mathrm{E}-10 \\
4.87 \mathrm{E}-10 \\
5.19 \mathrm{E}-10 \\
2.35 \mathrm{E}-10 \\
4.34 \mathrm{E}-10 \mathrm{E}-10 \\
5.30 \mathrm{E}-10 \\
\end{array}$ & $\begin{array}{l}\text { PA231 } \\
3.65 E-04 \\
2.40 E-04 \\
4.58 E-04 \\
5.91 E-04 \\
5.10 E-04 \\
5.27 E-04 \\
4.24 E-04 \\
5.15 E-04 \\
7.80 E-04 \\
5.20 E-04 \\
6.27 E-04 \\
6.74 E-04 \\
6.44 E-04 \\
5.77 E-04 \\
3.48 E-04 \\
5.27 E-04 \\
4.13 E-04 \\
4.72 E-04 \\
5.87 E-04 \\
4.62 E-04 \\
6.03 E-04 \\
2.93 E-04 \\
5.52 E-04 \\
3.74 E-04 \\
1.79 E-04 \\
7.84 E-05 \\
3.39 E-04 \\
2.97 E-04 \\
3.33 E-05 \\
1.47 E-04 \\
1.45 E-04 \\
2.91 E-04 \\
3.32 E-05 \\
2.82 E-04 \\
2.39 E-04 \\
1.94 E-04 \\
1.92 E-04 \\
3.43 E-04 \\
1.91 E-04 \\
2.04 E-04 \\
2.688 E-04 \\
1.83 E-04 \\
2.53 E-04 \\
3.61 E-04 \\
4.06 E-04 \\
3.50 E-04 \\
3.14 E-04 \\
3.54 E-04 \\
9.60 E-04 \\
1.12 E-03 \\
1.01 E-03 \\
1.12 E-03 \\
1.18 E-03 \\
1.47 E-03 \\
1.47 E-03 \\
1.71 E-03 \\
1.74 E-03 \\
1.75 E-03 \\
7.00 E-04 \\
8.84 E-04 \\
1.63 E-03 \\
1.77 E-03 \\
1.10 E-03 \\
1.20 E-03 \\
1.34 E-03\end{array}$ & $\begin{array}{l}\text { U232 } \\
2.13 \mathrm{E}-04 \\
1.93 \mathrm{E}-04 \\
3.20 \mathrm{E}-04 \\
3.88 \mathrm{E}-04 \\
3.58 \mathrm{E}-04 \\
3.48 \mathrm{E}-04 \\
4.16 \mathrm{E}-04 \\
1.82 \mathrm{E}-04 \\
3.11 \mathrm{E}-04 \\
3.47 \mathrm{E}-04 \\
4.72 \mathrm{E}-04 \\
4.53 \mathrm{E}-04 \\
4.34 \mathrm{E}-04 \\
3.90 \mathrm{E}-04 \\
3.31 \mathrm{E}-04 \\
6.12 \mathrm{E}-04 \\
4.56 \mathrm{E}-04 \\
5.23 \mathrm{E}-04 \\
6.87 \mathrm{E}-04 \\
5.15 \mathrm{E}-04 \\
6.74 \mathrm{E}-04 \\
3.46 \mathrm{E}-04 \\
6.54 \mathrm{E}-04 \\
4.03 \mathrm{E}-04 \\
2.24 \mathrm{E}-04 \\
1.02 \mathrm{E}-04 \\
4.05 \mathrm{E}-04 \\
3.56 \mathrm{E}-04 \\
4.36 \mathrm{E}-05 \\
1.54 \mathrm{E}-04 \\
1.83 \mathrm{E}-04 \\
3.86 \mathrm{E}-04 \\
4.40 \mathrm{E}-05 \\
3.43 \mathrm{E}-04 \\
2.77 \mathrm{E}-04 \\
1.09 \mathrm{E}-04 \\
1.33 \mathrm{E}-04 \\
2.38 \mathrm{E}-04 \\
1.46 \mathrm{E}-04 \\
1.27 \mathrm{E}-04 \\
2.21 \mathrm{E}-04 \\
2.39 \mathrm{E}-04 \\
3.31 \mathrm{E}-04 \\
4.72 \mathrm{E}-04 \\
5.34 \mathrm{E}-04 \\
4.61 \mathrm{E}-04 \\
4.15 \mathrm{E}-04 \\
1.05 \mathrm{E}-04 \\
2.85 \mathrm{E}-04 \\
3.34 \mathrm{E}-04 \\
3.01 \mathrm{E}-04 \\
3.36 \mathrm{E}-04 \\
3.53 \mathrm{E}-04 \\
4.19 \mathrm{E}-04 \\
4.06 \mathrm{E}-04 \\
4.75 \mathrm{E}-04 \\
5.13 \mathrm{E}-04 \\
2.34 \mathrm{E}-04 \\
2.71 \mathrm{E}-04 \\
5.17 \mathrm{E}-04 \\
6.37 \mathrm{E}-04 \\
4.68 \mathrm{E}-04 \\
5.07 \mathrm{E}-04 \\
\end{array}$ & 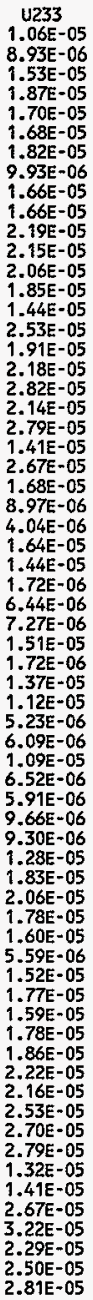 & $\begin{array}{l}\text { U234 } \\
8.90 \mathrm{E}+00 \\
5.93 \mathrm{E}+00 \\
1.13 \mathrm{E}+01 \\
1.46 \mathrm{E}+01 \\
1.26 \mathrm{E}+01 \\
1.30 \mathrm{E}+01 \\
1.07 \mathrm{E}+01 \\
1.25 \mathrm{E}+01 \\
1.91 \mathrm{E}+01 \\
1.30 \mathrm{E}+01 \\
1.57 \mathrm{E}+01 \\
1.69 \mathrm{E}+01 \\
1.62 \mathrm{E}+01 \\
1.45 \mathrm{E}+01 \\
8.89 \mathrm{E}+00 \\
1.36 \mathrm{E}+01 \\
1.07 \mathrm{E}+01 \\
1.22 \mathrm{E}+01 \\
1.52 \mathrm{E}+01 \\
1.20 \mathrm{E}+01 \\
1.57 \mathrm{E}+01 \\
7.66 \mathrm{E}+00 \\
1.45 \mathrm{E}+01 \\
9.77 \mathrm{E}+00 \\
4.73 \mathrm{E}+00 \\
2.07 \mathrm{E}+00 \\
8.93 \mathrm{E}+00 \\
7.84 \mathrm{E}+00 \\
8.83 \mathrm{E}-01 \\
3.87 \mathrm{E}+00 \\
3.85 \mathrm{E}+00 \\
7.79 \mathrm{E}+00 \\
8.89 \mathrm{E}-01 \\
7.52 \mathrm{E}+00 \\
6.38 \mathrm{E}+00 \\
5.06 \mathrm{E}+00 \\
5.05 \mathrm{E}+00 \\
9.03 \mathrm{E}+00 \\
5.05 \mathrm{E}+00 \\
5.36 \mathrm{E}+00 \\
7.13 \mathrm{E}+00 \\
4.99 \mathrm{E}+00 \\
6.90 \mathrm{E}+00 \\
9.85 \mathrm{E}+00 \\
1.11 \mathrm{E}+01 \\
9.59 \mathrm{E}+00 \\
8.62 \mathrm{E}+00 \\
9.27 \mathrm{E}+00 \\
2.52 \mathrm{E}+01 \\
2.95 \mathrm{E}+01 \\
2.65 \mathrm{E}+01 \\
2.96 \mathrm{E}+01 \\
3.11 \mathrm{E}+01 \\
3.89 \mathrm{E}+01 \\
3.89 \mathrm{E}+01 \\
4.55 \mathrm{E}+01 \\
4.64 \mathrm{E}+01 \\
4.68 \mathrm{E}+01 \\
1.88 \mathrm{E}+01 \\
2.37 \mathrm{E}+01 \\
4.39 \mathrm{E}+01 \\
4.78 \mathrm{E}+01 \\
2.99 \mathrm{E}+01 \\
3.24 \mathrm{E}+01 \\
3.66 \mathrm{E}+01\end{array}$ & $\begin{array}{l}\text { U235 } \\
3.92 \mathrm{E}-01 \\
2.58 \mathrm{E}-01 \\
4.94 \mathrm{E}-01 \\
6.39 \mathrm{E}-01 \\
5.52 \mathrm{E}-01 \\
5.72 \mathrm{E}-01 \\
4.60 \mathrm{E}-01 \\
5.61 \mathrm{E}-01 \\
8.52 \mathrm{E}-01 \\
5.69 \mathrm{E}-01 \\
6.87 \mathrm{E}-01 \\
7.41 \mathrm{E}-01 \\
7.09 \mathrm{E}-01 \\
6.37 \mathrm{E}-01 \\
3.84 \mathrm{E}-01 \\
5.82 \mathrm{E}-01 \\
4.57 \mathrm{E}-01 \\
5.24 \mathrm{E}-01 \\
6.53 \mathrm{E}-01 \\
5.15 \mathrm{E}-01 \\
6.73 \mathrm{E}-01 \\
3.28 \mathrm{E}-01 \\
6.19 \mathrm{E}-01 \\
4.20 \mathrm{E}-01 \\
2.02 \mathrm{E}-01 \\
8.81 \mathrm{E}-02 \\
3.82 \mathrm{E}-01 \\
3.36 \mathrm{E}-01 \\
3.76 \mathrm{E}-02 \\
1.67 \mathrm{E}-01 \\
1.65 \mathrm{E}-01 \\
3.32 \mathrm{E}-01 \\
3.79 \mathrm{E}-02 \\
3.22 \mathrm{E}-01 \\
2.74 \mathrm{E}-01 \\
2.24 \mathrm{E}-01 \\
2.22 \mathrm{E}-01 \\
3.97 \mathrm{E}-01 \\
2.21 \mathrm{E}-01 \\
2.36 \mathrm{E}-01 \\
3.11 \mathrm{E}-01 \\
2.13 \mathrm{E}-01 \\
2.95 \mathrm{E}-01 \\
4.21 \mathrm{E}-01 \\
4.75 \mathrm{E}-01 \\
4.10 \mathrm{E}-01 \\
3.68 \mathrm{E}-01 \\
4.18 \mathrm{E}-01 \\
1.14 \mathrm{E}+00 \\
1.33 \mathrm{E}+00 \\
1.20 \mathrm{E}+00 \\
1.34 \mathrm{E}+00 \\
1.40 \mathrm{E}+00 \\
1.76 \mathrm{E}+00 \\
1.76 \mathrm{E}+00 \\
2.05 \mathrm{E}+00 \\
2.09 \mathrm{E}+00 \\
8.11 \mathrm{E}+00 \\
1.44 \mathrm{E}-01 \\
1.97 \mathrm{E}+00 \\
2.15 \mathrm{E}+00 \\
1.34 \mathrm{E}+00 \\
1.35 \mathrm{~F}+00\end{array}$ & $\begin{array}{c}\text { U236 } \\
9.66 \mathrm{E}-02 \\
8.00 \mathrm{E}-02 \\
1.38 \mathrm{E}-01 \\
1.70 \mathrm{E}-01 \\
1.54 \mathrm{E}-01 \\
1.52 \mathrm{E}-01 \\
1.62 \mathrm{E}-01 \\
9.27 \mathrm{E}-02 \\
1.54 \mathrm{E}-01 \\
1.52 \mathrm{E}-01 \\
1.99 \mathrm{E}-01 \\
1.97 \mathrm{E}-01 \\
1.89 \mathrm{E}-01 \\
1.70 \mathrm{E}-01 \\
1.30 \mathrm{E}-01 \\
2.26 \mathrm{E}-01 \\
1.71 \mathrm{E}-01 \\
1.96 \mathrm{E}-01 \\
2.53 \mathrm{E}-01 \\
1.93 \mathrm{E}-01 \\
2.52 \mathrm{E}-01 \\
1.27 \mathrm{E}-01 \\
2.40 \mathrm{E}-01 \\
1.53 \mathrm{E}-01 \\
8.09 \mathrm{E}-02 \\
3.63 \mathrm{E}-02 \\
1.48 \mathrm{E}-01 \\
1.30 \mathrm{E}-01 \\
1.55 \mathrm{E}-02 \\
5.89 \mathrm{E}-02 \\
6.59 \mathrm{E}-02 \\
1.37 \mathrm{E}-01 \\
1.56 \mathrm{E}-02 \\
1.25 \mathrm{E}-01 \\
1.03 \mathrm{E}-01 \\
4.97 \mathrm{E}-02 \\
5.74 \mathrm{E}-02 \\
1.03 \mathrm{E}-01 \\
6.14 \mathrm{E}-02 \\
5.62 \mathrm{E}-02 \\
9.08 \mathrm{E}-02 \\
8.54 \mathrm{E}-02 \\
1.18 \mathrm{E}-01 \\
1.68 \mathrm{E}-01 \\
1.90 \mathrm{E}-01 \\
1.64 \mathrm{E}-01 \\
1.48 \mathrm{E}-01 \\
5.48 \mathrm{E}-02 \\
1.49 \mathrm{E}-01 \\
1.74 \mathrm{E}-01 \\
1.57 \mathrm{E}-01 \\
1.75 \mathrm{E}-01 \\
1.84 \mathrm{E}-01 \\
2.20 \mathrm{E}-01 \\
2.14 \mathrm{E}-01 \\
2.51 \mathrm{E}-01 \\
2.67 \mathrm{E}-01 \\
2.77 \mathrm{E}-01 \\
1.31 \mathrm{E}-01 \\
1.40 \mathrm{E}-01 \\
2.66 \mathrm{E}-01 \\
3.19 \mathrm{E}-01\end{array}$ & $\begin{array}{l}U 238 \\
9.05 E+00 \\
6.05 E+00 \\
1.15 E+01 \\
1.48 E+01 \\
1.29 E+01 \\
1.33 E+01 \\
1.09 E+01 \\
1.27 E+01 \\
1.94 E+01 \\
1.32 E+01 \\
1.60 E+01 \\
1.72 E+01 \\
1.65 E+01 \\
1.48 E+01 \\
9.08 E+00 \\
1.39 E+01 \\
1.09 E+01 \\
1.25 E+01 \\
1.56 E+01 \\
1.23 E+01 \\
1.60 E+01 \\
7.84 E+00 \\
1.48 E+01 \\
9.98 E+00 \\
4.84 E+00 \\
2.12 E+00 \\
9.14 E+00 \\
8.03 E+00 \\
9.05 E-01 \\
3.96 E+00 \\
3.95 E+00 \\
7.98 E+00 \\
9.10 E-01 \\
7.69 E+00 \\
6.53 E+00 \\
5.15 E+00 \\
5.14 E+00 \\
9.19 E+00 \\
5.14 E+00 \\
5.45 E+00 \\
7.26 E+00 \\
5.11 E+00 \\
7.06 E+00 \\
1.01 E+01 \\
1.14 E+01 \\
9.82 E+00 \\
8.83 E+00 \\
9.38 E+00 \\
2.55 E+01 \\
2.98 E+01 \\
2.68 E+01 \\
3.00 E+01 \\
3.15 E+01 \\
3.93 E+01 \\
3.93 E+01 \\
4.60 E+01 \\
4.69 E+01 \\
4.74 E+01 \\
1.91 E+01 \\
2.40 E+01 \\
4.45 E+01 \\
4.84 E+01 \\
3.03 E+01 \\
3.29 E+01 \\
3.71 E+01\end{array}$ & $\begin{array}{l}\text { NP237 } \\
3.11 E-02 \\
2.66 \mathrm{E}-02 \\
4.51 \mathrm{E}-02 \\
5.53 \mathrm{E}-02 \\
5.04 \mathrm{E}-02 \\
4.95 \mathrm{E}-02 \\
5.50 \mathrm{E}-02 \\
2.87 \mathrm{E}-02 \\
4.80 \mathrm{E}-02 \\
4.93 \mathrm{E}-02 \\
6.54 \mathrm{E}-02 \\
6.41 \mathrm{E}-02 \\
6.14 \mathrm{E}-02 \\
5.51 \mathrm{E}-02 \\
4.38 \mathrm{E}-02 \\
7.78 \mathrm{E}-02 \\
5.86 \mathrm{E}-02 \\
6.71 \mathrm{E}-02 \\
8.72 \mathrm{E}-02 \\
6.60 \mathrm{E}-02 \\
8.62 \mathrm{E}-02 \\
4.38 \mathrm{E}-02 \\
8.27 \mathrm{E}-02 \\
5.19 \mathrm{E}-02 \\
2.80 \mathrm{E}-02 \\
1.26 \mathrm{E}-02 \\
5.11 \mathrm{E}-02 \\
4.49 \mathrm{E}-02 \\
5.39 \mathrm{E}-03 \\
1.99 \mathrm{E}-02 \\
2.28 \mathrm{E}-02 \\
4.76 \mathrm{E}-02 \\
5.43 \mathrm{E}-03 \\
4.30 \mathrm{E}-02 \\
3.51 \mathrm{E}-02 \\
1.58 \mathrm{E}-02 \\
1.86 \mathrm{E}-02 \\
3.32 \mathrm{E}-02 \\
2.00 \mathrm{E}-02 \\
1.80 \mathrm{E}-02 \\
2.98 \mathrm{E}-02 \\
2.95 \mathrm{E}-02 \\
4.08 \mathrm{E}-02 \\
5.82 \mathrm{E}-02 \\
6.57 \mathrm{E}-02 \\
5.67 \mathrm{E}-02\end{array}$ \\
\hline
\end{tabular}


HNF-SD-WM-T1-794, ReV. 0

ACTIVITY IN UNSEPARATED FUEL (Curies decayed to $1 / 1 / 94$ )

\begin{tabular}{|c|c|c|c|c|c|c|c|c|c|}
\hline $\begin{array}{r}\text { isting } \# \text { ] } \\
66 \\
67 \\
68 \\
69 \\
70 \\
71 \\
72 \\
73 \\
74 \\
75 \\
76 \\
77 \\
78 \\
79 \\
80 \\
81 \\
82 \\
83 \\
84 \\
85 \\
86 \\
87 \\
88 \\
89 \\
90 \\
91 \\
92 \\
93 \\
94 \\
95 \\
96 \\
97 \\
98 \\
99 \\
100 \\
101 \\
102 \\
103 \\
104 \\
105 \\
106 \\
107 \\
108 \\
109 \\
110 \\
111 \\
112 \\
113 \\
114 \\
115 \\
116 \\
117 \\
118 \\
119 \\
120 \\
121 \\
122 \\
123 \\
124 \\
125 \\
126 \\
127 \\
128 \\
129 \\
130\end{array}$ & $\begin{array}{l}P U 238 \\
4.52 E+00 \\
5.09 E+00 \\
7.65 E+00 \\
8.88 E+00 \\
8.57 E+00 \\
7.96 E+00 \\
1.22 E+01 \\
2.39 E+00 \\
4.61 E+00 \\
7.94 E+00 \\
1.17 E+01 \\
1.04 E+01 \\
9.93 E+00 \\
8.92 E+00 \\
9.38 E+00 \\
1.93 E+01 \\
1.40 E+01 \\
1.61 E+01 \\
2.17 E+01 \\
1.58 E+01 \\
2.07 E+01 \\
1.09 E+01 \\
2.07 E+01 \\
1.21 E+01 \\
7.23 E+00 \\
3.36 E+00 \\
1.28 E+01 \\
1.12 E+01 \\
1.44 E+00 \\
4.51 E+00 \\
5.91 E+00 \\
1.27 E+01 \\
1.45 E+00 \\
1.08 E+01 \\
8.50 E+00 \\
2.06 E+00 \\
2.94 E+00 \\
5.25 E+00 \\
3.47 E+00 \\
2.57 E+00 \\
5.48 E+00 \\
7.71 E+00 \\
1.07 E+01 \\
1.52 E+01 \\
1.72 E+01 \\
1.49 E+01 \\
1.34 E+01 \\
9.57 E-01 \\
2.60 E+00 \\
3.05 E+00 \\
2.74 E+00 \\
3.07 E+00 \\
3.22 E+00 \\
3.52 E+00 \\
3.24 E+00 \\
3.80 E+00 \\
4.47 E+00 \\
4.87 E+00 \\
3.12 E+00 \\
2.47 E+00 \\
4.92 E+00 \\
7.14 E+00 \\
6.34 E+00 \\
6.88 E+00 \\
7.79 E+00\end{array}$ & 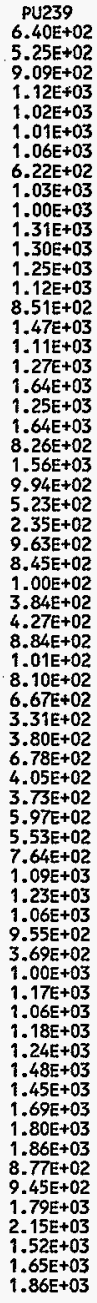 & $\begin{array}{l}\text { PU240 } \\
5.80 \mathrm{E}+01 \\
5.89 \mathrm{E}+01 \\
9.24 \mathrm{E}+01 \\
1.09 \mathrm{E}+02 \\
1.03 \mathrm{E}+02 \\
9.79 \mathrm{E}+01 \\
1.35 \mathrm{E}+02 \\
3.97 \mathrm{E}+01 \\
7.11 \mathrm{E}+01 \\
9.74 \mathrm{E}+01 \\
1.38 \mathrm{E}+02 \\
1.27 \mathrm{E}+02 \\
1.21 \mathrm{E}+02 \\
1.09 \mathrm{E}+02 \\
1.04 \mathrm{E}+02 \\
2.05 \mathrm{E}+02 \\
1.50 \mathrm{E}+02 \\
1.72 \mathrm{E}+02 \\
2.30 \mathrm{E}+02 \\
1.69 \mathrm{E}+02 \\
2.21 \mathrm{E}+02 \\
1.16 \mathrm{E}+02 \\
2.18 \mathrm{E}+02 \\
1.30 \mathrm{E}+02 \\
7.55 \mathrm{E}+01 \\
3.48 \mathrm{E}+01 \\
1.35 \mathrm{E}+02 \\
1.18 \mathrm{E}+02 \\
1.49 \mathrm{E}+01 \\
4.89 \mathrm{E}+01 \\
6.16 \mathrm{E}+01 \\
1.31 \mathrm{E}+02 \\
1.50 \mathrm{E}+01 \\
1.13 \mathrm{E}+02 \\
9.00 \mathrm{E}+01 \\
2.72 \mathrm{E}+01 \\
3.59 \mathrm{E}+01 \\
6.42 \mathrm{E}+01 \\
4.10 \mathrm{E}+01 \\
3.27 \mathrm{E}+01 \\
6.33 \mathrm{E}+01 \\
7.97 \mathrm{E}+01 \\
1.10 \mathrm{E}+02 \\
1.57 \mathrm{E}+02 \\
1.78 \mathrm{E}+02 \\
1.53 \mathrm{E}+02 \\
1.38 \mathrm{E}+02 \\
1.95 \mathrm{E}+01 \\
5.30 \mathrm{E}+01 \\
6.20 \mathrm{E}+01 \\
5.58 \mathrm{E}+01 \\
6.24 \mathrm{E}+01 \\
6.55 \mathrm{E}+01 \\
7.56 \mathrm{E}+01 \\
7.23 \mathrm{E}+01 \\
8.45 \mathrm{E}+01 \\
9.34 \mathrm{E}+01 \\
9.86 \mathrm{E}+01 \\
5.30 \mathrm{E}+01 \\
5.00 \mathrm{E}+01 \\
9.66 \mathrm{E}+01 \\
1.26 \mathrm{E}+02 \\
9.92 \mathrm{E}+01 \\
1.08 \mathrm{E}+02 \\
1.22 \mathrm{E}+02\end{array}$ & 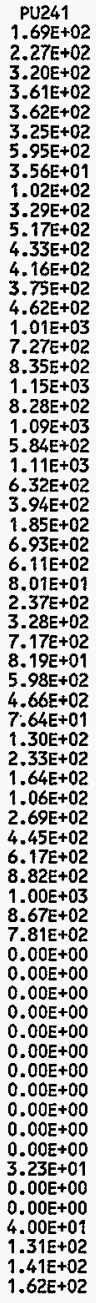 & $\begin{array}{l}\text { PU242 } \\
5.82 E-04 \\
1.10 E=03 \\
1.38 E-03 \\
1.44 E-03 \\
1.54 E-03 \\
1.29 E-03 \\
3.10 E-03 \\
0.00 E+00 \\
0.00 E+00 \\
1.28 E-03 \\
2.25 E-03 \\
1.67 E-03 \\
1.60 E-03 \\
1.44 E-03 \\
2.26 E-03 \\
5.31 E-03 \\
3.73 E-03 \\
4.28 E-03 \\
5.95 E-03 \\
4.21 E-03 \\
5.50 E-03 \\
2.99 E-03 \\
5.65 E-03 \\
3.11 E-03 \\
2.02 E-03 \\
9.61 E-04 \\
3.49 E-03 \\
3.06 E-03 \\
4.11 E-04 \\
1.13 E-03 \\
1.65 E-03 \\
3.62 E-03 \\
4.13 E-04 \\
2.94 E-03 \\
2.24 E-03 \\
1.52 E-04 \\
4.30 E-04 \\
7.68 E-04 \\
6.05 E-04 \\
2.80 E-04 \\
1.05 E-03 \\
2.14 E-03 \\
2.95 E-03 \\
4.21 E-03 \\
4.75 E-03 \\
4.111 E-03 \\
3.69 E-03 \\
0.00 E+00 \\
0.00 E+00 \\
0.00 E+00 \\
0.00 E+00 \\
0.00 E+00 \\
0.00 E+00 \\
0.00 E+00 \\
0.00 E+00 \\
0.00 E+00 \\
0.00 E+00 \\
0.00 E+00 \\
0.00 E+00 \\
0.00 E+00 \\
0.00 E+00 \\
0.00 E+00 \\
0.00 E+00 \\
0.00 E+00 \\
0.00 E+00\end{array}$ & 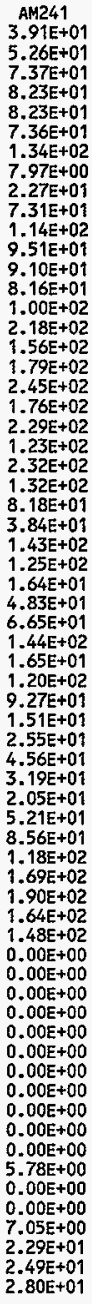 & $\begin{array}{c}\text { AM243 } \\
1.36 \mathrm{E}-04 \\
3.61 \mathrm{E}=04 \\
4.14 \mathrm{E}-04 \\
4.06 \mathrm{E}-04 \\
4.63 \mathrm{E}-04 \\
3.63 \mathrm{E}-04 \\
1.08 \mathrm{E}-03 \\
0.00 \mathrm{E}+00 \\
0.00 \mathrm{E}+00 \\
3.61 \mathrm{E}-04 \\
7.02 \mathrm{E}-04 \\
4.70 \mathrm{E}-04 \\
4.50 \mathrm{E}-04 \\
4.04 \mathrm{E}-04 \\
7.73 \mathrm{E}-04 \\
1.90 \mathrm{E}-03 \\
1.32 \mathrm{E}-03 \\
1.51 \mathrm{E}-03 \\
2.13 \mathrm{E}-03 \\
1.49 \mathrm{E}-03 \\
1.94 \mathrm{E}-03 \\
1.07 \mathrm{E}-03 \\
2.02 \mathrm{E}-03 \\
1.09 \mathrm{E}-03 \\
7.28 \mathrm{E}-04 \\
3.48 \mathrm{E}-04 \\
1.25 \mathrm{E}-03 \\
1.09 \mathrm{E}-03 \\
1.49 \mathrm{E}-04 \\
3.91 \mathrm{E}-04 \\
5.93 \mathrm{E}-04 \\
1.31 \mathrm{E}-03 \\
1.50 \mathrm{E}-04 \\
1.05 \mathrm{E}-03 \\
7.92 \mathrm{E}-04 \\
1.11 \mathrm{E}-05 \\
1.15 \mathrm{E}-04 \\
2.05 \mathrm{E}-04 \\
1.80 \mathrm{E}-04 \\
5.58 \mathrm{E}-05 \\
3.29 \mathrm{E}-04 \\
7.68 \mathrm{E}-04 \\
1.06 \mathrm{E}-03 \\
1.52 \mathrm{E}-03 \\
1.71 \mathrm{E}-03 \\
1.48 \mathrm{E}-03 \\
1.33 \mathrm{E}-03 \\
0.00 \mathrm{E}+00 \\
0.00 \mathrm{E}+00 \\
0.00 \mathrm{E}+00 \\
0.00 \mathrm{E}+00 \\
0.00 \mathrm{E}+00 \\
0.00 \mathrm{E}+00 \\
0.00 \mathrm{E}+00 \\
0.00 \mathrm{E}+00 \\
0.00 \mathrm{E}+00 \\
0.00 \mathrm{E}+00 \\
0.00 \mathrm{E}+00 \\
0.00 \mathrm{E}+00 \\
0.00 \mathrm{E}+00 \\
0.00 \mathrm{E}+00 \\
0.00 \mathrm{~s}+00\end{array}$ & $\begin{array}{l}\text { CM242 } \\
4.11 E-04 \\
1.08 E-03 \\
1.24 E-03 \\
1.22 E-03 \\
1.39 E-03 \\
1.09 E-03 \\
3.21 E-03 \\
0.00 E+00 \\
0.00 E+00 \\
1.09 E-03 \\
2.10 E-03 \\
1.42 E-03 \\
1.36 E-03 \\
1.22 E-03 \\
2.31 E-03 \\
5.66 E-03 \\
3.95 E-03 \\
4.52 E-03 \\
6.36 E-03 \\
4.45 E-03 \\
5.82 E-03 \\
3.20 E-03 \\
6.04 E-03 \\
3.27 E-03 \\
2.18 E-03 \\
1.04 E-03 \\
3.73 E-03 \\
3.28 E-03 \\
4.45 E-04 \\
1.17 E-03 \\
1.78 E-03 \\
3.93 E-03 \\
4.49 E-04 \\
3.15 E-03 \\
2.38 E-03 \\
3.78 E-05 \\
3.49 E-04 \\
6.23 E-04 \\
5.47 E-04 \\
1.72 E-04 \\
9.96 E-04 \\
2.32 E-03 \\
3.20 E-03 \\
4.57 E-03 \\
5.16 E-03 \\
4.46 E-03 \\
4.01 E-03 \\
0.00 E+00 \\
0.00 E+00 \\
0.00 E+00 \\
0.00 E+00 \\
0.00 E+00 \\
0.00 E+00 \\
0.00 E+00 \\
0.00 E+00 \\
0.00 E+00 \\
0.00 E+00 \\
0.00 E+00 \\
0.00 E+00 \\
0.00 E+00 \\
0.00 E+00 \\
0.00 E+00 \\
0.00 E+00 \\
0.00 E+00 \\
0.00 E+00\end{array}$ & $\begin{array}{c}\text { CM243 } \\
5.85 \mathrm{E}-06 \\
2.09 \mathrm{E}-05 \\
2.27 \mathrm{E}-05 \\
2.12 \mathrm{E}-05 \\
2.55 \mathrm{E}-05 \\
1.90 \mathrm{E}-05 \\
6.55 \mathrm{E}-05 \\
0.00 \mathrm{E}+00 \\
0.00 \mathrm{E}+00 \\
1.91 \mathrm{E}-05 \\
4.02 \mathrm{E}-05 \\
2.50 \mathrm{E}-05 \\
2.40 \mathrm{E}-05 \\
2.16 \mathrm{E}-05 \\
4.75 \mathrm{E}-05 \\
1.20 \mathrm{E}-04 \\
8.30 \mathrm{E}-05 \\
9.53 \mathrm{E}-05 \\
1.35 \mathrm{E}-04 \\
9.41 \mathrm{E}-05 \\
1.23 \mathrm{E}-04 \\
6.82 \mathrm{E}-05 \\
1.29 \mathrm{E}-04 \\
6.91 \mathrm{E}-05 \\
4.69 \mathrm{E}-05 \\
2.25 \mathrm{E}-05 \\
8.02 \mathrm{E}-05 \\
7.06 \mathrm{E}-05 \\
9.67 \mathrm{E}-06 \\
2.49 \mathrm{E}-05 \\
3.86 \mathrm{E}-05 \\
8.59 \mathrm{E}-05 \\
9.81 \mathrm{E}-06 \\
6.84 \mathrm{E}-05 \\
5.15 \mathrm{E}-05 \\
0.00 \mathrm{E}+00 \\
6.09 \mathrm{E}-06 \\
1.09 \mathrm{E}-05 \\
1.06 \mathrm{E}-05\end{array}$ \\
\hline
\end{tabular}


HNF-SD-WH-TI-794, ReV. 0

ACTIVITY IN UNSEPARATED FUEL (Curies decayed to $1 / 1 / 94$ )

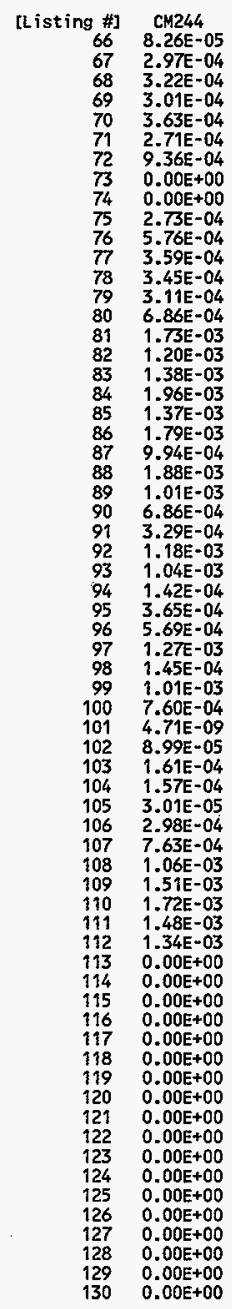


HNF-SD-WM-TI-794, ReV. 0

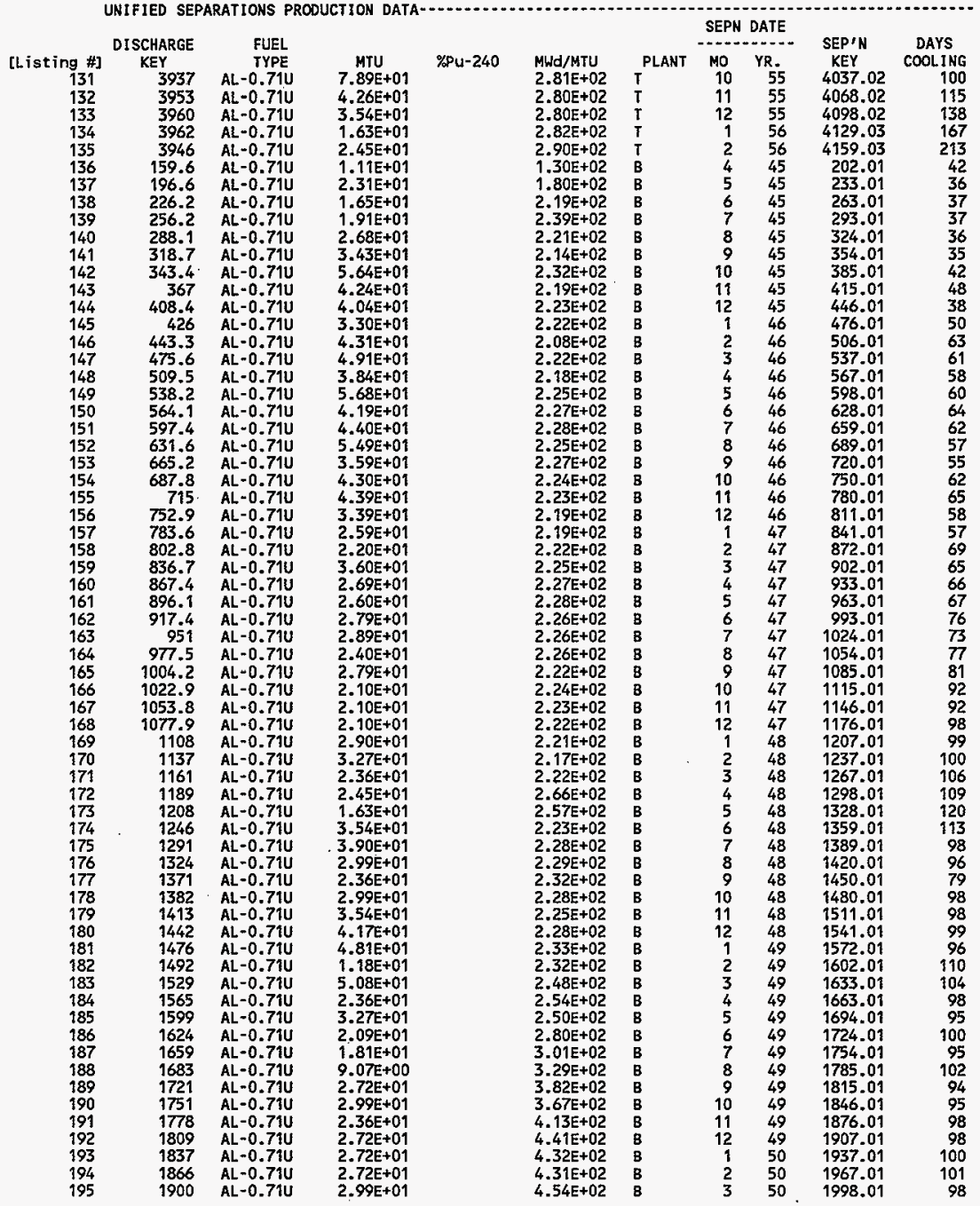


HNF-SD-WM-TI-794, ReV. 0

UNIFIED SEPARATIONS PRODUCTION DATA TOTAL WASTE LOSS FRACTION

[Listing \#] Fuel Data Reference

131 SPLANT.DAT 19348 9/30/93 1:19P

132 SPLANT.DAT 19348 9/30/93 1:19P

133 SPLANT.DAT 19348 9/30/93 1:19P

134 SPLANT.DAT 19348 9/30/93 1:19P

135 SPLANT.DAT 19348 9/30/93 1:19P

136 SPLANT.DAT $193489 / 30 / 93$ 1:19P

137 SPLANT.DAT 19348 9/30/93 1:19P

138 SPLANT.DAT 19348 9/30/93 1:19P

139 SPLANT.DAT 19348 9/30/93 1:19P

140 SPLANT,DAT 19348 \%/30/93 1:19P

141 SPLANT.DAT 19348 9/30/93 1:19P

142 SPLANT.DAT 19348 9/30/93 1:19P

143 SPLANT.DAT 19348 9/30/93 $1: 19 \mathrm{P}$

144 SPLANT.DAT 19348 9/30/93 $1: 19 \mathrm{P}$

145 SPLANT.DAT 19348 9/30/93 1:19P

146 SPLANT.DAT 19348 9/30/93 1:19P

147 SPLANT.DAT 19348 9/30/93 1:19P

148 SPLANT.DAT 19348 9/30/93 1:19P

149 SPLANT.DAT 19348 9/30/93 1:19P

150 SPLANT.DAT 19348 9/30/93 1:19P

151 SPLANT.DAT 19348 9/30/93 1:19P

152 SPLANT.DAT 19348 9/30/93 1:19P

153 SPLANT.DAT 19348 9/30/93 1:19P

154 SPLANT.DAT 19348 9/30/93 1:19P

155 SPLANT.DAT 19348 9/30/93 1:19P

156 SPLANT.DAT 19348 9/30/93 1:19P

157 SPLANT.DAT $19348 \% / 30 / 93$ 1:19P

158 SPLANT.DAT 19348 \%/30/93 1:19P

159 SPLANT.DAT 19348 9/30/93 1:19P

160 SPLANT.DAT 19348 9/30/93 1:19P

161 SPLANT.DAT 19348 9/30/93 1:19P

162 SPLANT.DAT 19348 9/30/93 $1: 19 \mathrm{P}$

163 SPLANT.DAT 19348 9/30/93 1:19P

164 SPLANT.DAT $193489 / 30 / 93$ 1:19p

165 SPLANT.DAT 19348 9/30/93 $1: 19 \mathrm{P}$

166 SPLANT.DAT 19348 9/30/93 $1: 19 \mathrm{P}$

167 SPLANT.DAT 19348 9/30/93 $1: 19 \mathrm{P}$

168 SPLANT.DAT 19348 \%/30/93 $1=19 \mathrm{P}$

169 SPLANT.DAT $193489 / 30 / 93$ 1: $19 \mathrm{P}$

170 SPLANT,DAT 19348 9/30/93 1:19P

171 SPLANT.OAT 19348 9/30/93 1:19P

172 SPLANT.OAT 19348 9/30/93 1:19P

173 SPLANT.DAT 19348 9/30/93 1:19P

174 SPLANT.DAT 19348 9/30/93 1:19P

175 SPLANT.DAT 19348 9/30/93 1:19P

176 SPLANT.DAT 19348 9/30/93 1:19P

177 SPLANT.DAT 19348 9/30/93 1:19P

178 SPLANT.DAT 19348 9/30/93 $1: 19 \mathrm{P}$

179 SPLANT.DAT 19348 9/30/93 1:19P

180 SPLANT.DAT $193489 / 30 / 93$ 1:19P

181 SPLANT.DAT 19348 9/30/93 1:19P

182 SPLANT.DAT 19348 9/30/93 1:19P

183 SPLANT.DAT 19348 9/30/93 1:199

184 SPLANT_DAT 19348 9/30/93 $1: 19 \mathrm{P}$

185 SPLANT.DAT 19348 9/30/93 1:19P

186 SPLANT.DAT $193489 / 30 / 93$ 1:19P

187 SPLANT.DAT 19348 9/30/93 $1: 19 \mathrm{P}$

188 SPLANT.DAT 19348 9/30/93 1:19P

189 SPLANT.DAT 19348 9/30/93 1:19P

190 SPLANT.DAT 19348 9/30/93 $1: 19 \mathrm{P}$

191 SPLANT.DAT 19348 \%/30/93 $i: 19 \mathrm{p}$

192 SPLANT.DAT 19348 9/30/93 $1: 19 \mathrm{P}$

193 SPLANT.DAT 19348 9/30/93 $1: 19 \mathrm{P}$

194 SPLANT.DAT 19348 9/30/93 $1: 19 \mathrm{P}$

195 SPLANT.DAT 19348 9/30/93 $1: 19 \mathrm{P}$
Th

1
1
1
1
1
1
1
1
1
1
1
1
1
1
1
1
1
1
1
1
1
1
1
1
1
1
1
1
1
1
1
1
1
1
1
1
1
1
1
1
1
1
1
1
1
1
1
1
1
1
1
1
1
1
1
1
1
1
1
1
1
1
1
1
1
1
1
1
1
1
1
1
1
1
1
1
1
1
1
1
1
1
1
1
1
1
1
1
1
1
1
1
1
1
1
1
1
1

Np

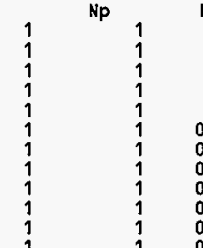

Pu

0.03

0.025

0.021

0.023

0.031

0.0288

0.0288

0.0288

0.0288

0.0288

0.0288

0.0288

0.0288

0.0288

0.0288

0.0288

0.0288

0.0288

0.0288

0.0288

0.0288

0.0288

0.0288

0.0288

0.0288

0.0288

0.0288

0.0288

0.0288

0.0288

0.0288

0.0288

0.0288

0.0288

0.0288

0.0288

0.0288

0.0288

0.0288

0.0288

0.0288

0.0288

0.0288

0.0288

0.0288

0.0288

0.0288

0.0288

0.0288

0.0288

0.0288

0.0288

0.0288

0.0288

0.0288

0.0288

0.0288

0.0288

0.0288

0.0288

0.0288

0.0288

0.0288

0.0288

0.0288
Waste Frac.

Reference

(b)

(b)

(b)

(b)

(a)

(a)

(a)

(a)

(a)

(a)

(a)

(a)

(a)

(a)

(a)

(a)

(a)

(a)

(a)

(a)

(a)

(a)

(a)

(a)

(a)

(a)

(a)

(a)

(a)

(a)

(a)

(a)

(a)

(a)

(a)

(a)

(a)

(a)

(a)

(a)

(a)

(a)

(a)

(a)

(a)

(a)

(a)

(a)

(a) 
HNF-SD-WM-TI-794, ReV. 0

ACTIVITY IN UNSEPARATED FUEL (Curies decayed to $1 / 1 / 94$ )

\begin{tabular}{|c|c|c|c|c|c|c|c|c|c|}
\hline $\begin{array}{r}\text { [Listing } \\
131 \\
132 \\
133 \\
134 \\
135 \\
136 \\
137 \\
138 \\
139 \\
140 \\
141 \\
142 \\
143 \\
144 \\
145 \\
146 \\
147 \\
148 \\
149 \\
150 \\
151 \\
152 \\
153 \\
154 \\
155 \\
156 \\
157 \\
158 \\
159 \\
160 \\
161 \\
162 \\
163 \\
164 \\
165 \\
166 \\
167 \\
168 \\
169 \\
170 \\
171 \\
172 \\
173 \\
174 \\
175 \\
176 \\
177 \\
178 \\
179 \\
180 \\
181 \\
182 \\
183 \\
184 \\
185 \\
186 \\
187 \\
188 \\
189 \\
190 \\
191 \\
192 \\
193 \\
194 \\
195\end{array}$ & $\begin{array}{l}H 03 \\
4.00 E+01 \\
2.16 E+01 \\
1.79 E+01 \\
8.35 E+00 \\
1.28 E+01 \\
1.45 E+00 \\
4.20 E+00 \\
3.68 E+00 \\
4.66 E+00 \\
6.06 E+00 \\
7.57 E+00 \\
1.35 E+01 \\
9.66 E+00 \\
9.40 E+00 \\
7.68 E+00 \\
9.44 E+00 \\
1.15 E+01 \\
8.90 E+00 \\
1.36 E+01 \\
1.02 E+01 \\
1.08 E+01 \\
1.34 E+01 \\
8.88 E+00 \\
1.05 E+01 \\
1.07 E+01 \\
8.21 E+00 \\
6.31 E+00 \\
5.41 E+00 \\
9.05 E+00 \\
6.87 E+00 \\
6.70 E+00 \\
7.14 E+00 \\
7.43 E+00 \\
6.18 E+00 \\
7.10 E+00 \\
5.39 E+00 \\
5.39 E+00 \\
5.38 E+00 \\
7.45 E+00 \\
8.30 E+00 \\
6.14 E+00 \\
7.69 E+00 \\
4.97 E+00 \\
9.37 E+00 \\
1.07 E+01 \\
8.27 E+00 \\
6.62 E+00 \\
8.30 E+00 \\
9.72 E+00 \\
1.17 E+01 \\
1.38 E+01 \\
3.38 E+00 \\
1.57 E+01 \\
7.48 E+00 \\
1.03 E+01 \\
7.38 E+00 \\
6.95 E+00 \\
3.81 E+00 \\
1.34 E+01 \\
1.42 E+01 \\
1.27 E+01 \\
1.57 E+01 \\
1.55 E+01 \\
1.55 E+01 \\
1.81 E+01\end{array}$ & $\begin{array}{l}\text { C14 } \\
1.42 \mathrm{E}+00 \\
7.66 \mathrm{E}-01 \\
6.36 \mathrm{E}-01 \\
2.96 \mathrm{E}-01 \\
4.56 \mathrm{E}-01 \\
9.27 \mathrm{E}-02 \\
2.67 \mathrm{E}-01 \\
2.33 \mathrm{E}-01 \\
2.92 \mathrm{E}-01 \\
3.79 \mathrm{E}-01 \\
4.71 \mathrm{E}-01 \\
8.38 \mathrm{E}-01 \\
5.97 \mathrm{E}-01 \\
5.77 \mathrm{E}-01 \\
4.70 \mathrm{E}-01 \\
5.77 \mathrm{E}-01 \\
6.98 \mathrm{E}-01 \\
5.38 \mathrm{E}-01 \\
8.20 \mathrm{E}-01 \\
6.11 \mathrm{E}-01 \\
6.44 \mathrm{E}-01 \\
7.92 \mathrm{E}-01 \\
5.24 \mathrm{E}-01 \\
6.18 \mathrm{E}-01 \\
6.28 \mathrm{E}-01 \\
4.78 \mathrm{E}-01 \\
3.65 \mathrm{E}-01 \\
3.12 \mathrm{E}-01 \\
5.20 \mathrm{E}-01 \\
3.93 \mathrm{E}-01 \\
3.81 \mathrm{E}-01 \\
4.05 \mathrm{E}-01 \\
4.20 \mathrm{E}-01 \\
3.48 \mathrm{E}-01 \\
3.98 \mathrm{E}-01 \\
3.01 \mathrm{E}-01 \\
3.00 \mathrm{E}-01 \\
2.98 \mathrm{E}-01 \\
4.11 \mathrm{E}-01 \\
4.56 \mathrm{E}-01 \\
3.36 \mathrm{E}-01 \\
4.17 \mathrm{E}-01 \\
2.69 \mathrm{E}-01 \\
5.06 \mathrm{E}-01 \\
5.71 \mathrm{E}-01 \\
4.41 \mathrm{E}-01 \\
3.51 \mathrm{E}-01 \\
4.39 \mathrm{E}-01 \\
5.11 \mathrm{E}-01 \\
6.11 \mathrm{E}-01 \\
7.18 \mathrm{E}-01 \\
1.75 \mathrm{E}-01 \\
8.09 \mathrm{E}-01 \\
3.84 \mathrm{E}-01 \\
5.25 \mathrm{E}-01 \\
3.75 \mathrm{E}-01 \\
3.50 \mathrm{E}-01 \\
1.91 \mathrm{E}-01 \\
6.64 \mathrm{E}-01 \\
7.03 \mathrm{E}-01 \\
6.23 \mathrm{E}-01 \\
7.67 \mathrm{E}-01 \\
7.51 \mathrm{E}-01 \\
7.49 \mathrm{E}-01 \\
8.68 \mathrm{E}-01\end{array}$ & $\begin{array}{l}\text { NI59 } \\
4.05 \mathrm{E}-01 \\
2.18 \mathrm{E}-01 \\
1.81 \mathrm{E}-01 \\
8.42 \mathrm{E}-02 \\
1.30 \mathrm{E}-01 \\
2.64 \mathrm{E}-02 \\
7.62 \mathrm{E}-02 \\
6.63 \mathrm{E}-02 \\
8.33 \mathrm{E}-02 \\
1.08 \mathrm{E}-01 \\
1.34 \mathrm{E}-01 \\
2.39 \mathrm{E}-01 \\
1.70 \mathrm{E}-01 \\
1.65 \mathrm{E}-01 \\
1.34 \mathrm{E}-01 \\
1.64 \mathrm{E}-01 \\
1.99 \mathrm{E}-01 \\
1.53 \mathrm{E}-01 \\
2.34 \mathrm{E}-01 \\
1.74 \mathrm{E}-01 \\
1.84 \mathrm{E}-01 \\
2.26 \mathrm{E}-01 \\
1.49 \mathrm{E}-01 \\
1.76 \mathrm{E}-01 \\
1.79 \mathrm{E}-01 \\
1.36 \mathrm{E}-01 \\
1.04 \mathrm{E}-01 \\
8.90 \mathrm{E}-02 \\
1.48 \mathrm{E}-01 \\
1.12 \mathrm{E}-01 \\
1.09 \mathrm{E}-01 \\
1.16 \mathrm{E}-01 \\
1.20 \mathrm{E}-01 \\
9.91 \mathrm{E}-02 \\
1.13 \mathrm{E}-01 \\
8.58 \mathrm{E}-02 \\
8.54 \mathrm{E}-02 \\
8.50 \mathrm{E}-02 \\
1.17 \mathrm{E}-01 \\
1.30 \mathrm{E}-01 \\
9.56 \mathrm{E}-02 \\
1.19 \mathrm{E}-01 \\
7.67 \mathrm{E}-02 \\
1.44 \mathrm{E}-01 \\
1.63 \mathrm{E}-01 \\
1.26 \mathrm{E}-01 \\
9.99 \mathrm{E}-02 \\
1.25 \mathrm{E}-01 \\
1.46 \mathrm{E}-01 \\
1.74 \mathrm{E}-01 \\
2.05 \mathrm{E}-01 \\
4.99 \mathrm{E}-02 \\
2.30 \mathrm{E}-01 \\
1.09 \mathrm{E}-01 \\
1.49 \mathrm{E}-01 \\
1.07 \mathrm{E}-01 \\
9.96 \mathrm{E}-02 \\
5.43 \mathrm{E}-02 \\
1.89 \mathrm{E}-01 \\
2.00 \mathrm{E}-01 \\
1.77 \mathrm{E}-01 \\
2.18 \mathrm{E}-01 \\
2.14 \mathrm{E}-01 \\
2.13 \mathrm{E}-01 \\
2.47 \mathrm{E}-01\end{array}$ & 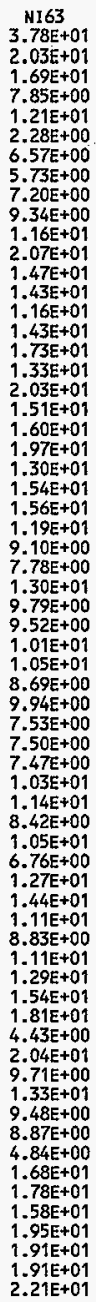 & $\begin{array}{l}\text { CO60 } \\
5.60 \mathrm{E}-01 \\
3.03 \mathrm{E}-01 \\
2.52 \mathrm{E}-01 \\
1.17 \mathrm{E}-01 \\
1.80 \mathrm{E}-01 \\
9.39 \mathrm{E}-03 \\
2.74 \mathrm{E}-02 \\
2.41 \mathrm{E}-02 \\
3.06 \mathrm{E}-02 \\
4.01 \mathrm{E}-02 \\
5.05 \mathrm{E}-02 \\
9.06 \mathrm{E}-02 \\
6.51 \mathrm{E}-02 \\
6.38 \mathrm{E}-02 \\
5.23 \mathrm{E}-02 \\
6.46 \mathrm{E}-02 \\
7.91 \mathrm{E}-02 \\
6.17 \mathrm{E}-02 \\
9.50 \mathrm{E}-02 \\
7.15 \mathrm{E}-02 \\
7.63 \mathrm{E}-02 \\
9.50 \mathrm{E}-02 \\
6.35 \mathrm{E}-02 \\
7.56 \mathrm{E}-02 \\
7.76 \mathrm{E}-02 \\
5.98 \mathrm{E}-02 \\
4.63 \mathrm{E}-02 \\
3.98 \mathrm{E}-02 \\
6.71 \mathrm{E}-02 \\
5.12 \mathrm{E}-02 \\
5.03 \mathrm{E}-02 \\
5.39 \mathrm{E}-02 \\
5.64 \mathrm{E}-02 \\
4.72 \mathrm{E}-02 \\
5.45 \mathrm{E}-02 \\
4.16 \mathrm{E}-02 \\
4.18 \mathrm{E}-02 \\
4.20 \mathrm{E}-02 \\
5.85 \mathrm{E}-02 \\
6.55 \mathrm{E}-02 \\
4.87 \mathrm{E}-02 \\
6.11 \mathrm{E}-02 \\
3.97 \mathrm{E}-02 \\
7.56 \mathrm{E}-02 \\
8.68 \mathrm{E}-02 \\
6.78 \mathrm{E}-02 \\
5.48 \mathrm{E}-02 \\
6.89 \mathrm{E}-02 \\
8.11 \mathrm{E}-02 \\
9.81 \mathrm{E}-02 \\
1.17 \mathrm{E}-01 \\
2.87 \mathrm{E}-02 \\
1.34 \mathrm{E}-01 \\
6.43 \mathrm{E}-02 \\
8.90 \mathrm{E}-02 \\
6.41 \mathrm{E}-02 \\
6.06 \mathrm{E}-02 \\
3.33 \mathrm{E}-02 \\
1.18 \mathrm{E}-01 \\
1.26 \mathrm{E}-01 \\
1.12 \mathrm{E}-01 \\
1.40 \mathrm{E}-01 \\
1.38 \mathrm{E}-01 \\
1.40 \mathrm{E}-01 \\
1.63 \mathrm{E}-01\end{array}$ & $\begin{array}{l}\text { SE779 } \\
3.00 \mathrm{E}-01 \\
1.61 \mathrm{E}-01 \\
1.34 \mathrm{E}-01 \\
6.22 \mathrm{E}-02 \\
9.59 \mathrm{E}-02 \\
1.95 \mathrm{E}-02 \\
5.62 \mathrm{E}-02 \\
4.89 \mathrm{E}-02 \\
6.15 \mathrm{E}-02 \\
7.97 \mathrm{E}-02 \\
9.91 \mathrm{E}-02 \\
1.76 \mathrm{E}-01 \\
1.26 \mathrm{E}-01 \\
1.21 \mathrm{E}-01 \\
9.88 \mathrm{E}-02 \\
1.21 \mathrm{E}-01 \\
1.47 \mathrm{E}-01 \\
1.13 \mathrm{E}-01 \\
1.73 \mathrm{E}-01 \\
1.29 \mathrm{E}-01 \\
1.36 \mathrm{E}-01 \\
1.67 \mathrm{E}-01 \\
1.10 \mathrm{E}-01 \\
1.30 \mathrm{E}-01 \\
1.32 \mathrm{E}-01 \\
1.01 \mathrm{E}-01 \\
7.69 \mathrm{E}-02 \\
6.57 \mathrm{E}-02 \\
1.09 \mathrm{E}-01 \\
8.26 \mathrm{E}-02 \\
8.02 \mathrm{E}-02 \\
8.53 \mathrm{E}-02 \\
8.83 \mathrm{E}-02 \\
7.31 \mathrm{E}-02 \\
8.36 \mathrm{E}-02 \\
6.33 \mathrm{E}-02 \\
6.30 \mathrm{E}-02 \\
6.27 \mathrm{E}-02 \\
8.64 \mathrm{E}-02 \\
9.58 \mathrm{E}-02 \\
7.06 \mathrm{E}-02 \\
8.79 \mathrm{E}-02 \\
5.67 \mathrm{E}-02 \\
1.06 \mathrm{E}-01 \\
1.20 \mathrm{E}-01 \\
9.27 \mathrm{E}-02 \\
7.37 \mathrm{E}-02 \\
9.22 \mathrm{E}-02 \\
1.07 \mathrm{E}-01 \\
1.29 \mathrm{E}-01 \\
1.51 \mathrm{E}-01 \\
3.69 \mathrm{E}-02 \\
1.70 \mathrm{E}-01 \\
8.08 \mathrm{E}-02 \\
1.10 \mathrm{E}-01 \\
7.89 \mathrm{E}-02 \\
7.37 \mathrm{E}-02 \\
4.02 \mathrm{E}-02 \\
1.40 \mathrm{E}-01 \\
1.48 \mathrm{E}-01 \\
1.32 \mathrm{E}-01 \\
1.62 \mathrm{E}-01 \\
1.59 \mathrm{E}-01 \\
1.58 \mathrm{E}-01 \\
1.83 \mathrm{E}-01\end{array}$ & $\begin{array}{l}\text { SR90 } \\
2.59 E+04 \\
1.39 E+04 \\
1.16 E+04 \\
5.38 E+03 \\
8.28 E+03 \\
1.32 E+03 \\
3.82 E+03 \\
3.33 E+03 \\
4.19 E+03 \\
5.44 E+03 \\
6.78 E+03 \\
1.21 E+04 \\
8.62 E+03 \\
8.35 E+03 \\
6.81 E+03 \\
8.37 E+03 \\
1.02 E+04 \\
7.84 E+03 \\
1.20 E+04 \\
8.93 E+03 \\
9.44 E+03 \\
1.16 E+04 \\
7.71 E+03 \\
9.11 E+03 \\
9.27 E+03 \\
7.07 E+03 \\
5.42 E+03 \\
4.64 E+03 \\
7.74 E+03 \\
5.86 E+03 \\
5.70 E+03 \\
6.07 E+03 \\
6.29 E+03 \\
5.22 E+03 \\
5.98 E+03 \\
4.54 E+03 \\
4.52 E+03 \\
4.51 E+03 \\
6.22 E+03 \\
6.91 E+03 \\
5.10 E+03 \\
6.35 E+03 \\
4.10 E+03 \\
7.73 E+03 \\
8.76 E+03 \\
6.77 E+03 \\
5.40 E+03 \\
6.76 E+03 \\
7.89 E+03 \\
9.46 E+03 \\
1.11 E+04 \\
2.72 E+03 \\
1.26 E+04 \\
5.98 E+03 \\
8.19 E+03 \\
5.86 E+03 \\
5.48 E+03 \\
2.99 E+03 \\
1.04 E+04 \\
1.11 E+04 \\
9.80 E+03 \\
1.21 E+04 \\
1.19 E+04 \\
1.18 E+04 \\
1.37 E+04\end{array}$ & 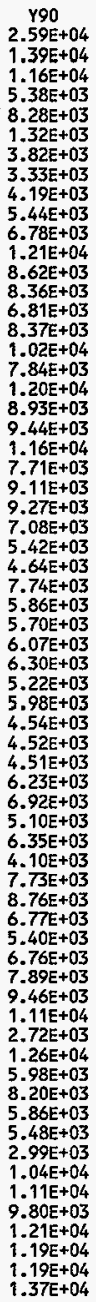 & 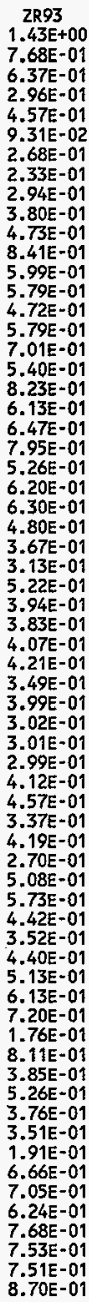 \\
\hline
\end{tabular}


HNF-SD-WM-TI-794, ReV. 0

ACTIVITY IN UNSEPARATED FUEL (Curies decayed to 1/1/94)

\begin{tabular}{|c|c|c|c|c|c|c|c|c|c|}
\hline $\begin{array}{c}\text { [Listing } 1] \\
131 \\
132 \\
133 \\
134 \\
135 \\
136 \\
137 \\
138 \\
139 \\
140 \\
141 \\
142 \\
143 \\
144 \\
145 \\
146 \\
147 \\
148 \\
149 \\
150 \\
151 \\
152 \\
153 \\
154 \\
155 \\
156 \\
157 \\
158 \\
159 \\
160 \\
161 \\
162 \\
163 \\
164 \\
165 \\
166 \\
167 \\
168 \\
169 \\
170 \\
171 \\
172 \\
173 \\
174 \\
175 \\
176 \\
177 \\
178 \\
179 \\
180 \\
181 \\
182 \\
183 \\
184 \\
185 \\
186 \\
187 \\
188 \\
189 \\
190 \\
191 \\
192 \\
193 \\
194 \\
195\end{array}$ & $\begin{array}{l}\text { NB93* } \\
1.17 \mathrm{E}+00 \\
6.27 \mathrm{E}-01 \\
5.20 \mathrm{E}-01 \\
2.42 \mathrm{E}-01 \\
3.73 \mathrm{E}-01 \\
8.11 \mathrm{E}-02 \\
2.34 \mathrm{E}-01 \\
2.03 \mathrm{E}-01 \\
2.55 \mathrm{E}-01 \\
3.31 \mathrm{E}-01 \\
4.11 \mathrm{E}-01 \\
7.32 \mathrm{E}-01 \\
5.21 \mathrm{E}-01 \\
5.03 \mathrm{E}-01 \\
4.10 \mathrm{E}-01 \\
5.03 \mathrm{E}-01 \\
6.08 \mathrm{E}-01 \\
4.68 \mathrm{E}-01 \\
7.14 \mathrm{E}-01 \\
5.31 \mathrm{E}-01 \\
5.60 \mathrm{E}-01 \\
6.89 \mathrm{E}-01 \\
4.55 \mathrm{E}-01 \\
5.37 \mathrm{E}-01 \\
5.45 \mathrm{E}-01 \\
4.15 \mathrm{E}-01 \\
3.17 \mathrm{E}-01 \\
2.71 \mathrm{E}-01 \\
4.51 \mathrm{E}-01 \\
3.40 \mathrm{E}-01 \\
3.30 \mathrm{E}-01 \\
3.51 \mathrm{E}-01 \\
3.63 \mathrm{E}-01 \\
3.01 \mathrm{E}-01 \\
3.44 \mathrm{E}-01 \\
2.60 \mathrm{E}-01 \\
2.59 \mathrm{E}-01 \\
2.58 \mathrm{E}-01 \\
3.55 \mathrm{E}-01 \\
3.93 \mathrm{E}-01 \\
2.90 \mathrm{E}-01 \\
3.60 \mathrm{E}-01 \\
2.32 \mathrm{E}-01 \\
4.36 \mathrm{E}-01 \\
4.92 \mathrm{E}-01 \\
3.79 \mathrm{E}-01 \\
3.01 \mathrm{E}-01 \\
3.77 \mathrm{E}-01 \\
4.39 \mathrm{E}-01 \\
5.25 \mathrm{E}-01 \\
6.16 \mathrm{E}-01 \\
1.50 \mathrm{E}-01 \\
6.94 \mathrm{E}-01 \\
3.29 \mathrm{E}-01 \\
4.49 \mathrm{E}-01 \\
3.21 \mathrm{E}-01 \\
2.99 \mathrm{E}-01 \\
1.63 \mathrm{E}-01 \\
5.67 \mathrm{E}-01 \\
6.00 \mathrm{E}-01 \\
5.32 \mathrm{E}-01 \\
6.54 \mathrm{E}-01 \\
6.41 \mathrm{E}-01 \\
6.39 \mathrm{E}-01 \\
7.39 \mathrm{E}-01\end{array}$ & $\begin{array}{l}7 C 99 \\
9.85 \mathrm{E}+00 \\
5.30 \mathrm{E}+00 \\
4.40 \mathrm{E}+00 \\
2.05 \mathrm{E}+00 \\
3.15 \mathrm{E}+00 \\
6.39 \mathrm{E}-01 \\
1.85 \mathrm{E}+00 \\
1.61 \mathrm{E}+00 \\
2.02 \mathrm{E}+00 \\
2.62 \mathrm{E}+00 \\
3.26 \mathrm{E}+00 \\
5.80 \mathrm{E}+00 \\
4.13 \mathrm{E}+00 \\
3.99 \mathrm{E}+00 \\
3.25 \mathrm{E}+00 \\
3.98 \mathrm{E}+00 \\
4.83 \mathrm{E}+00 \\
3.72 \mathrm{E}+00 \\
5.67 \mathrm{E}+00 \\
4.22 \mathrm{E}+00 \\
4.46 \mathrm{E}+00 \\
5.48 \mathrm{E}+00 \\
3.62 \mathrm{E}+00 \\
4.27 \mathrm{E}+00 \\
4.34 \mathrm{E}+00 \\
3.30 \mathrm{E}+00 \\
2.53 \mathrm{E}+00 \\
2.16 \mathrm{E}+00 \\
3.59 \mathrm{E}+00 \\
2.72 \mathrm{E}+00 \\
2.64 \mathrm{E}+00 \\
2.80 \mathrm{E}+00 \\
2.90 \mathrm{E}+00 \\
2.40 \mathrm{E}+00 \\
2.75 \mathrm{E}+00 \\
2.08 \mathrm{E}+00 \\
2.07 \mathrm{E}+00 \\
2.06 \mathrm{E}+00 \\
2.84 \mathrm{E}+00 \\
3.15 \mathrm{E}+00 \\
2.32 \mathrm{E}+00 \\
2.89 \mathrm{E}+00 \\
1.86 \mathrm{E}+00 \\
3.50 \mathrm{E}+00 \\
3.95 \mathrm{E}+00 \\
3.05 \mathrm{E}+00 \\
2.42 \mathrm{E}+00 \\
3.03 \mathrm{E}+00 \\
3.53 \mathrm{E}+00 \\
4.23 \mathrm{E}+00 \\
4.96 \mathrm{E}+00 \\
1.21 \mathrm{E}+00 \\
5.59 \mathrm{E}+00 \\
2.65 \mathrm{E}+00 \\
3.63 \mathrm{E}+00 \\
2.59 \mathrm{E}+00 \\
2.42 \mathrm{E}+00 \\
1.32 \mathrm{E}+00 \\
4.61 \mathrm{E}+00 \\
4.88 \mathrm{E}+00 \\
4.33 \mathrm{E}+00 \\
5.33 \mathrm{E}+00 \\
5.22 \mathrm{E}+00 \\
5.21 \mathrm{E}+00 \\
6.04 \mathrm{E}+00\end{array}$ & $\begin{array}{l}\text { RU106 } \\
7.66 \mathrm{E}-07 \\
4.25 \mathrm{E}-07 \\
3.57 \mathrm{E}-07 \\
1.67 \mathrm{E}-07 \\
2.51 \mathrm{E}-07 \\
3.82 \mathrm{E}-11 \\
1.20 \mathrm{E}-10 \\
1.13 \mathrm{E}-10 \\
1.51 \mathrm{E}-10 \\
2.07 \mathrm{E}-10 \\
2.72 \mathrm{E}-10 \\
5.09 \mathrm{E}-10 \\
3.78 \mathrm{E}-10 \\
3.94 \mathrm{E}-10 \\
3.33 \mathrm{E}-10 \\
4.21 \mathrm{E}-10 \\
5.42 \mathrm{E}-10 \\
4.45 \mathrm{E}-10 \\
7.15 \mathrm{E}-10 \\
5.62 \mathrm{E}-10 \\
6.30 \mathrm{E}-10 \\
8.27 \mathrm{E}-10 \\
5.83 \mathrm{E}-10 \\
7.16 \mathrm{E}-10 \\
7.67 \mathrm{E}-10 \\
6.26 \mathrm{E}-10 \\
5.08 \mathrm{E}-10 \\
4.50 \mathrm{E}-10 \\
7.96 \mathrm{E}-10 \\
6.39 \mathrm{E}-10 \\
6.55 \mathrm{E}-10 \\
7.23 \mathrm{E}-10 \\
7.97 \mathrm{E}-10 \\
6.94 \mathrm{E}-10 \\
8.35 \mathrm{E}-10 \\
6.56 \mathrm{E}-10 \\
6.92 \mathrm{E}-10 \\
7.20 \mathrm{E}-10 \\
1.05 \mathrm{E}-09 \\
1.23 \mathrm{E}-09 \\
9.47 \mathrm{E}-10 \\
1.27 \mathrm{E}-09 \\
8.41 \mathrm{E}-10 \\
1.68 \mathrm{E}-09 \\
2.07 \mathrm{E}-09 \\
1.70 \mathrm{E}-09 \\
1.48 \mathrm{E}-09 \\
1.89 \mathrm{E}-09 \\
2.33 \mathrm{E}-09 \\
2.94 \mathrm{E}-09 \\
3.68 \mathrm{E}-09 \\
9.29 \mathrm{E}-10 \\
4.61 \mathrm{E}-09 \\
2.35 \mathrm{E}-09 \\
3.41 \mathrm{E}-09 \\
2.59 \mathrm{E}-09 \\
2.61 \mathrm{E}-09 \\
1.50 \mathrm{E}-09 \\
5.75 \mathrm{E}-09 \\
6.39 \mathrm{E}-09 \\
6.08 \mathrm{E}-09 \\
8.00 \mathrm{E}-09 \\
8.25 \mathrm{E}-09 \\
8.71 \mathrm{E}-09 \\
1.08 \mathrm{E}-08\end{array}$ & $\begin{array}{l}\text { CD113* } \\
4.25 \mathrm{E}+00 \\
2.29 \mathrm{E}+00 \\
1.90 \mathrm{E}+00 \\
8.85 \mathrm{E}-01 \\
1.36 \mathrm{E}+00 \\
1.67 \mathrm{E}-01 \\
4.85 \mathrm{E}-01 \\
4.26 \mathrm{E}-01 \\
5.39 \mathrm{E}-01 \\
6.99 \mathrm{E}-01 \\
8.72 \mathrm{E}-01 \\
1.56 \mathrm{E}+00 \\
1.11 \mathrm{E}+00 \\
1.08 \mathrm{E}+00 \\
8.83 \mathrm{E}-01 \\
1.09 \mathrm{E}+00 \\
1.32 \mathrm{E}+00 \\
1.02 \mathrm{E}+00 \\
1.56 \mathrm{E}+00 \\
1.17 \mathrm{E}+00 \\
1.24 \mathrm{E}+00 \\
1.53 \mathrm{E}+00 \\
1.02 \mathrm{E}+00 \\
1.20 \mathrm{E}+00 \\
1.23 \mathrm{E}+00 \\
9.37 \mathrm{E}-01 \\
7.20 \mathrm{E}-01 \\
6.17 \mathrm{E}-01 \\
1.03 \mathrm{E}+00 \\
7.82 \mathrm{E}-01 \\
7.62 \mathrm{E}-01 \\
8.12 \mathrm{E}-01 \\
8.45 \mathrm{E}-01 \\
7.02 \mathrm{E}-01 \\
8.05 \mathrm{E}-01 \\
6.11 \mathrm{E}-01 \\
6.11 \mathrm{E}-01 \\
6.10 \mathrm{E}-01 \\
8.44 \mathrm{E}-01 \\
9.38 \mathrm{E}-01 \\
6.94 \mathrm{E}-01 \\
8.70 \mathrm{E}-01 \\
5.62 \mathrm{E}-01 \\
1.06 \mathrm{E}+00 \\
1.20 \mathrm{E}+00 \\
9.31 \mathrm{E}-01 \\
7.45 \mathrm{E}-01 \\
9.34 \mathrm{E}-01 \\
1.09 \mathrm{E}+00 \\
1.31 \mathrm{E}+00 \\
1.55 \mathrm{E}+00 \\
3.79 \mathrm{E}-01 \\
1.76 \mathrm{E}+00 \\
8.39 \mathrm{E}-01 \\
1.15 \mathrm{E}+00 \\
8.27 \mathrm{E}-01 \\
7.78 \mathrm{E}-01 \\
4.27 \mathrm{E}-01 \\
1.50 \mathrm{E}+00 \\
1.59 \mathrm{E}+00 \\
1.42 \mathrm{E}+00 \\
1.76 \mathrm{E}+00 \\
1.73 \mathrm{E}+00 \\
1.74 \mathrm{E}+00 \\
2.02 \mathrm{E}+00\end{array}$ & $\begin{array}{c}\text { SB125 } \\
7.52 \mathrm{E}-01 \\
4.09 \mathrm{E}-01 \\
3.41 \mathrm{E}-01 \\
1.59 \mathrm{E}-01 \\
2.42 \mathrm{E}-01 \\
3.64 \mathrm{E}-03 \\
1.08 \mathrm{E}-02 \\
9.63 \mathrm{E}-03 \\
1.24 \mathrm{E}-02 \\
1.64 \mathrm{E}-02 \\
2.08 \mathrm{E}-02 \\
3.76 \mathrm{E}-02 \\
2.72 \mathrm{E}-02 \\
2.71 \mathrm{E}-02 \\
2.23 \mathrm{E}-02 \\
2.77 \mathrm{E}-02 \\
3.43 \mathrm{E}-02 \\
2.70 \mathrm{E}-02 \\
4.20 \mathrm{E}-02 \\
3.19 \mathrm{E}-02 \\
3.44 \mathrm{E}-02 \\
4.33 \mathrm{E}-02 \\
2.93 \mathrm{E}-02 \\
3.51 \mathrm{E}-02 \\
3.64 \mathrm{E}-02 \\
2.84 \mathrm{E}-02 \\
2.22 \mathrm{E}-02 \\
1.92 \mathrm{E}-02 \\
3.27 \mathrm{E}-02 \\
2.52 \mathrm{E}-02 \\
2.50 \mathrm{E}-02 \\
2.69 \mathrm{E}-02 \\
2.85 \mathrm{E}-02 \\
2.41 \mathrm{E}-02 \\
2.80 \mathrm{E}-02 \\
2.15 \mathrm{E}-02 \\
2.19 \mathrm{E}-02 \\
2.21 \mathrm{E}-02 \\
3.11 \mathrm{E}-02 \\
3.52 \mathrm{E}-02 \\
2.64 \mathrm{E}-02 \\
3.35 \mathrm{E}-02 \\
2.19 \mathrm{E}-02 \\
4.21 \mathrm{E}-02 \\
4.91 \mathrm{E}-02 \\
3.87 \mathrm{E}-02 \\
3.18 \mathrm{E}-02 \\
4.01 \mathrm{E}-02 \\
4.77 \mathrm{E}-02 \\
5.83 \mathrm{E}-02 \\
7.00 \mathrm{E}-02 \\
1.73 \mathrm{E}-02 \\
8.18 \mathrm{E}-02 \\
3.98 \mathrm{E}-02 \\
5.57 \mathrm{E}-02 \\
4.06 \mathrm{E}-02 \\
3.89 \mathrm{E}-02 \\
2.16 \mathrm{E}-02 \\
7.75 \mathrm{E}-02 \\
8.36 \mathrm{E}-02 \\
7.57 \mathrm{E}-02 \\
9.53 \mathrm{E}-02 \\
9.52 \mathrm{E}-02 \\
9.70 \mathrm{E}-02 \\
.15 \mathrm{E}-01\end{array}$ & $\begin{array}{c}\text { SN126 } \\
4.46 \mathrm{E}-01 \\
2.40 \mathrm{E}-01 \\
1.99 \mathrm{E}-01 \\
9.26 \mathrm{E}-02 \\
1.43 \mathrm{E}-01 \\
2.86 \mathrm{E}-02 \\
8.28 \mathrm{E}-02 \\
7.24 \mathrm{E}-02 \\
9.12 \mathrm{E}-02 \\
1.18 \mathrm{E}-01 \\
1.47 \mathrm{E}-01 \\
2.61 \mathrm{E}-01 \\
1.86 \mathrm{E}-01 \\
1.80 \mathrm{E}-01 \\
1.46 \mathrm{E}-01 \\
1.79 \mathrm{E}-01 \\
2.17 \mathrm{E}-01 \\
1.68 \mathrm{E}-01 \\
2.55 \mathrm{E}-01 \\
1.90 \mathrm{E}-01 \\
2.01 \mathrm{E}-01 \\
2.47 \mathrm{E}-01 \\
1.63 \mathrm{E}-01 \\
1.92 \mathrm{E}-01 \\
1.96 \mathrm{E}-01 \\
1.49 \mathrm{E}-01 \\
1.14 \mathrm{E}-01 \\
9.73 \mathrm{E}-02 \\
1.62 \mathrm{E}-01 \\
1.22 \mathrm{E}-01 \\
1.19 \mathrm{E}-01 \\
1.26 \mathrm{E}-01 \\
1.31 \mathrm{E}-01 \\
1.08 \mathrm{E}-01 \\
1.24 \mathrm{E}-01 \\
9.37 \mathrm{E}-02 \\
9.33 \mathrm{E}-02 \\
9.28 \mathrm{E}-02 \\
1.28 \mathrm{E}-01 \\
1.42 \mathrm{E}-01 \\
1.05 \mathrm{E}-01 \\
1.31 \mathrm{E}-01 \\
8.41 \mathrm{E}-02 \\
1.58 \mathrm{E}-01 \\
1.78 \mathrm{E}-01 \\
1.37 \mathrm{E}-01 \\
1.09 \mathrm{E}-01 \\
1.37 \mathrm{E}-01 \\
1.59 \mathrm{E}-01 \\
1.91 \mathrm{E}-01 \\
2.24 \mathrm{E}-01 \\
5.46 \mathrm{E}-02 \\
2.53 \mathrm{E}-01 \\
1.20 \mathrm{E}-01 \\
1.64 \mathrm{E}-01 \\
1.17 \mathrm{E}-01 \\
1.10 \mathrm{E}-01 \\
6.01 \mathrm{E}-02 \\
2.10 \mathrm{E}-01 \\
2.22 \mathrm{E}-01 \\
1.98 \mathrm{E}-01 \\
2.44 \mathrm{E}-01 \\
2.39 \mathrm{E}-01 \\
2.39 \mathrm{E}-01 \\
2.77 \mathrm{E}-01\end{array}$ & $\begin{array}{l}1129 \\
1.84 \mathrm{E}-02 \\
9.89 \mathrm{E}-03 \\
8.20 \mathrm{E}-03 \\
3.82 \mathrm{E}-03 \\
5.89 \mathrm{E}-03 \\
1.18 \mathrm{E}-03 \\
3.41 \mathrm{E}-03 \\
2.98 \mathrm{E}-03 \\
3.76 \mathrm{E}-03 \\
4.86 \mathrm{E}-03 \\
6.04 \mathrm{E}-03 \\
1.08 \mathrm{E}-02 \\
7.66 \mathrm{E}-03 \\
7.41 \mathrm{E}-03 \\
6.03 \mathrm{E}-03 \\
7.39 \mathrm{E}-03 \\
8.96 \mathrm{E}-03 \\
6.90 \mathrm{E}-03 \\
1.05 \mathrm{E}-02 \\
7.85 \mathrm{E}-03 \\
8.28 \mathrm{E}-03 \\
1.02 \mathrm{E}-02 \\
6.73 \mathrm{E}-03 \\
7.93 \mathrm{E}-03 \\
8.06 \mathrm{E}-03 \\
6.13 \mathrm{E}-03 \\
4.69 \mathrm{E}-03 \\
4.01 \mathrm{E}-03 \\
6.68 \mathrm{E}-03 \\
5.05 \mathrm{E}-03 \\
4.90 \mathrm{E}-03 \\
5.20 \mathrm{E}-03 \\
5.39 \mathrm{E}-03 \\
4.46 \mathrm{E}-03 \\
5.10 \mathrm{E}-03 \\
3.86 \mathrm{E}-03 \\
3.84 \mathrm{E}-03 \\
3.83 \mathrm{E}-03 \\
5.27 \mathrm{E}-03 \\
5.84 \mathrm{E}-03 \\
4.31 \mathrm{E}-03 \\
5.38 \mathrm{E}-03 \\
3.47 \mathrm{E}-03 \\
6.49 \mathrm{E}-03 \\
7.34 \mathrm{E}-03 \\
5.66 \mathrm{E}-03 \\
4.50 \mathrm{E}-03 \\
5.63 \mathrm{E}-03 \\
6.56 \mathrm{E}-03 \\
7.85 \mathrm{E}-03 \\
9.22 \mathrm{E}-03 \\
2.25 \mathrm{E}-03 \\
1.04 \mathrm{E}-02 \\
4.94 \mathrm{E}-03 \\
6.75 \mathrm{E}-03 \\
4.84 \mathrm{E}-03 \\
4.53 \mathrm{E}-03 \\
2.48 \mathrm{E}-03 \\
8.67 \mathrm{E}-03 \\
9.17 \mathrm{E}-03 \\
8.16 \mathrm{E}-03 \\
1.01 \mathrm{E}-02 \\
9.86 \mathrm{E}-03 \\
9.84 \mathrm{E}-03 \\
1.14 \mathrm{E}-02\end{array}$ & $\begin{array}{l}\text { CS134 } \\
4.10 \mathrm{E}-03 \\
2.24 \mathrm{E}-03 \\
1.87 \mathrm{E}-03 \\
8.71 \mathrm{E}-04 \\
1.38 \mathrm{E}-03 \\
1.06 \mathrm{E}-06 \\
1.02 \mathrm{E}-05 \\
1.40 \mathrm{E}-05 \\
2.16 \mathrm{E}-05 \\
2.42 \mathrm{E}-05 \\
2.97 \mathrm{E}-05 \\
6.19 \mathrm{E}-05 \\
4.10 \mathrm{E}-05 \\
4.11 \mathrm{E}-05 \\
3.41 \mathrm{E}-05 \\
3.87 \mathrm{E}-05 \\
5.45 \mathrm{E}-05 \\
4.33 \mathrm{E}-05 \\
6.78 \mathrm{E}-05 \\
5.53 \mathrm{E}-05 \\
6.01 \mathrm{E}-05 \\
7.15 \mathrm{E}-05 \\
5.20 \mathrm{E}-05 \\
5.87 \mathrm{E}-05 \\
6.12 \mathrm{E}-05 \\
4.82 \mathrm{E}-05 \\
3.80 \mathrm{E}-05 \\
3.30 \mathrm{E}-05 \\
5.66 \mathrm{E}-05 \\
4.69 \mathrm{E}-05 \\
4.68 \mathrm{E}-05 \\
4.76 \mathrm{E}-05 \\
5.08 \mathrm{E}-05 \\
4.31 \mathrm{E}-05 \\
5.05 \mathrm{E}-05 \\
3.89 \mathrm{E}-05 \\
3.99 \mathrm{E}-05 \\
4.06 \mathrm{E}-05 \\
5.75 \mathrm{E}-05 \\
6.10 \mathrm{E}-05 \\
4.93 \mathrm{E}-05 \\
8.73 \mathrm{E}-05 \\
5.29 \mathrm{E}-05 \\
8.03 \mathrm{E}-05 \\
1.28 \mathrm{E}-04 \\
1.00 \mathrm{E}-04 \\
7.95 \mathrm{E}-04 \\
3.81 \mathrm{E}-05 \\
3.94 \mathrm{E}-05 \\
8.36 \mathrm{E}-05 \\
1.06 \mathrm{E}-04 \\
4.36 \mathrm{E}-04 \\
6.01 \mathrm{E}-04 \\
5.89 \mathrm{E}-04 \\
6.04 \mathrm{E}-04 \\
1.23 \mathrm{E}-04 \\
1.49 \mathrm{E}-04 \\
3.70 \mathrm{E}-05 \\
2.05 \mathrm{E}-04 \\
1.05 \mathrm{E}-04 \\
\end{array}$ & $\begin{array}{l}\text { CS137 } \\
2.90 \mathrm{E}+04 \\
1.56 \mathrm{E}+04 \\
1.30 \mathrm{E}+04 \\
6.04 \mathrm{E}+03 \\
9.30 \mathrm{E}+03 \\
1.48 \mathrm{E}+03 \\
4.29 \mathrm{E}+03 \\
3.75 \mathrm{E}+03 \\
4.72 \mathrm{E}+03 \\
6.13 \mathrm{E}+03 \\
7.63 \mathrm{E}+03 \\
1.36 \mathrm{E}+04 \\
9.70 \mathrm{E}+03 \\
9.41 \mathrm{E}+03 \\
7.67 \mathrm{E}+03 \\
9.42 \mathrm{E}+03 \\
1.14 \mathrm{E}+04 \\
8.82 \mathrm{E}+03 \\
1.35 \mathrm{E}+04 \\
1.01 \mathrm{E}+04 \\
1.06 \mathrm{E}+04 \\
1.31 \mathrm{E}+04 \\
8.68 \mathrm{E}+03 \\
1.03 \mathrm{E}+04 \\
1.04 \mathrm{E}+04 \\
7.96 \mathrm{E}+03 \\
6.10 \mathrm{E}+03 \\
5.22 \mathrm{E}+03 \\
8.71 \mathrm{E}+03 \\
6.59 \mathrm{E}+03 \\
6.41 \mathrm{E}+03 \\
6.82 \mathrm{E}+03 \\
7.08 \mathrm{E}+03 \\
1.35 \mathrm{E}+04 \\
1.37 \mathrm{E}+04 \\
6.73 \mathrm{E}+03 \\
5.10 \mathrm{E}+03 \\
5.09 \mathrm{E}+03 \\
5.07 \mathrm{E}+03 \\
7.00 \mathrm{E}+03 \\
7.77 \mathrm{E}+03 \\
5.73 \mathrm{E}+03 \\
7.16 \mathrm{E}+03 \\
4.62 \mathrm{E}+03 \\
8.69 \mathrm{E}+03 \\
9.85 \mathrm{E}+03 \\
7.61 \mathrm{E}+03 \\
6.07 \mathrm{E}+03 \\
7.60 \mathrm{E}+03 \\
8.87 \mathrm{E}+03 \\
1.06 \mathrm{E}+04 \\
1.25 \mathrm{E}+04 \\
3.06 \mathrm{E}+03 \\
1.42 \mathrm{E}+04 \\
6.73 \mathrm{E}+03 \\
9.22 \mathrm{E}+03 \\
6.60 \mathrm{E}+03 \\
6.19 \mathrm{E}+03 \\
3.38 \mathrm{E}+03 \\
1.18 \mathrm{E}+04 \\
1.35 \mathrm{E}\end{array}$ \\
\hline
\end{tabular}


HNF-SD-WM-TI-794, Rev, 0

ACTIVITY IN UNSEPARATED FUEL (Curies decayed to 1/1/94)

\begin{tabular}{|c|c|c|c|c|c|c|c|c|c|}
\hline $\begin{array}{r}\text { [Listing } \\
131 \\
132 \\
133 \\
134 \\
135 \\
136 \\
137 \\
138 \\
139 \\
140 \\
141 \\
142 \\
143 \\
144 \\
145 \\
146 \\
147 \\
148 \\
149 \\
150 \\
151 \\
152 \\
153 \\
154 \\
155 \\
156 \\
157 \\
158 \\
159 \\
160 \\
161 \\
162 \\
163 \\
164 \\
165 \\
166 \\
167 \\
168 \\
169 \\
170 \\
171 \\
172 \\
173 \\
174 \\
175 \\
176 \\
177 \\
178 \\
179 \\
180 \\
181 \\
182 \\
183 \\
184 \\
185 \\
186 \\
187 \\
188 \\
189 \\
190 \\
191 \\
192 \\
193 \\
194 \\
195\end{array}$ & $\begin{array}{l}B A 137 \\
2.75 \mathrm{E}+04 \\
1.48 \mathrm{E}+04 \\
1.23 \mathrm{E}+04 \\
5.72 \mathrm{E}+03 \\
8.80 \mathrm{E}+03 \\
1.40 \mathrm{E}+03 \\
4.06 \mathrm{E}+03 \\
3.54 \mathrm{E}+03 \\
4.47 \mathrm{E}+03 \\
5.80 \mathrm{E}+03 \\
7.22 \mathrm{E}+03 \\
1.29 \mathrm{E}+04 \\
9.18 \mathrm{E}+03 \\
8.90 \mathrm{E}+03 \\
7.25 \mathrm{E}+03 \\
8.91 \mathrm{E}+03 \\
1.08 \mathrm{E}+04 \\
8.35 \mathrm{E}+03 \\
1.27 \mathrm{E}+04 \\
9.5 \mathrm{E}+03 \\
1.01 \mathrm{E}+04 \\
1.24 \mathrm{E}+04 \\
8.21 \mathrm{E}+03 \\
9.69 \mathrm{E}+03 \\
9.87 \mathrm{E}+03 \\
7.53 \mathrm{E}+03 \\
5.77 \mathrm{E}+03 \\
4.94 \mathrm{E}+03 \\
8.24 \mathrm{E}+03 \\
6.24 \mathrm{E}+03 \\
6.06 \mathrm{E}+03 \\
6.46 \mathrm{E}+03 \\
6.70 \mathrm{E}+03 \\
5.55 \mathrm{E}+03 \\
6.36 \mathrm{E}+03 \\
4.83 \mathrm{E}+03 \\
4.81 \mathrm{E}+03 \\
4.79 \mathrm{E}+03 \\
6.62 \mathrm{E}+03 \\
7.35 \mathrm{E}+03 \\
5.43 \mathrm{E}+03 \\
6.77 \mathrm{E}+03 \\
4.37 \mathrm{E}+03 \\
8.22 \mathrm{E}+03 \\
9.32 \mathrm{E}+03 \\
7.20 \mathrm{E}+03 \\
5.74 \mathrm{E}+03 \\
7.19 \mathrm{E}+03 \\
8.39 \mathrm{E}+03 \\
1.01 \mathrm{E}+04 \\
1.18 \mathrm{E}+04 \\
2.89 \mathrm{E}+03 \\
1.34 \mathrm{E}+04 \\
6.37 \mathrm{E}+03 \\
8.72 \mathrm{E}+03 \\
6.25 \mathrm{E}+03 \\
5.85 \mathrm{E}+03 \\
3.20 \mathrm{E}+03 \\
1.12 \mathrm{E}+04 \\
1.19 \mathrm{E}+04 \\
1.05 \mathrm{E}+04 \\
1.30 \mathrm{E}+04 \\
1.28 \mathrm{E}+04 \\
1.28 \mathrm{E}+04 \\
1.48 \mathrm{E}+04\end{array}$ & $\begin{array}{l}\text { SH151 } \\
1.20 \mathrm{E}+03 \\
6.43 \mathrm{E}+02 \\
5.34 \mathrm{E}+02 \\
2.49 \mathrm{E}+02 \\
3.82 \mathrm{E}+02 \\
7.49 \mathrm{E}+01 \\
2.13 \mathrm{E}+02 \\
1.84 \mathrm{E}+02 \\
2.30 \mathrm{E}+02 \\
3.00 \mathrm{E}+02 \\
3.74 \mathrm{E}+02 \\
6.62 \mathrm{E}+02 \\
4.73 \mathrm{E}+02 \\
4.58 \mathrm{E}+02 \\
3.73 \mathrm{E}+02 \\
4.59 \mathrm{E}+02 \\
5.54 \mathrm{E}+02 \\
4.27 \mathrm{E}+02 \\
6.52 \mathrm{E}+02 \\
4.85 \mathrm{E}+02 \\
5.12 \mathrm{E}+02 \\
6.31 \mathrm{E}+02 \\
4.17 \mathrm{E}+02 \\
4.93 \mathrm{E}+02 \\
5.01 \mathrm{E}+02 \\
3.82 \mathrm{E}+02 \\
2.92 \mathrm{E}+02 \\
2.50 \mathrm{E}+02 \\
4.16 \mathrm{E}+02 \\
3.14 \mathrm{E}+02 \\
3.05 \mathrm{E}+02 \\
3.25 \mathrm{E}+02 \\
3.37 \mathrm{E}+02 \\
2.79 \mathrm{E}+02 \\
3.19 \mathrm{E}+02 \\
2.42 \mathrm{E}+02 \\
2.41 \mathrm{E}+02 \\
2.40 \mathrm{E}+02 \\
3.31 \mathrm{E}+02 \\
3.67 \mathrm{E}+02 \\
2.70 \mathrm{E}+02 \\
3.32 \mathrm{E}+02 \\
2.15 \mathrm{E}+02 \\
4.08 \mathrm{E}+02 \\
4.61 \mathrm{E}+02 \\
3.55 \mathrm{E}+02 \\
2.83 \mathrm{E}+02 \\
3.54 \mathrm{E}+02 \\
4.13 \mathrm{E}+02 \\
4.94 \mathrm{E}+02 \\
5.81 \mathrm{E}+02 \\
1.42 \mathrm{E}+02 \\
6.52 \mathrm{E}+02 \\
3.09 \mathrm{E}+02 \\
4.23 \mathrm{E}+02 \\
3.00 \mathrm{E}+02 \\
2.79 \mathrm{E}+02 \\
1.51 \mathrm{E}+02 \\
5.18 \mathrm{E}+02 \\
5.51 \mathrm{E}+02 \\
4.82 \mathrm{E}+02 \\
5.89 \mathrm{E}+02 \\
5.79 \mathrm{E}+02 \\
5.78 \mathrm{E}+02 \\
6.66 \mathrm{E}+02\end{array}$ & $\begin{array}{l}\text { EU152 } \\
4.88 \mathrm{E}-02 \\
2.63 \mathrm{E}-02 \\
2.19 \mathrm{E}-02 \\
1.02 \mathrm{E}-02 \\
1.68 \mathrm{E}-02 \\
0.00 \mathrm{E}+00 \\
4.37 \mathrm{E}-04 \\
2.05 \mathrm{E}-03 \\
3.72 \mathrm{E}-03 \\
3.36 \mathrm{E}-03 \\
3.81 \mathrm{E}-03 \\
9.24 \mathrm{E}-03 \\
5.36 \mathrm{E}-03 \\
5.21 \mathrm{E}-03 \\
4.25 \mathrm{E}-03 \\
4.14 \mathrm{E}-03 \\
6.80 \mathrm{E}-03 \\
5.26 \mathrm{E}-03 \\
8.06 \mathrm{E}-03 \\
6.94 \mathrm{E}-03 \\
7.35 \mathrm{E}-03 \\
7.89 \mathrm{E}-03 \\
6.03 \mathrm{E}-03 \\
6.20 \mathrm{E}-03 \\
6.32 \mathrm{E}-03 \\
4.84 \mathrm{E}-03 \\
3.72 \mathrm{E}-03 \\
3.18 \mathrm{E}-03 \\
5.33 \mathrm{E}-03 \\
4.65 \mathrm{E}-03 \\
4.54 \mathrm{E}-03 \\
4.20 \mathrm{E}-03 \\
4.37 \mathrm{E}-03 \\
3.63 \mathrm{E}-03 \\
4.17 \mathrm{E}-03 \\
3.17 \mathrm{E}-03 \\
3.16 \mathrm{E}-03 \\
3.16 \mathrm{E}-03 \\
4.37 \mathrm{E}-03 \\
4.13 \mathrm{E}-03 \\
3.60 \mathrm{E}-03 \\
8.40 \mathrm{E}-03 \\
4.74 \mathrm{E}-03 \\
5.49 \mathrm{E}-03 \\
7.05 \mathrm{E}-03 \\
5.46 \mathrm{E}-03 \\
4.37 \mathrm{E}-03 \\
5.48 \mathrm{E}-03 \\
6.41 \mathrm{E}-03 \\
7.70 \mathrm{E}-03 \\
9.09 \mathrm{E}-03 \\
2.22 \mathrm{E}-03 \\
1.39 \mathrm{E}-02 \\
7.10 \mathrm{E}-03 \\
9.10 \mathrm{E}-03 \\
9.22 \mathrm{E}-03 \\
1.03 \mathrm{E}-02 \\
6.85 \mathrm{E}-03 \\
3.21 \mathrm{E}-02 \\
3.17 \mathrm{E}-02 \\
3.49 \mathrm{E}-02 \\
4.81 \mathrm{E}-02 \\
4.58 \mathrm{E}-02 \\
4.59 \mathrm{E}-02 \\
5.79 \mathrm{E}-02\end{array}$ & $\begin{array}{l}\text { EU154 } \\
5.54 \mathrm{E}+00 \\
2.99 \mathrm{E}+00 \\
2.49 \mathrm{E}+00 \\
1.16 \mathrm{E}+00 \\
1.83 \mathrm{E}+00 \\
6.31 \mathrm{E}-02 \\
2.73 \mathrm{E}-01 \\
3.00 \mathrm{E}-01 \\
4.21 \mathrm{E}-01 \\
4.95 \mathrm{E}-01 \\
6.06 \mathrm{E}-01 \\
1.17 \mathrm{E}+00 \\
7.94 \mathrm{E}-01 \\
7.75 \mathrm{E}-01 \\
6.33 \mathrm{E}-01 \\
7.39 \mathrm{E}-01 \\
9.67 \mathrm{E}-01 \\
7.51 \mathrm{E}-01 \\
1.15 \mathrm{E}+00 \\
8.98 \mathrm{E}-01 \\
9.54 \mathrm{E}-01 \\
1.14 \mathrm{E}+00 \\
7.87 \mathrm{E}-01 \\
8.97 \mathrm{E}-01 \\
9.17 \mathrm{E}-01 \\
7.04 \mathrm{E}-01 \\
5.42 \mathrm{E}-01 \\
4.65 \mathrm{E}-01 \\
7.80 \mathrm{E}-01 \\
6.17 \mathrm{E}-01 \\
6.03 \mathrm{E}-01 \\
6.19 \mathrm{E}-01 \\
6.46 \mathrm{E}-01 \\
5.38 \mathrm{E}-01 \\
6.19 \mathrm{E}-01 \\
4.71 \mathrm{E}-01 \\
4.72 \mathrm{E}-01 \\
4.72 \mathrm{E}-01 \\
6.55 \mathrm{E}-01 \\
7.01 \mathrm{E}-01 \\
5.41 \mathrm{E}-01 \\
8.32 \mathrm{E}-01 \\
5.11 \mathrm{E}-01 \\
8.31 \mathrm{E}-01 \\
9.80 \mathrm{E}-01 \\
7.61 \mathrm{E}-01 \\
6.72 \mathrm{E}-01 \\
7.68 \mathrm{E}-01 \\
9.00 \mathrm{E}-01 \\
1.08 \mathrm{E}+00 \\
1.28 \mathrm{E}+00 \\
3.14 \mathrm{E}-01 \\
1.61 \mathrm{E}+00 \\
7.83 \mathrm{E}-01 \\
1.06 \mathrm{E}+00 \\
8.71 \mathrm{E}-01 \\
8.89 \mathrm{E}-01 \\
5.37 \mathrm{E}-01 \\
2.22 \mathrm{E}+00 \\
2.27 \mathrm{E}+00 \\
2.30 \mathrm{E}+00 \\
3.06 \mathrm{E}+00 \\
2.95 \mathrm{E}+00 \\
2.96 \mathrm{E}+00 \\
3.64 \mathrm{E}+00\end{array}$ & $\begin{array}{c}\mathrm{EU155} \\
1.17 \mathrm{E}+01 \\
6.31 \mathrm{E}+00 \\
5.25 \mathrm{E}+00 \\
2.44 \mathrm{E}+00 \\
3.73 \mathrm{E}+00 \\
1.90 \mathrm{E}-01 \\
5.46 \mathrm{E}-01 \\
4.73 \mathrm{E}-01 \\
5.96 \mathrm{E}-01 \\
7.89 \mathrm{E}-01 \\
9.94 \mathrm{E}-01 \\
1.77 \mathrm{E}+00 \\
1.28 \mathrm{E}+00 \\
1.26 \mathrm{E}+00 \\
1.03 \mathrm{E}+00 \\
1.28 \mathrm{E}+00 \\
1.56 \mathrm{E}+00 \\
1.22 \mathrm{E}+00 \\
1.88 \mathrm{E}+00 \\
1.41 \mathrm{E}+00 \\
1.50 \mathrm{E}+00 \\
1.88 \mathrm{E}+00 \\
1.25 \mathrm{E}+00 \\
1.50 \mathrm{E}+00 \\
1.54 \mathrm{E}+00 \\
1.19 \mathrm{E}+00 \\
9.19 \mathrm{E}-01 \\
7.91 \mathrm{E}-01 \\
1.33 \mathrm{E}+00 \\
1.02 \mathrm{E}+00 \\
9.97 \mathrm{E}-01 \\
1.07 \mathrm{E}+00 \\
1.12 \mathrm{E}+00 \\
9.40 \mathrm{E}-01 \\
1.09 \mathrm{E}+00 \\
8.29 \mathrm{E}-01 \\
8.35 \mathrm{E}-01 \\
8.38 \mathrm{E}-01 \\
1.17 \mathrm{E}+00 \\
1.32 \mathrm{E}+00 \\
9.74 \mathrm{E}-01 \\
1.20 \mathrm{E}+00 \\
7.85 \mathrm{E}-01 \\
1.52 \mathrm{E}+00 \\
1.74 \mathrm{E}+00 \\
1.36 \mathrm{E}+00 \\
1.10 \mathrm{E}+00 \\
1.38 \mathrm{E}+00 \\
1.63 \mathrm{E}+00 \\
1.97 \mathrm{E}+00 \\
2.35 \mathrm{E}+00 \\
5.76 \mathrm{E}-01 \\
2.67 \mathrm{E}+00 \\
1.28 \mathrm{E}+00 \\
1.78 \mathrm{E}+00 \\
1.27 \mathrm{E}+00 \\
1.19 \mathrm{E}+00 \\
6.46 \mathrm{E}-01 \\
2.23 \mathrm{E}+00 \\
2.40 \mathrm{E}+00 \\
2.11 \mathrm{E}+00 \\
2.59 \mathrm{E}+00 \\
2.58 \mathrm{E}+00 \\
2.60 \mathrm{E}+00 \\
3.03 \mathrm{E}+00\end{array}$ & $\begin{array}{l}\text { RA226 } \\
7.51 \mathrm{E}-05 \\
4.03 \mathrm{E}-05 \\
3.34 \mathrm{E}-05 \\
1.55 \mathrm{E}-05 \\
2.33 \mathrm{E}-05 \\
1.69 \mathrm{E}-05 \\
3.52 \mathrm{E}-05 \\
2.52 \mathrm{E}-05 \\
2.88 \mathrm{E}-05 \\
4.07 \mathrm{E}-05 \\
5.15 \mathrm{E}-05 \\
8.53 \mathrm{E}-05 \\
6.36 \mathrm{E}-05 \\
6.12 \mathrm{E}-05 \\
4.97 \mathrm{E}-05 \\
6.38 \mathrm{E}-05 \\
7.24 \mathrm{E}-05 \\
5.55 \mathrm{E}-05 \\
8.44 \mathrm{E}-05 \\
6.06 \mathrm{E}-05 \\
6.37 \mathrm{E}-05 \\
8.07 \mathrm{E}-05 \\
5.13 \mathrm{E}-05 \\
6.25 \mathrm{E}-05 \\
6.33 \mathrm{E}-05 \\
4.80 \mathrm{E}-05 \\
3.66 \mathrm{E}-05 \\
3.12 \mathrm{E}-05 \\
5.17 \mathrm{E}-05 \\
3.76 \mathrm{E}-05 \\
3.64 \mathrm{E}-05 \\
4.00 \mathrm{E}-05 \\
4.12 \mathrm{E}-05 \\
3.40 \mathrm{E}-05 \\
3.88 \mathrm{E}=05 \\
2.93 \mathrm{E}-05 \\
2.91 \mathrm{E}-05 \\
2.88 \mathrm{E}-05 \\
3.96 \mathrm{E}-05 \\
4.53 \mathrm{E}-05 \\
3.21 \mathrm{E}-05 \\
3.32 \mathrm{E}-05 \\
2.24 \mathrm{E}-05 \\
4.79 \mathrm{E}-05 \\
5.23 \mathrm{E}-05 \\
4.02 \mathrm{E}-05 \\
3.18 \mathrm{E}-05 \\
3.97 \mathrm{E}-05 \\
4.61 \mathrm{E}-05 \\
5.50 \mathrm{E}-05 \\
6.43 \mathrm{E}-05 \\
1.57 \mathrm{E}-05 \\
6.62 \mathrm{E}-05 \\
3.06 \mathrm{E}-05 \\
4.26 \mathrm{E}-05 \\
2.69 \mathrm{E}-05 \\
2.32 \mathrm{E}-05 \\
3.16 \mathrm{E}-05 \\
3.46 \mathrm{E}-05 \\
3.79 \mathrm{E}-05 \\
2.97 \mathrm{E}-05 \\
3.42 \mathrm{E}-05 \\
3.41 \mathrm{E}-05 \\
3.38 \mathrm{E}-05 \\
\end{array}$ & $\begin{array}{l}\text { RA228 } \\
2.75 \mathrm{E}-10 \\
1.48 \mathrm{E}-10 \\
1.23 \mathrm{E}-10 \\
5.71 \mathrm{E}-11 \\
8.80 \mathrm{E}-11 \\
2.45 \mathrm{E}-11 \\
7.03 \mathrm{E}-11 \\
6.09 \mathrm{E}-11 \\
7.63 \mathrm{E}-11 \\
9.87 \mathrm{E}-11 \\
1.22 \mathrm{E}-10 \\
2.17 \mathrm{E}-10 \\
1.55 \mathrm{E}-10 \\
1.49 \mathrm{E}-10 \\
1.21 \mathrm{E}-10 \\
1.49 \mathrm{E}-10 \\
1.80 \mathrm{E}-10 \\
1.38 \mathrm{E}-10 \\
2.10 \mathrm{E}-10 \\
1.56 \mathrm{E}-10 \\
1.64 \mathrm{E}-10 \\
2.02 \mathrm{E}-10 \\
1.33 \mathrm{E}-10 \\
1.57 \mathrm{E}-10 \\
1.59 \mathrm{E}-10 \\
1.20 \mathrm{E}-10 \\
9.19 \mathrm{E}-11 \\
7.84 \mathrm{E}-11 \\
1.30 \mathrm{E}-10 \\
9.82 \mathrm{E}-11 \\
9.51 \mathrm{E}-11 \\
1.01 \mathrm{E}-10 \\
1.04 \mathrm{E}-10 \\
8.62 \mathrm{E}-11 \\
9.84 \mathrm{E}-11 \\
7.44 \mathrm{E}-11 \\
7.39 \mathrm{E}-11 \\
7.34 \mathrm{E}-11 \\
1.01 \mathrm{E}-10 \\
1.12 \mathrm{E}-10 \\
8.21 \mathrm{E}-11 \\
1.02 \mathrm{E}-10 \\
6.55 \mathrm{E}-11 \\
1.23 \mathrm{E}-10 \\
1.38 \mathrm{E}-10 \\
1.06 \mathrm{E}-10 \\
8.44 \mathrm{E}-11 \\
1.05 \mathrm{E}-10 \\
1.22 \mathrm{E}-10 \\
1.46 \mathrm{E}-10 \\
1.77 \mathrm{E}-10 \\
4.18 \mathrm{E}-11 \\
1.92 \mathrm{E}-10 \\
9.09 \mathrm{E}-11 \\
1.24 \mathrm{E}-10 \\
8.81 \mathrm{E}-11 \\
8.20 \mathrm{E}-11 \\
4.45 \mathrm{E}-11 \\
1.54 \mathrm{E}-10 \\
1.63 \mathrm{E}-10 \\
1.43 \mathrm{E}-10 \\
1.76 \mathrm{E}-10 \\
1.72 \mathrm{E}-10 \\
1.71 \mathrm{E}-10 \\
1.97 \mathrm{E}-10\end{array}$ & $\begin{array}{c}\text { AC227 } \\
4.03 \mathrm{E}-04 \\
2.17 \mathrm{E}-04 \\
1.79 \mathrm{E}-04 \\
8.35 \mathrm{E}-05 \\
1.25 \mathrm{E}-04 \\
8.51 \mathrm{E}-05 \\
1.76 \mathrm{E}-04 \\
1.25 \mathrm{E}-04 \\
1.43 \mathrm{E}-04 \\
2.03 \mathrm{E}-04 \\
2.57 \mathrm{E}-04 \\
4.25 \mathrm{E}-04 \\
3.17 \mathrm{E}-04 \\
3.06 \mathrm{E}-04 \\
2.48 \mathrm{E}-04 \\
3.19 \mathrm{E}-04 \\
3.62 \mathrm{E}-04 \\
2.78 \mathrm{E}-04 \\
4.23 \mathrm{E}-04 \\
3.03 \mathrm{E}-04 \\
3.19 \mathrm{E}-04 \\
4.05 \mathrm{E}-04 \\
2.58 \mathrm{E}-04 \\
3.14 \mathrm{E}-04 \\
3.18 \mathrm{E}-04 \\
2.41 \mathrm{E}-04 \\
1.84 \mathrm{E}-04 \\
1.57 \mathrm{E}-04 \\
2.61 \mathrm{E}-04 \\
1.90 \mathrm{E}-04 \\
1.83 \mathrm{E}-04 \\
2.02 \mathrm{E}-04 \\
2.08 \mathrm{E}-04 \\
1.72 \mathrm{E}-04 \\
1.96 \mathrm{E}-04 \\
1.48 \mathrm{E}-04 \\
1.47 \mathrm{E}-04 \\
1.46 \mathrm{E}-04 \\
2.01 \mathrm{E}-04 \\
2.30 \mathrm{E}-04 \\
1.63 \mathrm{E}-04 \\
1.68 \mathrm{E}-04 \\
1.13 \mathrm{E}-04 \\
2.44 \mathrm{E}-04 \\
2.66 \mathrm{E}-04 \\
2.05 \mathrm{E}-04 \\
1.62 \mathrm{E}-04 \\
2.02 \mathrm{E}-04 \\
2.35 \mathrm{E}-04 \\
2.80 \mathrm{E}-04 \\
3.28 \mathrm{E}-04 \\
8.00 \mathrm{E}-05 \\
3.38 \mathrm{E}-04 \\
1.56 \mathrm{E}-04 \\
2.18 \mathrm{E}-04 \\
1.37 \mathrm{E}-04 \\
1.18 \mathrm{E}-04 \\
5.87 \mathrm{E}-05 \\
1.74 \mathrm{E}-04 \\
1.92 \mathrm{E}-04 \\
1.50 \mathrm{E}-04 \\
1.71 \mathrm{E}-04 \\
1.71 \mathrm{E}-04 \\
1.87 \mathrm{e}\end{array}$ & $\begin{array}{l}\text { TH229 } \\
5.24 \mathrm{E}-08 \\
2.81 \mathrm{E}-08 \\
2.33 \mathrm{E}-08 \\
1.08 \mathrm{E}-08 \\
1.68 \mathrm{E}-08 \\
4.70 \mathrm{E}-09 \\
1.35 \mathrm{E}-08 \\
1.18 \mathrm{E}-08 \\
1.48 \mathrm{E}-08 \\
1.91 \mathrm{E}-08 \\
2.37 \mathrm{E}-08 \\
4.21 \mathrm{E}-08 \\
2.99 \mathrm{E}-08 \\
2.88 \mathrm{E}-08 \\
2.34 \mathrm{E}-08 \\
2.87 \mathrm{E}-08 \\
3.46 \mathrm{E}-08 \\
2.66 \mathrm{E}-08 \\
4.05 \mathrm{E}-08 \\
3.01 \mathrm{E}-08 \\
3.17 \mathrm{E}-08 \\
3.88 \mathrm{E}-08 \\
2.56 \mathrm{E}-08 \\
3.01 \mathrm{E}-08 \\
3.05 \mathrm{E}-08 \\
2.32 \mathrm{E}-08 \\
1.77 \mathrm{E}-08 \\
1.51 \mathrm{E}-08 \\
2.51 \mathrm{E}-08 \\
1.89 \mathrm{E}-08 \\
1.83 \mathrm{E}-08 \\
1.94 \mathrm{E}-08 \\
2.01 \mathrm{E}-08 \\
1.66 \mathrm{E}-08 \\
1.89 \mathrm{E}-08 \\
1.59 \mathrm{E}-08 \\
1.63 \mathrm{E}-08 \\
1.49 \mathrm{E}-09 \\
3.99 \mathrm{E}-08 \\
3.16 \mathrm{E}-08 \\
2.80 \mathrm{E}-08 \\
3.43 \mathrm{E}-08 \mathrm{E}-08 \\
1.94 \mathrm{E}-08 \\
2.14 \mathrm{E}-08 \\
1.57 \mathrm{E}-08 \\
1.96 \mathrm{E}-08 \\
1.26 \mathrm{E}-08 \\
2.36 \mathrm{E}-08 \\
2.65 \mathrm{E}-08 \\
2.04 \mathrm{E}-08 \\
1.62 \mathrm{E}-08 \\
2.02 \mathrm{E}-08 \\
2.35 \mathrm{E}-08 \\
2.80 \mathrm{E}-08 \\
3.28 \mathrm{E}-08 \\
8.00 \mathrm{E}-09 \\
3.69 \mathrm{E}-08 \\
1.74 \mathrm{E}-08 \\
\end{array}$ \\
\hline
\end{tabular}


HNF-SD-WM-TI-794, ReV. 0

ACTIVITY IN UNSEPARATED FUEL (Curies decayed to 1/1/94)

\begin{tabular}{|c|c|c|c|c|c|c|c|c|c|}
\hline 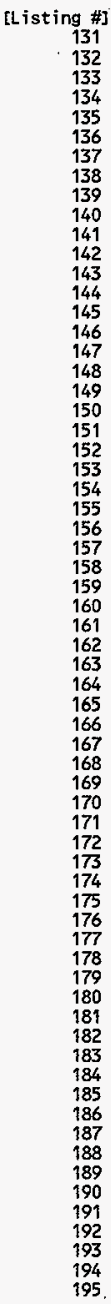 & $\begin{array}{l}\text { TH232 } \\
3.65 E-10 \\
1.96 E-10 \\
1.63 E-10 \\
7.58 E-11 \\
1.17 E-10 \\
3.05 E-11 \\
8.75 E-11 \\
7.58 E-11 \\
9.50 E-11 \\
1.23 E-10 \\
1.53 E-10 \\
2.71 E-10 \\
1.93 E-10 \\
1.86 E-10 \\
1.51 E-10 \\
1.86 E-10 \\
2.24 E-10 \\
1.72 E-10 \\
2.63 E-10 \\
1.95 E-10 \\
2.06 E-10 \\
2.52 E-10 \\
1.66 E-10 \\
1.96 E-10 \\
1.99 E-10 \\
1.51 E-10 \\
1.15 E-10 \\
9.85 E-11 \\
1.64 E-10 \\
1.23 E-10 \\
1.20 E-10 \\
1.27 E-10 \\
1.31 E-10 \\
1.09 E-10 \\
1.24 E-10 \\
9.37 E-11 \\
9.31 E-11 \\
9.25 E-11 \\
1.27 E-10 \\
1.41 E-10 \\
1.04 E-10 \\
1.28 E-10 \\
8.27 E-11 \\
1.55 E-10 \\
1.75 E-10 \\
1.35 E-10 \\
1.07 E-10 \\
1.34 E-10 \\
1.55 E-10 \\
1.86 E-10 \\
2.17 E-10 \\
5.30 E-11 \\
2.44 E-10 \\
1.15 E-10 \\
1.57 E-10 \\
1.12 E-10 \\
1.04 E-10 \\
5.67 E-11 \\
1.96 E-10 \\
2.07 E-10 \\
1.83 E-10 \\
2.24 E-10 \\
2.19 E-10 \\
2.18 E-10 \\
2.52 E-10\end{array}$ & $\begin{array}{l}\text { PA231 } \\
9.49 \mathrm{E}-04 \\
5.10 \mathrm{E}-04 \\
4.23 \mathrm{E}-04 \\
1.97 \mathrm{E}-04 \\
2.95 \mathrm{E}-04 \\
1.72 \mathrm{E}-04 \\
3.57 \mathrm{E}-04 \\
2.54 \mathrm{E}-04 \\
2.91 \mathrm{E}-04 \\
4.13 \mathrm{E}-04 \\
5.23 \mathrm{E}-04 \\
8.66 \mathrm{E}-04 \\
6.47 \mathrm{E}-04 \\
6.25 \mathrm{E}-04 \\
5.08 \mathrm{E}-04 \\
6.53 \mathrm{E}-04 \\
7.41 \mathrm{E}-04 \\
5.70 \mathrm{E}-04 \\
8.68 \mathrm{E}-04 \\
6.23 \mathrm{E}-04 \\
6.56 \mathrm{E}-04 \\
8.34 \mathrm{E}-04 \\
5.31 \mathrm{E}-04 \\
6.48 \mathrm{E}-04 \\
6.57 \mathrm{E}-04 \\
4.99 \mathrm{E}-04 \\
3.81 \mathrm{E}-04 \\
3.25 \mathrm{E}-04 \\
5.41 \mathrm{E}-04 \\
3.94 \mathrm{E}-04 \\
3.82 \mathrm{E}-04 \\
4.20 \mathrm{E}-04 \\
4.34 \mathrm{E}-04 \\
3.58 \mathrm{E}-04 \\
4.09 \mathrm{E}-04 \\
3.10 \mathrm{E}-04 \\
3.08 \mathrm{E}-04 \\
3.06 \mathrm{E}-04 \\
4.21 \mathrm{E}-04 \\
4.83 \mathrm{E}-04 \\
3.42 \mathrm{E}-04 \\
3.53 \mathrm{E}-04 \\
2.38 \mathrm{E}-04 \\
5.13 \mathrm{E}-04 \\
5.61 \mathrm{E}-04 \\
4.32 \mathrm{E}-04 \\
3.43 \mathrm{E}-04 \\
4.29 \mathrm{E}-04 \\
4.98 \mathrm{E}-04 \\
5.95 \mathrm{E}-04 \\
6.97 \mathrm{E}-04 \\
1.70 \mathrm{E}-04 \\
7.19 \mathrm{E}-04 \\
3.33 \mathrm{E}-04 \\
4.64 \mathrm{E}-04 \\
2.92 \mathrm{E}-04 \\
2.53 \mathrm{E}-04 \\
1.26 \mathrm{E}-04 \\
3.74 \mathrm{E}-04 \\
4.11 \mathrm{E}-04 \\
3.21 \mathrm{E}-04 \\
3.68 \mathrm{E}-04 \\
3.68 \mathrm{E}-04 \\
3.67 \mathrm{E}-04 \\
4.02 \mathrm{E}-04\end{array}$ & $\begin{array}{l}\text { U232 } \\
3.95 E-04 \\
2.12 E-04 \\
1.76 E-04 \\
8.20 E-05 \\
1.27 E-04 \\
2.02 E-05 \\
6.11 E-05 \\
5.53 E-05 \\
7.09 E-05 \\
9.01 E-05 \\
1.12 E-04 \\
2.02 E-04 \\
1.42 E-04 \\
1.38 E-04 \\
1.12 E-04 \\
1.37 E-04 \\
1.67 E-04 \\
1.29 E-04 \\
1.97 E-04 \\
1.48 E-04 \\
1.56 E-04 \\
1.91 E-04 \\
1.27 E-04 \\
1.49 E-04 \\
1.51 E-04 \\
1.15 E-04 \\
8.84 E-05 \\
7.56 E-05 \\
1.26 E-04 \\
9.59 E-05 \\
9.31 E-05 \\
9.84 E-05 \\
1.02 E-04 \\
8.45 E-05 \\
9.67 E-05 \\
7.33 E-05 \\
7.29 E-05 \\
7.26 E-05 \\
1.00 E-04 \\
1.10 E-04 \\
8.19 E-05 \\
1.06 E-04 \\
6.78 E-05 \\
1.24 E-04 \\
1.41 E-04 \\
1.09 E-04 \\
8.66 E-05 \\
1.08 E-04 \\
1.26 E-04 \\
1.51 E-04 \\
1.78 E-04 \\
4.34 E-05 \\
2.04 E-04 \\
9.75 E-05 \\
1.33 E-04 \\
9.77 E-05 \\
9.30 E-05 \\
5.20 E-05 \\
1.89 E-04 \\
1.98 E-04 \\
1.83 E-04 \\
2.30 E-04 \\
2.24 E-04 \\
2.24 E-04 \\
2.64 E-04\end{array}$ & $\begin{array}{c}\text { U233 } \\
1.93 \mathrm{E}-05 \\
1.04 \mathrm{E}-05 \\
8.63 \mathrm{E}-06 \\
4.01 \mathrm{E}-06 \\
6.19 \mathrm{E}-06 \\
1.42 \mathrm{E}-06 \\
4.10 \mathrm{E}-06 \\
3.58 \mathrm{E}-06 \\
4.50 \mathrm{E}-06 \\
5.82 \mathrm{E}-06 \\
7.22 \mathrm{E}-06 \\
1.29 \mathrm{E}-05 \\
9.14 \mathrm{E}-06 \\
8.83 \mathrm{E}-06 \\
7.18 \mathrm{E}-06 \\
8.80 \mathrm{E}-06 \\
1.07 \mathrm{E}-05 \\
8.20 \mathrm{E}-06 \\
1.25 \mathrm{E}-05 \\
9.30 \mathrm{E}-06 \\
9.80 \mathrm{E}-06 \\
1.20 \mathrm{E}-05 \\
7.95 \mathrm{E}-06 \\
9.36 \mathrm{E}-06 \\
9.51 \mathrm{E}-06 \\
7.23 \mathrm{E}-06 \\
5.52 \mathrm{E}-06 \\
4.72 \mathrm{E}-06 \\
7.85 \mathrm{E}-06 \\
5.92 \mathrm{E}-06 \\
5.74 \mathrm{E}-06 \\
6.10 \mathrm{E}-06 \\
6.31 \mathrm{E}-06 \\
5.22 \mathrm{E}-06 \\
5.96 \mathrm{E}-06 \\
4.51 \mathrm{E}-06 \\
4.49 \mathrm{E}-06 \\
4.46 \mathrm{E}-06 \\
6.14 \mathrm{E}-06 \\
6.80 \mathrm{E}-06 \\
5.01 \mathrm{E}-06 \\
6.24 \mathrm{E}-06 \\
4.02 \mathrm{E}-06 \\
7.53 \mathrm{E}-06 \\
8.49 \mathrm{E}-06 \\
6.54 \mathrm{E}-06 \\
5.20 \mathrm{E}-06 \\
6.50 \mathrm{E}-06 \\
7.56 \mathrm{E}-06 \\
9.04 \mathrm{E}-06 \\
1.06 \mathrm{E}-05 \\
2.59 \mathrm{E}-06 \\
1.19 \mathrm{E}-05 \\
5.66 \mathrm{E}-06 \\
7.72 \mathrm{E}-06 \\
5.52 \mathrm{E}-06 \\
5.16 \mathrm{E}-06 \\
2.82 \mathrm{E}-06 \\
9.83 \mathrm{E}-06 \\
1.04 \mathrm{E}-05 \\
9.23 \mathrm{E}-06 \\
1.14 \mathrm{E}-05 \\
1.11 \mathrm{E}-05 \\
1.11 \mathrm{E}-05 \\
.28 \mathrm{E}-05\end{array}$ & $\begin{array}{l}\text { U234 } \\
2.60 \mathrm{E}+01 \\
1.40 \mathrm{E}+01 \\
1.16 \mathrm{E}+01 \\
5.40 \mathrm{E}+00 \\
8.09 \mathrm{E}+00 \\
3.66 \mathrm{E}+00 \\
7.64 \mathrm{E}+00 \\
5.47 \mathrm{E}+00 \\
6.28 \mathrm{E}+00 \\
8.91 \mathrm{E}+00 \\
1.13 \mathrm{E}+01 \\
1.88 \mathrm{E}+01 \\
1.40 \mathrm{E}+01 \\
1.36 \mathrm{E}+01 \\
1.11 \mathrm{E}+01 \\
1.42 \mathrm{E}+01 \\
1.62 \mathrm{E}+01 \\
1.25 \mathrm{E}+01 \\
1.90 \mathrm{E}+01 \\
1.37 \mathrm{E}+01 \\
1.44 \mathrm{E}+01 \\
1.84 \mathrm{E}+01 \\
1.17 \mathrm{E}+01 \\
1.43 \mathrm{E}+01 \\
1.46 \mathrm{E}+01 \\
1.11 \mathrm{E}+01 \\
8.47 \mathrm{E}+00 \\
7.24 \mathrm{E}+00 \\
1.21 \mathrm{E}+01 \\
8.80 \mathrm{E}+00 \\
8.54 \mathrm{E}+00 \\
9.40 \mathrm{E}+00 \\
9.73 \mathrm{E}+00 \\
8.05 \mathrm{E}+00 \\
9.21 \mathrm{E}+00 \\
6.98 \mathrm{E}+00 \\
6.94 \mathrm{E}+00 \\
6.91 \mathrm{E}+00 \\
9.52 \mathrm{E}+00 \\
1.09 \mathrm{E}+01 \\
7.78 \mathrm{E}+00 \\
8.07 \mathrm{E}+00 \\
5.45 \mathrm{E}+00 \\
1.17 \mathrm{E}+01 \\
1.29 \mathrm{E}+01 \\
9.91 \mathrm{E}+00 \\
7.89 \mathrm{E}+00 \\
9.87 \mathrm{E}+00 \\
1.15 \mathrm{E}+01 \\
1.38 \mathrm{E}+01 \\
1.62 \mathrm{E}+01 \\
3.94 \mathrm{E}+00 \\
1.67 \mathrm{E}+01 \\
7.77 \mathrm{E}+00 \\
1.09 \mathrm{E}+01 \\
6.87 \mathrm{E}+00 \\
5.97 \mathrm{E}+00 \\
2.98 \mathrm{E}+00 \\
8.93 \mathrm{E}+00 \\
9.83 \mathrm{E}+00 \\
7.73 \mathrm{E}+00 \\
8.92 \mathrm{E}+00 \\
8.92 \mathrm{E}+00 \\
8.90 \mathrm{E}+00 \\
9.81 \mathrm{E}+00\end{array}$ & $\begin{array}{l}\text { U235 } \\
1.16 \mathrm{E}+00 \\
6.26 \mathrm{E}-01 \\
5.19 \mathrm{E}-01 \\
2.42 \mathrm{E}-01 \\
3.62 \mathrm{E}-01 \\
1.67 \mathrm{E}-01 \\
3.46 \mathrm{E}-01 \\
2.47 \mathrm{E}-01 \\
2.83 \mathrm{E}-01 \\
4.02 \mathrm{E}-01 \\
5.10 \mathrm{E}-01 \\
8.46 \mathrm{E}-01 \\
6.33 \mathrm{E}-01 \\
6.12 \mathrm{E}-01 \\
4.99 \mathrm{E}-01 \\
6.42 \mathrm{E}-01 \\
7.30 \mathrm{E}-01 \\
5.62 \mathrm{E}-01 \\
8.57 \mathrm{E}-01 \\
6.16 \mathrm{E}-01 \\
6.50 \mathrm{E}-01 \\
8.28 \mathrm{E}-01 \\
5.28 \mathrm{E}-01 \\
6.46 \mathrm{E}-01 \\
6.56 \mathrm{E}-01 \\
4.99 \mathrm{E}-01 \\
3.82 \mathrm{E}-01 \\
3.26 \mathrm{E}-01 \\
5.43 \mathrm{E}-01 \\
3.96 \mathrm{E}-01 \\
3.85 \mathrm{E}-01 \\
4.24 \mathrm{E}-01 \\
4.39 \mathrm{E}-01 \\
3.63 \mathrm{E}-01 \\
4.15 \mathrm{E}-01 \\
3.15 \mathrm{E}-01 \\
3.13 \mathrm{E}-01 \\
3.12 \mathrm{E}-01 \\
4.29 \mathrm{E}-01 \\
4.94 \mathrm{E}-01 \\
3.51 \mathrm{E}-01 \\
3.62 \mathrm{E}-01 \\
2.45 \mathrm{E}-01 \\
5.29 \mathrm{E}-01 \\
5.79 \mathrm{E}-01 \\
4.47 \mathrm{E}-01 \\
3.55 \mathrm{E}-01 \\
4.45 \mathrm{E}-01 \\
5.18 \mathrm{E}-01 \\
6.20 \mathrm{E}-01 \\
7.28 \mathrm{E}-01 \\
1.78 \mathrm{E}-01 \\
7.52 \mathrm{E}-01 \\
.3 .49 \mathrm{E}-01 \\
4.88 \mathrm{E}-01 \\
3.07 \mathrm{E}-01 \\
2.67 \mathrm{E}-01 \\
1.33 \mathrm{E}-01 \\
3.95 \mathrm{E}-01 \\
4.35 \mathrm{E}-01 \\
3.41 \mathrm{E}-01 \\
3.92 \mathrm{E}-01 \\
3.92 \mathrm{E}-01 \\
3.91 \mathrm{E}-01 \\
4.30 \mathrm{E}-01\end{array}$ & $\begin{array}{l}\text { U236 } \\
1.92 \mathrm{E}-01 \\
1.03 \mathrm{E}-01 \\
8.57 \mathrm{E}-02 \\
3.99 \mathrm{E}-02 \\
6.14 \mathrm{E}-02 \\
1.27 \mathrm{E}-02 \\
3.64 \mathrm{E}-02 \\
3.16 \mathrm{E}-02 \\
3.96 \mathrm{E}-02 \\
5.14 \mathrm{E}-02 \\
6.39 \mathrm{E}-02 \\
1.14 \mathrm{E}-01 \\
8.10 \mathrm{E}-02 \\
7.83 \mathrm{E}-02 \\
6.38 \mathrm{E}-02 \\
7.83 \mathrm{E}-02 \\
9.47 \mathrm{E}-02 \\
7.30 \mathrm{E}-02 \\
1.11 \mathrm{E}-01 \\
8.28 \mathrm{E}-02 \\
8.74 \mathrm{E}-02 \\
1.08 \mathrm{E}-01 \\
7.10 \mathrm{E}-02 \\
8.38 \mathrm{E}-02 \\
8.51 \mathrm{E}-02 \\
6.48 \mathrm{E}-02 \\
4.96 \mathrm{E}-02 \\
4.24 \mathrm{E}-02 \\
7.05 \mathrm{E}-02 \\
5.32 \mathrm{E}-02 \\
5.17 \mathrm{E}-02 \\
5.50 \mathrm{E}-02 \\
5.69 \mathrm{E}-02 \\
4.71 \mathrm{E}-02 \\
5.39 \mathrm{E}-02 \\
4.08 \mathrm{E}-02 \\
4.06 \mathrm{E}-02 \\
4.04 \mathrm{E}-02 \\
5.57 \mathrm{E}-02 \\
6.18 \mathrm{E}-02 \\
4.55 \mathrm{E}-02 \\
5.64 \mathrm{E}-02 \\
3.64 \mathrm{E}-02 \\
6.86 \mathrm{E}-02 \\
7.75 \mathrm{E}-02 \\
5.97 \mathrm{E}-02 \\
4.75 \mathrm{E}-02 \\
5.94 \mathrm{E}-02 \\
6.92 \mathrm{E}-02 \\
8.29 \mathrm{E}-02 \\
9.73 \mathrm{E}-02 \\
2.38 \mathrm{E}-02 \\
1.10 \mathrm{E}-01 \\
5.19 \mathrm{E}-02 \\
7.10 \mathrm{E}-02 \\
5.06 \mathrm{E}-02 \\
4.72 \mathrm{E}-02 \\
2.57 \mathrm{E}-02 \\
8.90 \mathrm{E}-02 \\
9.43 \mathrm{E}-02 \\
8.33 \mathrm{E}-02 \\
1.02 \mathrm{E}-01 \\
1.00 \mathrm{E}-01 \\
1.00 \mathrm{E}-01 \\
1.16 \mathrm{E}-01\end{array}$ & 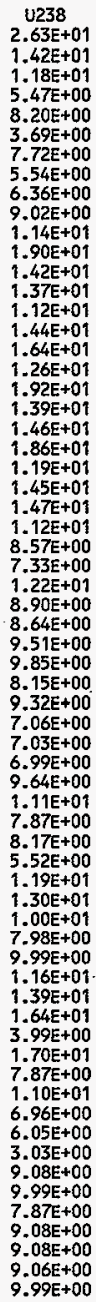 & 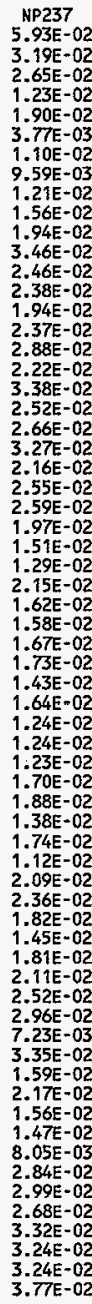 \\
\hline
\end{tabular}


HWF-SD-WM-TI-794, ReV. 0

ACTIVITY IN UNSEPARATED FUEL (Curies decayed to 1/1/94)

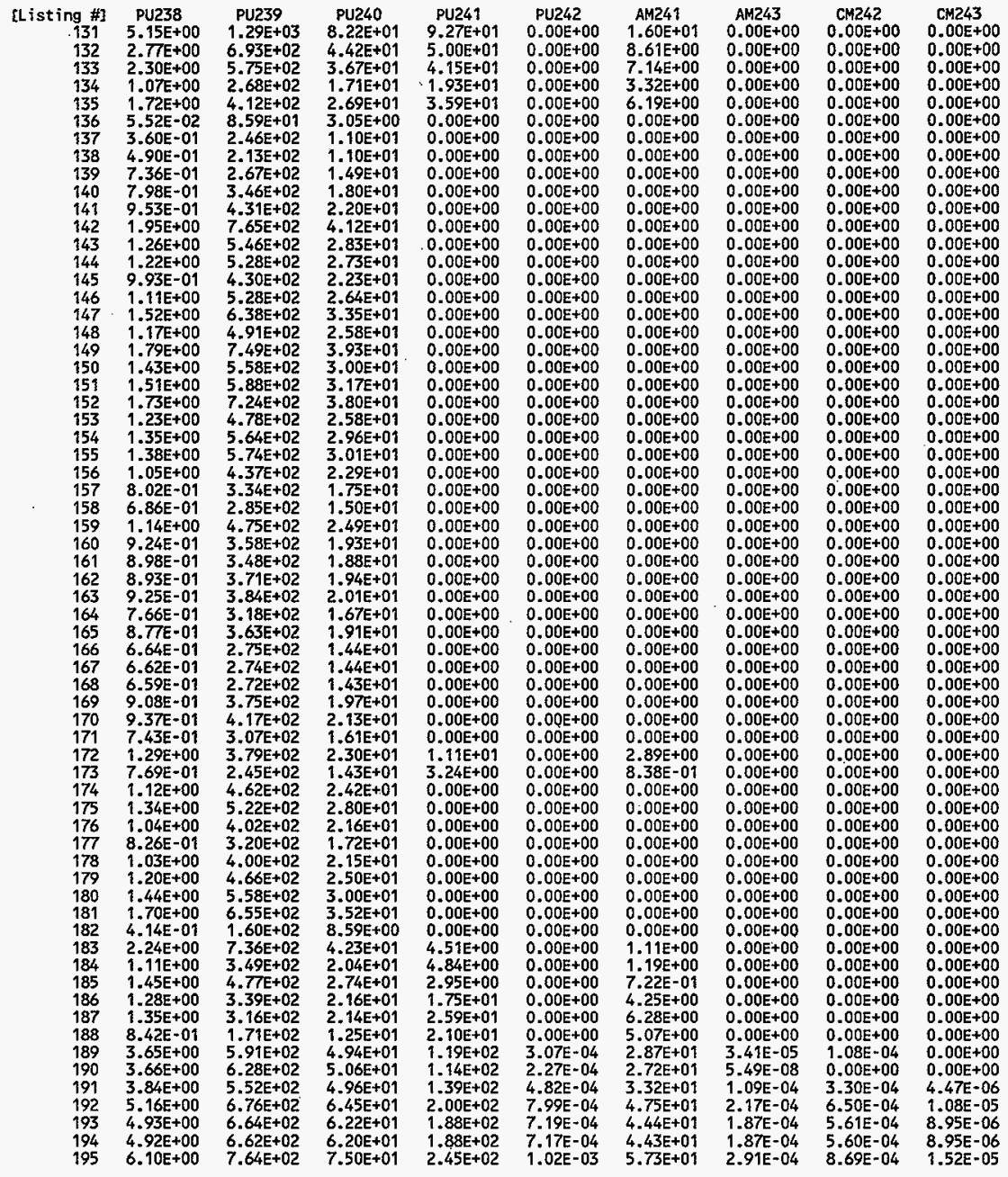


HNF-SD-WM-TI-794, Rev. 0

ACTIVITY IN UNSEPARATED FUEL (Curies decayed to 1/1/94)

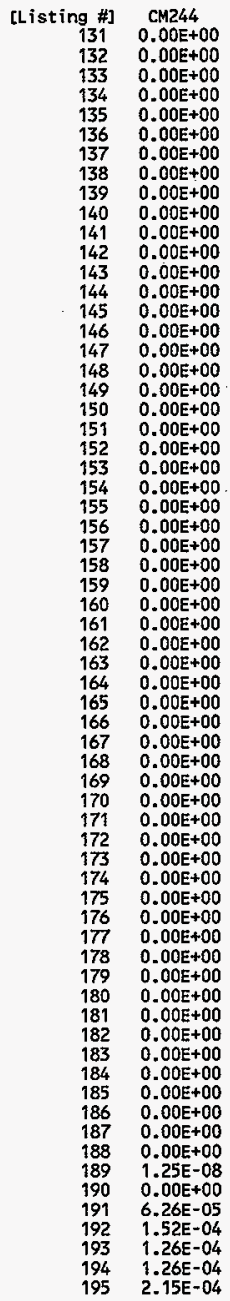


HNF-SD-WM-TI-794, ReV. 0

UNIFIED SEPARATIONS PRODUCTION DATA-

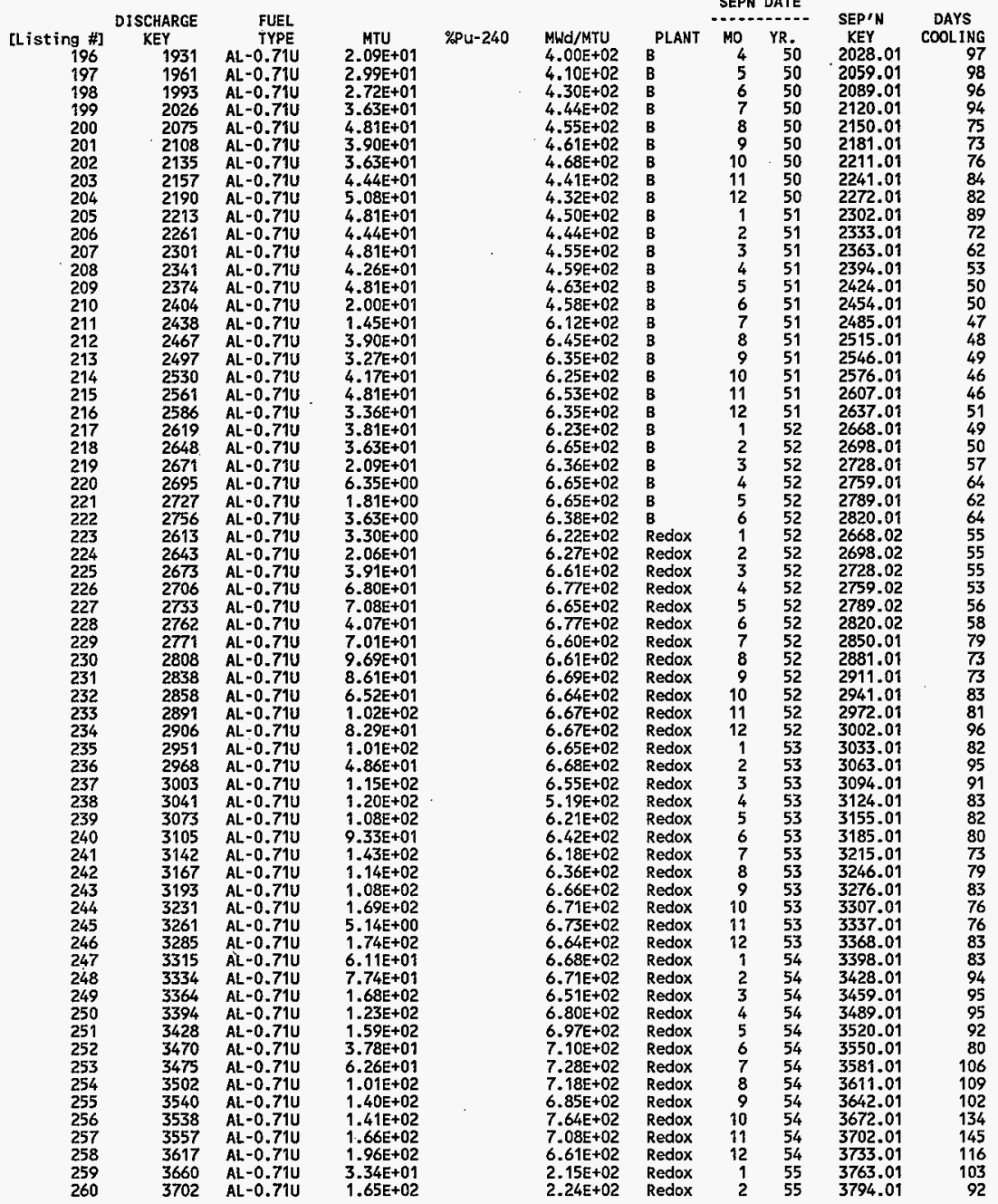


HNF-SD-WM-TI-794, ReV. O

UNIFIED SEPARATIONS PRODUCTION DATA TOTAL WASTE LOSS FRACTION

[Listing \#] Fuel Data Reference

196 SPLANT.DAT 19348 9/30/93 1:19P

197 SPLANT.DAT 19348 9/30/93 1:19P

198 SPLANT.DAT 19348 9/30/93 1:19P 199 SPLANT.DAT 19348 9/30/93 $1: 19 P$ 200 SPLANT.DAT 19348 9/30/93 1:19P 201 SPLANT.DAT 19348 9/30/93 1:19P 202 SPLANT.DAT 19348 9/30/93 1:19P 203 SPLANT.DAT $193489 / 30 / 931: 19 \mathrm{P}$ 204 SPLANT.DAT 19348 9/30/93 1:19P 205 SPLANT.DAT 19348 9/30/93 1:19P 206 SPLANT.DAT 19348 9/30/93 $1: 19 \mathrm{P}$ 207 SPLANT.DAT 19348 9/30/93 1:19P 208 SPLANT.DAT 19348 9/30/93 $1: 19 \mathrm{P}$ 209 SPLANT.DAT 19348 9/30/93 1:19P 210 SPLANT.DAT $19348 \% / 30 / 93$ 1:19P 211 SPLANT.DAT $193489 / 30 / 93 \quad 1: 19 \mathrm{P}$ 212 SPLANT.DAT $193489 / 30 / 93 \quad 1: 19 \mathrm{P}$ 213 SPLANT.DAT 19348 9/30/93 1:19P 214 SPLANT.DAT 19348 9/30/93 $1: 19 \mathrm{P}$ 215 SPLANT.DAT 19348 9/30/93 $1: 19 \mathrm{P}$ 216 SPLANT.DAT 19348 9/30/93 $1: 19 \mathrm{P}$ 217 SPLANT.DAT $19348 \% / 30 / 93 \quad 1: 19 \mathrm{P}$ 218 SPLANT.DAT 19348 9/30/93 $1: 19 P$ 219 SPLANT.DAT 19348 9/30/93 $1: 19 \mathrm{P}$ 220 SPLANT.DAT $19348 \quad 9 / 30 / 93 \quad 1: 19 \mathrm{P}$ 221 SPLANT.DAT 19348 9/30/93 1:19P 222 SPLANT.DAT 19348 9/30/93 1:19P 223 SPLANT.DAT 19348 9/30/93 1:19P 224 SPLANT.DAT 19348 9/30/93 1: $19 \mathrm{P}$ 225 SPLANT_OAT 19348 9/30/93 1: 19P 226 SPLANT.DAT 19348 9/30/93 1:19P 227 SPLANT.DAT 19348 \%/30/93 1:19p 228 SPLANT.DAT 19348 9/30/93 1:19P 229 SPLANT.DAT 19348 9/30/93 1:19P 230 SPLANT.DAT 19348 9/30/93 1:19P 231 SPLANT.DAT 19348 9/30/93 1:19P 232 SPLANT.DAT 19348 9/30/93 1:19P 233 SPLANT DAT 19348 9/30/93 1:19P 234 SPLANT.DAT 19348 9/30/93 1:19P 235 SPLANT.OAT 19348 9/30/93 1: 19P 236 SPLANT.DAT 19348 \%/30/93 1:19P 237 SPLANT.DAT 19348 9/30/93 1:19P 238 SPLANT.DAT 19348 9/30/93 1:19P 239 SPLANT.DAT 19348 9/30/93 1:19P 240 SPLANT.DAT $19348 \% / 30 / 931: 19 P$ 241 SPLANT.DAT 19348 9/30/93 $1: 19 P$ 242 SPLANT.DAT $193489 / 30 / 931: 19 \mathrm{P}$ 243 SPLANT.DAT 19348 9/30/93 1:19P 244 SPLANT.DAT 19348 9/30/93 $1: 19 \mathrm{P}$. 245 SPLANT.DAT $193489 / 30 / 93 \quad 1: 19 \mathrm{P}$ 246 SPLANT.DAT 19348 9/30/93 $1: 19 \mathrm{P}$ 247 SPLANT.DAT 19348 9/30/93 $1: 19 \mathrm{P}$ 248 SPLANT.DAT 19348 9/30/93 1:19P 249 SPLANT DAT 19348 \%/30/93 $1: 19 \mathrm{P}$ 250 SPLANT.DAT 19348 9/30/93 1:19P 251 SPLANT.DAT 19348 9/30/93 1:19P 252 SPLANT.DAT 19348 9/30/93 1:19P 253 SPLANT.DAT 19348 9/30/93 1:19P 254 SPLANT_DAT $19348 \% / 30 / 93$ i:19P 255 SPLANT.DAT 19348 9/30/93 1:19P 256 SPLANT.DAT 19348 9/30/93 1:19P 257 SPLANT.DAT 19348 9/30/93 1:19P 258 SPLANT.DAT 19348 9/30/93 1:19P 259 SPLANT.DAT 19348 9/30/93 1:19P 260 SPLANT_DAT 19348 9/30/93 1:19P $\cdots$

Th

(

u

Np

1

1

Np

p 1

Pu

0.0288

0.0288

0.0288

0.0288

0.0288

0.0288

0.0288

0.0288

0.0288

0.0288

0.0288

0.0288

0.0288

0.0288

0.0288

0.0288

0.0288

0.0288

0.0288

0.0288

0.0288

0.0288

0.0288

0.0288

0.0288

0.0288

0.0288

0.0456

0.0456

0.0373

0.0164

0.0223

0.0232

0.0109

0.0083

0.0112

0.0111

0.0086

0.013

0.0102

0.0117

0.0205

0.0162

0.0198

0.0105

0.0126

0.0122

0.0133

0.0079

0.021

0.0085

0.0117

0.004

0.0069

0.0053

0.0066

0.0215

0.0073

0.0084

0.006

0.0058

0.0067

0.0066

0.0201

0.0079
Waste Frac.

Reference

(a)

(a)

(a)

(a)

(a)

(a)

(a)

(a)

(a)

(a)

(a)

(a)

(a)

(a)

(a)

(a)

(a)

(a)

(a)

(b)

(b)

(b)

(b)

b)

(b)

(b)

(b)

(b)

(b)

(b)

(b)

(b)

(b)

(b)

(b)

(b)

(b)

(b)

(b)

b)

(b)

(b)

(b)

(b)

(b)

(b)

(b)

(b)

(b) 
$H N F-S D-H M-T I-794$, Rev. 0

ACTIVITY IN UNSEPARATED FUEL (Curies decayed to $1 / 1 / 94$ )

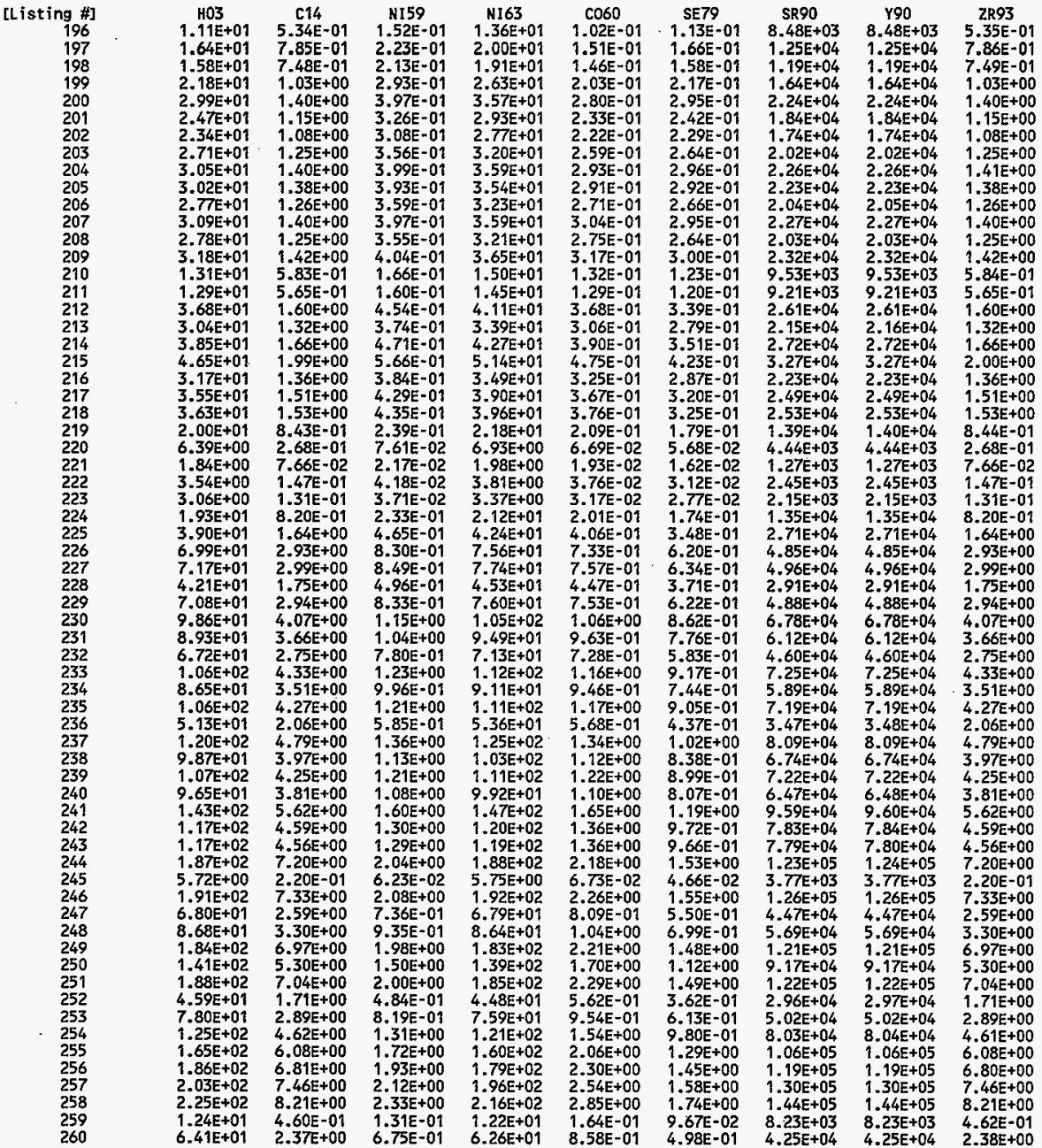


HNF-SD-WN-TI-794, ReV. 0

ACTIVITY IN UNSEPARATED FUEL (Curies decayed to 1/1/94)

\begin{tabular}{|c|c|c|c|c|c|c|c|c|c|}
\hline $\begin{array}{r}\text { isting } 4] \\
196 \\
197 \\
198 \\
199 \\
200 \\
201 \\
202 \\
203 \\
204 \\
205 \\
206 \\
207 \\
208 \\
209 \\
210 \\
211 \\
212 \\
213 \\
214 \\
215 \\
216 \\
217 \\
218 \\
219 \\
220 \\
221 \\
222 \\
223 \\
224 \\
225 \\
226 \\
227 \\
228 \\
229 \\
230 \\
231 \\
232 \\
233 \\
234 \\
235 \\
236 \\
237 \\
238 \\
239 \\
240 \\
241 \\
242 \\
243 \\
244 \\
245 \\
246 \\
247 \\
248 \\
249 \\
250 \\
251 \\
252 \\
253 \\
254 \\
255 \\
256 \\
257 \\
258 \\
259 \\
260\end{array}$ & $\begin{array}{l}\text { NB93* } \\
4.54 \mathrm{E}-01 \\
6.68 \mathrm{E}-01 \\
6.36 \mathrm{E}-01 \\
8.75 \mathrm{E}-01 \\
1.19 \mathrm{E}+00 \\
9.74 \mathrm{E}-01 \\
9.18 \mathrm{E}-01 \\
1.06 \mathrm{E}+00 \\
1.19 \mathrm{E}+00 \\
1.17 \mathrm{E}+00 \\
1.07 \mathrm{E}+00 \\
1.18 \mathrm{E}+00 \\
1.06 \mathrm{E}+00 \\
1.20 \mathrm{E}+00 \\
4.92 \mathrm{E}-01 \\
4.76 \mathrm{E}-01 \\
1.35 \mathrm{E}+00 \\
1.11 \mathrm{E}+00 \\
1.40 \mathrm{E}+00 \\
1.68 \mathrm{E}+00 \\
1.14 \mathrm{E}+00 \\
1.27 \mathrm{E}+00 \\
1.29 \mathrm{E}+00 \\
7.08 \mathrm{E}-01 \\
2.25 \mathrm{E}-01 \\
6.42 \mathrm{E}-02 \\
1.23 \mathrm{E}-01 \\
1.10 \mathrm{E}-01 \\
6.88 \mathrm{E}-01 \\
1.38 \mathrm{E}+00 \\
2.45 \mathrm{E}+00 \\
2.51 \mathrm{E}+00 \\
1.47 \mathrm{E}+00 \\
2.46 \mathrm{E}+00 \\
3.40 \mathrm{E}+00 \\
3.06 \mathrm{E}+00 \\
2.30 \mathrm{E}+00 \\
3.61 \mathrm{E}+00 \\
2.93 \mathrm{E}+00 \\
3.56 \mathrm{E}+00 \\
1.72 \mathrm{E}+00 \\
3.99 \mathrm{E}+00 \\
3.31 \mathrm{E}+00 \\
3.54 \mathrm{E}+00 \\
3.17 \mathrm{E}+00 \\
4.67 \mathrm{E}+00 \\
3.81 \mathrm{E}+00 \\
3.79 \mathrm{E}+00 \\
5.98 \mathrm{E}+00 \\
1.82 \mathrm{E}-01 \\
6.08 \mathrm{E}+00 \\
2.15 \mathrm{E}+00 \\
2.73 \mathrm{E}+00 \\
5.77 \mathrm{E}+00 \\
4.38 \mathrm{E}+00 \\
5.82 \mathrm{E}+00 \\
1.41 \mathrm{E}+00 \\
2.39 \mathrm{E}+00 \\
3.81 \mathrm{E}+00 \\
5.01 \mathrm{E}+00 \\
5.61 \mathrm{E}+00 \\
6.15 \mathrm{E}+00 \\
6.75 \mathrm{E}+00 \\
3.79 \mathrm{E}-01 \\
1.95 \mathrm{E}+00\end{array}$ & $\begin{array}{l}7 C 99 \\
3.71 E+00 \\
5.45 E+00 \\
5.19 E+00 \\
7.16 E+00 \\
9.72 E+00 \\
7.98 E+00 \\
7.53 E+00 \\
8.70 E+00 \\
9.75 E+00 \\
9.60 E+00 \\
8.77 E+00 \\
9.72 E+00 \\
8.68 E+00 \\
9.88 E+00 \\
4.05 E+00 \\
3.94 E+00 \\
1.12 E+01 \\
9.21 E+00 \\
1.16 E+01 \\
1.39 E+01 \\
9.46 E+00 \\
1.05 E+01 \\
1.07 E+01 \\
5.89 E+00 \\
1.87 E+00 \\
5.36 E-01 \\
1.03 E+00 \\
9.12 E-01 \\
5.72 E+00 \\
1.15 E+01 \\
2.05 E+01 \\
2.09 E+01 \\
1.22 E+01 \\
2.05 E+01 \\
2.84 E+01 \\
2.56 E+01 \\
1.92 E+01 \\
3.02 E+01 \\
2.45 E+01 \\
2.98 E+01 \\
1.44 E+01 \\
3.35 E+01 \\
2.76 E+01 \\
2.96 E+01 \\
2.66 E+01 \\
3.92 E+01 \\
3.21 E+01 \\
3.19 E+01 \\
5.04 E+01 \\
1.54 E+00 \\
5.12 E+01 \\
1.81 E+01 \\
2.31 E+01 \\
4.87 E+01 \\
3.70 E+01 \\
4.92 E+01 \\
1.19 E+01 \\
2.02 E+01 \\
3.23 E+01 \\
4.25 E+01 \\
4.77 E+01 \\
5.22 E+01 \\
5.74 E+01 \\
3.18 E+00 \\
1.64 E+01\end{array}$ & $\begin{array}{l}\text { RU106 } \\
6.90 E-09 \\
1.08 E-08 \\
1.10 E-08 \\
1.61 E-08 \\
2.42 E-08 \\
2.11 E-08 \\
2.11 E-08 \\
2.51 E-08 \\
2.99 E-08 \\
3.10 E-08 \\
3.09 E-08 \\
3.69 E-08 \\
3.56 E-08 \\
4.31 E-08 \\
1.87 E-08 \\
2.05 E-08 \\
6.21 E-08 \\
5.41 E-08 \\
7.20 E-08 \\
9.24 E-08 \\
6.58 E-08 \\
7.71 E-08 \\
8.44 E-08 \\
4.81 E-08 \\
1.61 E-08 \\
4.89 E-09 \\
9.85 E-09 \\
6.60 E-09 \\
4.41 E-08 \\
9.46 E-08 \\
1.80 E-07 \\
1.93 E-07 \\
1.19 E-07 \\
2.03 E-07 \\
3.02 E-07 \\
2.89 E-07 \\
2.25 E-07 \\
3.77 E-07 \\
3.14 E-07 \\
4.16 E-07 \\
2.08 E-07 \\
5.09 E-07 \\
4.33 E-07 \\
5.09 E-07 \\
4.91 E-07 \\
7.69 E-07 \\
6.66 E-07 \\
7.01 E-07 \\
1.19 E-06 \\
3.84 E-08 \\
1.34 E-06 \\
5.02 E-07 \\
6.62 E-07 \\
1.46 E-06 \\
1.19 E-06 \\
1.70 E-06 \\
4.46 E-07 \\
7.70 E-07 \\
1.30 E-06 \\
1.81 E-06 \\
2.07 E-06 \\
2.30 E-06 \\
2.80 E-06 \\
1.43 E-07 \\
8.02 E-07\end{array}$ & $\begin{array}{l}C D 113 * \\
1.24 E+00 \\
1.84 E+00 \\
1.76 E+00 \\
2.44 E+00 \\
3.33 E+00 \\
2.75 E+00 \\
2.61 E+00 \\
3.01 E+00 \\
3.39 E+00 \\
3.35 E+00 \\
3.08 E+00 \\
3.43 E+00 \\
3.08 E+00 \\
3.52 E+00 \\
1.45 E+00 \\
1.43 E+00 \\
4.09 E+00 \\
3.38 E+00 \\
4.27 E+00 \\
5.16 E+00 \\
3.52 E+00 \\
3.92 E+00 \\
4.02 E+00 \\
2.21 E+00 \\
7.08 E-01 \\
2.03 E-01 \\
3.91 E-01 \\
3.39 E-01 \\
2.14 E+00 \\
4.32 E+00 \\
7.73 E+00 \\
7.93 E+00 \\
4.66 E+00 \\
7.82 E+00 \\
1.09 E+01 \\
9.85 E+00 \\
7.41 E+00 \\
1.17 E+01 \\
9.52 E+00 \\
1.17 E+01 \\
5.64 E+00 \\
1.31 E+01 \\
1.08 E+01 \\
1.17 E+01 \\
1.06 E+01 \\
1.56 E+01 \\
1.29 E+01 \\
1.28 E+01 \\
2.04 E+01 \\
6.24 E-01 \\
2.09 E+01 \\
7.42 E+00 \\
9.46 E+00 \\
2.00 E+01 \\
1.53 E+01 \\
2.05 E+01 \\
4.99 E+00 \\
8.49 E+00 \\
1.36 E+01 \\
1.80 E+01 \\
2.03 E+01 \\
2.21 E+01 \\
2.44 E+01 \\
1.32 E+00 \\
6.82 E+00\end{array}$ & $\begin{array}{l}\text { SB125 } \\
7.19 \mathrm{E}-02 \\
1.08 \mathrm{E}-01 \\
1.05 \mathrm{E}-01 \\
1.48 \mathrm{E}-01 \\
2.09 \mathrm{E}-01 \\
1.75 \mathrm{E}-01 \\
1.69 \mathrm{E}-01 \\
1.98 \mathrm{E}-01 \\
2.26 \mathrm{E}-01 \\
2.27 \mathrm{E}-01 \\
2.14 \mathrm{E}-01 \\
2.43 \mathrm{E}-01 \\
2.24 \mathrm{E}-01 \\
2.60 \mathrm{E}-01 \\
1.09 \mathrm{E}-01 \\
1.09 \mathrm{E}-01 \\
3.16 \mathrm{E}-01 \\
2.66 \mathrm{E}-01 \\
3.42 \mathrm{E}-01 \\
4.21 \mathrm{E}-01 \\
2.91 \mathrm{E}-01 \\
3.31 \mathrm{E}-01 \\
3.44 \mathrm{E}-01 \\
1.92 \mathrm{E}-01 \\
6.21 \mathrm{E}-02 \\
1.81 \mathrm{E}-02 \\
3.55 \mathrm{E}-02 \\
2.85 \mathrm{E}-02 \\
1.83 \mathrm{E}-01 \\
3.74 \mathrm{E}-01 \\
6.83 \mathrm{E}-01 \\
7.11 \mathrm{E}-01 \\
4.24 \mathrm{E}-01 \\
7.17 \mathrm{E}-01 \\
1.02 \mathrm{E}+00 \\
9.36 \mathrm{E}-01 \\
7.13 \mathrm{E}-01 \\
1.15 \mathrm{E}+00 \\
9.40 \mathrm{E}-01 \\
1.18 \mathrm{E}+00 \\
5.77 \mathrm{E}-01 \\
1.37 \mathrm{E}+00 \\
1.15 \mathrm{E}+00 \\
1.27 \mathrm{E}+00 \\
1.17 \mathrm{E}+00 \\
1.76 \mathrm{E}+00 \\
1.47 \mathrm{E}+00 \\
1.49 \mathrm{E}+00 \\
2.41 \mathrm{E}+00 \\
7.51 \mathrm{E}-02 \\
2.54 \mathrm{E}+00 \\
9.19 \mathrm{E}-01 \\
1.19 \mathrm{E}+00 \\
2.55 \mathrm{E}+00 \\
1.98 \mathrm{E}+00 \\
2.70 \mathrm{E}+00 \\
6.73 \mathrm{E}-01 \\
1.15 \mathrm{E}+00 \\
1.87 \mathrm{E}+00 \\
2.52 \mathrm{E}+00 \\
2.83 \mathrm{E}+00 \\
3.13 \mathrm{E}+00 \\
3.58 \mathrm{E}+00 \\
2.00 \mathrm{E}-01 \\
1.06 \mathrm{E}+00\end{array}$ & $\begin{array}{c}\text { SN126 } \\
1.70 \mathrm{E}-01 \\
2.49 \mathrm{E}-01 \\
2.38 \mathrm{E}-01 \\
3.28 \mathrm{E}-01 \\
4.46 \mathrm{E}-01 \\
3.67 \mathrm{E}-01 \\
3.46 \mathrm{E}-01 \\
3.99 \mathrm{E}-01 \\
4.47 \mathrm{E}-01 \\
4.41 \mathrm{E}-01 \\
4.02 \mathrm{E}-01 \\
4.46 \mathrm{E}-01 \\
3.99 \mathrm{E}-01 \\
4.54 \mathrm{E}-01 \\
1.86 \mathrm{E}-01 \\
1.83 \mathrm{E}-01 \\
5.21 \mathrm{E}-01 \\
4.30 \mathrm{E}-01 \\
5.39 \mathrm{E}-01 \\
6.50 \mathrm{E}-01 \\
4.42 \mathrm{E}-01 \\
4.90 \mathrm{E}-01 \\
5.01 \mathrm{E}-01 \\
2.75 \mathrm{E}-01 \\
8.76 \mathrm{E}-02 \\
2.50 \mathrm{E}-02 \\
4.80 \mathrm{E}-02 \\
4.24 \mathrm{E}-02 \\
2.67 \mathrm{E}-01 \\
5.36 \mathrm{E}-01 \\
9.56 \mathrm{E}-01 \\
9.77 \mathrm{E}-01 \\
5.71 \mathrm{E}-01 \\
9.59 \mathrm{E}-01 \\
1.33 \mathrm{E}+00 \\
1.20 \mathrm{E}+00 \\
8.98 \mathrm{E}-01 \\
1.41 \mathrm{E}+00 \\
1.15 \mathrm{E}+00 \\
1.40 \mathrm{E}+00 \\
6.74 \mathrm{E}-01 \\
1.56 \mathrm{E}+00 \\
1.28 \mathrm{E}+00 \\
1.38 \mathrm{E}+00 \\
1.24 \mathrm{E}+00 \\
1.83 \mathrm{E}+00 \\
1.50 \mathrm{E}+00 \\
1.49 \mathrm{E}+00 \\
2.35 \mathrm{E}+00 \\
7.18 \mathrm{E}-02 \\
2.40 \mathrm{E}+00 \\
8.47 \mathrm{E}-01 \\
1.08 \mathrm{E}+00 \\
2.27 \mathrm{E}+00 \\
1.73 \mathrm{E}+00 \\
2.31 \mathrm{E}+00 \\
5.59 \mathrm{E}-01 \\
9.51 \mathrm{E}-01 \\
1.52 \mathrm{E}+00 \\
1.99 \mathrm{E}+00 \\
2.25 \mathrm{E}+00 \\
2.44 \mathrm{E}+00 \\
2.68 \mathrm{E}+00 \\
1.43 \mathrm{E}-01 \\
7.38 \mathrm{E}-01\end{array}$ & $\begin{array}{l}1129 \\
6.98 \mathrm{E}-03 \\
1.03 \mathrm{E}-02 \\
9.81 \mathrm{E}-03 \\
1.35 \mathrm{E}-02 \\
1.84 \mathrm{E}-02 \\
1.51 \mathrm{E}-02 \\
1.43 \mathrm{E}-02 \\
1.64 \mathrm{E}-02 \\
1.84 \mathrm{E}-02 \\
1.82 \mathrm{E}-02 \\
1.66 \mathrm{E}-02 \\
1.84 \mathrm{E}-02 \\
1.64 \mathrm{E}-02 \\
1.87 \mathrm{E}-02 \\
7.67 \mathrm{E}-03 \\
7.56 \mathrm{E}-03 \\
2.15 \mathrm{E}-02 \\
1.77 \mathrm{E}-02 \\
2.22 \mathrm{E}-02 \\
2.68 \mathrm{E}-02 \\
1.82 \mathrm{E}-02 \\
2.02 \mathrm{E}-02 \\
2.06 \mathrm{E}-02 \\
1.13 \mathrm{E}-02 \\
3.61 \mathrm{E}-03 \\
1.03 \mathrm{E}-03 \\
1.98 \mathrm{E}-03 \\
1.75 \mathrm{E}-03 \\
1.10 \mathrm{E}-02 \\
2.21 \mathrm{E}-02 \\
3.94 \mathrm{E}-02 \\
4.02 \mathrm{E}-02 \\
2.35 \mathrm{E}-02 \\
3.95 \mathrm{E}-02 \\
5.47 \mathrm{E}-02 \\
4.93 \mathrm{E}-02 \\
3.70 \mathrm{E}-02 \\
5.82 \mathrm{E}-02 \\
4.72 \mathrm{E}-02 \\
5.74 \mathrm{E}-02 \\
2.77 \mathrm{E}-02 \\
6.43 \mathrm{E}-02 \\
5.25 \mathrm{E}-02 \\
5.68 \mathrm{E}-02 \\
5.11 \mathrm{E}-02 \\
7.52 \mathrm{E}-02 \\
6.16 \mathrm{E}-02 \\
6.13 \mathrm{E}-02 \\
9.69 \mathrm{E}-02 \\
2.96 \mathrm{E}-03 \\
9.86 \mathrm{E}-02 \\
3.49 \mathrm{E}-02 \\
4.44 \mathrm{E}-02 \\
9.36 \mathrm{E}-02 \\
7.14 \mathrm{E}-02 \\
9.50 \mathrm{E}-02 \\
2.30 \mathrm{E}-02 \\
6.91 \mathrm{E}-02 \\
8.25 \mathrm{E}-02 \\
8.20 \mathrm{E}-02 \\
9.26 \mathrm{E}-02 \\
1.01 \mathrm{E}-01 \\
1.10 \mathrm{E}-01 \\
3.90 \mathrm{E}-03 \\
.04 \mathrm{E}-02\end{array}$ & $\begin{array}{l}\text { CS134 } \\
4.11 \mathrm{E}-04 \\
6.48 \mathrm{E}-04 \\
6.76 \mathrm{E}-04 \\
9.85 \mathrm{E}-04 \\
1.46 \mathrm{E}-03 \\
1.23 \mathrm{E}-03 \\
1.24 \mathrm{E}-03 \\
1.32 \mathrm{E}-03 \\
1.52 \mathrm{E}-03 \\
1.63 \mathrm{E}-03 \\
1.50 \mathrm{E}-03 \\
1.79 \mathrm{E}-03 \\
1.66 \mathrm{E}-03 \\
1.95 \mathrm{E}-03 \\
8.22 \mathrm{E}-04 \\
1.20 \mathrm{E}-03 \\
3.72 \mathrm{E}-03 \\
3.15 \mathrm{E}-03 \\
3.94 \mathrm{E}-03 \\
5.06 \mathrm{E}-03 \\
3.52 \mathrm{E}-03 \\
3.79 \mathrm{E}-03 \\
4.38 \mathrm{E}-03 \\
2.37 \mathrm{E}-03 \\
7.99 \mathrm{E}-04 \\
2.35 \mathrm{E}-04 \\
4.47 \mathrm{E}-04 \\
3.26 \mathrm{E}-04 \\
2.16 \mathrm{E}-03 \\
4.79 \mathrm{E}-03 \\
8.81 \mathrm{E}-03 \\
9.23 \mathrm{E}-03 \\
5.54 \mathrm{E}-03 \\
9.38 \mathrm{E}-03 \\
1.34 \mathrm{E}-02 \\
1.25 \mathrm{E}-02 \\
9.53 \mathrm{E}-03 \\
1.55 \mathrm{E}-02 \\
1.27 \mathrm{E}-02 \\
1.61 \mathrm{E}-02 \\
7.91 \mathrm{E}-03 \\
1.83 \mathrm{E}-02 \\
1.19 \mathrm{E}-02 \\
1.62 \mathrm{E}-02 \\
1.60 \mathrm{E}-02 \\
2.28 \mathrm{E}-02 \\
2.04 \mathrm{E}-02 \\
2.15 \mathrm{E}-02 \\
3.52 \mathrm{E}-02 \\
1.10 \mathrm{E}-03 \\
3.76 \mathrm{E}-02 \\
1.37 \mathrm{E}-02 \\
1.77 \mathrm{E}-02 \\
3.70 \mathrm{E}-02 \\
3.09 \mathrm{E}-02 \\
4.35 \mathrm{E}-02 \\
1.10 \mathrm{E}-02 \\
1.97 \mathrm{E}-02 \\
3.22 \mathrm{E}-02 \\
4.17 \mathrm{E}-02\end{array}$ & 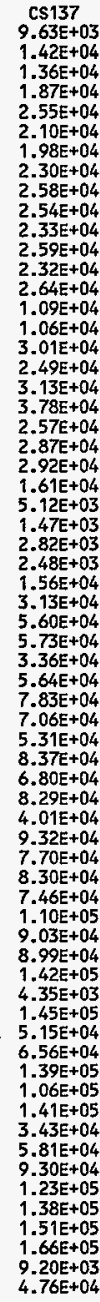 \\
\hline
\end{tabular}


HNF-SD-WH-TI-794, Rev, 0

ACTIVITY IN UNSEPARATED FUEL (Curies decayed to $1 / 1 / 94$ )

\begin{tabular}{|c|c|c|c|c|c|c|c|c|c|}
\hline $\begin{array}{r}\text { isting } \# \\
196 \\
197 \\
198 \\
199 \\
200 \\
201 \\
202 \\
203 \\
204 \\
205 \\
206 \\
207 \\
208 \\
209 \\
210 \\
211 \\
212 \\
213 \\
214 \\
215 \\
216 \\
217 \\
218 \\
219 \\
220 \\
221 \\
222 \\
223 \\
224 \\
225 \\
226 \\
227 \\
228 \\
229 \\
230 \\
231 \\
232 \\
233 \\
234 \\
235 \\
236 \\
237 \\
238 \\
239 \\
240 \\
241 \\
242 \\
243 \\
244 \\
245 \\
246 \\
247 \\
248 \\
249 \\
250 \\
251 \\
252 \\
253 \\
254 \\
255 \\
256 \\
257 \\
258 \\
259 \\
260\end{array}$ & $\begin{array}{l}8 A 137 \\
9.11 \mathrm{E}+03 \\
1.34 \mathrm{E}+04 \\
1.28 \mathrm{E}+04 \\
1.77 \mathrm{E}+04 \\
2.41 \mathrm{E}+04 \\
1.99 \mathrm{E}+04 \\
1.88 \mathrm{E}+04 \\
2.17 \mathrm{E}+04 \\
2.44 \mathrm{E}+04 \\
2.40 \mathrm{E}+04 \\
2.20 \mathrm{E}+04 \\
2.45 \mathrm{E}+04 \\
2.19 \mathrm{E}+04 \\
2.50 \mathrm{E}+04 \\
1.03 \mathrm{E}+04 \\
1.00 \mathrm{E}+04 \\
2.85 \mathrm{E}+04 \\
2.35 \mathrm{E}+04 \\
2.96 \mathrm{E}+04 \\
3.57 \mathrm{E}+04 \\
2.43 \mathrm{E}+04 \\
2.71 \mathrm{E}+04 \\
2.76 \mathrm{E}+04 \\
1.52 \mathrm{E}+04 \\
4.85 \mathrm{E}+03 \\
1.39 \mathrm{E}+03 \\
2.67 \mathrm{E}+03 \\
2.34 \mathrm{E}+03 \\
1.48 \mathrm{E}+04 \\
2.96 \mathrm{E}+04 \\
5.29 \mathrm{E}+04 \\
5.42 \mathrm{E}+04 \\
3.18 \mathrm{E}+04 \\
5.33 \mathrm{E}+04 \\
7.40 \mathrm{E}+04 \\
6.68 \mathrm{E}+04 \\
5.02 \mathrm{E}+04 \\
7.92 \mathrm{E}+04 \\
6.43 \mathrm{E}+04 \\
7.85 \mathrm{E}+04 \\
3.79 \mathrm{E}+04 \\
8.82 \mathrm{E}+04 \\
7.29 \mathrm{E}+04 \\
7.85 \mathrm{E}+04 \\
7.06 \mathrm{E}+04 \\
1.04 \mathrm{E}+05 \\
8.54 \mathrm{E}+04 \\
8.51 \mathrm{E}+04 \\
1.35 \mathrm{E}+05 \\
4.12 \mathrm{E}+03 \\
1.38 \mathrm{E}+05 \\
4.88 \mathrm{E}+04 \\
6.21 \mathrm{E}+04 \\
1.31 \mathrm{E}+05 \\
1.00 \mathrm{E}+05 \\
1.33 \mathrm{E}+05 \\
3.24 \mathrm{E}+04 \\
8.50 \mathrm{E}+04 \\
1.16 \mathrm{E}+05 \\
1.30 \mathrm{E}+05 \\
1.43 \mathrm{E}+05 \\
1.57 \mathrm{E}+05 \\
8.71 \mathrm{E}+03 \\
4.50 \mathrm{E}+04\end{array}$ & 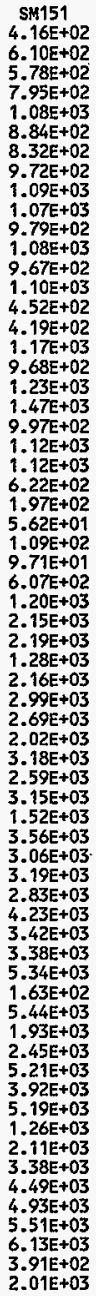 & $\begin{array}{l}\text { EU152 } \\
2.89 \mathrm{E}-02 \\
4.51 \mathrm{E}-02 \\
4.66 \mathrm{E}-02 \\
6.66 \mathrm{E}-02 \\
9.55 \mathrm{E}-02 \\
7.88 \mathrm{E}-02 \\
7.81 \mathrm{E}-02 \\
7.98 \mathrm{E}-02 \\
8.98 \mathrm{E}-02 \\
9.62 \mathrm{E}-02 \\
8.43 \mathrm{E}-02 \\
9.86 \mathrm{E}-02 \\
8.85 \mathrm{E}-02 \\
1.01 \mathrm{E}-01 \\
4.17 \mathrm{E}-02 \\
6.34 \mathrm{E}-02 \\
1.94 \mathrm{E}-01 \\
1.61 \mathrm{E}-01 \\
1.94 \mathrm{E}-01 \\
2.45 \mathrm{E}-01 \\
1.67 \mathrm{E}-01 \\
1.74 \mathrm{E}-01 \\
1.99 \mathrm{E}-01 \\
1.05 \mathrm{E}-01 \\
3.50 \mathrm{E}-02 \\
1.01 \mathrm{E}-02 \\
1.86 \mathrm{E}-02 \\
1.50 \mathrm{E}-02 \\
9.76 \mathrm{E}-02 \\
2.14 \mathrm{E}-01 \\
3.83 \mathrm{E}-01 \\
3.93 \mathrm{E}-01 \\
2.31 \mathrm{E}-01 \\
3.87 \mathrm{E}-01 \\
5.39 \mathrm{E}-01 \\
4.88 \mathrm{E}-01 \\
3.67 \mathrm{E}-01 \\
5.81 \mathrm{E}-01 \\
4.72 \mathrm{E}-01 \\
5.78 \mathrm{E}-01 \\
2.80 \mathrm{E}-01 \\
6.26 \mathrm{E}-01 \\
3.81 \mathrm{E}-01 \\
5.21 \mathrm{E}-01 \\
5.05 \mathrm{E}-01 \\
6.97 \mathrm{E}-01 \\
6.14 \mathrm{E}-01 \\
6.38 \mathrm{E}-01 \\
1.01 \mathrm{E}+00 \\
3.11 \mathrm{E}-02 \\
1.04 \mathrm{E}+00 \\
3.69 \mathrm{E}-01 \\
4.71 \mathrm{E}-01 \\
9.58 \mathrm{E}-01 \\
7.86 \mathrm{E}-01 \\
1.08 \mathrm{E}+00 \\
2.64 \mathrm{E}-01 \\
4.75 \mathrm{E}-01 \\
7.62 \mathrm{E}-01 \\
9.51 \mathrm{E}-01 \\
1.20 \mathrm{E}+00 \\
1.17 \mathrm{E}+00 \\
1.22 \mathrm{E}+00 \\
6.04 \mathrm{E}-03 \\
3.79 \mathrm{E}-02\end{array}$ & $\begin{array}{l}\mathrm{EU154} \\
1.97 \mathrm{E}+00 \\
3.02 \mathrm{E}+00 \\
3.04 \mathrm{E}+00 \\
4.31 \mathrm{E}+00 \\
6.10 \mathrm{E}+00 \\
5.04 \mathrm{E}+00 \\
4.94 \mathrm{E}+00 \\
5.28 \mathrm{E}+00 \\
5.95 \mathrm{E}+00 \\
6.21 \mathrm{E}+00 \\
5.56 \mathrm{E}+00 \\
6.41 \mathrm{E}+00 \\
5.78 \mathrm{E}+00 \\
6.62 \mathrm{E}+00 \\
2.74 \mathrm{E}+00 \\
3.67 \mathrm{E}+00 \\
1.11 \mathrm{E}+01 \\
9.18 \mathrm{E}+00 \\
1.13 \mathrm{E}+01 \\
1.41 \mathrm{E}+01 \\
9.62 \mathrm{E}+00 \\
1.02 \mathrm{E}+01 \\
1.14 \mathrm{E}+01 \\
6.10 \mathrm{E}+00 \\
2.02 \mathrm{E}+00 \\
5.80 \mathrm{E}-01 \\
1.09 \mathrm{E}+00 \\
8.82 \mathrm{E}-01 \\
5.70 \mathrm{E}+00 \\
1.23 \mathrm{E}+01 \\
2.20 \mathrm{E}+01 \\
2.27 \mathrm{E}+01 \\
1.33 \mathrm{E}+01 \\
2.24 \mathrm{E}+01 \\
3.13 \mathrm{E}+01 \\
2.84 \mathrm{E}+01 \\
2.14 \mathrm{E}+01 \\
3.39 \mathrm{E}+01 \\
2.76 \mathrm{E}+01 \\
3.40 \mathrm{E}+01 \\
1.65 \mathrm{E}+01 \\
3.73 \mathrm{E}+01 \\
2.47 \mathrm{E}+01 \\
3.18 \mathrm{E}+01 \\
3.03 \mathrm{E}+01 \\
4.27 \mathrm{E}+01 \\
3.70 \mathrm{E}+01 \\
3.82 \mathrm{E}+01 \\
6.09 \mathrm{E}+01 \\
1.87 \mathrm{E}+00 \\
6.27 \mathrm{E}+01 \\
2.23 \mathrm{E}+01 \\
2.85 \mathrm{E}+01 \\
5.88 \mathrm{E}+01 \\
4.76 \mathrm{E}+01 \\
6.53 \mathrm{E}+01 \\
1.60 \mathrm{E}+01 \\
2.84 \mathrm{E}+01 \\
4.56 \mathrm{E}+01 \\
5.78 \mathrm{E}+01 \\
7.13 \mathrm{E}+01 \\
7.12 \mathrm{E}+01 \\
7.56 \mathrm{E}+01 \\
1.24 \mathrm{E}+00 \\
6.77 \mathrm{E}+00\end{array}$ & $\begin{array}{c}E U 155 \\
1.92 \mathrm{E}+00 \\
2.84 \mathrm{E}+00 \\
2.72 \mathrm{E}+00 \\
3.78 \mathrm{E}+00 \\
5.19 \mathrm{E}+00 \\
4.32 \mathrm{E}+00 \\
4.09 \mathrm{E}+00 \\
4.85 \mathrm{E}+00 \\
5.50 \mathrm{E}+00 \\
5.41 \mathrm{E}+00 \\
5.06 \mathrm{E}+00 \\
5.66 \mathrm{E}+00 \\
5.13 \mathrm{E}+00 \\
5.92 \mathrm{E}+00 \\
2.46 \mathrm{E}+00 \\
2.23 \mathrm{E}+00 \\
6.28 \mathrm{E}+00 \\
5.23 \mathrm{E}+00 \\
6.74 \mathrm{E}+00 \\
8.12 \mathrm{E}+00 \\
5.57 \mathrm{E}+00 \\
6.39 \mathrm{E}+00 \\
6.38 \mathrm{E}+00 \\
3.58 \mathrm{E}+00 \\
1.14 \mathrm{E}+00 \\
3.29 \mathrm{E}-01 \\
6.45 \mathrm{E}+01 \\
5.51 \mathrm{E}-01 \\
3.48 \mathrm{E}+00 \\
6.90 \mathrm{E}+00 \\
1.25 \mathrm{E}+01 \\
1.29 \mathrm{E}+01 \\
7.61 \mathrm{E}+00 \\
1.28 \mathrm{E}+01 \\
1.80 \mathrm{E}+01 \\
1.64 \mathrm{E}+01 \\
1.24 \mathrm{E}+01 \\
1.98 \mathrm{E}+01 \\
1.61 \mathrm{E}+01 \\
2.00 \mathrm{E}+01 \\
9.71 \mathrm{E}+00 \\
2.31 \mathrm{E}+01 \\
2.07 \mathrm{E}+01 \\
2.14 \mathrm{E}+01 \\
1.91 \mathrm{E}+01 \\
2.91 \mathrm{E}+01 \\
2.35 \mathrm{E}+01 \\
2.34 \mathrm{E}+01 \\
3.75 \mathrm{E}+01 \\
1.16 \mathrm{E}+00 \\
3.90 \mathrm{E}+01 \\
1.39 \mathrm{E}+01 \\
1.79 \mathrm{E}+01 \\
3.85 \mathrm{E}+01 \\
2.91 \mathrm{E}+01 \\
3.88 \mathrm{E}+01 \\
9.56 \mathrm{E}+00 \\
1.60 \mathrm{E}+01 \\
2.58 \mathrm{E}+01 \\
3.50 \mathrm{E}+01 \\
3.78 \mathrm{E}+01 \\
4.33 \mathrm{E}+01 \\
4.95 \mathrm{E}+01 \\
3.19 \mathrm{~F}+00\end{array}$ & 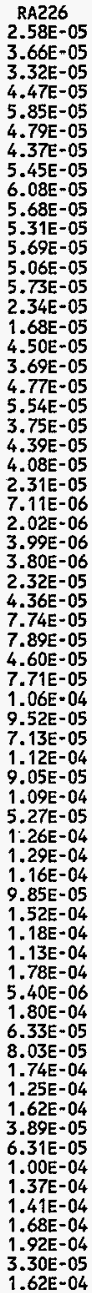 & $\begin{array}{l}\text { RA228 } \\
1.22 \mathrm{E}-10 \\
1.78 \mathrm{E}-10 \\
1.69 \mathrm{E}-10 \\
2.32 \mathrm{E}-10 \\
3.14 \mathrm{E}-10 \\
2.57 \mathrm{E}-10 \\
2.41 \mathrm{E}-10 \\
2.79 \mathrm{E}-10 \\
3.12 \mathrm{E}-10 \\
3.06 \mathrm{E}-10 \\
2.79 \mathrm{E}-10 \\
3.08 \mathrm{E}-10 \\
2.74 \mathrm{E}-10 \\
3.11 \mathrm{E}-10 \\
1.27 \mathrm{E}-10 \\
1.22 \mathrm{E}-10 \\
3.43 \mathrm{E}-10 \\
2.82 \mathrm{E}-10 \\
3.54 \mathrm{E}-10 \\
4.24 \mathrm{E}-10 \\
2.87 \mathrm{E}-10 \\
3.20 \mathrm{E}-10 \\
3.23 \mathrm{E}-10 \\
1.78 \mathrm{E}-10 \\
5.63 \mathrm{E}-11 \\
1.60 \mathrm{E}-11 \\
3.08 \mathrm{E}-11 \\
2.77 \mathrm{E}-11 \\
1.73 \mathrm{E}-10 \\
3.45 \mathrm{E}-10 \\
6.14 \mathrm{E}-10 \\
6.26 \mathrm{E}-10 \\
3.65 \mathrm{E}-10 \\
6.13 \mathrm{E}-10 \\
8.46 \mathrm{E}-10 \\
7.60 \mathrm{E}-10 \\
5.70 \mathrm{E}-10 \\
8.93 \mathrm{E}-10 \\
7.24 \mathrm{E}-10 \\
8.78 \mathrm{E}-10 \\
4.23 \mathrm{E}-10 \\
9.81 \mathrm{E}-10 \\
8.17 \mathrm{E}-10 \\
8.67 \mathrm{E}-10 \\
7.73 \mathrm{E}-10 \\
1.14 \mathrm{E}-09 \\
9.27 \mathrm{E}-10 \\
9.18 \mathrm{E}-10 \\
1.45 \mathrm{E}-09 \\
4.40 \mathrm{E}-11 \\
1.46 \mathrm{E}-09 \\
5.16 \mathrm{E}-10 \\
6.56 \mathrm{E}-10 \\
1.38 \mathrm{E}-09 \\
1.05 \mathrm{E}-09 \\
1.39 \mathrm{E}-09 \\
3.34 \mathrm{E}-10 \\
5.65 \mathrm{E}-10 \\
9.00 \mathrm{E}-10 \\
1.40 \mathrm{~s}\end{array}$ & $\begin{array}{l}\text { AC227 } \\
1.31 E-04 \\
1.85 E-04 \\
1.68 E-04 \\
2.25 E-04 \\
2.95 E-04 \\
2.41 E-04 \\
2.20 E-04 \\
2.76 E-04 \\
3.08 E-04 \\
2.87 E-04 \\
2.69 E-04 \\
2.88 E-04 \\
2.56 E-04 \\
2.91 E-04 \\
1.19 E-04 \\
8.38 E-05 \\
2.24 E-04 \\
1.84 E-04 \\
2.38 E-04 \\
2.76 E-04 \\
1.87 E-04 \\
2.20 E-04 \\
2.04 E-04 \\
1.15 E-04 \\
3.54 E-05 \\
1.01 E-05 \\
2.00 E-05 \\
1.90 E-05 \\
1.16 E-04 \\
2.17 E-04 \\
3.86 E-04 \\
3.94 E-04 \\
2.29 E-04 \\
3.85 E-04 \\
5.31 E-04 \\
4.777 E-04 \\
3.57 E-04 \\
5.60 E-04 \\
4.54 E-04 \\
5.49 E-04 \\
2.65 E-04 \\
6.32 E-04 \\
6.57 E-04 \\
5.89 E-04 \\
4.975-04 \\
7.73 E-04 \\
5.95 E-04 \\
5.71 E-04 \\
8.98 E-04 \\
2.73 E-05 \\
9.08 E-04 \\
3.20 E-04 \\
4.06 E-04 \\
8.84 E-04 \\
6.33 E-04 \\
8.17 E-04 \\
1.97 E-04 \\
3.18 E-04 \\
5.07 E-04 \\
6.96 E-04 \\
7.07 E-04 \\
8.52 E-04 \\
9.79 E-04 \\
1.78 E-04 \\
8.71 E-04\end{array}$ & $\begin{array}{l}\text { TH229 } \\
2.36 \mathrm{E}-08 \\
3.47 \mathrm{E}-08 \\
3.30 \mathrm{E}-08 \\
4.53 \mathrm{E}-08 \\
6.12 \mathrm{E}-08 \\
5.01 \mathrm{E}-08 \\
4.72 \mathrm{E}-08 \\
5.44 \mathrm{E}-08 \\
6.08 \mathrm{E}-08 \\
5.98 \mathrm{E}-08 \\
5.43 \mathrm{E}-08 \\
6.00 \mathrm{E}-08 \\
5.34 \mathrm{E}-08 \\
6.07 \mathrm{E}-08 \\
2.48 \mathrm{E}-08 \\
2.41 \mathrm{E}-08 \\
6.81 \mathrm{E}-08 \\
5.60 \mathrm{E}-08 \\
7.02 \mathrm{E}-08 \\
8.42 \mathrm{E}-08 \\
5.71 \mathrm{E}-08 \\
6.33 \mathrm{E}-08 \\
6.42 \mathrm{E}-08 \\
3.53 \mathrm{E}-08 \\
1.12 \mathrm{E}-08 \\
3.19 \mathrm{E}-09 \\
6.11 \mathrm{E}-09 \\
5.48 \mathrm{E}-09 \\
3.43 \mathrm{E}-08 \\
6.86 \mathrm{E}-08 \\
1.22 \mathrm{E}-07 \\
1.24 \mathrm{E}-07 \\
7.26 \mathrm{E}-08 \\
1.22 \mathrm{E}-07 \\
1.68 \mathrm{E}-07 \\
1.51 \mathrm{E}-07 \\
1.13 \mathrm{E}-07 \\
1.77 \mathrm{E}-07 \\
1.44 \mathrm{E}-07 \\
1.74 \mathrm{E}-07 \\
8.40 \mathrm{E}-08 \\
1.94 \mathrm{E}-07 \\
1.60 \mathrm{E}-07 \\
1.71 \mathrm{E}-07 \\
1.53 \mathrm{E}-07 \\
2.25 \mathrm{E}-07 \\
1.83 \mathrm{E}-07 \\
1.82 \mathrm{E}-07 \\
2.86 \mathrm{E}-07 \\
8.71 \mathrm{E}-09 \\
2.90 \mathrm{E}-07 \\
1.02 \mathrm{E}-07 \\
1.30 \mathrm{E}-07 \\
2.74 \mathrm{E}-07 \\
2.07 \mathrm{E}-07 \\
2.75 \mathrm{E}-07\end{array}$ \\
\hline
\end{tabular}


HNF-SD-WH-TI-794, ReV. 0

ACTIVITY IN UNSEPARATED FUEL (Curies decayed to $1 / 1 / 94$ )

\begin{tabular}{|c|c|c|c|c|c|c|c|c|c|}
\hline $\begin{array}{r}\text { [Listing } \# \text { ] } \\
196 \\
197 \\
198 \\
199 \\
200 \\
201 \\
202 \\
203 \\
204 \\
205 \\
206 \\
207 \\
208 \\
209 \\
210 \\
211 \\
212 \\
213 \\
214 \\
215 \\
216 \\
217 \\
218 \\
219 \\
220 \\
221 \\
222 \\
223 \\
224 \\
225 \\
226 \\
227 \\
228 \\
229 \\
230 \\
231 \\
232 \\
233 \\
234 \\
235 \\
236 \\
237 \\
238 \\
239 \\
240 \\
241 \\
242 \\
243 \\
244 \\
245 \\
246 \\
247 \\
248 \\
249 \\
250 \\
251 \\
252 \\
253 \\
254 \\
255 \\
256 \\
257 \\
258 \\
260 \\
\end{array}$ & $\begin{array}{l}\text { TH232 } \\
1.55 \mathrm{E}-10 \\
2.28 \mathrm{E}-10 \\
2.16 \mathrm{E}-10 \\
2.97 \mathrm{E}-10 \\
4.01 \mathrm{E}-10 \\
3.29 \mathrm{E}-10 \\
3.09 \mathrm{E}-10 \\
3.58 \mathrm{E}-10 \\
4.01 \mathrm{E}-10 \\
3.93 \mathrm{E}-10 \\
3.58 \mathrm{E}-10 \\
3.96 \mathrm{E}-10 \\
3.53 \mathrm{E}-10 \\
4.01 \mathrm{E}-10 \\
1.64 \mathrm{E}-10 \\
1.57 \mathrm{E}-10 \\
4.41 \mathrm{E}-10 \\
3.63 \mathrm{E}-10 \\
4.57 \mathrm{E}-10 \\
5.47 \mathrm{E}-10 \\
3.71 \mathrm{E}-10 \\
4.14 \mathrm{E}-10 \\
4.18 \mathrm{E}-10 \\
2.30 \mathrm{E}-10 \\
7.29 \mathrm{E}-11 \\
2.08 \mathrm{E}-11 \\
3.99 \mathrm{E}-11 \\
3.58 \mathrm{E}-11 \\
2.24 \mathrm{E}-10 \\
4.46 \mathrm{E}-10 \\
7.94 \mathrm{E}-10 \\
8.11 \mathrm{E}-10 \\
4.73 \mathrm{E}-10 \\
7.94 \mathrm{E}-10 \\
1.10 \mathrm{E}-09 \\
9.86 \mathrm{E}-10 \\
7.39 \mathrm{E}-10 \\
1.16 \mathrm{E}-09 \\
9.41 \mathrm{E}-10 \\
1.14 \mathrm{E}-09 \\
5.50 \mathrm{E}-10 \\
1.28 \mathrm{E}-09 \\
1.06 \mathrm{E}-09 \\
1.13 \mathrm{E}-09 \\
1.01 \mathrm{E}-09 \\
1.49 \mathrm{E}-09 \\
1.21 \mathrm{E}-09 \\
1.20 \mathrm{E}-09 \\
1.89 \mathrm{E}-09 \\
5.75 \mathrm{E}-11 \\
1.92 \mathrm{E}-09 \\
6.76 \mathrm{E}-10 \\
8.58 \mathrm{E}-10 \\
1.81 \mathrm{E}-09 \\
1.37 \mathrm{E}-09 \\
1.82 \mathrm{E}-09 \\
4.39 \mathrm{E}-10 \\
7.42 \mathrm{E}-10 \\
1.18 \mathrm{E}-09 \\
1.56 \mathrm{E}-09 \\
1.73 \mathrm{E}-09 \\
1.91 \mathrm{E}-09 \\
2.09 \mathrm{E}-09 \\
1.21 \mathrm{E}-10 \\
6.21 \mathrm{E}-10\end{array}$ & $\begin{array}{l}\text { PA231 } \\
2.82 E-04 \\
4.00 E-04 \\
3.63 E-04 \\
4.88 E-04 \\
6.41 E-04 \\
5.25 E-04 \\
4.80 E-04 \\
6.02 E-04 \\
6.72 E-04 \\
6.27 E-04 \\
5.89 E-04 \\
6.32 E-04 \\
5.63 E-04 \\
6.39 E-04 \\
2.62 E-04 \\
1.85 E-04 \\
4.93 E-04 \\
4.05 E-04 \\
5.26 E-04 \\
6.11 E-04 \\
4.14 E-04 \\
4.87 E-04 \\
4.52 E-04 \\
2.57 E-04 \\
7.88 E-05 \\
2.25 E-05 \\
4.45 E-05 \\
4.22 E-05 \\
2.58 E-04 \\
4.83 E-04 \\
8.59 E-04 \\
8.77 E-04 \\
5.12 E-04 \\
8.59 E-04 \\
1.19 E-03 \\
1.07 E-03 \\
8.00 E-04 \\
1.26 E-03 \\
1.02 E-03 \\
1.23 E-03 \\
5.95 E-04 \\
1.43 E-03 \\
1.48 E-03 \\
1.33 E-03 \\
1.12 E-03 \\
1.75 E-03 \\
1.35 E-03 \\
1.30 E-03 \\
2.04 E-03 \\
6.22 E-05 \\
2.07 E-03 \\
7.31 E-04 \\
9.29 E-04 \\
2.02 E-03 \\
1.45 E-03 \\
1.87 E-03 \\
4.53 E-04 \\
7.31 E-04 \\
1.17 E-03 \\
1.61 E-03 \\
1.63 E-03 \\
1.97 E-03 \\
2.27 E-03 \\
4.13 E-04 \\
2.03 E-03\end{array}$ & $\begin{array}{l}\text { U232 } \\
1.55 E-04 \\
2.31 E-04 \\
2.24 E-04 \\
3.10 E-04 \\
4.26 E-04 \\
3.50 E-04 \\
3.34 E-04 \\
3.76 E-04 \\
4.22 E-04 \\
4.23 E-04 \\
3.83 E-04 \\
4.29 E-04 \\
3.83 E-04 \\
4.37 E-04 \\
1.79 E-04 \\
1.95 E-04 \\
5.66 E-04 \\
4.67 E-04 \\
5.80 E-04 \\
7.08 E-04 \\
4.81 E-04 \\
5.25 E-04 \\
5.53 E-04 \\
3.00 E-04 \\
9.68 E-05 \\
2.77 E-05 \\
5.25 E-05 \\
4.54 E-05 \\
2.88 E-04 \\
5.92 E-04 \\
1.06 E-03 \\
1.08 E-03 \\
6.33 E-04 \\
1.06 E-03 \\
1.47 E-03 \\
1.33 E-03 \\
9.97 E-04 \\
1.57 E-03 \\
1.28 E-03 \\
1.55 E-03 \\
7.50 E-04 \\
1.72 E-03 \\
1.30 E-03 \\
1.49 E-03 \\
1.37 E-03 \\
1.98 E-03 \\
1.66 E-03 \\
1.67 E-03 \\
2.64 E-03 \\
8.05 E-05 \\
2.69 E-03 \\
9.52 E-04 \\
1.21 E-03 \\
2.53 E-03 \\
1.97 E-03 \\
2.65 E-03 \\
6.42 E-04 \\
1.11 E-03 \\
1.78 E-03 \\
2.29 E-03 \\
2.68 E-03 \\
2.81 E-03 \\
3.04 E-03 \\
1.19 E-04 \\
6.20 E-04\end{array}$ & 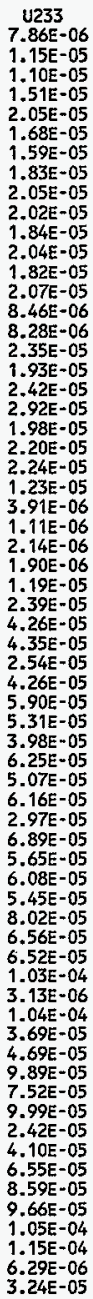 & 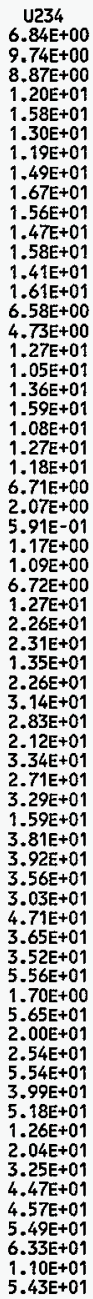 & $\begin{array}{l}\text { U235 } \\
3.02 \mathrm{E}-01 \\
4.29 \mathrm{E}-01 \\
3.90 \mathrm{E}-01 \\
5.26 \mathrm{E}-01 \\
6.92 \mathrm{E}-01 \\
5.68 \mathrm{E}-01 \\
5.20 \mathrm{E}-01 \\
6.53 \mathrm{E}-01 \\
7.32 \mathrm{E}-01 \\
6.84 \mathrm{E}-01 \\
6.44 \mathrm{E}-01 \\
6.92 \mathrm{E}-01 \\
6.18 \mathrm{E}-01 \\
7.04 \mathrm{E}-01 \\
2.89 \mathrm{E}-01 \\
2.04 \mathrm{E}-01 \\
5.45 \mathrm{E}-01 \\
4.49 \mathrm{E}-01 \\
5.84 \mathrm{E}-01 \\
6.79 \mathrm{E}-01 \\
4.61 \mathrm{E}-01 \\
5.44 \mathrm{E}-01 \\
5.05 \mathrm{E}-01 \\
2.87 \mathrm{E}-01 \\
8.84 \mathrm{E}-02 \\
2.53 \mathrm{E}-02 \\
5.01 \mathrm{E}-02 \\
4.71 \mathrm{E}-02 \\
2.89 \mathrm{E}-01 \\
5.41 \mathrm{E}-01 \\
9.65 \mathrm{E}-01 \\
9.86 \mathrm{E}-01 \\
5.77 \mathrm{E}-01 \\
9.68 \mathrm{E}-01 \\
1.34 \mathrm{E}+00 \\
1.21 \mathrm{E}+00 \\
9.07 \mathrm{E}-01 \\
1.43 \mathrm{E}+00 \\
1.16 \mathrm{E}+00 \\
1.41 \mathrm{E}+00 \\
6.80 \mathrm{E}-01 \\
1.63 \mathrm{E}+00 \\
1.71 \mathrm{E}+00 \\
1.53 \mathrm{E}+00 \\
1.30 \mathrm{E}+00 \\
2.03 \mathrm{E}+00 \\
1.56 \mathrm{E}+00 \\
1.50 \mathrm{E}+00 \\
2.38 \mathrm{E}+00 \\
7.25 \mathrm{E}-02 \\
2.42 \mathrm{E}+00 \\
8.55 \mathrm{E}-01 \\
1.09 \mathrm{E}+00 \\
2.37 \mathrm{E}+00 \\
1.70 \mathrm{E}+00 \\
2.21 \mathrm{E}+00 \\
5.35 \mathrm{E}-01 \\
8.64 \mathrm{E}-01 \\
1.38 \mathrm{E}+00 \\
1.91 \mathrm{E}+00 \\
1.93 \mathrm{E}+00 \\
2.34 \mathrm{E}+00 \\
2.71 \mathrm{E}+00 \\
4.96 \mathrm{E}-01\end{array}$ & $\begin{array}{l}\text { U236 } \\
7.14 \mathrm{E}-02 \\
1.05 \mathrm{E}-01 \\
9.98 \mathrm{E}-02 \\
1.37 \mathrm{E}-01 \\
1.86 \mathrm{E}-01 \\
1.53 \mathrm{E}-01 \\
1.44 \mathrm{E}-01 \\
1.67 \mathrm{E}-01 \\
1.87 \mathrm{E}-01 \\
1.84 \mathrm{E}-01 \\
1.68 \mathrm{E}-01 \\
1.86 \mathrm{E}-01 \\
1.66 \mathrm{E}-01 \\
1.90 \mathrm{E}-01 \\
7.77 \mathrm{E}-02 \\
7.44 \mathrm{E}-02 \\
2.10 \mathrm{E}-01 \\
1.73 \mathrm{E}-01 \\
2.18 \mathrm{E}-01 \\
2.62 \mathrm{E}-01 \\
1.78 \mathrm{E}-01 \\
1.99 \mathrm{E}-01 \\
2.01 \mathrm{E}-01 \\
1.11 \mathrm{E}-01 \\
3.52 \mathrm{E}-02 \\
1.01 \mathrm{E}-02 \\
1.93 \mathrm{E}-02 \\
1.72 \mathrm{E}-02 \\
1.08 \mathrm{E}-01 \\
2.15 \mathrm{E}-01 \\
3.84 \mathrm{E}-01 \\
3.92 \mathrm{E}-01 \\
2.29 \mathrm{E}-01 \\
3.85 \mathrm{E}-01 \\
5.33 \mathrm{E}-01 \\
4.80 \mathrm{E}-01 \\
3.61 \mathrm{E}-01 \\
5.67 \mathrm{E}-01 \\
4.60 \mathrm{E}-01 \\
5.60 \mathrm{E}-01 \\
2.70 \mathrm{E}-01 \\
6.29 \mathrm{E}-01 \\
5.26 \mathrm{E}-01 \\
5.59 \mathrm{E}-01 \\
5.00 \mathrm{E}-01 \\
7.40 \mathrm{E}-01 \\
6.03 \mathrm{E}-01 \\
5.98 \mathrm{E}-01 \\
9.45 \mathrm{E}-01 \\
2.88 \mathrm{E}-02 \\
9.61 \mathrm{E}-01 \\
3.40 \mathrm{E}-01 \\
4.32 \mathrm{E}-01 \\
9.15 \mathrm{E}-01 \\
6.94 \mathrm{E}-01 \\
9.21 \mathrm{E}-01 \\
2.23 \mathrm{E}-01 \\
3.77 \mathrm{E}-01 \\
6.02 \mathrm{E}-01 \\
7.95 \mathrm{E}-01 \\
8.86 \mathrm{E}-01 \\
9.76 \mathrm{E}-01 \\
1.08 \mathrm{E}+00 \\
6.24 \mathrm{E}-02 \\
3.21 \mathrm{E}-01\end{array}$ & $\begin{array}{l}U 238 \\
6.96 E+00 \\
9.91 E+00 \\
9.03 E+00 \\
1.22 E+01 \\
1.61 E+01 \\
1.32 E+01 \\
1.21 E+01 \\
1.51 E+01 \\
1.69 E+01 \\
1.59 E+01 \\
1.49 E+01 \\
1.61 E+01 \\
1.44 E+01 \\
1.64 E+01 \\
6.70 E+00 \\
4.84 E+00 \\
1.30 E+01 \\
1.07 E+01 \\
1.39 E+01 \\
1.62 E+01 \\
1.10 E+01 \\
1.29 E+01 \\
1.21 E+01 \\
6.86 E+00 \\
2.12 E+00 \\
6.05 E-01 \\
1.20 E+00 \\
1.12 E+00 \\
6.88 E+00 \\
1.30 E+01 \\
2.31 E+01 \\
2.36 E+01 \\
1.38 E+01 \\
2.32 E+01 \\
3.21 E+01 \\
2.89 E+01 \\
2.17 E+01 \\
3.42 E+01 \\
2.77 E+01 \\
3.37 E+01 \\
1.63 E+01 \\
3.90 E+01 \\
4.00 E+01 \\
3.64 E+01 \\
3.10 E+01 \\
4.82 E+01 \\
3.73 E+01 \\
3.60 E+01 \\
5.69 E+01 \\
1.74 E+00 \\
5.79 E+01 \\
2.05 E+01 \\
2.60 E+01 \\
5.67 E+01 \\
4.09 E+01 \\
5.31 E+01 \\
1.29 E+01 \\
2.09 E+01 \\
3.33 E+01 \\
4.58 E+01 \\
4.69 E+01 \\
5.63 E+01 \\
6.48 E+01 \\
1.11 E+01\end{array}$ & $\begin{array}{l}\text { HP237 } \\
2.29 \mathrm{E}-02 \\
3.37 \mathrm{E}-02 \\
3.23 \mathrm{E}-02 \\
4.45 \mathrm{E}-02 \\
6.06 \mathrm{E}-02 \\
4.98 \mathrm{E}-02 \\
4.71 \mathrm{E}-02 \\
5.41 \mathrm{E}-02 \\
6.05 \mathrm{E}-02 \\
5.99 \mathrm{E}-02 \\
5.45 \mathrm{E}-02 \\
6.06 \mathrm{E}-02 \\
5.41 \mathrm{E}-02 \\
6.16 \mathrm{E}-02 \\
2.53 \mathrm{E}-02 \\
2.53 \mathrm{E}-02 \\
7.22 \mathrm{E}-02 \\
5.95 \mathrm{E}-02 \\
7.46 \mathrm{E}-02 \\
9.00 \mathrm{E}-02 \\
6.12 \mathrm{E}-02 \\
6.77 \mathrm{E}-02 \\
6.95 \mathrm{E}-02 \\
3.81 \mathrm{E}-02 \\
1.22 \mathrm{E}-02 \\
3.47 \mathrm{E}-03 \\
6.64 \mathrm{E}-03 \\
5.85 \mathrm{E}-03 \\
3.68 \mathrm{E}-02 \\
7.44 \mathrm{E}-02 \\
1.33 \mathrm{E}-01 \\
1.36 \mathrm{E}-01 \\
7.93 \mathrm{E}-02 \\
1.33 \mathrm{E}-01 \\
1.84 \mathrm{E}-01 \\
1.66 \mathrm{E}-01 \\
1.25 \mathrm{E}-01 \\
1.96 \mathrm{E}-01 \\
1.59 \mathrm{E}-01 \\
1.93 \mathrm{E}-01 \\
9.34 \mathrm{E}-02 \\
2.16 \mathrm{E}-01 \\
1.74 \mathrm{E}-01 \\
1.90 \mathrm{E}-01 \\
1.72 \mathrm{E}-01 \\
2.52 \mathrm{E}-01 \\
2.07 \mathrm{E}-01 \\
2.06 \mathrm{E}-01 \\
3.26 \mathrm{E}-01 \\
9.94 \mathrm{E}-03 \\
3.32 \mathrm{E}-01 \\
1.17 \mathrm{~T}-01 \\
1.49 \mathrm{E}-01 \\
3.14 \mathrm{E}-01 \\
2.40 \mathrm{E}-01 \\
3.21 \mathrm{E}-01 \\
7.76 \mathrm{E}-02 \\
1.32 \mathrm{E}-01 \\
2.12 \mathrm{E}-01 \\
2.77 \mathrm{E}-01 \\
3.14 \mathrm{E}-01 \\
3.39 \mathrm{E}-01 \\
3.71 \mathrm{E}-01 \\
1.89 \mathrm{E}-02 \\
9.77 \mathrm{E}-02\end{array}$ \\
\hline
\end{tabular}


HNF-SD-WM-TI-794, ReV. 0

ACTIVITY IN UHSEPARATED FUEL (CUries decayed to $1 / 1 / 94$ )

\begin{tabular}{|c|c|c|c|c|c|c|c|c|c|}
\hline 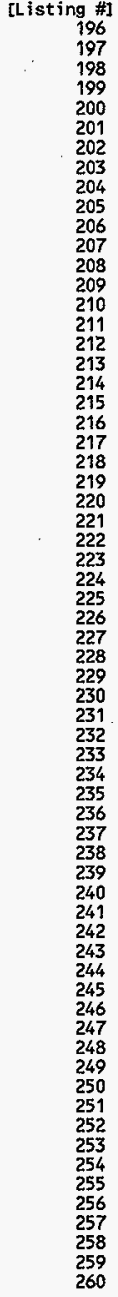 & $\begin{array}{l}P U 238 \\
3.15 \mathrm{E}+00 \\
4.85 \mathrm{E}+00 \\
4.92 \mathrm{E}+00 \\
6.97 \mathrm{E}+00 \\
9.85 \mathrm{E}+00 \\
8.10 \mathrm{E}+00 \\
7.94 \mathrm{E}+00 \\
8.27 \mathrm{E}+00 \\
9.27 \mathrm{E}+00 \\
9.76 \mathrm{E}+00 \\
8.58 \mathrm{E}+00 \\
9.90 \mathrm{E}+00 \\
8.85 \mathrm{E}+00 \\
1.01 \mathrm{E}+01 \\
4.14 \mathrm{E}+00 \\
5.89 \mathrm{E}+00 \\
1.78 \mathrm{E}+01 \\
1.47 \mathrm{E}+01 \\
1.78 \mathrm{E}+01 \\
2.23 \mathrm{E}+01 \\
1.51 \mathrm{E}+01 \\
1.58 \mathrm{E}+01 \\
1.78 \mathrm{E}+01 \\
9.43 \mathrm{E}+00 \\
3.11 \mathrm{E}+00 \\
8.90 \mathrm{E}-01 \\
1.65 \mathrm{E}+00 \\
1.37 \mathrm{E}+00 \\
8.81 \mathrm{E}+00 \\
1.90 \mathrm{E}+01 \\
3.40 \mathrm{E}+01 \\
3.48 \mathrm{E}+01 \\
2.03 \mathrm{E}+01 \\
3.42 \mathrm{E}+01 \\
4.73 \mathrm{E}+01 \\
4.27 \mathrm{E}+01 \\
3.20 \mathrm{E}+01 \\
5.05 \mathrm{E}+01 \\
4.10 \mathrm{E}+01 \\
4.99 \mathrm{E}+01 \\
2.41 \mathrm{E}+01 \\
5.40 \mathrm{E}+01 \\
3.40 \mathrm{E}+01 \\
4.49 \mathrm{E}+01 \\
4.30 \mathrm{E}+01 \\
5.95 \mathrm{E}+01 \\
5.19 \mathrm{E}+01 \\
5.35 \mathrm{E}+01 \\
8.46 \mathrm{E}+01 \\
2.58 \mathrm{E}+00 \\
8.62 \mathrm{E}+01 \\
3.05 \mathrm{E}+01 \\
3.88 \mathrm{E}+01 \\
7.91 \mathrm{E}+01 \\
6.42 \mathrm{E}+01 \\
8.79 \mathrm{E}+01 \\
2.13 \mathrm{E}+01 \\
3.81 \mathrm{E}+01 \\
6.09 \mathrm{E}+01 \\
7.61 \mathrm{E}+01 \\
9.52 \mathrm{E}+01 \\
9.34 \mathrm{E}+01 \\
9.72 \mathrm{E}+01 \\
1.01 \mathrm{E}+00 \\
5.66 \mathrm{E}+00\end{array}$ & $\begin{array}{l}\text { PU239 } \\
4.74 \mathrm{E}+02 \\
6.95 \mathrm{E}+02 \\
6.60 \mathrm{E}+02 \\
9.08 \mathrm{E}+02 \\
1.23 \mathrm{E}+03 \\
1.01 \mathrm{E}+03 \\
9.51 \mathrm{E}+02 \\
1.11 \mathrm{E}+03 \\
1.24 \mathrm{E}+03 \\
1.22 \mathrm{E}+03 \\
1.11 \mathrm{E}+03 \\
1.23 \mathrm{E}+03 \\
1.10 \mathrm{E}+03 \\
1.25 \mathrm{E}+03 \\
5.13 \mathrm{E}+02 \\
4.84 \mathrm{E}+02 \\
1.36 \mathrm{E}+03 \\
1.12 \mathrm{E}+03 \\
1.42 \mathrm{E}+03 \\
1.70 \mathrm{E}+03 \\
1.15 \mathrm{E}+03 \\
1.29 \mathrm{E}+03 \\
1.30 \mathrm{E}+03 \\
7.19 \mathrm{E}+02 \\
2.28 \mathrm{E}+02 \\
6.51 \mathrm{E}+01 \\
1.25 \mathrm{E}+02 \\
1.12 \mathrm{E}+02 \\
7.01 \mathrm{E}+02 \\
1.39 \mathrm{E}+03 \\
2.48 \mathrm{E}+03 \\
2.54 \mathrm{E}+03 \\
1.49 \mathrm{E}+03 \\
2.49 \mathrm{E}+03 \\
3.45 \mathrm{E}+03 \\
3.11 \mathrm{E}+03 \\
2.34 \mathrm{E}+03 \\
3.67 \mathrm{E}+03 \\
2.98 \mathrm{E}+03 \\
3.63 \mathrm{E}+03 \\
1.75 \mathrm{E}+03 \\
4.08 \mathrm{E}+03 \\
3.45 \mathrm{E}+03 \\
3.64 \mathrm{E}+03 \\
3.24 \mathrm{E}+03 \\
4.82 \mathrm{E}+03 \\
3.91 \mathrm{E}+03 \\
3.87 \mathrm{E}+03 \\
6.12 \mathrm{E}+03 \\
1.87 \mathrm{E}+02 \\
6.22 \mathrm{E}+03 \\
2.20 \mathrm{E}+03 \\
2.80 \mathrm{E}+03 \\
5.94 \mathrm{E}+03 \\
4.48 \mathrm{E}+03 \\
5.94 \mathrm{E}+03 \\
1.44 \mathrm{E}+03 \\
2.43 \mathrm{E}+03 \\
3.88 \mathrm{E}+03 \\
5.13 \mathrm{E}+03 \\
5.68 \mathrm{E}+03 \\
6.30 \mathrm{E}+03 \\
6.97 \mathrm{E}+03 \\
4.21 \mathrm{E}+02 \\
2.16 \mathrm{E}+03\end{array}$ & $\begin{array}{l}\text { PU240 } \\
4.13 \mathrm{E}+01 \\
6.25 \mathrm{E}+01 \\
6.19 \mathrm{E}+01 \\
8.67 \mathrm{E}+01 \\
1.21 \mathrm{E}+02 \\
9.92 \mathrm{E}+01 \\
9.60 \mathrm{E}+01 \\
1.04 \mathrm{E}+02 \\
1.16 \mathrm{E}+02 \\
1.19 \mathrm{E}+02 \\
1.06 \mathrm{E}+02 \\
1.21 \mathrm{E}+02 \\
1.08 \mathrm{E}+02 \\
1.23 \mathrm{E}+02 \\
5.04 \mathrm{E}+01 \\
6.37 \mathrm{E}+01 \\
1.89 \mathrm{E}+02 \\
1.56 \mathrm{E}+02 \\
1.91 \mathrm{E}+02 \\
2.36 \mathrm{E}+02 \\
1.60 \mathrm{E}+02 \\
1.70 \mathrm{E}+02 \\
1.86 \mathrm{E}+02 \\
9.98 \mathrm{E}+01 \\
3.26 \mathrm{E}+01 \\
9.32 \mathrm{E}+00 \\
1.74 \mathrm{E}+01 \\
1.47 \mathrm{E}+01 \\
9.42 \mathrm{E}+01 \\
2.00 \mathrm{E}+02 \\
3.56 \mathrm{E}+02 \\
3.64 \mathrm{E}+02 \\
2.13 \mathrm{E}+02 \\
3.57 \mathrm{E}+02 \\
4.95 \mathrm{E}+02 \\
4.46 \mathrm{E}+02 \\
3.35 \mathrm{E}+02 \\
5.26 \mathrm{E}+02 \\
4.27 \mathrm{E}+02 \\
5.20 \mathrm{E}+02 \\
2.51 \mathrm{E}+02 \\
5.67 \mathrm{E}+02 \\
3.85 \mathrm{E}+02 \\
4.79 \mathrm{E}+02 \\
4.51 \mathrm{E}+02 \\
6.34 \mathrm{E}+02 \\
5.43 \mathrm{E}+02 \\
5.55 \mathrm{E}+02 \\
8.76 \mathrm{E}+02 \\
2.67 \mathrm{E}+01 \\
8.92 \mathrm{E}+02 \\
3.16 \mathrm{E}+02 \\
4.01 \mathrm{E}+02 \\
8.25 \mathrm{E}+02 \\
6.58 \mathrm{E}+02 \\
8.94 \mathrm{E}+02 \\
2.17 \mathrm{E}+02 \\
3.83 \mathrm{E}+02 \\
6.11 \mathrm{E}+02 \\
7.72 \mathrm{E}+02 \\
9.42 \mathrm{E}+02 \\
9.47 \mathrm{E}+02 \\
9.98 \mathrm{E}+02 \\
2.15 \mathrm{E}+01 \\
1.15 \mathrm{E}+02\end{array}$ & $\begin{array}{l}\text { PU241 } \\
1.12 \mathrm{E}+02 \\
1.79 \mathrm{E}+02 \\
1.91 \mathrm{E}+02 \\
2.76 \mathrm{E}+02 \\
4.03 \mathrm{E}+02 \\
3.32 \mathrm{E}+02 \\
3.35 \mathrm{E}+02 \\
3.27 \mathrm{E}+02 \\
3.67 \mathrm{E}+02 \\
4.05 \mathrm{E}+02 \\
3.49 \mathrm{E}+02 \\
4.15 \mathrm{E}+02 \\
3.73 \mathrm{E}+02 \\
4.26 \mathrm{E}+02 \\
1.76 \mathrm{E}+02 \\
3.00 \mathrm{E}+02 \\
9.30 \mathrm{E}+02 \\
7.69 \mathrm{E}+02 \\
9.24 \mathrm{E}+02 \\
1.17 \mathrm{E}+03 \\
8.00 \mathrm{E}+02 \\
8.21 \mathrm{E}+02 \\
9.58 \mathrm{E}+02 \\
5.04 \mathrm{E}+02 \\
1.69 \mathrm{E}+02 \\
4.84 \mathrm{E}+01 \\
8.89 \mathrm{E}+01 \\
7.10 \mathrm{E}+01 \\
4.63 \mathrm{E}+02 \\
1.03 \mathrm{E}+03 \\
1.84 \mathrm{E}+03 \\
1.89 \mathrm{E}+03 \\
1.11 \mathrm{E}+03 \\
1.87 \mathrm{E}+03 \\
2.60 \mathrm{E}+03 \\
2.35 \mathrm{E}+03 \\
1.77 \mathrm{E}+03 \\
2.79 \mathrm{E}+03 \\
2.27 \mathrm{E}+03 \\
2.78 \mathrm{E}+03 \\
1.35 \mathrm{E}+03 \\
2.99 \mathrm{E}+03 \\
1.70 \mathrm{E}+03 \\
2.45 \mathrm{E}+03 \\
2.41 \mathrm{E}+03 \\
3.28 \mathrm{E}+03 \\
2.93 \mathrm{E}+03 \\
3.06 \mathrm{E}+03 \\
4.86 \mathrm{E}+03 \\
1.49 \mathrm{E}+02 \\
4.98 \mathrm{E}+03 \\
1.77 \mathrm{E}+03 \\
2.26 \mathrm{E}+03 \\
4.56 \mathrm{E}+03 \\
3.79 \mathrm{E}+03 \\
5.25 \mathrm{E}+03 \\
1.28 \mathrm{E}+03 \\
2.32 \mathrm{E}+03 \\
3.72 \mathrm{E}+03 \\
4.60 \mathrm{E}+03 \\
5.92 \mathrm{E}+03 \\
5.66 \mathrm{E}+03 \\
5.83 \mathrm{E}+03 \\
0.00 \mathrm{E}+00 \\
0.00 \mathrm{E}+00\end{array}$ & $\begin{array}{l}\text { PU242 } \\
3.44 E-04 \\
6.07 E-04 \\
7.15 E-04 \\
1.07 E-03 \\
1.64 E-03 \\
1.34 E-03 \\
1.41 E-03 \\
1.20 E-03 \\
1.34 E-03 \\
1.62 E-03 \\
1.31 E-03 \\
1.64 E-03 \\
1.46 E-03 \\
1.67 E-03 \\
6.82 E-04 \\
1.53 E-03 \\
4.86 E-03 \\
4.01 E-03 \\
4.72 E-03 \\
6.06 E-03 \\
4.12 E-03 \\
4.10 E-03 \\
4.96 E-03 \\
2.57 E-03 \\
8.68 E-04 \\
2.48 E-04 \\
4.48 E-04 \\
3.55 E-04 \\
2.33 E-03 \\
5.31 E-03 \\
9.47 E-03 \\
9.68 E-03 \\
5.66 E-03 \\
9.50 E-03 \\
1.32 E-02 \\
1.19 E-02 \\
8.90 E-03 \\
1.40 E-02 \\
1.14 E-02 \\
1.38 E-02 \\
6.67 E-03 \\
1.46 E-02 \\
7.13 E-03 \\
1.15 E-02 \\
1.16 E-02 \\
1.53 E-02 \\
1.40 E-02 \\
1.48 E-02 \\
2.33 E-02 \\
7.11 E-02 \\
2.37 E-02 \\
8.39 E-03 \\
1.07 E-02 \\
2.12 E-02 \\
1.79 E-02 \\
2.50 E-02 \\
6.05 E-03 \\
1.12 E-03 \\
1.78 E-02 \\
2.16 E-02 \\
2.87 E-02 \\
2.65 E-02 \\
2.66 E-02 \\
0.00 E+00 \\
0.00 E+00\end{array}$ & $\begin{array}{l}\text { AM241 } \\
2.61 E+01 \\
4.17 E+01 \\
4.41 E+01 \\
6.36 E+01 \\
9.21 E+01 \\
7.55 E+01 \\
7.58 E+01 \\
7.37 E+01 \\
8.25 E+01 \\
9.07 E+01 \\
7.76 E+01 \\
9.17 E+01 \\
8.19 E+01 \\
9.32 E+01 \\
3.82 E+01 \\
6.50 E+01 \\
2.01 E+02 \\
1.65 E+02 \\
1.98 E+02 \\
2.50 E+02 \\
1.70 E+02 \\
1.73 E+02 \\
2.01 E+02 \\
1.06 E+02 \\
3.52 E+01 \\
1.01 E+01 \\
1.84 E+01 \\
1.50 E+01 \\
9.74 E+01 \\
2.15 E+02 \\
3.84 E+02 \\
3.92 E+02 \\
2.29 E+02 \\
3.85 E+02 \\
5.33 E+02 \\
4.79 E+02 \\
3.60 E+02 \\
5.66 E+02 \\
4.59 E+02 \\
5.58 E+02 \\
2.69 E+02 \\
5.96 E+02 \\
3.36 E+02 \\
4.84 E+02 \\
4.73 E+02 \\
6.39 E+02 \\
5.69 E+02 \\
5.93 E+02 \\
9.37 E+02 \\
2.86 E+01 \\
9.52 E+02 \\
3.37 E+02 \\
4.28 E+02 \\
8.61 E+02 \\
7.12 E+02 \\
9.82 E+02 \\
2.38 E+02 \\
4.31 E+02 \\
6.88 E+02 \\
8.46 E+02 \\
1.09 E+03 \\
1.04 E+03 \\
1.06 E+03 \\
0.00 E+00 \\
0.00 E+00\end{array}$ & $\begin{array}{c}\text { AM243 } \\
6.63 \mathrm{E}-05 \\
1.38 \mathrm{E}-04 \\
1.86 \mathrm{E}-04 \\
2.92 \mathrm{E}-04 \\
4.68 \mathrm{E}-04 \\
3.84 \mathrm{E}-04 \\
4.19 \mathrm{E}-04 \\
3.12 \mathrm{E}-04 \\
3.49 \mathrm{E}-04 \\
4.62 \mathrm{E}-04 \\
3.57 \mathrm{E}-04 \\
4.68 \mathrm{E}-04 \\
4.18 \mathrm{E}-04 \\
4.76 \mathrm{E}-04 \\
1.95 \mathrm{E}-04 \\
5.39 \mathrm{E}-04 \\
1.73 \mathrm{E}-03 \\
1.43 \mathrm{E}-03 \\
1.67 \mathrm{E}-03 \\
2.16 \mathrm{E}-03 \\
1.47 \mathrm{E}-03 \\
1.44 \mathrm{E}-03 \\
1.78 \mathrm{E}-03 \\
9.14 \mathrm{E}-04 \\
3.12 \mathrm{E}-04 \\
8.90 \mathrm{E}-05 \\
1.60 \mathrm{E}-04 \\
1.24 \mathrm{E}-04 \\
8.24 \mathrm{E}-04 \\
1.91 \mathrm{E}-03 \\
3.40 \mathrm{E}-03 \\
3.48 \mathrm{E}-03 \\
2.03 \mathrm{E}-03 \\
3.41 \mathrm{E}-03 \\
4.73 \mathrm{E}-03 \\
4.26 \mathrm{E}-03 \\
3.20 \mathrm{E}-03 \\
5.03 \mathrm{E}-03 \\
4.08 \mathrm{E}-03 \\
4.96 \mathrm{E}-03 \\
2.40 \mathrm{E}-03 \\
5.19 \mathrm{E}-03 \\
2.32 \mathrm{E}-03 \\
4.05 \mathrm{E}-03 \\
4.13 \mathrm{E}-03 \\
5.36 \mathrm{E}-03 \\
4.97 \mathrm{E}-03 \\
5.30 \mathrm{E}-03 \\
8.37 \mathrm{E}-03 \\
2.55 \mathrm{E}-04 \\
8.52 \mathrm{E}-03 \\
3.01 \mathrm{E}-03 \\
3.83 \mathrm{E}-03 \\
7.56 \mathrm{E}-03 \\
6.47 \mathrm{E}-03 \\
9.07 \mathrm{E}-03 \\
2.20 \mathrm{E}-03 \\
4.08 \mathrm{E}-03 \\
6.52 \mathrm{E}-03 \\
7.83 \mathrm{E}-03 \\
1.06 \mathrm{E}-02 \\
9.61 \mathrm{E}-03 \\
9.54 \mathrm{E}-03 \\
0.00 \mathrm{E}+00 \\
0\end{array}$ & $\begin{array}{c}\text { CM242 } \\
2.02 \mathrm{E}-04 \\
4.17 \mathrm{E}-04 \\
5.59 \mathrm{E}-04 \\
8.76 \mathrm{E}-04 \\
1.40 \mathrm{E}-03 \\
1.15 \mathrm{E}-03 \\
1.25 \mathrm{E}-03 \\
9.39 \mathrm{E}-04 \\
1.05 \mathrm{E}-03 \\
1.39 \mathrm{E}-03 \\
1.08 \mathrm{E}-03 \\
1.41 \mathrm{E}-03 \\
1.26 \mathrm{E}-03 \\
1.43 \mathrm{E}-03 \\
5.87 \mathrm{E}-04 \\
1.61 \mathrm{E}-03 \\
5.18 \mathrm{E}-03 \\
4.27 \mathrm{E}-03 \\
4.99 \mathrm{E}-03 \\
6.46 \mathrm{E}-03 \\
4.39 \mathrm{E}-03 \\
4.31 \mathrm{E}-03 \\
5.33 \mathrm{E}-03 \\
2.74 \mathrm{E}-03 \\
9.33 \mathrm{E}-04 \\
2.67 \mathrm{E}-04 \\
4.78 \mathrm{E}-04 \\
3.73 \mathrm{E}-04 \\
2.47 \mathrm{E}-03 \\
5.71 \mathrm{E}-03 \\
1.02 \mathrm{E}-02 \\
1.04 \mathrm{E}-02 \\
6.09 \mathrm{E}-03 \\
1.02 \mathrm{E}-02 \\
1.42 \mathrm{E}-02 \\
1.28 \mathrm{E}-02 \\
9.59 \mathrm{E}-03 \\
1.51 \mathrm{E}-02 \\
1.23 \mathrm{E}-02 \\
1.49 \mathrm{E}-02 \\
7.20 \mathrm{E}-03 \\
1.56 \mathrm{E}-02 \\
7.02 \mathrm{E}-03 \\
1.22 \mathrm{E}-02 \\
1.24 \mathrm{E}-02 \\
1.62 \mathrm{E}-02 \\
1.50 \mathrm{E}-02 \\
1.60 \mathrm{E}-02 \\
2.52 \mathrm{E}-02 \\
7.70 \mathrm{E}-04 \\
2.57 \mathrm{E}-02 \\
9.09 \mathrm{E}-03 \\
1.16 \mathrm{E}-02 \\
2.28 \mathrm{E}-02 \\
1.96 \mathrm{E}-02 \\
2.74 \mathrm{E}-02 \\
6.64 \mathrm{E}-03 \\
1.23 \mathrm{E}-02 \\
1.97 \mathrm{E}-02 \\
2.37 \mathrm{E}-02 \\
3.20 \mathrm{E}-02 \\
2.91 \mathrm{E}-02 \\
2.89 \mathrm{E}-02 \\
0.00 \mathrm{E}+00 \\
0.00 \mathrm{E}+00\end{array}$ & $\begin{array}{l}\text { CM243 } \\
2.10 \mathrm{E}-06 \\
5.70 \mathrm{E}-06 \\
9.00 \mathrm{E}-06 \\
1.47 \mathrm{E}-05 \\
2.47 \mathrm{E}-05 \\
2.04 \mathrm{E}-05 \\
2.29 \mathrm{E}-05 \\
1.52 \mathrm{E}-05 \\
1.71 \mathrm{E}-05 \\
2.47 \mathrm{E}-05 \\
1.83 \mathrm{E}-05 \\
2.51 \mathrm{E}-05 \\
2.25 \mathrm{E}-05 \\
2.57 \mathrm{E}-05 \\
1.06 \mathrm{E}-05 \\
3.36 \mathrm{E}-05 \\
1.09 \mathrm{E}-04 \\
9.02 \mathrm{E}-05 \\
1.05 \mathrm{E}-04 \\
1.37 \mathrm{E}-04 \\
9.33 \mathrm{E}-05 \\
9.09 \mathrm{E}-05 \\
1.14 \mathrm{E}-04 \\
5.84 \mathrm{E}-05 \\
2.00 \mathrm{E}-05 \\
5.73 \mathrm{E}-06 \\
1.03 \mathrm{E}-05 \\
7.86 \mathrm{E}-06 \\
5.23 \mathrm{E}-05 \\
1.22 \mathrm{E}-04 \\
2.19 \mathrm{E}-04 \\
2.24 \mathrm{E}-04 \\
1.31 \mathrm{E}-04 \\
2.20 \mathrm{E}-04 \\
3.06 \mathrm{E}-04 \\
2.76 \mathrm{E}-04 \\
2.08 \mathrm{E}-04 \\
3.27 \mathrm{E}-04 \\
2.66 \mathrm{E}-04 \\
3.24 \mathrm{E}-04 \\
1.57 \mathrm{E}-04 \\
3.39 \mathrm{E}-04 \\
1.44 \mathrm{E}-04 \\
2.63 \mathrm{E}-04 \\
2.71 \mathrm{E}-04 \\
3.50 \mathrm{E}-04 \\
3.28 \mathrm{E}-04 \\
3.52 \mathrm{E}-04 \\
5.57 \mathrm{E}-04 \\
1.70 \mathrm{E}-05 \\
5.69 \mathrm{E}-04 \\
2.02 \mathrm{E}-04 \\
2.57 \mathrm{E}-04 \\
5.05 \mathrm{E}-04 \\
4.37 \mathrm{E}-04 \\
6.15 \mathrm{E}-04 \\
1.49 \mathrm{E}-04 \\
2.79 \mathrm{E}-04 \\
4.46 \mathrm{E}-04 \\
5.35 \mathrm{E}-04 \\
7.29 \mathrm{E}-04 \\
6.57 \mathrm{E}-04 \\
6.51 \mathrm{E}-04 \\
0.00 \mathrm{E}+00 \\
0.00 \mathrm{E}+00\end{array}$ \\
\hline
\end{tabular}


HNF-SD-WM-TI-794, ReV. 0

ACTIVITY IN UNSEPARATED FUEL (Curies decayed to 1/1/94)

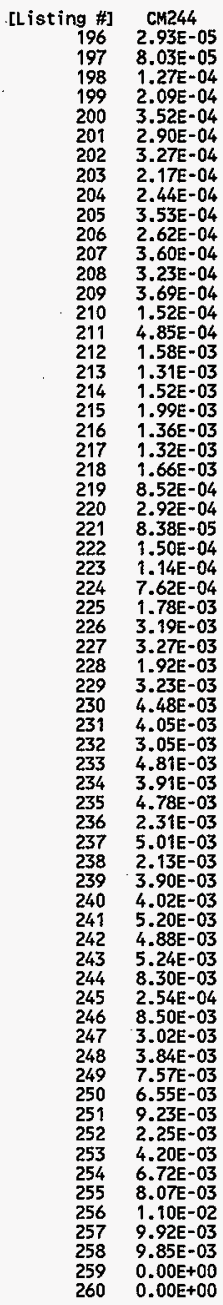


HNF-SD-WM-TI-794, ReV: 0

UNIFIED SEPARATIONS PROOUCTION DATA

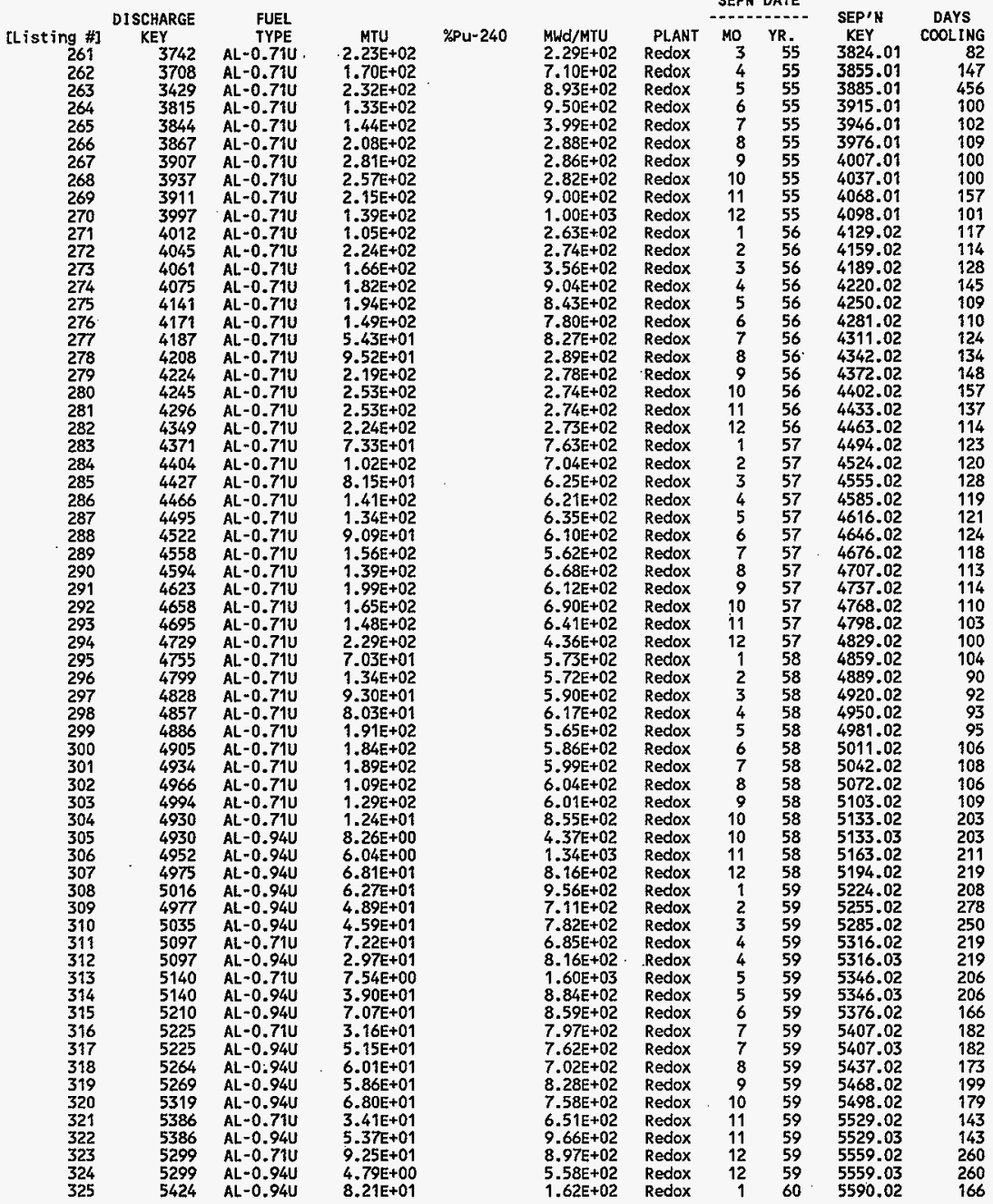


HNF-SD-WM-TI-794, Rev, 0

UNIFIED SEPARATIONS PRODUCTION DATA TOTAL WASTE LOSS FRACTION

[Listing \#] Fuel Data Reference

261 SPLANT.DAT 19348 9/30/93 1:19P 262 SPLANT.DAT 19348 9/30/93 1:19P 263 SPLANT.DAT 19348 9/30/93 1:19P 264 SPLANT.DAT $193489 / 30 / 93$ 1:19P 265 SPLANT.DAT 19348 9/30/93 $1: 19 \mathrm{P}$ 266 SPLANT.DAT 19348 \%, $130 / 93$ 1:19P 267 SPLANT.DAT 19348 9/30/93 1:19P 268 SPLANT.DAT 19348 9/30/93 1:19P 269 SPLANT.DAT 19348 9/30/93 1:19P 270 SPLANT.DAT 19348 9/30/93 1:19P 271 SPLANT.DAT $19348 \% / 30 / 93 \quad 1: 19 P$ 272 SPLANT.DAT $19348 \% / 30 / 93 \quad 1: 19 \mathrm{P}$ 273 SPLANT.DAT 19348 9/30/93 $1: 19 \mathrm{P}$ 274 SPLANT.DAT 19348 9/30/93 $1: 19 P$ 275 SPLANT.DAT 19348 9/30/93 1:19P 276 SPLANT.DAT 19348 9/30/93 1:19P 277 SPLANT.DAT 19348 9/30/93 1:19P 278 SPLANT.DAT 19348 9/30/93 1:19P 279 SPLANT.DAT 19348 9/30/93 1:19p 280 SPLANT.DAT 19348 9/30/93 1:19P 281 SPLANT.DAT 19348 9/30/93 1:19P 282 SPLANT.DAT 19348 9/30/93 1:19P 283 SPLANT.DAT 19348 9/30/93 1:19P 284 SPLANT.DAT 19348 9/30/93 1:19P 285 SPLANT.DAT 19348 9/30/93 1:19P 286 SPLANT.DAT 19348 9/30/93 1:19P 287 SPLANT.DAT 19348 9/30/93 1:19P 288 SPLANT.DAT 19348 9/30/93 1:19P 289 SPLANT.DAT 19348 9/30/93 1:19P 290 SPLANT.DAT 19348 9/30/93 1:19P 291 SPLANT.DAT 19348 9/30/93 1:19P 292 SPL.ANT.DAT 19348 9/30/93 1:19P 293 SPLANT.DAT 19348 9/30/93 $1: 19 \mathrm{P}$ 294 SPLANT.DAT 19348 9/30/93 1:19P 295 SPLANT.DAT 19348 9/30/93 $1: 19 \mathrm{p}$ 296 SPLANT.DAT 19348 9/30/93 1:19P 297 SPLANT.DAT 19348 9/30/93 1:19P 298 SPLANT.DAT 19348 9/30/93 1:19P 299 SPLANT.DAT 19348 9/30/93 1:19P 300 SPLANT.DAT 19348 9/30/93 1:19P 301 SPLANT.DAT 19348 9/30/93 1:19P 302 SPLANT.DAT 19348 9/30/93 $1: 19 \mathrm{P}$ 303 SPLANT.DAT $193489 / 30 / 93 \quad 1: 19 P$ 304 SPLANT.DAT 19348 9/30/93 $1: 19 P$ 305 SPLANT.DAT 19348 9/30/93 1:19P 306 SPLANT.DAT 19348 9/30/93 1:19P 307 SPLANT.OAT 19348 9/30/93 1:19P 308 SPLANT.DAT 99348 9/30/93 1:19P 309 SPLANT_DAT 19348 9/30/93 1:19P 310 SPLANT.DAT 19348 9/30/93 1:19P 311 SPLANT.DAT 19348 9/30/93 1:19P 312 SPLANT.DAT 19348 9/30/93 1:19P 313 SPLANT.DAT 19348 9/30/93 1:19P 314 SPLANT.DAT 19348 9/30/93 1:19P 315 SPLANT.DAT 19348 9/30/93 $1: 19 \mathrm{P}$ 316. SPLANT.DAT 19348 9/30/93 $1: 19 \mathrm{P}$ 317 SPLANT.DAT $193489 / 30 / 93 \quad 1: 19 \mathrm{P}$ 318 SPLANT.DAT 19348 9/30/93 $\imath: 19 \mathrm{P}$ 319 SPLANT.DAT 19348 9/30/93 $1: 19 \mathrm{PP}$ 320 SPLANT.DAT 19348 9/30/93 1:19P 321 SPLANT.DAT 19348 9/30/93 1: 19P 322 SPLANT.DAT 19348 9/30/93 1:19P 323 SPLANT.DAT 19348 9/30/93 1:19p 324 SPLANT.DAT 19348 9/30/93 1:19P 325 SPLANT.DAT 19348 9/30/93 1:19P

\author{
Th
}

0.0046

0.009

0.0043

0.0099

0.0096

0.0079

0.0043

0.0043

0.0063

0.0087

0.0081

0.0008

0.0048

0.0018

0.0025

0.0044

0.0049

0.007

0.0067

0.0033

0.0026

0.0023

0.0039

0.0017

0.0023

0.004

0.001

0.0018

0.0023

0.0009

0.001

0.0024

0.0017

0.0016

0.0075

0.0022

0.0027

0.0031

0.0024

0.0015

0.0016

0.0014

0.0025

0.0059

0.0059

0.0023

0.0023

0.0024

0.0036

0.0021

0.0016

0.0016

0.0018

0.0018

0.0011

0.001

0.001

0.0029

0.0009

0.0014

0.0011

0.0011

0.0012

0.0012

0.0019
Np $1 \mathrm{Pu}$ Pu

0.0131

0.0087

0.0096

0.0062

0.0071

0.0076

0.0088

0.0092

0.0182

0.0112

0.0043

0.0086

0.0021

0.0075

0.0108

0.0139

0.0216

0.0056

0.0025

0.0027

0.0026

0.0067

0.0043

0.0087

0.0083

0.0039

0.0069

0.0057

0.0039

0.0042

0.0085

0.0078

0.0067

0.0068

0.0058

0.0039

0.0033

0.0044

0.0028

0.0031

0.0032

0.0051

0.0074

0.0074

0.0038

0.0038

0.0051

0.0055

0.0053

0.0042

0.0042

0.0051

0.0051

0.0042

0.0045

0.0045

0.0072

0.0049

0.0064

0.003

0.003

0.0052

$\begin{array}{rr}0.3 & 0.0052 \\ 0.3 & 0.005\end{array}$
Waste Frac.

Reference

(b)

(b)

(b)

(b)

(b)

(b)

(b)

(b)

(b)

(b)

(b)

(b)

(b)

(b)

(b)

(b)

(b)

(b)

(b)

(b)

(b)

(b)

(b)

(b)

(b)

(b)

(b)

(b) (b)

(b)

(b)

(b)

(b)

(b)

(b)

(b)

(b)

(b)

(b)

(b)

(b)

(b)

(b)

(b)

(b)

(b)

(b) 
HAF-SD-HM-TI-794, ReV. 0

ACTIVITY IN UNSEPARATED FUEL (Curies decayed to $1 / 1 / 94$ )

\begin{tabular}{|c|c|c|c|c|c|c|c|c|c|}
\hline 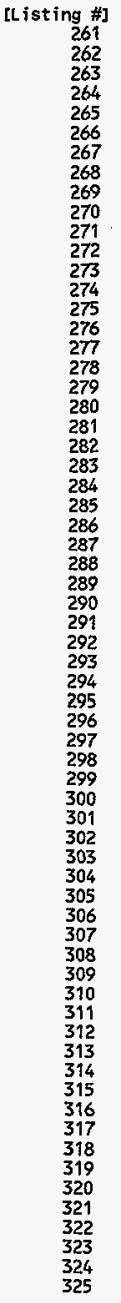 & $\begin{array}{c}H 03 \\
8.90 E+01 \\
2.14 E+02 \\
3.55 E+02 \\
2.30 E+02 \\
1.03 E+02 \\
1.07 E+02 \\
1.44 E+02 \\
1.30 E+02 \\
3.57 E+02 \\
2.61 E+02 \\
5.03 E+01 \\
1.12 E+02 \\
1.09 E+02 \\
3.11 E+02 \\
3.12 E+02 \\
2.22 E+02 \\
8.61 E+01 \\
5.17 E+01 \\
1.15 E+02 \\
1.31 E+02 \\
1.32 E+02 \\
1.18 E+02 \\
1.10 E+02 \\
1.42 E+02 \\
1.01 E+02 \\
1.73 E+02 \\
1.70 E+02 \\
1.11 E+02 \\
1.76 E+02 \\
1.88 E+02 \\
2.48 E+02 \\
2.33 E+02 \\
1.95 E+02 \\
2.05 E+02 \\
8.34 E+01 \\
1.60 E+02 \\
1.15 E+02 \\
1.04 E+02 \\
2.28 E+02 \\
2.29 E+02 \\
2.41 E+02 \\
1.41 E+02 \\
1.67 E+02 \\
2.28 E+01 \\
7.43 E+00 \\
1.71 E+01 \\
1.16 E+02 \\
1.27 E+02 \\
7.27 E+01 \\
7.58 E+01 \\
1.08 E+02 \\
5.16 E+01 \\
2.77 E+01 \\
7.41 E+01 \\
1.32 E+02 \\
5.66 E+01 \\
8.53 E+01 \\
9.20 E+01 \\
1.06 E+02 \\
1.14 E+02 \\
5.07 E+01 \\
1.16 E+02 \\
1.89 E+02 \\
5.84 E+00 \\
2.94 E+01\end{array}$ & 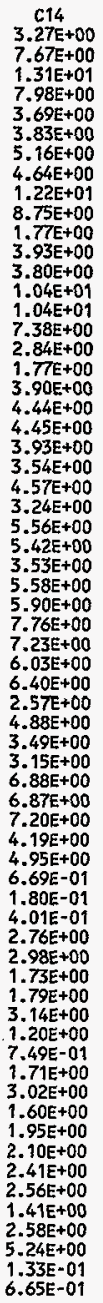 & $\begin{array}{l}\text { N159 } \\
9.32 \mathrm{E}-01 \\
2.18 \mathrm{E}+00 \\
3.71 \mathrm{E}+00 \\
2.26 \mathrm{E}+00 \\
1.05 \mathrm{E}+00 \\
1.09 \mathrm{E}+00 \\
1.47 \mathrm{E}+00 \\
1.32 \mathrm{E}+00 \\
3.46 \mathrm{E}+00 \\
2.47 \mathrm{E}+00 \\
5.04 \mathrm{E}-01 \\
1.12 \mathrm{E}+00 \\
1.08 \mathrm{E}+00 \\
2.94 \mathrm{E}+00 \\
2.93 \mathrm{E}+00 \\
2.09 \mathrm{E}+00 \\
8.03 \mathrm{E}-01 \\
5.02 \mathrm{E}-01 \\
1.11 \mathrm{E}+00 \\
1.26 \mathrm{E}+00 \\
1.27 \mathrm{E}+00 \\
1.12 \mathrm{E}+00 \\
1.00 \mathrm{E}+00 \\
1.30 \mathrm{E}+00 \\
9.20 \mathrm{E}-01 \\
1.58 \mathrm{E}+00 \\
1.54 \mathrm{E}+00 \\
1.00 \mathrm{E}+00 \\
1.58 \mathrm{E}+00 \\
1.67 \mathrm{E}+00 \\
2.20 \mathrm{E}+00 \\
2.05 \mathrm{E}+00 \\
1.71 \mathrm{E}+00 \\
1.82 \mathrm{E}+00 \\
7.28 \mathrm{E}-01 \\
1.38 \mathrm{E}+00 \\
9.91 \mathrm{E}-01 \\
8.94 \mathrm{E}-01 \\
1.95 \mathrm{E}+00 \\
1.95 \mathrm{E}+00 \\
2.04 \mathrm{E}+00 \\
1.19 \mathrm{E}+00 \\
1.40 \mathrm{E}+00 \\
1.89 \mathrm{E}-01 \\
5.11 \mathrm{E}-02 \\
1.13 \mathrm{E}-01 \\
7.83 \mathrm{E}-01 \\
8.44 \mathrm{E}-01 \\
4.91 \mathrm{E}-01 \\
5.07 \mathrm{E}-01 \\
8.90 \mathrm{E}-01 \\
3.41 \mathrm{E}-01 \\
2.10 \mathrm{E}-01 \\
4.85 \mathrm{E}-01 \\
8.56 \mathrm{E}-01 \\
4.52 \mathrm{0}-01 \\
5.54 \mathrm{E}-01 \\
5.95 \mathrm{E}-01 \\
6.84 \mathrm{E}-01 \\
7.27 \mathrm{E}-01 \\
4.00 \mathrm{E}-01 \\
7.30 \mathrm{E}-01 \\
1.48 \mathrm{E}+00 \\
3.78 \mathrm{E}-02 \\
1.90 \mathrm{E}-01\end{array}$ & $\begin{array}{l}N 163 \\
8.66 \mathrm{E}+01 \\
2.02 \mathrm{E}+02 \\
3.44 \mathrm{E}+02 \\
2.11 \mathrm{E}+02 \\
9.76 \mathrm{E}+01 \\
1.02 \mathrm{E}+02 \\
1.37 \mathrm{E}+02 \\
1.23 \mathrm{E}+02 \\
3.24 \mathrm{E}+02 \\
2.32 \mathrm{E}+02 \\
4.71 \mathrm{E}+01 \\
1.05 \mathrm{E}+02 \\
1.01 \mathrm{E}+02 \\
2.76 \mathrm{E}+02 \\
2.76 \mathrm{E}+02 \\
1.96 \mathrm{E}+02 \\
7.56 \mathrm{E}+01 \\
4.71 \mathrm{E}+01 \\
1.04 \mathrm{E}+02 \\
1.19 \mathrm{E}+02 \\
1.19 \mathrm{E}+02 \\
1.05 \mathrm{E}+02 \\
9.47 \mathrm{E}+01 \\
1.22 \mathrm{E}+02 \\
8.68 \mathrm{E}+01 \\
1.49 \mathrm{E}+02 \\
1.45 \mathrm{E}+02 \\
9.47 \mathrm{E}+01 \\
1.50 \mathrm{E}+02 \\
1.58 \mathrm{E}+02 \\
2.09 \mathrm{E}+02 \\
1.94 \mathrm{E}+02 \\
1.62 \mathrm{E}+02 \\
1.73 \mathrm{E}+02 \\
6.92 \mathrm{E}+01 \\
1.32 \mathrm{E}+02 \\
9.43 \mathrm{E}+01 \\
8.52 \mathrm{E}+01 \\
1.86 \mathrm{E}+02 \\
1.86 \mathrm{E}+02 \\
1.95 \mathrm{E}+02 \\
1.13 \mathrm{E}+02 \\
1.34 \mathrm{E}+02 \\
1.81 \mathrm{E}+01 \\
4.87 \mathrm{E}+00 \\
1.08 \mathrm{E}+01 \\
7.48 \mathrm{E}+01 \\
8.077 \mathrm{E}+01 \\
4.69 \mathrm{E}+01 \\
4.84 \mathrm{E}+01 \\
8.52 \mathrm{E}+01 \\
3.27 \mathrm{E}+01 \\
2.03 \mathrm{E}+01 \\
4.65 \mathrm{E}+01 \\
8.21 \mathrm{E}+01 \\
4.34 \mathrm{E}+01 \\
5.32 \mathrm{E}+01 \\
5.71 \mathrm{E}+01 \\
6.57 \mathrm{E}+01 \\
6.98 \mathrm{E}+01 \\
3.85 \mathrm{E}+01 \\
7.03 \mathrm{E}+01 \\
1.43 \mathrm{E}+02 \\
3.62 \mathrm{E}+00 \\
1.82 \mathrm{E}+01\end{array}$ & $\begin{array}{l}\text { CO60 } \\
1.20 E+00 \\
2.76 E+00 \\
4.24 E+00 \\
2.96 E+00 \\
1.40 E+00 \\
1.47 E+00 \\
2.01 E+00 \\
1.83 E+00 \\
4.70 E+00 \\
3.47 E+00 \\
7.16 E-01 \\
1.61 E+00 \\
1.56 E+00 \\
4.24 E+00 \\
4.34 E+00 \\
3.13 E+00 \\
1.21 E+00 \\
7.65 E-01 \\
1.70 E+00 \\
1.95 E+00 \\
1.99 E+00 \\
1.79 E+00 \\
1.61 E+00 \\
2.11 E+00 \\
1.51 E+00 \\
2.63 E+00 \\
2.59 E+00 \\
1.70 E+00 \\
2.73 E+00 \\
2.91 E+00 \\
3.88 E+00 \\
3.65 E+00 \\
3.10 E+00 \\
3.34 E+00 \\
1.35 E+00 \\
2.60 E+00 \\
1.88 E+00 \\
1.72 E+00 \\
3.78 E+00 \\
3.81 E+00 \\
4.03 E+00 \\
2.37 E+00 \\
2.83 E+00 \\
3.72 E=01 \\
1.01 E E-01 \\
2.23 E-01 \\
1.56 E+00 \\
1.711 E+00 \\
9.82 E-01 \\
1.03 E+00 \\
1.86 E+00 \\
7.11 E-01 \\
4.42 E-01 \\
1.03 E+00 \\
1.86 E+00 \\
9.88 E-01 \\
1.21 E+00 \\
1.32 E+00 \\
1.52 E+00 \\
1.64 E+00 \\
9.27 E-01 \\
1.68 E+00 \\
3.32 E+00 \\
8.49 E=02 \\
4.48 E-01\end{array}$ & $\begin{array}{l}\text { SE79 } \\
6.88 E-01 \\
1.63 E+00 \\
2.79 E+00 \\
1.70 E+00 \\
7.77 E-01 \\
8.06 E-01 \\
1.09 E+00 \\
9.76 E-01 \\
2.60 E+00 \\
1.87 E+00 \\
3.72 E-01 \\
8.28 E-01 \\
8.00 E-01 \\
2.21 E+00 \\
2.20 E+00 \\
1.57 E+00 \\
6.03 E-01 \\
3.71 E-01 \\
8.21 E-01 \\
9.34 E-01 \\
9.36 E-01 \\
8.27 E-01 \\
7.52 E-01 \\
9.69 E-01 \\
6.86 E-01 \\
1.18 E+00 \\
1.15 E+00 \\
7.47 E-01 \\
1.18 E+00 \\
1.25 E+00 \\
1.64 E+00 \\
1.53 E+00 \\
1.28 E+00 \\
1.35 E+00 \\
5.43 E-01 \\
1.03 E+00 \\
7.39 E-01 \\
6.67 E-01 \\
1.45 E+00 \\
1.45 E+00 \\
1.52 E+00 \\
8.86 E-01 \\
1.05 E+00 \\
1.42 E-01 \\
4.83 E=02 \\
1.08 E-01 \\
7.43 E=01 \\
8.01 E-01 \\
4.65 E-01 \\
4.80 E-01 \\
6.65 E-01 \\
3.24 E-01 \\
1.61 E-01 \\
4.60 E-01 \\
8.11 E-01 \\
3.39 E-01 \\
5.25 E-01 \\
5.64 E-01 \\
6.49 E-01 \\
6.89 E-01 \\
2.99 E-01 \\
6.93 E-01 \\
1.12 E+00 \\
3.57 E-02 \\
1.79 E-01\end{array}$ & $\begin{array}{l}\text { SR90 } \\
5.88 E+04 \\
1.35 E+05 \\
2.26 E+05 \\
1.41 E+05 \\
6.63 E+04 \\
6.93 E+04 \\
9.35 E+04 \\
8.43 E+04 \\
2.17 E+05 \\
1.56 E+05 \\
3.23 E+04 \\
7.20 E+04 \\
6.94 E+04 \\
1.87 E+05 \\
1.87 E+05 \\
1.34 E+05 \\
5.14 E+04 \\
3.26 E+04 \\
7.22 E+04 \\
8.23 E+04 \\
8.27 E+04 \\
7.33 E+04 \\
6.52 E+04 \\
8.44 E+04 \\
6.01 E+04 \\
1.03 E+05 \\
1.01 E+05 \\
6.59 E+04 \\
1.05 E+05 \\
1.10 E+05 \\
1.46 E+05 \\
1.36 E+05 \\
1.14 E+05 \\
1.22 E+05 \\
4.87 E+04 \\
9.29 E+04 \\
6.65 E+04 \\
6.01 E+04 \\
1.32 E+05 \\
1.32 E+05 \\
1.38 E+05 \\
8.05 E+04 \\
9.52 E+04 \\
1.27 E+04 \\
4.55 E+03 \\
9.86 E+03 \\
6.92 E+04 \\
7.45 E+04 \\
4.35 E+04 \\
4.50 E+04 \\
6.07 E+04 \\
3.04 E+04 \\
1.41 E+04 \\
4.33 E+04 \\
7.67 E+04 \\
3.10 E+04 \\
4.99 E+04 \\
5.38 E+04 \\
6.16 E+04 \\
6.58 E+04 \\
2.78 E+04 \\
6.60 E+04 \\
1.02 E+05 \\
3.43 E+03 \\
1.76 E+04\end{array}$ & $\begin{array}{c}Y 90 \\
5.88 E+04 \\
1.36 E+05 \\
2.26 E+05 \\
1.41 E+05 \\
6.63 E+04 \\
6.93 E+04 \\
9.35 E+04 \\
8.43 E+04 \\
2.17 E+05 \\
1.56 E+05 \\
3.23 E+04 \\
7.20 E+04 \\
6.94 E+04 \\
1.87 E+05 \\
1.87 E+05 \\
1.34 E+05 \\
5.14 E+04 \\
3.26 E+04 \\
7.22 E+04 \\
8.23 E+04 \\
8.27 E+04 \\
7.34 E+04 \\
6.52 E+04 \\
8.44 E+04 \\
6.01 E+04 \\
1.03 E+05 \\
1.01 E+05 \\
6.59 E+04 \\
1.05 E+05 \\
1.10 E+05 \\
1.46 E+05 \\
1.36 E+05 \\
1.14 E+05 \\
1.22 E+05 \\
4.87 E+04 \\
9.29 E+04 \\
6.66 E+04 \\
6.02 E+04 \\
1.32 E+05 \\
1.32 E+05 \\
1.38 E+05 \\
8.05 E+04 \\
9.53 E+04 \\
1.27 E+04 \\
4.55 E+03 \\
9.86 E+03 \\
6.92 E+04 \\
7.45 E+04 \\
4.36 E+04 \\
4.50 E+04 \\
6.07 E+04 \\
3.04 E+04 \\
1.41 E+04 \\
4.33 E+04 \\
7.67 E+04 \\
3.10 E+04 \\
4.99 E+04 \\
5.38 E+04 \\
6.16 E+04 \\
6.58 E+04 \\
2.78 E+04 \\
6.60 E+04 \\
1.02 E+05 \\
3.43 E+03 \\
1.76 E+04\end{array}$ & $\begin{array}{l}2 R 93 \\
3.28 E+00 \\
7.67 E+00 \\
1.31 E+01 \\
7.97 E+00 \\
3.69 E+00 \\
3.84 E+00 \\
5.17 E+00 \\
4.65 E+00 \\
1.22 E+01 \\
8.73 E+00 \\
1.78 E+00 \\
3.94 E+00 \\
3.81 E+00 \\
1.04 E+01 \\
1.03 E+01 \\
7.37 E+00 \\
2.83 E+00 \\
1.77 E+00 \\
3.91 E+00 \\
4.45 E+00 \\
4.46 E+00 \\
3.94 E+00 \\
3.54 E+00 \\
4.57 E+00 \\
3.24 E+00 \\
5.56 E+00 \\
5.42 E+00 \\
3.53 E+00 \\
5.58 E+00 \\
5.89 E+00 \\
7.76 E+00 \\
7.22 E+00 \\
6.03 E+00 \\
6.40 E+00 \\
2.57 E+00 \\
4.88 E+00 \\
3.49 E+00 \\
3.15 E+00 \\
6.88 E+00 \\
6.87 E+00 \\
7.20 E+00 \\
4.19 E+00 \\
4.95 E+00 \\
6.67 \mathrm{E}-01 \\
2.34 E-01 \\
5.15 E-01 \\
3.58 E+00 \\
3.85 E+00 \\
2.25 E+00 \\
2.32 E+00 \\
3.14 E+00 \\
1.56 E+00 \\
7.45 E-01 \\
2.22 E+00 \\
3.91 E+00 \\
1.59 E+00 \\
2.53 E+00 \\
2.72 E+00 \\
3.12 E+00 \\
3.32 E+00 \\
1.41 E+00 \\
3.33 E+00 \\
5.23 E+00 \\
1.73 E-01 \\
8.71 E-01\end{array}$ \\
\hline
\end{tabular}


ACTIVITY IN UNSEPARATED FUEL (Curies decayed to $1 / 1 / 94$ )

\begin{tabular}{|c|c|c|c|c|c|c|c|c|c|}
\hline $\begin{array}{r}\text { isting } 7 \text { ] } \\
261 \\
262 \\
263 \\
264 \\
265 \\
266 \\
267 \\
268 \\
269 \\
270 \\
271 \\
272 \\
273 \\
274 \\
275 \\
276 \\
277 \\
278 \\
279 \\
280 \\
281 \\
282 \\
283 \\
284 \\
285 \\
286 \\
287 \\
288 \\
289 \\
290 \\
291 \\
292 \\
293 \\
294 \\
295 \\
296 \\
297 \\
298 \\
299 \\
300 \\
301 \\
302 \\
303 \\
304 \\
305 \\
306 \\
307 \\
308 \\
309 \\
310 \\
311 \\
312 \\
313 \\
314 \\
315 \\
316 \\
317 \\
318 \\
319 \\
320 \\
321 \\
322 \\
323 \\
324 \\
325\end{array}$ & 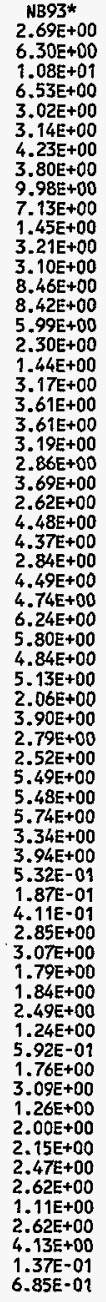 & 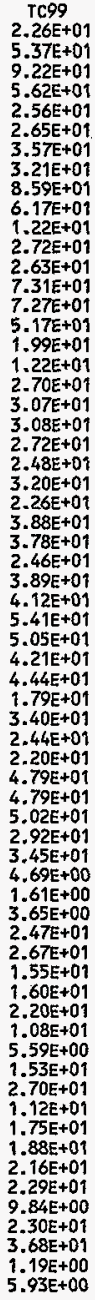 & $\begin{array}{l}\text { RU106 } \\
1.19 \mathrm{E}-06 \\
3.13 \mathrm{E}-06 \\
3.39 \mathrm{E}-06 \\
4.35 \mathrm{E}-06 \\
1.74 \mathrm{E}-06 \\
1.81 \mathrm{E}-06 \\
2.63 \mathrm{E}-06 \\
2.50 \mathrm{E}-06 \\
7.84 \mathrm{E}-06 \\
6.83 \mathrm{E}-06 \\
1.09 \mathrm{E}-06 \\
2.59 \mathrm{E}-06 \\
2.66 \mathrm{E}-06 \\
9.08 \mathrm{E}-06 \\
1.01 \mathrm{E}-05 \\
7.43 \mathrm{E}-06 \\
3.01 \mathrm{E}-06 \\
1.59 \mathrm{E}-06 \\
3.61 \mathrm{E}-06 \\
4.26 \mathrm{E}-06 \\
4.70 \mathrm{E}-06 \\
4.59 \mathrm{E}-06 \\
5.18 \mathrm{E}-06 \\
6.97 \mathrm{E}-06 \\
4.99 \mathrm{E}-06 \\
9.23 \mathrm{E}-06 \\
9.49 \mathrm{E}-06 \\
6.48 \mathrm{E}-06 \\
1.08 \mathrm{E}-05 \\
1.26 \mathrm{E}-05 \\
1.73 \mathrm{E}-05 \\
1.77 \mathrm{E}-05 \\
1.55 \mathrm{E}-05 \\
1.62 \mathrm{E}-05 \\
7.22 \mathrm{E}-06 \\
1.49 \mathrm{E}-05 \\
1.13 \mathrm{E}-05 \\
1.09 \mathrm{E}-05 \\
2.46 \mathrm{E}-05 \\
2.57 \mathrm{E}-05 \\
2.87 \mathrm{E}-05 \\
1.77 \mathrm{E}-05 \\
2.21 \mathrm{E}-05 \\
2.88 \mathrm{E}-06 \\
7.39 \mathrm{E}-07 \\
2.12 \mathrm{E}-06 \\
1.35 \mathrm{E}-05 \\
1.63 \mathrm{E}-05 \\
8.33 \mathrm{E}-06 \\
9.71 \mathrm{E}-06 \\
1.74 \mathrm{E}-05 \\
7.41 \mathrm{E}-06 \\
6.01 \mathrm{E}-06 \\
1.16 \mathrm{E}-05 \\
2.33 \mathrm{E}-05 \\
1.17 \mathrm{E}-05 \\
1.51 \mathrm{E}-05 \\
1.73 \mathrm{E}-05 \\
2.06 \mathrm{E}-05 \\
2.37 \mathrm{~s}-05 \\
1.33 \mathrm{E}-05 \\
2.83 \mathrm{E}-05 \\
4.59 \mathrm{E}-05 \\
1.13 \mathrm{E}-06 \\
6.45 \mathrm{E}-06\end{array}$ & $\begin{array}{l}C D 113 * \\
9.47 E+00 \\
2.32 E+01 \\
3.89 E+01 \\
2.51 E+01 \\
1.10 E+01 \\
1.13 E+01 \\
1.53 E+01 \\
1.38 E+01 \\
3.86 E+01 \\
2.83 E+01 \\
5.32 E+00 \\
1.19 E+01 \\
1.16 E+01 \\
3.36 E+01 \\
3.36 E+01 \\
2.39 E+01 \\
9.25 E+00 \\
5.46 E+00 \\
1.21 E+01 \\
1.38 E+01 \\
1.39 E+01 \\
1.24 E+01 \\
1.17 E+01 \\
1.51 E+01 \\
1.07 E+01 \\
1.84 E+01 \\
1.80 E+01 \\
1.17 E+01 \\
1.86 E+01 \\
1.99 E+01 \\
2.62 E+01 \\
2.47 E+01 \\
2.06 E+01 \\
2.15 E+01 \\
8.77 E+00 \\
1.67 E+01 \\
1.21 E+01 \\
1.10 E+01 \\
2.39 E+01 \\
2.40 E+01 \\
2.53 E+01 \\
1.48 E+01 \\
1.75 E+01 \\
2.40 E+00 \\
6.71 E=01 \\
1.60 E+00 \\
1.06 E+01 \\
1.17 E+01 \\
6.62 E+00 \\
6.92 E+00 \\
1.13 E+01 \\
4.71 E+00 \\
2.97 E+00 \\
6.77 E+00 \\
1.20 E+01 \\
5.93 E+00 \\
7.75 E+00 \\
8.33 E+00 \\
9.66 E+00 \\
1.03 E+01 \\
5.27 E+00 \\
1.06 E+01 \\
1.99 E+01 \\
5.25 E-01 \\
2.59 E+00\end{array}$ & 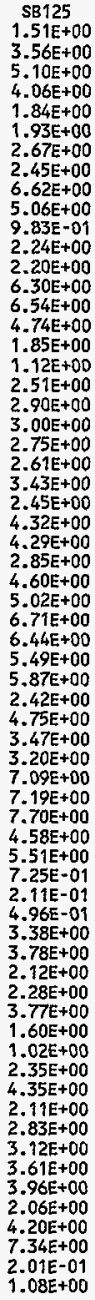 & $\begin{array}{c}\text { SN126 } \\
1.02 E+00 \\
2.51 \mathrm{E}+00 \\
4.39 \mathrm{E}+00 \\
2.69 \mathrm{E}+00 \\
1.17 \mathrm{E}+00 \\
1.20 \mathrm{E}+00 \\
1.62 \mathrm{E}+00 \\
1.45 \mathrm{E}+00 \\
4.09 \mathrm{E}+00 \\
2.96 \mathrm{E}+00 \\
5.53 \mathrm{E}-01 \\
1.23 \mathrm{E}+00 \\
1.20 \mathrm{E}+00 \\
3.48 \mathrm{E}+00 \\
3.45 \mathrm{E}+00 \\
2.44 \mathrm{E}+00 \\
9.45 \mathrm{E}-01 \\
5.53 \mathrm{E}-01 \\
1.22 \mathrm{E}+00 \\
1.39 \mathrm{E}+00 \\
1.39 \mathrm{E}+00 \\
1.23 \mathrm{E}+00 \\
1.17 \mathrm{E}+00 \\
1.50 \mathrm{E}+00 \\
1.05 \mathrm{E}+00 \\
1.81 \mathrm{E}+00 \\
1.76 \mathrm{E}+00 \\
1.15 \mathrm{E}+00 \\
1.80 \mathrm{E}+00 \\
1.93 \mathrm{E}+00 \\
2.52 \mathrm{E}+00 \\
2.37 \mathrm{E}+00 \\
1.97 \mathrm{E}+00 \\
2.04 \mathrm{E}+00 \\
8.30 \mathrm{E}-01 \\
1.58 \mathrm{E}+00 \\
1.13 \mathrm{E}+00 \\
1.02 \mathrm{E}+00 \\
2.22 \mathrm{E}+00 \\
2.23 \mathrm{E}+00 \\
2.34 \mathrm{E}+00 \\
1.36 \mathrm{E}+00 \\
1.61 \mathrm{E}+00 \\
2.23 \mathrm{E}-01 \\
6.59 \mathrm{E}-02 \\
1.56 \mathrm{E}-01 \\
1.04 \mathrm{E}+00 \\
1.13 \mathrm{E}+00 \\
6.46 \mathrm{E}-01 \\
6.71 \mathrm{E}-01 \\
1.03 \mathrm{E}+00 \\
4.53 \mathrm{E}-01 \\
2.70 \mathrm{E}-01 \\
6.47 \mathrm{E}-01 \\
1.14 \mathrm{E}+00 \\
5.29 \mathrm{E}-01 \\
7.32 \mathrm{E}-01 \\
7.84 \mathrm{E}-01 \\
9.08 \mathrm{E}-01 \\
9.60 \mathrm{E}-01 \\
4.59 \mathrm{E}-01 \\
9.78 \mathrm{E}-01 \\
1.75 \mathrm{E}+00 \\
4.91 \mathrm{E}-02\end{array}$ & $\begin{array}{l}1129 \\
4.20 \mathrm{E}-02 \\
1.04 \mathrm{E}-01 \\
1.81 \mathrm{E}-01 \\
1.11 \mathrm{E}-01 \\
4.82 \mathrm{E}-02 \\
4.95 \mathrm{E}-02 \\
6.66 \mathrm{E}-02 \\
5.99 \mathrm{E}-02 \\
1.68 \mathrm{E}-01 \\
1.22 \mathrm{E}-01 \\
2.28 \mathrm{E}-02 \\
5.07 \mathrm{E}-02 \\
4.94 \mathrm{E}-02 \\
1.43 \mathrm{E}-01 \\
1.42 \mathrm{E}-01 \\
1.01 \mathrm{E}-01 \\
3.89 \mathrm{E}-02 \\
2.28 \mathrm{E}-02 \\
5.03 \mathrm{E}-02 \\
5.73 \mathrm{E}-02 \\
5.74 \mathrm{E}-02 \\
5.07 \mathrm{E}-02 \\
4.81 \mathrm{E}-02 \\
6.17 \mathrm{E}-02 \\
4.34 \mathrm{E}-02 \\
7.44 \mathrm{E}-02 \\
7.26 \mathrm{E}-02 \\
4.72 \mathrm{E}-02 \\
7.42 \mathrm{E}-02 \\
7.93 \mathrm{E}-02 \\
1.04 \mathrm{E}-01 \\
9.75 \mathrm{E}-02 \\
8.09 \mathrm{E}-02 \\
8.40 \mathrm{E}-02 \\
3.42 \mathrm{E}-02 \\
6.49 \mathrm{E}-02 \\
4.66 \mathrm{E}-02 \\
4.21 \mathrm{E}-02 \\
9.14 \mathrm{E}-02 \\
9.17 \mathrm{E}-02 \\
9.63 \mathrm{E}-02 \\
5.60 \mathrm{E}-02 \\
6.62 \mathrm{E}-02 \\
9.17 \mathrm{E}-03 \\
2.92 \mathrm{E}-03 \\
6.87 \mathrm{E}-03 \\
4.59 \mathrm{E}-02 \\
4.99 \mathrm{E}-02 \\
2.86 \mathrm{E}-02 \\
2.96 \mathrm{E}-02 \\
4.23 \mathrm{E}-02 \\
2.00 \mathrm{E}-02 \\
1.11 \mathrm{E}-02 \\
2.85 \mathrm{E}-02 \\
5.02 \mathrm{E}-02 \\
2.18 \mathrm{E}-02 \\
3.23 \mathrm{E}-02 \\
3.46 \mathrm{E}-02 \\
4.01 \mathrm{E}-02 \\
4.24 \mathrm{E}-02 \\
1.89 \mathrm{E}-02 \\
4.31 \mathrm{E}-02 \\
7.22 \mathrm{E}-02 \\
2.17 \mathrm{E}-03 \\
.06 \mathrm{E}-02\end{array}$ & $\begin{array}{l}\text { CS134 } \\
5.49 E-03 \\
6.14 E-02 \\
1.09 E-01 \\
1.02 E-01 \\
1.64 E-02 \\
1.07 E-02 \\
1.50 E-02 \\
1.34 E-02 \\
1.58 E-01 \\
1.40 E-01 \\
4.90 E-03 \\
1.20 E-02 \\
1.76 E-02 \\
1.56 E-01 \\
1.55 E-01 \\
1.04 E-01 \\
4.42 E-02 \\
6.77 E-03 \\
1.47 E-02 \\
1.63 E-02 \\
1.71 E-02 \\
1.58 E-02 \\
5.82 E-02 \\
7.04 E-02 \\
4.41 E-02 \\
7.83 E-02 \\
7.84 E-02 \\
5.09 E-02 \\
7.50 E-02 \\
1.01 E-01 \\
1.23 E-01 \\
1.40 E-01 \\
1.09 E-01 \\
7.27 E-02 \\
4.25 E-02 \\
8.20 E-02 \\
6.39 E-02 \\
6.19 E-02 \\
1.25 E-01 \\
1.35 E-01 \\
1.52 E-01 \\
9.09 E-02 \\
1.10 E-01 \\
2.10 E-02 \\
2.49 E-03 \\
2.16 E-02 \\
8.66 E-02 \\
1.17 \mathrm{E}-01 \\
4.63 E-02 \\
5.63 E-02 \\
8.83 E-02 \\
4.22 E-02 \\
5.81 E-02 \\
6.85 E-02 \\
1.25 E-01 \\
6.05 E-02 \\
7.12 E E-02 \\
7.30 E-02 \\
9.92 E-02 \\
1.02 E-01 \\
4.87 E-02 \\
1.42 E-01 \\
2.44 E-01 \\
3.55 E-03 \\
2.19 E-03\end{array}$ & $\begin{array}{l}\text { CS137 } \\
6.58 E+04 \\
1.56 \mathrm{E}+05 \\
2.65 \mathrm{E}+05 \\
1.65 \mathrm{E}+05 \\
7.50 \mathrm{E}+04 \\
7.78 \mathrm{E}+04 \\
1.05 \mathrm{E}+05 \\
9.46 \mathrm{E}+04 \\
2.54 \mathrm{E}+05 \\
1.84 \mathrm{E}+05 \\
3.63 \mathrm{E}+04 \\
8.08 \mathrm{E}+04 \\
7.83 \mathrm{E}+04 \\
2.18 \mathrm{E}+05 \\
2.18 \mathrm{E}+05 \\
1.55 \mathrm{E}+05 \\
5.99 \mathrm{E}+04 \\
3.66 \mathrm{E}+04 \\
8.10 \mathrm{E}+04 \\
9.23 \mathrm{E}+04 \\
9.28 \mathrm{E}+04 \\
8.22 \mathrm{E}+04 \\
7.55 \mathrm{E}+04 \\
9.74 \mathrm{E}+04 \\
6.90 \mathrm{E}+04 \\
1.19 \mathrm{E}+05 \\
1.16 \mathrm{E}+05 \\
7.55 \mathrm{E}+04 \\
1.19 \mathrm{E}+05 \\
1.27 \mathrm{E}+05 \\
1.67 \mathrm{E}+05 \\
1.56 \mathrm{E}+05 \\
1.31 \mathrm{E}+05 \\
1.38 \mathrm{E}+05 \\
5.57 \mathrm{E}+04 \\
1.06 \mathrm{E}+05 \\
7.61 \mathrm{E}+04 \\
6.89 \mathrm{E}+04 \\
1.50 \mathrm{E}+05 \\
1.51 \mathrm{E}+05 \\
1.58 \mathrm{E}+05 \\
9.22 \mathrm{E}+04 \\
1.09 \mathrm{E}+05 \\
1.48 \mathrm{E}+04 \\
5.06 \mathrm{E}+03 \\
1.14 \mathrm{E}+04 \\
7.81 \mathrm{E}+04 \\
8.46 \mathrm{E}+04 \\
4.89 \mathrm{E}+04 \\
5.07 \mathrm{E}+04 \\
6.99 \mathrm{E}+04 \\
3.43 \mathrm{E}+04 \\
1.72 \mathrm{E}+04 \\
4.90 \mathrm{E}+04 \\
8.67 \mathrm{E}+04 \\
3.59 \mathrm{E}+04 \\
5.61 \mathrm{E}+04 \\
6.04 \mathrm{E}+04\end{array}$ \\
\hline
\end{tabular}


HNF-SD-WM-TI-794, ReV, 0

ACTIVITY IN UNSEPARATED FUEL (Curies decayed to $1 / 1 / 94$ )

\begin{tabular}{|c|c|c|c|c|c|c|c|c|c|}
\hline $\begin{array}{c}\text { Listing \#] } \\
261 \\
262 \\
263 \\
264 \\
265 \\
266 \\
267 \\
268 \\
269 \\
270 \\
271 \\
272 \\
273 \\
274 \\
275 \\
276 \\
277 \\
278 \\
279 \\
280 \\
287 \\
282 \\
283 \\
284 \\
285 \\
286 \\
287 \\
288 \\
289 \\
290 \\
291 \\
292 \\
293 \\
294 \\
295 \\
296 \\
297 \\
298 \\
299 \\
300 \\
301 \\
302 \\
303 \\
304 \\
305 \\
306 \\
307 \\
308 \\
309 \\
310 \\
311 \\
312 \\
313 \\
314 \\
315 \\
316 \\
317 \\
318 \\
319 \\
320 \\
321 \\
322 \\
323 \\
324 \\
325\end{array}$ & $\begin{array}{l}B A 137 \\
6.23 \mathrm{E}+04 \\
1.48 \mathrm{E}+05 \\
2.50 \mathrm{E}+05 \\
1.56 \mathrm{E}+05 \\
7.10 \mathrm{E}+04 \\
7.36 \mathrm{E}+04 \\
9.94 \mathrm{E}+04 \\
8.95 \mathrm{E}+04 \\
2.40 \mathrm{E}+05 \\
1.74 \mathrm{E}+05 \\
3.43 \mathrm{E}+04 \\
7.64 \mathrm{E}+04 \\
7.41 \mathrm{E}+04 \\
2.07 \mathrm{E}+05 \\
2.06 \mathrm{E}+05 \\
1.47 \mathrm{E}+05 \\
5.67 \mathrm{E}+04 \\
3.46 \mathrm{E}+04 \\
7.66 \mathrm{E}+04 \\
8.73 \mathrm{E}+04 \\
8.78 \mathrm{E}+04 \\
7.78 \mathrm{E}+04 \\
7.14 \mathrm{E}+04 \\
9.21 \mathrm{E}+04 \\
6.53 \mathrm{E}+04 \\
1.12 \mathrm{E}+05 \\
1.10 \mathrm{E}+05 \\
7.14 \mathrm{E}+04 \\
1.13 \mathrm{E}+05 \\
1.20 \mathrm{E}+05 \\
1.58 \mathrm{E}+05 \\
1.48 \mathrm{E}+05 \\
1.24 \mathrm{E}+05 \\
1.30 \mathrm{E}+05 \\
5.27 \mathrm{E}+04 \\
1.00 \mathrm{E}+05 \\
7.20 \mathrm{E}+04 \\
6.52 \mathrm{E}+04 \\
1.42 \mathrm{E}+05 \\
1.42 \mathrm{E}+05 \\
1.50 \mathrm{E}+05 \\
8.72 \mathrm{E}+04 \\
1.03 \mathrm{E}+05 \\
1.40 \mathrm{E}+04 \\
4.78 \mathrm{E}+03 \\
1.08 \mathrm{E}+04 \\
7.39 \mathrm{E}+04 \\
8.00 \mathrm{E}+04 \\
4.63 \mathrm{E}+04 \\
4.80 \mathrm{E}+04 \\
6.61 \mathrm{E}+04 \\
3.25 \mathrm{E}+04 \\
1.63 \mathrm{E}+04 \\
4.63 \mathrm{E}+04 \\
8.20 \mathrm{E}+04 \\
3.40 \mathrm{E}+04 \\
5.31 \mathrm{E}+04 \\
5.71 \mathrm{E}+04 \\
6.58 \mathrm{E}+04 \\
7.00 \mathrm{E}+04 \\
3.02 \mathrm{E}+04 \\
7.08 \mathrm{E}+04 \\
1.13 \mathrm{E}+05 \\
3.62 \mathrm{E}+03 \\
1.82 \mathrm{E}+04\end{array}$ & 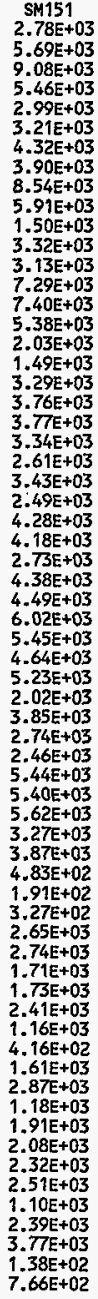 & $\begin{array}{l}\text { EU152 } \\
5.75 \mathrm{E}-02 \\
1.23 \mathrm{E}+00 \\
2.79 \mathrm{E}+00 \\
1.94 \mathrm{E}+00 \\
2.59 \mathrm{E}-01 \\
1.38 \mathrm{E}-01 \\
1.87 \mathrm{E}-01 \\
1.60 \mathrm{E}-01 \\
2.78 \mathrm{E}+00 \\
2.32 \mathrm{E}+00 \\
5.15 \mathrm{E}-02 \\
1.28 \mathrm{E}-01 \\
2.24 \mathrm{E}-01 \\
2.42 \mathrm{E}+00 \\
2.26 \mathrm{E}+00 \\
1.46 \mathrm{E}+00 \\
6.23 \mathrm{E}-01 \\
6.66 \mathrm{E}-02 \\
1.40 \mathrm{E}-01 \\
1.48 \mathrm{E}-01 \\
1.50 \mathrm{E}-01 \\
1.33 \mathrm{E}-01 \\
7.02 \mathrm{E}-01 \\
8.18 \mathrm{E}-01 \\
4.95 \mathrm{E}-01 \\
8.53 \mathrm{E}-01 \\
8.35 \mathrm{E}-01 \\
5.28 \mathrm{E}-01 \\
7.45 \mathrm{E}-01 \\
1.01 \mathrm{E}+00 \\
1.18 \mathrm{E}+00 \\
1.34 \mathrm{E}+00 \\
9.91 \mathrm{E}-01 \\
5.93 \mathrm{E}-01 \\
3.63 \mathrm{E}-01 \\
6.74 \mathrm{E}-01 \\
5.19 \mathrm{E}-01 \\
4.94 \mathrm{E}-01 \\
9.61 \mathrm{E}-01 \\
1.03 \mathrm{E}+00 \\
1.14 \mathrm{E}+00 \\
6.66 \mathrm{E}-01 \\
7.90 \mathrm{E}-01 \\
1.66 \mathrm{E}-01 \\
1.83 \mathrm{E}-02 \\
1.90 \mathrm{E}-01 \\
7.13 \mathrm{E}-01 \\
9.47 \mathrm{E}-01 \\
3.72 \mathrm{E}-01 \\
4.40 \mathrm{E}-01 \\
5.96 \mathrm{E}-01 \\
3.16 \mathrm{E}-01 \\
4.08 \mathrm{E}-01 \\
5.01 \mathrm{E}-01 \\
8.60 \mathrm{E}-01 \\
3.77 \mathrm{E}-01 \\
4.77 \mathrm{E}-01 \\
4.69 \mathrm{E}-01 \\
6.49 \mathrm{E}-01 \\
6.34 \mathrm{E}-01 \\
2.60 \mathrm{E}-01 \\
8.62 \mathrm{E}-01 \\
1.45 \mathrm{E}+00 \\
2.11 \mathrm{E}-02 \\
0.00 \mathrm{E}+00\end{array}$ & $\begin{array}{l}\mathrm{EU154} \\
9.67 \mathrm{E}+00 \\
7.57 \mathrm{E}+01 \\
1.58 \mathrm{E}+02 \\
1.12 \mathrm{E}+02 \\
2.06 \mathrm{E}+01 \\
1.51 \mathrm{E}+01 \\
2.05 \mathrm{E}+01 \\
1.81 \mathrm{E}+01 \\
1.64 \mathrm{E}+02 \\
1.35 \mathrm{E}+02 \\
6.51 \mathrm{E}+00 \\
1.52 \mathrm{E}+01 \\
1.97 \mathrm{E}+01 \\
1.45 \mathrm{E}+02 \\
1.38 \mathrm{E}+02 \\
9.09 \mathrm{E}+01 \\
3.81 \mathrm{E}+01 \\
7.48 \mathrm{E}+00 \\
1.62 \mathrm{E}+01 \\
1.79 \mathrm{E}+01 \\
1.82 \mathrm{E}+01 \\
1.63 \mathrm{E}+01 \\
4.46 \mathrm{E}+01 \\
5.32 \mathrm{E}+01 \\
3.34 \mathrm{E}+01 \\
5.78 \mathrm{E}+01 \\
5.67 \mathrm{E}+01 \\
3.62 \mathrm{E}+01 \\
5.28 \mathrm{E}+01 \\
6.76 \mathrm{E}+01 \\
8.14 \mathrm{E}+01 \\
8.89 \mathrm{E}+01 \\
6.78 \mathrm{E}+01 \\
4.80 \mathrm{E}+01 \\
2.60 \mathrm{E}+01 \\
4.87 \mathrm{E}+01 \\
3.70 \mathrm{E}+01 \\
3.48 \mathrm{E}+01 \\
7.00 \mathrm{E}+01 \\
7.40 \mathrm{E}+01 \\
8.09 \mathrm{E}+01 \\
4.74 \mathrm{E}+01 \\
5.63 \mathrm{E}+01 \\
1.07 \mathrm{E}+01 \\
1.37 \mathrm{E}+00 \\
9.94 \mathrm{E}+00 \\
4.12 \mathrm{E}+01 \\
5.29 \mathrm{E}+01 \\
2.24 \mathrm{E}+01 \\
2.58 \mathrm{E}+01 \\
4.13 \mathrm{E}+01 \\
1.84 \mathrm{E}+01 \\
2.44 \mathrm{E}+01 \\
2.88 \mathrm{E}+01 \\
5.00 \mathrm{E}+01 \\
2.54 \mathrm{E}+01 \\
2.87 \mathrm{E}+01 \\
2.89 \mathrm{E}+01 \\
3.84 \mathrm{E}+01 \\
3.84 \mathrm{E}+01 \\
1.87 \mathrm{E}+01 \\
4.96 \mathrm{E}+01 \\
9.60 \mathrm{E}+01 \\
1.43 \mathrm{E}+00 \\
1.84 \mathrm{E}+00\end{array}$ & $\begin{array}{c}E U 155 \\
2.54 E+01 \\
4.71 E+01 \\
6.47 E+01 \\
4.41 E+01 \\
2.76 E+01 \\
3.04 E+01 \\
4.16 E+01 \\
3.80 E+01 \\
7.25 E+01 \\
5.02 E+01 \\
1.50 E+01 \\
3.37 E+01 \\
3.15 E+01 \\
6.56 E+01 \\
6.92 E+01 \\
5.17 E+01 \\
1.93 E+01 \\
1.60 E+01 \\
3.56 E+01 \\
4.10 E+01 \\
4.19 E+01 \\
3.78 E+01 \\
2.71 E+01 \\
3.65 E+01 \\
2.72 E+01 \\
4.73 E+01 \\
4.67 E+01 \\
3.10 E+01 \\
5.08 E+01 \\
5.16 E+01 \\
7.08 E+01 \\
6.35 E+01 \\
5.57 E+01 \\
6.61 E+01 \\
2.51 E+01 \\
4.88 E+01 \\
3.48 E+01 \\
3.14 E+01 \\
7.10 E+01 \\
7.05 E+01 \\
7.39 E+01 \\
4.35 E+01 \\
5.20 E+01 \\
5.99 E+00 \\
2.56 E+00 \\
3.33 E+00 \\
3.29 E+01 \\
3.32 E+01 \\
2.19 E+01 \\
2.22 E+01 \\
3.30 E+01 \\
1.50 E+01 \\
4.00 E+00 \\
2.09 E+01 \\
3.84 E+01 \\
1.66 E+01 \\
2.64 E+01 \\
2.96 E+01 \\
3.22 E+01 \\
3.59 E+01 \\
1.69 E+01 \\
3.31 E+01 \\
5.28 E+01 \\
2.06 E+00 \\
1.29 E+01\end{array}$ & $\begin{array}{l}\text { RA.226 } \\
2.18 E-04 \\
1.70 E-04 \\
2.35 E-04 \\
1.28 E-04 \\
1.39 E-04 \\
1.99 E-04 \\
2.67 E-04 \\
2.44 E-04 \\
2.05 E-04 \\
1.30 E-04 \\
9.86 E-05 \\
2.10 E-04 \\
1.55 E-04 \\
1.70 E-04 \\
1.78 E-04 \\
1.37 E-04 \\
4.85 E-05 \\
8.75 E-05 \\
1.97 E-04 \\
2.30 E-04 \\
2.29 E-04 \\
2.01 E-04 \\
6.51 E-05 \\
9.04 E-05 \\
7.20 E-05 \\
1.23 E-04 \\
1.19 E-04 \\
7.91 E-05 \\
1.35 E-04 \\
1.20 E-04 \\
1.71 E-04 \\
1.38 E-04 \\
1.25 E-04 \\
1.94 E-04 \\
5.91 E-05 \\
1.14 E-04 \\
7.74 E-05 \\
6.72 E-05 \\
1.59 E-04 \\
1.50 E-04 \\
1.52 E-04 \\
8.78 E-05 \\
1.03 E-04 \\
1.01 E-05 \\
1.02 E-05 \\
7.42 E-05 \\
8.36 E-05 \\
7.65 E-05 \\
6.01 E-05 \\
5.59 E-05 \\
5.76 E-05 \\
3.58 E-05 \\
5.91 E-06 \\
4.67 E-05 \\
8.37 E-05 \\
2.47 E-05 \\
6.09 E-05 \\
6.97 E-05 \\
6.98 E-05 \\
7.877 E-05 \\
2.60 E-05 \\
6.25 E-05 \\
7.13 E-05 \\
5.60 E-06 \\
9.45 E-05\end{array}$ & $\begin{array}{l}\text { RA228 } \\
6.47 \mathrm{E}-10 \\
1.47 \mathrm{E}-09 \\
2.54 \mathrm{E}-09 \\
1.49 \mathrm{E}-09 \\
7.13 \mathrm{E}-10 \\
7.46 \mathrm{E}-10 \\
1.00 \mathrm{E}-09 \\
8.98 \mathrm{E}-10 \\
2.27 \mathrm{E}-09 \\
1.60 \mathrm{E}-09 \\
3.41 \mathrm{E}-10 \\
7.54 \mathrm{E}-10 \\
7.23 \mathrm{E}-10 \\
1.90 \mathrm{E}-09 \\
1.89 \mathrm{E}-09 \\
1.35 \mathrm{E}-09 \\
5.15 \mathrm{E}-10 \\
3.33 \mathrm{E}-10 \\
7.34 \mathrm{E}-10 \\
8.35 \mathrm{E}-10 \\
8.32 \mathrm{E}-10 \\
7.32 \mathrm{E}-10 \\
6.36 \mathrm{E}-10 \\
8.21 \mathrm{E}-10 \\
5.84 \mathrm{E}-10 \\
9.98 \mathrm{E}-10 \\
9.71 \mathrm{E}-10 \\
6.31 \mathrm{E}-10 \\
9.97 \mathrm{E}-10 \\
1.04 \mathrm{E}-09 \\
1.37 \mathrm{E}-09 \\
1.27 \mathrm{E}-09 \\
1.06 \mathrm{E}-09 \\
1.13 \mathrm{E}-09 \\
4.50 \mathrm{E}-10 \\
8.51 \mathrm{E}-10 \\
6.07 \mathrm{E}-10 \\
5.45 \mathrm{E}-10 \\
1.19 \mathrm{E}-09 \\
1.18 \mathrm{E}-09 \\
1.24 \mathrm{E}-09 \\
7.17 \mathrm{E}-10 \\
8.44 \mathrm{E}-10 \\
1.13 \mathrm{E}-10 \\
3.20 \mathrm{E}-10 \\
2.92 \mathrm{E}-10 \\
2.91 \mathrm{E}-09 \\
2.76 \mathrm{E}-09 \\
2.04 \mathrm{E}-09 \\
1.94 \mathrm{E}-09 \\
5.27 \mathrm{E}-10 \\
1.25 \mathrm{E}-09 \\
1.17 \mathrm{E}-10 \\
1.67 \mathrm{E}-09 \\
2.98 \mathrm{E}-09 \\
2.63 \mathrm{E}-10 \\
2.12 \mathrm{E}-09 \\
2.40 \mathrm{E}-09 \\
2.47 \mathrm{E}-09 \\
2.75 \mathrm{E}-09 \\
2.30 \mathrm{E}-10 \\
2.30 \mathrm{E}-09 \\
8.49 \mathrm{E}-10 \\
1.85 \mathrm{E}-10 \\
2.78 \mathrm{E}-09\end{array}$ & $\begin{array}{l}\text { AC227 } \\
1.17 E-03 \\
8.62 E-04 \\
1.16 E-03 \\
6.33 E-04 \\
7.34 E-04 \\
1.07 E-03 \\
1.43 E-03 \\
1.31 E-03 \\
1.03 E-03 \\
6.43 E-04 \\
5.31 E-04 \\
1.13 E-03 \\
8.29 E-04 \\
8.55 E-04 \\
9.01 E-04 \\
6.95 E-04 \\
2.45 E-04 \\
4.72 E-04 \\
1.06 E-03 \\
1.25 E-03 \\
1.24 E-03 \\
1.09 E-03 \\
3.34 E-04 \\
4.67 E-04 \\
3.75 E-04 \\
6.40 E-04 \\
6.22 E-04 \\
4.14 E-04 \\
7.11 E-04 \\
6.23 E-04 \\
8.99 E-04 \\
7.16 E-04 \\
6.57 E-04 \\
1.04 E-03 \\
3.12 E-04 \\
6.03 E-04 \\
4.09 E-04 \\
3.55 E-04 \\
8.42 E-04 \\
7.97 E-04 \\
8.03 E-04 \\
4.65 E-04 \\
5.47 E-04 \\
5.19 E-05 \\
4.94 E-05 \\
3.31 E E-05 \\
3.90 E=04 \\
3.53 E E-04 \\
2.83 E-04 \\
2.62 E-04 \\
3.03 E-04 \\
1.67 E-04 \\
2.83 E-05 \\
2.17 E-04 \\
3.91 E-04 \\
1.29 E-04 \\
2.87 E-04 \\
3.31 E-04 \\
3.28 E-04 \\
3.72 E-04 \\
1.38 E-04 \\
2.91 E-04 \\
3.68 E-04 \\
2.70 E-05 \\
4.73 E-04\end{array}$ & $\begin{array}{l}\text { TH229 } \\
1.22 \mathrm{E}-07 \\
2.92 \mathrm{E}-07 \\
5.15 \mathrm{E}-07 \\
3.03 \mathrm{E}-07 \\
1.37 \mathrm{E}-07 \\
1.42 \mathrm{E}-07 \\
1.90 \mathrm{E}-07 \\
1.71 \mathrm{E}-07 \\
4.59 \mathrm{E}-07 \\
3.27 \mathrm{E}-07 \\
6.46 \mathrm{E}-08 \\
1.43 \mathrm{E}-07 \\
1.38 \mathrm{E}-07 \\
3.84 \mathrm{E}-07 \\
3.79 \mathrm{E}-07 \\
2.69 \mathrm{E}-07 \\
1.03 \mathrm{E}-07 \\
6.32 \mathrm{E}-08 \\
1.39 \mathrm{E}-07 \\
1.58 \mathrm{E}-07 \\
1.58 \mathrm{E}-07 \\
1.39 \mathrm{E}-07 \\
1.27 \mathrm{E}-07 \\
1.62 \mathrm{E}-07 \\
1.15 \mathrm{E}-07 \\
1.96 \mathrm{E}-07 \\
1.91 \mathrm{E}-07 \\
1.24 \mathrm{E}-07 \\
1.95 \mathrm{E}-07 \\
2.06 \mathrm{E}-07 \\
2.69 \mathrm{E}-07 \\
2.50 \mathrm{E}-07 \\
2.08 \mathrm{E}-07 \\
2.19 \mathrm{E}-07 \\
8.78 \mathrm{E}-08 \\
1.66 \mathrm{E}-07 \\
1.19 \mathrm{E}-07 \\
1.07 \mathrm{E}-07 \\
2.32 \mathrm{E}-07 \\
2.32 \mathrm{E}-07 \\
2.42 \mathrm{E}-07 \\
1.40 \mathrm{E}-07 \\
1.65 \mathrm{E}-07 \\
2.26 \mathrm{E}-08 \\
1.03 \mathrm{E}-08 \\
2.34 \mathrm{E}-08 \\
1.58 \mathrm{E}-07 \\
1.70 \mathrm{E}-07 \\
9.89 \mathrm{E}-08 \\
1.02 \mathrm{E}-07 \\
1.04 \mathrm{E}-07 \\
6.80 \mathrm{E}-07 \\
1.53 \mathrm{E}-08 \\
1.41 \mathrm{E}-07 \\
7.38 \mathrm{E}-07 \\
3.58 \mathrm{E}-09 \\
9.64 \mathrm{E}-08 \\
1.69 \mathrm{E}-07 \\
5.24 \mathrm{E}-08 \\
1.09 \mathrm{E}-07 \\
\end{array}$ \\
\hline
\end{tabular}


HNF-SD-WM-TI-794, Rev. 0

ACTIVITY IN UNSEPARATED FUEL (Curies decayed to 1/1/94)

\begin{tabular}{|c|c|c|c|c|c|c|c|c|c|}
\hline $\begin{array}{r}\text { isting \#3 } \\
261 \\
262 \\
263 \\
264 \\
265 \\
266 \\
267 \\
268 \\
269 \\
270 \\
271 \\
272 \\
273 \\
274 \\
275 \\
276 \\
277 \\
278 \\
279 \\
280 \\
281 \\
282 \\
283 \\
284 \\
285 \\
286 \\
287 \\
288 \\
289 \\
290 \\
291 \\
292 \\
293 \\
294 \\
295 \\
296 \\
297 \\
298 \\
299 \\
300 \\
301 \\
302 \\
303 \\
304 \\
305 \\
306 \\
307 \\
308 \\
309 \\
323 \\
325 \\
310 \\
311 \\
312 \\
313 \\
314 \\
315 \\
316 \\
317 \\
318 \\
319 \\
320 \\
321 \\
322 \\
325\end{array}$ & $\begin{array}{l}\text { TH232 } \\
8.55 \mathrm{E}-10 \\
1.94 \mathrm{E}-09 \\
3.34 \mathrm{E}-09 \\
1.97 \mathrm{E}-09 \\
9.44 \mathrm{E}-10 \\
9.87 \mathrm{E}-10 \\
1.33 \mathrm{E}-09 \\
1.19 \mathrm{E}-09 \\
3.00 \mathrm{E}-09 \\
2.12 \mathrm{E}-09 \\
4.52 \mathrm{E}-10 \\
1.00 \mathrm{E}-09 \\
9.61 \mathrm{E}-10 \\
2.53 \mathrm{E}-09 \\
2.52 \mathrm{E}-09 \\
1.80 \mathrm{E}-09 \\
6.87 \mathrm{E}-10 \\
4.44 \mathrm{E}-10 \\
9.80 \mathrm{E}-10 \\
1.11 \mathrm{E}-09 \\
1.11 \mathrm{E}-09 \\
9.79 \mathrm{E}-10 \\
8.51 \mathrm{E}-10 \\
1.10 \mathrm{E}-09 \\
7.83 \mathrm{E}-10 \\
1.34 \mathrm{E}-09 \\
1.30 \mathrm{E}-09 \\
8.48 \mathrm{E}-10 \\
1.34 \mathrm{E}-09 \\
1.40 \mathrm{E}-09 \\
1.85 \mathrm{E}-09 \\
1.71 \mathrm{E}-09 \\
1.43 \mathrm{E}-09 \\
1.53 \mathrm{E}-09 \\
6.07 \mathrm{E}-10 \\
1.15 \mathrm{E}-09 \\
8.21 \mathrm{E}-10 \\
7.38 \mathrm{E}-10 \\
1.61 \mathrm{E}-09 \\
1.61 \mathrm{E}-09 \\
1.68 \mathrm{E}-09 \\
9.73 \mathrm{E}-10 \\
1.15 \mathrm{E}-09 \\
1.53 \mathrm{E}-10 \\
4.34 \mathrm{E}-10 \\
3.95 \mathrm{E}-10 \\
3.95 \mathrm{E}-09 \\
3.75 \mathrm{E}-09 \\
3.15 \mathrm{E}-09 \\
1.16 \mathrm{E}-09 \\
2.53 \mathrm{E}-10 \\
3.82 \mathrm{E}-09 \\
2.63 \mathrm{E}-09 \\
7.18 \mathrm{E}-10 \\
1.71 \mathrm{E}-09 \\
1.59 \mathrm{E}-10 \\
2.27 \mathrm{E}-09 \\
4.07 \mathrm{E}-09 \\
3.59 \mathrm{E}-10 \\
3.39 \mathrm{E}-09-09\end{array}$ & 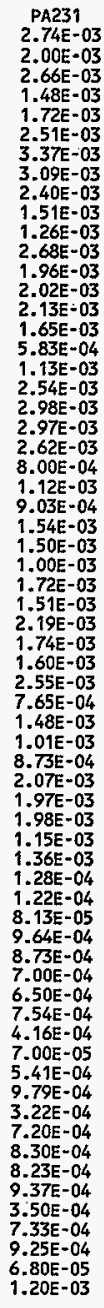 & $\begin{array}{c}\text { U232 } \\
8.61 \mathrm{E}-04 \\
2.91 \mathrm{E}-03 \\
5.58 \mathrm{E}-03 \\
3.54 \mathrm{E}-03 \\
1.13 \mathrm{E}-03 \\
1.07 \mathrm{E}-03 \\
1.44 \mathrm{E}-03 \\
1.29 \mathrm{E}-03 \\
5.26 \mathrm{E}-03 \\
4.02 \mathrm{E}-03 \\
4.84 \mathrm{E}-04 \\
1.09 \mathrm{E}-03 \\
1.13 \mathrm{E}-03 \\
4.50 \mathrm{E}-03 \\
4.35 \mathrm{E}-03 \\
2.99 \mathrm{E}-03 \\
1.19 \mathrm{E}-03 \\
4.95 \mathrm{E}-04 \\
1.09 \mathrm{E}-03 \\
1.23 \mathrm{E}-03 \\
1.24 \mathrm{E}-03 \\
1.09 \mathrm{E}-03 \\
1.43 \mathrm{E}-03 \\
1.77 \mathrm{E}-03 \\
1.19 \mathrm{E}-03 \\
2.04 \mathrm{E}-03 \\
2.00 \mathrm{E}-03 \\
1.29 \mathrm{E}-03 \\
1.97 \mathrm{E}-03 \\
2.24 \mathrm{E}-03 \\
2.84 \mathrm{E}-03 \\
2.82 \mathrm{E}-03 \\
2.26 \mathrm{E}-03 \\
2.06 \mathrm{E}-03 \\
9.18 \mathrm{E}-04 \\
1.73 \mathrm{E}-03 \\
1.27 \mathrm{E}-03 \\
1.16 \mathrm{E}-03 \\
2.45 \mathrm{E}-03 \\
2.49 \mathrm{E}-03 \\
2.66 \mathrm{E}-03 \\
1.55 \mathrm{E}-03 \\
1.83 \mathrm{E}-03 \\
2.89 \mathrm{E}-04 \\
9.07 \mathrm{E}-05 \\
3.59 \mathrm{E}-04 \\
1.85 \mathrm{E}-03 \\
2.18 \mathrm{E}-03 \\
1.08 \mathrm{E}-03 \\
1.17 \mathrm{E}-03 \\
1.22 \mathrm{E}-03 \\
8.08 \mathrm{E}-04 \\
4.75 \mathrm{E}-04 \\
1.20 \mathrm{E}-03 \\
2.09 \mathrm{E}-03 \\
6.71 \mathrm{E}-04 \\
1.27 \mathrm{E}-03 \\
1.32 \mathrm{E}-03 \\
1.63 \mathrm{E}-03 \\
1.97 \mathrm{E}-03 \\
2.35 \mathrm{E}-03-03 \\
7.46 \mathrm{E}-03 \\
2.60 \mathrm{E}-04\end{array}$ & $\begin{array}{l}\text { U233 } \\
4.47 \mathrm{E}-05 \\
1.08 \mathrm{E}-04 \\
1.88 \mathrm{E}-04 \\
1.13 \mathrm{E}-04 \\
5.06 \mathrm{E}-05 \\
5.22 \mathrm{E}-05 \\
7.02 \mathrm{E}-05 \\
6.30 \mathrm{E}-05 \\
1.72 \mathrm{E}-04 \\
1.24 \mathrm{E}-04 \\
2.40 \mathrm{E}-05 \\
5.32 \mathrm{E}-05 \\
5.16 \mathrm{E}-05 \\
1.45 \mathrm{E}-04 \\
1.44 \mathrm{E}-04 \\
1.02 \mathrm{E}-04 \\
3.94 \mathrm{E}-05 \\
2.37 \mathrm{E}-05 \\
5.24 \mathrm{E}-05 \\
5.96 \mathrm{E}-05 \\
5.96 \mathrm{E}-05 \\
5.25 \mathrm{E}-05 \\
4.86 \mathrm{E}-05 \\
6.24 \mathrm{E}-05 \\
4.41 \mathrm{E}-05 \\
7.54 \mathrm{E}-05 \\
7.35 \mathrm{E}-05 \\
4.77 \mathrm{E}-05 \\
7.51 \mathrm{E}-05 \\
7.98 \mathrm{E}-05 \\
1.05 \mathrm{E}-04 \\
9.77 \mathrm{E}-05 \\
8.12 \mathrm{E}-05 \\
8.51 \mathrm{E}-05 \\
3.43 \mathrm{E}-05 \\
6.51 \mathrm{E}-05 \\
4.66 \mathrm{E}-05 \\
4.21 \mathrm{E}-05 \\
9.15 \mathrm{E}-05 \\
9.15 \mathrm{E}-05 \\
9.59 \mathrm{E}-05 \\
5.57 \mathrm{E}-05 \\
6.57 \mathrm{E}-05 \\
9.01 \mathrm{E}-06 \\
4.34 \mathrm{E}-06 \\
9.95 \mathrm{E}-06 \\
6.70 \mathrm{E}-05 \\
7.23 \mathrm{E}-05 \\
4.19 \mathrm{E}-05 \\
4.32 \mathrm{E}-05 \\
4.17 \mathrm{E}-05 \\
2.90 \mathrm{E}-05 \\
1.07 \mathrm{E}-05 \\
4.13 \mathrm{E}-05 \\
7.24 \mathrm{E}-05 \\
2.12 \mathrm{E}-05 \\
4.68 \mathrm{E}-05 \\
5.01 \mathrm{E}-05 \\
6.10 \mathrm{E}-05 \\
1.85 \mathrm{E}-05 \\
6.14 \mathrm{E}-05 \\
6.97 \mathrm{E}-05 \\
3.16 \mathrm{E}-06 \\
1.56 \mathrm{E}-05\end{array}$ & $\begin{array}{c}\text { U234 } \\
7.34 \mathrm{E}+01 \\
5.65 \mathrm{E}+01 \\
7.53 \mathrm{E}+01 \\
4.31 \mathrm{E}+01 \\
4.74 \mathrm{E}+01 \\
6.83 \mathrm{E}+01 \\
9.20 \mathrm{E}+01 \\
8.45 \mathrm{E}+01 \\
7.01 \mathrm{E}+01 \\
4.49 \mathrm{E}+01 \\
3.45 \mathrm{E}+01 \\
7.38 \mathrm{0}+01 \\
5.47 \mathrm{E}+01 \\
5.96 \mathrm{E}+01 \\
6.30 \mathrm{E}+01 \\
4.85 \mathrm{E}+01 \\
1.73 \mathrm{E}+01 \\
3.15 \mathrm{E}+01 \\
7.10 \mathrm{E}+01 \\
8.33 \mathrm{E}+01 \\
8.34 \mathrm{E}+01 \\
7.37 \mathrm{E}+01 \\
2.38 \mathrm{E}+01 \\
3.33 \mathrm{E}+01 \\
2.66 \mathrm{E}+01 \\
4.56 \mathrm{E}+01 \\
4.45 \mathrm{E}+01 \\
2.96 \mathrm{E}+01 \\
5.09 \mathrm{E}+01 \\
4.52 \mathrm{E}+01 \\
6.52 \mathrm{E}+01 \\
5.26 \mathrm{E}+01 \\
4.82 \mathrm{E}+01 \\
7.52 \mathrm{E}+01 \\
2.29 \mathrm{E}+01 \\
4.45 \mathrm{E}+01 \\
3.04 \mathrm{E}+01 \\
2.65 \mathrm{E}+01 \\
6.27 \mathrm{E}+01 \\
5.97 \mathrm{E}+01 \\
6.05 \mathrm{E}+01 \\
3.52 \mathrm{E}+01 \\
4.16 \mathrm{E}+01 \\
4.01 \mathrm{E}+00 \\
4.09 \mathrm{E}+00 \\
2.94 \mathrm{E}+00 \\
3.34 \mathrm{E}+01 \\
3.07 \mathrm{E}+01 \\
2.41 \mathrm{E}+01 \\
2.26 \mathrm{E}+01 \\
2.35 \mathrm{E}+01 \\
1.46 \mathrm{E}+01 \\
2.40 \mathrm{E}+00 \\
1.91 \mathrm{E}+01 \\
3.47 \mathrm{E}+01 \\
1.03 \mathrm{E}+01 \\
2.53 \mathrm{E}+01 \\
2.92 \mathrm{E}+01 \\
2.92 \mathrm{E}+01 \\
3.32 \mathrm{E}+01 \\
1.11 \mathrm{E}+01 \\
2.66 \mathrm{E}+01 \\
2.99 \mathrm{E}+01 \\
2.37 \mathrm{E}+00 \\
4.09 \mathrm{E}+01\end{array}$ & $\begin{array}{c}\mathrm{U235} \\
3.31 \mathrm{E}+00 \\
2.40 \mathrm{E}+00 \\
3.13 \mathrm{E}+00 \\
1.78 \mathrm{E}+00 \\
2.09 \mathrm{E}+00 \\
3.06 \mathrm{E}+00 \\
4.12 \mathrm{E}+00 \\
3.78 \mathrm{E}+00 \\
2.92 \mathrm{E}+00 \\
1.84 \mathrm{E}+00 \\
1.55 \mathrm{E}+00 \\
3.30 \mathrm{E}+00 \\
2.43 \mathrm{E}+00 \\
2.48 \mathrm{E}+00 \\
2.64 \mathrm{E}+00 \\
2.05 \mathrm{E}+00 \\
7.22 \mathrm{E}-01 \\
1.41 \mathrm{E}+00 \\
3.18 \mathrm{E}+00 \\
3.73 \mathrm{E}+00 \\
3.74 \mathrm{E}+00 \\
3.30 \mathrm{E}+00 \\
1.01 \mathrm{E}+00 \\
1.42 \mathrm{E}+00 \\
1.14 \mathrm{E}+00 \\
1.96 \mathrm{E}+00 \\
1.91 \mathrm{E}+00 \\
1.28 \mathrm{E}+00 \\
2.20 \mathrm{E}+00 \\
1.93 \mathrm{E}+00 \\
2.81 \mathrm{E}+00 \\
2.24 \mathrm{E}+00 \\
2.07 \mathrm{E}+00 \\
3.30 \mathrm{E}+00 \\
9.91 \mathrm{E}-01 \\
1.93 \mathrm{E}+00 \\
1.31 \mathrm{E}+00 \\
1.14 \mathrm{E}+00 \\
2.72 \mathrm{E}+00 \\
2.58 \mathrm{E}+00 \\
2.60 \mathrm{E}+00 \\
1.51 \mathrm{E}+00 \\
1.79 \mathrm{E}+00 \\
1.67 \mathrm{E}-01 \\
1.61 \mathrm{E}-01 \\
1.06 \mathrm{E}-01 \\
1.27 \mathrm{E}+00 \\
1.15 \mathrm{E}+00 \\
9.22 \mathrm{E}-01 \\
8.59 \mathrm{E}-01 \\
1.00 \mathrm{E}+00 \\
5.52 \mathrm{E}-01 \\
9.20 \mathrm{E}-02 \\
7.20 \mathrm{E}-01 \\
1.31 \mathrm{E}+00 \\
4.32 \mathrm{E}-01 \\
9.65 \mathrm{E}-01 \\
1.12 \mathrm{E}+00 \\
1.11 \mathrm{E}+00 \\
1.27 \mathrm{E}+00 \\
4.75 \mathrm{E}-01 \\
1.93 \mathrm{E}-01 \\
9.19 \mathrm{E}+00 \\
1.65 \mathrm{E}+02 \\
\end{array}$ & $\begin{array}{c}\text { U236 } \\
4.43 \mathrm{E}-01 \\
1.00 \mathrm{E}+00 \\
1.69 \mathrm{E}+00 \\
1.02 \mathrm{E}+00 \\
4.93 \mathrm{E}-01 \\
5.17 \mathrm{E}-01 \\
6.96 \mathrm{E}-01 \\
6.26 \mathrm{E}-01 \\
1.57 \mathrm{E}+00 \\
1.12 \mathrm{E}+00 \\
2.39 \mathrm{E}-01 \\
5.31 \mathrm{E}-01 \\
5.10 \mathrm{E}-01 \\
1.34 \mathrm{E}+00 \\
1.34 \mathrm{E}+00 \\
9.59 \mathrm{E}-01 \\
3.67 \mathrm{E}-01 \\
2.38 \mathrm{E}-01 \\
5.26 \mathrm{E}-01 \\
5.99 \mathrm{E}-01 \\
6.00 \mathrm{E}-01 \\
5.30 \mathrm{E}-01 \\
4.61 \mathrm{E}-01 \\
5.97 \mathrm{E}-01 \\
4.26 \mathrm{E}-01 \\
7.31 \mathrm{E}-01 \\
7.12 \mathrm{E}-01 \\
4.64 \mathrm{E}-01 \\
7.37 \mathrm{E}-01 \\
7.72 \mathrm{E}-01 \\
1.02 \mathrm{E}+00 \\
9.44 \mathrm{E}-01 \\
7.92 \mathrm{E}-01 \\
8.53 \mathrm{E}-01 \\
3.39 \mathrm{E}-01 \\
6.44 \mathrm{E}-01 \\
4.60 \mathrm{E}-01 \\
4.15 \mathrm{E}-01 \\
9.08 \mathrm{E}-01 \\
9.06 \mathrm{E}-01 \\
9.48 \mathrm{E}-01 \\
5.51 \mathrm{E}-01 \\
6.51 \mathrm{E}-01 \\
8.63 \mathrm{E}-02 \\
2.45 \mathrm{E}-01 \\
2.22 \mathrm{E}-01 \\
2.23 \mathrm{E}+00 \\
2.13 \mathrm{E}+00 \\
1.56 \mathrm{E}+00 \\
1.49 \mathrm{E}+00 \\
4.11 \mathrm{E}-01 \\
9.72 \mathrm{E}-01 \\
9.09 \mathrm{E}-02 \\
1.30 \mathrm{E}+00 \\
2.34 \mathrm{E}+00 \\
2.07 \mathrm{E}-01 \\
1.67 \mathrm{E}+00 \\
1.89 \mathrm{E}+00 \\
1.95 \mathrm{E}+00 \\
2.19 \mathrm{E}+00 \\
1.85 \mathrm{E}-01 \\
1.84 \mathrm{E}+00 \\
6.75 \mathrm{E}-01 \\
1.47 \mathrm{E}-01 \\
2.24 \mathrm{E}+00\end{array}$ & $\begin{array}{c}\text { U238 } \\
7.43 E+01 \\
5.79 E+01 \\
7.75 E+01 \\
4.44 E+01 \\
4.82 E+01 \\
6.93 E+01 \\
9.33 E+01 \\
8.57 E+01 \\
7.22 E+01 \\
4.63 E+01 \\
3.49 E+01 \\
7.47 E+01 \\
5.55 E+01 \\
6.14 E+01 \\
6.48 E+01 \\
4.98 E+01 \\
1.78 E+01 \\
3.19 E+01 \\
7.20 E+01 \\
8.44 E+01 \\
8.45 E+01 \\
7.47 E+01 \\
2.44 E+01 \\
3.41 E+01 \\
2.72 E+01 \\
4.66 E+01 \\
4.55 E+01 \\
3.03 E+01 \\
5.20 E+01 \\
4.63 E+01 \\
6.67 E+01 \\
5.39 E+01 \\
4.93 E+01 \\
7.65 E+01 \\
2.34 E+01 \\
4.55 E+01 \\
3.10 E+01 \\
2.71 E+01 \\
6.41 E+01 \\
6.10 E+01 \\
6.19 E+01 \\
3.60 E+01 \\
4.25 E+01 \\
4.12 E+00 \\
2.75 E+00 \\
2.01 E+00 \\
2.26 E+01 \\
2.09 E+01 \\
1.63 E+01 \\
1.53 E+01 \\
2.41 E+01 \\
9.87 E+00 \\
2.51 E+00 \\
1.30 E+01 \\
2.35 E+01 \\
1.06 E+01 \\
1.71 E+01 \\
1.97 E+01 \\
1.98 E+01 \\
2.25 E+01 \\
1.14 E+01 \\
1.80 E+01 \\
3.08 E+01 \\
1.59 E+00 \\
2.73 E+01\end{array}$ & $\begin{array}{l}\text { NP237 } \\
1.35 \mathrm{E}-01 \\
3.49 \mathrm{E}-0 \\
6.21 \mathrm{E}-01 \\
3.82 \mathrm{E}-01 \\
1.58 \mathrm{E}-01 \\
1.60 \mathrm{E}-01 \\
2.15 \mathrm{E}-01 \\
1.94 \mathrm{E}-01 \\
5.78 \mathrm{E}-01 \\
4.22 \mathrm{E}-01 \\
7.35 \mathrm{E}-02 \\
1.64 \mathrm{E}-01 \\
1.61 \mathrm{E}-01 \\
4.91 \mathrm{E}-01 \\
4.84 \mathrm{E}-01 \\
3.41 \mathrm{E}-01 \\
1.33 \mathrm{E}-01 \\
7.37 \mathrm{E}-02 \\
1.63 \mathrm{E}-01 \\
1.85 \mathrm{E}-01 \\
1.85 \mathrm{E}-01 \\
1.64 \mathrm{E}-01 \\
1.63 \mathrm{E}-01 \\
2.08 \mathrm{E}-01 \\
1.45 \mathrm{E}-01 \\
2.49 \mathrm{E}-01 \\
2.42 \mathrm{E}-01 \\
1.57 \mathrm{E}-01 \\
2.46 \mathrm{E}-01 \\
2.66 \mathrm{E}-01 \\
3.46 \mathrm{E}-01 \\
3.28 \mathrm{E}-01 \\
2.71 \mathrm{E}-01 \\
2.75 \mathrm{E}-01 \\
1.14 \mathrm{E}-01 \\
2.15 \mathrm{E}-01 \\
1.55 \mathrm{E}-01 \\
1.41 \mathrm{E}-01 \\
3.03 \mathrm{E}-01 \\
3.05 \mathrm{E}-01 \\
3.21 \mathrm{E}-01 \\
1.87 \mathrm{E}-01 \\
2.20 \mathrm{E}-01 \\
3.13 \mathrm{E}-02 \\
1.68 \mathrm{E}-02 \\
4.08 \mathrm{E}-02 \\
2.65 \mathrm{E}-01 \\
2.89 \mathrm{E}-01 \\
1.65 \mathrm{E}-01 \\
1.71 \mathrm{E}-01 \\
1.42 \mathrm{E}-01 \\
1.16 \mathrm{E}-01 \\
4.23 \mathrm{E}-02 \\
1.65 \mathrm{E}-01 \\
2.91 \mathrm{E}-01 \\
7.37 \mathrm{E}-02 \\
1.87 \mathrm{E}-01 \\
2.00 \mathrm{E}-01 \\
2.32 \mathrm{E}-01 \\
2.45 \mathrm{E}-01 \\
6.32 \mathrm{E}-02 \\
2.50 \mathrm{E}-01 \\
2.47 \mathrm{E}-01 \\
1.35 \mathrm{n}\end{array}$ \\
\hline
\end{tabular}


HNF-SD-WM-TI-794, ReV, 0

ACTIVITY IN UNSEPARATED FUEL (Curies decayed to $1 / 1 / 94$ )

\begin{tabular}{|c|c|c|c|c|c|c|c|c|c|}
\hline $\begin{array}{r}\text { [Listing \#] } \\
261 \\
262 \\
263 \\
264 \\
265 \\
266 \\
267 \\
268 \\
269 \\
270 \\
271 \\
272 \\
273 \\
274 \\
275 \\
276 \\
277 \\
278 \\
279 \\
280 \\
281 \\
282 \\
283 \\
284 \\
285 \\
286 \\
287 \\
288 \\
289 \\
290 \\
291 \\
292 \\
293 \\
294 \\
295 \\
296 \\
297 \\
298 \\
299 \\
300 \\
301 \\
302 \\
303 \\
304 \\
305 \\
306 \\
307 \\
308 \\
309 \\
310 \\
311 \\
312 \\
313 \\
314 \\
315 \\
316 \\
317 \\
318 \\
319 \\
320 \\
321 \\
322 \\
323 \\
324 \\
325\end{array}$ & $\begin{array}{l}P U 238 \\
8.16 \mathrm{E}+00 \\
9.64 \mathrm{E}+01 \\
2.20 \mathrm{E}+02 \\
1.45 \mathrm{E}+02 \\
2.26 \mathrm{E}+01 \\
1.44 \mathrm{E}+01 \\
1.94 \mathrm{E}+01 \\
1.68 \mathrm{E}+01 \\
2.07 \mathrm{E}+02 \\
1.69 \mathrm{E}+02 \\
5.74 \mathrm{E}+00 \\
1.36 \mathrm{E}+01 \\
1.99 \mathrm{E}+01 \\
1.77 \mathrm{E}+02 \\
1.65 \mathrm{E}+02 \\
1.07 \mathrm{E}+02 \\
4.52 \mathrm{E}+01 \\
6.66 \mathrm{E}+00 \\
1.42 \mathrm{E}+01 \\
1.55 \mathrm{E}+01 \\
1.55 \mathrm{E}+01 \\
1.37 \mathrm{E}+01 \\
5.03 \mathrm{E}+01 \\
5.90 \mathrm{E}+01 \\
3.62 \mathrm{E}+01 \\
6.21 \mathrm{E}+01 \\
6.06 \mathrm{E}+01 \\
3.83 \mathrm{E}+01 \\
5.47 \mathrm{E}+01 \\
7.17 \mathrm{E}+01 \\
8.45 \mathrm{E}+01 \\
9.38 \mathrm{E}+01 \\
7.00 \mathrm{E}+01 \\
4.52 \mathrm{E}+01 \\
2.59 \mathrm{E}+01 \\
4.81 \mathrm{E}+01 \\
3.66 \mathrm{E}+01 \\
3.45 \mathrm{E}+01 \\
6.79 \mathrm{E}+01 \\
7.21 \mathrm{E}+01 \\
7.90 \mathrm{E}+01 \\
4.59 \mathrm{E}+01 \\
5.43 \mathrm{E}+01 \\
1.10 \mathrm{E}+01 \\
2.20 \mathrm{E}+00 \\
1.85 \mathrm{E}+01 \\
7.31 \mathrm{E}+01 \\
9.47 \mathrm{E}+01 \\
3.90 \mathrm{E}+01 \\
4.51 \mathrm{E}+01 \\
3.98 \mathrm{E}+01 \\
3.20 \mathrm{E}+01 \\
2.53 \mathrm{E}+01 \\
4.99 \mathrm{E}+01 \\
8.52 \mathrm{E}+01 \\
2.43 \mathrm{E}+01 \\
4.80 \mathrm{E}+01 \\
4.76 \mathrm{E}+01 \\
6.43 \mathrm{E}+01 \\
6.31 \mathrm{E}+01 \\
1.69 \mathrm{E}+01 \\
8.25 \mathrm{E}+01 \\
9.20 \mathrm{E}+01 \\
2.25 \mathrm{E}+00 \\
1.35 \mathrm{E}+00\end{array}$ & 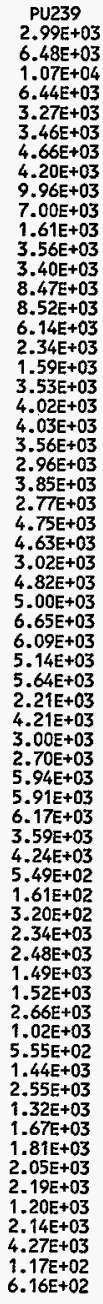 & 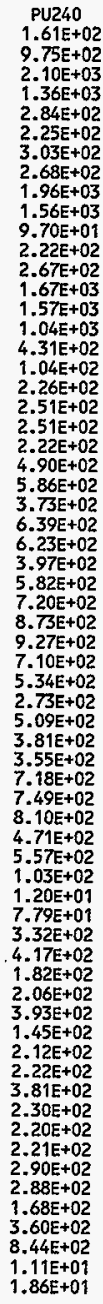 & 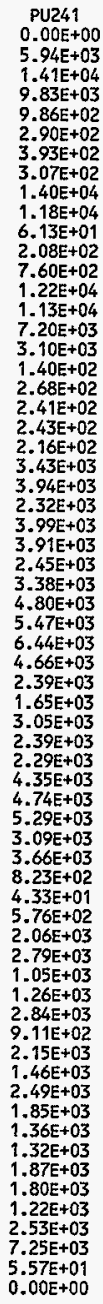 & $\begin{array}{l}\text { PU242 } \\
0.00 \mathrm{E}+00 \\
2.72 \mathrm{E}-02 \\
7.22 \mathrm{E}-02 \\
4.84 \mathrm{E}-02 \\
2.32 \mathrm{E}-03 \\
0.00 \mathrm{E}+00 \\
0.00 \mathrm{E}+00 \\
0.00 \mathrm{E}+00 \\
6.73 \mathrm{E}-02 \\
5.75 \mathrm{E}-02 \\
0.00 \mathrm{E}+00 \\
0.00 \mathrm{E}+00 \\
8.32 \mathrm{E}-04 \\
5.72 \mathrm{E}-02 \\
5.18 \mathrm{E}-02 \\
3.23 \mathrm{E}-02 \\
1.42 \mathrm{E}-02 \\
0.00 \mathrm{E}+00 \\
0.00 \mathrm{E}+00 \\
0.00 \mathrm{E}+00 \\
0.00 \mathrm{E}+00 \\
0.00 \mathrm{E}+00 \\
1.49 \mathrm{E}-02 \\
1.65 \mathrm{E}-02 \\
9.21 \mathrm{E}-03 \\
1.58 \mathrm{E}-02 \\
1.54 \mathrm{E}-02 \\
9.52 \mathrm{E}-03 \\
1.24 \mathrm{E}-02 \\
1.92 \mathrm{E}-02 \\
2.09 \mathrm{E}-02 \\
2.62 \mathrm{E}-02 \\
1.81 \mathrm{E}-02 \\
6.38 \mathrm{E}-03 \\
5.99 \mathrm{E}-03 \\
1.08 \mathrm{E}-02 \\
8.72 \mathrm{E}-03 \\
8.50 \mathrm{E}-03 \\
1.53 \mathrm{E}-02 \\
1.72 \mathrm{E}-02 \\
1.94 \mathrm{E}-02 \\
1.13 \mathrm{E}-02 \\
1.33 \mathrm{E}-02 \\
3.42 \mathrm{E}-03 \\
8.81 \mathrm{E}-05 \\
1.99 \mathrm{E}-03 \\
6.54 \mathrm{E}-03 \\
9.12 \mathrm{E}-03 \\
3.20 \mathrm{E}-03 \\
3.93 \mathrm{E}-03 \\
1.08 \mathrm{E}-02 \\
2.85 \mathrm{E}-03 \\
9.50 \mathrm{E}-03 \\
4.63 \mathrm{E}-03 \\
7.79 \mathrm{E}-03 \\
7.25 \mathrm{E}-03 \\
4.11 \mathrm{E}-03 \\
3.87 \mathrm{E}-03 \\
5.71 \mathrm{E}-03 \\
5.38 \mathrm{E}-03 \\
4.36 \mathrm{E}-03 \\
7.89 \mathrm{E}-03 \\
2.90 \mathrm{E}-02 \\
1.44 \mathrm{E}-04 \\
0.00 \mathrm{E}+00\end{array}$ & 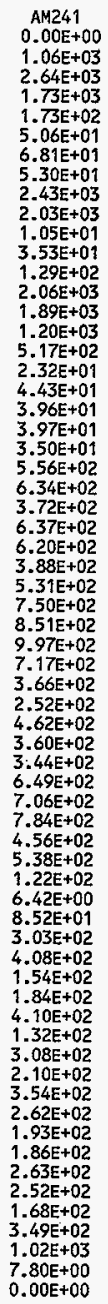 & $\begin{array}{l}\text { AM243 } \\
0.00 \mathrm{E}+00 \\
9.88 \mathrm{E}-03 \\
2.72 \mathrm{E}-02 \\
1.83 \mathrm{E}-02 \\
4.37 \mathrm{E}-04 \\
0.00 \mathrm{E}+00 \\
0.00 \mathrm{E}+00 \\
0.00 \mathrm{E}+00 \\
2.53 \mathrm{E}-02 \\
2.19 \mathrm{E}-02 \\
0.00 \mathrm{E}+00 \\
0.00 \mathrm{E}+00 \\
2.06 \mathrm{E}-07 \\
2.15 \mathrm{E}-02 \\
1.94 \mathrm{E}-02 \\
1.19 \mathrm{E}-02 \\
5.30 \mathrm{E}-03 \\
0.00 \mathrm{E}+00 \\
0.00 \mathrm{E}+00 \\
0.00 \mathrm{E}+00 \\
0.00 \mathrm{E}+00 \\
0.00 \mathrm{E}+00 \\
5.49 \mathrm{E}-03 \\
6.02 \mathrm{E}-03 \\
3.26 \mathrm{E}-03 \\
5.58 \mathrm{E}-03 \\
5.45 \mathrm{E}-03 \\
3.34 \mathrm{E}-03 \\
4.22 \mathrm{E}-03 \\
6.92 \mathrm{E}-03 \\
7.35 \mathrm{E}-03 \\
9.51 \mathrm{E}-03 \\
6.45 \mathrm{E}-03 \\
1.70 \mathrm{E}-03 \\
2.06 \mathrm{E}-03 \\
3.69 \mathrm{E}-03 \\
3.02 \mathrm{E}-03 \\
2.98 \mathrm{E}-03 \\
5.20 \mathrm{E}-03 \\
5.95 \mathrm{E}-03 \\
6.82 \mathrm{E}-03 \\
3.97 \mathrm{E}-03 \\
4.68 \mathrm{E}-03 \\
1.28 \mathrm{E}-03 \\
1.85 \mathrm{E}-05 \\
6.03 \mathrm{E}-04 \\
1.91 \mathrm{E}-03 \\
2.70 \mathrm{E}-03 \\
9.13 \mathrm{E}-04 \\
1.14 \mathrm{E}-03 \\
3.90 \mathrm{E}-03 \\
.8 .30 \mathrm{E}-04 \\
3.71 \mathrm{E}-03 \\
1.36 \mathrm{E}-03 \\
2.28 \mathrm{E}-03 \\
2.69 \mathrm{E}-03 \\
1.19 \mathrm{E}-03 \\
1.11 \mathrm{E}-03 \\
1.66 \mathrm{E}-03 \\
1.55 \mathrm{E}-03 \\
1.56 \mathrm{E}-03 \\
2.34 \mathrm{E}-03 \\
1.09 \mathrm{E}-02 \\
3.85 \mathrm{E}-05 \\
0.00 \mathrm{E}+00\end{array}$ & $\begin{array}{l}\text { CH242 } \\
0.00 \mathrm{E}+00 \\
3.00 \mathrm{E}-02 \\
8.19 \mathrm{E}-02 \\
5.56 \mathrm{E}-02 \\
1.37 \mathrm{E}-03 \\
0.00 \mathrm{E}+00 \\
0.00 \mathrm{E}+00 \\
0.00 \mathrm{E}+00 \\
7.68 \mathrm{E}-02 \\
6.64 \mathrm{E}-02 \\
0.00 \mathrm{E}+00 \\
0.00 \mathrm{E}+00 \\
1.39 \mathrm{E}-27 \\
6.54 \mathrm{E}-02 \\
5.89 \mathrm{E}-02 \\
3.64 \mathrm{E}-02 \\
1.62 \mathrm{E}-02 \\
0.00 \mathrm{E}+00 \\
0.00 \mathrm{E}+00 \\
0.00 \mathrm{E}+00 \\
0.00 \mathrm{E}+00 \\
0.00 \mathrm{E}+00 \\
1.68 \mathrm{E}-02 \\
1.84 \mathrm{E}-02 \\
9.97 \mathrm{E}-03 \\
1.71 \mathrm{E}-02 \\
1.67 \mathrm{E}-02 \\
1.02 \mathrm{E}-02 \\
1.29 \mathrm{E}-02 \\
2.12 \mathrm{E}-02 \\
2.26 \mathrm{E}-02 \\
2.92 \mathrm{E}-02 \\
1.98 \mathrm{E}-02 \\
5.27 \mathrm{E}-03 \\
6.33 \mathrm{E}-03 \\
1.14 \mathrm{E}-02 \\
9.31 \mathrm{E}-03 \\
9.19 \mathrm{E}-03 \\
1.60 \mathrm{E}-02 \\
1.83 \mathrm{E}-02 \\
2.10 \mathrm{E}-02 \\
1.22 \mathrm{E}-02 \\
1.44 \mathrm{E}-02 \\
3.94 \mathrm{E}-03 \\
7.50 \mathrm{E}-05 \\
2.41 \mathrm{E}-03 \\
7.63 \mathrm{E}-03 \\
1.08 \mathrm{E}-02 \\
3.66 \mathrm{E}-03 \\
4.56 \mathrm{E}-03 \\
1.20 \mathrm{E}-02 \\
3.33 \mathrm{E}-03 \\
1.14 \mathrm{E}-02 \\
5.46 \mathrm{E}-03 \\
9.16 \mathrm{E}-03 \\
8.30 \mathrm{E}-03 \\
4.76 \mathrm{E}-03 \\
4.45 \mathrm{E}-03 \\
6.69 \mathrm{E}-03 \\
6.25 \mathrm{E}-03 \\
4.82 \mathrm{E}-03 \\
9.40 \mathrm{E}-03 \\
3.37 \mathrm{E}-02 \\
1.55 \mathrm{E}-04 \\
0.00 \mathrm{E}+00\end{array}$ & $\begin{array}{l}\text { CM243 } \\
0.00 \mathrm{E}+0 \\
6.83 \mathrm{E}-0 \\
1.88 \mathrm{E}-0 \\
1.30 \mathrm{E}-0 \\
1.50 \mathrm{E}-0 \\
0.00 \mathrm{E}+0 \\
0.00 \mathrm{E}+0 \\
0.00 \mathrm{E}+0 \\
1.81 \mathrm{E}-0 \\
1.58 \mathrm{E}-0 \\
0.00 \mathrm{E}+0 \\
0.00 \mathrm{E}+0 \\
0.00 \mathrm{E}+0 \\
1.55 \mathrm{E}-0 \\
1.40 \mathrm{E}-0 \\
8.60 \mathrm{E}-0 \\
3.84 \mathrm{E}-0 \\
0.00 \mathrm{E}+0 \\
0.00 \mathrm{E}+0 \\
0.00 \mathrm{E}+0 \\
0.00 \mathrm{E}+0 \\
0.00 \mathrm{E}+0 \\
4.00 \mathrm{E}-0 \\
4.36 \mathrm{E}-04 \\
2.33 \mathrm{E}-0 \\
4.00 \mathrm{E}-04 \\
3.91 \mathrm{E}-04 \\
2.39 \mathrm{E}-04 \\
2.97 \mathrm{E}-04 \\
5.05 \mathrm{E}-04 \\
5.30 \mathrm{E}-04 \\
7.01 \mathrm{E}-04 \\
4.71 \mathrm{E}-04 \\
1.01 \mathrm{E}-04 \\
1.48 \mathrm{E}-04 \\
2.64 \mathrm{E}-04 \\
2.19 \mathrm{E}-04 \\
2.18 \mathrm{E}-04 \\
3.74 \mathrm{E}-04 \\
4.34 \mathrm{E}-04 \\
5.02 \mathrm{E}-04 \\
2.92 \mathrm{E}-04 \\
3.46 \mathrm{E}-04 \\
9.777 \mathrm{E}-05 \\
1.13 \mathrm{E}-06 \\
4.68 \mathrm{E}-05 \\
1.46 \mathrm{E}-04 \\
2.09 \mathrm{E}-04 \\
6.89 \mathrm{E}-05 \\
8.70 \mathrm{E}-05 \\
2.95 \mathrm{E}-04 \\
6.39 \mathrm{E}-05 \\
2.92 \mathrm{E}-04 \\
1.06 \mathrm{E}-04 \\
1.78 \mathrm{E}-04 \\
2.08 \mathrm{E}-04 \\
9.15 \mathrm{E}-05 \\
8.51 \mathrm{E}-05 \\
1.30 \mathrm{E}-04 \\
1.21 \mathrm{E}-04 \\
1.19 \mathrm{E}-04 \\
1.85 \mathrm{E}-04 \\
8.56 \mathrm{E}-04 \\
2.85 \mathrm{E}-06 \\
0.00 \mathrm{E}+00\end{array}$ \\
\hline
\end{tabular}


HNF-SD-WM-TI-794, ReV. O

ACTIVITY IN UNSEPARATED FUEL (Curies decayed to $1 / 1 / 94$ )

\begin{tabular}{|c|c|}
\hline $\begin{array}{c}\text { Listing } \# 1 \\
261 \\
262 \\
263 \\
264 \\
265 \\
266 \\
267 \\
268 \\
269 \\
270 \\
271 \\
272 \\
273 \\
274 \\
275 \\
276 \\
277 \\
278 \\
279 \\
280 \\
281 \\
282 \\
283 \\
284 \\
285 \\
286 \\
287 \\
288 \\
289 \\
290 \\
291 \\
292 \\
293 \\
294 \\
295 \\
296 \\
297 \\
298 \\
299 \\
300 \\
301 \\
302 \\
303 \\
304 \\
305 \\
306 \\
307 \\
308 \\
309 \\
310 \\
311 \\
312 \\
313 \\
314 \\
315 \\
316 \\
317 \\
318 \\
319 \\
320 \\
321 \\
322 \\
323 \\
324 \\
325\end{array}$ & $\begin{array}{l}\text { CM244 } \\
0.00 \mathrm{E}+00 \\
1.04 \mathrm{E}-02 \\
2.82 \mathrm{E}-02 \\
1.99 \mathrm{E}-02 \\
2.25 \mathrm{E}-04 \\
0.00 \mathrm{E}+00 \\
0.00 \mathrm{E}+00 \\
0.00 \mathrm{E}+00 \\
2.76 \mathrm{E}-02 \\
2.42 \mathrm{E}-02 \\
0.00 \mathrm{E}+00 \\
0.00 \mathrm{E}+00 \\
0.00 \mathrm{E}+00 \\
2.39 \mathrm{E}-02 \\
2.16 \mathrm{E}-02 \\
1.33 \mathrm{E}-02 \\
5.94 \mathrm{E}-03 \\
0.00 \mathrm{E}+00 \\
0.00 \mathrm{E}+00 \\
0.00 \mathrm{E}+00 \\
0.00 \mathrm{E}+00 \\
0.00 \mathrm{E}+00 \\
6.23 \mathrm{E}-03 \\
6.79 \mathrm{E}-03 \\
3.63 \mathrm{E}-03 \\
6.24 \mathrm{E}-03 \\
6.11 \mathrm{E}-03 \\
3.74 \mathrm{E}-03 \\
4.65 \mathrm{E}-03 \\
7.92 \mathrm{E}-03 \\
8.32 \mathrm{E}-03 \\
1.10 \mathrm{E}-02 \\
7.42 \mathrm{E}-03 \\
1.58 \mathrm{E}-03 \\
2.33 \mathrm{E}-03 \\
4.17 \mathrm{E}-03 \\
3.47 \mathrm{E}-03 \\
3.46 \mathrm{E}-03 \\
5.94 \mathrm{E}-03 \\
6.89 \mathrm{E}-03 \\
7.97 \mathrm{E}-03 \\
4.65 \mathrm{E}-03 \\
5.51 \mathrm{E}-03 \\
1.55 \mathrm{E}-03 \\
1.39 \mathrm{E}-05 \\
5.81 \mathrm{E}-04 \\
1.81 \mathrm{E}-03 \\
2.60 \mathrm{E}-03 \\
8.56 \mathrm{E}-04 \\
1.08 \mathrm{E}-03 \\
4.711 \mathrm{E}-03 \\
7.98 \mathrm{E}-04 \\
4.69 \mathrm{E}-03 \\
1.32 \mathrm{E}-03 \\
2.23 \mathrm{E}-03 \\
3.35 \mathrm{E}-03 \\
1.15 \mathrm{E}-03 \\
1.07 \mathrm{E}-03 \\
1.63 \mathrm{E}-03 \\
1.52 \mathrm{E}-03 \\
1.93 \mathrm{E}-03 \\
2.33 \mathrm{E}-03 \\
1.38 \mathrm{E}-03 \\
3.58 \mathrm{E}-02 \\
0.00 \mathrm{E}+00\end{array}$ \\
\hline
\end{tabular}


HNF-SD-WM-TI-794, ReV. 0

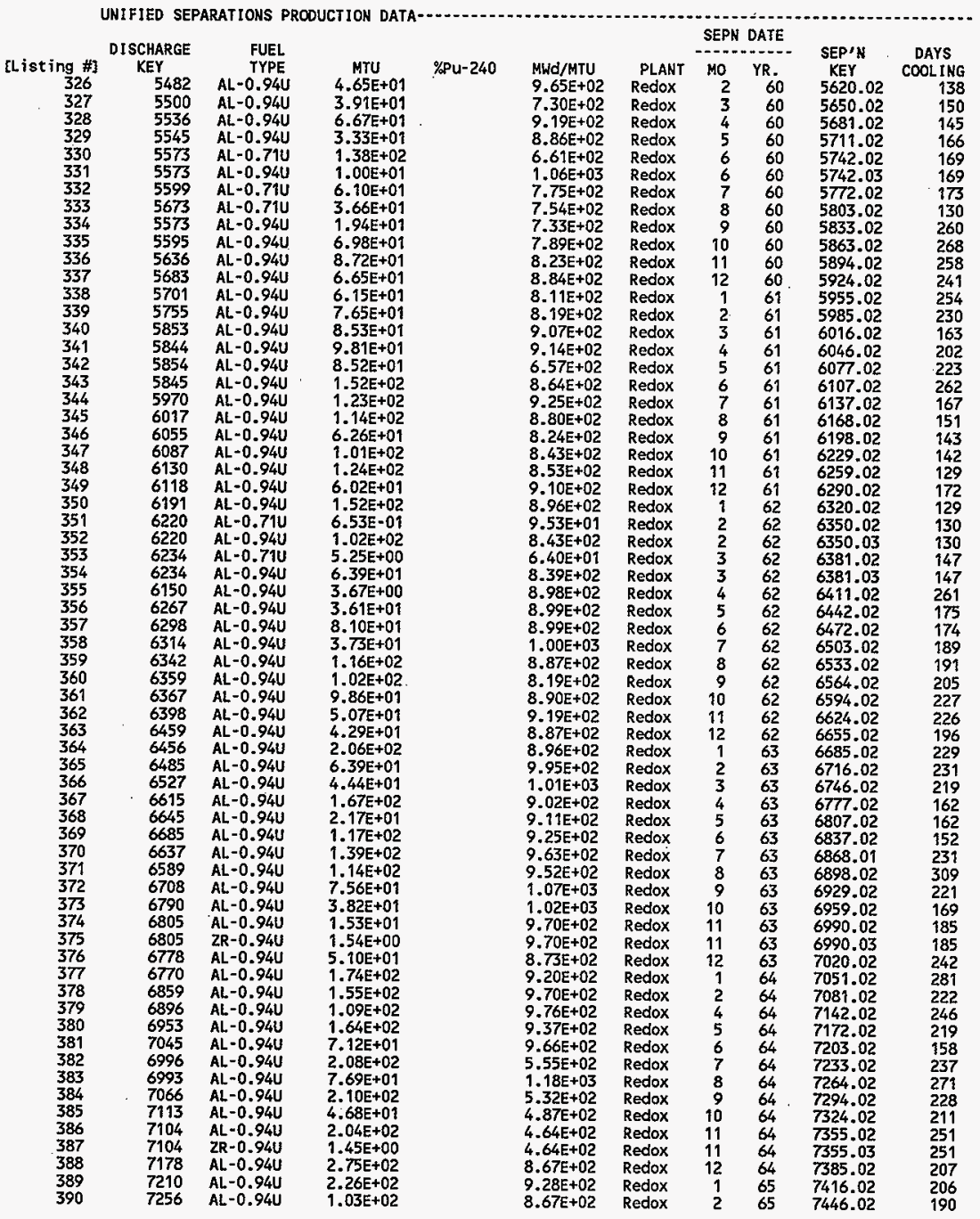


HNF-SD - WM-TI-794, ReV. 0

UNIFIED SEPARATIONS PRODUCTION DATA TOTAL WASTE LOSS FRACTION

[Listing \#]fuel Data Reference

326 SPLANT.DAT 19348 9/30/93 1:19P

327 SPLAHT.DAT $19348 \% / 30 / 93$ 1:19P

328 SPLANT.DAT 19348 9/30/93 1:19P

329 SPLANT.DAT 19348 9/30/93 1:19P

330 SPLANT.DAT 19348 9/30/93 1:19P

331 SPLANT.DAT 19348 9/30/93 1:19P

332 SPLANT.DAT $193489 / 30 / 931: 19 \mathrm{P}$

333 SPLANT.DAT 19348 9/30/93 1:19P

334 SPLANT.DAT $193489 / 30 / 93$ 1:19P

335 SPLANT.DAT $193489 / 30 / 93$ 1:19P

336 SPLANT.DAT 19348 9/30/93 $1: 19 \mathrm{p}$

337 SPLANT.DAT $193489 / 30 / 93 \quad 1: 19 \mathrm{P}$

338 SPLANT.DAT $193489 / 30 / 93 \quad 1: 19 \mathrm{P}$

339 SPLANT.DAT $193489 / 30 / 931: 19 \mathrm{P}$

340 SPLANT.DAT 19348 9/30/93 1:19P

341 SPLANT.DAT 19348 9/30/93 1:19P

342 SPLANT.DAT 19348 9/30/93 1:19P

343 SPLANT.DAT 19348 9/30/93 1:19P

344 SPLANT.DAT 19348 9/30/93 1:19P

345 SPLANT.DAT 19348 9/30/93 1:19P

346 SPLANT.DAT 19348 9/30/93 1:19P

347 SPLANT.DAT 19348 9/30/93 1:19P

348 SPLANT.DAT 19348 9/30/93 1:19P

349 SPLANT.DAT $193489 / 30 / 93$ 1:19P

350 SPLANT.DAT 19348 9/30/93 1:19P

351 SPLANT.DAT 19348 9/30/93 1:19P

352 SPLANT.DAT 19348 9/30/93 1:19P

353 SPLANT.DAT 19348 9/30/93 1:19P

354 SPLANT.DAT 19348 9/30/93 1:19P

355 SPLANT.DAT 19348 9/30/93 1:19P

356 SPLANT.DAT 19348 9/30/93 1:19P

357 SPLANT.DAT 19348 9/30/93 1:19P

358 SPLANT.DAT 19348 9/30/93 1:19P

359 SPLANT.DAT 19348 9/30/93 $1: 19 \mathrm{P}$

360 SPLANT.DAT 19348 9/30/93 $1: 19 \mathrm{P}$

361 SPLANT.DAT 19348 9/30/93 1:19P

362 SPLANT.DAT 19348 9/30/93 $1: 19 \mathrm{P}$

363 SPLANT.DAT 19348 \%/30/93 $1: 19 \mathrm{P}$

364 Reconciled to Jenkins and foster (1

365 SPLANT.DAT 19348 9/30/93 $1: 19 \mathrm{P}$

366 SPLANT.DAT 19348 9/30/93 $1: 19 \mathrm{P}$

367 SPLANT.DAT 19348 9/30/93 1:19P

368 SPLANT.DAT 19348 9/30/93 1: $19 \mathrm{P}$

369 SPLANT.DAT 19348 9/30/93 1:19P

370 SPLANT.DAT 19348 9/30/93 1:19P

371 SPLANT_DAT 19348 9/30/93 1:19P

372 SPLANT.DAT 19348 9/30/93 1:19P

373 SPLANT.DAT 19348 9/30/93 1:19P

374 Added 1.54 MTU PRTR to Zr clad

375 Added 1.54 MTU PRTR to $\mathrm{Zr}$ clad

376 SPLANT.DAT 19348 9/30/93 1:19P

377 Reconciled to Jenkins and Foster ( 1

378 SPLANT.DAT 19348 9/30/93 $1: 19 \mathrm{P}$

379 SPLANT_DAT $193489 / 30 / 93$ i:19P

380 SPLANT.DAT 19348 9/30/93 1:19P

381 SPLANT.DAT 19348 9/30/93 $1: 19 \mathrm{p}$

382 SPLANT.DAT 19348 9/30/93 1:19P

383 SPLANT.DAT 19348 9/30/93 1:19P

384 SPLANT.DAT 19348 9/30/93 1:19P

385 SPLANT.DAT 19348 9/30/93 1:19P

386 Added 1.45 MTU PRTR to $\mathrm{Zr} \mathrm{clad}$

387 Added 1.45 MTU PRTR to $\mathrm{Zr}$ clad

388 SPLANT.DAT 19348 9/30/93 1:19P

389 Reconciled to Jenkins and Foster (1

390 SPLANT.DAT 19348 9/30/93 1:19P

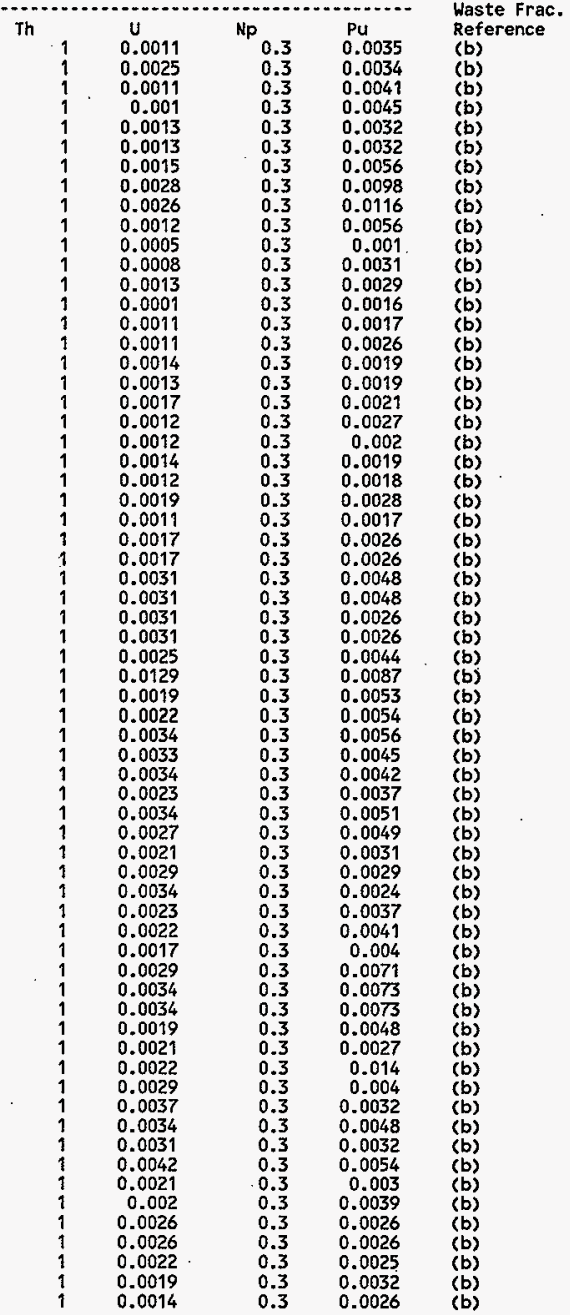


HNF-SD-WM-TI-794, ReV. 0

ACTIVITY IN UNSEPARATED FUEL (Curies decayed to 1/1/94)

\begin{tabular}{|c|c|c|c|c|c|c|c|c|c|}
\hline 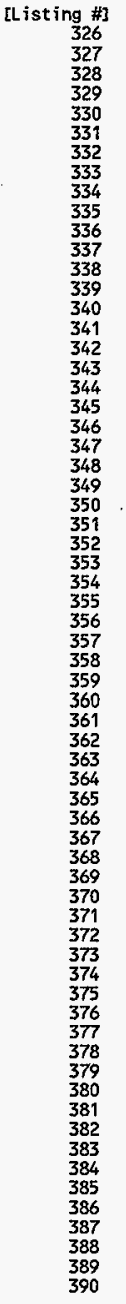 & $\begin{array}{l}403 \\
1.02 E+02 \\
6.46 E+01 \\
1.40 E+02 \\
6.75 E+01 \\
2.14 E+02 \\
2.45 E+01 \\
1.13 E+02 \\
6.63 E+01 \\
3.26 E+01 \\
1.27 E+02 \\
1.66 E+02 \\
1.38 E+02 \\
1.17 E+02 \\
1.48 E+02 \\
1.86 E+02 \\
2.15 E+02 \\
1.34 E+02 \\
3.15 E+02 \\
2.79 E+02 \\
2.47 E+02 \\
1.28 E+02 \\
2.13 E+02 \\
2.65 E+02 \\
1.37 E+02 \\
3.45 E+02 \\
1.58 E-01 \\
2.18 E+02 \\
8.55 E-01 \\
1.36 E+02 \\
8.29 E+00 \\
8.29 E+01 \\
1.87 E+02 \\
9.64 E+01 \\
2.66 E+02 \\
2.17 E+02 \\
2.28 E+02 \\
1.22 E+02 \\
1.00 E+02 \\
4.86 E+02 \\
1.69 E+02 \\
1.20 E+02 \\
4.07 E+02 \\
5.35 E+01 \\
2.96 E+02 \\
3.63 E+02 \\
2.93 E+02 \\
2.22 E+02 \\
1.08 E+02 \\
4.13 E+01 \\
4.34 E+00 \\
1.23 E+02 \\
4.42 E+02 \\
4.21 E+02 \\
3.00 E+02 \\
4.37 E+02 \\
1.99 E+02 \\
3.27 E+02 \\
2.61 E+02 \\
3.21 E+02 \\
6.58 E+01 \\
2.73 E+02 \\
2.01 E+00 \\
7.02 E+02 \\
6.20 E+02 \\
2.66 E+02\end{array}$ & $\begin{array}{l}\text { C14 } \\
2.23 E+00 \\
1.42 E+00 \\
3.05 E+00 \\
1.47 E+00 \\
5.78 E+00 \\
5.27 E-01 \\
3.00 E+00 \\
1.75 E+00 \\
7.08 E-01 \\
2.74 E+00 \\
3.57 E+00 \\
2.92 E+00 \\
2.48 E+00 \\
3.12 E+00 \\
3.84 E+00 \\
4.46 E+00 \\
2.79 E+00 \\
6.54 E+00 \\
5.67 E+00 \\
5.00 E+00 \\
2.57 E+00 \\
4.25 E+00 \\
5.26 E+00 \\
2.72 E+00 \\
6.78 E+00 \\
4.02 E-03 \\
4.27 E+00 \\
2.17 E-02 \\
2.67 E+00 \\
1.64 E-01 \\
1.61 E+00 \\
3.62 E+00 \\
1.86 E+00 \\
5.11 E+00 \\
4.17 E+00 \\
4.36 E+00 \\
2.32 E+00 \\
1.89 E+00 \\
9.17 E+00 \\
3.16 E+00 \\
2.23 E+00 \\
7.50 E+00 \\
9.81 E-01 \\
5.39 E+00 \\
6.66 E+00 \\
5.41 E+00 \\
4.00 E+00 \\
1.94 E+00 \\
7.37 E-01 \\
1.61 E-01 \\
2.21 E+00 \\
7.95 E+00 \\
7.46 E+00 \\
5.28 E+00 \\
7.64 E+00 \\
3.42 E+00 \\
5.74 E+00 \\
4.50 E+00 \\
5.58 E+00 \\
1.14 E+00 \\
4.72 E+00 \\
7.16 E-02 \\
1.19 E+01 \\
1.04 E+01 \\
4.45 E+00\end{array}$ & $\begin{array}{l}\text { NI59 } \\
6.32 \mathrm{E}-01 \\
4.03 \mathrm{E}-01 \\
8.62 \mathrm{E}-01 \\
4.15 \mathrm{E}-01 \\
1.64 \mathrm{E}+00 \\
1.49 \mathrm{E}-01 \\
8.49 \mathrm{E}-01 \\
4.96 \mathrm{E}-01 \\
2.01 \mathrm{E}-01 \\
7.77 \mathrm{E}-01 \\
1.01 \mathrm{E}+00 \\
8.28 \mathrm{E}-01 \\
7.03 \mathrm{E}-01 \\
8.83 \mathrm{E}-01 \\
1.09 \mathrm{E}+00 \\
1.26 \mathrm{E}+00 \\
7.91 \mathrm{E}-01 \\
1.85 \mathrm{E}+00 \\
1.61 \mathrm{E}+00 \\
1.42 \mathrm{E}+00 \\
7.27 \mathrm{E}-01 \\
1.21 \mathrm{E}+00 \\
1.49 \mathrm{E}+00 \\
7.71 \mathrm{E}-01 \\
1.92 \mathrm{E}+00 \\
1.14 \mathrm{E}-03 \\
1.21 \mathrm{E}+00 \\
6.18 \mathrm{E}-03 \\
7.56 \mathrm{E}-01 \\
4.65 \mathrm{E}-02 \\
4.56 \mathrm{E}-01 \\
1.03 \mathrm{E}+00 \\
5.25 \mathrm{E}-01 \\
1.45 \mathrm{E}+00 \\
1.18 \mathrm{E}+00 \\
1.24 \mathrm{E}+00 \\
6.57 \mathrm{E}-01 \\
5.36 \mathrm{E}-01 \\
2.60 \mathrm{E}+00 \\
8.95 \mathrm{E}-01 \\
6.31 \mathrm{E}-01 \\
2.12 \mathrm{E}+00 \\
2.78 \mathrm{E}-01 \\
1.53 \mathrm{E}+00 \\
1.88 \mathrm{E}+00 \\
1.53 \mathrm{E}+00 \\
1.13 \mathrm{E}+00 \\
5.48 \mathrm{E}-01 \\
2.09 \mathrm{E}-01 \\
9.61 \mathrm{E}-03 \\
6.27 \mathrm{E}-01 \\
2.25 \mathrm{E}+00 \\
2.11 \mathrm{E}+00 \\
1.49 \mathrm{E}+00 \\
2.16 \mathrm{E}+00 \\
9.68 \mathrm{E}-01 \\
1.63 \mathrm{E}+00 \\
1.27 \mathrm{E}+00 \\
1.58 \mathrm{E}+00 \\
3.23 \mathrm{E}-01 \\
1.34 \mathrm{E}+00 \\
4.30 \mathrm{E}-03 \\
3.36 \mathrm{E}+00 \\
2.95 \mathrm{E}+00 \\
1.26 \mathrm{E}+00\end{array}$ & $\begin{array}{l}N 163 \\
6.10 E+01 \\
3.89 E+01 \\
8.33 E+01 \\
4.01 E+01 \\
1.58 E+02 \\
1.44 E+01 \\
8.22 E+01 \\
4.81 E+01 \\
1.94 E+01 \\
7.52 E+01 \\
9.79 E+01 \\
8.03 E+01 \\
6.81 E+01 \\
8.57 E+01 \\
1.06 E+02 \\
1.23 E+02 \\
7.68 E+01 \\
1.80 E+02 \\
1.57 E+02 \\
1.38 E+02 \\
7.10 E+01 \\
1.18 E+02 \\
1.46 E+02 \\
7.54 E+01 \\
1.88 E+02 \\
1.12 E-01 \\
1.19 E+02 \\
6.04 E-01 \\
7.41 E+01 \\
4.55 E+00 \\
4.48 E+01 \\
1.01 E+02 \\
5.16 E+01 \\
1.42 E+02 \\
1.16 E+02 \\
1.21 E+02 \\
6.46 E+01 \\
5.27 E+01 \\
2.56 E+02 \\
8.82 E+01 \\
6.22 E+01 \\
2.10 E+02 \\
2.75 E+01 \\
1.51 E+02 \\
1.86 E+02 \\
1.51 E+02 \\
1.12 E+02 \\
5.44 E+01 \\
2.07 E+01 \\
9.61 E-01 \\
6.22 E+01 \\
2.23 E+02 \\
2.10 E+02 \\
1.49 E+02 \\
2.15 E+02 \\
9.65 E+01 \\
1.62 E+02 \\
1.27 E+02 \\
1.58 E+02 \\
3.22 E+01 \\
1.34 E+02 \\
4.32 E=01 \\
3.36 E+02 \\
2.95 E+02 \\
1.26 E+02\end{array}$ & $\begin{array}{l}C 060 \\
1.51 E+00 \\
9.72 E-01 \\
2.10 E+00 \\
1.02 E+00 \\
4.06 E+00 \\
3.68 E-01 \\
2.12 E+00 \\
1.27 E+00 \\
4.97 E-01 \\
1.94 E+00 \\
2.56 E+00 \\
2.13 E+00 \\
1.82 E+00 \\
2.33 E+00 \\
2.97 E+00 \\
3.44 E+00 \\
2.17 E+00 \\
5.05 E+00 \\
4.58 E+00 \\
4.11 E+00 \\
2.14 E+00 \\
3.59 E+00 \\
4.51 E+00 \\
2.32 E+00 \\
5.93 E+00 \\
3.61 E-03 \\
3.78 E+00 \\
1.96 E-02 \\
2.37 E+00 \\
1.41 E-01 \\
1.45 E+00 \\
3.29 E+00 \\
1.69 E+00 \\
4.71 E+00 \\
3.88 E+00 \\
4.06 E+00 \\
2.18 E+00 \\
1.82 E+00 \\
8.82 E+00 \\
3.07 E+00 \\
2.19 E+00 \\
7.63 E+00 \\
1.01 E+00 \\
5.62 E+00 \\
6.82 E+00 \\
5.45 E+00 \\
4.20 E+00 \\
2.10 E+00 \\
8.03 E-01 \\
1.80 E-01 \\
2.39 E+00 \\
8.56 E+00 \\
8.28 E+00 \\
5.94 E+00 \\
8.79 E+00 \\
4.06 E+00 \\
6.75 E+00 \\
5.21 E+00 \\
6.73 E+00 \\
1.40 E+00 \\
5.79 E+00 \\
9.06 E-00 \\
1.48 E+01 \\
1.31 E+01 \\
5.72 E+00\end{array}$ & $\begin{array}{l}\text { SE79 } \\
6.00 \mathrm{E}-01 \\
3.82 \mathrm{E}-01 \\
8.18 \mathrm{E}-01 \\
3.94 \mathrm{E}-01 \\
1.22 \mathrm{E}+00 \\
1.42 \mathrm{E}-01 \\
6.37 \mathrm{E}-01 \\
3.72 \mathrm{E}-01 \\
1.90 \mathrm{E}-01 \\
7.37 \mathrm{E}-01 \\
9.59 \mathrm{E}-01 \\
7.86 \mathrm{E}-01 \\
6.66 \mathrm{E}-01 \\
8.37 \mathrm{E}-01 \\
1.03 \mathrm{E}+00 \\
1.20 \mathrm{E}+00 \\
7.49 \mathrm{E}-01 \\
1.76 \mathrm{E}+00 \\
1.52 \mathrm{E}+00 \\
1.35 \mathrm{E}+00 \\
6.89 \mathrm{E}-01 \\
1.14 \mathrm{E}+00 \\
1.41 \mathrm{E}+00 \\
7.32 \mathrm{E}-01 \\
1.82 \mathrm{E}+00 \\
8.41 \mathrm{E}-04 \\
1.15 \mathrm{E}+00 \\
4.55 \mathrm{E}-03 \\
7.17 \mathrm{E}-01 \\
4.41 \mathrm{E}-02 \\
4.33 \mathrm{E}-01 \\
9.73 \mathrm{E}-01 \\
4.99 \mathrm{E}-01 \\
1.37 \mathrm{E}+00 \\
1.12 \mathrm{E}+00 \\
1.17 \mathrm{E}+00 \\
6.23 \mathrm{E}-01 \\
5.08 \mathrm{E}-01 \\
2.46 \mathrm{E}+00 \\
8.49 \mathrm{E}-01 \\
5.99 \mathrm{E}-01 \\
2.02 \mathrm{E}+00 \\
2.64 \mathrm{E}-01 \\
1.45 \mathrm{E}+00 \\
1.79 \mathrm{E}+00 \\
1.45 \mathrm{E}+00 \\
1.08 \mathrm{E}+00 \\
5.21 \mathrm{E}-01 \\
1.98 \mathrm{E}-01 \\
2.00 \mathrm{E}-02 \\
5.95 \mathrm{E}-01 \\
2.14 \mathrm{E}+00 \\
2.00 \mathrm{E}+00 \\
1.42 \mathrm{E}+00 \\
2.05 \mathrm{E}+00 \\
9.19 \mathrm{E}-01 \\
1.54 \mathrm{E}+00 \\
1.21 \mathrm{E}+00 \\
1.50 \mathrm{E}+00 \\
3.05 \mathrm{E}-01 \\
1.27 \mathrm{E}+00 \\
9.04 \mathrm{E}-03 \\
3.19 \mathrm{E}+00 \\
2.80 \mathrm{E}+00 \\
1.20 \mathrm{E}+00\end{array}$ & $\begin{array}{l}\text { SR90 } \\
5.75 E+04 \\
3.69 E+04 \\
7.88 E+04 \\
3.80 E+04 \\
1.15 E+05 \\
1.36 E+04 \\
5.97 E+04 \\
3.51 E+04 \\
1.85 E+04 \\
7.16 E+04 \\
9.33 E+04 \\
7.65 E+04 \\
6.51 E+04 \\
8.21 E+04 \\
1.02 E+05 \\
1.18 E+05 \\
7.44 E+04 \\
1.73 E+05 \\
1.51 E+05 \\
1.34 E+05 \\
6.89 E+04 \\
1.14 E+05 \\
1.42 E+05 \\
7.32 E+04 \\
1.83 E+05 \\
8.51 E+01 \\
1.16 E+05 \\
4.61 E+02 \\
7.25 E+04 \\
4.42 E+03 \\
4.38 E+04 \\
9.85 E+04 \\
5.04 E+04 \\
1.39 E+05 \\
1.14 E+05 \\
1.19 E+05 \\
6.35 E+04 \\
5.20 E+04 \\
2.52 E+05 \\
8.68 E+04 \\
6.14 E+04 \\
2.08 E+05 \\
2.73 E+04 \\
1.50 E+05 \\
1.85 E+05 \\
1.50 E+05 \\
1.11 E+05 \\
5.43 E+04 \\
2.07 E+04 \\
2.02 E+03 \\
6.23 E+04 \\
2.23 E+05 \\
2.10 E+05 \\
1.49 E+05 \\
2.17 E+05 \\
9.75 E+04 \\
1.65 E+05 \\
1.27 E+05 \\
1.62 E+05 \\
3.31 E+04 \\
1.38 E+05 \\
9.52 E+02 \\
3.42 E+05 \\
3.01 E+05 \\
1.29 E+05\end{array}$ & $\begin{array}{l}Y 90 \\
5.75 E+04 \\
3.69 E+04 \\
7.88 E+04 \\
3.80 E+04 \\
1.15 E+05 \\
1.36 E+04 \\
5.97 E+04 \\
3.51 E+04 \\
1.85 E+04 \\
7.16 E+04 \\
9.33 E+04 \\
7.65 E+04 \\
6.51 E+04 \\
8.21 E+04 \\
1.02 E+05 \\
1.18 E+05 \\
7.44 E+04 \\
1.73 E+05 \\
1.51 E+05 \\
1.34 E+05 \\
6.89 E+04 \\
1.14 E+05 \\
1.42 E+05 \\
7.32 E+04 \\
1.83 E+05 \\
8.51 E+01 \\
1.16 E+05 \\
4.61 E+02 \\
7.25 E+04 \\
4.43 E+03 \\
4.38 E+04 \\
9.86 E+04 \\
5.04 E+04 \\
1.39 E+05 \\
1.14 E+05 \\
1.19 E+05 \\
6.35 E+04 \\
5.20 E+04 \\
2.52 E+05 \\
8.68 E+04 \\
6.14 E+04 \\
2.08 E+05 \\
2.73 E+04 \\
1.50 E+05 \\
1.85 E+05 \\
1.50 E+05 \\
1.11 E+05 \\
5.43 E+04 \\
2.07 E+04 \\
2.02 E+03 \\
6.23 E+04 \\
2.23 E+05 \\
2.10 E+05 \\
1.49 E+05 \\
2.17 E+05 \\
9.75 E+04 \\
1.65 E+05 \\
1.27 E+05 \\
1.62 E+05 \\
3.31 E+04 \\
1.38 E+05 \\
9.53 E+02 \\
3.43 E+05 \\
3.01 E+05 \\
1.29 E+05\end{array}$ & $\begin{array}{l}2 R 93 \\
2.88 \mathrm{E}+00 \\
1.84 \mathrm{E}+00 \\
3.94 \mathrm{E}+00 \\
1.90 \mathrm{E}+00 \\
5.77 \mathrm{E}+00 \\
6.79 \mathrm{E}-01 \\
3.00 \mathrm{E}+00 \\
1.75 \mathrm{E}+00 \\
9.17 \mathrm{E}-01 \\
3.55 \mathrm{E}+00 \\
4.62 \mathrm{E}+00 \\
3.78 \mathrm{E}+00 \\
3.21 \mathrm{E}+00 \\
4.03 \mathrm{E}+00 \\
4.97 \mathrm{E}+00 \\
5.76 \mathrm{E}+00 \\
3.62 \mathrm{E}+00 \\
8.46 \mathrm{E}+00 \\
7.33 \mathrm{E}+00 \\
6.47 \mathrm{E}+00 \\
3.32 \mathrm{E}+00 \\
5.50 \mathrm{E}+00 \\
6.81 \mathrm{E}+00 \\
3.52 \mathrm{E}+00 \\
8.76 \mathrm{E}+00 \\
4.03 \mathrm{E}-03 \\
5.53 \mathrm{E}+00 \\
2.18 \mathrm{E}-02 \\
3.45 \mathrm{E}+00 \\
2.12 \mathrm{E}-01 \\
2.08 \mathrm{E}+00 \\
4.68 \mathrm{E}+00 \\
2.39 \mathrm{E}+00 \\
6.60 \mathrm{E}+00 \\
5.40 \mathrm{E}+00 \\
5.64 \mathrm{E}+00 \\
3.00 \mathrm{E}+00 \\
2.45 \mathrm{E}+00 \\
1.19 \mathrm{E}+01 \\
4.08 \mathrm{E}+00 \\
2.88 \mathrm{E}+00 \\
9.69 \mathrm{E}+00 \\
1.27 \mathrm{E}+00 \\
6.96 \mathrm{E}+00 \\
8.59 \mathrm{E}+00 \\
6.98 \mathrm{E}+00 \\
5.16 \mathrm{E}+00 \\
2.50 \mathrm{E}+00 \\
9.52 \mathrm{E}-01 \\
9.71 \mathrm{E}-02 \\
2.86 \mathrm{E}+00 \\
1.03 \mathrm{E}+01 \\
9.62 \mathrm{E}+00 \\
6.81 \mathrm{E}+00 \\
9.87 \mathrm{E}+00 \\
4.41 \mathrm{E}+00 \\
7.46 \mathrm{E}+00 \\
5.78 \mathrm{E}+00 \\
7.25 \mathrm{E}+00 \\
1.48 \mathrm{E}+00 \\
6.15 \mathrm{E}+00 \\
4.44 \mathrm{E}-02 \\
1.53 \mathrm{E}+01 \\
1.35 \mathrm{E}+01 \\
5.76 \mathrm{E}+00\end{array}$ \\
\hline
\end{tabular}


HNF-SD-WH-TI-794, ReV. 0

ACTIVITY IN UNSEPARATED FUEL (Curies decayed to $1 / 1 / 94$ )

\begin{tabular}{|c|c|c|c|c|c|c|c|c|c|}
\hline 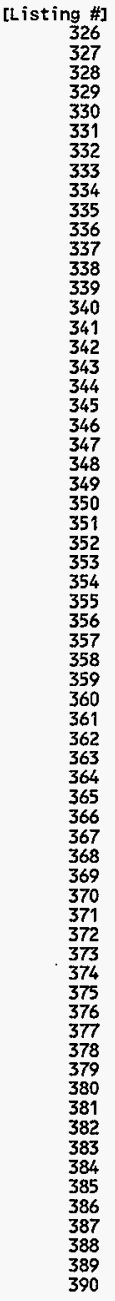 & $\begin{array}{l}\text { NB93* } \\
2.27 E+00 \\
1.45 E+00 \\
3.09 E+00 \\
1.49 E+00 \\
4.52 E+00 \\
5.32 E-01 \\
2.34 E+00 \\
1.37 E+00 \\
7.19 E-01 \\
2.78 E+00 \\
3.61 E+00 \\
2.95 E+00 \\
2.50 E+00 \\
3.14 E+00 \\
3.86 E+00 \\
4.48 E+00 \\
2.81 E+00 \\
6.58 E+00 \\
5.67 E+00 \\
5.00 E+00 \\
2.56 E+00 \\
4.24 E+00 \\
5.24 E+00 \\
2.71 E+00 \\
6.73 E+00 \\
3.09 E-03 \\
4.25 E+00 \\
1.67 E-02 \\
2.65 E+00 \\
1.63 E-01 \\
1.60 E+00 \\
3.58 E+00 \\
1.83 E+00 \\
5.05 E+00 \\
4.12 E+00 \\
4.31 E+00 \\
2.29 E+00 \\
1.86 E+00 \\
9.02 E+00 \\
3.10 E+00 \\
2.19 E+00 \\
7.34 E+00 \\
9.59 E-01 \\
5.26 E+00 \\
6.51 E+00 \\
5.29 E+00 \\
3.90 E+00 \\
1.88 E+00 \\
7.16 E-01 \\
7.30 E-00 \\
2.16 E+00 \\
7.74 E+00 \\
7.23 E+00 \\
5.11 E+00 \\
7.39 E+00 \\
3.29 E+00 \\
5.57 E+00 \\
4.32 E+00 \\
5.40 E+00 \\
1.10 E+00 \\
4.57 E+00 \\
3.30 E=02 \\
1.14 E+01 \\
9.97 E+00 \\
4.26 E+00\end{array}$ & $\begin{array}{l}\text { TC99 } \\
2.00 \mathrm{E}+01 \\
1.27 \mathrm{E}+01 \\
2.72 \mathrm{E}+01 \\
1.31 \mathrm{E}+01 \\
4.03 \mathrm{E}+01 \\
4.71 \mathrm{E}+00 \\
2.10 \mathrm{E}+01 \\
1.23 \mathrm{E}+01 \\
6.32 \mathrm{E}+00 \\
2.45 \mathrm{E}+01 \\
3.19 \mathrm{E}+01 \\
2.61 \mathrm{E}+01 \\
2.22 \mathrm{E}+01 \\
2.78 \mathrm{E}+01 \\
3.44 \mathrm{E}+01 \\
3.99 \mathrm{E}+01 \\
2.49 \mathrm{E}+01 \\
5.85 \mathrm{E}+01 \\
5.07 \mathrm{E}+01 \\
4.47 \mathrm{E}+01 \\
2.29 \mathrm{E}+01 \\
3.80 \mathrm{E}+01 \\
4.70 \mathrm{E}+01 \\
2.43 \mathrm{E}+01 \\
6.06 \mathrm{E}+01 \\
2.76 \mathrm{E}-02 \\
3.82 \mathrm{E}+01 \\
1.49 \mathrm{E}-01 \\
2.38 \mathrm{E}+01 \\
1.47 \mathrm{E}+00 \\
1.44 \mathrm{E}+01 \\
3.24 \mathrm{E}+01 \\
1.66 \mathrm{E}+01 \\
4.56 \mathrm{E}+01 \\
3.73 \mathrm{E}+01 \\
3.90 \mathrm{E}+01 \\
2.07 \mathrm{E}+01 \\
1.69 \mathrm{E}+01 \\
8.19 \mathrm{E}+01 \\
2.83 \mathrm{E}+01 \\
1.99 \mathrm{E}+01 \\
6.70 \mathrm{E}+01 \\
8.77 \mathrm{E}+00 \\
4.81 \mathrm{E}+01 \\
5.95 \mathrm{E}+01 \\
4.83 \mathrm{E}+01 \\
3.58 \mathrm{E}+01 \\
1.73 \mathrm{E}+01 \\
6.59 \mathrm{E}+00 \\
6.67 \mathrm{E}-01 \\
1.98 \mathrm{E}+01 \\
7.11 \mathrm{E}+01 \\
6.66 \mathrm{E}+01 \\
4.72 \mathrm{E}+01 \\
6.83 \mathrm{E}+01 \\
3.06 \mathrm{E}+01 \\
5.12 \mathrm{E}+01 \\
4.02 \mathrm{E}+01 \\
4.97 \mathrm{E}+01 \\
1.01 \mathrm{E}+01 \\
4.21 \mathrm{E}+01 \\
3.02 \mathrm{E}-01 \\
1.06 \mathrm{E}+02 \\
9.31 \mathrm{E}+01 \\
3.98 \mathrm{E}+01\end{array}$ & $\begin{array}{l}\text { RU106 } \\
2.95 \mathrm{E}-05 \\
1.83 \mathrm{E}=05 \\
4.39 \mathrm{E}-05 \\
2.14 \mathrm{E}-05 \\
7.78 \mathrm{E}-05 \\
8.40 \mathrm{E}-06 \\
4.43 \mathrm{E}-05 \\
2.95 \mathrm{E}-05 \\
1.05 \mathrm{E}-05 \\
4.30 \mathrm{E}-05 \\
6.09 \mathrm{E}-05 \\
5.53 \mathrm{E}-05 \\
4.78 \mathrm{E}-05 \\
6.66 \mathrm{E}-05 \\
1.01 \mathrm{E}-04 \\
1.15 \mathrm{E}-04 \\
6.87 \mathrm{E}-05 \\
1.67 \mathrm{E}-04 \\
1.85 \mathrm{E}-04 \\
1.76 \mathrm{E}-04 \\
9.64 \mathrm{E}-05 \\
1.70 \mathrm{E}-04 \\
2.30 \mathrm{E}-04 \\
1.17 \mathrm{E}-04 \\
3.33 \mathrm{E}-04 \\
1.47 \mathrm{E}-07 \\
2.20 \mathrm{E}-04 \\
8.03 \mathrm{E}-04 \\
1.40 \mathrm{E}-04 \\
7.48 \mathrm{E}-06 \\
9.14 \mathrm{E}-05 \\
2.18 \mathrm{E}-04 \\
1.18 \mathrm{E}-04 \\
3.34 \mathrm{E}-04 \\
2.77 \mathrm{E}-04 \\
2.99 \mathrm{E}-04 \\
1.70 \mathrm{E}-04 \\
1.54 \mathrm{E}-04 \\
7.45 \mathrm{E}-04 \\
2.78 \mathrm{E}-04 \\
2.11 \mathrm{E}-04 \\
8.23 \mathrm{E}-04 \\
1.14 \mathrm{E}-04 \\
6.75 \mathrm{E}-04 \\
7.69 \mathrm{E}-04 \\
5.72 \mathrm{E}-04 \\
5.42 \mathrm{E}-04 \\
3.02 \mathrm{E}-04 \\
1.17 \mathrm{E}-04 \\
1.49 \mathrm{E}-05 \\
3.27 \mathrm{E}-04 \\
1.17 \mathrm{E}-03 \\
1.31 \mathrm{E}-03 \\
9.95 \mathrm{E}-04 \\
1.59 \mathrm{E}-03 \\
8.53 \mathrm{E}-04 \\
1.19 \mathrm{E}-03 \\
1.06 \mathrm{E}-03 \\
1.31 \mathrm{E}-03 \\
2.89 \mathrm{E}-04 \\
1.17 \mathrm{E}-03 \\
1.08 \mathrm{E}-05 \\
3.73 \mathrm{E}-03 \\
1.62 \mathrm{E}-03\end{array}$ & $\begin{array}{l}\text { CD113* } \\
9.28 \mathrm{E}+00 \\
5.82 \mathrm{E}+00 \\
1.27 \mathrm{E}+01 \\
6.11 \mathrm{E}+00 \\
2.21 \mathrm{E}+01 \\
2.23 \mathrm{E}+00 \\
1.17 \mathrm{E}+01 \\
6.86 \mathrm{E}+00 \\
2.93 \mathrm{E}+00 \\
1.14 \mathrm{E}+01 \\
1.50 \mathrm{E}+01 \\
1.24 \mathrm{E}+01 \\
1.05 \mathrm{E}+01 \\
1.33 \mathrm{E}+01 \\
1.67 \mathrm{E}+01 \\
1.94 \mathrm{E}+01 \\
1.19 \mathrm{E}+01 \\
2.83 \mathrm{E}+01 \\
2.50 \mathrm{E}+01 \\
2.21 \mathrm{E}+01 \\
1.14 \mathrm{E}+01 \\
1.90 \mathrm{E}+01 \\
2.36 \mathrm{E}+01 \\
1.23 \mathrm{E}+01 \\
3.07 \mathrm{E}+01 \\
1.58 \mathrm{E}-02 \\
1.94 \mathrm{E}+01 \\
8.53 \mathrm{E}-02 \\
1.21 \mathrm{E}+01 \\
7.40 \mathrm{E}-01 \\
7.38 \mathrm{E}+00 \\
1.67 \mathrm{E}+01 \\
8.61 \mathrm{E}+00 \\
2.36 \mathrm{E}+01 \\
1.92 \mathrm{E}+01 \\
2.03 \mathrm{E}+01 \\
1.08 \mathrm{E}+01 \\
8.88 \mathrm{E}+00 \\
4.30 \mathrm{E}+01 \\
1.50 \mathrm{E}+01 \\
1.06 \mathrm{E}+01 \\
3.60 \mathrm{E}+01 \\
4.72 \mathrm{E}+00 \\
2.61 \mathrm{E}+01 \\
3.21 \mathrm{E}+01 \\
2.59 \mathrm{E}+01 \\
1.96 \mathrm{E}+01 \\
9.57 \mathrm{E}+00 \\
3.64 \mathrm{E}+00 \\
4.08 \mathrm{E}-01 \\
1.08 \mathrm{E}+01 \\
3.89 \mathrm{E}+01 \\
3.70 \mathrm{E}+01 \\
2.64 \mathrm{E}+01 \\
3.83 \mathrm{E}+01 \\
1.74 \mathrm{E}+01 \\
2.82 \mathrm{E}+01 \\
2.31 \mathrm{E}+01 \\
2.76 \mathrm{E}+01 \\
5.65 \mathrm{E}+00 \\
2.34 \mathrm{E}+01 \\
1.82 \mathrm{E}-01 \\
6.11 \mathrm{E}+01 \\
5.41 \mathrm{E}+01 \\
2.31 \mathrm{E}+01\end{array}$ & $\begin{array}{l}5 B 125 \\
3.89 \mathrm{E}+00 \\
2.48 \mathrm{E}+00 \\
5.50 \mathrm{E}+00 \\
2.66 \mathrm{E}+00 \\
9.60 \mathrm{E}+00 \\
9.80 \mathrm{E}-01 \\
5.12 \mathrm{E}+00 \\
3.14 \mathrm{E}+00 \\
1.30 \mathrm{E}+00 \\
5.13 \mathrm{E}+00 \\
6.88 \mathrm{E}+00 \\
5.83 \mathrm{E}+00 \\
5.00 \mathrm{E}+00 \\
6.52 \mathrm{E}+00 \\
8.62 \mathrm{E}+00 \\
9.94 \mathrm{E}+00 \\
6.19 \mathrm{E}+00 \\
1.46 \mathrm{E}+01 \\
1.38 \mathrm{E}+01 \\
1.25 \mathrm{E}+01 \\
6.59 \mathrm{E}+00 \\
1.12 \mathrm{E}+01 \\
1.43 \mathrm{E}+01 \\
7.32 \mathrm{E}+00 \\
1.92 \mathrm{E}+01 \\
1.00 \mathrm{E}-02 \\
1.23 \mathrm{E}+01 \\
5.44 \mathrm{E}-02 \\
7.74 \mathrm{E}+00 \\
4.51 \mathrm{E}-01 \\
4.79 \mathrm{E}+00 \\
1.10 \mathrm{E}+01 \\
5.72 \mathrm{E}+00 \\
1.60 \mathrm{E}+01 \\
1.32 \mathrm{E}+01 \\
1.39 \mathrm{E}+01 \\
7.56 \mathrm{E}+00 \\
6.42 \mathrm{E}+00 \\
3.11 \mathrm{E}+01 \\
1.10 \mathrm{E}+01 \\
7.95 \mathrm{E}+00 \\
2.83 \mathrm{E}+01 \\
3.78 \mathrm{E}+00 \\
2.14 \mathrm{E}+01 \\
2.56 \mathrm{E}+01 \\
2.01 \mathrm{E}+01 \\
1.62 \mathrm{E}+01 \\
8.28 \mathrm{E}+00 \\
3.18 \mathrm{E}+00 \\
3.70 \mathrm{E}-01 \\
9.34 \mathrm{E}+00 \\
3.34 \mathrm{E}+01 \\
3.34 \mathrm{E}+01 \\
2.42 \mathrm{E}+01 \\
3.64 \mathrm{E}+01 \\
1.74 \mathrm{E}+01 \\
2.78 \mathrm{E}+01 \\
2.22 \mathrm{E}+01 \\
2.83 \mathrm{E}+01 \\
5.95 \mathrm{E}+00 \\
2.46 \mathrm{E}+01 \\
2.00 \mathrm{E}-01 \\
6.59 \mathrm{E}+01 \\
5.93 \mathrm{E}+01 \\
2.61 \mathrm{E}+01\end{array}$ & $\begin{array}{l}\text { SN126 } \\
8.48 \mathrm{E}-01 \\
5.31 \mathrm{E}-01 \\
1.15 \mathrm{E}+00 \\
5.54 \mathrm{E}-01 \\
1.88 \mathrm{E}+00 \\
2.01 \mathrm{E}-01 \\
9.91 \mathrm{E}-01 \\
5.77 \mathrm{E}-01 \\
2.65 \mathrm{E}-01 \\
1.03 \mathrm{E}+00 \\
1.34 \mathrm{E}+00 \\
1.11 \mathrm{E}+00 \\
9.33 \mathrm{E}-01 \\
1.17 \mathrm{E}+00 \\
1.46 \mathrm{E}+00 \\
1.69 \mathrm{E}+00 \\
1.04 \mathrm{E}+00 \\
2.47 \mathrm{E}+00 \\
2.15 \mathrm{E}+00 \\
1.89 \mathrm{E}+00 \\
9.65 \mathrm{E}-01 \\
1.60 \mathrm{E}+00 \\
1.99 \mathrm{E}+00 \\
1.03 \mathrm{E}+00 \\
2.56 \mathrm{E}+00 \\
1.23 \mathrm{E}-03 \\
1.61 \mathrm{E}+00 \\
6.63 \mathrm{E}-03 \\
1.00 \mathrm{E}+00 \\
6.21 \mathrm{E}-02 \\
6.09 \mathrm{E}-01 \\
1.37 \mathrm{E}+00 \\
7.06 \mathrm{E}-01 \\
1.93 \mathrm{E}+00 \\
1.57 \mathrm{E}+00 \\
1.65 \mathrm{E}+00 \\
8.78 \mathrm{E}-01 \\
7.15 \mathrm{E}-01 \\
3.47 \mathrm{E}+00 \\
1.20 \mathrm{E}+00 \\
8.48 \mathrm{E}-01 \\
2.84 \mathrm{E}+00 \\
3.71 \mathrm{E}-01 \\
2.04 \mathrm{E}+00 \\
2.53 \mathrm{E}+00 \\
2.05 \mathrm{E}+00 \\
1.53 \mathrm{E}+00 \\
7.38 \mathrm{E}-01 \\
2.80 \mathrm{E}-01 \\
3.07 \mathrm{E}-02 \\
8.35 \mathrm{E}-01 \\
3.01 \mathrm{E}+00 \\
2.83 \mathrm{E}+00 \\
2.00 \mathrm{E}+00 \\
2.89 \mathrm{E}+00 \\
1.30 \mathrm{E}+00 \\
2.12 \mathrm{E}+00 \\
1.73 \mathrm{E}+00 \\
2.06 \mathrm{E}+00 \\
4.18 \mathrm{E}-01 \\
1.73 \mathrm{E}+00 \\
1.33 \mathrm{E}-02 \\
4.48 \mathrm{E}+00 \\
3.95 \mathrm{E}+00 \\
1.68 \mathrm{E}+00\end{array}$ & $\begin{array}{l}1129 \\
3.74 \mathrm{E}-02 \\
2.35 \mathrm{E}-02 \\
5.08 \mathrm{E}-02 \\
2.44 \mathrm{E}-02 \\
7.75 \mathrm{E}-02 \\
8.87 \mathrm{E}-03 \\
4.08 \mathrm{E}-02 \\
2.38 \mathrm{E}-02 \\
1.17 \mathrm{E}-02 \\
4.54 \mathrm{E}-02 \\
5.93 \mathrm{E}-02 \\
4.87 \mathrm{E}-02 \\
4.12 \mathrm{E}-02 \\
5.17 \mathrm{E}-02 \\
6.42 \mathrm{E}-02 \\
7.44 \mathrm{E}-02 \\
4.58 \mathrm{E}-02 \\
1.09 \mathrm{E}-01 \\
9.47 \mathrm{E}-02 \\
8.33 \mathrm{E}-02 \\
4.26 \mathrm{E}-02 \\
7.07 \mathrm{E}-02 \\
8.76 \mathrm{E}-02 \\
4.54 \mathrm{E}-02 \\
1.13 \mathrm{E}-01 \\
5.08 \mathrm{E}-05 \\
7.10 \mathrm{E}-02 \\
2.73 \mathrm{E}-04 \\
4.43 \mathrm{E}-02 \\
2.74 \mathrm{E}-03 \\
2.69 \mathrm{E}-02 \\
6.04 \mathrm{E}-02 \\
3.11 \mathrm{E}-02 \\
8.51 \mathrm{E}-02 \\
6.92 \mathrm{E}-02 \\
7.28 \mathrm{E}-02 \\
3.87 \mathrm{E}-02 \\
3.15 \mathrm{E}-02 \\
1.53 \mathrm{E}-01 \\
5.30 \mathrm{E}-02 \\
3.74 \mathrm{E}-02 \\
1.25 \mathrm{E}-01 \\
1.64 \mathrm{E}-02 \\
8.99 \mathrm{E}-02 \\
1.11 \mathrm{E}-01 \\
9.05 \mathrm{E}-02 \\
6.74 \mathrm{E}-02 \\
3.25 \mathrm{E}-02 \\
1.23 \mathrm{E}-02 \\
1.31 \mathrm{E}-03 \\
3.68 \mathrm{E}-02 \\
1.33 \mathrm{E}-01 \\
1.25 \mathrm{E}-01 \\
8.83 \mathrm{E}-02 \\
1.28 \mathrm{E}-01 \\
5.72 \mathrm{E}-02 \\
9.37 \mathrm{E}-02 \\
7.61 \mathrm{E}-02 \\
9.10 \mathrm{E}-02 \\
1.85 \mathrm{E}-02 \\
7.68 \mathrm{E}-02 \\
5.73 \mathrm{E}-02 \\
1.97 \mathrm{E}-01 \\
1.74 \mathrm{E}-01 \\
7.41 \mathrm{E}-02\end{array}$ & $\begin{array}{l}\text { CS134 } \\
1.36 \mathrm{E}-01 \\
6.33 \mathrm{E}-02 \\
1.83 \mathrm{E}-01 \\
8.54 \mathrm{E}-02 \\
2.37 \mathrm{E}-01 \\
3.86 \mathrm{E}-02 \\
1.55 \mathrm{E}-01 \\
9.39 \mathrm{E}-02 \\
3.38 \mathrm{E}-02 \\
1.46 \mathrm{E}-01 \\
2.08 \mathrm{E}-01 \\
1.94 \mathrm{E}-01 \\
1.53 \mathrm{E}-01 \\
2.03 \mathrm{E}-01 \\
3.10 \mathrm{E}-01 \\
3.57 \mathrm{E}-01 \\
1.52 \mathrm{E}-01 \\
4.88 \mathrm{E}-01 \\
5.09 \mathrm{E}-01 \\
4.37 \mathrm{E}-01 \\
2.20 \mathrm{E}-01 \\
3.85 \mathrm{E}-01 \\
5.11 \mathrm{E}-01 \\
2.80 \mathrm{E}-01 \\
7.16 \mathrm{E}-01 \\
4.48 \mathrm{E}-10 \\
4.38 \mathrm{E}-01 \\
2.42 \mathrm{E}-09 \\
2.69 \mathrm{E}-01 \\
1.70 \mathrm{E}-02 \\
1.85 \mathrm{E}-01 \\
4.28 \mathrm{E}-01 \\
2.52 \mathrm{E}-01 \\
6.29 \mathrm{E}-01 \\
4.72 \mathrm{E}-01 \\
5.50 \mathrm{E}-01 \\
3.09 \mathrm{E}-01 \\
2.60 \mathrm{E}-01 \\
1.26 \mathrm{E}+00 \\
5.03 \mathrm{E}-01 \\
3.68 \mathrm{E}-01 \\
1.22 \mathrm{E}+00 \\
1.64 \mathrm{E}-01 \\
9.34 \mathrm{E}-01 \\
1.16 \mathrm{E}+00 \\
9.05 \mathrm{E}-01 \\
8.39 \mathrm{E}-01 \\
4.08 \mathrm{E}-01 \\
1.51 \mathrm{E}-01 \\
2.15 \mathrm{E}-02 \\
3.90 \mathrm{E}-01 \\
1.49 \mathrm{E}+00 \\
1.60 \mathrm{E}+00 \\
1.17 \mathrm{E}+00 \\
1.70 \mathrm{E}+00 \\
8.71 \mathrm{E}-01 \\
7.25 \mathrm{E}-01 \\
1.37 \mathrm{E}+00 \\
7.13 \mathrm{E}-01 \\
1.35 \mathrm{E}-01 \\
5.23 \mathrm{E}-01 \\
5.48 \mathrm{E}-03 \\
3.03 \mathrm{E}+00 \\
2.97 \mathrm{E}+00 \\
1.22 \mathrm{E}+00\end{array}$ & $\begin{array}{l}\mathrm{CS137} \\
6.53 \mathrm{E}+04 \\
4.15 \mathrm{E}+04 \\
8.93 \mathrm{E}+04 \\
4.30 \mathrm{E}+04 \\
1.32 \mathrm{E}+05 \\
1.55 \mathrm{E}+04 \\
6.91 \mathrm{E}+04 \\
4.05 \mathrm{E}+04 \\
2.08 \mathrm{E}+04 \\
8.06 \mathrm{E}+04 \\
1.05 \mathrm{E}+05 \\
8.65 \mathrm{E}+04 \\
7.34 \mathrm{E}+04 \\
9.25 \mathrm{E}+04 \\
1.15 \mathrm{E}+05 \\
1.33 \mathrm{E}+05 \\
8.32 \mathrm{E}+04 \\
1.96 \mathrm{E}+05 \\
1.71 \mathrm{E}+05 \\
1.51 \mathrm{E}+05 \\
7.77 \mathrm{E}+04 \\
1.29 \mathrm{E}+05 \\
1.60 \mathrm{E}+05 \\
8.28 \mathrm{E}+04 \\
2.07 \mathrm{E}+05 \\
9.40 \mathrm{E}+01 \\
1.31 \mathrm{E}+05 \\
5.08 \mathrm{E}+02 \\
8.17 \mathrm{E}+04 \\
5.00 \mathrm{E}+03 \\
4.95 \mathrm{E}+04 \\
1.11 \mathrm{E}+05 \\
5.72 \mathrm{E}+04 \\
1.58 \mathrm{E}+05 \\
1.29 \mathrm{E}+05 \\
1.35 \mathrm{E}+05 \\
7.18 \mathrm{E}+04 \\
5.88 \mathrm{E}+04 \\
2.85 \mathrm{E}+05 \\
9.85 \mathrm{E}+04 \\
6.96 \mathrm{E}+04 \\
2.35 \mathrm{E}+05 \\
3.08 \mathrm{E}+04 \\
1.70 \mathrm{E}+05 \\
2.09 \mathrm{E}+05 \\
1.65 \mathrm{E}+05 \\
1.69 \mathrm{E}+05 \\
1.27 \mathrm{E}+05 \\
1.57 \mathrm{E}+04 \\
1.52 \mathrm{E}+05 \\
1.09 \mathrm{E}+03 \\
3.86 \mathrm{E}+05 \\
3.39 \mathrm{E}+05 \\
2.34 \mathrm{E}+04 \\
2.37 \mathrm{E}+03 \\
7.02 \mathrm{E}+04 \\
2.52 \mathrm{E}+05 \\
2.38 \mathrm{E}+05 \\
1.69 \mathrm{E}+05 \\
2.45 \mathrm{E}+05 \\
1.10 \mathrm{E}+05\end{array}$ \\
\hline
\end{tabular}


HNF-SD-WH-TI-794, ReV. 0

ACTIVITY IN UNSEPARATED FUEL (Curies decayed to 1/1/94)

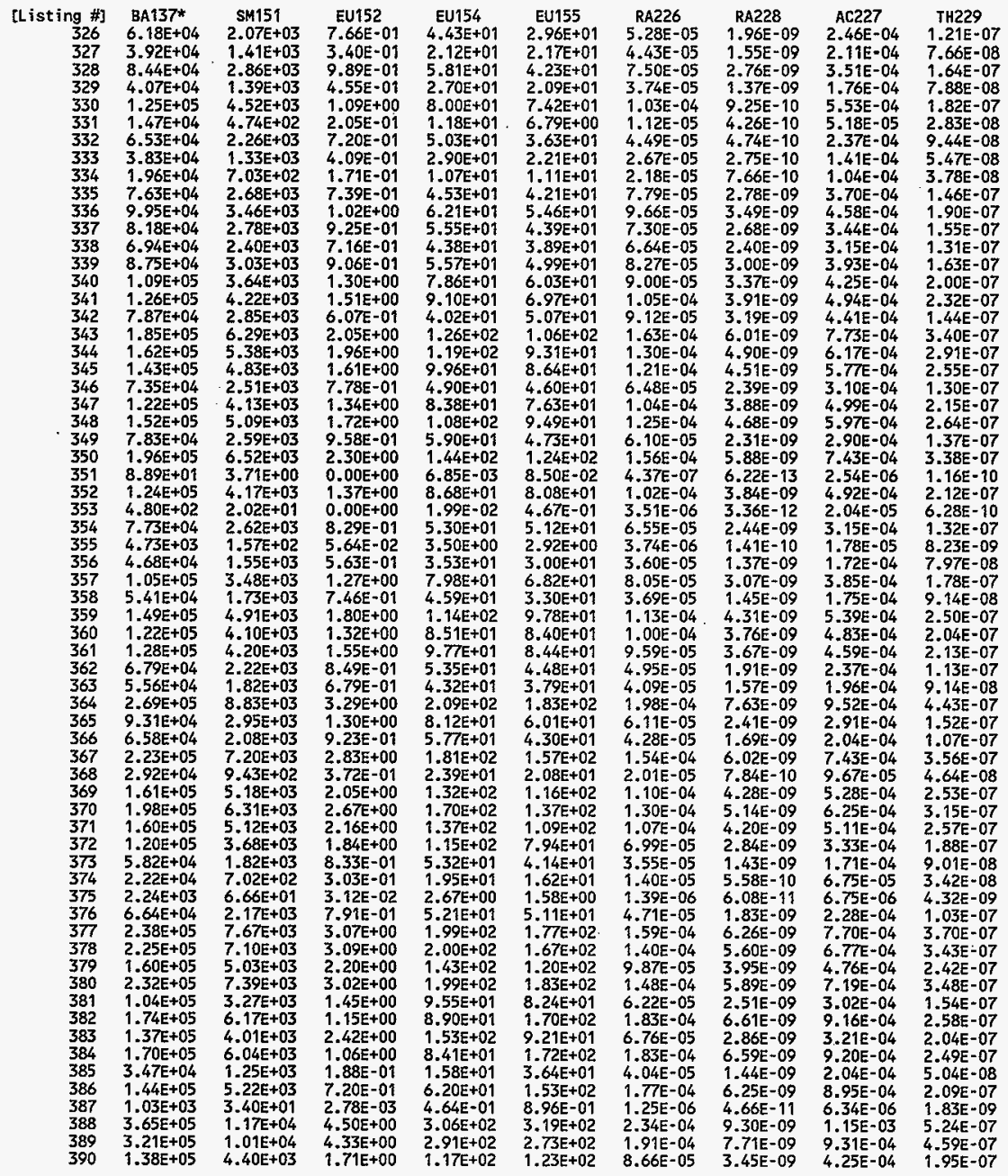


HNF-SD-WM-TI-794, ReV. 0

ACTIVITY IN UNSEPARATED FUEL (Curies decayed to 1/1/94)

\begin{tabular}{|c|c|c|c|c|c|c|c|c|c|}
\hline 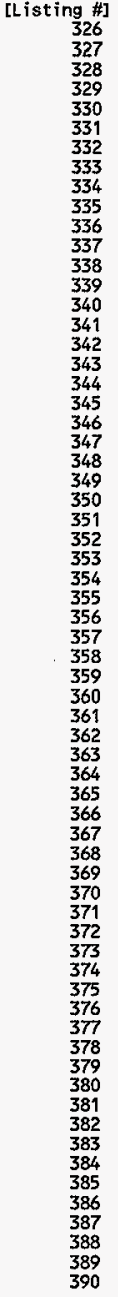 & $\begin{array}{l}\text { JH232 } \\
2.69 E-09 \\
2.13 E-09 \\
3.80 E-09 \\
1.88 E-09 \\
1.28 E-09 \\
5.87 E-10 \\
6.55 E-10 \\
3.81 E-10 \\
1.06 E-09 \\
3.83 E-09 \\
4.81 E-09 \\
3.71 E-09 \\
3.32 E-09 \\
4.16 E-09 \\
4.67 E-09 \\
5.42 E-09 \\
4.43 E-09 \\
8.34 E-09 \\
6.83 E-09 \\
6.29 E-09 \\
3.34 E-09 \\
5.42 E-09 \\
6.55 E-09 \\
3.24 E-09 \\
8.24 E-09 \\
8.74 E-13 \\
5.39 E-09 \\
4.73 E-12 \\
3.42 E-09 \\
1.98 E-10 \\
1.92 E-09 \\
4.31 E-09 \\
2.03 E-09 \\
6.06 E-09 \\
5.29 E-09 \\
5.17 E-09 \\
2.69 E-09 \\
2.22 E-09 \\
1.08 E-08 \\
3.41 E-09 \\
2.40 E-09 \\
8.54 E-09 \\
1.11 E-09 \\
6.10 E-09 \\
7.30 E-09 \\
5.95 E-09 \\
4.04 E-09 \\
2.04 E-09 \\
7.97 E-10 \\
8.69 E-11 \\
2.61 E-09 \\
8.94 E-09 \\
8.02 E-09 \\
5.66 E-09 \\
8.45 E-09 \\
3.62 E-09 \\
9.51 E-09 \\
4.11 E-09 \\
9.51 E-09 \\
2.08 E-09 \\
9.03 E-09 \\
6.74 E-11 \\
1.34 E-08 \\
1.12 E-08 \\
5.01 E-09\end{array}$ & $\begin{array}{l}\text { PA231 } \\
6.24 \mathrm{E}-04 \\
5.37 \mathrm{E}=04 \\
8.95 \mathrm{E}-04 \\
4.48 \mathrm{E}-04 \\
1.41 \mathrm{E}-03 \\
1.32 \mathrm{E}-04 \\
6.06 \mathrm{E}-04 \\
3.62 \mathrm{E}-04 \\
2.66 \mathrm{E}-04 \\
9.45 \mathrm{E}-04 \\
1.17 \mathrm{E}-03 \\
8.83 \mathrm{E}-04 \\
8.10 \mathrm{E}-04 \\
1.01 \mathrm{E}-03 \\
1.10 \mathrm{E}-03 \\
1.28 \mathrm{E}-03 \\
1.14 \mathrm{E}-03 \\
2.00 \mathrm{E}-03 \\
1.61 \mathrm{E}-03 \\
1.51 \mathrm{E}-03 \\
8.34 \mathrm{E}-04 \\
1.31 \mathrm{E}-03 \\
1.57 \mathrm{E}-03 \\
7.63 \mathrm{E}-04 \\
1.96 \mathrm{E}-03 \\
6.76 \mathrm{E}-06 \\
1.30 \mathrm{E}-03 \\
5.45 \mathrm{E}-05 \\
8.34 \mathrm{E}-04 \\
4.70 \mathrm{E}-05 \\
4.57 \mathrm{E}-04 \\
1.02 \mathrm{E}-03 \\
4.66 \mathrm{E}-04 \\
1.44 \mathrm{E}-03 \\
1.29 \mathrm{E}-03 \\
1.23 \mathrm{E}-03 \\
6.34 \mathrm{E}-04 \\
5.27 \mathrm{E}-04 \\
2.56 \mathrm{E}-03 \\
7.82 \mathrm{E}-04 \\
5.50 \mathrm{E}-04 \\
2.01 \mathrm{E}-03 \\
2.63 \mathrm{E}-04 \\
1.44 \mathrm{E}-03 \\
1.69 \mathrm{E}-03 \\
1.38 \mathrm{E}-03 \\
9.06 \mathrm{E}-04 \\
4.67 \mathrm{E}-04 \\
1.85 \mathrm{E}-04 \\
1.85 \mathrm{E}-05 \\
6.26 \mathrm{E}-04 \\
2.11 \mathrm{E}-03 \\
1.86 \mathrm{E}-03 \\
1.31 \mathrm{E}-03 \\
1.99 \mathrm{E}-03 \\
8.40 \mathrm{E}-04 \\
2.55 \mathrm{E}-03 \\
8.90 \mathrm{E}-04 \\
2.58 \mathrm{E}-03 \\
5.75 \mathrm{E}-04 \\
2.51 \mathrm{E}-03 \\
1.78 \mathrm{E}-05 \\
3.23 \mathrm{E}-03 \\
2.62 \mathrm{E}-03 \\
1.20 \mathrm{E}-03\end{array}$ & $\begin{array}{l}\text { U232 } \\
1.66 \mathrm{E}-03 \\
9.09 \mathrm{E}-04 \\
2.20 \mathrm{E}-03 \\
1.04 \mathrm{E}-03 \\
2.23 \mathrm{E}-03 \\
4.14 \mathrm{E}-04 \\
1.26 \mathrm{E}-03 \\
7.25 \mathrm{E}-04 \\
4.54 \mathrm{E}-04 \\
1.83 \mathrm{E}-03 \\
2.44 \mathrm{E}-03 \\
2.08 \mathrm{E}-03 \\
1.70 \mathrm{E}-03 \\
2.14 \mathrm{E}-03 \\
2.80 \mathrm{E}-03 \\
3.25 \mathrm{E}-03 \\
1.71 \mathrm{E}-03 \\
4.62 \mathrm{E}-03 \\
4.15 \mathrm{E}-03 \\
3.54 \mathrm{E}-03 \\
1.77 \mathrm{E}-03 \\
2.98 \mathrm{E}-03 \\
3.74 \mathrm{E}-03 \\
2.00 \mathrm{E}-03 \\
4.89 \mathrm{E}-03 \\
1.02 \mathrm{E}-06 \\
3.00 \mathrm{E}-03 \\
5.50 \mathrm{E}-06 \\
1.85 \mathrm{E}-03 \\
1.19 \mathrm{E}-04 \\
1.17 \mathrm{E}-03 \\
2.64 \mathrm{E}-03 \\
1.44 \mathrm{E}-03 \\
3.73 \mathrm{E}-03 \\
2.90 \mathrm{E}-03 \\
3.19 \mathrm{E}-03 \\
1.72 \mathrm{E}-03 \\
1.38 \mathrm{E}-03 \\
6.71 \mathrm{E}-03 \\
2.46 \mathrm{E}-03 \\
1.74 \mathrm{E}-03 \\
5.58 \mathrm{E}-03 \\
7.30 \mathrm{E}-04 \\
4.02 \mathrm{E}-03 \\
5.09 \mathrm{E}-03 \\
4.13 \mathrm{E}-03 \\
3.25 \mathrm{E}-03 \\
1.52 \mathrm{E}-03 \\
5.66 \mathrm{E}-04 \\
8.28 \mathrm{E}-05 \\
1.60 \mathrm{E}-03 \\
5.94 \mathrm{E}-03 \\
5.73 \mathrm{E}-03 \\
4.06 \mathrm{E}-03 \\
5.74 \mathrm{E}-03 \\
2.64 \mathrm{E}-03 \\
3.36 \mathrm{E}-03 \\
3.91 \mathrm{E}-03 \\
6.31 \mathrm{E}-03 \\
2.58 \mathrm{E}-03 \\
2.20 \mathrm{E}-03 \\
8.68 \mathrm{E}-05 \\
7.92 \mathrm{E}-03 \\
3.26 \mathrm{E}-03\end{array}$ & $\begin{array}{l}\text { U233 } \\
5.30 \mathrm{E}-05 \\
3.35 \mathrm{E}=05 \\
7.20 \mathrm{E}-05 \\
3.46 \mathrm{E}-05 \\
7.50 \mathrm{E}-05 \\
1.25 \mathrm{E}-05 \\
3.92 \mathrm{E}-05 \\
2.28 \mathrm{E}-05 \\
1.66 \mathrm{E}-05 \\
6.45 \mathrm{E}-05 \\
8.38 \mathrm{E}-05 \\
6.86 \mathrm{E}-05 \\
5.80 \mathrm{E}-05 \\
7.27 \mathrm{E}-05 \\
8.95 \mathrm{E}-05 \\
1.04 \mathrm{E}-04 \\
6.46 \mathrm{E}-05 \\
1.52 \mathrm{E}-04 \\
1.31 \mathrm{E}-04 \\
1.15 \mathrm{E}-04 \\
5.90 \mathrm{E}-05 \\
9.77 \mathrm{E}-05 \\
1.21 \mathrm{E}-04 \\
6.25 \mathrm{E}-05 \\
1.55 \mathrm{E}-04 \\
4.96 \mathrm{E}-08 \\
9.75 \mathrm{E}-05 \\
2.68 \mathrm{E}-07 \\
6.08 \mathrm{E}-05 \\
3.76 \mathrm{E}-06 \\
3.67 \mathrm{E}-05 \\
8.24 \mathrm{E}-05 \\
4.22 \mathrm{E}-05 \\
1.16 \mathrm{E}-04 \\
9.45 \mathrm{E}-05 \\
9.89 \mathrm{E}-05 \\
5.25 \mathrm{E}-05 \\
4.27 \mathrm{E}-05 \\
2.07 \mathrm{E}-04 \\
7.13 \mathrm{E}-05 \\
5.02 \mathrm{E}-05 \\
1.68 \mathrm{E}-04 \\
2.19 \mathrm{E}-05 \\
1.20 \mathrm{E}-04 \\
1.49 \mathrm{E}=04 \\
1.21 \mathrm{E}-04 \\
8.94 \mathrm{E}-05 \\
4.31 \mathrm{E}-05 \\
1.64 \mathrm{E}-05 \\
2.11 \mathrm{E}-06 \\
4.91 \mathrm{E}-05 \\
1.77 \mathrm{E}-04 \\
1.65 \mathrm{E}-04 \\
1.17 \mathrm{E}-04 \\
1.68 \mathrm{E}-04 \\
1.68 \mathrm{E}-04 \\
7.49 \mathrm{E}-04 \\
1.25 \mathrm{E}-05 \\
9.90 \mathrm{E}-05 \\
1.21 \mathrm{E}-04 \\
2.46 \mathrm{E}-05 \\
\end{array}$ & $\begin{array}{l}\text { U234 } \\
2.28 E+01 \\
1.92 E+01 \\
3.27 E+01 \\
1.63 E+01 \\
4.55 E+01 \\
4.89 E+00 \\
1.98 E+01 \\
1.19 E+01 \\
9.59 E+00 \\
3.43 E+01 \\
4.28 E+01 \\
3.26 E+01 \\
2.97 E+01 \\
3.74 E+01 \\
4.12 E+01 \\
4.78 E+01 \\
4.20 E+01 \\
7.47 E+01 \\
6.08 E+01 \\
5.71 E+01 \\
3.08 E+01 \\
4.98 E+01 \\
6.01 E+01 \\
2.92 E+01 \\
7.55 E+01 \\
2.16 E-01 \\
5.00 E+01 \\
1.74 E+00 \\
3.20 E+01 \\
1.80 E+00 \\
1.77 E+01 \\
3.97 E+01 \\
1.83 E+01 \\
5.60 E+01 \\
5.00 E+01 \\
4.79 E+01 \\
2.49 E+01 \\
2.08 E+01 \\
1.01 E+02 \\
3.11 E+01 \\
2.19 E+01 \\
8.04 E+01 \\
1.05 E+01 \\
5.78 E+01 \\
6.81 E+01 \\
5.53 E+01 \\
3.69 E+01 \\
1.91 E+01 \\
7.54 E+00 \\
7.52 E-01 \\
2.53 E+01 \\
8.53 E+01 \\
7.62 E+01 \\
5.40 E+01 \\
8.20 E+01 \\
3.50 E+01 \\
1.03 E+02 \\
3.75 E+01 \\
1.04 E+02 \\
2.32 E+01 \\
1.01 E+02 \\
7.17 E=01 \\
1.35 E+02 \\
1.11 E+02 \\
5.07 E+01\end{array}$ & $\begin{array}{l}\text { U235 } \\
8.52 E-01 \\
7.36 E-01 \\
1.23 E+00 \\
6.15 E-01 \\
1.95 E+00 \\
1.81 E-01 \\
8.36 E-01 \\
5.03 E-01 \\
3.67 E-01 \\
1.30 E+00 \\
1.62 E+00 \\
1.23 E+00 \\
1.13 E+00 \\
1.42 E+00 \\
1.55 E+00 \\
1.80 E+00 \\
1.62 E+00 \\
2.82 E+00 \\
2.28 E+00 \\
2.16 E+00 \\
1.17 E+00 \\
1.88 E+00 \\
2.27 E+00 \\
1.10 E+00 \\
2.84 E+00 \\
9.89 E-03 \\
1.89 E+00 \\
7.99 E-02 \\
1.21 E+00 \\
6.77 E-02 \\
6.65 E-01 \\
1.49 E+00 \\
6.80 E-01 \\
2.11 E+00 \\
1.90 E+00 \\
1.80 E+00 \\
9.34 E-01 \\
7.81 E-01 \\
3.78 E+00 \\
1.16 E+00 \\
8.17 E-01 \\
3.02 E+00 \\
3.95 E-01 \\
2.17 E+00 \\
2.55 E+00 \\
2.07 E+00 \\
1.37 E+00 \\
7.111 E-01 \\
2.82 E-01 \\
2.82 E-02 \\
9.53 E-01 \\
3.20 E+00 \\
2.85 E+00 \\
2.02 E+00 \\
3.08 E+00 \\
1.31 E+00 \\
3.98 E+00 \\
1.37 E+00 \\
4.05 E+00 \\
9.06 E-01 \\
3.96 E+00 \\
2.81 E-02 \\
5.09 E+00 \\
4.15 E+00 \\
1.91 E+00\end{array}$ & $\begin{array}{l}\text { U236 } \\
1.58 E+00 \\
1.26 E+00 \\
2.24 E+00 \\
1.11 E+00 \\
7.58 E-01 \\
3.47 E-01 \\
3.90 E-01 \\
2.28 E-01 \\
6.25 E-01 \\
2.27 E+00 \\
2.86 E+00 \\
2.21 E+00 \\
1.99 E+00 \\
2.50 E+00 \\
2.83 E+00 \\
3.28 E+00 \\
2.68 E+00 \\
5.05 E+00 \\
4.17 E+00 \\
3.86 E+00 \\
2.06 E+00 \\
3.35 E+00 \\
4.06 E+00 \\
2.00 E+00 \\
5.13 E+00 \\
5.49 E-04 \\
3.36 E+00 \\
2.97 E-03 \\
2.14 E+00 \\
1.23 E-01 \\
1.21 E+00 \\
2.71 E+00 \\
1.28 E+00 \\
3.82 E+00 \\
3.34 E+00 \\
3.27 E+00 \\
1.71 E+00 \\
1.42 E+00 \\
6.86 E+00 \\
2.18 E+00 \\
1.53 E+00 \\
5.51 E+00 \\
7.21 E-01 \\
3.96 E+00 \\
4.72 E+00 \\
3.83 E+00 \\
2.63 E+00 \\
1.33 E+00 \\
5.23 E-01 \\
5.70 E-02 \\
1.71 E+00 \\
5.85 E+00 \\
5.28 E+00 \\
3.74 E+00 \\
5.62 E+00 \\
2.42 E+00 \\
6.36 E+00 \\
2.74 E+00 \\
6.40 E+00 \\
1.41 E+00 \\
6.11 E+00 \\
4.56 E-02 \\
9.13 E+00 \\
7.60 E+00 \\
3.43 E+00\end{array}$ & $\begin{array}{c}\text { U238 } \\
1.55 E+01 \\
1.30 E+01 \\
2.22 E+01 \\
1.11 E+01 \\
4.66 E+01 \\
3.33 E+00 \\
2.04 E+01 \\
1.22 E+01 \\
6.48 E+00 \\
2.32 E+01 \\
2.90 E+01 \\
2.21 E+01 \\
2.01 E+01 \\
2.53 E+01 \\
2.80 E+01 \\
3.25 E+01 \\
2.83 E+01 \\
5.06 E+01 \\
4.13 E+01 \\
3.87 E+01 \\
2.08 E+01 \\
3.37 E+01 \\
4.07 E+01 \\
1.98 E+01 \\
5.12 E+01 \\
2.18 E-01 \\
3.39 E+01 \\
1.75 E+00 \\
2.17 E+01 \\
1.22 E+00 \\
1.20 E+01 \\
2.70 E+01 \\
1.24 E+01 \\
3.80 E+01 \\
3.39 E+01 \\
3.25 E+01 \\
1.69 E+01 \\
1.41 E+01 \\
6.82 E+01 \\
2.12 E+01 \\
1.49 E+01 \\
5.46 E+01 \\
7.14 E+00 \\
3.92 E+01 \\
4.62 E+01 \\
3.76 E+01 \\
2.51 E+01 \\
1.30 E+01 \\
5.12 E+00 \\
5.13 E-01 \\
1.71 E+01 \\
5.79 E+01 \\
5.18 E+01 \\
3.67 E+01 \\
5.56 E+01 \\
2.38 E+01 \\
6.90 E+01 \\
2.56 E+01 \\
6.99 E+01 \\
1.56 E+01 \\
6.80 E+01 \\
4.83 E-01 \\
9.15 E+01 \\
7.50 E+01 \\
3.43 E+01\end{array}$ & $\begin{array}{c}\text { NP237 } \\
2.17 \mathrm{E}-01 \\
1.35 \mathrm{E}-01 \\
2.94 \mathrm{E}-01 \\
1.41 \mathrm{E}-01 \\
2.59 \mathrm{E}-01 \\
5.15 \mathrm{E}-02 \\
1.38 \mathrm{E}-01 \\
8.01 \mathrm{E}-02 \\
6.74 \mathrm{E}-02 \\
2.62 \mathrm{E}-01 \\
3.42 \mathrm{E}-01 \\
2.82 \mathrm{E}-01 \\
2.38 \mathrm{E}-01 \\
2.99 \mathrm{E}-01 \\
3.71 \mathrm{E}-01 \\
4.31 \mathrm{E}-01 \\
2.64 \mathrm{E}-01 \\
6.30 \mathrm{E}-01 \\
5.48 \mathrm{E}-01 \\
4.81 \mathrm{E}-01 \\
2.46 \mathrm{E}-01 \\
4.08 \mathrm{E}-01 \\
5.06 \mathrm{E}-01 \\
2.63 \mathrm{E}-01 \\
6.53 \mathrm{E}-01 \\
1.62 \mathrm{E}-04 \\
4.10 \mathrm{E}-01 \\
8.71 \mathrm{E}-04 \\
2.56 \mathrm{E}-01 \\
1.58 \mathrm{E}-02 \\
1.55 \mathrm{E}-01 \\
3.49 \mathrm{E}-01 \\
1.80 \mathrm{E}-01 \\
4.92 \mathrm{E}-01 \\
4.00 \mathrm{E}-01 \\
4.21 \mathrm{E}-01 \\
2.24 \mathrm{E}-01 \\
1.82 \mathrm{E}-01 \\
8.83 \mathrm{E}-01 \\
3.07 \mathrm{E}-01 \\
2.16 \mathrm{E}-01 \\
7.24 \mathrm{E}-01 \\
9.47 \mathrm{E}-02 \\
5.20 \mathrm{E}-01 \\
6.44 \mathrm{E}-01 \\
5.24 \mathrm{E}-01 \\
3.91 \mathrm{E}-01 \\
1.88 \mathrm{E}-01 \\
7.14 \mathrm{E}-02 \\
9.62 \mathrm{E}-03 \\
2.13 \mathrm{E}-01 \\
7.67 \mathrm{E}-01 \\
7.22 \mathrm{E}-01 \\
5.11 \mathrm{E}-01 \\
7.37 \mathrm{E}-01 \\
3.31 \mathrm{E}-01 \\
5.38 \mathrm{E}-01 \\
4.42 \mathrm{E}-01 \\
5.22 \mathrm{E}-01 \\
1.06 \mathrm{E}-01 \\
4.40 \mathrm{E}-01 \\
4.05 \mathrm{E}-03 \\
1.14 \mathrm{E}+00 \\
1.01 \mathrm{E}+00 \\
4.28 \mathrm{E}-01\end{array}$ \\
\hline
\end{tabular}


SNF-SD-WM-TI-794, ReV. 0

ACTIVITY IN UNSEPARATED FUEL (Curies decayed to 1/1/94)

\begin{tabular}{|c|c|c|c|c|c|c|c|c|c|}
\hline $\begin{array}{r}\text { isting } \\
326 \\
327 \\
328 \\
329 \\
330 \\
331 \\
332 \\
333 \\
334 \\
335 \\
336 \\
337 \\
338 \\
339 \\
340 \\
341 \\
342 \\
343 \\
344 \\
345 \\
346 \\
347 \\
348 \\
349 \\
350 \\
351 \\
352 \\
353 \\
354 \\
355 \\
356 \\
357 \\
358 \\
359 \\
360 \\
361 \\
362 \\
363 \\
364 \\
365 \\
366 \\
367 \\
368 \\
369 \\
370 \\
371 \\
372 \\
373 \\
374 \\
375 \\
376 \\
377 \\
378 \\
379 \\
380 \\
381 \\
382 \\
383 \\
384 \\
385 \\
386 \\
387 \\
388 \\
389 \\
390\end{array}$ & $\begin{array}{l}P U 238 \\
7.24 \mathrm{E}+01 \\
3.34 \mathrm{E}+01 \\
9.34 \mathrm{E}+01 \\
4.32 \mathrm{E}+01 \\
6.95 \mathrm{E}+01 \\
1.90 \mathrm{E}+01 \\
4.46 \mathrm{E}+01 \\
2.52 \mathrm{E}+01 \\
1.67 \mathrm{E}+01 \\
7.08 \mathrm{E}+01 \\
9.68 \mathrm{E}+01 \\
8.63 \mathrm{E}+01 \\
6.73 \mathrm{E}+01 \\
8.47 \mathrm{E}+01 \\
1.19 \mathrm{E}+02 \\
1.38 \mathrm{E}+02 \\
5.83 \mathrm{E}+01 \\
1.89 \mathrm{E}+02 \\
1.76 \mathrm{E}+02 \\
1.45 \mathrm{E}+02 \\
7.02 \mathrm{E}+01 \\
1.20 \mathrm{E}+02 \\
1.53 \mathrm{E}+02 \\
8.46 \mathrm{E}+01 \\
2.02 \mathrm{E}+02 \\
2.67 \mathrm{E}-03 \\
1.21 \mathrm{E}+02 \\
1.42 \mathrm{E}-02 \\
7.33 \mathrm{E}+01 \\
4.97 \mathrm{E}+00 \\
4.90 \mathrm{E}+01 \\
1.10 \mathrm{E}+02 \\
6.37 \mathrm{E}+01 \\
1.55 \mathrm{E}+02 \\
1.15 \mathrm{E}+02 \\
1.33 \mathrm{E}+02 \\
7.25 \mathrm{E}+01 \\
5.77 \mathrm{E}+01 \\
2.80 \mathrm{E}+02 \\
1.09 \mathrm{E}+02 \\
7.69 \mathrm{E}+01 \\
2.36 \mathrm{E}+02 \\
3.08 \mathrm{E}+01 \\
1.69 \mathrm{E}+02 \\
2.21 \mathrm{E}+02 \\
1.79 \mathrm{E}+02 \\
1.49 \mathrm{E}+02 \\
6.72 \mathrm{E}+01 \\
2.45 \mathrm{E}+01 \\
4.73 \mathrm{E}+00 \\
6.51 \mathrm{E}+01 \\
2.51 \mathrm{E}+02 \\
2.48 \mathrm{E}+02 \\
1.76 \mathrm{E}+02 \\
2.42 \mathrm{E}+02 \\
1.14 \mathrm{E}+02 \\
9.99 \mathrm{E}+01 \\
1.88 \mathrm{E}+02 \\
9.23 \mathrm{E}+01 \\
1.68 \mathrm{E}+01 \\
6.55 \mathrm{E}+01 \\
7.73 \mathrm{E}-01 \\
3.53 \mathrm{E}+02 \\
3.35 \mathrm{E}+02 \\
1.33 \mathrm{E}+02\end{array}$ & $\begin{array}{l}\text { PU239 } \\
1.85 E+03 \\
1.22 E+03 \\
2.55 E+03 \\
1.23 E+03 \\
4.91 E+03 \\
4.31 E+02 \\
2.50 E+03 \\
1.46 E+03 \\
6.07 E+02 \\
2.33 E+03 \\
3.02 E+03 \\
2.46 E+03 \\
2.10 E+03 \\
2.64 E+03 \\
3.21 E+03 \\
3.73 E+03 \\
2.42 E+03 \\
5.51 E+03 \\
4.74 E+03 \\
4.21 E+03 \\
2.17 E+03 \\
3.59 E+03 \\
4.43 E+03 \\
2.28 E+03 \\
5.69 E+03 \\
3.74 E+00 \\
3.61 E+03 \\
2.03 E+01 \\
2.26 E+03 \\
1.38 E+02 \\
1.35 E+03 \\
3.03 E+03 \\
1.53 E+03 \\
4.28 E+03 \\
3.53 E+03 \\
3.66 E+03 \\
1.94 E+03 \\
1.59 E+03 \\
7.68 E+03 \\
2.61 E+03 \\
1.84 E+03 \\
6.27 E+03 \\
8.20 E+02 \\
4.50 E+03 \\
5.53 E+03 \\
4.49 E+03 \\
3.28 E+03 \\
1.60 E+03 \\
6.12 E+02 \\
7.68 E+01 \\
1.86 E+03 \\
6.65 E+03 \\
6.19 E+03 \\
4.38 E+03 \\
6.39 E+03 \\
2.84 E+03 \\
5.04 E+03 \\
3.62 E+03 \\
4.91 E+03 \\
1.01 E+03 \\
4.20 E+03 \\
3.68 E+01 \\
9.99 E+03 \\
8.69 E+03 \\
3\end{array}$ & $\begin{array}{l}\text { PU240 } \\
3.15 E+02 \\
1.53 E+02 \\
4.09 E+02 \\
1.90 E+02 \\
6.89 E+02 \\
8.14 E+01 \\
4.21 E+02 \\
2.39 E+02 \\
7.63 E+01 \\
3.19 E+02 \\
4.32 E+02 \\
3.80 E+02 \\
3.00 E+02 \\
3.77 E+02 \\
5.17 E+02 \\
5.99 E+02 \\
2.72 E+02 \\
8.30 E+02 \\
7.62 E+02 \\
6.34 E+02 \\
3.10 E+02 \\
5.26 E+02 \\
6.67 E+02 \\
3.66 E+02 \\
8.80 E+02 \\
1.09 E-01 \\
5.29 E+02 \\
4.79 E-01 \\
3.23 E+02 \\
2.16 E+01 \\
2.12 E+02 \\
4.76 E+02 \\
2.71 E+02 \\
6.72 E+02 \\
5.04 E+02 \\
5.74 E+02 \\
3.12 E+02 \\
2.49 E+02 \\
1.21 E+03 \\
4.61 E+02 \\
3.25 E+02 \\
1.01 E+03 \\
1.32 E+02 \\
7.24 E+02 \\
9.36 E+02 \\
7.61 E+02 \\
6.22 E+02 \\
2.83 E+02 \\
1.04 E+02 \\
1.85 E+01 \\
2.81 E+02 \\
1.07 E+03 \\
1.05 E+03 \\
7.43 E+02 \\
1.03 E+03 \\
4.81 E+02 \\
4.77 E+02 \\
7.68 E+02 \\
4.46 E+02 \\
8.36 E+01 \\
3.32 E+02 \\
6.49 E+00 \\
1.51 E+03 \\
1.42 E+03\end{array}$ & $\begin{array}{l}P U 241 \\
2.25 E+03 \\
9.58 E+02 \\
2.88 E+03 \\
1.32 E+03 \\
5.12 E+03 \\
6.08 E+02 \\
3.50 E+03 \\
1.98 E+03 \\
4.82 E+02 \\
2.11 E+03 \\
2.93 E+03 \\
2.68 E+03 \\
2.05 E+03 \\
2.60 E+03 \\
3.80 E+03 \\
4.40 E+03 \\
1.67 E+03 \\
5.93 E+03 \\
5.69 E+03 \\
4.64 E+03 \\
2.23 E+03 \\
3.84 E+03 \\
4.95 E+03 \\
2.78 E+03 \\
6.65 E+03 \\
0.00 E+00 \\
3.93 E+03 \\
0.00 E+00 \\
2.37 E+03 \\
1.63 E+02 \\
1.63 E+03 \\
3.68 E+03 \\
2.19 E+03 \\
5.21 E+03 \\
3.777 E+03 \\
4.47 E+03 \\
2.46 E+03 \\
1.96 E+03 \\
9.50 E+03 \\
3.81 E+03 \\
2.70 E+03 \\
8.19 E+03 \\
1.08 E+03 \\
5.94 E+03 \\
7.77 E E+03 \\
6.27 E+03 \\
5.41 E+03 \\
2.43 E+03 \\
8.80 E+02 \\
2.21 E+02 \\
2.27 E+03 \\
8.86 E+03 \\
8.97 E+03 \\
6.38 E+03 \\
8.73 E+03 \\
4.21 E+03 \\
2.98 E+03 \\
7.16 E+03 \\
2.70 E+03 \\
4.64 E+02 \\
1.74 E+03 \\
1.21 E+01 \\
1.29 E+04 \\
1.25 E+04 \\
4.88 E+03\end{array}$ & 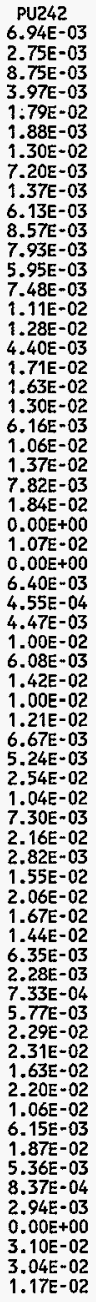 & 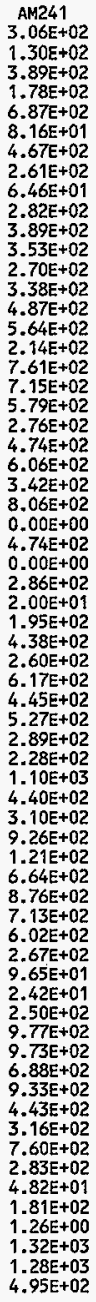 & $\begin{array}{l}\text { AM243 } \\
2.06 E-03 \\
7.89 E-04 \\
2.58 E-03 \\
1.17 E-03 \\
6.39 E-03 \\
5.62 E-04 \\
4.80 E-03 \\
2.65 E-03 \\
3.93 E-04 \\
1.78 E-03 \\
2.50 E-03 \\
2.33 E-03 \\
1.74 E-03 \\
2.18 E-03 \\
3.26 E-03 \\
3.78 E-03 \\
1.24 E-03 \\
5.00 E-03 \\
4.81 E-03 \\
3.82 E-03 \\
1.80 E-03 \\
3.11 E-03 \\
4.02 E-03 \\
2.31 E-03 \\
5.40 E-03 \\
0.00 E+00 \\
3.13 E-03 \\
0.00 E+00 \\
1.87 E-03 \\
1.34 E-04 \\
1.32 E-03 \\
2.96 E-03 \\
1.81 E-03 \\
4.17 E-03 \\
2.92 E-03 \\
3.57 E-03 \\
1.97 E-03 \\
1.54 E-03 \\
7.49 E-03 \\
3.08 E-03 \\
2.17 E-03 \\
6.36 E-03 \\
8.32 E-04 \\
4.57 E-03 \\
6.10 E-03 \\
4.96 E-03 \\
4.31 E-03 \\
1.89 E-03 \\
6.76 E-04 \\
1.80 E-07 \\
1.69 E-03 \\
6.75 E-03 \\
6.84 E-03 \\
4.84 E-03 \\
6.49 E-03 \\
3.14 E-03 \\
1.64 E-03 \\
5.61 E-03 \\
1.399 E-03 \\
2.05 E-04 \\
6.82 E-04 \\
0.00 E+00 \\
9.11 E-03 \\
9.00 E-03\end{array}$ & $\begin{array}{l}\text { CM242 } \\
8.28 \mathrm{E}-03 \\
3.18 \mathrm{E}-03 \\
1.04 \mathrm{E}-02 \\
4.71 \mathrm{E}-03 \\
1.98 \mathrm{E}-02 \\
2.27 \mathrm{E}-03 \\
1.49 \mathrm{E}-02 \\
8.23 \mathrm{E}-03 \\
1.59 \mathrm{E}-03 \\
7.17 \mathrm{E}-03 \\
1.01 \mathrm{E}-02 \\
9.41 \mathrm{E}-03 \\
7.01 \mathrm{E}-03 \\
8.82 \mathrm{E}-03 \\
1.32 \mathrm{E}-02 \\
1.53 \mathrm{E}-02 \\
5.01 \mathrm{E}-03 \\
2.02 \mathrm{E}-02 \\
1.95 \mathrm{E}-02 \\
1.55 \mathrm{E}-02 \\
7.29 \mathrm{E}-03 \\
1.26 \mathrm{E}-02 \\
1.63 \mathrm{E}-02 \\
9.37 \mathrm{E}-03 \\
2.20 \mathrm{E}-02 \\
0.00 \mathrm{E}+00 \\
1.27 \mathrm{E}-02 \\
0.00 \mathrm{E}+00 \\
7.60 \mathrm{E}-03 \\
5.44 \mathrm{E}-04 \\
5.35 \mathrm{E}-03 \\
1.20 \mathrm{E}-02 \\
7.35 \mathrm{E}-03 \\
1.70 \mathrm{E}-02 \\
1.19 \mathrm{E}-02 \\
1.45 \mathrm{E}-02 \\
8.01 \mathrm{E}-03 \\
6.30 \mathrm{E}-03 \\
3.05 \mathrm{E}-02 \\
1.26 \mathrm{E}-02 \\
8.86 \mathrm{E}-03 \\
2.60 \mathrm{E}-02 \\
3.40 \mathrm{E}-03 \\
1.87 \mathrm{E}-02 \\
2.49 \mathrm{E}-02 \\
2.02 \mathrm{E}-02 \\
1.76 \mathrm{E}-02 \\
7.73 \mathrm{E}-03 \\
2.77 \mathrm{E}-03 \\
1.06 \mathrm{E}-22 \\
6.92 \mathrm{E}-03 \\
2.76 \mathrm{E}-02 \\
2.80 \mathrm{E}-02 \\
1.98 \mathrm{E}-02 \\
2.66 \mathrm{E}-02 \\
1.29 \mathrm{E}-02 \\
6.74 \mathrm{E}-03 \\
2.30 \mathrm{E}-02 \\
5.75 \mathrm{E}-03 \\
8.46 \mathrm{E}-04 \\
2.82 \mathrm{E}-03 \\
0.00 \mathrm{E}+00 \\
3.75 \mathrm{E}-02 \\
3.70 \mathrm{E}-02 \\
1.41 \mathrm{E}-02\end{array}$ & $\begin{array}{l}\text { CH243 } \\
1.64 \mathrm{E}-04 \\
6.19 \mathrm{E}-05 \\
2.06 \mathrm{E}-04 \\
9.31 \mathrm{E}-05 \\
4.95 \mathrm{E}-04 \\
4.52 \mathrm{E}-05 \\
3.80 \mathrm{E}-04 \\
2.10 \mathrm{E}-04 \\
3.10 \mathrm{E}-05 \\
1.41 \mathrm{E}-04 \\
1.99 \mathrm{E}-04 \\
1.87 \mathrm{E}-04 \\
1.39 \mathrm{E}-04 \\
1.76 \mathrm{E}-04 \\
2.66 \mathrm{E}-04 \\
3.08 \mathrm{E}-04 \\
9.81 \mathrm{E}-05 \\
4.06 \mathrm{E}-04 \\
3.95 \mathrm{E}-04 \\
3.14 \mathrm{E}-04 \\
1.47 \mathrm{E}-04 \\
2.56 \mathrm{E}-04 \\
3.33 \mathrm{E}-04 \\
1.91 \mathrm{E}-04 \\
4.49 \mathrm{E}-04 \\
0.00 \mathrm{E}+00 \\
2.60 \mathrm{E}-04 \\
0.00 \mathrm{E}+00 \\
1.55 \mathrm{E}-04 \\
1.11 \mathrm{E}-05 \\
1.10 \mathrm{E}-04 \\
2.48 \mathrm{E}-04 \\
1.52 \mathrm{E}-04 \\
3.51 \mathrm{E}-04 \\
2.44 \mathrm{E}-04 \\
3.00 \mathrm{E}-04 \\
1.66 \mathrm{E}-04 \\
1.31 \mathrm{E}-04 \\
6.34 \mathrm{E}-04 \\
2.63 \mathrm{E}-04 \\
1.86 \mathrm{E}-04 \\
5.45 \mathrm{E}-04 \\
7.15 \mathrm{E}-05 \\
3.93 \mathrm{E}-04 \\
5.25 \mathrm{E}-04 \\
4.25 \mathrm{E}-04 \\
3.74 \mathrm{E}-04 \\
1.64 \mathrm{E}-04 \\
5.88 \mathrm{E}-05 \\
0.00 \mathrm{E}+00 \\
1.46 \mathrm{E}-04 \\
5.84 \mathrm{E}-04 \\
5.97 \mathrm{E}-04 \\
4.23 \mathrm{E}-04 \\
5.68 \mathrm{E}-04 \\
2.77 \mathrm{E}-04 \\
1.36 \mathrm{E}-04 \\
4.97 \mathrm{E}-04 \\
1.15 \mathrm{E}-04 \\
1.62 \mathrm{E}-05 \\
5.20 \mathrm{E}-05 \\
0.00 \mathrm{E}+00 \\
8.08 \mathrm{E}-04 \\
8.02 \mathrm{E}-04 \\
3.05 \mathrm{E}-04\end{array}$ \\
\hline
\end{tabular}


HNF-SD-WM-TI-794, REV. O

ACTIVITY IN UNSEPARATED FUEL (Curies decayed to $1 / 1 / 94$ )

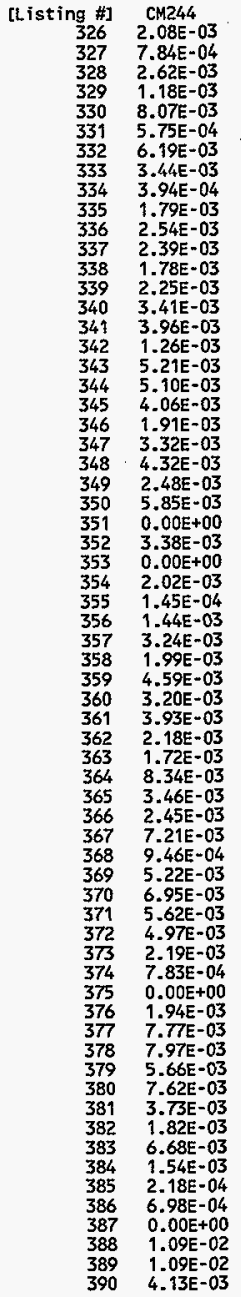


HNF-SD-WM-T1-794, Rev. 0

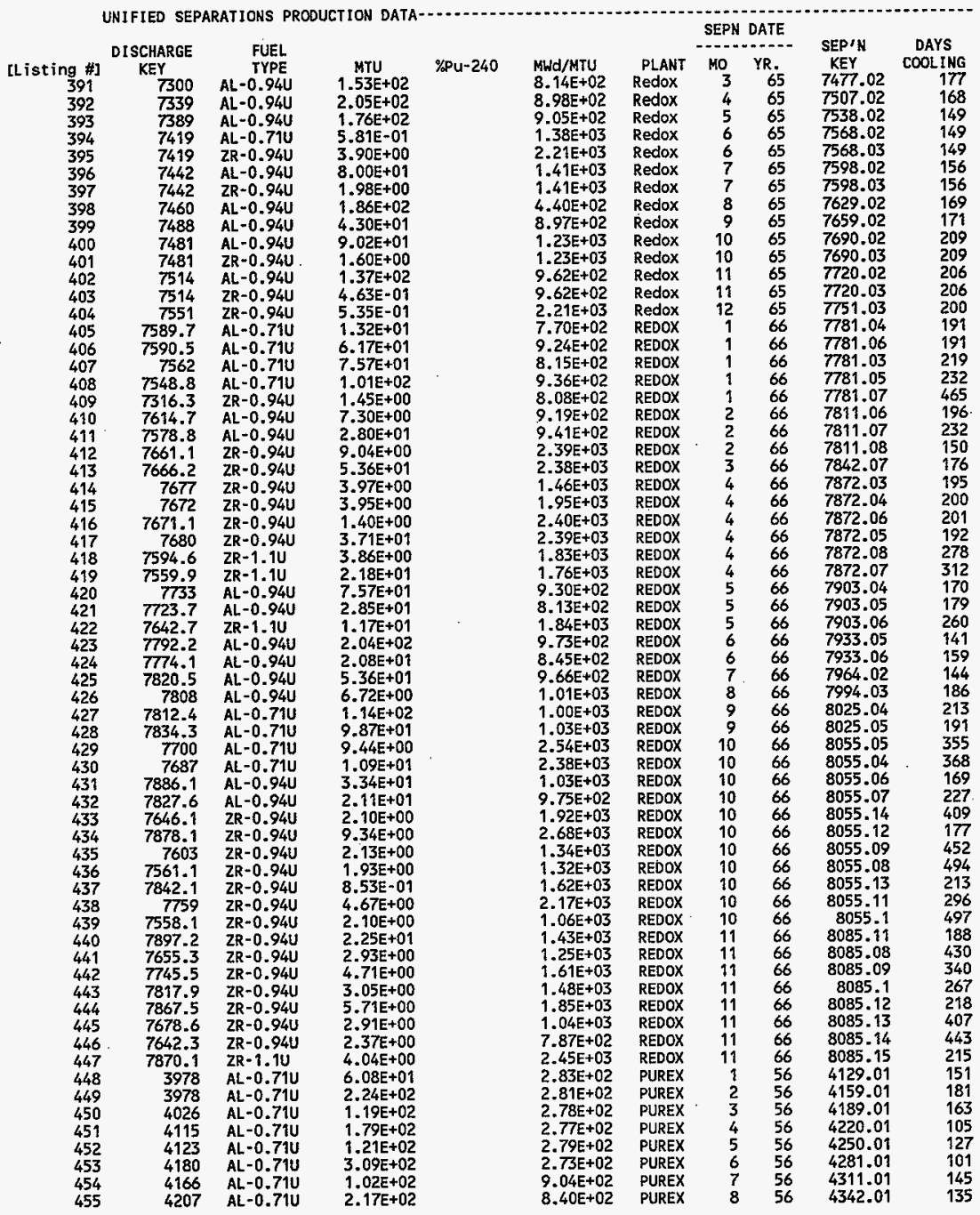


HNF-SD-WM-TI-794, ReV. O

\begin{tabular}{|c|c|c|c|c|c|c|c|c|}
\hline $\begin{array}{c}\text { [Listing \#] } \\
391 \\
392 \\
393 \\
394 \\
395 \\
396 \\
397 \\
398 \\
399 \\
400 \\
401 \\
402 \\
403 \\
404 \\
405 \\
406 \\
407 \\
408 \\
409 \\
410 \\
411 \\
412 \\
413 \\
414 \\
415 \\
416 \\
417 \\
418 \\
419 \\
420 \\
421 \\
422 \\
423 \\
424 \\
425 \\
426 \\
427 \\
428 \\
429 \\
430 \\
431 \\
432 \\
433 \\
434 \\
435 \\
436 \\
437 \\
438 \\
439 \\
440 \\
441 \\
442 \\
443 \\
444 \\
445 \\
446 \\
447 \\
448 \\
449 \\
450 \\
451 \\
452 \\
453 \\
454 \\
455\end{array}$ & 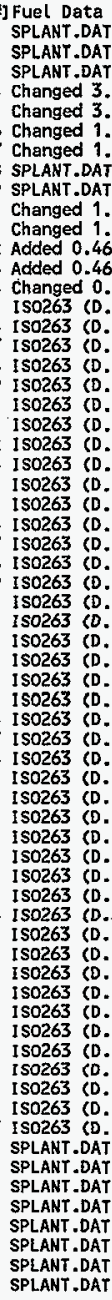 & 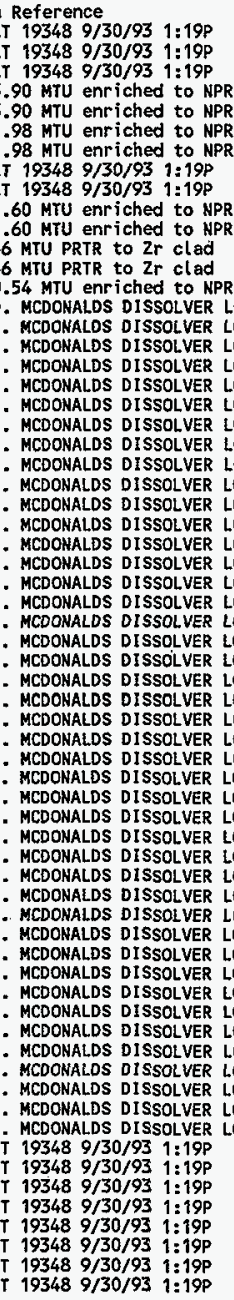 & $\begin{array}{l}\text { R (2 } \\
\text { LOG) } \\
\text { LOG) } \\
\text { LOG) } \\
\text { LOG) } \\
\text { LOG) } \\
\text { LOG) } \\
\text { LOG) } \\
\text { LOG) } \\
\text { LOG) } \\
\text { LOG) } \\
\text { LOG) } \\
\text { LOG) } \\
\text { LOG) } \\
\text { LOG) } \\
\text { LOG) } \\
\text { LOG) } \\
\text { LOG) } \\
\text { LOG) } \\
\text { LOG) } \\
\text { LOG) } \\
\text { LOG) } \\
\text { LOG) } \\
\text { LOG) } \\
\text { LOG) } \\
\text { LOG) } \\
\text { LOG) } \\
\text { LOG) } \\
\text { LOG) } \\
\text { LOG) } \\
\text { LOG) } \\
\text { LOG) } \\
\text { LOG) } \\
\text { LOG) } \\
\text { LOG) } \\
\text { LOG) } \\
\text { LOG) } \\
\text { LOG) } \\
\text { LOG) } \\
\text { LOG) } \\
\text { LOG) } \\
\text { LOG) } \\
\text { LOG) } \\
\text { LOG) }\end{array}$ & 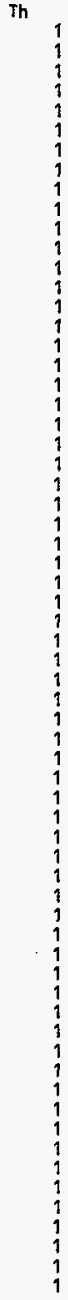 & $\begin{array}{l}\text { J } \\
0.0018 \\
0.0022 \\
0.0027 \\
0.0028 \\
0.0028 \\
0.0025 \\
0.0025 \\
0.002 \\
0.0027 \\
0.0025 \\
0.0025 \\
0.0026 \\
0.0026 \\
0.0024 \\
0.0025 \\
0.0025 \\
0.0025 \\
0.0025 \\
0.0025 \\
0.0027 \\
0.0027 \\
0.0027 \\
0.0099 \\
0.0088 \\
0.0088 \\
0.0088 \\
0.0088 \\
0.0088 \\
0.0088 \\
0.0047 \\
0.0047 \\
0.0047 \\
0.0019 \\
0.0019 \\
0.0018 \\
0.0018 \\
0.0012 \\
0.0012 \\
0.0043 \\
0.0043 \\
0.0043 \\
0.0043 \\
0.0043 \\
0.0043 \\
0.0043 \\
0.0043 \\
0.0043 \\
0.0043 \\
0.0043 \\
0.0048 \\
0.0048 \\
0.0048 \\
0.0048 \\
0.0048 \\
0.0048 \\
0.0048 \\
0.0048 \\
0.0046 \\
0.0099 \\
0.0065 \\
0.0136 \\
0.0052 \\
0.0061 \\
0.0116 \\
0.0095\end{array}$ & $\begin{array}{c}\text { Ap } \\
0.3 \\
0.3 \\
0.3 \\
0.3 \\
0.3 \\
0.3 \\
0.3 \\
0.3 \\
0.3 \\
0.3 \\
0.3 \\
0.3 \\
0.3 \\
0.3 \\
0.3 \\
0.3 \\
0.3 \\
0.3 \\
0.3 \\
0.3 \\
0.3 \\
0.3 \\
0.3 \\
0.3 \\
0.3 \\
0.3 \\
0.3 \\
0.3 \\
0.3 \\
0.3 \\
0.3 \\
0.3 \\
0.3 \\
0.3 \\
0.3 \\
0.3 \\
0.3 \\
0.3 \\
0.3 \\
0.3 \\
0.3 \\
0.3 \\
0.3 \\
0.3 \\
0.3 \\
0.3 \\
0.3 \\
0.3 \\
0.3 \\
0.3 \\
0.3 \\
0.3 \\
0.3 \\
0.3 \\
0.3 \\
0.3 \\
0.3 \\
1 \\
1 \\
1 \\
1 \\
0\end{array}$ & $\begin{array}{l}\text { Pu } \\
0.0032 \\
0.0044 \\
0.0038 \\
0.0219 \\
0.0219 \\
0.0107 \\
0.0107 \\
0.0065 \\
0.0054 \\
0.0075 \\
0.0075 \\
0.0052 \\
0.0052 \\
0.0047 \\
0.0031 \\
0.0031 \\
0.0031 \\
0.0031 \\
0.0031 \\
0.0051 \\
0.0051 \\
0.0051 \\
0.0071 \\
0.0103 \\
0.0103 \\
0.0103 \\
0.0103 \\
0.0103 \\
0.0103 \\
0.0069 \\
0.0069 \\
0.0069 \\
0.0028 \\
0.0028 \\
0.0038 \\
0.0038 \\
0.0039 \\
0.0039 \\
0.003 \\
0.003 \\
0.003 \\
0.003 \\
0.003 \\
0.003 \\
0.003 \\
0.003 \\
0.003 \\
0.003 \\
0.003 \\
0.01 \\
0.01 \\
0.01 \\
0.01 \\
0.01 \\
0.01 \\
0.01 \\
0.01 \\
0.0183 \\
0.051 \\
0.041 \\
0.028 \\
0.035 \\
0.014 \\
0.023 \\
0.0126\end{array}$ & 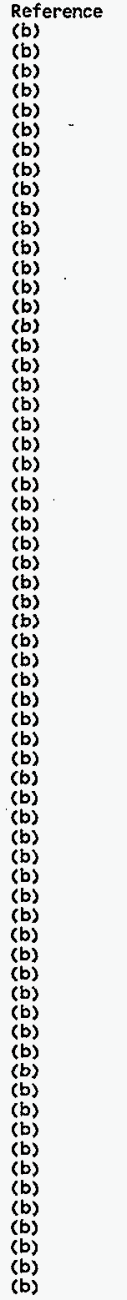 \\
\hline
\end{tabular}


HNF-SD-WM-TI-794, Rev. 0

ACTIVITY IN UNSEPARATED FUEL (Curies decayed to $1 / 1 / 94$ )

\begin{tabular}{|c|c|c|c|c|c|c|c|c|c|}
\hline $\begin{array}{c}\text { [tisting } \# \\
391 \\
392 \\
393 \\
394 \\
395 \\
396 \\
397 \\
398 \\
399 \\
400 \\
401 \\
402 \\
403 \\
404 \\
405 \\
406 \\
407 \\
408 \\
409 \\
410 \\
411 \\
412 \\
413 \\
414 \\
415 \\
416 \\
417 \\
418 \\
419 \\
420 \\
421 \\
422 \\
423 \\
424 \\
425 \\
426 \\
427 \\
428 \\
429 \\
430 \\
431 \\
432 \\
433 \\
434 \\
435 \\
436 \\
437 \\
438 \\
439 \\
440 \\
441 \\
442 \\
443 \\
453 \\
444 \\
455 \\
445 \\
446 \\
447 \\
448 \\
449 \\
450 \\
451 \\
453 \\
40\end{array}$ & $\begin{array}{l}403 \\
3.72 E+02 \\
5.56 E+02 \\
4.83 E+02 \\
2.58 E+00 \\
2.84 E+01 \\
3.50 E+02 \\
9.04 E+00 \\
2.48 E+02 \\
1.19 E+02 \\
3.46 E+02 \\
6.38 E+00 \\
4.10 E+02 \\
1.44 E+00 \\
3.98 E+00 \\
3.27 E+01 \\
1.85 E+02 \\
1.99 E+02 \\
3.05 E+02 \\
3.66 E+00 \\
2.12 E+01 \\
8.27 E+01 \\
7.45 E+01 \\
4.39 E+02 \\
1.95 E+01 \\
2.63 E+01 \\
1.16 E+01 \\
3.07 E+02 \\
2.29 E+01 \\
1.24 E+02 \\
2.26 E+02 \\
7.40 E+01 \\
7.01 E+01 \\
6.44 E+02 \\
5.66 E+01 \\
1.69 E+02 \\
2.21 E+01 \\
3.83 E+02 \\
3.43 E+02 \\
8.44 E+01 \\
9.06 E+01 \\
1.13 E+02 \\
6.70 E+01 \\
1.36 E+01 \\
8.98 E+01 \\
9.44 E+00 \\
8.40 E+00 \\
4.77 E+00 \\
3.54 E+01 \\
7.25 E+00 \\
1.12 E+02 \\
1.22 E+01 \\
2.59 E+01 \\
1.55 E+01 \\
3.70 E+01 \\
1.01 E+01 \\
6.11 E+00 \\
3.40 E+01 \\
3.12 E+01 \\
1.14 E+02 \\
6.03 E+01 \\
9.16 E+01 \\
6.25 E+01 \\
1.58 E+02 \\
1.77 E+02 \\
3.51 E+02\end{array}$ & $\begin{array}{l}114 \\
6.18 \mathrm{E}+00 \\
9.17 \mathrm{E}+00 \\
7.91 \mathrm{E}+00 \\
5.00 \mathrm{E}-02 \\
9.45 \mathrm{E}-01 \\
5.60 \mathrm{E}+00 \\
3.02 \mathrm{E}-01 \\
4.07 \mathrm{E}+00 \\
1.92 \mathrm{E}+00 \\
5.53 \mathrm{E}+00 \\
2.12 \mathrm{E}-01 \\
6.57 \mathrm{E}+00 \\
4.78 \mathrm{E}-02 \\
1.30 \mathrm{E}-01 \\
6.43 \mathrm{E}-01 \\
3.60 \mathrm{E}+00 \\
3.91 \mathrm{E}+00 \\
5.97 \mathrm{E}+00 \\
1.25 \mathrm{E}-01 \\
3.34 \mathrm{E}-01 \\
1.31 \mathrm{E}+00 \\
2.38 \mathrm{E}+00 \\
1.40 \mathrm{E}+01 \\
6.29 \mathrm{E}-01 \\
8.42 \mathrm{E}-01 \\
3.69 \mathrm{E}-01 \\
9.77 \mathrm{E}+00 \\
6.70 \mathrm{E}-01 \\
3.64 \mathrm{E}+00 \\
3.50 \mathrm{E}+00 \\
1.15 \mathrm{E}+00 \\
2.04 \mathrm{E}+00 \\
9.89 \mathrm{E}+00 \\
8.74 \mathrm{E}-01 \\
2.58 \mathrm{E}+00 \\
3.39 \mathrm{E}-01 \\
7.17 \mathrm{E}+00 \\
6.38 \mathrm{E}+00 \\
1.45 \mathrm{E}+00 \\
1.58 \mathrm{E}+00 \\
1.71 \mathrm{E}+00 \\
1.02 \mathrm{E}+00 \\
4.39 \mathrm{E}-01 \\
2.77 \mathrm{E}+00 \\
3.08 \mathrm{E}-01 \\
2.76 \mathrm{E}-01 \\
1.50 \mathrm{E}-01 \\
1.12 \mathrm{E}+00 \\
2.39 \mathrm{E}-01 \\
3.48 \mathrm{E}+00 \\
3.94 \mathrm{E}-01 \\
8.23 \mathrm{E}-01 \\
4.89 \mathrm{E}-01 \\
1.15 \mathrm{E}+00 \\
3.25 \mathrm{E}-01 \\
1.99 \mathrm{E}-01 \\
9.51 \mathrm{E}-01 \\
1.10 \mathrm{E}+00 \\
4.04 \mathrm{E}+00 \\
2.12 \mathrm{E}+00 \\
3.17 \mathrm{E}+00 \\
2.16 \mathrm{E}+00 \\
5.42 \mathrm{E}+00 \\
5.84 \mathrm{E}+00 \\
1.16 \mathrm{E}+01\end{array}$ & $\begin{array}{l}\text { NIS9 } \\
1.75 \mathrm{E}+00 \\
2.60 \mathrm{E}+00 \\
2.24 \mathrm{E}+00 \\
1.41 \mathrm{E}-02 \\
5.58 \mathrm{E}-02 \\
1.58 \mathrm{E}+00 \\
1.80 \mathrm{E}-02 \\
1.16 \mathrm{E}+00 \\
5.44 \mathrm{E}-01 \\
1.56 \mathrm{E}+00 \\
1.27 \mathrm{E}-02 \\
1.86 \mathrm{E}+00 \\
2.86 \mathrm{E}-03 \\
7.65 \mathrm{E}-03 \\
1.82 \mathrm{E}-01 \\
1.02 \mathrm{E}+00 \\
1.11 \mathrm{E}+00 \\
1.69 \mathrm{E}+00 \\
7.52 \mathrm{E}-03 \\
9.46 \mathrm{E}-02 \\
3.72 \mathrm{E}-01 \\
1.40 \mathrm{E}-01 \\
8.26 \mathrm{E}-01 \\
3.74 \mathrm{E}-02 \\
4.98 \mathrm{E}-02 \\
2.17 \mathrm{E}-02 \\
5.75 \mathrm{E}-01 \\
4.17 \mathrm{E}-02 \\
2.26 \mathrm{E}-01 \\
9.91 \mathrm{E}-01 \\
3.27 \mathrm{E}-01 \\
1.27 \mathrm{E}-01 \\
2.80 \mathrm{E}+00 \\
2.48 \mathrm{E}-01 \\
7.29 \mathrm{E}-01 \\
9.58 \mathrm{E}-02 \\
2.02 \mathrm{E}+00 \\
1.80 \mathrm{E}+00 \\
4.03 \mathrm{E}-01 \\
4.39 \mathrm{E}-01 \\
4.85 \mathrm{E}-01 \\
2.89 \mathrm{E}-01 \\
2.60 \mathrm{E}-02 \\
1.62 \mathrm{E}-01 \\
1.84 \mathrm{E}-02 \\
1.64 \mathrm{E}-02 \\
8.89 \mathrm{E}-03 \\
6.58 \mathrm{E}-02 \\
1.43 \mathrm{E}-02 \\
2.07 \mathrm{E}-01 \\
2.35 \mathrm{E}-02 \\
4.89 \mathrm{E}-02 \\
2.91 \mathrm{E}-02 \\
6.83 \mathrm{E}-02 \\
1.94 \mathrm{E}-02 \\
1.20 \mathrm{E}-02 \\
5.88 \mathrm{E}-02 \\
3.14 \mathrm{E}-01 \\
1.15 \mathrm{E}+00 \\
6.02 \mathrm{E}-01 \\
9.03 \mathrm{E}-01 \\
6.15 \mathrm{E}-01 \\
1.54 \mathrm{E}+00 \\
1.65 \mathrm{E}+00 \\
3.27 \mathrm{E}+00\end{array}$ & $\begin{array}{c}\text { NI } 63 \\
1.75 \mathrm{E}+02 \\
2.60 \mathrm{E}+02 \\
2.25 \mathrm{E}+02 \\
1.42 \mathrm{E}+00 \\
5.69 \mathrm{E}+00 \\
1.59 \mathrm{E}+02 \\
1.83 \mathrm{E}+00 \\
1.16 \mathrm{E}+02 \\
5.47 \mathrm{E}+01 \\
1.57 \mathrm{E}+02 \\
1.29 \mathrm{E}+00 \\
1.87 \mathrm{E}+02 \\
2.90 \mathrm{E}-01 \\
7.83 \mathrm{E}-01 \\
1.83 \mathrm{E}+01 \\
1.03 \mathrm{E}+02 \\
1.12 \mathrm{E}+02 \\
1.70 \mathrm{E}+02 \\
7.59 \mathrm{E}-01 \\
9.54 \mathrm{E}+00 \\
3.75 \mathrm{E}+01 \\
1.44 \mathrm{E}+01 \\
8.49 \mathrm{E}+01 \\
3.82 \mathrm{E}+00 \\
5.11 \mathrm{E}+00 \\
2.23 \mathrm{E}+00 \\
5.91 \mathrm{E}+01 \\
4.25 \mathrm{E}+00 \\
2.30 \mathrm{E}+01 \\
1.00 \mathrm{E}+02 \\
3.30 \mathrm{E}+01 \\
1.29 \mathrm{E}+01 \\
2.83 \mathrm{E}+02 \\
2.50 \mathrm{E}+01 \\
7.38 \mathrm{E}+01 \\
9.70 \mathrm{E}+00 \\
2.05 \mathrm{E}+02 \\
1.83 \mathrm{E}+02 \\
4.12 \mathrm{E}+01 \\
4.48 \mathrm{E}+01 \\
4.92 \mathrm{E}+01 \\
2.93 \mathrm{E}+01 \\
2.66 \mathrm{E}+00 \\
1.68 \mathrm{E}+01 \\
1.87 \mathrm{E}+00 \\
1.67 \mathrm{E}+00 \\
9.12 \mathrm{E}-01 \\
6.77 \mathrm{E}+00 \\
1.45 \mathrm{E}+00 \\
2.13 \mathrm{E}+01 \\
2.40 \mathrm{E}+00 \\
5.01 \mathrm{E}+00 \\
2.98 \mathrm{E}+00 \\
7.02 \mathrm{E}+00 \\
1.98 \mathrm{E}+00 \\
1.22 \mathrm{E}+00 \\
6.05 \mathrm{E}+00 \\
2.93 \mathrm{E}+01 \\
1.07 \mathrm{E}+02 \\
5.63 \mathrm{E}+01 \\
8.45 \mathrm{E}+01 \\
5.76 \mathrm{E}+01 \\
1.45 \mathrm{E}+02 \\
1.55 \mathrm{E}+02 \\
3.08 \mathrm{E}+02\end{array}$ & $\begin{array}{l}\text { CO60 } \\
8.06 \mathrm{E}+00 \\
1.21 \mathrm{E}+01 \\
1.06 \mathrm{E}+01 \\
6.72 \mathrm{E}-02 \\
1.29 \mathrm{E}+00 \\
7.60 \mathrm{E}+00 \\
4.22 \mathrm{E}-01 \\
5.67 \mathrm{E}+00 \\
2.68 \mathrm{E}+00 \\
7.63 \mathrm{E}+00 \\
3.02 \mathrm{E}-01 \\
9.24 \mathrm{E}+00 \\
6.93 \mathrm{E}-02 \\
1.85 \mathrm{E}-01 \\
9.32 \mathrm{E}-01 \\
5.21 \mathrm{E}+00 \\
5.61 \mathrm{E}+00 \\
8.50 \mathrm{E}+00 \\
1.70 \mathrm{E}-01 \\
4.88 \mathrm{E}-01 \\
1.89 \mathrm{E}+00 \\
3.52 \mathrm{E}+00 \\
2.08 \mathrm{E}+01 \\
9.56 \mathrm{E}-01 \\
1.26 \mathrm{E}+00 \\
5.47 \mathrm{E}-01 \\
1.45 \mathrm{E}+01 \\
1.16 \mathrm{E}+00 \\
6.24 \mathrm{E}+00 \\
5.33 \mathrm{E}+00 \\
1.75 \mathrm{E}+00 \\
3.60 \mathrm{E}+00 \\
1.54 \mathrm{E}+01 \\
1.35 \mathrm{E}+00 \\
4.04 \mathrm{E}+00 \\
5.29 \mathrm{E}-01 \\
1.12 \mathrm{E}+01 \\
1.00 \mathrm{E}+01 \\
2.10 \mathrm{E}+00 \\
2.28 \mathrm{E}+00 \\
2.75 \mathrm{E}+00 \\
1.61 \mathrm{E}+00 \\
6.53 \mathrm{E}-01 \\
4.39 \mathrm{E}+00 \\
4.57 \mathrm{E}-01 \\
4.03 \mathrm{E}-01 \\
2.41 \mathrm{E}-01 \\
1.72 \mathrm{E}+00 \\
3.52 \mathrm{E}-01 \\
5.74 \mathrm{E}+00 \\
5.97 \mathrm{E}-01 \\
1.28 \mathrm{E}+00 \\
7.82 \mathrm{E}-01 \\
1.86 \mathrm{E}+00 \\
5.00 \mathrm{E}-01 \\
3.04 \mathrm{E}-01 \\
1.80 \mathrm{E}+00 \\
4.40 \mathrm{E}-01 \\
1.61 \mathrm{E}+00 \\
8.59 \mathrm{E}-01 \\
1.33 \mathrm{E}+00 \\
9.09 \mathrm{E}-01 \\
2.33 \mathrm{E}+00 \\
2.46 \mathrm{E}+00 \\
4.95 \mathrm{E}+00\end{array}$ & $\begin{array}{c}\text { SE79 } \\
1.66 \mathrm{E}+00 \\
2.46 \mathrm{E}+00 \\
2.13 \mathrm{E}+00 \\
1.07 \mathrm{E}-02 \\
1.13 \mathrm{E}-01 \\
1.51 \mathrm{E}+00 \\
3.71 \mathrm{E}-02 \\
1.09 \mathrm{E}+00 \\
5.16 \mathrm{E}-01 \\
1.48 \mathrm{E}+00 \\
2.62 \mathrm{E}-02 \\
1.77 \mathrm{E}+00 \\
5.94 \mathrm{E}-03 \\
1.55 \mathrm{E}-02 \\
1.36 \mathrm{E}-01 \\
7.66 \mathrm{E}-01 \\
8.30 \mathrm{E}-01 \\
1.27 \mathrm{E}+00 \\
1.57 \mathrm{E}-02 \\
8.97 \mathrm{E}-02 \\
3.53 \mathrm{E}-01 \\
2.84 \mathrm{E}-01 \\
1.67 \mathrm{E}+00 \\
7.71 \mathrm{E}-02 \\
1.02 \mathrm{E}-01 \\
4.40 \mathrm{E}-02 \\
1.17 \mathrm{E}+00 \\
9.31 \mathrm{E}-02 \\
5.06 \mathrm{E}-01 \\
9.40 \mathrm{E}-01 \\
3.10 \mathrm{E}-01 \\
2.83 \mathrm{E}-01 \\
2.66 \mathrm{E}+00 \\
2.35 \mathrm{E}-01 \\
6.92 \mathrm{E}-01 \\
9.10 \mathrm{E}-02 \\
1.53 \mathrm{E}+00 \\
1.36 \mathrm{E}+00 \\
3.18 \mathrm{E}-01 \\
3.45 \mathrm{E}-01 \\
4.60 \mathrm{E}-01 \\
2.75 \mathrm{E}-01 \\
5.31 \mathrm{E}-02 \\
3.28 \mathrm{E}-01 \\
3.79 \mathrm{E}-02 \\
3.39 \mathrm{E}-02 \\
1.83 \mathrm{E}-02 \\
1.34 \mathrm{E}-01 \\
2.97 \mathrm{E}-02 \\
4.27 \mathrm{E}-01 \\
4.86 \mathrm{E}-02 \\
1.00 \mathrm{E}-01 \\
5.99 \mathrm{E}-02 \\
1.40 \mathrm{E}-01 \\
4.04 \mathrm{E}-02 \\
2.49 \mathrm{E}-02 \\
1.30 \mathrm{E}-01 \\
2.32 \mathrm{E}-01 \\
8.50 \mathrm{E}-01 \\
4.45 \mathrm{E}-01 \\
6.67 \mathrm{E}-01 \\
4.55 \mathrm{E}-01 \\
1.14 \mathrm{E}+00 \\
1.24 \mathrm{E}+00 \\
2.46 \mathrm{E}+00\end{array}$ & 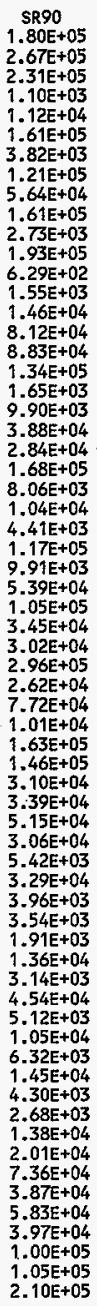 & $\begin{array}{l}Y 90 \\
1.80 E+05 \\
2.67 E+05 \\
2.31 E+05 \\
1.10 E+03 \\
1.12 E+04 \\
1.61 E+05 \\
3.83 E+03 \\
1.21 E+05 \\
5.65 E+04 \\
1.61 E+05 \\
2.73 E+03 \\
1.93 E+05 \\
6.29 E+02 \\
1.55 E+03 \\
1.46 E+04 \\
8.12 E+04 \\
8.83 E+04 \\
1.34 E+05 \\
1.65 E+03 \\
9.90 E+03 \\
3.88 E+04 \\
2.84 E+04 \\
1.68 E+05 \\
8.06 E+03 \\
1.04 E+04 \\
4.41 E+03 \\
1.17 E+05 \\
9.91 E+03 \\
5.39 E+04 \\
1.05 E+05 \\
3.45 E+04 \\
3.02 E+04 \\
2.96 E+05 \\
2.62 E+04 \\
7.72 E+04 \\
1.01 E+04 \\
1.64 E+05 \\
1.46 E+05 \\
3.11 E+04 \\
3.39 E+04 \\
5.15 E+04 \\
3.07 E+04 \\
5.42 E+03 \\
3.29 E+04 \\
3.96 E+03 \\
3.54 E+03 \\
1.92 E+03 \\
1.36 E+04 \\
3.14 E+03 \\
4.54 E+04 \\
5.13 E+03 \\
1.05 E+04 \\
6.32 E+03 \\
1.45 E+04 \\
4.30 E+03 \\
2.68 E+03 \\
1.38 E+04 \\
2.01 E+04 \\
7.36 E+04 \\
3.87 E+04 \\
5.83 E+04 \\
3.98 E+04 \\
1.00 E+05 \\
1.05 E+05 \\
2.10 E+05\end{array}$ & $\begin{array}{l}2 R 93 \\
8.00 \mathrm{E}+00 \\
1.19 \mathrm{E}+01 \\
1.02 \mathrm{E}+01 \\
4.97 \mathrm{E}-02 \\
5.35 \mathrm{E}-01 \\
7.18 \mathrm{E}+00 \\
1.78 \mathrm{E}-01 \\
5.30 \mathrm{E}+00 \\
2.48 \mathrm{E}+00 \\
7.10 \mathrm{E}+00 \\
1.27 \mathrm{E}-01 \\
8.48 \mathrm{E}+00 \\
2.89 \mathrm{E}-02 \\
7.34 \mathrm{E}-02 \\
6.41 \mathrm{E}-01 \\
3.59 \mathrm{E}+00 \\
3.90 \mathrm{E}+00 \\
5.95 \mathrm{E}+00 \\
7.64 \mathrm{E}-02 \\
4.31 \mathrm{E}-01 \\
1.70 \mathrm{E}+00 \\
1.34 \mathrm{E}+00 \\
7.88 \mathrm{E}+00 \\
3.70 \mathrm{E}-01 \\
4.83 \mathrm{E}-01 \\
2.07 \mathrm{E}-01 \\
5.49 \mathrm{E}+00 \\
4.55 \mathrm{E}-01 \\
2.47 \mathrm{E}+00 \\
4.52 \mathrm{E}+00 \\
1.49 \mathrm{E}+00 \\
1.38 \mathrm{E}+00 \\
1.28 \mathrm{E}+01 \\
1.13 \mathrm{E}+00 \\
3.32 \mathrm{E}+00 \\
4.37 \mathrm{E}-01 \\
7.14 \mathrm{E}+00 \\
6.36 \mathrm{E}+00 \\
1.44 \mathrm{E}+00 \\
1.56 \mathrm{E}+00 \\
2.21 \mathrm{E}+00 \\
1.32 \mathrm{E}+00 \\
2.52 \mathrm{E}-01 \\
1.54 \mathrm{E}+00 \\
1.83 \mathrm{E}-01 \\
1.64 \mathrm{E}-01 \\
8.74 \mathrm{E}-02 \\
6.32 \mathrm{E}-01 \\
1.44 \mathrm{E}-01 \\
2.05 \mathrm{E}+00 \\
2.35 \mathrm{E}-01 \\
4.81 \mathrm{E}-01 \\
2.88 \mathrm{E}-01 \\
6.65 \mathrm{E}-01 \\
1.96 \mathrm{E}-01 \\
1.22 \mathrm{E}-01 \\
6.28 \mathrm{E}-01 \\
1.11 \mathrm{E}+00 \\
4.05 \mathrm{E}+00 \\
2.12 \mathrm{E}+00 \\
3.18 \mathrm{E}+00 \\
2.17 \mathrm{E}+00 \\
5.43 \mathrm{E}+00 \\
1.15 \mathrm{E}+00\end{array}$ \\
\hline
\end{tabular}


ACTIVITY IN UNSEPARATED FUEL (Curies decayed to $1 / 1 / 94$ )

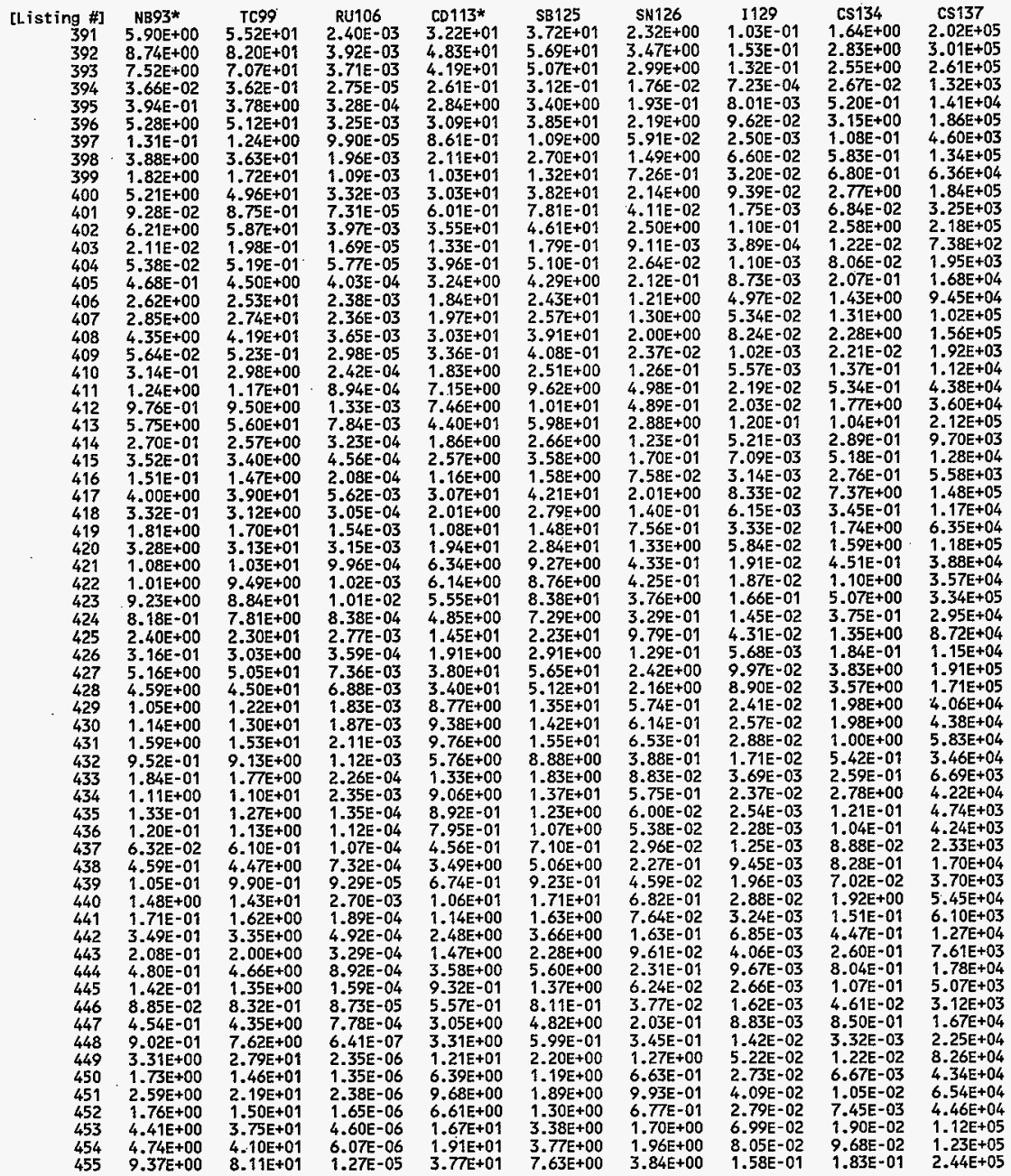


HNF-SD-HM-TI-794, ReV. O

ACIIVITY IN UNSEPARATED FUEL (CUries decayed to $1 / 1 / 94$ )

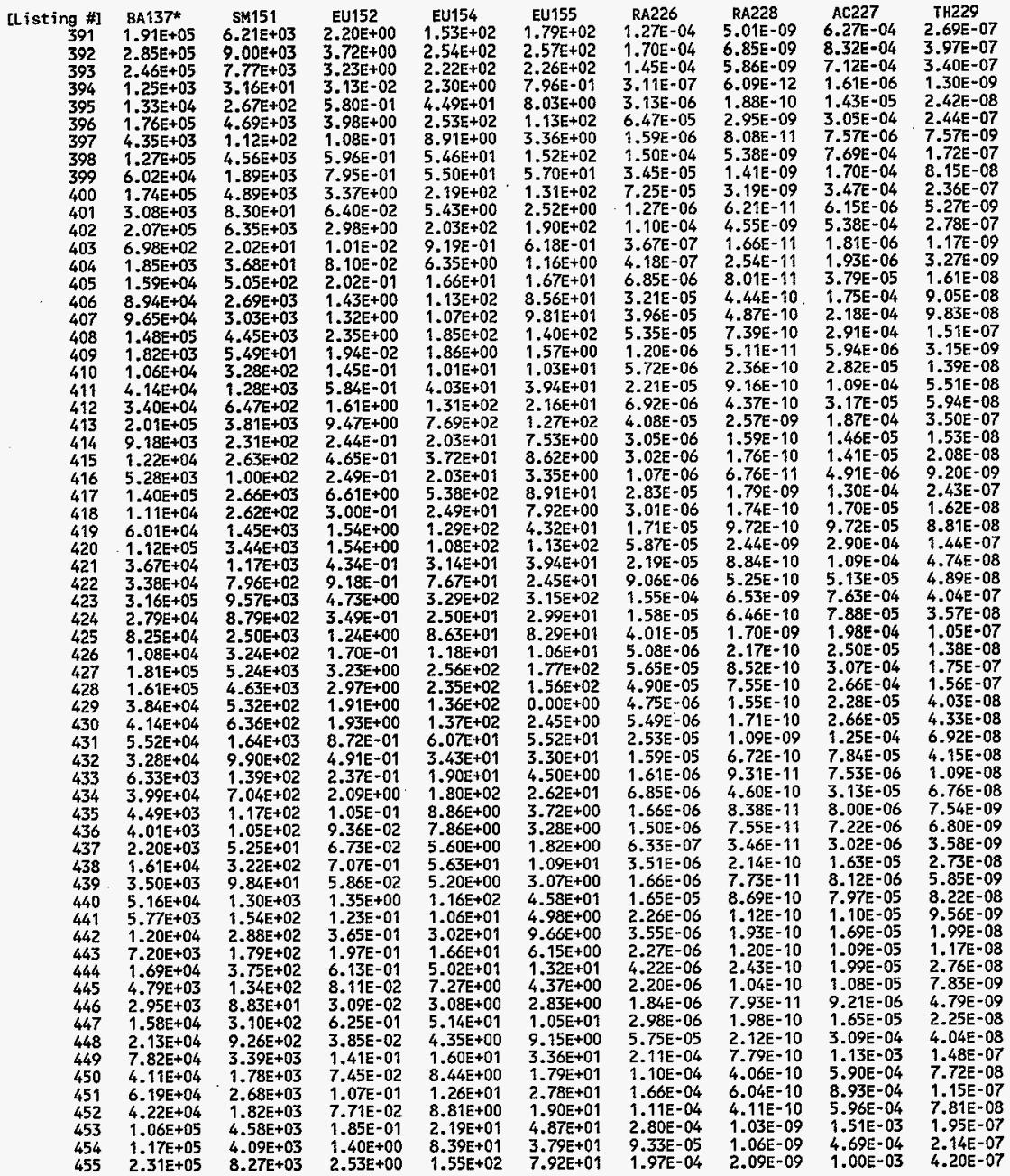


HNF-SD-WH-TI-794, REV. 0

ACTIVITY IN UNSEPARATED FUEL (Curjes decayed to 1/1/94)

\begin{tabular}{|c|c|c|c|c|c|c|c|c|c|}
\hline 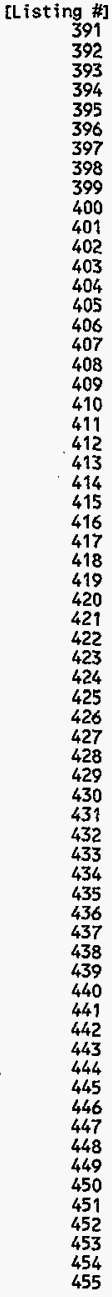 & $\begin{array}{l}\text { TH232 } \\
7.28 \mathrm{E}-09 \\
9.96 \mathrm{E}-09 \\
8.55 \mathrm{E}-09 \\
8.89 \mathrm{E}-12 \\
2.74 \mathrm{E}-10 \\
4.31 \mathrm{E}-09 \\
1.18 \mathrm{E}-10 \\
7.88 \mathrm{E}-09 \\
2.06 \mathrm{E}-09 \\
4.66 \mathrm{E}-09 \\
9.08 \mathrm{E}-11 \\
6.66 \mathrm{E}-09 \\
2.44 \mathrm{E}-11 \\
3.71 \mathrm{E}-11 \\
1.18 \mathrm{E}-10 \\
6.53 \mathrm{E}-10 \\
7.16 \mathrm{E}-10 \\
1.09 \mathrm{E}-09 \\
7.43 \mathrm{E}-11 \\
3.47 \mathrm{E}-10 \\
1.34 \mathrm{E}-09 \\
6.41 \mathrm{E}-10 \\
3.77 \mathrm{E}-09 \\
2.35 \mathrm{E}-10 \\
2.58 \mathrm{E}-10 \\
9.92 \mathrm{E}-11 \\
2.62 \mathrm{E}-09 \\
2.55 \mathrm{E}-10 \\
1.42 \mathrm{E}-09 \\
3.60 \mathrm{E}-09 \\
1.31 \mathrm{E}-09 \\
7.72 \mathrm{E}-10 \\
9.67 \mathrm{E}-09 \\
9.55 \mathrm{E}-10 \\
2.51 \mathrm{E}-09 \\
3.21 \mathrm{E}-10 \\
1.26 \mathrm{E}-09 \\
1.12 \mathrm{E}-09 \\
2.27 \mathrm{E}-10 \\
2.51 \mathrm{E}-10 \\
1.61 \mathrm{E}-09 \\
9.96 \mathrm{E}-10 \\
1.37 \mathrm{E}-10 \\
6.79 \mathrm{E}-10 \\
1.23 \mathrm{E}-10 \\
1.11 \mathrm{E}-10 \\
5.13 \mathrm{E}-11 \\
3.16 \mathrm{E}-10 \\
1.13 \mathrm{E}-10 \\
1.29 \mathrm{E}-09 \\
1.65 \mathrm{E}-10 \\
2.85 \mathrm{E}-10 \\
1.78 \mathrm{E}-10 \\
3.60 \mathrm{E}-10 \\
1.52 \mathrm{E}-10 \\
1.17 \mathrm{E}-10 \\
2.94 \mathrm{E}-10 \\
2.82 \mathrm{E}-10 \\
1.03 \mathrm{~B}-09 \\
8.40 \mathrm{E}-10 \\
5.47 \mathrm{E}-10-10 \\
1.37 \mathrm{E}-09 \\
1.41 \mathrm{E}-09 \\
2.79 \mathrm{E}-09\end{array}$ & $\begin{array}{l}\text { PA231 } \\
1.78 E-03 \\
2.36 E-03 \\
2.03 E-03 \\
4.57 E-06 \\
4.01 E-05 \\
8.68 E-04 \\
2.15 E-05 \\
2.21 E-03 \\
4.88 E-04 \\
9.92 E-04 \\
1.76 E-05 \\
1.55 E-03 \\
5.21 E-06 \\
5.44 E-06 \\
1.10 E-04 \\
5.05 E-04 \\
6.30 E-04 \\
8.40 E-04 \\
1.69 E-05 \\
8.18 E-05 \\
3.15 E-04 \\
8.98 E-05 \\
5.29 E-04 \\
4.22 E-05 \\
4.03 E-05 \\
1.39 E-05 \\
3.68 E-04 \\
4.88 E-05 \\
2.78 E-04 \\
8.48 E-04 \\
3.19 E-04 \\
1.48 E-04 \\
2.24 E-03 \\
2.31 E-04 \\
5.81 E-04 \\
7.32 E-05 \\
9.01 E-04 \\
7.80 E-04 \\
6.46 E-05 \\
7.55 E-05 \\
3.68 E-04 \\
2.30 E-04 \\
2.15 E-05 \\
8.93 E-05 \\
2.30 E-05 \\
2.07 E-05 \\
8.79 E-06 \\
4.67 E-05 \\
2.34 E-05 \\
2.34 E-04 \\
3.17 E-05 \\
4.88 E-05 \\
3.19 E-05 \\
5.778 E-05 \\
3.15 E-05 \\
2.68 E-05 \\
4.79 E-05 \\
7.29 E-04 \\
2.67 E-03 \\
1.39 E-03 \\
2.12 E-03 \\
1.42 E-03 \\
3.61 E-03 \\
1.11 E-03 \\
2.38 E-03\end{array}$ & $\begin{array}{l}\text { U232 } \\
4.38 E-03 \\
6.87 E-03 \\
5.93 E-03 \\
3.04 E-05 \\
9.74 E-04 \\
5.53 E-03 \\
2.14 E-04 \\
2.20 E-03 \\
1.44 E-03 \\
5.01 E-03 \\
1.35 E-04 \\
5.13 E-03 \\
2.49 E-05 \\
1.34 E-04 \\
2.82 E-04 \\
1.74 E-03 \\
1.77 E-03 \\
2.87 E-03 \\
5.70 E-05 \\
2.55 E-04 \\
1.02 E-03 \\
2.66 E-03 \\
1.57 E-02 \\
4.61 E-04 \\
7.87 E-04 \\
4.12 E-04 \\
1.09 E-02 \\
4.86 E-04 \\
2.54 E-03 \\
2.68 E-03 \\
8.26 E-04 \\
1.48 E-03 \\
7.84 E-03 \\
6.40 E-04 \\
2.04 E-03 \\
2.75 E-04 \\
3.63 E-03 \\
3.28 E-03 \\
1.39 E-03 \\
1.43 E-03 \\
1.39 E-03 \\
8.11 E-04 \\
4.04 E-04 \\
3.47 E-03 \\
2.09 E-04 \\
1.87 E-04 \\
1.20 E-04 \\
1.14 E-03 \\
1.34 E-04 \\
2.52 E-03 \\
2.53 E-04 \\
6.57 E-04 \\
3.64 E-04 \\
1.03 E-03 \\
1.84 E-04 \\
8.97 E-05 \\
8.95 E-04 \\
3.06 E-04 \\
1.12 E-03 \\
5.89 E-03 \\
8.80 E-04 \\
6.03 E-04 \\
1.51 E-04 \\
2.55 E-03 \\
4.85 E-03\end{array}$ & $\begin{array}{l}\text { U233 } \\
1.33 E-04 \\
1.98 E-04 \\
1.70 E-04 \\
6.19 E-07 \\
1.24 E-05 \\
1.23 E-04 \\
3.87 E-06 \\
8.66 E-05 \\
4.10 E-05 \\
1.19 E-04 \\
2.70 E-06 \\
1.40 E-04 \\
6.03 E-07 \\
1.68 E-06 \\
7.68 E-06 \\
4.33 E-05 \\
4.69 E-05 \\
7.18 E-05 \\
1.59 E-06 \\
7.09 E-06 \\
2.79 E-05 \\
3.08 E-05 \\
1.82 E-04 \\
7.97 E-06 \\
1.08 E-05 \\
4.77 E-06 \\
1.26 E-04 \\
8.39 E-06 \\
4.55 E-05 \\
7.38 E-05 \\
2.43 E-05 \\
2.54 E-05 \\
2.08 E-04 \\
1.84 E-05 \\
5.41 E-05 \\
7.12 E-06 \\
8.55 E-05 \\
7.61 E-05 \\
2.07 E-05 \\
2.21 E-05 \\
3.59 E-05 \\
2.15 E-05 \\
5.64 E-06 \\
3.55 E-05 \\
3.90 E-06 \\
3.51 E-06 \\
1.88 E-06 \\
1.43 E-05 \\
3.02 E-06 \\
4.35 E-05 \\
4.97 F-06 \\
1.04 E-05 \\
6.15 E-06 \\
1.46 E-05 \\
4.08 E-06 \\
2.49 E-06 \\
1.19 E-05 \\
1.49 E-05 \\
5.48 E-05 \\
2.86 E-05 \\
4.28 E-05 \\
2.92 E-05 \\
7.29 E-05 \\
8.14 E-05 \\
1.60 E-04\end{array}$ & $\begin{array}{l}\text { U234 } \\
7.49 E+01 \\
1.01 E+02 \\
8.68 E+01 \\
1.86 E-01 \\
1.84 E+00 \\
3.88 E+01 \\
9.53 E-01 \\
9.18 E+01 \\
2.11 E+01 \\
4.39 E+01 \\
7.73 E-01 \\
6.73 E+01 \\
2.26 E-01 \\
2.53 E-01 \\
4.27 E+00 \\
2.00 E+01 \\
2.46 E+01 \\
3.30 E+01 \\
7.10 E-01 \\
3.58 E+00 \\
1.37 E+01 \\
4.25 E+00 \\
2.51 E+01 \\
1.91 E+00 \\
1.88 E+00 \\
6.59 E-01 \\
1.75 E+01 \\
1.85 E+00 \\
1.05 E+01 \\
3.75 E+01 \\
1.40 E+01 \\
5.64 E+00 \\
1.00 E+02 \\
1.02 E+01 \\
2.60 E+01 \\
3.29 E+00 \\
3.66 E+01 \\
3.19 E+01 \\
2.94 E+00 \\
3.40 E+00 \\
1.66 E+01 \\
1.03 E+01 \\
9.97 E-01 \\
4.36 E+00 \\
1.03 E+00 \\
9.22 E-01 \\
4.09 E-01 \\
2.21 E+00 \\
1.02 E+00 \\
1.08 E+01 \\
1.42 E+00 \\
2.25 E+00 \\
1.47 E+00 \\
2.72 E+00 \\
1.39 E+00 \\
1.16 E+00 \\
1.92 E+00 \\
2.00 E+01 \\
7.34 E+01 \\
3.84 E+01 \\
5.88 E+01 \\
3.93 E+01 \\
1.00 E+02 \\
3.31 E+01 \\
7.05 E+01\end{array}$ & $\begin{array}{l}\text { U235 } \\
2.84 E+00 \\
3.79 E+00 \\
3.26 E+00 \\
7.30 E-03 \\
6.27 E-02 \\
1.39 E+00 \\
3.45 E-02 \\
3.61 E+00 \\
7.92 E-01 \\
1.60 E+00 \\
2.84 E-02 \\
2.52 E+00 \\
8.48 E-03 \\
8.61 E-03 \\
1.80 E-01 \\
8.27 E-01 \\
1.03 E+00 \\
1.37 E+00 \\
2.71 E-02 \\
1.34 E-01 \\
5.15 E-01 \\
1.43 E-01 \\
8.42 E-01 \\
6.89 E-02 \\
6.51 E E-02 \\
2.21 E-02 \\
5.86 E-01 \\
7.92 E-02 \\
4.51 E-01 \\
1.41 E+00 \\
5.31 E-01 \\
2.41 E-01 \\
3.73 E+00 \\
3.86 E-01 \\
9.73 E-01 \\
1.22 E-01 \\
1.51 E+00 \\
1.31 E+00 \\
1.03 E-01 \\
1.20 E-01 \\
6.19 E-01 \\
3.86 E-01 \\
3.47 E-02 \\
1.43 E-01 \\
3.75 E-02 \\
3.36 E-02 \\
1.46 E-02 \\
7.54 E-02 \\
3.81 E-02 \\
3.91 E-01 \\
5.20 E-02 \\
8.01 E-02 \\
5.28 E-02 \\
9.52 E-02 \\
5.19 E-02 \\
4.42 E-02 \\
7.88 E-02 \\
8.96 E-01 \\
3.28 E+00 \\
1.72 E+00 \\
2.63 E+00 \\
1.76 E+00 \\
4.49 E+00 \\
1.38 E+00 \\
2.95 E+00\end{array}$ & $\begin{array}{l}\text { U236 } \\
5.00 \mathrm{E}+00 \\
6.86 \mathrm{E}+00 \\
5.92 \mathrm{E}+00 \\
6.17 \mathrm{E}-03 \\
1.88 \mathrm{E}-01 \\
2.99 \mathrm{E}+00 \\
8.18 \mathrm{E}-02 \\
5.51 \mathrm{E}+00 \\
1.44 \mathrm{E}+00 \\
3.25 \mathrm{E}+00 \\
6.33 \mathrm{E}-02 \\
4.66 \mathrm{E}+00 \\
1.71 \mathrm{E}-02 \\
2.58 \mathrm{E}-02 \\
8.35 \mathrm{E}-02 \\
4.62 \mathrm{E}-01 \\
5.06 \mathrm{E}-01 \\
7.65 \mathrm{E}-01 \\
5.12 \mathrm{E}-02 \\
2.45 \mathrm{E}-01 \\
9.47 \mathrm{E}-01 \\
4.50 \mathrm{E}-01 \\
2.66 \mathrm{E}+00 \\
1.66 \mathrm{E}-01 \\
1.82 \mathrm{E}-01 \\
6.98 \mathrm{E}-02 \\
1.85 \mathrm{E}+00 \\
1.79 \mathrm{E}-01 \\
9.99 \mathrm{E}-01 \\
2.57 \mathrm{E}+00 \\
9.33 \mathrm{E}-01 \\
5.45 \mathrm{E}-01 \\
6.95 \mathrm{E}+00 \\
6.86 \mathrm{E}-01 \\
1.81 \mathrm{E}+00 \\
2.31 \mathrm{E}-01 \\
9.14 \mathrm{E}-01 \\
8.12 \mathrm{E}-01 \\
1.61 \mathrm{E}-01 \\
1.78 \mathrm{E}-01 \\
1.17 \mathrm{E}+00 \\
7.18 \mathrm{E}-01 \\
9.63 \mathrm{E}-02 \\
4.87 \mathrm{E}-01 \\
8.67 \mathrm{E}-02 \\
7.77 \mathrm{E}-02 \\
3.69 \mathrm{E}-02 \\
2.24 \mathrm{E}-01 \\
7.97 \mathrm{E}-02 \\
9.34 \mathrm{E}-01 \\
1.17 \mathrm{E}-01 \\
2.03 \mathrm{E}-01 \\
1.28 \mathrm{E}-01 \\
2.59 \mathrm{E}-01 \\
1.08 \mathrm{E}-01 \\
8.29 \mathrm{E}-02 \\
2.11 \mathrm{E}-01 \\
1.49 \mathrm{E}-01 \\
5.45 \mathrm{E}-01 \\
2.85 \mathrm{E}-01 \\
4.28 \mathrm{E}-01 \\
2.92 \mathrm{E}-01 \\
7.31 \mathrm{E}-01 \\
7.51 \mathrm{E}-01 \\
1.49 \mathrm{E}+00\end{array}$ & $\begin{array}{l}\text { U238 } \\
5.07 \mathrm{E}+01 \\
6.83 \mathrm{E}+01 \\
5.89 \mathrm{E}+01 \\
1.94 \mathrm{E}-01 \\
1.30 \mathrm{E}+00 \\
2.66 \mathrm{E}+01 \\
6.57 \mathrm{E}-01 \\
6.17 \mathrm{E}+01 \\
1.43 \mathrm{E}+01 \\
3.00 \mathrm{E}+01 \\
5.31 \mathrm{E}-01 \\
4.57 \mathrm{E}+01 \\
1.54 \mathrm{E}-01 \\
1.78 \mathrm{E}-01 \\
4.39 \mathrm{E}+00 \\
2.06 \mathrm{E}+01 \\
2.52 \mathrm{E}+01 \\
3.41 \mathrm{E}+01 \\
4.83 \mathrm{E}-01 \\
2.43 \mathrm{E}+00 \\
9.33 \mathrm{E}+00 \\
3.00 \mathrm{E}+00 \\
1.77 \mathrm{E}+01 \\
1.32 \mathrm{E}+00 \\
1.31 \mathrm{E}+00 \\
4.65 \mathrm{E}-01 \\
1.23 \mathrm{E}+01 \\
1.28 \mathrm{E}+00 \\
7.23 \mathrm{E}+00 \\
2.55 \mathrm{E}+01 \\
9.48 \mathrm{E}+00 \\
3.89 \mathrm{E}+00 \\
6.79 \mathrm{E}+01 \\
6.91 \mathrm{E}+00 \\
1.77 \mathrm{E}+01 \\
2.24 \mathrm{E}+00 \\
3.78 \mathrm{E}+01 \\
3.29 \mathrm{E}+01 \\
3.14 \mathrm{E}+00 \\
3.62 \mathrm{E}+00 \\
1.13 \mathrm{E}+01 \\
7.02 \mathrm{E}+00 \\
6.96 \mathrm{E}-01 \\
3.10 \mathrm{E}+00 \\
7.09 \mathrm{E}-01 \\
6.35 \mathrm{E}-01 \\
2.83 \mathrm{E}-01 \\
1.55 \mathrm{E}+00 \\
7.00 \mathrm{E}-01 \\
7.47 \mathrm{E}+00 \\
9.74 \mathrm{E}-01 \\
1.56 \mathrm{E}+00 \\
1.01 \mathrm{E}+00 \\
1.90 \mathrm{E}+00 \\
9.52 \mathrm{E}-01 \\
7.87 \mathrm{E}-01 \\
1.34 \mathrm{E}+00 \\
2.03 \mathrm{E}+01 \\
7.44 \mathrm{E}+01 \\
3.90 \mathrm{E}+01 \\
5.96 \mathrm{E}+01 \\
3.98 \mathrm{E}+01 \\
1.02 \mathrm{E}+02 \\
3.41 \mathrm{E}+01 \\
7.25 \mathrm{E}+01\end{array}$ & $\begin{array}{l}\text { NP237 } \\
5.91 \mathrm{E}-01 \\
8.83 \mathrm{E}-01 \\
7.61 \mathrm{E}-01 \\
2.56 \mathrm{E}-03 \\
6.36 \mathrm{E}-02 \\
5.78 \mathrm{E}-01 \\
1.89 \mathrm{E}-02 \\
3.79 \mathrm{E}-01 \\
1.85 \mathrm{E}-01 \\
5.45 \mathrm{E}-01 \\
1.31 \mathrm{E}-02 \\
6.35 \mathrm{E}-01 \\
2.85 \mathrm{E}-03 \\
8.72 \mathrm{E}-03 \\
2.93 \mathrm{E}-02 \\
1.69 \mathrm{E}-01 \\
1.80 \mathrm{E}-01 \\
2.80 \mathrm{E}-01 \\
7.38 \mathrm{E}-03 \\
3.22 \mathrm{E}-02 \\
1.27 \mathrm{E}-01 \\
1.62 \mathrm{E}-01 \\
9.56 \mathrm{E}-01 \\
3.95 \mathrm{E}-02 \\
5.53 \mathrm{E}-02 \\
2.51 \mathrm{E}-02 \\
6.65 \mathrm{E}-01 \\
4.16 \mathrm{E}-02 \\
2.24 \mathrm{E}-01 \\
3.37 \mathrm{E}-01 \\
1.10 \mathrm{E}-01 \\
1.27 \mathrm{E}-01 \\
9.57 \mathrm{E}-01 \\
8.38 \mathrm{E}-02 \\
2.49 \mathrm{E}-01 \\
3.29 \mathrm{E}-02 \\
3.40 \mathrm{E}-01 \\
3.04 \mathrm{E}-01 \\
1.08 \mathrm{E}-01 \\
1.12 \mathrm{E}-01 \\
1.66 \mathrm{E}-01 \\
9.89 \mathrm{E}-02 \\
2.88 \mathrm{E}-02 \\
1.92 \mathrm{E}-01 \\
1.91 \mathrm{E}-02 \\
1.71 \mathrm{E}-02 \\
9.53 \mathrm{E}-03 \\
7.47 \mathrm{E}-02 \\
1.44 \mathrm{E}-02 \\
2.18 \mathrm{E}-01 \\
2.42 \mathrm{E}-02 \\
5.24 \mathrm{E}-02 \\
3.08 \mathrm{E}-02 \\
7.50 \mathrm{E}-02 \\
1.96 \mathrm{E}-02 \\
1.17 \mathrm{E}-02 \\
6.25 \mathrm{E}-02 \\
4.60 \mathrm{E}-02 \\
1.68 \mathrm{E}-01 \\
8.82 \mathrm{E}-02 \\
1.32 \mathrm{E}-01 \\
9.01 \mathrm{E}-02 \\
2.26 \mathrm{E}-01 \\
2.76 \mathrm{E}-01 \\
5.40 \mathrm{E}-01\end{array}$ \\
\hline
\end{tabular}


HNF-SD-WM-TI-794, ReV. 0

ACTIVITY IN UNSEPARATED FUEL (Curies decayed to 1/1/94)

\begin{tabular}{|c|c|c|c|c|c|c|c|c|c|}
\hline $\begin{array}{r}\text { [Listing } 71 \\
391 \\
392 \\
393 \\
394 \\
395 \\
396 \\
397 \\
398 \\
399 \\
400 \\
401 \\
402 \\
403 \\
404 \\
405 \\
406 \\
407 \\
408 \\
409 \\
410 \\
411 \\
412 \\
413 \\
414 \\
415 \\
416 \\
417 \\
418 \\
419 \\
420 \\
421 \\
422 \\
423 \\
424 \\
425 \\
426 \\
427 \\
428 \\
429 \\
430 \\
431 \\
432 \\
433 \\
434 \\
435 \\
436 \\
437 \\
438 \\
439 \\
440 \\
441 \\
442 \\
443 \\
444 \\
445 \\
446 \\
447 \\
448 \\
449 \\
450 \\
451 \\
452 \\
453 \\
454 \\
455\end{array}$ & $\begin{array}{c}\text { PU238 } \\
1.71 E+02 \\
2.85 E+02 \\
2.46 E+02 \\
1.50 E+00 \\
7.24 E+01 \\
2.89 E+02 \\
1.41 E+01 \\
5.31 E+01 \\
5.99 E+01 \\
2.46 E+02 \\
8.50 E+00 \\
2.22 E+02 \\
1.41 E+00 \\
9.96 E+00 \\
9.90 E+00 \\
6.87 E+01 \\
6.43 E+01 \\
1.14 E+02 \\
2.98 E+00 \\
1.07 E+01 \\
4.33 E+01 \\
2.02 E+02 \\
1.19 E+03 \\
3.08 E+01 \\
5.69 E+01 \\
3.13 E+01 \\
8.29 E+02 \\
3.37 E+01 \\
1.74 E+02 \\
1.13 E+02 \\
3.22 E+01 \\
1.03 E+02 \\
3.40 E+02 \\
2.56 E+01 \\
8.86 E+01 \\
1.22 E+01 \\
1.51 E+02 \\
1.38 E+02 \\
8.64 E+01 \\
8.75 E+01 \\
6.18 E+01 \\
3.52 E+01 \\
2.91 E+01 \\
2.70 E+02 \\
1.36 E+01 \\
1.22 E+01 \\
8.23 E+00 \\
8.48 E+01 \\
7.96 E+00 \\
1.67 E+02 \\
1.60 E+01 \\
4.52 E+01 \\
2.44 E+01 \\
7.37 E+01 \\
1.09 E+01 \\
4.62 E+00 \\
6.71 E+01 \\
4.02 E+00 \\
1.47 E+01 \\
7.73 E+00 \\
1.12 E+01 \\
7.91 E+00 \\
1.92 E+01 \\
1.01 E+02 \\
1.83 E+02\end{array}$ & $\begin{array}{l}\text { PU239 } \\
5.24 E+03 \\
7.69 E+03 \\
6.63 E+03 \\
3.78 E+01 \\
3.70 E+02 \\
4.44 E+03 \\
1.35 E+02 \\
3.63 E+03 \\
1.61 E+03 \\
4.42 E+03 \\
9.75 E+01 \\
5.46 E+03 \\
2.28 E+01 \\
5.08 E+01 \\
5.35 E+02 \\
2.92 E+03 \\
3.23 E+03 \\
4.83 E+03 \\
6.14 E+01 \\
2.79 E+02 \\
1.09 E+03 \\
9.10 E+02 \\
5.36 E+03 \\
2.79 E+02 \\
3.45 E+02 \\
1.41 E+02 \\
3.74 E+03 \\
2.79 E+02 \\
1.53 E+03 \\
2.92 E+03 \\
9.78 E+02 \\
8.48 E+02 \\
8.20 E+03 \\
7.38 E+02 \\
2.14 E+03 \\
2.79 E+02 \\
5.72 E+03 \\
5.07 E+03 \\
9.73 E+02 \\
1.08 E+03 \\
1.41 E+03 \\
8.47 E+02 \\
1.81 E+02 \\
1.02 E+03 \\
1.39 E+02 \\
1.25 E+02 \\
6.47 E+01 \\
4.40 E+02 \\
1.13 E+02 \\
1.55 E+03 \\
1.81 E+02 \\
3.56 E+02 \\
2.16 E+02 \\
4.80 E+02 \\
1.54 E+02 \\
9.79 E+01 \\
3.63 E+02 \\
9.97 E+02 \\
3.65 E+03 \\
1.91 E+03 \\
2.87 E+03 \\
1.96 E+03 \\
4.90 E+03 \\
4.75 E+03 \\
9.51 E+03\end{array}$ & $\begin{array}{l}\text { PU240 } \\
7.40 E+02 \\
1.21 E+03 \\
1.04 E+03 \\
1.22 E+01 \\
1.63 E+02 \\
1.14 E+03 \\
4.11 E+01 \\
2.73 E+02 \\
2.53 E+02 \\
9.88 E+02 \\
2.71 E+01 \\
9.23 E+02 \\
5.48 E+00 \\
2.24 E+01 \\
8.96 E+01 \\
5.97 E+02 \\
5.75 E+02 \\
9.88 E+02 \\
1.35 E+01 \\
4.49 E+01 \\
1.81 E+02 \\
4.25 E+02 \\
2.51 E+03 \\
8.71 E+01 \\
1.36 E+02 \\
6.59 E+01 \\
1.75 E+03 \\
9.11 E+01 \\
4.83 E+02 \\
4.71 E+02 \\
1.38 E+02 \\
2.77 E+02 \\
1.41 E+03 \\
1.09 E+02 \\
3.66 E+02 \\
5.00 E+01 \\
1.28 E+03 \\
1.17 E+03 \\
6.56 E+02 \\
6.68 E+02 \\
2.53 E+02 \\
1.45 E+02 \\
7.02 E E+01 \\
5.17 E+02 \\
4.08 E+01 \\
3.66 E+01 \\
2.18 E+01 \\
1.91 E+02 \\
2.85 E+01 \\
4.77 E+02 \\
5.06 E+01 \\
1.20 E+02 \\
6.82 E+01 \\
1.81 E+02 \\
3.88 E+01 \\
2.12 E+01 \\
1.53 E+02 \\
6.39 E+01 \\
2.34 E+02 \\
1.23 E+02 \\
1.81 E+02 \\
1.25 E+02 \\
3.09 E+02 \\
9.46 E+02 \\
1.75 E+03\end{array}$ & $\begin{array}{l}P U 241 \\
6.22 E+03 \\
1.07 E+04 \\
9.27 E+03 \\
1.61 E+02 \\
4.33 E+03 \\
1.19 E+04 \\
7.91 E+02 \\
1.39 E+03 \\
2.28 E+03 \\
9.97 E+03 \\
4.63 E+02 \\
8.60 E+03 \\
7.09 E+01 \\
6.05 E+02 \\
9.65 E+02 \\
7.02 E+03 \\
6.35 E+03 \\
1.16 E+04 \\
1.33 E+02 \\
4.17 E+02 \\
1.68 E+03 \\
1.22 E+04 \\
7.18 E+04 \\
1.78 E+03 \\
3.45 E+03 \\
1.89 E+03 \\
5.01 E+04 \\
1.89 E+03 \\
9.66 E+03 \\
4.43 E+03 \\
1.22 E+03 \\
5.78 E+03 \\
1.36 E+04 \\
9.89 E+02 \\
3.57 E+03 \\
4.94 E+02 \\
1.60 E+04 \\
1.48 E+04 \\
1.01 E+04 \\
1.02 E+04 \\
2.53 E+03 \\
1.42 E+03 \\
1.76 E+03 \\
1.62 E+04 \\
7.64 E+02 \\
6.81 E+02 \\
4.95 E+02 \\
5.26 E+03 \\
4.17 E+02 \\
9.88 E+03 \\
8.92 E+02 \\
2.69 E+03 \\
1.44 E+03 \\
4.53 E+03 \\
5.777 E+02 \\
2.09 E+02 \\
4.06 E+03 \\
7.49 E+01 \\
2.75 E+02 \\
1.45 E+02 \\
1.88 E+02 \\
1.50 E+02 \\
3.244 E+02 \\
7.03 E+03 \\
1.26 E+04\end{array}$ & $\begin{array}{l}\text { PU242 } \\
1.46 E-02 \\
2.54 E-02 \\
2.19 E-02 \\
5.19 E-04 \\
3.93 E-02 \\
2.98 E-02 \\
5.42 E-03 \\
2.06 E-03 \\
5.32 E-03 \\
2.45 E-02 \\
2.67 E-03 \\
2.03 E-02 \\
2.05 E-04 \\
5.39 E-03 \\
2.75 E-03 \\
2.09 E-02 \\
1.84 E-02 \\
3.46 E-02 \\
0.00 E+00 \\
9.61 E-04 \\
3.92 E-03 \\
1.18 E-01 \\
6.95 E-01 \\
1.22 E-02 \\
2.87 E-02 \\
1.83 E-02 \\
4.84 E-01 \\
1.40 E-02 \\
6.94 E-02 \\
1.01 E-02 \\
2.71 E-03 \\
4.26 E-02 \\
3.11 E-02 \\
2.19 E-03 \\
8.40 E-03 \\
1.13 E-03 \\
4.71 E-02 \\
4.35 E-02 \\
3.28 E-02 \\
3.29 E-02 \\
5.73 E-03 \\
3.21 E-03 \\
1.45 E-03 \\
1.73 E-01 \\
4.83 E-03 \\
4.33 E-03 \\
3.60 E-03 \\
4.53 E-02 \\
1.72 E-03 \\
6.46 E-02 \\
5.11 E-03 \\
1.98 E-02 \\
9.81 E-03 \\
3.57 F-02 \\
2.35 E-03 \\
0.000+00 \\
3.45 E-02 \\
0.00 E+00 \\
0.00 E+00 \\
0.00 E+00 \\
0.00 E+00 \\
0.00 E+00 \\
0.00 E+00 \\
3.277 E-02 \\
5.74 E-02\end{array}$ & $\begin{array}{l}\text { AM241 } \\
6.27 \mathrm{E}+02 \\
1.07 \mathrm{E}+03 \\
9.20 \mathrm{E}+02 \\
1.59 \mathrm{E}+01 \\
4.30 \mathrm{E}+02 \\
1.17 \mathrm{E}+03 \\
7.81 \mathrm{E}+01 \\
1.36 \mathrm{E}+02 \\
2.22 \mathrm{E}+02 \\
9.74 \mathrm{E}+02 \\
4.53 \mathrm{E}+01 \\
8.35 \mathrm{E}+02 \\
6.88 \mathrm{E}+00 \\
5.87 \mathrm{E}+01 \\
9.25 \mathrm{E}+01 \\
6.73 \mathrm{E}+02 \\
6.12 \mathrm{E}+02 \\
1.12 \mathrm{E}+03 \\
1.33 \mathrm{E}+01 \\
3.97 \mathrm{E}+01 \\
1.62 \mathrm{E}+02 \\
1.16 \mathrm{E}+03 \\
6.84 \mathrm{E}+03 \\
1.69 \mathrm{E}+02 \\
3.28 \mathrm{E}+02 \\
1.80 \mathrm{E}+02 \\
4.76 \mathrm{E}+03 \\
1.82 \mathrm{E}+02 \\
9.34 \mathrm{E}+02 \\
4.14 \mathrm{E}+02 \\
1.15 \mathrm{E}+02 \\
5.52 \mathrm{E}+02 \\
1.26 \mathrm{E}+03 \\
9.18 \mathrm{E}+01 \\
3.29 \mathrm{E}+02 \\
4.56 \mathrm{E}+01 \\
1.48 \mathrm{E}+03 \\
1.36 \mathrm{E}+03 \\
9.48 \mathrm{E}+02 \\
9.57 \mathrm{E}+02 \\
2.30 \mathrm{E}+02 \\
1.30 \mathrm{E}+02 \\
1.68 \mathrm{E}+02 \\
1.49 \mathrm{E}+03 \\
7.33 \mathrm{E}+01 \\
6.58 \mathrm{E}+01 \\
4.56 \mathrm{E}+01 \\
4.93 \mathrm{E}+02 \\
4.02 \mathrm{E}+01 \\
9.00 \mathrm{E}+02 \\
8.47 \mathrm{E}+01 \\
2.52 \mathrm{E}+02 \\
1.33 \mathrm{E}+02 \\
4.16 \mathrm{E}+02 \\
5.44 \mathrm{E}+01 \\
1.98 \mathrm{E}+01 \\
3.72 \mathrm{E}+02 \\
1.28 \mathrm{E}+01 \\
4.71 \mathrm{E}+01 \\
2.46 \mathrm{E}+01 \\
3.15 \mathrm{E}+01 \\
2.51 \mathrm{E}+01 \\
5.38 \mathrm{E}+01 \\
1.18 \mathrm{E}+03 \\
2.09 \mathrm{E}+03\end{array}$ & $\begin{array}{l}\text { AM243 } \\
4.24 E-03 \\
7.49 E-03 \\
6.46 E-03 \\
2.01 E-04 \\
4.80 E-02 \\
9.04 E-03 \\
5.34 E-03 \\
4.39 E-04 \\
1.57 E-03 \\
7.38 E-03 \\
2.16 E-03 \\
6.01 E-03 \\
4.97 E-08 \\
6.58 E-03 \\
1.02 E-03 \\
7.90 E-03 \\
6.87 E-03 \\
1.31 E-02 \\
0.00 E+00 \\
2.84 E-04 \\
1.16 E-03 \\
1.66 E-01 \\
9.80 E-01 \\
1.24 E-02 \\
3.38 E-02 \\
2.58 E-02 \\
6.83 E-01 \\
1.45 E-02 \\
6.96 E-02 \\
2.97 E-03 \\
7.89 E-04 \\
4.40 E-02 \\
9.23 E-03 \\
6.42 E-04 \\
2.40 E-03 \\
3.37 E-04 \\
1.79 E-02 \\
1.66 E-02 \\
1.29 E-02 \\
1.30 E-02 \\
1.70 E-03 \\
9.54 E-04 \\
1.70 E-02 \\
2.83 E-01 \\
4.47 E-03 \\
4.01 E-03 \\
3.92 E-03 \\
5.49 E-02 \\
6.52 E-04 \\
6.45 E-02 \\
4.23 E-03 \\
2.16 E-03 \\
1.01 E-02 \\
4.13 E-02 \\
8.87 E-04 \\
0.00 E+00 \\
4.02 E-02 \\
0.00 E+00 \\
0.00 E+00 \\
0.00 E+00 \\
0.00 E+00 \\
0.00 E+00 \\
0.00 E+00 \\
1.23 E-02 \\
2.15 E-02\end{array}$ & 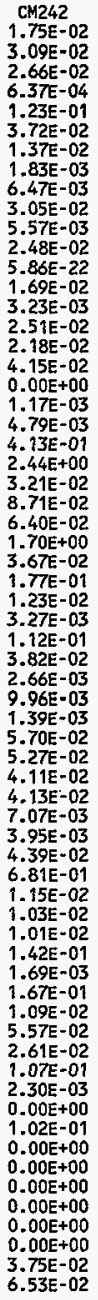 & $\begin{array}{l}\text { CM243 } \\
3.78 \mathrm{E}-04 \\
6.73 \mathrm{E}-04 \\
5.82 \mathrm{E}-04 \\
1.84 \mathrm{E}-05 \\
1.23 \mathrm{E}-02 \\
8.29 \mathrm{E}-04 \\
1.21 \mathrm{E}-03 \\
3.20 \mathrm{E}-05 \\
1.42 \mathrm{E}-04 \\
6.77 \mathrm{E}-04 \\
4.24 \mathrm{E}-04 \\
5.48 \mathrm{E}-04 \\
0.00 \mathrm{E}+00 \\
1.71 \mathrm{E}-03 \\
9.16 \mathrm{E}-05 \\
7.22 \mathrm{E}-04 \\
6.21 \mathrm{E}-04 \\
1.19 \mathrm{E}-03 \\
0.00 \mathrm{E}+00 \\
2.60 \mathrm{E}-05 \\
1.06 \mathrm{E}-04 \\
5.04 \mathrm{E}-02 \\
2.97 \mathrm{E}-01 \\
2.93 \mathrm{E}-03 \\
8.64 \mathrm{E}-03 \\
7.82 \mathrm{E}-03 \\
2.07 \mathrm{E}-01 \\
3.34 \mathrm{E}-03 \\
1.58 \mathrm{E}-02 \\
2.74 \mathrm{E}-04 \\
7.23 \mathrm{E}-05 \\
1.02 \mathrm{E}-02 \\
8.57 \mathrm{E}-04 \\
5.92 \mathrm{E}-05 \\
2.24 \mathrm{E}-04 \\
3.14 \mathrm{E}-05 \\
1.67 \mathrm{E}-03 \\
1.54 \mathrm{E}-03 \\
1.22 \mathrm{E}-03 \\
1.22 \mathrm{E}-03 \\
1.60 \mathrm{E}-04 \\
8.88 \mathrm{E}-05 \\
4.34 \mathrm{E}-03 \\
9.79 \mathrm{E}-02 \\
9.86 \mathrm{E}-04 \\
8.81 \mathrm{E}-04 \\
9.74 \mathrm{E}-04 \\
3.43 \mathrm{E}-02 \\
0.00 \mathrm{E}+00 \\
1.53 \mathrm{E}-00 \\
8.56 \mathrm{E}-04 \\
5.32 \mathrm{E}-03 \\
2.42 \mathrm{E}-03 \\
1.06 \mathrm{E}-02 \\
0.00 \mathrm{E}+00 \\
0.00 \mathrm{E}+00 \\
1.01 \mathrm{E}-02 \\
0.00 \mathrm{E}+00 \\
0.00 \mathrm{E}+00 \\
0.00 \mathrm{E}+00 \\
0.00 \mathrm{E}+00 \\
0.00 \mathrm{E}+00 \\
0.00 \mathrm{E}+00 \\
.96 \mathrm{E}-04\end{array}$ \\
\hline
\end{tabular}


HNF-SD-WM-TI-794, ReV. O

ACTIVITY IN UNSEPARATED FUEL (Curies decayed to 1/1/94)

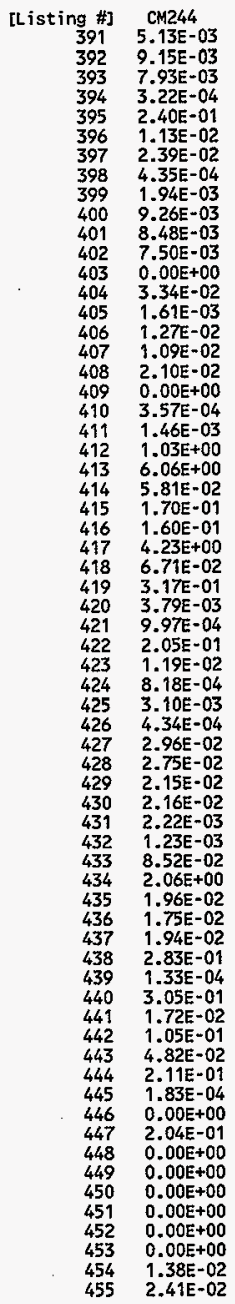


HNF-SD-WN-T1-794, Rev, O

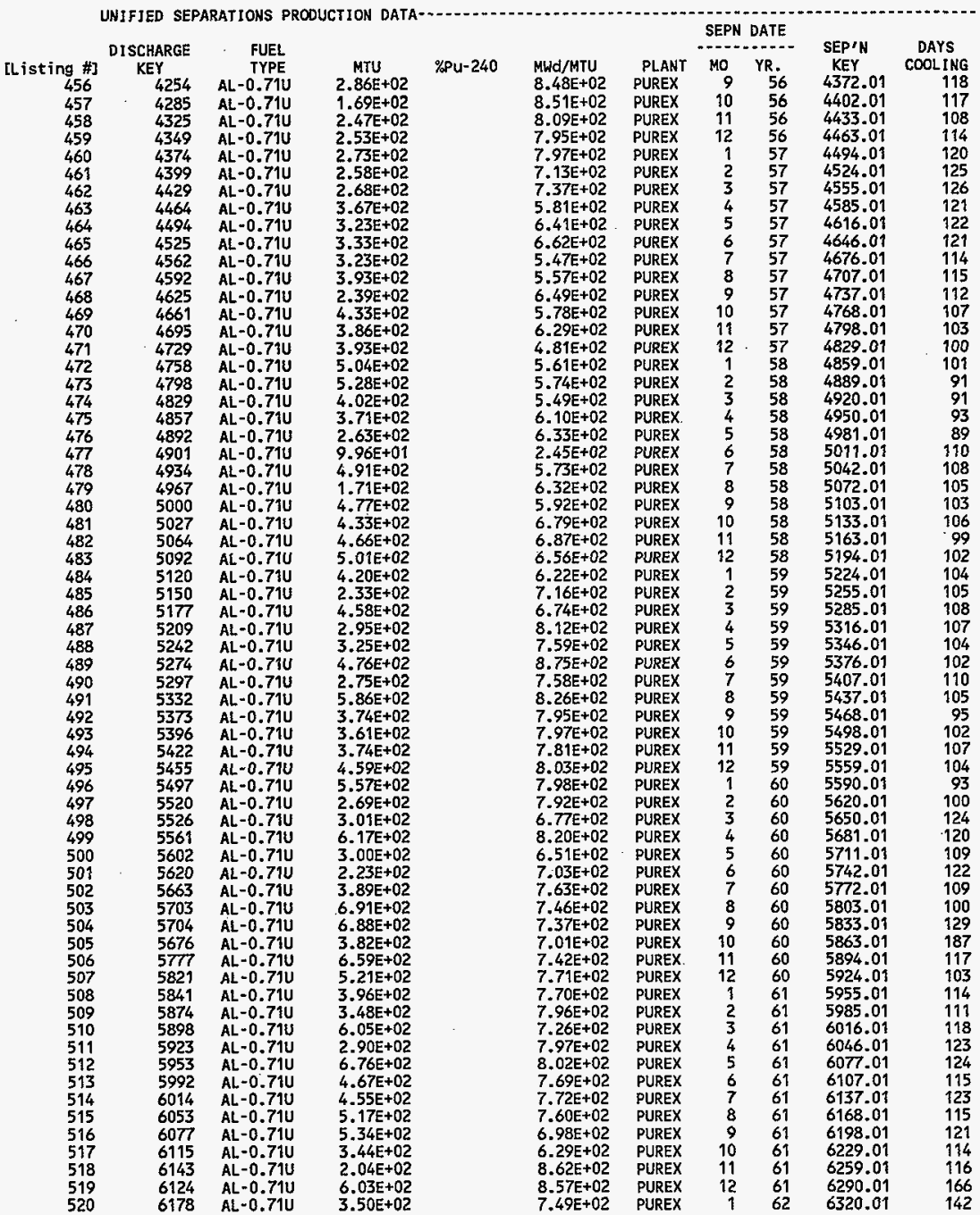


HNF-SD-WM-TI-794, REV. 0

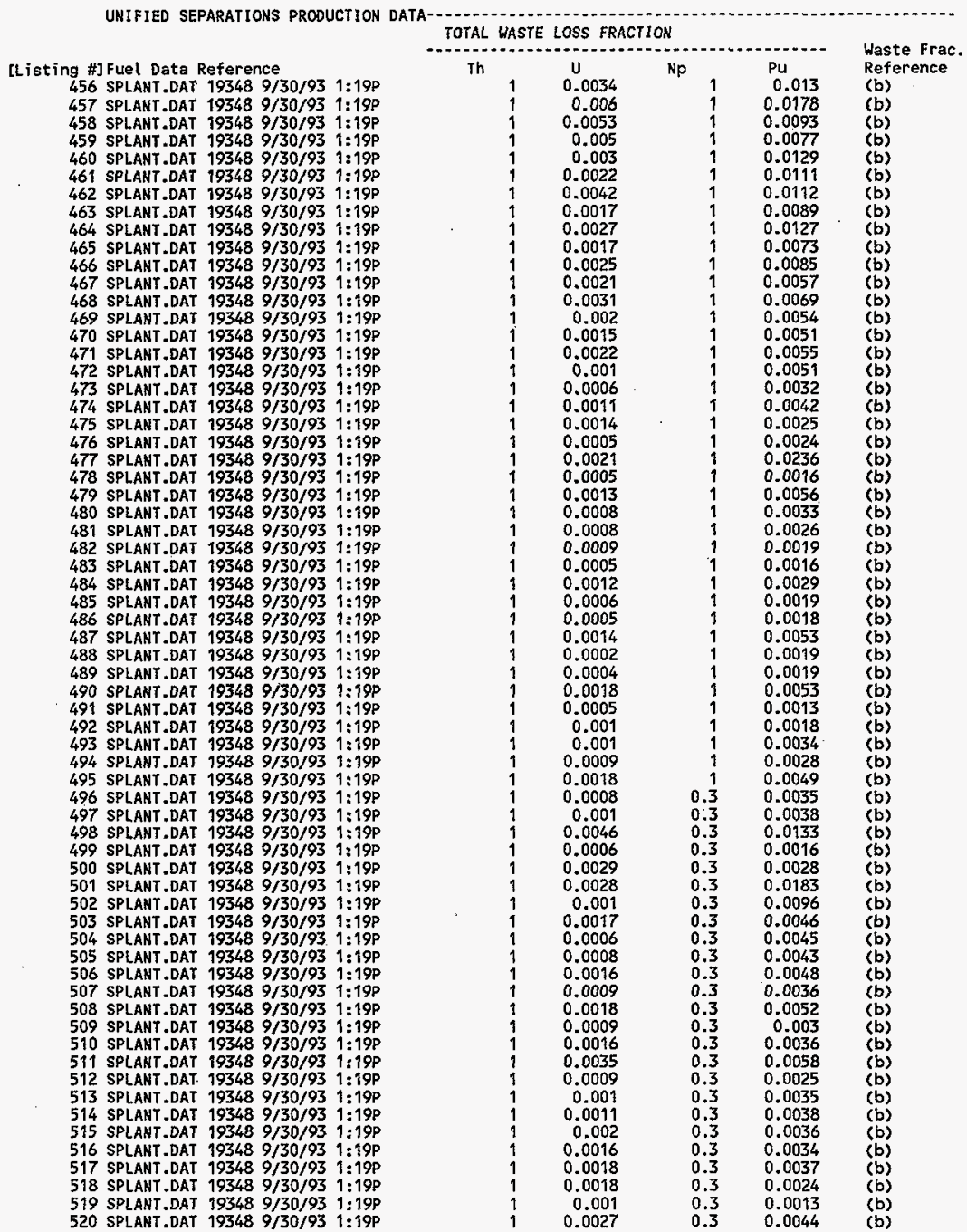


HNF-SD-WH-TI-794, ReV. O

ACTIVITY IN UNSEPARATED FUEL (Curies decayed to $1 / 1 / 94$ )

\begin{tabular}{|c|c|c|c|c|c|c|c|c|c|}
\hline $\begin{array}{c}\text { isting } \# \\
456 \\
457 \\
458 \\
459 \\
460 \\
461 \\
462 \\
463 \\
464 \\
465 \\
466 \\
467 \\
468 \\
469 \\
470 \\
471 \\
472 \\
473 \\
474 \\
475 \\
476 \\
477 \\
478 \\
479 \\
480 \\
481 \\
482 \\
483 \\
484 \\
485 \\
486 \\
487 \\
488 \\
489 \\
490 \\
491 \\
492 \\
493 \\
494 \\
495 \\
496 \\
497 \\
498 \\
499 \\
500 \\
501 \\
502 \\
503 \\
504 \\
505 \\
506 \\
507 \\
508 \\
509 \\
510 \\
511 \\
512 \\
513 \\
514 \\
515 \\
516 \\
517 \\
518 \\
519 \\
520\end{array}$ & $\begin{array}{l}\text { H03 } \\
4.70 E+02 \\
2.80 E+02 \\
3.91 E+02 \\
3.95 E+02 \\
4.29 E+02 \\
3.62 E+02 \\
3.91 E+02 \\
4.23 E+02 \\
4.13 E+02 \\
4.43 E+02 \\
3.55 E+02 \\
4.41 E+02 \\
3.16 E+02 \\
5.12 E+02 \\
5.00 E+02 \\
3.88 E+02 \\
5.86 E+02 \\
6.32 E+02 \\
4.61 E+02 \\
4.76 E+02 \\
3.53 E+02 \\
5.10 E+01 \\
5.99 E+02 \\
2.32 E+02 \\
6.07 E+02 \\
6.37 E+02 \\
6.98 E+02 \\
7.19 E+02 \\
5.73 E+02 \\
3.69 E+02 \\
6.84 E+02 \\
5.37 E+02 \\
5.55 E+02 \\
9.46 E+02 \\
4.72 E+02 \\
1.11 E+03 \\
6.82 E+02 \\
6.63 E+02 \\
6.76 E+02 \\
8.58 E+02 \\
1.04 E+03 \\
5.02 E+02 \\
4.77 E+02 \\
1.20 E+03 \\
4.62 E+02 \\
3.73 E+02 \\
7.11 E+02 \\
1.24 E+03 \\
1.22 E+03 \\
6.42 E+02 \\
1.19 E+03 \\
9.88 E+02 \\
7.52 E+02 \\
6.88 E+02 \\
1.09 E+03 \\
5.77 E+02 \\
1.36 E+03 \\
9.05 E+02 \\
8.89 E+02 \\
1.00 E+03 \\
9.50 E+02 \\
5.52 E+02 \\
4.55 E+02 \\
1.34 E+03 \\
6.81 E+02\end{array}$ & $\begin{array}{l}C 14 \\
1.53 E+01 \\
9.09 E+00 \\
1.27 E+01 \\
1.27 E+01 \\
1.38 E+01 \\
1.17 E+01 \\
1.25 E+01 \\
1.36 E+01 \\
1.32 E+01 \\
1.40 E+01 \\
1.13 E+01 \\
1.40 E+01 \\
9.87 E+00 \\
1.60 E+01 \\
1.54 E+01 \\
1.21 E+01 \\
1.80 E+01 \\
1.93 E+01 \\
1.41 E+01 \\
1.44 E+01 \\
1.06 E+01 \\
1.57 E+00 \\
1.79 E+01 \\
6.88 E+00 \\
1.80 E+01 \\
1.87 E+01 \\
2.04 E+01 \\
2.09 E+01 \\
1.66 E+01 \\
1.06 E+01 \\
1.96 E+01 \\
1.52 E+01 \\
1.57 E+01 \\
2.63 E+01 \\
1.32 E+01 \\
3.07 E+01 \\
1.88 E+01 \\
1.82 E+01 \\
1.85 E+01 \\
2.33 E+01 \\
2.82 E+01 \\
1.35 E+01 \\
1.30 E+01 \\
3.21 E+01 \\
1.24 E+01 \\
9.97 E+00 \\
1.88 E+01 \\
3.27 E+01 \\
3.22 E+01 \\
1.70 E+01 \\
3.10 E+01 \\
2.55 E+01 \\
1.94 E+01 \\
1.76 E+01 \\
2.79 E+01 \\
1.46 E+01 \\
3.44 E+01 \\
2.28 E+01 \\
2.23 E+01 \\
2.49 E+01 \\
2.37 E+01 \\
1.37 E+01 \\
1.11 E+01 \\
3.27 E+01 \\
1.66 E+01\end{array}$ & $\begin{array}{l}N 159 \\
4.34 E+00 \\
2.57 E+00 \\
3.58 E+00 \\
3.61 E+00 \\
3.90 E+00 \\
3.31 E+00 \\
3.55 E+00 \\
3.86 E+00 \\
3.73 E+00 \\
3.98 E+00 \\
3.20 E+00 \\
3.96 E+00 \\
2.80 E+00 \\
4.53 E+00 \\
4.38 E+00 \\
3.43 E+00 \\
5.11 E+00 \\
5.47 E+00 \\
3.99 E+00 \\
4.08 E+00 \\
3.00 E+00 \\
4.46 E-01 \\
5.09 E+00 \\
1.95 E+00 \\
5.10 E+00 \\
5.29 E+00 \\
5.77 E+00 \\
5.92 E+00 \\
4.72 E+00 \\
3.00 E+00 \\
5.56 E+00 \\
4.29 E+00 \\
4.43 E+00 \\
7.45 E+00 \\
3.74 E+00 \\
8.68 E+00 \\
5.33 E+00 \\
5.15 E+00 \\
5.24 E+00 \\
6.61 E+00 \\
7.98 E+00 \\
3.83 E+00 \\
3.67 E+00 \\
9.08 E+00 \\
3.52 E+00 \\
2.82 E+00 \\
5.33 E+00 \\
9.25 E+00 \\
9.11 E+00 \\
4.82 E+00 \\
8.78 E+00 \\
7.22 E+00 \\
5.48 E+00 \\
4.97 E+00 \\
7.90 E+00 \\
4.13 E+00 \\
9.72 E+00 \\
6.44 E+00 \\
6.31 E+00 \\
7.05 E+00 \\
6.71 E+00 \\
3.90 E+00 \\
3.14 E+00 \\
9.25 E+00 \\
4.71 E+00\end{array}$ & 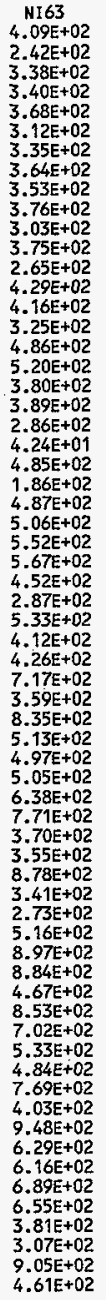 & $\begin{array}{c}\text { CO60 } \\
6.69 E+00 \\
4.01 E+00 \\
5.66 E+00 \\
5.75 E+00 \\
6.27 E+00 \\
5.37 E+00 \\
5.82 E+00 \\
6.42 E+00 \\
6.28 E+00 \\
6.77 E+00 \\
5.52 E+00 \\
6.91 E+00 \\
4.94 E+00 \\
8.10 E+00 \\
7.92 E+00 \\
6.28 E+00 \\
9.47 E+00 \\
1.03 E+01 \\
7.58 E+00 \\
7.83 E+00 \\
5.83 E+00 \\
8.73 E-01 \\
1.00 E+01 \\
3.89 E+00 \\
1.03 E+01 \\
1.08 E+01 \\
1.19 E+01 \\
1.24 E+01 \\
9.95 E+00 \\
6.38 E+00 \\
1.20 E+01 \\
9.32 E+00 \\
9.74 E+00 \\
1.66 E+01 \\
8.38 E+00 \\
1.97 E+01 \\
1.23 E+01 \\
1.20 E+01 \\
1.23 E+01 \\
1.57 E+01 \\
1.92 E+01 \\
9.31 E+00 \\
8.95 E+00 \\
2.24 E+01 \\
8.83 E+00 \\
7.12 E+00 \\
1.36 E+01 \\
2.40 E+01 \\
2.37 E+01 \\
1.24 E+01 \\
2.34 E+01 \\
1.96 E+01 \\
1.50 E+01 \\
1.37 E+01 \\
2.20 E+01 \\
1.16 E+01 \\
2.76 E+01 \\
1.86 E+01 \\
1.83 E+01 \\
2.08 E+01 \\
1.99 E+01 \\
1.18 E+01 \\
9.54 E+00 \\
2.79 E+01 \\
1.45 E+01\end{array}$ & $\begin{array}{l}\text { SE79 } \\
3.26 E+00 \\
1.93 E+00 \\
2.69 E+00 \\
2.71 E+00 \\
2.92 E+00 \\
2.47 E+00 \\
2.66 E+00 \\
2.87 E+00 \\
2.79 E+00 \\
2.97 E+00 \\
2.38 E+00 \\
2.95 E+00 \\
2.09 E+00 \\
3.38 E+00 \\
3.27 E+00 \\
2.55 E+00 \\
3.81 E+00 \\
4.08 E+00 \\
2.97 E+00 \\
3.05 E+00 \\
2.24 E+00 \\
3.30 E-01 \\
3.79 E+00 \\
1.46 E+00 \\
3.80 E+00 \\
3.96 E+00 \\
4.31 E+00 \\
4.43 E+00 \\
3.52 E+00 \\
2.24 E+00 \\
4.16 E+00 \\
3.22 E+00 \\
3.32 E+00 \\
5.60 E+00 \\
2.80 E+00 \\
6.51 E+00 \\
3.99 E+00 \\
3.87 E+00 \\
3.93 E+00 \\
4.96 E+00 \\
5.99 E+00 \\
2.87 E+00 \\
2.75 E+00 \\
6.81 E+00 \\
2.63 E+00 \\
2.11 E+00 \\
3.99 E+00 \\
6.93 E+00 \\
6.82 E+00 \\
3.61 E+00 \\
6.58 E+00 \\
5.41 E+00 \\
4.11 E+00 \\
3.73 E+00 \\
5.91 E+00 \\
3.10 E+00 \\
7.30 E+00 \\
4.83 E+00 \\
4.72 E+00 \\
5.28 E+00 \\
5.02 E+00 \\
2.91 E+00 \\
2.36 E+00 \\
6.95 E+00 \\
3.53 E+00\end{array}$ & 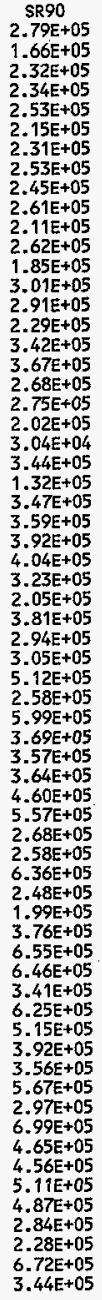 & $\begin{array}{l}Y 90 \\
2.79 E+05 \\
1.66 E+05 \\
2.32 E+05 \\
2.34 E+05 \\
2.53 E+05 \\
2.15 E+05 \\
2.31 E+05 \\
2.53 E+05 \\
2.45 E+05 \\
2.62 E+05 \\
2.11 E+05 \\
2.62 E+05 \\
1.85 E+05 \\
3.01 E+05 \\
2.91 E+05 \\
2.29 E+05 \\
3.42 E+05 \\
3.67 E+05 \\
2.68 E+05 \\
2.75 E+05 \\
2.02 E+05 \\
3.04 E+04 \\
3.44 E+05 \\
1.32 E+05 \\
3.47 E+05 \\
3.59 E+05 \\
3.93 E+05 \\
4.04 E+05 \\
3.23 E+05 \\
2.05 E+05 \\
3.81 E+05 \\
2.94 E+05 \\
3.05 E+05 \\
5.12 E+05 \\
2.58 E+05 \\
5.99 E+05 \\
3.69 E+05 \\
3.57 E+05 \\
3.64 E+05 \\
4.60 E+05 \\
5.57 E+05 \\
2.68 E+05 \\
2.58 E+05 \\
6.36 E+05 \\
2.48 E+05 \\
1.99 E+05 \\
3.76 E+05 \\
6.55 E+05 \\
6.46 E+05 \\
3.41 E+05 \\
6.25 E+05 \\
5.15 E+05 \\
3.92 E+05 \\
3.56 E+05 \\
5.67 E+05 \\
2.97 E+05 \\
6.99 E+05 \\
4.65 E+05 \\
4.56 E+05 \\
5.11 E+05 \\
4.87 E+05 \\
2.84 E+05 \\
2.28 E+05 \\
6.72 E+05 \\
3.44 E+05\end{array}$ & $\begin{array}{l}2 R 93 \\
1.53 \mathrm{E}+01 \\
9.07 \mathrm{E}+00 \\
1.26 \mathrm{E}+01 \\
1.27 \mathrm{E}+01 \\
1.37 \mathrm{E}+01 \\
1.17 \mathrm{E}+01 \\
1.25 \mathrm{E}+01 \\
1.36 \mathrm{E}+01 \\
1.32 \mathrm{E}+01 \\
1.40 \mathrm{E}+01 \\
1.13 \mathrm{E}+01 \\
1.40 \mathrm{E}+01 \\
9.87 \mathrm{E}+00 \\
1.60 \mathrm{E}+01 \\
1.54 \mathrm{E}+01 \\
1.21 \mathrm{E}+01 \\
1.80 \mathrm{E}+01 \\
1.93 \mathrm{E}+01 \\
1.41 \mathrm{E}+01 \\
1.44 \mathrm{E}+01 \\
1.06 \mathrm{E}+01 \\
1.57 \mathrm{E}+00 \\
1.79 \mathrm{E}+01 \\
6.88 \mathrm{E}+00 \\
1.80 \mathrm{E}+01 \\
1.87 \mathrm{E}+01 \\
2.03 \mathrm{E}+01 \\
2.09 \mathrm{E}+01 \\
1.66 \mathrm{E}+01 \\
1.06 \mathrm{E}+01 \\
1.96 \mathrm{E}+01 \\
1.51 \mathrm{E}+01 \\
1.56 \mathrm{E}+01 \\
2.63 \mathrm{E}+01 \\
1.32 \mathrm{E}+01 \\
3.06 \mathrm{E}+01 \\
1.88 \mathrm{E}+01 \\
1.82 \mathrm{E}+01 \\
1.85 \mathrm{E}+01 \\
2.33 \mathrm{E}+01 \\
2.81 \mathrm{E}+01 \\
1.35 \mathrm{E}+01 \\
1.29 \mathrm{E}+01 \\
3.20 \mathrm{E}+01 \\
1.24 \mathrm{E}+01 \\
9.95 \mathrm{E}+00 \\
1.88 \mathrm{E}+01 \\
3.26 \mathrm{E}+01 \\
3.21 \mathrm{E}+01 \\
1.70 \mathrm{E}+01 \\
3.10 \mathrm{E}+01 \\
2.55 \mathrm{E}+01 \\
1.93 \mathrm{E}+01 \\
1.75 \mathrm{E}+01 \\
2.79 \mathrm{E}+01 \\
1.46 \mathrm{E}+01 \\
3.43 \mathrm{E}+01 \\
2.27 \mathrm{E}+01 \\
2.22 \mathrm{E}+01 \\
2.49 \mathrm{E}+01 \\
2.36 \mathrm{E}+01 \\
1.37 \mathrm{E}+01 \\
1.11 \mathrm{E}+01 \\
3.26 \mathrm{E}+01 \\
1.66 \mathrm{E}+01\end{array}$ \\
\hline
\end{tabular}


HNF-SD-WM-TI-794, ReV. 0

ACTIVITY IN UNSEPARATED FUEL (Curies decayed to $1 / 1 / 94$ )

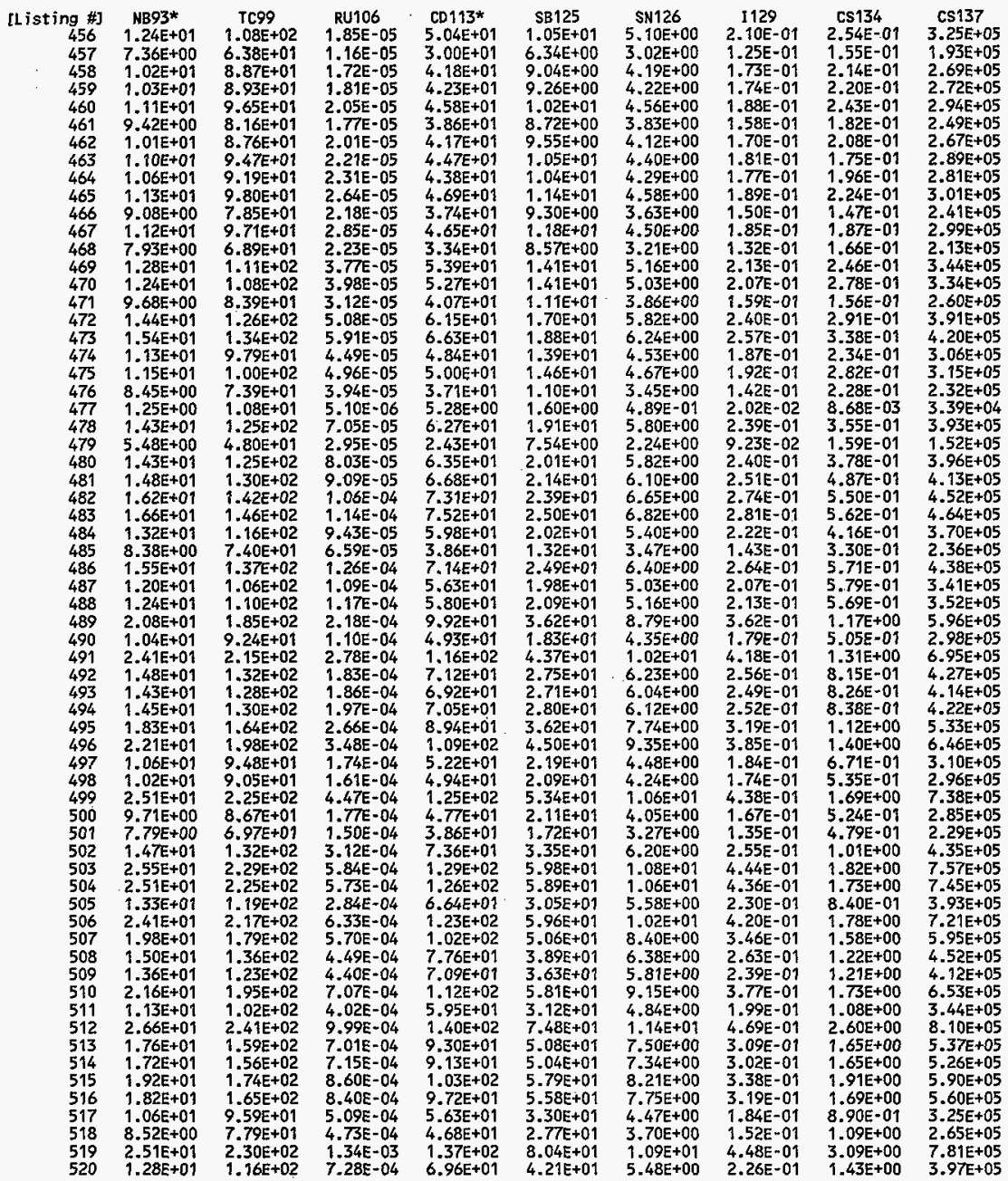


HNF-SD-WM-TI-794, ReV. O

ACTIVITY IN UNSEPARATED FUEL (Curies decayed to 1/1/94)

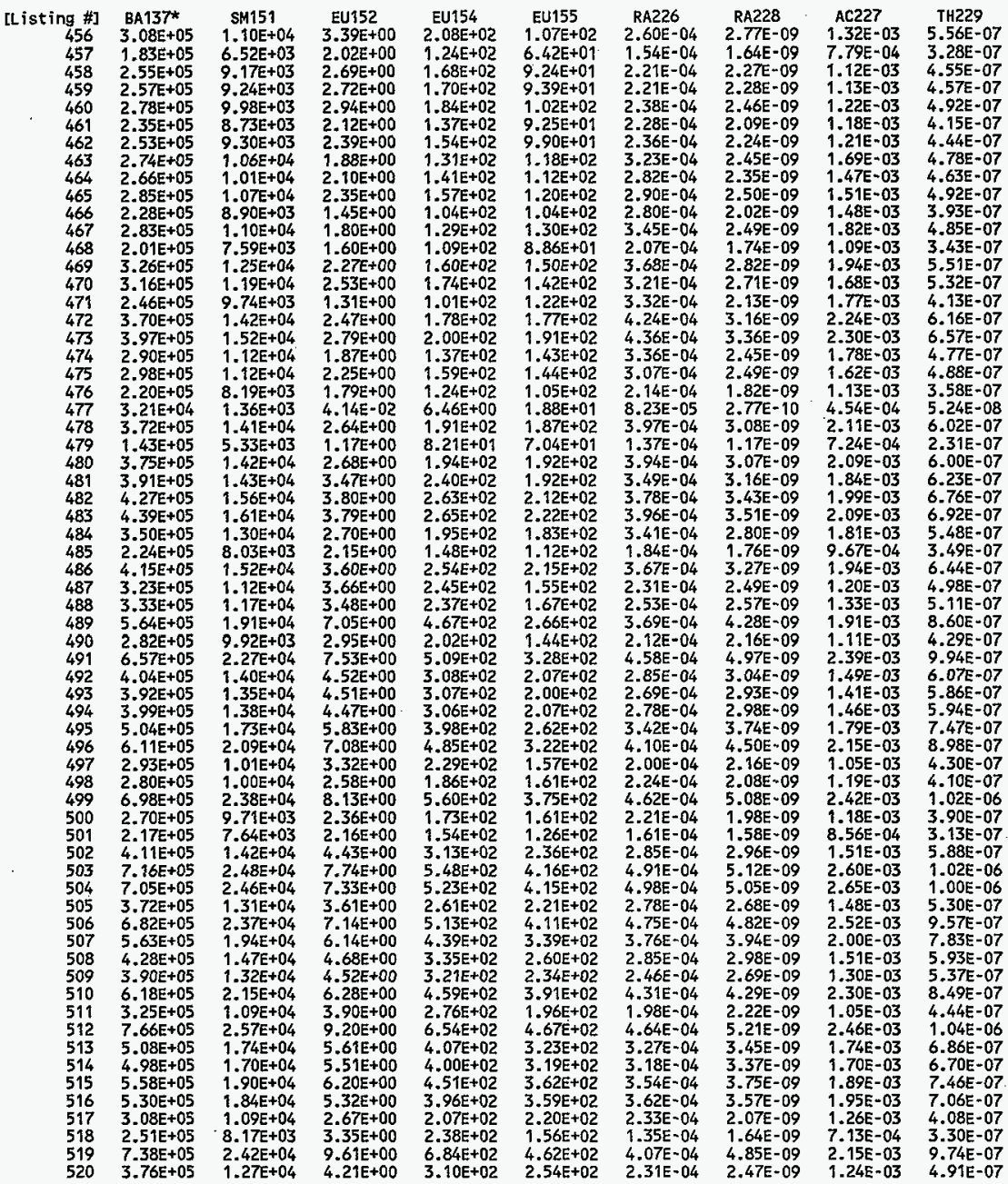


ACTIVITY IN UNSEPARATED FUEL (Curies decayed to $1 / 1 / 94$ )

\begin{tabular}{|c|c|c|c|c|c|c|c|c|c|}
\hline $\begin{array}{r}\text { isting } \# \\
456 \\
457 \\
458 \\
459 \\
460 \\
461 \\
462 \\
463 \\
464 \\
465 \\
466 \\
467 \\
468 \\
469 \\
470 \\
471 \\
472 \\
473 \\
474 \\
475 \\
476 \\
477 \\
478 \\
479 \\
480 \\
481 \\
482 \\
483 \\
484 \\
485 \\
486 \\
487 \\
488 \\
489 \\
490 \\
491 \\
492 \\
493 \\
494 \\
495 \\
496 \\
497 \\
498 \\
499 \\
500 \\
501 \\
502 \\
503 \\
504 \\
505 \\
506 \\
507 \\
508 \\
509 \\
510 \\
511 \\
512 \\
513 \\
514 \\
515 \\
516 \\
517 \\
518 \\
519 \\
520\end{array}$ & $\begin{array}{l}\text { TH232 } \\
3.69 \mathrm{E}-09 \\
2.18 \mathrm{E}-09 \\
3.04 \mathrm{E}-09 \\
3.05 \mathrm{E}-09 \\
3.29 \mathrm{E}-09 \\
2.81 \mathrm{E}-09 \\
3.00 \mathrm{E}-09 \\
3.28 \mathrm{E}-09 \\
3.16 \mathrm{E}-09 \\
3.36 \mathrm{E}-09 \\
2.71 \mathrm{E}-09 \\
3.35 \mathrm{E}-09 \\
2.35 \mathrm{E}-09 \\
3.80 \mathrm{E}-09 \\
3.65 \mathrm{E}-09 \\
2.88 \mathrm{E}-09 \\
4.27 \mathrm{E}-09 \\
4.55 \mathrm{E}-09 \\
3.32 \mathrm{E}-09 \\
3.37 \mathrm{E}-09 \\
2.47 \mathrm{E}-09 \\
3.76 \mathrm{E}-10 \\
4.18 \mathrm{E}-09 \\
1.59 \mathrm{E}-09 \\
4.18 \mathrm{E}-09 \\
4.29 \mathrm{E}-09 \\
4.67 \mathrm{E}-09 \\
4.79 \mathrm{E}-09 \\
3.82 \mathrm{E}-09 \\
2.40 \mathrm{E}-09 \\
4.47 \mathrm{E}-09 \\
3.41 \mathrm{E}-09 \\
3.52 \mathrm{E}-09 \\
5.86 \mathrm{E}-09 \\
2.95 \mathrm{E}-09 \\
6.82 \mathrm{E}-09 \\
4.18 \mathrm{E}-09 \\
4.03 \mathrm{E}-09 \\
4.09 \mathrm{E}-09 \\
5.14 \mathrm{E}-09 \\
6.19 \mathrm{E}-09 \\
2.97 \mathrm{E}-09 \\
2.86 \mathrm{E}-09 \\
7.00 \mathrm{E}-09 \\
2.74 \mathrm{E}-09 \\
2.18 \mathrm{E}-09 \\
4.09 \mathrm{E}-09 \\
7.08 \mathrm{E}-09 \\
6.98 \mathrm{E}-09 \\
3.71 \mathrm{E}-09 \\
6.69 \mathrm{E}-09 \\
5.47 \mathrm{E}-09 \\
4.15 \mathrm{E}-09 \\
3.74 \mathrm{E}-09 \\
5.97 \mathrm{E}-09 \\
3.10 \mathrm{E}-09 \\
7.27 \mathrm{E}-09 \\
4.82 \mathrm{E}-09 \\
4.70 \mathrm{E}-09 \\
5.24 \mathrm{E}-09 \\
4.99 \mathrm{E}-09 \\
2.91 \mathrm{E}-09 \\
2.30 \mathrm{E}-09 \\
6.79 \mathrm{E}-09 \\
3.46 \mathrm{E}-09\end{array}$ & $\begin{array}{l}\text { PA231 } \\
3.14 \mathrm{E}-03 \\
1.86 \mathrm{E}-03 \\
2.69 \mathrm{E}-03 \\
2.70 \mathrm{E}-03 \\
2.91 \mathrm{E}-03 \\
2.82 \mathrm{E}-03 \\
2.92 \mathrm{E}-03 \\
4.08 \mathrm{E}-03 \\
3.55 \mathrm{E}-03 \\
3.65 \mathrm{E}-03 \\
3.59 \mathrm{E}-03 \\
4.43 \mathrm{E}-03 \\
2.64 \mathrm{E}-03 \\
4.73 \mathrm{E}-03 \\
4.10 \mathrm{E}-03 \\
4.34 \mathrm{E}-03 \\
5.49 \mathrm{E}-03 \\
5.65 \mathrm{E}-03 \\
4.39 \mathrm{E}-03 \\
3.98 \mathrm{E}-03 \\
2.77 \mathrm{E}-03 \\
1.12 \mathrm{E}-03 \\
5.20 \mathrm{E}-03 \\
1.79 \mathrm{E}-03 \\
5.19 \mathrm{E}-03 \\
4.55 \mathrm{E}-03 \\
4.95 \mathrm{E}-03 \\
5.20 \mathrm{E}-03 \\
4.51 \mathrm{E}-03 \\
2.41 \mathrm{E}-03 \\
4.85 \mathrm{E}-03 \\
3.00 \mathrm{E}-03 \\
3.33 \mathrm{E}-03 \\
4.79 \mathrm{E}-03 \\
2.79 \mathrm{E}-03 \\
6.01 \mathrm{E}-03 \\
3.77 \mathrm{E}-03 \\
3.55 \mathrm{E}-03 \\
3.69 \mathrm{E}-03 \\
4.53 \mathrm{E}-03 \\
5.46 \mathrm{E}-03 \\
2.68 \mathrm{E}-03 \\
3.04 \mathrm{E}-03 \\
6.18 \mathrm{E}-03 \\
3.02 \mathrm{E}-03 \\
2.19 \mathrm{E}-03 \\
3.86 \mathrm{E}-03 \\
6.69 \mathrm{E}-03 \\
6.80 \mathrm{E}-03 \\
3.81 \mathrm{E}-03 \\
6.52 \mathrm{E}-03 \\
5.17 \mathrm{E}-03 \\
3.92 \mathrm{E}-03 \\
3.38 \mathrm{E}-03 \\
5.99 \mathrm{E}-03 \\
2.73 \mathrm{E}-03 \\
6.41 \mathrm{E}-03 \\
4.55 \mathrm{E}-03 \\
4.45 \mathrm{E}-03 \\
4.96 \mathrm{E}-03 \\
5.12 \mathrm{E}-03 \\
3.33 \mathrm{E}-03 \\
1.88 \mathrm{E}-03 \\
5.67 \mathrm{E}-03 \\
3.28 \mathrm{E}-03\end{array}$ & $\begin{array}{l}\text { U232 } \\
6.45 \mathrm{E}-03 \\
3.83 \mathrm{E}-03 \\
5.23 \mathrm{E}-03 \\
5.27 \mathrm{E}-03 \\
5.70 \mathrm{E}-03 \\
4.55 \mathrm{E}-03 \\
4.96 \mathrm{E}-03 \\
4.85 \mathrm{E}-03 \\
4.90 \mathrm{E}-03 \\
5.30 \mathrm{E}-03 \\
3.93 \mathrm{E}-03 \\
4.87 \mathrm{E}-03 \\
3.69 \mathrm{E}-03 \\
5.72 \mathrm{E}-03 \\
5.78 \mathrm{E}-03 \\
4.03 \mathrm{E}-03 \\
6.39 \mathrm{E}-03 \\
6.94 \mathrm{E}-03 \\
4.94 \mathrm{E}-03 \\
5.29 \mathrm{E}-03 \\
3.98 \mathrm{E}-03 \\
4.31 \mathrm{E}-04 \\
6.47 \mathrm{E}-03 \\
2.59 \mathrm{E}-03 \\
6.50 \mathrm{E}-03 \\
7.23 \mathrm{E}-03 \\
7.89 \mathrm{E}-03 \\
8.02 \mathrm{E}-03 \\
6.16 \mathrm{E}-03 \\
4.21 \mathrm{E}-03 \\
7.54 \mathrm{E}-03 \\
6.43 \mathrm{E}-03 \\
6.42 \mathrm{E}-03 \\
1.16 \mathrm{E}-02 \\
5.42 \mathrm{E}-03 \\
1.30 \mathrm{E}-02 \\
7.92 \mathrm{E}-03 \\
7.76 \mathrm{E}-03 \\
7.80 \mathrm{E}-03 \\
9.96 \mathrm{E}-03 \\
1.20 \mathrm{E}-02 \\
5.72 \mathrm{E}-03 \\
5.08 \mathrm{E}-03 \\
1.37 \mathrm{E}-02 \\
4.79 \mathrm{E}-03 \\
4.02 \mathrm{E}-03 \\
7.80 \mathrm{E}-03 \\
1.36 \mathrm{E}-03 \\
1.32 \mathrm{E}-02 \\
6.80 \mathrm{E}-03 \\
1.27 \mathrm{E}-02 \\
1.06 \mathrm{E}-02 \\
8.07 \mathrm{E}-03 \\
7.49 \mathrm{E}-03 \\
1.13 \mathrm{E}-02 \\
6.31 \mathrm{E}-03 \\
1.49 \mathrm{E}-02 \\
9.53 \mathrm{E}-03 \\
9.33 \mathrm{E}-03 \\
1.04 \mathrm{E}-02 \\
9.56 \mathrm{E}-03 \\
5.29 \mathrm{E}-03 \\
5.01 \mathrm{E}-03 \\
1.46 \mathrm{E}-03 \\
6.99 \mathrm{E}-03\end{array}$ & $\begin{array}{c}\text { U233 } \\
2.12 \mathrm{E}-04 \\
1.26 \mathrm{E}-04 \\
1.74 \mathrm{E}-04 \\
1.75 \mathrm{E}-04 \\
1.89 \mathrm{E}-04 \\
1.59 \mathrm{E}-04 \\
1.71 \mathrm{E}-04 \\
1.84 \mathrm{E}-04 \\
1.79 \mathrm{E}-04 \\
1.90 \mathrm{E}-04 \\
1.52 \mathrm{E}-04 \\
1.88 \mathrm{E}-04 \\
1.33 \mathrm{E}-04 \\
2.14 \mathrm{E}-04 \\
2.08 \mathrm{E}-04 \\
1.61 \mathrm{E}-04 \\
2.41 \mathrm{E}-04 \\
2.58 \mathrm{E}-04 \\
1.87 \mathrm{E}-04 \\
1.92 \mathrm{E}-04 \\
1.41 \mathrm{E}-04 \\
2.05 \mathrm{E}-05 \\
2.38 \mathrm{E}-04 \\
9.16 \mathrm{E}-05 \\
2.38 \mathrm{E}-04 \\
2.48 \mathrm{E}-04 \\
2.70 \mathrm{E}-04 \\
2.77 \mathrm{E}-04 \\
2.20 \mathrm{E}-04 \\
1.40 \mathrm{E}-04 \\
2.59 \mathrm{E}-04 \\
2.02 \mathrm{E}-04 \\
2.07 \mathrm{E}-04 \\
3.50 \mathrm{E}-04 \\
1.74 \mathrm{E}-04 \\
4.05 \mathrm{E}-04 \\
2.48 \mathrm{E}-04 \\
2.40 \mathrm{E}-04 \\
2.44 \mathrm{E}-04 \\
3.07 \mathrm{E}-04 \\
3.70 \mathrm{E}-04 \\
1.77 \mathrm{E}-04 \\
1.69 \mathrm{E}-04 \\
4.20 \mathrm{E}-04 \\
1.61 \mathrm{E}-04 \\
1.30 \mathrm{E}-04 \\
2.45 \mathrm{E}-04 \\
4.24 \mathrm{E}-04 \\
4.17 \mathrm{E}-04 \\
2.21 \mathrm{E}-04 \\
4.01 \mathrm{E}-04 \\
3.29 \mathrm{E}-04 \\
2.50 \mathrm{E}-04 \\
2.27 \mathrm{E}-04 \\
3.58 \mathrm{E}-04 \\
1.88 \mathrm{E}-04 \\
4.43 \mathrm{E}-04 \\
2.92 \mathrm{E}-04 \\
2.85 \mathrm{E}-04 \\
3.19 \mathrm{E}-04 \\
3.02 \mathrm{E}-04 \\
1.74 \mathrm{E}-04 \\
1.42 \mathrm{E}-04 \\
4.19 \mathrm{E}-04 \\
2.11 \mathrm{E}-04\end{array}$ & $\begin{array}{l}\text { U234 } \\
9.36 \mathrm{E}+01 \\
5.54 \mathrm{E}+01 \\
8.01 \mathrm{E}+01 \\
8.07 \mathrm{E}+01 \\
8.72 \mathrm{E}+01 \\
8.38 \mathrm{~B}+01 \\
8.70 \mathrm{0}+01 \\
1.20 \mathrm{E}+02 \\
1.05 \mathrm{E}+02 \\
1.09 \mathrm{E}+02 \\
1.06 \mathrm{E}+02 \\
1.31 \mathrm{E}+02 \\
7.89 \mathrm{E}+01 \\
1.41 \mathrm{E}+02 \\
1.23 \mathrm{E}+02 \\
1.29 \mathrm{E}+02 \\
1.65 \mathrm{E}+02 \\
1.70 \mathrm{E}+02 \\
1.32 \mathrm{E}+02 \\
1.21 \mathrm{E}+02 \\
8.47 \mathrm{E}+01 \\
3.28 \mathrm{E}+01 \\
1.58 \mathrm{E}+02 \\
5.50 \mathrm{E}+01 \\
1.59 \mathrm{E}+02 \\
1.41 \mathrm{E}+02 \\
1.54 \mathrm{E}+02 \\
1.62 \mathrm{E}+02 \\
1.40 \mathrm{E}+02 \\
7.57 \mathrm{E}+01 \\
1.52 \mathrm{E}+02 \\
9.57 \mathrm{E}+01 \\
1.06 \mathrm{E}+02 \\
1.54 \mathrm{E}+02 \\
8.91 \mathrm{E}+01 \\
1.93 \mathrm{E}+02 \\
1.21 \mathrm{E}+02 \\
1.15 \mathrm{E}+02 \\
1.19 \mathrm{E}+02 \\
1.47 \mathrm{E}+02 \\
1.78 \mathrm{E}+02 \\
8.72 \mathrm{E}+01 \\
9.78 \mathrm{E}+01 \\
2.02 \mathrm{E}+02 \\
9.77 \mathrm{E}+01 \\
7.14 \mathrm{E}+01 \\
1.27 \mathrm{E}+02 \\
2.20 \mathrm{E}+02 \\
2.24 \mathrm{E}+02 \\
1.24 \mathrm{E}+02 \\
2.16 \mathrm{E}+02 \\
1.72 \mathrm{E}+02 \\
1.31 \mathrm{E}+02 \\
1.13 \mathrm{E}+02 \\
2.00 \mathrm{E}+02 \\
9.21 \mathrm{E}+01 \\
2.17 \mathrm{E}+02 \\
1.54 \mathrm{E}+02 \\
1.50 \mathrm{E}+02 \\
1.68 \mathrm{E}+02 \\
1.73 \mathrm{E}+02 \\
1.12 \mathrm{E}+02 \\
6.50 \mathrm{E}+01 \\
1.95 \mathrm{E}+02 \\
1.12 \mathrm{E}+02\end{array}$ & $\begin{array}{l}\text { U235 } \\
3.92 \mathrm{E}+00 \\
2.32 \mathrm{E}+00 \\
3.37 \mathrm{E}+00 \\
3.39 \mathrm{E}+00 \\
3.66 \mathrm{E}+00 \\
3.56 \mathrm{E}+00 \\
3.69 \mathrm{E}+00 \\
5.18 \mathrm{E}+00 \\
4.51 \mathrm{E}+00 \\
4.64 \mathrm{E}+00 \\
4.58 \mathrm{E}+00 \\
5.67 \mathrm{E}+00 \\
3.38 \mathrm{E}+00 \\
6.09 \mathrm{E}+00 \\
5.29 \mathrm{E}+00 \\
5.62 \mathrm{E}+00 \\
7.12 \mathrm{E}+00 \\
7.35 \mathrm{E}+00 \\
5.72 \mathrm{E}+00 \\
5.20 \mathrm{E}+00 \\
3.63 \mathrm{E}+00 \\
1.48 \mathrm{E}+00 \\
6.83 \mathrm{E}+00 \\
2.36 \mathrm{E}+00 \\
6.86 \mathrm{E}+00 \\
6.02 \mathrm{E}+00 \\
6.56 \mathrm{E}+00 \\
6.91 \mathrm{E}+00 \\
6.01 \mathrm{E}+00 \\
3.22 \mathrm{E}+00 \\
6.49 \mathrm{E}+00 \\
4.02 \mathrm{E}+00 \\
4.46 \mathrm{E}+00 \\
6.43 \mathrm{E}+00 \\
3.76 \mathrm{E}+00 \\
8.12 \mathrm{E}+00 \\
5.11 \mathrm{E}+00 \\
4.82 \mathrm{E}+00 \\
5.02 \mathrm{E}+00 \\
6.18 \mathrm{E}+00 \\
7.47 \mathrm{E}+00 \\
3.67 \mathrm{E}+00 \\
4.17 \mathrm{E}+00 \\
8.50 \mathrm{E}+00 \\
4.18 \mathrm{E}+00 \\
3.03 \mathrm{E}+00 \\
5.36 \mathrm{E}+00 \\
9.32 \mathrm{E}+00 \\
9.48 \mathrm{E}+00 \\
5.29 \mathrm{E}+00 \\
9.13 \mathrm{E}+00 \\
7.27 \mathrm{E}+00 \\
5.52 \mathrm{E}+00 \\
4.77 \mathrm{E}+00 \\
8.48 \mathrm{E}+00 \\
3.87 \mathrm{E}+00 \\
9.10 \mathrm{E}+00 \\
6.49 \mathrm{E}+00 \\
6.35 \mathrm{E}+00 \\
7.10 \mathrm{E}+00 \\
7.36 \mathrm{E}+00 \\
4.81 \mathrm{E}+00 \\
2.71 \mathrm{E}+00 \\
8.16 \mathrm{E}+00 \\
4.74 \mathrm{E}+00\end{array}$ & $\begin{array}{l}U 236 \\
1.98 \mathrm{E}+00 \\
1.18 \mathrm{E}+00 \\
1.64 \mathrm{E}+00 \\
1.65 \mathrm{E}+00 \\
1.78 \mathrm{E}+00 \\
1.52 \mathrm{E}+00 \\
1.63 \mathrm{E}+00 \\
1.79 \mathrm{E}+00 \\
1.73 \mathrm{E}+00 \\
1.84 \mathrm{E}+00 \\
1.49 \mathrm{E}+00 \\
1.84 \mathrm{E}+00 \\
1.30 \mathrm{E}+00 \\
2.11 \mathrm{E}+00 \\
2.03 \mathrm{E}+00 \\
1.60 \mathrm{E}+00 \\
2.38 \mathrm{E}+00 \\
2.54 \mathrm{E}+00 \\
1.86 \mathrm{E}+00 \\
1.89 \mathrm{E}+00 \\
1.39 \mathrm{E}+00 \\
2.12 \mathrm{E}-01 \\
2.36 \mathrm{E}+00 \\
9.03 \mathrm{E}-01 \\
2.37 \mathrm{E}+00 \\
2.44 \mathrm{E}+00 \\
2.66 \mathrm{E}+00 \\
2.74 \mathrm{E}+00 \\
2.19 \mathrm{E}+00 \\
1.38 \mathrm{E}+00 \\
2.57 \mathrm{E}+00 \\
1.96 \mathrm{E}+00 \\
2.04 \mathrm{E}+00 \\
3.40 \mathrm{E}+00 \\
1.72 \mathrm{E}+00 \\
3.97 \mathrm{E}+00 \\
2.44 \mathrm{E}+00 \\
2.36 \mathrm{E}+00 \\
2.40 \mathrm{E}+00 \\
3.02 \mathrm{E}+00 \\
3.65 \mathrm{E}+00 \\
1.75 \mathrm{E}+00 \\
1.70 \mathrm{E}+00 \\
4.15 \mathrm{E}+00 \\
1.63 \mathrm{E}+00 \\
1.30 \mathrm{E}+00 \\
2.45 \mathrm{E}+00 \\
4.25 \mathrm{E}+00 \\
4.19 \mathrm{E}+00 \\
2.22 \mathrm{E}+00 \\
4.04 \mathrm{E}+00 \\
3.32 \mathrm{E}+00 \\
2.52 \mathrm{E}+00 \\
2.28 \mathrm{E}+00 \\
3.64 \mathrm{E}+00 \\
1.89 \mathrm{E}+00 \\
4.45 \mathrm{E}+00 \\
2.96 \mathrm{E}+00 \\
2.90 \mathrm{E}+00 \\
3.24 \mathrm{E}+00 \\
3.09 \mathrm{E}+00 \\
1.81 \mathrm{E}+00 \\
1.43 \mathrm{E}+00 \\
4.22 \mathrm{E}+00 \\
2.16 \mathrm{E}+00\end{array}$ & $\begin{array}{l}\mathrm{U} 238 \\
9.62 \mathrm{E}+01 \\
5.70 \mathrm{E}+01 \\
8.23 \mathrm{E}+01 \\
8.29 \mathrm{E}+01 \\
8.96 \mathrm{E}+01 \\
8.59 \mathrm{E}+01 \\
8.92 \mathrm{E}+01 \\
1.23 \mathrm{E}+02 \\
1.08 \mathrm{E}+02 \\
1.31 \mathrm{E}+02 \\
1.08 \mathrm{E}+02 \\
1.34 \mathrm{E}+02 \\
8.07 \mathrm{E}+01 \\
1.44 \mathrm{E}+02 \\
1.26 \mathrm{E}+02 \\
1.31 \mathrm{E}+02 \\
1.68 \mathrm{E}+02 \\
1.74 \mathrm{E}+02 \\
1.35 \mathrm{E}+02 \\
1.24 \mathrm{E}+02 \\
8.66 \mathrm{E}+01 \\
3.32 \mathrm{E}+01 \\
1.62 \mathrm{E}+02 \\
5.63 \mathrm{E}+01 \\
1.62 \mathrm{E}+02 \\
1.44 \mathrm{E}+02 \\
1.57 \mathrm{E}+02 \\
1.66 \mathrm{E}+02 \\
1.43 \mathrm{E}+02 \\
7.76 \mathrm{E}+01 \\
1.55 \mathrm{E}+02 \\
9.83 \mathrm{E}+01 \\
1.08 \mathrm{E}+02 \\
1.59 \mathrm{E}+02 \\
9.14 \mathrm{E}+01 \\
1.99 \mathrm{E}+02 \\
1.25 \mathrm{E}+02 \\
1.18 \mathrm{E}+02 \\
1.23 \mathrm{E}+02 \\
1.51 \mathrm{E}+02 \\
1.83 \mathrm{E}+02 \\
8.96 \mathrm{E}+01 \\
1.00 \mathrm{E}+02 \\
2.08 \mathrm{E}+02 \\
1.00 \mathrm{E}+02 \\
7.32 \mathrm{E}+01 \\
1.30 \mathrm{E}+02 \\
2.26 \mathrm{E}+02 \\
2.29 \mathrm{E}+02 \\
1.27 \mathrm{E}+02 \\
2.21 \mathrm{E}+02 \\
1.77 \mathrm{E}+02 \\
1.34 \mathrm{E}+02 \\
1.16 \mathrm{E}+02 \\
2.05 \mathrm{E}+02 \\
9.47 \mathrm{E}+01 \\
2.23 \mathrm{E}+02 \\
1.58 \mathrm{E}+02 \\
1.54 \mathrm{E}+02 \\
1.73 \mathrm{E}+02 \\
1.77 \mathrm{E}+02 \\
1.15 \mathrm{E}+02 \\
6.69 \mathrm{E}+01 \\
2.01 \mathrm{E}+02 \\
1.15 \mathrm{E}+02\end{array}$ & 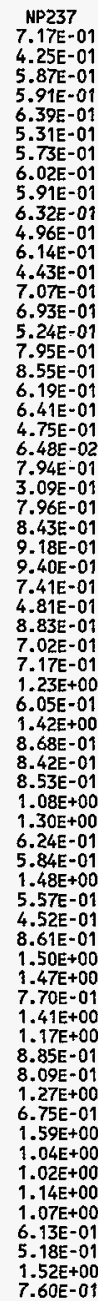 \\
\hline
\end{tabular}


HNF-SD-WM-TI-794, ReV. 0

ACTIVITY IN UNSEPARATED FUEL (Curies decayed to $1 / 1 / 94$ )

\begin{tabular}{|c|c|c|c|c|c|c|c|c|c|}
\hline 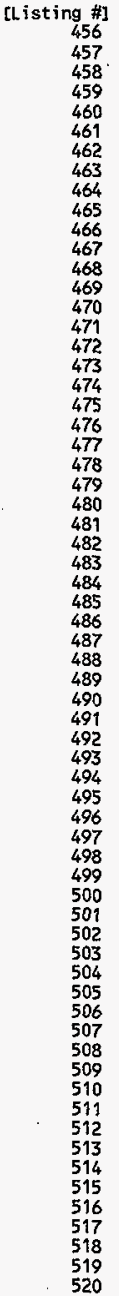 & 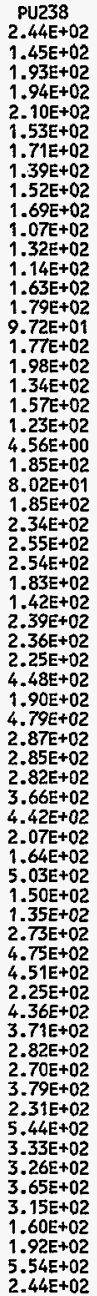 & 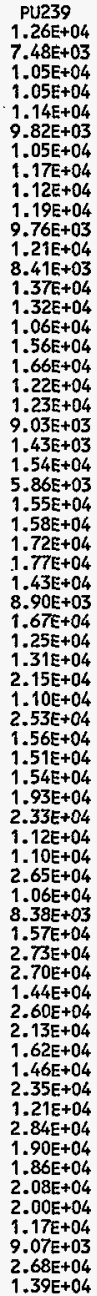 & 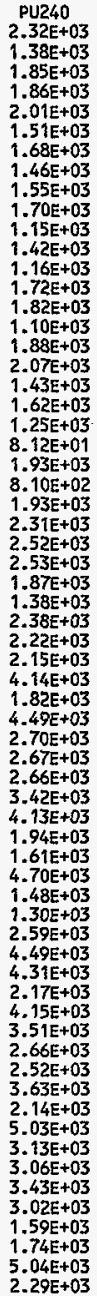 & 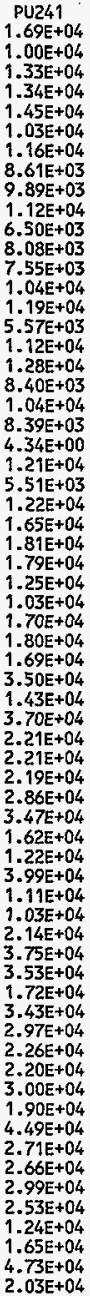 & 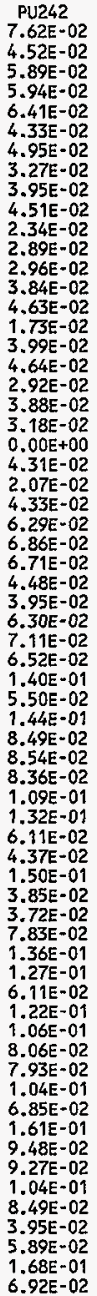 & 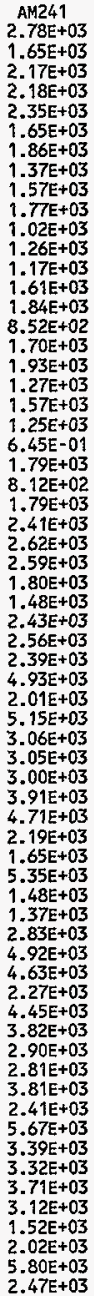 & 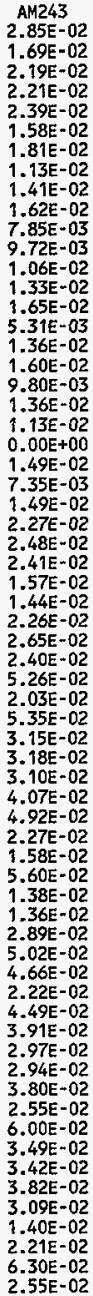 & 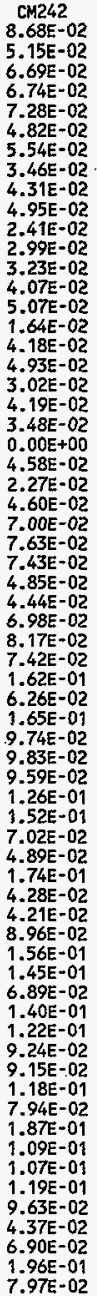 & 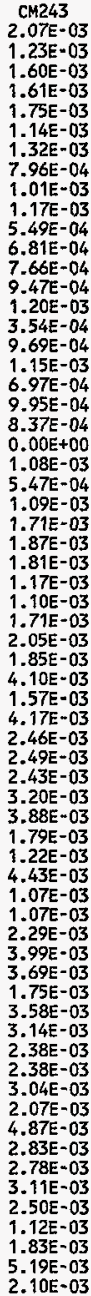 \\
\hline
\end{tabular}


HNF-SD-WM-TI-794, ReV. 0

ACTIVITY IN UNSEPARATED FUEL (Curies decayed to $1 / 1 / 94$ )

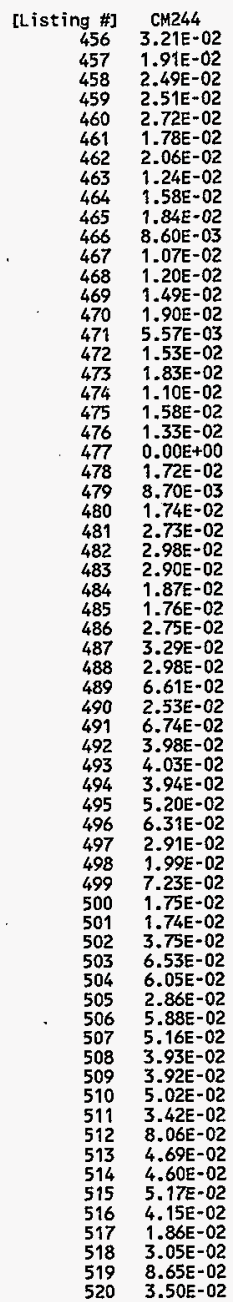


HNF-SD-WM-TI-794, ReV, O

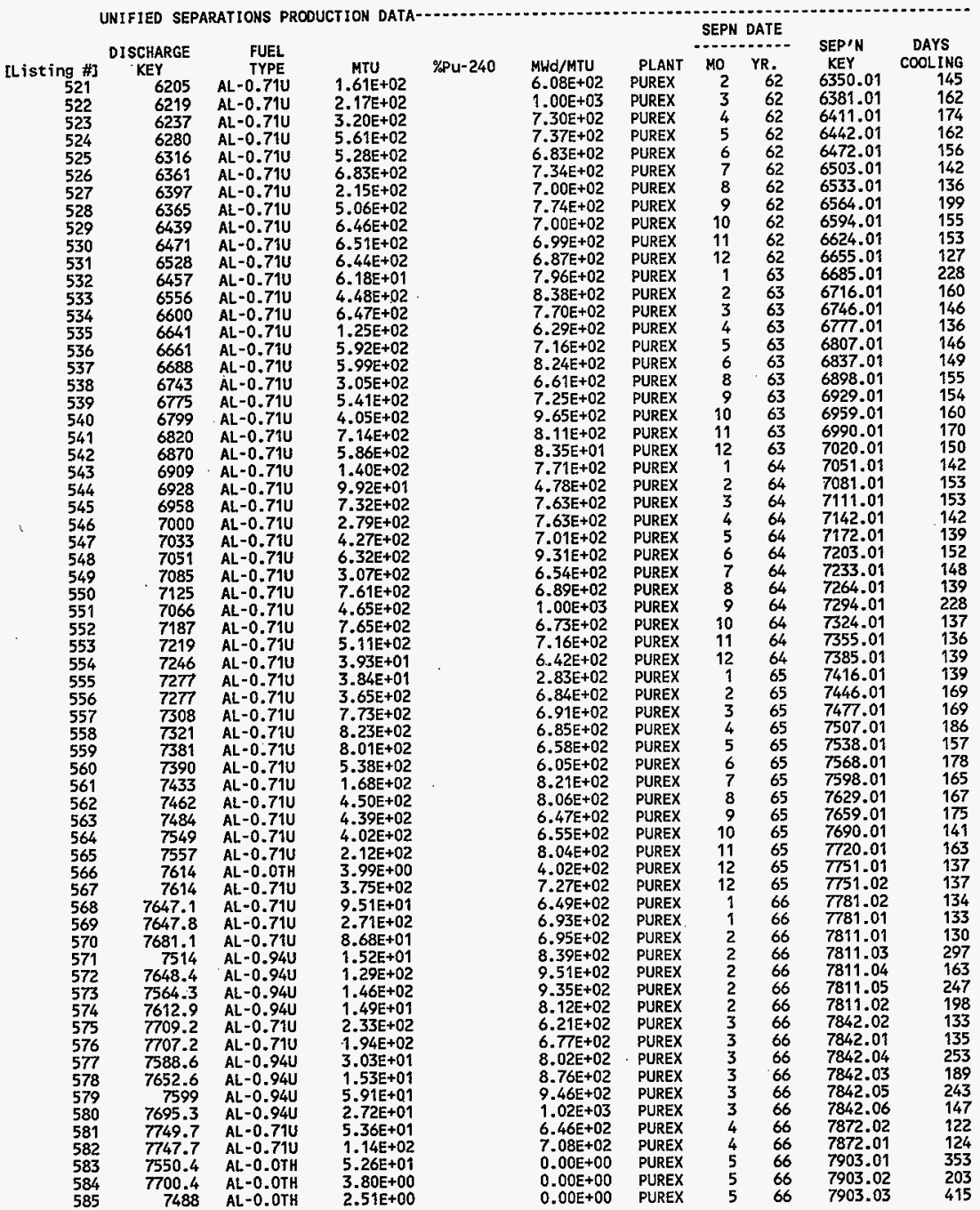


HNF-SD-WM-II-794, Rev, 0

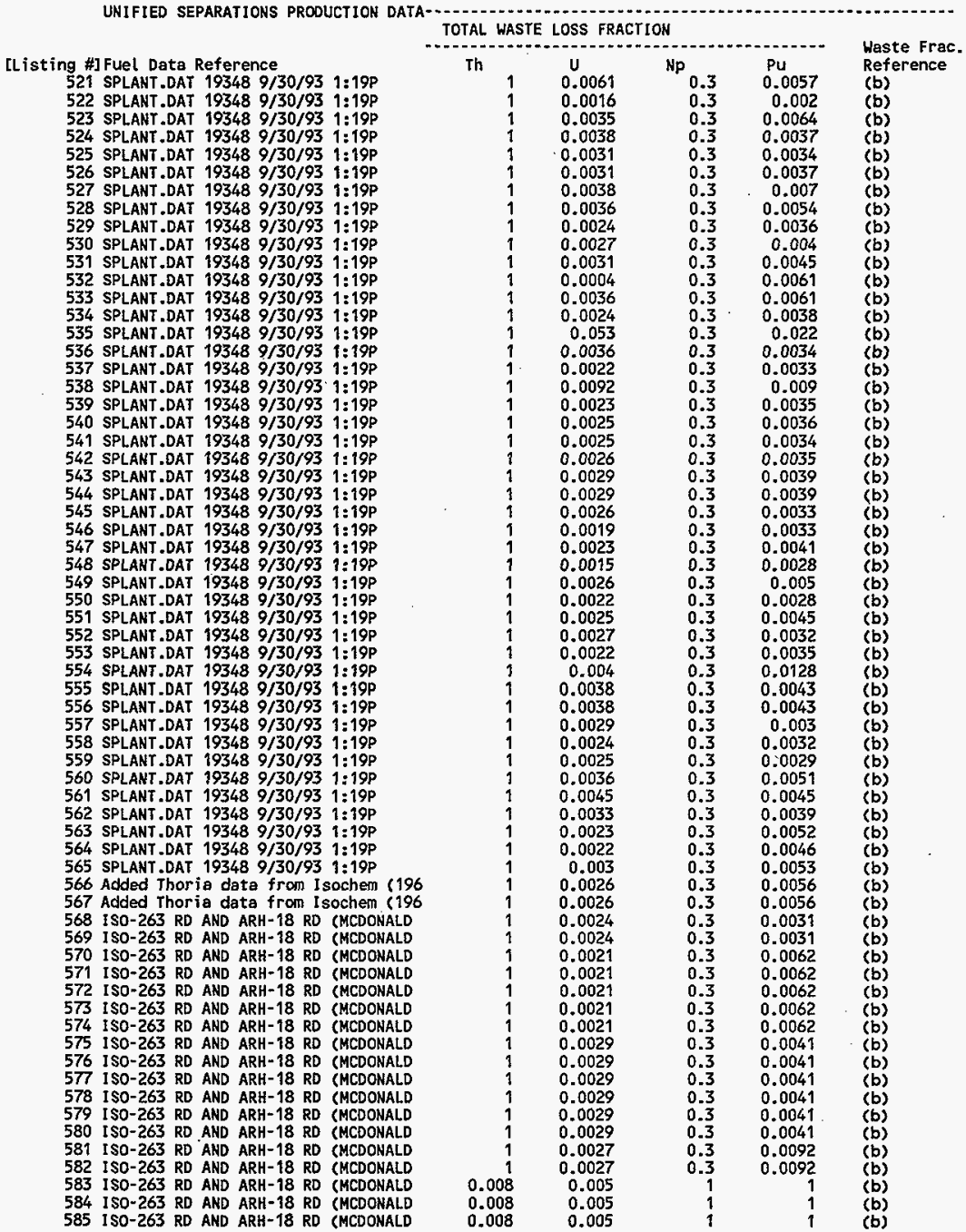


HNF-SD-WW-TI-794, ReV. O

ACTIVITY IN UNSEPARATED FUEL (Curies decayed to 1/1/94)

\begin{tabular}{|c|c|c|c|c|c|c|c|c|c|}
\hline 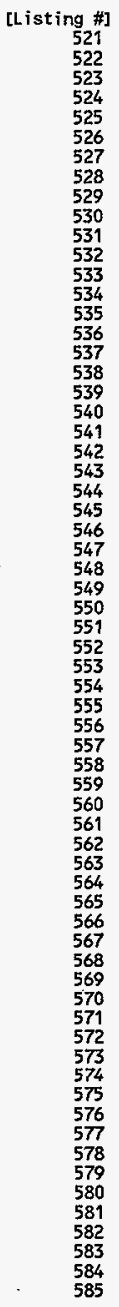 & $\begin{array}{l}H 03 \\
2.54 E+02 \\
5.75 E+02 \\
6.12 E+02 \\
1.09 E+03 \\
9.54 E+02 \\
1.34 E+03 \\
4.03 E+02 \\
1.05 E+03 \\
1.22 E+03 \\
1.23 E+03 \\
1.21 E+03 \\
1.34 E+02 \\
1.04 E+03 \\
1.38 E+03 \\
2.17 E+02 \\
1.18 E+03 \\
1.39 E+03 \\
5.69 E+02 \\
1.11 E+03 \\
1.13 E+03 \\
1.66 E+03 \\
1.38 E+02 \\
3.15 E+02 \\
1.37 E+02 \\
1.64 E+03 \\
6.28 E+02 \\
8.84 E+02 \\
1.76 E+03 \\
5.96 E+02 \\
1.57 E+03 \\
1.40 E+03 \\
1.56 E+03 \\
1.11 E+03 \\
7.68 E+01 \\
3.27 E+01 \\
7.66 E+02 \\
1.65 E+03 \\
1.74 E+03 \\
1.64 E+03 \\
1.01 E+03 \\
4.36 E+02 \\
1.15 E+03 \\
8.97 E+02 \\
8.40 E+02 \\
5.47 E+02 \\
1.12 E+00 \\
8.81 E+02 \\
2.00 E+02 \\
6.09 E+02 \\
1.97 E+02 \\
3.94 E+01 \\
3.88 E+02 \\
4.26 E+02 \\
3.80 E+01 \\
4.73 E+02 \\
4.30 E+02 \\
7.59 E+01 \\
4.24 E+01 \\
1.76 E+02 \\
8.85 E+01 \\
1.14 E+02 \\
2.65 E+02 \\
1.46 E+01 \\
1.08 E+00 \\
6.91 E-01\end{array}$ & $\begin{array}{l}c 14 \\
6.25 \mathrm{E}+00 \\
1.37 \mathrm{E}+01 \\
1.48 \mathrm{E}+01 \\
2.62 \mathrm{E}+01 \\
2.29 \mathrm{E}+01 \\
3.18 \mathrm{E}+01 \\
9.55 \mathrm{E}+00 \\
2.48 \mathrm{E}+01 \\
2.87 \mathrm{E}+01 \\
2.89 \mathrm{E}+01 \\
2.81 \mathrm{E}+01 \\
3.12 \mathrm{E}+00 \\
2.38 \mathrm{E}+01 \\
3.16 \mathrm{E}+01 \\
4.99 \mathrm{E}+00 \\
2.69 \mathrm{E}+01 \\
3.13 \mathrm{E}+01 \\
1.28 \mathrm{E}+01 \\
2.49 \mathrm{E}+01 \\
2.47 \mathrm{E}+01 \\
3.67 \mathrm{E}+01 \\
3.16 \mathrm{E}+00 \\
6.86 \mathrm{E}+00 \\
3.03 \mathrm{E}+00 \\
3.54 \mathrm{E}+01 \\
1.35 \mathrm{E}+01 \\
1.90 \mathrm{E}+01 \\
3.72 \mathrm{E}+01 \\
1.28 \mathrm{E}+01 \\
3.34 \mathrm{E}+01 \\
2.93 \mathrm{E}+01 \\
3.28 \mathrm{E}+01 \\
2.32 \mathrm{E}+01 \\
1.61 \mathrm{E}+00 \\
6.97 \mathrm{E}-01 \\
1.59 \mathrm{E}+01 \\
3.40 \mathrm{E}+01 \\
3.59 \mathrm{E}+01 \\
3.36 \mathrm{E}+01 \\
2.08 \mathrm{E}+01 \\
8.74 \mathrm{E}+00 \\
2.30 \mathrm{E}+01 \\
1.81 \mathrm{E}+01 \\
1.68 \mathrm{E}+01 \\
1.08 \mathrm{E}+01 \\
9.54 \mathrm{E}-07 \\
1.73 \mathrm{E}+01 \\
3.93 \mathrm{E}+00 \\
1.19 \mathrm{E}+01 \\
3.84 \mathrm{E}+00 \\
6.34 \mathrm{E}-01 \\
6.09 \mathrm{E}+00 \\
6.78 \mathrm{E}+00 \\
6.02 \mathrm{E}-01 \\
9.22 \mathrm{E}+00 \\
8.36 \mathrm{E}+00 \\
1.21 \mathrm{E}+00 \\
6.67 \mathrm{E}-01 \\
2.78 \mathrm{E}+00 \\
1.38 \mathrm{E}+00 \\
2.20 \mathrm{E}+00 \\
5.11 \mathrm{E}+00 \\
1.26 \mathrm{E}-05 \\
9.10 \mathrm{E}-07 \\
6.01 \mathrm{E}-07\end{array}$ & $\begin{array}{l}\text { N159 } \\
1.77 \mathrm{E}+00 \\
3.88 \mathrm{E}+00 \\
4.20 \mathrm{E}+00 \\
7.43 \mathrm{E}+00 \\
6.50 \mathrm{E}+00 \\
9.01 \mathrm{E}+00 \\
2.70 \mathrm{E}+00 \\
7.03 \mathrm{E}+00 \\
8.14 \mathrm{E}+00 \\
8.19 \mathrm{E}+00 \\
7.97 \mathrm{E}+00 \\
8.83 \mathrm{E}-01 \\
6.73 \mathrm{E}+00 \\
8.93 \mathrm{E}+00 \\
1.41 \mathrm{E}+00 \\
7.63 \mathrm{E}+00 \\
8.85 \mathrm{E}+00 \\
3.63 \mathrm{E}+00 \\
7.05 \mathrm{E}+00 \\
6.97 \mathrm{E}+00 \\
1.04 \mathrm{E}+01 \\
9.01 \mathrm{E}-01 \\
1.94 \mathrm{E}+00 \\
8.60 \mathrm{E}-01 \\
1.00 \mathrm{E}+01 \\
3.82 \mathrm{E}+00 \\
5.39 \mathrm{E}+00 \\
1.05 \mathrm{E}+01 \\
3.62 \mathrm{E}+00 \\
9.44 \mathrm{E}+00 \\
8.28 \mathrm{E}+00 \\
9.29 \mathrm{E}+00 \\
6.58 \mathrm{E}+00 \\
4.55 \mathrm{E}-01 \\
1.98 \mathrm{E}-01 \\
4.50 \mathrm{E}+00 \\
9.61 \mathrm{E}+00 \\
1.02 \mathrm{E}+01 \\
9.51 \mathrm{E}+00 \\
5.88 \mathrm{E}+00 \\
2.47 \mathrm{E}+00 \\
6.50 \mathrm{E}+00 \\
5.12 \mathrm{E}+00 \\
4.74 \mathrm{E}+00 \\
3.05 \mathrm{E}+00 \\
0.00 \mathrm{E}+00 \\
4.90 \mathrm{E}+00 \\
1.11 \mathrm{E}+00 \\
3.38 \mathrm{E}+00 \\
1.09 \mathrm{E}+00 \\
1.80 \mathrm{E}-01 \\
1.72 \mathrm{E}+00 \\
1.92 \mathrm{E}+00 \\
1.71 \mathrm{E}-01 \\
2.61 \mathrm{E}+00 \\
2.37 \mathrm{E}+00 \\
3.42 \mathrm{E}-01 \\
1.89 \mathrm{E}-01 \\
7.87 \mathrm{E}-01 \\
3.90 \mathrm{E}-01 \\
6.24 \mathrm{E}-01 \\
1.45 \mathrm{E}+00 \\
0.00 \mathrm{E}+00 \\
0.00 \mathrm{E}+00 \\
0.00 \mathrm{E}+00\end{array}$ & $\begin{array}{l}1163 \\
1.73 \mathrm{E}+02 \\
3.81 \mathrm{E}+02 \\
4.12 \mathrm{E}+02 \\
7.29 \mathrm{E}+02 \\
6.38 \mathrm{E}+02 \\
8.86 \mathrm{E}+02 \\
2.66 \mathrm{E}+02 \\
6.91 \mathrm{E}+02 \\
8.01 \mathrm{E}+02 \\
8.07 \mathrm{E}+02 \\
7.86 \mathrm{E}+02 \\
8.70 \mathrm{E}+01 \\
6.65 \mathrm{E}+02 \\
8.83 \mathrm{E}+02 \\
1.40 \mathrm{E}+02 \\
7.54 \mathrm{E}+02 \\
8.76 \mathrm{E}+02 \\
3.60 \mathrm{E}+02 \\
6.99 \mathrm{E}+02 \\
6.92 \mathrm{E}+02 \\
1.03 \mathrm{E}+03 \\
8.91 \mathrm{E}+01 \\
1.93 \mathrm{E}+02 \\
8.54 \mathrm{E}+01 \\
9.97 \mathrm{E}+02 \\
3.81 \mathrm{E}+02 \\
5.37 \mathrm{E}+02 \\
1.05 \mathrm{E}+03 \\
3.61 \mathrm{E}+02 \\
9.43 \mathrm{E}+02 \\
8.27 \mathrm{E}+02 \\
9.28 \mathrm{E}+02 \\
6.58 \mathrm{E}+02 \\
4.55 \mathrm{E}+01 \\
1.98 \mathrm{E}+01 \\
4.51 \mathrm{E}+02 \\
9.63 \mathrm{E}+02 \\
1.02 \mathrm{E}+03 \\
9.54 \mathrm{E}+02 \\
5.90 \mathrm{E}+02 \\
2.49 \mathrm{E}+02 \\
6.54 \mathrm{E}+02 \\
5.15 \mathrm{E}+02 \\
4.78 \mathrm{E}+02 \\
3.07 \mathrm{E}+02 \\
0.00 \mathrm{E}+00 \\
4.94 \mathrm{E}+02 \\
1.12 \mathrm{E}+02 \\
3.41 \mathrm{E}+02 \\
1.10 \mathrm{E}+02 \\
1.81 \mathrm{E}+01 \\
1.74 \mathrm{E}+02 \\
1.93 \mathrm{E}+02 \\
1.72 \mathrm{E}+01 \\
2.64 \mathrm{E}+02 \\
2.39 \mathrm{E}+02 \\
3.45 \mathrm{E}+01 \\
1.90 \mathrm{E}+01 \\
7.93 \mathrm{E}+01 \\
3.94 \mathrm{E}+01 \\
6.31 \mathrm{E}+01 \\
1.46 \mathrm{E}+02 \\
0.00 \mathrm{E}+00 \\
0.00 \mathrm{E}+00 \\
0.00 \mathrm{E}+00\end{array}$ & $\begin{array}{l}\text { CO60 } \\
5.52 \mathrm{E}+00 \\
1.21 \mathrm{E}+01 \\
1.32 \mathrm{E}+01 \\
2.38 \mathrm{E}+01 \\
2.11 \mathrm{E}+01 \\
2.96 \mathrm{E}+01 \\
9.02 \mathrm{E}+00 \\
2.32 \mathrm{E}+01 \\
2.76 \mathrm{E}+01 \\
2.81 \mathrm{E}+01 \\
2.79 \mathrm{E}+01 \\
3.01 \mathrm{E}+00 \\
2.38 \mathrm{E}+01 \\
3.20 \mathrm{E}+01 \\
5.15 \mathrm{E}+00 \\
2.80 \mathrm{E}+01 \\
3.27 \mathrm{E}+01 \\
1.37 \mathrm{E}+01 \\
2.70 \mathrm{E}+01 \\
2.68 \mathrm{E}+01 \\
4.03 \mathrm{E}+01 \\
3.59 \mathrm{E}+00 \\
7.78 \mathrm{E}+00 \\
3.48 \mathrm{E}+00 \\
4.09 \mathrm{E}+01 \\
1.58 \mathrm{E}+01 \\
2.26 \mathrm{E}+01 \\
4.42 \mathrm{E}+01 \\
1.55 \mathrm{E}+01 \\
4.09 \mathrm{E}+01 \\
3.50 \mathrm{E}+01 \\
4.12 \mathrm{E}+01 \\
2.95 \mathrm{E}+01 \\
2.06 \mathrm{E}+00 \\
9.12 \mathrm{E}-01 \\
2.06 \mathrm{E}+01 \\
4.45 \mathrm{E}+01 \\
4.73 \mathrm{E}+01 \\
4.52 \mathrm{E}+01 \\
2.81 \mathrm{E}+01 \\
1.20 \mathrm{E}+01 \\
3.18 \mathrm{E}+01 \\
2.53 \mathrm{E}+01 \\
2.40 \mathrm{E}+01 \\
1.54 \mathrm{E}+01 \\
0.00 \mathrm{E}+00 \\
2.53 \mathrm{E}+01 \\
5.83 \mathrm{E}+00 \\
1.77 \mathrm{E}+01 \\
5.76 \mathrm{E}+00 \\
8.93 \mathrm{E}-01 \\
8.98 \mathrm{E}+00 \\
9.71 \mathrm{E}+00 \\
8.80 \mathrm{E}-01 \\
1.40 \mathrm{E}+01 \\
1.27 \mathrm{E}+01 \\
1.75 \mathrm{E}+00 \\
9.86 \mathrm{E}-01 \\
4.03 \mathrm{E}+00 \\
2.06 \mathrm{E}+00 \\
3.39 \mathrm{E}+00 \\
7.85 \mathrm{E}+00 \\
0.00 \mathrm{E}+00 \\
0.00 \mathrm{E}+00 \\
0.00 \mathrm{E}+00\end{array}$ & $\begin{array}{l}\text { SE79 } \\
1.32 E+00 \\
2.92 \mathrm{E}+00 \\
3.15 \mathrm{E}+00 \\
5.56 \mathrm{E}+00 \\
4.86 \mathrm{E}+00 \\
6.75 \mathrm{E}+00 \\
2.02 \mathrm{E}+00 \\
5.27 \mathrm{E}+00 \\
6.09 \mathrm{E}+00 \\
6.13 \mathrm{E}+00 \\
5.96 \mathrm{E}+00 \\
6.62 \mathrm{E}-01 \\
5.05 \mathrm{E}+00 \\
6.70 \mathrm{E}+00 \\
1.06 \mathrm{E}+00 \\
5.71 \mathrm{E}+00 \\
6.64 \mathrm{E}+00 \\
2.72 \mathrm{E}+00 \\
5.28 \mathrm{E}+00 \\
5.25 \mathrm{E}+00 \\
7.79 \mathrm{E}+00 \\
6.62 \mathrm{E}-01 \\
1.46 \mathrm{E}+00 \\
6.39 \mathrm{E}-01 \\
7.51 \mathrm{E}+00 \\
2.86 \mathrm{E}+00 \\
4.03 \mathrm{E}+00 \\
7.91 \mathrm{E}+00 \\
2.70 \mathrm{E}+00 \\
7.06 \mathrm{E}+00 \\
6.24 \mathrm{E}+00 \\
6.94 \mathrm{E}+00 \\
4.92 \mathrm{E}+00 \\
3.40 \mathrm{E}-01 \\
1.47 \mathrm{E}-01 \\
3.36 \mathrm{E}+00 \\
7.19 \mathrm{E}+00 \\
7.59 \mathrm{E}+00 \\
7.11 \mathrm{E}+00 \\
4.39 \mathrm{E}+00 \\
1.86 \mathrm{E}+00 \\
4.88 \mathrm{E}+00 \\
3.82 \mathrm{E}+00 \\
3.54 \mathrm{E}+00 \\
2.29 \mathrm{E}+00 \\
7.81 \mathrm{E}-03 \\
3.67 \mathrm{E}+00 \\
8.32 \mathrm{E}-01 \\
2.53 \mathrm{E}+00 \\
8.12 \mathrm{E}-01 \\
1.70 \mathrm{E}-01 \\
1.64 \mathrm{E}+00 \\
1.82 \mathrm{E}+00 \\
1.62 \mathrm{E}-01 \\
1.95 \mathrm{E}+00 \\
1.77 \mathrm{E}+00 \\
3.24 \mathrm{E}-01 \\
1.79 \mathrm{E}-01 \\
7.47 \mathrm{E}-01 \\
3.70 \mathrm{E}-01 \\
4.66 \mathrm{E}-01 \\
1.08 \mathrm{E}+00 \\
1.03 \mathrm{E}-01 \\
7.45 \mathrm{E}-00 \\
4.92 \mathrm{E}-03\end{array}$ & $\begin{array}{l}\text { SR90 } \\
1.30 \mathrm{E}+05 \\
2.82 \mathrm{E}+05 \\
3.08 \mathrm{E}+05 \\
5.47 \mathrm{E}+05 \\
4.80 \mathrm{E}+05 \\
6.66 \mathrm{E}+05 \\
2.01 \mathrm{E}+05 \\
5.19 \mathrm{E}+05 \\
6.05 \mathrm{E}+05 \\
6.11 \mathrm{E}+05 \\
5.97 \mathrm{E}+05 \\
6.56 \mathrm{E}+04 \\
5.03 \mathrm{E}+05 \\
6.70 \mathrm{E}+05 \\
1.07 \mathrm{E}+05 \\
5.75 \mathrm{E}+05 \\
6.67 \mathrm{E}+05 \\
2.76 \mathrm{E}+05 \\
5.36 \mathrm{E}+05 \\
5.27 \mathrm{E}+05 \\
7.89 \mathrm{E}+05 \\
6.99 \mathrm{E}+04 \\
1.49 \mathrm{E}+05 \\
6.64 \mathrm{E}+04 \\
7.70 \mathrm{E}+05 \\
2.94 \mathrm{E}+05 \\
4.16 \mathrm{E}+05 \\
8.08 \mathrm{E}+05 \\
2.81 \mathrm{E}+05 \\
7.35 \mathrm{E}+05 \\
6.37 \mathrm{E}+05 \\
7.26 \mathrm{E}+05 \\
5.15 \mathrm{E}+05 \\
3.57 \mathrm{E}+04 \\
1.57 \mathrm{E}+04 \\
3.54 \mathrm{E}+05 \\
7.57 \mathrm{E}+05 \\
8.00 \mathrm{E}+05 \\
7.53 \mathrm{E}+05 \\
4.66 \mathrm{E}+05 \\
1.96 \mathrm{E}+05 \\
5.15 \mathrm{E}+05 \\
4.08 \mathrm{E}+05 \\
3.80 \mathrm{E}+05 \\
2.43 \mathrm{E}+05 \\
5.31 \mathrm{E}+02 \\
3.93 \mathrm{E}+05 \\
8.97 \mathrm{E}+04 \\
2.72 \mathrm{E}+05 \\
8.77 \mathrm{E}+04 \\
1.87 \mathrm{E}+04 \\
1.81 \mathrm{E}+05 \\
2.00 \mathrm{E}+05 \\
1.79 \mathrm{E}+04 \\
2.12 \mathrm{E}+05 \\
1.91 \mathrm{E}+05 \\
3.59 \mathrm{E}+04 \\
1.98 \mathrm{E}+04 \\
8.22 \mathrm{E}+04 \\
4.09 \mathrm{E}+04 \\
5.06 \mathrm{E}+04 \\
1.17 \mathrm{E}+05 \\
6.99 \mathrm{E}+03 \\
5.09 \mathrm{E}+02 \\
3.32 \mathrm{E}+02\end{array}$ & $\begin{array}{c}Y 90 \\
1.30 E+05 \\
2.82 E+05 \\
3.08 \mathrm{E}+05 \\
5.47 \mathrm{E}+05 \\
4.80 \mathrm{E}+05 \\
6.66 \mathrm{E}+05 \\
2.01 \mathrm{E}+05 \\
5.19 \mathrm{E}+05 \\
6.05 \mathrm{E}+05 \\
6.11 \mathrm{E}+05 \\
5.97 \mathrm{E}+05 \\
6.56 \mathrm{E}+04 \\
5.03 \mathrm{E}+05 \\
6.70 \mathrm{E}+05 \\
1.07 \mathrm{E}+05 \\
5.76 \mathrm{E}+05 \\
6.67 \mathrm{E}+05 \\
2.76 \mathrm{E}+05 \\
5.36 \mathrm{E}+05 \\
5.27 \mathrm{E}+05 \\
7.90 \mathrm{E}+05 \\
6.99 \mathrm{E}+04 \\
1.49 \mathrm{E}+05 \\
6.64 \mathrm{E}+04 \\
7.70 \mathrm{E}+05 \\
2.94 \mathrm{E}+05 \\
4.16 \mathrm{E}+05 \\
8.09 \mathrm{E}+05 \\
2.81 \mathrm{E}+05 \\
7.35 \mathrm{E}+05 \\
6.37 \mathrm{E}+05 \\
7.26 \mathrm{E}+05 \\
5.15 \mathrm{E}+05 \\
3.57 \mathrm{E}+04 \\
1.57 \mathrm{E}+04 \\
3.54 \mathrm{E}+05 \\
7.57 \mathrm{E}+05 \\
8.00 \mathrm{E}+05 \\
7.53 \mathrm{E}+05 \\
4.67 \mathrm{E}+05 \\
1.96 \mathrm{E}+05 \\
5.16 \mathrm{E}+05 \\
4.09 \mathrm{E}+05 \\
3.80 \mathrm{E}+05 \\
2.43 \mathrm{E}+05 \\
5.31 \mathrm{E}+02 \\
3.93 \mathrm{E}+05 \\
8.98 \mathrm{E}+04 \\
2.72 \mathrm{E}+05 \\
8.77 \mathrm{E}+04 \\
1.87 \mathrm{E}+04 \\
1.81 \mathrm{E}+05 \\
2.00 \mathrm{E}+05 \\
1.79 \mathrm{E}+04 \\
2.12 \mathrm{E}+05 \\
1.92 \mathrm{E}+05 \\
3.59 \mathrm{E}+04 \\
1.98 \mathrm{E}+04 \\
8.22 \mathrm{E}+04 \\
4.09 \mathrm{E}+04 \\
5.07 \mathrm{E}+04 \\
1.17 \mathrm{E}+05 \\
6.99 \mathrm{E}+03 \\
5.10 \mathrm{E}+02 \\
3.32 \mathrm{E}+02\end{array}$ & 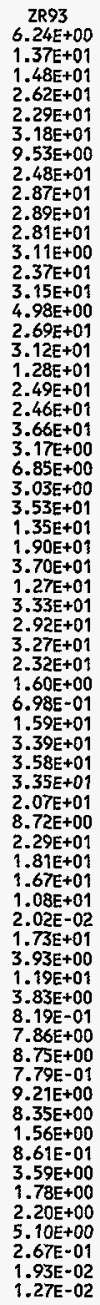 \\
\hline
\end{tabular}


HNF-SD-WM-TI-794, ReV. 0

ACTIVITY IN UNSEPARATED FUEL (Curies decayed to 1/1/94)

\begin{tabular}{|c|c|c|c|c|c|c|c|c|c|}
\hline $\begin{array}{r}\text { [Listing } \mathbf{~}] \\
\mathbf{5 2 1} \\
\mathbf{5 2 2} \\
\mathbf{5 2 3} \\
\mathbf{5 2 4} \\
\mathbf{5 2 5} \\
\mathbf{5 2 6} \\
\mathbf{5 2 7} \\
\mathbf{5 2 8} \\
\mathbf{5 2 9} \\
\mathbf{5 3 0} \\
\mathbf{5 3 1} \\
\mathbf{5 3 2} \\
\mathbf{5 3 3} \\
\mathbf{5 3 4} \\
\mathbf{5 3 5} \\
\mathbf{5 3 6} \\
\mathbf{5 3 7} \\
\mathbf{5 3 8} \\
\mathbf{5 3 9} \\
\mathbf{5 4 0} \\
\mathbf{5 4 1} \\
\mathbf{5 4 2} \\
\mathbf{5 4 3} \\
\mathbf{5 4 4} \\
\mathbf{5 4 5} \\
\mathbf{5 4 6} \\
\mathbf{5 4 7} \\
\mathbf{5 4 8} \\
\mathbf{5 4 9} \\
\mathbf{5 5 0} \\
\mathbf{5 5 1} \\
\mathbf{5 5 2} \\
\mathbf{5 5 3} \\
\mathbf{5 5 4} \\
\mathbf{5 5 5} \\
\mathbf{5 5 6} \\
\mathbf{5 5 7} \\
\mathbf{5 5 8} \\
\mathbf{5 5 9} \\
\mathbf{5 6 0} \\
\mathbf{5 6 1} \\
\mathbf{5 6 2} \\
\mathbf{5 6 3} \\
\mathbf{5 6 4} \\
\mathbf{5 6 5} \\
\mathbf{5 6 6} \\
\mathbf{5 6 7} \\
\mathbf{5 6 8} \\
\mathbf{5 6 9} \\
\mathbf{5 7 0} \\
\mathbf{5 7 1} \\
\mathbf{5 7 2} \\
\mathbf{5 7 3} \\
\mathbf{5 7 4} \\
\mathbf{5 7 5} \\
\mathbf{5 7 6} \\
\mathbf{5 7 7} \\
\mathbf{5 7 8} \\
\mathbf{5 7 9} \\
\mathbf{5 8 0} \\
\mathbf{5 8 1} \\
\mathbf{5 8 2} \\
\mathbf{5 8 3} \\
\mathbf{5 8 4} \\
\mathbf{5 8 5}\end{array}$ & $\begin{array}{l}\text { NB93* } \\
4.79 \mathrm{E}+00 \\
1.05 \mathrm{E}+01 \\
1.14 \mathrm{E}+01 \\
2.01 \mathrm{E}+01 \\
1.75 \mathrm{E}+01 \\
2.43 \mathrm{E}+01 \\
7.28 \mathrm{E}+00 \\
1.89 \mathrm{E}+01 \\
2.19 \mathrm{E}+01 \\
2.20 \mathrm{E}+01 \\
2.13 \mathrm{E}+01 \\
2.37 \mathrm{E}+00 \\
1.80 \mathrm{E}+01 \\
2.39 \mathrm{E}+01 \\
3.77 \mathrm{E}+00 \\
2.03 \mathrm{E}+01 \\
2.36 \mathrm{E}+01 \\
9.65 \mathrm{E}+00 \\
1.87 \mathrm{E}+01 \\
1.85 \mathrm{E}+01 \\
2.75 \mathrm{E}+01 \\
2.38 \mathrm{E}+00 \\
5.13 \mathrm{E}+00 \\
2.27 \mathrm{E}+00 \\
2.64 \mathrm{E}+01 \\
1.01 \mathrm{E}+01 \\
1.42 \mathrm{E}+01 \\
2.76 \mathrm{E}+01 \\
9.48 \mathrm{E}+00 \\
2.47 \mathrm{E}+01 \\
2.18 \mathrm{E}+01 \\
2.43 \mathrm{E}+01 \\
1.72 \mathrm{E}+01 \\
1.19 \mathrm{E}+00 \\
5.15 \mathrm{E}-01 \\
1.17 \mathrm{E}+01 \\
2.50 \mathrm{E}+01 \\
2.64 \mathrm{E}+01 \\
2.47 \mathrm{E}+01 \\
1.52 \mathrm{E}+01 \\
6.40 \mathrm{E}+00 \\
1.68 \mathrm{E}+01 \\
1.32 \mathrm{E}+01 \\
1.22 \mathrm{E}+01 \\
7.85 \mathrm{E}+00 \\
1.47 \mathrm{E}-02 \\
1.26 \mathrm{E}+01 \\
2.86 \mathrm{E}+00 \\
8.66 \mathrm{E}+00 \\
2.78 \mathrm{E}+00 \\
6.00 \mathrm{E}-01 \\
5.72 \mathrm{E}+00 \\
6.39 \mathrm{E}+00 \\
5.67 \mathrm{E}-01 \\
6.68 \mathrm{E}+00 \\
6.06 \mathrm{E}+00 \\
1.14 \mathrm{E}+00 \\
6.27 \mathrm{E}-01 \\
2.62 \mathrm{E}+00 \\
1.29 \mathrm{E}+00 \\
1.59 \mathrm{E}+00 \\
3.69 \mathrm{E}+00 \\
1.95 \mathrm{E}-01 \\
1.40 \mathrm{E}-02 \\
9.33 \mathrm{E}-03\end{array}$ & $\begin{array}{l}1 C 99 \\
4.36 \mathrm{E}+01 \\
9.67 \mathrm{E}+01 \\
1.04 \mathrm{E}+02 \\
1.84 \mathrm{E}+02 \\
1.60 \mathrm{E}+02 \\
2.23 \mathrm{E}+02 \\
6.67 \mathrm{E}+01 \\
1.74 \mathrm{E}+02 \\
2.01 \mathrm{E}+02 \\
2.02 \mathrm{E}+02 \\
1.97 \mathrm{E}+02 \\
2.19 \mathrm{E}+01 \\
1.67 \mathrm{E}+02 \\
2.21 \mathrm{E}+02 \\
3.48 \mathrm{E}+01 \\
1.88 \mathrm{E}+02 \\
2.19 \mathrm{E}+02 \\
8.95 \mathrm{E}+01 \\
1.74 \mathrm{E}+02 \\
1.74 \mathrm{E}+02 \\
2.57 \mathrm{E}+02 \\
2.17 \mathrm{E}+01 \\
4.80 \mathrm{E}+01 \\
2.10 \mathrm{E}+01 \\
2.48 \mathrm{E}+02 \\
9.45 \mathrm{E}+01 \\
1.33 \mathrm{E}+02 \\
2.61 \mathrm{E}+02 \\
8.91 \mathrm{E}+01 \\
2.33 \mathrm{E}+02 \\
2.06 \mathrm{E}+02 \\
2.29 \mathrm{E}+02 \\
1.62 \mathrm{E}+02 \\
1.12 \mathrm{E}+01 \\
4.81 \mathrm{E}+00 \\
1.11 \mathrm{E}+02 \\
2.37 \mathrm{E}+02 \\
2.50 \mathrm{E}+02 \\
2.34 \mathrm{E}+02 \\
1.45 \mathrm{E}+02 \\
6.13 \mathrm{E}+01 \\
1.61 \mathrm{E}+02 \\
1.26 \mathrm{E}+02 \\
1.17 \mathrm{E}+02 \\
7.55 \mathrm{E}+01 \\
8.39 \mathrm{E}-02 \\
1.21 \mathrm{E}+02 \\
2.74 \mathrm{E}+01 \\
8.33 \mathrm{E}+01 \\
2.68 \mathrm{E}+01 \\
5.66 \mathrm{E}+00 \\
5.44 \mathrm{E}+01 \\
6.06 \mathrm{E}+01 \\
5.37 \mathrm{E}+00 \\
6.43 \mathrm{E}+01 \\
5.84 \mathrm{E}+01 \\
1.08 \mathrm{E}+01 \\
5.95 \mathrm{E}+00 \\
2.48 \mathrm{E}+01 \\
1.23 \mathrm{E}+01 \\
1.54 \mathrm{E}+01 \\
3.57 \mathrm{E}+01 \\
1.11 \mathrm{E}+00 \\
8.00 \mathrm{E}-02 \\
5.29 \mathrm{E}-02\end{array}$ & $\begin{array}{l}\text { RU106 } \\
2.73 E-04 \\
7.02 E-04 \\
7.19 E-04 \\
1.38 E-03 \\
1.27 E-03 \\
1.94 E-03 \\
6.17 E-04 \\
1.55 E-03 \\
2.01 E-03 \\
2.15 E-03 \\
2.31 E-03 \\
2.34 E-04 \\
2.18 E-03 \\
3.06 E-03 \\
4.97 E-04 \\
2.87 E-03 \\
3.67 E-03 \\
1.57 E-03 \\
3.30 E-03 \\
3.72 E-03 \\
5.46 E-03 \\
3.91 E-04 \\
1.19 E-03 \\
4.92 E-04 \\
6.75 E-03 \\
2.79 E-03 \\
4.07 E-03 \\
8.90 E-03 \\
2.97 E-03 \\
8.49 E-03 \\
7.40 E-03 \\
9.26 E-03 \\
7.11 E-03 \\
5.03 E-04 \\
2.02 E-04 \\
5.37 E-03 \\
1.22 E-00 \\
1.31 E-02 \\
1.36 E-02 \\
8.40 E-03 \\
4.14 E-03 \\
1.15 E-00 \\
8.87 E-03 \\
9.33 E-03 \\
6.43 E-03 \\
2.43 E-06 \\
1.12 E-02 \\
2.62 E-03 \\
8.10 E-03 \\
2.78 E-03 \\
3.73 E-04 \\
4.73 E-03 \\
4.50 E-03 \\
4.24 E-03 \\
6.83 E-03 \\
6.30 E-03 \\
8.09 E-04 \\
5.11 E-04 \\
1.96 E-03 \\
1.18 E-03 \\
1.78 E-03 \\
4.21 E-03 \\
2.84 E-05 \\
2.72 E-06 \\
1.20 E-06\end{array}$ & $\begin{array}{l}\text { CD113 } \\
2.59 \mathrm{E}+01 \\
5.92 \mathrm{E}+01 \\
6.25 \mathrm{E}+01 \\
1.11 \mathrm{E}+02 \\
9.70 \mathrm{E}+01 \\
1.36 \mathrm{E}+02 \\
4.09 \mathrm{E}+01 \\
1.07 \mathrm{E}+02 \\
1.24 \mathrm{E}+02 \\
1.25 \mathrm{E}+02 \\
1.22 \mathrm{E}+02 \\
1.36 \mathrm{E}+01 \\
1.05 \mathrm{E}+02 \\
1.40 \mathrm{E}+02 \\
2.19 \mathrm{E}+01 \\
1.20 \mathrm{E}+02 \\
1.41 \mathrm{E}+02 \\
5.72 \mathrm{E}+01 \\
1.12 \mathrm{E}+02 \\
1.14 \mathrm{E}+02 \\
1.68 \mathrm{E}+02 \\
1.35 \mathrm{E}+01 \\
3.16 \mathrm{E}+01 \\
1.36 \mathrm{E}+01 \\
1.64 \mathrm{E}+02 \\
6.30 \mathrm{E}+01 \\
8.84 \mathrm{E}+01 \\
1.77 \mathrm{E}+02 \\
5.95 \mathrm{E}+01 \\
1.57 \mathrm{E}+02 \\
1.41 \mathrm{E}+02 \\
1.55 \mathrm{E}+02 \\
1.11 \mathrm{E}+02 \\
7.63 \mathrm{E}+00 \\
3.21 \mathrm{E}+00 \\
7.61 \mathrm{E}+01 \\
1.64 \mathrm{E}+02 \\
1.73 \mathrm{E}+02 \\
1.63 \mathrm{E}+02 \\
1.00 \mathrm{E}+02 \\
4.33 \mathrm{E}+01 \\
1.14 \mathrm{E}+02 \\
8.86 \mathrm{E}+01 \\
8.29 \mathrm{E}+01 \\
5.42 \mathrm{E}+01 \\
4.08 \mathrm{E}-02 \\
8.69 \mathrm{E}+01 \\
1.97 \mathrm{E}+01 \\
6.00 \mathrm{E}+01 \\
1.94 \mathrm{E}+01 \\
3.40 \mathrm{E}+00 \\
3.35 \mathrm{E}+01 \\
3.69 \mathrm{E}+01 \\
3.26 \mathrm{E}+00 \\
4.64 \mathrm{E}+01 \\
4.23 \mathrm{E}+01 \\
6.53 \mathrm{E}+00 \\
3.65 \mathrm{E}+00 \\
1.52 \mathrm{E}+01 \\
7.65 \mathrm{E}+00 \\
1.12 \mathrm{E}+01 \\
2.61 \mathrm{E}+01 \\
5.34 \mathrm{E}-01 \\
3.93 \mathrm{E}-02 \\
2.53 \mathrm{E}-02\end{array}$ & $\begin{array}{c}\text { SB125 } \\
1.60 \mathrm{E}+01 \\
3.63 \mathrm{E}+01 \\
3.91 \mathrm{E}+01 \\
7.11 \mathrm{E}+01 \\
6.35 \mathrm{E}+01 \\
9.11 \mathrm{E}+01 \\
2.80 \mathrm{E}+01 \\
7.15 \mathrm{E}+01 \\
8.66 \mathrm{E}+01 \\
8.92 \mathrm{E}+01 \\
9.01 \mathrm{E}+01 \\
9.59 \mathrm{E}+00 \\
7.86 \mathrm{E}+01 \\
1.07 \mathrm{E}+02 \\
1.72 \mathrm{E}+01 \\
9.46 \mathrm{E}+01 \\
1.13 \mathrm{E}+02 \\
4.75 \mathrm{E}+01 \\
9.47 \mathrm{E}+01 \\
9.70 \mathrm{E}+01 \\
1.45 \mathrm{E}+02 \\
1.23 \mathrm{E}+01 \\
2.87 \mathrm{E}+01 \\
1.26 \mathrm{E}+01 \\
1.53 \mathrm{E}+02 \\
6.01 \mathrm{E}+01 \\
8.61 \mathrm{E}+01 \\
1.73 \mathrm{E}+02 \\
5.97 \mathrm{E}+01 \\
1.61 \mathrm{E}+02 \\
1.39 \mathrm{E}+02 \\
1.65 \mathrm{E}+02 \\
1.20 \mathrm{E}+02 \\
8.39 \mathrm{E}+00 \\
3.63 \mathrm{E}+00 \\
8.51 \mathrm{E}+01 \\
1.86 \mathrm{E}+02 \\
1.98 \mathrm{E}+02 \\
1.92 \mathrm{E}+02 \\
1.19 \mathrm{E}+02 \\
5.25 \mathrm{E}+01 \\
1.41 \mathrm{E}+02 \\
1.11 \mathrm{E}+02 \\
1.08 \mathrm{E}+02 \\
7.05 \mathrm{E}+01 \\
1.19 \mathrm{E}-01 \\
1.17 \mathrm{E}+02 \\
2.70 \mathrm{E}+01 \\
8.23 \mathrm{E}+01 \\
2.71 \mathrm{E}+01 \\
4.43 \mathrm{E}+00 \\
4.68 \mathrm{E}+01 \\
4.92 \mathrm{E}+01 \\
4.49 \mathrm{E}+00 \\
6.59 \mathrm{E}+01 \\
6.00 \mathrm{E}+01 \\
8.85 \mathrm{E}+00 \\
5.12 \mathrm{E}+00 \\
2.06 \mathrm{E}+01 \\
1.09 \mathrm{E}+01 \\
1.63 \mathrm{E}+01 \\
3.78 \mathrm{E}+01 \\
1.51 \mathrm{E}+00 \\
1.21 \mathrm{E}-01 \\
6.89 \mathrm{E}+02\end{array}$ & 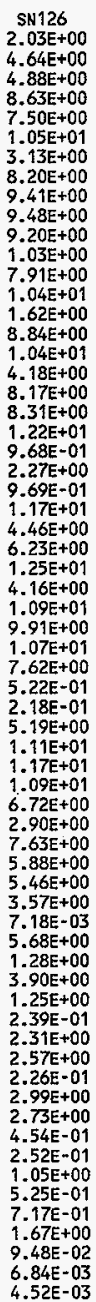 & $\begin{array}{l}1129 \\
8.35 \mathrm{E}-02 \\
1.91 \mathrm{E}-01 \\
2.01 \mathrm{E}-01 \\
3.55 \mathrm{E}-01 \\
3.09 \mathrm{E}-01 \\
4.31 \mathrm{E}-01 \\
1.29 \mathrm{E}-01 \\
3.37 \mathrm{E}-01 \\
3.87 \mathrm{E}-01 \\
3.90 \mathrm{E}-01 \\
3.79 \mathrm{E}-01 \\
4.25 \mathrm{E}-02 \\
3.26 \mathrm{E}-01 \\
4.29 \mathrm{E}-01 \\
6.68 \mathrm{E}-02 \\
3.64 \mathrm{E}-01 \\
4.28 \mathrm{E}-01 \\
1.72 \mathrm{E}-01 \\
3.36 \mathrm{E}-01 \\
3.42 \mathrm{E}-01 \\
5.01 \mathrm{E}-01 \\
3.99 \mathrm{E}-02 \\
9.33 \mathrm{E}-02 \\
3.99 \mathrm{E}-02 \\
4.81 \mathrm{E}-01 \\
1.84 \mathrm{E}-01 \\
2.56 \mathrm{E}-01 \\
5.13 \mathrm{E}-01 \\
1.71 \mathrm{E}-01 \\
4.50 \mathrm{E}-01 \\
4.08 \mathrm{E}-01 \\
4.40 \mathrm{E}-01 \\
3.14 \mathrm{E}-01 \\
2.15 \mathrm{E}-02 \\
8.98 \mathrm{E}-03 \\
2.14 \mathrm{E}-01 \\
4.58 \mathrm{E}-01 \\
4.83 \mathrm{E}-01 \\
4.51 \mathrm{E}-01 \\
2.77 \mathrm{E}-01 \\
1.19 \mathrm{E}-01 \\
3.14 \mathrm{E}-01 \\
2.42 \mathrm{E}-01 \\
2.25 \mathrm{E}-01 \\
1.47 \mathrm{E}-01 \\
3.07 \mathrm{E}-04 \\
2.34 \mathrm{E}-01 \\
5.27 \mathrm{E}-02 \\
1.61 \mathrm{E}-01 \\
5.16 \mathrm{E}-02 \\
1.05 \mathrm{E}-02 \\
1.02 \mathrm{E}-01 \\
1.13 \mathrm{E}-01 \\
9.98 \mathrm{E}-03 \\
1.23 \mathrm{E}-01 \\
1.13 \mathrm{E}-01 \\
2.00 \mathrm{E}-02 \\
1.11 \mathrm{E}-02 \\
4.65 \mathrm{E}-02 \\
2.31 \mathrm{E}-02 \\
2.95 \mathrm{E}-02 \\
6.89 \mathrm{E}-02 \\
4.05 \mathrm{E}-03 \\
2.93 \mathrm{E}-04 \\
1.93 \mathrm{E}-04\end{array}$ & $\begin{array}{l}\text { CS134 } \\
4.23 \mathrm{E}-01 \\
1.70 \mathrm{E}+00 \\
1.30 \mathrm{E}+00 \\
2.39 \mathrm{E}+00 \\
1.97 \mathrm{E}+00 \\
3.13 \mathrm{E}+00 \\
9.14 \mathrm{E}-01 \\
2.60 \mathrm{E}+00 \\
2.86 \mathrm{E}+00 \\
2.97 \mathrm{E}+00 \\
2.94 \mathrm{E}+00 \\
3.67 \mathrm{E}-01 \\
3.26 \mathrm{E}+00 \\
4.10 \mathrm{E}+00 \\
5.23 \mathrm{E}-01 \\
3.37 \mathrm{E}+00 \\
4.83 \mathrm{E}+00 \\
1.57 \mathrm{E}+00 \\
3.46 \mathrm{E}+00 \\
4.98 \mathrm{E}+00 \\
6.16 \mathrm{E}+00 \\
6.38 \mathrm{E}-07 \\
1.18 \mathrm{E}+00 \\
2.94 \mathrm{E}-01 \\
6.39 \mathrm{E}+00 \\
2.53 \mathrm{E}+00 \\
3.27 \mathrm{E}+00 \\
9.06 \mathrm{E}+00 \\
2.14 \mathrm{E}+00 \\
6.24 \mathrm{E}+00 \\
7.88 \mathrm{E}+00 \\
6.06 \mathrm{E}+00 \\
4.87 \mathrm{E}+00 \\
3.02 \mathrm{E}-01 \\
4.38 \mathrm{E}-02 \\
3.33 \mathrm{E}+00 \\
7.51 \mathrm{E}+00 \\
7.80 \mathrm{E}+00 \\
7.36 \mathrm{E}+00 \\
4.14 \mathrm{E}+00 \\
2.62 \mathrm{E}+00 \\
7.08 \mathrm{E}+00 \\
4.23 \mathrm{E}+00 \\
4.29 \mathrm{E}+00 \\
3.53 \mathrm{E}+00 \\
4.05 \mathrm{E}-03 \\
5.30 \mathrm{E}+00 \\
1.07 \mathrm{E}+00 \\
3.51 \mathrm{E}+00 \\
1.17 \mathrm{E}+00 \\
2.13 \mathrm{E}-01 \\
2.67 \mathrm{E}+00 \\
2.75 \mathrm{E}+00 \\
2.13 \mathrm{E}-01 \\
2.55 \mathrm{E}+00 \\
2.56 \mathrm{E}+00 \\
4.17 \mathrm{E}-01 \\
2.66 \mathrm{E}-01 \\
1.16 \mathrm{E}+00 \\
6.78 \mathrm{E}-01 \\
6.63 \mathrm{E}-01 \\
1.72 \mathrm{E}+00 \\
5.04 \mathrm{E}-02 \\
4.18 \mathrm{E}-03 \\
2.27 \mathrm{E}-03\end{array}$ & $\begin{array}{l}\text { CS137 } \\
1.49 \mathrm{E}+05 \\
3.31 \mathrm{E}+05 \\
3.55 \mathrm{E}+05 \\
6.30 \mathrm{E}+05 \\
5.51 \mathrm{E}+05 \\
7.68 \mathrm{E}+05 \\
2.31 \mathrm{E}+05 \\
6.00 \mathrm{E}+05 \\
6.96 \mathrm{E}+05 \\
7.02 \mathrm{E}+05 \\
6.85 \mathrm{E}+05 \\
7.59 \mathrm{E}+04 \\
5.83 \mathrm{E}+05 \\
7.74 \mathrm{E}+05 \\
1.22 \mathrm{E}+05 \\
6.62 \mathrm{E}+05 \\
7.73 \mathrm{E}+05 \\
3.16 \mathrm{E}+05 \\
6.16 \mathrm{E}+05 \\
6.17 \mathrm{E}+05 \\
9.13 \mathrm{E}+05 \\
7.71 \mathrm{E}+04 \\
1.72 \mathrm{E}+05 \\
7.51 \mathrm{E}+04 \\
8.88 \mathrm{E}+05 \\
3.40 \mathrm{E}+05 \\
4.78 \mathrm{E}+05 \\
9.43 \mathrm{E}+05 \\
3.21 \mathrm{E}+05 \\
8.43 \mathrm{E}+05 \\
7.46 \mathrm{E}+05 \\
8.31 \mathrm{E}+05 \\
5.91 \mathrm{E}+05 \\
4.08 \mathrm{E}+04 \\
1.75 \mathrm{E}+04 \\
4.05 \mathrm{E}+05 \\
8.68 \mathrm{E}+05 \\
9.17 \mathrm{E}+05 \\
8.61 \mathrm{E}+05 \\
5.32 \mathrm{E}+05 \\
2.26 \mathrm{E}+05 \\
5.96 \mathrm{E}+05 \\
4.67 \mathrm{E}+05 \\
4.34 \mathrm{E}+05 \\
2.81 \mathrm{E}+05 \\
4.89 \mathrm{E}+02 \\
4.51 \mathrm{E}+05 \\
1.03 \mathrm{E}+05 \\
3.12 \mathrm{E}+05 \\
1.00 \mathrm{E}+05 \\
2.10 \mathrm{E}+04 \\
2.04 \mathrm{E}+05 \\
2.26 \mathrm{E}+05 \\
2.01 \mathrm{E}+04 \\
2.41 \mathrm{E}+05 \\
2.19 \mathrm{E}+05 \\
4.03 \mathrm{E}+04 \\
2.23 \mathrm{E}+04 \\
9.28 \mathrm{E}+04 \\
4.63 \mathrm{E}+04 \\
5.78 \mathrm{0}+04 \\
1.34 \mathrm{E}+05 \\
6.43 \mathrm{E}+03 \\
4.69 \mathrm{E}+02 \\
3.06 \mathrm{E}+02\end{array}$ \\
\hline
\end{tabular}


ACTIVITY IN UNSEPARATED FUEL (Curies decayed to 1/1/94)

\begin{tabular}{|c|c|c|c|c|c|c|c|c|c|}
\hline $\begin{array}{c}\text { tListing } \# \text { ] } \\
\mathbf{5 2 1} \\
\mathbf{5 2 2} \\
\mathbf{5 2 3} \\
\mathbf{5 2 4} \\
\mathbf{5 2 5} \\
\mathbf{5 2 6} \\
\mathbf{5 2 7} \\
\mathbf{5 2 8} \\
\mathbf{5 2 9} \\
\mathbf{5 3 0} \\
\mathbf{5 3 1} \\
\mathbf{5 3 2} \\
\mathbf{5 3 3} \\
\mathbf{5 3 4} \\
\mathbf{5 3 5} \\
\mathbf{5 3 6} \\
\mathbf{5 3 7} \\
\mathbf{5 3 8} \\
\mathbf{5 3 9} \\
\mathbf{5 4 0} \\
\mathbf{5 4 1} \\
\mathbf{5 4 2} \\
\mathbf{5 4 3} \\
\mathbf{5 4 4} \\
\mathbf{5 4 5} \\
\mathbf{5 4 6} \\
\mathbf{5 4 7} \\
\mathbf{5 4 8} \\
\mathbf{5 4 9} \\
\mathbf{5 5 0} \\
\mathbf{5 5 1} \\
\mathbf{5 5 2} \\
\mathbf{5 5 3} \\
\mathbf{5 5 4} \\
\mathbf{5 5 5} \\
\mathbf{5 5 6} \\
\mathbf{5 5 7} \\
\mathbf{5 5 8} \\
\mathbf{5 5 9} \\
\mathbf{5 6 0} \\
\mathbf{5 6 1} \\
\mathbf{5 6 2} \\
\mathbf{5 6 3} \\
\mathbf{5 6 4} \\
\mathbf{5 6 5} \\
\mathbf{5 6 6} \\
\mathbf{5 6 7} \\
568 \\
569 \\
\mathbf{5 7 0} \\
\mathbf{5 7 1} \\
\mathbf{5 7 2} \\
\mathbf{5 7 3} \\
\mathbf{5 7 4} \\
\mathbf{5 7 5} \\
\mathbf{5 7 6} \\
\mathbf{5 7 7} \\
\mathbf{5 7 8} \\
\mathbf{5 7 9} \\
\mathbf{5 8 0} \\
\mathbf{5 8 1} \\
\mathbf{5 8 2} \\
583 \\
\mathbf{5 8 4} \\
585\end{array}$ & $\begin{array}{l}8 A 137 * \\
1.41 \mathrm{E}+05 \\
3.13 \mathrm{E}+05 \\
3.36 \mathrm{E}+05 \\
5.96 \mathrm{E}+05 \\
5.21 \mathrm{E}+05 \\
7.26 \mathrm{E}+05 \\
2.18 \mathrm{E}+05 \\
5.67 \mathrm{E}+05 \\
6.58 \mathrm{E}+05 \\
6.64 \mathrm{E}+05 \\
6.48 \mathrm{E}+05 \\
7.18 \mathrm{E}+04 \\
5.52 \mathrm{E}+05 \\
7.32 \mathrm{E}+05 \\
1.15 \mathrm{E}+05 \\
6.26 \mathrm{E}+05 \\
7.31 \mathrm{E}+05 \\
2.99 \mathrm{E}+05 \\
5.83 \mathrm{E}+05 \\
5.83 \mathrm{E}+05 \\
8.64 \mathrm{E}+05 \\
7.29 \mathrm{E}+04 \\
1.62 \mathrm{E}+05 \\
7.10 \mathrm{E}+04 \\
8.40 \mathrm{E}+05 \\
3.21 \mathrm{E}+05 \\
4.52 \mathrm{E}+05 \\
8.92 \mathrm{E}+05 \\
3.04 \mathrm{E}+05 \\
7.98 \mathrm{E}+05 \\
7.06 \mathrm{E}+05 \\
7.86 \mathrm{E}+05 \\
5.60 \mathrm{E}+05 \\
3.86 \mathrm{E}+04 \\
1.66 \mathrm{E}+04 \\
3.84 \mathrm{E}+05 \\
8.21 \mathrm{E}+05 \\
8.68 \mathrm{E}+05 \\
8.15 \mathrm{E}+05 \\
5.03 \mathrm{E}+05 \\
2.14 \mathrm{E}+05 \\
5.64 \mathrm{E}+05 \\
4.41 \mathrm{E}+05 \\
4.11 \mathrm{E}+05 \\
2.66 \mathrm{E}+05 \\
4.62 \mathrm{E}+02 \\
4.27 \mathrm{E}+05 \\
9.70 \mathrm{E}+04 \\
2.95 \mathrm{E}+05 \\
9.50 \mathrm{E}+04 \\
1.99 \mathrm{E}+04 \\
1.93 \mathrm{E}+05 \\
2.14 \mathrm{E}+05 \\
1.90 \mathrm{E}+04 \\
2.28 \mathrm{E}+05 \\
2.07 \mathrm{0}+05 \\
3.81 \mathrm{E}+04 \\
2.11 \mathrm{E}+04 \\
8.78 \mathrm{E}+04 \\
4.38 \mathrm{E}+04 \\
5.47 \mathrm{E}+04 \\
1.27 \mathrm{E}+05 \\
6.08 \mathrm{E}+03 \\
4.43 \mathrm{E}+02 \\
2.89 \mathrm{E}+02\end{array}$ & $\begin{array}{l}5 M 151 \\
5.01 E+03 \\
9.69 E+03 \\
1.15 E+04 \\
2.03 E+04 \\
1.80 E+04 \\
2.46 E+04 \\
7.48 E+03 \\
1.90 E+04 \\
2.25 E+04 \\
2.27 E+04 \\
2.22 E+04 \\
2.37 E+03 \\
1.79 E+04 \\
2.43 E+04 \\
4.01 E+03 \\
2.11 E+04 \\
2.36 E+04 \\
1.02 E+04 \\
1.96 E+04 \\
1.79 E+04 \\
2.80 E+04 \\
2.97 E+03 \\
5.31 E+03 \\
2.56 E+03 \\
2.74 E+04 \\
1.05 E+04 \\
1.51 E+04 \\
2.74 E+04 \\
1.03 E+04 \\
2.65 E+04 \\
2.11 E+04 \\
2.64 E+04 \\
1.84 E+04 \\
1.30 E+03 \\
6.27 E+02 \\
1.27 E+04 \\
2.71 E+04 \\
2.88 E+04 \\
2.72 E+04 \\
1.71 E+04 \\
6.73 E+03 \\
1.77 E+04 \\
1.47 E+04 \\
1.36 E+04 \\
8.37 E+03 \\
7.53 E+00 \\
1.38 E+04 \\
3.22 E+03 \\
9.63 E+03 \\
3.10 E+03 \\
6.35 E+02 \\
5.91 E+03 \\
6.58 E+03 \\
6.09 E+02 \\
7.60 E+03 \\
6.78 E+03 \\
1.22 E+03 \\
6.63 E+02 \\
2.70 E+03 \\
1.31 E+03 \\
1.80 E+03 \\
4.10 E+03 \\
9.93 E+01 \\
7.19 E+00 \\
4.73 E+00\end{array}$ & $\begin{array}{l}\text { EU152 } \\
1.18 \mathrm{E}+00 \\
4.97 \mathrm{E}+00 \\
3.64 \mathrm{E}+00 \\
6.48 \mathrm{E}+00 \\
5.14 \mathrm{E}+00 \\
7.94 \mathrm{E}+00 \\
2.24 \mathrm{E}+00 \\
6.62 \mathrm{E}+00 \\
6.78 \mathrm{E}+00 \\
6.86 \mathrm{E}+00 \\
6.49 \mathrm{E}+00 \\
8.71 \mathrm{E}-01 \\
7.22 \mathrm{E}+00 \\
8.69 \mathrm{E}+00 \\
1.04 \mathrm{E}+00 \\
6.75 \mathrm{E}+00 \\
9.66 \mathrm{E}+00 \\
2.92 \mathrm{E}+00 \\
6.34 \mathrm{E}+00 \\
9.24 \mathrm{E}+00 \\
1.11 \mathrm{E}+01 \\
0.00 \mathrm{E}+00 \\
1.97 \mathrm{E}+00 \\
4.41 \mathrm{E}-01 \\
1.03 \mathrm{E}+01 \\
3.93 \mathrm{E}+00 \\
4.88 \mathrm{E}+00 \\
1.38 \mathrm{E}+01 \\
3.05 \mathrm{E}+00 \\
8.67 \mathrm{E}+00 \\
1.19 \mathrm{E}+01 \\
7.94 \mathrm{E}+00 \\
6.29 \mathrm{E}+00 \\
3.76 \mathrm{E}-01 \\
3.86 \mathrm{E}-02 \\
4.08 \mathrm{E}+00 \\
9.05 \mathrm{E}+00 \\
9.27 \mathrm{E}+00 \\
8.30 \mathrm{E}+00 \\
4.57 \mathrm{E}+00 \\
2.92 \mathrm{E}+00 \\
7.71 \mathrm{E}+00 \\
4.38 \mathrm{E}+00 \\
4.24 \mathrm{E}+00 \\
3.56 \mathrm{E}+00 \\
1.99 \mathrm{E}-03 \\
5.05 \mathrm{E}+00 \\
9.74 \mathrm{E}-01 \\
3.23 \mathrm{E}+00 \\
1.04 \mathrm{E}+00 \\
2.41 \mathrm{E}-01 \\
2.77 \mathrm{E}+00 \\
3.05 \mathrm{E}+00 \\
2.23 \mathrm{E}-01 \\
2.20 \mathrm{E}+00 \\
2.25 \mathrm{E}+00 \\
4.45 \mathrm{E}-01 \\
2.74 \mathrm{E}-01 \\
1.26 \mathrm{E}+00 \\
6.85 \mathrm{E}-01 \\
5.59 \mathrm{E}-01 \\
1.47 \mathrm{E}+00 \\
2.60 \mathrm{E}-02 \\
1.92 \mathrm{E}-03 \\
1.23 \mathrm{E}-03\end{array}$ & $\begin{array}{l}E U 154 \\
9.26 \mathrm{E}+01 \\
3.46 \mathrm{E}+02 \\
2.71 \mathrm{E}+02 \\
4.84 \mathrm{E}+02 \\
3.93 \mathrm{E}+02 \\
5.98 \mathrm{E}+02 \\
1.72 \mathrm{E}+02 \\
4.92 \mathrm{E}+02 \\
5.21 \mathrm{E}+02 \\
5.28 \mathrm{E}+02 \\
5.06 \mathrm{E}+02 \\
6.48 \mathrm{E}+01 \\
5.35 \mathrm{E}+02 \\
6.59 \mathrm{E}+02 \\
8.42 \mathrm{E}+01 \\
5.24 \mathrm{E}+02 \\
7.24 \mathrm{E}+02 \\
2.34 \mathrm{E}+02 \\
4.97 \mathrm{E}+02 \\
6.79 \mathrm{E}+02 \\
8.43 \mathrm{E}+02 \\
5.14 \mathrm{E}+00 \\
1.53 \mathrm{E}+02 \\
4.07 \mathrm{E}+01 \\
7.99 \mathrm{E}+02 \\
3.08 \mathrm{E}+02 \\
3.93 \mathrm{E}+02 \\
1.04 \mathrm{E}+03 \\
2.51 \mathrm{E}+02 \\
7.04 \mathrm{E}+02 \\
8.88 \mathrm{E}+02 \\
6.59 \mathrm{E}+02 \\
5.12 \mathrm{E}+02 \\
3.17 \mathrm{E}+01 \\
5.69 \mathrm{E}+00 \\
3.38 \mathrm{E}+02 \\
7.46 \mathrm{E}+02 \\
7.70 \mathrm{E}+02 \\
7.01 \mathrm{E}+02 \\
3.97 \mathrm{E}+02 \\
2.33 \mathrm{E}+02 \\
6.18 \mathrm{E}+02 \\
3.76 \mathrm{E}+02 \\
3.63 \mathrm{E}+02 \\
2.89 \mathrm{E}+02 \\
1.30 \mathrm{E}-01 \\
4.22 \mathrm{E}+02 \\
8.47 \mathrm{E}+01 \\
2.75 \mathrm{E}+02 \\
8.92 \mathrm{E}+01 \\
1.70 \mathrm{E}+01 \\
1.92 \mathrm{E}+02 \\
2.10 \mathrm{E}+02 \\
1.60 \mathrm{E}+01 \\
1.95 \mathrm{E}+02 \\
1.93 \mathrm{E}+02 \\
3.19 \mathrm{E}+01 \\
1.93 \mathrm{E}+01 \\
8.66 \mathrm{E}+01 \\
4.70 \mathrm{E}+01 \\
4.89 \mathrm{E}+01 \\
1.25 \mathrm{E}+02 \\
1.69 \mathrm{E}+00 \\
1.26 \mathrm{E}-01 \\
7.97 \mathrm{E}-02\end{array}$ & $\begin{array}{l}\mathrm{EU155} \\
1.05 \mathrm{E}+02 \\
1.84 \mathrm{E}+02 \\
2.34 \mathrm{E}+02 \\
4.21 \mathrm{E}+02 \\
3.85 \mathrm{E}+02 \\
5.27 \mathrm{E}+02 \\
1.64 \mathrm{E}+02 \\
4.03 \mathrm{E}+02 \\
5.00 \mathrm{E}+02 \\
5.10 \mathrm{E}+02 \\
5.12 \mathrm{E}+02 \\
5.19 \mathrm{E}+01 \\
4.01 \mathrm{E}+02 \\
5.61 \mathrm{E}+02 \\
9.74 \mathrm{E}+01 \\
5.06 \mathrm{E}+02 \\
5.54 \mathrm{E}+02 \\
2.56 \mathrm{E}+02 \\
4.89 \mathrm{E}+02 \\
4.22 \mathrm{E}+02 \\
6.95 \mathrm{E}+02 \\
8.61 \mathrm{E}+01 \\
1.37 \mathrm{E}+02 \\
7.12 \mathrm{E}+01 \\
7.22 \mathrm{E}+02 \\
2.80 \mathrm{E}+02 \\
4.15 \mathrm{E}+02 \\
7.16 \mathrm{E}+02 \\
2.90 \mathrm{E}+02 \\
7.54 \mathrm{E}+02 \\
5.43 \mathrm{E}+02 \\
7.76 \mathrm{E}+02 \\
5.41 \mathrm{E}+02 \\
3.93 \mathrm{E}+01 \\
2.04 \mathrm{E}+01 \\
3.85 \mathrm{E}+02 \\
8.23 \mathrm{E}+02 \\
8.82 \mathrm{E}+02 \\
8.56 \mathrm{E}+02 \\
5.46 \mathrm{E}+02 \\
2.08 \mathrm{E}+02 \\
5.53 \mathrm{E}+02 \\
4.84 \mathrm{E}+02 \\
4.56 \mathrm{E}+02 \\
2.72 \mathrm{E}+02 \\
1.40 \mathrm{E}-01 \\
4.65 \mathrm{E}+02 \\
1.12 \mathrm{E}+02 \\
3.33 \mathrm{E}+02 \\
1.08 \mathrm{E}+02 \\
1.97 \mathrm{E}+01 \\
1.86 \mathrm{E}+02 \\
2.01 \mathrm{E}+02 \\
1.97 \mathrm{E}+01 \\
2.72 \mathrm{E}+02 \\
2.39 \mathrm{E}+02 \\
3.92 \mathrm{E}+01 \\
2.14 \mathrm{E}+01 \\
8.34 \mathrm{E}+01 \\
4.11 \mathrm{E}+01 \\
6.52 \mathrm{E}+01 \\
1.46 \mathrm{E}+02 \\
1.81 \mathrm{E}+00 \\
1.38 \mathrm{E}-01 \\
8.41 \mathrm{E}-02\end{array}$ & $\begin{array}{l}\text { RA226 } \\
1.08 \mathrm{E}-04 \\
1.44 \mathrm{E}-04 \\
2.10 \mathrm{E}-04 \\
3.69 \mathrm{E}-04 \\
3.46 \mathrm{E}-04 \\
4.42 \mathrm{E}-04 \\
1.39 \mathrm{E}-04 \\
3.28 \mathrm{E}-04 \\
4.14 \mathrm{E}-04 \\
4.14 \mathrm{E}-04 \\
4.09 \mathrm{E}-04 \\
3.95 \mathrm{E}-05 \\
2.81 \mathrm{E}-04 \\
4.01 \mathrm{E}-04 \\
7.73 \mathrm{E}-05 \\
3.66 \mathrm{E}-04 \\
3.62 \mathrm{E}-04 \\
1.86 \mathrm{E}-04 \\
3.31 \mathrm{E}-04 \\
2.43 \mathrm{E}-04 \\
4.28 \mathrm{E}-04 \\
3.51 \mathrm{E}-04 \\
8.25 \mathrm{E}-05 \\
5.85 \mathrm{E}-05 \\
4.22 \mathrm{E}-04 \\
1.60 \mathrm{E}-04 \\
2.46 \mathrm{E}-04 \\
3.63 \mathrm{E}-04 \\
1.74 \mathrm{E}-04 \\
4.25 \mathrm{E}-04 \\
2.66 \mathrm{E}-04 \\
4.38 \mathrm{E}-04 \\
2.85 \mathrm{E}-04 \\
2.19 \mathrm{E}-05 \\
2.13 \mathrm{E}-05 \\
2.02 \mathrm{E}-04 \\
4.18 \mathrm{E}-04 \\
4.52 \mathrm{E}-04 \\
4.35 \mathrm{E}-04 \\
2.92 \mathrm{E}-04 \\
9.00 \mathrm{E}-05 \\
2.36 \mathrm{E}-04 \\
2.35 \mathrm{E}-04 \\
2.10 \mathrm{E}-04 \\
1.11 \mathrm{E}-04 \\
3.47 \mathrm{E}-05 \\
1.94 \mathrm{E}-04 \\
4.96 \mathrm{E}-05 \\
1.41 \mathrm{E}-04 \\
4.51 \mathrm{E}-05 \\
1.21 \mathrm{E}-05 \\
1.00 \mathrm{E}-04 \\
1.13 \mathrm{E}-04 \\
1.17 \mathrm{E}-05 \\
1.19 \mathrm{E}-04 \\
9.90 \mathrm{E}-05 \\
2.36 \mathrm{E}-05 \\
1.19 \mathrm{E}-05 \\
4.62 \mathrm{E}-05 \\
2.10 \mathrm{E}-05 \\
2.71 \mathrm{E}-05 \\
5.74 \mathrm{E}-05 \\
4.61 \mathrm{E}-04 \\
3.28 \mathrm{E}-05 \\
2.22 \mathrm{E}-05\end{array}$ & $\begin{array}{l}\text { RA228 } \\
9.35 \mathrm{E}-10 \\
1.99 \mathrm{E}-09 \\
2.19 \mathrm{E}-09 \\
3.86 \mathrm{E}-09 \\
3.37 \mathrm{E}-09 \\
4.63 \mathrm{E}-09 \\
1.39 \mathrm{E}-09 \\
3.60 \mathrm{E}-09 \\
4.16 \mathrm{E}-09 \\
4.17 \mathrm{E}-09 \\
4.03 \mathrm{E}-09 \\
4.47 \mathrm{E}-10 \\
3.36 \mathrm{E}-09 \\
4.46 \mathrm{E}-09 \\
7.08 \mathrm{E}-10 \\
3.79 \mathrm{E}-09 \\
4.35 \mathrm{E}-09 \\
1.79 \mathrm{E}-09 \\
3.46 \mathrm{E}-09 \\
3.35 \mathrm{E}-09 \\
5.03 \mathrm{E}-09 \\
4.53 \mathrm{E}-10 \\
9.33 \mathrm{E}-10 \\
4.20 \mathrm{E}-10 \\
4.79 \mathrm{E}-09 \\
1.82 \mathrm{E}-09 \\
2.56 \mathrm{E}-09 \\
4.91 \mathrm{E}-09 \\
1.71 \mathrm{E}-09 \\
4.44 \mathrm{E}-09 \\
3.84 \mathrm{E}-09 \\
4.34 \mathrm{E}-09 \\
3.05 \mathrm{E}-09 \\
2.11 \mathrm{E}-10 \\
9.36 \mathrm{E}-11 \\
2.07 \mathrm{E}-09 \\
4.41 \mathrm{E}-09 \\
4.66 \mathrm{E}-09 \\
4.34 \mathrm{E}-09 \\
2.69 \mathrm{E}-09 \\
1.11 \mathrm{E}-09 \\
2.90 \mathrm{E}-09 \\
2.30 \mathrm{E}-09 \\
2.11 \mathrm{E}-09 \\
1.35 \mathrm{E}-09 \\
4.14 \mathrm{E}-01 \\
2.16 \mathrm{E}-09 \\
4.91 \mathrm{E}-10 \\
1.48 \mathrm{E}-09 \\
4.75 \mathrm{E}-10 \\
4.87 \mathrm{E}-10 \\
4.17 \mathrm{E}-09 \\
4.70 \mathrm{E}-09 \\
4.69 \mathrm{E}-10 \\
1.14 \mathrm{E}-09 \\
1.03 \mathrm{E}-09 \\
9.44 \mathrm{E}-10 \\
4.87 \mathrm{E}-10 \\
1.92 \mathrm{E}-09 \\
8.92 \mathrm{E}-10 \\
2.71 \mathrm{E}-10 \\
6.26 \mathrm{E}-10 \\
5.47 \mathrm{E}+00 \\
3.94 \mathrm{E}-01 \\
2.61 \mathrm{E}-01\end{array}$ & $\begin{array}{l}\text { AC227 } \\
5.87 \mathrm{E}-04 \\
7.52 \mathrm{E}-04 \\
1.13 \mathrm{E}-03 \\
1.99 \mathrm{E}-03 \\
1.88 \mathrm{E}-03 \\
2.38 \mathrm{E}-03 \\
7.51 \mathrm{E}-04 \\
1.76 \mathrm{E}-03 \\
2.25 \mathrm{E}-03 \\
2.25 \mathrm{E}-03 \\
2.23 \mathrm{E}-03 \\
2.12 \mathrm{E}-04 \\
1.51 \mathrm{E}-03 \\
2.16 \mathrm{E}-03 \\
4.25 \mathrm{E}-04 \\
1.99 \mathrm{E}-03 \\
1.94 \mathrm{E}-03 \\
1.02 \mathrm{E}-03 \\
1.81 \mathrm{E}-03 \\
1.29 \mathrm{E}-03 \\
2.32 \mathrm{E}-03 \\
2.07 \mathrm{E}-03 \\
4.49 \mathrm{E}-04 \\
3.30 \mathrm{E}-04 \\
2.30 \mathrm{E}-03 \\
8.71 \mathrm{E}-04 \\
1.36 \mathrm{E}-03 \\
1.95 \mathrm{E}-03 \\
9.62 \mathrm{E}-04 \\
2.34 \mathrm{E}-03 \\
1.42 \mathrm{E}-03 \\
2.43 \mathrm{E}-03 \\
1.58 \mathrm{E}-03 \\
1.22 \mathrm{E}-04 \\
1.24 \mathrm{E}-04 \\
1.12 \mathrm{E}-03 \\
2.32 \mathrm{E}-03 \\
2.51 \mathrm{E}-03 \\
2.43 \mathrm{E}-03 \\
1.64 \mathrm{E}-03 \\
4.94 \mathrm{E}-04 \\
1.29 \mathrm{E}-03 \\
1.32 \mathrm{E}-03 \\
1.18 \mathrm{E}-03 \\
6.11 \mathrm{E}-04 \\
7.19 \mathrm{E}-01 \\
1.08 \mathrm{E}-03 \\
2.79 \mathrm{E}-04 \\
7.91 \mathrm{E}-04 \\
2.53 \mathrm{E}-04 \\
6.01 \mathrm{E}-05 \\
4.93 \mathrm{E}-04 \\
5.57 \mathrm{E}-04 \\
5.81 \mathrm{E}-05 \\
6.71 \mathrm{E}-04 \\
5.56 \mathrm{E}-04 \\
1.17 \mathrm{E}-04 \\
5.90 \mathrm{E}-05 \\
2.27 \mathrm{E}-04 \\
1.03 \mathrm{E}-04 \\
1.53 \mathrm{E}-04 \\
3.21 \mathrm{E}-04 \\
9.53 \mathrm{E}+00 \\
6.82 \mathrm{E}-01 \\
4.57 \mathrm{E}-01\end{array}$ & $\begin{array}{l}\text { TH229 } \\
1.83 \mathrm{E}-07 \\
4.06 \mathrm{E}-07 \\
4.35 \mathrm{E}-07 \\
7.66 \mathrm{E}-07 \\
6.66 \mathrm{E}-07 \\
9.20 \mathrm{E}-07 \\
2.75 \mathrm{E}-07 \\
7.18 \mathrm{E}-07 \\
8.23 \mathrm{E}-07 \\
8.26 \mathrm{E}-07 \\
7.97 \mathrm{E}-07 \\
8.94 \mathrm{E}-08 \\
6.75 \mathrm{E}-07 \\
8.89 \mathrm{E}-07 \\
1.39 \mathrm{E}-07 \\
7.53 \mathrm{E}-07 \\
8.73 \mathrm{E}-07 \\
3.55 \mathrm{E}-07 \\
6.87 \mathrm{E}-07 \\
6.82 \mathrm{E}-07 \\
1.01 \mathrm{E}-06 \\
8.52 \mathrm{E}-08 \\
1.87 \mathrm{E}-07 \\
8.17 \mathrm{E}-08 \\
9.57 \mathrm{E}-07 \\
3.63 \mathrm{E}-07 \\
5.09 \mathrm{E}-07 \\
9.97 \mathrm{E}-07 \\
3.39 \mathrm{E}-07 \\
8.82 \mathrm{E}-07 \\
7.86 \mathrm{E}-07 \\
8.60 \mathrm{E}-07 \\
6.08 \mathrm{E}-07 \\
1.27 \mathrm{E}-07 \mathrm{E} \\
1.25 \mathrm{E}-07 \\
1.18 \mathrm{E}-08 \\
1.79 \mathrm{E}-08 \\
4.13 \mathrm{E}-07 \\
8.79 \mathrm{E}-07 \\
9.26 \mathrm{E}-07 \\
8.61 \mathrm{E}-07 \\
5.31 \mathrm{E}-07 \\
2.00 \mathrm{E}-07 \\
4.32 \mathrm{E}-07 \\
9.75 \mathrm{E}-08 \\
2.96 \mathrm{E}-07 \\
9.48 \mathrm{E}-08 \\
2.68 \mathrm{E}-08 \\
2.53 \mathrm{E}-07 \\
2.85 \mathrm{E}-07 \\
2.51 \mathrm{E}-07 \\
2.27 \mathrm{E}-08 \\
2.06 \mathrm{E}-07 \\
5.06 \mathrm{E}-08 \\
2.77 \mathrm{E}-08 \\
5.71 \mathrm{E}-07 \\
\end{array}$ \\
\hline
\end{tabular}


HNF-SD-WM-TI-794, ReV. 0

ACTIVITY IN UNSEPARATED FUEL. (Curies decayed to $1 / 1 / 94$ )

\begin{tabular}{|c|c|c|c|c|c|c|c|c|c|}
\hline $\begin{array}{c}\text { [Listing \#] } \\
\mathbf{5 2 1} \\
\mathbf{5 2 2} \\
\mathbf{5 2 3} \\
\mathbf{5 2 4} \\
\mathbf{5 2 5} \\
\mathbf{5 2 6} \\
\mathbf{5 2 7} \\
\mathbf{5 2 8} \\
\mathbf{5 2 9} \\
530 \\
\mathbf{5 3 1} \\
\mathbf{5 3 2} \\
\mathbf{5 3 3} \\
\mathbf{5 3 4} \\
\mathbf{5 3 5} \\
\mathbf{5 3 6} \\
\mathbf{5 3 7} \\
\mathbf{5 3 8} \\
\mathbf{5 3 9} \\
\mathbf{5 4 0} \\
\mathbf{5 4 1} \\
542 \\
\mathbf{5 4 3} \\
\mathbf{5 4 4} \\
\mathbf{5 4 5} \\
\mathbf{5 4 6} \\
\mathbf{5 4 7} \\
\mathbf{5 4 8} \\
\mathbf{5 4 9} \\
\mathbf{5 5 0} \\
\mathbf{5 5 1} \\
\mathbf{5 5 2} \\
\mathbf{5 5 3} \\
\mathbf{5 5 4} \\
\mathbf{5 5 5} \\
\mathbf{5 5 6} \\
\mathbf{5 5 7} \\
\mathbf{5 5 8} \\
\mathbf{5 5 9} \\
\mathbf{5 6 0} \\
\mathbf{5 6 1} \\
\mathbf{5 6 2} \\
\mathbf{5 6 3} \\
\mathbf{5 6 4} \\
\mathbf{5 6 5} \\
\mathbf{5 6 6} \\
\mathbf{5 6 7} \\
\mathbf{5 6 8} \\
\mathbf{5 6 9} \\
\mathbf{5 7 0} \\
\mathbf{5 7 1} \\
\mathbf{5 7 2} \\
\mathbf{5 7 3} \\
\mathbf{5 7 4} \\
\mathbf{5 7 5} \\
\mathbf{5 7 6} \\
\mathbf{5 7 7} \\
\mathbf{5 7 8} \\
\mathbf{5 7 9} \\
\mathbf{5 8 0} \\
\mathbf{5 8 1} \\
\mathbf{5 8 2} \\
\mathbf{5 8 3} \\
\mathbf{5 8 4} \\
\mathbf{5 8 5}\end{array}$ & $\begin{array}{l}\text { TH232 } \\
1.31 \mathrm{E}-09 \\
2.80 \mathrm{E}-09 \\
3.08 \mathrm{E}-09 \\
5.43 \mathrm{E}-09 \\
4.75 \mathrm{E}-09 \\
6.54 \mathrm{E}-09 \\
1.96 \mathrm{E}-09 \\
5.08 \mathrm{E}-09 \\
5.88 \mathrm{E}-09 \\
5.90 \mathrm{E}-09 \\
5.72 \mathrm{E}-09 \\
6.33 \mathrm{E}-10 \\
4.77 \mathrm{E}-09 \\
6.33 \mathrm{E}-09 \\
1.01 \mathrm{E}-09 \\
5.40 \mathrm{E}-09 \\
6.20 \mathrm{E}-09 \\
2.56 \mathrm{E}-09 \\
4.94 \mathrm{E}-09 \\
4.79 \mathrm{E}-09 \\
7.20 \mathrm{E}-09 \\
6.51 \mathrm{E}-10 \\
1.34 \mathrm{E}-09 \\
6.03 \mathrm{E}-10 \\
6.88 \mathrm{E}-09 \\
2.61 \mathrm{E}-09 \\
3.69 \mathrm{E}-09 \\
7.08 \mathrm{E}-09 \\
2.47 \mathrm{E}-09 \\
6.42 \mathrm{E}-09 \\
5.55 \mathrm{E}-09 \\
6.29 \mathrm{E}-09 \\
4.43 \mathrm{E}-09 \\
3.07 \mathrm{E}-10 \\
1.36 \mathrm{E}-10 \\
3.02 \mathrm{E}-09 \\
6.42 \mathrm{E}-09 \\
6.78 \mathrm{E}-09 \\
6.33 \mathrm{E}-09 \\
3.92 \mathrm{E}-09 \\
1.62 \mathrm{E}-09 \\
4.25 \mathrm{E}-09 \\
3.38 \mathrm{E}-09 \\
3.11 \mathrm{E}-09 \\
1.98 \mathrm{E}-09 \\
4.36 \mathrm{E}-01 \\
3.17 \mathrm{E}-09 \\
7.23 \mathrm{E}-10 \\
2.19 \mathrm{E}-09 \\
7.02 \mathrm{E}-10 \\
7.14 \mathrm{E}-10 \\
6.14 \mathrm{E}-09 \\
6.89 \mathrm{E}-09 \\
6.89 \mathrm{E}-10 \\
1.69 \mathrm{E}-09 \\
1.53 \mathrm{E}-09 \\
1.39 \mathrm{E}-09 \\
7.17 \mathrm{E}-10 \\
2.82 \mathrm{E}-09 \\
1.31 \mathrm{E}-09 \\
4.01 \mathrm{E}-10 \\
9.26 \mathrm{E}-10 \\
5.76 \mathrm{E}+00 \\
4.16 \mathrm{E}-01 \\
2.75 \mathrm{E}-01\end{array}$ & $\begin{array}{l}\text { PA231 } \\
1.56 \mathrm{E}-03 \\
1.99 \mathrm{E}-03 \\
3.00 \mathrm{E}-03 \\
5.29 \mathrm{E}-03 \\
5.01 \mathrm{E}-03 \\
6.37 \mathrm{E}-03 \\
2.01 \mathrm{E}-03 \\
4.71 \mathrm{E}-03 \\
6.03 \mathrm{E}-03 \\
6.06 \mathrm{E}-03 \\
6.03 \mathrm{E}-03 \\
5.71 \mathrm{E}-04 \\
4.08 \mathrm{E}-03 \\
5.87 \mathrm{E}-03 \\
1.16 \mathrm{E}-03 \\
5.42 \mathrm{E}-03 \\
5.30 \mathrm{E}-03 \\
2.79 \mathrm{E}-03 \\
4.96 \mathrm{E}-03 \\
3.54 \mathrm{E}-03 \\
6.37 \mathrm{E}-03 \\
5.74 \mathrm{E}-03 \\
1.24 \mathrm{E}-03 \\
9.16 \mathrm{E}-04 \\
6.38 \mathrm{E}-03 \\
2.42 \mathrm{E}-03 \\
3.79 \mathrm{E}-03 \\
5.43 \mathrm{E}-03 \\
2.69 \mathrm{E}-03 \\
6.58 \mathrm{E}-03 \\
3.96 \mathrm{E}-03 \\
6.85 \mathrm{E}-03 \\
4.45 \mathrm{E}-03 \\
3.45 \mathrm{E}-04 \\
3.52 \mathrm{E}-04 \\
3.17 \mathrm{E}-03 \\
6.59 \mathrm{E}-03 \\
7.14 \mathrm{E}-03 \\
6.93 \mathrm{E}-03 \\
4.68 \mathrm{E}-03 \\
1.41 \mathrm{E}-03 \\
3.71 \mathrm{E}-03 \\
3.80 \mathrm{E}-03 \\
3.40 \mathrm{E}-03 \\
1.76 \mathrm{E}-03 \\
1.20 \mathrm{E}+00 \\
3.14 \mathrm{E}-03 \\
8.13 \mathrm{E}-04 \\
2.30 \mathrm{E}-03 \\
7.38 \mathrm{E}-04 \\
1.73 \mathrm{E}-04 \\
1.43 \mathrm{E}-03 \\
1.61 \mathrm{E}-03 \\
1.69 \mathrm{E}-04 \\
1.96 \mathrm{E}-03 \\
1.62 \mathrm{E}-03 \\
3.39 \mathrm{E}-04 \\
1.71 \mathrm{E}-04 \\
6.57 \mathrm{E}-04 \\
3.00 \mathrm{E}-04 \\
4.48 \mathrm{E}-04 \\
9.41 \mathrm{E}-04 \\
1.59 \mathrm{E}+01 \\
1.15 \mathrm{E}+00 \\
7.58 \mathrm{E}-01\end{array}$ & $\begin{array}{l}\text { U232 } \\
2.38 \mathrm{E}-03 \\
6.68 \mathrm{E}-03 \\
6.16 \mathrm{E}-03 \\
1.09 \mathrm{E}-02 \\
9.21 \mathrm{E}-03 \\
1.33 \mathrm{E}-02 \\
3.89 \mathrm{E}-03 \\
1.06 \mathrm{E}-02 \\
1.17 \mathrm{E}-02 \\
1.18 \mathrm{E}-02 \\
1.14 \mathrm{E}-02 \\
1.35 \mathrm{E}-03 \\
1.06 \mathrm{E}-02 \\
1.36 \mathrm{E}-02 \\
1.95 \mathrm{E}-03 \\
1.12 \mathrm{E}-02 \\
1.40 \mathrm{E}-02 \\
5.13 \mathrm{E}-03 \\
1.03 \mathrm{E}-02 \\
1.19 \mathrm{E}-02 \\
1.62 \mathrm{E}-02 \\
8.17 \mathrm{E}-04 \\
2.97 \mathrm{E}-03 \\
1.07 \mathrm{E}-03 \\
1.53 \mathrm{E}-02 \\
5.86 \mathrm{E}-03 \\
7.88 \mathrm{E}-03 \\
1.78 \mathrm{E}-02 \\
5.15 \mathrm{E}-03 \\
1.38 \mathrm{E}-02 \\
1.46 \mathrm{E}-02 \\
1.33 \mathrm{E}-02 \\
9.76 \mathrm{E}-03 \\
6.42 \mathrm{E}-04 \\
2.11 \mathrm{E}-04 \\
6.55 \mathrm{E}-03 \\
1.42 \mathrm{E}-02 \\
1.48 \mathrm{E}-02 \\
1.36 \mathrm{E}-02 \\
8.12 \mathrm{E}-03 \\
3.95 \mathrm{E}-03 \\
1.04 \mathrm{E}-02 \\
7.28 \mathrm{E}-03 \\
6.83 \mathrm{E}-03 \\
4.83 \mathrm{E}-03 \\
1.87 \mathrm{E}+01 \\
7.39 \mathrm{E}-03 \\
1.59 \mathrm{E}-03 \\
4.96 \mathrm{E}-03 \\
1.60 \mathrm{E}-03 \\
4.59 \mathrm{E}-04 \\
4.74 \mathrm{E}-03 \\
5.27 \mathrm{E}-03 \\
4.30 \mathrm{E}-04 \\
3.68 \mathrm{E}-03 \\
3.47 \mathrm{E}-03 \\
8.62 \mathrm{E}-04 \\
4.96 \mathrm{E}-04 \\
2.16 \mathrm{E}-03 \\
1.12 \mathrm{E}-03 \\
8.96 \mathrm{E}-04 \\
2.16 \mathrm{E}-03 \\
2.47 \mathrm{E}+02 \\
1.79 \mathrm{E}+01 \\
1.18 \mathrm{E}+01\end{array}$ & $\begin{array}{c}\text { U233 } \\
7.89 \mathrm{E}-05 \\
1.76 \mathrm{E}-04 \\
1.88 \mathrm{E}-04 \\
3.32 \mathrm{E}-04 \\
2.89 \mathrm{E}-04 \\
4.01 \mathrm{E}-04 \\
1.20 \mathrm{E}-04 \\
3.13 \mathrm{E}-04 \\
3.60 \mathrm{E}-04 \\
3.62 \mathrm{E}-04 \\
3.51 \mathrm{E}-04 \\
3.93 \mathrm{E}-05 \\
2.99 \mathrm{E}-04 \\
3.94 \mathrm{E}-04 \\
6.19 \mathrm{E}-05 \\
3.35 \mathrm{E}-04 \\
3.90 \mathrm{E}-04 \\
1.59 \mathrm{E}-04 \\
3.08 \mathrm{E}-04 \\
3.08 \mathrm{E}-04 \\
4.55 \mathrm{E}-04 \\
3.80 \mathrm{E}-05 \\
8.46 \mathrm{E}-05 \\
3.69 \mathrm{E}-05 \\
4.35 \mathrm{E}-04 \\
1.66 \mathrm{E}-04 \\
2.32 \mathrm{E}-04 \\
4.58 \mathrm{E}-04 \\
1.55 \mathrm{E}-04 \\
4.06 \mathrm{E}-04 \\
3.62 \mathrm{E}-04 \\
3.97 \mathrm{E}-04 \\
2.82 \mathrm{E}-04 \\
1.94 \mathrm{E}-05 \\
8.30 \mathrm{E}-06 \\
1.92 \mathrm{E}-04 \\
4.10 \mathrm{E}-04 \\
4.32 \mathrm{E}-04 \\
4.03 \mathrm{E}-04 \\
2.49 \mathrm{E}-04 \\
1.05 \mathrm{E}-04 \\
2.77 \mathrm{E}-04 \\
2.16 \mathrm{E}-04 \\
2.00 \mathrm{E}-04 \\
1.29 \mathrm{E}-04 \\
7.42 \mathrm{E}+01 \\
2.06 \mathrm{E}-04 \\
4.66 \mathrm{E}-05 \\
1.42 \mathrm{E}-04 \\
4.55 \mathrm{E}-05 \\
1.35 \mathrm{E}-05 \\
1.29 \mathrm{E}-04 \\
1.44 \mathrm{E}-04 \\
1.28 \mathrm{E}-05 \\
1.09 \mathrm{E}-04 \\
9.91 \mathrm{E}-05 \\
2.56 \mathrm{E}-05 \\
1.41 \mathrm{E}-05 \\
5.91 \mathrm{E}-05 \\
2.91 \mathrm{E}-05 \\
2.60 \mathrm{E}-05 \\
6.05 \mathrm{E}-05 \\
9.81 \mathrm{E}+02 \\
7.08 \mathrm{E}+01 \\
4.68 \mathrm{E}+01\end{array}$ & $\begin{array}{c}\text { U234 } \\
5.26 \mathrm{E}+01 \\
7.01 \mathrm{E}+01 \\
1.03 \mathrm{E}+02 \\
1.82 \mathrm{E}+02 \\
1.72 \mathrm{E}+02 \\
2.21 \mathrm{E}+02 \\
6.98 \mathrm{E}+01 \\
1.64 \mathrm{E}+02 \\
2.10 \mathrm{E}+02 \\
2.11 \mathrm{E}+02 \\
2.11 \mathrm{E}+02 \\
2.01 \mathrm{E}+01 \\
1.45 \mathrm{E}+02 \\
2.09 \mathrm{E}+02 \\
4.07 \mathrm{E}+01 \\
1.93 \mathrm{E}+02 \\
1.91 \mathrm{E}+02 \\
9.93 \mathrm{E}+01 \\
1.78 \mathrm{E}+02 \\
1.31 \mathrm{E}+02 \\
2.32 \mathrm{E}+02 \\
1.94 \mathrm{E}+02 \\
4.54 \mathrm{E}+01 \\
3.25 \mathrm{E}+01 \\
2.34 \mathrm{E}+02 \\
8.93 \mathrm{E}+01 \\
1.39 \mathrm{E}+02 \\
2.04 \mathrm{E}+02 \\
9.88 \mathrm{E}+01 \\
2.43 \mathrm{E}+02 \\
1.50 \mathrm{E}+02 \\
2.54 \mathrm{E}+02 \\
1.66 \mathrm{E}+02 \\
1.28 \mathrm{E}+01 \\
1.26 \mathrm{E}+01 \\
1.19 \mathrm{E}+02 \\
2.48 \mathrm{E}+02 \\
2.68 \mathrm{E}+02 \\
2.61 \mathrm{E}+02 \\
1.76 \mathrm{E}+02 \\
5.45 \mathrm{E}+01 \\
1.43 \mathrm{E}+02 \\
1.44 \mathrm{E}+02 \\
1.30 \mathrm{x}+02 \\
6.86 \mathrm{E}+01 \\
5.19 \mathrm{E}-01 \\
1.22 \mathrm{E}+02 \\
3.14 \mathrm{E}+01 \\
8.92 \mathrm{E}+01 \\
2.87 \mathrm{E}+01 \\
7.45 \mathrm{E}+00 \\
6.30 \mathrm{E}+01 \\
7.02 \mathrm{E}+01 \\
7.31 \mathrm{E}+00 \\
7.60 \mathrm{E}+01 \\
6.32 \mathrm{E}+01 \\
1.47 \mathrm{E}+01 \\
7.50 \mathrm{E}+00 \\
2.88 \mathrm{E}+01 \\
1.33 \mathrm{E}+01 \\
1.75 \mathrm{E}+01 \\
3.69 \mathrm{E}+01 \\
6.85 \mathrm{E}+00 \\
4.94 \mathrm{E}-01 \\
3.27 \mathrm{E}-01\end{array}$ & $\begin{array}{c}\text { U235 } \\
2.27 \mathrm{E}+00 \\
2.88 \mathrm{E}+00 \\
4.37 \mathrm{E}+00 \\
7.73 \mathrm{E}+00 \\
7.34 \mathrm{E}+00 \\
9.37 \mathrm{E}+00 \\
2.97 \mathrm{E}+00 \\
6.93 \mathrm{E}+00 \\
8.93 \mathrm{E}+00 \\
9.00 \mathrm{E}+00 \\
9.00 \mathrm{E}+00 \\
8.46 \mathrm{E}-01 \\
6.09 \mathrm{E}+00 \\
8.81 \mathrm{E}+00 \\
1.75 \mathrm{E}+00 \\
8.19 \mathrm{E}+00 \\
8.00 \mathrm{E}+00 \\
4.25 \mathrm{E}+00 \\
7.57 \mathrm{E}+00 \\
5.40 \mathrm{E}+00 \\
9.74 \mathrm{E}+00 \\
8.90 \mathrm{E}+00 \\
1.92 \mathrm{E}+00 \\
1.42 \mathrm{E}+00 \\
9.88 \mathrm{E}+00 \\
3.77 \mathrm{E}+00 \\
5.91 \mathrm{E}+00 \\
8.47 \mathrm{E}+00 \\
4.23 \mathrm{E}+00 \\
1.04 \mathrm{E}+01 \\
6.16 \mathrm{E}+00 \\
1.09 \mathrm{E}+01 \\
7.06 \mathrm{E}+00 \\
5.49 \mathrm{E}-01 \\
5.65 \mathrm{E}-01 \\
5.07 \mathrm{E}+00 \\
1.06 \mathrm{E}+01 \\
1.14 \mathrm{E}+01 \\
1.12 \mathrm{E}+01 \\
7.56 \mathrm{E}+00 \\
2.29 \mathrm{E}+00 \\
6.01 \mathrm{E}+00 \\
6.18 \mathrm{E}+00 \\
5.57 \mathrm{E}+00 \\
2.89 \mathrm{E}+00 \\
1.61 \mathrm{E}=06 \\
5.17 \mathrm{E}+00 \\
1.35 \mathrm{E}+00 \\
3.81 \mathrm{E}+00 \\
1.22 \mathrm{E}+00 \\
2.82 \mathrm{E}-01 \\
2.36 \mathrm{E}+00 \\
2.63 \mathrm{E}+00 \\
2.77 \mathrm{E}-01 \\
3.27 \mathrm{E}+00 \\
2.70 \mathrm{E}+00 \\
5.57 \mathrm{E}=01 \\
2.83 \mathrm{E}=01 \\
1.08 \mathrm{E}+00 \\
4.95 \mathrm{E}-01 \\
7.48 \mathrm{E}-01 \\
1.57 \mathrm{E}+00 \\
2.12 \mathrm{E}-05 \\
1.53 \mathrm{E}-06 \\
1.01 \mathrm{E}-06\end{array}$ & $\begin{array}{l}\text { U236 } \\
8.22 \mathrm{E}-01 \\
1.75 \mathrm{E}+00 \\
1.93 \mathrm{E}+00 \\
3.42 \mathrm{E}+00 \\
3.00 \mathrm{E}+00 \\
4.14 \mathrm{E}+00 \\
1.25 \mathrm{E}+00 \\
3.22 \mathrm{E}+00 \\
3.75 \mathrm{E}+00 \\
3.78 \mathrm{E}+00 \\
3.68 \mathrm{E}+00 \\
4.04 \mathrm{E}-01 \\
3.07 \mathrm{E}+00 \\
4.10 \mathrm{E}+00 \\
6.55 \mathrm{E}-01 \\
3.51 \mathrm{E}+00 \\
4.04 \mathrm{E}+00 \\
1.68 \mathrm{E}+00 \\
3.25 \mathrm{E}+00 \\
3.15 \mathrm{E}+00 \\
4.75 \mathrm{E}+00 \\
4.32 \mathrm{E}-01 \\
8.91 \mathrm{E}-01 \\
4.02 \mathrm{E}-01 \\
4.60 \mathrm{E}+00 \\
1.75 \mathrm{E}+00 \\
2.48 \mathrm{E}+00 \\
4.76 \mathrm{E}+00 \\
1.67 \mathrm{E}+00 \\
4.35 \mathrm{E}+00 \\
3.74 \mathrm{E}+00 \\
4.29 \mathrm{E}+00 \\
3.03 \mathrm{E}+00 \\
2.11 \mathrm{E}-01 \\
9.39 \mathrm{E}-02 \\
2.08 \mathrm{E}+00 \\
4.43 \mathrm{E}+00 \\
4.69 \mathrm{E}+00 \\
4.40 \mathrm{E}+00 \\
2.73 \mathrm{E}+00 \\
1.13 \mathrm{E}+00 \\
2.97 \mathrm{E}+00 \\
2.37 \mathrm{E}+00 \\
2.19 \mathrm{E}+00 \\
1.40 \mathrm{E}+00 \\
2.24 \mathrm{E}-07 \\
2.25 \mathrm{E}+00 \\
5.15 \mathrm{E}-01 \\
1.56 \mathrm{E}+00 \\
5.02 \mathrm{E}-01 \\
5.00 \mathrm{E}-01 \\
4.35 \mathrm{E}+00 \\
4.85 \mathrm{E}+00 \\
4.88 \mathrm{E}-01 \\
1.21 \mathrm{E}+00 \\
1.09 \mathrm{E}+00 \\
9.79 \mathrm{E}-01 \\
5.09 \mathrm{E}-01 \\
1.99 \mathrm{E}+00 \\
9.35 \mathrm{E}-01 \\
2.89 \mathrm{E}-01 \\
6.66 \mathrm{E}-01 \\
2.96 \mathrm{E}-06 \\
2.14 \mathrm{E}-07 \\
1.41 \mathrm{E}-07\end{array}$ & $\begin{array}{l}\mathrm{U} 238 \\
5.38 \mathrm{E}+01 \\
7.24 \mathrm{E}+01 \\
1.06 \mathrm{E}+02 \\
1.87 \mathrm{E}+02 \\
1.76 \mathrm{E}+02 \\
2.27 \mathrm{E}+02 \\
7.15 \mathrm{E}+01 \\
1.69 \mathrm{E}+02 \\
2.15 \mathrm{E}+02 \\
2.17 \mathrm{E}+02 \\
2.16 \mathrm{E}+02 \\
2.07 \mathrm{E}+01 \\
1.50 \mathrm{E}+02 \\
2.14 \mathrm{E}+02 \\
4.16 \mathrm{E}+01 \\
1.98 \mathrm{E}+02 \\
1.97 \mathrm{E}+02 \\
1.02 \mathrm{E}+02 \\
1.83 \mathrm{E}+02 \\
1.35 \mathrm{E}+02 \\
2.38 \mathrm{E}+02 \\
1.96 \mathrm{E}+02 \\
4.66 \mathrm{E}+01 \\
3.31 \mathrm{E}+01 \\
2.40 \mathrm{E}+02 \\
9.17 \mathrm{E}+01 \\
1.42 \mathrm{E}+02 \\
2.11 \mathrm{E}+02 \\
1.01 \mathrm{E}+02 \\
2.50 \mathrm{E}+02 \\
1.55 \mathrm{E}+02 \\
2.60 \mathrm{E}+02 \\
1.70 \mathrm{E}+02 \\
1.31 \mathrm{E}+01 \\
1.28 \mathrm{E}+01 \\
1.22 \mathrm{E}+02 \\
2.54 \mathrm{E}+02 \\
2.75 \mathrm{E}+02 \\
2.67 \mathrm{E}+02 \\
1.80 \mathrm{E}+02 \\
5.60 \mathrm{E}+01 \\
1.47 \mathrm{E}+02 \\
1.48 \mathrm{E}+02 \\
1.33 \mathrm{E}+02 \\
7.05 \mathrm{E}+01 \\
1.31 \mathrm{E}-14 \\
1.25 \mathrm{E}+02 \\
3.21 \mathrm{E}+01 \\
9.14 \mathrm{E}+01 \\
2.94 \mathrm{E}+01 \\
5.05 \mathrm{E}+00 \\
4.28 \mathrm{E}+01 \\
4.76 \mathrm{E}+01 \\
4.95 \mathrm{E}+00 \\
7.77 \mathrm{E}+01 \\
6.48 \mathrm{E}+01 \\
9.94 \mathrm{E}+00 \\
5.09 \mathrm{E}+00 \\
1.95 \mathrm{E}+01 \\
9.05 \mathrm{E}+00 \\
1.79 \mathrm{E}+01 \\
3.79 \mathrm{E}+01 \\
1.73 \mathrm{E}-13 \\
1.25 \mathrm{E}-14 \\
8.27 \mathrm{E}-15\end{array}$ & $\begin{array}{l}\text { NP237 } \\
2.77 \mathrm{E}-01 \\
6.56 \mathrm{E}-01 \\
6.75 \mathrm{E}-01 \\
1.19 \mathrm{E}+00 \\
1.03 \mathrm{E}+00 \\
1.45 \mathrm{E}+00 \\
4.31 \mathrm{E}-01 \\
1.14 \mathrm{E}+00 \\
1.30 \mathrm{E}+00 \\
1.31 \mathrm{E}+00 \\
1.27 \mathrm{E}+00 \\
1.43 \mathrm{E}-01 \\
1.10 \mathrm{E}+00 \\
1.44 \mathrm{E}+00 \\
2.22 \mathrm{E}-01 \\
1.22 \mathrm{E}+00 \\
1.45 \mathrm{E}+00 \\
5.74 \mathrm{E}-01 \\
1.13 \mathrm{E}+00 \\
1.17 \mathrm{E}+00 \\
1.69 \mathrm{E}+00 \\
1.27 \mathrm{E}-01 \\
3.14 \mathrm{E}-01 \\
1.31 \mathrm{E}-01 \\
1.62 \mathrm{E}+00 \\
6.17 \mathrm{E}-01 \\
8.57 \mathrm{E}-01 \\
1.75 \mathrm{E}+00 \\
5.71 \mathrm{E}-01 \\
1.50 \mathrm{E}+00 \\
1.40 \mathrm{E}+00 \\
1.47 \mathrm{E}+00 \\
1.05 \mathrm{E}+00 \\
7.15 \mathrm{E}-02 \\
2.90 \mathrm{E}-02 \\
7.14 \mathrm{E}-01 \\
1.53 \mathrm{E}+00 \\
1.61 \mathrm{E}+00 \\
1.50 \mathrm{E}+00 \\
9.18 \mathrm{E}-01 \\
4.02 \mathrm{E}-01 \\
1.06 \mathrm{E}+00 \\
8.05 \mathrm{E}-01 \\
7.48 \mathrm{E}-01 \\
4.94 \mathrm{E}-01 \\
7.25 \mathrm{E}-09 \\
7.82 \mathrm{E}-01 \\
1.75 \mathrm{E}-01 \\
5.35 \mathrm{E}-01 \\
1.72 \mathrm{E}-01 \\
6.07 \mathrm{E}-02 \\
5.88 \mathrm{E}-01 \\
6.55 \mathrm{E}-01 \\
5.75 \mathrm{E}-02 \\
4.09 \mathrm{E}-01 \\
3.75 \mathrm{E}-01 \\
1.16 \mathrm{E}-01 \\
6.40 \mathrm{E}-02 \\
2.39 \mathrm{E}-01 \\
9.57 \mathrm{E}-01 \\
4.57 \mathrm{E}-08 \\
\end{array}$ \\
\hline
\end{tabular}


HNF-SD-WM-TI-794, ReV. 0

ACTIVITY IN UNSEPARATEO FUEL (Curies decayed to 1/1/94)

\begin{tabular}{|c|c|c|c|c|c|c|c|c|c|}
\hline $\begin{aligned} \text { [Listing \#] } \\
\mathbf{5 2 1} \\
\mathbf{5 2 2} \\
\mathbf{5 2 3} \\
\mathbf{5 2 4} \\
\mathbf{5 2 5} \\
\mathbf{5 2 6} \\
\mathbf{5 2 7} \\
\mathbf{5 2 8} \\
\mathbf{5 2 9} \\
\mathbf{5 3 0} \\
\mathbf{5 3 1} \\
\mathbf{5 3 2} \\
\mathbf{5 3 3} \\
\mathbf{5 3 4} \\
\mathbf{5 3 5} \\
\mathbf{5 3 6} \\
\mathbf{5 3 7} \\
\mathbf{5 3 8} \\
\mathbf{5 3 9} \\
\mathbf{5 4 0} \\
\mathbf{5 4 1} \\
\mathbf{5 4 2} \\
\mathbf{5 4 3} \\
\mathbf{5 4 4} \\
\mathbf{5 4 5} \\
\mathbf{5 4 6} \\
\mathbf{5 4 7} \\
\mathbf{5 4 8} \\
\mathbf{5 4 9} \\
\mathbf{5 5 0} \\
\mathbf{5 5 1} \\
\mathbf{5 5 2} \\
\mathbf{5 5 3} \\
\mathbf{5 5 4} \\
\mathbf{5 5 5} \\
\mathbf{5 5 6} \\
\mathbf{5 5 7} \\
\mathbf{5 5 8} \\
\mathbf{5 5 9} \\
\mathbf{5 6 0} \\
\mathbf{5 6 1} \\
\mathbf{5 6 2} \\
\mathbf{5 6 3} \\
\mathbf{5 6 4} \\
\mathbf{5 6 5} \\
\mathbf{5 6 6} \\
\mathbf{5 6 7} \\
\mathbf{5 6 8} \\
\mathbf{5 6 9} \\
\mathbf{5 7 0} \\
\mathbf{5 7 1} \\
\mathbf{5 7 2} \\
\mathbf{5 7 3} \\
\mathbf{5 7 4} \\
\mathbf{5 7 3} \\
\mathbf{5 7 6} \\
\mathbf{5 7 7} \\
\mathbf{5 7 8} \\
\mathbf{5 7 9} \\
\mathbf{5 8 0} \\
\mathbf{5 8 1} \\
\mathbf{5 8 2} \\
\mathbf{5 8 3} \\
\mathbf{5 8 4} \\
585\end{aligned}$ & $\begin{array}{l}\text { PU238 } \\
7.01 \mathrm{E}+01 \\
2.79 \mathrm{E}+02 \\
2.11 \mathrm{E}+02 \\
3.73 \mathrm{E}+02 \\
2.97 \mathrm{E}+02 \\
4.53 \mathrm{E}+02 \\
1.28 \mathrm{E}+02 \\
3.75 \mathrm{E}+02 \\
3.85 \mathrm{E}+02 \\
3.88 \mathrm{E}+02 \\
3.66 \mathrm{E}+02 \\
4.86 \mathrm{E}+01 \\
3.96 \mathrm{E}+02 \\
4.79 \mathrm{E}+02 \\
5.88 \mathrm{E}+01 \\
3.72 \mathrm{E}+02 \\
5.22 \mathrm{E}+02 \\
1.61 \mathrm{E}+02 \\
3.45 \mathrm{E}+02 \\
4.87 \mathrm{E}+02 \\
5.90 \mathrm{E}+02 \\
2.11 \mathrm{E}+00 \\
1.05 \mathrm{E}+02 \\
2.53 \mathrm{E}+01 \\
5.41 \mathrm{E}+02 \\
2.06 \mathrm{E}+02 \\
2.59 \mathrm{E}+02 \\
7.06 \mathrm{E}+02 \\
1.62 \mathrm{E}+02 \\
4.54 \mathrm{E}+02 \\
6.06 \mathrm{E}+02 \\
4.16 \mathrm{E}+02 \\
3.25 \mathrm{E}+02 \\
1.97 \mathrm{E}+01 \\
2.73 \mathrm{E}+00 \\
2.11 \mathrm{E}+02 \\
4.64 \mathrm{E}+02 \\
4.76 \mathrm{E}+02 \\
4.26 \mathrm{E}+02 \\
2.37 \mathrm{E}+02 \\
1.45 \mathrm{E}+02 \\
3.81 \mathrm{E}+02 \\
2.23 \mathrm{E}+02 \\
2.13 \mathrm{E}+02 \\
1.74 \mathrm{E}+02 \\
9.90 \mathrm{E}-07 \\
2.49 \mathrm{E}+02 \\
4.86 \mathrm{E}+01 \\
1.60 \mathrm{E}+02 \\
5.13 \mathrm{E}+01 \\
1.83 \mathrm{E}+01 \\
2.03 \mathrm{E}+02 \\
2.26 \mathrm{E}+02 \\
1.68 \mathrm{E}+01 \\
1.10 \mathrm{E}+02 \\
1.10 \mathrm{E}+02 \\
3.36 \mathrm{E}+01 \\
2.03 \mathrm{E}+01 \\
9.28 \mathrm{E}+01 \\
4.96 \mathrm{E}+01 \\
2.75 \mathrm{E}+01 \\
7.13 \mathrm{E}+01 \\
1.31 \mathrm{E}-05 \\
9.46 \mathrm{E}-07 \\
6.22 \mathrm{E}-07\end{array}$ & $\begin{array}{l}P U 239 \\
5.35 E+03 \\
1.10 E+04 \\
1.24 E+04 \\
2.20 E+04 \\
1.94 E+04 \\
2.67 E+04 \\
8.05 E+03 \\
2.07 E+04 \\
2.42 E+04 \\
2.44 E+04 \\
2.38 E+04 \\
2.59 E+03 \\
1.96 E+04 \\
2.63 E+04 \\
4.26 E+03 \\
2.26 E+04 \\
2.57 E+04 \\
1.09 E+04 \\
2.09 E+04 \\
1.98 E+04 \\
3.03 E+04 \\
2.95 E+03 \\
5.71 E+03 \\
2.65 E+03 \\
2.95 E+04 \\
1.12 E+04 \\
1.60 E+04 \\
3.01 E+04 \\
1.08 E+04 \\
2.81 E+04 \\
2.34 E+04 \\
2.78 E+04 \\
1.95 E+04 \\
1.37 E+03 \\
6.30 E+02 \\
1.34 E+04 \\
2.86 E+04 \\
3.03 E+04 \\
2.85 E+04 \\
1.78 E+04 \\
7.21 E+03 \\
1.90 E+04 \\
1.54 E+04 \\
1.42 E+04 \\
8.92 E+03 \\
2.82 E-11 \\
1.45 E+04 \\
3.35 E+03 \\
1.01 E+04 \\
3.24 E+03 \\
5.35 E+02 \\
5.06 E+03 \\
5.64 E+03 \\
5.10 E+02 \\
7.88 E+03 \\
7.08 E+03 \\
1.02 E+03 \\
5.60 E+02 \\
2.31 E+03 \\
1.13 E+03 \\
1.88 E+03 \\
4.30 E+03 \\
3.72 E=10 \\
2.69 E-11 \\
1.78 E-11\end{array}$ & $\begin{array}{l}\text { PU240 } \\
7.00 E+02 \\
2.46 E+03 \\
1.99 E+03 \\
3.51 E+03 \\
2.86 E+03 \\
4.26 E+03 \\
1.22 E+03 \\
3.48 E+03 \\
3.67 E+03 \\
3.69 E+03 \\
3.51 E+03 \\
4.48 E+02 \\
3.59 E+03 \\
4.42 E+03 \\
5.76 E+02 \\
3.51 E+03 \\
4.72 E+03 \\
1.55 E+03 \\
3.24 E+03 \\
4.26 E+03 \\
5.36 E+03 \\
7.99 E+01 \\
9.61 E+02 \\
2.74 E+02 \\
4.96 E+03 \\
1.89 E+03 \\
2.43 E+03 \\
6.19 E+03 \\
1.54 E+03 \\
4.26 E+03 \\
5.23 E+03 \\
3.97 E+03 \\
3.03 E+03 \\
1.89 E+02 \\
4.04 E+01 \\
1.99 E+03 \\
4.34 E+03 \\
4.48 E+03 \\
4.05 E+03 \\
2.31 E+03 \\
1.29 E+03 \\
3.40 E+03 \\
2.13 E+03 \\
2.02 E+03 \\
1.56 E+03 \\
1.51 E-12 \\
2.29 E+03 \\
4.63 E+02 \\
1.49 E+03 \\
4.79 E+02 \\
7.81 E+01 \\
8.46 E+02 \\
9.43 E+02 \\
7.18 E+01 \\
1.05 E+03 \\
1.03 E+03 \\
1.44 E+02 \\
8.56 E+01 \\
3.87 E+02 \\
2.04 E+02 \\
2.61 E+02 \\
6.58 E+02 \\
1.99 E-11 \\
1.44 E-12 \\
9.52 E-13\end{array}$ & $\begin{array}{l}\text { PU241 } \\
5.39 \mathrm{E}+03 \\
2.50 \mathrm{E}+04 \\
1.75 \mathrm{E}+04 \\
3.10 \mathrm{E}+04 \\
2.42 \mathrm{E}+04 \\
3.80 \mathrm{E}+04 \\
1.06 \mathrm{E}+04 \\
3.20 \mathrm{E}+04 \\
3.21 \mathrm{E}+04 \\
3.25 \mathrm{E}+04 \\
3.05 \mathrm{E}+04 \\
4.22 \mathrm{E}+03 \\
3.53 \mathrm{E}+04 \\
4.19 \mathrm{E}+04 \\
4.81 \mathrm{E}+03 \\
3.20 \mathrm{E}+04 \\
4.71 \mathrm{E}+04 \\
1.36 \mathrm{E}+04 \\
3.01 \mathrm{E}+04 \\
4.60 \mathrm{E}+04 \\
5.36 \mathrm{E}+04 \\
0.00 \mathrm{E}+00 \\
9.49 \mathrm{E}+03 \\
1.84 \mathrm{E}+03 \\
4.93 \mathrm{E}+04 \\
1.89 \mathrm{E}+04 \\
2.30 \mathrm{E}+04 \\
6.81 \mathrm{E}+04 \\
1.42 \mathrm{E}+04 \\
4.09 \mathrm{E}+04 \\
5.93 \mathrm{E}+04 \\
3.69 \mathrm{E}+04 \\
2.98 \mathrm{E}+04 \\
1.74 \mathrm{E}+03 \\
7.33 \mathrm{E}+01 \\
1.91 \mathrm{E}+04 \\
4.26 \mathrm{E}+04 \\
4.34 \mathrm{E}+04 \\
3.85 \mathrm{E}+04 \\
2.07 \mathrm{E}+04 \\
1.41 \mathrm{E}+04 \\
3.73 \mathrm{E}+04 \\
2.02 \mathrm{E}+04 \\
1.96 \mathrm{E}+04 \\
1.72 \mathrm{E}+04 \\
6.60 \mathrm{E}-12 \\
2.39 \mathrm{E}+04 \\
4.48 \mathrm{E}+03 \\
1.51 \mathrm{E}+04 \\
4.87 \mathrm{E}+03 \\
6.85 \mathrm{E}+02 \\
7.99 \mathrm{E}+03 \\
8.80 \mathrm{E}+03 \\
6.29 \mathrm{E}+02 \\
1.00 \mathrm{E}+04 \\
1.05 \mathrm{E}+04 \\
1.26 \mathrm{E}+03 \\
7.80 \mathrm{E}+02 \\
3.62 \mathrm{E}+03 \\
1.99 \mathrm{E}+03 \\
2.57 \mathrm{E}+03 \\
6.89 \mathrm{E}+03 \\
8.64 \mathrm{E}-11 \\
6.36 \mathrm{E}-12 \\
4.09 \mathrm{E}-12\end{array}$ & $\begin{array}{l}\text { PU242 } \\
1.67 \mathrm{E}-02 \\
9.05 \mathrm{E}-02 \\
5.85 \mathrm{E}-02 \\
1.04 \mathrm{E}-01 \\
7.81 \mathrm{E}-02 \\
1.26 \mathrm{E}-01 \\
3.43 \mathrm{E}-02 \\
1.07 \mathrm{E}-01 \\
1.03 \mathrm{E}-01 \\
1.04 \mathrm{E}-01 \\
9.59 \mathrm{E}-02 \\
1.41 \mathrm{E}-02 \\
1.18 \mathrm{E}-01 \\
1.36 \mathrm{E}-01 \\
1.43 \mathrm{E}-02 \\
1.01 \mathrm{E}-01 \\
1.55 \mathrm{E}-01 \\
4.10 \mathrm{E}-02 \\
9.30 \mathrm{E}-02 \\
1.53 \mathrm{E}-01 \\
1.71 \mathrm{E}-01 \\
0.00 \mathrm{E}+00 \\
2.96 \mathrm{E}-02 \\
4.24 \mathrm{E}-03 \\
1.53 \mathrm{E}-01 \\
5.82 \mathrm{E}-02 \\
6.83 \mathrm{E}-02 \\
2.18 \mathrm{E}-01 \\
4.08 \mathrm{E}-02 \\
1.20 \mathrm{E}-01 \\
1.92 \mathrm{E}-01 \\
1.05 \mathrm{E}-01 \\
8.68 \mathrm{E}-02 \\
4.82 \mathrm{E}-03 \\
0.00 \mathrm{E}+00 \\
5.44 \mathrm{E}-02 \\
1.22 \mathrm{E}-01 \\
1.23 \mathrm{E}-01 \\
1.06 \mathrm{E}-01 \\
5.48 \mathrm{E}-02 \\
4.18 \mathrm{E}-02 \\
1.10 \mathrm{E}-01 \\
5.43 \mathrm{E}-02 \\
5.30 \mathrm{E}-02 \\
4.96 \mathrm{E}-02 \\
8.74 \mathrm{E}-18 \\
6.65 \mathrm{E}-02 \\
1.18 \mathrm{E}-02 \\
4.08 \mathrm{E}-02 \\
1.31 \mathrm{E}-02 \\
1.57 \mathrm{E}-03 \\
1.85 \mathrm{E}-02 \\
2.06 \mathrm{E}-02 \\
1.41 \mathrm{E}-03 \\
2.58 \mathrm{E}-02 \\
2.80 \mathrm{E}-02 \\
2.83 \mathrm{E}-03 \\
1.77 \mathrm{E}-03 \\
8.44 \mathrm{E}-03 \\
4.63 \mathrm{E}-03 \\
6.71 \mathrm{E}-03 \\
1.87 \mathrm{E}-02 \\
1.16 \mathrm{E}-16 \\
8.34 \mathrm{E}-18 \\
5.51 \mathrm{E}-18\end{array}$ & $\begin{array}{l}A M 241 \\
6.52 \mathrm{E}+02 \\
3.01 \mathrm{E}+03 \\
2.10 \mathrm{E}+03 \\
3.71 \mathrm{E}+03 \\
2.88 \mathrm{E}+03 \\
4.49 \mathrm{E}+03 \\
1.25 \mathrm{E}+03 \\
3.77 \mathrm{E}+03 \\
3.74 \mathrm{E}+03 \\
3.76 \mathrm{E}+03 \\
3.50 \mathrm{E}+03 \\
4.90 \mathrm{E}+02 \\
4.03 \mathrm{E}+03 \\
4.75 \mathrm{E}+03 \\
5.42 \mathrm{E}+02 \\
3.60 \mathrm{E}+03 \\
5.27 \mathrm{E}+03 \\
1.51 \mathrm{E}+03 \\
3.31 \mathrm{E}+03 \\
5.04 \mathrm{E}+03 \\
5.86 \mathrm{E}+03 \\
0.00 \mathrm{E}+00 \\
1.02 \mathrm{E}+03 \\
1.97 \mathrm{E}+02 \\
5.26 \mathrm{E}+03 \\
2.00 \mathrm{E}+03 \\
2.43 \mathrm{E}+03 \\
7.16 \mathrm{E}+03 \\
1.48 \mathrm{E}+03 \\
4.24 \mathrm{E}+03 \\
6.22 \mathrm{E}+03 \\
3.79 \mathrm{E}+03 \\
3.04 \mathrm{E}+03 \\
1.76 \mathrm{E}+02 \\
7.38 \mathrm{E}+00 \\
1.93 \mathrm{E}+03 \\
4.29 \mathrm{E}+03 \\
4.35 \mathrm{E}+03 \\
3.83 \mathrm{E}+03 \\
2.05 \mathrm{E}+03 \\
1.39 \mathrm{E}+03 \\
3.65 \mathrm{E}+03 \\
1.97 \mathrm{E}+03 \\
1.90 \mathrm{E}+03 \\
1.65 \mathrm{E}+03 \\
6.29 \mathrm{E}-13 \\
2.28 \mathrm{E}+03 \\
4.25 \mathrm{E}+02 \\
1.43 \mathrm{E}+03 \\
4.59 \mathrm{E}+02 \\
6.65 \mathrm{E}+01 \\
7.58 \mathrm{E}+02 \\
8.47 \mathrm{E}+02 \\
6.00 \mathrm{E}+01 \\
9.42 \mathrm{E}+02 \\
9.83 \mathrm{E}+02 \\
1.21 \mathrm{E}+02 \\
7.40 \mathrm{E}+01 \\
3.47 \mathrm{E}+02 \\
1.87 \mathrm{E}+02 \\
2.40 \mathrm{E}+02 \\
6.42 \mathrm{E}+02 \\
8.33 \mathrm{E}-12 \\
5.97 \mathrm{E}-13 \\
3.98 \mathrm{E}-13\end{array}$ & $\begin{array}{l}\text { AM243 } \\
5.86 \mathrm{E}-03 \\
3.44 \mathrm{E}-02 \\
2.15 \mathrm{E}-02 \\
3.80 \mathrm{E}-02 \\
2.83 \mathrm{E}-02 \\
4.61 \mathrm{E}-02 \\
1.25 \mathrm{E}-02 \\
3.96 \mathrm{E}-02 \\
3.75 \mathrm{E}-02 \\
3.77 \mathrm{E}-02 \\
3.47 \mathrm{E}-02 \\
5.23 \mathrm{E}-03 \\
4.40 \mathrm{E}-02 \\
5.03 \mathrm{E}-02 \\
5.08 \mathrm{E}-03 \\
3.67 \mathrm{E}-02 \\
5.78 \mathrm{E}-02 \\
1.47 \mathrm{E}-02 \\
3.40 \mathrm{E}-02 \\
5.82 \mathrm{E}-02 \\
6.38 \mathrm{E}-02 \\
0.00 \mathrm{E}+00 \\
1.09 \mathrm{E}-02 \\
1.29 \mathrm{E}-03 \\
5.65 \mathrm{E}-02 \\
2.15 \mathrm{E}-02 \\
2.48 \mathrm{E}-02 \\
8.25 \mathrm{E}-02 \\
1.46 \mathrm{E}-02 \\
4.36 \mathrm{E}-02 \\
7.32 \mathrm{E}-02 \\
3.76 \mathrm{E}-02 \\
3.17 \mathrm{E}-02 \\
1.72 \mathrm{E}-03 \\
0.00 \mathrm{E}+00 \\
1.97 \mathrm{E}-02 \\
4.44 \mathrm{E}-02 \\
4.44 \mathrm{E}-02 \\
3.82 \mathrm{E}-02 \\
1.92 \mathrm{E}-02 \\
1.56 \mathrm{E}-02 \\
4.10 \mathrm{E}-02 \\
1.93 \mathrm{E}-02 \\
1.90 \mathrm{E}-02 \\
1.85 \mathrm{E}-02 \\
1.75 \mathrm{E}-18 \\
2.43 \mathrm{E}-02 \\
4.21 \mathrm{E}-03 \\
1.48 \mathrm{E}-02 \\
4.75 \mathrm{E}-03 \\
4.59 \mathrm{E}-04 \\
5.47 \mathrm{E}-03 \\
6.09 \mathrm{E}-03 \\
4.11 \mathrm{E}-04 \\
9.12 \mathrm{E}-03 \\
1.01 \mathrm{E}-02 \\
8.25 \mathrm{E}-04 \\
5.21 \mathrm{E}-04 \\
2.50 \mathrm{E}-03 \\
1.38 \mathrm{E}-03 \\
2.39 \mathrm{E}-03 \\
6.80 \mathrm{E}-03 \\
2.31 \mathrm{E}-17 \\
1.67 \mathrm{E}-18 \\
1.10 \mathrm{E}-18\end{array}$ & $\begin{array}{l}\text { CM242 } \\
1.83 \mathrm{E}-02 \\
1.07 \mathrm{E}=01 \\
6.71 \mathrm{E}-02 \\
1.19 \mathrm{E}-01 \\
8.84 \mathrm{E}-02 \\
1.44 \mathrm{E}-01 \\
3.90 \mathrm{E}-02 \\
1.24 \mathrm{E}-01 \\
1.17 \mathrm{E}-01 \\
1.18 \mathrm{E}-01 \\
1.09 \mathrm{E}-01 \\
1.64 \mathrm{E}-02 \\
1.38 \mathrm{E}-01 \\
1.58 \mathrm{E}-01 \\
1.60 \mathrm{E}-02 \\
1.15 \mathrm{E}-01 \\
1.82 \mathrm{E}-01 \\
4.63 \mathrm{E}-02 \\
1.07 \mathrm{E}-01 \\
1.83 \mathrm{E}-01 \\
2.01 \mathrm{E}-01 \\
0.00 \mathrm{E}+00 \\
3.45 \mathrm{E}-02 \\
4.10 \mathrm{E}-03 \\
1.78 \mathrm{E}-01 \\
6.79 \mathrm{E}-02 \\
7.84 \mathrm{E}-02 \\
2.60 \mathrm{E}-01 \\
4.63 \mathrm{E}-02 \\
1.38 \mathrm{E}-01 \\
2.31 \mathrm{E}-01 \\
1.19 \mathrm{E}-01 \\
1.00 \mathrm{E}-01 \\
5.44 \mathrm{E}-03 \\
0.00 \mathrm{E}+00 \\
6.23 \mathrm{E}-02 \\
1.41 \mathrm{E}-01 \\
1.41 \mathrm{E}-01 \\
1.21 \mathrm{E}-01 \\
6.09 \mathrm{E}-02 \\
4.94 \mathrm{E}-02 \\
1.30 \mathrm{E}-01 \\
6.14 \mathrm{E}-02 \\
6.05 \mathrm{E}-02 \\
5.86 \mathrm{E}-02 \\
0.00 \mathrm{E}+00 \\
7.73 \mathrm{E}-02 \\
1.34 \mathrm{E}-02 \\
4.70 \mathrm{E}-02 \\
1.51 \mathrm{E}-02 \\
1.90 \mathrm{E}-03 \\
2.26 \mathrm{E}-02 \\
2.52 \mathrm{E}-02 \\
1.70 \mathrm{E}-03 \\
2.91 \mathrm{E}-02 \\
3.22 \mathrm{E}-02 \\
3.41 \mathrm{E}-03 \\
2.16 \mathrm{E}-03 \\
1.03 \mathrm{E}-02 \\
5.70 \mathrm{E}-03 \\
7.63 \mathrm{E}-03 \\
2.17 \mathrm{E}-02 \\
0.00 \mathrm{E}+00 \\
0.00 \mathrm{E}+00 \\
0.00 \mathrm{E}+00\end{array}$ & $\begin{array}{l}\text { CM243 } \\
4.69 \mathrm{E}-04 \\
2.88 \mathrm{E}-03 \\
1.77 \mathrm{E}-03 \\
3.13 \mathrm{E}-03 \\
2.32 \mathrm{E}-03 \\
3.82 \mathrm{E}-03 \\
1.03 \mathrm{E}-03 \\
3.30 \mathrm{E}-03 \\
3.11 \mathrm{E}-03 \\
3.13 \mathrm{E}-03 \\
2.88 \mathrm{E}-03 \\
4.39 \mathrm{E}-04 \\
3.73 \mathrm{E}-03 \\
4.26 \mathrm{E}-03 \\
4.21 \mathrm{E}-04 \\
3.10 \mathrm{E}-03 \\
4.94 \mathrm{E}-03 \\
1.23 \mathrm{E}-03 \\
2.88 \mathrm{E}-03 \\
5.05 \mathrm{E}-03 \\
5.49 \mathrm{E}-03 \\
0.00 \mathrm{E}+00 \\
9.44 \mathrm{E}-04 \\
9.90 \mathrm{E}-05 \\
4.89 \mathrm{E}-03 \\
1.87 \mathrm{E}-03 \\
2.14 \mathrm{E}-03 \\
7.27 \mathrm{E}-03 \\
1.26 \mathrm{E}-03 \\
3.78 \mathrm{E}-03 \\
6.48 \mathrm{E}-03 \\
3.25 \mathrm{E}-03 \\
2.77 \mathrm{E}-03 \\
1.49 \mathrm{E}-04 \\
0.00 \mathrm{E}+00 \\
1.72 \mathrm{E}-03 \\
3.89 \mathrm{E}-03 \\
3.89 \mathrm{E}-03 \\
3.34 \mathrm{E}-03 \\
1.66 \mathrm{E}-03 \\
1.40 \mathrm{E}-03 \\
3.68 \mathrm{E}-03 \\
1.70 \mathrm{E}-03 \\
1.68 \mathrm{E}-03 \\
1.67 \mathrm{E}-03 \\
1.02 \mathrm{E}-19 \\
2.19 \mathrm{E}-03 \\
3.73 \mathrm{E}-04 \\
1.32 \mathrm{E}-03 \\
4.27 \mathrm{E}-04 \\
4.16 \mathrm{E}-05 \\
5.03 \mathrm{E}-04 \\
5.57 \mathrm{E}-04 \\
3.74 \mathrm{E}-05 \\
8.09 \mathrm{E}-04 \\
9.08 \mathrm{E}-04 \\
7.49 \mathrm{E}-05 \\
4.77 \mathrm{E}-05 \\
2.29 \mathrm{E}-04 \\
1.27 \mathrm{E}-04 \\
2.14 \mathrm{E}-04 \\
6.16 \mathrm{E}-04 \\
1.35 \mathrm{E}-18 \\
9.81 \mathrm{E}-20 \\
6.40 \mathrm{E}-20\end{array}$ \\
\hline
\end{tabular}


HNF-SD-WM-TI-794, ReV. 0

ACTIVITY IN UNSEPARATED FUEL (Curies decayed to $1 / 1 / 94$ )

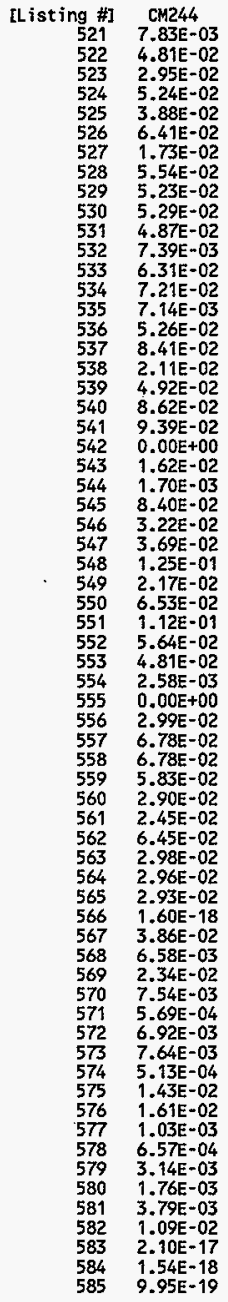

Page $A-74$ 
HNF-SD-WM-TI-794, ReV. 0

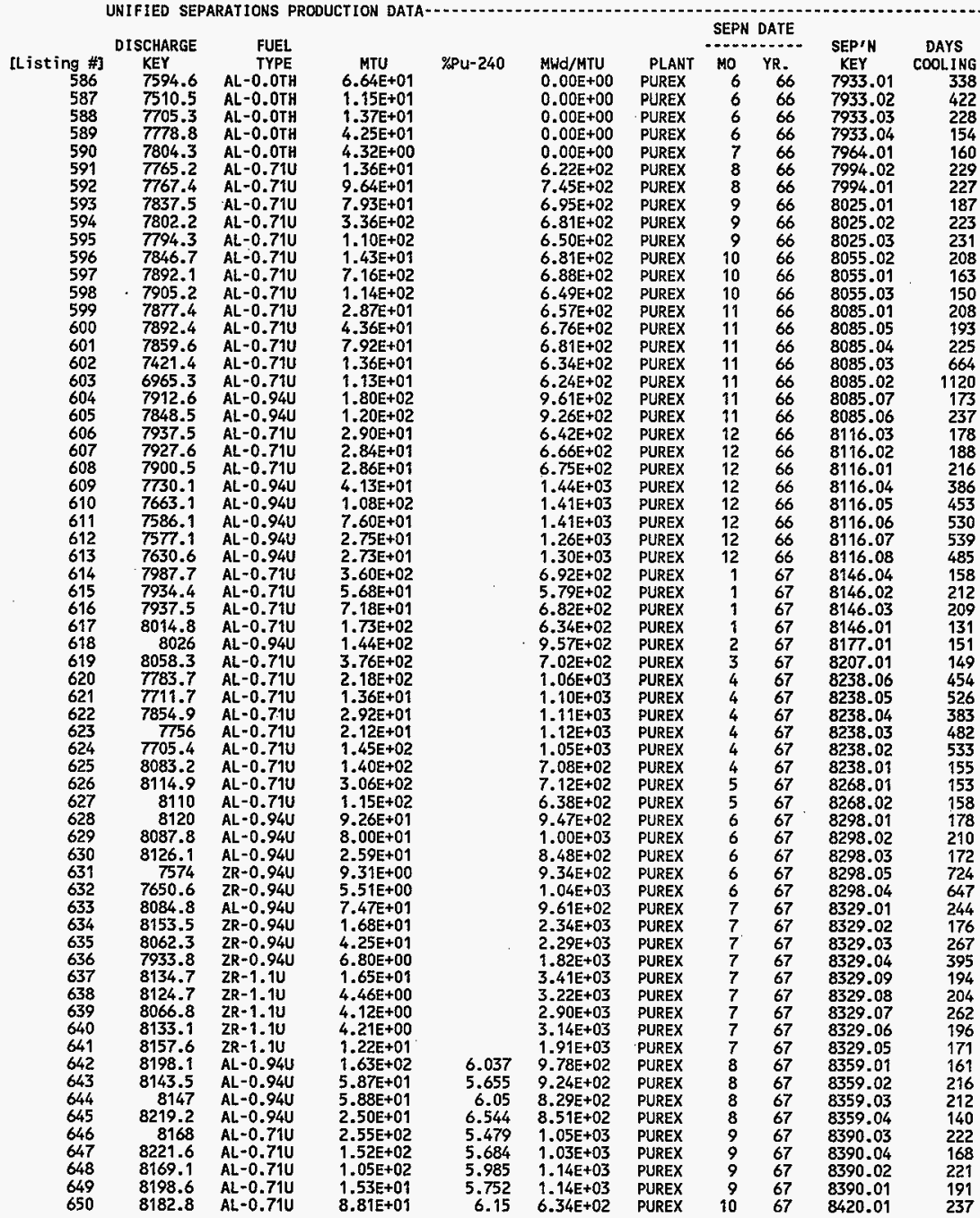


HNF-SD-WN-TI-794, Rev. 0

UNIFIED SEPARATIONS PRODUCTION DATA TOTAL WASTE LOSS FRACTIOH

[Listing \#] Fuel Data Reference

586 ISO-263 RD AND ARH-18 RD (MCDONALD

587 ISO-263 RD AND ARH-18 RD (MCDONALD

588 ISO-263 RD AND ARH-18 RD (MCDONALD

589 ISO-263 RD AND ARH-18 RD (MCDONALD

590 ISO-263 RD AND ARH-18 RD (MCDONALD

591 ISO-263 RD AND ARH-18 RD (MCDONALD

592 ISO-263 RD AND ARH-18 RD (MCDONALD

593 ISO-263 RD AND ARH-18 RD (MCDONALD

594 ISO-263 RD AND ARH-18 RD (MCDONALD

595 ISO-263 RD AND ARH-18 RD (MCDONALD

596 ISO-263 RD AND ARH-18 RD (MCDONALO

597 ISO-263 RD AND ARH-18 RD (MCDONALD

598 ISO-2.63 RD AND ARH-18 RD (MCDONALD

599 ISO-263 RD AND ARH-18 RD (MCDONALD

600 ISO-263 RD AND ARH-18 RD (MCDONALD

601 ISO-263 RD AND ARH-18 RD (MCDONALD

602 ISO-263 RD AND ARH-18 RD (MCDONALD

603 ISO-263 RD AND ARH-18 RD (MCDONALD

604 iso-263 RD AND ARH-18 RD (MCDONALD

605 ISO-263 RD AND ARH-18 RD (MCDONALD

606 ISO-263 RD AND ARH-18 RD (MCDONALD

607 ISO-263 RD AND ARH-18 RD (MCDONALD

608 ISO-263 RD AND ARH-18 RD (MCDONALD

609 ISO-263 RD AND ARH-18 RD (MCDONALD

610 ISO-263 RD AND ARH-18 RD (MCDONALD

611 I SO-263 RD AND ARH-18 RD (MCDONALD

612 1SO-263 RD AND ARH-18 RD (MCDONALD

613 ISO-263 RD AND ARH-18 RD (MCDONALD

614 15O-263 RD AND ARH-18 RD (MCDONALD

615 ISO-263 RD AND ARH-18 RD (MCDONALD

616 ISO-263 RD AND ARH-18 RD (MCDONALD

617 ISO-263 RD AND ARH-18 RD (MCDONALD

618 ISO-263 RD AND ARH-18 RD (MCDONALD

619 ISO-263 RD AND ARH-18 RD (MCDONALD

620 J $50-263$ RD AND ARH-18 RD (MCDONALD

621 ISO-263 RD AND ARH-18 RD (HCDONALD

622 1SO-263 RD AND ARH-18 RD (MCDONALD

623 ISO-263 RD AND ARH- 18 RD (MCDONALD

624 iso-263 RD AND ARH-18 RD (MCDONALD

625 1SO-263 RD AND ARH-18 RD (MCDONALD

626 ISO-263 RD AND ARH-18 RD (MCDONALD

627 150-263 RD AND ARH-18 RD (MCDONALD

628 ISO-263 RD AND ARH-18 RD (MCDONALD

629 ISO-263 RD AND ARH-18 RD (MCDONALD

630 1SO-263 RD AND ARH-18 RD (MCDONALD

631 ISO-263 RD AND ARH-18 RD (MCDONALD

632 150-263 RD AND ARH-18 RD (MCDONALD

633 150-263 RD AND ARH-18 RD (MCDONALD

634 150-263 RD AND ARH 18 RD (MCDONALD

635 ISO-263 RD AND ARH-18 RD (MCDONALD

636 1SO-263 RD AND ARH-18 RD (MCDONALD

637 ISO-263 RD AND ARH-18 RD (MCDONALD

638 ISO-263 RD AND ARH- 18 RD (MCDONALD

639 ISO-263 RD AND ARH-18 RD (MCDONALD

640 ISO-263 RD AND ARH-18 RD (MCDONALD

641 ISO-263 RD AND ARH-18 RD (MCDONALD

642 ISO-263 RD AND ARH-18 RD (MCDONALD

643 ISO-263 RD AND ARH-18 RD (MCDONALD

644 ISO-263 RD AND ARH-18 RD (MCDONALD

645 ISO-263 RD AND ARH-18 RD (MCDONALD

646 ISO-263 RD AND ARH-18 RD (MCDONALD

647 ISO-263 RD AND ARH-18 RD (MCDONALD

648 ISO-263 RD AND ARH-18 RD (MCDONALD

649 ISO-263 RD AND ARH-18 RD (MCDONALD 650 I SO-263 RD AND ARH-18 RD (MCDONALD
TOTAL WASTE LOSS FRACTION

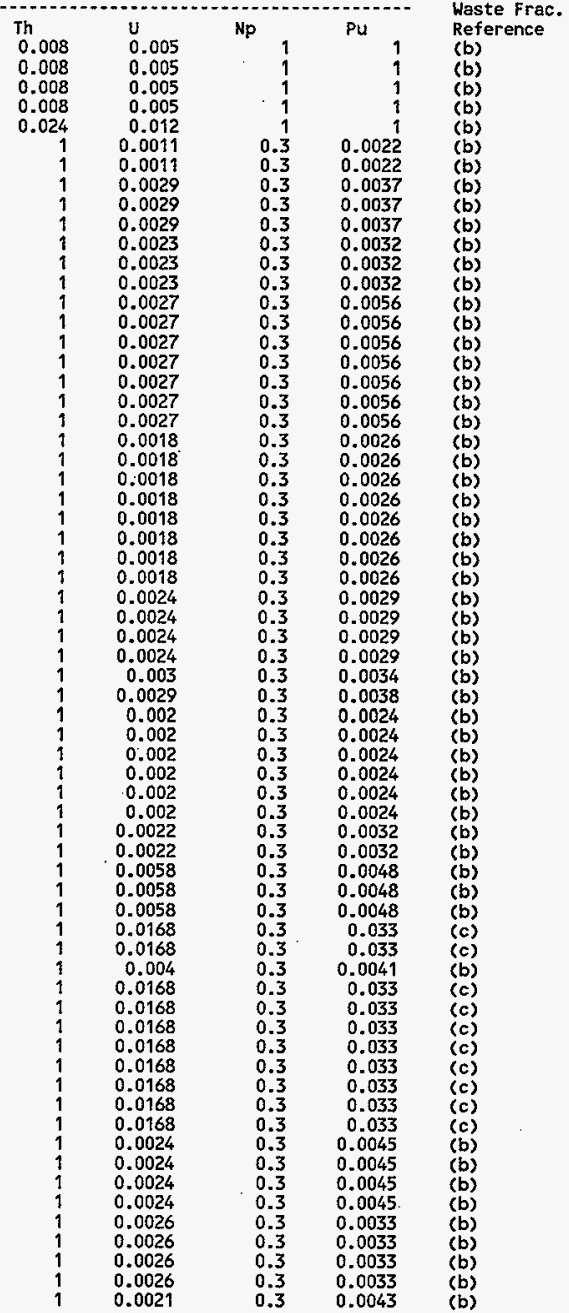


HNF-SD-WM-TI-794, ReV. 0

ACTIVITY IN UNSEPARATED FUEL (Curies decayed to $1 / 1 / 94$ )

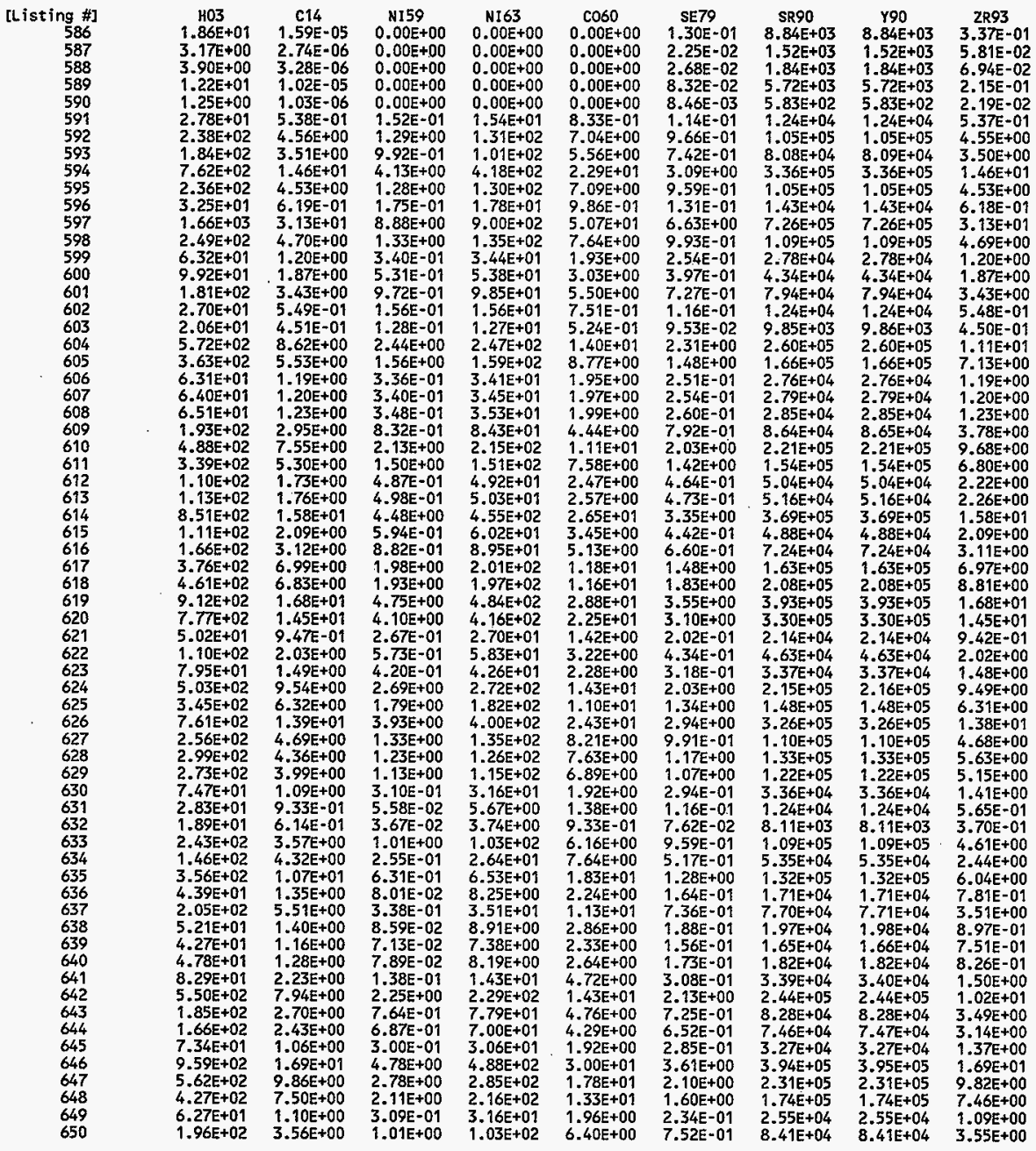


ACTIVITY IN UNSEPARATED FUEL (Curies decayed to $1 / 1 / 94$ )

\begin{tabular}{|c|c|c|c|c|c|c|c|c|c|}
\hline 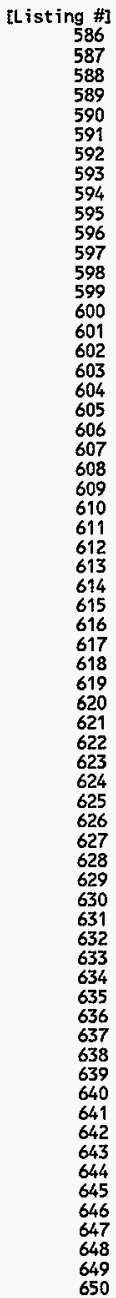 & 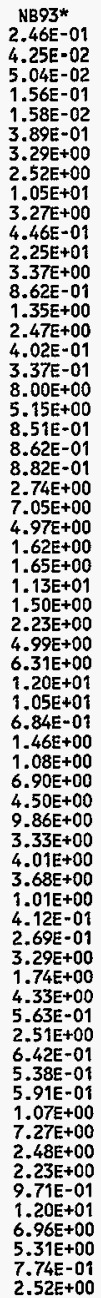 & 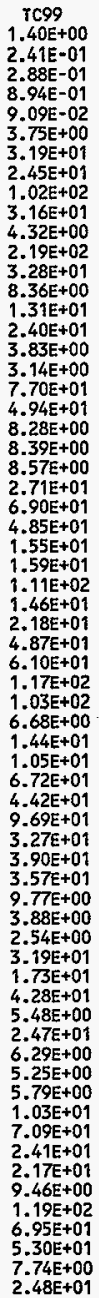 & 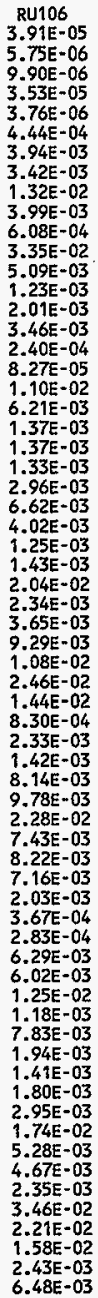 & 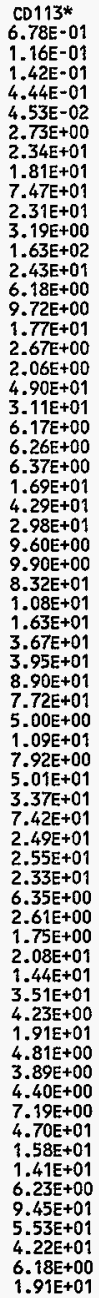 & 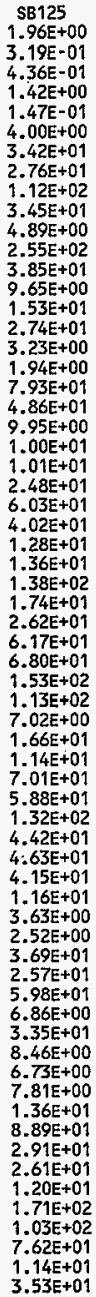 & 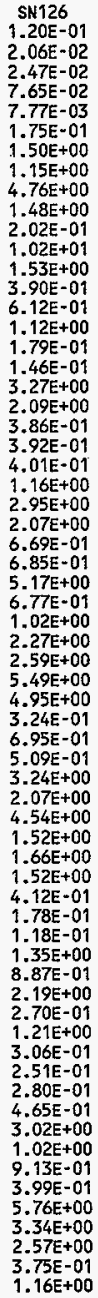 & 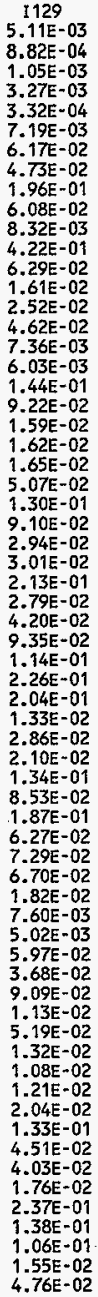 & 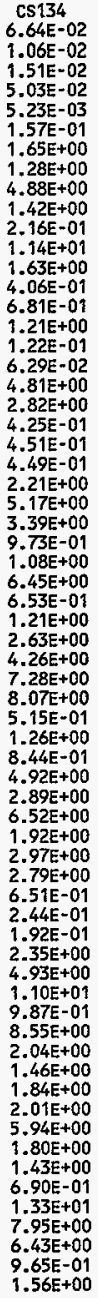 & 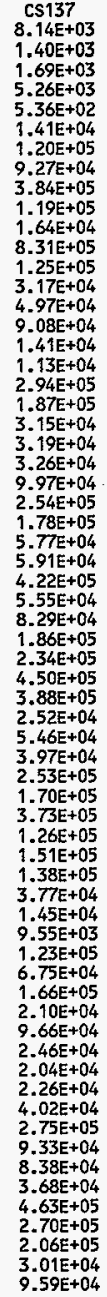 \\
\hline
\end{tabular}


ACTIVITY IN UNSEPARATED FUEL (Curies decayed to $1 / 1 / 94$ )

\begin{tabular}{|c|c|c|c|c|c|c|c|c|c|}
\hline 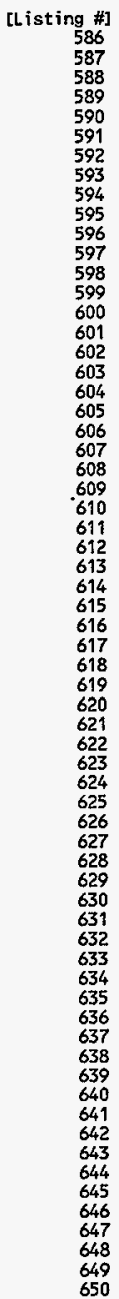 & 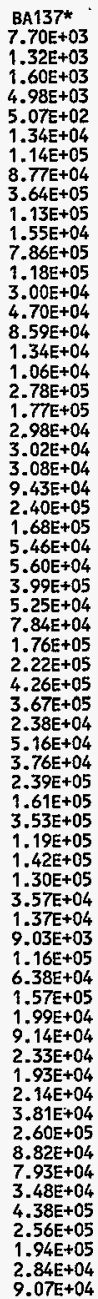 & 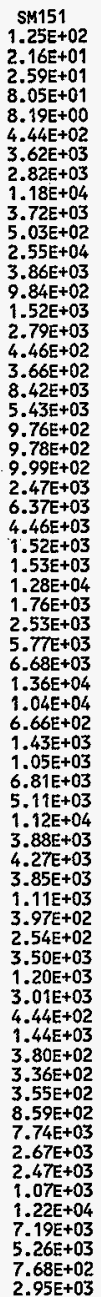 & 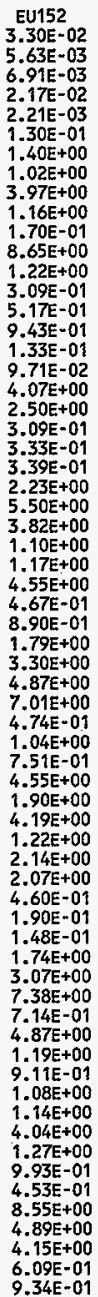 & 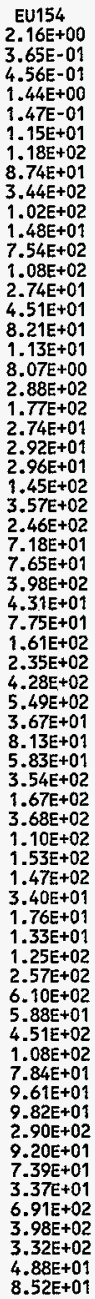 & 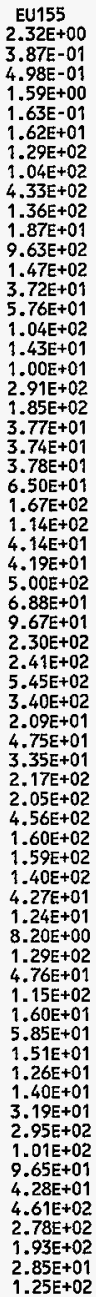 & 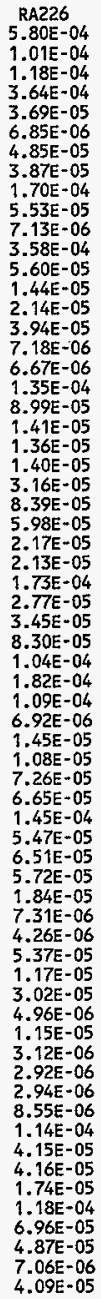 & 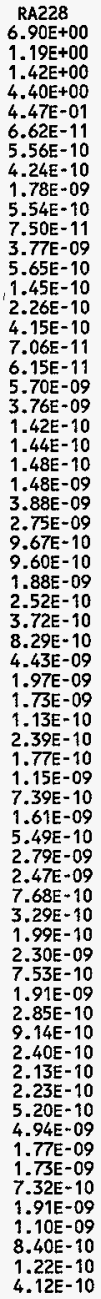 & 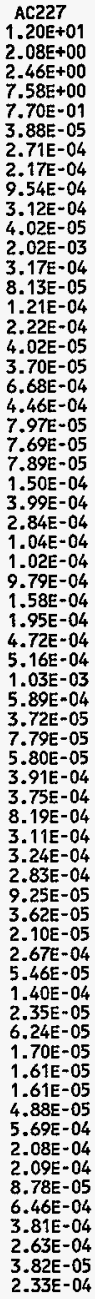 & 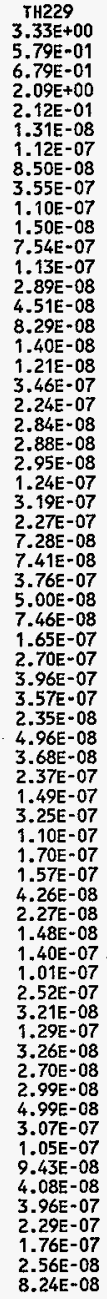 \\
\hline
\end{tabular}


ACTIVITY IN UNSEPARATED FUEL (Curies decayed to 1/1/94)

\begin{tabular}{|c|c|c|c|c|c|c|c|c|c|}
\hline $\begin{array}{r}\text { [Listing } \\
586 \\
587 \\
588 \\
589 \\
590 \\
591 \\
592 \\
593 \\
594 \\
595 \\
596 \\
597 \\
598 \\
599 \\
600 \\
601 \\
602 \\
603 \\
604 \\
605 \\
606 \\
607 \\
608 \\
609 \\
610 \\
611 \\
612 \\
613 \\
614 \\
615 \\
616 \\
617 \\
618 \\
619 \\
620 \\
621 \\
622 \\
623 \\
624 \\
625 \\
626 \\
627 \\
628 \\
629 \\
630 \\
631 \\
632 \\
633 \\
634 \\
635 \\
636 \\
637 \\
638 \\
639 \\
640 \\
641 \\
642 \\
643 \\
644 \\
645 \\
646 \\
647 \\
648 \\
649 \\
650\end{array}$ & $\begin{array}{l}\text { TH232 } \\
7.27 \mathrm{E}+00 \\
1.25 \mathrm{E}+00 \\
1.50 \mathrm{E}+00 \\
4.65 \mathrm{E}+00 \\
4.73 \mathrm{E}-01 \\
9.80 \mathrm{E}-11 \\
8.23 \mathrm{E}-10 \\
6.30 \mathrm{E}-10 \\
2.64 \mathrm{E}-09 \\
8.22 \mathrm{E}-10 \\
1.11 \mathrm{E}-10 \\
5.62 \mathrm{E}-09 \\
8.42 \mathrm{E}-10 \\
2.16 \mathrm{E}-10 \\
3.36 \mathrm{E}-10 \\
6.17 \mathrm{E}-10 \\
1.03 \mathrm{E}-10 \\
8.85 \mathrm{E}-11 \\
8.47 \mathrm{E}=09 \\
5.58 \mathrm{E}-09 \\
2.12 \mathrm{E}-10 \\
2.15 \mathrm{E}-10 \\
2.20 \mathrm{E}-10 \\
2.18 \mathrm{E}-09 \\
5.70 \mathrm{E}-09 \\
4.04 \mathrm{E}-09 \\
1.42 \mathrm{E}-09 \\
1.41 \mathrm{E}-09 \\
2.81 \mathrm{E}-09 \\
3.76 \mathrm{E}-10 \\
5.55 \mathrm{E}-10 \\
1.24 \mathrm{E}-09 \\
6.61 \mathrm{E}-09 \\
2.95 \mathrm{E}-09 \\
2.56 \mathrm{E}-09 \\
1.67 \mathrm{E}-10 \\
3.54 \mathrm{E}-10 \\
2.62 \mathrm{E}-10 \\
1.69 \mathrm{E}-09 \\
1.11 \mathrm{E}-09 \\
2.42 \mathrm{E}-09 \\
8.24 \mathrm{E}-10 \\
4.18 \mathrm{E}-09 \\
3.70 \mathrm{E}-09 \\
1.15 \mathrm{E}-09 \\
4.83 \mathrm{E}-10 \\
2.92 \mathrm{E}-10 \\
3.44 \mathrm{E}-09 \\
1.13 \mathrm{E}-09 \\
2.85 \mathrm{E}-09 \\
4.23 \mathrm{E}-10 \\
1.36 \mathrm{E}-09 \\
3.58 \mathrm{E}-10 \\
3.17 \mathrm{E}-10 \\
3.34 \mathrm{E}-10 \\
7.80 \mathrm{E}-10 \\
7.43 \mathrm{E}-09 \\
2.65 \mathrm{E}-09 \\
2.59 \mathrm{E}-09 \\
1.10 \mathrm{E}-09 \\
2.87 \mathrm{E}-09 \\
1.66 \mathrm{E}-09 \\
1.26 \mathrm{E}-09 \\
1.84 \mathrm{E}-10 \\
6.21 \mathrm{E}-10\end{array}$ & $\begin{array}{l}\text { PA231 } \\
2.00 \mathrm{E}+01 \\
3.46 \mathrm{E}+00 \\
4.13 \mathrm{E}+00 \\
1.28 \mathrm{E}+01 \\
1.30 \mathrm{E}+00 \\
1.14 \mathrm{E}-04 \\
7.94 \mathrm{E}-04 \\
6.40 \mathrm{E}-04 \\
2.81 \mathrm{E}-03 \\
9.18 \mathrm{E}-04 \\
1.18 \mathrm{E}-04 \\
5.98 \mathrm{E}-03 \\
9.40 \mathrm{E}-04 \\
2.41 \mathrm{E}-04 \\
3.57 \mathrm{E}-04 \\
6.57 \mathrm{E}-04 \\
1.15 \mathrm{E}-04 \\
1.03 \mathrm{E}-04 \\
1.98 \mathrm{E}-03 \\
1.31 \mathrm{E}-03 \\
2.37 \mathrm{E}-04 \\
2.28 \mathrm{E}-04 \\
2.34 \mathrm{E}-04 \\
4.35 \mathrm{E}-04 \\
1.15 \mathrm{E}-03 \\
8.15 \mathrm{E}-04 \\
2.99 \mathrm{E}-04 \\
2.94 \mathrm{E}-04 \\
2.92 \mathrm{E}-03 \\
4.70 \mathrm{E}-04 \\
5.78 \mathrm{E}-04 \\
1.41 \mathrm{E}-03 \\
1.54 \mathrm{E}-03 \\
3.08 \mathrm{E}-03 \\
1.72 \mathrm{E}-03 \\
1.08 \mathrm{E}-04 \\
2.29 \mathrm{E}-04 \\
1.69 \mathrm{E}-04 \\
1.14 \mathrm{E}-03 \\
1.13 \mathrm{E}-03 \\
2.46 \mathrm{E}-03 \\
9.38 \mathrm{E}-04 \\
9.74 \mathrm{E}-04 \\
8.48 \mathrm{E}-04 \\
2.78 \mathrm{E}-04 \\
1.05 \mathrm{E}-04 \\
6.08 \mathrm{E}-05 \\
8.00 \mathrm{E}-04 \\
1.60 \mathrm{E}-04 \\
4.10 \mathrm{E}-04 \\
6.85 \mathrm{E}-05 \\
1.81 \mathrm{E}-04 \\
4.95 \mathrm{E}-05 \\
4.69 \mathrm{E}-05 \\
4.69 \mathrm{E}-05 \\
1.46 \mathrm{E}-04 \\
1.72 \mathrm{E}-03 \\
6.25 \mathrm{E}-04 \\
6.31 \mathrm{E}-04 \\
2.66 \mathrm{E}-04 \\
1.94 \mathrm{E}-03 \\
1.15 \mathrm{E}-03 \\
7.91 \mathrm{E}-04 \\
1.15 \mathrm{E}-04 \\
7.06 \mathrm{E}-04\end{array}$ & $\begin{array}{l}\text { U232 } \\
3.12 \mathrm{E}+02 \\
5.37 \mathrm{E}+01 \\
6.44 \mathrm{E}+01 \\
2.00 \mathrm{E}+02 \\
2.04 \mathrm{E}+01 \\
2.15 \mathrm{E}-04 \\
1.98 \mathrm{E}-03 \\
1.49 \mathrm{E}-03 \\
6.06 \mathrm{E}-03 \\
1.85 \mathrm{E}-03 \\
2.58 \mathrm{E}-04 \\
1.31 \mathrm{E}-02 \\
1.92 \mathrm{E}-03 \\
4.89 \mathrm{E}-04 \\
7.81 \mathrm{E}-04 \\
1.43 \mathrm{E}-03 \\
2.21 \mathrm{E}-04 \\
1.77 \mathrm{E}-04 \\
6.76 \mathrm{E}-03 \\
4.26 \mathrm{E}-03 \\
4.85 \mathrm{E}-04 \\
5.01 \mathrm{E}-04 \\
5.11 \mathrm{E}-04 \\
2.97 \mathrm{E}-03 \\
7.48 \mathrm{E}-03 \\
5.24 \mathrm{E}-03 \\
1.59 \mathrm{E}-03 \\
1.66 \mathrm{E}-03 \\
6.67 \mathrm{E}-03 \\
8.16 \mathrm{E}-04 \\
1.31 \mathrm{E}-03 \\
2.84 \mathrm{E}-03 \\
5.39 \mathrm{E}-03 \\
7.09 \mathrm{E}-03 \\
7.60 \mathrm{E}-03 \\
5.06 \mathrm{E}-04 \\
1.09 \mathrm{E}-03 \\
7.97 \mathrm{E}-04 \\
4.98 \mathrm{E}-03 \\
2.70 \mathrm{E}-03 \\
5.93 \mathrm{E}-03 \\
1.91 \mathrm{E}-03 \\
3.45 \mathrm{E}-03 \\
3.25 \mathrm{E}-03 \\
8.09 \mathrm{E}-04 \\
4.77 \mathrm{E}-04 \\
3.41 \mathrm{E}-04 \\
2.82 \mathrm{E}-03 \\
4.79 \mathrm{E}-03 \\
1.16 \mathrm{E}-02 \\
1.20 \mathrm{E}-03 \\
7.19 \mathrm{E}-03 \\
1.72 \mathrm{E}-03 \\
1.28 \mathrm{E}-03 \\
1.54 \mathrm{E}-03 \\
1.70 \mathrm{E}-03 \\
6.37 \mathrm{E}-03 \\
2.10 \mathrm{E}-03 \\
1.78 \mathrm{E}-03 \\
7.88 \mathrm{E}-04 \\
8.92 \mathrm{E}-03 \\
5.14 \mathrm{E}-03 \\
4.13 \mathrm{E}-03 \\
6.03 \mathrm{E}-04 \\
1.45 \mathrm{E}-03\end{array}$ & $\begin{array}{l}\text { U233 } \\
1.24 \mathrm{E}+03 \\
2.14 \mathrm{E}+02 \\
2.55 \mathrm{E}+02 \\
7.91 \mathrm{E}+02 \\
8.04 \mathrm{E}+01 \\
6.34 \mathrm{E}-06 \\
5.40 \mathrm{E}-05 \\
4.13 \mathrm{E}-05 \\
1.72 \mathrm{E}-04 \\
5.34 \mathrm{E}-05 \\
7.29 \mathrm{E}-06 \\
3.68 \mathrm{E}-04 \\
5.51 \mathrm{E}-05 \\
1.41 \mathrm{E}-05 \\
2.20 \mathrm{E}-05 \\
4.04 \mathrm{E}-05 \\
6.58 \mathrm{E}-06 \\
5.51 \mathrm{E}-06 \\
1.80 \mathrm{E}-04 \\
1.16 \mathrm{E}-04 \\
1.39 \mathrm{E}-05 \\
1.41 \mathrm{E}-05 \\
1.44 \mathrm{E}-05 \\
6.41 \mathrm{E}-05 \\
1.64 \mathrm{E}-04 \\
1.16 \mathrm{E}-04 \\
3.69 \mathrm{E}-05 \\
3.77 \mathrm{E}-05 \\
1.85 \mathrm{E}-04 \\
2.45 \mathrm{E}-05 \\
3.65 \mathrm{E}-05 \\
8.15 \mathrm{E}-05 \\
1.42 \mathrm{E}-04 \\
1.96 \mathrm{E}-04 \\
1.74 \mathrm{E}-04 \\
1.14 \mathrm{E}-05 \\
2.43 \mathrm{E}-05 \\
1.79 \mathrm{E}-05 \\
1.15 \mathrm{E}-04 \\
7.37 \mathrm{E}-05 \\
1.61 \mathrm{E}-04 \\
5.44 \mathrm{E}-05 \\
9.01 \mathrm{E}-05 \\
8.27 \mathrm{E}-05 \\
2.25 \mathrm{E}-05 \\
1.17 \mathrm{E}-05 \\
7.70 \mathrm{E}-06 \\
7.39 \mathrm{E}-05 \\
5.42 \mathrm{E}-05 \\
1.35 \mathrm{E}-04 \\
1.70 \mathrm{E}-05 \\
6.94 \mathrm{E}-05 \\
1.76 \mathrm{E}-05 \\
1.45 \mathrm{E}-05 \\
1.61 \mathrm{E}-05 \\
2.70 \mathrm{E}-05 \\
1.63 \mathrm{E}-04 \\
5.57 \mathrm{E}-05 \\
5.00 \mathrm{E}-05 \\
2.17 \mathrm{E}-05 \\
1.99 \mathrm{E}-04 \\
1.15 \mathrm{E}-04 \\
8.83 \mathrm{E}-05 \\
1.29 \mathrm{E}-05 \\
4.32 \mathrm{E}-05\end{array}$ & $\begin{array}{l}\text { U234 } \\
8.64 \mathrm{E}+00 \\
1.49 \mathrm{E}+00 \\
1.78 \mathrm{E}+00 \\
5.52 \mathrm{E}+00 \\
5.62 \mathrm{E}-01 \\
4.43 \mathrm{E}+00 \\
3.13 \mathrm{E}+01 \\
2.53 \mathrm{E}+01 \\
1.10 \mathrm{E}+02 \\
3.59 \mathrm{E}+01 \\
4.68 \mathrm{E}+00 \\
2.37 \mathrm{E}+02 \\
3.72 \mathrm{E}+01 \\
9.50 \mathrm{E}+00 \\
1.42 \mathrm{E}+01 \\
2.60 \mathrm{E}+01 \\
4.35 \mathrm{E}+00 \\
3.72 \mathrm{E}+00 \\
8.92 \mathrm{E}+01 \\
5.88 \mathrm{E}+01 \\
9.41 \mathrm{E}+00 \\
9.09 \mathrm{E}+00 \\
9.28 \mathrm{E}+00 \\
2.00 \mathrm{E}+01 \\
5.25 \mathrm{E}+01 \\
3.69 \mathrm{E}+01 \\
1.34 \mathrm{E}+01 \\
1.33 \mathrm{E}+01 \\
1.17 \mathrm{E}+02 \\
1.85 \mathrm{E}+01 \\
2.31 \mathrm{E}+01 \\
5.64 \mathrm{E}+01 \\
7.03 \mathrm{E}+01 \\
1.24 \mathrm{E}+02 \\
7.02 \mathrm{E}+01 \\
4.39 \mathrm{E}+00 \\
9.43 \mathrm{E}+00 \\
6.91 \mathrm{E}+00 \\
4.60 \mathrm{E}+01 \\
4.57 \mathrm{E}+01 \\
1.00 \mathrm{E}+02 \\
3.78 \mathrm{E}+01 \\
4.49 \mathrm{E}+01 \\
3.91 \mathrm{E}+01 \\
1.27 \mathrm{E}+01 \\
4.54 \mathrm{E}+00 \\
2.68 \mathrm{E}+00 \\
3.67 \mathrm{E}+01 \\
7.92 \mathrm{E}+00 \\
2.01 \mathrm{E}+01 \\
3.24 \mathrm{E}+00 \\
7.71 \mathrm{E}+00 \\
2.09 \mathrm{E}+00 \\
1.94 \mathrm{E}+00 \\
1.97 \mathrm{E}+00 \\
5.88 \mathrm{E}+00 \\
7.99 \mathrm{E}+01 \\
2.88 \mathrm{E}+01 \\
2.89 \mathrm{E}+01 \\
1.23 \mathrm{E}+01 \\
8.22 \mathrm{E}+01 \\
4.89 \mathrm{E}+01 \\
3.38 \mathrm{E}+01 \\
4.92 \mathrm{E}+00\end{array}$ & $\begin{array}{l}\text { U235 } \\
2.67 \mathrm{E}-05 \\
4.61 \mathrm{E}=06 \\
5.51 \mathrm{E}-06 \\
1.71 \mathrm{E}-05 \\
1.74 \mathrm{E}-06 \\
1.91 \mathrm{E}-01 \\
1.33 \mathrm{E}+00 \\
1.08 \mathrm{E}+00 \\
4.71 \mathrm{E}+00 \\
1.54 \mathrm{E}+00 \\
2.00 \mathrm{E}-01 \\
1.01 \mathrm{E}+01 \\
1.60 \mathrm{E}+00 \\
4.07 \mathrm{E}-01 \\
6.05 \mathrm{E}-01 \\
1.11 \mathrm{E}+00 \\
1.86 \mathrm{E}-01 \\
1.60 \mathrm{E}-01 \\
3.34 \mathrm{E}+00 \\
2.20 \mathrm{E}+00 \\
4.03 \mathrm{E}-01 \\
3.88 \mathrm{E}-01 \\
3.96 \mathrm{E}-01 \\
7.16 \mathrm{E}-01 \\
1.88 \mathrm{E}+00 \\
1.32 \mathrm{E}+00 \\
4.86 \mathrm{E}-01 \\
4.80 \mathrm{E}-01 \\
4.99 \mathrm{E}+00 \\
8.00 \mathrm{E}-01 \\
9.83 \mathrm{E}-01 \\
2.42 \mathrm{E}+00 \\
2.63 \mathrm{E}+00 \\
5.29 \mathrm{E}+00 \\
2.86 \mathrm{E}+00 \\
1.78 \mathrm{E}-01 \\
3.83 \mathrm{E}-01 \\
2.81 \mathrm{E}-01 \\
1.88 \mathrm{E}+00 \\
1.94 \mathrm{E}+00 \\
4.26 \mathrm{E}+00 \\
1.62 \mathrm{E}+00 \\
1.68 \mathrm{E}+00 \\
1.46 \mathrm{E}+00 \\
4.81 \mathrm{E}-01 \\
1.71 \mathrm{E}-01 \\
1.00 \mathrm{E}-01 \\
1.37 \mathrm{E}+00 \\
2.67 \mathrm{E}-01 \\
6.79 \mathrm{E}-01 \\
1.14 \mathrm{E}-01 \\
2.98 \mathrm{E}-01 \\
8.18 \mathrm{E}-02 \\
7.77 \mathrm{E}-02 \\
7.77 \mathrm{E}-02 \\
2.49 \mathrm{E}-01 \\
2.98 \mathrm{E}+00 \\
1.08 \mathrm{E}+00 \\
1.09 \mathrm{E}+00 \\
4.64 \mathrm{E}-01 \\
3.36 \mathrm{E}+00 \\
2.00 \mathrm{E}+00 \\
1.37 \mathrm{E}+00 \\
1.99 \mathrm{E}-01 \\
1.23 \mathrm{E}+00\end{array}$ & $\begin{array}{l}\text { U236 } \\
3.73 \mathrm{E}-06 \\
6.44 \mathrm{E}-07 \\
7.69 \mathrm{E}-07 \\
2.39 \mathrm{E}-06 \\
2.43 \mathrm{E}-07 \\
7.06 \mathrm{E}-02 \\
5.93 \mathrm{E}-01 \\
4.57 \mathrm{E}-01 \\
1.91 \mathrm{E}+00 \\
5.94 \mathrm{E}-01 \\
8.09 \mathrm{E}-02 \\
4.10 \mathrm{E}+00 \\
6.15 \mathrm{E}-01 \\
1.57 \mathrm{E}-01 \\
2.45 \mathrm{E}-01 \\
4.49 \mathrm{E}-01 \\
7.20 \mathrm{E}-02 \\
5.92 \mathrm{E}-02 \\
6.16 \mathrm{E}+00 \\
4.04 \mathrm{E}+00 \\
1.56 \mathrm{E}-01 \\
1.57 \mathrm{E}-01 \\
1.61 \mathrm{E}-01 \\
1.55 \mathrm{E}+00 \\
4.03 \mathrm{E}+00 \\
2.83 \mathrm{E}+00 \\
9.96 \mathrm{E}-01 \\
9.95 \mathrm{E}-01 \\
2.07 \mathrm{E}+00 \\
2.76 \mathrm{E}-01 \\
4.07 \mathrm{E}-01 \\
9.16 \mathrm{E}-01 \\
4.86 \mathrm{E}+00 \\
2.19 \mathrm{E}+00 \\
1.84 \mathrm{E}+00 \\
1.20 \mathrm{E}-01 \\
2.57 \mathrm{E}-01 \\
1.88 \mathrm{E}-01 \\
1.21 \mathrm{E}+00 \\
8.25 \mathrm{E}-01 \\
1.81 \mathrm{E}+00 \\
6.15 \mathrm{E}-01 \\
3.11 \mathrm{E}+00 \\
2.74 \mathrm{E}+00 \\
8.56 \mathrm{E}-01 \\
3.41 \mathrm{E}-01 \\
2.07 \mathrm{E}-01 \\
2.54 \mathrm{E}+00 \\
8.31 \mathrm{E}-01 \\
2.08 \mathrm{E}+00 \\
3.07 \mathrm{E}-01 \\
1.00 \mathrm{E}+00 \\
2.63 \mathrm{E}-01 \\
2.32 \mathrm{E}-01 \\
2.45 \mathrm{E}-01 \\
5.79 \mathrm{E}-01 \\
5.56 \mathrm{E}+00 \\
1.98 \mathrm{E}+00 \\
1.93 \mathrm{E}+00 \\
8.27 \mathrm{E}-01 \\
2.15 \mathrm{E}+00 \\
1.25 \mathrm{E}+00 \\
9.45 \mathrm{E}-01 \\
1.38 \mathrm{E}-01 \\
4.67 \mathrm{E}-01\end{array}$ & $\begin{array}{l}\text { U238 } \\
2.19 \mathrm{E}-13 \\
3.77 \mathrm{E}-14 \\
4.51 \mathrm{E}-14 \\
1.40 \mathrm{E}-13 \\
1.42 \mathrm{E}-14 \\
4.53 \mathrm{E}+00 \\
3.22 \mathrm{E}+01 \\
2.60 \mathrm{E}+01 \\
1.13 \mathrm{E}+02 \\
3.68 \mathrm{E}+01 \\
4.79 \mathrm{E}+00 \\
2.43 \mathrm{E}+02 \\
3.81 \mathrm{E}+01 \\
9.72 \mathrm{E}+00 \\
1.45 \mathrm{E}+01 \\
2.66 \mathrm{E}+01 \\
4.45 \mathrm{E}+00 \\
3.80 \mathrm{E}+00 \\
6.06 \mathrm{E}+01 \\
3.99 \mathrm{E}+01 \\
9.63 \mathrm{E}+00 \\
9.31 \mathrm{E}+00 \\
9.51 \mathrm{E}+00 \\
1.37 \mathrm{E}+01 \\
3.60 \mathrm{E}+01 \\
2.53 \mathrm{E}+01 \\
9.14 \mathrm{E}+00 \\
9.06 \mathrm{E}+00 \\
1.20 \mathrm{E}+02 \\
1.89 \mathrm{E}+01 \\
2.36 \mathrm{E}+01 \\
5.77 \mathrm{E}+01 \\
4.77 \mathrm{E}+01 \\
1.27 \mathrm{E}+02 \\
7.26 \mathrm{E}+01 \\
4.55 \mathrm{E}+00 \\
9.76 \mathrm{E}+00 \\
7.16 \mathrm{E}+00 \\
4.76 \mathrm{E}+01 \\
4.68 \mathrm{E}+01 \\
1.03 \mathrm{E}+02 \\
3.87 \mathrm{E}+01 \\
3.05 \mathrm{E}+01 \\
2.66 \mathrm{E}+01 \\
8.62 \mathrm{E}+00 \\
3.09 \mathrm{E}+00 \\
1.83 \mathrm{E}+00 \\
2.49 \mathrm{E}+01 \\
5.59 \mathrm{E}+00 \\
1.41 \mathrm{E}+01 \\
2.26 \mathrm{E}+00 \\
5.48 \mathrm{E}+00 \\
1.48 \mathrm{E}+00 \\
1.36 \mathrm{E}+00 \\
1.39 \mathrm{E}+00 \\
4.06 \mathrm{E}+00 \\
5.43 \mathrm{E}+01 \\
1.95 \mathrm{E}+01 \\
1.96 \mathrm{E}+01 \\
8.32 \mathrm{E}+00 \\
8.50 \mathrm{E}+01 \\
5.05 \mathrm{E}+01 \\
3.50 \mathrm{E}+01 \\
5.11 \mathrm{E}+00 \\
2.94 \mathrm{E}+01\end{array}$ & $\begin{array}{l}\text { NP237 } \\
1.21 \mathrm{E}-07 \\
2.09 \mathrm{E}-08 \\
2.49 \mathrm{E}-08 \\
7.72 \mathrm{E}-08 \\
7.85 \mathrm{E}-09 \\
2.39 \mathrm{E}-02 \\
2.07 \mathrm{E}-01 \\
1.58 \mathrm{E}-01 \\
6.53 \mathrm{E}-01 \\
2.02 \mathrm{E}-01 \\
2.77 \mathrm{E}-02 \\
1.40 \mathrm{E}+00 \\
2.09 \mathrm{E}-01 \\
5.34 \mathrm{E}-02 \\
8.39 \mathrm{E}-02 \\
1.54 \mathrm{E}-01 \\
2.45 \mathrm{E}-02 \\
2.00 \mathrm{E}-02 \\
8.32 \mathrm{E}-01 \\
5.33 \mathrm{E}-01 \\
5.28 \mathrm{E}-02 \\
5.38 \mathrm{E}-02 \\
5.50 \mathrm{E}-02 \\
3.07 \mathrm{E}-01 \\
7.77 \mathrm{E}-01 \\
5.46 \mathrm{E}-01 \\
1.71 \mathrm{E}-01 \\
1.76 \mathrm{E}-01 \\
7.10 \mathrm{E}-01 \\
9.21 \mathrm{E}-02 \\
1.40 \mathrm{E}-01 \\
3.10 \mathrm{E}-01 \\
6.60 \mathrm{E}-01 \\
7.53 \mathrm{E}-01 \\
6.97 \mathrm{E}-01 \\
4.57 \mathrm{E}-02 \\
9.82 \mathrm{E}-02 \\
7.20 \mathrm{E}-02 \\
4.57 \mathrm{E}-01 \\
2.85 \mathrm{E}-01 \\
6.24 \mathrm{E}-01 \\
2.68 \mathrm{E}-01 \\
2.32 \mathrm{E}-01 \\
1.02 \mathrm{E}-01 \\
8.10 \mathrm{E}-01 \\
4.69 \mathrm{E}-01 \\
3.64 \mathrm{E}-01 \\
5.31 \mathrm{E}-02 \\
1.58 \mathrm{E}-01 \\
3.87 \mathrm{E}-01 \\
1.05 \mathrm{E}-01 \\
5.56 \mathrm{E}-02 \\
3.70 \mathrm{E}-02 \\
3.45 \mathrm{E}-01 \\
2.93 \mathrm{E}-01 \\
7.22 \mathrm{E}-01 \\
3.78 \mathrm{E}-02 \\
9.73 \mathrm{E}-02 \\
3.88 \mathrm{E}-02 \\
\end{array}$ \\
\hline
\end{tabular}


HNF-SD-WH-II-794, ReV, O

ACTIVITY IN UNSEPARATED FUEL (Curies decayed to $1 / 1 / 94$ )

\begin{tabular}{|c|c|c|c|c|c|c|c|c|c|}
\hline 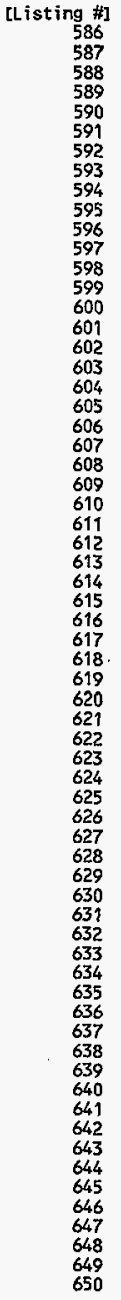 & 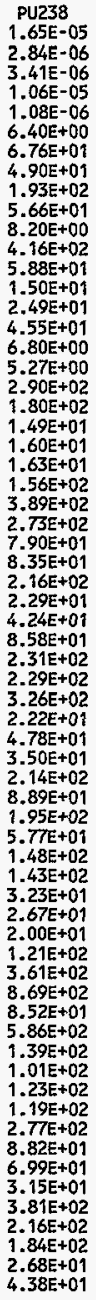 & 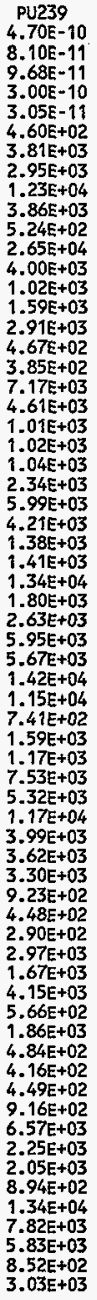 & 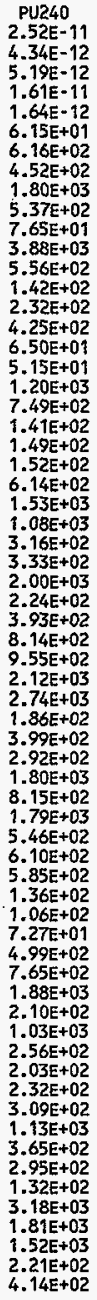 & 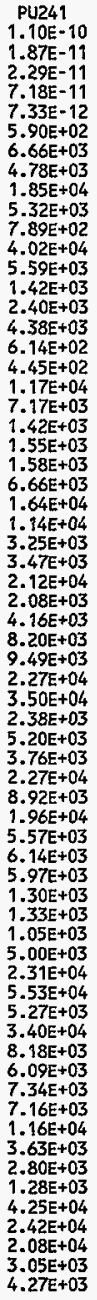 & 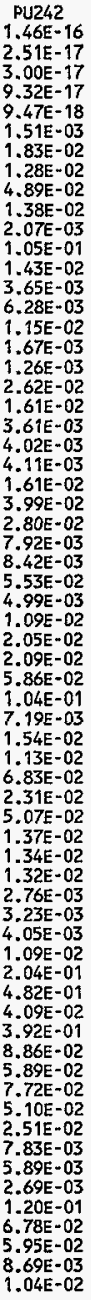 & 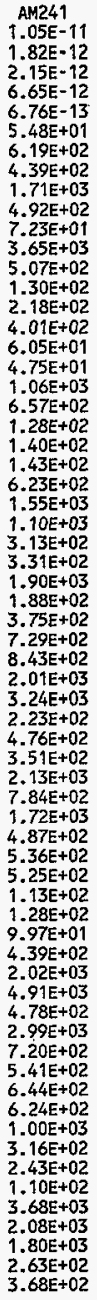 & 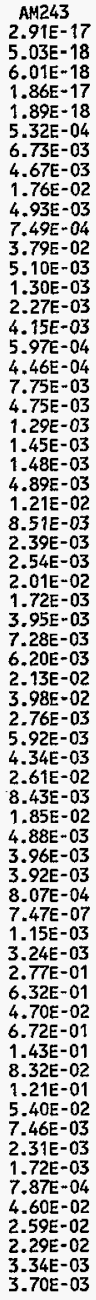 & 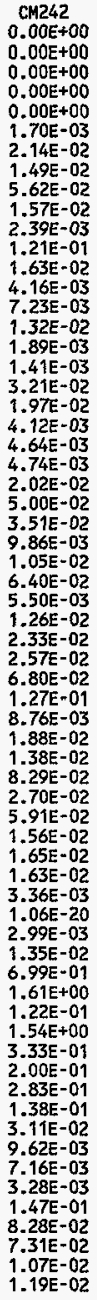 & $\begin{array}{l}C M 243 \\
1.70 E-18 \\
2.92 E-19 \\
3.54 E-19 \\
1.10 E-18 \\
1.12 E-19 \\
4.74 E-05 \\
6.13 E-04 \\
4.25 E-04 \\
1.59 E-03 \\
4.42 E-04 \\
6.78 E-05 \\
3.45 E-03 \\
4.61 E-04 \\
1.17 E-04 \\
2.06 E-04 \\
3.77 E-04 \\
5.22 E-05 \\
3.77 E-05 \\
7.25 E-04 \\
4.42 E-04 \\
1.17 E-04 \\
1.32 E-04 \\
1.35 E-04 \\
4.57 E-04 \\
1.13 E-03 \\
7.88 E-04 \\
2.21 E-04 \\
2.36 E-04 \\
1.84 E-03 \\
1.53 E-04 \\
3.61 E-04 \\
6.61 E-04 \\
5.84 E-04 \\
1.96 E-03 \\
3.70 E-03 \\
2.55 E-04 \\
5.54 E-04 \\
4.03 E-04 \\
2.42 E-03 \\
7.80 E-04 \\
1.71 E-03 \\
4.46 E-04 \\
3.75 E-04 \\
3.71 E-04 \\
7.61 E-05 \\
0.00 E+00 \\
0.00 E+00 \\
3.07 E-04 \\
8.38 E-02 \\
1.83 E-01 \\
1.21 E-02 \\
2.35 E-01 \\
4.83 E-02 \\
2.53 E-02\end{array}$ \\
\hline
\end{tabular}


HNF-SD-WM-TI-794, ReV. 0

ACTIVITY IN UNSEPARATED FUEL (Curies decayed to $1 / 1 / 94$ )

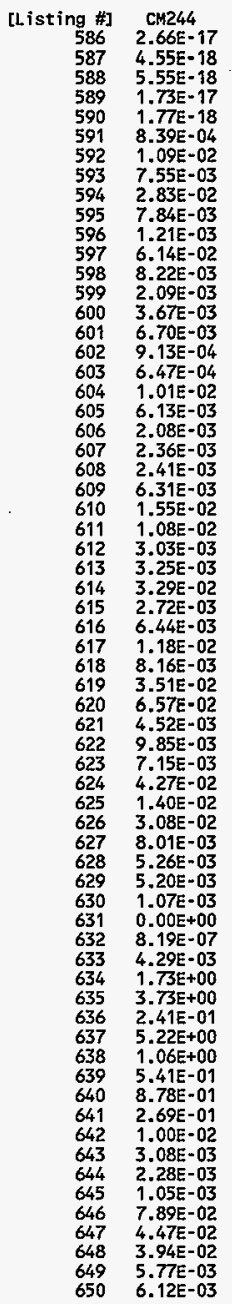


HNF-SD-WN-II-794, ReV. O

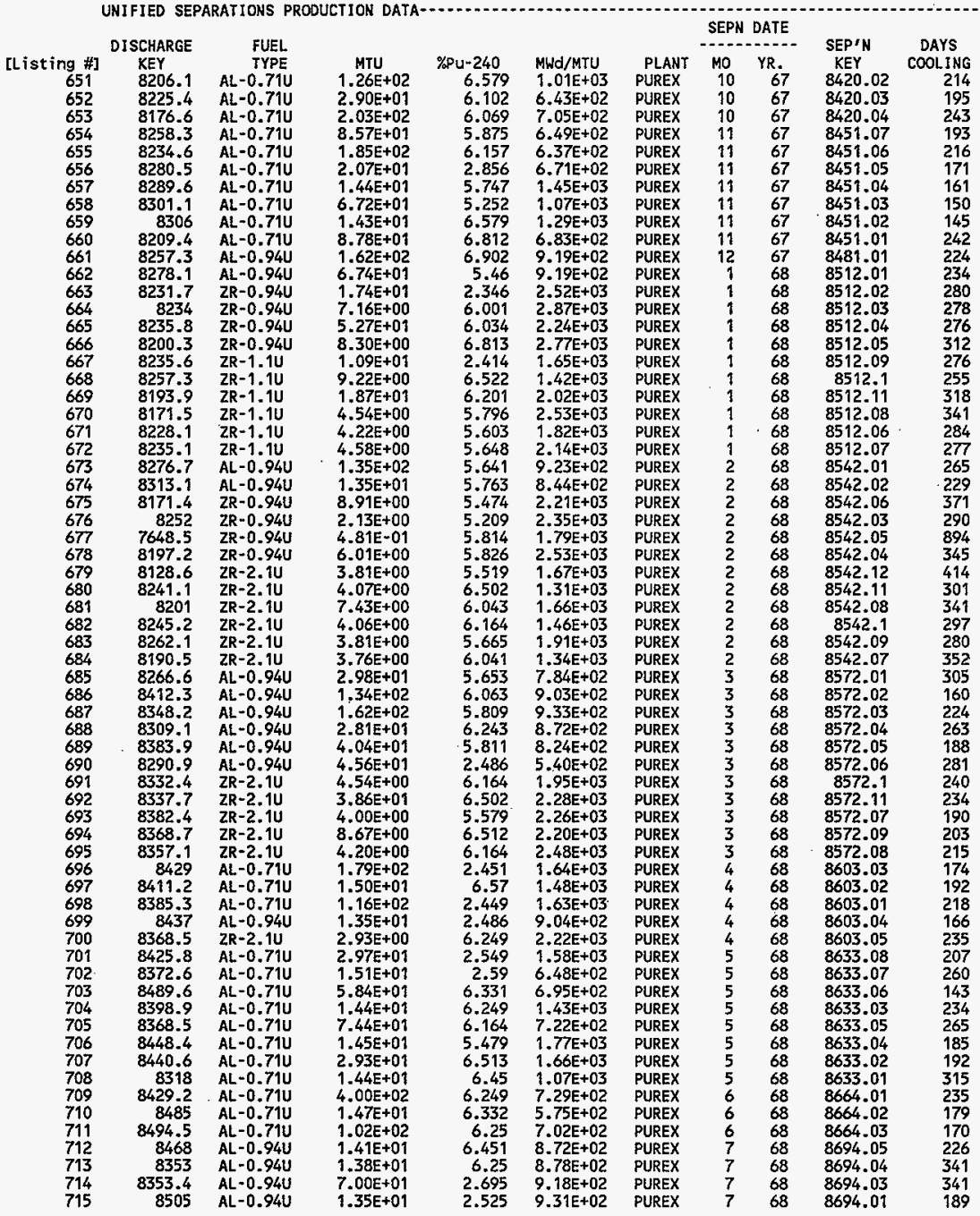


HNF-SD-WN-TI-794, Rev. 0

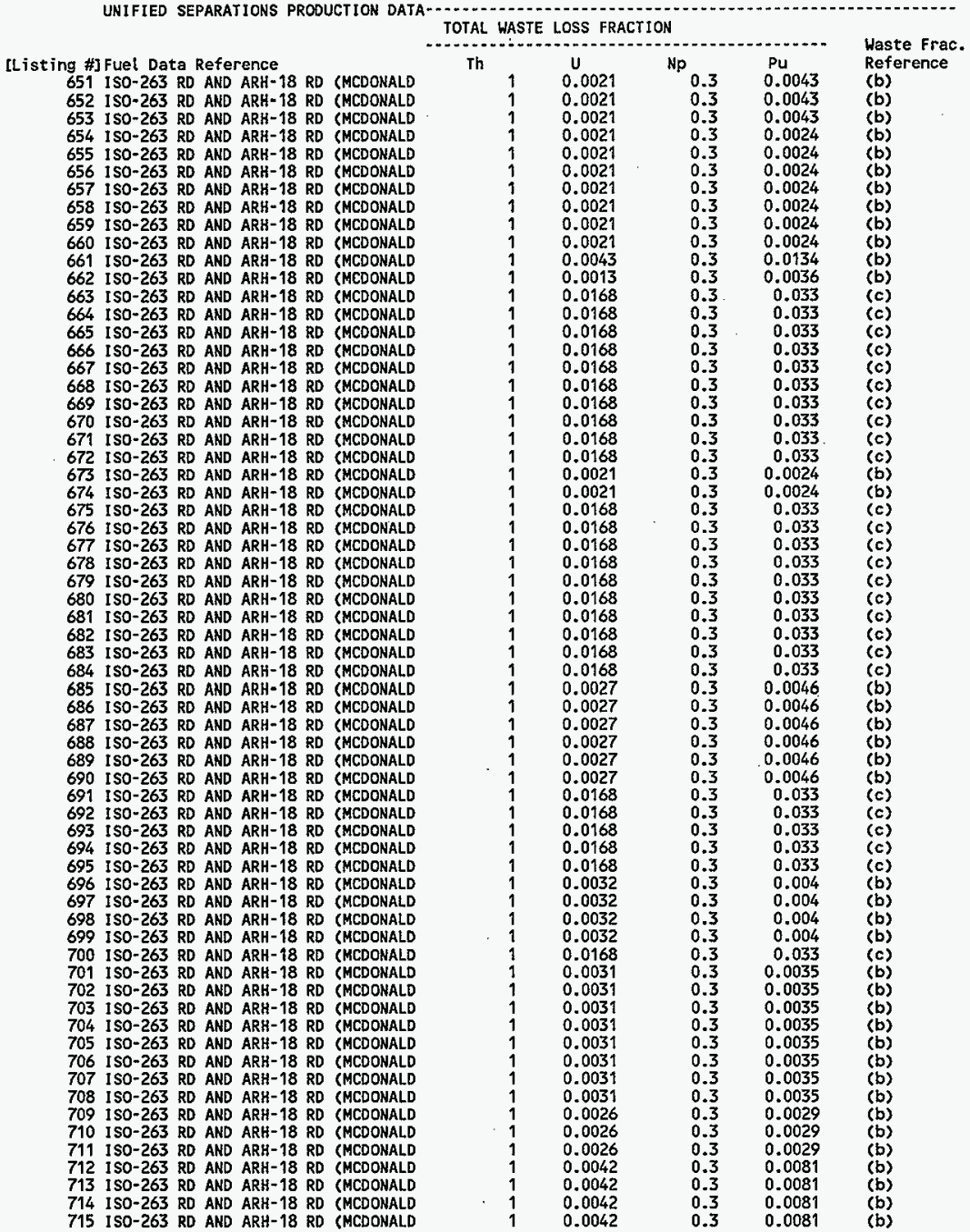


HNF-SD-WN-TI-794, ReV. 0

ACTIVITY IN UNSEPARATED FUEL (Curies decayed to 1/1/94)

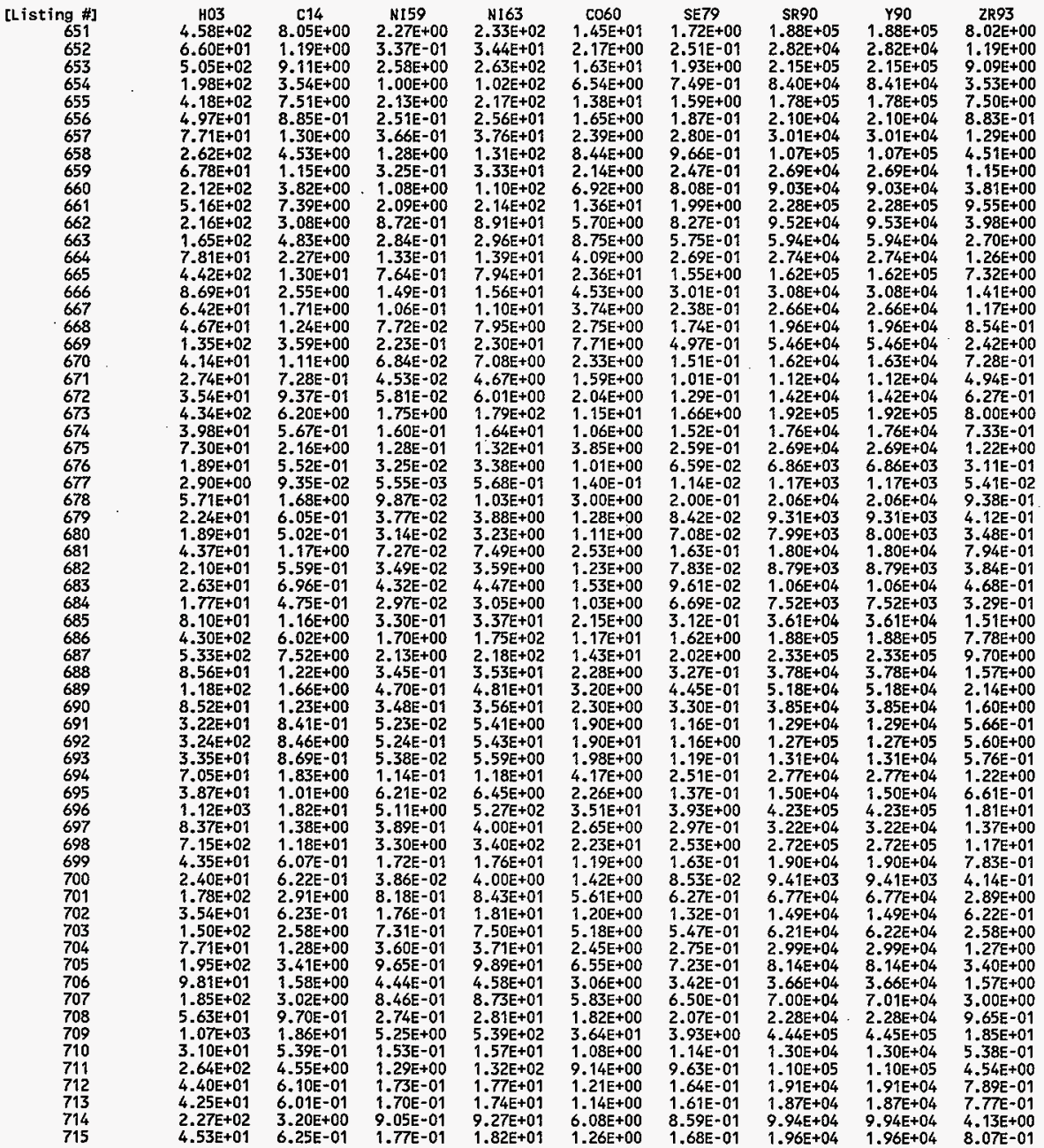


HNF-SD-WM-II-794, Rev. 0

ACTIVITY IN UNSEPARATED FUEL (Curies decayed to 1/9/94)

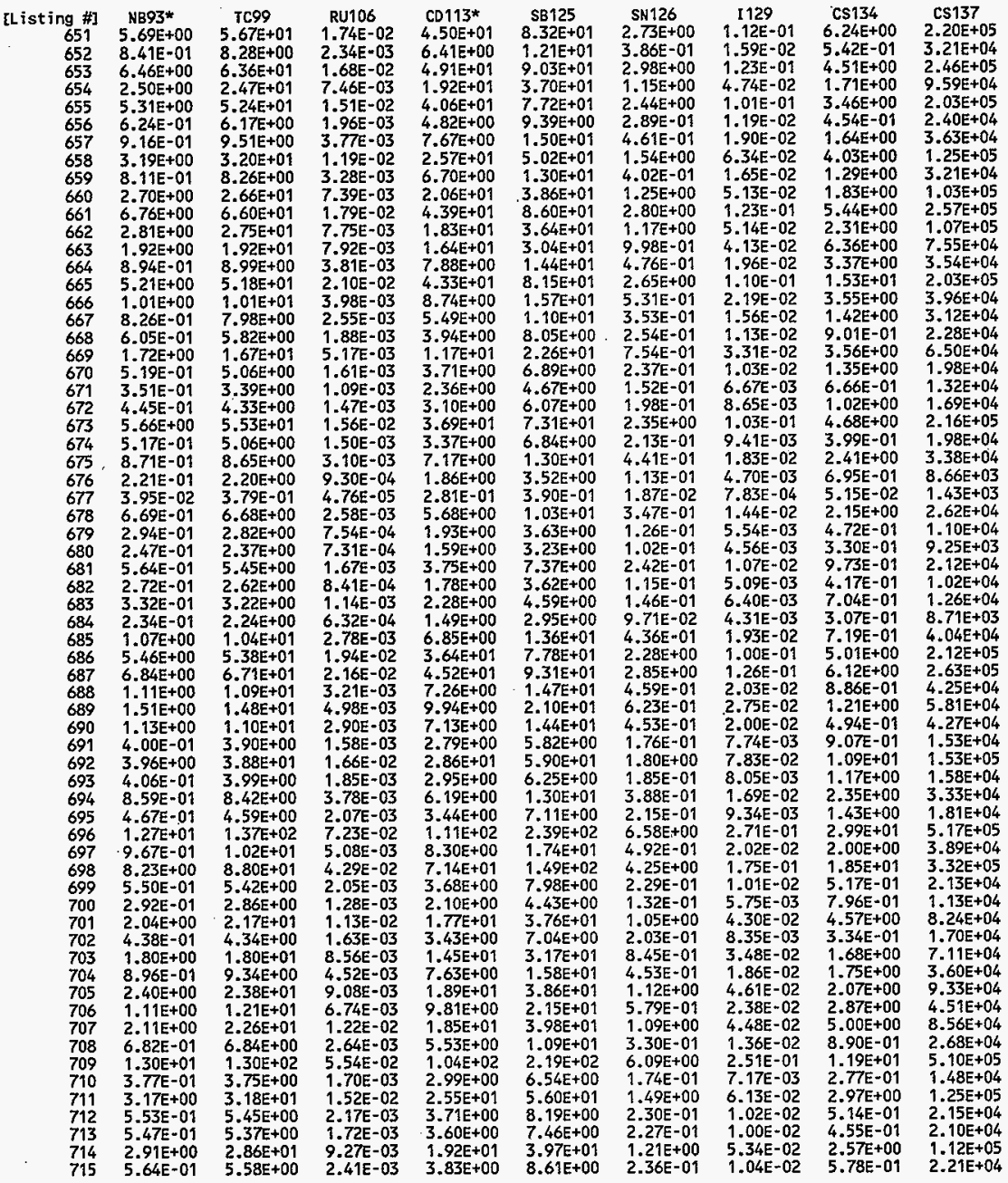


HNF-SD-WH-TI-794, ReV. 0

ACTIVITY IN UNSEPARATED FUEL (Curies decayed to $1 / 1 / 94$ )

\begin{tabular}{|c|c|c|c|c|c|c|c|c|c|}
\hline 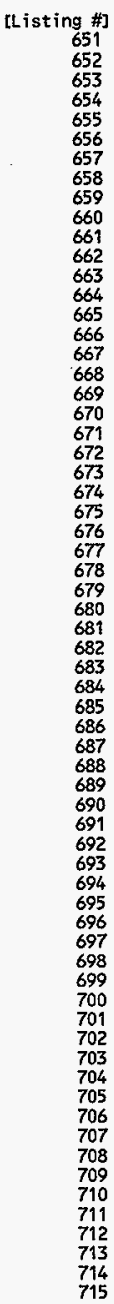 & $\begin{array}{l}B A 137 * \\
2.08 E+05 \\
3.04 E+04 \\
2.33 E+05 \\
9.07 E+04 \\
1.92 E+05 \\
2.27 E+04 \\
3.44 E+04 \\
1.18 E+05 \\
3.03 E+04 \\
9.76 E+04 \\
2.43 E+05 \\
1.02 E+05 \\
7.14 E+04 \\
3.35 E+04 \\
1.92 E+05 \\
3.74 E+04 \\
2.95 E+04 \\
2.15 E+04 \\
6.15 E+04 \\
1.87 E+04 \\
1.25 E+04 \\
1.60 E+04 \\
2.04 E+05 \\
1.87 E+04 \\
3.20 E+04 \\
8.19 E+03 \\
1.35 E+03 \\
2.48 E+04 \\
1.04 E+04 \\
8.75 E+03 \\
2.01 E+04 \\
9.69 E+03 \\
1.19 E+04 \\
8.24 E+03 \\
3.83 E+04 \\
2.00 E+05 \\
2.49 E+05 \\
4.02 E+04 \\
5.50 E+04 \\
4.04 E+04 \\
1.45 E+04 \\
1.45 E+05 \\
1.49 E+04 \\
3.15 E+04 \\
1.72 E+04 \\
4.89 E+05 \\
3.68 E+04 \\
3.14 E+05 \\
2.02 E+04 \\
1.07 E+04 \\
7.79 E+04 \\
1.61 E+04 \\
6.73 E+04 \\
3.40 E+04 \\
8.83 E+04 \\
4.27 E+04 \\
8.09 E+04 \\
2.53 E+04 \\
4.82 E+05 \\
1.40 E+04 \\
1.19 E+05 \\
2.04 E+04 \\
1.99 E+04 \\
1.06 E+05 \\
2.09 E+04\end{array}$ & $\begin{array}{l}S M 151 \\
5.91 E+03 \\
9.86 E+02 \\
7.39 E+03 \\
2.93 E+03 \\
6.24 E+03 \\
7.27 E+02 \\
8.19 E+02 \\
3.28 E+03 \\
7.69 E+02 \\
3.13 E+03 \\
7.35 E+03 \\
3.06 E+03 \\
1.29 E+03 \\
5.58 E+02 \\
3.70 E+03 \\
6.38 E+02 \\
7.08 E+02 \\
5.45 E+02 \\
1.35 E+03 \\
3.54 E+02 \\
2.89 E+02 \\
3.39 E+02 \\
6.15 E+03 \\
5.77 E+02 \\
6.16 E+02 \\
1.53 E+02 \\
3.09 E+01 \\
4.46 E+02 \\
2.40 E+02 \\
2.28 E+02 \\
4.64 E+02 \\
2.39 E+02 \\
2.57 E+02 \\
2.12 E+02 \\
1.20 E+03 \\
6.03 E+03 \\
7.45 E+03 \\
1.23 E+03 \\
1.70 E+03 \\
1.36 E+03 \\
3.12 E+02 \\
2.85 E+03 \\
2.93 E+02 \\
6.29 E+02 \\
3.22 E+02 \\
1.07 E+04 \\
8.60 E+02 \\
6.89 E+03 \\
6.08 E+02 \\
2.14 E+02 \\
1.75 E+03 \\
5.16 E+02 \\
2.12 E+03 \\
8.14 E+02 \\
2.76 E+03 \\
8.82 E+02 \\
1.77 E+03 \\
6.99 E+02 \\
1.51 E+04 \\
4.58 E+02 \\
3.73 E+03 \\
6.18 E+02 \\
6.07 E+02 \\
3.19 E+03 \\
6.24 E+02\end{array}$ & $\begin{array}{l}\text { EU152 } \\
3.88 \mathrm{E}+00 \\
3.14 \mathrm{E}-01 \\
2.76 \mathrm{E}+00 \\
9.69 \mathrm{E}-01 \\
1.99 \mathrm{E}+00 \\
2.54 \mathrm{E}-01 \\
9.76 \mathrm{E}-01 \\
2.33 \mathrm{E}+00 \\
7.54 \mathrm{E}-01 \\
1.09 \mathrm{E}+00 \\
3.51 \mathrm{E}+00 \\
1.47 \mathrm{E}+00 \\
3.67 \mathrm{E}+00 \\
1.89 \mathrm{E}+00 \\
9.01 \mathrm{E}+00 \\
2.06 \mathrm{E}+00 \\
7.31 \mathrm{E}-01 \\
4.38 \mathrm{E}-01 \\
1.97 \mathrm{E}+00 \\
7.88 \mathrm{E}-01 \\
3.52 \mathrm{E}-01 \\
5.54 \mathrm{E}-01 \\
2.97 \mathrm{E}+00 \\
2.43 \mathrm{E}-01 \\
1.49 \mathrm{E}+00 \\
3.99 \mathrm{E}-01 \\
4.63 \mathrm{E}-02 \\
1.27 \mathrm{E}+00 \\
2.88 \mathrm{E}-01 \\
1.58 \mathrm{E}-01 \\
5.61 \mathrm{E}-01 \\
2.18 \mathrm{E}-01 \\
3.95 \mathrm{E}-01 \\
1.57 \mathrm{E}-01 \\
4.50 \mathrm{E}-01 \\
2.85 \mathrm{E}+00 \\
3.68 \mathrm{E}+00 \\
5.45 \mathrm{E}-01 \\
6.96 \mathrm{E}-01 \\
2.82 \mathrm{E}-01 \\
4.83 \mathrm{E}-01 \\
5.75 \mathrm{E}+00 \\
5.94 \mathrm{E}-01 \\
1.21 \mathrm{E}+00 \\
7.34 \mathrm{E}-01 \\
1.61 \mathrm{E}+01 \\
1.09 \mathrm{E}+00 \\
1.03 \mathrm{E}+01 \\
2.89 \mathrm{E}-01 \\
4.12 \mathrm{E}-01 \\
2.46 \mathrm{E}+00 \\
1.73 \mathrm{E}-01 \\
8.02 \mathrm{E}-01 \\
9.59 \mathrm{E}-01 \\
1.10 \mathrm{E}+00 \\
1.53 \mathrm{E}+00 \\
2.67 \mathrm{E}+00 \\
5.09 \mathrm{E}-01 \\
6.01 \mathrm{E}+00 \\
1.29 \mathrm{E}-01 \\
1.41 \mathrm{E}+00 \\
2.79 \mathrm{E}-01 \\
2.70 \mathrm{E}-01 \\
1.54 \mathrm{E}+00 \\
3.06 \mathrm{E}-01\end{array}$ & $\begin{array}{l}E \cup 154 \\
3.17 \mathrm{E}+02 \\
2.87 \mathrm{E}+01 \\
2.44 \mathrm{E}+02 \\
8.83 \mathrm{E}+01 \\
1.82 \mathrm{E}+02 \\
2.30 \mathrm{E}+01 \\
7.63 \mathrm{E}+01 \\
1.91 \mathrm{E}+02 \\
5.98 \mathrm{E}+01 \\
9.75 \mathrm{E}+01 \\
2.57 \mathrm{E}+02 \\
1.08 \mathrm{E}+02 \\
3.19 \mathrm{E}+02 \\
1.73 \mathrm{E}+02 \\
7.49 \mathrm{E}+02 \\
1.85 \mathrm{E}+02 \\
6.51 \mathrm{E}+01 \\
4.01 \mathrm{E}+01 \\
1.70 \mathrm{E}+02 \\
6.62 \mathrm{E}+01 \\
3.09 \mathrm{E}+01 \\
4.76 \mathrm{E}+01 \\
2.17 \mathrm{E}+02 \\
1.83 \mathrm{E}+01 \\
1.23 \mathrm{E}+02 \\
3.38 \mathrm{E}+01 \\
3.74 \mathrm{E}+00 \\
1.10 \mathrm{E}+02 \\
2.26 \mathrm{E}+01 \\
1.48 \mathrm{E}+01 \\
4.43 \mathrm{E}+01 \\
1.84 \mathrm{E}+01 \\
3.10 \mathrm{E}+01 \\
1.42 \mathrm{E}+01 \\
3.43 \mathrm{E}+01 \\
2.13 \mathrm{E}+02 \\
2.71 \mathrm{E}+02 \\
4.06 \mathrm{E}+01 \\
5.29 \mathrm{E}+01 \\
2.46 \mathrm{E}+01 \\
3.81 \mathrm{E}+01 \\
4.66 \mathrm{E}+02 \\
4.84 \mathrm{E}+01 \\
9.77 \mathrm{E}+01 \\
6.11 \mathrm{E}+01 \\
1.26 \mathrm{E}+03 \\
8.54 \mathrm{E}+01 \\
8.02 \mathrm{E}+02 \\
2.15 \mathrm{E}+01 \\
3.32 \mathrm{E}+01 \\
1.93 \mathrm{E}+02 \\
1.59 \mathrm{E}+01 \\
7.29 \mathrm{E}+01 \\
7.57 \mathrm{E}+01 \\
9.76 \mathrm{E}+01 \\
1.19 \mathrm{E}+02 \\
2.08 \mathrm{E}+02 \\
4.16 \mathrm{E}+01 \\
5.38 \mathrm{E}+02 \\
1.24 \mathrm{E}+01 \\
1.29 \mathrm{E}+02 \\
2.10 \mathrm{E}+01 \\
2.02 \mathrm{E}+01 \\
1.13 \mathrm{E}+02 \\
2.29 \mathrm{E}+01\end{array}$ & $\begin{array}{l}E \cup 155 \\
2.29 \mathrm{E}+02 \\
4.24 \mathrm{E}+01 \\
3.07 \mathrm{E}+02 \\
1.27 \mathrm{E}+02 \\
2.69 \mathrm{E}+02 \\
3.16 \mathrm{E}+01 \\
2.72 \mathrm{E}+01 \\
1.30 \mathrm{E}+02 \\
2.79 \mathrm{E}+01 \\
1.33 \mathrm{E}+02 \\
2.91 \mathrm{E}+02 \\
1.22 \mathrm{E}+02 \\
5.34 \mathrm{E}+01 \\
2.42 \mathrm{E}+01 \\
1.50 \mathrm{E}+02 \\
2.70 \mathrm{E}+01 \\
2.69 \mathrm{E}+01 \\
2.08 \mathrm{E}+01 \\
5.08 \mathrm{E}+01 \\
1.34 \mathrm{E}+01 \\
1.10 \mathrm{E}+01 \\
1.30 \mathrm{E}+01 \\
2.45 \mathrm{E}+02 \\
2.38 \mathrm{E}+01 \\
2.44 \mathrm{E}+01 \\
6.30 \mathrm{E}+00 \\
1.00 \mathrm{E}+00 \\
1.83 \mathrm{E}+01 \\
8.63 \mathrm{E}+00 \\
8.64 \mathrm{E}+00 \\
1.71 \mathrm{E}+01 \\
9.03 \mathrm{E}+00 \\
9.73 \mathrm{E}+00 \\
7.89 \mathrm{E}+00 \\
4.97 \mathrm{E}+01 \\
2.54 \mathrm{E}+02 \\
3.04 \mathrm{E}+02 \\
5.03 \mathrm{E}+01 \\
7.24 \mathrm{E}+01 \\
6.01 \mathrm{E}+01 \\
1.21 \mathrm{E}+01 \\
1.12 \mathrm{E}+02 \\
1.17 \mathrm{E}+01 \\
2.50 \mathrm{E}+01 \\
1.30 \mathrm{E}+01 \\
3.28 \mathrm{E}+02 \\
2.92 \mathrm{E}+01 \\
2.08 \mathrm{E}+02 \\
2.58 \mathrm{E}+01 \\
8.48 \mathrm{E}+00 \\
5.62 \mathrm{E}+01 \\
2.34 \mathrm{E}+01 \\
9.87 \mathrm{E}+01 \\
2.84 \mathrm{E}+01 \\
1.23 \mathrm{E}+02 \\
2.44 \mathrm{E}+01 \\
5.46 \mathrm{E}+01 \\
2.77 \mathrm{E}+01 \\
6.83 \mathrm{E}+02 \\
2.19 \mathrm{E}+01 \\
1.74 \mathrm{E}+02 \\
2.68 \mathrm{E}+01 \\
2.52 \mathrm{E}+01 \\
1.31 \mathrm{E}+02 \\
2.70 \mathrm{E}+01\end{array}$ & $\begin{array}{l}\text { RA226 } \\
5.82 E-05 \\
1.35 E=05 \\
9.44 E-05 \\
3.92 E-05 \\
8.54 E-05 \\
9.43 E-06 \\
6.51 E-06 \\
3.08 E-05 \\
6.46 E-06 \\
4.13 E-05 \\
1.12 E-04 \\
4.64 E-05 \\
1.19 E-05 \\
4.89 E-06 \\
3.61 E-05 \\
5.71 E-06 \\
7.52 E-06 \\
6.33 E-06 \\
1.29 E-05 \\
3.15 E-06 \\
2.91 E-06 \\
3.15 E-06 \\
9.28 E-05 \\
9.22 E-06 \\
6.10 E-06 \\
1.46 E-06 \\
3.70 E-07 \\
4.14 E-06 \\
2.68 E-06 \\
2.80 E-06 \\
5.10 E-06 \\
2.79 E-06 \\
2.61 E-06 \\
2.61 E-06 \\
2.06 E-05 \\
8.97 E-05 \\
1.10 E-04 \\
1.92 E-05 \\
2.72 E-05 \\
3.13 E-05 \\
3.11 E-06 \\
2.60 E-05 \\
2.65 E-06 \\
5.80 E-06 \\
2.81 E-06 \\
7.86 E-05 \\
6.59 E-06 \\
5.12 E-05 \\
9.00 E-06 \\
1.97 E-06 \\
1.31 E-05 \\
6.74 E-06 \\
2.54 E-05 \\
6.36 E-06 \\
3.32 E=05 \\
6.33 E-06 \\
1.30 E-06 \\
6.48 E-06 \\
1.78 E-04 \\
6.41 E-06 \\
4.48 E-05 \\
9.31 E-06 \\
9.39 E-06 \\
4.75 E-05 \\
8.98 E-06\end{array}$ & $\begin{array}{l}\text { RA228 } \\
9.06 \mathrm{E}-10 \\
1.37 \mathrm{E}-10 \\
1.05 \mathrm{E}-09 \\
4.05 \mathrm{E}-10 \\
8.64 \mathrm{E}-10 \\
1.01 \mathrm{E}-10 \\
1.40 \mathrm{E}-10 \\
5.01 \mathrm{E}-10 \\
1.25 \mathrm{E}-10 \\
4.39 \mathrm{E}-10 \\
4.78 \mathrm{E}-09 \\
1.99 \mathrm{E}-09 \\
7.93 \mathrm{E}-10 \\
3.45 \mathrm{E}-10 \\
2.29 \mathrm{E}-09 \\
3.96 \mathrm{E}-10 \\
4.32 \mathrm{E}-10 \\
3.44 \mathrm{E}-10 \\
8.07 \mathrm{E}-10 \\
2.17 \mathrm{E}-10 \\
1.74 \mathrm{E}-10 \\
2.02 \mathrm{E}-10 \\
3.98 \mathrm{E}-09 \\
3.89 \mathrm{E}-10 \\
3.85 \mathrm{E}-10 \\
9.43 \mathrm{E}-11 \\
2.08 \mathrm{E}-11 \\
2.76 \mathrm{E}-10 \\
1.54 \mathrm{E}-10 \\
1.48 \mathrm{E}-10 \\
2.94 \mathrm{E}-10 \\
1.53 \mathrm{E}-10 \\
1.59 \mathrm{E}-10 \\
1.39 \mathrm{E}-10 \\
8.51 \mathrm{E}-10 \\
3.86 \mathrm{E}-09 \\
4.74 \mathrm{E}-09 \\
8.14 \mathrm{E}-10 \\
1.15 \mathrm{E}-09 \\
1.21 \mathrm{E}-09 \\
1.91 \mathrm{E}-10 \\
1.72 \mathrm{E}-09 \\
1.76 \mathrm{E}-10 \\
3.79 \mathrm{E}-10 \\
1.94 \mathrm{E}-10 \\
1.89 \mathrm{E}-09 \\
1.46 \mathrm{E}-10 \\
1.23 \mathrm{E}-09 \\
3.88 \mathrm{E}-10 \\
1.29 \mathrm{E}-10 \\
3.04 \mathrm{E}-10 \\
7.02 \mathrm{E}-11 \\
2.85 \mathrm{E}-10 \\
1.36 \mathrm{E}-10 \\
3.82 \mathrm{E}-10 \\
1.62 \mathrm{E}-10 \\
3.12 \mathrm{E}-10 \\
1.07 \mathrm{E}-10 \\
2.06 \mathrm{E}-09 \\
6.00 \mathrm{E}-11 \\
5.01 \mathrm{E}-10 \\
3.99 \mathrm{E}-10 \\
3.99 \mathrm{E}-10 \\
2.04 \mathrm{E}-09 \\
3.90 \mathrm{E}-10\end{array}$ & $\begin{array}{l}\text { AC227 } \\
3.20 \mathrm{E}-04 \\
7.74 \mathrm{E}-05 \\
5.35 \mathrm{E}-04 \\
2.24 \mathrm{E}-04 \\
4.88 \mathrm{E}-04 \\
5.37 \mathrm{E}-05 \\
3.43 \mathrm{E}-05 \\
1.69 \mathrm{E}-04 \\
3.46 \mathrm{E}-05 \\
2.35 \mathrm{E}-04 \\
5.61 \mathrm{E}-04 \\
2.33 \mathrm{E}-04 \\
5.52 \mathrm{E}-05 \\
2.25 \mathrm{E}-05 \\
1.69 \mathrm{E}-04 \\
2.63 \mathrm{E}-05 \\
4.38 \mathrm{E}-05 \\
3.76 \mathrm{E}-05 \\
7.35 \mathrm{E}-05 \\
1.76 \mathrm{E}-05 \\
1.67 \mathrm{E}-05 \\
1.78 \mathrm{E}-05 \\
4.65 \mathrm{E}-04 \\
4.66 \mathrm{E}-05 \\
2.85 \mathrm{E}-05 \\
6.80 \mathrm{E}-05 \\
1.74 \mathrm{E}-06 \\
1.92 \mathrm{E}-05 \\
1.57 \mathrm{E}-05 \\
1.68 \mathrm{E}-05 \\
2.99 \mathrm{E}-05 \\
1.66 \mathrm{E}-05 \\
1.51 \mathrm{E}-05 \\
1.56 \mathrm{E}-05 \\
1.04 \mathrm{E}-04 \\
4.52 \mathrm{E}-04 \\
5.52 \mathrm{E}-04 \\
9.67 \mathrm{E}-05 \\
1.38 \mathrm{E}-04 \\
1.62 \mathrm{E}-04 \\
1.81 \mathrm{E}-05 \\
1.48 \mathrm{E}-04 \\
1.51 \mathrm{E}-05 \\
3.33 \mathrm{E}-05 \\
1.59 \mathrm{E}-05 \\
4.09 \mathrm{E}-04 \\
3.47 \mathrm{E}-05 \\
2.66 \mathrm{E}-04 \\
4.54 \mathrm{E}-05 \\
1.13 \mathrm{E}-05 \\
6.83 \mathrm{E}-05 \\
3.86 \mathrm{E}-05 \\
1.45 \mathrm{E}-04 \\
3.37 \mathrm{E}-05 \\
1.89 \mathrm{E}-04 \\
3.27 \mathrm{E}-05 \\
6.76 \mathrm{E}-05 \\
3.54 \mathrm{E}-05 \\
1.02 \mathrm{E}-03 \\
3.71 \mathrm{E}-05 \\
2.56 \mathrm{E}-04 \\
4.71 \mathrm{E}-05 \\
4.74 \mathrm{E}-05 \\
2.38 \mathrm{E}-04 \\
4.53 \mathrm{0}-05\end{array}$ & $\begin{array}{l}\text { TH2229 } \\
1.87 \mathrm{E}-07 \\
2.74 \mathrm{E}-08 \\
2.12 \mathrm{E}-07 \\
8.12 \mathrm{E}-08 \\
1.73 \mathrm{E}-07 \\
2.03 \mathrm{E}-08 \\
3.04 \mathrm{E}-08 \\
1.04 \mathrm{E}-07 \\
2.66 \mathrm{E}-08 \\
8.82 \mathrm{E}-08 \\
2.83 \mathrm{E}-07 \\
1.18 \mathrm{E}-07 \\
1.12 \mathrm{E}-07 \\
5.32 \mathrm{E}-08 \\
2.98 \mathrm{E}-07 \\
5.97 \mathrm{E}-08 \\
3.75 \mathrm{E}-08 \\
2.70 \mathrm{E}-08 \\
8.04 \mathrm{E}-08 \\
2.52 \mathrm{E}-08 \\
1.61 \mathrm{E}-08 \\
2.09 \mathrm{E}-08 \\
2.37 \mathrm{E}-07 \\
2.15 \mathrm{E}-08 \\
5.01 \mathrm{E}-08 \\
1.27 \mathrm{E}-08 \\
2.30 \mathrm{E}-09 \\
3.91 \mathrm{E}-08 \\
1.35 \mathrm{E}-08 \\
1.09 \mathrm{E}-08 \\
2.58 \mathrm{E}-08 \\
1.22 \mathrm{E}-08 \\
1.53 \mathrm{E}-08 \\
1.04 \mathrm{E}-08 \\
4.44 \mathrm{E}-08 \\
2.26 \mathrm{E}-07 \\
2.84 \mathrm{E}-07 \\
4.63 \mathrm{E}-08 \\
6.23 \mathrm{E}-08 \\
4.66 \mathrm{E}-08 \\
1.84 \mathrm{E}-08 \\
1.87 \mathrm{E}-07 \\
1.90 \mathrm{E}-08 \\
4.01 \mathrm{E}-08 \\
2.22 \mathrm{E}-08 \\
4.25 \mathrm{E}-07 \\
3.19 \mathrm{E}-08 \\
2.76 \mathrm{E}-07 \\
2.27 \mathrm{E}-08 \\
1.36 \mathrm{E}-08 \\
6.77 \mathrm{E}-08 \\
1.41 \mathrm{E}-08 \\
5.75 \mathrm{E}-08 \\
2.95 \mathrm{E}-08 \\
7.73 \mathrm{E}-08 \\
3.72 \mathrm{E}-08 \\
7.03 \mathrm{E}-08 \\
2.23 \mathrm{E}-08 \\
4.17 \mathrm{E}-07 \\
1.20 \mathrm{E}-08 \\
1.01 \mathrm{E}-07 \\
2.27 \mathrm{E}-08 \\
2.27 \mathrm{E}-08 \\
1.21 \mathrm{E}-07 \\
2.31 \mathrm{E}-08\end{array}$ \\
\hline
\end{tabular}


HNF-SO-WH-TI-794, ReV. 0

ACTIVITY IN UNSEPARATED FUEL (Curies decayed to 1/1/94)

\begin{tabular}{|c|c|c|c|c|c|c|c|c|c|}
\hline $\begin{array}{c}\text { Listing } 7] \\
651 \\
652 \\
653 \\
654 \\
655 \\
656 \\
657 \\
658 \\
659 \\
660 \\
661 \\
662 \\
663 \\
664 \\
665 \\
666 \\
667 \\
668 \\
669 \\
670 \\
671 \\
672 \\
673 \\
674 \\
675 \\
676 \\
677 \\
678 \\
679 \\
680 \\
681 \\
682 \\
683 \\
684 \\
685 \\
686 \\
687 \\
688 \\
689 \\
690 \\
691 \\
692 \\
693 \\
694 \\
695 \\
696 \\
697 \\
698 \\
699 \\
700 \\
701 \\
702 \\
703 \\
704 \\
705 \\
706 \\
707 \\
708 \\
709 \\
710 \\
711 \\
712 \\
713 \\
714 \\
715\end{array}$ & $\begin{array}{l}\text { TH232 } \\
1.36 \mathrm{E}-09 \\
2.07 \mathrm{E}-10 \\
1.58 \mathrm{E}-09 \\
6.13 \mathrm{E}-10 \\
1.30 \mathrm{E}-09 \\
1.52 \mathrm{E}-10 \\
2.11 \mathrm{E}-10 \\
7.58 \mathrm{E}-10 \\
1.89 \mathrm{E}-10 \\
6.63 \mathrm{E}-10 \\
7.21 \mathrm{E}-09 \\
3.00 \mathrm{E}-09 \\
1.19 \mathrm{E}-09 \\
5.17 \mathrm{E}-10 \\
3.44 \mathrm{E}-09 \\
5.93 \mathrm{E}-10 \\
6.51 \mathrm{E}-10 \\
5.19 \mathrm{E}-10 \\
1.21 \mathrm{E}-09 \\
3.25 \mathrm{E}-10 \\
2.61 \mathrm{E}-10 \\
3.03 \mathrm{E}-10 \\
6.01 \mathrm{E}-09 \\
5.87 \mathrm{E}-10 \\
5.77 \mathrm{E}-10 \\
1.42 \mathrm{E}-10 \\
3.05 \mathrm{E}-11 \\
4.14 \mathrm{E}-10 \\
2.31 \mathrm{E}-10 \\
2.23 \mathrm{E}-10 \\
4.41 \mathrm{E}-10 \\
2.31 \mathrm{E}-10 \\
2.40 \mathrm{E}-10 \\
2.09 \mathrm{E}-10 \\
1.28 \mathrm{E}-09 \\
5.86 \mathrm{E}-09 \\
7.18 \mathrm{E}-09 \\
1.23 \mathrm{E}-09 \\
1.74 \mathrm{E}-09 \\
1.84 \mathrm{E}-09 \\
2.88 \mathrm{E}-10 \\
2.59 \mathrm{E}-09 \\
2.65 \mathrm{E}-10 \\
5.72 \mathrm{E}-10 \\
2.93 \mathrm{E}-10 \\
2.87 \mathrm{E}-09 \\
2.21 \mathrm{E}-10 \\
1.86 \mathrm{E}-09 \\
5.89 \mathrm{E}-10 \\
1.95 \mathrm{E}-10 \\
4.62 \mathrm{E}-10 \\
1.07 \mathrm{E}-10 \\
4.35 \mathrm{E}-10 \\
2.06 \mathrm{E}-10 \\
5.81 \mathrm{E}-10 \\
2.46 \mathrm{E}-10 \\
4.75 \mathrm{E}-10 \\
1.62 \mathrm{E}-10 \\
3.14 \mathrm{E}-09 \\
9.16 \mathrm{E}-11 \\
7.65 \mathrm{E}-10 \\
6.07 \mathrm{E}-10 \\
6.05 \mathrm{E}-10 \\
3.09 \mathrm{E}-09 \\
5.94 \mathrm{E}-10\end{array}$ & $\begin{array}{l}\text { PA231 } \\
9.64 \mathrm{E}-04 \\
2.35 \mathrm{E}-04 \\
1.62 \mathrm{E}-03 \\
6.81 \mathrm{E}-04 \\
1.48 \mathrm{E}-03 \\
1.64 \mathrm{E}-04 \\
1.04 \mathrm{E}-04 \\
5.13 \mathrm{E}-04 \\
1.05 \mathrm{E}-04 \\
7.12 \mathrm{E}-04 \\
1.70 \mathrm{E}-03 \\
7.07 \mathrm{E}-04 \\
1.62 \mathrm{E}-04 \\
6.54 \mathrm{E}-05 \\
5.00 \mathrm{E}-04 \\
7.65 \mathrm{E}-05 \\
1.32 \mathrm{E}-04 \\
1.14 \mathrm{E}-04 \\
2.20 \mathrm{E}-04 \\
5.21 \mathrm{E}-05 \\
5.03 \mathrm{E}-05 \\
5.33 \mathrm{E}-05 \\
1.41 \mathrm{E}-03 \\
1.42 \mathrm{E}-04 \\
8.40 \mathrm{E}-05 \\
2.01 \mathrm{E}-05 \\
4.98 \mathrm{E}-06 \\
5.63 \mathrm{E}-05 \\
4.70 \mathrm{E}-05 \\
5.10 \mathrm{E}-05 \\
8.99 \mathrm{E}-05 \\
5.03 \mathrm{E}-05 \\
4.55 \mathrm{E}-05 \\
4.72 \mathrm{E}-05 \\
3.17 \mathrm{E}-04 \\
1.39 \mathrm{E}-03 \\
1.69 \mathrm{E}-03 \\
2.95 \mathrm{E}-04 \\
4.24 \mathrm{E}-04 \\
4.96 \mathrm{E}-04 \\
5.47 \mathrm{E}-05 \\
4.46 \mathrm{E}-04 \\
4.56 \mathrm{E}-05 \\
1.00 \mathrm{E}-04 \\
4.78 \mathrm{E}-05 \\
1.24 \mathrm{E}-03 \\
1.06 \mathrm{E}-04 \\
8.06 \mathrm{E}-04 \\
1.40 \mathrm{E}-04 \\
3.41 \mathrm{E}-05 \\
2.08 \mathrm{E}-04 \\
1.19 \mathrm{E}-04 \\
4.50 \mathrm{E}-04 \\
1.02 \mathrm{E}-04 \\
5.79 \mathrm{E}-04 \\
9.91 \mathrm{E}-05 \\
2.06 \mathrm{E}-04 \\
1.08 \mathrm{E}-04 \\
3.13 \mathrm{E}-03 \\
1.15 \mathrm{E}-04 \\
7.93 \mathrm{E}-04 \\
1.45 \mathrm{E}-04 \\
1.45 \mathrm{E}-04 \\
7.29 \mathrm{E}-04 \\
.40 \mathrm{E}-04\end{array}$ & $\begin{array}{l}\text { U232 } \\
4.14 \mathrm{E}-03 \\
4.85 \mathrm{E}-04 \\
3.89 \mathrm{E}-03 \\
1.46 \mathrm{E}-03 \\
3.07 \mathrm{E}-03 \\
3.71 \mathrm{E}-04 \\
8.36 \mathrm{E}-04 \\
2.39 \mathrm{E}-03 \\
6.87 \mathrm{E}-04 \\
1.60 \mathrm{E}-03 \\
5.74 \mathrm{E}-03 \\
2.39 \mathrm{E}-03 \\
5.77 \mathrm{E}-03 \\
3.07 \mathrm{E}-03 \\
1.38 \mathrm{E}-02 \\
3.32 \mathrm{E}-03 \\
1.15 \mathrm{E}-03 \\
7.35 \mathrm{E}-04 \\
2.88 \mathrm{E}-03 \\
1.08 \mathrm{E}-03 \\
5.33 \mathrm{E}-04 \\
7.91 \mathrm{E}-04 \\
4.82 \mathrm{E}-03 \\
4.20 \mathrm{E}-04 \\
2.30 \mathrm{E}-03 \\
6.17 \mathrm{E}-04 \\
8.10 \mathrm{E}-05 \\
2.01 \mathrm{E}-03 \\
4.02 \mathrm{E}-04 \\
2.78 \mathrm{E}-04 \\
7.76 \mathrm{E}-04 \\
3.33 \mathrm{E}-04 \\
5.20 \mathrm{E}-04 \\
2.67 \mathrm{E}-04 \\
8.29 \mathrm{E}-04 \\
4.64 \mathrm{E}-03 \\
5.90 \mathrm{E}-03 \\
9.19 \mathrm{E}-04 \\
1.22 \mathrm{E}-03 \\
7.35 \mathrm{E}-04 \\
6.30 \mathrm{E}-04 \\
7.46 \mathrm{E}-03 \\
7.68 \mathrm{E}-04 \\
1.56 \mathrm{E}-03 \\
9.62 \mathrm{E}-04 \\
1.28 \mathrm{E}-02 \\
9.06 \mathrm{E}-04 \\
8.23 \mathrm{E}-03 \\
4.68 \mathrm{E}-04 \\
5.31 \mathrm{E}-04 \\
1.99 \mathrm{E}-03 \\
2.58 \mathrm{E}-04 \\
1.11 \mathrm{E}-03 \\
8.17 \mathrm{E}-04 \\
1.48 \mathrm{E}-03 \\
1.17 \mathrm{E}-03 \\
2.12 \mathrm{E}-03 \\
5.17 \mathrm{E}-04 \\
8.07 \mathrm{E}-03 \\
2.12 \mathrm{E}-04 \\
1.95 \mathrm{E}-03 \\
4.63 \mathrm{E}-04 \\
4.54 \mathrm{E}-04 \\
2.49 \mathrm{E}-03 \\
4.88 \mathrm{E}-04\end{array}$ & $\begin{array}{l}\text { U233 } \\
9.42 E-05 \\
1.37 \mathrm{E}-05 \\
1.06 \mathrm{E}-04 \\
4.08 \mathrm{E}-05 \\
8.68 \mathrm{E}-05 \\
1.02 \mathrm{E}-05 \\
1.56 \mathrm{E}-05 \\
5.29 \mathrm{E}-05 \\
1.35 \mathrm{E}-05 \\
4.42 \mathrm{E}-05 \\
1.51 \mathrm{E}-04 \\
6.30 \mathrm{E}-05 \\
6.04 \mathrm{E}-05 \\
2.85 \mathrm{E}-05 \\
1.61 \mathrm{E}-04 \\
3.20 \mathrm{E}-05 \\
2.04 \mathrm{E}-05 \\
1.47 \mathrm{E}-05 \\
4.36 \mathrm{E}-05 \\
1.36 \mathrm{E}-05 \\
8.77 \mathrm{E}-06 \\
1.14 \mathrm{E}-05 \\
1.27 \mathrm{E}-04 \\
1.16 \mathrm{E}-05 \\
2.70 \mathrm{E}-05 \\
6.87 \mathrm{E}-06 \\
1.20 \mathrm{E}-06 \\
2.10 \mathrm{E}-05 \\
7.32 \mathrm{E}-06 \\
5.95 \mathrm{E}-06 \\
1.41 \mathrm{E}-05 \\
6.66 \mathrm{E}-06 \\
8.40 \mathrm{E}-06 \\
5.66 \mathrm{E}-06 \\
2.38 \mathrm{E}-05 \\
1.22 \mathrm{E}-04 \\
1.53 \mathrm{E}-04 \\
2.49 \mathrm{E}-05 \\
3.37 \mathrm{E}-05 \\
2.50 \mathrm{E}-05 \\
1.01 \mathrm{E}-05 \\
1.03 \mathrm{E}-04 \\
1.05 \mathrm{E}-05 \\
2.21 \mathrm{E}-05 \\
1.22 \mathrm{E}-05 \\
2.22 \mathrm{E}-04 \\
1.65 \mathrm{E}-05 \\
1.43 \mathrm{E}-04 \\
1.23 \mathrm{E}-05 \\
7.52 \mathrm{E}-06 \\
3.52 \mathrm{E}-05 \\
7.15 \mathrm{E}-06 \\
2.95 \mathrm{E}-05 \\
1.52 \mathrm{E}-05 \\
3.92 \mathrm{E}-05 \\
1.96 \mathrm{E}-05 \\
3.67 \mathrm{E}-05 \\
1.13 \mathrm{E}-05 \\
2.13 \mathrm{E}-04 \\
6.14 \mathrm{E}-06 \\
5.20 \mathrm{E}-05 \\
1.24 \mathrm{E}-05 \\
1.22 \mathrm{E}-05 \\
6.52 \mathrm{E}-05 \\
1.26 \mathrm{E}-05\end{array}$ & $\begin{array}{l}\text { U233 } \\
4.08 E+01 \\
9.59 E+00 \\
6.62 E+01 \\
2.79 E+01 \\
6.06 E+01 \\
6.75 E+00 \\
4.60 E+00 \\
2.20 E+01 \\
4.59 E+00 \\
2.91 E+01 \\
7.92 E+01 \\
3.30 E+01 \\
8.14 E+00 \\
3.33 E+00 \\
2.49 E+01 \\
3.87 E+00 \\
5.27 E+00 \\
4.47 E+00 \\
8.94 E+00 \\
2.15 E+00 \\
2.03 E+00 \\
2.19 E+00 \\
6.61 E+01 \\
6.62 E+00 \\
4.15 E+00 \\
1.00 E+00 \\
2.30 E-01 \\
2.82 E+00 \\
1.84 E+00 \\
1.98 E+00 \\
3.55 E+00 \\
1.97 E+00 \\
1.83 E+00 \\
1.82 E+00 \\
1.47 E+01 \\
6.57 E+01 \\
7.93 E+01 \\
1.38 E+01 \\
1.99 E+01 \\
2.25 E+01 \\
2.21 E+00 \\
1.84 E+01 \\
1.89 E+00 \\
4.14 E+00 \\
1.99 E+00 \\
5.69 E+01 \\
4.77 E+00 \\
3.67 E+01 \\
6.62 E+00 \\
1.41 E+00 \\
9.46 E+00 \\
4.92 E+00 \\
1.90 E+01 \\
4.60 E+00 \\
2.42 E+01 \\
4.59 E+00 \\
9.42 E+00 \\
4.64 E+00 \\
1.32 E+02 \\
4.80 E+00 \\
3.35 E+01 \\
6.90 E+00 \\
6.79 E+00 \\
3.43 E+01 \\
6.70 E+00\end{array}$ & $\begin{array}{c}\text { U235 } \\
1.68 \mathrm{E}+00 \\
4.11 \mathrm{E}-01 \\
2.82 \mathrm{E}+00 \\
1.20 \mathrm{E}+00 \\
2.60 \mathrm{E}+00 \\
2.88 \mathrm{E}-01 \\
1.80 \mathrm{E}-01 \\
8.99 \mathrm{E}-01 \\
1.83 \mathrm{E}-01 \\
1.24 \mathrm{E}+00 \\
2.98 \mathrm{E}+00 \\
1.24 \mathrm{E}+00 \\
2.71 \mathrm{E}-01 \\
1.08 \mathrm{E}-01 \\
8.45 \mathrm{E}-01 \\
1.26 \mathrm{E}-01 \\
2.29 \mathrm{E}-01 \\
1.99 \mathrm{E}-01 \\
3.76 \mathrm{E}-01 \\
8.81 \mathrm{E}-02 \\
8.67 \mathrm{E}-02 \\
9.14 \mathrm{E}-02 \\
2.48 \mathrm{E}+00 \\
2.50 \mathrm{E}-01 \\
1.41 \mathrm{E}-01 \\
3.38 \mathrm{E}-02 \\
8.06 \mathrm{E}-03 \\
9.36 \mathrm{E}-02 \\
8.05 \mathrm{E}-02 \\
8.90 \mathrm{E}-02 \\
1.55 \mathrm{E}-01 \\
8.76 \mathrm{E}-02 \\
7.87 \mathrm{E}-02 \\
8.18 \mathrm{E}-02 \\
5.57 \mathrm{E}-01 \\
2.47 \mathrm{E}+00 \\
2.97 \mathrm{E}+00 \\
5.19 \mathrm{E}-01 \\
7.52 \mathrm{E}-01 \\
8.76 \mathrm{E}-01 \\
9.52 \mathrm{E}-02 \\
7.70 \mathrm{E}-01 \\
7.92 \mathrm{E}-02 \\
1.74 \mathrm{E}-01 \\
8.23 \mathrm{E}-02 \\
2.18 \mathrm{E}+00 \\
1.86 \mathrm{E}-01 \\
1.40 \mathrm{E}+00 \\
2.49 \mathrm{E}-01 \\
5.92 \mathrm{E}-00 \\
3.64 \mathrm{E}-01 \\
2.11 \mathrm{E}-01 \\
8.09 \mathrm{E}-01 \\
1.80 \mathrm{E}-01 \\
1.03 \mathrm{E}+00 \\
1.73 \mathrm{E}-01 \\
3.60 \mathrm{E}-01 \\
1.89 \mathrm{E}-01 \\
5.59 \mathrm{E}+00 \\
2.07 \mathrm{E}-01 \\
1.43 \mathrm{E}+00 \\
2.60 \mathrm{E}-01 \\
2.56 \mathrm{E}-01 \\
2.29 \mathrm{E}+00 \\
\end{array}$ & $\begin{array}{l}\text { U236 } \\
1.03 \mathrm{E}+00 \\
1.56 \mathrm{E}-01 \\
1.19 \mathrm{E}+00 \\
4.64 \mathrm{E}-01 \\
9.86 \mathrm{E}-01 \\
1.16 \mathrm{E}-01 \\
1.60 \mathrm{E}-01 \\
5.75 \mathrm{E}-01 \\
1.44 \mathrm{E}-01 \\
4.99 \mathrm{E}-01 \\
5.43 \mathrm{E}+00 \\
2.26 \mathrm{E}+00 \\
8.83 \mathrm{E}-01 \\
3.83 \mathrm{E}-01 \\
2.56 \mathrm{E}+00 \\
4.38 \mathrm{E}-01 \\
4.88 \mathrm{E}-01 \\
3.91 \mathrm{E}-01 \\
9.02 \mathrm{E}-01 \\
2.41 \mathrm{E}-01 \\
1.96 \mathrm{E}-01 \\
2.27 \mathrm{E}-01 \\
4.53 \mathrm{E}+00 \\
4.45 \mathrm{E}-01 \\
4.27 \mathrm{E}-01 \\
1.06 \mathrm{E}-01 \\
2.15 \mathrm{E}-02 \\
3.06 \mathrm{E}-01 \\
1.71 \mathrm{E}-01 \\
1.68 \mathrm{E}-01 \\
3.30 \mathrm{E}-01 \\
1.73 \mathrm{E}-01 \\
1.80 \mathrm{E}-01 \\
1.56 \mathrm{E}-01 \\
9.69 \mathrm{E}-01 \\
4.48 \mathrm{E}+00 \\
5.46 \mathrm{E}+00 \\
9.32 \mathrm{E}-01 \\
1.33 \mathrm{E}+00 \\
1.39 \mathrm{E}+00 \\
2.18 \mathrm{E}-01 \\
1.96 \mathrm{E}+00 \\
2.01 \mathrm{E}-01 \\
4.34 \mathrm{E}-01 \\
2.21 \mathrm{E}-01 \\
2.20 \mathrm{E}+00 \\
1.69 \mathrm{E}-01 \\
1.42 \mathrm{E}+00 \\
4.52 \mathrm{E}-01 \\
1.47 \mathrm{E}-01 \\
3.54 \mathrm{E}-01 \\
8.16 \mathrm{E}-02 \\
3.37 \mathrm{E}-01 \\
1.58 \mathrm{E}-01 \\
4.44 \mathrm{E}-01 \\
1.89 \mathrm{E}-01 \\
3.64 \mathrm{E}-01 \\
1.23 \mathrm{E}-01 \\
2.42 \mathrm{E}+00 \\
7.10 \mathrm{E}-02 \\
5.94 \mathrm{E}-01 \\
4.67 \mathrm{E}-01 \\
4.60 \mathrm{E}-01 \\
4.35 \mathrm{E}+00 \\
\end{array}$ & $\begin{array}{l}\text { U238 } \\
4.21 \mathrm{E}+01 \\
9.81 \mathrm{E}+00 \\
6.78 \mathrm{E}+01 \\
2.86 \mathrm{E}+01 \\
6.20 \mathrm{E}+01 \\
6.91 \mathrm{E}+00 \\
4.81 \mathrm{E}+00 \\
2.28 \mathrm{E}+01 \\
4.78 \mathrm{E}+00 \\
2.98 \mathrm{E}+01 \\
5.38 \mathrm{E}+01 \\
2.24 \mathrm{E}+01 \\
5.76 \mathrm{E}+00 \\
2.37 \mathrm{E}+00 \\
1.75 \mathrm{E}+01 \\
2.75 \mathrm{E}+00 \\
3.62 \mathrm{E}+00 \\
3.06 \mathrm{E}+00 \\
6.19 \mathrm{E}+00 \\
1.50 \mathrm{E}+00 \\
1.40 \mathrm{E}+00 \\
1.52 \mathrm{E}+00 \\
4.48 \mathrm{E}+01 \\
4.49 \mathrm{E}+00 \\
2.92 \mathrm{E}+00 \\
7.08 \mathrm{E}+01 \\
1.60 \mathrm{E}-01 \\
2.00 \mathrm{E}+00 \\
1.26 \mathrm{E}+00 \\
1.35 \mathrm{E}+00 \\
2.44 \mathrm{E}+00 \\
1.35 \mathrm{E}+00 \\
1.26 \mathrm{E}+00 \\
1.25 \mathrm{E}+00 \\
9.91 \mathrm{E}+00 \\
4.46 \mathrm{E}+01 \\
5.38 \mathrm{E}+01 \\
9.33 \mathrm{E}+00 \\
1.35 \mathrm{E}+01 \\
1.52 \mathrm{E}+01 \\
1.53 \mathrm{E}+00 \\
1.28 \mathrm{E}+01 \\
1.31 \mathrm{E}+00 \\
2.87 \mathrm{E}+00 \\
1.39 \mathrm{E}+00 \\
5.97 \mathrm{E}+01 \\
4.99 \mathrm{E}+00 \\
3.85 \mathrm{E}+01 \\
4.49 \mathrm{E}+00 \\
9.77 \mathrm{E}-01 \\
9.91 \mathrm{E}+00 \\
5.04 \mathrm{E}+00 \\
1.95 \mathrm{E}+01 \\
4.80 \mathrm{E}+00 \\
2.48 \mathrm{E}+01 \\
4.83 \mathrm{E}+00 \\
9.88 \mathrm{E}+00 \\
4.80 \mathrm{E}+00 \\
1.35 \mathrm{E}+02 \\
4.90 \mathrm{E}+00 \\
3.43 \mathrm{E}+01 \\
4.68 \mathrm{E}+00 \\
4.61 \mathrm{E}+00 \\
2.33 \mathrm{E}+01 \\
4.55 \mathrm{E}+00\end{array}$ & $\begin{array}{l}\text { NP237 } \\
3.82 \mathrm{E}-01 \\
5.28 \mathrm{E}-02 \\
4.10 \mathrm{E}-01 \\
1.58 \mathrm{E}-01 \\
3.34 \mathrm{E}-01 \\
3.95 \mathrm{E}-02 \\
6.82 \mathrm{E}-02 \\
2.17 \mathrm{E}-01 \\
5.73 \mathrm{E}-02 \\
1.71 \mathrm{E}-01 \\
7.12 \mathrm{E}-01 \\
2.97 \mathrm{E}-01 \\
3.31 \mathrm{E}-01 \\
1.59 \mathrm{E}-01 \\
8.70 \mathrm{E}-01 \\
1.77 \mathrm{E}-01 \\
1.04 \mathrm{E}-01 \\
7.36 \mathrm{E}-02 \\
2.27 \mathrm{E}-01 \\
7.31 \mathrm{E}-02 \\
4.50 \mathrm{E}-02 \\
5.98 \mathrm{E}-02 \\
5.97 \mathrm{E}-01 \\
5.43 \mathrm{E}-02 \\
1.45 \mathrm{E}-01 \\
3.73 \mathrm{E}-02 \\
6.06 \mathrm{E}-03 \\
1.15 \mathrm{E}-01 \\
3.71 \mathrm{E}-02 \\
2.95 \mathrm{E}-02 \\
7.16 \mathrm{E}-02 \\
3.34 \mathrm{E}-02 \\
4.37 \mathrm{E}-02 \\
2.80 \mathrm{E}-02 \\
1.11 \mathrm{E}-01 \\
5.79 \mathrm{E}-01 \\
7.24 \mathrm{E}-01 \\
1.17 \mathrm{E}-01 \\
1.59 \mathrm{E}-01 \\
1.15 \mathrm{E}-01 \\
5.28 \mathrm{E}-02 \\
5.36 \mathrm{E}-02 \\
2.37 \mathrm{E}-01 \\
2.04 \mathrm{E}-01 \\
5.85 \mathrm{E}-02 \\
5.77 \mathrm{E}-02 \\
3.08 \mathrm{E}-01 \\
6.01 \mathrm{E}-02 \\
1.63 \mathrm{E}-02 \\
1.18 \mathrm{E}-01 \\
6.616 \mathrm{E}-02 \\
1.02 \mathrm{E}-02 \\
7.33 \mathrm{E}-00 \\
6.58 \mathrm{E}-02 \\
5.83 \mathrm{E}-02 \\
1.00 \mathrm{E}-02 \\
2.60 \mathrm{E}-01 \\
\end{array}$ \\
\hline
\end{tabular}


ACTIVITY IN UNSEPARATED FUEL (Curies decayed to 1/1/94)

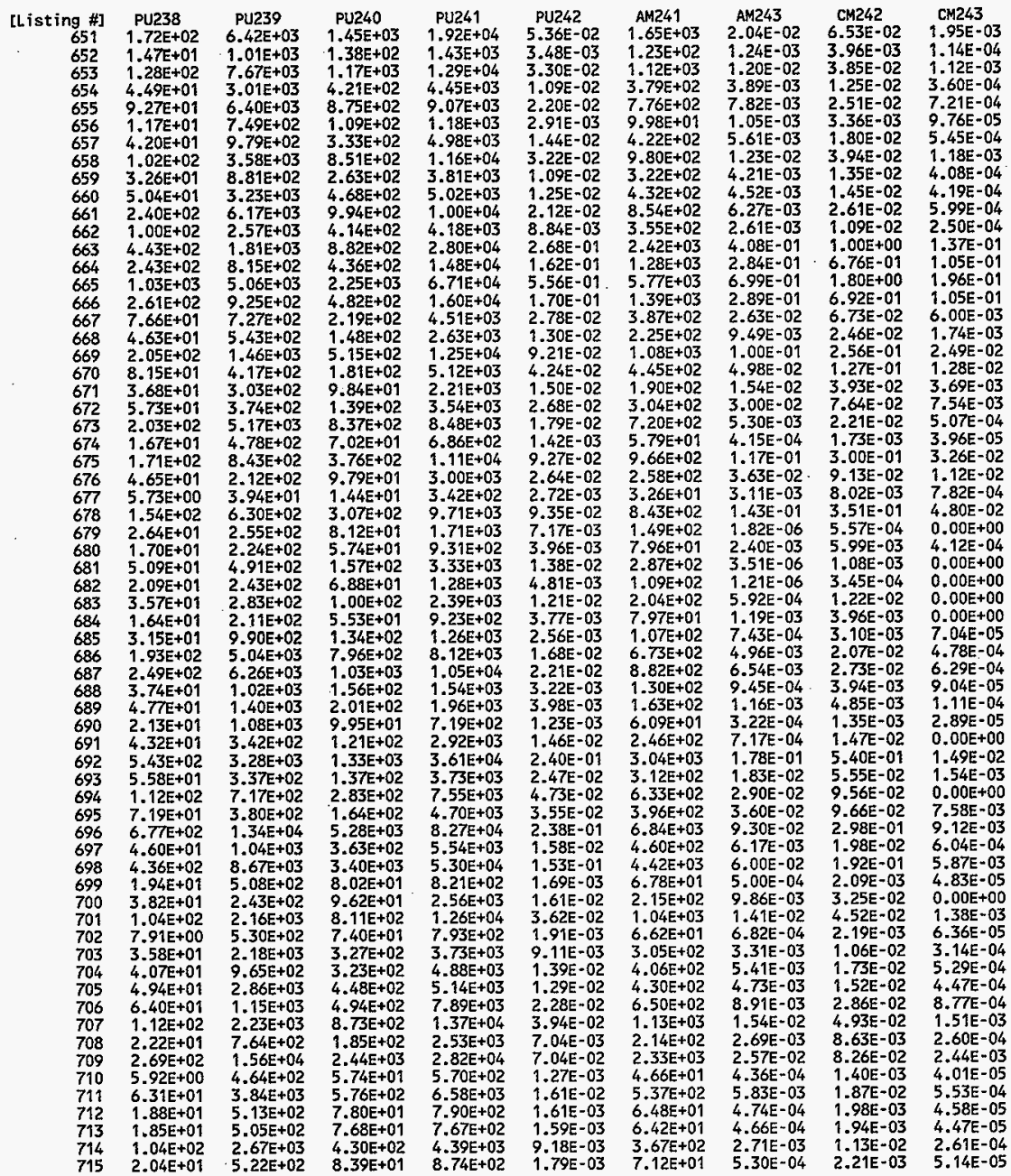


HNF-SD-WM-TI-794, ReV. O

ACTIVITY IN UNSEPARATED FUEL (Curies decayed to 1/1/94)

\begin{tabular}{|c|c|}
\hline 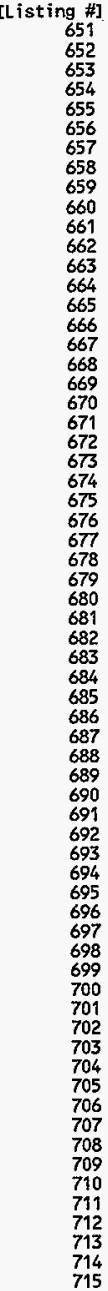 & $\begin{array}{l}\text { CM244 } \\
3.52 \mathrm{E}-02 \\
2.05 \mathrm{E}-03 \\
2.01 \mathrm{E}-02 \\
6.50 \mathrm{E}-03 \\
1.30 \mathrm{E}-02 \\
1.76 \mathrm{E}-03 \\
9.85 \mathrm{E}-03 \\
2.14 \mathrm{E}-02 \\
7.38 \mathrm{E}-03 \\
7.55 \mathrm{E}-03 \\
8.44 \mathrm{E}-03 \\
3.52 \mathrm{E}-03 \\
2.89 \mathrm{E}+00 \\
2.27 \mathrm{E}+00 \\
3.98 \mathrm{E}+00 \\
2.24 \mathrm{E}+00 \\
1.24 \mathrm{E}-01 \\
3.66 \mathrm{E}-02 \\
5.10 \mathrm{E}-01 \\
2.61 \mathrm{E}-01 \\
7.59 \mathrm{E}-02 \\
1.55 \mathrm{E}-01 \\
7.15 \mathrm{E}-03 \\
5.59 \mathrm{E}-04 \\
6.59 \mathrm{E}-01 \\
2.32 \mathrm{E}-01 \\
1.54 \mathrm{E}-02 \\
1.01 \mathrm{E}+00 \\
0.00 \mathrm{E}+00 \\
9.65 \mathrm{E}-03 \\
0.00 \mathrm{E}+00 \\
0.00 \mathrm{E}+00 \\
0.00 \mathrm{E}+00 \\
8.47 \mathrm{E}-07 \\
9.92 \mathrm{E}-04 \\
6.77 \mathrm{E}-03 \\
8.89 \mathrm{E}-03 \\
1.28 \mathrm{E}-03 \\
1.58 \mathrm{E}-03 \\
4.07 \mathrm{E}-04 \\
0.00 \mathrm{E}+00 \\
8.58 \mathrm{E}-02 \\
8.86 \mathrm{E}-03 \\
2.32 \mathrm{E}-05 \\
1.43 \mathrm{E}-01 \\
1.66 \mathrm{E}-01 \\
1.10 \mathrm{E}-02 \\
1.07 \mathrm{E}-01 \\
6.84 \mathrm{E}-04 \\
7.89 \mathrm{E}-06 \\
2.52 \mathrm{E}-02 \\
1.15 \mathrm{E}-03 \\
5.72 \mathrm{E}-03 \\
9.61 \mathrm{E}-03 \\
8.10 \mathrm{E}-03 \\
1.60 \mathrm{E}-02 \\
2.75 \mathrm{E}-02 \\
4.70 \mathrm{E}-03 \\
4.44 \mathrm{E}-02 \\
7.29 \mathrm{E}-04 \\
1.01 \mathrm{E}-02 \\
6.50 \mathrm{E}-04 \\
6.32 \mathrm{E}-04 \\
3.68 \mathrm{E}-03 \\
7.31 \mathrm{E}-04 \\
\end{array}$ \\
\hline
\end{tabular}


HNF-SD-WN-TI-794, ReV, 0

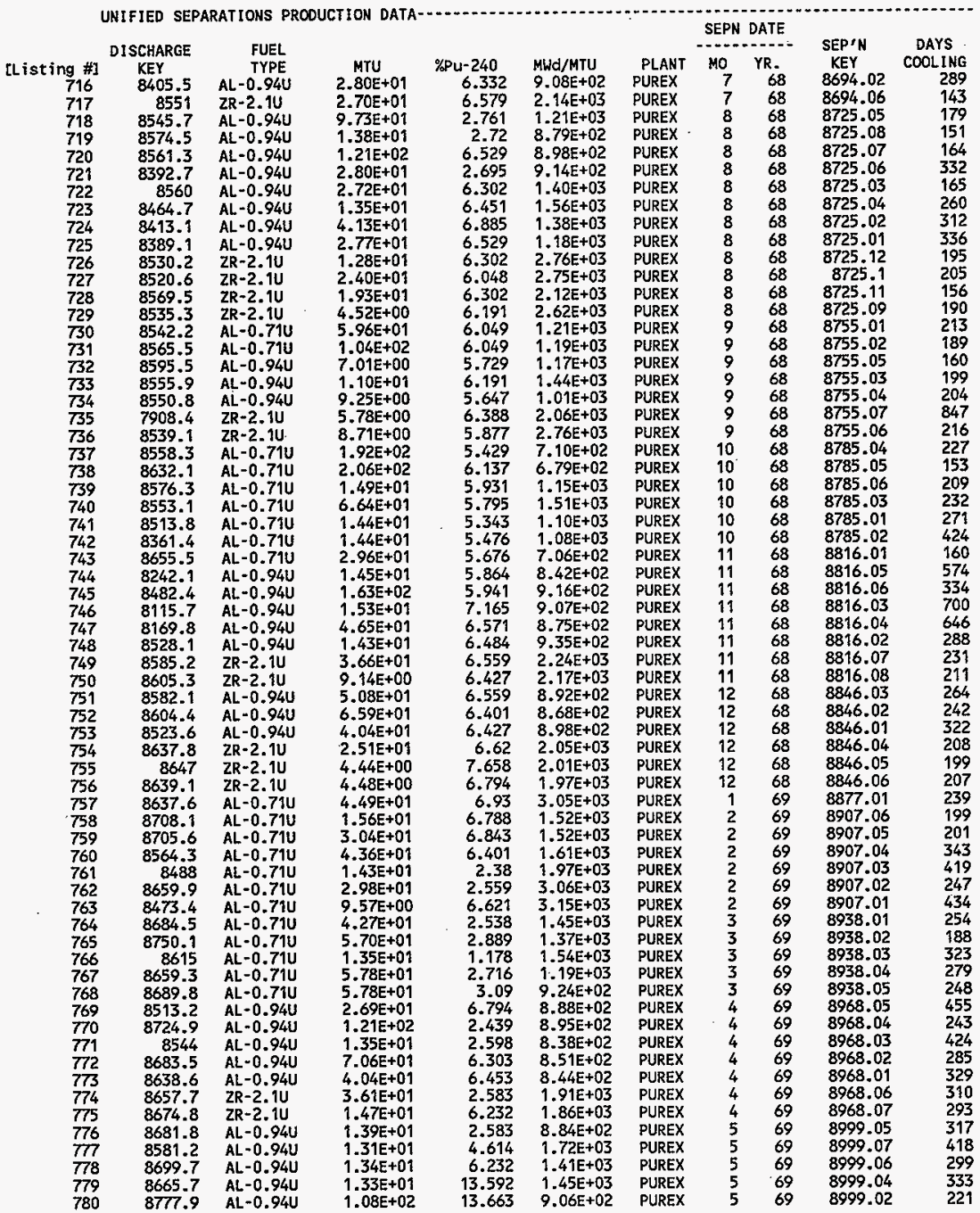


HNF-SD-WM-TI-794, ReV. 0

UNIFIED SEPARATIONS PRODUCTION DATA TOTAL WASTE LOSS FRACTION
TON

[Listing \#] Fuel Data Reference

716 ISO-263 RD AND ARH-18 RD (MCDONALD 717 ISO-263 RD AND ARH-18 RD (MCDONALD 718 ISO-263 RD AND ARH-18 RD (MCDONALD 719 ISO-263 RD AND ARH-18 RD (MCDONALD 720 ISO-263 RD AND ARH-18 RD (HCOONALO 721 ISO-263 RD AND ARH-18 RD (MCDONALD 722 ISO-263 RD AND ARH-18 RD (MCDONALD 723 ISO-263 RD AND ARH-18 RD (MCDONALD 724 ISO-263 RD AND ARH-18 RD (MCDONALD 725 ISO-263 RD AND ARH-18 RD (MCDONALD 726 ISO-263 RD AND ARH-18 RD (MCDONALD 727 ISO-263 RD AND ARH-18 RD (MCDONALD 728 ISO-263 RD AND ARH-18 RD (MCDONALD 729 ISO-263 RD AND ARH-18 RD (MCDONALD 730 ISO-263 RD AND ARH-18 RD (MCDONALD 731 ISO-263 RD AND ARH-18 RD (MCDONALD 732 ISO-263 RD AND ARH-18 RD (MCDONALD 733 ISO-263 RD AND ARH-18 RO (MCDONALD 734 ISO-263 RD AND ARH-18 RD (MCDONALD 735 ISO-263 RD AND ARH-18 RD (MCDONALD 736 ISO-263 RD AND ARH-18 RD (MCDONALD 737 ISO-263 RD AND ARH-18 RD (MCDONALD 738 ISO-263 RO AND ARH-18 RD (MCDONALD 739 ISO-263 RD AND ARH-18 RD (MCDONALD 740 ISO-263 RD AND ARH-18 RD (MCDONALD 741 ISO-263 RD AND ARH-18 RD (MCDONALD 742 ISO-263 RD AND ARH-18 RD (MCDONALD 743 ISO-263 RD AND ARH-18 RD (MCDONALD 744 ISO-263 RD AND ARH-18 RD (MCDONALD 745 ISO-263 RD AND ARH-18 RD (MCOONALD 746 ISO-263 RD AND ARH-18 RD (MCOONALD 747 ISO-263 RD AND ARH-18 RD (MCDONALD 748 ISO-263 RD AND ARH-18 RD (MCDONALD 749 ISO-263 RD AND ARH-18 RD (MCDONALD 750 ISO-263 RD AND ARH-18 RD (MCDONALD 751 ISO-263 RD AND ARH-18 RD (MCDONALD 752 ISO-263 RD AND ARH-18 RD (MCDONALD 753 ISO-263 RD AND ARH-18 RD (MCDONALD 754 ISO-263 RD AND ARH-18 RD (MCDONALD 755 ISO-263 RD AND ARH-18 RD (MCDONALD 756 ISO-263 RD AND ARH-18 RD (MCDONALD 757 ISO-263 RD AND ARH-18 RD (MCDONALD 758 ISO-263 RD AND ARH-18 RD (MCDONALD 759 ISO-263 RD AND ARH-18 RO (MCDONALD 760 ISO-263 RD AND ARH-18 RD (MCDONALD 761 ISO-263 RD AND ARH-18 RD (MCDONALD 762 ISO-263 RD AND ARH-18 RD (MCDONALD 763 ISO-263 RD AND ARH-18 RD (MCDONALD 764 ISO-263 RD AND ARH-18 RD (MCDONALD 765 ISO-263 RD AND ARH-18 RD (MCDONALD 766 ISO-263 RD AND ARH-18 RD (MCDONALD 767 ISO-263 RD AND ARH-18 RD (MCOONALD 768 IS0-263 RD AND ARH-18 RD (MCDONALD 769 ISO-263 RD AND ARH-18 RD (MCDONALD 770 1SO-263 RD AND ARH-18 RD (MCDONALD 771 ISO-263 RD AND ARH-18 RD (MCDONALD 772 ISO-263 RD AND ARH-18 RD (MCDONALD 773 ISO-263 RD AND ARH-18 RD (MCDONALD 774 ISO-263 RD AND ARH-18 RD (MCDONALD 775 ISO-263 RD AND ARH-18 RD (MCDONALD 776 ISO-263 RD AND ARH-18 RD (MCDONALD 777 ISO-263 RD AND ARH-18 RD (MCDONALD 778 ISO-263 RD AND ARH-18 RD (MCDONALD 779 ISO-263 RD AND ARH-18 RD (MCDONALD 780 ISO-263 RD AND ARH-18 RD (MCDONALD

.

0.

0.0168

0.002

0.002

0.002

0.002

0.002

0.002

0.002

0.002

0.0168

0.0168

0.0168

0.0168

0.0032

0.0032

0.0032

0.0032

0.0032

0.0168

0.0168

0.0021

0.0021

0.0021

0.0021

0.0021

0.0021

0.0022

0.0022

0.0022

0.0022

0.0022

0.0022

0.0168

0.0168

0.0023

0.0023

0.0023

0.0168

0.0168

0.0168

0.0034

0.0034

0.0034

0.0034

0.0034

0.0034

0.0034

0.0019

0.0019

0.0019

0.0019

0.0019

0.0028

0.0028

0.0028

0.0028

0.0028

0.0168

0.0168

0.0022

0.0022

0.0022

0.0022

0.0022
Np

0.

0.3

0.3

0.3

0.3

0.3

0.3

0.3

0.3

0.3

0.3

0.3

0.3

0.3

0.3

0.3

0.3

0.3

0.3

0.3

0.3

0.3

0.3

0.3

0.3

0.3

0.3

0.3

0.3

0.3

0.3

0.3

0.3

0.3

0.3

0.3

0.3

0.3

0.3

0.3

0.3

0.3

0.3

0.3

0.3

0.3

0.3

0.3

0.3

0.3

0.3

0.3

0.3

0.3

0.3

0.3

0.3

0.3

0.3

0.3

0.3

0.3

0.3

0.3

0.3
Pu

0.008

0.033

0.003

0.003

0.003

0.003

0.003

0.003

0.003

0.003

0.033

0.033

0.033

0.033

0.0046

0.0046

0.0046

0.0046

0.0046

0.033

0.033

0.003

0.003

0.003

0.003

0.003

0.003

0.0029

0.0029

0.0029

0.0029

0.0029

0.0029

0.033

0.033

0.0041

0.0041

0.0041

0.033

0.033

0.033

0.0046

0.0046

0.0046

0.0046

0.0046

0.0046

0.0046

0.0024

0.0024

0.0024

0.0024

0.0024

0.0065

0.0065

0.0065

0.0065

0.0065

0.033

0.033

0.0039

0.0039

0.0039

0.0039

0.0039

Waste Frac.

Reference

(b)

(c)

(b)

(b)

(b)

(b)

(b)

(b)

(c)

(c)

(b)

(b)

(b)

(b)

(b)

(c)

(b)

(b)

(b) 
HNF-SD - WM-TI-794, ReV. 0

ACTIVITY IN UNSEPARATED FUEL (Curies decayed to $1 / 1 / 94$ )

\begin{tabular}{|c|c|c|c|c|c|c|c|c|c|}
\hline 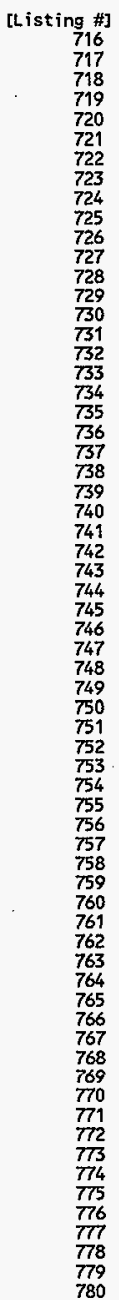 & 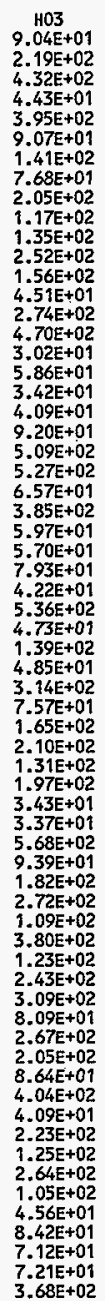 & $\begin{array}{l}C 14 \\
1.27 E+00 \\
5.54 E+00 \\
5.87 E+00 \\
6.05 E-01 \\
5.41 E+00 \\
1.27 E+00 \\
1.89 E+00 \\
1.05 E+00 \\
2.82 E+00 \\
1.62 E+00 \\
3.42 E+00 \\
6.38 E+00 \\
3.92 E+00 \\
1.14 E+00 \\
4.52 E+00 \\
7.73 E+00 \\
4.08 E-01 \\
7.89 E-01 \\
4.66 E-01 \\
1.14 E+00 \\
2.32 E+00 \\
8.66 E+00 \\
8.88 E+00 \\
1.08 E+00 \\
6.22 E+00 \\
9.96 E-01 \\
9.75 E-01 \\
1.33 E+00 \\
6.06 E-01 \\
7.41 E+00 \\
6.92 E-01 \\
2.02 E+00 \\
6.65 E-01 \\
7.88 E+00 \\
1.90 E+00 \\
2.25 E+00 \\
2.85 E+00 \\
1.81 E+00 \\
4.92 E+00 \\
8.53 E-01 \\
8.42 E-01 \\
8.18 E+00 \\
1.48 E+00 \\
2.83 E+00 \\
4.35 E+00 \\
1.73 E+00 \\
5.45 E+00 \\
1.80 E+00 \\
3.85 E+00 \\
4.89 E+00 \\
1.29 E+00 \\
4.33 E+00 \\
3.38 E+00 \\
1.19 E+00 \\
5.39 E+00 \\
5.62 E-01 \\
2.99 E+00 \\
1.70 E+00 \\
6.56 E+00 \\
2.60 E+00 \\
6.12 E-01 \\
1.12 E+00 \\
9.39 E-01 \\
9.55 E-01 \\
4.87 E+00\end{array}$ & $\begin{array}{l}\text { NI59 } \\
3.58 \mathrm{E}-01 \\
3.44 \mathrm{E}-01 \\
1.66 \mathrm{E}+00 \\
1.71 \mathrm{E}-01 \\
1.53 \mathrm{E}+00 \\
3.60 \mathrm{E}-01 \\
5.34 \mathrm{E}-01 \\
2.94 \mathrm{E}-01 \\
7.95 \mathrm{E}-01 \\
4.59 \mathrm{E}-01 \\
2.11 \mathrm{E}-01 \\
3.93 \mathrm{E}-01 \\
2.43 \mathrm{E}-01 \\
7.04 \mathrm{E}-02 \\
1.27 \mathrm{E}+00 \\
2.18 \mathrm{E}+00 \\
1.15 \mathrm{E}-01 \\
2.22 \mathrm{E}-01 \\
1.32 \mathrm{E}-01 \\
7.08 \mathrm{E}-02 \\
1.43 \mathrm{E}-01 \\
2.45 \mathrm{E}+00 \\
2.51 \mathrm{E}+00 \\
3.05 \mathrm{E}-01 \\
1.75 \mathrm{E}+00 \\
2.81 \mathrm{E}-01 \\
2.75 \mathrm{E}-01 \\
3.76 \mathrm{E}-01 \\
1.72 \mathrm{E}-01 \\
2.10 \mathrm{E}+00 \\
1.96 \mathrm{E}-01 \\
5.73 \mathrm{E}-01 \\
1.88 \mathrm{E}-01 \\
4.88 \mathrm{E}-01 \\
1.18 \mathrm{E}-01 \\
6.38 \mathrm{E}-01 \\
8.06 \mathrm{E}-01 \\
5.11 \mathrm{E}-01 \\
3.05 \mathrm{E}-01 \\
5.30 \mathrm{E}-02 \\
5.23 \mathrm{E}-02 \\
2.26 \mathrm{E}+00 \\
4.15 \mathrm{E}-01 \\
8.07 \mathrm{E}-01 \\
1.22 \mathrm{E}+00 \\
4.84 \mathrm{E}-01 \\
1.51 \mathrm{E}+00 \\
4.96 \mathrm{E}-01 \\
1.08 \mathrm{E}+00 \\
1.38 \mathrm{E}+00 \\
3.63 \mathrm{E}-01 \\
1.22 \mathrm{E}+00 \\
9.54 \mathrm{E}-01 \\
3.37 \mathrm{E}-01 \\
1.53 \mathrm{E}+00 \\
1.59 \mathrm{E}-01 \\
8.47 \mathrm{E}-01 \\
4.81 \mathrm{E}-01 \\
4.08 \mathrm{E}-01 \\
1.62 \mathrm{E}-01 \\
1.73 \mathrm{E}-01 \\
3.15 \mathrm{E}-01 \\
2.65 \mathrm{E}-01 \\
2.69 \mathrm{E}-01 \\
1.38 \mathrm{E}+00\end{array}$ & $\begin{array}{c}N 163 \\
3.67 E+01 \\
3.58 E+01 \\
1.71 E+02 \\
1.76 E+01 \\
1.57 E+02 \\
3.69 E+01 \\
5.50 E+01 \\
3.03 E+01 \\
8.16 E+01 \\
4.71 E+01 \\
2.20 E+01 \\
4.10 E+01 \\
2.53 E+01 \\
7.35 E+00 \\
1.31 E+02 \\
2.25 E+02 \\
1.19 E+01 \\
2.29 E+01 \\
1.36 E+01 \\
7.27 E+00 \\
1.50 E+01 \\
2.52 E+02 \\
2.59 E+02 \\
3.14 E+01 \\
1.81 E+02 \\
2.89 E+01 \\
2.82 E+01 \\
3.88 E+01 \\
1.75 E+01 \\
2.15 E+02 \\
2.00 E+01 \\
5.84 E+01 \\
1.94 E+01 \\
5.09 E+01 \\
1.23 E+01 \\
6.56 E+01 \\
8.30 E+01 \\
5.25 E+01 \\
3.18 E+01 \\
5.52 E+00 \\
5.44 E+00 \\
2.36 E+02 \\
4.30 E+01 \\
8.37 E+01 \\
1.26 E+02 \\
5.01 E+01 \\
1.58 E+02 \\
5.18 E+01 \\
1.12 E+02 \\
1.43 E+02 \\
3.76 E+01 \\
1.26 E+02 \\
9.85 E+01 \\
3.46 E+01 \\
1.57 E+02 \\
1.64 E+01 \\
8.73 E+01 \\
4.95 E+01 \\
4.25 E+01 \\
1.68 E+01 \\
1.79 E+01 \\
3.25 E+01 \\
2.74 E+01 \\
2.78 E+01 \\
1.42 E+02\end{array}$ & $\begin{array}{c}C 060 \\
2.45 E+00 \\
1.35 E+01 \\
1.19 E+01 \\
1.25 E+00 \\
1.11 E+01 \\
2.46 E+00 \\
3.84 E+00 \\
2.04 E+00 \\
5.42 E+00 \\
3.11 E+00 \\
8.15 E+00 \\
1.52 E+01 \\
9.60 E+00 \\
2.73 E+00 \\
9.14 E+00 \\
1.58 E+01 \\
8.42 E-01 \\
1.60 E+00 \\
9.51 E-01 \\
2.21 E+00 \\
5.56 E+00 \\
1.78 E+01 \\
1.88 E+01 \\
2.22 E+00 \\
1.26 E+01 \\
2.00 E+00 \\
1.85 E+00 \\
2.83 E+00 \\
1.11 E+00 \\
1.48 E+01 \\
1.21 E+00 \\
3.61 E+00 \\
1.35 E+00 \\
1.94 E+01 \\
4.71 E+00 \\
4.66 E+00 \\
5.94 E+00 \\
3.65 E+00 \\
1.24 E+01 \\
2.15 E+00 \\
2.12 E+00 \\
1.64 E+01 \\
3.16 E+00 \\
6.13 E+00 \\
8.81 E+00 \\
3.38 E+00 \\
1.10 E+01 \\
3.39 E+00 \\
8.17 E+00 \\
1.06 E+01 \\
2.67 E+00 \\
9.13 E+00 \\
7.24 E+00 \\
2.40 E+00 \\
1.17 E+01 \\
1.15 E+00 \\
6.42 E+00 \\
3.58 E+00 \\
1.67 E+01 \\
6.64 E+00 \\
1.31 E+00 \\
2.27 E+00 \\
2.00 E+00 \\
2.01 E+00 \\
1.08 E+01\end{array}$ & $\begin{array}{l}\text { SE79 } \\
3.40 \mathrm{E}-01 \\
7.61 \mathrm{E}-01 \\
1.58 \mathrm{E}+00 \\
1.63 \mathrm{E}-01 \\
1.45 \mathrm{E}+00 \\
3.42 \mathrm{E}-01 \\
5.09 \mathrm{E}-01 \\
2.81 \mathrm{E}-01 \\
7.57 \mathrm{E}-01 \\
4.36 \mathrm{E}-01 \\
4.63 \mathrm{E}-01 \\
8.63 \mathrm{E}-01 \\
5.39 \mathrm{E}-01 \\
1.55 \mathrm{E}-01 \\
9.66 \mathrm{E}-01 \\
1.65 \mathrm{E}+00 \\
1.10 \mathrm{E}-01 \\
2.12 \mathrm{E}-01 \\
1.25 \mathrm{E}-01 \\
1.57 \mathrm{E}-01 \\
3.15 \mathrm{E}-01 \\
1.83 \mathrm{E}+00 \\
1.88 \mathrm{E}+00 \\
2.31 \mathrm{E}-01 \\
1.34 \mathrm{E}+00 \\
2.12 \mathrm{E}-01 \\
2.08 \mathrm{E}-01 \\
2.81 \mathrm{E}-01 \\
1.63 \mathrm{E}-01 \\
1.99 \mathrm{E}+00 \\
1.86 \mathrm{E}-01 \\
5.44 \mathrm{E}-01 \\
1.79 \mathrm{E}-01 \\
1.08 \mathrm{E}+00 \\
2.61 \mathrm{E}-01 \\
6.05 \mathrm{E}-01 \\
7.65 \mathrm{E}-01 \\
4.85 \mathrm{E}-01 \\
6.77 \mathrm{E}-01 \\
1.18 \mathrm{E}-01 \\
1.16 \mathrm{E}-01 \\
1.81 \mathrm{E}+00 \\
3.18 \mathrm{E}-01 \\
6.18 \mathrm{E}-01 \\
9.38 \mathrm{E}-01 \\
3.75 \mathrm{E}-01 \\
1.21 \mathrm{E}+00 \\
3.99 \mathrm{E}-01 \\
8.28 \mathrm{E}-01 \\
1.05 \mathrm{E}+00 \\
2.78 \mathrm{E}-01 \\
9.25 \mathrm{E}-01 \\
7.18 \mathrm{E}-01 \\
3.20 \mathrm{E}-01 \\
1.45 \mathrm{E}+00 \\
1.51 \mathrm{E}-01 \\
8.03 \mathrm{E}-01 \\
4.56 \mathrm{E}-01 \\
9.07 \mathrm{E}-01 \\
3.59 \mathrm{E}-01 \\
1.64 \mathrm{E}-01 \\
3.01 \mathrm{E}-01 \\
2.52 \mathrm{E}-01 \\
2.56 \mathrm{E}-01 \\
1.31 \mathrm{E}+00\end{array}$ & $\begin{array}{l}\text { SR90 } \\
3.94 \mathrm{E}+04 \\
8.50 \mathrm{E}+04 \\
1.83 \mathrm{E}+05 \\
1.91 \mathrm{E}+04 \\
1.70 \mathrm{E}+05 \\
3.96 \mathrm{E}+04 \\
5.86 \mathrm{E}+04 \\
3.20 \mathrm{E}+04 \\
8.64 \mathrm{E}+04 \\
5.01 \mathrm{E}+04 \\
5.07 \mathrm{E}+04 \\
9.45 \mathrm{E}+04 \\
6.03 \mathrm{E}+04 \\
1.71 \mathrm{E}+04 \\
1.07 \mathrm{E}+05 \\
1.84 \mathrm{E}+05 \\
1.28 \mathrm{E}+04 \\
2.44 \mathrm{E}+04 \\
1.46 \mathrm{E}+04 \\
1.69 \mathrm{E}+04 \\
3.45 \mathrm{E}+04 \\
2.09 \mathrm{E}+05 \\
2.16 \mathrm{E}+05 \\
2.58 \mathrm{E}+04 \\
1.46 \mathrm{E}+05 \\
2.37 \mathrm{E}+04 \\
2.30 \mathrm{E}+04 \\
3.23 \mathrm{E}+04 \\
1.88 \mathrm{E}+04 \\
2.32 \mathrm{E}+05 \\
2.12 \mathrm{E}+04 \\
6.23 \mathrm{E}+04 \\
2.09 \mathrm{E}+04 \\
1.21 \mathrm{E}+05 \\
2.92 \mathrm{E}+04 \\
7.12 \mathrm{E}+04 \\
9.01 \mathrm{E}+04 \\
5.67 \mathrm{E}+04 \\
7.64 \mathrm{E}+04 \\
1.33 \mathrm{E}+04 \\
1.31 \mathrm{E}+04 \\
1.82 \mathrm{E}+05 \\
3.51 \mathrm{E}+04 \\
6.82 \mathrm{E}+04 \\
1.02 \mathrm{E}+05 \\
3.98 \mathrm{E}+04 \\
1.22 \mathrm{E}+05 \\
3.95 \mathrm{E}+04 \\
9.16 \mathrm{E}+04 \\
1.17 \mathrm{E}+05 \\
3.05 \mathrm{E}+04 \\
1.04 \mathrm{E}+05 \\
8.17 \mathrm{E}+04 \\
3.74 \mathrm{E}+04 \\
1.72 \mathrm{E}+05 \\
1.77 \mathrm{E}+04 \\
9.53 \mathrm{E}+04 \\
5.39 \mathrm{E}+04 \\
1.03 \mathrm{E}+05 \\
4.09 \mathrm{E}+04 \\
1.95 \mathrm{E}+04 \\
3.43 \mathrm{E}+04 \\
2.93 \mathrm{E}+04 \\
2.97 \mathrm{E}+04 \\
1.56 \mathrm{E}+05\end{array}$ & $\begin{array}{l}Y 90 \\
3.94 \mathrm{E}+04 \\
8.51 \mathrm{E}+04 \\
1.83 \mathrm{E}+05 \\
1.91 \mathrm{E}+04 \\
1.70 \mathrm{E}+05 \\
3.97 \mathrm{E}+04 \\
5.86 \mathrm{E}+04 \\
3.20 \mathrm{E}+04 \\
8.64 \mathrm{E}+04 \\
5.01 \mathrm{E}+04 \\
5.07 \mathrm{E}+04 \\
9.45 \mathrm{E}+04 \\
6.03 \mathrm{E}+04 \\
1.71 \mathrm{E}+04 \\
1.07 \mathrm{E}+05 \\
1.84 \mathrm{E}+05 \\
1.28 \mathrm{E}+04 \\
2.44 \mathrm{E}+04 \\
1.46 \mathrm{E}+04 \\
1.69 \mathrm{E}+04 \\
3.45 \mathrm{E}+04 \\
2.09 \mathrm{E}+05 \\
2.16 \mathrm{E}+05 \\
2.58 \mathrm{E}+04 \\
1.46 \mathrm{E}+05 \\
2.37 \mathrm{E}+04 \\
2.30 \mathrm{E}+04 \\
3.23 \mathrm{E}+04 \\
1.88 \mathrm{E}+04 \\
2.32 \mathrm{E}+05 \\
2.12 \mathrm{E}+04 \\
6.23 \mathrm{E}+04 \\
2.09 \mathrm{E}+04 \\
1.21 \mathrm{E}+05 \\
2.92 \mathrm{E}+04 \\
7.12 \mathrm{E}+04 \\
9.01 \mathrm{E}+04 \\
5.68 \mathrm{E}+04 \\
7.64 \mathrm{E}+04 \\
1.33 \mathrm{E}+04 \\
1.31 \mathrm{E}+04 \\
1.82 \mathrm{E}+05 \\
3.51 \mathrm{E}+04 \\
6.82 \mathrm{E}+04 \\
1.02 \mathrm{E}+05 \\
3.99 \mathrm{E}+04 \\
1.22 \mathrm{E}+05 \\
3.95 \mathrm{E}+04 \\
9.16 \mathrm{E}+04 \\
1.17 \mathrm{E}+05 \\
3.05 \mathrm{E}+04 \\
1.04 \mathrm{E}+05 \\
8.17 \mathrm{E}+04 \\
3.74 \mathrm{E}+04 \\
1.72 \mathrm{E}+05 \\
1.77 \mathrm{E}+04 \\
9.53 \mathrm{E}+04 \\
5.39 \mathrm{E}+04 \\
1.03 \mathrm{E}+05 \\
4.09 \mathrm{E}+04 \\
1.95 \mathrm{E}+04 \\
3.43 \mathrm{E}+04 \\
2.93 \mathrm{E}+04 \\
2.97 \mathrm{E}+04 \\
1.56 \mathrm{E}+05\end{array}$ & $\begin{array}{l}2 R 93 \\
1.63 \mathrm{E}+00 \\
3.69 \mathrm{E}+00 \\
7.54 \mathrm{E}+00 \\
7.82 \mathrm{E}-01 \\
6.98 \mathrm{E}+00 \\
1.64 \mathrm{E}+00 \\
2.43 \mathrm{E}+00 \\
1.34 \mathrm{E}+00 \\
3.61 \mathrm{E}+00 \\
2.09 \mathrm{E}+00 \\
2.23 \mathrm{E}+00 \\
4.15 \mathrm{E}+00 \\
2.61 \mathrm{E}+00 \\
7.47 \mathrm{E}-01 \\
4.49 \mathrm{E}+00 \\
7.69 \mathrm{E}+00 \\
5.24 \mathrm{E}-01 \\
1.01 \mathrm{E}+00 \\
6.01 \mathrm{E}-01 \\
7.63 \mathrm{E}-01 \\
1.51 \mathrm{E}+00 \\
8.64 \mathrm{E}+00 \\
8.86 \mathrm{E}+00 \\
1.08 \mathrm{E}+00 \\
6.18 \mathrm{E}+00 \\
9.91 \mathrm{E}-01 \\
9.71 \mathrm{E}-01 \\
1.33 \mathrm{E}+00 \\
7.84 \mathrm{E}-01 \\
9.56 \mathrm{E}+00 \\
8.93 \mathrm{E}-01 \\
2.62 \mathrm{E}+00 \\
8.59 \mathrm{E}-01 \\
5.23 \mathrm{E}+00 \\
1.26 \mathrm{E}+00 \\
2.91 \mathrm{E}+00 \\
3.68 \mathrm{E}+00 \\
2.33 \mathrm{E}+00 \\
3.29 \mathrm{E}+00 \\
5.71 \mathrm{E}-01 \\
5.65 \mathrm{E}-01 \\
8.08 \mathrm{E}+00 \\
1.47 \mathrm{E}+00 \\
2.86 \mathrm{E}+00 \\
4.33 \mathrm{E}+00 \\
1.72 \mathrm{E}+00 \\
5.39 \mathrm{E}+00 \\
1.78 \mathrm{E}+00 \\
3.83 \mathrm{E}+00 \\
4.86 \mathrm{E}+00 \\
1.28 \mathrm{E}+00 \\
4.31 \mathrm{E}+00 \\
3.36 \mathrm{E}+00 \\
1.54 \mathrm{E}+00 \\
6.97 \mathrm{E}+00 \\
7.27 \mathrm{E}-01 \\
3.87 \mathrm{E}+00 \\
2.19 \mathrm{E}+00 \\
4.42 \mathrm{E}+00 \\
1.75 \mathrm{E}+00 \\
7.91 \mathrm{E}-01 \\
1.43 \mathrm{E}+00 \\
1.20 \mathrm{E}+00 \\
1.22 \mathrm{E}+00 \\
6.28 \mathrm{E}+00\end{array}$ \\
\hline
\end{tabular}


HNF-SD-WM-T1-794, Rev, O

ACTIVITY IN UNSEPARATED FUEL (Curies decayed to $1 / 1 / 94$ )

\begin{tabular}{|c|c|c|c|c|c|c|c|c|c|}
\hline $\begin{array}{r}\text { isting } 71 \\
716 \\
717 \\
718 \\
719 \\
720 \\
721 \\
722 \\
723 \\
724 \\
725 \\
726 \\
727 \\
728 \\
729 \\
730 \\
731 \\
732 \\
733 \\
734 \\
735 \\
736 \\
737 \\
738 \\
739 \\
740 \\
741 \\
742 \\
743 \\
744 \\
745 \\
746 \\
747 \\
748 \\
749 \\
750 \\
751 \\
752 \\
753 \\
754 \\
755 \\
756 \\
757 \\
758 \\
759 \\
760 \\
761 \\
762 \\
763 \\
764 \\
765 \\
766 \\
767 \\
768 \\
769 \\
770 \\
771 \\
772 \\
773 \\
774 \\
775 \\
776 \\
7777 \\
778 \\
779 \\
780\end{array}$ & $\begin{array}{l}\text { NB93* } \\
1.15 E+00 \\
2.58 E+00 \\
5.27 E+00 \\
5.45 E-01 \\
4.87 E+00 \\
1.16 E+00 \\
1.70 E+00 \\
9.38 E-01 \\
2.54 E+00 \\
1.47 E+00 \\
1.56 E+00 \\
2.91 E+00 \\
1.83 E+00 \\
5.23 E-01 \\
3.14 E+00 \\
5.37 E+00 \\
3.65 E-01 \\
7.06 E-01 \\
4.20 E-01 \\
5.50 E-01 \\
1.06 E+00 \\
6.02 E+00 \\
6.15 E+00 \\
7.50 E-01 \\
4.32 E+00 \\
6.93 E-01 \\
6.84 E-01 \\
9.20 E-01 \\
5.55 E-01 \\
6.70 E+00 \\
6.37 E-01 \\
1.86 E+00 \\
6.00 E-01 \\
3.65 E+00 \\
8.81 E-01 \\
2.03 E+00 \\
2.56 E+00 \\
1.63 E+00 \\
2.29 E+00 \\
3.97 E-01 \\
3.93 E-01 \\
5.65 E+00 \\
1.02 E+00 \\
1.98 E+00 \\
3.02 E+00 \\
1.21 E+00 \\
3.77 E+00 \\
1.25 E+00 \\
2.66 E+00 \\
3.36 E+00 \\
8.95 E-01 \\
2.99 E+00 \\
2.33 E+00 \\
1.08 E+00 \\
4.82 E+00 \\
5.07 E-01 \\
2.68 E+00 \\
1.52 E+00 \\
3.07 E+00 \\
1.22 E+00 \\
5.48 E-01 \\
9.98 E-01 \\
8.34 E=01 \\
8.49 E=01 \\
4.34 E+00\end{array}$ & $\begin{array}{l}7 C 99 \\
1.13 E+01 \\
2.55 E+01 \\
5.25 E+01 \\
5.40 E+00 \\
4.83 E+01 \\
1.14 E+01 \\
1.73 E+01 \\
9.70 E+00 \\
2.58 E+01 \\
1.45 E+01 \\
1.55 E+01 \\
2.90 E+01 \\
1.81 E+01 \\
5.20 E+00 \\
3.20 E+01 \\
5.48 E+01 \\
3.65 E+00 \\
7.23 E+00 \\
4.17 E+00 \\
5.26 E+00 \\
1.06 E+01 \\
6.05 E+01 \\
6.20 E+01 \\
7.65 E+00 \\
4.58 E+01 \\
7.03 E+00 \\
6.88 E+00 \\
9.28 E+00 \\
5.41 E+00 \\
6.62 E+01 \\
6.18 E+00 \\
1.81 E+01 \\
5.94 E+00 \\
3.62 E+01 \\
8.73 E+00 \\
2.01 E+01 \\
2.54 E+01 \\
1.61 E+01 \\
2.27 E+01 \\
3.94 E+00 \\
3.89 E+00 \\
7.27 E+01 \\
1.09 E+01 \\
2.12 E+01 \\
3.25 E+01 \\
1.35 E+01 \\
4.85 E+01 \\
1.62 E+01 \\
2.82 E+01 \\
3.54 E+01 \\
9.54 E+00 \\
3.06 E+01 \\
2.37 E+01 \\
1.06 E+01 \\
4.82 E+01 \\
5.02 E+00 \\
2.67 E+01 \\
1.52 E+01 \\
3.04 E+01 \\
1.21 E+01 \\
5.47 E+00 \\
1.06 E+01 \\
8.58 E+00 \\
8.76 E+00 \\
4.35 E+01\end{array}$ & 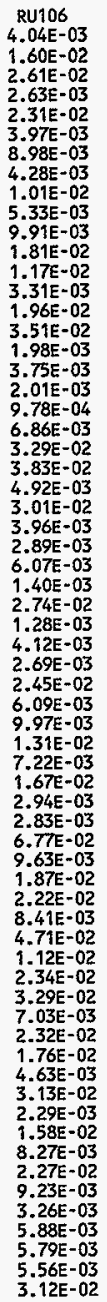 & $\begin{array}{l}C D 113 * \\
7.65 E+00 \\
1.91 E+01 \\
3.69 E+01 \\
3.73 E+00 \\
3.33 E+01 \\
7.69 E+00 \\
1.21 E+01 \\
6.65 E+00 \\
1.76 E+01 \\
9.98 E+00 \\
1.21 E+01 \\
2.26 E+01 \\
1.36 E+01 \\
4.02 E+00 \\
2.69 E+01 \\
4.61 E+01 \\
2.58 E+00 \\
5.04 E+00 \\
2.90 E+00 \\
3.61 E+00 \\
8.25 E+00 \\
4.91 E+01 \\
5.07 E+01 \\
6.43 E+00 \\
3.81 E+01 \\
5.84 E+00 \\
5.59 E+00 \\
7.63 E+00 \\
3.58 E+00 \\
4.53 E+01 \\
4.03 E+00 \\
1.19 E+01 \\
4.10 E+00 \\
2.74 E+01 \\
6.60 E+00 \\
1.39 E+01 \\
1.76 E+01 \\
1.11 E+01 \\
1.71 E+01 \\
2.97 E+00 \\
2.91 E+00 \\
5.85 E+01 \\
9.26 E+00 \\
1.80 E+01 \\
2.70 E+01 \\
1.10 E+01 \\
3.91 E+01 \\
1.27 E+01 \\
2.39 E+01 \\
3.04 E+01 \\
8.00 E+00 \\
2.61 E+01 \\
1.99 E+01 \\
7.29 E+00 \\
3.40 E+01 \\
3.44 E+00 \\
1.87 E+01 \\
1.05 E+01 \\
2.27 E+01 \\
8.97 E+00 \\
3.83 E+00 \\
7.32 E+00 \\
6.10 E+00 \\
6.19 E+00 \\
3.09 E+01\end{array}$ & $\begin{array}{c}\text { SB125 } \\
1.63 E+01 \\
4.47 E+01 \\
8.41 E+01 \\
8.73 E+00 \\
7.74 E+01 \\
1.63 E+01 \\
2.80 E+01 \\
1.48 E+01 \\
3.76 E+01 \\
2.09 E+01 \\
2.73 E+01 \\
5.05 E+01 \\
3.20 E+01 \\
9.13 E+00 \\
5.96 E+01 \\
1.04 E+02 \\
6.04 E+00 \\
1.17 E+01 \\
6.66 E+00 \\
5.92 E+00 \\
1.87 E+01 \\
1.12 E+02 \\
1.20 E+02 \\
1.46 E+01 \\
8.66 E+01 \\
1.28 E+01 \\
1.13 E+01 \\
1.83 E+01 \\
6.97 E+00 \\
1.01 E+02 \\
7.31 E+00 \\
2.22 E+01 \\
9.33 E+00 \\
6.51 E+01 \\
1.59 E+01 \\
3.27 E+01 \\
4.20 E+01 \\
2.52 E+01 \\
4.21 E+01 \\
7.35 E+00 \\
7.20 E+00 \\
1.58 E+02 \\
2.30 E+01 \\
4.46 E+01 \\
6.23 E+01 \\
2.51 E+01 \\
1.07 E+02 \\
3.15 E+01 \\
5.80 E+01 \\
7.58 E+01 \\
1.88 E+01 \\
6.18 E+01 \\
4.83 E+01 \\
1.65 E+01 \\
8.64 E+01 \\
7.94 E+00 \\
4.65 E+01 \\
2.56 E+01 \\
5.68 E+01 \\
2.27 E+01 \\
9.51 E+00 \\
1.76 E+01 \\
1.53 E+01 \\
1.53 E+01 \\
8.08 E+01\end{array}$ & $\begin{array}{l}\text { SN126 } \\
4.79 E-01 \\
1.17 E+00 \\
2.27 E+00 \\
2.28 E-01 \\
2.04 E+00 \\
4.81 E-01 \\
7.40 E-01 \\
4.13 E-01 \\
1.10 E+00 \\
6.25 E-01 \\
7.37 E-01 \\
1.37 E+00 \\
8.28 E-01 \\
2.45 E-01 \\
1.56 E+00 \\
2.67 E+00 \\
1.57 E-01 \\
3.09 E-01 \\
1.78 E-01 \\
2.40 E-01 \\
5.01 E-01 \\
2.84 E+00 \\
2.90 E+00 \\
3.72 E-01 \\
2.22 E+00 \\
3.40 E-01 \\
3.32 E-01 \\
4.35 E-01 \\
2.28 E-01 \\
2.80 E+00 \\
2.62 E-01 \\
7.64 E-01 \\
2.52 E-01 \\
1.67 E+00 \\
4.01 E-01 \\
8.50 E-01 \\
1.07 E+00 \\
6.83 E-01 \\
1.04 E+00 \\
1.80 E-01 \\
1.77 E-01 \\
3.40 E+00 \\
5.27 E-01 \\
1.03 E+00 \\
1.57 E+00 \\
.6 .46 E-01 \\
2.26 E+00 \\
7.54 E-01 \\
1.37 E+00 \\
1.72 E+00 \\
4.61 E-01 \\
1.49 E+00 \\
1.13 E+00 \\
4.49 E-01 \\
2.04 E+00 \\
2.11 E-01 \\
1.13 E+00 \\
6.39 E-01 \\
1.37 E+00 \\
5.43 E-01 \\
2.31 E-01 \\
4.47 E-01 \\
3.67 E-01 \\
3.74 E-01 \\
1.84 E+00\end{array}$ & $\begin{array}{l}1129 \\
2.11 \mathrm{E}-02 \\
5.12 \mathrm{E}-02 \\
9.96 \mathrm{E}-02 \\
1.01 \mathrm{E}-02 \\
9.01 \mathrm{E}-02 \\
2.12 \mathrm{E}-02 \\
3.25 \mathrm{E}-02 \\
1.81 \mathrm{E}-02 \\
4.83 \mathrm{E}-02 \\
2.75 \mathrm{E}-02 \\
3.19 \mathrm{E}-02 \\
5.95 \mathrm{E}-02 \\
3.62 \mathrm{E}-02 \\
1.06 \mathrm{E}-02 \\
6.42 \mathrm{E}-02 \\
1.10 \mathrm{E}-01 \\
6.91 \mathrm{E}-03 \\
1.36 \mathrm{E}-02 \\
7.82 \mathrm{E}-03 \\
1.05 \mathrm{E}-02 \\
2.17 \mathrm{E}-02 \\
1.17 \mathrm{E}-01 \\
1.19 \mathrm{E}-01 \\
1.53 \mathrm{E}-02 \\
9.12 \mathrm{E}-02 \\
1.40 \mathrm{E}-02 \\
1.37 \mathrm{E}-02 \\
1.79 \mathrm{E}-02 \\
1.01 \mathrm{E}-02 \\
1.24 \mathrm{E}-01 \\
1.15 \mathrm{E}-02 \\
3.37 \mathrm{E}-02 \\
1.11 \mathrm{E}-02 \\
7.29 \mathrm{E}-02 \\
1.75 \mathrm{E}-02 \\
3.75 \mathrm{E}-02 \\
4.74 \mathrm{E}-02 \\
3.01 \mathrm{E}-02 \\
4.54 \mathrm{E}-02 \\
7.87 \mathrm{E}-03 \\
7.75 \mathrm{E}-03 \\
1.46 \mathrm{E}-01 \\
2.17 \mathrm{E}-02 \\
4.22 \mathrm{E}-02 \\
6.44 \mathrm{E}-02 \\
2.66 \mathrm{E}-02 \\
9.74 \mathrm{E}-02 \\
3.26 \mathrm{E}-02 \\
5.62 \mathrm{E}-02 \\
7.07 \mathrm{E}-02 \\
1.90 \mathrm{E}-02 \\
6.14 \mathrm{E}-02 \\
4.66 \mathrm{E}-02 \\
1.98 \mathrm{E}-02 \\
8.98 \mathrm{E}-02 \\
9.33 \mathrm{E}-03 \\
4.97 \mathrm{E}-02 \\
2.82 \mathrm{E}-02 \\
6.03 \mathrm{E}-02 \\
2.39 \mathrm{E}-02 \\
1.02 \mathrm{E}-02 \\
1.96 \mathrm{E}-02 \\
1.61 \mathrm{E}-02 \\
1.64 \mathrm{E}-02 \\
8.11 \mathrm{E}-02\end{array}$ & $\begin{array}{l}C S 134 \\
1.07 E+00 \\
8.17 E+00 \\
7.71 E+00 \\
5.62 E-01 \\
5.13 E+00 \\
1.06 E+00 \\
2.95 E+00 \\
1.68 E+00 \\
3.84 E+00 \\
1.79 E+00 \\
6.28 E+00 \\
1.16 E+01 \\
5.88 E+00 \\
2.01 E+00 \\
5.85 E+00 \\
1.02 E+01 \\
5.44 E-01 \\
1.27 E+00 \\
5.03 E-01 \\
8.98 E-01 \\
4.31 E+00 \\
6.15 E+00 \\
6.40 E+00 \\
1.37 E+00 \\
1.04 E+01 \\
1.13 E+00 \\
9.38 E-01 \\
1.03 E+00 \\
3.99 E-01 \\
6.69 E+00 \\
4.46 E-01 \\
1.30 E+00 \\
6.42 E-01 \\
1.25 E+01 \\
2.97 E+00 \\
2.12 E+00 \\
2.73 E+00 \\
1.70 E+00 \\
7.53 E+00 \\
1.32 E+00 \\
1.24 E+00 \\
3.25 E+01 \\
2.89 E+00 \\
5.61 E+00 \\
7.93 E+00 \\
3.68 E+00 \\
2.21 E+01 \\
6.37 E+00 \\
6.98 E+00 \\
8.85 E+00 \\
2.31 E+00 \\
6.15 E+00 \\
3.68 E+00 \\
1.07 E+00 \\
5.87 E+00 \\
4.85 E-01 \\
2.93 E+00 \\
1.60 E+00 \\
9.50 E+00 \\
3.72 E+00 \\
6.40 E=01 \\
2.22 E+00 \\
1.67 E+00 \\
1.70 E+00 \\
5.69 E+00\end{array}$ & $\begin{array}{l}C 5137 \\
4.44 E+04 \\
1.02 E+05 \\
2.08 E+05 \\
2.15 E+04 \\
1.92 E+05 \\
4.47 E+04 \\
6.74 E+04 \\
3.70 E+04 \\
9.94 E+04 \\
5.71 E+04 \\
6.21 E+04 \\
1.16 E+05 \\
7.22 E+04 \\
2.08 E+04 \\
1.27 E+05 \\
2.18 E+05 \\
1.45 E+04 \\
2.81 E+04 \\
1.65 E+04 \\
2.02 E+04 \\
4.23 E+04 \\
2.40 E+05 \\
2.47 E+05 \\
3.04 E+04 \\
1.77 E+05 \\
2.79 E+04 \\
2.70 E+04 \\
3.70 E+04 \\
2.11 E+04 \\
2.61 E+05 \\
2.39 E+04 \\
7.00 E+04 \\
2.36 E+04 \\
1.45 E+05 \\
3.50 E+04 \\
8.00 E+04 \\
1.01 E+05 \\
6.39 E+04 \\
9.12 E+04 \\
1.58 E+04 \\
1.56 E+04 \\
2.47 E+05 \\
4.25 E+04 \\
8.26 E+04 \\
1.24 E+05 \\
4.98 E+04 \\
1.65 E+05 \\
5.39 E+04 \\
1.10 E+05 \\
1.40 E+05 \\
3.69 E+04 \\
1.23 E+05 \\
9.49 E+04 \\
4.21 E+04 \\
1.93 E+05 \\
1.99 E+04 \\
1.07 E+05 \\
6.05 E+04 \\
1.22 E+05 \\
4.85 E+04 \\
2.19 E+04 \\
4.00 E+04 \\
3.37 E+04 \\
3.42 E+04 \\
1.75 E+05\end{array}$ \\
\hline
\end{tabular}


ACTIVITY IN UNSEPARATED FUEL (Curies decayed to 1/1/94)

\begin{tabular}{|c|c|c|c|c|c|c|c|c|c|}
\hline $\begin{array}{r}\text { Listing } \\
716 \\
717 \\
718 \\
719 \\
720 \\
721 \\
722 \\
723 \\
724 \\
725 \\
726 \\
727 \\
728 \\
729 \\
730 \\
731 \\
732 \\
733 \\
734 \\
735 \\
736 \\
737 \\
738 \\
739 \\
740 \\
741 \\
742 \\
743 \\
744 \\
745 \\
746 \\
747 \\
748 \\
749 \\
750 \\
751 \\
752 \\
753 \\
754 \\
755 \\
756 \\
757 \\
758 \\
759 \\
760 \\
761 \\
762 \\
763 \\
764 \\
765 \\
766 \\
767 \\
768 \\
769 \\
770 \\
771 \\
772 \\
773 \\
774 \\
775 \\
776 \\
777 \\
778 \\
779 \\
780\end{array}$ & $\begin{array}{l}B A 137 \\
4.20 E+04 \\
9.64 E+04 \\
1.97 E+05 \\
2.03 E+04 \\
1.81 E+05 \\
4.22 E+04 \\
6.38 E+04 \\
3.50 E+04 \\
9.40 E+04 \\
5.40 E+04 \\
5.87 E+04 \\
1.10 E+05 \\
6.83 E+04 \\
1.97 E+04 \\
1.20 E+05 \\
2.06 E+05 \\
1.37 E+04 \\
2.66 E+04 \\
1.57 E+04 \\
1.91 E+04 \\
4.00 E+04 \\
2.27 E+05 \\
2.33 E+05 \\
2.88 E+04 \\
1.67 E+05 \\
2.64 E+04 \\
2.55 E+04 \\
3.50 E+04 \\
1.99 E+04 \\
2.47 E+05 \\
2.26 E+04 \\
6.62 E+04 \\
2.23 E+04 \\
1.37 E+05 \\
3.31 E+04 \\
7.57 E+04 \\
9.58 E+04 \\
6.04 E+04 \\
8.63 E+04 \\
1.50 E+04 \\
1.48 E+04 \\
2.33 E+05 \\
4.02 E+04 \\
7.81 E+04 \\
1.18 E+05 \\
4.71 E+04 \\
1.56 E+05 \\
5.10 E+04 \\
1.04 E+05 \\
1.33 E+05 \\
3.49 E+04 \\
1.16 E+05 \\
8.98 E+04 \\
3.98 E+04 \\
1.83 E+05 \\
1.88 E+04 \\
1.01 E+05 \\
5.72 E+04 \\
1.16 E+05 \\
4.58 E+04 \\
2.07 E+04 \\
3.79 E+04 \\
3.19 E+04 \\
3.24 E+04 \\
1.66 E+05\end{array}$ & $\begin{array}{l}54151 \\
1.26 \mathrm{E}+03 \\
1.94 \mathrm{E}+03 \\
5.34 \mathrm{E}+03 \\
6.14 \mathrm{E}+02 \\
5.44 \mathrm{E}+03 \\
1.27 \mathrm{E}+03 \\
1.63 \mathrm{E}+03 \\
8.54 \mathrm{E}+02 \\
2.42 \mathrm{E}+03 \\
1.49 \mathrm{E}+03 \\
1.03 \mathrm{E}+03 \\
1.93 \mathrm{E}+03 \\
1.37 \mathrm{E}+03 \\
3.56 \mathrm{E}+02 \\
3.11 \mathrm{E}+03 \\
5.32 \mathrm{E}+03 \\
3.76 \mathrm{E}+02 \\
6.70 \mathrm{E}+02 \\
4.53 \mathrm{E}+02 \\
4.02 \mathrm{E}+02 \\
7.04 \mathrm{E}+02 \\
7.07 \mathrm{E}+03 \\
7.33 \mathrm{E}+03 \\
7.60 \mathrm{E}+02 \\
3.85 \mathrm{E}+03 \\
7.13 \mathrm{E}+02 \\
7.02 \mathrm{E}+02 \\
1.09 \mathrm{E}+03 \\
6.16 \mathrm{E}+02 \\
7.40 \mathrm{E}+03 \\
6.86 \mathrm{E}+02 \\
2.04 \mathrm{E}+03 \\
6.62 \mathrm{E}+02 \\
2.69 \mathrm{E}+03 \\
6.61 \mathrm{E}+02 \\
2.29 \mathrm{E}+03 \\
2.89 \mathrm{E}+03 \\
1.81 \mathrm{E}+03 \\
1.77 \mathrm{E}+03 \\
3.07 \mathrm{E}+02 \\
3.09 \mathrm{E}+02 \\
2.02 \mathrm{E}+03 \\
9.13 \mathrm{E}+02 \\
1.78 \mathrm{E}+03 \\
2.59 \mathrm{E}+03 \\
8.82 \mathrm{E}+02 \\
1.35 \mathrm{E}+03 \\
3.95 \mathrm{E}+02 \\
2.44 \mathrm{E}+03 \\
3.19 \mathrm{E}+03 \\
7.97 \mathrm{E}+02 \\
3.00 \mathrm{E}+03 \\
2.58 \mathrm{E}+03 \\
1.20 \mathrm{E}+03 \\
5.46 \mathrm{E}+03 \\
5.76 \mathrm{0}+02 \\
3.07 \mathrm{E}+03 \\
1.74 \mathrm{E}+03 \\
2.45 \mathrm{E}+03 \\
9.85 \mathrm{E}+02 \\
6.20 \mathrm{E}+02 \\
8.69 \mathrm{E}+02 \\
8.08 \mathrm{0}+02 \\
8.12 \mathrm{E}+02 \\
4.91 \mathrm{E}+03\end{array}$ & $\begin{array}{l}E U 152 \\
6.12 E-01 \\
3.67 E+00 \\
4.07 E+00 \\
2.81 E-01 \\
2.60 E+00 \\
6.14 E-01 \\
1.56 E+00 \\
9.58 E-01 \\
2.27 E+00 \\
1.07 E+00 \\
2.77 E+00 \\
5.15 E+00 \\
2.60 E+00 \\
8.89 E-01 \\
2.83 E+00 \\
4.87 E+00 \\
2.76 E-01 \\
6.70 E-01 \\
2.60 E-01 \\
6.66 E-01 \\
1.88 E+00 \\
2.79 E+00 \\
2.73 E+00 \\
6.45 E-01 \\
5.07 E+00 \\
5.55 E-01 \\
5.19 E-01 \\
4.34 E-01 \\
2.57 E-01 \\
3.61 E+00 \\
3.20 E-01 \\
8.92 E-01 \\
3.35 E-01 \\
5.45 E+00 \\
1.28 E+00 \\
1.05 E+00 \\
1.33 E+00 \\
8.85 E-01 \\
3.16 E+00 \\
5.49 E-01 \\
5.19 E-01 \\
1.51 E+01 \\
1.24 E+00 \\
2.42 E+00 \\
3.83 E+00 \\
1.90 E+00 \\
1.01 E+01 \\
3.38 E+00 \\
3.06 E+00 \\
3.68 E+00 \\
1.07 E+00 \\
2.72 E+00 \\
1.56 E+00 \\
5.60 E-01 \\
2.61 E+00 \\
2.47 E-01 \\
1.34 E+00 \\
7.55 E-01 \\
3.92 E+00 \\
1.51 E+00 \\
2.95 E-01 \\
1.17 E+00 \\
7.92 E-01 \\
8.26 E-01 \\
2.44 E+00\end{array}$ & 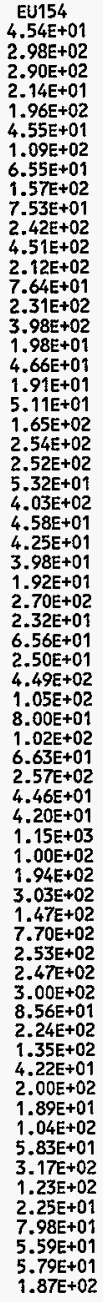 & $\begin{array}{c}E U 155 \\
5.27 E+01 \\
8.20 E+01 \\
2.12 E+02 \\
2.76 E+01 \\
2.42 E+02 \\
5.28 E+01 \\
5.92 E+01 \\
2.73 E+01 \\
8.32 E+01 \\
5.66 E+01 \\
4.52 E+01 \\
8.40 E+01 \\
5.84 E+01 \\
1.54 E+01 \\
1.27 E+02 \\
2.20 E+02 \\
1.54 E+01 \\
2.38 E+01 \\
1.94 E+01 \\
1.34 E+01 \\
3.09 E+01 \\
3.37 E+02 \\
3.62 E+02 \\
3.21 E+01 \\
1.36 E+02 \\
3.01 E+01 \\
2.82 E+01 \\
5.37 E+01 \\
2.48 E+01 \\
3.18 E+02 \\
2.59 E+01 \\
7.91 E+01 \\
2.88 E+01 \\
1.16 E+02 \\
2.85 E+01 \\
1.03 E+02 \\
1.31 E+02 \\
7.88 E+01 \\
7.69 E+01 \\
1.34 E+01 \\
1.34 E+01 \\
0.00 E+00 \\
3.37 E+01 \\
6.56 E+01 \\
8.57 E+01 \\
1.95 E+01 \\
0.00 E+00 \\
0.00 E+00 \\
9.33 E+01 \\
1.30 E+02 \\
2.84 E+01 \\
1.29 E+02 \\
1.22 E+02 \\
5.26 E+01 \\
2.59 E+02 \\
2.59 E+01 \\
1.45 E+02 \\
8.11 E+01 \\
1.07 E+02 \\
4.32 E+01 \\
2.89 E+01 \\
2.56 E+01 \\
3.07 E+01 \\
2.99 E+01 \\
2.35 E+02\end{array}$ & $\begin{array}{l}\text { RA226 } \\
1.86 E-05 \\
1.74 E-05 \\
6.34 E-05 \\
9.03 E-06 \\
7.85 E-05 \\
1.87 E-05 \\
1.76 E-05 \\
8.95 E-06 \\
2.71 E-05 \\
1.86 E-05 \\
8.28 E-06 \\
1.55 E-05 \\
1.23 E-05 \\
2.92 E-06 \\
2.56 E-05 \\
4.36 E-05 \\
4.48 E-06 \\
7.15 E-06 \\
6.02 E-06 \\
4.24 E-06 \\
5.62 E-06 \\
8.24 E-05 \\
8.69 E-05 \\
6.36 E-06 \\
2.84 E-05 \\
6.23 E-06 \\
6.42 E-06 \\
1.24 E-05 \\
1.00 E-05 \\
1.07 E-04 \\
1.08 E-05 \\
3.27 E-05 \\
9.35 E-06 \\
2.34 E-05 \\
5.83 E-06 \\
3.34 E-05 \\
4.21 E-05 \\
2.59 E-05 \\
1.59 E-05 \\
2.75 E-06 \\
2.84 E-06 \\
1.87 E-05 \\
6.47 E-06 \\
1.26 E-05 \\
1.86 E-05 \\
6.18 E-06 \\
1.24 E-05 \\
4.12 E-06 \\
1.78 E-05 \\
2.34 E-05 \\
5.77 E-06 \\
2.42 E-05 \\
2.41 E-05 \\
1.77 E-05 \\
7.66 E-05 \\
8.78 E-06 \\
4.54 E-05 \\
2.60 E-05 \\
2.28 E-05 \\
9.23 E-06 \\
8.77 E-06 \\
8.47 E-06 \\
8.45 E-06 \\
8.44 E-06 \\
6.69 E-05\end{array}$ & $\begin{array}{l}\text { RA228 } \\
8.01 \mathrm{E}-10 \\
1.13 \mathrm{E}-09 \\
2.96 \mathrm{E}-09 \\
3.89 \mathrm{E}-10 \\
3.40 \mathrm{E}-09 \\
8.08 \mathrm{E}-10 \\
8.58 \mathrm{E}-10 \\
4.47 \mathrm{E}-10 \\
1.30 \mathrm{E}-09 \\
8.54 \mathrm{E}-10 \\
6.05 \mathrm{E}-10 \\
1.13 \mathrm{E}-09 \\
8.00 \mathrm{E}-10 \\
2.08 \mathrm{E}-10 \\
4.76 \mathrm{E}-10 \\
8.12 \mathrm{E}-10 \\
2.08 \mathrm{E}-10 \\
3.51 \mathrm{E}-10 \\
2.68 \mathrm{E}-10 \\
2.62 \mathrm{E}-10 \\
4.11 \mathrm{E}-10 \\
9.44 \mathrm{E}-10 \\
9.60 \mathrm{E}-10 \\
1.14 \mathrm{E}-10 \\
6.40 \mathrm{E}-10 \\
1.06 \mathrm{E}-10 \\
1.07 \mathrm{E}-10 \\
1.43 \mathrm{E}-10 \\
4.21 \mathrm{E}-10 \\
4.65 \mathrm{E}-09 \\
4.58 \mathrm{E}-10 \\
1.38 \mathrm{E}-09 \\
4.08 \mathrm{E}-10 \\
1.56 \mathrm{E}-09 \\
3.83 \mathrm{E}-10 \\
1.44 \mathrm{E}-09 \\
1.82 \mathrm{E}-09 \\
1.13 \mathrm{E}-09 \\
1.02 \mathrm{E}-09 \\
1.77 \mathrm{E}-10 \\
1.79 \mathrm{E}-10 \\
7.27 \mathrm{E}-10 \\
1.48 \mathrm{E}-10 \\
2.89 \mathrm{E}-10 \\
4.44 \mathrm{E}-10 \\
1.73 \mathrm{E}-10 \\
4.83 \mathrm{E}-10 \\
1.62 \mathrm{E}-10 \\
3.91 \mathrm{E}-10 \\
4.94 \mathrm{E}-10 \\
1.32 \mathrm{E}-10 \\
4.49 \mathrm{E}-10 \\
3.56 \mathrm{E}-10 \\
7.62 \mathrm{E}-10 \\
3.34 \mathrm{E}-09 \\
3.74 \mathrm{E}-10 \\
1.95 \mathrm{E}-09 \\
1.11 \mathrm{E}-09 \\
1.42 \mathrm{E}-09 \\
5.70 \mathrm{E}-10 \\
3.82 \mathrm{E}-10 \\
4.40 \mathrm{E}-10 \\
4.15 \mathrm{E}-10 \\
4.17 \mathrm{E}-10 \\
2.95 \mathrm{E}-09\end{array}$ & 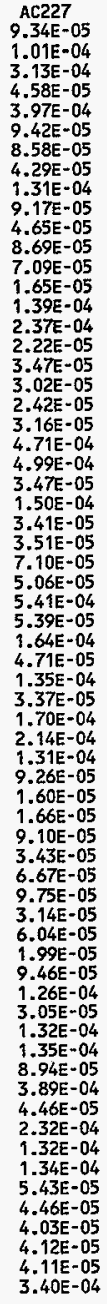 & $\begin{array}{l}\text { TH229 } \\
4.75 E-08 \\
1.18 E-07 \\
2.17 E-07 \\
2.22 E-08 \\
1.98 E-07 \\
4.78 E-08 \\
7.08 E-08 \\
4.02 E-08 \\
1.08 E-07 \\
6.13 E-08 \\
7.43 E-08 \\
1.39 E-07 \\
8.34 E-08 \\
2.47 E-08 \\
1.01 E-07 \\
1.72 E-07 \\
1.50 E-08 \\
2.96 E-08 \\
1.72 E-08 \\
2.66 E-08 \\
5.05 E-08 \\
1.91 E-07 \\
1.94 E-07 \\
2.40 E-08 \\
1.41 E-07 \\
2.23 E-08 \\
2.23 E-08 \\
2.90 E-08 \\
2.32 E-08 \\
2.75 E-07 \\
2.70 E-08 \\
7.84 E-08 \\
2.46 E-08 \\
1.68 E-07 \\
4.01 E-08 \\
8.25 E-08 \\
1.04 E-07 \\
6.66 E-08 \\
1.03 E-07 \\
1.79 E-08 \\
1.76 E-08 \\
2.08 E-07 \\
3.29 E-08 \\
6.40 E-08 \\
9.95 E-08 \\
4.12 E-08 \\
1.38 E-07 \\
4.72 E-08 \\
8.56 E-08 \\
1.07 E-07 \\
2.91 E-08 \\
9.52 E-08 \\
7.36 E-08 \\
4.40 E-08 \\
1.93 E-07 \\
2.07 E-08 \\
1.08 E-07 \\
6.16 E-08 \\
1.37 E-07 \\
5.39 E-08 \\
2.21 E-08 \\
4.31 E-08 \\
3.44 E-08 \\
3.53 E-08 \\
1.73 E-07\end{array}$ \\
\hline
\end{tabular}


HNF-SD-WH-TI-794, Rev, O

ACTIVITY IN UNSEPARATED FUEL (Curies decayed to 1/1/94)

\begin{tabular}{|c|c|c|c|c|c|c|c|c|c|}
\hline $\begin{array}{r}\text { [Listing } 7] \\
716 \\
717 \\
718 \\
719 \\
720 \\
721 \\
722 \\
723 \\
724 \\
725 \\
726 \\
727 \\
728 \\
729 \\
730 \\
731 \\
732 \\
733 \\
734 \\
735 \\
736 \\
737 \\
738 \\
739 \\
740 \\
741 \\
742 \\
743 \\
744 \\
745 \\
746 \\
747 \\
748 \\
749 \\
750 \\
751 \\
752 \\
753 \\
754 \\
755 \\
756 \\
757 \\
758 \\
759 \\
760 \\
761 \\
762 \\
763 \\
764 \\
765 \\
766 \\
767 \\
768 \\
769 \\
770 \\
771 \\
772 \\
773 \\
774 \\
775 \\
776 \\
7777 \\
778 \\
779 \\
780\end{array}$ & $\begin{array}{l}1 H 232 \\
1.22 \mathrm{E}-09 \\
1.73 \mathrm{E}-09 \\
4.51 \mathrm{E}-09 \\
5.95 \mathrm{E}-10 \\
5.20 \mathrm{E}-09 \\
1.23 \mathrm{E}-09 \\
1.31 \mathrm{E}-09 \\
6.78 \mathrm{E}-10 \\
1.98 \mathrm{E}-09 \\
1.29 \mathrm{E}-09 \\
9.19 \mathrm{E}-10 \\
1.72 \mathrm{E}-09 \\
1.22 \mathrm{E}-09 \\
3.16 \mathrm{E}-10 \\
7.28 \mathrm{E}-10 \\
1.24 \mathrm{E}-09 \\
3.18 \mathrm{E}-10 \\
5.35 \mathrm{E}-10 \\
4.09 \mathrm{E}-10 \\
3.89 \mathrm{E}-10 \\
6.24 \mathrm{E}-10 \\
1.45 \mathrm{E}-09 \\
1.47 \mathrm{E}-09 \\
1.74 \mathrm{E}-10 \\
9.78 \mathrm{E}-10 \\
1.62 \mathrm{E}-10 \\
1.62 \mathrm{E}-10 \\
2.20 \mathrm{E}-10 \\
6.34 \mathrm{E}-10 \\
7.08 \mathrm{E}-09 \\
6.86 \mathrm{E}-10 \\
2.07 \mathrm{E}-09 \\
6.23 \mathrm{E}-10 \\
2.38 \mathrm{E}-09 \\
5.85 \mathrm{E}-10 \\
2.21 \mathrm{E}-09 \\
2.78 \mathrm{E}-09 \\
1.72 \mathrm{E}-09 \\
1.56 \mathrm{E}-09 \\
2.71 \mathrm{E}-10 \\
2.74 \mathrm{E}-10 \\
1.13 \mathrm{E}-09 \\
2.28 \mathrm{E}-10 \\
4.44 \mathrm{E}-10 \\
6.78 \mathrm{E}-10 \\
2.64 \mathrm{E}-10 \\
7.38 \mathrm{E}-10 \\
2.45 \mathrm{E}-10 \\
6.01 \mathrm{E}-10 \\
7.61 \mathrm{E}-10 \\
2.02 \mathrm{E}-10 \\
6.90 \mathrm{E}-10 \\
5.47 \mathrm{E}-10 \\
1.16 \mathrm{E}-09 \\
5.15 \mathrm{E}-09 \\
5.72 \mathrm{E}-10 \\
3.00 \mathrm{E}-09 \\
1.71 \mathrm{E}-09 \\
2.18 \mathrm{E}-09 \\
8.75 \mathrm{E}-10 \\
5.87 \mathrm{E}-10 \\
6.71 \mathrm{E}-10 \\
6.37 \mathrm{E}-10 \\
6.39 \mathrm{E}-10 \\
4.55 \mathrm{E}-09\end{array}$ & $\begin{array}{l}\text { PA231 } \\
2.87 \mathrm{E}-04 \\
3.08 \mathrm{E}-04 \\
9.66 \mathrm{E}-04 \\
1.43 \mathrm{E}-04 \\
1.23 \mathrm{E}-03 \\
2.89 \mathrm{E}-04 \\
2.65 \mathrm{E}-04 \\
1.31 \mathrm{E}-04 \\
4.00 \mathrm{E}-04 \\
2.80 \mathrm{E}-04 \\
1.41 \mathrm{E}-04 \\
2.63 \mathrm{E}-04 \\
2.18 \mathrm{E}-04 \\
5.00 \mathrm{E}-05 \\
4.29 \mathrm{E}-04 \\
7.32 \mathrm{E}-04 \\
6.89 \mathrm{E}-05 \\
1.07 \mathrm{E}-04 \\
9.36 \mathrm{E}-05 \\
7.07 \mathrm{E}-05 \\
9.56 \mathrm{E}-05 \\
1.47 \mathrm{E}-03 \\
1.56 \mathrm{E}-03 \\
1.08 \mathrm{E}-04 \\
4.61 \mathrm{E}-04 \\
1.05 \mathrm{E}-04 \\
1.07 \mathrm{E}-04 \\
2.23 \mathrm{E}-04 \\
1.53 \mathrm{E}-04 \\
1.67 \mathrm{E}-03 \\
1.62 \mathrm{E}-04 \\
4.95 \mathrm{E}-04 \\
1.46 \mathrm{E}-04 \\
4.14 \mathrm{E}-04 \\
1.04 \mathrm{E}-04 \\
5.28 \mathrm{E}-04 \\
6.66 \mathrm{E}-04 \\
4.05 \mathrm{E}-04 \\
2.86 \mathrm{E}-04 \\
4.96 \mathrm{E}-05 \\
5.13 \mathrm{E}-05 \\
2.71 \mathrm{E}-04 \\
1.07 \mathrm{E}-04 \\
2.08 \mathrm{E}-04 \\
3.00 \mathrm{E}-04 \\
9.54 \mathrm{E}-05 \\
1.80 \mathrm{E}-04 \\
5.84 \mathrm{E}-05 \\
2.94 \mathrm{E}-04 \\
3.94 \mathrm{E}-04 \\
9.43 \mathrm{E}-05 \\
4.12 \mathrm{E}-04 \\
4.24 \mathrm{E}-04 \\
2.77 \mathrm{E}-04 \\
1.23 \mathrm{E}-03 \\
1.39 \mathrm{E}-04 \\
7.28 \mathrm{E}-04 \\
4.15 \mathrm{E}-04 \\
4.15 \mathrm{E}-04 \\
1.69 \mathrm{E}-04 \\
1.40 \mathrm{E}-04 \\
1.24 \mathrm{E}-04 \\
1.29 \mathrm{E}-04 \\
1.28 \mathrm{E}-04 \\
1.08 \mathrm{E}-03\end{array}$ & $\begin{array}{l}\text { U232 } \\
9.85 \mathrm{E}-04 \\
4.63 \mathrm{E}-03 \\
5.41 \mathrm{E}-03 \\
4.60 \mathrm{E}-04 \\
4.18 \mathrm{E}-03 \\
9.91 \mathrm{E}-04 \\
1.92 \mathrm{E}-03 \\
1.13 \mathrm{E}-03 \\
2.84 \mathrm{E}-03 \\
1.46 \mathrm{E}-03 \\
3.64 \mathrm{E}-03 \\
6.79 \mathrm{E}-03 \\
3.28 \mathrm{E}-03 \\
1.15 \mathrm{E}-03 \\
2.60 \mathrm{E}-03 \\
4.46 \mathrm{E}-03 \\
3.70 \mathrm{E}-04 \\
8.13 \mathrm{E}-04 \\
3.85 \mathrm{E}-04 \\
9.06 \mathrm{E}-04 \\
2.48 \mathrm{E}-03 \\
3.75 \mathrm{E}-03 \\
3.77 \mathrm{E}-03 \\
6.07 \mathrm{E}-04 \\
4.13 \mathrm{E}-03 \\
5.42 \mathrm{E}-04 \\
5.22 \mathrm{E}-04 \\
5.77 \mathrm{E}-04 \\
4.49 \mathrm{E}-04 \\
5.77 \mathrm{E}-03 \\
5.34 \mathrm{E}-04 \\
1.53 \mathrm{E}-03 \\
5.25 \mathrm{E}-04 \\
6.87 \mathrm{E}-03 \\
1.60 \mathrm{E}-03 \\
1.72 \mathrm{E}-03 \\
2.17 \mathrm{E}-03 \\
1.41 \mathrm{E}-03 \\
3.95 \mathrm{E}-03 \\
6.86 \mathrm{E}-04 \\
6.51 \mathrm{E}-04 \\
9.34 \mathrm{E}-03 \\
9.91 \mathrm{E}-04 \\
1.93 \mathrm{E}-03 \\
3.02 \mathrm{E}-03 \\
1.39 \mathrm{E}-03 \\
6.23 \mathrm{E}-03 \\
2.11 \mathrm{E}-03 \\
2.51 \mathrm{E}-03 \\
3.07 \mathrm{E}-03 \\
8.65 \mathrm{E}-04 \\
2.48 \mathrm{E}-03 \\
1.68 \mathrm{E}-03 \\
9.12 \mathrm{E}-04 \\
4.15 \mathrm{E}-03 \\
4.18 \mathrm{E}-04 \\
2.23 \mathrm{E}-03 \\
1.27 \mathrm{E}-03 \\
4.94 \mathrm{E}-03 \\
1.91 \mathrm{E}-03 \\
4.71 \mathrm{E}-04 \\
1.30 \mathrm{E}-03 \\
9.56 \mathrm{E}-04 \\
9.88 \mathrm{E}-04 \\
3.80 \mathrm{E}-03\end{array}$ & $\begin{array}{l}\text { U233 } \\
2.57 E-05 \\
6.61 E-05 \\
1.19 E-04 \\
1.22 E-05 \\
1.09 E-04 \\
2.59 E-05 \\
3.90 E-05 \\
2.21 E-05 \\
5.85 E-05 \\
3.31 E-05 \\
4.14 E-05 \\
7.72 E-05 \\
4.67 E-05 \\
1.38 E-05 \\
5.23 E-05 \\
8.94 E-05 \\
8.22 E-06 \\
1.63 E-05 \\
9.40 E-06 \\
1.41 E-05 \\
2.81 E-05 \\
9.87 E-05 \\
1.01 E-04 \\
1.25 E-05 \\
7.40 E-05 \\
1.15 E-05 \\
1.13 E-05 \\
1.51 E-05 \\
1.24 E-05 \\
1.50 E-04 \\
1.43 E-05 \\
4.16 E-05 \\
1.34 E-05 \\
9.40 E-05 \\
2.26 E-05 \\
4.53 E-05 \\
5.72 E-05 \\
3.64 E-05 \\
5.82 E-05 \\
1.01 E-05 \\
9.94 E-06 \\
1.17 E-04 \\
1.75 E-05 \\
3.39 E-05 \\
5.24 E-05 \\
2.19 E-05 \\
7.78 E-05 \\
2.63 E-05 \\
4.52 E-05 \\
5.66 E-05 \\
1.53 E-05 \\
4.98 E-05 \\
3.85 E-05 \\
2.40 E-05 \\
1.07 E-04 \\
1.13 E-05 \\
5.98 E-05 \\
3.40 E-05 \\
7.73 E-05 \\
3.05 E-05 \\
1.22 E-05 \\
2.40 E-05 \\
1.92 E-05 \\
1.96 E-05 \\
9.68 E-05\end{array}$ & $\begin{array}{l}\text { U234 } \\
1.36 E+01 \\
1.29 E+01 \\
4.74 E+01 \\
6.84 E+00 \\
5.93 E+01 \\
1.37 E+01 \\
1.32 E+01 \\
6.54 E+00 \\
1.96 E+01 \\
1.35 E+01 \\
6.05 E+00 \\
1.13 E+01 \\
9.12 E+00 \\
2.14 E+00 \\
1.91 E+01 \\
3.27 E+01 \\
3.39 E+00 \\
5.34 E+00 \\
4.53 E+00 \\
2.77 E+00 \\
4.11 E+00 \\
6.24 E+01 \\
6.69 E+01 \\
4.80 E+00 \\
2.12 E+01 \\
4.64 E+00 \\
4.64 E+00 \\
9.58 E+00 \\
7.10 E+00 \\
7.96 E+01 \\
7.44 E+00 \\
2.28 E+01 \\
7.01 E+00 \\
1.75 E+01 \\
4.37 E+00 \\
2.54 E+01 \\
3.21 E+01 \\
1.94 E+01 \\
1.20 E+01 \\
2.09 E+00 \\
2.15 E+00 \\
1.38 E+01 \\
4.98 E+00 \\
9.68 E+00 \\
1.39 E+01 \\
4.51 E+00 \\
9.21 E+00 \\
2.94 E+00 \\
1.36 E+01 \\
1.82 E+01 \\
4.36 E+00 \\
1.86 E+01 \\
1.87 E+01 \\
1.32 E+01 \\
5.99 E+01 \\
6.61 E+00 \\
3.52 E+01 \\
2.00 E+01 \\
1.73 E+01 \\
7.06 E+00 \\
6.79 E+00 \\
6.32 E+00 \\
6.51 E+00 \\
6.45 E+00 \\
5.29 E+01\end{array}$ & $\begin{array}{l}\text { U235 } \\
5.09 \mathrm{E}-01 \\
5.46 \mathrm{E}-01 \\
1.73 \mathrm{E}+00 \\
2.58 \mathrm{E}-01 \\
2.23 \mathrm{E}+00 \\
5.12 \mathrm{E}-01 \\
4.73 \mathrm{E}-01 \\
2.31 \mathrm{E}-01 \\
7.04 \mathrm{E}-01 \\
4.94 \mathrm{E}-01 \\
2.45 \mathrm{E}-01 \\
4.57 \mathrm{E}-01 \\
3.86 \mathrm{E}-01 \\
8.75 \mathrm{E}-02 \\
7.68 \mathrm{E}-01 \\
1.31 \mathrm{E}+00 \\
1.24 \mathrm{E}-01 \\
1.91 \mathrm{E}-01 \\
1.68 \mathrm{E}-01 \\
1.18 \mathrm{E}-01 \\
1.67 \mathrm{E}-01 \\
2.66 \mathrm{E}+00 \\
2.86 \mathrm{E}+00 \\
1.94 \mathrm{E}-01 \\
8.21 \mathrm{E}-01 \\
1.89 \mathrm{E}-01 \\
1.89 \mathrm{E}-01 \\
4.08 \mathrm{E}-01 \\
2.68 \mathrm{E}-01 \\
2.99 \mathrm{E}+00 \\
2.79 \mathrm{E}-01 \\
8.59 \mathrm{E}-01 \\
2.63 \mathrm{E}-01 \\
7.34 \mathrm{E}-01 \\
1.85 \mathrm{E}-01 \\
9.57 \mathrm{E}-01 \\
1.21 \mathrm{E}+00 \\
7.29 \mathrm{E}-01 \\
5.12 \mathrm{E}-01 \\
8.88 \mathrm{E}-02 \\
9.21 \mathrm{E}-02 \\
4.61 \mathrm{E}-01 \\
1.93 \mathrm{E}-01 \\
3.75 \mathrm{E}-01 \\
5.32 \mathrm{E}-01 \\
1.66 \mathrm{E}-01 \\
3.08 \mathrm{E}-01 \\
9.74 \mathrm{E}-02 \\
5.31 \mathrm{E}-01 \\
7.17 \mathrm{E}-01 \\
1.69 \mathrm{E}-01 \\
7.46 \mathrm{E}-01 \\
7.75 \mathrm{E}-01 \\
4.98 \mathrm{E}-01 \\
2.25 \mathrm{E}+00 \\
2.50 \mathrm{E}-01 \\
1.33 \mathrm{E}+00 \\
7.56 \mathrm{E}-01 \\
7.46 \mathrm{E}-01 \\
3.05 \mathrm{E}-01 \\
2.56 \mathrm{E}-01 \\
2.20 \mathrm{E}-01 \\
2\end{array}$ & $\begin{array}{l}\text { U236 } \\
9.30 \mathrm{E}-01 \\
1.33 \mathrm{E}+00 \\
3.49 \mathrm{E}+00 \\
4.63 \mathrm{E}-01 \\
4.04 \mathrm{E}+00 \\
9.35 \mathrm{E}-01 \\
1.01 \mathrm{E}+00 \\
5.19 \mathrm{E}-01 \\
1.51 \mathrm{E}+00 \\
9.86 \mathrm{E}-01 \\
7.05 \mathrm{E}-01 \\
1.32 \mathrm{E}+00 \\
9.44 \mathrm{E}-01 \\
2.43 \mathrm{E}-01 \\
5.66 \mathrm{E}-01 \\
9.68 \mathrm{E}-01 \\
2.48 \mathrm{E}-01 \\
4.14 \mathrm{E}-01 \\
3.18 \mathrm{E}-01 \\
2.81 \mathrm{E}-01 \\
4.80 \mathrm{E}-01 \\
1.13 \mathrm{E}+00 \\
1.16 \mathrm{E}+00 \\
1.36 \mathrm{E}-01 \\
7.60 \mathrm{E}-01 \\
1.26 \mathrm{E}-01 \\
1.24 \mathrm{E}-01 \\
1.73 \mathrm{E}-01 \\
4.77 \mathrm{E}-01 \\
5.46 \mathrm{E}+00 \\
5.10 \mathrm{E}-01 \\
1.55 \mathrm{E}+00 \\
4.82 \mathrm{E}-01 \\
1.85 \mathrm{E}+00 \\
4.54 \mathrm{E}-01 \\
1.72 \mathrm{E}+00 \\
2.17 \mathrm{E}+00 \\
1.33 \mathrm{E}+00 \\
1.22 \mathrm{E}+00 \\
2.11 \mathrm{E}-01 \\
2.14 \mathrm{E}-01 \\
8.60 \mathrm{E}-01 \\
1.80 \mathrm{E}-01 \\
3.51 \mathrm{E}-01 \\
5.27 \mathrm{E}-01 \\
2.03 \mathrm{E}-01 \\
5.73 \mathrm{E}-01 \\
1.87 \mathrm{E}-01 \\
4.73 \mathrm{E}-01 \\
6.04 \mathrm{E}-01 \\
1.58 \mathrm{E}-01 \\
5.43 \mathrm{E}-01 \\
4.33 \mathrm{E}-01 \\
8.98 \mathrm{E}-01 \\
4.07 \mathrm{E}+00 \\
4.44 \mathrm{E}-01 \\
2.36 \mathrm{E}+00 \\
1.34 \mathrm{E}+00 \\
1.70 \mathrm{E}+00 \\
6.85 \mathrm{E}-01 \\
4.62 \mathrm{E}-01 \\
5.19 \mathrm{E}-01 \\
5.00 \mathrm{E}-01\end{array}$ & $\begin{array}{l}\text { U238 } \\
9.21 E+00 \\
8.94 E+00 \\
3.24 E+01 \\
4.64 E+00 \\
4.02 E+01 \\
9.26 E+00 \\
9.04 E+00 \\
4.49 E+00 \\
1.35 E+01 \\
9.20 E+00 \\
4.25 E+00 \\
7.92 E+00 \\
6.33 E+00 \\
1.50 E+00 \\
1.99 E+01 \\
3.40 E+01 \\
2.31 E+00 \\
3.66 E+00 \\
3.08 E+00 \\
1.92 E+00 \\
2.89 E+00 \\
6.40 E+01 \\
6.85 E+01 \\
4.97 E+00 \\
2.21 E+01 \\
4.80 E+00 \\
4.80 E+00 \\
9.82 E+00 \\
4.81 E+00 \\
5.40 E+01 \\
5.05 E+00 \\
1.55 E+01 \\
4.76 E+00 \\
1.21 E+01 \\
3.03 E+00 \\
1.72 E+01 \\
2.17 E+01 \\
1.32 E+01 \\
8.32 E+00 \\
1.44 E+00 \\
1.49 E+00 \\
1.49 E+01 \\
5.21 E+00 \\
1.03 E+01 \\
1.45 E+01 \\
4.76 E+00 \\
9.96 E+00 \\
3.18 E+00 \\
1.42 E+01 \\
1.90 E+01 \\
4.55 E+00 \\
1.93 E+01 \\
1.93 E+01 \\
8.96 E+00 \\
4.06 E+01 \\
4.48 E+00 \\
2.39 E+01 \\
1.35 E+01 \\
1.20 E+01 \\
4.87 E+00 \\
4.61 E+00 \\
4.36 E+00 \\
4.46 E+00 \\
4.42 E+00 \\
3.59 E+01\end{array}$ & $\begin{array}{l}\text { NP237 } \\
1.22 \mathrm{E}-01 \\
3.54 \mathrm{E}-01 \\
5.77 \mathrm{E}-01 \\
5.80 \mathrm{E}-02 \\
5.20 \mathrm{E}-01 \\
1.23 \mathrm{E}-01 \\
1.95 \mathrm{E}-01 \\
1.12 \mathrm{E}-01 \\
2.90 \mathrm{E}-01 \\
1.59 \mathrm{E}-01 \\
2.29 \mathrm{E}-01 \\
4.28 \mathrm{E}-01 \\
2.51 \mathrm{E}-01 \\
7.58 \mathrm{E}-02 \\
2.21 \mathrm{E}-01 \\
3.79 \mathrm{E}-01 \\
4.00 \mathrm{E}-02 \\
8.19 \mathrm{E}-02 \\
4.52 \mathrm{E}-02 \\
7.27 \mathrm{E}-02 \\
1.56 \mathrm{E}-01 \\
3.89 \mathrm{E}-01 \\
3.97 \mathrm{E}-01 \\
5.25 \mathrm{E}-02 \\
3.32 \mathrm{E}-01 \\
4.79 \mathrm{E}-02 \\
4.68 \mathrm{E}-02 \\
5.97 \mathrm{E}-02 \\
5.81 \mathrm{E}-02 \\
7.13 \mathrm{E}-01 \\
6.66 \mathrm{E}-02 \\
1.94 \mathrm{E}-01 \\
6.41 \mathrm{E}-02 \\
5.08 \mathrm{E}-01 \\
1.22 \mathrm{E}-01 \\
2.16 \mathrm{E}-01 \\
2.73 \mathrm{E}-01 \\
1.74 \mathrm{E}-01 \\
3.12 \mathrm{E}-01 \\
5.41 \mathrm{E}-02 \\
5.30 \mathrm{E}-02 \\
6.86 \mathrm{E}-01 \\
7.92 \mathrm{E}-02 \\
1.54 \mathrm{E}-01 \\
2.41 \mathrm{E}-01 \\
1.08 \mathrm{E}-01 \\
4.58 \mathrm{E}-01 \\
1.55 \mathrm{E}-01 \\
2.02 \mathrm{E}-01 \\
2.49 \mathrm{E}-01 \\
6.93 \mathrm{E}-02 \\
2.11 \mathrm{E}-01 \\
1.58 \mathrm{E}-01 \\
1.14 \mathrm{E}-01 \\
5.18 \mathrm{E}-01 \\
5.38 \mathrm{E}-02 \\
2.86 \mathrm{E}-01 \\
1.62 \mathrm{E}-01 \\
4.11 \mathrm{E}-01 \\
1.62 \mathrm{E}-01 \\
5.88 \mathrm{E}-02 \\
1.26 \mathrm{E}-01 \\
9.66 \mathrm{E}-02 \\
9.93 \mathrm{E}-02 \\
4.68 \mathrm{E}-01\end{array}$ \\
\hline
\end{tabular}


ACTIVITY IN UNSEPARATED FUEL (Curies decayed to 1/1/94)

\begin{tabular}{|c|c|c|c|c|c|c|c|c|c|}
\hline $\begin{array}{r}\text { isting } \# 1 \\
716 \\
717 \\
718 \\
719 \\
720 \\
721 \\
722 \\
723 \\
724 \\
725 \\
726 \\
727 \\
728 \\
729 \\
730 \\
731 \\
732 \\
733 \\
734 \\
735 \\
736 \\
737 \\
738 \\
739 \\
740 \\
741 \\
742 \\
743 \\
744 \\
745 \\
746 \\
747 \\
748 \\
749 \\
750 \\
751 \\
752 \\
753 \\
754 \\
755 \\
756 \\
757 \\
758 \\
759 \\
760 \\
761 \\
762 \\
763 \\
764 \\
765 \\
766 \\
767 \\
768 \\
769 \\
770 \\
771 \\
772 \\
773 \\
774 \\
775 \\
776 \\
777 \\
778 \\
779 \\
780\end{array}$ & $\begin{array}{l}\text { PU238 } \\
4.12 \mathrm{E}+01 \\
3.30 \mathrm{E}+02 \\
2.62 \mathrm{E}+02 \\
1.87 \mathrm{E}+01 \\
1.73 \mathrm{E}+02 \\
4.15 \mathrm{E}+01 \\
9.92 \mathrm{E}+01 \\
6.12 \mathrm{E}+01 \\
1.47 \mathrm{E}+02 \\
7.03 \mathrm{E}+01 \\
2.80 \mathrm{E}+02 \\
5.23 \mathrm{E}+02 \\
2.33 \mathrm{E}+02 \\
8.76 \mathrm{E}+01 \\
1.20 \mathrm{E}+02 \\
2.05 \mathrm{E}+02 \\
1.77 \mathrm{E}+01 \\
4.26 \mathrm{E}+01 \\
1.70 \mathrm{E}+01 \\
6.38 \mathrm{E}+01 \\
1.91 \mathrm{E}+02 \\
1.23 \mathrm{E}+02 \\
1.20 \mathrm{E}+02 \\
2.72 \mathrm{E}+01 \\
2.11 \mathrm{E}+02 \\
2.36 \mathrm{E}+01 \\
2.25 \mathrm{E}+01 \\
1.90 \mathrm{E}+01 \\
1.78 \mathrm{E}+01 \\
2.41 \mathrm{E}+02 \\
2.24 \mathrm{E}+01 \\
6.22 \mathrm{E}+01 \\
2.22 \mathrm{E}+01 \\
4.96 \mathrm{E}+02 \\
1.15 \mathrm{E}+02 \\
6.98 \mathrm{E}+01 \\
8.83 \mathrm{E}+01 \\
5.88 \mathrm{E}+01 \\
2.77 \mathrm{E}+02 \\
4.81 \mathrm{E}+01 \\
4.50 \mathrm{E}+01 \\
6.10 \mathrm{E}+02 \\
5.08 \mathrm{E}+01 \\
9.88 \mathrm{E}+01 \\
1.59 \mathrm{E}+02 \\
7.90 \mathrm{E}+01 \\
4.07 \mathrm{E}+02 \\
1.39 \mathrm{E}+02 \\
1.26 \mathrm{E}+02 \\
1.50 \mathrm{E}+02 \\
4.43 \mathrm{E}+01 \\
1.13 \mathrm{E}+02 \\
6.59 \mathrm{E}+01 \\
3.74 \mathrm{E}+01 \\
1.70 \mathrm{E}+02 \\
1.66 \mathrm{E}+01 \\
8.84 \mathrm{E}+01 \\
5.01 \mathrm{E}+01 \\
3.37 \mathrm{E}+02 \\
1.29 \mathrm{E}+02 \\
1.93 \mathrm{E}+01 \\
7.33 \mathrm{E}+01 \\
4.96 \mathrm{E}+01 \\
5.18 \mathrm{E}+01 \\
1.58 \mathrm{E}+02\end{array}$ & $\begin{array}{c}P U 239 \\
1.06 E+03 \\
2.18 E+03 \\
4.70 E+03 \\
5.09 E+02 \\
4.53 E+03 \\
1.06 E+03 \\
1.50 E+03 \\
8.22 E+02 \\
2.24 E+03 \\
1.31 E+03 \\
1.25 E+03 \\
2.33 E+03 \\
1.55 E+03 \\
4.24 E+02 \\
3.47 E+03 \\
5.95 E+03 \\
3.28 E+02 \\
6.24 E+02 \\
3.84 E+02 \\
4.54 E+02 \\
8.50 E+02 \\
7.28 E+03 \\
7.51 E+03 \\
8.39 E+02 \\
4.65 E+03 \\
7.81 E+02 \\
7.68 E+02 \\
1.12 E+03 \\
5.12 E+02 \\
6.19 E+03 \\
5.78 E+02 \\
1.70 E+03 \\
5.54 E+02 \\
3.07 E+03 \\
7.46 E+02 \\
1.89 E+03 \\
2.39 E+03 \\
1.51 E+03 \\
1.96 E+03 \\
3.41 E+02 \\
3.40 E+02 \\
5.15 E+03 \\
1.10 E+03 \\
2.15 E+03 \\
3.22 E+03 \\
1.24 E+03 \\
3.44 E+03 \\
1.12 E+03 \\
2.90 E+03 \\
3.70 E+03 \\
9.65 E+02 \\
3.33 E+03 \\
2.73 E+03 \\
9.98 E+02 \\
4.52 E+03 \\
4.75 E+002 \\
2.53 E+03 \\
1.43 E+03 \\
2.67 E+03 \\
1.06 E+03 \\
5.13 E+02 \\
8.73 E+02 \\
7.44 E+02 \\
7.55 E+02 \\
4.07 E+03\end{array}$ & 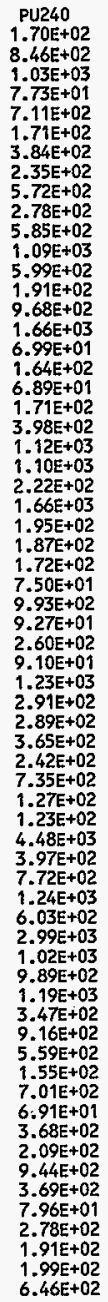 & $\begin{array}{l}P U 241 \\
1.75 E+03 \\
2.27 E+04 \\
1.19 E+04 \\
7.94 E+02 \\
7.39 E+03 \\
1.75 E+03 \\
4.62 E+03 \\
2.86 E+03 \\
6.74 E+03 \\
3.12 E+03 \\
1.82 E+04 \\
3.38 E+04 \\
1.61 E+04 \\
5.76 E+03 \\
1.42 E+04 \\
2.44 E+04 \\
8.06 E+02 \\
1.99 E+03 \\
7.50 E+02 \\
4.11 E+03 \\
1.24 E+04 \\
1.30 E+04 \\
1.26 E+04 \\
3.23 E+03 \\
2.59 E+04 \\
2.76 E+03 \\
2.58 E+03 \\
2.03 E+03 \\
7.25 E+02 \\
1.03 E+04 \\
9.16 E+02 \\
2.53 E+03 \\
9.57 E+02 \\
3.40 E+04 \\
7.91 E+03 \\
2.98 E+03 \\
3.78 E+03 \\
2.52 E+03 \\
1.93 E+04 \\
3.36 E+03 \\
3.15 E+03 \\
7.96 E+04 \\
6.35 E+03 \\
1.23 E+04 \\
1.96 E+04 \\
9.87 E+03 \\
5.33 E+04 \\
1.78 E+04 \\
1.56 E+04 \\
1.86 E+04 \\
5.48 E+03 \\
1.36 E+04 \\
7.59 E+03 \\
1.59 E+03 \\
7.41 E+03 \\
6.94 E+02 \\
3.77 E+03 \\
2.12 E+03 \\
2.36 E+04 \\
9.08 E+03 \\
8.36 E+02 \\
3.51 E+03 \\
2.35 E+03 \\
2.45 E+03 \\
6.93 E+03\end{array}$ & 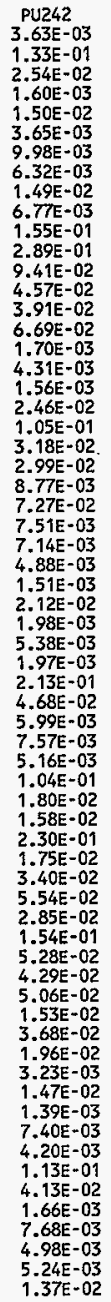 & $\begin{array}{l}A M 241 \\
1.45 \mathrm{E}+02 \\
1.84 \mathrm{E}+03 \\
9.65 \mathrm{E}+02 \\
6.39 \mathrm{E}+01 \\
5.96 \mathrm{E}+02 \\
1.46 \mathrm{E}+02 \\
3.73 \mathrm{E}+02 \\
2.35 \mathrm{E}+02 \\
5.59 \mathrm{E}+02 \\
2.60 \mathrm{E}+02 \\
1.48 \mathrm{E}+03 \\
2.77 \mathrm{E}+03 \\
1.30 \mathrm{E}+03 \\
4.70 \mathrm{E}+02 \\
1.15 \mathrm{E}+03 \\
1.97 \mathrm{E}+03 \\
6.46 \mathrm{E}+01 \\
1.61 \mathrm{E}+02 \\
6.06 \mathrm{E}+01 \\
3.73 \mathrm{E}+02 \\
1.01 \mathrm{E}+03 \\
1.05 \mathrm{E}+03 \\
1.00 \mathrm{E}+03 \\
2.60 \mathrm{E}+02 \\
2.09 \mathrm{E}+03 \\
2.25 \mathrm{E}+02 \\
2.16 \mathrm{E}+02 \\
1.61 \mathrm{E}+02 \\
6.20 \mathrm{E}+01 \\
8.42 \mathrm{E}+02 \\
8.01 \mathrm{E}+01 \\
2.19 \mathrm{E}+02 \\
7.77 \mathrm{E}+01 \\
2.74 \mathrm{E}+03 \\
6.36 \mathrm{E}+02 \\
2.39 \mathrm{E}+02 \\
3.02 \mathrm{E}+02 \\
2.05 \mathrm{E}+02 \\
1.54 \mathrm{E}+03 \\
2.67 \mathrm{E}+02 \\
2.51 \mathrm{E}+02 \\
6.34 \mathrm{E}+03 \\
4.99 \mathrm{E}+02 \\
9.70 \mathrm{E}+02 \\
1.58 \mathrm{E}+03 \\
8.07 \mathrm{E}+02 \\
4.22 \mathrm{E}+03 \\
1.46 \mathrm{E}+03 \\
1.23 \mathrm{E}+03 \\
1.45 \mathrm{E}+03 \\
4.38 \mathrm{E}+02 \\
1.08 \mathrm{E}+03 \\
5.99 \mathrm{E}+02 \\
1.29 \mathrm{E}+02 \\
5.80 \mathrm{E}+02 \\
5.62 \mathrm{E}+01 \\
2.97 \mathrm{E}+02 \\
1.69 \mathrm{E}+02 \\
1.88 \mathrm{E}+03 \\
7.19 \mathrm{E}+02 \\
6.60 \mathrm{E}+01 \\
2.82 \mathrm{E}+02 \\
1.85 \mathrm{E}+02 \\
1.94 \mathrm{E}+02 \\
5.37 \mathrm{E}+02\end{array}$ & $\begin{array}{l}\text { AML243 } \\
1.07 \mathrm{E}-03 \\
6.79 \mathrm{E}-02 \\
7.66 \mathrm{E}-03 \\
4.69 \mathrm{E}-04 \\
4.41 \mathrm{E}-03 \\
1.08 \mathrm{E}-03 \\
3.03 \mathrm{E}-03 \\
1.92 \mathrm{E}-03 \\
4.51 \mathrm{E}-03 \\
2.04 \mathrm{E}-03 \\
2.00 \mathrm{E}-01 \\
3.74 \mathrm{E}-01 \\
4.81 \mathrm{E}-02 \\
5.31 \mathrm{E}-02 \\
1.51 \mathrm{E}-02 \\
2.58 \mathrm{E}-02 \\
5.11 \mathrm{E}-04 \\
1.31 \mathrm{E}-03 \\
4.64 \mathrm{E}-04 \\
8.88 \mathrm{E}-03 \\
1.36 \mathrm{E}-01 \\
1.16 \mathrm{E}-02 \\
1.08 \mathrm{E}-02 \\
3.37 \mathrm{E}-03 \\
2.83 \mathrm{E}-02 \\
2.88 \mathrm{E}-03 \\
2.73 \mathrm{E}-03 \\
1.78 \mathrm{E}-03 \\
4.43 \mathrm{E}-04 \\
6.25 \mathrm{E}-03 \\
5.84 \mathrm{E}-04 \\
1.58 \mathrm{E}-03 \\
5.81 \mathrm{E}-04 \\
1.44 \mathrm{E}-01 \\
2.58 \mathrm{E}-02 \\
1.76 \mathrm{E}-03 \\
2.22 \mathrm{E}-03 \\
1.52 \mathrm{E}-03 \\
3.45 \mathrm{E}-02 \\
5.98 \mathrm{E}-03 \\
2.58 \mathrm{E}-03 \\
9.13 \mathrm{E}-02 \\
6.81 \mathrm{E}-03 \\
1.33 \mathrm{E}-02 \\
2.16 \mathrm{E}-02 \\
1.12 \mathrm{E}-02 \\
6.09 \mathrm{E}-02 \\
2.09 \mathrm{E}-02 \\
1.67 \mathrm{E}-02 \\
1.96 \mathrm{E}-02 \\
5.96 \mathrm{E}-03 \\
1.42 \mathrm{E}-02 \\
7.40 \mathrm{E}-03 \\
9.52 \mathrm{E}-04 \\
4.31 \mathrm{E}-03 \\
4.06 \mathrm{E}-04 \\
2.16 \mathrm{E}-03 \\
1.23 \mathrm{E}-03 \\
3.30 \mathrm{E}-03 \\
1.08 \mathrm{E}-05 \\
4.90 \mathrm{E}-04 \\
2.35 \mathrm{E}-03 \\
1.51 \mathrm{E}-03 \\
1.59 \mathrm{E}-03 \\
4.03 \mathrm{E}-03\end{array}$ & $\begin{array}{l}\text { CM242 } \\
4.47 \mathrm{E}-03 \\
2.45 \mathrm{E}-01 \\
3.20 \mathrm{E}-02 \\
1.96 \mathrm{E}-03 \\
1.85 \mathrm{E}-02 \\
4.50 \mathrm{E}-03 \\
1.27 \mathrm{E}-02 \\
8.02 \mathrm{E}-03 \\
1.88 \mathrm{E}-02 \\
8.49 \mathrm{E}-03 \\
4.97 \mathrm{E}-01 \\
9.28 \mathrm{E}-01 \\
1.74 \mathrm{E}-01 \\
1.37 \mathrm{E}-01 \\
4.84 \mathrm{E}-02 \\
8.28 \mathrm{E}-02 \\
2.14 \mathrm{E}-03 \\
5.47 \mathrm{E}-03 \\
1.94 \mathrm{E}-03 \\
3.85 \mathrm{E}-02 \\
3.38 \mathrm{E}-01 \\
3.73 \mathrm{E}-02 \\
3.48 \mathrm{E}-02 \\
1.08 \mathrm{E}-02 \\
9.09 \mathrm{E}-02 \\
9.24 \mathrm{E}-03 \\
8.75 \mathrm{E}-03 \\
5.73 \mathrm{E}-03 \\
1.84 \mathrm{E}-03 \\
2.61 \mathrm{E}-02 \\
2.43 \mathrm{E}-03 \\
6.58 \mathrm{E}-03 \\
2.43 \mathrm{E}-03 \\
4.55 \mathrm{E}-01 \\
8.96 \mathrm{E}-02 \\
7.36 \mathrm{E}-03 \\
9.31 \mathrm{E}-03 \\
6.37 \mathrm{E}-03 \\
1.59 \mathrm{E}-01 \\
2.75 \mathrm{E}-02 \\
1.93 \mathrm{E}-02 \\
2.93 \mathrm{E}-01 \\
2.19 \mathrm{E}-02 \\
4.26 \mathrm{E}-02 \\
6.94 \mathrm{E}-02 \\
3.60 \mathrm{E}-02 \\
1.96 \mathrm{E}-01 \\
6.70 \mathrm{E}-02 \\
5.36 \mathrm{E}-02 \\
6.31 \mathrm{E}-02 \\
1.91 \mathrm{E}-02 \\
4.56 \mathrm{E}-02 \\
2.38 \mathrm{E}-02 \\
3.98 \mathrm{E}-03 \\
1.81 \mathrm{E}-02 \\
1.70 \mathrm{E}-03 \\
9.06 \mathrm{E}-03 \\
5.14 \mathrm{E}-03 \\
1.10 \mathrm{E}-01 \\
3.23 \mathrm{E}-02 \\
2.05 \mathrm{E}-03 \\
9.81 \mathrm{E}-03 \\
6.32 \mathrm{E}-03 \\
6.65 \mathrm{E}-03 \\
1.69 \mathrm{E}-02\end{array}$ & 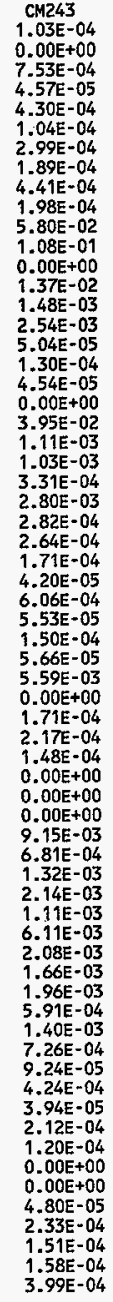 \\
\hline
\end{tabular}


ACTIVITY IN UNSEPARATED FUEL (Curies decayed to 1/1/94)

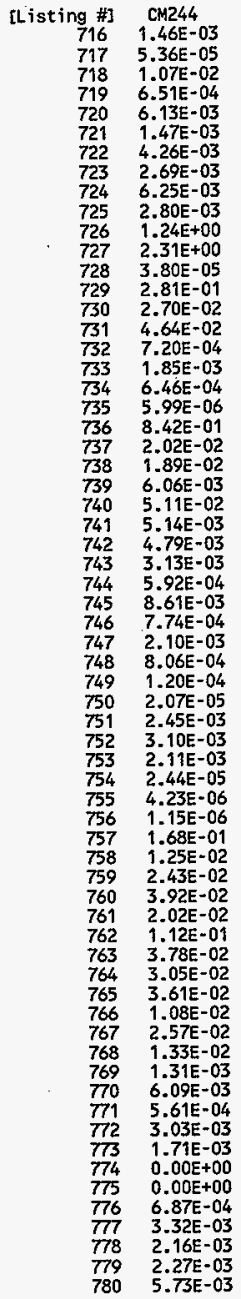


HNF-SD-WN-YI-794, Rev. 0

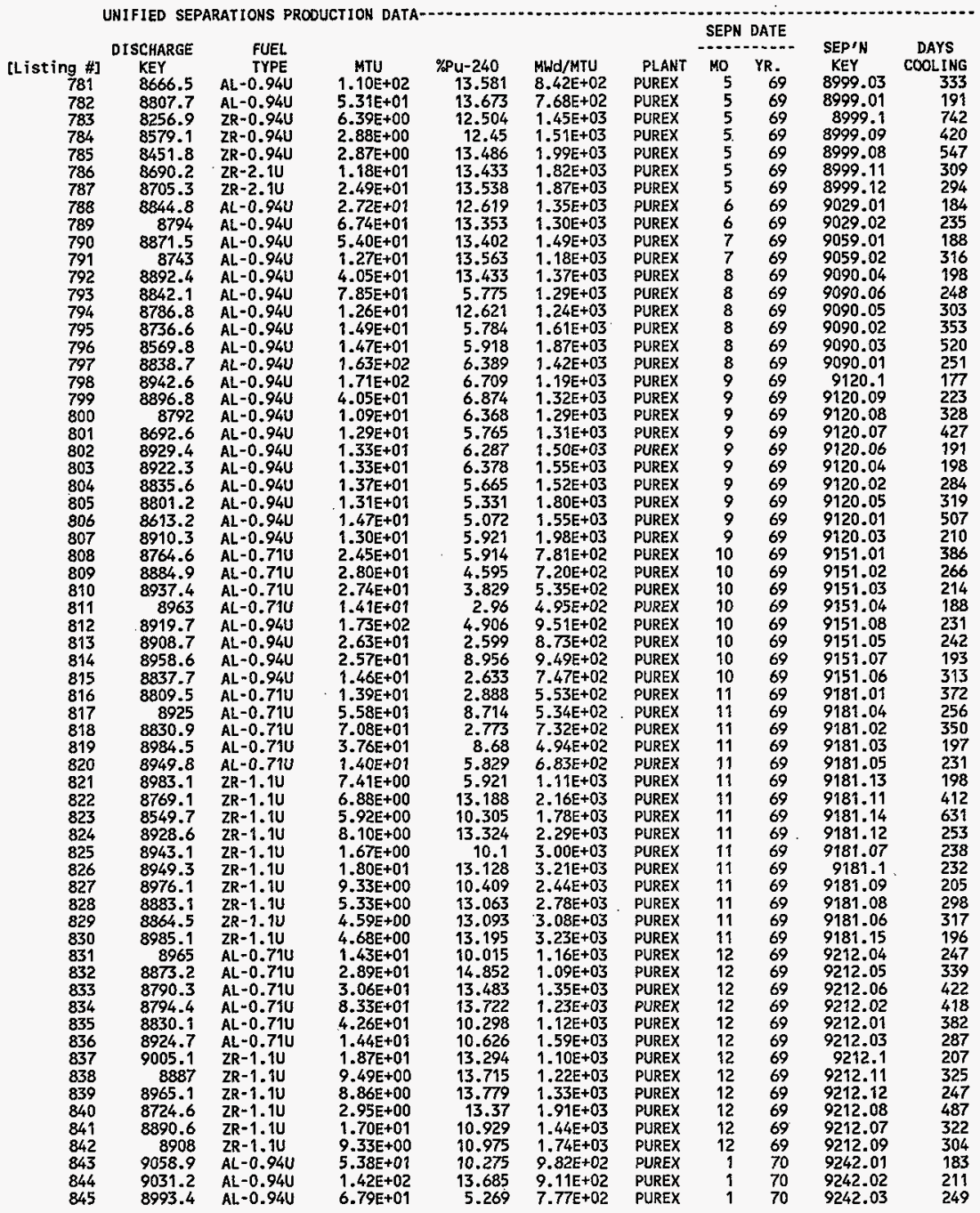


HNF-SD-WM-TI-794, ReV. 0

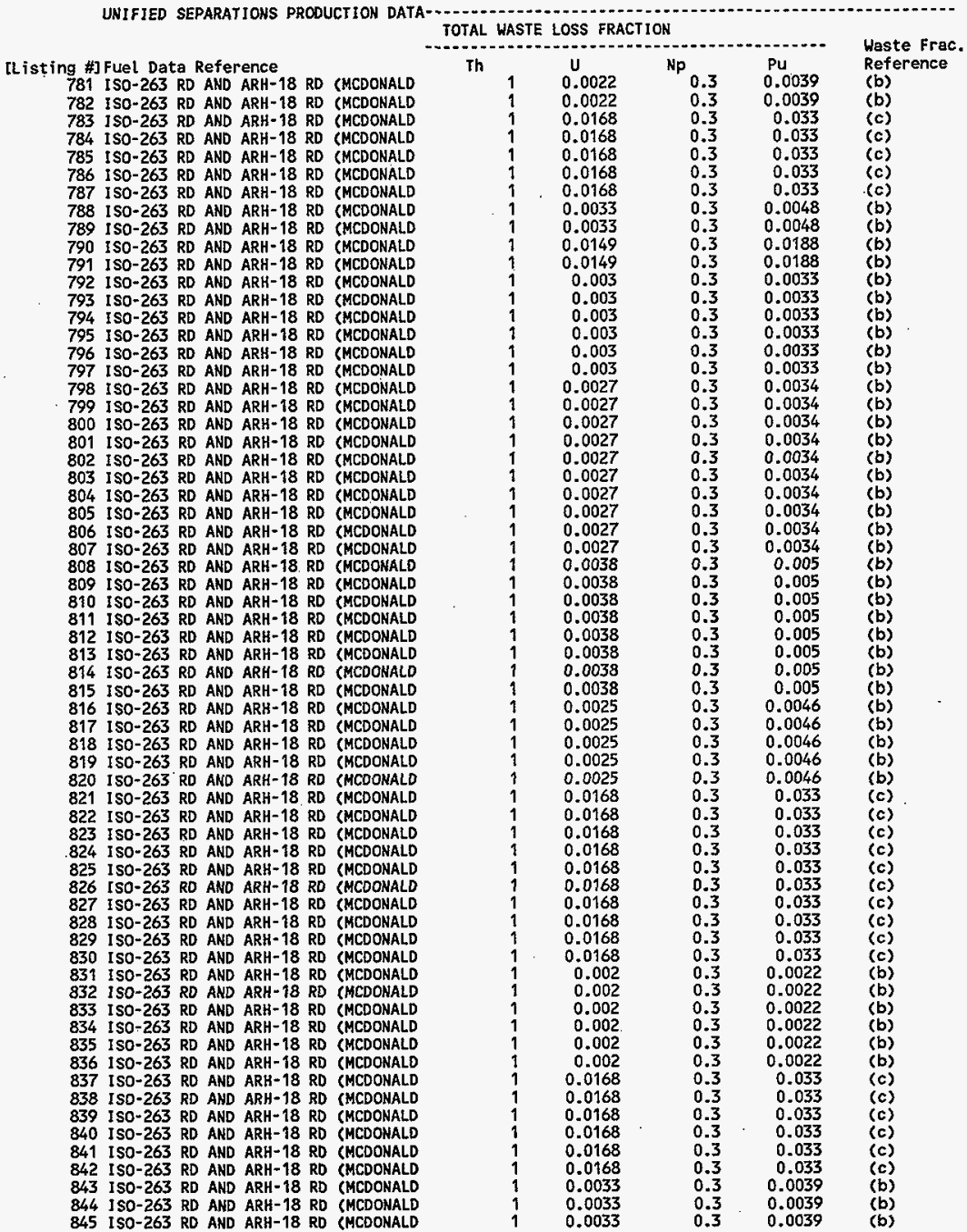


HNF-SD-WM-TI-794, ReV. 0

ACTIVITY IN UNSEPARATED FUEL (Curies decayed to $1 / 1 / 94$ )

\begin{tabular}{|c|c|c|c|c|c|c|c|c|c|}
\hline $\begin{array}{r}\text { [Listing \#] } \\
781 \\
782 \\
783 \\
784 \\
785 \\
786 \\
787 \\
788 \\
788 \\
789 \\
790 \\
791 \\
792 \\
793 \\
794 \\
795 \\
796 \\
797 \\
798 \\
799 \\
800 \\
801 \\
802 \\
803 \\
804 \\
805 \\
806 \\
807 \\
808 \\
809 \\
810 \\
811 \\
812 \\
813 \\
814 \\
815 \\
816 \\
817 \\
818 \\
819 \\
820 \\
821 \\
822 \\
823 \\
824 \\
825 \\
826 \\
827 \\
828 \\
829 \\
830 \\
831 \\
832 \\
833 \\
834 \\
835 \\
836 \\
837 \\
838 \\
839 \\
840 \\
841 \\
842 \\
843 \\
844 \\
845\end{array}$ & $\begin{array}{l}\text { H03 } \\
3.42 E+02 \\
1.54 E+02 \\
3.41 E+01 \\
1.68 E+01 \\
2.20 E+01 \\
8.24 E+01 \\
1.79 E+02 \\
1.42 E+02 \\
3.33 E+02 \\
3.11 E+02 \\
5.64 E+01 \\
2.15 E+02 \\
3.90 E+02 \\
5.95 E+01 \\
9.14 E+01 \\
1.03 E+02 \\
8.38 E+02 \\
7.90 E+02 \\
2.06 E+02 \\
5.35 E+01 \\
6.37 E+01 \\
7.77 E+01 \\
8.03 E+01 \\
8.04 E+01 \\
9.14 E+01 \\
8.49 E+01 \\
1.01 E+02 \\
7.40 E+01 \\
7.90 E+01 \\
5.76 E+01 \\
2.73 E+01 \\
6.34 E+02 \\
8.81 E+01 \\
9.46 E+01 \\
4.11 E+01 \\
2.97 E+01 \\
1.17 E+02 \\
2.02 E+02 \\
7.34 E+01 \\
3.79 E+01 \\
3.23 E+01 \\
5.83 E+01 \\
3.96 E+01 \\
7.47 E+01 \\
2.04 E+01 \\
2.37 E+02 \\
9.25 E+01 \\
5.98 E+01 \\
5.73 E+01 \\
6.24 E+01 \\
6.70 E+01 \\
1.25 E+02 \\
1.64 E+02 \\
4.04 E+02 \\
1.89 E+02 \\
9.38 E+01 \\
8.15 E+01 \\
4.51 E+01 \\
4.65 E+01 \\
2.17 E+01 \\
9.54 E+01 \\
6.42 E+01 \\
2.08 E+02 \\
5.07 E+02 \\
2.05 E+02\end{array}$ & $\begin{array}{l}\text { C14 } \\
4.62 E+00 \\
2.03 E+00 \\
1.00 E+00 \\
4.70 E-01 \\
6.25 E-01 \\
2.04 E+00 \\
4.43 E+00 \\
1.83 E+00 \\
4.34 E+00 \\
3.98 E+00 \\
7.44 E-01 \\
2.76 E+00 \\
5.04 E+00 \\
7.77 E-01 \\
1.19 E+00 \\
1.36 E+00 \\
1.15 E+01 \\
1.01 E+01 \\
2.65 E+00 \\
6.97 E-01 \\
8.43 E-01 \\
9.86 E-01 \\
1.02 E+00 \\
1.03 E+00 \\
1.17 E+00 \\
1.13 E+00 \\
1.27 E+00 \\
1.21 E+00 \\
1.28 E+00 \\
9.36 E=01 \\
4.46 E-01 \\
8.20 E+00 \\
1.14 E+00 \\
1.22 E+00 \\
5.41 E-01 \\
4.91 E-01 \\
1.90 E+00 \\
3.30 E+00 \\
1.19 E+00 \\
6.09 E-01 \\
7.67 E-01 \\
1.42 E+00 \\
1.00 E+00 \\
1.78 E+00 \\
4.85 E-01 \\
5.61 E+00 \\
2.19 E+00 \\
1.43 E+00 \\
1.38 E+00 \\
1.47 E+00 \\
1.04 E+00 \\
1.97 E+00 \\
2.57 E+00 \\
6.40 E+00 \\
3.00 E+00 \\
1.42 E+00 \\
1.93 E+00 \\
1.09 E+00 \\
1.11 E+00 \\
5.35 E-01 \\
2.29 E+00 \\
1.54 E+00 \\
2.63 E+00 \\
6.44 E+00 \\
2.63 E+00\end{array}$ & $\begin{array}{l}\text { NI59 } \\
1.31 \mathrm{E}+00 \\
5.75 \mathrm{E}-01 \\
5.97 \mathrm{E}-02 \\
2.79 \mathrm{E}-02 \\
3.70 \mathrm{E}-02 \\
1.27 \mathrm{E}-01 \\
2.75 \mathrm{E}-01 \\
5.16 \mathrm{E}-01 \\
1.23 \mathrm{E}+00 \\
1.12 \mathrm{E}+00 \\
2.10 \mathrm{E}-01 \\
7.78 \mathrm{E}-01 \\
1.42 \mathrm{E}+00 \\
2.19 \mathrm{E}-01 \\
3.36 \mathrm{E}-01 \\
3.83 \mathrm{E}-01 \\
3.23 \mathrm{E}+00 \\
2.85 \mathrm{E}+00 \\
7.47 \mathrm{E}-01 \\
1.97 \mathrm{E}-01 \\
2.38 \mathrm{E}-01 \\
2.78 \mathrm{E}-01 \\
2.87 \mathrm{E}-01 \\
2.91 \mathrm{E}-01 \\
3.30 \mathrm{E}-01 \\
3.18 \mathrm{E}-01 \\
3.57 \mathrm{E}-01 \\
3.43 \mathrm{E}-01 \\
3.62 \mathrm{E}-01 \\
2.65 \mathrm{E}-01 \\
1.26 \mathrm{E}-01 \\
2.32 \mathrm{E}+00 \\
3.24 \mathrm{E}-01 \\
3.44 \mathrm{E}-01 \\
1.53 \mathrm{E}-01 \\
1.39 \mathrm{E}-01 \\
5.40 \mathrm{E}-01 \\
9.32 \mathrm{E}-01 \\
3.37 \mathrm{E}-01 \\
1.72 \mathrm{E}-01 \\
4.80 \mathrm{E}-02 \\
8.82 \mathrm{E}-02 \\
6.23 \mathrm{E}-02 \\
1.10 \mathrm{E}-01 \\
2.98 \mathrm{E}-02 \\
3.44 \mathrm{E}-01 \\
1.35 \mathrm{E}-01 \\
8.83 \mathrm{E}-02 \\
8.45 \mathrm{E}-02 \\
9.02 \mathrm{E}-02 \\
2.93 \mathrm{E}-01 \\
5.56 \mathrm{E}-01 \\
7.24 \mathrm{E}-01 \\
1.80 \mathrm{E}+00 \\
8.466 \mathrm{E}-01 \\
3.99 \mathrm{E}-01 \\
1.21 \mathrm{E}-01 \\
6.79 \mathrm{E}-02 \\
6.91 \mathrm{E}-02 \\
3.33 \mathrm{E}-02 \\
1.43 \mathrm{E}-01 \\
9.56 \mathrm{E}-02 \\
7.43 \mathrm{E}-01 \\
1.82 \mathrm{E}+00 \\
7.45 \mathrm{E}-01\end{array}$ & $\begin{array}{l}\text { NI } 63 \\
1.35 E+02 \\
5.94 E+01 \\
6.18 E+00 \\
2.91 E+00 \\
3.85 E+00 \\
1.32 E+01 \\
2.87 E+01 \\
5.35 E+01 \\
1.27 E+02 \\
1.16 E+02 \\
2.17 E+01 \\
8.07 E+01 \\
1.47 E+02 \\
2.27 E+01 \\
3.47 E+01 \\
3.95 E+01 \\
3.35 E+02 \\
2.96 E+02 \\
7.75 E+01 \\
2.04 E+01 \\
2.46 E+01 \\
2.89 E+01 \\
2.98 E+01 \\
3.02 E+01 \\
3.42 E+01 \\
3.29 E+01 \\
3.71 E+01 \\
3.55 E+01 \\
3.75 E+01 \\
2.75 E+01 \\
1.31 E+01 \\
2.40 E+02 \\
3.35 E+01 \\
3.56 E+01 \\
1.58 E+01 \\
1.44 E+01 \\
5.58 E+01 \\
9.64 E+01 \\
3.49 E+01 \\
1.79 E+01 \\
5.01 E+00 \\
9.22 E+00 \\
6.47 E+00 \\
1.16 E+01 \\
3.14 E+00 \\
3.63 E+01 \\
1.42 E+01 \\
9.28 E+00 \\
8.90 E+00 \\
9.53 E+00 \\
3.05 E+01 \\
5.77 E+01 \\
7.51 E+01 \\
1.87 E+02 \\
8.77 E+01 \\
4.16 E+01 \\
1.26 E+01 \\
7.07 E+00 \\
7.21 E+00 \\
3.47 E+00 \\
1.49 E+01 \\
1.00 E+01 \\
7.73 E+01 \\
1.89 E+02 \\
7.72 E+01\end{array}$ & $\begin{array}{l}C 060 \\
9.84 E+00 \\
4.56 E+00 \\
1.88 E+00 \\
9.86 E-01 \\
1.24 E+00 \\
5.25 E+00 \\
1.14 E+01 \\
4.12 E+00 \\
9.61 E+00 \\
9.01 E+00 \\
1.62 E+00 \\
6.31 E+00 \\
1.14 E+01 \\
1.72 E+00 \\
2.57 E+00 \\
2.75 E+00 \\
2.57 E+01 \\
2.37 E+01 \\
6.08 E+00 \\
1.54 E+00 \\
1.80 E+00 \\
2.28 E+00 \\
2.35 E+00 \\
2.31 E+00 \\
2.57 E+00 \\
2.33 E+00 \\
2.89 E+00 \\
2.69 E+00 \\
2.96 E+00 \\
2.21 E+00 \\
1.06 E+00 \\
1.91 E+01 \\
2.66 E+00 \\
2.87 E+00 \\
1.23 E+00 \\
1.11 E+00 \\
4.48 E+00 \\
7.47 E+00 \\
2.86 E+00 \\
1.44 E+00 \\
2.23 E+00 \\
3.74 E+00 \\
2.45 E+00 \\
4.95 E+00 \\
1.34 E+00 \\
1.54 E+01 \\
6.16 E+00 \\
3.88 E+00 \\
3.68 E+00 \\
4.10 E+00 \\
2.45 E+00 \\
4.50 E+00 \\
5.68 E+00 \\
1.42 E+01 \\
6.75 E+00 \\
3.28 E+00 \\
5.63 E+00 \\
3.03 E+00 \\
3.17 E+00 \\
1.39 E+00 \\
6.39 E+00 \\
4.28 E+00 \\
6.44 E+00 \\
1.57 E+01 \\
6.32 E+00\end{array}$ & $\begin{array}{c}\text { SE79 } \\
1.24 \mathrm{E}+00 \\
5.45 \mathrm{E}-01 \\
1.23 \mathrm{E}-01 \\
5.75 \mathrm{E}-02 \\
7.54 \mathrm{E}-02 \\
2.83 \mathrm{E}-01 \\
6.12 \mathrm{E}-01 \\
4.91 \mathrm{E}-01 \\
1.17 \mathrm{E}+00 \\
1.07 \mathrm{E}+00 \\
2.00 \mathrm{E}-01 \\
7.41 \mathrm{E}-01 \\
1.35 \mathrm{E}+00 \\
2.09 \mathrm{E}-01 \\
3.20 \mathrm{E}-01 \\
3.66 \mathrm{E}-01 \\
3.08 \mathrm{E}+00 \\
2.71 \mathrm{E}+00 \\
7.11 \mathrm{E}-01 \\
1.87 \mathrm{E}-01 \\
2.26 \mathrm{E}-01 \\
2.65 \mathrm{E}-01 \\
2.74 \mathrm{E}-01 \\
2.78 \mathrm{E}-01 \\
3.15 \mathrm{E}-01 \\
3.03 \mathrm{E}-01 \\
3.41 \mathrm{E}-01 \\
2.57 \mathrm{E}-01 \\
2.71 \mathrm{E}-01 \\
1.97 \mathrm{E}-01 \\
9.40 \mathrm{E}-02 \\
2.20 \mathrm{E}+00 \\
3.07 \mathrm{E}-01 \\
3.26 \mathrm{E}-01 \\
1.45 \mathrm{E}-01 \\
1.04 \mathrm{E}-01 \\
4.02 \mathrm{E}-01 \\
6.98 \mathrm{E}-01 \\
2.51 \mathrm{E}-01 \\
1.29 \mathrm{E}-01 \\
1.09 \mathrm{E}-01 \\
1.96 \mathrm{E}-01 \\
1.39 \mathrm{E}-01 \\
2.44 \mathrm{E}-01 \\
6.53 \mathrm{E}-02 \\
7.52 \mathrm{E}-01 \\
2.99 \mathrm{E}-01 \\
1.94 \mathrm{E}-01 \\
1.85 \mathrm{E}-01 \\
1.97 \mathrm{E}-01 \\
2.22 \mathrm{E}-01 \\
4.20 \mathrm{E}-01 \\
5.52 \mathrm{E}-01 \\
1.37 \mathrm{E}+00 \\
6.41 \mathrm{E}-01 \\
3.06 \mathrm{E}-01 \\
2.73 \mathrm{E}-01 \\
1.53 \mathrm{E}-01 \\
1.56 \mathrm{E}-01 \\
7.41 \mathrm{E}-02 \\
3.22 \mathrm{E}-01 \\
2.14 \mathrm{E}-01 \\
7.06 \mathrm{E}-01 \\
1.73 \mathrm{E}+00 \\
7.06 \mathrm{E}-01\end{array}$ & $\begin{array}{l}\text { SR90 } \\
1.47 \mathrm{E}+05 \\
6.53 \mathrm{E}+04 \\
1.34 \mathrm{E}+04 \\
6.36 \mathrm{E}+03 \\
8.08 \mathrm{E}+03 \\
3.22 \mathrm{E}+04 \\
6.98 \mathrm{E}+04 \\
5.78 \mathrm{E}+04 \\
1.37 \mathrm{E}+05 \\
1.25 \mathrm{E}+05 \\
2.35 \mathrm{E}+04 \\
8.74 \mathrm{E}+04 \\
1.60 \mathrm{E}+05 \\
2.46 \mathrm{E}+04 \\
3.70 \mathrm{E}+04 \\
4.15 \mathrm{E}+04 \\
3.61 \mathrm{E}+05 \\
3.23 \mathrm{E}+05 \\
8.41 \mathrm{E}+04 \\
2.20 \mathrm{E}+04 \\
2.64 \mathrm{E}+04 \\
3.12 \mathrm{E}+04 \\
3.22 \mathrm{E}+04 \\
3.24 \mathrm{E}+04 \\
3.63 \mathrm{E}+04 \\
3.49 \mathrm{E}+04 \\
3.94 \mathrm{E}+04 \\
2.97 \mathrm{E}+04 \\
3.15 \mathrm{E}+04 \\
2.33 \mathrm{E}+04 \\
1.11 \mathrm{E}+04 \\
2.64 \mathrm{E}+05 \\
3.69 \mathrm{E}+04 \\
3.92 \mathrm{E}+04 \\
1.75 \mathrm{E}+04 \\
1.21 \mathrm{E}+04 \\
4.74 \mathrm{E}+04 \\
8.10 \mathrm{E}+04 \\
2.97 \mathrm{E}+04 \\
1.51 \mathrm{E}+04 \\
1.30 \mathrm{E}+04 \\
2.22 \mathrm{E}+04 \\
1.58 \mathrm{E}+04 \\
2.79 \mathrm{E}+04 \\
7.29 \mathrm{E}+03 \\
8.36 \mathrm{E}+04 \\
3.40 \mathrm{E}+04 \\
2.17 \mathrm{E}+04 \\
2.05 \mathrm{E}+04 \\
2.20 \mathrm{E}+04 \\
2.54 \mathrm{E}+04 \\
4.80 \mathrm{E}+04 \\
6.18 \mathrm{E}+04 \\
1.54 \mathrm{E}+05 \\
7.28 \mathrm{E}+04 \\
3.41 \mathrm{E}+04 \\
3.26 \mathrm{E}+04 \\
1.81 \mathrm{E}+04 \\
1.84 \mathrm{E}+04 \\
8.47 \mathrm{E}+03 \\
3.78 \mathrm{E}+04 \\
2.49 \mathrm{E}+04 \\
8.53 \mathrm{E}+04 \\
2.09 \mathrm{E}+05 \\
8.56 \mathrm{E}+04\end{array}$ & $\begin{array}{l}Y 90 \\
1.47 E+05 \\
6.53 E+04 \\
1.34 E+04 \\
6.36 E+03 \\
8.08 E+03 \\
3.22 E+04 \\
6.98 E+04 \\
5.78 E+04 \\
1.37 E+05 \\
1.25 E+05 \\
2.35 E+04 \\
8.74 E+04 \\
1.60 E+05 \\
2.46 E+04 \\
3.71 E+04 \\
4.15 E+04 \\
3.61 E+05 \\
3.24 E+05 \\
8.41 E+04 \\
2.20 E+04 \\
2.64 E+04 \\
3.12 E+04 \\
3.22 E+04 \\
3.24 E+04 \\
3.63 E+04 \\
3.49 E+04 \\
3.94 E+04 \\
2.97 E+04 \\
3.16 E+04 \\
2.33 E+04 \\
1.11 E+04 \\
2.64 E+05 \\
3.69 E+04 \\
3.92 E+04 \\
1.75 E+04 \\
1.21 E+04 \\
4.74 E+04 \\
8.10 E+04 \\
2.97 E+04 \\
1.51 E+04 \\
1.30 E+04 \\
2.22 E+04 \\
1.58 E+04 \\
2.79 E+04 \\
7.30 E+03 \\
8.36 E+04 \\
3.40 E+04 \\
2.17 E+04 \\
2.05 E+04 \\
2.20 E+04 \\
2.54 E+04 \\
4.80 E+04 \\
6.18 E+04 \\
1.54 E+05 \\
7.28 E+04 \\
3.42 E+04 \\
3.27 E+04 \\
1.81 E+04 \\
1.84 E+04 \\
8.47 E+03 \\
3.78 E+04 \\
2.49 E+04 \\
8.53 E+04 \\
2.09 E+05 \\
8.56 E+04\end{array}$ & 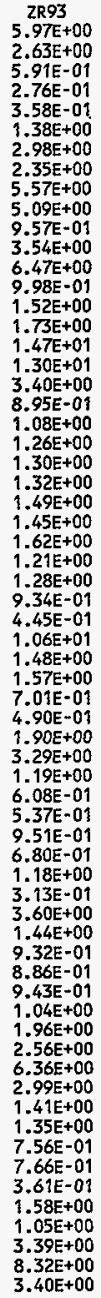 \\
\hline
\end{tabular}


HNF-SD-WM-TI-794, ReV. 0

ACTIVITY IN UNSEPARATED FUEL (Curies decayed to $1 / 1 / 94$ )

\begin{tabular}{|c|c|c|c|c|c|c|c|c|c|}
\hline $\begin{array}{r}\text { [Listing \#] } \\
781 \\
782 \\
783 \\
784 \\
785 \\
786 \\
787 \\
788 \\
789 \\
790 \\
791 \\
792 \\
793 \\
794 \\
795 \\
796 \\
797 \\
798 \\
799 \\
800 \\
801 \\
802 \\
803 \\
804 \\
805 \\
806 \\
807 \\
808 \\
809 \\
810 \\
811 \\
812 \\
813 \\
814 \\
815 \\
816 \\
817 \\
818 \\
819 \\
820 \\
821 \\
822 \\
823 \\
824 \\
825 \\
826 \\
827 \\
828 \\
829 \\
830 \\
831 \\
832 \\
833 \\
834 \\
835 \\
836 \\
837 \\
838 \\
839 \\
840 \\
841 \\
842 \\
843 \\
844 \\
845\end{array}$ & 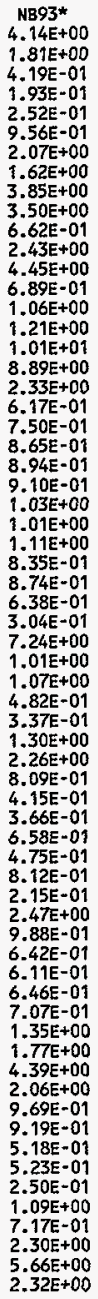 & $\begin{array}{l}\text { TC99 } \\
4.12 E+01 \\
1.81 E+01 \\
4.11 E+00 \\
1.92 E+00 \\
2.52 E+00 \\
9.47 E+00 \\
2.05 E+01 \\
1.66 E+01 \\
3.92 E+01 \\
3.67 E+01 \\
6.65 E+00 \\
2.51 E+01 \\
4.56 E+01 \\
6.98 E+00 \\
1.11 E+01 \\
1.31 E+01 \\
1.05 E+02 \\
9.03 E+01 \\
2.39 E+01 \\
6.30 E+00 \\
7.62 E+00 \\
9.09 E+00 \\
9.45 E+00 \\
9.58 E+00 \\
1.12 E+01 \\
1.05 E+01 \\
1.23 E+01 \\
8.49 E+00 \\
8.93 E+00 \\
6.50 E+00 \\
3.09 E+00 \\
7.32 E+01 \\
1.02 E+01 \\
1.09 E+01 \\
4.83 E+00 \\
3.42 E+00 \\
1.32 E+01 \\
2.30 E+01 \\
8.25 E+00 \\
4.25 E+00 \\
3.64 E+00 \\
6.56 E+00 \\
4.66 E+00 \\
8.18 E+00 \\
2.19 E+00 \\
2.52 E+01 \\
1.00 E+01 \\
6.50 E+00 \\
6.20 E+00 \\
6.61 E+00 \\
7.35 E+00 \\
1.39 E+01 \\
1.86 E+01 \\
4.54 E+01 \\
2.12 E+01 \\
1.06 E+01 \\
9.15 E+00 \\
5.14 E+00 \\
5.22 E+00 \\
2.49 E+00 \\
1.08 E+01 \\
7.17 E+00 \\
2.35 E+01 \\
5.75 E+01 \\
2.35 E+01\end{array}$ & $\begin{array}{l}\text { RU106 } \\
2.36 \mathrm{E}-02 \\
1.34 \mathrm{E}-02 \\
1.53 \mathrm{E}-03 \\
1.32 \mathrm{E}-03 \\
1.48 \mathrm{E}-03 \\
7.45 \mathrm{E}-03 \\
1.66 \mathrm{E}-02 \\
1.47 \mathrm{E}-02 \\
3.13 \mathrm{E}-02 \\
3.45 \mathrm{E}-02 \\
4.75 \mathrm{E}-03 \\
2.42 \mathrm{E}-02 \\
3.98 \mathrm{E}-02 \\
5.47 \mathrm{E}-03 \\
8.22 \mathrm{E}-03 \\
7.21 \mathrm{E}-03 \\
9.21 \mathrm{E}-02 \\
9.42 \mathrm{E}-02 \\
2.32 \mathrm{E}-02 \\
5.02 \mathrm{E}-03 \\
5.04 \mathrm{E}-03 \\
9.54 \mathrm{E}-03 \\
9.85 \mathrm{E}-03 \\
8.50 \mathrm{E}-03 \\
9.49 \mathrm{E}-03 \\
6.11 \mathrm{E}-03 \\
1.31 \mathrm{E}-02 \\
6.96 \mathrm{E}-03 \\
9.00 \mathrm{E}-03 \\
6.79 \mathrm{E}-03 \\
3.35 \mathrm{E}-03 \\
6.95 \mathrm{E}-02 \\
9.33 \mathrm{E}-03 \\
1.11 \mathrm{E}-02 \\
3.76 \mathrm{E}-03 \\
2.82 \mathrm{E}-03 \\
1.35 \mathrm{E}-02 \\
2.10 \mathrm{E}-02 \\
9.30 \mathrm{E}-03 \\
4.79 \mathrm{E}-03 \\
4.41 \mathrm{E}-03 \\
6.14 \mathrm{E}-03 \\
2.75 \mathrm{E}-03 \\
1.05 \mathrm{E}-02 \\
3.10 \mathrm{E}-03 \\
3.67 \mathrm{E}-02 \\
1.43 \mathrm{E}-02 \\
8.09 \mathrm{E}-03 \\
7.62 \mathrm{E}-03 \\
1.03 \mathrm{E}-02 \\
9.84 \mathrm{E}-03 \\
1.53 \mathrm{E}-02 \\
1.85 \mathrm{E}-02 \\
4.48 \mathrm{E}-02 \\
2.18 \mathrm{E}-02 \\
1.42 \mathrm{E}-02 \\
1.15 \mathrm{E}-02 \\
5.26 \mathrm{E}-03 \\
6.29 \mathrm{E}-03 \\
2.07 \mathrm{E}-03 \\
1.15 \mathrm{E}-02 \\
8.22 \mathrm{E}-03 \\
2.92 \mathrm{E}-02 \\
6.69 \mathrm{E}-02 \\
2.46 \mathrm{E}-02\end{array}$ & $\begin{array}{l}C D 113 \\
2.87 E+01 \\
1.28 E+01 \\
3.19 E+00 \\
1.56 E+00 \\
2.12 E+00 \\
7.06 E+00 \\
1.53 E+01 \\
1.21 E+01 \\
2.84 E+01 \\
2.66 E+01 \\
4.79 E+00 \\
1.83 E+01 \\
3.32 E+01 \\
5.05 E+00 \\
7.89 E+00 \\
8.97 E+00 \\
7.58 E+01 \\
6.68 E+01 \\
1.75 E+01 \\
4.55 E+00 \\
5.44 E+00 \\
6.64 E+00 \\
6.88 E+00 \\
6.90 E+00 \\
7.92 E+00 \\
7.33 E+00 \\
8.82 E+00 \\
7.12 E+00 \\
7.57 E+00 \\
5.48 E+00 \\
2.61 E+00 \\
5.32 E+01 \\
7.36 E+00 \\
7.92 E+00 \\
3.42 E+00 \\
2.83 E+00 \\
1.11 E+01 \\
1.94 E+01 \\
6.97 E+00 \\
3.62 E+00 \\
2.64 E+00 \\
5.05 E+00 \\
3.38 E+00 \\
6.50 E+00 \\
1.83 E+00 \\
2.14 E+01 \\
8.09 E+00 \\
5.32 E+00 \\
5.17 E+00 \\
5.64 E+00 \\
6.50 E+00 \\
1.21 E+01 \\
1.60 E+01 \\
3.94 E+01 \\
1.84 E+01 \\
9.22 E+00 \\
6.64 E+00 \\
3.71 E+00 \\
3.84 E+00 \\
1.86 E+00 \\
7.94 E+00 \\
5.42 E+00 \\
1.74 E+01 \\
4.23 E+01 \\
1.70 E+01\end{array}$ & $\begin{array}{l}\text { SB125 } \\
7.08 E+01 \\
3.42 E+01 \\
6.31 E+00 \\
3.68 E+00 \\
4.54 E+00 \\
1.80 E+01 \\
3.95 E+01 \\
3.26 E+01 \\
7.40 E+01 \\
7.37 E+01 \\
1.22 E+01 \\
5.07 E+01 \\
8.88 E+01 \\
1.32 E+01 \\
2.05 E+01 \\
2.18 E+01 \\
2.05 E+02 \\
1.90 E+02 \\
4.85 E+01 \\
1.19 E+01 \\
1.34 E+01 \\
1.90 E+01 \\
1.98 E+01 \\
1.89 E+01 \\
2.18 E+01 \\
1.77 E+01 \\
2.61 E+01 \\
1.81 E+01 \\
2.06 E+01 \\
1.54 E+01 \\
7.46 E+00 \\
1.50 E+02 \\
2.08 E+01 \\
2.29 E+01 \\
9.32 E+00 \\
7.43 E+00 \\
3.12 E+01 \\
5.12 E+01 \\
2.02 E+01 \\
1.02 E+01 \\
8.18 E+00 \\
1.33 E+01 \\
8.01 E+00 \\
1.86 E+01 \\
5.14 E+00 \\
5.97 E+01 \\
2.36 E+01 \\
1.46 E+01 \\
1.38 E+01 \\
1.60 E+01 \\
1.83 E+01 \\
3.23 E+01 \\
4.08 E+01 \\
1.00 E+02 \\
4.79 E+01 \\
2.59 E+01 \\
2.08 E+01 \\
1.08 E+01 \\
1.17 E+01 \\
4.83 E+00 \\
2.30 E+01 \\
1.57 E+01 \\
5.31 E+01\end{array}$ & 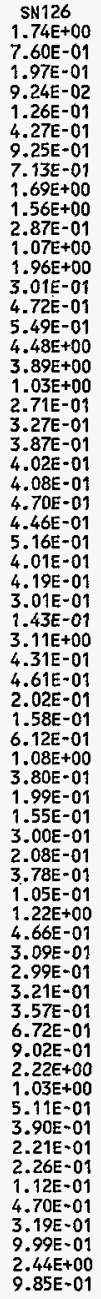 & $\begin{array}{c}1129 \\
7.66 \mathrm{E}-02 \\
3.36 \mathrm{E}-02 \\
8.31 \mathrm{E}-03 \\
3.90 \mathrm{E}-03 \\
5.27 \mathrm{E}-03 \\
1.88 \mathrm{E}-02 \\
4.06 \mathrm{E}-02 \\
3.13 \mathrm{E}-02 \\
7.41 \mathrm{E}-02 \\
6.86 \mathrm{E}-02 \\
1.26 \mathrm{E}-02 \\
4.72 \mathrm{E}-02 \\
8.60 \mathrm{E}-02 \\
1.32 \mathrm{E}-02 \\
2.07 \mathrm{E}-02 \\
2.40 \mathrm{E}-02 \\
1.97 \mathrm{E}-01 \\
1.71 \mathrm{E}-01 \\
4.51 \mathrm{E}-02 \\
1.19 \mathrm{E}-02 \\
1.44 \mathrm{E}-02 \\
1.70 \mathrm{E}-02 \\
1.76 \mathrm{E}-02 \\
1.79 \mathrm{E}-02 \\
2.06 \mathrm{E}-02 \\
1.95 \mathrm{E}-02 \\
2.26 \mathrm{E}-02 \\
1.65 \mathrm{E}-02 \\
1.73 \mathrm{E}-02 \\
1.24 \mathrm{E}-02 \\
5.87 \mathrm{E}-03 \\
1.37 \mathrm{E}-01 \\
1.90 \mathrm{E}-02 \\
2.03 \mathrm{E}-02 \\
8.94 \mathrm{E}-03 \\
6.51 \mathrm{E}-03 \\
2.52 \mathrm{E}-02 \\
4.45 \mathrm{E}-02 \\
1.57 \mathrm{E}-02 \\
8.19 \mathrm{E}-03 \\
6.93 \mathrm{E}-03 \\
1.31 \mathrm{E}-02 \\
9.17 \mathrm{E}-03 \\
1.65 \mathrm{E}-02 \\
4.54 \mathrm{E}-03 \\
5.27 \mathrm{E}-02 \\
2.03 \mathrm{E}-02 \\
1.34 \mathrm{E}-02 \\
1.29 \mathrm{E}-02 \\
1.38 \mathrm{E}-02 \\
1.47 \mathrm{E}-02 \\
2.77 \mathrm{E}-02 \\
3.71 \mathrm{E}-02 \\
9.11 \mathrm{E}-02 \\
4.23 \mathrm{E}-02 \\
2.10 \mathrm{E}-02 \\
1.74 \mathrm{E}-02 \\
9.82 \mathrm{E}-03 \\
1.00 \mathrm{E}-02 \\
4.91 \mathrm{E}-03 \\
2.09 \mathrm{E}-02 \\
1.41 \mathrm{E}-02 \\
4.40 \mathrm{E}-02 \\
1.07 \mathrm{E}-01 \\
4.35 \mathrm{E}-02\end{array}$ & $\begin{array}{l}C 5134 \\
4.45 E+00 \\
2.02 E+00 \\
7.81 E-01 \\
5.11 E-01 \\
8.05 E-01 \\
2.97 E+00 \\
6.51 E+00 \\
3.57 E+00 \\
7.71 E+00 \\
8.80 E+00 \\
1.13 E+00 \\
5.61 E+00 \\
9.36 E+00 \\
1.31 E+00 \\
2.54 E+00 \\
2.93 E+00 \\
2.34 E+01 \\
1.85 E+01 \\
5.17 E+00 \\
1.24 E+00 \\
1.37 E+00 \\
2.30 E+00 \\
2.47 E+00 \\
2.31 E+00 \\
3.00 E+00 \\
2.06 E+00 \\
3.97 E+00 \\
1.17 E+00 \\
1.25 E+00 \\
6.65 E-01 \\
2.93 E-01 \\
1.16 E+01 \\
1.45 E+00 \\
1.77 E+00 \\
5.35 E-01 \\
3.23 E-01 \\
1.34 E+00 \\
3.06 E+00 \\
7.98 E-01 \\
5.92 E-01 \\
8.32 E-01 \\
2.56 E+00 \\
1.21 E+00 \\
3.94 E+00 \\
1.41 E+00 \\
1.75 E+01 \\
5.38 E+00 \\
3.67 E+00 \\
3.81 E+00 \\
4.73 E+00 \\
1.89 E+00 \\
3.06 E+00 \\
4.72 E+00 \\
1.06 E+01 \\
4.65 E+00 \\
3.56 E+00 \\
2.13 E+00 \\
1.20 E+00 \\
1.43 E+00 \\
8.13 E-01 \\
3.02 E+00 \\
2.51 E+00 \\
4.37 E+00 \\
9.59 E+00 \\
3.14 E+00\end{array}$ & $\begin{array}{c}\text { CS137 } \\
1.65 \mathrm{E}+05 \\
7.30 \mathrm{E}+04 \\
1.61 \mathrm{E}+04 \\
7.66 \mathrm{E}+03 \\
1.00 \mathrm{E}+04 \\
3.81 \mathrm{E}+04 \\
8.27 \mathrm{E}+04 \\
6.63 \mathrm{E}+04 \\
1.57 \mathrm{E}+05 \\
1.45 \mathrm{E}+05 \\
2.68 \mathrm{E}+04 \\
1.00 \mathrm{E}+05 \\
1.83 \mathrm{E}+05 \\
2.80 \mathrm{E}+04 \\
4.30 \mathrm{E}+04 \\
4.87 \mathrm{E}+04 \\
4.15 \mathrm{E}+05 \\
3.68 \mathrm{E}+05 \\
9.62 \mathrm{E}+04 \\
2.52 \mathrm{E}+04 \\
3.02 \mathrm{E}+04 \\
3.60 \mathrm{E}+04 \\
3.72 \mathrm{E}+04 \\
3.75 \mathrm{E}+04 \\
4.25 \mathrm{E}+04 \\
4.04 \mathrm{E}+04 \\
4.65 \mathrm{E}+04 \\
3.41 \mathrm{E}+04 \\
3.61 \mathrm{E}+04 \\
2.64 \mathrm{E}+04 \\
1.26 \mathrm{E}+04 \\
2.97 \mathrm{E}+05 \\
4.14 \mathrm{E}+04 \\
4.42 \mathrm{E}+04 \\
1.95 \mathrm{E}+04 \\
1.37 \mathrm{E}+04 \\
5.36 \mathrm{E}+04 \\
9.28 \mathrm{E}+04 \\
3.35 \mathrm{E}+04 \\
1.73 \mathrm{E}+04 \\
1.49 \mathrm{E}+04 \\
2.66 \mathrm{E}+04 \\
1.86 \mathrm{E}+04 \\
3.35 \mathrm{E}+04 \\
9.00 \mathrm{E}+03 \\
1.04 \mathrm{E}+05 \\
4.11 \mathrm{E}+04 \\
2.66 \mathrm{E}+04 \\
2.54 \mathrm{E}+04 \\
2.73 \mathrm{E}+04 \\
3.00 \mathrm{E}+04 \\
5.64 \mathrm{E}+04 \\
7.39 \mathrm{E}+04 \\
1.83 \mathrm{E}+05 \\
8.57 \mathrm{E}+04 \\
4.15 \mathrm{E}+04 \\
3.74 \mathrm{E}+04 \\
2.09 \mathrm{E}+04 \\
2.13 \mathrm{E}+04 \\
1.00 \mathrm{E}+04 \\
4.39 \mathrm{E}+04 \\
2.92 \mathrm{E}+04 \\
9.62 \mathrm{E}+04 \\
2.35 \mathrm{E}+05 \\
9.57 \mathrm{E}+04\end{array}$ \\
\hline
\end{tabular}


HNF-SD-WN-TI-794, ReV, O

ACTIVITY IN UNSEPARATED FUEL (Curies decayed to $1 / 1 / 94$ )

\begin{tabular}{|c|c|c|c|c|c|c|c|c|c|}
\hline 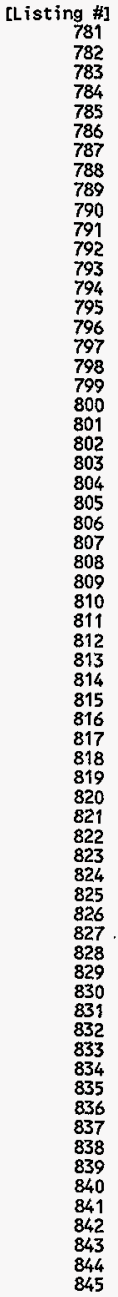 & $\begin{array}{l}8 \mathrm{~B} 137 \\
1.56 \mathrm{E}+05 \\
6.91 \mathrm{E}+04 \\
1.52 \mathrm{E}+04 \\
7.25 \mathrm{E}+03 \\
9.47 \mathrm{E}+03 \\
3.61 \mathrm{E}+04 \\
7.82 \mathrm{E}+04 \\
6.27 \mathrm{E}+04 \\
1.48 \mathrm{E}+05 \\
1.37 \mathrm{E}+05 \\
2.53 \mathrm{E}+04 \\
9.48 \mathrm{E}+04 \\
1.73 \mathrm{E}+05 \\
2.65 \mathrm{E}+04 \\
4.07 \mathrm{E}+04 \\
4.61 \mathrm{E}+04 \\
3.93 \mathrm{E}+05 \\
3.48 \mathrm{E}+05 \\
9.10 \mathrm{E}+04 \\
2.38 \mathrm{E}+04 \\
2.86 \mathrm{E}+04 \\
3.40 \mathrm{E}+04 \\
3.52 \mathrm{E}+04 \\
3.55 \mathrm{E}+04 \\
4.02 \mathrm{E}+04 \\
3.82 \mathrm{E}+04 \\
4.40 \mathrm{E}+04 \\
3.23 \mathrm{E}+04 \\
3.42 \mathrm{E}+04 \\
2.49 \mathrm{E}+04 \\
1.19 \mathrm{E}+04 \\
2.81 \mathrm{E}+05 \\
3.92 \mathrm{E}+04 \\
4.18 \mathrm{E}+04 \\
1.85 \mathrm{E}+04 \\
1.30 \mathrm{E}+04 \\
5.07 \mathrm{E}+04 \\
8.78 \mathrm{E}+04 \\
3.17 \mathrm{E}+04 \\
1.63 \mathrm{E}+04 \\
1.41 \mathrm{E}+04 \\
2.51 \mathrm{E}+04 \\
1.76 \mathrm{E}+04 \\
3.17 \mathrm{E}+04 \\
8.52 \mathrm{E}+03 \\
9.83 \mathrm{E}+04 \\
3.89 \mathrm{E}+04 \\
2.52 \mathrm{E}+04 \\
2.40 \mathrm{E}+04 \\
2.58 \mathrm{E}+04 \\
2.84 \mathrm{E}+04 \\
5.33 \mathrm{E}+04 \\
6.99 \mathrm{E}+04 \\
1.73 \mathrm{E}+05 \\
8.11 \mathrm{E}+04 \\
3.93 \mathrm{E}+04 \\
3.54 \mathrm{E}+04 \\
1.97 \mathrm{E}+04 \\
2.02 \mathrm{E}+04 \\
9.48 \mathrm{E}+03 \\
4.15 \mathrm{E}+04 \\
2.77 \mathrm{E}+04 \\
9.10 \mathrm{E}+04 \\
2.23 \mathrm{E}+05 \\
9.05 \mathrm{E}+04\end{array}$ & $\begin{array}{l}\text { SH151 } \\
4.74 \mathrm{E}+03 \\
2.13 \mathrm{E}+03 \\
3.74 \mathrm{E}+02 \\
1.73 \mathrm{E}+02 \\
1.96 \mathrm{E}+02 \\
7.75 \mathrm{E}+02 \\
1.68 \mathrm{E}+03 \\
1.61 \mathrm{E}+03 \\
3.88 \mathrm{E}+03 \\
3.36 \mathrm{E}+03 \\
6.89 \mathrm{E}+02 \\
2.43 \mathrm{E}+03 \\
4.51 \mathrm{E}+03 \\
7.05 \mathrm{E}+02 \\
9.63 \mathrm{E}+02 \\
1.00 \mathrm{E}+03 \\
9.86 \mathrm{E}+03 \\
9.39 \mathrm{E}+03 \\
2.37 \mathrm{E}+03 \\
6.22 \mathrm{E}+02 \\
7.51 \mathrm{E}+02 \\
8.33 \mathrm{0}+02 \\
8.44 \mathrm{E}+02 \\
8.54 \mathrm{E}+02 \\
8.87 \mathrm{E}+02 \\
9.30 \mathrm{E}+02 \\
9.05 \mathrm{E}+02 \\
9.73 \mathrm{E}+02 \\
1.05 \mathrm{E}+03 \\
8.12 \mathrm{E}+02 \\
3.91 \mathrm{E}+02 \\
8.18 \mathrm{E}+03 \\
1.17 \mathrm{E}+03 \\
1.21 \mathrm{E}+03 \\
5.73 \mathrm{E}+02 \\
4.23 \mathrm{E}+02 \\
1.65 \mathrm{E}+03 \\
2.70 \mathrm{E}+03 \\
1.04 \mathrm{E}+03 \\
5.05 \mathrm{E}+02 \\
3.70 \mathrm{~N}+02 \\
5.17 \mathrm{E}+02 \\
4.04 \mathrm{E}+02 \\
6.24 \mathrm{E}+02 \\
1.40 \mathrm{E}+02 \\
1.55 \mathrm{E}+03 \\
7.33 \mathrm{E}+02 \\
4.35 \mathrm{E}+02 \\
3.91 \mathrm{E}+02 \\
4.08 \mathrm{E}+02 \\
7.36 \mathrm{E}+02 \\
1.43 \mathrm{E}+03 \\
1.70 \mathrm{E}+03 \\
4.40 \mathrm{E}+03 \\
2.15 \mathrm{E}+03 \\
8.59 \mathrm{E}+02 \\
9.31 \mathrm{E}+02 \\
5.09 \mathrm{E}+02 \\
5.06 \mathrm{E}+02 \\
2.09 \mathrm{E}+02 \\
1.02 \mathrm{E}+03 \\
6.32 \mathrm{E}+02 \\
2.61 \mathrm{E}+03 \\
6.52 \mathrm{E}+03 \\
2.77 \mathrm{E}+03\end{array}$ & $\begin{array}{l}\text { EU152 } \\
2.06 \mathrm{E}+00 \\
8.25 \mathrm{E}-01 \\
4.18 \mathrm{E}-01 \\
2.14 \mathrm{E}-01 \\
3.94 \mathrm{E}-01 \\
1.19 \mathrm{E}+00 \\
2.59 \mathrm{E}+00 \\
1.50 \mathrm{E}+00 \\
3.37 \mathrm{E}+00 \\
3.66 \mathrm{E}+00 \\
5.11 \mathrm{E}-01 \\
2.28 \mathrm{E}+00 \\
3.94 \mathrm{E}+00 \\
5.73 \mathrm{E}-01 \\
1.18 \mathrm{E}+00 \\
1.56 \mathrm{E}+00 \\
9.92 \mathrm{E}+00 \\
7.14 \mathrm{E}+00 \\
2.09 \mathrm{E}+00 \\
5.41 \mathrm{E}-01 \\
6.45 \mathrm{E}-01 \\
9.13 \mathrm{E}-01 \\
9.87 \mathrm{E}-01 \\
9.89 \mathrm{E}-01 \\
1.33 \mathrm{E}+00 \\
1.05 \mathrm{E}+00 \\
1.62 \mathrm{E}+00 \\
4.57 \mathrm{E}-01 \\
4.39 \mathrm{E}-01 \\
2.14 \mathrm{E}-01 \\
9.08 \mathrm{E}-02 \\
4.45 \mathrm{E}+00 \\
5.56 \mathrm{E}-01 \\
6.63 \mathrm{E}-01 \\
2.13 \mathrm{E}-01 \\
1.16 \mathrm{E}-01 \\
4.35 \mathrm{E}-01 \\
1.12 \mathrm{E}+00 \\
2.43 \mathrm{E}-01 \\
1.97 \mathrm{E}-01 \\
2.09 \mathrm{E}-01 \\
9.16 \mathrm{E}-01 \\
4.94 \mathrm{E}-01 \\
1.26 \mathrm{E}+00 \\
4.41 \mathrm{E}-01 \\
5.34 \mathrm{E}+00 \\
1.67 \mathrm{E}+00 \\
1.22 \mathrm{E}+00 \\
1.26 \mathrm{E}+00 \\
1.41 \mathrm{E}+00 \\
6.58 \mathrm{E}-01 \\
1.14 \mathrm{E}+00 \\
1.90 \mathrm{E}+00 \\
4.23 \mathrm{E}+00 \\
1.79 \mathrm{E}+00 \\
1.30 \mathrm{E}+00 \\
5.25 \mathrm{E}-01 \\
3.37 \mathrm{E}-01 \\
3.92 \mathrm{E}-01 \\
2.95 \mathrm{E}-01 \\
8.97 \mathrm{E}-01 \\
7.72 \mathrm{E}-01 \\
1.51 \mathrm{E}+00 \\
3.37 \mathrm{E}+00 \\
1.11 \mathrm{E}+00\end{array}$ & $\begin{array}{l}\mathrm{EU15} \\
1.60 \mathrm{E}+02 \\
6.61 \mathrm{E}+01 \\
3.66 \mathrm{E}+01 \\
1.91 \mathrm{E}+01 \\
3.36 \mathrm{E}+01 \\
9.66 \mathrm{E}+01 \\
2.10 \mathrm{E}+02 \\
1.08 \mathrm{E}+02 \\
2.42 \mathrm{E}+02 \\
2.60 \mathrm{E}+02 \\
3.72 \mathrm{E}+01 \\
1.64 \mathrm{E}+02 \\
2.85 \mathrm{E}+02 \\
4.15 \mathrm{E}+01 \\
8.20 \mathrm{E}+01 \\
1.05 \mathrm{E}+02 \\
7.08 \mathrm{E}+02 \\
5.28 \mathrm{E}+02 \\
1.51 \mathrm{E}+02 \\
3.89 \mathrm{E}+01 \\
4.60 \mathrm{E}+01 \\
6.52 \mathrm{E}+01 \\
7.01 \mathrm{E}+01 \\
6.97 \mathrm{E}+01 \\
9.20 \mathrm{E}+01 \\
7.26 \mathrm{E}+01 \\
1.12 \mathrm{E}+02 \\
4.12 \mathrm{E}+01 \\
4.09 \mathrm{E}+01 \\
2.21 \mathrm{E}+01 \\
9.72 \mathrm{E}+00 \\
3.42 \mathrm{E}+02 \\
4.35 \mathrm{E}+01 \\
5.11 \mathrm{E}+01 \\
1.72 \mathrm{E}+01 \\
1.17 \mathrm{E}+01 \\
4.48 \mathrm{E}+01 \\
1.04 \mathrm{E}+02 \\
2.60 \mathrm{E}+01 \\
1.87 \mathrm{E}+01 \\
2.17 \mathrm{E}+01 \\
8.21 \mathrm{E}+01 \\
4.46 \mathrm{E}+01 \\
1.13 \mathrm{E}+02 \\
4.13 \mathrm{E}+01 \\
5.15 \mathrm{E}+02 \\
1.50 \mathrm{E}+02 \\
1.11 \mathrm{E}+02 \\
1.19 \mathrm{E}+02 \\
1.36 \mathrm{E}+02 \\
5.59 \mathrm{E}+01 \\
9.69 \mathrm{E}+01 \\
1.56 \mathrm{E}+02 \\
3.52 \mathrm{E}+02 \\
1.51 \mathrm{E}+02 \\
1.06 \mathrm{E}+02 \\
5.45 \mathrm{E}+01 \\
3.37 \mathrm{E}+01 \\
3.86 \mathrm{E}+01 \\
2.67 \mathrm{E}+01 \\
8.64 \mathrm{E}+01 \\
7.20 \mathrm{E}+01 \\
1.17 \mathrm{E}+02 \\
2.63 \mathrm{E}+02 \\
9.03 \mathrm{E}+01\end{array}$ & $\begin{array}{l}E \cup 155 \\
2.23 E+02 \\
1.08 E+02 \\
1.51 E+01 \\
7.83 E+00 \\
8.50 E+00 \\
3.41 E+01 \\
7.44 E+01 \\
6.65 E+01 \\
1.62 E+02 \\
1.30 E+02 \\
2.98 E+01 \\
1.02 E+02 \\
1.92 E+02 \\
3.01 E+01 \\
3.28 E+01 \\
2.55 E+01 \\
3.93 E+02 \\
4.37 E+02 \\
1.03 E+02 \\
2.60 E+01 \\
3.03 E+01 \\
3.30 E+01 \\
3.21 E+01 \\
3.15 E+01 \\
2.63 E+01 \\
3.16 E+01 \\
2.29 E+01 \\
4.92 E+01 \\
5.61 E+01 \\
4.61 E+01 \\
2.26 E+01 \\
4.08 E+02 \\
5.93 E+01 \\
6.13 E+01 \\
2.94 E+01 \\
2.28 E+01 \\
9.33 E+01 \\
1.42 E+02 \\
6.08 E+01 \\
2.80 E+01 \\
1.83 E+01 \\
2.41 E+01 \\
1.73 E+01 \\
3.09 E+01 \\
7.30 E+00 \\
8.28 E+01 \\
3.71 E+01 \\
2.16 E+01 \\
1.99 E+01 \\
2.20 E+01 \\
3.57 E+01 \\
6.88 E+01 \\
7.13 E+01 \\
1.96 E+02 \\
1.01 E+02 \\
3.27 E+01 \\
4.64 E+01 \\
2.44 E+01 \\
2.49 E+01 \\
9.55 E+00 \\
4.90 E+01 \\
3.07 E+01 \\
1.35 E+02 \\
3.42 E+02 \\
1.49 E+02\end{array}$ & 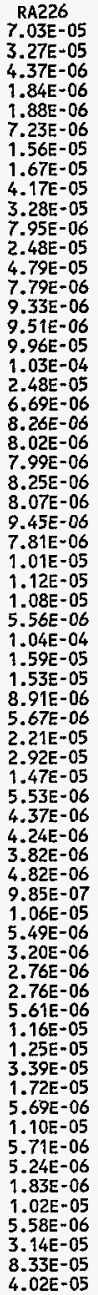 & $\begin{array}{l}\text { RA228 } \\
3.02 E-09 \\
1.39 E-09 \\
2.36 E-10 \\
1.03 E-10 \\
1.16 E-10 \\
4.47 E-10 \\
9.67 E-10 \\
8.15 E-10 \\
2.01 E-09 \\
1.65 E-09 \\
3.72 E-10 \\
1.22 E-09 \\
2.32 E-09 \\
3.71 E-10 \\
4.78 E-10 \\
5.06 E-10 \\
4.94 E-09 \\
4.90 E-09 \\
1.21 E-09 \\
3.23 E-10 \\
3.96 E-10 \\
4.06 E-10 \\
4.09 E-10 \\
4.20 E-10 \\
4.31 E-10 \\
4.75 E-10 \\
4.33 E-10 \\
1.28 E-10 \\
1.33 E-10 \\
9.74 E-11 \\
4.63 E-11 \\
4.67 E-09 \\
6.98 E-10 \\
6.89 E-10 \\
3.78 E-10 \\
5.21 E-11 \\
1.99 E-10 \\
3.44 E-10 \\
1.23 E-10 \\
6.26 E-11 \\
2.27 E-10 \\
2.818-10 \\
2.31 E-10 \\
3.31 E-10 \\
7.68 E-11 \\
8.54 E-10 \\
3.89 E-10 \\
2.39 E-10 \\
2.17 E-10 \\
2.23 E-10 \\
1.03 E-10 \\
1.99 E-10 \\
2.59 E-10 \\
6.49 E-10 \\
3.05 E-10 \\
1.37 E-10 \\
5.70 E-10 \\
3.05 E-10 \\
2.90 E-10 \\
1.15 E-10 \\
5.78 E-10 \\
3.41 E-10 \\
1.43 E-09 \\
3.73 E-09 \\
1.73 E-09\end{array}$ & $\begin{array}{l}\text { AC227 } \\
3.59 E-04 \\
1.68 E-04 \\
2.13 E-05 \\
9.01 E-06 \\
8.96 E-06 \\
4.26 E-05 \\
9.21 E-05 \\
8.19 E-05 \\
2.06 E-04 \\
1.60 E-04 \\
3.95 E-05 \\
1.22 E-04 \\
2.37 E-04 \\
3.86 E-05 \\
4.50 E-05 \\
4.48 E-05 \\
4.87 E-04 \\
5.17 E-04 \\
1.23 E-04 \\
3.30 E-05 \\
4.06 E-05 \\
3.92 E-05 \\
3.88 E-05 \\
4.00 E-05 \\
3.85 E-05 \\
4.56 E-05 \\
3.69 E-05 \\
5.74 E-05 \\
6.44 E-05 \\
6.38 E-05 \\
3.28 E-05 \\
5.30 E-04 \\
8.11 E-05 \\
7.79 E-05 \\
4.60 E-05 \\
3.32 E-05 \\
1.30 E-04 \\
1.68 E-04 \\
8.72 E-05 \\
3.20 E-05 \\
2.74 E-05 \\
2.43 E-05 \\
2.22 E-05 \\
2.76 E-05 \\
5.55 E-06 \\
5.92 E-05 \\
3.14 E-05 \\
1.81 E-05 \\
1.55 E-05 \\
1.54 E-05 \\
3.09 E-05 \\
6.40 E-05 \\
6.72 E-05 \\
1.85 E-04 \\
9.49 E-05 \\
3.02 E-05 \\
6.86 E-05 \\
3.52 E-05 \\
3.20 E-05 \\
1.06 E-05 \\
6.15 E-05 \\
3.29 E-05 \\
1.60 E-04 \\
4.27 E-04 \\
2.08 E-04\end{array}$ & $\begin{array}{l}\text { TH229 } \\
1.67 \mathrm{E}-07 \\
7.18 \mathrm{E}-08 \\
2.26 \mathrm{E}-08 \\
1.01 \mathrm{E}-08 \\
1.38 \mathrm{E}-08 \\
4.23 \mathrm{E}-08 \\
9.15 \mathrm{E}-08 \\
6.53 \mathrm{E}-08 \\
1.55 \mathrm{E}-07 \\
1.43 \mathrm{E}-07 \\
2.67 \mathrm{E}-08 \\
9.78 \mathrm{E}-08 \\
1.79 \mathrm{E}-07 \\
2.78 \mathrm{E}-08 \\
4.43 \mathrm{E}-08 \\
5.32 \mathrm{E}-08 \\
4.12 \mathrm{E}-07 \\
3.52 \mathrm{E}-07 \\
9.33 \mathrm{E}-08 \\
2.49 \mathrm{E}-08 \\
3.06 \mathrm{E}-08 \\
3.52 \mathrm{E}-08 \\
3.67 \mathrm{E}-08 \\
3.77 \mathrm{E}-08 \\
4.40 \mathrm{E}-08 \\
4.25 \mathrm{E}-08 \\
4.77 \mathrm{E}-08 \\
2.61 \mathrm{E}-08 \\
2.70 \mathrm{E}-08 \\
1.96 \mathrm{E}-08 \\
9.28 \mathrm{E}-09 \\
2.86 \mathrm{E}-07 \\
3.99 \mathrm{E}-08 \\
4.21 \mathrm{E}-08 \\
1.90 \mathrm{E}-08 \\
1.04 \mathrm{E}-08 \\
3.99 \mathrm{E}-08 \\
7.01 \mathrm{E}-08 \\
2.47 \mathrm{E}-08 \\
1.27 \mathrm{E}-08 \\
1.49 \mathrm{E}-08 \\
2.94 \mathrm{E}-08 \\
2.11 \mathrm{E}-08 \\
3.61 \mathrm{E}-08 \\
9.99 \mathrm{E}-09 \\
1.16 \mathrm{E}-07 \\
4.42 \mathrm{E}-08 \\
2.96 \mathrm{E}-08 \\
2.87 \mathrm{E}-08 \\
3.03 \mathrm{E}-08 \\
2.19 \mathrm{E}-08 \\
4.20 \mathrm{E}-08 \\
5.58 \mathrm{E}-08 \\
1.11 \mathrm{E}-08 \\
1.38 \mathrm{E}-07 \\
3.44 \mathrm{E}-08 \\
3.09 \mathrm{E}-08 \\
3.08 \mathrm{E}-08 \\
2.21 \mathrm{E}-08-08 \\
9.05 \mathrm{E}-08 \\
\end{array}$ \\
\hline
\end{tabular}


HNF-SD-WM-TI-794, ReV, 0

ACTIVITY IN UNSEPARATED FUEL (Curies decayed to 1/1/94)

\begin{tabular}{|c|c|c|c|c|c|c|c|c|c|}
\hline $\begin{array}{c}\text { Listing } \# \text { ] } \\
781 \\
782 \\
783 \\
784 \\
785 \\
786 \\
787 \\
788 \\
789 \\
790 \\
791 \\
792 \\
793 \\
794 \\
795 \\
796 \\
797 \\
798 \\
799 \\
800 \\
801 \\
802 \\
803 \\
804 \\
805 \\
806 \\
807 \\
808 \\
809 \\
810 \\
811 \\
812 \\
813 \\
814 \\
815 \\
816 \\
817 \\
818 \\
819 \\
820 \\
821 \\
822 \\
823 \\
824 \\
825 \\
826 \\
827 \\
828 \\
829 \\
830 \\
831 \\
832 \\
833 \\
834 \\
835 \\
836 \\
837 \\
838 \\
839 \\
840 \\
841 \\
842 \\
843 \\
844 \\
845\end{array}$ & $\begin{array}{l}\text { TH232 } \\
4.64 \mathrm{E}-09 \\
2.15 \mathrm{E}-09 \\
3.55 \mathrm{E}-10 \\
1.57 \mathrm{E}-10 \\
1.75 \mathrm{E}-10 \\
6.86 \mathrm{E}-10 \\
1.48 \mathrm{E}-09 \\
1.26 \mathrm{E}-09 \\
3.10 \mathrm{E}-09 \\
2.56 \mathrm{E}-09 \\
5.73 \mathrm{E}-10 \\
1.89 \mathrm{E}-09 \\
3.58 \mathrm{E}-09 \\
5.72 \mathrm{E}-10 \\
7.35 \mathrm{E}-10 \\
7.72 \mathrm{E}-10 \\
7.63 \mathrm{E}-09 \\
7.61 \mathrm{E}-09 \\
1.87 \mathrm{E}-09 \\
4.97 \mathrm{E}-10 \\
6.08 \mathrm{E}-10 \\
6.29 \mathrm{E}-10 \\
6.33 \mathrm{E}-10 \\
6.48 \mathrm{E}-10 \\
6.64 \mathrm{E}-10 \\
7.26 \mathrm{E}-10 \\
6.70 \mathrm{E}-10 \\
1.97 \mathrm{E}-10 \\
2.06 \mathrm{E}-10 \\
1.52 \mathrm{E}-10 \\
7.23 \mathrm{E}-11 \\
7.26 \mathrm{E}-09 \\
1.08 \mathrm{E}-09 \\
1.07 \mathrm{E}-09 \\
5.85 \mathrm{E}-10 \\
8.07 \mathrm{E}-11 \\
3.09 \mathrm{E}-10 \\
5.34 \mathrm{E}-10 \\
1.92 \mathrm{E}-10 \\
9.76 \mathrm{E}-11 \\
3.55 \mathrm{E}-10 \\
4.33 \mathrm{E}-10 \\
3.52 \mathrm{E}-10 \\
5.14 \mathrm{E}-10 \\
1.19 \mathrm{E}-10 \\
1.32 \mathrm{E}-09 \\
6.04 \mathrm{E}-10 \\
3.69 \mathrm{E}-10 \\
3.35 \mathrm{E}-10 \\
3.45 \mathrm{E}-10 \\
1.61 \mathrm{E}-10 \\
3.10 \mathrm{E}-10 \\
4.00 \mathrm{E}-10 \\
1.00 \mathrm{E}-09 \\
4.72 \mathrm{E}-10 \\
2.13 \mathrm{E}-10 \\
8.90 \mathrm{E}-10 \\
4.74 \mathrm{E}-10 \\
4.52 \mathrm{E}-10 \\
1.77 \mathrm{E}-10 \\
8.96 \mathrm{E}-10 \\
5.29 \mathrm{E}-10 \\
2.24 \mathrm{E}-09 \\
5.83 \mathrm{E}-09 \\
2.71 \mathrm{E}-09\end{array}$ & $\begin{array}{l}\text { PA231 } \\
1.12 \mathrm{E}-03 \\
5.35 \mathrm{E}-04 \\
6.41 \mathrm{E}-05 \\
2.78 \mathrm{E}-05 \\
2.71 \mathrm{E}-05 \\
1.33 \mathrm{E}-04 \\
2.87 \mathrm{E}-04 \\
2.59 \mathrm{E}-04 \\
6.47 \mathrm{E}-04 \\
5.05 \mathrm{E}-04 \\
1.24 \mathrm{E}-04 \\
3.88 \mathrm{E}-04 \\
7.48 \mathrm{E}-04 \\
1.21 \mathrm{E}-04 \\
1.40 \mathrm{E}-04 \\
1.37 \mathrm{E}-04 \\
1.54 \mathrm{E}-03 \\
1.65 \mathrm{E}-03 \\
3.90 \mathrm{E}-04 \\
1.04 \mathrm{E}-04 \\
1.27 \mathrm{E}-04 \\
1.24 \mathrm{E}-04 \\
1.23 \mathrm{E}-04 \\
1.26 \mathrm{E}-04 \\
1.20 \mathrm{E}-04 \\
1.41 \mathrm{E}-04 \\
1.16 \mathrm{E}-04 \\
1.81 \mathrm{E}-04 \\
2.06 \mathrm{E}-04 \\
2.06 \mathrm{E}-04 \\
1.06 \mathrm{E}-04 \\
1.69 \mathrm{E}-03 \\
2.60 \mathrm{E}-04 \\
2.50 \mathrm{E}-04 \\
1.47 \mathrm{E}-04 \\
1.06 \mathrm{E}-04 \\
4.19 \mathrm{E}-04 \\
5.34 \mathrm{E}-04 \\
2.82 \mathrm{E}-04 \\
1.03 \mathrm{E}-04 \\
8.81 \mathrm{E}-05 \\
7.57 \mathrm{E}-05 \\
6.84 \mathrm{E}-05 \\
8.70 \mathrm{E}-05 \\
1.72 \mathrm{E}-05 \\
1.83 \mathrm{E}-04 \\
9.91 \mathrm{E}-05 \\
5.63 \mathrm{E}-05 \\
4.77 \mathrm{E}-05 \\
4.79 \mathrm{E}-05 \\
9.89 \mathrm{E}-05 \\
2.04 \mathrm{E}-04 \\
2.11 \mathrm{E}-04 \\
5.83 \mathrm{E}-04 \\
3.00 \mathrm{E}-04 \\
9.54 \mathrm{E}-05 \\
2.21 \mathrm{E}-04 \\
1.12 \mathrm{E}-04 \\
1.03 \mathrm{E}-04 \\
3.31 \mathrm{E}-05 \\
1.96 \mathrm{E}-04 \\
1.04 \mathrm{E}-04 \\
5.17 \mathrm{E}-04 \\
1.38 \mathrm{E}-03 \\
6.70 \mathrm{E}-04\end{array}$ & $\begin{array}{c}\text { U232 } \\
3.44 \mathrm{E}-03 \\
1.45 \mathrm{E}-03 \\
7.43 \mathrm{E}-04 \\
3.61 \mathrm{E}-04 \\
6.07 \mathrm{E}-04 \\
1.50 \mathrm{E}-03 \\
3.26 \mathrm{E}-03 \\
1.82 \mathrm{E}-03 \\
4.20 \mathrm{E}-03 \\
4.22 \mathrm{E}-03 \\
6.76 \mathrm{E}-04 \\
2.75 \mathrm{E}-03 \\
4.88 \mathrm{E}-03 \\
7.32 \mathrm{E}-04 \\
1.33 \mathrm{E}-03 \\
1.68 \mathrm{E}-03 \\
1.18 \mathrm{E}-02 \\
9.23 \mathrm{E}-03 \\
2.57 \mathrm{E}-03 \\
6.74 \mathrm{E}-04 \\
8.13 \mathrm{E}-04 \\
1.05 \mathrm{E}-03 \\
1.11 \mathrm{E}-03 \\
1.12 \mathrm{E}-03 \\
1.42 \mathrm{E}-03 \\
1.22 \mathrm{E}-03 \\
1.65 \mathrm{E}-03 \\
5.53 \mathrm{E}-04 \\
5.62 \mathrm{E}-04 \\
3.62 \mathrm{E}-04 \\
1.68 \mathrm{E}-04 \\
6.60 \mathrm{E}-03 \\
8.77 \mathrm{E}-04 \\
9.78 \mathrm{E}-04 \\
3.82 \mathrm{E}-04 \\
1.92 \mathrm{E}-04 \\
7.37 \mathrm{E}-04 \\
1.45 \mathrm{E}-03 \\
4.47 \mathrm{E}-04 \\
2.62 \mathrm{E}-04 \\
3.76 \mathrm{E}-04 \\
1.23 \mathrm{E}-03 \\
7.26 \mathrm{E}-04 \\
1.62 \mathrm{E}-03 \\
5.68 \mathrm{E}-04 \\
7.03 \mathrm{E}-03 \\
2.11 \mathrm{E}-03 \\
1.55 \mathrm{E}-03 \\
1.65 \mathrm{E}-03 \\
1.84 \mathrm{E}-03 \\
5.91 \mathrm{E}-04 \\
1.08 \mathrm{E}-03 \\
1.60 \mathrm{E}-03 \\
3.74 \mathrm{E}-03 \\
1.67 \mathrm{E}-03 \\
9.89 \mathrm{E}-04 \\
9.42 \mathrm{E}-04 \\
5.74 \mathrm{E}-04 \\
6.30 \mathrm{E}-04 \\
4.13 \mathrm{E}-04 \\
1.39 \mathrm{E}-03 \\
1.10 \mathrm{E}-03 \\
2.16 \mathrm{E}-03 \\
5.08 \mathrm{E}-03 \\
1.90 \mathrm{E}-03\end{array}$ & $\begin{array}{l}\text { U233 } \\
9.23 \mathrm{E}-05 \\
4.03 \mathrm{E}-05 \\
1.23 \mathrm{E}-05 \\
5.64 \mathrm{E}-06 \\
7.64 \mathrm{E}-06 \\
2.40 \mathrm{E}-05 \\
5.19 \mathrm{E}-05 \\
3.68 \mathrm{E}-05 \\
8.68 \mathrm{E}-05 \\
8.12 \mathrm{E}-05 \\
1.49 \mathrm{E}-05 \\
5.53 \mathrm{E}-05 \\
1.01 \mathrm{E}-04 \\
1.55 \mathrm{E}-05 \\
2.49 \mathrm{E}-05 \\
2.98 \mathrm{E}-05 \\
2.32 \mathrm{E}-04 \\
1.99 \mathrm{E}-04 \\
5.26 \mathrm{E}-05 \\
1.39 \mathrm{E}-05 \\
1.70 \mathrm{E}-05 \\
2.01 \mathrm{E}-05 \\
2.09 \mathrm{E}-05 \\
2.13 \mathrm{E}-05 \\
2.51 \mathrm{E}-05 \\
2.36 \mathrm{E}-05 \\
2.76 \mathrm{E}-05 \\
1.37 \mathrm{E}-05 \\
1.43 \mathrm{E}-05 \\
1.04 \mathrm{E}-05 \\
4.95 \mathrm{E}-06 \\
1.62 \mathrm{E}-04 \\
2.25 \mathrm{E}-05 \\
2.39 \mathrm{E}-05 \\
1.07 \mathrm{E}-05 \\
5.51 \mathrm{E}-06 \\
2.12 \mathrm{E}-05 \\
3.71 \mathrm{E}-05 \\
1.32 \mathrm{E}-05 \\
6.81 \mathrm{E}-05 \\
8.65 \mathrm{E}-06 \\
1.67 \mathrm{E}-05 \\
1.18 \mathrm{E}-05 \\
2.08 \mathrm{E}-05 \\
5.73 \mathrm{E}-06 \\
6.66 \mathrm{E}-05 \\
2.55 \mathrm{E}-05 \\
1.69 \mathrm{E}-05 \\
1.64 \mathrm{E}-05 \\
1.74 \mathrm{E}-05 \\
1.18 \mathrm{E}-05 \\
2.24 \mathrm{E}-05 \\
2.96 \mathrm{E}-05 \\
7.32 \mathrm{E}-05 \\
3.42 \mathrm{E}-05 \\
1.67 \mathrm{E}-05 \\
2.17 \mathrm{E}-05 \\
1.23 \mathrm{E}-05 \\
1.26 \mathrm{E}-05 \\
6.26 \mathrm{E}-00 \\
2.62 \mathrm{E}-05 \\
1.77 \mathrm{E}-05 \\
5.14 \mathrm{E}-05 \\
1.26 \mathrm{E}-04 \\
5.15 \mathrm{E}-05\end{array}$ & $\begin{array}{l}\text { U234 } \\
5.43 \mathrm{E}+01 \\
2.61 \mathrm{E}+01 \\
3.07 \mathrm{E}+00 \\
1.38 \mathrm{E}+00 \\
1.36 \mathrm{E}+00 \\
5.55 \mathrm{E}+00 \\
1.20 \mathrm{E}+01 \\
1.32 \mathrm{E}+01 \\
3.28 \mathrm{E}+01 \\
2.61 \mathrm{E}+01 \\
6.21 \mathrm{E}+00 \\
1.99 \mathrm{E}+01 \\
3.81 \mathrm{E}+01 \\
6.13 \mathrm{E}+00 \\
7.21 \mathrm{E}+00 \\
7.06 \mathrm{E}+00 \\
7.89 \mathrm{E}+01 \\
8.42 \mathrm{E}+01 \\
2.00 \mathrm{E}+01 \\
5.26 \mathrm{E}+00 \\
6.36 \mathrm{E}+00 \\
6.48 \mathrm{E}+00 \\
6.43 \mathrm{E}+00 \\
6.52 \mathrm{E}+00 \\
6.30 \mathrm{E}+00 \\
7.13 \mathrm{E}+00 \\
6.22 \mathrm{E}+00 \\
7.95 \mathrm{E}+00 \\
9.09 \mathrm{E}+00 \\
8.95 \mathrm{E}+00 \\
4.61 \mathrm{E}+00 \\
8.48 \mathrm{E}+01 \\
1.29 \mathrm{E}+01 \\
1.26 \mathrm{E}+01 \\
7.16 \mathrm{E}+00 \\
4.55 \mathrm{E}+00 \\
1.82 \mathrm{E}+01 \\
2.34 \mathrm{E}+01 \\
1.23 \mathrm{E}+01 \\
4.56 \mathrm{E}+00 \\
3.62 \mathrm{E}+00 \\
3.29 \mathrm{E}+00 \\
2.85 \mathrm{E}+00 \\
3.86 \mathrm{E}+00 \\
7.82 \mathrm{E}-01 \\
8.39 \mathrm{E}+00 \\
4.43 \mathrm{E}+00 \\
2.51 \mathrm{E}+00 \\
2.15 \mathrm{E}+00 \\
2.20 \mathrm{E}+00 \\
4.60 \mathrm{E}+00 \\
9.29 \mathrm{E}+00 \\
9.79 \mathrm{E}+00 \\
2.67 \mathrm{E}+01 \\
1.37 \mathrm{E}+01 \\
4.58 \mathrm{E}+00 \\
9.11 \mathrm{E}+00 \\
4.62 \mathrm{E}+00 \\
4.31 \mathrm{E}+00 \\
1.42 \mathrm{E}+00 \\
8.22 \mathrm{E}+00 \\
4.50 \mathrm{E}+00 \\
2.63 \mathrm{E}+01 \\
6.96 \mathrm{E}+01 \\
3.34 \mathrm{E}+01\end{array}$ & $\begin{array}{l}\text { U235 } \\
2.06 \mathrm{E}+00 \\
9.93 \mathrm{E}-01 \\
1.11 \mathrm{E}-01 \\
4.97 \mathrm{E}-02 \\
4.71 \mathrm{E}-02 \\
2.40 \mathrm{E}-01 \\
5.19 \mathrm{E}-01 \\
4.77 \mathrm{E}-01 \\
1.19 \mathrm{E}+00 \\
9.30 \mathrm{E}-01 \\
2.27 \mathrm{E}-01 \\
7.19 \mathrm{E}-01 \\
1.38 \mathrm{E}+00 \\
2.23 \mathrm{E}-01 \\
2.54 \mathrm{E}-01 \\
2.43 \mathrm{E}-01 \\
2.83 \mathrm{E}+00 \\
3.09 \mathrm{E}+00 \\
7.24 \mathrm{E}-01 \\
1.91 \mathrm{E}-01 \\
2.31 \mathrm{E}-01 \\
2.31 \mathrm{E}-01 \\
2.28 \mathrm{E}-01 \\
2.31 \mathrm{E}-01 \\
2.18 \mathrm{E}-01 \\
2.52 \mathrm{E}-01 \\
2.12 \mathrm{E}-01 \\
3.35 \mathrm{E}-01 \\
3.86 \mathrm{E}-01 \\
3.88 \mathrm{E}-01 \\
2.01 \mathrm{E}-01 \\
3.18 \mathrm{E}+00 \\
4.87 \mathrm{E}-01 \\
4.71 \mathrm{E}-01 \\
2.73 \mathrm{E}-01 \\
1.97 \mathrm{E}-01 \\
7.90 \mathrm{E}-01 \\
9.95 \mathrm{E}-01 \\
5.36 \mathrm{E}-01 \\
1.95 \mathrm{E}-01 \\
1.67 \mathrm{E}-01 \\
1.37 \mathrm{E}-01 \\
1.22 \mathrm{E}-01 \\
1.60 \mathrm{E}-01 \\
3.11 \mathrm{E}-02 \\
3.29 \mathrm{E}-01 \\
1.82 \mathrm{E}-01 \\
1.02 \mathrm{E}-01 \\
8.52 \mathrm{E}-02 \\
8.63 \mathrm{E}-02 \\
1.85 \mathrm{E}-01 \\
3.78 \mathrm{E}-01 \\
3.87 \mathrm{E}-01 \\
1.07 \mathrm{E}+00 \\
5.55 \mathrm{E}-01 \\
1.76 \mathrm{E}-01 \\
4.20 \mathrm{E}-01 \\
2.10 \mathrm{E}-01 \\
1.93 \mathrm{E}-01 \\
6.01 \mathrm{E}-02 \\
3.64 \mathrm{E}-01 \\
1.94 \mathrm{E}-01 \\
9.83 \mathrm{E}-01 \\
2.62 \mathrm{E}+00 \\
1.27 \mathrm{E}+00\end{array}$ & $\begin{array}{l}\text { U236 } \\
3.65 \mathrm{E}+00 \\
1.72 \mathrm{E}+00 \\
2.67 \mathrm{E}-01 \\
1.22 \mathrm{E}-01 \\
1.34 \mathrm{E}-01 \\
5.39 \mathrm{E}-01 \\
1.17 \mathrm{E}+00 \\
1.01 \mathrm{E}+00 \\
2.46 \mathrm{E}+00 \\
2.04 \mathrm{E}+00 \\
4.53 \mathrm{E}-01 \\
1.52 \mathrm{E}+00 \\
2.86 \mathrm{E}+00 \\
4.54 \mathrm{E}-01 \\
5.79 \mathrm{E}-01 \\
5.96 \mathrm{E}-01 \\
6.08 \mathrm{E}+00 \\
6.15 \mathrm{E}+00 \\
1.50 \mathrm{E}+00 \\
3.95 \mathrm{E}-01 \\
4.78 \mathrm{E}-01 \\
5.06 \mathrm{E}-01 \\
5.09 \mathrm{E}-01 \\
5.16 \mathrm{E}-01 \\
5.26 \mathrm{E}-01 \\
5.64 \mathrm{E}-01 \\
5.36 \mathrm{E}-01 \\
1.57 \mathrm{E}-01 \\
1.67 \mathrm{E}-01 \\
1.24 \mathrm{E}-01 \\
5.90 \mathrm{E}-02 \\
5.86 \mathrm{E}+00 \\
8.74 \mathrm{E}-01 \\
8.69 \mathrm{E}-01 \\
4.69 \mathrm{E}-01 \\
6.48 \mathrm{E}-02 \\
2.51 \mathrm{E}-01 \\
4.29 \mathrm{E}-01 \\
1.57 \mathrm{E}-01 \\
7.95 \mathrm{E}-02 \\
2.89 \mathrm{E}-01 \\
3.42 \mathrm{E}-01 \\
2.72 \mathrm{E}-01 \\
4.12 \mathrm{E}-01 \\
9.51 \mathrm{E}-02 \\
1.06 \mathrm{E}+00 \\
4.87 \mathrm{E}-01 \\
2.94 \mathrm{E}-01 \\
2.65 \mathrm{E}-01 \\
2.77 \mathrm{E}-01 \\
1.31 \mathrm{E}-01 \\
2.49 \mathrm{E}-01 \\
3.19 \mathrm{E}-01 \\
8.00 \mathrm{E}-01 \\
3.79 \mathrm{E}-01 \\
1.72 \mathrm{E}-01 \\
7.26 \mathrm{E}-01 \\
3.81 \mathrm{E}-01 \\
3.66 \mathrm{E}-01 \\
1.39 \mathrm{E}-01 \\
7.20 \mathrm{E}-01 \\
4.25 \mathrm{E}-01 \\
1.83 \mathrm{E}+00 \\
4.76 \mathrm{E}+00 \\
2.20 \mathrm{E}+00\end{array}$ & $\begin{array}{l}\text { U238 } \\
3.68 \mathrm{E}+01 \\
1.77 \mathrm{E}+01 \\
2.12 \mathrm{E}+00 \\
9.55 \mathrm{E}-01 \\
9.52 \mathrm{E}-01 \\
3.83 \mathrm{E}+00 \\
8.29 \mathrm{E}+00 \\
9.06 \mathrm{E}+00 \\
2.24 \mathrm{E}+01 \\
1.79 \mathrm{E}+01 \\
4.23 \mathrm{E}+00 \\
1.37 \mathrm{E}+01 \\
2.60 \mathrm{E}+01 \\
4.19 \mathrm{E}+00 \\
4.96 \mathrm{E}+00 \\
4.88 \mathrm{E}+00 \\
5.41 \mathrm{E}+01 \\
5.75 \mathrm{E}+01 \\
1.37 \mathrm{E}+01 \\
3.60 \mathrm{E}+00 \\
4.35 \mathrm{E}+00 \\
4.44 \mathrm{E}+00 \\
4.42 \mathrm{E}+00 \\
4.48 \mathrm{E}+00 \\
4.35 \mathrm{E}+00 \\
4.90 \mathrm{E}+00 \\
4.31 \mathrm{E}+00 \\
8.17 \mathrm{E}+00 \\
9.32 \mathrm{E}+00 \\
9.13 \mathrm{E}+00 \\
4.70 \mathrm{E}+00 \\
5.76 \mathrm{E}+01 \\
8.75 \mathrm{E}+00 \\
8.54 \mathrm{E}+00 \\
4.84 \mathrm{E}+00 \\
4.64 \mathrm{E}+00 \\
1.86 \mathrm{E}+01 \\
2.40 \mathrm{E}+01 \\
1.25 \mathrm{E}+01 \\
4.67 \mathrm{E}+00 \\
2.46 \mathrm{E}+00 \\
2.28 \mathrm{E}+00 \\
1.96 \mathrm{E}+00 \\
2.69 \mathrm{E}+00 \\
5.52 \mathrm{E}=01 \\
5.94 \mathrm{E}+00 \\
3.09 \mathrm{E}+00 \\
1.77 \mathrm{E}+00 \\
1.52 \mathrm{E}+00 \\
1.56 \mathrm{E}+00 \\
4.76 \mathrm{E}+00 \\
9.62 \mathrm{E}+00 \\
1.02 \mathrm{E}+01 \\
2.78 \mathrm{E}+01 \\
1.42 \mathrm{E}+01 \\
4.80 \mathrm{E}+00 \\
6.19 \mathrm{E}+00 \\
3.15 \mathrm{E}+00 \\
2.94 \mathrm{E}+00 \\
9.78 \mathrm{E}-01 \\
5.62 \mathrm{E}+00 \\
3.10 \mathrm{E}+00 \\
1.79 \mathrm{E}+01 \\
4.72 \mathrm{E}+01 \\
2.26 \mathrm{E}+01\end{array}$ & $\begin{array}{l}\text { NP237 } \\
4.42 \mathrm{E}-01 \\
1.93 \mathrm{E}-01 \\
6.28 \mathrm{E}-02 \\
2.95 \mathrm{E}-02 \\
4.11 \mathrm{E}-02 \\
1.27 \mathrm{E}-01 \\
2.76 \mathrm{E}-01 \\
1.85 \mathrm{E}-01 \\
4.32 \mathrm{E}-01 \\
4.18 \mathrm{E}-01 \\
7.29 \mathrm{E}-02 \\
2.79 \mathrm{E}-01 \\
5.01 \mathrm{E}-01 \\
7.65 \mathrm{E}-02 \\
1.30 \mathrm{E}-01 \\
1.59 \mathrm{E}-01 \\
1.18 \mathrm{E}+00 \\
9.90 \mathrm{E}-01 \\
2.63 \mathrm{E}-01 \\
6.93 \mathrm{E}-02 \\
8.38 \mathrm{E}-02 \\
1.04 \mathrm{E}-01 \\
1.09 \mathrm{E}-01 \\
1.11 \mathrm{E}-01 \\
1.35 \mathrm{E}-01 \\
1.21 \mathrm{E}-01 \\
1.53 \mathrm{E}-01 \\
5.52 \mathrm{E}-02 \\
5.75 \mathrm{E}-02 \\
4.07 \mathrm{E}-02 \\
1.93 \mathrm{E}-02 \\
7.90 \mathrm{E}-01 \\
1.10 \mathrm{E}-01 \\
1.17 \mathrm{E}-01 \\
5.14 \mathrm{E}-02 \\
2.15 \mathrm{E}-02 \\
8.29 \mathrm{E}-02 \\
1.48 \mathrm{E}-01 \\
5.13 \mathrm{E}-02 \\
2.72 \mathrm{E}-02 \\
4.41 \mathrm{E}-02 \\
9.06 \mathrm{E}-02 \\
6.16 \mathrm{E}-02 \\
1.15 \mathrm{E}-01 \\
3.30 \mathrm{E}-02 \\
3.88 \mathrm{E}-01 \\
1.43 \mathrm{E}-01 \\
9.61 \mathrm{E}-02 \\
9.43 \mathrm{E}-02 \\
1.31 \mathrm{E}-01 \\
6.50 \mathrm{E}-02 \\
3.30 \mathrm{E}-02 \\
1.33 \mathrm{E}-02 \\
1.36 \mathrm{E}-01 \\
9.40 \mathrm{E}-02 \\
2.54 \mathrm{E}-01 \\
6.19 \mathrm{E}-01 \\
2.50 \mathrm{E}-02 \\
1.29 \mathrm{E}-02 \\
3.14 \mathrm{E}-01 \\
\end{array}$ \\
\hline
\end{tabular}


HWF-SD-WM-TI-794, ReV. O

ACTIVITY IN UNSEPARATED FUEL (Curies decayed to $1 / 1 / 94$ )

\begin{tabular}{|c|c|c|c|c|c|c|c|c|c|}
\hline $\begin{array}{r}\text { ILting } \# \text { \#3 } \\
781 \\
782 \\
783 \\
784 \\
785 \\
786 \\
787 \\
788 \\
789 \\
790 \\
791 \\
792 \\
793 \\
794 \\
795 \\
796 \\
797 \\
798 \\
799 \\
800 \\
801 \\
802 \\
803 \\
804 \\
805 \\
806 \\
807 \\
808 \\
809 \\
810 \\
811 \\
812 \\
813 \\
814 \\
815 \\
816 \\
817 \\
818 \\
819 \\
820 \\
821 \\
822 \\
823 \\
824 \\
825 \\
826 \\
827 \\
828 \\
829 \\
830 \\
831 \\
832 \\
833 \\
834 \\
835 \\
836 \\
837 \\
838 \\
839 \\
840 \\
841 \\
842 \\
843 \\
844 \\
845\end{array}$ & 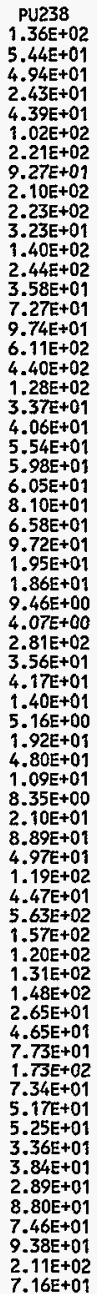 & $\begin{array}{l}P U 239 \\
3.90 E+03 \\
1.73 E+03 \\
4.46 E+02 \\
2.07 E+02 \\
2.54 E+02 \\
8.36 E+02 \\
1.81 E+03 \\
1.46 E+03 \\
3.47 E+03 \\
3.14 E+03 \\
5.99 E+02 \\
2.19 E+03 \\
4.02 E+03 \\
6.22 E+02 \\
9.34 E+02 \\
1.05 E+03 \\
9.08 E+03 \\
8.13 E+03 \\
2.11 E+03 \\
5.56 E+02 \\
6.73 E+02 \\
7.78 E+02 \\
8.02 E+02 \\
8.13 E+02 \\
9.09 E+02 \\
8.89 E+02 \\
9.76 E+02 \\
1.01 E+03 \\
1.07 E+03 \\
8.11 E+02 \\
3.89 E+02 \\
6.81 E+03 \\
9.61 E+02 \\
1.01 E+03 \\
4.63 E+02 \\
4.24 E+02 \\
1.65 E+03 \\
2.77 E+03 \\
1.04 E+03 \\
5.15 E+02 \\
3.51 E+02 \\
5.65 E+02 \\
4.19 E+02 \\
6.94 E+02 \\
1.72 E+02 \\
1.94 E+03 \\
8.35 E+02 \\
5.22 E+02 \\
4.84 E+02 \\
5.09 E+02 \\
8.06 E+02 \\
1.55 E+03 \\
1.95 E+03 \\
4.91 E+03 \\
2.34 E+03 \\
1.05 E+03 \\
8.82 E+02 \\
4.89 E+02 \\
4.92 E+02 \\
2.20 E+02 \\
1.01 E+03 \\
6.47 E+02 \\
2.18 E+03 \\
5.39 E+03 \\
2.24 E+03\end{array}$ & $\begin{array}{l}P U 240 \\
5.68 \mathrm{E}+02 \\
2.30 \mathrm{E}+02 \\
1.39 \mathrm{E}+02 \\
6.60 \mathrm{E}+01 \\
1.02 \mathrm{E}+02 \\
2.90 \mathrm{E}+02 \\
6.29 \mathrm{E}+02 \\
3.58 \mathrm{E}+02 \\
8.16 \mathrm{E}+02 \\
8.54 \mathrm{E}+02 \\
1.27 \mathrm{E}+02 \\
5.40 \mathrm{E}+02 \\
9.47 \mathrm{E}+02 \\
1.40 \mathrm{E}+02 \\
2.77 \mathrm{E}+02 \\
3.67 \mathrm{E}+02 \\
2.35 \mathrm{E}+03 \\
1.73 \mathrm{E}+03 \\
4.97 \mathrm{E}+02 \\
1.31 \mathrm{E}+02 \\
1.58 \mathrm{E}+02 \\
2.12 \mathrm{E}+02 \\
2.28 \mathrm{E}+02 \\
2.31 \mathrm{E}+02 \\
3.05 \mathrm{E}+02 \\
2.52 \mathrm{E}+02 \\
3.62 \mathrm{E}+02 \\
1.71 \mathrm{E}+02 \\
1.67 \mathrm{E}+02 \\
9.34 \mathrm{E}+01 \\
4.15 \mathrm{E}+01 \\
1.14 \mathrm{E}+03 \\
1.46 \mathrm{E}+02 \\
1.69 \mathrm{E}+02 \\
5.96 \mathrm{E}+01 \\
5.05 \mathrm{E}+01 \\
1.90 \mathrm{E}+02 \\
4.31 \mathrm{E}+02 \\
1.11 \mathrm{E}+02 \\
7.59 \mathrm{E}+01 \\
8.14 \mathrm{E}+01 \\
2.12 \mathrm{E}+02 \\
1.34 \mathrm{E}+02 \\
2.75 \mathrm{E}+02 \\
8.60 \mathrm{E}+01 \\
1.02 \mathrm{E}+03 \\
3.50 \mathrm{E}+02 \\
2.46 \mathrm{E}+02 \\
2.47 \mathrm{E}+02 \\
2.68 \mathrm{E}+02 \\
2.14 \mathrm{E}+02 \\
3.81 \mathrm{E}+02 \\
6.13 \mathrm{E}+02 \\
1.39 \mathrm{E}+03 \\
5.98 \mathrm{0}+02 \\
3.99 \mathrm{E}+02 \\
2.04 \mathrm{E}+02 \\
1.20 \mathrm{E}+02 \\
1.27 \mathrm{E}+02 \\
7.43 \mathrm{E}+01 \\
2.75 \mathrm{E}+02 \\
2.03 \mathrm{E}+02 \\
3.77 \mathrm{E}+02 \\
8.60 \mathrm{E}+02 \\
3.01 \mathrm{E}+02\end{array}$ & $\begin{array}{l}P U 241 \\
5.80 \mathrm{E}+03 \\
2.29 \mathrm{E}+03 \\
3.04 \mathrm{E}+03 \\
1.56 \mathrm{E}+03 \\
2.91 \mathrm{E}+03 \\
7.15 \mathrm{E}+03 \\
1.55 \mathrm{E}+04 \\
4.43 \mathrm{E}+03 \\
9.91 \mathrm{E}+03 \\
1.08 \mathrm{E}+04 \\
1.49 \mathrm{E}+03 \\
6.72 \mathrm{E}+03 \\
1.16 \mathrm{E}+04 \\
1.68 \mathrm{E}+03 \\
3.51 \mathrm{E}+03 \\
4.69 \mathrm{E}+03 \\
2.94 \mathrm{E}+04 \\
2.08 \mathrm{E}+04 \\
6.13 \mathrm{E}+03 \\
1.59 \mathrm{E}+03 \\
1.90 \mathrm{E}+03 \\
2.71 \mathrm{E}+03 \\
2.93 \mathrm{E}+03 \\
2.94 \mathrm{E}+03 \\
3.99 \mathrm{E}+03 \\
3.12 \mathrm{E}+03 \\
4.89 \mathrm{E}+03 \\
2.17 \mathrm{E}+03 \\
2.05 \mathrm{E}+03 \\
9.22 \mathrm{E}+02 \\
3.79 \mathrm{E}+02 \\
1.27 \mathrm{E}+04 \\
1.57 \mathrm{E}+03 \\
1.89 \mathrm{E}+03 \\
5.86 \mathrm{E}+02 \\
5.05 \mathrm{E}+02 \\
1.87 \mathrm{E}+03 \\
5.25 \mathrm{E}+03 \\
1.01 \mathrm{E}+03 \\
9.10 \mathrm{E}+02 \\
1.16 \mathrm{E}+03 \\
5.83 \mathrm{E}+03 \\
3.08 \mathrm{E}+03 \\
8.04 \mathrm{E}+03 \\
2.95 \mathrm{E}+03 \\
3.64 \mathrm{E}+04 \\
1.07 \mathrm{E}+04 \\
8.03 \mathrm{E}+03 \\
8.50 \mathrm{E}+03 \\
9.60 \mathrm{E}+03 \\
3.28 \mathrm{E}+03 \\
5.64 \mathrm{E}+03 \\
9.60 \mathrm{E}+03 \\
2.12 \mathrm{E}+04 \\
8.92 \mathrm{E}+03 \\
6.64 \mathrm{E}+03 \\
2.92 \mathrm{E}+03 \\
1.94 \mathrm{E}+03 \\
2.31 \mathrm{E}+03 \\
1.85 \mathrm{E}+03 \\
5.37 \mathrm{E}+03 \\
4.78 \mathrm{E}+03 \\
4.33 \mathrm{E}+03 \\
9.56 \mathrm{E}+03 \\
3.09 \mathrm{E}+03\end{array}$ & $\begin{array}{l}\text { PU242 } \\
1.14 E-02 \\
4.32 \mathrm{E}-03 \\
1.92 \mathrm{E}-02 \\
9.77 \mathrm{E}-03 \\
2.21 \mathrm{E}-02 \\
3.25 \mathrm{E}-02 \\
7.04 \mathrm{E}-02 \\
9.19 \mathrm{E}-03 \\
2.06 \mathrm{E}-02 \\
2.26 \mathrm{E}-02 \\
3.09 \mathrm{E}-03 \\
1.39 \mathrm{E}-02 \\
2.39 \mathrm{E}-02 \\
3.48 \mathrm{E}-03 \\
7.50 \mathrm{E}-03 \\
1.04 \mathrm{E}-02 \\
6.12 \mathrm{E}-02 \\
4.19 \mathrm{E}-02 \\
1.26 \mathrm{E}-02 \\
3.31 \mathrm{E}-03 \\
4.00 \mathrm{E}-03 \\
5.60 \mathrm{E}-03 \\
6.10 \mathrm{E}-03 \\
6.18 \mathrm{E}-03 \\
8.53 \mathrm{E}-03 \\
6.76 \mathrm{E}-03 \\
1.04 \mathrm{E}-02 \\
5.31 \mathrm{E}-03 \\
4.82 \mathrm{E}-03 \\
1.83 \mathrm{E}-03 \\
6.95 \mathrm{E}-04 \\
2.48 \mathrm{E}-02 \\
3.02 \mathrm{E}-03 \\
3.68 \mathrm{E}-03 \\
1.09 \mathrm{E}-03 \\
1.04 \mathrm{E}-03 \\
3.73 \mathrm{E}-03 \\
1.24 \mathrm{E}-02 \\
1.85 \mathrm{E}-03 \\
2.08 \mathrm{E}-03 \\
1.80 \mathrm{E}-03 \\
4.15 \mathrm{E}-02 \\
1.97 \mathrm{E}-02 \\
5.77 \mathrm{E}-02 \\
2.65 \mathrm{E}-02 \\
3.53 \mathrm{E}-01 \\
7.87 \mathrm{E}-02 \\
6.60 \mathrm{E}-02 \\
7.96 \mathrm{E}-02 \\
9.25 \mathrm{E}-02 \\
8.47 \mathrm{E}-03 \\
1.46 \mathrm{E}-02 \\
2.58 \mathrm{E}-02 \\
5.65 \mathrm{E}-02 \\
2.33 \mathrm{E}-02 \\
1.78 \mathrm{E}-02 \\
4.43 \mathrm{E}-03 \\
5.72 \mathrm{E}-03 \\
8.90 \mathrm{E}-03 \\
1.22 \mathrm{E}-02 \\
2.49 \mathrm{E}-02 \\
2.84 \mathrm{E}-02 \\
8.37 \mathrm{E}-03 \\
1.83 \mathrm{E}-02 \\
5.71 \mathrm{E}-03\end{array}$ & $\begin{array}{l}\text { AM241 } \\
4.59 E+02 \\
1.76 E+02 \\
2.60 E+02 \\
1.26 E+02 \\
2.41 E+02 \\
5.65 E+02 \\
1.22 E+03 \\
3.40 E+02 \\
7.67 E+02 \\
8.27 E+02 \\
1.17 E+02 \\
5.11 E+02 \\
8.88 E+02 \\
1.30 E+02 \\
2.74 E+02 \\
3.78 E+02 \\
2.25 E+03 \\
1.57 E+03 \\
4.65 E+02 \\
1.23 E+02 \\
1.50 E+02 \\
2.04 E+02 \\
2.22 E+02 \\
2.26 E+02 \\
3.08 E+02 \\
2.50 E+02 \\
3.70 E+02 \\
1.69 E+02 \\
1.56 E+02 \\
6.94 E+01 \\
2.84 E+01 \\
9.60 E+02 \\
1.19 E+02 \\
1.42 E+02 \\
4.50 E+01 \\
3.89 E+01 \\
1.41 E+02 \\
4.03 E+02 \\
7.55 E+01 \\
6.83 E+01 \\
8.66 E+01 \\
4.55 E+02 \\
2.50 E+02 \\
6.09 E+02 \\
2.24 E+02 \\
2.76 E+03 \\
8.06 E+02 \\
6.15 E+02 \\
6.54 E+02 \\
7.22 E+02 \\
2.46 E+02 \\
4.30 E+02 \\
7.43 E+02 \\
1.64 E+03 \\
6.85 E+02 \\
5.01 E+02 \\
2.17 E+02 \\
1.47 E+02 \\
1.73 E+02 \\
1.46 E+02 \\
4.09 E+02 \\
3.63 E+02 \\
3.19 E+02 \\
7.07 E+02 \\
2.30 E+02\end{array}$ & $\begin{array}{l}\text { AM243 } \\
3.34 \mathrm{E}-03 \\
1.25 \mathrm{E}-03 \\
1.94 \mathrm{E}-02 \\
1.02 \mathrm{E}-02 \\
2.61 \mathrm{E}-02 \\
8.48 \mathrm{E}-06 \\
1.84 \mathrm{E}-05 \\
2.78 \mathrm{E}-03 \\
6.23 \mathrm{E}-03 \\
6.88 \mathrm{E}-03 \\
9.29 \mathrm{E}-04 \\
4.20 \mathrm{E}-03 \\
7.23 \mathrm{E}-03 \\
1.05 \mathrm{E}-03 \\
2.29 \mathrm{E}-03 \\
3.17 \mathrm{E}-03 \\
1.86 \mathrm{E}-02 \\
1.26 \mathrm{E}-02 \\
3.80 \mathrm{E}-03 \\
1.00 \mathrm{E}-03 \\
1.21 \mathrm{E}-03 \\
1.70 \mathrm{E}-03 \\
1.86 \mathrm{E}-03 \\
1.88 \mathrm{E}-03 \\
2.61 \mathrm{E}-03 \\
2.06 \mathrm{E}-03 \\
3.18 \mathrm{E}-03 \\
1.97 \mathrm{E}-03 \\
1.76 \mathrm{E}-03 \\
6.09 \mathrm{E}-04 \\
2.19 \mathrm{E}-04 \\
7.36 \mathrm{E}-03 \\
8.88 \mathrm{E}-04 \\
1.09 \mathrm{E}-03 \\
3.15 \mathrm{E}-04 \\
3.53 \mathrm{E}-04 \\
1.24 \mathrm{E}-03 \\
4.54 \mathrm{E}-03 \\
5.85 \mathrm{E}-04 \\
7.51 \mathrm{E}-04 \\
3.51 \mathrm{E}-07 \\
4.65 \mathrm{E}-02 \\
1.99 \mathrm{E}-02 \\
6.60 \mathrm{E}-02 \\
3.93 \mathrm{E}-02 \\
5.70 \mathrm{E}-01 \\
9.15 \mathrm{E}-02 \\
8.66 \mathrm{E}-02 \\
1.22 \mathrm{E}-01 \\
1.49 \mathrm{E}-01 \\
3.26 \mathrm{E}-03 \\
5.60 \mathrm{E}-03 \\
1.00 \mathrm{E}-02 \\
2.18 \mathrm{E}-02 \\
8.96 \mathrm{E}-03 \\
6.97 \mathrm{E}-03 \\
8.54 \mathrm{E}-07 \\
6.47 \mathrm{E}-04 \\
4.79 \mathrm{E}-03 \\
1.29 \mathrm{E}-02 \\
1.85 \mathrm{E}-02 \\
2.82 \mathrm{E}-02 \\
2.49 \mathrm{E}-03 \\
5.39 \mathrm{E}-03 \\
1.65 \mathrm{E}-03\end{array}$ & $\begin{array}{l}\text { CM242 } \\
1.40 \mathrm{E}-02 \\
5.24 \mathrm{E}-03 \\
5.05 \mathrm{E}-02 \\
2.65 \mathrm{E}-02 \\
6.81 \mathrm{E}-02 \\
2.54 \mathrm{E}-02 \\
5.50 \mathrm{E}-02 \\
1.17 \mathrm{E}-02 \\
2.61 \mathrm{E}-02 \\
2.88 \mathrm{E}-02 \\
3.89 \mathrm{E}-03 \\
1.76 \mathrm{E}-02 \\
3.03 \mathrm{E}-02 \\
4.40 \mathrm{E}-03 \\
9.58 \mathrm{E}-03 \\
1.33 \mathrm{E}-02 \\
7.79 \mathrm{E}-02 \\
5.29 \mathrm{E}-02 \\
1.59 \mathrm{E}-02 \\
4.19 \mathrm{E}-03 \\
5.06 \mathrm{E}-03 \\
7.15 \mathrm{E}-03 \\
7.79 \mathrm{E}-03 \\
7.89 \mathrm{E}-03 \\
1.09 \mathrm{E}-02 \\
8.60 \mathrm{E}-03 \\
1.33 \mathrm{E}-02 \\
6.34 \mathrm{E}-03 \\
5.69 \mathrm{E}-03 \\
1.97 \mathrm{E}-03 \\
7.12 \mathrm{E}-04 \\
3.09 \mathrm{E}-02 \\
3.73 \mathrm{E}-03 \\
4.58 \mathrm{E}-03 \\
1.32 \mathrm{E}-03 \\
1.14 \mathrm{E}-03 \\
4.02 \mathrm{E}-03 \\
1.46 \mathrm{E}-02 \\
1.90 \mathrm{E}-03 \\
2.43 \mathrm{E}-03 \\
2.13 \mathrm{E}-18 \\
1.19 \mathrm{E}-01 \\
5.12 \mathrm{E}-02 \\
1.69 \mathrm{E}-01 \\
9.41 \mathrm{E}-02 \\
1.34 \mathrm{E}+00 \\
2.35 \mathrm{E}-01 \\
2.15 \mathrm{E}-01 \\
2.90 \mathrm{E}-01 \\
3.50 \mathrm{E}-01 \\
1.05 \mathrm{E}-02 \\
1.80 \mathrm{E}-02 \\
3.23 \mathrm{E}-02 \\
7.02 \mathrm{E}-02 \\
2.89 \mathrm{E}-02 \\
2.25 \mathrm{E}-02 \\
5.64 \mathrm{E}-18 \\
2.14 \mathrm{E}-03 \\
1.27 \mathrm{E}-02 \\
3.32 \mathrm{E}-02 \\
4.84 \mathrm{E}-02 \\
7.28 \mathrm{E}-02 \\
1.05 \mathrm{E}-02 \\
2.27 \mathrm{E}-02 \\
6.96 \mathrm{E}-02\end{array}$ & $\begin{array}{l}\text { CN243 } \\
3.26 E-04 \\
1.22 E-04 \\
4.75 E-03 \\
2.58 E-03 \\
7.07 E-03 \\
0.00 E+00 \\
0.00 E+00 \\
2.80 E-04 \\
6.24 E-04 \\
6.94 E-04 \\
9.25 E-05 \\
4.23 E-04 \\
7.27 E-04 \\
1.05 E-04 \\
2.29 E-04 \\
3.15 E-04 \\
1.87 E-03 \\
1.27 E-03 \\
3.83 E-04 \\
1.00 E-04 \\
1.20 E-04 \\
1.73 E-04 \\
1.88 E-04 \\
1.90 E-04 \\
2.63 E-04 \\
2.04 E-04 \\
3.23 E-04 \\
1.92 E-04 \\
1.72 E-04 \\
5.65 E-05 \\
1.97 E-05 \\
7.36 E-04 \\
8.84 E-05 \\
1.09 E-04 \\
3.09 E=05 \\
3.28 E-05 \\
1.15 E-04 \\
4.42 E-04 \\
5.25 E-05 \\
7.33 E-05 \\
0.00 E+00 \\
1.21 E-02 \\
4.85 E-03 \\
1.76 E-02 \\
1.31 E-02 \\
2.02 E-01 \\
2.47 E-02 \\
2.60 E-02 \\
4.17 E-02 \\
5.31 E-02 \\
3.29 E-04 \\
5.60 E-04 \\
1.00 E-03 \\
2.18 E-03 \\
8.95 E-04 \\
7.06 E-04 \\
0.00 E+00 \\
0.00 E+00 \\
5.73 E-04 \\
3.26 E-03 \\
3.64 E-03 \\
6.95 E-03 \\
2.51 E-04 \\
5.42 E-04 \\
1.64 E-04\end{array}$ \\
\hline
\end{tabular}


ACTIVITY IN UNSEPARATED FUEL (Curies decayed to 1/1/94)

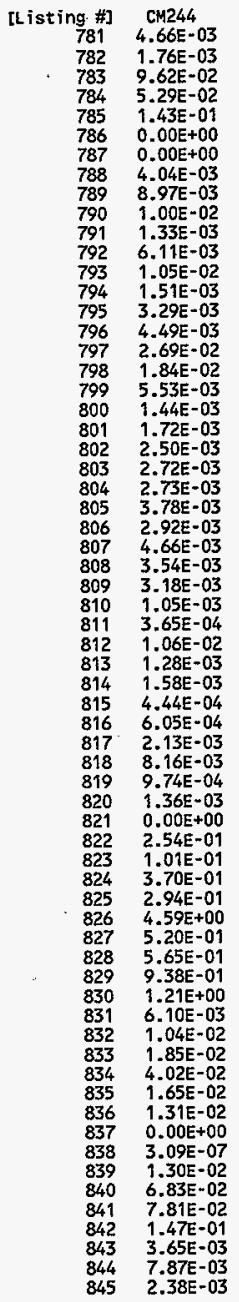


HNF-SO-WM-TI-794, ReV. O

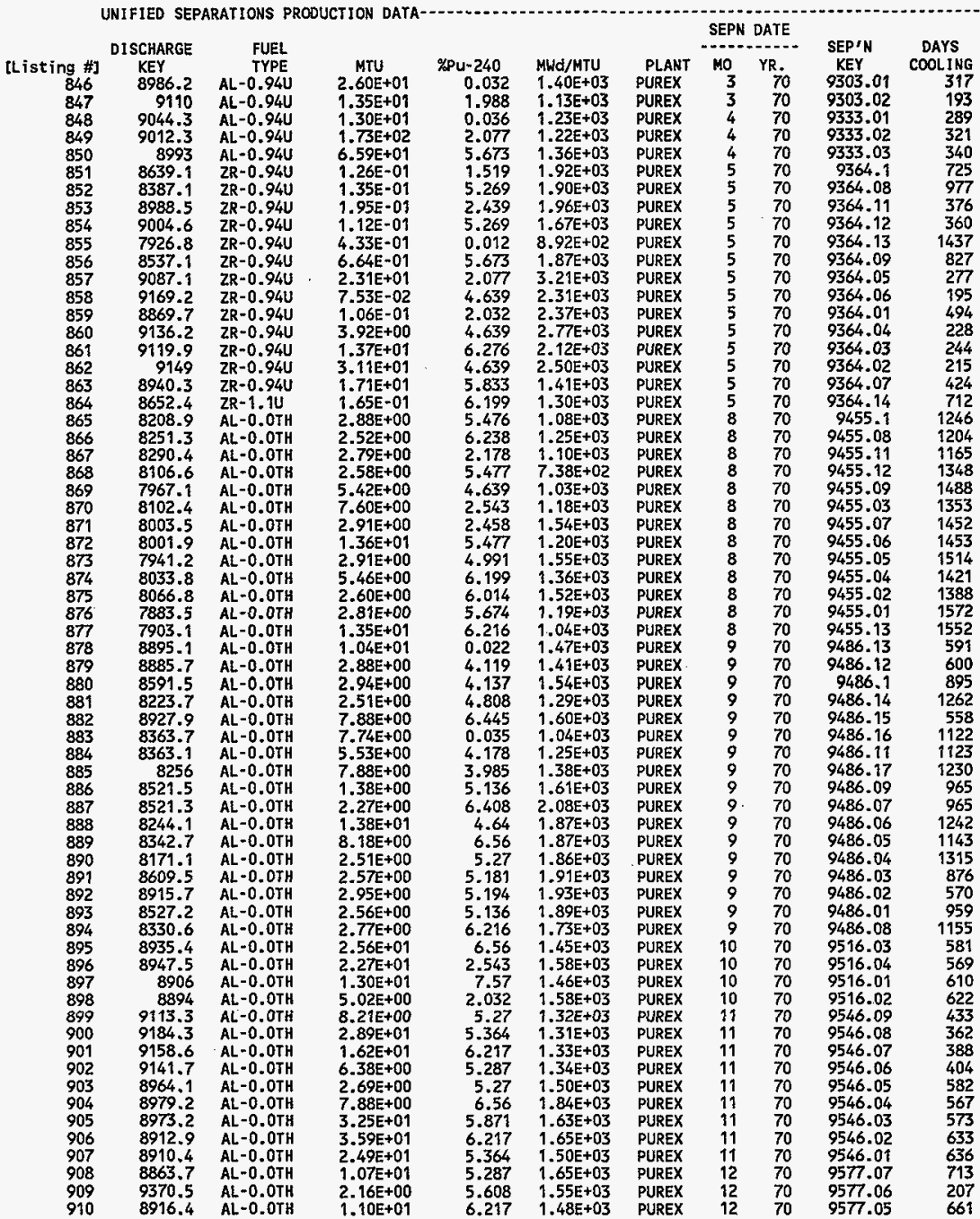


HNF-SD-HIH-TI-794, ReV. O

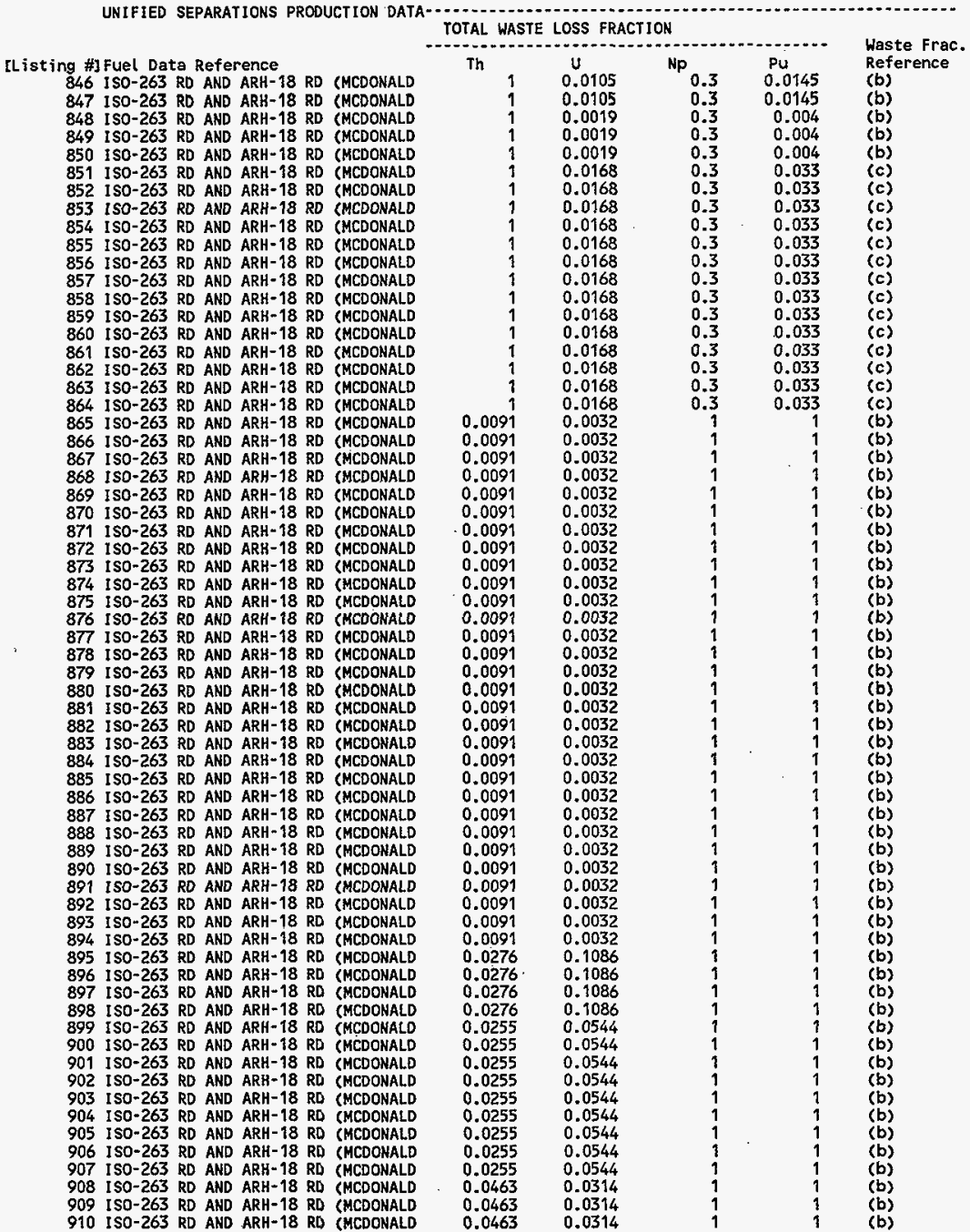


HNF-SD-WIM-TI-794, ReV. O

ACTIVITY IN UNSEPARATED FUEL (Curies decayed to 1/1/94)

\begin{tabular}{|c|c|c|c|c|c|c|c|c|c|}
\hline $\begin{array}{r}\text { [Listing \#] } \\
846 \\
847 \\
848 \\
849 \\
850 \\
851 \\
852 \\
853 \\
854 \\
855 \\
856 \\
857 \\
858 \\
859 \\
860 \\
861 \\
862 \\
863 \\
864 \\
865 \\
866 \\
867 \\
868 \\
869 \\
870 \\
871 \\
872 \\
873 \\
874 \\
875 \\
876 \\
877 \\
878 \\
879 \\
880 \\
881 \\
882 \\
883 \\
884 \\
885 \\
886 \\
887 \\
888 \\
889 \\
890 \\
891 \\
892 \\
893 \\
894 \\
895 \\
896 \\
897 \\
898 \\
899 \\
900 \\
901 \\
902 \\
903 \\
904 \\
905 \\
906 \\
907 \\
908 \\
909 \\
910\end{array}$ & $\begin{array}{l}\text { H03 } \\
1.44 E+02 \\
6.08 E+01 \\
6.35 E+01 \\
8.29 E+02 \\
3.52 E+02 \\
9.57 E-01 \\
9.77 E-01 \\
1.60 E+00 \\
7.72 E-01 \\
1.33 E+00 \\
4.84 E+00 \\
3.24 E+02 \\
7.55 E=01 \\
1.04 E+00 \\
4.73 E+01 \\
1.24 E+02 \\
3.38 E+02 \\
9.80 E+01 \\
8.10 E-01 \\
8.84 E-01 \\
7.80 E-01 \\
8.80 E-01 \\
7.80 E-01 \\
1.60 E+00 \\
2.30 E+00 \\
8.67 E-01 \\
4.11 E+00 \\
8.65 E-01 \\
1.63 E+00 \\
7.71 E-01 \\
8.30 E-01 \\
4.01 E+00 \\
3.57 E+00 \\
9.81 E-01 \\
9.58 E-01 \\
7.74 E-01 \\
2.71 E+00 \\
2.46 E+00 \\
1.73 E+00 \\
2.47 E+00 \\
4.47 E-01 \\
7.32 E-01 \\
4.26 E+00 \\
2.57 E+00 \\
7.64 E-01 \\
8.39 E-01 \\
1.02 E+00 \\
8.38 E-01 \\
8.66 E-01 \\
8.69 E+00 \\
7.73 E+00 \\
4.43 E+00 \\
1.69 E+00 \\
2.90 E+00 \\
1.05 E+01 \\
5.81 E+00 \\
2.30 E+00 \\
9.27 E-01 \\
2.70 E+00 \\
1.12 E+01 \\
1.25 E+01 \\
8.51 E+00 \\
3.69 E+00 \\
7.94 E-01 \\
3.72 E+00\end{array}$ & $\begin{array}{l}\text { C14 } \\
1.81 \mathrm{E}+00 \\
7.58 \mathrm{E}-01 \\
7.98 \mathrm{E}-01 \\
1.05 \mathrm{E}+01 \\
4.44 \mathrm{E}+00 \\
2.65 \mathrm{E}-02 \\
2.81 \mathrm{E}-02 \\
4.19 \mathrm{E}-02 \\
2.02 \mathrm{E}-02 \\
4.14 \mathrm{E}-02 \\
1.36 \mathrm{E}-01 \\
8.24 \mathrm{E}+00 \\
1.91 \mathrm{E}-02 \\
2.76 \mathrm{E}-02 \\
1.20 \mathrm{E}+00 \\
3.19 \mathrm{E}+00 \\
8.58 \mathrm{E}+00 \\
2.60 \mathrm{E}+00 \\
2.02 \mathrm{E}-02 \\
6.88 \mathrm{E}-07 \\
6.04 \mathrm{E}-07 \\
6.77 \mathrm{E}-07 \\
6.17 \mathrm{E}-07 \\
1.30 \mathrm{E}-06 \\
1.82 \mathrm{E}-06 \\
6.97 \mathrm{E}-07 \\
3.31 \mathrm{E}-06 \\
7.02 \mathrm{E}-07 \\
1.31 \mathrm{E}-06 \\
6.14 \mathrm{E}-07 \\
6.80 \mathrm{E}-07 \\
3.27 \mathrm{E}-06 \\
2.50 \mathrm{E}-06 \\
6.89 \mathrm{E}-07 \\
7.04 \mathrm{E}-07 \\
6.02 \mathrm{E}-07 \\
1.89 \mathrm{E}-06 \\
1.87 \mathrm{E}-06 \\
1.32 \mathrm{E}-06 \\
1.91 \mathrm{E}-06 \\
3.32 \mathrm{E}-07 \\
5.43 \mathrm{E}-07 \\
3.30 \mathrm{E}-06 \\
1.96 \mathrm{E}-06 \\
5.99 \mathrm{E}-07 \\
6.15 \mathrm{E}-07 \\
7.14 \mathrm{E}-07 \\
6.22 \mathrm{E}-07 \\
6.62 \mathrm{E}-07 \\
6.06 \mathrm{E}-06 \\
5.38 \mathrm{E}-06 \\
3.10 \mathrm{E}-06 \\
1.19 \mathrm{E}-06 \\
1.97 \mathrm{E}-06 \\
7.02 \mathrm{E}-06 \\
3.91 \mathrm{E}-06 \\
1.55 \mathrm{E}-06 \\
6.43 \mathrm{E}-07 \\
1.87 \mathrm{E}-06 \\
7.79 \mathrm{E}-06 \\
8.71 \mathrm{E}-06 \\
5.95 \mathrm{E}-06 \\
2.60 \mathrm{E}-06 \\
5.18 \mathrm{E}-07 \\
2.60 \mathrm{E}-06\end{array}$ & $\begin{array}{l}\text { NI59 } \\
5.11 \mathrm{E}-01 \\
2.14 \mathrm{E}-01 \\
2.25 \mathrm{E}-01 \\
2.96 \mathrm{E}+00 \\
1.25 \mathrm{E}+00 \\
1.57 \mathrm{E}-03 \\
1.66 \mathrm{E}-03 \\
2.48 \mathrm{E}-03 \\
1.20 \mathrm{E}-03 \\
2.48 \mathrm{E}-03 \\
8.05 \mathrm{E}-03 \\
4.81 \mathrm{E}-01 \\
1.13 \mathrm{E}-03 \\
1.63 \mathrm{E}-03 \\
7.03 \mathrm{E}-02 \\
1.88 \mathrm{E}-01 \\
5.05 \mathrm{E}-01 \\
1.55 \mathrm{E}-01 \\
1.26 \mathrm{E}-03 \\
0.00 \mathrm{E}+00 \\
0.00 \mathrm{E}+00 \\
0.00 \mathrm{E}+00 \\
0.00 \mathrm{E}+00 \\
0.00 \mathrm{E}+00 \\
0.00 \mathrm{E}+00 \\
0.00 \mathrm{E}+00 \\
0.00 \mathrm{E}+00 \\
0.00 \mathrm{E}+00 \\
0.00 \mathrm{E}+00 \\
0.00 \mathrm{E}+00 \\
0.00 \mathrm{E}+00 \\
0.00 \mathrm{E}+00 \\
0.00 \mathrm{E}+00 \\
0.00 \mathrm{E}+00 \\
0.00 \mathrm{E}+00 \\
0.00 \mathrm{E}+00 \\
0.00 \mathrm{E}+00 \\
0.00 \mathrm{E}+00 \\
0.00 \mathrm{E}+00 \\
0.00 \mathrm{E}+00 \\
0.00 \mathrm{E}+00 \\
0.00 \mathrm{E}+00 \\
0.00 \mathrm{E}+00 \\
0.00 \mathrm{E}+00 \\
0.00 \mathrm{E}+00 \\
0.00 \mathrm{E}+00 \\
0.00 \mathrm{E}+00 \\
0.00 \mathrm{E}+00 \\
0.00 \mathrm{E}+00 \\
0.00 \mathrm{E}+00 \\
0.00 \mathrm{E}+00 \\
0.00 \mathrm{E}+00 \\
0.00 \mathrm{E}+00 \\
0.00 \mathrm{E}+00 \\
0.00 \mathrm{E}+00 \\
0.00 \mathrm{E}+00 \\
0.00 \mathrm{E}+00 \\
0.00 \mathrm{E}+00 \\
0.00 \mathrm{E}+00 \\
0.00 \mathrm{E}+00 \\
0.00 \mathrm{E}+00 \\
0.00 \mathrm{E}+00 \\
0.00 \mathrm{E}+00 \\
0.00 \mathrm{E}+00 \\
0.00 \mathrm{E}+00\end{array}$ & $\begin{array}{l}\text { NI } 63 \\
5.31 \mathrm{E}+01 \\
2.23 \mathrm{E}+01 \\
2.34 \mathrm{E}+01 \\
3.07 \mathrm{E}+02 \\
1.30 \mathrm{E}+02 \\
1.64 \mathrm{E}-01 \\
1.73 \mathrm{E}+01 \\
2.61 \mathrm{E}=01 \\
1.26 \mathrm{E}-01 \\
2.54 \mathrm{E}-01 \\
8.39 \mathrm{E}-01 \\
5.12 \mathrm{E}+01 \\
1.20 \mathrm{E}=01 \\
1.71 \mathrm{E}-01 \\
7.46 \mathrm{E}+00 \\
1.99 \mathrm{E}+01 \\
5.35 \mathrm{E}+01 \\
1.62 \mathrm{E}+01 \\
1.31 \mathrm{E}-01 \\
0.00 \mathrm{E}+00 \\
0.00 \mathrm{E}+00 \\
0.00 \mathrm{E}+00 \\
0.00 \mathrm{E}+00 \\
0.00 \mathrm{E}+00 \\
0.00 \mathrm{E}+00 \\
0.00 \mathrm{E}+00 \\
0.00 \mathrm{E}+00 \\
0.00 \mathrm{E}+00 \\
0.00 \mathrm{E}+00 \\
0.00 \mathrm{E}+00 \\
0.00 \mathrm{E}+00 \\
0.00 \mathrm{E}+00 \\
0.00 \mathrm{E}+00 \\
0.00 \mathrm{E}+00 \\
0.00 \mathrm{E}+00 \\
0.00 \mathrm{E}+00 \\
0.00 \mathrm{E}+00 \\
0.00 \mathrm{E}+00 \\
0.00 \mathrm{E}+00 \\
0.00 \mathrm{E}+00 \\
0.00 \mathrm{E}+00 \\
0.00 \mathrm{E}+00 \\
0.00 \mathrm{E}+00 \\
0.00 \mathrm{E}+00 \\
0.00 \mathrm{E}+00 \\
0.00 \mathrm{E}+00 \\
0.00 \mathrm{E}+00 \\
0.00 \mathrm{E}+00 \\
0.00 \mathrm{E}+00 \\
0.00 \mathrm{E}+00 \\
0.00 \mathrm{E}+00 \\
0.00 \mathrm{E}+00 \\
0.00 \mathrm{E}+00 \\
0.00 \mathrm{E}+00 \\
0.00 \mathrm{E}+00 \\
0.00 \mathrm{E}+00 \\
0.00 \mathrm{E}+00 \\
0.00 \mathrm{E}+00 \\
0.00 \mathrm{E}+00 \\
0.00 \mathrm{E}+00 \\
0.00 \mathrm{E}+00 \\
0.00 \mathrm{E}+00 \\
0.00 \mathrm{E}+00 \\
0.00 \mathrm{E}+00 \\
0.00 \mathrm{E}+00\end{array}$ & $\begin{array}{l}\text { CO60 } \\
4.28 \mathrm{E}+00 \\
1.89 \mathrm{E}+00 \\
1.93 \mathrm{E}+00 \\
2.51 \mathrm{E}+01 \\
1.06 \mathrm{E}+01 \\
5.62 \mathrm{E}-02 \\
5.45 \mathrm{E}-02 \\
1.01 \mathrm{E}=01 \\
4.93 \mathrm{E}-02 \\
6.97 \mathrm{E}-02 \\
2.79 \mathrm{E}-01 \\
2.00 \mathrm{E}+01 \\
4.88 \mathrm{E}-02 \\
6.31 \mathrm{E}-02 \\
2.99 \mathrm{E}+00 \\
8.02 \mathrm{E}+00 \\
2.16 \mathrm{E}+01 \\
6.23 \mathrm{E}+00 \\
5.18 \mathrm{E}-02 \\
0.00 \mathrm{E}+00 \\
0.00 \mathrm{E}+00 \\
0.00 \mathrm{E}+00 \\
0.00 \mathrm{E}+00 \\
0.00 \mathrm{E}+00 \\
0.00 \mathrm{E}+00 \\
0.00 \mathrm{E}+00 \\
0.00 \mathrm{E}+00 \\
0.00 \mathrm{E}+00 \\
0.00 \mathrm{E}+00 \\
0.00 \mathrm{E}+00 \\
0.00 \mathrm{E}+00 \\
0.00 \mathrm{E}+00 \\
0.00 \mathrm{E}+00 \\
0.00 \mathrm{E}+00 \\
0.00 \mathrm{E}+00 \\
0.00 \mathrm{E}+00 \\
0.00 \mathrm{E}+00 \\
0.00 \mathrm{E}+00 \\
0.00 \mathrm{E}+00 \\
0.00 \mathrm{E}+00 \\
0.00 \mathrm{E}+00 \\
0.00 \mathrm{E}+00 \\
0.00 \mathrm{E}+00 \\
0.00 \mathrm{E}+00 \\
0.00 \mathrm{E}+00 \\
0.00 \mathrm{E}+00 \\
0.00 \mathrm{E}+00 \\
0.00 \mathrm{E}+00 \\
0.00 \mathrm{E}+00 \\
0.00 \mathrm{E}+00 \\
0.00 \mathrm{E}+00 \\
0.00 \mathrm{E}+00 \\
0.00 \mathrm{E}+00 \\
0.00 \mathrm{E}+00 \\
0.00 \mathrm{E}+00 \\
0.00 \mathrm{E}+00 \\
0.00 \mathrm{E}+00 \\
0.00 \mathrm{E}+00 \\
0.00 \mathrm{E}+00 \\
0.00 \mathrm{E}+00 \\
0.00 \mathrm{E}+00 \\
0.00 \mathrm{E}+00 \\
0.00 \mathrm{E}+00 \\
0.00 \mathrm{E}+00 \\
0.00 \mathrm{E}+00\end{array}$ & $\begin{array}{l}\text { SE79 } \\
4.86 \mathrm{E}-01 \\
2.04 \mathrm{E}-01 \\
2.14 \mathrm{E}-01 \\
2.81 \mathrm{E}+00 \\
1.19 \mathrm{E}+00 \\
3.20 \mathrm{E}-03 \\
3.39 \mathrm{E}-03 \\
5.05 \mathrm{E}-03 \\
2.46 \mathrm{E}-03 \\
5.16 \mathrm{E}-03 \\
1.64 \mathrm{E}-02 \\
9.67 \mathrm{E}-01 \\
2.29 \mathrm{E}-03 \\
3.30 \mathrm{E}-03 \\
1.42 \mathrm{E}-01 \\
3.83 \mathrm{E}-01 \\
1.02 \mathrm{E}+00 \\
3.19 \mathrm{E}-01 \\
2.85 \mathrm{E}-03 \\
5.64 \mathrm{E}-03 \\
4.94 \mathrm{E}-03 \\
5.54 \mathrm{E}-03 \\
5.05 \mathrm{E}-03 \\
1.06 \mathrm{E}-02 \\
1.49 \mathrm{E}-02 \\
5.71 \mathrm{E}-03 \\
2.71 \mathrm{E}-02 \\
5.74 \mathrm{E}-03 \\
1.07 \mathrm{E}-02 \\
5.02 \mathrm{E}-03 \\
5.56 \mathrm{E}-03 \\
2.68 \mathrm{E}-02 \\
2.05 \mathrm{E}-02 \\
5.64 \mathrm{E}-03 \\
5.76 \mathrm{E}-03 \\
4.92 \mathrm{E}-03 \\
1.55 \mathrm{E}-02 \\
1.53 \mathrm{E}-02 \\
1.08 \mathrm{E}-02 \\
1.56 \mathrm{E}-02 \\
2.72 \mathrm{E}-03 \\
4.45 \mathrm{E}-03 \\
2.70 \mathrm{E}-02 \\
1.61 \mathrm{E}-02 \\
4.90 \mathrm{E}-03 \\
5.03 \mathrm{E}-03 \\
5.84 \mathrm{E}-03 \\
5.09 \mathrm{E}-03 \\
5.42 \mathrm{E}-03 \\
4.96 \mathrm{E}-02 \\
4.40 \mathrm{E}-02 \\
2.54 \mathrm{E}-02 \\
9.72 \mathrm{E}-03 \\
1.61 \mathrm{E}-02 \\
5.74 \mathrm{E}-02 \\
3.20 \mathrm{E}-02 \\
1.27 \mathrm{E}-02 \\
5.26 \mathrm{E}-03 \\
1.53 \mathrm{E}-02 \\
6.37 \mathrm{E}-02 \\
7.12 \mathrm{E}-02 \\
4.87 \mathrm{E}-02 \\
2.13 \mathrm{E}-02 \\
2.24 \mathrm{E}-03 \\
.13 \mathrm{E}-02\end{array}$ & $\begin{array}{l}\text { SR90 } \\
5.76 \mathrm{E}+04 \\
2.46 \mathrm{E}+04 \\
2.57 \mathrm{E}+04 \\
3.36 \mathrm{E}+05 \\
1.42 \mathrm{E}+05 \\
3.48 \mathrm{E}+02 \\
3.64 \mathrm{E}+02 \\
5.62 \mathrm{E}+02 \\
2.78 \mathrm{E}+02 \\
5.63 \mathrm{E}+02 \\
1.78 \mathrm{E}+03 \\
1.03 \mathrm{E}+05 \\
2.53 \mathrm{E}+02 \\
3.57 \mathrm{E}+02 \\
1.54 \mathrm{E}+04 \\
4.26 \mathrm{E}+04 \\
1.12 \mathrm{E}+05 \\
3.63 \mathrm{E}+04 \\
3.31 \mathrm{E}+02 \\
3.98 \mathrm{E}+02 \\
3.51 \mathrm{E}+02 \\
3.94 \mathrm{E}+02 \\
3.55 \mathrm{E}+02 \\
7.39 \mathrm{E}+02 \\
1.05 \mathrm{E}+03 \\
3.98 \mathrm{E}+02 \\
1.89 \mathrm{E}+03 \\
3.99 \mathrm{E}+02 \\
7.48 \mathrm{E}+02 \\
3.52 \mathrm{E}+02 \\
3.85 \mathrm{E}+02 \\
1.86 \mathrm{E}+03 \\
1.51 \mathrm{E}+03 \\
4.16 \mathrm{E}+02 \\
4.18 \mathrm{E}+02 \\
3.49 \mathrm{E}+02 \\
1.15 \mathrm{E}+03 \\
1.09 \mathrm{E}+03 \\
7.70 \mathrm{E}+02 \\
1.11 \mathrm{E}+03 \\
1.96 \mathrm{E}+02 \\
3.21 \mathrm{E}+02 \\
1.91 \mathrm{E}+03 \\
1.15 \mathrm{E}+03 \\
3.46 \mathrm{E}+02 \\
3.65 \mathrm{E}+02 \\
4.33 \mathrm{E}+02 \\
3.67 \mathrm{E}+02 \\
3.86 \mathrm{E}+02 \\
3.68 \mathrm{E}+03 \\
3.27 \mathrm{E}+03 \\
1.88 \mathrm{E}+03 \\
7.19 \mathrm{E}+02 \\
1.21 \mathrm{E}+03 \\
4.33 \mathrm{E}+03 \\
2.41 \mathrm{E}+03 \\
9.55 \mathrm{E}+02 \\
3.91 \mathrm{E}+02 \\
1.14 \mathrm{E}+03 \\
4.74 \mathrm{E}+03 \\
5.27 \mathrm{E}+03 \\
3.60 \mathrm{E}+03 \\
1.57 \mathrm{E}+03 \\
3.23 \mathrm{E}+02 \\
1.58 \mathrm{E}+03\end{array}$ & $\begin{array}{l}\text { Y90 } \\
5.76 \mathrm{E}+04 \\
2.46 \mathrm{E}+04 \\
2.57 \mathrm{E}+04 \\
3.36 \mathrm{E}+05 \\
1.42 \mathrm{E}+05 \\
3.48 \mathrm{E}+02 \\
3.64 \mathrm{E}+02 \\
5.62 \mathrm{E}+02 \\
2.78 \mathrm{E}+02 \\
5.63 \mathrm{E}+02 \\
1.78 \mathrm{E}+03 \\
1.03 \mathrm{E}+05 \\
2.53 \mathrm{E}+02 \\
3.57 \mathrm{E}+02 \\
1.54 \mathrm{E}+04 \\
4.26 \mathrm{E}+04 \\
1.12 \mathrm{E}+05 \\
3.63 \mathrm{E}+04 \\
3.31 \mathrm{E}+02 \\
3.99 \mathrm{E}+02 \\
3.51 \mathrm{E}+02 \\
3.94 \mathrm{E}+02 \\
3.55 \mathrm{E}+02 \\
7.39 \mathrm{E}+02 \\
1.05 \mathrm{E}+03 \\
3.98 \mathrm{E}+02 \\
1.89 \mathrm{E}+03 \\
3.99 \mathrm{E}+02 \\
7.48 \mathrm{E}+02 \\
3.52 \mathrm{E}+02 \\
3.85 \mathrm{E}+02 \\
1.86 \mathrm{E}+03 \\
1.51 \mathrm{E}+03 \\
4.17 \mathrm{E}+02 \\
4.18 \mathrm{E}+02 \\
3.49 \mathrm{E}+02 \\
1.15 \mathrm{E}+03 \\
1.09 \mathrm{E}+03 \\
7.70 \mathrm{E}+02 \\
1.11 \mathrm{E}+03 \\
1.96 \mathrm{E}+02 \\
3.21 \mathrm{E}+02 \\
1.91 \mathrm{E}+03 \\
1.15 \mathrm{E}+03 \\
3.46 \mathrm{E}+02 \\
3.65 \mathrm{E}+02 \\
4.33 \mathrm{E}+02 \\
3.67 \mathrm{E}+02 \\
3.87 \mathrm{E}+02 \\
3.68 \mathrm{E}+03 \\
3.27 \mathrm{E}+03 \\
1.88 \mathrm{0}+03 \\
7.19 \mathrm{E}+02 \\
1.21 \mathrm{E}+03 \\
4.33 \mathrm{E}+03 \\
2.41 \mathrm{E}+03 \\
9.56 \mathrm{E}+02 \\
3.91 \mathrm{E}+02 \\
1.14 \mathrm{E}+03 \\
4.74 \mathrm{E}+03 \\
5.27 \mathrm{E}+03 \\
3.61 \mathrm{E}+03 \\
1.57 \mathrm{E}+03 \\
3.23 \mathrm{E}+02 \\
1.58 \mathrm{E}+03\end{array}$ & $\begin{array}{l}\text { 2R93 } \\
2.32 \mathrm{E}+00 \\
9.75 \mathrm{E}-01 \\
1.03 \mathrm{E}+00 \\
1.35 \mathrm{E}+01 \\
5.70 \mathrm{E}+00 \\
1.52 \mathrm{E}-02 \\
1.61 \mathrm{E}-02 \\
2.40 \mathrm{E}-02 \\
1.18 \mathrm{E}-02 \\
2.51 \mathrm{E}-02 \\
7.83 \mathrm{E}-02 \\
4.49 \mathrm{E}+00 \\
1.08 \mathrm{E}-02 \\
1.55 \mathrm{E}-02 \\
6.64 \mathrm{E}-01 \\
1.81 \mathrm{E}+00 \\
4.80 \mathrm{E}+00 \\
1.54 \mathrm{E}+00 \\
1.40 \mathrm{E}-02 \\
1.46 \mathrm{E}-02 \\
1.28 \mathrm{E}-02 \\
1.43 \mathrm{E}-02 \\
1.31 \mathrm{E}-02 \\
2.74 \mathrm{E}-02 \\
3.85 \mathrm{E}-02 \\
1.48 \mathrm{E}-02 \\
7.00 \mathrm{E}-02 \\
1.49 \mathrm{E}-02 \\
2.77 \mathrm{E}-02 \\
1.30 \mathrm{E}-02 \\
1.44 \mathrm{E}-02 \\
6.93 \mathrm{E}-02 \\
5.29 \mathrm{E}-02 \\
1.46 \mathrm{E}-02 \\
1.49 \mathrm{E}-02 \\
1.27 \mathrm{E}-02 \\
3.99 \mathrm{E}-02 \\
3.96 \mathrm{E}-02 \\
2.79 \mathrm{E}-02 \\
4.04 \mathrm{E}-02 \\
7.03 \mathrm{E}-03 \\
1.15 \mathrm{E}-02 \\
6.98 \mathrm{E}-02 \\
4.16 \mathrm{E}-02 \\
1.27 \mathrm{E}-02 \\
1.30 \mathrm{E}-02 \\
1.51 \mathrm{E}-02 \\
1.32 \mathrm{E}-02 \\
1.40 \mathrm{E}-02 \\
1.28 \mathrm{E}-01 \\
1.14 \mathrm{E}-01 \\
6.56 \mathrm{E}-02 \\
2.51 \mathrm{E}-02 \\
4.16 \mathrm{E}-02 \\
1.49 \mathrm{E}-01 \\
8.28 \mathrm{E}-02 \\
3.29 \mathrm{E}-02 \\
1.36 \mathrm{E}-02 \\
3.95 \mathrm{E}-02 \\
1.65 \mathrm{E}-01 \\
1.84 \mathrm{E}-01 \\
1.26 \mathrm{E}-01 \\
5.49 \mathrm{E}-02\end{array}$ \\
\hline
\end{tabular}


HNF-SD-WM-TI-794, ReV. 0

ACTIVITY IN UNSEPARATED FUEL (Curies decayed to $1 / 1 / 94$ )

\begin{tabular}{|c|c|c|c|c|c|c|c|c|c|}
\hline $\begin{array}{c}\text { isting } \mathbf{\#}] \\
846 \\
847 \\
848 \\
849 \\
850 \\
851 \\
852 \\
853 \\
854 \\
855 \\
856 \\
857 \\
858 \\
859 \\
860 \\
861 \\
862 \\
863 \\
864 \\
865 \\
866 \\
867 \\
868 \\
869 \\
870 \\
871 \\
872 \\
873 \\
874 \\
875 \\
876 \\
877 \\
878 \\
879 \\
880 \\
881 \\
882 \\
883 \\
884 \\
885 \\
886 \\
887 \\
888 \\
889 \\
890 \\
891 \\
892 \\
893 \\
894 \\
895 \\
896 \\
897 \\
898 \\
899 \\
900 \\
901 \\
902 \\
903 \\
904 \\
905 \\
906 \\
907 \\
908 \\
000\end{array}$ & $\begin{array}{l}\text { NB93* } \\
1.59 \mathrm{E}+00 \\
6.61 \mathrm{E}-01 \\
6.98 \mathrm{E}-01 \\
9.17 \mathrm{E}+00 \\
3.89 \mathrm{E}+00 \\
1.06 \mathrm{E}-02 \\
1.14 \mathrm{E}-02 \\
1.64 \mathrm{E}-02 \\
8.05 \mathrm{E}-03 \\
1.81 \mathrm{E}-02 \\
5.48 \mathrm{E}-02 \\
3.07 \mathrm{E}+00 \\
7.32 \mathrm{E}-03 \\
1.07 \mathrm{E}-02 \\
4.52 \mathrm{E}-01 \\
1.23 \mathrm{E}+00 \\
3.26 \mathrm{E}+00 \\
1.05 \mathrm{E}+00 \\
9.74 \mathrm{E}-03 \\
1.03 \mathrm{E}-02 \\
9.06 \mathrm{E}-03 \\
1.01 \mathrm{E}-02 \\
9.31 \mathrm{E}-03 \\
1.97 \mathrm{E}-02 \\
2.75 \mathrm{E}-02 \\
1.06 \mathrm{E}-02 \\
5.01 \mathrm{E}-02 \\
1.07 \mathrm{E}-02 \\
1.98 \mathrm{E}-02 \\
9.28 \mathrm{E}-03 \\
1.04 \mathrm{E}-02 \\
4.99 \mathrm{E}-02 \\
3.63 \mathrm{E}-02 \\
1.00 \mathrm{E}-02 \\
1.04 \mathrm{E}-02 \\
9.03 \mathrm{E}-03 \\
2.73 \mathrm{E}-02 \\
2.79 \mathrm{E}-02 \\
1.97 \mathrm{E}-02 \\
2.87 \mathrm{E}-02 \\
4.91 \mathrm{E}-03 \\
8.04 \mathrm{E}-03 \\
4.95 \mathrm{E}-02 \\
2.93 \mathrm{E}-02 \\
9.01 \mathrm{E}-03 \\
9.06 \mathrm{E}-03 \\
1.04 \mathrm{E}-02 \\
9.20 \mathrm{E}-03 \\
9.89 \mathrm{E}-03 \\
8.77 \mathrm{E}-02 \\
7.78 \mathrm{E}-02 \\
4.50 \mathrm{E}-02 \\
1.72 \mathrm{E}-02 \\
2.82 \mathrm{E}-02 \\
1.00 \mathrm{E}-02 \\
5.60 \mathrm{E}-02 \\
2.22 \mathrm{E}-02 \\
9.30 \mathrm{E}-03 \\
2.69 \mathrm{E}-02 \\
1.13 \mathrm{E}-01 \\
1.26 \mathrm{E}-01 \\
8.63 \mathrm{E}-02 \\
3.77 \mathrm{E}-02 \\
7.31 \mathrm{E}-03 \\
3.77 \mathrm{E}-02\end{array}$ & $\begin{array}{l}\text { TC99 } \\
1.65 \mathrm{E}+01 \\
6.78 \mathrm{E}+00 \\
7.16 \mathrm{E}+00 \\
9.39 \mathrm{E}+01 \\
4.04 \mathrm{E}+01 \\
1.07 \mathrm{E}-01 \\
1.13 \mathrm{E}-01 \\
1.69 \mathrm{E}-01 \\
8.23 \mathrm{E}-02 \\
1.72 \mathrm{E}-01 \\
5.49 \mathrm{E}-01 \\
3.24 \mathrm{E}+01 \\
7.65 \mathrm{E}-02 \\
1.10 \mathrm{E}-01 \\
4.75 \mathrm{E}+00 \\
1.28 \mathrm{E}+01 \\
3.42 \mathrm{E}+01 \\
1.07 \mathrm{E}+01 \\
9.54 \mathrm{E}-02 \\
6.05 \mathrm{E}-02 \\
5.31 \mathrm{E}-02 \\
5.95 \mathrm{E}-02 \\
5.42 \mathrm{E}-02 \\
1.14 \mathrm{E}-01 \\
1.60 \mathrm{E}-01 \\
6.13 \mathrm{E}-02 \\
2.91 \mathrm{E}-01 \\
6.17 \mathrm{E}-02 \\
1.15 \mathrm{E}-01 \\
5.39 \mathrm{E}-02 \\
5.98 \mathrm{E}-02 \\
2.88 \mathrm{E}-01 \\
2.20 \mathrm{E}-01 \\
6.05 \mathrm{E}-02 \\
6.19 \mathrm{E}-02 \\
5.29 \mathrm{E}-02 \\
1.66 \mathrm{E}-01 \\
1.65 \mathrm{E}-01 \\
1.16 \mathrm{E}-01 \\
1.68 \mathrm{E}-01 \\
2.92 \mathrm{E}-02 \\
4.77 \mathrm{E}-02 \\
2.90 \mathrm{E}-01 \\
1.73 \mathrm{E}-01 \\
5.27 \mathrm{E}-02 \\
5.40 \mathrm{E}-02 \\
6.27 \mathrm{E}-02 \\
5.47 \mathrm{E}-02 \\
5.82 \mathrm{E}-02 \\
5.33 \mathrm{E}-01 \\
4.73 \mathrm{E}-01 \\
2.73 \mathrm{E}-01 \\
1.04 \mathrm{E}-01 \\
1.73 \mathrm{E}-01 \\
6.17 \mathrm{E}-01 \\
3.44 \mathrm{E}-01 \\
1.37 \mathrm{E}-01 \\
5.65 \mathrm{E}-02 \\
1.64 \mathrm{E}-01 \\
6.85 \mathrm{E}-01 \\
7.65 \mathrm{E}-01 \\
5.23 \mathrm{E}-01 \\
2.28 \mathrm{E}-01 \\
4.55 \mathrm{E}-02 \\
2.29 \mathrm{E}-01\end{array}$ & $\begin{array}{l}\text { RU106 } \\
1.91 \mathrm{E}-02 \\
9.55 \mathrm{E}-03 \\
9.09 \mathrm{E}-03 \\
1.12 \mathrm{E}-01 \\
4.72 \mathrm{E}-02 \\
8.80 \mathrm{E}-05 \\
5.82 \mathrm{E}-05 \\
2.71 \mathrm{E}-04 \\
1.30 \mathrm{E}-04 \\
3.14 \mathrm{E}-05 \\
3.71 \mathrm{E}-04 \\
6.99 \mathrm{E}-02 \\
1.80 \mathrm{E}-04 \\
1.49 \mathrm{E}-04 \\
1.09 \mathrm{E}-02 \\
2.69 \mathrm{E}-02 \\
7.88 \mathrm{E}-02 \\
1.43 \mathrm{E}-02 \\
6.35 \mathrm{E}-05 \\
5.37 \mathrm{E}-06 \\
5.10 \mathrm{E}-06 \\
6.15 \mathrm{E}-06 \\
3.97 \mathrm{E}-06 \\
6.42 \mathrm{E}-06 \\
1.16 \mathrm{E}-05 \\
3.69 \mathrm{E}-06 \\
1.75 \mathrm{E}-05 \\
3.31 \mathrm{E}-06 \\
7.34 \mathrm{E}-06 \\
3.66 \mathrm{E}-06 \\
2.87 \mathrm{E}-06 \\
1.43 \mathrm{E}-05 \\
7.10 \mathrm{E}-05 \\
1.92 \mathrm{E}-05 \\
1.13 \mathrm{E}-05 \\
4.83 \mathrm{E}-06 \\
5.70 \mathrm{E}-05 \\
1.95 \mathrm{E}-05 \\
1.37 \mathrm{E}-05 \\
1.63 \mathrm{E}-05 \\
4.66 \mathrm{E}-06 \\
7.62 \mathrm{E}-06 \\
2.75 \mathrm{E}-05 \\
1.97 \mathrm{E}-05 \\
4.35 \mathrm{E}-06 \\
1.02 \mathrm{E}-05 \\
2.11 \mathrm{E}-05 \\
8.83 \mathrm{E}-06 \\
6.50 \mathrm{E}-06 \\
1.85 \mathrm{E}-04 \\
1.68 \mathrm{E}-04 \\
8.99 \mathrm{E}-05 \\
3.36 \mathrm{E}-05 \\
8.43 \mathrm{E}-05 \\
3.44 \mathrm{E}-05 \\
1.82 \mathrm{E}-04 \\
7.03 \mathrm{E}-05 \\
2.08 \mathrm{E}-05 \\
6.21 \mathrm{E}-05 \\
2.57 \mathrm{E}-04 \\
2.56 \mathrm{E}-04 \\
1.74 \mathrm{E}-04 \\
6.95 \mathrm{E}-04 \\
3.59 \mathrm{E}-05 \\
7.68 \mathrm{E}-05\end{array}$ & $\begin{array}{l}\text { CD113* } \\
1.22 \mathrm{E}+01 \\
5.10 \mathrm{E}+00 \\
5.36 \mathrm{E}+00 \\
7.00 \mathrm{E}+01 \\
2.99 \mathrm{E}+01 \\
9.13 \mathrm{E}-02 \\
9.38 \mathrm{E}-02 \\
1.52 \mathrm{E}-01 \\
7.20 \mathrm{E}-02 \\
1.21 \mathrm{E}-01 \\
4.61 \mathrm{E}-01 \\
3.25 \mathrm{E}+01 \\
7.26 \mathrm{E}-02 \\
1.01 \mathrm{E}-01 \\
4.65 \mathrm{E}+00 \\
1.19 \mathrm{E}+01 \\
3.28 \mathrm{E}+01 \\
9.01 \mathrm{E}+00 \\
6.73 \mathrm{E}-02 \\
3.18 \mathrm{E}-02 \\
2.80 \mathrm{E}-02 \\
3.16 \mathrm{E}-02 \\
2.81 \mathrm{E}-02 \\
5.80 \mathrm{E}-02 \\
8.29 \mathrm{E}-02 \\
3.13 \mathrm{E}-02 \\
1.49 \mathrm{E}-01 \\
3.13 \mathrm{E}-02 \\
5.90 \mathrm{E}-02 \\
2.78 \mathrm{E}-02 \\
3.01 \mathrm{E}-02 \\
1.45 \mathrm{E}-01 \\
1.26 \mathrm{E}-01 \\
3.47 \mathrm{E}-02 \\
3.42 \mathrm{E}-02 \\
2.78 \mathrm{E}-02 \\
9.57 \mathrm{E}-02 \\
8.81 \mathrm{E}-02 \\
6.21 \mathrm{E}-02 \\
8.88 \mathrm{E}-02 \\
1.60 \mathrm{E}-02 \\
2.61 \mathrm{E}-02 \\
1.53 \mathrm{E}-01 \\
9.22 \mathrm{E}-02 \\
2.75 \mathrm{E}-02 \\
2.99 \mathrm{E}-02 \\
3.61 \mathrm{E}-02 \\
2.99 \mathrm{E}-02 \\
3.11 \mathrm{E}-02 \\
3.07 \mathrm{E}-01 \\
2.73 \mathrm{E}-01 \\
1.57 \mathrm{E}-01 \\
5.99 \mathrm{E}-02 \\
1.02 \mathrm{E}-01 \\
3.68 \mathrm{E}-01 \\
2.04 \mathrm{E}-01 \\
8.10 \mathrm{E}-02 \\
3.28 \mathrm{E}-02 \\
9.52 \mathrm{E}-02 \\
3.97 \mathrm{E}-01 \\
4.40 \mathrm{E}-01 \\
3.01 \mathrm{E}-01 \\
1.31 \mathrm{E}-01 \\
2.78 \mathrm{E}-02 \\
1.32 \mathrm{E}-01\end{array}$ & $\begin{array}{l}\text { SB125 } \\
3.58 \mathrm{E}+01 \\
1.59 \mathrm{E}+01 \\
1.61 \mathrm{E}+01 \\
2.07 \mathrm{E}+02 \\
8.76 \mathrm{E}+01 \\
2.18 \mathrm{E}-01 \\
1.95 \mathrm{E}-01 \\
4.39 \mathrm{E}-01 \\
2.13 \mathrm{E}-01 \\
2.05 \mathrm{E}-01 \\
1.04 \mathrm{E}+00 \\
9.33 \mathrm{E}+01 \\
2.28 \mathrm{E}-01 \\
2.69 \mathrm{E}-01 \\
1.40 \mathrm{E}+01 \\
3.67 \mathrm{E}+01 \\
1.01 \mathrm{E}+02 \\
2.61 \mathrm{E}+01 \\
1.72 \mathrm{E}-01 \\
1.29 \mathrm{E}-01 \\
1.17 \mathrm{E}-01 \\
1.34 \mathrm{E}-01 \\
1.08 \mathrm{E}-01 \\
2.06 \mathrm{E}-01 \\
3.18 \mathrm{E}-01 \\
1.14 \mathrm{E}-01 \\
5.39 \mathrm{E}-01 \\
1.10 \mathrm{E}-01 \\
2.18 \mathrm{E}-01 \\
1.05 \mathrm{E}-01 \\
1.02 \mathrm{E}-01 \\
4.98 \mathrm{E}-01 \\
7.51 \mathrm{E}-01 \\
2.06 \mathrm{E}-01 \\
1.72 \mathrm{E}-01 \\
1.14 \mathrm{E}-01 \\
5.80 \mathrm{E}-01 \\
3.91 \mathrm{E}-01 \\
2.75 \mathrm{E}-01 \\
3.71 \mathrm{E}-01 \\
7.72 \mathrm{E}-02 \\
1.26 \mathrm{E}-01 \\
6.34 \mathrm{E}-01 \\
4.04 \mathrm{E}-01 \\
1.10 \mathrm{E}-01 \\
1.52 \mathrm{E}-01 \\
2.18 \mathrm{E}-01 \\
1.45 \mathrm{E}-01 \\
1.35 \mathrm{E}-01 \\
1.87 \mathrm{E}+00 \\
1.67 \mathrm{E}+00 \\
9.39 \mathrm{E}-01 \\
3.57 \mathrm{E}-01 \\
6.86 \mathrm{E}-01 \\
2.57 \mathrm{E}+00 \\
1.41 \mathrm{E}+00 \\
5.53 \mathrm{E}-01 \\
2.03 \mathrm{E}-01 \\
2.94 \mathrm{E}-01\end{array}$ & $\begin{array}{l}\text { SN126 } \\
7.08 \mathrm{E}-01 \\
2.91 \mathrm{E}-01 \\
3.08 \mathrm{E}-01 \\
4.05 \mathrm{E}+00 \\
1.73 \mathrm{E}+00 \\
5.32 \mathrm{E}-03 \\
5.64 \mathrm{E}-03 \\
8.44 \mathrm{E}-03 \\
4.01 \mathrm{E}-03 \\
7.87 \mathrm{E}-03 \\
2.72 \mathrm{E}-02 \\
1.75 \mathrm{E}+00 \\
3.92 \mathrm{E}-03 \\
5.67 \mathrm{E}-03 \\
2.50 \mathrm{E}-01 \\
6.47 \mathrm{E}-01 \\
1.77 \mathrm{E}+00 \\
5.09 \mathrm{E}-01 \\
4.12 \mathrm{E}-03 \\
5.18 \mathrm{E}-03 \\
4.54 \mathrm{E}-03 \\
5.09 \mathrm{E}-03 \\
4.64 \mathrm{E}-03 \\
9.75 \mathrm{E}-03 \\
1.37 \mathrm{E}-02 \\
5.24 \mathrm{E}-03 \\
2.49 \mathrm{E}-02 \\
5.28 \mathrm{E}-03 \\
9.83 \mathrm{E}-03 \\
4.62 \mathrm{E}-03 \\
5.11 \mathrm{E}-03 \\
2.46 \mathrm{E}-02 \\
1.88 \mathrm{E}-02 \\
5.18 \mathrm{E}-03 \\
5.29 \mathrm{E}-03 \\
4.52 \mathrm{E}-03 \\
1.42 \mathrm{E}-02 \\
1.41 \mathrm{E}-02 \\
9.91 \mathrm{E}-03 \\
1.44 \mathrm{E}-02 \\
2.50 \mathrm{E}-03 \\
4.08 \mathrm{E}-03 \\
2.48 \mathrm{E}-02 \\
1.48 \mathrm{E}-02 \\
4.50 \mathrm{E}-03 \\
4.62 \mathrm{E}-03 \\
5.37 \mathrm{E}-03 \\
4.68 \mathrm{E}-03 \\
4.98 \mathrm{E}-03 \\
4.56 \mathrm{E}-02 \\
4.04 \mathrm{E}-02 \\
2.33 \mathrm{E}-02 \\
8.93 \mathrm{E}-03 \\
1.48 \mathrm{E}-02 \\
5.28 \mathrm{E}-02 \\
2.94 \mathrm{E}-02 \\
1.17 \mathrm{E}-02 \\
4.83 \mathrm{E}-03 \\
1.40 \mathrm{E}-02 \\
5.86 \mathrm{E}-02 \\
6.55 \mathrm{E}-02 \\
4.48 \mathrm{E}-02 \\
1.95 \mathrm{E}-02 \\
3.89 \mathrm{E}-03 \\
1.96 \mathrm{E}-03\end{array}$ & $\begin{array}{l}1129 \\
3.11 \mathrm{E}-02 \\
1.28 \mathrm{E}-02 \\
1.36 \mathrm{E}-02 \\
1.78 \mathrm{E}-01 \\
7.60 \mathrm{E}-02 \\
2.22 \mathrm{E}-04 \\
2.36 \mathrm{E}-04 \\
3.53 \mathrm{E}-04 \\
1.69 \mathrm{E}-04 \\
3.36 \mathrm{E}-04 \\
1.14 \mathrm{E}-03 \\
7.16 \mathrm{E}-02 \\
1.63 \mathrm{E}-04 \\
2.35 \mathrm{E}-04 \\
1.03 \mathrm{E}-02 \\
2.70 \mathrm{E}-02 \\
7.33 \mathrm{E}-02 \\
2.15 \mathrm{E}-02 \\
1.83 \mathrm{E}-04 \\
2.21 \mathrm{E}-04 \\
1.94 \mathrm{E}-04 \\
2.18 \mathrm{E}-04 \\
1.98 \mathrm{E}-04 \\
4.17 \mathrm{E}-04 \\
5.85 \mathrm{E}-04 \\
2.24 \mathrm{E}-04 \\
1.06 \mathrm{E}-03 \\
2.26 \mathrm{E}-04 \\
4.20 \mathrm{E}-04 \\
1.97 \mathrm{E}-04 \\
2.18 \mathrm{E}-04 \\
1.05 \mathrm{E}-03 \\
8.04 \mathrm{E}-04 \\
2.21 \mathrm{E}-04 \\
2.26 \mathrm{E}-04 \\
1.93 \mathrm{E}-04 \\
6.07 \mathrm{E}-04 \\
6.02 \mathrm{E}-04 \\
4.24 \mathrm{E}-04 \\
6.14 \mathrm{E}-04 \\
1.07 \mathrm{E}-04 \\
1.75 \mathrm{E}-04 \\
1.06 \mathrm{E}-03 \\
6.31 \mathrm{E}-04 \\
1.93 \mathrm{E}-04 \\
1.98 \mathrm{E}-04 \\
2.29 \mathrm{E}-04 \\
2.00 \mathrm{E}-04 \\
2.13 \mathrm{E}-04 \\
1.95 \mathrm{E}-03 \\
1.73 \mathrm{E}-03 \\
9.97 \mathrm{E}-04 \\
3.82 \mathrm{E}-04 \\
6.32 \mathrm{E}-04 \\
2.26 \mathrm{E}-03 \\
1.26 \mathrm{E}-03 \\
4.99 \mathrm{E}-04 \\
2.07 \mathrm{E}-04 \\
6.00 \mathrm{E}-04 \\
2.50 \mathrm{E}-03 \\
2.80 \mathrm{E}-03 \\
1.91 \mathrm{E}-03 \\
8.35 \mathrm{E}-04 \\
1.66 \mathrm{E}-04 \\
8.36 \mathrm{E}-04\end{array}$ & $\begin{array}{l}\text { CS134 } \\
4.18 \mathrm{E}+00 \\
1.55 \mathrm{E}+00 \\
1.69 \mathrm{E}+00 \\
2.15 \mathrm{E}+01 \\
9.97 \mathrm{E}+00 \\
3.89 \mathrm{E}-02 \\
3.28 \mathrm{E}-02 \\
8.70 \mathrm{E}-02 \\
3.61 \mathrm{E}-02 \\
1.42 \mathrm{E}-02 \\
1.78 \mathrm{E}-01 \\
2.97 \mathrm{E}+01 \\
5.51 \mathrm{E}-02 \\
6.17 \mathrm{E}-02 \\
3.94 \mathrm{E}+00 \\
8.07 \mathrm{E}+00 \\
2.60 \mathrm{E}+01 \\
3.68 \mathrm{E}+00 \\
1.93 \mathrm{E}-02 \\
5.06 \mathrm{E}-03 \\
4.61 \mathrm{E}-03 \\
5.36 \mathrm{E}-03 \\
4.13 \mathrm{E}-03 \\
7.62 \mathrm{E}-03 \\
1.21 \mathrm{E}-02 \\
4.24 \mathrm{E}-03 \\
2.01 \mathrm{E}-02 \\
4.03 \mathrm{E}-03 \\
8.17 \mathrm{E}-03 \\
3.96 \mathrm{E}-03 \\
3.70 \mathrm{E}-03 \\
1.81 \mathrm{E}-02 \\
3.45 \mathrm{E}-02 \\
9.43 \mathrm{E}-03 \\
7.35 \mathrm{E}-03 \\
4.48 \mathrm{E}-03 \\
2.69 \mathrm{E}-02 \\
1.59 \mathrm{E}-02 \\
1.12 \mathrm{E}-02 \\
1.47 \mathrm{E}-02 \\
3.25 \mathrm{E}-03 \\
5.32 \mathrm{E}-03 \\
2.50 \mathrm{E}-02 \\
1.63 \mathrm{E}-02 \\
4.25 \mathrm{E}-03 \\
6.53 \mathrm{E}-03 \\
1.01 \mathrm{E}-02 \\
6.12 \mathrm{E}-03 \\
5.44 \mathrm{E}-03 \\
8.68 \mathrm{E}-02 \\
7.79 \mathrm{E}-02 \\
4.33 \mathrm{E}-02 \\
1.64 \mathrm{E}-02 \\
3.32 \mathrm{E}-02 \\
1.27 \mathrm{E}-01 \\
6.89 \mathrm{E}-02 \\
2.70 \mathrm{E}-02 \\
9.47 \mathrm{E}-03 \\
2.79 \mathrm{E}-02 \\
1.16 \mathrm{E}-01 \\
1.22 \mathrm{E}-01 \\
8.34 \mathrm{E}-02 \\
3.49 \mathrm{E}-02 \\
1.11 \mathrm{E}-02 \\
3.66 \mathrm{E}-02\end{array}$ & $\begin{array}{l}\mathrm{CS137} \\
6.62 \mathrm{E}+04 \\
2.79 \mathrm{E}+04 \\
2.93 \mathrm{E}+04 \\
3.83 \mathrm{E}+05 \\
1.63 \mathrm{E}+05 \\
4.29 \mathrm{E}+02 \\
4.48 \mathrm{E}+02 \\
6.94 \mathrm{E}+02 \\
3.38 \mathrm{E}+02 \\
6.57 \mathrm{E}+02 \\
2.19 \mathrm{E}+03 \\
1.35 \mathrm{E}+05 \\
3.19 \mathrm{E}+02 \\
4.51 \mathrm{E}+02 \\
1.98 \mathrm{E}+04 \\
5.31 \mathrm{E}+04 \\
1.42 \mathrm{E}+05 \\
4.35 \mathrm{E}+04 \\
3.82 \mathrm{E}+02 \\
3.66 \mathrm{E}+02 \\
3.22 \mathrm{E}+02 \\
3.62 \mathrm{E}+02 \\
3.26 \mathrm{E}+02 \\
6.79 \mathrm{E}+02 \\
9.62 \mathrm{E}+02 \\
3.66 \mathrm{E}+02 \\
1.74 \mathrm{E}+03 \\
3.67 \mathrm{E}+02 \\
6.88 \mathrm{E}+02 \\
3.23 \mathrm{E}+02 \\
3.54 \mathrm{E}+02 \\
1.71 \mathrm{E}+03 \\
1.39 \mathrm{E}+03 \\
3.82 \mathrm{E}+02 \\
3.84 \mathrm{E}+02 \\
3.20 \mathrm{E}+02 \\
1.05 \mathrm{E}+03 \\
1.01 \mathrm{E}+03 \\
7.08 \mathrm{E}+02 \\
1.02 \mathrm{E} \\
1.59 \mathrm{E}+03 \\
1.80 \mathrm{E}+02 \\
2.95 \mathrm{E}+02 \\
1.35 \mathrm{E}+03 \\
4.85 \mathrm{E}+03 \\
3.31 \mathrm{E}+03 \\
1.44 \mathrm{E}+03 \\
3.05 \mathrm{E}+03 \\
3.18 \mathrm{E}+03 \\
3.35 \mathrm{E}+02 \\
3.97 \mathrm{E}+02 \\
3.37 \mathrm{E}+02 \\
3.55 \mathrm{E}+02 \\
3.37 \mathrm{E}+03 \\
3.00 \mathrm{E}+03 \\
1.72 \mathrm{E}+03 \\
6.60 \mathrm{E}+02 \\
3.11 \mathrm{E}+03 \\
\end{array}$ \\
\hline
\end{tabular}


ACTIVITY IN UNSEPARATED FUEL (Curies decayed to $1 / 1 / 94$ )

\begin{tabular}{|c|c|c|c|c|c|c|c|c|c|}
\hline $\begin{aligned} \text { [Listing \#] } \\
846 \\
847 \\
848 \\
849 \\
850 \\
851 \\
852 \\
853 \\
854 \\
855 \\
856 \\
857 \\
858 \\
859 \\
860 \\
861 \\
862 \\
863 \\
864 \\
865 \\
866 \\
867 \\
868 \\
869 \\
870 \\
871 \\
872 \\
873 \\
874 \\
875 \\
876 \\
877 \\
878 \\
879 \\
880 \\
881 \\
882 \\
883 \\
884 \\
885 \\
806 \\
907 \\
908 \\
909 \\
910\end{aligned}$ & $\begin{array}{l}8 A 137 \\
6.26 E+04 \\
2.64 E+04 \\
2.77 E+04 \\
3.62 E+05 \\
1.54 E+05 \\
4.06 E+02 \\
4.24 E+02 \\
6.57 E+02 \\
3.20 E+02 \\
6.22 E+02 \\
2.07 E+03 \\
1.27 E+05 \\
3.01 E+02 \\
4.26 E+02 \\
1.87 E+04 \\
5.02 E+04 \\
1.35 E+05 \\
4.12 E+04 \\
3.62 E+02 \\
3.46 E+02 \\
3.05 E+02 \\
3.42 E+02 \\
3.08 E+02 \\
6.43 E+02 \\
9.10 E+02 \\
3.46 E+02 \\
1.64 E+03 \\
3.47 E+02 \\
6.51 E+02 \\
3.06 E+02 \\
3.35 E+02 \\
1.62 E+03 \\
1.31 E+03 \\
3.62 E+02 \\
3.63 E+02 \\
3.03 E+02 \\
9.94 E+02 \\
9.51 E+02 \\
6.69 E+02 \\
9.64 E+02 \\
1.70 E+02 \\
2.79 E+02 \\
1.66 E+03 \\
9.96 E+02 \\
3.01 E+02 \\
3.17 E+02 \\
3.76 E+02 \\
3.19 E+02 \\
3.36 E+02 \\
3.19 E+03 \\
2.83 E+03 \\
1.63 E+03 \\
6.24 E+02 \\
1.05 E+03 \\
3.76 E+03 \\
2.09 E+03 \\
8.29 E+02 \\
3.39 E+02 \\
9.85 E+02 \\
4.11 E+03 \\
4.58 E+03 \\
3.13 E+03 \\
1.36 E+03 \\
2.80 E+02 \\
1.37 E+03\end{array}$ & $\begin{array}{l}S M 151 \\
1.57 E+03 \\
7.18 E+02 \\
7.29 E+02 \\
9.56 E+03 \\
3.91 E+03 \\
8.52 E+00 \\
9.00 E+00 \\
1.34 E+01 \\
7.15 E+00 \\
1.79 E+01 \\
4.44 E+01 \\
1.90 E+03 \\
5.47 E+00 \\
7.75 E+00 \\
3.07 E+02 \\
9.65 E+02 \\
2.34 E+03 \\
9.95 E+02 \\
9.24 E+00 \\
5.50 E+00 \\
4.83 E+00 \\
5.42 E+00 \\
4.92 E+00 \\
1.03 E+01 \\
1.45 E+01 \\
5.55 E+00 \\
2.63 E+01 \\
5.58 E+00 \\
1.04 E+01 \\
4.89 E+00 \\
5.39 E+00 \\
2.60 E+01 \\
2.03 E+01 \\
5.58 E+00 \\
5.67 E+00 \\
4.81 E+00 \\
1.53 E+01 \\
1.50 E+01 \\
1.06 E+01 \\
1.53 E+01 \\
2.67 E+00 \\
4.37 E+00 \\
2.64 E+01 \\
1.57 E+01 \\
4.78 E+00 \\
4.95 E+00 \\
5.79 E+00 \\
5.00 E+00 \\
5.31 E+00 \\
4.92 E+01 \\
4.36 E+01 \\
2.52 E+01 \\
9.63 E+00 \\
1.60 E+01 \\
5.72 E+01 \\
3.19 E+01 \\
1.27 E+01 \\
5.22 E+00 \\
1.52 E+01 \\
6.32 E+01 \\
7.06 E+01 \\
4.83 E+01 \\
2.10 E+01 \\
4.24 E+00 \\
2.11 E+01\end{array}$ & $\begin{array}{c}E U 152 \\
1.58 \mathrm{E}+00 \\
5.24 \mathrm{E}-01 \\
6.05 \mathrm{E}-01 \\
7.90 \mathrm{E}+00 \\
3.74 \mathrm{E}+00 \\
1.64 \mathrm{E}-02 \\
1.68 \mathrm{E}-02 \\
2.80 \mathrm{E}-02 \\
1.11 \mathrm{E}-02 \\
8.23 \mathrm{E}-03 \\
8.07 \mathrm{E}-02 \\
8.30 \mathrm{E}+00 \\
1.55 \mathrm{E}-02 \\
2.19 \mathrm{E}-02 \\
1.10 \mathrm{E}+00 \\
2.37 \mathrm{E}+00 \\
7.36 \mathrm{E}+00 \\
1.15 \mathrm{E}+00 \\
6.69 \mathrm{E}-03 \\
1.56 \mathrm{E}-03 \\
1.37 \mathrm{E}-03 \\
1.55 \mathrm{E}-03 \\
1.37 \mathrm{E}-03 \\
2.83 \mathrm{E}-03 \\
4.05 \mathrm{E}-03 \\
1.53 \mathrm{E}-03 \\
7.26 \mathrm{E}-03 \\
1.53 \mathrm{E}-03 \\
2.88 \mathrm{E}-03 \\
1.36 \mathrm{E}-03 \\
1.47 \mathrm{E}-03 \\
7.09 \mathrm{E}-03 \\
6.22 \mathrm{E}-03 \\
1.71 \mathrm{E}-03 \\
1.68 \mathrm{E}-03 \\
1.36 \mathrm{E}-03 \\
4.72 \mathrm{E}-03 \\
4.32 \mathrm{E}-03 \\
3.04 \mathrm{E}-03 \\
4.35 \mathrm{E}-03 \\
7.84 \mathrm{E}-04 \\
1.28 \mathrm{E}-03 \\
7.49 \mathrm{E}-03 \\
4.52 \mathrm{E}-03 \\
1.35 \mathrm{E}-03 \\
1.47 \mathrm{E}-03 \\
1.78 \mathrm{E}-03 \\
1.47 \mathrm{E}-03 \\
1.52 \mathrm{E}-03 \\
1.52 \mathrm{E}-02 \\
1.35 \mathrm{E}-02 \\
7.73 \mathrm{E}-03 \\
2.96 \mathrm{E}-03 \\
5.04 \mathrm{E}-03 \\
1.82 \mathrm{E}-02 \\
1.00 \mathrm{E}-02 \\
4.72 \mathrm{E}-03 \\
1.96 \mathrm{E}-03 \\
2.17 \mathrm{E}-02 \\
1.48 \mathrm{E}-02 \\
6.43 \mathrm{E}-03 \\
1.38 \mathrm{E}-03 \\
6.49 \mathrm{E}-03\end{array}$ & $\begin{array}{c}E U 154 \\
1.14 \mathrm{E}+02 \\
3.95 \mathrm{E}+01 \\
4.47 \mathrm{E}+01 \\
5.82 \mathrm{E}+02 \\
2.72 \mathrm{E}+02 \\
1.42 \mathrm{E}+00 \\
1.43 \mathrm{E}+00 \\
2.49 \mathrm{E}+00 \\
1.01 \mathrm{E}+00 \\
7.96 \mathrm{E}-01 \\
6.96 \mathrm{E}+00 \\
8.51 \mathrm{E}+02 \\
1.41 \mathrm{E}+00 \\
1.95 \mathrm{E}+00 \\
1.07 \mathrm{E}+02 \\
2.12 \mathrm{E}+02 \\
6.85 \mathrm{E}+02 \\
1.07 \mathrm{E}+02 \\
6.45 \mathrm{E}-01 \\
1.07 \mathrm{E}-01 \\
9.47 \mathrm{E}-02 \\
1.07 \mathrm{E}-01 \\
9.37 \mathrm{E}-02 \\
1.91 \mathrm{E}-01 \\
2.76 \mathrm{E}-01 \\
1.04 \mathrm{E}-01 \\
4.91 \mathrm{E}-01 \\
1.03 \mathrm{E}-01 \\
1.95 \mathrm{E}-01 \\
9.24 \mathrm{E}-02 \\
9.82 \mathrm{E}-02 \\
4.75 \mathrm{E}-01 \\
4.52 \mathrm{E}-01 \\
1.24 \mathrm{E}-01 \\
1.19 \mathrm{E}-01 \\
9.37 \mathrm{E}-02 \\
3.44 \mathrm{E}-01 \\
3.01 \mathrm{E}-01 \\
2.12 \mathrm{E}-01 \\
3.00 \mathrm{E}-01 \\
5.53 \mathrm{E}-02 \\
9.04 \mathrm{E}-02 \\
5.16 \mathrm{E}-01 \\
3.14 \mathrm{E}-01 \\
9.23 \mathrm{E}-02 \\
1.04 \mathrm{E}-01 \\
1.30 \mathrm{E}-01 \\
1.04 \mathrm{E}-01 \\
1.06 \mathrm{E}-01 \\
1.10 \mathrm{E}+00 \\
9.83 \mathrm{E}-01 \\
5.62 \mathrm{E}-01 \\
2.15 \mathrm{E}-01 \\
3.73 \mathrm{E}-01 \\
1.35 \mathrm{E}+00 \\
7.49 \mathrm{E}-01 \\
2.97 \mathrm{E}-01 \\
1.18 \mathrm{E}-01 \\
3.43 \mathrm{E}-01 \\
1.43 \mathrm{E}+00 \\
1.58 \mathrm{E}+00 \\
1.08 \mathrm{E}+00 \\
4.66 \mathrm{E}-01 \\
1.04 \mathrm{E}-01 \\
4.72 \mathrm{E}-01\end{array}$ & $\begin{array}{l}\text { EU155 } \\
6.65 \mathrm{E}+01 \\
3.60 \mathrm{E}+01 \\
3.44 \mathrm{E}+01 \\
4.45 \mathrm{E}+02 \\
1.70 \mathrm{E}+02 \\
3.96 \mathrm{E}-01 \\
3.82 \mathrm{E}-01 \\
7.06 \mathrm{E}-01 \\
3.78 \mathrm{E}-01 \\
6.38 \mathrm{E}-01 \\
1.99 \mathrm{E}+00 \\
1.18 \mathrm{E}+02 \\
3.12 \mathrm{E}-01 \\
3.99 \mathrm{E}-01 \\
1.82 \mathrm{E}+01 \\
5.35 \mathrm{E}+01 \\
1.35 \mathrm{E}+02 \\
5.13 \mathrm{E}+01 \\
4.07 \mathrm{E}-01 \\
1.27 \mathrm{E}-01 \\
1.13 \mathrm{E}-01 \\
1.29 \mathrm{E}-01 \\
1.09 \mathrm{E}-01 \\
2.18 \mathrm{E}-01 \\
3.22 \mathrm{E}-01 \\
1.19 \mathrm{E}-01 \\
5.63 \mathrm{E}-01 \\
1.17 \mathrm{E}-01 \\
2.25 \mathrm{E}-01 \\
1.07 \mathrm{E}-01 \\
1.11 \mathrm{E}-01 \\
5.37 \mathrm{E}-01 \\
5.99 \mathrm{E}-01 \\
1.65 \mathrm{E}-01 \\
1.50 \mathrm{E}-01 \\
1.12 \mathrm{E}-01 \\
4.58 \mathrm{E}-01 \\
3.66 \mathrm{E}-01 \\
2.58 \mathrm{E}-01 \\
3.59 \mathrm{E}-01 \\
6.90 \mathrm{E}-02 \\
1.13 \mathrm{E}-01 \\
6.16 \mathrm{E}-01 \\
3.81 \mathrm{E}-01 \\
1.09 \mathrm{E}-01 \\
1.32 \mathrm{E}-01 \\
1.73 \mathrm{E}-01 \\
1.30 \mathrm{E}-01 \\
1.28 \mathrm{E}-01 \\
1.47 \mathrm{E}+00 \\
1.32 \mathrm{E}+00 \\
7.47 \mathrm{E}-01 \\
2.85 \mathrm{E}-01 \\
5.12 \mathrm{E}-01 \\
1.88 \mathrm{E}+00 \\
1.04 \mathrm{E}+00 \\
4.09 \mathrm{E}-01 \\
1.58 \mathrm{E}-01 \\
4.62 \mathrm{E}-01 \\
1.92 \mathrm{E}+00 \\
2.10 \mathrm{E}+00 \\
1.44 \mathrm{E}+00 \\
6.15 \mathrm{E}-01 \\
1.49 \mathrm{E}-01 \\
6.28 \mathrm{E}-01\end{array}$ & 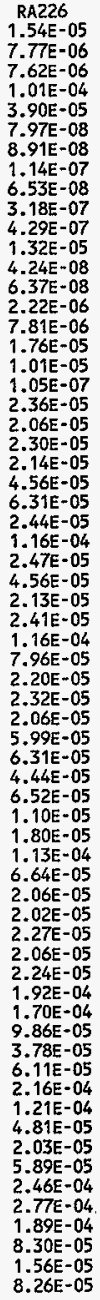 & $\begin{array}{l}\text { RA228 } \\
7.69 \mathrm{E}-10 \\
3.68 \mathrm{E}-10 \\
3.68 \mathrm{E}-10 \\
4.85 \mathrm{E}-09 \\
1.93 \mathrm{E}-09 \\
4.87 \mathrm{E}-12 \\
5.37 \mathrm{E}-12 \\
7.21 \mathrm{E}-12 \\
3.87 \mathrm{E}-12 \\
1.44 \mathrm{E}-11 \\
2.58 \mathrm{E}-11 \\
1.03 \mathrm{E}-09 \\
2.88 \mathrm{E}-12 \\
4.30 \mathrm{E}-12 \\
1.63 \mathrm{E}-10 \\
5.11 \mathrm{E}-10 \\
1.23 \mathrm{E}-09 \\
5.63 \mathrm{E}-10 \\
5.64 \mathrm{E}-12 \\
2.96 \mathrm{E}-01 \\
2.59 \mathrm{E}-01 \\
2.90 \mathrm{E}-01 \\
2.65 \mathrm{E}-01 \\
5.59 \mathrm{E}-01 \\
7.83 \mathrm{E}-01 \\
3.01 \mathrm{E}-01 \\
1.43 \mathrm{E}+00 \\
3.03 \mathrm{E}-01 \\
5.63 \mathrm{E}-01 \\
2.64 \mathrm{E}-01 \\
2.94 \mathrm{E}-01 \\
1.41 \mathrm{E}+00 \\
1.06 \mathrm{E}+00 \\
2.92 \mathrm{E}-01 \\
3.00 \mathrm{E}-01 \\
2.58 \mathrm{E}-01 \\
7.99 \mathrm{E}-01 \\
8.01 \mathrm{E}-01 \\
5.64 \mathrm{E}-01 \\
8.20 \mathrm{E}-01 \\
1.42 \mathrm{E}-01 \\
2.32 \mathrm{E}-01 \\
1.42 \mathrm{E}+00 \\
8.41 \mathrm{E}-01 \\
2.57 \mathrm{E}-01 \\
2.62 \mathrm{E}-01 \\
3.02 \mathrm{E}-01 \\
2.65 \mathrm{E}-01 \\
2.84 \mathrm{E}-01 \\
2.56 \mathrm{E}+00 \\
2.27 \mathrm{E}+00 \\
1.31 \mathrm{E}+00 \\
5.03 \mathrm{E}-01 \\
8.28 \mathrm{E}-01 \\
2.95 \mathrm{E}+00 \\
1.65 \mathrm{E}+00 \\
6.54 \mathrm{E}-01 \\
2.72 \mathrm{E}-01 \\
7.88 \mathrm{E}-01 \\
3.29 \mathrm{E}+00 \\
3.68 \mathrm{E}+00 \\
2.52 \mathrm{E}+00 \\
1.10 \mathrm{E}+00 \\
2.17 \mathrm{E}-01 \\
1.10 \mathrm{E}+00\end{array}$ & $\begin{array}{l}\text { AC227 } \\
7.58 \mathrm{E}-05 \\
3.92 \mathrm{E}=05 \\
3.80 \mathrm{E}-05 \\
5.02 \mathrm{E}-04 \\
1.92 \mathrm{E}-04 \\
3.83 \mathrm{E}-07 \\
4.25 \mathrm{E}-07 \\
5.54 \mathrm{E}-07 \\
3.20 \mathrm{E}-07 \\
1.59 \mathrm{E}-06 \\
2.06 \mathrm{E}-06 \\
6.22 \mathrm{E}-05 \\
2.04 \mathrm{E}-07 \\
3.03 \mathrm{E}-07 \\
1.05 \mathrm{E}-05 \\
3.78 \mathrm{E}-05 \\
8.42 \mathrm{E}-05 \\
5.03 \mathrm{E}-05 \\
6.35 \mathrm{E}-07 \\
5.00 \mathrm{E}-01 \\
4.38 \mathrm{E}-01 \\
4.89 \mathrm{E}-01 \\
4.51 \mathrm{E}-01 \\
9.56 \mathrm{E}-01 \\
1.33 \mathrm{E}+00 \\
5.13 \mathrm{E}-01 \\
2.43 \mathrm{E}+00 \\
5.19 \mathrm{E}-01 \\
9.60 \mathrm{E}-01 \\
4.50 \mathrm{E}-01 \\
5.04 \mathrm{E}-01 \\
2.42 \mathrm{E}+00 \\
1.73 \mathrm{E}+00 \\
4.78 \mathrm{E}-01 \\
4.99 \mathrm{E}-01 \\
4.37 \mathrm{E}-01 \\
1.31 \mathrm{E}+00 \\
1.35 \mathrm{E}+00 \\
9.48 \mathrm{E}-01 \\
1.38 \mathrm{E}+00 \\
2.36 \mathrm{E}-01 \\
3.87 \mathrm{E}-01 \\
2.39 \mathrm{E}+00 \\
1.41 \mathrm{E}+00 \\
4.36 \mathrm{E}-01 \\
4.35 \mathrm{E}-01 \\
4.94 \mathrm{E}-01 \\
4.42 \mathrm{E}-01 \\
4.77 \mathrm{E}-01 \\
4.19 \mathrm{E}+00 \\
3.72 \mathrm{E}+00 \\
2.15 \mathrm{E}+00 \\
8.24 \mathrm{E}-01 \\
1.34 \mathrm{E}+00 \\
4.76 \mathrm{E}+00 \\
2.66 \mathrm{E}+00 \\
1.06 \mathrm{E}+00 \\
4.44 \mathrm{E}-01 \\
1.29 \mathrm{E}+00 \\
5.37 \mathrm{E}+00 \\
6.03 \mathrm{E}+00 \\
4.12 \mathrm{E}+00 \\
3.80 \mathrm{E}+00 \\
1.47 \mathrm{E}-01 \\
\end{array}$ & $\begin{array}{l}\text { TH229 } \\
6.37 \mathrm{E}-08 \\
2.58 \mathrm{E}-08 \\
2.75 \mathrm{E}-08 \\
3.62 \mathrm{E}-07 \\
1.55 \mathrm{E}-07 \\
5.70 \mathrm{E}-10 \\
6.27 \mathrm{E}-10 \\
8.58 \mathrm{E}-10 \\
4.10 \mathrm{E}-10 \\
9.59 \mathrm{E}-10 \\
2.97 \mathrm{E}-09 \\
1.72 \mathrm{E}-07 \\
3.85 \mathrm{E}-10 \\
5.82 \mathrm{E}-10 \\
2.46 \mathrm{E}-08 \\
6.43 \mathrm{E}-08 \\
1.74 \mathrm{E}-07 \\
5.29 \mathrm{E}-08 \\
4.15 \mathrm{E}-10 \\
1.36 \mathrm{E}-01 \\
1.19 \mathrm{E}-01 \\
1.32 \mathrm{E}-01 \\
1.23 \mathrm{E}-01 \\
2.62 \mathrm{E}-01 \\
3.63 \mathrm{E}-01 \\
1.40 \mathrm{E}-01 \\
6.65 \mathrm{E}-01 \\
1.42 \mathrm{E}-01 \\
2.62 \mathrm{E}-01 \\
1.23 \mathrm{E}-01 \\
1.38 \mathrm{E}-01 \\
6.65 \mathrm{E}-01 \\
4.58 \mathrm{E}-01 \\
1.26 \mathrm{E}-01 \\
1.33 \mathrm{E}-01 \\
1.18 \mathrm{E}-01 \\
3.45 \mathrm{E}-01 \\
3.63 \mathrm{E}-01 \\
2.56 \mathrm{E}-01 \\
3.75 \mathrm{E}-01 \\
6.34 \mathrm{E}-02 \\
1.04 \mathrm{E}-01 \\
6.48 \mathrm{E}-01 \\
3.82 \mathrm{E}-01 \\
1.19 \mathrm{E}-01 \\
1.16 \mathrm{E}-01 \\
1.31 \mathrm{E}-01 \\
1.19 \mathrm{E}-01 \\
1.29 \mathrm{E}-01 \\
1.11 \mathrm{E}+00 \\
9.80 \mathrm{E}-01 \\
5.68 \mathrm{E}-01 \\
2.18 \mathrm{E}-01 \\
3.52 \mathrm{E}-01 \\
1.25 \mathrm{E}+00 \\
6.97 \mathrm{E}-01 \\
2.77 \mathrm{E}-01 \\
1.17 \mathrm{E}-01 \\
3.39 \mathrm{E}-01 \\
1.42 \mathrm{E}+00 \\
1.59 \mathrm{E}+00 \\
1.09 \mathrm{E}+00 \\
4.78 \mathrm{E}-01 \\
9.00 \mathrm{E}-02 \\
4.76 \mathrm{E}-01\end{array}$ \\
\hline
\end{tabular}


HNF-SD-WM-TI-794, ReV. 0

ACTIVITY IN UNSEPARATED FUEL (Curies decayed to $1 / 1 / 94$ )

\begin{tabular}{|c|c|c|c|c|c|c|c|c|c|}
\hline $\begin{array}{r}\text { isting } \\
846 \\
847 \\
848 \\
849 \\
850 \\
851 \\
852 \\
853 \\
854 \\
855 \\
856 \\
857 \\
858 \\
859 \\
860 \\
861 \\
862 \\
863 \\
864 \\
865 \\
866 \\
867 \\
868 \\
869 \\
870 \\
871 \\
872 \\
873 \\
874 \\
875 \\
876 \\
877 \\
878 \\
879 \\
880 \\
881 \\
882 \\
883 \\
884 \\
885 \\
886 \\
887 \\
888 \\
889 \\
890 \\
891 \\
892 \\
893 \\
894 \\
895 \\
896 \\
897 \\
898 \\
899 \\
900 \\
901 \\
902 \\
903 \\
904 \\
905 \\
906 \\
907 \\
908 \\
909 \\
910\end{array}$ & 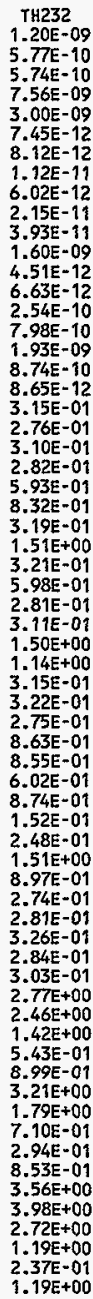 & $\begin{array}{c}\text { PA231 } \\
2.42 \mathrm{E}-04 \\
1.27 \mathrm{E}-04 \\
1.22 \mathrm{E}-04 \\
1.61 \mathrm{E}-03 \\
6.15 \mathrm{E}-04 \\
1.17 \mathrm{E}-06 \\
1.28 \mathrm{E}-06 \\
1.75 \mathrm{E}-06 \\
1.02 \mathrm{E}-06 \\
4.71 \mathrm{E}-06 \\
6.27 \mathrm{E}-06 \\
1.91 \mathrm{E}-04 \\
6.47 \mathrm{E}-07 \\
9.38 \mathrm{E}-07 \\
3.29 \mathrm{E}-05 \\
1.20 \mathrm{E}-04 \\
2.65 \mathrm{E}-04 \\
1.60 \mathrm{E}-04 \\
1.99 \mathrm{E}-06 \\
8.68 \mathrm{E}-01 \\
7.61 \mathrm{E}-01 \\
8.53 \mathrm{E}-01 \\
7.78 \mathrm{E}-01 \\
1.63 \mathrm{E}+00 \\
2.29 \mathrm{E}+00 \\
8.79 \mathrm{E}-01 \\
4.17 \mathrm{E}+00 \\
8.85 \mathrm{E}-01 \\
1.65 \mathrm{E}+00 \\
7.73 \mathrm{E}-01 \\
8.57 \mathrm{E}-01 \\
4.13 \mathrm{E}+00 \\
3.15 \mathrm{E}+00 \\
8.68 \mathrm{E}-01 \\
8.87 \mathrm{E}-01 \\
7.58 \mathrm{E}-01 \\
2.38 \mathrm{E}+00 \\
2.36 \mathrm{E}+00 \\
1.66 \mathrm{E}+00 \\
2.41 \mathrm{E}+00 \\
4.19 \mathrm{E}-01 \\
6.85 \mathrm{E}-01 \\
4.16 \mathrm{E}+00 \\
2.47 \mathrm{E}+00 \\
7.55 \mathrm{E}-01 \\
7.75 \mathrm{E}-01 \\
9.00 \mathrm{E}-01 \\
7.84 \mathrm{E}-01 \\
8.35 \mathrm{E}-01 \\
7.63 \mathrm{E}+00 \\
6.78 \mathrm{E}+00 \\
3.91 \mathrm{E}+00 \\
1.50 \mathrm{E}+00 \\
2.48 \mathrm{E}+00 \\
8.84 \mathrm{E}+00 \\
4.93 \mathrm{E}+00 \\
1.96 \mathrm{E}+00 \\
8.10 \mathrm{E}-01 \\
2.35 \mathrm{E}+00 \\
9.82 \mathrm{E}+00 \\
1.10 \mathrm{E}+01 \\
7.50 \mathrm{E}+00 \\
3.27 \mathrm{E}+00 \\
6.52 \mathrm{E}-01 \\
3.28 \mathrm{E}+00\end{array}$ & $\begin{array}{c}\text { U232 } \\
1.85 \mathrm{E}-03 \\
6.79 \mathrm{E}-04 \\
7.53 \mathrm{E}-04 \\
9.87 \mathrm{E}-03 \\
4.45 \mathrm{E}-03 \\
2.50 \mathrm{E}-05 \\
2.64 \mathrm{E}-05 \\
4.07 \mathrm{E}-05 \\
1.71 \mathrm{E}-05 \\
2.06 \mathrm{E}-05 \\
1.25 \mathrm{E}-04 \\
1.27 \mathrm{E}-02 \\
2.15 \mathrm{E}-05 \\
3.15 \mathrm{E}-05 \\
1.60 \mathrm{E}-03 \\
3.31 \mathrm{E}-03 \\
1.04 \mathrm{E}-02 \\
1.91 \mathrm{E}-03 \\
1.13 \mathrm{E}-05 \\
1.37 \mathrm{E}+01 \\
1.20 \mathrm{E}+01 \\
1.35 \mathrm{E}+01 \\
1.23 \mathrm{E}+01 \\
2.57 \mathrm{E}+01 \\
3.62 \mathrm{E}+01 \\
1.38 \mathrm{E}+01 \\
6.55 \mathrm{E}+01 \\
1.39 \mathrm{E}+01 \\
2.59 \mathrm{E}+01 \\
1.22 \mathrm{E}+01 \\
1.34 \mathrm{E}+01 \\
6.47 \mathrm{E}+01 \\
5.07 \mathrm{E}+01 \\
1.40 \mathrm{E}+01 \\
1.42 \mathrm{E}+01 \\
1.20 \mathrm{E}+01 \\
3.83 \mathrm{E}+01 \\
3.74 \mathrm{E}+01 \\
2.64 \mathrm{E}+01 \\
3.81 \mathrm{E}+01 \\
6.67 \mathrm{E}+00 \\
1.09 \mathrm{E}+01 \\
6.58 \mathrm{E}+01 \\
3.93 \mathrm{E}+01 \\
1.19 \mathrm{E}+01 \\
1.24 \mathrm{E}+01 \\
1.45 \mathrm{E}+01 \\
1.25 \mathrm{E}+01 \\
1.32 \mathrm{E}+01 \\
1.23 \mathrm{E}+02 \\
1.09 \mathrm{E}+02 \\
6.29 \mathrm{E}+01 \\
2.41 \mathrm{E}+01 \\
4.01 \mathrm{E}+01 \\
1.43 \mathrm{E}+02 \\
7.99 \mathrm{E}+01 \\
3.17 \mathrm{E}+01 \\
1.31 \mathrm{E}+01 \\
3.79 \mathrm{E}+01 \\
1.58 \mathrm{E}+02 \\
1.77 \mathrm{E}+02 \\
1.21 \mathrm{E}+02 \\
5.26 \mathrm{E}+01 \\
1.06 \mathrm{E}+01 \\
5.28 \mathrm{E}+01\end{array}$ & $\begin{array}{l}\text { U233 } \\
3.63 \mathrm{E}-05 \\
1.48 \mathrm{E}-05 \\
1.57 \mathrm{E}-05 \\
2.06 \mathrm{E}-04 \\
8.85 \mathrm{E}-05 \\
3.19 \mathrm{E}-07 \\
3.44 \mathrm{E}-07 \\
4.94 \mathrm{E}-07 \\
2.37 \mathrm{E}-07 \\
5.09 \mathrm{E}-07 \\
1.65 \mathrm{E}-06 \\
9.79 \mathrm{E}-05 \\
2.24 \mathrm{E}-07 \\
3.30 \mathrm{E}-07 \\
1.41 \mathrm{E}-05 \\
3.74 \mathrm{E}-05 \\
1.01 \mathrm{E}-04 \\
3.05 \mathrm{E}-05 \\
2.34 \mathrm{E}-07 \\
5.36 \mathrm{E}+01 \\
4.70 \mathrm{E}+01 \\
5.27 \mathrm{E}+01 \\
4.80 \mathrm{E}+01 \\
1.01 \mathrm{E}+02 \\
1.42 \mathrm{E}+02 \\
5.43 \mathrm{E}+01 \\
2.57 \mathrm{E}+02 \\
5.46 \mathrm{E}+01 \\
1.02 \mathrm{E}+02 \\
4.77 \mathrm{E}+01 \\
5.29 \mathrm{E}+01 \\
2.55 \mathrm{E}+02 \\
1.95 \mathrm{E}+02 \\
5.36 \mathrm{E}+01 \\
5.48 \mathrm{E}+01 \\
4.68 \mathrm{E}+01 \\
1.47 \mathrm{E}+02 \\
1.46 \mathrm{E}+02 \\
1.03 \mathrm{E}+02 \\
1.49 \mathrm{E}+02 \\
2.58 \mathrm{E}+01 \\
4.23 \mathrm{E}+01 \\
2.57 \mathrm{E}+02 \\
1.53 \mathrm{E}+02 \\
4.66 \mathrm{E}+01 \\
4.78 \mathrm{E}+01 \\
5.55 \mathrm{E}+01 \\
4.84 \mathrm{E}+01 \\
5.15 \mathrm{E}+01 \\
4.71 \mathrm{E}+02 \\
4.18 \mathrm{E}+02 \\
2.41 \mathrm{E}+02 \\
9.24 \mathrm{E}+01 \\
1.53 \mathrm{E}+02 \\
5.46 \mathrm{E}+02 \\
3.04 \mathrm{E}+02 \\
1.21 \mathrm{E}+02 \\
5.00 \mathrm{E}+01 \\
1.45 \mathrm{E}+02 \\
6.06 \mathrm{E}+02 \\
6.77 \mathrm{E}+02 \\
4.63 \mathrm{E}+02 \\
2.02 \mathrm{E}+02 \\
4.03 \mathrm{E}+01 \\
2.02 \mathrm{E}+02\end{array}$ & 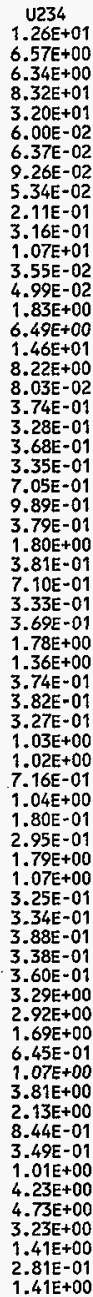 & 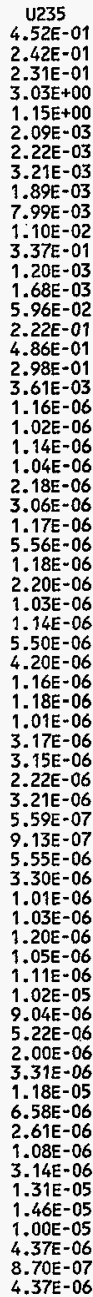 & 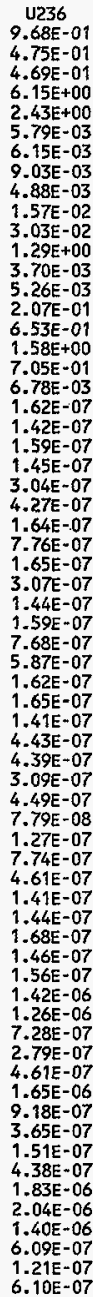 & $\begin{array}{l}\text { U238 } \\
8.64 \mathrm{E}+00 \\
4.48 \mathrm{E}+00 \\
4.33 \mathrm{E}+00 \\
5.68 \mathrm{E}+01 \\
2.19 \mathrm{E}+01 \\
4.19 \mathrm{E}-02 \\
4.45 \mathrm{E}-02 \\
6.48 \mathrm{E}-02 \\
3.71 \mathrm{E}-02 \\
1.44 \mathrm{E}-01 \\
2.21 \mathrm{E}-01 \\
7.66 \mathrm{E}+00 \\
2.50 \mathrm{E}-02 \\
3.52 \mathrm{E}-02 \\
1.30 \mathrm{E}+00 \\
4.55 \mathrm{E}+00 \\
1.03 \mathrm{E}+01 \\
5.67 \mathrm{E}+00 \\
5.48 \mathrm{E}-02 \\
9.46 \mathrm{E}-15 \\
8.30 \mathrm{E}-15 \\
9.31 \mathrm{E}-15 \\
8.48 \mathrm{E}-15 \\
1.78 \mathrm{E}-14 \\
2.50 \mathrm{E}-14 \\
9.58 \mathrm{E}-15 \\
4.54 \mathrm{E}-14 \\
9.65 \mathrm{E}-15 \\
1.80 \mathrm{E}-14 \\
8.44 \mathrm{E}-15 \\
9.34 \mathrm{E}-15 \\
4.50 \mathrm{E}-14 \\
3.44 \mathrm{E}-14 \\
9.46 \mathrm{E}-15 \\
9.67 \mathrm{E}-15 \\
8.27 \mathrm{E}-15 \\
2.59 \mathrm{E}-14 \\
2.57 \mathrm{E}-14 \\
1.81 \mathrm{E}-14 \\
2.63 \mathrm{E}-14 \\
4.56 \mathrm{E}-15 \\
7.46 \mathrm{E}-15 \\
4.53 \mathrm{E}-14 \\
2.70 \mathrm{E}-14 \\
8.23 \mathrm{E}-15 \\
8.45 \mathrm{E}-15 \\
9.81 \mathrm{E}-15 \\
8.55 \mathrm{E}-15 \\
9.10 \mathrm{E}-15 \\
8.33 \mathrm{E}-14 \\
7.39 \mathrm{E}-14 \\
4.26 \mathrm{E}-14 \\
1.63 \mathrm{E}-14 \\
2.70 \mathrm{E}-14 \\
9.64 \mathrm{E}-14 \\
5.38 \mathrm{E}-14 \\
2.13 \mathrm{E}-14 \\
8.84 \mathrm{E}-15 \\
2.56 \mathrm{E}-14 \\
1.07 \mathrm{E}-13 \\
1.20 \mathrm{E}-13 \\
8.18 \mathrm{E}-14 \\
3.57 \mathrm{E}-14 \\
7.11 \mathrm{E}-15 \\
3.57 \mathrm{E}-14\end{array}$ & 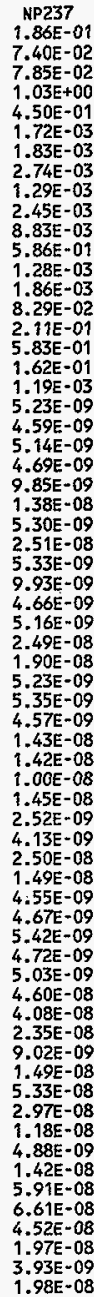 \\
\hline
\end{tabular}


HNF-SD-WM-TI-794, ReV. 0

ACTIVITY IN UNSEPARATED FUEL (Curies decayed to 1/1/94)

\begin{tabular}{|c|c|c|c|c|c|c|c|c|c|}
\hline 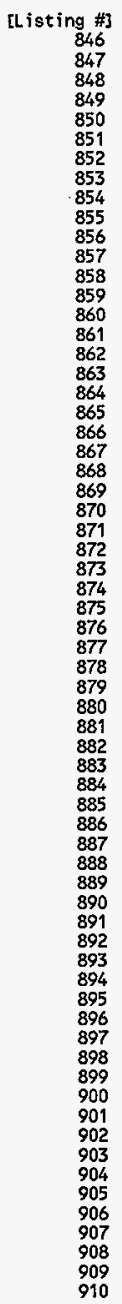 & $\begin{array}{l}\text { PU238 } \\
9.58 \mathrm{E}+01 \\
3.18 \mathrm{E}+01 \\
3.67 \mathrm{E}+01 \\
4.81 \mathrm{E}+02 \\
2.27 \mathrm{E}+02 \\
1.79 \mathrm{E}+00 \\
1.89 \mathrm{E}+00 \\
2.93 \mathrm{E}+00 \\
1.18 \mathrm{E}+00 \\
1.13 \mathrm{E}+00 \\
8.95 \mathrm{E}+00 \\
1.02 \mathrm{E}+03 \\
1.61 \mathrm{E}+00 \\
2.38 \mathrm{E}+00 \\
1.25 \mathrm{E}+02 \\
2.43 \mathrm{E}+02 \\
7.93 \mathrm{E}+02 \\
1.26 \mathrm{E}+02 \\
6.81 \mathrm{E}-01 \\
7.24 \mathrm{E}-07 \\
6.35 \mathrm{E}-07 \\
7.13 \mathrm{E}-07 \\
6.47 \mathrm{E}-07 \\
1.36 \mathrm{E}-06 \\
1.91 \mathrm{E}-06 \\
7.30 \mathrm{E}-07 \\
3.46 \mathrm{E}-06 \\
7.33 \mathrm{E}-07 \\
1.37 \mathrm{E}-06 \\
6.43 \mathrm{E}-07 \\
7.09 \mathrm{E}-07 \\
3.42 \mathrm{E}-06 \\
2.67 \mathrm{E}-06 \\
7.34 \mathrm{E}-07 \\
7.46 \mathrm{E}-07 \\
6.32 \mathrm{E}-07 \\
2.02 \mathrm{E}-06 \\
1.97 \mathrm{E}-06 \\
1.39 \mathrm{E}-06 \\
2.01 \mathrm{E}-06 \\
3.51 \mathrm{E}-07 \\
5.75 \mathrm{E}-07 \\
3.47 \mathrm{E}-06 \\
2.07 \mathrm{E}-06 \\
6.29 \mathrm{E}-07 \\
6.52 \mathrm{E}-07 \\
7.62 \mathrm{E}-07 \\
6.58 \mathrm{E}-07 \\
6.98 \mathrm{E}-07 \\
6.47 \mathrm{E}-06 \\
5.74 \mathrm{E}-06 \\
3.31 \mathrm{E}-06 \\
1.27 \mathrm{E}-06 \\
2.11 \mathrm{E}-06 \\
7.53 \mathrm{E}-06 \\
4.20 \mathrm{E}-06 \\
1.67 \mathrm{E}-06 \\
6.87 \mathrm{E}-07 \\
1.99 \mathrm{E}-06 \\
8.32 \mathrm{E}-06 \\
9.29 \mathrm{E}-06 \\
6.35 \mathrm{E}-06 \\
2.77 \mathrm{E}-06 \\
5.58 \mathrm{E}-07 \\
2.78 \mathrm{E}-06\end{array}$ & $\begin{array}{l}\text { PU239 } \\
1.44 \mathrm{E}+03 \\
6.14 \mathrm{E}+02 \\
6.39 \mathrm{E}+02 \\
8.38 \mathrm{E}+03 \\
3.53 \mathrm{E}+03 \\
1.09 \mathrm{E}+01 \\
1.16 \mathrm{E}+01 \\
1.71 \mathrm{E}+01 \\
8.68 \mathrm{E}+00 \\
2.00 \mathrm{E}+01 \\
5.63 \mathrm{E}+01 \\
2.83 \mathrm{E}+03 \\
7.40 \mathrm{E}+00 \\
1.06 \mathrm{E}+01 \\
4.36 \mathrm{E}+02 \\
1.27 \mathrm{E}+03 \\
3.23 \mathrm{E}+03 \\
1.16 \mathrm{E}+03 \\
9.02 \mathrm{E}+00 \\
2.03 \mathrm{E}-11 \\
1.78 \mathrm{E}-11 \\
2.00 \mathrm{E}-11 \\
1.82 \mathrm{E}-11 \\
3.83 \mathrm{E}-11 \\
5.38 \mathrm{E}-11 \\
2.06 \mathrm{E}-11 \\
9.76 \mathrm{E}-11 \\
2.07 \mathrm{E}-11 \\
3.86 \mathrm{E}-11 \\
1.81 \mathrm{E}-11 \\
2.01 \mathrm{E}-11 \\
9.66 \mathrm{E}-11 \\
7.38 \mathrm{E}-11 \\
2.03 \mathrm{E}-11 \\
2.08 \mathrm{E}-11 \\
1.78 \mathrm{E}-11 \\
5.57 \mathrm{E}-11 \\
5.52 \mathrm{E}-11 \\
3.89 \mathrm{E}-11 \\
5.64 \mathrm{E}-11 \\
9.81 \mathrm{E}-12 \\
1.60 \mathrm{E}-11 \\
9.74 \mathrm{E}-11 \\
5.80 \mathrm{E}-11 \\
1.77 \mathrm{E}-11 \\
1.82 \mathrm{E}-11 \\
2.11 \mathrm{E}-11 \\
1.84 \mathrm{E}-11 \\
1.96 \mathrm{E}-11 \\
1.79 \mathrm{E}-10 \\
1.59 \mathrm{E}-10 \\
9.16 \mathrm{E}-11 \\
3.51 \mathrm{E}-11 \\
5.81 \mathrm{E}-11 \\
2.07 \mathrm{E}-10 \\
1.16 \mathrm{E}-10 \\
4.59 \mathrm{E}-11 \\
1.90 \mathrm{E}-11 \\
5.51 \mathrm{E}-11 \\
2.30 \mathrm{E}-10 \\
2.57 \mathrm{E}-10 \\
1.76 \mathrm{E}-10 \\
7.67 \mathrm{E}-11 \\
1.53 \mathrm{E}-11 \\
7.68 \mathrm{E}-11\end{array}$ & $\begin{array}{l}\text { PU240 } \\
3.68 \mathrm{E}+02 \\
1.25 \mathrm{E}+02 \\
1.43 \mathrm{E}+02 \\
1.87 \mathrm{E}+03 \\
8.74 \mathrm{E}+02 \\
4.23 \mathrm{E}+00 \\
4.49 \mathrm{E}+00 \\
6.79 \mathrm{E}+00 \\
3.00 \mathrm{E}+00 \\
4.61 \mathrm{E}+00 \\
2.14 \mathrm{E}+01 \\
1.65 \mathrm{E}+03 \\
3.38 \mathrm{E}+00 \\
4.92 \mathrm{E}+00 \\
2.27 \mathrm{E}+02 \\
5.39 \mathrm{E}+02 \\
1.56 \mathrm{E}+03 \\
3.54 \mathrm{E}+02 \\
2.31 \mathrm{E}+00 \\
1.09 \mathrm{E}-12 \\
9.56 \mathrm{E}-13 \\
1.07 \mathrm{E}-12 \\
9.76 \mathrm{E}-13 \\
2.05 \mathrm{E}-12 \\
2.88 \mathrm{E}-12 \\
1.10 \mathrm{E}-12 \\
5.23 \mathrm{E}-12 \\
1.11 \mathrm{E}-12 \\
2.07 \mathrm{E}-12 \\
9.71 \mathrm{E}-13 \\
1.08 \mathrm{E}-12 \\
5.18 \mathrm{E}-12 \\
3.96 \mathrm{E}-12 \\
1.09 \mathrm{E}-12 \\
1.11 \mathrm{E}-12 \\
9.52 \mathrm{E}-13 \\
2.99 \mathrm{E}-12 \\
2.96 \mathrm{E}-12 \\
2.09 \mathrm{E}-12 \\
3.03 \mathrm{E}-12 \\
5.26 \mathrm{E}-13 \\
8.60 \mathrm{E}-13 \\
5.22 \mathrm{E}-12 \\
3.11 \mathrm{E}-12 \\
9.48 \mathrm{E}-13 \\
9.73 \mathrm{E}-13 \\
1.13 \mathrm{E}-12 \\
9.84 \mathrm{E}-13 \\
1.05 \mathrm{E}-12 \\
9.59 \mathrm{E}-12 \\
8.51 \mathrm{E}-12 \\
4.91 \mathrm{E}-12 \\
1.88 \mathrm{E}-12 \\
3.11 \mathrm{E}-12 \\
1.11 \mathrm{E}-11 \\
6.19 \mathrm{E}-12 \\
2.46 \mathrm{E}-12 \\
1.02 \mathrm{E}-12 \\
2.95 \mathrm{E}-12 \\
1.23 \mathrm{E}-11 \\
1.38 \mathrm{E}-111 \\
9.42 \mathrm{E}-12 \\
4.11 \mathrm{E}-12 \\
8.19 \mathrm{E}-13 \\
4.12 \mathrm{E}-12\end{array}$ & $\begin{array}{l}\text { PU241 } \\
4.68 \mathrm{E}+03 \\
1.52 \mathrm{E}+03 \\
1.77 \mathrm{E}+03 \\
2.31 \mathrm{E}+04 \\
1.10 \mathrm{E}+04 \\
1.21 \mathrm{E}+02 \\
1.24 \mathrm{E}+02 \\
2.06 \mathrm{E}+02 \\
8.12 \mathrm{E}+01 \\
5.70 \mathrm{E}+01 \\
5.94 \mathrm{E}+02 \\
6.64 \mathrm{E}+04 \\
1.16 \mathrm{E}+02 \\
1.64 \mathrm{E}+02 \\
8.51 \mathrm{E}+03 \\
1.75 \mathrm{E}+04 \\
5.56 \mathrm{E}+04 \\
8.28 \mathrm{E}+03 \\
3.92 \mathrm{E}+01 \\
5.15 \mathrm{E}-12 \\
4.54 \mathrm{E}-12 \\
5.12 \mathrm{E}-12 \\
4.55 \mathrm{E}-12 \\
9.39 \mathrm{E}-12 \\
1.34 \mathrm{E}-11 \\
5.08 \mathrm{E}-12 \\
2.41 \mathrm{E}-11 \\
5.07 \mathrm{E}-12 \\
9.55 \mathrm{E}-12 \\
4.50 \mathrm{E}-12 \\
4.87 \mathrm{E}-12 \\
2.35 \mathrm{E}-11 \\
2.05 \mathrm{E}-11 \\
5.63 \mathrm{E}-12 \\
5.54 \mathrm{E}-12 \\
4.51 \mathrm{E}-12 \\
1.55 \mathrm{E}-11 \\
1.43 \mathrm{E}-11 \\
1.01 \mathrm{E}-11 \\
1.44 \mathrm{E}-11 \\
2.59 \mathrm{E}-12 \\
4.23 \mathrm{E}-12 \\
2.48 \mathrm{E}-11 \\
1.49 \mathrm{E}-11 \\
4.46 \mathrm{E}-12 \\
4.85 \mathrm{E}-12 \\
5.86 \mathrm{E}-12 \\
4.85 \mathrm{E}-12 \\
5.03 \mathrm{E}-12 \\
4.98 \mathrm{E}-11 \\
4.43 \mathrm{E}-11 \\
2.54 \mathrm{E}-11 \\
9.72 \mathrm{E}-12 \\
1.66 \mathrm{E}-11 \\
5.97 \mathrm{E}-11 \\
3.32 \mathrm{E}-11 \\
1.31 \mathrm{E}-11 \\
5.31 \mathrm{E}-12 \\
1.54 \mathrm{E}-11 \\
6.44 \mathrm{E}-11 \\
7.14 \mathrm{E}-11 \\
4.88 \mathrm{E}-11 \\
2.12 \mathrm{E}-11 \\
4.51 \mathrm{E}-12 \\
2.13 \mathrm{E}-11\end{array}$ & $\begin{array}{l}\text { PU242 } \\
9.55 \mathrm{E}-03 \\
2.98 \mathrm{E}-03 \\
3.54 \mathrm{E}-03 \\
4.64 \mathrm{E}-02 \\
2.25 \mathrm{E}-02 \\
8.75 \mathrm{E}-04 \\
9.29 \mathrm{E}-04 \\
1.44 \mathrm{E}-03 \\
5.18 \mathrm{E}-04 \\
8.97 \mathrm{E}-05 \\
4.32 \mathrm{E}-03 \\
7.21 \mathrm{E}-01 \\
8.82 \mathrm{E}-04 \\
1.34 \mathrm{E}-03 \\
7.97 \mathrm{E}-02 \\
1.24 \mathrm{E}-01 \\
4.66 \mathrm{E}-01 \\
4.65 \mathrm{E}-02 \\
1.50 \mathrm{E}-04 \\
6.31 \mathrm{E}-18 \\
5.54 \mathrm{E}-18 \\
6.20 \mathrm{E}-18 \\
5.65 \mathrm{E}-18 \\
1.19 \mathrm{E}-17 \\
1.67 \mathrm{E}-17 \\
6.39 \mathrm{E}-18 \\
3.03 \mathrm{E}-17 \\
6.43 \mathrm{E}-18 \\
1.20 \mathrm{E}-17 \\
5.62 \mathrm{E}-18 \\
6.23 \mathrm{E}-18 \\
3.00 \mathrm{E}-17 \\
2.29 \mathrm{E}-17 \\
6.31 \mathrm{E}-18 \\
6.45 \mathrm{E}-18 \\
5.51 \mathrm{E}-18 \\
1.73 \mathrm{E}-17 \\
1.71 \mathrm{E}-17 \\
1.21 \mathrm{E}-17 \\
1.75 \mathrm{E}-17 \\
3.04 \mathrm{E}-18 \\
4.98 \mathrm{E}-18 \\
3.02 \mathrm{E}-17 \\
1.80 \mathrm{E}-17 \\
5.49 \mathrm{E}-18 \\
5.63 \mathrm{E}-18 \\
6.54 \mathrm{E}-18 \\
5.70 \mathrm{E}-18 \\
6.07 \mathrm{E}-18 \\
5.55 \mathrm{E}-17 \\
4.93 \mathrm{E}-17 \\
2.84 \mathrm{E}-17 \\
1.09 \mathrm{E}-17 \\
1.80 \mathrm{E}-17 \\
6.43 \mathrm{E}-17 \\
3.58 \mathrm{E}-17 \\
1.42 \mathrm{E}-17 \\
5.89 \mathrm{E}-18 \\
1.71 \mathrm{E}-17 \\
7.14 \mathrm{E}-17 \\
7.98 \mathrm{E}-17 \\
5.45 \mathrm{E}-17 \\
2.38 \mathrm{E}-17 \\
4.74 \mathrm{E}-18 \\
2.38 \mathrm{E}-17\end{array}$ & $\begin{array}{l}\text { AM241 } \\
3.49 E+02 \\
1.11 E+02 \\
1.31 E+02 \\
1.72 E+03 \\
8.22 E+02 \\
9.65 E+00 \\
1.04 E+01 \\
1.54 E+01 \\
6.07 E+00 \\
5.14 E+00 \\
4.84 E+01 \\
4.92 E+03 \\
8.40 E+00 \\
1.26 E+01 \\
6.24 E+02 \\
1.28 E+03 \\
4.06 E+03 \\
6.26 E+02 \\
3.12 E+00 \\
4.42 E-13 \\
3.87 E-13 \\
4.33 E-13 \\
3.98 E-13 \\
8.41 E-13 \\
1.17 E-12 \\
4.52 E-13 \\
2.14 E-12 \\
4.56 E-13 \\
8.46 E-13 \\
3.96 E-13 \\
4.43 E-13 \\
2.13 E-12 \\
1.55 E-12 \\
4.27 E-13 \\
4.43 E-13 \\
3.86 E-13 \\
1.17 E-12 \\
1.19 E-12 \\
8.39 E-13 \\
1.22 E-12 \\
2.10 E-13 \\
3.43 E-13 \\
2.11 E-12 \\
1.25 E-12 \\
3.85 E-13 \\
3.87 E-13 \\
4.42 E-13 \\
3.93 E-13 \\
4.23 E-13 \\
3.75 E-12 \\
3.32 E-12 \\
1.92 E-12 \\
7.36 E-13 \\
1.20 E-12 \\
4.28 E-12 \\
2.39 E-12 \\
9.50 E-13 \\
3.97 E-13 \\
1.15 E-12 \\
4.81 E-12 \\
5.39 E-12 \\
3.69 E-12 \\
1.61 E-12 \\
3.12 E-13 \\
1.61 E-12\end{array}$ & $\begin{array}{l}\text { AM243 } \\
2.90 \mathrm{E}-03 \\
8.94 \mathrm{E}-04 \\
1.07 \mathrm{E}-03 \\
1.40 \mathrm{E}-02 \\
6.80 \mathrm{E}-03 \\
1.03 \mathrm{E}-03 \\
1.09 \mathrm{E}-03 \\
1.70 \mathrm{E}-03 \\
5.74 \mathrm{E}-04 \\
1.78 \mathrm{E}-08 \\
5.02 \mathrm{E}-03 \\
1.38 \mathrm{E}-00 \\
1.18 \mathrm{E}-03 \\
1.86 \mathrm{E}-03 \\
1.35 \mathrm{E}-01 \\
1.50 \mathrm{E}-01 \\
7.02 \mathrm{E}-01 \\
4.58 \mathrm{E}-02 \\
7.08 \mathrm{E}-05 \\
1.26 \mathrm{E}-18 \\
1.11 \mathrm{E}-18 \\
1.24 \mathrm{E}-18 \\
1.13 \mathrm{E}-18 \\
2.38 \mathrm{E}-18 \\
3.34 \mathrm{E}-18 \\
1.28 \mathrm{E}-18 \\
6.06 \mathrm{E}-18 \\
1.29 \mathrm{E}-18 \\
2.40 \mathrm{E}-18 \\
1.12 \mathrm{E}-18 \\
1.25 \mathrm{E}-18 \\
6.00 \mathrm{E}-18 \\
4.58 \mathrm{E}-18 \\
1.26 \mathrm{E}-18 \\
1.29 \mathrm{E}-18 \\
1.10 \mathrm{E}-18 \\
3.46 \mathrm{E}-18 \\
3.43 \mathrm{E}-18 \\
2.41 \mathrm{E}-18 \\
3.50 \mathrm{E}-18 \\
6.09 \mathrm{E}-19 \\
9.96 \mathrm{E}-19 \\
6.04 \mathrm{E}-18 \\
3.60 \mathrm{E}-18 \\
1.10 \mathrm{E}-18 \\
1.13 \mathrm{E}-18 \\
1.31 \mathrm{E}-18 \\
1.14 \mathrm{E}-18 \\
1.21 \mathrm{E}-18 \\
1.11 \mathrm{E}-17 \\
9.86 \mathrm{E}-18 \\
5.68 \mathrm{E}-18 \\
2.18 \mathrm{E}-18 \\
3.60 \mathrm{E}-18 \\
1.29 \mathrm{E}-17 \\
7.17 \mathrm{E}-18 \\
2.85 \mathrm{E}-18 \\
1.18 \mathrm{E}-18 \\
3.42 \mathrm{E}-18 \\
1.43 \mathrm{E}-17 \\
1.59 \mathrm{E}-17 \\
1.09 \mathrm{E}-17 \\
4.76 \mathrm{E}-18 \\
9.49 \mathrm{E}-19 \\
4.77 \mathrm{E}-18\end{array}$ & $\begin{array}{l}\text { CM242 } \\
1.22 \mathrm{E}-02 \\
3.76 \mathrm{E}-03 \\
4.48 \mathrm{E}-03 \\
5.88 \mathrm{E}-02 \\
2.86 \mathrm{E}-02 \\
2.68 \mathrm{E}-03 \\
2.84 \mathrm{E}-03 \\
4.47 \mathrm{E}-03 \\
1.51 \mathrm{E}-03 \\
8.30 \mathrm{E}-22 \\
1.31 \mathrm{E}-02 \\
3.26 \mathrm{E}+00 \\
3.02 \mathrm{E}-03 \\
4.70 \mathrm{E}-03 \\
3.27 \mathrm{E}-01 \\
3.93 \mathrm{E}-01 \\
1.75 \mathrm{E}+00 \\
1.20 \mathrm{E}-01 \\
1.89 \mathrm{E}-04 \\
0.00 \mathrm{E}+00 \\
0.00 \mathrm{E}+00 \\
0.00 \mathrm{E}+00 \\
0.00 \mathrm{E}+00 \\
0.00 \mathrm{E}+00 \\
0.00 \mathrm{E}+00 \\
0.00 \mathrm{E}+00 \\
0.00 \mathrm{E}+00 \\
0.00 \mathrm{E}+00 \\
0.00 \mathrm{E}+00 \\
0.00 \mathrm{E}+00 \\
0.00 \mathrm{E}+00 \\
0.00 \mathrm{E}+00 \\
8.48 \mathrm{E}-31 \\
2.25 \mathrm{E}-31 \\
0.00 \mathrm{E}+00 \\
0.00 \mathrm{E}+00 \\
7.37 \mathrm{E}-31 \\
0.00 \mathrm{E}+00 \\
0.00 \mathrm{E}+00 \\
0.00 \mathrm{E}+00 \\
0.00 \mathrm{E}+00 \\
0.00 \mathrm{E}+00 \\
0.00 \mathrm{E}+00 \\
0.00 \mathrm{E}+00 \\
0.00 \mathrm{E}+00 \\
0.00 \mathrm{E}+00 \\
2.65 \mathrm{E}-31 \\
0.00 \mathrm{E}+00 \\
0.00 \mathrm{E}+00 \\
2.44 \mathrm{E}-30 \\
2.27 \mathrm{E}-30 \\
1.10 \mathrm{E}-30 \\
4.01 \mathrm{E}-31 \\
1.69 \mathrm{E}-30 \\
8.16 \mathrm{E}-30 \\
4.07 \mathrm{E}-30 \\
1.51 \mathrm{E}-30 \\
2.94 \mathrm{E}-31 \\
9.08 \mathrm{E}-31 \\
3.70 \mathrm{E}-30 \\
3.20 \mathrm{E}-30 \\
2.16 \mathrm{E}-30 \\
7.72 \mathrm{E}-31 \\
1.32 \mathrm{E}-30 \\
9.64 \mathrm{E}-31\end{array}$ & $\begin{array}{c}\text { CH243 } \\
2.95 \mathrm{E}-04 \\
9.12 \mathrm{E}-05 \\
1.08 \mathrm{E}-04 \\
1.42 \mathrm{E}-03 \\
6.91 \mathrm{E}-04 \\
2.79 \mathrm{E}-04 \\
2.91 \mathrm{E}-04 \\
4.76 \mathrm{E}-04 \\
1.55 \mathrm{E}-04 \\
0.00 \mathrm{E}+00 \\
1.35 \mathrm{E}-03 \\
5.69 \mathrm{E}-01 \\
3.75 \mathrm{E}-04 \\
6.01 \mathrm{E}-04 \\
5.18 \mathrm{E}-02 \\
4.27 \mathrm{E}-02 \\
2.48 \mathrm{E}-01 \\
1.15 \mathrm{E}-02 \\
5.57 \mathrm{E}-06 \\
7.68 \mathrm{E}-20 \\
6.76 \mathrm{E}-20 \\
7.59 \mathrm{E}-20 \\
6.83 \mathrm{E}-20 \\
1.42 \mathrm{E}-19 \\
2.02 \mathrm{E}-19 \\
7.67 \mathrm{E}-20 \\
3.64 \mathrm{E}-19 \\
7.69 \mathrm{E}-20 \\
1.44 \mathrm{E}-19 \\
6.78 \mathrm{E}-20 \\
7.42 \mathrm{E}-20 \\
3.58 \mathrm{E}-19 \\
2.92 \mathrm{E}-19 \\
8.03 \mathrm{E}-20 \\
8.05 \mathrm{E}-20 \\
6.72 \mathrm{E}-20 \\
2.21 \mathrm{E}-19 \\
2.11 \mathrm{E}-19 \\
1.48 \mathrm{E}-19 \\
2.14 \mathrm{E}-19 \\
3.78 \mathrm{E}-20 \\
6.18 \mathrm{E}-20 \\
3.69 \mathrm{E}-19 \\
2.21 \mathrm{E}-19 \\
6.66 \mathrm{E}-20 \\
7.04 \mathrm{E}-20 \\
8.34 \mathrm{E}-20 \\
7.08 \mathrm{E}-20 \\
7.45 \mathrm{E}-20 \\
7.09 \mathrm{E}-19 \\
6.30 \mathrm{E}-19 \\
3.62 \mathrm{E}-19 \\
1.39 \mathrm{E}-19 \\
2.33 \mathrm{~B}-19 \mathrm{E}-18 \\
3.35 \mathrm{E}-19 \\
3.65 \mathrm{E}-19 \\
7.54 \mathrm{E}-19 \\
2.19 \mathrm{E}-20-19 \\
3.04 \mathrm{E}-19 \\
\end{array}$ \\
\hline
\end{tabular}


HNF-SD-HM-TI-794, Rev. 0

ACTIVITY IN UNSEPARATED FUEL (Curies decayed to $1 / 1 / 94$ )

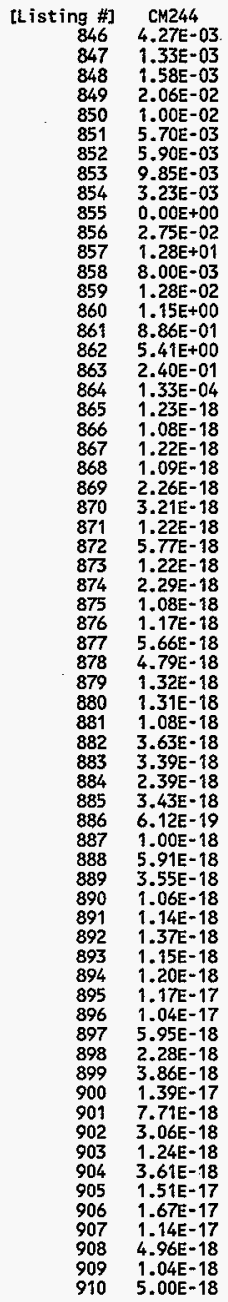


HNF-SD-WH-TI-794, ReV. 0

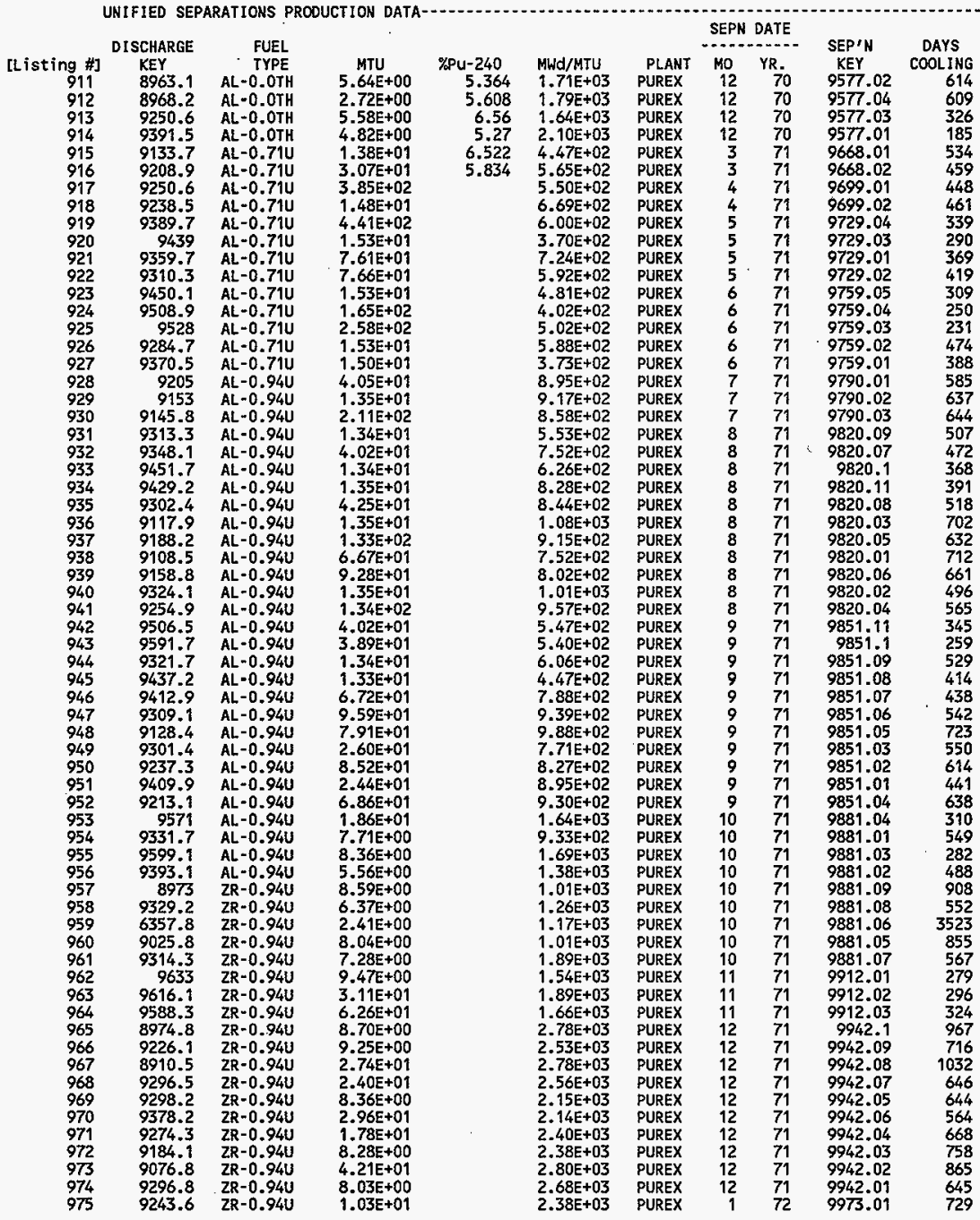




\section{[L.isting \#] Fuel Data Reference}

911 ISO-263 RD AND ARH-18 RD (MCDONALD 912 IS0-263 RD AND ARH-18 RD (MCDONALD 913 ISO-263 RD AND ARH-18 RD (MCDONALD 914 ISO-263 RD AND ARH-18 RD (MCDONALD 915 ISO-263 RD AND ARH-18 RD (MCDONALD 916 ISO-263 RD AND ARH-18 RD (MCDONALD 917 ISO-263 RD AND ARH-18 RD (MCDONALD 918 ISO-263 RD AND ARH-18 RD (MCDONALD 919 ISO-263 RD AND ARH-18 RO (MCDONALD 920 ISO-263 RD AND ARH-18 RD (MCDONALD 921 ISO-263 RD AND ARH-18 RD (MCDONALD 922 ISO-263 RD AND ARH-18 RD (MCDONALD 923 ISO-263 RD AND ARH-18 RD (MCDONALD 924 ISO-263 RD AND ARH-18 RD (MCDONALD 925 ISO-263 RD AND ARH-18 RD (MCDONALD 926 ISO-263 RD AND ARH-18 RD (MCDONALD 927 ISO-263 RD AND ARH-18 RD (HCDONALD 928 ISO-263 RD AND ARH-18 RD (MCDONALD 929 ISO-263 RD AND ARH-18 RD (MCDONALD 930 ISO-263 RD AND ARH-18 RD (MCDONALD 931 ISO-263 RD AND ARH-18 RD (MCDONALD 932 ISO-263 RD AND ARH-18 RD (MCDONALD 933 IsO-263 RD AND ARH-18 RD (MCDONALD 934 ISO-263 RD AND ARH-18 RD (MCDONALD 935 ISO-263 RD AND ARH-18 RD (MCDONALD 936 ISO-263 RD AND ARH-18 RD (MCDONALD 937 ISO-263 RD AND ARH-18 RO (MCDONALD 938 ISO-263 RD AND ARH-18 RD (MCDONALD 939 ISO-263 RD AND ARH-18 RD (MCDONALD 940 ISO-263 RO AND ARH-18 RD (MCDONALD 941 ISO-263 RD AND ARH-18 RD (MCDONALD 942 ISO-263 RD AND ARH-18 RD (MCDONALD 943 ISO-263 RD AND ARH-18 RD (MCDONALD 944 1SO-263 RD AND ARH-18 RD (MCDONALD 945 ISO-263 RD AND ARH-18 RD (MCDONALD 946 ISO-263 RD AND ARH-18 RD (MCDONALD 947 ISO-263 RD AND ARH-18 RD (MCDONALD 948 ISO-263 RD AND ARH-18 RD (MCDONALD 949 ISO-263 RD AND ARH-18 RD (MCDONALD 950 ISO-263 RD AND ARH-18 RD (MCDONALD 951 1SO-263 RD AND ARH-18 RD (MCDONAID 952 ISO-263 RD AND ARH-18 RD (MCDONALD 953 ISO-263 RD AND ARH-18 RD (MCDONALD 954 150-263 RD AND ARH-18 RD (MCDONALD 955 I\$O-263 RD AND ARH-18 RD (MCDONALD 956 ISO-263 RD AND ARH - 18 RD (MCDONALD 957 ISO-263 RD AND ARH-18 RD (MCDONALD 958 ISO-263 RD AND ARH-18 RD (MCDONALD 959 ISO-263 RD AND ARH-18 RD (MCDONAID 960 ISO-263 RD AND ARH-18 RD (MCDONALD 961 ISO-263 RD AND ARH-18 RD (MCDONALD 962 ISO-263 RD AND ARH-18 RD (MCDONALD 963 ISO-263 RD AND ARH-18 RD (NCDONALD 964 ISO-263 RD AND ARH-18 RD (MCDONALD 965 ISO-263 RD AND ARH-18 RD (MCDONALD 966 ISO-263 RD AND ARH-18 RD (MCDONALD 967 ISO-263 RD AND ARH-18 RD (MCDONALD 968 ISO-263 RD AND ARH-18 RD (MCDONALD 969 ISO-263 RD AND ARH-18 RD (MCDONALD 970 ISO-263 RD AND ARH-18 RD (MCDONALD 971 ISO-263 RD AND ARH-18 RD (MCDONALD 972 ISO-263 RD AND ARH-18 RD (MCDONALD 973 150-263 RD AND ARH-18 RD (MCDONALD 974 ISO-263 RD AND ARH-18 RD (MCDONALD 975 ISO-263 RD AND ARH-18 RD (MCDONALD
TOTAL WASTE LOSS FRACTION

\begin{tabular}{|c|c|c|c|c|}
\hline $\begin{array}{r}\text { Th } \\
0.0463 \\
0.0463 \\
0.0463 \\
0.0463 \\
1 \\
1 \\
1 \\
1 \\
1 \\
1 \\
1 \\
1 \\
1 \\
1 \\
1 \\
1 \\
1 \\
1 \\
1 \\
1 \\
1 \\
1 \\
1 \\
1 \\
1 \\
1 \\
1 \\
1 \\
1 \\
1 \\
1 \\
1 \\
1 \\
1 \\
1 \\
1 \\
1 \\
1 \\
1 \\
1 \\
1 \\
1 \\
1 \\
1 \\
1 \\
1 \\
1 \\
1 \\
1 \\
1 \\
1 \\
1 \\
1 \\
1 \\
1 \\
1 \\
1 \\
1 \\
1 \\
1 \\
1 \\
1 \\
1 \\
1 \\
1 \\
1 \\
1 \\
1 \\
1 \\
1 \\
1 \\
1 \\
1 \\
1 \\
1\end{array}$ & $\begin{array}{l}U \\
0.0314 \\
0.0314 \\
0.0314 \\
0.0314 \\
0.0035 \\
0.0035 \\
0.0035 \\
0.0035 \\
0.0023 \\
0.0023 \\
0.0023 \\
0.0023 \\
0.0022 \\
0.0022 \\
0.0022 \\
0.0022 \\
0.0022 \\
0.0042 \\
0.0042 \\
0.0042 \\
0.0025 \\
0.0025 \\
0.0025 \\
0.0025 \\
0.0025 \\
0.0025 \\
0.0025 \\
0.0025 \\
0.0025 \\
0.0025 \\
0.0025 \\
0.0026 \\
0.0026 \\
0.0026 \\
0.0026 \\
0.0026 \\
0.0026 \\
0.0026 \\
0.0026 \\
0.0026 \\
0.0026 \\
0.0026 \\
0.0098 \\
0.0098 \\
0.0098 \\
0.0098 \\
0.0168 \\
0.0168 \\
0.0168 \\
0.0168 \\
0.0168 \\
0.0168 \\
0.0168 \\
0.0168 \\
0.0168 \\
0.0168 \\
0.0168 \\
0.0168 \\
0.0168 \\
0.0168 \\
0.0168 \\
0.0168 \\
0.0168 \\
0.0168 \\
0.0168\end{array}$ & $\begin{array}{l}\text { Np } \\
1 \\
1 \\
1 \\
1 \\
0.3 \\
0.3 \\
0.3 \\
0.3 \\
0.3 \\
0.3 \\
0.3 \\
0.3 \\
0.3 \\
0.3 \\
0.3 \\
0.3 \\
0.3 \\
0.3 \\
0.3 \\
0.3 \\
0.3 \\
0.3 \\
0.3 \\
0.3 \\
0.3 \\
0.3 \\
0.3 \\
0.3 \\
0.3 \\
0.3 \\
0.3 \\
0.3 \\
0.3 \\
0.3 \\
0.3 \\
0.3 \\
0.3 \\
0.3 \\
0.3 \\
0.3 \\
0.3 \\
0.3 \\
0.3 \\
0.3 \\
0.3 \\
0.3 \\
0.3 \\
0.3 \\
0.3 \\
0.3 \\
0.3 \\
0.3 \\
0.3 \\
0.3 \\
0.3 \\
0.3 \\
0.3 \\
0.3 \\
0.3 \\
0.3 \\
0.3 \\
0.3 \\
0.3 \\
0\end{array}$ & $\begin{array}{r}\text { Pu } \\
1 \\
1 \\
1 \\
0.0049 \\
0.0049 \\
0.0049 \\
0.0049 \\
0.0049 \\
0.0064 \\
0.0064 \\
0.0064 \\
0.0064 \\
0.0051 \\
0.0051 \\
0.0051 \\
0.0051 \\
0.0051 \\
0.0069 \\
0.0069 \\
0.0069 \\
0.0027 \\
0.0027 \\
0.0027 \\
0.0027 \\
0.0027 \\
0.0027 \\
0.0027 \\
0.0027 \\
0.0027 \\
0.0027 \\
0.0027 \\
0.0028 \\
0.0028 \\
0.0028 \\
0.0028 \\
0.0028 \\
0.0028 \\
0.0028 \\
0.0028 \\
0.0028 \\
0.0028 \\
0.0028 \\
0.0109 \\
0.0109 \\
0.0109 \\
0.0109 \\
0.033 \\
0.033 \\
0.033 \\
0.033 \\
0.033 \\
0.033 \\
0.033 \\
0.033 \\
0.033 \\
0.033 \\
0.033 \\
0.033 \\
0.033 \\
0.033 \\
0.033 \\
0.033 \\
0.033 \\
0.033 \\
0.033\end{array}$ & $\begin{array}{l}\text { Reference } \\
\text { (b) } \\
\text { (b) } \\
\text { (b) } \\
\text { (b) } \\
\text { (b) } \\
\text { (b) } \\
\text { (b) } \\
\text { (b) } \\
\text { (b) } \\
\text { (b) } \\
\text { (b) } \\
\text { (b) } \\
\text { (b) } \\
\text { (b) } \\
\text { (b) } \\
\text { (b) } \\
\text { (b) } \\
\text { (b) } \\
\text { (b) } \\
\text { (b) } \\
\text { (b) } \\
\text { (b) } \\
\text { (b) } \\
\text { (b) } \\
\text { (b) } \\
\text { (b) } \\
\text { (b) } \\
\text { (b) } \\
\text { (b) } \\
\text { (b) } \\
\text { (b) } \\
\text { (b) } \\
\text { (b) } \\
\text { (b) } \\
\text { (b) } \\
\text { (b) } \\
\text { (b) } \\
\text { (b) } \\
\text { (b) } \\
\text { (b) } \\
\text { (b) } \\
\text { (b) } \\
\text { (b) } \\
\text { (b) } \\
\text { (b) } \\
\text { (b) } \\
\text { (c) } \\
\text { (c) } \\
\text { (c) } \\
\text { (c) } \\
\text { (c) } \\
\text { (c) } \\
\text { (c) } \\
\text { (c) } \\
\text { (c) } \\
\text { (c) } \\
\text { (c) } \\
\text { (c) } \\
\text { (c) } \\
\text { (c) } \\
\text { (c) } \\
\text { (c) } \\
\text { (c) } \\
\text { (c) } \\
\text { (c) } \\
\text { (c) }\end{array}$ \\
\hline
\end{tabular}


HNF-SD-HM-T1-794, ReV. 0

ACTIVITY IN UNSEPARATED FUEL (Curies decayed to $1 / 1 / 94$ )

\begin{tabular}{|c|c|c|c|c|c|c|c|c|c|}
\hline $\begin{array}{c}\text { [Listing } \# 3 \\
911 \\
912 \\
913 \\
914 \\
915 \\
916 \\
917 \\
918 \\
919 \\
920 \\
921 \\
922 \\
923 \\
924 \\
925 \\
926 \\
927 \\
928 \\
929 \\
930 \\
931 \\
932 \\
933 \\
934 \\
935 \\
936 \\
937 \\
938 \\
939 \\
940 \\
941 \\
942 \\
943 \\
944 \\
945 \\
946 \\
947 \\
948 \\
949 \\
950 \\
951 \\
952 \\
953 \\
954 \\
955 \\
956 \\
957 \\
958 \\
959 \\
960 \\
961 \\
962 \\
963 \\
964 \\
965 \\
966 \\
967 \\
968 \\
969 \\
970 \\
971 \\
972 \\
973 \\
974 \\
975\end{array}$ & $\begin{array}{l}103 \\
1.93 E+00 \\
9.40 E-01 \\
2.02 E+00 \\
1.80 E+00 \\
2.49 E+01 \\
7.12 E+01 \\
8.74 E+02 \\
4.10 E+01 \\
1.12 E+03 \\
2.38 E+01 \\
2.33 E+02 \\
1.89 E+02 \\
3.12 E+01 \\
2.83 E+02 \\
5.58 E+02 \\
3.75 E+01 \\
2.34 E+01 \\
1.45 E+02 \\
4.93 E+01 \\
7.19 E+02 \\
3.00 E+01 \\
1.24 E+02 \\
3.46 E+01 \\
4.64 E+01 \\
1.46 E+02 \\
5.81 E+01 \\
4.87 E+02 \\
1.98 E+02 \\
2.96 E+02 \\
5.62 E+01 \\
5.18 E+02 \\
9.17 E+01 \\
8.87 E+01 \\
3.30 E+01 \\
2.44 E+01 \\
2.19 E+02 \\
3.68 E+02 \\
3.11 E+02 \\
8.16 E+01 \\
2.84 E+02 \\
9.06 E+01 \\
2.57 E+02 \\
1.33 E+02 \\
2.95 E+01 \\
6.16 E+01 \\
3.21 E+01 \\
3.53 E+01 \\
3.45 E+01 \\
7.65 E+00 \\
3.32 E+01 \\
6.03 E+01 \\
6.65 E+01 \\
2.70 E+02 \\
4.71 E+02 \\
1.03 E+02 \\
1.03 E+02 \\
3.22 E+02 \\
2.73 E+02 \\
7.90 E+01 \\
2.83 E+02 \\
1.89 E+02 \\
8.58 E+01 \\
5.10 E+02 \\
9.58 E+01 \\
1.08 E+02\end{array}$ & $\begin{array}{l}\text { C14 } \\
1.34 E-06 \\
6.52 E-07 \\
1.34 E-06 \\
1.17 E-06 \\
3.95 E-01 \\
1.11 E+00 \\
1.35 E+01 \\
6.31 E-01 \\
1.69 E+01 \\
3.63 E-01 \\
3.50 E+00 \\
2.89 E+00 \\
4.70 E-01 \\
4.25 E+00 \\
8.30 E+00 \\
5.75 E-01 \\
3.60 E-01 \\
1.80 E+00 \\
6.16 E-01 \\
9.00 E+00 \\
3.69 E-01 \\
1.50 E+00 \\
4.16 E-01 \\
5.57 E-01 \\
1.79 E+00 \\
7.25 E-01 \\
6.05 E+00 \\
2.50 E+00 \\
3.71 E+00 \\
6.81 E-01 \\
6.36 E+00 \\
1.10 E+00 \\
1.05 E+00 \\
4.05 E-01 \\
2.96 E-01 \\
2.64 E+00 \\
4.48 E+00 \\
3.89 E+00 \\
1.00 E+00 \\
3.51 E+00 \\
1.09 E+00 \\
3.17 E+00 \\
1.52 E+00 \\
3.58 E-01 \\
7.02 E-01 \\
3.80 E-01 \\
9.36 E=01 \\
8.65 E-01 \\
3.03 E-01 \\
8.75 E-01 \\
1.50 E+00 \\
1.59 E+00 \\
6.42 E+00 \\
1.13 E+01 \\
2.68 E+00 \\
2.58 E+00 \\
8.44 E+00 \\
6.77 E+00 \\
1.97 E+00 \\
6.96 E+00 \\
4.70 E+00 \\
2.17 E+00 \\
1.30 E+01 \\
2.37 E+00 \\
2.71 E+00\end{array}$ & 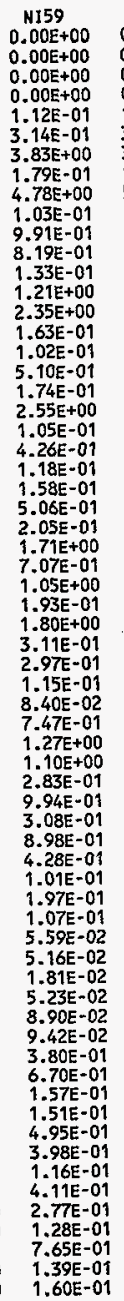 & $\begin{array}{l}H 163 \\
0.00 E+00 \\
0.00 E+00 \\
0.00 E+00 \\
0.00 E+00 \\
1.17 E+01 \\
3.27 E+01 \\
3.99 E+02 \\
1.86 E+01 \\
5.00 E+02 \\
1.08 E+01 \\
1.04 E+02 \\
8.55 E+01 \\
1.39 E+01 \\
1.26 E+02 \\
2.47 E+02 \\
1.70 E+01 \\
1.07 E+01 \\
5.31 E+01 \\
1.81 E+01 \\
2.65 E+02 \\
1.09 E+01 \\
4.45 E+01 \\
1.24 E+01 \\
1.65 E+01 \\
5.28 E+01 \\
2.13 E+01 \\
1.78 E+02 \\
7.35 E+01 \\
1.09 E+02 \\
2.01 E+01 \\
1.88 E+02 \\
3.25 E+01 \\
3.11 E+01 \\
1.20 E+01 \\
8.78 E+00 \\
7.81 E+01 \\
1.32 E+02 \\
1.15 E+02 \\
2.96 E+01 \\
1.04 E+02 \\
3.22 E+01 \\
9.36 E+01 \\
4.51 E+01 \\
1.06 E+01 \\
2.08 E+01 \\
1.12 E+01 \\
5.85 E+00 \\
5.44 E+00 \\
1.79 E+00 \\
5.48 E+00 \\
9.43 E+00 \\
1.00 E+01 \\
4.05 E+01 \\
7.12 E+01 \\
1.66 E+01 \\
1.61 E+01 \\
5.23 E+01 \\
4.23 E+01 \\
1.23 E+01 \\
4.37 E+01 \\
2.94 E+01 \\
1.35 E+01 \\
8.11 E+01 \\
1.48 E+01 \\
1.69 E+01\end{array}$ & $\begin{array}{l}C 060 \\
0.00 E+00 \\
0.00 E+00 \\
0.00 E+00 \\
0.00 E+00 \\
1.01 E+00 \\
2.89 E+00 \\
3.58 E+01 \\
1.66 E+00 \\
4.69 E+01 \\
1.03 E+00 \\
9.60 E+00 \\
7.81 E+00 \\
1.34 E+00 \\
1.24 E+01 \\
2.43 E+01 \\
1.54 E+00 \\
9.99 E-01 \\
4.66 E+00 \\
1.56 E+00 \\
2.28 E+01 \\
1.00 E+00 \\
4.11 E+00 \\
1.18 E+00 \\
1.56 E+00 \\
4.79 E+00 \\
1.81 E+00 \\
1.55 E+01 \\
6.25 E+00 \\
9.45 E+00 \\
1.83 E+00 \\
1.67 E+01 \\
3.18 E+00 \\
3.13 E+00 \\
1.10 E+00 \\
8.40 E-01 \\
7.37 E+00 \\
1.20 E+01 \\
9.77 E+00 \\
2.68 E+00 \\
9.20 E+00 \\
3.03 E+00 \\
8.23 E+00 \\
4.42 E+00 \\
9.69 E-01 \\
2.06 E+00 \\
1.04 E+00 \\
2.29 E+00 \\
2.39 E+00 \\
2.88 E-01 \\
2.18 E+00 \\
4.08 E+00 \\
4.86 E+00 \\
1.94 E+01 \\
3.40 E+01 \\
6.29 E+00 \\
6.67 E+00 \\
1.94 E+01 \\
1.80 E+01 \\
5.28 E+00 \\
1.92 E+01 \\
1.24 E+01 \\
5.55 E+00 \\
3.18 E+01 \\
6.28 E+00 \\
7.08 E+00\end{array}$ & $\begin{array}{c}\text { SE79 } \\
1.10 E-02 \\
5.33 E-03 \\
1.10 E-02 \\
9.56 E-03 \\
8.33 E-02 \\
2.34 E-01 \\
2.85 E+00 \\
1.33 E-01 \\
3.57 E+00 \\
7.63 E-02 \\
7.41 E-01 \\
6.11 E-01 \\
9.91 E-02 \\
8.95 E-01 \\
1.75 E+00 \\
1.21 E-01 \\
7.58 E-02 \\
4.84 E-01 \\
1.65 E-01 \\
2.42 E+00 \\
9.92 E-02 \\
4.04 E-01 \\
1.12 E-01 \\
1.49 E-01 \\
4.80 E-01 \\
1.95 E-01 \\
1.62 E+00 \\
6.70 E-01 \\
9.95 E-01 \\
1.83 E-01 \\
1.71 E+00 \\
2.94 E-01 \\
2.81 E-01 \\
1.09 E-01 \\
7.95 E-02 \\
7.08 E-01 \\
1.20 E+00 \\
1.04 E+00 \\
2.69 E-01 \\
9.43 E-01 \\
2.92 E-01 \\
8.52 E-01 \\
4.08 E-01 \\
9.62 E-02 \\
1.88 E-01 \\
1.02 E-01 \\
1.16 E-01 \\
1.07 E-01 \\
3.74 E-02 \\
1.09 E-01 \\
1.82 E-01 \\
1.94 E-01 \\
7.76 E-01 \\
1.37 E+00 \\
3.17 E-01 \\
3.06 E-01 \\
9.99 E-01 \\
8.05 E-01 \\
2.36 E-01 \\
8.35 E-01 \\
5.61 E-01 \\
2.59 E-01 \\
1.54 E+00 \\
2.81 E-01 \\
3.23 E-01\end{array}$ & $\begin{array}{l}\text { SR90 } \\
8.13 E+02 \\
3.96 E+02 \\
8.32 E+02 \\
7.30 E+02 \\
1.00 E+04 \\
2.80 E+04 \\
3.43 E+05 \\
1.60 E+04 \\
4.32 E+05 \\
9.38 E+03 \\
8.91 E+04 \\
7.36 E+04 \\
1.21 E+04 \\
1.10 E+05 \\
2.15 E+05 \\
1.46 E+04 \\
9.28 E+03 \\
5.92 E+04 \\
2.01 E+04 \\
2.95 E+05 \\
1.24 E+04 \\
5.02 E+04 \\
1.40 E+04 \\
1.86 E+04 \\
5.92 E+04 \\
2.35 E+04 \\
1.98 E+05 \\
8.20 E+04 \\
1.22 E+05 \\
2.25 E+04 \\
2.09 E+05 \\
3.72 E+04 \\
3.57 E+04 \\
1.36 E+04 \\
1.00 E+04 \\
8.82 E+04 \\
1.48 E+05 \\
1.27 E+05 \\
3.32 E+04 \\
1.16 E+05 \\
3.62 E+04 \\
1.04 E+05 \\
4.98 E+04 \\
1.19 E+04 \\
2.30 E+04 \\
1.24 E+04 \\
1.35 E+04 \\
1.25 E+04 \\
3.64 E+03 \\
1.27 E+04 \\
2.07 E+04 \\
2.29 E+04 \\
9.02 E+04 \\
1.61 E+05 \\
3.40 E+04 \\
3.38 E+04 \\
1.07 E+05 \\
8.91 E+04 \\
2.65 E+04 \\
9.43 E+04 \\
6.22 E+04 \\
2.85 E+04 \\
1.67 E+05 \\
3.10 E+04 \\
3.58 E+04\end{array}$ & $\begin{array}{l}Y 90 \\
8.13 E+02 \\
3.96 E+02 \\
8.32 E+02 \\
7.30 E+02 \\
1.00 E+04 \\
2.81 E+04 \\
3.44 E+05 \\
1.60 E+04 \\
4.32 E+05 \\
9.38 E+03 \\
8.91 E+04 \\
7.37 E+04 \\
1.21 E+04 \\
1.11 E+05 \\
2.15 E+05 \\
1.46 E+04 \\
9.28 E+03 \\
5.93 E+04 \\
2.02 E+04 \\
2.95 E+05 \\
1.24 E+04 \\
5.02 E+04 \\
1.40 E+04 \\
1.86 E+04 \\
5.92 E+04 \\
2.36 E+04 \\
1.98 E+05 \\
8.20 E+04 \\
1.22 E+05 \\
2.25 E+04 \\
2.09 E+05 \\
3.72 E+04 \\
3.57 E+04 \\
1.36 E+04 \\
1.00 E+04 \\
8.82 E+04 \\
1.48 E+05 \\
1.27 E+05 \\
3.32 E+04 \\
1.16 E+05 \\
3.62 E+04 \\
1.04 E+05 \\
4.98 E+04 \\
1.19 E+04 \\
2.30 E+04 \\
1.24 E+04 \\
1.35 E+04 \\
1.25 E+04 \\
3.64 E+03 \\
1.27 E+04 \\
2.07 E+04 \\
2.29 E+04 \\
9.02 E+04 \\
1.61 E+05 \\
3.40 E+04 \\
3.38 E+04 \\
1.07 E+05 \\
8.91 E+04 \\
2.65 E+04 \\
9.44 E+04 \\
6.23 E+04 \\
2.86 E+04 \\
1.67 E+05 \\
3.10 E+04 \\
3.58 E+04\end{array}$ & $\begin{array}{l}2 R 93 \\
2.83 E-02 \\
1.38 E-02 \\
2.84 E-02 \\
2.47 E-02 \\
3.95 E-01 \\
1.11 E+00 \\
1.35 E+01 \\
6.29 E-01 \\
1.69 E+01 \\
3.62 E-01 \\
3.49 E+00 \\
2.89 E+00 \\
4.70 E-01 \\
4.25 E+00 \\
8.29 E+00 \\
5.74 E-01 \\
3.60 E-01 \\
2.33 E+00 \\
7.95 E-01 \\
1.16 E+01 \\
4.80 E-01 \\
1.95 E+00 \\
5.40 E-01 \\
7.20 E-01 \\
2.31 E+00 \\
9.33 E-01 \\
7.80 E+00 \\
3.23 E+00 \\
4.80 E+00 \\
8.77 E-01 \\
8.21 E+00 \\
1.42 E+00 \\
1.36 E+00 \\
5.26 E-01 \\
3.85 E-01 \\
3.41 E+00 \\
5.78 E+00 \\
5.02 E+00 \\
1.29 E+00 \\
4.54 E+00 \\
1.40 E+00 \\
4.10 E+00 \\
1.94 E+00 \\
4.62 E-01 \\
8.95 E-01 \\
4.87 E-01 \\
5.64 E-01 \\
5.15 E-01 \\
1.81 E-01 \\
5.27 E-01 \\
8.65 E-01 \\
9.29 E-01 \\
3.69 E+00 \\
6.57 E+00 \\
1.48 E+00 \\
1.44 E+00 \\
4.67 E+00 \\
3.78 E+00 \\
1.12 E+00 \\
3.95 E+00 \\
2.64 E+00 \\
1.22 E+00 \\
7.22 E+00 \\
1.32 E+00 \\
1.52 E+00\end{array}$ \\
\hline
\end{tabular}


HNF-SD-WM-TI-794, ReV. O

ACTIVITY IN UNSEPARATED FUEL (Curies decayed to $1 / 1 / 94$ )

\begin{tabular}{|c|c|c|c|c|c|c|c|c|c|}
\hline $\begin{array}{c}\text { [Listing } \# \text { ] } \\
911 \\
912 \\
913 \\
914 \\
915 \\
916 \\
917 \\
918 \\
919 \\
920 \\
921 \\
922 \\
923 \\
924 \\
925 \\
926 \\
927 \\
928 \\
929 \\
930 \\
931 \\
932 \\
933 \\
934 \\
935 \\
936 \\
937 \\
938 \\
939 \\
940 \\
941 \\
942 \\
943 \\
944 \\
945 \\
946 \\
947 \\
948 \\
949 \\
950 \\
951 \\
952 \\
953 \\
954 \\
955 \\
956 \\
957 \\
958 \\
959 \\
960 \\
961 \\
962 \\
963 \\
964 \\
965 \\
966 \\
967 \\
968 \\
969 \\
970 \\
971 \\
972 \\
973 \\
974 \\
975\end{array}$ & $\begin{array}{l}\text { NB93* } \\
1.93 E-02 \\
9.42 E-03 \\
1.91 E-02 \\
1.65 E-02 \\
2.67 E-01 \\
7.44 E-01 \\
9.06 E+00 \\
4.23 E-01 \\
1.12 E+01 \\
2.41 E-01 \\
2.33 E+00 \\
1.93 E+00 \\
3.11 E-01 \\
2.81 E+00 \\
5.47 E+00 \\
3.84 E-01 \\
2.40 E-01 \\
1.57 E+00 \\
5.37 E-01 \\
7.86 E+00 \\
3.21 E-01 \\
1.30 E+00 \\
3.58 E-01 \\
4.78 E-01 \\
1.55 E+00 \\
6.32 E-01 \\
5.26 E+00 \\
2.19 E+00 \\
3.24 E+00 \\
5.87 E-01 \\
5.51 E+00 \\
9.41 E-01 \\
8.94 E-01 \\
3.51 E-01 \\
2.56 E-01 \\
2.27 E+00 \\
3.87 E+00 \\
3.40 E+00 \\
8.67 E-01 \\
3.05 E+00 \\
9.35 E-01 \\
2.76 E+00 \\
1.28 E+00 \\
3.09 E-01 \\
5.91 E-01 \\
3.25 E-01 \\
3.85 E-01 \\
3.45 E-01 \\
1.38 E-01 \\
3.59 E-01 \\
5.81 E-01 \\
6.11 E-01 \\
2.44 E+00 \\
4.34 E+00 \\
1.02 E+00 \\
9.74 E-01 \\
3.22 E+00 \\
2.55 E+00 \\
7.52 E-01 \\
2.65 E+00 \\
1.78 E+00 \\
8.25 E=01 \\
4.93 E+00 \\
8.89 E=01 \\
1.03 E+00\end{array}$ & $\begin{array}{l}\text { TC99 } \\
1.18 E-01 \\
5.73 E-02 \\
1.18 E-01 \\
1.03 E-01 \\
2.74 E+00 \\
7.70 E+00 \\
9.40 E+01 \\
4.40 E+00 \\
1.18 E+02 \\
2.51 E+00 \\
2.45 E+01 \\
2.01 E+01 \\
3.26 E+00 \\
2.95 E+01 \\
5.76 E+01 \\
4.00 E+00 \\
2.49 E+00 \\
1.61 E+01 \\
5.50 E+00 \\
8.04 E+01 \\
3.29 E+00 \\
1.34 E+01 \\
3.71 E+00 \\
4.97 E+00 \\
1.60 E+01 \\
6.48 E+00 \\
5.40 E+01 \\
2.23 E+01 \\
3.31 E+01 \\
6.08 E+00 \\
5.68 E+01 \\
9.77 E+00 \\
9.33 E+00 \\
3.61 E+00 \\
2.64 E+00 \\
2.36 E+01 \\
4.00 E+01 \\
3.47 E+01 \\
8.92 E+00 \\
3.13 E+01 \\
9.71 E+00 \\
2.83 E+01 \\
1.42 E+01 \\
3.20 E+00 \\
6.60 E+00 \\
3.46 E+00 \\
3.88 E+00 \\
3.56 E+00 \\
1.25 E+00 \\
3.63 E+00 \\
6.07 E+00 \\
6.47 E+00 \\
2.59 E+01 \\
4.59 E+01 \\
1.06 E+01 \\
1.03 E+01 \\
3.34 E+01 \\
2.69 E+01 \\
7.89 E+00 \\
2.79 E+01 \\
1.88 E+01 \\
8.65 E+00 \\
5.17 E+01 \\
9.41 E+00 \\
1.08 E+01\end{array}$ & $\begin{array}{l}\text { RU106 } \\
4.32 \mathrm{E}-05 \\
2.12 \mathrm{E}-05 \\
7.45 \mathrm{E}-05 \\
8.45 \mathrm{E}-05 \\
4.02 \mathrm{E}-03 \\
1.35 \mathrm{E}-02 \\
1.78 \mathrm{E}-01 \\
8.46 \mathrm{E}-03 \\
2.94 \mathrm{E}-01 \\
6.34 \mathrm{E}-03 \\
6.02 \mathrm{E}-02 \\
4.33 \mathrm{E}-02 \\
8.78 \mathrm{E}-03 \\
8.62 \mathrm{E}-02 \\
1.81 \mathrm{E}-01 \\
8.23 \mathrm{E}-03 \\
5.56 \mathrm{E}-03 \\
2.58 \mathrm{E}-02 \\
8.02 \mathrm{E}-03 \\
1.14 \mathrm{E}-01 \\
5.98 \mathrm{E}-03 \\
2.73 \mathrm{E}-02 \\
8.92 \mathrm{E}-03 \\
1.20 \mathrm{E}-02 \\
3.05 \mathrm{E}-02 \\
9.19 \mathrm{E}-03 \\
8.43 \mathrm{E}-02 \\
2.88 \mathrm{E}-02 \\
4.77 \mathrm{E}-02 \\
1.25 \mathrm{E}-02 \\
1.02 \mathrm{E}-01 \\
2.55 \mathrm{E}-02 \\
2.86 \mathrm{E}-02 \\
6.76 \mathrm{E}-03 \\
5.90 \mathrm{E}-03 \\
5.47 \mathrm{E}-02 \\
7.92 \mathrm{E}-02 \\
4.92 \mathrm{E}-02 \\
1.67 \mathrm{E}-02 \\
5.26 \mathrm{E}-02 \\
2.29 \mathrm{E}-02 \\
4.65 \mathrm{E}-02 \\
5.08 \mathrm{E}-02 \\
6.57 \mathrm{E}-03 \\
2.49 \mathrm{E}-02 \\
8.61 \mathrm{E}-03 \\
5.18 \mathrm{E}-03 \\
9.69 \mathrm{E}-03 \\
1.25 \mathrm{E}-05 \\
5.35 \mathrm{E}-03 \\
1.78 \mathrm{E}-02 \\
3.27 \mathrm{E}-02 \\
1.34 \mathrm{E}-01 \\
2.17 \mathrm{E}-01 \\
1.80 \mathrm{E}-02 \\
2.74 \mathrm{E}-02 \\
5.03 \mathrm{E}-02 \\
8.22 \mathrm{E}-02 \\
2.33 \mathrm{E}-02 \\
9.59 \mathrm{E}-02 \\
5.44 \mathrm{E}-02 \\
2.12 \mathrm{E}-02 \\
1.07 \mathrm{E}-01 \\
2.91 \mathrm{E}-02 \\
2.96 \mathrm{E}-02\end{array}$ & $\begin{array}{l}\text { CD113* } \\
6.81 E-02 \\
3.32 E+02 \\
7.10 E-02 \\
6.29 E-02 \\
2.35 E+00 \\
6.73 E+00 \\
8.25 E+01 \\
3.89 E+00 \\
1.05 E+02 \\
2.23 E+00 \\
2.21 E+01 \\
1.79 E+01 \\
2.92 E+00 \\
2.65 E+01 \\
5.23 E+01 \\
3.54 E+00 \\
2.19 E+00 \\
1.21 E+01 \\
4.10 E+00 \\
5.97 E+01 \\
2.45 E+00 \\
1.02 E+01 \\
2.83 E+00 \\
3.82 E+00 \\
1.21 E+01 \\
4.87 E+00 \\
4.05 E+01 \\
1.64 E+01 \\
2.46 E+01 \\
4.67 E+00 \\
4.31 E+01 \\
7.46 E+00 \\
7.20 E+00 \\
2.70 E+00 \\
1.98 E+00 \\
1.81 E+01 \\
3.06 E+01 \\
2.60 E+01 \\
6.73 E+00 \\
2.35 E+01 \\
7.49 E+00 \\
2.14 E+01 \\
1.12 E+01 \\
2.45 E+00 \\
5.22 E+00 \\
2.70 E+00 \\
3.16 E+00 \\
3.12 E+00 \\
7.36 E-01 \\
2.98 E+00 \\
5.65 E+00 \\
6.06 E+00 \\
2.51 E+01 \\
4.33 E+01 \\
1.02 E+01 \\
9.96 E+00 \\
3.18 E+01 \\
2.64 E+01 \\
7.51 E+00 \\
2.69 E+01 \\
1.82 E+01 \\
8.29 E+00 \\
5.02 E+01 \\
9.34 E+00 \\
1.04 E+01\end{array}$ & 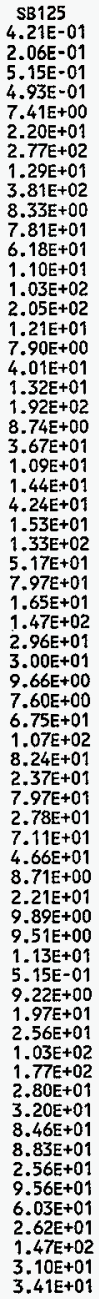 & $\begin{array}{c}\text { SN126 } \\
1.01 \mathrm{E}-02 \\
4.90 \mathrm{E}-03 \\
1.01 \mathrm{E}-02 \\
8.78 \mathrm{E}-03 \\
1.26 \mathrm{E}-01 \\
3.57 \mathrm{E}-01 \\
4.35 \mathrm{E}+00 \\
2.06 \mathrm{E}-01 \\
5.46 \mathrm{E}+00 \\
1.14 \mathrm{E}-01 \\
1.15 \mathrm{E}+00 \\
9.36 \mathrm{E}-01 \\
1.50 \mathrm{E}-01 \\
1.35 \mathrm{E}+00 \\
2.66 \mathrm{E}+00 \\
1.86 \mathrm{E}-01 \\
1.14 \mathrm{E}-01 \\
6.81 \mathrm{E}-01 \\
2.33 \mathrm{E}-01 \\
3.39 \mathrm{E}+00 \\
1.36 \mathrm{E}-01 \\
5.63 \mathrm{E}-01 \\
1.54 \mathrm{E}-01 \\
2.09 \mathrm{E}-01 \\
6.73 \mathrm{E}-01 \\
2.77 \mathrm{E}-01 \\
2.29 \mathrm{E}+00 \\
9.34 \mathrm{E}-01 \\
1.39 \mathrm{E}+00 \\
2.59 \mathrm{E}-01 \\
2.41 \mathrm{E}+00 \\
4.04 \mathrm{E}-01 \\
3.86 \mathrm{E}-01 \\
1.50 \mathrm{E}-01 \\
1.08 \mathrm{E}-01 \\
9.90 \mathrm{E}-01 \\
1.70 \mathrm{E}+00 \\
1.48 \mathrm{E}+00 \\
3.75 \mathrm{E}-01 \\
1.32 \mathrm{E}+00 \\
4.11 \mathrm{E}-01 \\
1.20 \mathrm{E}+00 \\
6.04 \mathrm{E}-01 \\
1.36 \mathrm{E}-01 \\
2.79 \mathrm{E}-01 \\
1.48 \mathrm{E}-01 \\
1.79 \mathrm{E}-01 \\
1.68 \mathrm{E}-01 \\
5.84 \mathrm{E}-02 \\
1.67 \mathrm{E}-01 \\
3.01 \mathrm{E}-01 \\
3.12 \mathrm{E}-01 \\
1.29 \mathrm{E}+00 \\
2.24 \mathrm{E}+00 \\
5.58 \mathrm{E}-01 \\
5.32 \mathrm{E}-01 \\
1.76 \mathrm{E}+00 \\
1.40 \mathrm{E}+00 \\
4.00 \mathrm{E}-01 \\
1.41 \mathrm{E}+00 \\
9.66 \mathrm{E}-01 \\
4.46 \mathrm{E}-01 \\
2.72 \mathrm{E}+00 \\
4.93 \mathrm{E}-01 \\
5.57 \mathrm{E}-01\end{array}$ & $\begin{array}{l}1129 \\
4.30 \mathrm{E}-04 \\
2.09 \mathrm{E}-04 \\
4.32 \mathrm{E}-04 \\
3.75 \mathrm{E}-04 \\
5.19 \mathrm{E}-03 \\
1.47 \mathrm{E}-02 \\
1.79 \mathrm{E}-01 \\
8.47 \mathrm{E}-03 \\
2.25 \mathrm{E}-01 \\
4.71 \mathrm{E}-03 \\
4.73 \mathrm{E}-02 \\
3.86 \mathrm{E}-02 \\
6.19 \mathrm{E}-03 \\
5.55 \mathrm{E}-02 \\
1.10 \mathrm{E}-01 \\
7.66 \mathrm{E}-03 \\
4.68 \mathrm{E}-03 \\
3.00 \mathrm{E}-02 \\
1.03 \mathrm{E}-02 \\
1.50 \mathrm{E}-01 \\
6.03 \mathrm{E}-03 \\
2.48 \mathrm{E}-02 \\
6.83 \mathrm{E}-03 \\
9.23 \mathrm{E}-03 \\
2.97 \mathrm{E}-02 \\
1.22 \mathrm{E}-02 \\
1.01 \mathrm{E}-01 \\
4.12 \mathrm{E}-02 \\
6.14 \mathrm{E}-02 \\
1.14 \mathrm{E}-02 \\
1.06 \mathrm{E}-01 \\
1.79 \mathrm{E}-02 \\
1.71 \mathrm{E}-02 \\
6.63 \mathrm{E}-03 \\
4.80 \mathrm{E}-03 \\
4.37 \mathrm{E}-02 \\
7.49 \mathrm{E}-02 \\
6.51 \mathrm{E}-02 \\
1.65 \mathrm{E}-02 \\
5.82 \mathrm{E}-02 \\
1.81 \mathrm{E}-02 \\
5.30 \mathrm{E}-02 \\
2.64 \mathrm{E}-02 \\
5.98 \mathrm{E}-03 \\
1.22 \mathrm{E}-02 \\
6.51 \mathrm{E}-03 \\
7.64 \mathrm{E}-03 \\
7.12 \mathrm{E}-03 \\
2.48 \mathrm{E}-03 \\
7.14 \mathrm{E}-03 \\
1.26 \mathrm{E}-02 \\
1.32 \mathrm{E}-02 \\
5.39 \mathrm{E}-02 \\
9.40 \mathrm{E}-02 \\
2.30 \mathrm{E}-02 \\
2.20 \mathrm{E}-02 \\
7.27 \mathrm{E}-02 \\
5.79 \mathrm{E}-02 \\
1.67 \mathrm{E}-02 \\
5.89 \mathrm{E}-02 \\
4.01 \mathrm{E}-02 \\
1.85 \mathrm{E}-02 \\
1.12 \mathrm{E}-01 \\
2.04 \mathrm{E}-02 \\
2.31 \mathrm{E}-02\end{array}$ & $\begin{array}{c}\text { CS134 } \\
1.97 E-02 \\
9.62 E-03 \\
2.57 E-02 \\
2.55 E-02 \\
2.67 E-01 \\
1.08 E+00 \\
1.33 E+01 \\
7.78 E-01 \\
2.10 E+01 \\
2.49 E-01 \\
5.31 E+00 \\
3.34 E+00 \\
4.67 E-01 \\
3.51 E+00 \\
9.38 E+00 \\
6.49 E-01 \\
2.33 E-01 \\
3.08 E+00 \\
1.03 E+00 \\
1.39 E+01 \\
3.92 E-01 \\
2.40 E+00 \\
5.85 E-01 \\
1.07 E+00 \\
3.18 E+00 \\
1.42 E+00 \\
1.05 E+01 \\
3.19 E+00 \\
5.35 E+00 \\
1.50 E+00 \\
1.23 E+01 \\
1.39 E+00 \\
1.39 E+00 \\
4.85 E-01 \\
2.67 E-01 \\
4.81 E+00 \\
9.10 E+00 \\
6.93 E+00 \\
1.57 E+00 \\
5.65 E+00 \\
2.24 E+00 \\
5.71 E+00 \\
7.15 E+00 \\
7.19 E-01 \\
3.49 E+00 \\
1.25 E+00 \\
9.66 E-01 \\
1.56 E+00 \\
3.27 E-02 \\
9.48 E-01 \\
4.05 E+00 \\
4.66 E+00 \\
2.28 E+01 \\
3.43 E+01 \\
7.64 E+00 \\
8.48 E+00 \\
2.27 E+01 \\
2.38 E+01 \\
5.94 E+00 \\
2.26 E+01 \\
1.55 E+01 \\
6.56 E+00 \\
4.09 E+01 \\
8.78 E+00 \\
8.67 E+00\end{array}$ & $\begin{array}{l}\text { CS137 } \\
7.46 E+02 \\
3.64 E+02 \\
7.63 E+02 \\
6.69 E+02 \\
1.12 E+04 \\
3.18 E+04 \\
3.89 E+05 \\
1.82 E+04 \\
4.90 E+05 \\
1.05 E+04 \\
1.02 E+05 \\
8.36 E+04 \\
1.37 E+04 \\
1.24 E+05 \\
2.43 E+05 \\
1.66 E+04 \\
1.04 E+04 \\
6.65 E+04 \\
2.27 E+04 \\
3.31 E+05 \\
1.37 E+04 \\
5.60 E+04 \\
1.56 E+04 \\
2.08 E+04 \\
6.64 E+04 \\
2.67 E+04 \\
2.23 E+05 \\
9.15 E+04 \\
1.36 E+05 \\
2.54 E+04 \\
2.36 E+05 \\
4.11 E+04 \\
3.95 E+04 \\
1.50 E+04 \\
1.11 E+04 \\
9.86 E+04 \\
1.67 E+05 \\
1.43 E+05 \\
3.71 E+04 \\
1.30 E+05 \\
4.07 E+04 \\
1.17 E+05 \\
5.78 E+04 \\
1.33 E+04 \\
2.67 E+04 \\
1.43 E+04 \\
1.58 E+04 \\
1.49 E+04 \\
4.33 E+03 \\
1.49 E+04 \\
2.55 E+04 \\
2.76 E+04 \\
1.11 E+05 \\
1.95 E+05 \\
4.37 E+04 \\
4.29 E+04 \\
1.37 E+05 \\
1.13 E+05 \\
3.31 E+04 \\
1.18 E+05 \\
7.86 E+04 \\
3.61 E+04 \\
2.14 E+05 \\
3.96 E+04 \\
4.53 E+04\end{array}$ \\
\hline
\end{tabular}


HNF-SD-WM-TI-794, ReV. 0

ACTIVITY IN UNSEPARATED FUEL (Curies decayed to 1/1/94)

\begin{tabular}{|c|c|c|c|c|c|c|c|c|c|}
\hline $\begin{aligned} \text { [Listing } \\
911 \\
912 \\
913 \\
914 \\
914 \\
915 \\
916 \\
917 \\
918 \\
919 \\
920 \\
921 \\
922 \\
923 \\
924 \\
925 \\
926 \\
927 \\
928 \\
929 \\
930 \\
931 \\
932 \\
933 \\
934 \\
935 \\
936 \\
937 \\
938 \\
939 \\
940 \\
941 \\
942 \\
943 \\
944 \\
945 \\
946 \\
947 \\
948 \\
949 \\
950 \\
951 \\
952 \\
953 \\
954 \\
955 \\
956 \\
957 \\
958 \\
959 \\
960 \\
961 \\
962 \\
963 \\
964 \\
965 \\
966 \\
967 \\
968 \\
969 \\
970 \\
971 \\
972 \\
973 \\
974 \\
975\end{aligned}$ & $\begin{array}{l}B A 137 * \\
7.06 E+02 \\
3.44 E+02 \\
7.22 E+02 \\
6.33 E+02 \\
1.06 E+04 \\
3.01 E+04 \\
3.68 E+05 \\
1.72 E+04 \\
4.64 E+05 \\
9.92 E+03 \\
9.65 E+04 \\
7.91 E+04 \\
1.29 E+04 \\
1.17 E+05 \\
2.29 E+05 \\
1.57 E+04 \\
9.81 E+03 \\
6.30 E+04 \\
2.14 E+04 \\
3.13 E+05 \\
1.30 E+04 \\
5.30 E+04 \\
1.47 E+04 \\
1.97 E+04 \\
6.28 E+04 \\
2.52 E+04 \\
2.11 E+05 \\
8.66 E+04 \\
1.29 E+05 \\
2.40 E+04 \\
2.23 E+05 \\
3.89 E+04 \\
3.74 E+04 \\
1.42 E+04 \\
1.05 E+04 \\
9.33 E+04 \\
1.58 E+05 \\
1.35 E+05 \\
3.51 E+04 \\
1.23 E+05 \\
3.85 E+04 \\
1.11 E+05 \\
5.47 E+04 \\
1.26 E+04 \\
2.53 E+04 \\
1.35 E+04 \\
1.50 E+04 \\
1.41 E+04 \\
4.09 E+03 \\
1.40 E+04 \\
2.41 E+04 \\
2.61 E+04 \\
1.05 E+05 \\
1.85 E+05 \\
4.13 E+04 \\
4.06 E+04 \\
1.30 E+05 \\
1.07 E+05 \\
3.13 E+04 \\
1.11 E+05 \\
7.44 E+04 \\
3.41 E+04 \\
2.03 E+05 \\
3.74 E+04 \\
4.28 E+04\end{array}$ & $\begin{array}{l}34151 \\
1.09 E+01 \\
5.29 E+00 \\
1.10 E+01 \\
9.57 E+00 \\
3.53 E+02 \\
9.58 E+02 \\
1.18 E+04 \\
5.29 E+02 \\
1.45 E+04 \\
3.33 E+02 \\
2.89 E+03 \\
2.48 E+03 \\
4.19 E+02 \\
3.88 E+03 \\
7.36 E+03 \\
4.92 E+02 \\
3.30 E+02 \\
1.84 E+03 \\
6.24 E+02 \\
9.28 E+03 \\
4.17 E+02 \\
1.61 E+03 \\
4.62 E+02 \\
5.83 E+02 \\
1.85 E+03 \\
6.97 E+02 \\
6.13 E+03 \\
2.65 E+03 \\
3.89 E+03 \\
6.72 E+02 \\
6.38 E+03 \\
1.24 E+03 \\
1.19 E+03 \\
4.51 E+02 \\
3.45 E+02 \\
2.78 E+03 \\
4.50 E+03 \\
3.85 E+03 \\
1.06 E+03 \\
3.66 E+03 \\
1.12 E+03 \\
3.21 E+03 \\
1.24 E+03 \\
3.63 E+02 \\
5.61 E+02 \\
3.35 E+02 \\
4.01 E+02 \\
3.49 E+02 \\
1.18 E+02 \\
3.76 E+02 \\
4.96 E+02 \\
5.91 E+02 \\
2.13 E+03 \\
4.05 E+03 \\
6.81 E+02 \\
7.00 E+02 \\
2.15 E+03 \\
1.84 E+03 \\
5.92 E+02 \\
2.10 E+03 \\
1.32 E+03 \\
6.06 E+02 \\
3.33 E+03 \\
6.23 E+02 \\
7.59 E+02\end{array}$ & 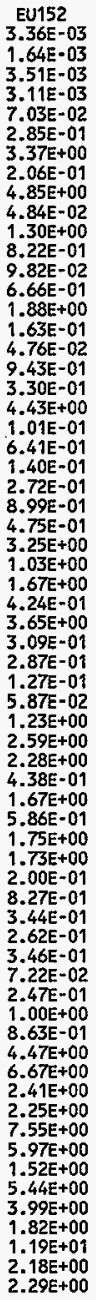 & 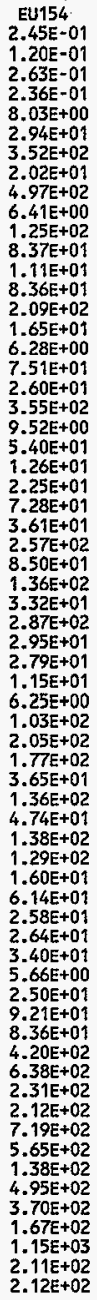 & $\begin{array}{l}E U 155 \\
3.29 E-01 \\
1.61 E=01 \\
3.69 E-01 \\
3.39 E-01 \\
2.19 E+01 \\
5.96 E+01 \\
7.45 E+02 \\
3.26 E+01 \\
9.56 E+02 \\
2.34 E+01 \\
1.84 E+02 \\
1.59 E+02 \\
2.89 E+01 \\
2.78 E+02 \\
5.21 E+02 \\
3.13 E+01 \\
2.26 E+01 \\
1.03 E+02 \\
3.41 E+01 \\
5.16 E+02 \\
2.66 E+01 \\
9.89 E+01 \\
3.05 E+01 \\
3.62 E+01 \\
1.09 E+02 \\
3.57 E+01 \\
3.40 E+02 \\
1.50 E+02 \\
2.21 E+02 \\
3.80 E+01 \\
3.58 E+02 \\
8.49 E+01 \\
8.43 E+01 \\
2.85 E+01 \\
2.35 E+01 \\
1.73 E+02 \\
2.58 E+02 \\
2.05 E+02 \\
6.39 E+01 \\
2.12 E+02 \\
6.75 E+01 \\
1.79 E+02 \\
5.54 E+01 \\
2.11 E+01 \\
2.45 E+01 \\
1.67 E+01 \\
2.09 E+01 \\
2.07 E+01 \\
2.38 E+00 \\
1.99 E+01 \\
2.94 E+01 \\
3.92 E+01 \\
1.41 E+02 \\
2.65 E+02 \\
3.81 E+01 \\
4.16 E+01 \\
1.17 E+01 \\
1.12 E+02 \\
3.51 E+01 \\
1.28 E+02 \\
7.87 E+01 \\
3.51 E+01 \\
1.93 E+01 \\
3.87 E+01 \\
4.49 E+01\end{array}$ & $\begin{array}{l}\text { RA226 } \\
4.23 \mathrm{E}-05 \\
2.06 \mathrm{E}-05 \\
4.11 \mathrm{E}-05 \\
3.52 \mathrm{E}-05 \\
5.24 \mathrm{E}-06 \\
1.15 \mathrm{E}-05 \\
1.42 \mathrm{E}-04 \\
5.49 \mathrm{E}-06 \\
1.58 \mathrm{E}-04 \\
5.42 \mathrm{E}-06 \\
2.74 \mathrm{E}-05 \\
2.76 \mathrm{E}-05 \\
5.40 \mathrm{E}-06 \\
5.76 \mathrm{E}-05 \\
8.96 \mathrm{E}-05 \\
5.51 \mathrm{E}-06 \\
5.46 \mathrm{E}-06 \\
2.28 \mathrm{E}-05 \\
7.71 \mathrm{E}-06 \\
1.21 \mathrm{E}-04 \\
7.38 \mathrm{E}-06 \\
2.20 \mathrm{E}-05 \\
7.13 \mathrm{E}-06 \\
7.25 \mathrm{E}-06 \\
2.31 \mathrm{E}-05 \\
7.76 \mathrm{E}-06 \\
7.51 \mathrm{E}-05 \\
3.85 \mathrm{E}-05 \\
5.30 \mathrm{E}-05 \\
7.42 \mathrm{E}-06 \\
7.46 \mathrm{E}-05 \\
2.09 \mathrm{E}-05 \\
2.01 \mathrm{E}-05 \\
7.37 \mathrm{E}-06 \\
7.10 \mathrm{E}-06 \\
3.56 \mathrm{E}-05 \\
5.19 \mathrm{E}-05 \\
4.55 \mathrm{E}-05 \\
1.44 \mathrm{E}-05 \\
4.78 \mathrm{E}-05 \\
1.32 \mathrm{E}-05 \\
3.87 \mathrm{E}-05 \\
9.68 \mathrm{E}-06 \\
4.25 \mathrm{E}-06 \\
4.32 \mathrm{E}-06 \\
3.01 \mathrm{E}-06 \\
5.07 \mathrm{E}-06 \\
3.47 \mathrm{E}-06 \\
2.35 \mathrm{E}-06 \\
4.69 \mathrm{E}-06 \\
3.98 \mathrm{E}-06 \\
4.81 \mathrm{E}-06 \\
1.58 \mathrm{E}-05 \\
3.21 \mathrm{E}-05 \\
5.10 \mathrm{E}-06 \\
5.14 \mathrm{E}-06 \\
1.63 \mathrm{E}-05 \\
4.33 \mathrm{E}-05 \\
1.59 \mathrm{E}-05 \\
9.76 \mathrm{E}-06 \\
2.59 \mathrm{E}-06 \\
4.39 \mathrm{E}-05-06 \\
5.67 \mathrm{E}-06\end{array}$ & $\begin{array}{l}\text { RA228 } \\
5.66 \mathrm{E}-01 \\
2.76 \mathrm{E}-01 \\
5.64 \mathrm{E}-01 \\
4.89 \mathrm{E}-01 \\
4.01 \mathrm{E}-11 \\
1.10 \mathrm{E}-10 \\
1.34 \mathrm{E}-09 \\
6.20 \mathrm{E}-11 \\
1.63 \mathrm{E}-09 \\
3.52 \mathrm{E}-11 \\
3.36 \mathrm{E}-10 \\
2.82 \mathrm{E}-10 \\
4.52 \mathrm{E}-11 \\
4.07 \mathrm{E}-10 \\
7.86 \mathrm{E}-10 \\
5.63 \mathrm{E}-11 \\
3.53 \mathrm{E}-11 \\
1.03 \mathrm{E}-09 \\
3.48 \mathrm{E}-10 \\
5.36 \mathrm{E}-09 \\
3.05 \mathrm{E}-10 \\
9.62 \mathrm{E}-10 \\
3.04 \mathrm{E}-10 \\
3.25 \mathrm{E}-10 \\
1.04 \mathrm{E}-09 \\
3.63 \mathrm{E}-10 \\
3.40 \mathrm{E}-09 \\
1.66 \mathrm{E}-09 \\
2.32 \mathrm{E}-09 \\
3.46 \mathrm{E}-10 \\
3.42 \mathrm{E}-09 \\
8.78 \mathrm{E}-10 \\
8.44 \mathrm{E}-10 \\
3.10 \mathrm{E}-10 \\
2.87 \mathrm{E}-10 \\
1.59 \mathrm{E}-09 \\
2.39 \mathrm{E}-09 \\
2.09 \mathrm{E}-09 \\
6.32 \mathrm{E}-10 \\
2.12 \mathrm{E}-09 \\
6.00 \mathrm{E}-10 \\
1.76 \mathrm{E}-09 \\
5.25 \mathrm{E}-10 \\
1.95 \mathrm{E}-10 \\
2.37 \mathrm{E}-10 \\
1.53 \mathrm{E}-10 \\
2.54 \mathrm{E}-10 \\
1.90 \mathrm{E}-10 \\
1.06 \mathrm{E}-10 \\
2.36 \mathrm{E}-10 \\
2.52 \mathrm{E}-10 \\
2.88 \mathrm{E}-10 \\
1.02 \mathrm{E}-09 \\
1.97 \mathrm{E}-09 \\
3.71 \mathrm{E}-10 \\
3.64 \mathrm{E}-10 \\
1.18 \mathrm{E}-09 \\
9.45 \mathrm{E}-10 \\
3.04 \mathrm{E}-10 \\
1.06 \mathrm{E}-09 \\
6.79 \mathrm{E}-10 \\
3.18 \mathrm{E}-10 \\
1.78 \mathrm{E}-09 \\
3.20 \mathrm{E}-10 \\
3.93 \mathrm{E}-10\end{array}$ & $\begin{array}{l}\text { AC227 } \\
9.23 \mathrm{E}-01 \\
4.50 \mathrm{E}-01 \\
9.08 \mathrm{E}-01 \\
7.81 \mathrm{E}-01 \\
3.13 \mathrm{E}-05 \\
6.77 \mathrm{E}-05 \\
8.42 \mathrm{E}-04 \\
3.21 \mathrm{E}-05 \\
9.33 \mathrm{E}-04 \\
3.29 \mathrm{E}-05 \\
1.60 \mathrm{E}-04 \\
1.62 \mathrm{E}-04 \\
3.24 \mathrm{E}-05 \\
3.49 \mathrm{E}-04 \\
5.37 \mathrm{E}-04 \\
3.24 \mathrm{E}-05 \\
3.31 \mathrm{E}-05 \\
1.18 \mathrm{E}-04 \\
3.96 \mathrm{E}-05 \\
6.21 \mathrm{E}-04 \\
3.92 \mathrm{E}-05 \\
1.15 \mathrm{E}-04 \\
3.78 \mathrm{E}-05 \\
3.77 \mathrm{E}-05 \\
1.20 \mathrm{E}-04 \\
3.92 \mathrm{E}-05 \\
3.86 \mathrm{E}-04 \\
2.00 \mathrm{E}-04 \\
2.75 \mathrm{E}-04 \\
3.79 \mathrm{E}-05 \\
3.83 \mathrm{E}-04 \\
1.12 \mathrm{E}-04 \\
1.08 \mathrm{E}-04 \\
3.90 \mathrm{E}-05 \\
3.82 \mathrm{E}-05 \\
1.86 \mathrm{E}-04 \\
2.67 \mathrm{E}-04 \\
2.32 \mathrm{E}-04 \\
7.50 \mathrm{E}-05 \\
2.47 \mathrm{E}-04 \\
6.81 \mathrm{E}-05 \\
1.99 \mathrm{E}-04 \\
4.76 \mathrm{E}-05 \\
2.19 \mathrm{E}-05 \\
2.12 \mathrm{E}-05 \\
1.50 \mathrm{E}-05 \\
2.59 \mathrm{E}-05 \\
1.76 \mathrm{E}-05 \\
1.11 \mathrm{E}-05 \\
2.39 \mathrm{E}-05 \\
1.95 \mathrm{E}-05 \\
2.42 \mathrm{E}-05 \\
7.84 \mathrm{E}-05 \\
1.60 \mathrm{E}-04 \\
2.41 \mathrm{E}-05 \\
2.46 \mathrm{E}-05 \\
7.69 \mathrm{E}-05 \\
6.38 \mathrm{E}-05 \\
2.22 \mathrm{E}-05 \\
7.74 \mathrm{E}-05 \\
.70 \mathrm{~s}-05\end{array}$ & $\begin{array}{l}\text { TH229 } \\
2.44 \mathrm{E}-01 \\
1.19 \mathrm{E}-01 \\
2.37 \mathrm{E}-01 \\
2.03 \mathrm{E}-01 \\
8.03 \mathrm{E}-09 \\
2.23 \mathrm{E}-08 \\
2.71 \mathrm{E}-07 \\
1.27 \mathrm{E}-08 \\
3.32 \mathrm{E}-07 \\
7.05 \mathrm{E}-09 \\
6.92 \mathrm{E}-08 \\
5.75 \mathrm{E}-08 \\
9.14 \mathrm{E}-09 \\
8.19 \mathrm{E}-08 \\
1.60 \mathrm{E}-07 \\
1.15 \mathrm{E}-08 \\
7.07 \mathrm{E}-09 \\
6.02 \mathrm{E}-08 \\
2.07 \mathrm{E}-08 \\
3.03 \mathrm{E}-07 \\
1.21 \mathrm{E}-08 \\
4.91 \mathrm{E}-08 \\
1.34 \mathrm{E}-08 \\
1.80 \mathrm{E}-08 \\
5.88 \mathrm{E}-08 \\
2.46 \mathrm{E}-08 \\
2.03 \mathrm{E}-07 \\
8.45 \mathrm{E}-08 \\
1.25 \mathrm{E}-07 \\
2.24 \mathrm{E}-08 \\
2.11 \mathrm{E}-07 \\
3.48 \mathrm{E}-08 \\
3.28 \mathrm{E}-08 \\
1.32 \mathrm{E}-08 \\
9.49 \mathrm{E}-09 \\
8.53 \mathrm{E}-08 \\
1.48 \mathrm{E}-07 \\
1.32 \mathrm{E}-07 \\
3.29 \mathrm{E}-08 \\
1.17 \mathrm{E}-07 \\
3.52 \mathrm{E}-08 \\
1.06 \mathrm{E}-07 \\
5.00 \mathrm{E}-08 \\
1.17 \mathrm{E}-08 \\
2.31 \mathrm{E}-08 \\
1.25 \mathrm{E}-08 \\
1.87 \mathrm{E}-08 \\
1.65 \mathrm{E}-08 \\
8.65 \mathrm{E}-09 \\
1.74 \mathrm{E}-08 \\
2.92 \mathrm{E}-08 \\
2.91 \mathrm{E}-08 \\
1.19 \mathrm{E}-07 \\
2.09 \mathrm{E}-07 \\
5.62 \mathrm{E}-08 \\
5.17 \mathrm{E}-08 \\
1.79 \mathrm{E}-07 \\
1.34 \mathrm{E}-07 \\
3.86 \mathrm{E}-08 \\
1.35 \mathrm{E}-07 \\
9.33 \mathrm{E}-08 \\
4.36 \mathrm{E}-08 \\
.30 \mathrm{~s}\end{array}$ \\
\hline
\end{tabular}


HNF-SD-WM-II-794, ReV. 0

ACTIVITY IN UNSEPARATED FUEL (Curies decayed to $1 / 1 / 94$ )

\begin{tabular}{|c|c|c|c|c|c|c|c|c|c|}
\hline $\begin{array}{c}\text { [Listing } \#] \\
911 \\
912 \\
913 \\
914 \\
915 \\
916 \\
917 \\
918 \\
919 \\
920 \\
921 \\
922 \\
923 \\
924 \\
925 \\
926 \\
927 \\
928 \\
929 \\
930 \\
931 \\
932 \\
933 \\
934 \\
935 \\
936 \\
937 \\
938 \\
939 \\
940 \\
941 \\
942 \\
943 \\
944 \\
945 \\
946 \\
947 \\
948 \\
949 \\
950 \\
951 \\
952 \\
953 \\
954 \\
955 \\
956 \\
957 \\
958 \\
959 \\
960 \\
961 \\
962 \\
963 \\
964 \\
965 \\
966 \\
967 \\
968 \\
969 \\
970 \\
971 \\
972 \\
973 \\
974 \\
975\end{array}$ & $\begin{array}{l}\text { TH232 } \\
6.11 \mathrm{E}-01 \\
2.98 \mathrm{E}-01 \\
6.14 \mathrm{E}-01 \\
5.34 \mathrm{E}-01 \\
6.32 \mathrm{E}-11 \\
1.74 \mathrm{E}-10 \\
2.12 \mathrm{E}-09 \\
9.81 \mathrm{E}-11 \\
2.59 \mathrm{E}-09 \\
5.62 \mathrm{E}-11 \\
5.35 \mathrm{E}-10 \\
4.48 \mathrm{E}-10 \\
7.22 \mathrm{E}-11 \\
6.53 \mathrm{E}-10 \\
1.26 \mathrm{E}-09 \\
8.93 \mathrm{E}-11 \\
5.63 \mathrm{E}-11 \\
1.62 \mathrm{E}-09 \\
5.47 \mathrm{E}-10 \\
8.42 \mathrm{E}-09 \\
4.85 \mathrm{E}-10 \\
1.53 \mathrm{E}-09 \\
4.85 \mathrm{E}-10 \\
5.19 \mathrm{E}-10 \\
1.64 \mathrm{E}-09 \\
5.70 \mathrm{E}-10 \\
5.35 \mathrm{E}-09 \\
2.60 \mathrm{E}-09 \\
3.65 \mathrm{E}-09 \\
5.49 \mathrm{E}-10 \\
5.41 \mathrm{E}-09 \\
1.41 \mathrm{E}-09 \\
1.36 \mathrm{E}-09 \\
4.92 \mathrm{E}-10 \\
4.59 \mathrm{E}-10 \\
2.53 \mathrm{E}-09 \\
3.78 \mathrm{E}-09 \\
3.27 \mathrm{E}-09 \\
1.00 \mathrm{E}-09 \\
3.34 \mathrm{E}-09 \\
9.56 \mathrm{E}-10 \\
2.77 \mathrm{E}-09 \\
8.41 \mathrm{E}-10 \\
3.09 \mathrm{E}-10 \\
3.80 \mathrm{E}-10 \\
2.43 \mathrm{E}-10 \\
3.96 \mathrm{E}-10 \\
3.01 \mathrm{E}-10 \\
1.49 \mathrm{E}-10 \\
3.68 \mathrm{E}-10 \\
3.97 \mathrm{E}-10 \\
4.63 \mathrm{E}-10 \\
1.64 \mathrm{E}-09 \\
3.15 \mathrm{E}-09 \\
5.74 \mathrm{E}-10 \\
5.70 \mathrm{E}-10 \\
1.82 \mathrm{E}-09 \\
1.49 \mathrm{E}-09 \\
4.79 \mathrm{E}-10 \\
1.68 \mathrm{E}-09 \\
3.07 \mathrm{E}-09 \\
4.97 \mathrm{E}-10 \\
2.77 \mathrm{E}-09 \\
5.03 \mathrm{E}-10 \\
6.18 \mathrm{E}-10\end{array}$ & 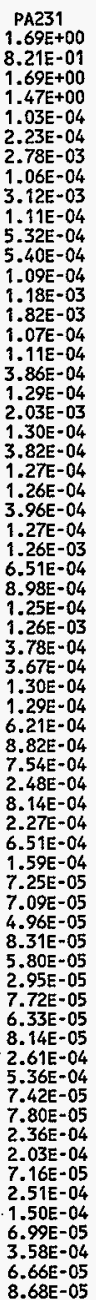 & $\begin{array}{l}\text { U232 } \\
2.72 E+01 \\
1.32 E+01 \\
2.75 E+01 \\
2.40 E+01 \\
1.44 E-04 \\
4.41 E-04 \\
5.34 E-03 \\
2.70 E-04 \\
6.93 E-03 \\
1.25 E-04 \\
1.56 E-03 \\
1.19 E-03 \\
1.77 E-04 \\
1.51 E-03 \\
3.19 E-03 \\
2.35 E-04 \\
1.24 E-04 \\
1.41 E-03 \\
4.88 E-04 \\
6.88 E-03 \\
2.29 E-04 \\
1.08 E-03 \\
2.74 E-04 \\
4.20 E-04 \\
1.37 E-03 \\
6.32 E-04 \\
4.80 E-03 \\
1.78 E-03 \\
2.74 E-03 \\
5.74 E-04 \\
5.18 E-03 \\
6.84 E-04 \\
6.47 E-04 \\
2.62 E-04 \\
1.69 E-04 \\
1.96 E-03 \\
3.65 E-03 \\
3.21 E-03 \\
7.26 E-04 \\
2.64 E-03 \\
8.56 E-04 \\
2.54 E-03 \\
1.76 E-03 \\
2.88 E-04 \\
8.27 E-04 \\
3.88 E-04 \\
5.28 E-04 \\
5.84 E-04 \\
1.78 E-04 \\
4.94 E-04 \\
1.42 E-03 \\
1.28 E-03 \\
6.12 E-03 \\
9.63 E-03 \\
3.57 E-03 \\
3.15 E-03 \\
1.13 E-03 \\
8.30 E-03 \\
2.08 E-03 \\
7.36 E-03 \\
5.50 E-03 \\
2.53 E-03 \\
1.75 E-03 \\
3.07 E-03 \\
3.17 E-03\end{array}$ & 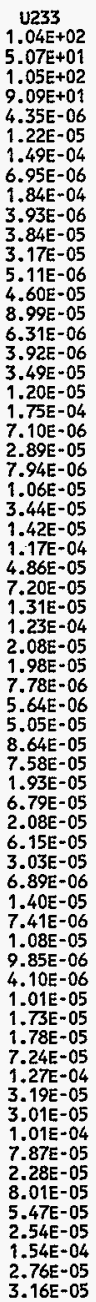 & $\begin{array}{l}\text { U234 } \\
7.27 E-01 \\
3.54 E-01 \\
7.30 E-01 \\
6.35 E-01 \\
4.53 E+00 \\
1.00 E+01 \\
1.26 E+02 \\
4.83 E+00 \\
1.44 E+02 \\
5.02 E+00 \\
2.47 E+01 \\
2.47 E+01 \\
5.00 E+00 \\
5.42 E+01 \\
8.45 E+01 \\
4.90 E+00 \\
4.98 E+00 \\
1.98 E+01 \\
6.61 E+00 \\
1.03 E+02 \\
6.61 E+00 \\
1.97 E+01 \\
6.58 E+00 \\
6.63 E+00 \\
2.05 E+01 \\
6.58 E+00 \\
6.49 E+01 \\
3.28 E+01 \\
4.56 E+01 \\
6.60 E+00 \\
6.54 E+01 \\
1.96 E+01 \\
1.92 E+01 \\
6.62 E+00 \\
6.57 E+00 \\
3.25 E+01 \\
4.61 E+01 \\
3.87 E+01 \\
1.28 E+01 \\
4.18 E+01 \\
1.20 E+01 \\
3.36 E+01 \\
8.99 E+00 \\
3.79 E+00 \\
4.03 E+00 \\
2.70 E+00 \\
4.18 E+00 \\
3.08 E+00 \\
1.17 E+00 \\
3.91 E+00 \\
3.47 E+00 \\
4.55 E+00 \\
1.48 E+01 \\
3.00 E+01 \\
4.05 E+00 \\
4.33 E+00 \\
1.28 E+01 \\
1.14 E+01 \\
3.95 E+00 \\
1.40 E+01 \\
8.34 E+00 \\
3.85 E+00 \\
1.97 E+01 \\
3.75 E+00 \\
4.81 E+00\end{array}$ & 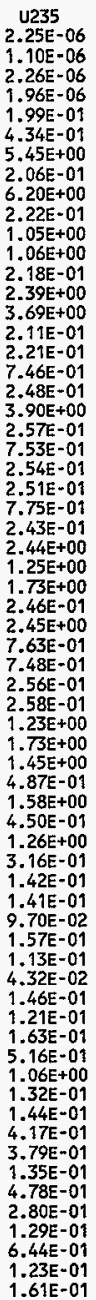 & $\begin{array}{l}\text { U236 } \\
3.14 \mathrm{E}-07 \\
1.53 \mathrm{E}-07 \\
3.15 \mathrm{E}-07 \\
2.74 \mathrm{E}-07 \\
5.26 \mathrm{E}-02 \\
1.46 \mathrm{E}-01 \\
1.78 \mathrm{E}+00 \\
8.25 \mathrm{E}-02 \\
2.22 \mathrm{E}+00 \\
4.85 \mathrm{E}-02 \\
4.56 \mathrm{E}-01 \\
3.80 \mathrm{E}-01 \\
6.23 \mathrm{E}-02 \\
5.68 \mathrm{E}-01 \\
1.10 \mathrm{E}+00 \\
7.55 \mathrm{E}-02 \\
4.82 \mathrm{E}-02 \\
1.35 \mathrm{E}+00 \\
4.53 \mathrm{E}-01 \\
6.97 \mathrm{E}+00 \\
4.10 \mathrm{E}-01 \\
1.30 \mathrm{E}+00 \\
4.17 \mathrm{E}-01 \\
4.44 \mathrm{E}-01 \\
1.38 \mathrm{E}+00 \\
4.70 \mathrm{E}-01 \\
4.45 \mathrm{E}+00 \\
2.15 \mathrm{E}+00 \\
3.03 \mathrm{E}+00 \\
4.63 \mathrm{E}-01 \\
4.53 \mathrm{E}+00 \\
1.22 \mathrm{E}+00 \\
1.19 \mathrm{E}+00 \\
4.17 \mathrm{E}-01 \\
3.95 \mathrm{E}-01 \\
2.16 \mathrm{E}+00 \\
3.19 \mathrm{E}+00 \\
2.70 \mathrm{E}+00 \\
8.44 \mathrm{E}-01 \\
2.80 \mathrm{E}+00 \\
8.15 \mathrm{E}-01 \\
2.31 \mathrm{E}+00 \\
7.27 \mathrm{E}-01 \\
2.61 \mathrm{E}-01 \\
3.29 \mathrm{E}-01 \\
2.06 \mathrm{E}-01 \\
3.22 \mathrm{E}-01 \\
2.54 \mathrm{E}-01 \\
9.39 \mathrm{E}-02 \\
3.01 \mathrm{E}-01 \\
3.33 \mathrm{E}-01 \\
4.04 \mathrm{E}-01 \\
1.42 \mathrm{E}+00 \\
2.73 \mathrm{E}+00 \\
4.60 \mathrm{E}-01 \\
4.70 \mathrm{E}-01 \\
1.45 \mathrm{E}+00 \\
1.24 \mathrm{E}+00 \\
4.00 \mathrm{E}-01 \\
1.41 \mathrm{E}+00 \\
8.86 \mathrm{E}-01 \\
4.09 \mathrm{E}-01 \\
2.24 \mathrm{E}+00 \\
4.18 \mathrm{E}-01 \\
5.11 \mathrm{E}-01\end{array}$ & $\begin{array}{l}\text { U238 } \\
1.84 E-14 \\
8.96 E=15 \\
1.85 E-14 \\
1.60 E-14 \\
4.61 E+00 \\
1.03 E+01 \\
1.28 E+02 \\
4.94 E+00 \\
1.47 E+02 \\
5.10 E+00 \\
2.54 E+01 \\
2.52 E+01 \\
5.09 E+00 \\
5.51 E+01 \\
8.62 E+01 \\
5.01 E+00 \\
5.06 E+00 \\
1.35 E+01 \\
4.49 E+00 \\
7.00 E+01 \\
4.46 E+00 \\
1.34 E+01 \\
4.44 E+00 \\
4.49 E+00 \\
1.39 E+01 \\
4.48 E+00 \\
4.41 E+01 \\
2.22 E+01 \\
3.09 E+01 \\
4.49 E+00 \\
4.44 E+01 \\
1.32 E+01 \\
1.29 E+01 \\
4.46 E+00 \\
4.42 E+00 \\
2.20 E+01 \\
3.13 E+01 \\
2.63 E+01 \\
8.66 E+00 \\
2.83 E+01 \\
8.12 E+00 \\
2.28 E+01 \\
6.19 E+00 \\
2.58 E+00 \\
2.78 E+00 \\
1.85 E+00 \\
2.86 E+00 \\
2.12 E+00 \\
8.01 E-01 \\
2.67 E+00 \\
2.42 E+00 \\
3.15 E+00 \\
1.03 E+01 \\
2.08 E+01 \\
2.89 E+00 \\
3.07 E+00 \\
9.11 E+00 \\
8.07 E+00 \\
2.78 E+00 \\
9.81 E+00 \\
5.89 E+00 \\
2.72 E+00 \\
1.41 E+01 \\
2.66 E+00 \\
3.40 E+00\end{array}$ & $\begin{array}{l}\text { NP237 } \\
1.02 \mathrm{E}-08 \\
4.95 \mathrm{E}=09 \\
1.02 \mathrm{E}-08 \\
8.87 \mathrm{E}-09 \\
1.69 \mathrm{E}-02 \\
4.84 \mathrm{E}-02 \\
5.90 \mathrm{E}-01 \\
2.81 \mathrm{E}-02 \\
7.43 \mathrm{E}-01 \\
1.53 \mathrm{E}-02 \\
1.57 \mathrm{E}-01 \\
1.27 \mathrm{E}-01 \\
2.03 \mathrm{E}-02 \\
1.81 \mathrm{E}-01 \\
3.59 \mathrm{E}-01 \\
2.53 \mathrm{E}-02 \\
1.52 \mathrm{E}-02 \\
1.73 \mathrm{E}-01 \\
5.92 \mathrm{E}-02 \\
8.62 \mathrm{E}-01 \\
3.46 \mathrm{E}-02 \\
1.43 \mathrm{E}-01 \\
3.92 \mathrm{E}-02 \\
5.32 \mathrm{E}-02 \\
1.71 \mathrm{E}-01 \\
7.05 \mathrm{E}-02 \\
5.81 \mathrm{E}-01 \\
2.37 \mathrm{E}-01 \\
3.54 \mathrm{E}-01 \\
6.59 \mathrm{E}-02 \\
6.13 \mathrm{E}-01 \\
1.03 \mathrm{E}-01 \\
9.79 \mathrm{E}-02 \\
3.81 \mathrm{E}-02 \\
2.75 \mathrm{E}-02 \\
2.52 \mathrm{E}-01 \\
4.32 \mathrm{E}-01 \\
3.76 \mathrm{E}-01 \\
9.51 \mathrm{E}-02 \\
3.35 \mathrm{E}-01 \\
1.04 \mathrm{E}-01 \\
3.05 \mathrm{E}-01 \\
1.67 \mathrm{E}-01 \\
3.45 \mathrm{E}-02 \\
7.80 \mathrm{E}-02 \\
3.87 \mathrm{E}-02 \\
5.60 \mathrm{E}-02 \\
5.30 \mathrm{E}-02 \\
1.85 \mathrm{E}-02 \\
5.24 \mathrm{E}-02 \\
9.74 \mathrm{E}-02 \\
9.95 \mathrm{E}-02 \\
4.15 \mathrm{E}-01 \\
7.16 \mathrm{E}-01 \\
1.85 \mathrm{E}-01 \\
1.75 \mathrm{E}-01\end{array}$ \\
\hline
\end{tabular}


HNF-SD-WM-TI-794, ReV. 0

ACTIVITY IN UNSEPARATED FUEL (Curies decayed to $1 / 1 / 94$ )

\begin{tabular}{|c|c|c|c|c|c|c|c|c|c|}
\hline $\begin{array}{r}\text { Listing \#] } \\
911 \\
912 \\
913 \\
914 \\
915 \\
916 \\
917 \\
918 \\
919 \\
920 \\
921 \\
922 \\
923 \\
924 \\
925 \\
926 \\
927 \\
928 \\
929 \\
930 \\
931 \\
932 \\
933 \\
934 \\
935 \\
936 \\
937 \\
938 \\
939 \\
940 \\
941 \\
942 \\
943 \\
944 \\
945 \\
946 \\
947 \\
948 \\
949 \\
950 \\
951 \\
952 \\
953 \\
954 \\
955 \\
956 \\
957 \\
958 \\
959 \\
960 \\
961 \\
962 \\
963 \\
964 \\
965 \\
966 \\
967 \\
968 \\
969 \\
970 \\
971 \\
972 \\
973 \\
974 \\
975\end{array}$ & $\begin{array}{c}\text { PU238 } \\
1.43 \mathrm{E}-06 \\
6.96 \mathrm{E}-07 \\
1.44 \mathrm{E}-06 \\
1.26 \mathrm{E}-06 \\
3.17 \mathrm{E}+00 \\
1.21 \mathrm{E}+01 \\
1.43 \mathrm{E}+02 \\
8.48 \mathrm{E}+00 \\
1.99 \mathrm{E}+02 \\
2.25 \mathrm{E}+00 \\
5.19 \mathrm{E}+01 \\
3.41 \mathrm{E}+01 \\
4.19 \mathrm{E}+00 \\
2.97 \mathrm{E}+01 \\
7.84 \mathrm{E}+01 \\
6.76 \mathrm{E}+00 \\
2.23 \mathrm{E}+00 \\
5.80 \mathrm{E}+01 \\
2.04 \mathrm{E}+01 \\
2.76 \mathrm{E}+02 \\
6.73 \mathrm{E}+00 \\
3.97 \mathrm{E}+01 \\
8.85 \mathrm{E}+00 \\
1.65 \mathrm{E}+01 \\
5.50 \mathrm{E}+01 \\
2.89 \mathrm{E}+01 \\
2.00 \mathrm{E}+02 \\
6.56 \mathrm{E}+01 \\
1.05 \mathrm{E}+02 \\
2.53 \mathrm{E}+01 \\
2.21 \mathrm{E}+02 \\
2.01 \mathrm{E}+01 \\
1.86 \mathrm{E}+01 \\
8.26 \mathrm{E}+00 \\
4.12 \mathrm{E}+00 \\
7.52 \mathrm{E}+01 \\
1.56 \mathrm{E}+02 \\
1.40 \mathrm{E}+02 \\
2.72 \mathrm{E}+01 \\
1.04 \mathrm{E}+02 \\
3.52 \mathrm{E}+01 \\
1.07 \mathrm{E}+02 \\
9.67 \mathrm{E}+01 \\
1.21 \mathrm{E}+01 \\
4.60 \mathrm{E}+01 \\
1.99 \mathrm{E}+01 \\
3.04 \mathrm{E}+01 \\
3.68 \mathrm{E}+01 \\
1.11 \mathrm{E}+01 \\
2.85 \mathrm{E}+01 \\
1.01 \mathrm{E}+02 \\
8.59 \mathrm{E}+01 \\
4.36 \mathrm{E}+02 \\
6.63 \mathrm{E}+02 \\
2.80 \mathrm{E}+02 \\
2.41 \mathrm{E}+02 \\
8.82 \mathrm{E}+02 \\
6.35 \mathrm{E}+02 \\
1.52 \mathrm{E}+02 \\
5.40 \mathrm{E}+02 \\
4.16 \mathrm{E}+02 \\
1.91 \mathrm{E}+02 \\
1.37 \mathrm{E}+03 \\
2.38 \mathrm{~B}+02 \\
2.40 \mathrm{E}+02\end{array}$ & $\begin{array}{l}\text { PU239 } \\
3.95 \mathrm{E}-11 \\
1.92 \mathrm{E}-11 \\
3.97 \mathrm{E}-11 \\
3.45 \mathrm{E}-11 \\
3.48 \mathrm{E}+02 \\
9.55 \mathrm{E}+02 \\
1.17 \mathrm{E}+04 \\
5.34 \mathrm{E}+02 \\
1.45 \mathrm{E}+04 \\
3.23 \mathrm{E}+02 \\
2.94 \mathrm{E}+03 \\
2.48 \mathrm{E}+03 \\
4.11 \mathrm{E}+02 \\
3.77 \mathrm{E}+03 \\
7.23 \mathrm{E}+03 \\
4.92 \mathrm{E}+02 \\
3.21 \mathrm{E}+02 \\
1.51 \mathrm{E}+03 \\
5.14 \mathrm{E}+02 \\
7.58 \mathrm{E}+03 \\
3.25 \mathrm{E}+02 \\
1.29 \mathrm{E}+03 \\
3.62 \mathrm{E}+02 \\
4.71 \mathrm{E}+02 \\
1.51 \mathrm{E}+03 \\
5.91 \mathrm{E}+02 \\
5.05 \mathrm{E}+03 \\
2.13 \mathrm{E}+03 \\
3.15 \mathrm{E}+03 \\
5.61 \mathrm{E}+02 \\
5.28 \mathrm{E}+03 \\
9.63 \mathrm{E}+02 \\
9.21 \mathrm{E}+02 \\
3.53 \mathrm{E}+02 \\
2.64 \mathrm{E}+02 \\
2.24 \mathrm{E}+03 \\
3.72 \mathrm{E}+03 \\
3.22 \mathrm{E}+03 \\
8.53 \mathrm{E}+02 \\
2.97 \mathrm{E}+03 \\
9.11 \mathrm{E}+02 \\
2.65 \mathrm{E}+03 \\
1.19 \mathrm{E}+03 \\
2.99 \mathrm{E}+02 \\
5.47 \mathrm{E}+02 \\
3.02 \mathrm{E}+02 \\
4.44 \mathrm{E}+02 \\
3.96 \mathrm{E}+02 \\
1.40 \mathrm{E}+02 \\
4.15 \mathrm{E}+02 \\
6.21 \mathrm{E}+02 \\
6.93 \mathrm{E}+02 \\
2.65 \mathrm{E}+03 \\
4.85 \mathrm{E}+03 \\
9.71 \mathrm{E}+02 \\
9.67 \mathrm{E}+02 \\
3.06 \mathrm{E}+03 \\
2.54 \mathrm{E}+03 \\
7.79 \mathrm{E}+02 \\
2.76 \mathrm{E}+03 \\
1.79 \mathrm{E}+03 \\
8.27 \mathrm{E}+02 \\
4.73 \mathrm{E}+03 \\
8.73 \mathrm{E}+02 \\
1.03 \mathrm{E}+03\end{array}$ & $\begin{array}{l}\text { PU240 } \\
2.12 \mathrm{E}-12 \\
1.03 \mathrm{E}-12 \\
2.13 \mathrm{E}-12 \\
1.85 \mathrm{E}-12 \\
3.37 \mathrm{E}+01 \\
1.16 \mathrm{E}+02 \\
1.39 \mathrm{E}+03 \\
7.71 \mathrm{E}+01 \\
1.87 \mathrm{E}+03 \\
2.62 \mathrm{E}+01 \\
4.61 \mathrm{E}+02 \\
3.20 \mathrm{E}+02 \\
4.27 \mathrm{E}+01 \\
3.30 \mathrm{E}+02 \\
7.84 \mathrm{E}+02 \\
6.36 \mathrm{E}+01 \\
2.61 \mathrm{E}+01 \\
2.36 \mathrm{E}+02 \\
8.26 \mathrm{E}+01 \\
1.13 \mathrm{E}+03 \\
3.06 \mathrm{E}+01 \\
1.67 \mathrm{E}+02 \\
3.88 \mathrm{E}+01 \\
6.77 \mathrm{E}+01 \\
2.25 \mathrm{E}+02 \\
1.14 \mathrm{E}+02 \\
8.11 \mathrm{E}+02 \\
2.77 \mathrm{E}+02 \\
4.37 \mathrm{E}+02 \\
1.01 \mathrm{E}+02 \\
8.89 \mathrm{E}+02 \\
9.09 \mathrm{E}+01 \\
8.48 \mathrm{E}+01 \\
3.66 \mathrm{E}+01 \\
2.01 \mathrm{E}+01 \\
3.11 \mathrm{E}+02 \\
6.27 \mathrm{E}+02 \\
5.61 \mathrm{E}+02 \\
1.14 \mathrm{E}+02 \\
4.27 \mathrm{E}+02 \\
1.43 \mathrm{E}+02 \\
4.32 \mathrm{E}+02 \\
3.61 \mathrm{E}+02 \\
4.87 \mathrm{E}+01 \\
1.71 \mathrm{E}+02 \\
7.58 \mathrm{E}+01 \\
1.10 \mathrm{E}+02 \\
1.12 \mathrm{E}+02 \\
3.77 \mathrm{E}+01 \\
1.03 \mathrm{E}+02 \\
2.38 \mathrm{E}+02 \\
2.25 \mathrm{E}+02 \\
1.02 \mathrm{E}+03 \\
1.67 \mathrm{E}+03 \\
5.07 \mathrm{E}+02 \\
4.70 \mathrm{E}+02 \\
1.60 \mathrm{E}+03 \\
1.24 \mathrm{E}+03 \\
3.35 \mathrm{E}+02 \\
1.19 \mathrm{E}+03 \\
8.42 \mathrm{E}+02 \\
3.88 \mathrm{E}+02 \\
2.47 \mathrm{E}+03 \\
4.43 \mathrm{E}+02 \\
4.85 \mathrm{E}+02\end{array}$ & $\begin{array}{l}P U 241 \\
1.10 \mathrm{E}-11 \\
5.39 \mathrm{E}-12 \\
1.15 \mathrm{E}-11 \\
1.02 \mathrm{E}-11 \\
2.79 \mathrm{E}+02 \\
1.25 \mathrm{E}+03 \\
1.46 \mathrm{E}+04 \\
9.46 \mathrm{E}+02 \\
2.16 \mathrm{E}+04 \\
1.65 \mathrm{E}+02 \\
6.04 \mathrm{E}+03 \\
3.67 \mathrm{E}+03 \\
4.03 \mathrm{E}+02 \\
2.44 \mathrm{E}+03 \\
7.84 \mathrm{E}+03 \\
7.26 \mathrm{E}+02 \\
1.62 \mathrm{E}+02 \\
2.67 \mathrm{E}+03 \\
9.35 \mathrm{E}+02 \\
1.25 \mathrm{E}+04 \\
2.59 \mathrm{E}+02 \\
1.76 \mathrm{E}+03 \\
3.69 \mathrm{E}+02 \\
7.59 \mathrm{E}+02 \\
2.53 \mathrm{E}+03 \\
1.37 \mathrm{E}+03 \\
9.23 \mathrm{E}+03 \\
2.83 \mathrm{E}+03 \\
4.66 \mathrm{E}+03 \\
1.21 \mathrm{E}+03 \\
1.04 \mathrm{E}+04 \\
7.88 \mathrm{E}+02 \\
7.26 \mathrm{E}+02 \\
3.34 \mathrm{E}+02 \\
1.36 \mathrm{E}+02 \\
3.43 \mathrm{E}+03 \\
7.37 \mathrm{E}+03 \\
6.52 \mathrm{E}+03 \\
1.21 \mathrm{E}+03 \\
4.67 \mathrm{E}+03 \\
1.65 \mathrm{E}+03 \\
4.96 \mathrm{E}+03 \\
5.13 \mathrm{E}+03 \\
5.68 \mathrm{E}+02 \\
2.46 \mathrm{E}+03 \\
1.01 \mathrm{E}+03 \\
1.84 \mathrm{E}+03 \\
2.47 \mathrm{E}+03 \\
5.24 \mathrm{E}+02 \\
1.73 \mathrm{E}+03 \\
7.34 \mathrm{E}+03 \\
6.24 \mathrm{E}+03 \\
3.26 \mathrm{E}+04 \\
4.85 \mathrm{E}+04 \\
1.87 \mathrm{E}+04 \\
1.70 \mathrm{E}+04 \\
5.85 \mathrm{E}+04 \\
4.52 \mathrm{E}+04 \\
1.12 \mathrm{E}+04 \\
4.00 \mathrm{E}+04 \\
2.99 \mathrm{E}+04 \\
1.36 \mathrm{E}+04 \\
9.24 \mathrm{E}+04 \\
1.67 \mathrm{E}+00\end{array}$ & 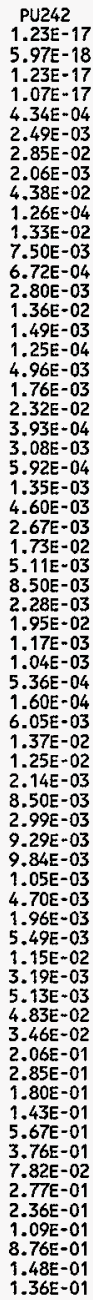 & 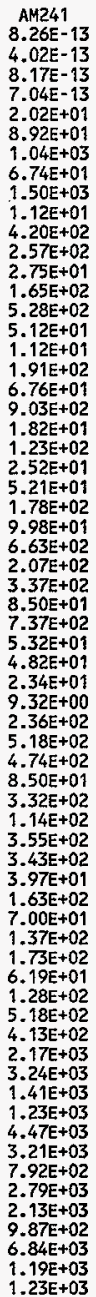 & $\begin{array}{l}\text { AM243 } \\
2.45 \mathrm{E}-18 \\
1.19 \mathrm{E}-18 \\
2.46 \mathrm{E}-18 \\
2.14 \mathrm{E}-18 \\
1.21 \mathrm{E}-04 \\
8.50 \mathrm{E}-04 \\
9.62 \mathrm{E}-03 \\
7.42 \mathrm{E}-04 \\
1.53 \mathrm{E}-02 \\
2.10 \mathrm{E}-06 \\
4.88 \mathrm{E}-03 \\
2.62 \mathrm{E}-03 \\
2.07 \mathrm{E}-04 \\
5.53 \mathrm{E}-04 \\
4.34 \mathrm{E}-03 \\
5.21 \mathrm{E}-04 \\
2.09 \mathrm{E}-06 \\
1.46 \mathrm{E}-03 \\
5.20 \mathrm{E}-04 \\
6.79 \mathrm{E}-03 \\
1.04 \mathrm{E}-04 \\
8.88 \mathrm{E}-04 \\
1.64 \mathrm{E}-04 \\
3.94 \mathrm{E}-04 \\
1.35 \mathrm{E}-03 \\
7.99 \mathrm{E}-04 \\
5.11 \mathrm{E}-03 \\
1.47 \mathrm{E}-03 \\
2.47 \mathrm{E}-03 \\
6.78 \mathrm{E}-04 \\
5.77 \mathrm{E}-03 \\
3.10 \mathrm{E}-04 \\
2.74 \mathrm{E}-04 \\
1.47 \mathrm{E}-04 \\
3.52 \mathrm{E}-05 \\
1.76 \mathrm{E}-03 \\
4.07 \mathrm{E}-03 \\
3.71 \mathrm{E}-03 \\
6.20 \mathrm{E}-04 \\
2.48 \mathrm{E}-03 \\
8.82 \mathrm{E}-04 \\
2.75 \mathrm{E}-03 \\
3.00 \mathrm{E}-03 \\
3.10 \mathrm{E}-04 \\
1.44 \mathrm{E}-03 \\
5.93 \mathrm{E}-04 \\
8.00 \mathrm{E}-04 \\
9.68 \mathrm{E}-03 \\
2.22 \mathrm{E}-03 \\
7.48 \mathrm{E}-04 \\
5.664 \mathrm{E}-02 \\
3.66 \mathrm{E}-02 \\
2.41 \mathrm{E}-01 \\
3.15 \mathrm{E}-01 \\
3.06 \mathrm{E}-01 \\
2.18 \mathrm{E}-01 \\
9.66 \mathrm{E}-01 \\
5.73 \mathrm{E}-01 \\
9.45 \mathrm{E}-02 \\
3.34 \mathrm{E}-01 \\
3.36 \mathrm{E}-01 \\
1.55 \mathrm{E}-01 \\
1.49 \mathrm{E}+00 \\
2.42 \mathrm{E}-01 \\
1.94 \mathrm{E}-01\end{array}$ & $\begin{array}{l}\text { CM242 } \\
6.06 \mathrm{E}-31 \\
3.01 \mathrm{E}-31 \\
2.07 \mathrm{E}-30 \\
3.27 \mathrm{E}-30 \\
3.97 \mathrm{E}-04 \\
2.76 \mathrm{E}-03 \\
3.13 \mathrm{E}-02 \\
2.41 \mathrm{E}-03 \\
4.98 \mathrm{E}-02 \\
1.15 \mathrm{E}-05 \\
1.59 \mathrm{E}-02 \\
8.52 \mathrm{E}-03 \\
6.76 \mathrm{E}-04 \\
1.85 \mathrm{E}-03 \\
1.42 \mathrm{E}-02 \\
1.69 \mathrm{E}-03 \\
1.14 \mathrm{E}-05 \\
6.16 \mathrm{E}-03 \\
2.19 \mathrm{E}-03 \\
2.86 \mathrm{E}-02 \\
4.42 \mathrm{E}-04 \\
3.75 \mathrm{E}-03 \\
6.96 \mathrm{E}-04 \\
1.67 \mathrm{E}-03 \\
5.69 \mathrm{E}-03 \\
3.36 \mathrm{E}-03 \\
2.15 \mathrm{E}-02 \\
6.21 \mathrm{E}-03 \\
1.04 \mathrm{E}-02 \\
2.86 \mathrm{E}-03 \\
2.43 \mathrm{E}-02 \\
1.31 \mathrm{E}-03 \\
1.16 \mathrm{E}-03 \\
6.23 \mathrm{E}-04 \\
1.50 \mathrm{E}-04 \\
7.44 \mathrm{E}-03 \\
1.72 \mathrm{E}-02 \\
1.56 \mathrm{E}-02 \\
2.62 \mathrm{E}-03 \\
1.05 \mathrm{E}-02 \\
3.73 \mathrm{E}-03 \\
1.16 \mathrm{E}-02 \\
1.27 \mathrm{E}-02 \\
1.31 \mathrm{E}-03 \\
6.08 \mathrm{E}-03 \\
2.50 \mathrm{E}-03 \\
2.13 \mathrm{E}-03 \\
2.55 \mathrm{E}-02 \\
5.66 \mathrm{E}-03 \\
1.99 \mathrm{E}-03 \\
1.48 \mathrm{E}-01 \\
9.68 \mathrm{E}-02 \\
6.36 \mathrm{E}-01 \\
8.32 \mathrm{E}-01 \\
7.40 \mathrm{E}-01 \\
5.42 \mathrm{E}-01 \\
2.33 \mathrm{E}+00 \\
1.43 \mathrm{E}+00 \\
2.49 \mathrm{E}-01 \\
8.81 \mathrm{E}-01 \\
8.48 \mathrm{E}-01 \\
3.91 \mathrm{E}-01 \\
3.61 \mathrm{E}+00 \\
5.91 \mathrm{E}-01 \\
4.89 \mathrm{E}-01\end{array}$ & $\begin{array}{c}\text { CM243 } \\
1.57 \mathrm{E}-19 \\
7.64 \mathrm{E}-20 \\
1.61 \mathrm{E}-19 \\
1.41 \mathrm{E}-19 \\
1.00 \mathrm{E}-05 \\
8.16 \mathrm{E}-05 \\
9.19 \mathrm{E}-04 \\
7.36 \mathrm{E}-05 \\
1.51 \mathrm{E}-03 \\
0.00 \mathrm{E}+00 \\
4.93 \mathrm{E}-04 \\
2.57 \mathrm{E}-04 \\
1.88 \mathrm{E}-05 \\
3.03 \mathrm{E}-05 \\
4.08 \mathrm{E}-04 \\
5.09 \mathrm{E}-05 \\
0.00 \mathrm{E}+00 \\
1.49 \mathrm{E}-04 \\
5.27 \mathrm{E}-05 \\
6.86 \mathrm{E}-04 \\
1.01 \mathrm{E}-05 \\
9.01 \mathrm{E}-05 \\
1.64 \mathrm{E}-05 \\
4.04 \mathrm{E}-05 \\
1.38 \mathrm{E}-04 \\
8.13 \mathrm{E}-05 \\
5.19 \mathrm{E}-04 \\
1.47 \mathrm{E}-04 \\
2.49 \mathrm{E}-04 \\
6.98 \mathrm{E}-05 \\
5.90 \mathrm{E}-04 \\
3.03 \mathrm{E}-05 \\
2.67 \mathrm{E}-05 \\
1.45 \mathrm{E}-05 \\
3.00 \mathrm{E}-06 \\
1.80 \mathrm{E}-04 \\
4.17 \mathrm{E}-04 \\
3.77 \mathrm{E}-04 \\
6.28 \mathrm{E}-05 \\
2.52 \mathrm{E}-04 \\
9.09 \mathrm{E}-05 \\
2.80 \mathrm{E}-04 \\
3.18 \mathrm{E}-04 \\
3.18 \mathrm{E}-05 \\
1.52 \mathrm{E}-04 \\
6.19 \mathrm{E}-05 \\
0.00 \mathrm{E}+00 \\
2.22 \mathrm{E}-03 \\
3.45 \mathrm{E}-04 \\
0.00 \mathrm{E}+00 \\
1.60 \mathrm{E}-02 \\
1.01 \mathrm{E}-02 \\
6.97 \mathrm{E}-02 \\
8.84 \mathrm{E}-02 \\
1.17 \mathrm{E}-01 \\
7.83 \mathrm{E}-02 \\
3.67 \mathrm{E}-01 \\
2.07 \mathrm{E}-01 \\
2.73 \mathrm{E}-02 \\
9.70 \mathrm{E}-02 \\
1.14 \mathrm{E}-01 \\
5.23 \mathrm{E}-02 \\
5.73 \mathrm{E}-01 \\
9.16 \mathrm{E}-02 \\
6.57 \mathrm{E}-02\end{array}$ \\
\hline
\end{tabular}


HNF-SD-WN-TI-794, Rev, O

ACTIVITY IN UNSEPARATED FUEL (Curies decayed to $1 / 1 / 94$ )

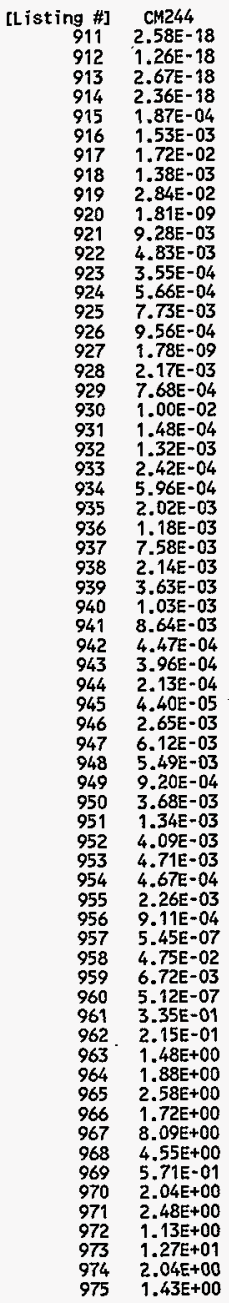


HNF-SD-WM-TI-794, ReV. O

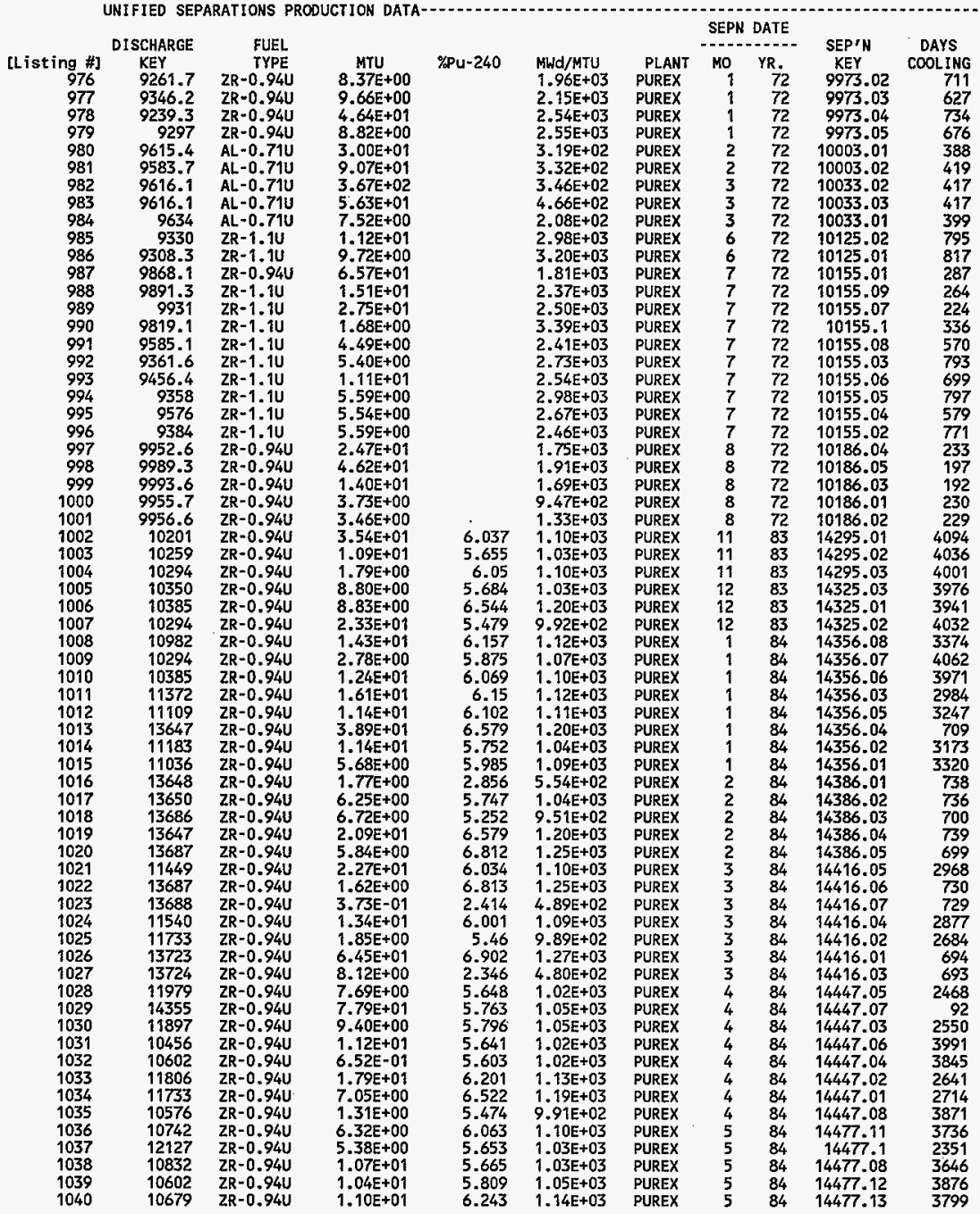


HNF-SD-WM-TI-794, Rev. O

UNIFIED SEPARATIONS PRODUCTION DATA

\section{[Listing \#] Fuel Data Reference}

976 I S0-263 RD AND ARH-18 RD (MCDONALD

977 ISO-263 RD AND ARH -18 RD (MCDONALD

978 IS0-263 RD AND ARH-18 RD (MCDONALD

979 ISO-263 RD AND ARH-18 RD (MCDONALD

980 ISO-263 RD AND ARH-18 RD (MCDONALD

981 ISO-263 RD AND ARH-18 RD (MCDONALD

982 ISO-263 RD AND ARH-18 RD (MCDONALD

983 ISO-263 RD AND ARH-18 RD (MCDONALD

984 I $50-263$ RD AND ARH-18 RD (MCDONALD

985 ISO-263 RD AND ARH-18 RD (MCDONALO

986 ISO-263 RD AND ARH-18 RD (MCDONALD

987 ISO-263 RD AND ARH-18 RD (MCDONALD

988 ISO-263 RD AND ARH-18 RD (MCDONALD

989 ISO-263 RD AND ARH-18 RD (MCDONALD

990 ISO-263 RD AND ARH-18 RD (MCDONALD

991 ISO-263 RD AND ARH-18 RD (MCDONALD

992 ISO-263 RD AND ARH-18 RD (MCDONALD

993 ISO-263 RD AND ARH-18 RD (MCDONALD

994 ISO-263 RD AND ARH-18 RD (MCDONALD

995 ISO-263 RD AND ARH-18 RD (MCOONALD

996 ISO-263 RD AND ARH-18 RD (MCDONALD

997 ISO-263 RD AND ARH-18 RD (MCDONALD

998 ISO-263 RD AND ARH-18 RD (MCDONALD

999 150-263 RD AND ARH-18 RD (MCDONALO 1000 ISO-263 RD AND ARH-18 RD (MCDONALD 1001 ISO-263 RD AND ARH-18 RD (MCDONALD $1002 \mathrm{~J}$. SCHLOSSER FILE 'SHIP PX.DAT' 01/ $1003 \mathrm{~J}$. SCHLOSSER FILE 'SHIP-PX.DAT' 01/ $1004 \mathrm{~J}$. SCHLOSSER FILE 'SHIP.PX.DAT' 01/ 1005 J. SCHLOSSER FILE 'SHIP PX.DAT' 01/ $1006 \mathrm{~J}$. SCHLOSSER FILE 'SHIP'PX.DAT' 01/ 1007 J. SCHLOSSER FILE 'SHIP-PX.DAT' 01/ $1008 \mathrm{~J}$. SCHLOSSER FILE 'SHIP PX.DAT' 01/ $1009 \mathrm{3}$. SCHLOSSER FILE 'SHIP_PX.DAT' 01/ $1010 \mathrm{~J}$. SCHLOSSER FILE 'SHIP-PX.DAT' 01/ $1011 \mathrm{~J}$. SCHLOSSER FILE 'SHIP'PX.DAT' 01/ 1012 J. SCHLOSSER FILE 'SHIP-PX.DAT' 01/ $1013 \mathrm{~J}$. SCHLOSSER FILE 'SHIPTPX.DAT' 01/ 1014 J. SCHLOSSER FILE 'SHIP-PX.DAT' O1/ $1015 \mathrm{~J}$. SCHLOSSER FILE 'SHIP_PX.DAT' 01/ $1016 \mathrm{~J}$. SCHLOSSER FILE 'SHIP-PX.DAT' 01/ $1017 \mathrm{~J}$. SCHLOSSER FILE 'SHIP_PX.DAT' O1/ $1018 \mathrm{~J}$. SCHLOSSER FILE 'SHIP'PX.DAT' O1/ $1019 \mathrm{~J}$. SCHLOSSER FILE 'SHIPPX.DAT' O1/ $1020 \mathrm{~J}$. SCHLOSSER FILE 'SHIP'PX.DAT' 01/ $1021 \mathrm{~J}$. SCHLOSSER FILE 'SHIP'PX.DAT' 01/ $1022 \mathrm{~J}$. SCHLOSSER FILE 'SHIP_PX.DAT' 01/ $1023 \mathrm{~J}$. SCHLOSSER FILE 'SHIP_PX.DAT' 01/ $1024 \mathrm{~J}$. SCHLOSSER FILE 'SHIP PX.DAT' O1/ $1025 \mathrm{~J}$. SCHLOSSER FILE 'SHIP PX.DAT' 01/ $1026 \mathrm{~J}$. SCHLOSSER FILE 'SHIP-PX.DAT' 01/ $1027 \mathrm{~J}$. SCHLOSSER FILE 'SHIP-PX.DAT' 01/ $1028 \mathrm{~J}$. SCHLOSSER FILE 'SHIP_PX.DAT' 01/ $1029 \mathrm{~J}$. SCHLOSSER FILE 'SHIP-PX.DAT' 01/ $1030 \mathrm{~J}$. SCHLOSSER FILE 'SHIP_PX.DAT' 01/ $1031 \mathrm{~J}$. SCHLOSSER FILE 'SHIP_PX.DAT' 01/ 1032 J. SCHLOSSER FILE 'SHIP'PX.DAT' 01/ 1033 J. SCHLOSSER FILE 'SHIP_PX.DAT' 01/ $1034 \mathrm{~J}$. SCHLOSSER FILE 'SHIP'PX.DAT' 01/ $1035 \mathrm{~J}$. SCHLOSSER FILE 'SHIP-PX.DAT' 01/ $1036 \mathrm{~J}$. SCHLOSSER FILE 'SHIP-PX.DAT' O1/ $1037 \mathrm{~J}$. SCHLOSSER FILE 'SHIP'PX.DAT' O1/ $1038 \mathrm{~J}$. SCHLOSSER FILE 'SHIP_PX.DAT' O1/ $1039 \mathrm{~J}$. SCHLOSSER FILE 'SHIP_PX.DAT' 01/ $1040 \mathrm{~J}$. SCHLOSSER FILE 'SHIP_PX.DAT' 01/

TOTAL WASTE LOSS FRACTION

Th

1

110.

u

0.0168

0.0168

0.0168

0.0062

0.0062

0.0023

0.0023

0.0023

0.0168

0.0168

0.0168

0.0168

0.0168

0.0168

0.0168

0.0168

0.0168

0.0168

0.0168

0.0168

0.0168

0.0168

0.0168

0.0168

0.0168

0.0168

0.0168

0.0168

0.0168

0.0168

0.0168

0.0168

0.0168

0.0168

0.0168

0.0168

0.0168

0.0168

0.0168

0.0168

0.0168

0.0168

0.0168

0.0168

0.0168

0.0168

0.0168

0.0168

0.0168

0.0168

0.0168

0.0168

0.0168

0.0168

0.0168

0.0168

0.0168

0.0168

0.0168

0.0168

0.0168

0.0168

0.0168

0.0168
Np

0.

0.3

0.3

0.3

0.3

0.3

0.3

0.3

0.3

0.3

0.3

0.3

0.3

0.3

0.3

0.3

0.3

0.3

0.3

0.3

0.3

0.3

0.3

0.3

0.3

0.3

.

\section{1}

1

-1--.--

Pu

0.033

0.033

0.033

0.0082

0.0082

0.004

0.004

0.004

0.033

0.033

0.033

0.033

0.033

0.033

0.033

0.033

0.033

0.033

0.033

0.033

0.033

0.033

0.033

0.033

0.033

0.033

0.033

0.033

0.033

0.033

0.033

0.033

0.033

0.033

0.033

0.033

0.033

0.033

0.033

0.033

0.033

0.033

0.033

0.033

0.033

0.033

0.033

0.033

0.033

0.033

0.033

0.033

0.033

0.033

0.033

0.033

0.033

0.033

0.033

0.033

0.033

0.033

0.033

0.033

Waste Frac. Reference

(c)

(c)

(c)

(b)

(b)

(b)

(b)

(b)

(c)

(c)

(c)

(c)

(c)

(c)

(c)

(c)

(c)

(c)

(c)

(c)

(c)

(c)

(c)

(c)

(c)

(c)

(c)

(c)

(c)

(c)

(c)

(c)

(c)

(c)

(c)

(c)

(c)

(c)

(c)

(c)

(c)

(c)

(c) 
HNF-SD-WM-TI-794, ReV. 0

ACTIVITY IN UNSEPARATED FUEL (Curies decayed to $1 / 1 / 94$ )

\begin{tabular}{|c|c|c|c|c|c|c|c|c|c|}
\hline $\begin{array}{c}\text { [Listing } \$ \\
976 \\
977 \\
978 \\
979 \\
980 \\
981 \\
982 \\
983 \\
984 \\
985 \\
986 \\
987 \\
988 \\
989 \\
990 \\
991 \\
992 \\
993 \\
994 \\
995 \\
996 \\
997 \\
998 \\
999 \\
1000 \\
1001 \\
1002 \\
1003 \\
1004 \\
1005 \\
1006 \\
1007 \\
1008 \\
1009 \\
1010 \\
1011 \\
1012 \\
1013 \\
1014 \\
1015 \\
1016 \\
1017 \\
1018 \\
1019 \\
1020 \\
1021 \\
1022 \\
1023 \\
1024 \\
1025 \\
1026 \\
1027 \\
1028 \\
1029 \\
1030 \\
1031 \\
1032 \\
1033 \\
1034 \\
1035 \\
1036 \\
1037 \\
1038 \\
1039 \\
1040\end{array}$ & $\begin{array}{l}103 \\
7.15 \mathrm{E}+01 \\
9.22 \mathrm{E}+01 \\
5.19 \mathrm{E}+02 \\
1.00 \mathrm{E}+02 \\
4.14 \mathrm{E}+01 \\
1.29 \mathrm{E}+02 \\
5.51 \mathrm{E}+02 \\
1.14 \mathrm{E}+02 \\
6.76 \mathrm{E}+00 \\
1.44 \mathrm{E}+02 \\
1.35 \mathrm{E}+02 \\
5.67 \mathrm{E}+02 \\
1.67 \mathrm{E}+02 \\
3.25 \mathrm{E}+02 \\
2.69 \mathrm{E}+01 \\
4.83 \mathrm{E}+01 \\
6.41 \mathrm{E}+01 \\
1.24 \mathrm{E}+02 \\
7.25 \mathrm{E}+01 \\
6.63 \mathrm{E}+01 \\
5.97 \mathrm{E}+01 \\
2.09 \mathrm{E}+02 \\
4.28 \mathrm{E}+02 \\
1.15 \mathrm{E}+02 \\
1.66 \mathrm{E}+01 \\
2.19 \mathrm{E}+01 \\
1.91 \mathrm{E}+02 \\
5.53 \mathrm{E}+01 \\
9.81 \mathrm{E}+00 \\
4.54 \mathrm{E}+01 \\
5.35 \mathrm{E}+01 \\
1.14 \mathrm{E}+02 \\
8.86 \mathrm{E}+01 \\
1.47 \mathrm{E}+01 \\
6.88 \mathrm{E}+01 \\
1.06 \mathrm{E}+02 \\
7.10 \mathrm{E}+01 \\
3.91 \mathrm{E}+02 \\
6.73 \mathrm{E}+01 \\
3.45 \mathrm{E}+01 \\
8.05 \mathrm{E}+00 \\
5.41 \mathrm{E}+01 \\
5.32 \mathrm{E}+01 \\
2.10 \mathrm{E}+02 \\
6.15 \mathrm{E}+01 \\
1.48 \mathrm{E}+02 \\
1.71 \mathrm{E}+01 \\
1.50 \mathrm{E}+00 \\
8.82 \mathrm{E}+01 \\
1.13 \mathrm{E}+01 \\
6.93 \mathrm{E}+02 \\
3.22 \mathrm{E}+01 \\
5.06 \mathrm{E}+01 \\
7.54 \mathrm{E}+02 \\
6.29 \mathrm{E}+01 \\
5.83 \mathrm{E}+01 \\
3.44 \mathrm{E}+00 \\
1.27 \mathrm{E}+02 \\
5.23 \mathrm{E}+01 \\
6.71 \mathrm{E}+00 \\
3.71 \mathrm{E}+01 \\
3.62 \mathrm{E}+01 \\
5.93 \mathrm{E}+01 \\
5.69 \mathrm{E}+01 \\
6.62 \mathrm{E}+01\end{array}$ & $\begin{array}{l}\text { C14 } \\
1.80 \mathrm{E}+00 \\
2.28 \mathrm{E}+00 \\
1.30 \mathrm{E}+01 \\
2.48 \mathrm{E}+00 \\
6.15 \mathrm{E}-01 \\
1.93 \mathrm{E}+00 \\
8.17 \mathrm{E}+00 \\
1.68 \mathrm{E}+00 \\
1.01 \mathrm{E}-01 \\
3.22 \mathrm{E}+00 \\
3.02 \mathrm{E}+00 \\
1.30 \mathrm{E}+04 \\
3.43 \mathrm{E}+00 \\
6.63 \mathrm{E}+00 \\
5.57 \mathrm{E}-01 \\
1.04 \mathrm{E}+00 \\
1.43 \mathrm{E}+00 \\
2.72 \mathrm{E}+00 \\
1.61 \mathrm{E}+00 \\
1.43 \mathrm{E}+00 \\
1.33 \mathrm{E}+00 \\
4.73 \mathrm{E}+00 \\
9.63 \mathrm{E}+00 \\
2.58 \mathrm{E}+00 \\
3.79 \mathrm{E}-01 \\
4.97 \mathrm{E}-01 \\
4.19 \mathrm{E}+00 \\
1.21 \mathrm{E}+00 \\
2.13 \mathrm{E}-01 \\
9.75 \mathrm{E}-01 \\
1.14 \mathrm{E}+00 \\
2.48 \mathrm{E}+00 \\
1.73 \mathrm{E}+00 \\
3.19 \mathrm{E}-01 \\
1.47 \mathrm{E}+00 \\
1.95 \mathrm{E}+00 \\
1.36 \mathrm{E}+00 \\
5.06 \mathrm{E}+00 \\
1.27 \mathrm{E}+00 \\
6.66 \mathrm{E}-01 \\
1.05 \mathrm{E}-01 \\
7.01 \mathrm{E}-01 \\
6.87 \mathrm{E}-01 \\
2.72 \mathrm{E}+00 \\
7.90 \mathrm{E}-01 \\
2.69 \mathrm{E}+00 \\
2.19 \mathrm{E}-01 \\
1.95 \mathrm{E}-02 \\
1.58 \mathrm{E}+00 \\
1.96 \mathrm{E}-01 \\
8.85 \mathrm{E}+00 \\
4.15 \mathrm{E}-01 \\
8.47 \mathrm{E}-01 \\
8.77 \mathrm{E}+00 \\
1.07 \mathrm{E}+00 \\
1.23 \mathrm{E}+00 \\
7.12 \mathrm{E}-02 \\
2.18 \mathrm{E}+00 \\
9.08 \mathrm{E}-01 \\
1.39 \mathrm{E}-01 \\
7.51 \mathrm{E}-01 \\
5.93 \mathrm{E}-01 \\
1.19 \mathrm{E}+00 \\
1.18 \mathrm{E}+00 \\
1.35 \mathrm{E}+00\end{array}$ & $\begin{array}{l}\text { N159 } \\
1.06 \mathrm{E}-01 \\
1.35 \mathrm{E}-01 \\
7.64 \mathrm{E}-01 \\
1.46 \mathrm{E}-01 \\
1.75 \mathrm{E}-01 \\
5.48 \mathrm{E}-01 \\
2.32 \mathrm{E}+00 \\
4.76 \mathrm{E}-01 \\
2.87 \mathrm{E}-02 \\
1.98 \mathrm{E}-01 \\
1.86 \mathrm{E}-01 \\
7.70 \mathrm{E}-01 \\
2.12 \mathrm{E}-01 \\
4.10 \mathrm{E}-01 \\
3.42 \mathrm{E}-02 \\
6.44 \mathrm{E}-02 \\
8.80 \mathrm{E}-02 \\
1.68 \mathrm{E}-01 \\
9.92 \mathrm{E}-02 \\
8.81 \mathrm{E}-02 \\
8.19 \mathrm{E}-02 \\
2.80 \mathrm{E}-01 \\
5.70 \mathrm{E}-01 \\
1.53 \mathrm{E}-01 \\
2.27 \mathrm{E}-02 \\
2.96 \mathrm{E}-02 \\
2.50 \mathrm{E}-01 \\
7.21 \mathrm{E}-02 \\
1.27 \mathrm{E}-02 \\
5.83 \mathrm{E}-02 \\
6.80 \mathrm{E}-02 \\
1.48 \mathrm{E}-01 \\
1.03 \mathrm{E}-01 \\
1.90 \mathrm{E}-02 \\
8.77 \mathrm{E}-02 \\
1.16 \mathrm{E}-01 \\
8.10 \mathrm{E}-02 \\
3.01 \mathrm{E}-01 \\
7.61 \mathrm{E}-02 \\
3.98 \mathrm{E}-02 \\
6.29 \mathrm{E}-03 \\
4.18 \mathrm{E}-02 \\
4.30 \mathrm{E}-02 \\
1.62 \mathrm{E}-01 \\
4.70 \mathrm{E}-02 \\
1.60 \mathrm{E}-01 \\
1.31 \mathrm{E}-02 \\
1.17 \mathrm{E}-03 \\
9.42 \mathrm{E}-02 \\
1.17 \mathrm{E}-02 \\
5.27 \mathrm{E}-01 \\
2.49 \mathrm{E}-02 \\
5.06 \mathrm{E}-02 \\
5.23 \mathrm{E}-01 \\
6.36 \mathrm{E}-02 \\
7.36 \mathrm{E}-02 \\
4.25 \mathrm{E}-03 \\
1.30 \mathrm{E}-01 \\
5.41 \mathrm{E}-02 \\
8.32 \mathrm{E}-03 \\
4.48 \mathrm{E}-02 \\
3.54 \mathrm{E}-02 \\
7.07 \mathrm{E}-02 \\
7.03 \mathrm{E}-02 \\
8.06 \mathrm{E}-02\end{array}$ & $\begin{array}{l}\text { NI63 } \\
1.13 E+01 \\
1.43 E+01 \\
8.12 E+01 \\
1.55 E+01 \\
1.83 E+01 \\
5.74 E+01 \\
2.43 E+02 \\
5.00 E+01 \\
3.01 E+00 \\
2.11 E+01 \\
1.97 E+01 \\
8.25 E+01 \\
2.28 E+01 \\
4.40 E+01 \\
3.67 E+00 \\
6.85 E+00 \\
9.34 E+00 \\
1.79 E+01 \\
1.06 E+01 \\
9.39 E+00 \\
8.69 E+00 \\
3.01 E+01 \\
6.12 E+01 \\
1.64 E+01 \\
2.42 E+00 \\
3.17 E+00 \\
2.68 E+01 \\
7.74 E+00 \\
1.37 E+00 \\
6.27 E+00 \\
7.33 E+00 \\
1.59 E+01 \\
1.12 E+01 \\
2.05 E+00 \\
9.45 E+00 \\
1.28 E+01 \\
8.86 E+00 \\
3.48 E+01 \\
8.33 E+00 \\
4.34 E+00 \\
7.23 E-01 \\
4.82 E+00 \\
4.73 E+00 \\
1.87 E+01 \\
5.43 E+00 \\
1.77 E+01 \\
1.51 E+00 \\
1.34 E-01 \\
1.04 E+01 \\
1.30 E+00 \\
6.09 E+01 \\
2.87 E+00 \\
5.63 E+00 \\
6.11 E+01 \\
7.06 E+00 \\
7.94 E+00 \\
4.60 E-01 \\
1.44 E+01 \\
6.00 E+00 \\
9.00 E-01 \\
4.87 E+00 \\
3.95 E+00 \\
7.69 E+00 \\
7.60 E+00 \\
8.74 E+00\end{array}$ & $\begin{array}{l}\text { CO60 } \\
4.77 E+00 \\
6.22 E+00 \\
3.38 E+01 \\
6.59 E+00 \\
1.87 E+00 \\
5.79 E+00 \\
2.47 E+01 \\
5.08 E+00 \\
3.09 E-01 \\
1.02 E+01 \\
9.46 E+00 \\
4.31 E+01 \\
1.35 E+01 \\
2.63 E+01 \\
2.09 E+00 \\
3.66 E+00 \\
4.59 E+00 \\
9.10 E+00 \\
5.16 E+00 \\
4.97 E+00 \\
4.32 E+00 \\
1.62 E+01 \\
3.33 E+01 \\
8.98 E+00 \\
1.33 E+00 \\
1.72 E+00 \\
1.59 E+01 \\
4.69 E+00 \\
8.35 E-01 \\
3.92 E+00 \\
4.62 E+00 \\
9.77 E+00 \\
8.68 E+00 \\
1.25 E+00 \\
5.97 E+00 \\
1.13 E+01 \\
7.15 E+00 \\
6.63 E+01 \\
6.91 E+00 \\
3.42 E+00 \\
1.40 E+00 \\
9.24 E+00 \\
9.18 E+00 \\
3.56 E+01 \\
1.05 E+01 \\
1.60 E+01 \\
2.91 E+00 \\
2.63 E-01 \\
9.71 E+00 \\
1.30 E+00 \\
1.19 E+02 \\
5.69 E+00 \\
6.12 E+00 \\
1.49 E+02 \\
7.46 E+00 \\
5.14 E+00 \\
3.13 E-01 \\
1.48 E+01 \\
5.97 E+00 \\
6.07 E-01 \\
3.47 E+00 \\
4.51 E+00 \\
5.66 E+00 \\
5.17 E+00 \\
6.09 E+00\end{array}$ & $\begin{array}{l}\text { SE79 } \\
2.17 E-01 \\
2.74 E-01 \\
1.55 E+00 \\
2.95 E-01 \\
1.29 E-01 \\
4.06 E-01 \\
1.72 E+00 \\
3.54 E-01 \\
2.12 E-02 \\
4.34 E-01 \\
4.06 E-01 \\
1.58 E+00 \\
4.69 E-01 \\
9.03 E-01 \\
7.45 E-02 \\
1.42 E-01 \\
1.93 E-01 \\
3.71 E-01 \\
2.17 E-01 \\
1.94 E-01 \\
1.81 E-01 \\
5.74 E-01 \\
1.16 E+00 \\
3.14 E-01 \\
4.72 E-02 \\
6.11 E-02 \\
5.19 E-01 \\
1.50 E-01 \\
2.63 E-02 \\
1.21 E-01 \\
1.41 E-01 \\
3.08 E-01 \\
2.13 E-01 \\
3.95 E-02 \\
1.82 E-01 \\
2.41 E-01 \\
1.68 E-01 \\
6.24 E-01 \\
1.58 E-01 \\
8.25 E-02 \\
1.32 E-02 \\
8.69 E-02 \\
8.53 E-02 \\
3.35 E-01 \\
9.72 E-02 \\
3.32 E-01 \\
2.70 E-02 \\
2.45 E-03 \\
1.95 E-01 \\
2.44 E-02 \\
1.09 E+00 \\
5.23 E-02 \\
1.05 E-01 \\
1.09 E+00 \\
1.32 E-01 \\
1.53 E-01 \\
8.83 E-03 \\
2.69 E-01 \\
1.12 E-01 \\
1.733 E-02 \\
9.30 E-02 \\
7.35 E-02 \\
1.47 E-01 \\
1.46 E-01 \\
1.67 E-01\end{array}$ & $\begin{array}{l}\text { SR90 } \\
2.45 \mathrm{E}+04 \\
3.09 \mathrm{E}+04 \\
1.70 \mathrm{E}+05 \\
3.27 \mathrm{E}+04 \\
1.61 \mathrm{E}+04 \\
5.05 \mathrm{E}+04 \\
2.14 \mathrm{E}+05 \\
4.38 \mathrm{E}+04 \\
2.66 \mathrm{E}+03 \\
4.98 \mathrm{E}+04 \\
4.61 \mathrm{E}+04 \\
1.87 \mathrm{E}+05 \\
5.69 \mathrm{E}+04 \\
1.09 \mathrm{E}+05 \\
8.71 \mathrm{E}+03 \\
1.69 \mathrm{E}+04 \\
2.24 \mathrm{E}+04 \\
4.35 \mathrm{E}+04 \\
2.50 \mathrm{E}+04 \\
2.28 \mathrm{E}+04 \\
2.11 \mathrm{E}+04 \\
6.86 \mathrm{E}+04 \\
1.38 \mathrm{E}+05 \\
3.77 \mathrm{E}+04 \\
5.86 \mathrm{E}+03 \\
7.46 \mathrm{E}+03 \\
6.50 \mathrm{E}+04 \\
1.89 \mathrm{E}+04 \\
3.32 \mathrm{E}+03 \\
1.54 \mathrm{E}+04 \\
1.78 \mathrm{E}+04 \\
3.90 \mathrm{E}+04 \\
2.81 \mathrm{E}+04 \\
4.98 \mathrm{E}+03 \\
2.31 \mathrm{E}+04 \\
3.26 \mathrm{E}+04 \\
2.23 \mathrm{E}+04 \\
9.74 \mathrm{E}+04 \\
2.12 \mathrm{E}+04 \\
1.09 \mathrm{E}+04 \\
2.12 \mathrm{E}+03 \\
1.37 \mathrm{E}+04 \\
1.35 \mathrm{E}+04 \\
5.23 \mathrm{E}+04 \\
1.52 \mathrm{E}+04 \\
4.52 \mathrm{E}+04 \\
4.22 \mathrm{E}+03 \\
3.96 \mathrm{E}+02 \\
2.67 \mathrm{E}+04 \\
3.39 \mathrm{E}+03 \\
1.71 \mathrm{E}+05 \\
8.48 \mathrm{E}+03 \\
1.48 \mathrm{E}+04 \\
1.79 \mathrm{E}+05 \\
1.85 \mathrm{E}+04 \\
1.95 \mathrm{E}+04 \\
1.14 \mathrm{E}+03 \\
3.74 \mathrm{E}+04 \\
1.54 \mathrm{E}+04 \\
2.23 \mathrm{E}+03 \\
1.21 \mathrm{E}+04 \\
1.05 \mathrm{E}+04 \\
1.93 \mathrm{E}+04 \\
1.88 \mathrm{E}+04 \\
2.16 \mathrm{E}+04\end{array}$ & $\begin{array}{l}Y 90 \\
2.45 \mathrm{E}+04 \\
3.09 \mathrm{E}+04 \\
1.71 \mathrm{E}+05 \\
3.27 \mathrm{E}+04 \\
1.61 \mathrm{E}+04 \\
5.05 \mathrm{E}+04 \\
2.14 \mathrm{E}+05 \\
4.38 \mathrm{E}+04 \\
2.66 \mathrm{E}+03 \\
4.98 \mathrm{E}+04 \\
4.61 \mathrm{E}+04 \\
1.87 \mathrm{E}+05 \\
5.69 \mathrm{E}+04 \\
1.09 \mathrm{E}+05 \\
8.71 \mathrm{E}+03 \\
1.69 \mathrm{E}+04 \\
2.24 \mathrm{E}+04 \\
4.35 \mathrm{E}+04 \\
2.50 \mathrm{E}+04 \\
2.28 \mathrm{E}+04 \\
2.11 \mathrm{E}+04 \\
6.86 \mathrm{E}+04 \\
1.38 \mathrm{E}+05 \\
3.77 \mathrm{E}+04 \\
5.86 \mathrm{E}+03 \\
7.46 \mathrm{E}+03 \\
6.50 \mathrm{E}+04 \\
1.89 \mathrm{E}+04 \\
3.32 \mathrm{E}+03 \\
1.54 \mathrm{E}+04 \\
1.78 \mathrm{E}+04 \\
3.90 \mathrm{E}+04 \\
2.81 \mathrm{E}+04 \\
4.99 \mathrm{E}+03 \\
2.31 \mathrm{E}+04 \\
3.26 \mathrm{E}+04 \\
2.23 \mathrm{E}+04 \\
9.74 \mathrm{E}+04 \\
2.12 \mathrm{E}+04 \\
1.09 \mathrm{E}+04 \\
2.12 \mathrm{E}+03 \\
1.37 \mathrm{E}+04 \\
1.35 \mathrm{E}+04 \\
5.23 \mathrm{E}+04 \\
1.52 \mathrm{E}+04 \\
4.52 \mathrm{E}+04 \\
4.22 \mathrm{E}+03 \\
3.96 \mathrm{E}+02 \\
2.67 \mathrm{E}+04 \\
3.39 \mathrm{E}+03 \\
1.71 \mathrm{E}+05 \\
8.48 \mathrm{E}+03 \\
1.48 \mathrm{E}+04 \\
1.79 \mathrm{E}+05 \\
1.85 \mathrm{E}+04 \\
1.96 \mathrm{E}+04 \\
1.14 \mathrm{E}+03 \\
3.75 \mathrm{E}+04 \\
1.54 \mathrm{E}+04 \\
2.23 \mathrm{E}+03 \\
1.21 \mathrm{E}+04 \\
1.05 \mathrm{E}+04 \\
1.93 \mathrm{E}+04 \\
1.88 \mathrm{E}+04 \\
2.16 \mathrm{E}+04\end{array}$ & $\begin{array}{l}\text { 2R93 } \\
1.03 \mathrm{E}+00 \\
1.30 \mathrm{E}+00 \\
7.26 \mathrm{E}+00 \\
1.39 \mathrm{E}+00 \\
6.15 \mathrm{E}-01 \\
1.93 \mathrm{E}+00 \\
8.16 \mathrm{E}+00 \\
1.68 \mathrm{E}+00 \\
1.01 \mathrm{E}-01 \\
2.08 \mathrm{E}+00 \\
1.94 \mathrm{E}+00 \\
7.51 \mathrm{E}+00 \\
2.27 \mathrm{E}+00 \\
4.36 \mathrm{E}+00 \\
3.55 \mathrm{E}-01 \\
6.89 \mathrm{E}-01 \\
9.30 \mathrm{E}-01 \\
1.79 \mathrm{E}+00 \\
1.04 \mathrm{E}+00 \\
9.33 \mathrm{E}-01 \\
8.72 \mathrm{E}-01 \\
2.74 \mathrm{E}+00 \\
5.54 \mathrm{E}+00 \\
1.50 \mathrm{E}+00 \\
2.29 \mathrm{E}-01 \\
2.95 \mathrm{E}-01 \\
2.51 \mathrm{E}+00 \\
7.26 \mathrm{E}-01 \\
1.28 \mathrm{E}-01 \\
5.87 \mathrm{E}-01 \\
6.80 \mathrm{E}-01 \\
1.50 \mathrm{E}+00 \\
1.03 \mathrm{E}+00 \\
1.91 \mathrm{E}-01 \\
8.81 \mathrm{E}-01 \\
1.17 \mathrm{E}+00 \\
8.14 \mathrm{E}-01 \\
3.01 \mathrm{E}+00 \\
7.67 \mathrm{E}-01 \\
3.99 \mathrm{E}-01 \\
6.47 \mathrm{E}-02 \\
4.22 \mathrm{E}-01 \\
4.14 \mathrm{E}-01 \\
1.62 \mathrm{E}+00 \\
4.69 \mathrm{E}-01 \\
1.61 \mathrm{E}+00 \\
1.30 \mathrm{E}-01 \\
1.20 \mathrm{E}-02 \\
9.46 \mathrm{E}-01 \\
1.18 \mathrm{E}-01 \\
5.26 \mathrm{E}+00 \\
2.57 \mathrm{E}-01 \\
5.10 \mathrm{E}-01 \\
5.27 \mathrm{E}+00 \\
6.39 \mathrm{E}-01 \\
7.42 \mathrm{E}-01 \\
4.29 \mathrm{E}-02 \\
1.30 \mathrm{E}+00 \\
5.41 \mathrm{E}-01 \\
8.40 \mathrm{E}-02 \\
4.50 \mathrm{E}-01 \\
3.57 \mathrm{E}-01 \\
7.13 \mathrm{E}-01 \\
7.07 \mathrm{E}-01 \\
8.09 \mathrm{E}-01\end{array}$ \\
\hline
\end{tabular}


HNF-SD-WM-TI-794, Rev, 0

ACTIVITY IN UNSEPARATED FUEL (Curies decayed to 1/1/94)

\begin{tabular}{|c|c|c|c|c|c|c|c|c|c|}
\hline $\begin{array}{r}\text { Listing } \$ \\
976 \\
977 \\
978 \\
979 \\
980 \\
981 \\
982 \\
983 \\
984 \\
985 \\
986 \\
987 \\
988 \\
989 \\
990 \\
991 \\
992 \\
993 \\
994 \\
995 \\
996 \\
997 \\
998 \\
999 \\
1000 \\
1001 \\
1002 \\
1003 \\
1004 \\
1005 \\
1006 \\
1007 \\
1008 \\
1009 \\
1010 \\
1011 \\
1012 \\
1013 \\
1014 \\
1015 \\
1016 \\
1017 \\
1018 \\
1019 \\
1020 \\
1021 \\
1022 \\
1023 \\
1024 \\
1025 \\
1026 \\
1027 \\
1028 \\
1029 \\
1030 \\
1031 \\
1032 \\
1033 \\
1034 \\
1035 \\
1036 \\
1037 \\
1038 \\
1039 \\
1040\end{array}$ & $\begin{array}{l}\text { NB93* } \\
6.94 \mathrm{E}-01 \\
8.69 \mathrm{E}-01 \\
4.91 \mathrm{E}+00 \\
9.34 \mathrm{E}-01 \\
4.04 \mathrm{E}-01 \\
1.27 \mathrm{E}+00 \\
5.36 \mathrm{E}+00 \\
1.10 \mathrm{E}+00 \\
6.62 \mathrm{E}-02 \\
1.40 \mathrm{E}+00 \\
1.31 \mathrm{E}+00 \\
4.87 \mathrm{E}+00 \\
1.47 \mathrm{E}+00 \\
2.82 \mathrm{E}+00 \\
2.32 \mathrm{E}-01 \\
4.55 \mathrm{E}-01 \\
6.24 \mathrm{E}-01 \\
1.19 \mathrm{E}+00 \\
7.00 \mathrm{E}-01 \\
6.17 \mathrm{E}-01 \\
5.84 \mathrm{E}-01 \\
1.77 \mathrm{E}+00 \\
3.57 \mathrm{E}+00 \\
9.65 \mathrm{E}-01 \\
1.47 \mathrm{E}-01 \\
1.90 \mathrm{E}-01 \\
1.59 \mathrm{E}+00 \\
4.58 \mathrm{E}-01 \\
8.01 \mathrm{E}-02 \\
3.68 \mathrm{E}-01 \\
4.25 \mathrm{E}-01 \\
9.40 \mathrm{E}-01 \\
6.16 \mathrm{E}-01 \\
1.20 \mathrm{E}-01 \\
5.51 \mathrm{E}-01 \\
6.73 \mathrm{E}-01 \\
4.80 \mathrm{E}-01 \\
1.32 \mathrm{E}+00 \\
4.49 \mathrm{E}-01 \\
2.37 \mathrm{E}-01 \\
2.81 \mathrm{E}-02 \\
1.84 \mathrm{E}-01 \\
1.80 \mathrm{E}-01 \\
7.08 \mathrm{E}-01 \\
2.04 \mathrm{E}-01 \\
9.22 \mathrm{E}-01 \\
5.67 \mathrm{E}-02 \\
5.19 \mathrm{E}-03 \\
5.37 \mathrm{E}-01 \\
6.59 \mathrm{E}-02 \\
2.27 \mathrm{E}+00 \\
1.10 \mathrm{E}-01 \\
2.77 \mathrm{E}-01 \\
2.02 \mathrm{E}+00 \\
3.50 \mathrm{E}-01 \\
4.61 \mathrm{E}-01 \\
2.63 \mathrm{E}-02 \\
7.22 \mathrm{E}-01 \\
3.02 \mathrm{E}-01 \\
5.17 \mathrm{E}-02 \\
2.74 \mathrm{E}-01 \\
1.91 \mathrm{E}-01 \\
4.30 \mathrm{E}-01 \\
4.34 \mathrm{E}-01 \\
4.94 \mathrm{E}-01\end{array}$ & $\begin{array}{l}7 C 99 \\
7.24 E+00 \\
9.15 E+00 \\
5.17 E+01 \\
9.87 E+00 \\
4.25 E+00 \\
1.33 E+01 \\
5.65 E+01 \\
1.17 E+01 \\
6.95 E-01 \\
1.46 E+01 \\
1.36 E+01 \\
5.26 E+01 \\
1.57 E+01 \\
3.03 E+01 \\
2.50 E+00 \\
4.77 E+00 \\
6.48 E+00 \\
1.24 E+01 \\
7.29 E+00 \\
6.50 E+00 \\
6.06 E+00 \\
1.92 E+01 \\
3.89 E+01 \\
1.05 E+01 \\
1.57 E+00 \\
2.04 E+00 \\
1.73 E+01 \\
5.00 E+00 \\
8.79 E-01 \\
4.04 E+00 \\
4.70 E+00 \\
1.03 E+01 \\
7.12 E+00 \\
1.32 E+00 \\
6.07 E+00 \\
8.04 E+00 \\
5.61 E+00 \\
2.08 E+01 \\
5.27 E+00 \\
2.75 E+00 \\
4.40 E-01 \\
2.90 E+00 \\
2.85 E+00 \\
1.12 E+01 \\
3.24 E+00 \\
1.11 E+01 \\
9.01 E-01 \\
8.18 E-02 \\
6.52 E+00 \\
8.14 E-01 \\
3.64 E+01 \\
1.75 E+00 \\
3.51 E+00 \\
3.63 E+01 \\
4.40 E+00 \\
5.10 E+00 \\
2.95 E-01 \\
8.99 E+00 \\
3.74 E+00 \\
5.77 E-01 \\
3.10 E+00 \\
2.45 E+00 \\
4.90 E+00 \\
4.87 E+00 \\
5.58 E+00\end{array}$ & $\begin{array}{l}\text { RU106 } \\
1.95 E-02 \\
2.96 E-02 \\
1.42 E-01 \\
3.02 E-02 \\
1.48 E-02 \\
4.39 E-02 \\
1.98 E-01 \\
4.27 E-02 \\
2.39 E-03 \\
4.26 E-02 \\
3.88 E-02 \\
4.33 E-01 \\
1.25 E-01 \\
2.63 E-01 \\
1.89 E-02 \\
2.13 E-02 \\
1.98 E-02 \\
4.42 E-02 \\
2.25 E-02 \\
2.95 E-02 \\
1.88 E-02 \\
1.83 E-01 \\
4.07 E-01 \\
1.07 E-01 \\
1.32 E-02 \\
1.83 E-02 \\
2.37 E-01 \\
7.53 E-02 \\
1.43 E-02 \\
7.21 E-02 \\
9.23 E-02 \\
1.64 E-01 \\
4.26 E-01 \\
2.14 E-02 \\
1.18 E-01 \\
9.97 E-01 \\
4.24 E-01 \\
1.90 E+02 \\
4.53 E-01 \\
1.81 E-01 \\
3.60 E+00 \\
2.59 E+01 \\
2.69 E+01 \\
1.03 E+02 \\
3.23 E+01 \\
1.59 E+00 \\
8.95 E+00 \\
7.10 E-01 \\
1.11 E+00 \\
1.95 E-01 \\
3.87 E+02 \\
1.62 E+01 \\
1.35 E+00 \\
1.22 E+03 \\
1.46 E+00 \\
1.12 E-01 \\
8.47 E-03 \\
2.54 E+00 \\
9.31 E-01 \\
1.57 E-02 \\
1.17 E-01 \\
1.24 E+00 \\
2.17 E-01 \\
1.41 E-01 \\
1.88 E-01\end{array}$ & $\begin{array}{l}\text { CD113* } \\
6.74 \mathrm{E}+00 \\
8.76 \mathrm{E}+00 \\
5.04 \mathrm{E}+01 \\
9.69 \mathrm{E}+00 \\
3.85 \mathrm{E}+00 \\
1.20 \mathrm{E}+01 \\
5.12 \mathrm{E}+01 \\
1.07 \mathrm{E}+01 \\
6.25 \mathrm{E}-01 \\
1.28 \mathrm{E}+01 \\
1.21 \mathrm{E}+01 \\
5.23 \mathrm{E}+01 \\
1.43 \mathrm{E}+01 \\
2.79 \mathrm{E}+01 \\
2.40 \mathrm{E}+00 \\
4.15 \mathrm{E}+00 \\
5.63 \mathrm{E}+00 \\
1.07 \mathrm{E}+01 \\
6.43 \mathrm{E}+00 \\
5.77 \mathrm{E}+00 \\
5.19 \mathrm{E}+00 \\
1.91 \mathrm{E}+01 \\
3.96 \mathrm{E}+01 \\
1.05 \mathrm{E}+01 \\
1.45 \mathrm{E}+00 \\
1.95 \mathrm{E}+00 \\
1.67 \mathrm{E}+01 \\
4.82 \mathrm{E}+00 \\
8.57 \mathrm{E}-01 \\
3.94 \mathrm{E}+00 \\
4.69 \mathrm{E}+00 \\
9.92 \mathrm{E}+00 \\
7.62 \mathrm{E}+00 \\
1.28 \mathrm{E}+00 \\
6.00 \mathrm{E}+00 \\
9.03 \mathrm{E}+00 \\
6.09 \mathrm{E}+00 \\
3.18 \mathrm{E}+01 \\
5.74 \mathrm{E}+00 \\
2.96 \mathrm{E}+00 \\
6.27 \mathrm{E}-01 \\
4.35 \mathrm{E}+00 \\
4.25 \mathrm{E}+00 \\
1.71 \mathrm{E}+01 \\
5.00 \mathrm{E}+00 \\
1.26 \mathrm{E}+01 \\
1.39 \mathrm{E}+00 \\
1.16 \mathrm{E}-01 \\
7.49 \mathrm{E}+00 \\
9.47 \mathrm{E}-01 \\
5.64 \mathrm{E}+01 \\
2.49 \mathrm{E}+00 \\
4.23 \mathrm{E}+00 \\
5.96 \mathrm{E}+01 \\
5.28 \mathrm{E}+00 \\
5.05 \mathrm{E}+00 \\
2.97 \mathrm{E}-01 \\
1.07 \mathrm{E}+01 \\
4.45 \mathrm{E}+00 \\
5.78 \mathrm{E}-01 \\
3.21 \mathrm{E}+00 \\
3.02 \mathrm{E}+00 \\
5.10 \mathrm{E}+00 \\
4.93 \mathrm{E}+00 \\
5.74 \mathrm{E}+00\end{array}$ & $\begin{array}{l}\text { SB125 } \\
2.27 E+01 \\
3.07 E+01 \\
1.63 E+02 \\
3.24 E+01 \\
1.59 E+01 \\
4.89 E+01 \\
2.12 E+02 \\
4.39 E+01 \\
2.62 E+00 \\
4.45 E+01 \\
4.11 E+01 \\
2.48 E+02 \\
6.93 E+01 \\
1.38 E+02 \\
1.08 E+01 \\
1.70 E+01 \\
2.01 E+01 \\
4.08 E+01 \\
2.27 E+01 \\
2.33 E+01 \\
1.89 E+01 \\
9.56 E+01 \\
2.00 E+02 \\
5.35 E+01 \\
7.55 E+00 \\
9.98 E+00 \\
9.89 E+01 \\
2.96 E+01 \\
5.35 E+00 \\
2.54 E+01 \\
3.06 E+01 \\
6.22 E+01 \\
6.96 E+01 \\
8.01 E+00 \\
3.94 E+01 \\
1.03 E+02 \\
5.97 E+01 \\
1.27 E+03 \\
5.88 E+01 \\
2.79 E+01 \\
2.60 E+01 \\
1.76 E+02 \\
1.76 E+02 \\
6.81 E+02 \\
2.04 E+02 \\
1.49 E+02 \\
5.65 E+01 \\
4.93 E+00 \\
9.32 E+01 \\
1.32 E+01 \\
2.34 E+03 \\
1.08 E+02 \\
6.75 E+01 \\
3.55 E+03 \\
8.03 E+01 \\
3.46 E+01 \\
2.21 E+00 \\
1.55 E+02 \\
6.13 E+01 \\
4.24 E+00 \\
2.57 E+01 \\
5.22 E+01 \\
4.30 E+01 \\
3.65 E+01 \\
4.42 E+01\end{array}$ & $\begin{array}{l}\text { SN126 } \\
3.62 E-01 \\
4.64 E-01 \\
2.68 E+00 \\
5.13 E-01 \\
1.93 E-01 \\
6.07 E-01 \\
2.57 E+00 \\
5.36 E-01 \\
3.13 E-02 \\
6.99 E-01 \\
6.60 E-01 \\
2.60 E+00 \\
7.30 E-01 \\
1.42 E+00 \\
1.22 E-01 \\
2.21 E-01 \\
3.07 E-01 \\
5.81 E-01 \\
3.50 E-01 \\
3.07 E-01 \\
2.83 E-01 \\
9.42 E-01 \\
1.93 E+00 \\
5.12 E-01 \\
7.23 E-02 \\
9.68 E-02 \\
8.05 E-01 \\
2.31 E-01 \\
4.08 E-02 \\
1.87 E-01 \\
2.20 E-01 \\
4.74 E-01 \\
3.32 E-01 \\
6.11 E-02 \\
2.82 E-01 \\
3.74 E-01 \\
2.61 E-01 \\
9.76 E-01 \\
2.44 E-01 \\
1.28 E-01 \\
1.95 E-02 \\
1.34 E-01 \\
1.31 E-01 \\
5.25 E-01 \\
1.53 E-01 \\
5.76 E-01 \\
4.25 E-02 \\
3.61 E-03 \\
3.03 E-01 \\
3.75 E-02 \\
1.71 E+00 \\
7.69 E-02 \\
1.62 E-01 \\
1.68 E+00 \\
2.04 E-01 \\
2.36 E-01 \\
1.36 E-02 \\
4.19 E-01 \\
1.75 E-01 \\
2.66 E-02 \\
1.44 E-01 \\
1.133 E-01 \\
2.27 E-01 \\
2.26 E-01 \\
2.60 E-01\end{array}$ & $\begin{array}{l}1129 \\
1.51 E-02 \\
1.93 E-02 \\
1.11 E-01 \\
2.12 E-02 \\
7.96 E-03 \\
2.50 E-02 \\
1.06 E-01 \\
2.21 E-02 \\
1.29 E-03 \\
3.02 E-02 \\
2.84 E-02 \\
1.09 E-01 \\
3.18 E-02 \\
6.16 E-02 \\
5.25 E-03 \\
9.64 E-03 \\
1.33 E-02 \\
2.53 E-02 \\
1.51 E-02 \\
1.33 E-02 \\
1.23 E-02 \\
3.95 E-02 \\
8.09 E-02 \\
2.15 E-02 \\
3.09 E-03 \\
4.10 E-03 \\
3.43 E-02 \\
9.84 E-03 \\
1.74 E-03 \\
7.96 E-03 \\
9.36 E-03 \\
2.02 E-02 \\
1.41 E-02 \\
2.60 E-03 \\
1.20 E-02 \\
1.59 E-02 \\
1.11 E-02 \\
4.15 E-02 \\
1.04 E-02 \\
5.45 E-03 \\
8.42 E-04 \\
5.71 E-03 \\
5.58 E-03 \\
2.23 E-02 \\
6.48 E-03 \\
2.20 E-02 \\
1.80 E-03 \\
1.56 E-04 \\
1.29 E-02 \\
1.60 E-03 \\
7.27 E-02 \\
3.32 E-03 \\
6.91 E-03 \\
7.14 E-02 \\
8.70 E-03 \\
1.01 E-02 \\
5.81 E-04 \\
1.78 E-02 \\
7.45 E-03 \\
1.13 E-03 \\
6.15 E-03 \\
4.84 E-03 \\
9.66 E-03 \\
9.62 E-03 \\
1.11 E-02\end{array}$ & 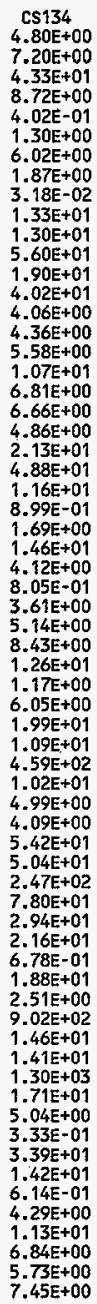 & $\begin{array}{l}\text { CS137 } \\
3.03 E+04 \\
3.85 E+04 \\
2.16 E+05 \\
4.15 E+04 \\
1.80 E+04 \\
5.63 E+04 \\
2.39 E+05 \\
4.93 E+04 \\
2.94 E+03 \\
6.13 E+04 \\
5.73 E+04 \\
2.28 E+05 \\
6.85 E+04 \\
1.32 E+05 \\
1.09 E+04 \\
2.04 E+04 \\
2.73 E+04 \\
5.27 E+04 \\
3.08 E+04 \\
2.78 E+04 \\
2.55 E+04 \\
8.36 E+04 \\
1.70 E+05 \\
4.58 E+04 \\
6.83 E+03 \\
8.88 E+03 \\
7.64 E+04 \\
2.21 E+04 \\
3.90 E+03 \\
1.80 E+04 \\
2.10 E+04 \\
4.56 E+04 \\
3.30 E+04 \\
5.85 E+03 \\
2.71 E+04 \\
3.82 E+04 \\
2.62 E+04 \\
1.14 E+05 \\
2.48 E+04 \\
1.28 E+04 \\
2.41 E+03 \\
1.59 E+04 \\
1.56 E+04 \\
6.14 E+04 \\
1.79 E+04 \\
5.30 E+04 \\
4.96 E+03 \\
4.48 E+02 \\
3.13 E+04 \\
3.95 E+03 \\
2.01 E+05 \\
9.59 E+03 \\
1.73 E+04 \\
2.08 E+05 \\
2.16 E+04 \\
2.29 E+04 \\
1.33 E+03\end{array}$ \\
\hline
\end{tabular}


HWF-SD-WM-TI-794, Rev, 0

ACTIVITY IN UNSEPARATED FUEL (Curies decayed to 1/1/94)

\begin{tabular}{|c|c|c|c|c|c|c|c|c|c|}
\hline $\begin{array}{r}\text { isting \#] } \\
976 \\
977 \\
978 \\
979 \\
980 \\
981 \\
982 \\
983 \\
984 \\
985 \\
986 \\
987 \\
988 \\
989 \\
990 \\
991 \\
992 \\
993 \\
994 \\
995 \\
996 \\
997 \\
998 \\
999 \\
1000 \\
1001 \\
1002 \\
1003 \\
1004 \\
1005 \\
1006 \\
1007 \\
1008 \\
1009 \\
1010 \\
1011 \\
1012 \\
1013 \\
1014 \\
1015 \\
1016 \\
1017 \\
1018 \\
1019 \\
1020 \\
1021 \\
1022 \\
1023 \\
1024 \\
1025 \\
1026 \\
1027 \\
1028 \\
1029 \\
1030 \\
1031 \\
1032 \\
1033 \\
1034 \\
1035 \\
1036 \\
1037 \\
1038 \\
1039 \\
1040\end{array}$ & $\begin{array}{l}B A 137 \\
2.86 \mathrm{E}+04 \\
3.64 \mathrm{E}+04 \\
2.05 \mathrm{E}+05 \\
3.93 \mathrm{E}+04 \\
3.70 \mathrm{E}+04 \\
5.32 \mathrm{E}+04 \\
2.26 \mathrm{E}+05 \\
4.66 \mathrm{E}+04 \\
2.78 \mathrm{E}+03 \\
5.80 \mathrm{E}+04 \\
5.42 \mathrm{E}+04 \\
2.16 \mathrm{E}+05 \\
6.48 \mathrm{E}+04 \\
1.25 \mathrm{E}+05 \\
1.03 \mathrm{E}+04 \\
1.93 \mathrm{E}+04 \\
2.59 \mathrm{E}+04 \\
4.98 \mathrm{E}+04 \\
2.91 \mathrm{E}+04 \\
2.63 \mathrm{E}+04 \\
2.42 \mathrm{E}+04 \\
7.91 \mathrm{E}+04 \\
1.61 \mathrm{E}+05 \\
4.33 \mathrm{E}+04 \\
6.47 \mathrm{E}+03 \\
8.40 \mathrm{E}+03 \\
7.22 \mathrm{E}+04 \\
2.09 \mathrm{E}+04 \\
3.69 \mathrm{E}+03 \\
1.70 \mathrm{E}+04 \\
1.99 \mathrm{E}+04 \\
4.31 \mathrm{E}+04 \\
3.13 \mathrm{E}+04 \\
5.53 \mathrm{E}+03 \\
2.57 \mathrm{E}+04 \\
3.61 \mathrm{E}+04 \\
2.48 \mathrm{E}+04 \\
1.08 \mathrm{E}+05 \\
2.34 \mathrm{E}+04 \\
1.21 \mathrm{E}+04 \\
2.28 \mathrm{E}+03 \\
1.51 \mathrm{E}+04 \\
1.48 \mathrm{E}+04 \\
5.81 \mathrm{E}+04 \\
1.69 \mathrm{E}+04 \\
5.01 \mathrm{E}+04 \\
4.69 \mathrm{E}+03 \\
4.24 \mathrm{E}+02 \\
2.96 \mathrm{E}+04 \\
3.74 \mathrm{E}+03 \\
1.90 \mathrm{E}+05 \\
9.07 \mathrm{E}+03 \\
1.64 \mathrm{E}+04 \\
1.97 \mathrm{E}+05 \\
2.05 \mathrm{E}+04 \\
2.16 \mathrm{E}+04 \\
1.26 \mathrm{E}+03 \\
4.16 \mathrm{E}+04 \\
1.72 \mathrm{E}+04 \\
2.47 \mathrm{E}+03 \\
1.34 \mathrm{E}+04 \\
1.16 \mathrm{E}+04 \\
2.13 \mathrm{E}+04 \\
2.08 \mathrm{E}+04 \\
2.40 \mathrm{E}+04\end{array}$ & $\begin{array}{l}\text { SM151 } \\
5.78 E+02 \\
6.87 E+02 \\
3.53 E+03 \\
6.75 E+02 \\
5.75 E+02 \\
1.80 E+03 \\
7.58 E+03 \\
1.51 E+03 \\
9.72 E+01 \\
9.46 E+02 \\
8.47 E+02 \\
4.46 E+03 \\
1.20 E+03 \\
2.22 E+03 \\
1.51 E+02 \\
3.61 E+02 \\
4.42 E+02 \\
9.02 E+02 \\
4.74 E+02 \\
4.50 E+02 \\
4.39 E+02 \\
1.66 E+03 \\
3.21 E+03 \\
9.23 E+02 \\
1.69 E+02 \\
1.99 E+02 \\
1.80 E+03 \\
5.30 E+02 \\
9.16 E+01 \\
4.29 E+02 \\
4.78 E+02 \\
1.10 E+03 \\
7.49 E+02 \\
1.38 E+02 \\
6.34 E+02 \\
8.57 E+02 \\
5.95 E+02 \\
2.27 E+03 \\
5.70 E+02 \\
2.91 E+02 \\
5.59 E+01 \\
3.30 E+02 \\
3.31 E+02 \\
1.22 E+03 \\
3.49 E+02 \\
1.19 E+03 \\
9.70 E+01 \\
1.05 E+01 \\
6.98 E+02 \\
8.97 E+01 \\
3.92 E+03 \\
2.25 E+02 \\
3.86 E+02 \\
4.19 E+03 \\
4.78 E+02 \\
5.43 E+02 \\
3.15 E+01 \\
9.62 E+02 \\
3.92 E+02 \\
6.21 E+01 \\
3.27 E+02 \\
2.71 E+02 \\
5.26 E+02 \\
5.15 E+02 \\
5.82 E+02\end{array}$ & $\begin{array}{l}\text { EU152 } \\
1.25 \mathrm{E}+00 \\
1.78 \mathrm{E}+00 \\
1.14 \mathrm{E}+01 \\
2.19 \mathrm{E}+00 \\
6.26 \mathrm{E}-02 \\
2.12 \mathrm{E}-01 \\
9.84 \mathrm{E}-01 \\
3.42 \mathrm{E}=01 \\
2.60 \mathrm{E}-03 \\
3.07 \mathrm{E}+00 \\
3.02 \mathrm{E}+00 \\
8.93 \mathrm{E}+00 \\
2.88 \mathrm{E}+00 \\
5.94 \mathrm{E}+00 \\
6.20 \mathrm{E}-01 \\
8.37 \mathrm{E}-01 \\
1.29 \mathrm{E}+00 \\
2.28 \mathrm{E}+00 \\
1.55 \mathrm{E}+00 \\
1.31 \mathrm{E}+00 \\
1.10 \mathrm{E}+00 \\
3.16 \mathrm{E}+00 \\
7.14 \mathrm{E}+00 \\
1.66 \mathrm{E}+00 \\
1.10 \mathrm{E}-01 \\
2.34 \mathrm{E}-01 \\
1.56 \mathrm{E}+00 \\
4.11 \mathrm{E}-01 \\
8.04 \mathrm{E}-02 \\
3.37 \mathrm{E}-01 \\
4.94 \mathrm{E}-01 \\
8.09 \mathrm{E}-01 \\
7.41 \mathrm{E}-01 \\
1.16 \mathrm{E}-01 \\
5.63 \mathrm{E}-01 \\
8.56 \mathrm{E}-01 \\
5.75 \mathrm{E}-01 \\
3.45 \mathrm{E}+00 \\
4.94 \mathrm{E}-01 \\
2.80 \mathrm{E}-01 \\
1.78 \mathrm{E}-02 \\
3.83 \mathrm{E}-01 \\
3.34 \mathrm{E}-01 \\
1.85 \mathrm{E}+00 \\
5.76 \mathrm{E}-01 \\
1.19 \mathrm{E}+00 \\
1.60 \mathrm{E}-01 \\
2.30 \mathrm{E}-03 \\
7.10 \mathrm{E}-01 \\
7.83 \mathrm{E}-02 \\
6.48 \mathrm{E}+00 \\
4.59 \mathrm{E}-02 \\
3.67 \mathrm{E}-01 \\
5.29 \mathrm{E}+00 \\
4.83 \mathrm{E}-01 \\
4.32 \mathrm{E}-01 \\
2.55 \mathrm{E}-00 \\
1.05 \mathrm{E}+00 \\
4.75 \mathrm{E}-01 \\
4.73 \mathrm{E}-02 \\
3.03 \mathrm{E}-01 \\
2.62 \mathrm{E}-01 \\
4.37 \mathrm{E}-01 \\
4.46 \mathrm{E}-01 \\
5.56 \mathrm{E}-01\end{array}$ & $\begin{array}{l}\text { EU154 } \\
1.13 E+02 \\
1.61 E+02 \\
1.07 E+03 \\
2.07 E+02 \\
9.63 E+00 \\
3.13 E+01 \\
1.40 E+02 \\
3.97 E+01 \\
9.79 E-01 \\
2.96 E+02 \\
2.99 E+02 \\
8.62 E+02 \\
2.80 E+02 \\
5.77 E+02 \\
6.58 E+01 \\
7.94 E+01 \\
1.20 E+02 \\
2.13 E+02 \\
1.49 E+02 \\
1.23 E+02 \\
1.02 E+02 \\
3.08 E+02 \\
6.92 E+02 \\
1.63 E+02 \\
1.23 E+01 \\
2.39 E+01 \\
1.70 E+02 \\
4.59 E+01 \\
8.82 E+00 \\
3.78 E+01 \\
5.34 E+01 \\
9.14 E+01 \\
8.55 E+01 \\
1.28 E+01 \\
6.22 E+01 \\
1.02 E+02 \\
6.74 E+01 \\
4.86 E+02 \\
5.94 E+01 \\
3.26 E+01 \\
3.78 E+00 \\
5.63 E+01 \\
5.05 E+01 \\
2.61 E+02 \\
8.06 E+01 \\
1.44 E+02 \\
2.24 E+01 \\
5.86 E-01 \\
8.62 E+01 \\
9.94 E+00 \\
9.10 E+02 \\
1.22 E+01 \\
4.71 E+01 \\
8.22 E+02 \\
6.08 E+01 \\
4.89 E+01 \\
2.92 E+00 \\
1.29 E+02 \\
5.733 E+01 \\
5.46 E+00 \\
3.44 E+01 \\
3.40 E+01 \\
5.11 E+01 \\
5.05 E+01 \\
6.26 E+01\end{array}$ & $\begin{array}{l}E U 155 \\
3.37 \mathrm{E}+01 \\
4.14 \mathrm{E}+01 \\
2.11 \mathrm{E}+02 \\
4.12 \mathrm{E}+01 \\
4.34 \mathrm{E}+01 \\
1.34 \mathrm{E}+02 \\
5.69 \mathrm{E}+02 \\
1.11 \mathrm{E}+02 \\
7.52 \mathrm{E}+00 \\
5.64 \mathrm{E}+01 \\
5.13 \mathrm{E}+01 \\
3.23 \mathrm{E}+02 \\
8.42 \mathrm{E}+01 \\
1.59 \mathrm{E}+02 \\
1.13 \mathrm{E}+01 \\
2.27 \mathrm{E}+01 \\
2.60 \mathrm{E}+01 \\
5.44 \mathrm{E}+01 \\
2.85 \mathrm{E}+01 \\
2.85 \mathrm{E}+01 \\
2.58 \mathrm{E}+01 \\
1.24 \mathrm{E}+02 \\
2.43 \mathrm{E}+02 \\
6.98 \mathrm{E}+01 \\
1.25 \mathrm{E}+01 \\
1.48 \mathrm{E}+01 \\
1.46 \mathrm{E}+02 \\
4.38 \mathrm{E}+01 \\
7.68 \mathrm{E}+00 \\
3.67 \mathrm{E}+01 \\
4.15 \mathrm{E}+01 \\
9.21 \mathrm{E}+01 \\
8.05 \mathrm{E}+01 \\
1.16 \mathrm{E}+01 \\
5.50 \mathrm{E}+01 \\
1.06 \mathrm{E}+02 \\
6.69 \mathrm{E}+01 \\
6.41 \mathrm{E}+02 \\
6.59 \mathrm{E}+01 \\
3.20 \mathrm{E}+01 \\
1.57 \mathrm{E}+01 \\
9.32 \mathrm{E}+01 \\
9.44 \mathrm{E}+01 \\
3.44 \mathrm{E}+02 \\
1.00 \mathrm{E}+02 \\
1.51 \mathrm{E}+02 \\
2.78 \mathrm{E}+01 \\
3.00 \mathrm{E}+00 \\
9.18 \mathrm{E}+01 \\
1.26 \mathrm{E}+01 \\
1.14 \mathrm{E}+03 \\
6.50 \mathrm{E}+01 \\
5.94 \mathrm{E}+01 \\
1.53 \mathrm{E}+03 \\
7.16 \mathrm{E}+01 \\
4.83 \mathrm{E}+01 \\
2.95 \mathrm{E}+00 \\
1.39 \mathrm{E}+02 \\
5.53 \mathrm{E}+01 \\
5.76 \mathrm{E}+00 \\
3.22 \mathrm{E}+01 \\
4.40 \mathrm{E}+01 \\
5.36 \mathrm{E}+01 \\
4.82 \mathrm{E}+01 \\
5.61 \mathrm{E}+01\end{array}$ & $\begin{array}{l}\text { RA226 } \\
4.62 E-06 \\
5.25 E-06 \\
2.59 \mathrm{E}-05 \\
4.87 \mathrm{E}-06 \\
1.02 \mathrm{E}-05 \\
3.11 \mathrm{E}-05 \\
1.25 \mathrm{E}-04 \\
1.91 \mathrm{E}-05 \\
2.55 \mathrm{E}-06 \\
6.07 \mathrm{E}-06 \\
5.31 \mathrm{E}-06 \\
3.15 \mathrm{E}-05 \\
7.19 \mathrm{E}-06 \\
1.30 \mathrm{E}-05 \\
8.17 \mathrm{E}-07 \\
2.34 \mathrm{E}-06 \\
2.92 \mathrm{E}-06 \\
5.97 \mathrm{E}-06 \\
3.02 \mathrm{E}-06 \\
2.85 \mathrm{E}-06 \\
2.96 \mathrm{E}-06 \\
1.16 \mathrm{E}-05 \\
2.15 \mathrm{E}-05 \\
6.51 \mathrm{E}-06 \\
1.75 \mathrm{E}-06 \\
1.62 \mathrm{E}-06 \\
1.57 \mathrm{E}-05 \\
4.77 \mathrm{E}-06 \\
7.77 \mathrm{E}-07 \\
3.77 \mathrm{E}-06 \\
3.73 \mathrm{E}-06 \\
1.01 \mathrm{E}-05 \\
5.13 \mathrm{E}-06 \\
1.20 \mathrm{E}-06 \\
5.25 \mathrm{E}-06 \\
5.29 \mathrm{E}-06 \\
3.98 \mathrm{E}-06 \\
5.53 \mathrm{E}-06 \\
3.92 \mathrm{E}-06 \\
2.00 \mathrm{E}-06 \\
2.48 \mathrm{E}-07 \\
8.93 \mathrm{E}-07 \\
9.31 \mathrm{E}-07 \\
2.97 \mathrm{E}-06 \\
8.10 \mathrm{E}-07 \\
7.14 \mathrm{E}-06 \\
2.25 \mathrm{E}-07 \\
5.12 \mathrm{E}-08 \\
4.08 \mathrm{E}-06 \\
5.30 \mathrm{E}-07 \\
8.95 \mathrm{E}-06 \\
1.10 \mathrm{E}-06 \\
2.04 \mathrm{E}-06 \\
7.99 \mathrm{E}-06 \\
2.53 \mathrm{E}-06 \\
4.63 \mathrm{E}-06 \\
2.58 \mathrm{E}-07 \\
5.07 \mathrm{E}-06 \\
2.02 \mathrm{E}-06 \\
2.24 \mathrm{~s}-07 \mathrm{E}-06 \\
1.36 \mathrm{E}-06\end{array}$ & $\begin{array}{l}\text { RA228 } \\
2.96 \mathrm{E}-10 \\
3.50 \mathrm{E}-10 \\
1.83 \mathrm{E}-09 \\
3.46 \mathrm{E}-10 \\
5.81 \mathrm{E}-11 \\
1.83 \mathrm{E}-10 \\
7.69 \mathrm{E}-10 \\
1.57 \mathrm{E}-10 \\
9.56 \mathrm{E}-12 \\
4.82 \mathrm{E}-10 \\
4.36 \mathrm{E}-10 \\
2.03 \mathrm{E}-10 \\
5.33 \mathrm{E}-10 \\
9.90 \mathrm{E}-10 \\
7.13 \mathrm{E}-11 \\
1.70 \mathrm{E}-10 \\
2.23 \mathrm{E}-10 \\
4.41 \mathrm{E}-10 \\
2.40 \mathrm{E}-10 \\
2.18 \mathrm{E}-10 \\
2.17 \mathrm{E}-10 \\
7.45 \mathrm{E}-10 \\
1.43 \mathrm{E}-09 \\
4.13 \mathrm{E}-10 \\
9.16 \mathrm{E}-11 \\
9.43 \mathrm{E}-11 \\
8.69 \mathrm{E}-10 \\
2.60 \mathrm{E}-10 \\
4.33 \mathrm{E}-11 \\
2.06 \mathrm{E}-10 \\
2.15 \mathrm{E}-10 \\
5.43 \mathrm{E}-10 \\
3.01 \mathrm{E}-10 \\
6.63 \mathrm{E}-11 \\
2.94 \mathrm{E}-10 \\
3.16 \mathrm{E}-10 \\
2.34 \mathrm{E}-10 \\
3.99 \mathrm{E}-10 \\
2.27 \mathrm{E}-10 \\
1.17 \mathrm{E}-10 \\
1.47 \mathrm{E}-11 \\
6.16 \mathrm{E}-11 \\
6.29 \mathrm{E}-11 \\
2.14 \mathrm{E}-10 \\
5.95 \mathrm{E}-11 \\
4.29 \mathrm{E}-10 \\
1.66 \mathrm{E}-11 \\
2.97 \mathrm{E}-12 \\
2.47 \mathrm{E}-10 \\
3.15 \mathrm{E}-11 \\
6.59 \mathrm{E}-10 \\
6.35 \mathrm{E}-11 \\
1.25 \mathrm{E}-10 \\
5.75 \mathrm{E}-10 \\
1.56 \mathrm{E}-10 \\
2.55 \mathrm{E}-10 \\
1.43 \mathrm{E}-11 \\
3.15 \mathrm{E}-10 \\
1.27 \mathrm{E}-10 \\
2.89 \mathrm{E}-11 \\
1.40 \mathrm{E}-10 \\
2.28 \mathrm{E}-11 \\
2.30 \mathrm{E}-10 \\
2.51 \mathrm{E}-10\end{array}$ & $\begin{array}{l}\text { AC227 } \\
2.26 \mathrm{E}-05 \\
2.55 \mathrm{E}-05 \\
1.24 \mathrm{E}-04 \\
2.34 \mathrm{E}-05 \\
6.27 \mathrm{E}-05 \\
1.90 \mathrm{E}-04 \\
7.64 \mathrm{E}-04 \\
1.16 \mathrm{E}-04 \\
1.58 \mathrm{E}-05 \\
3.46 \mathrm{E}-05 \\
3.00 \mathrm{E}-05 \\
1.58 \mathrm{E}-04 \\
4.23 \mathrm{E}-05 \\
7.66 \mathrm{E}-05 \\
4.67 \mathrm{E}-06 \\
1.37 \mathrm{E}-05 \\
1.68 \mathrm{E}-05 \\
3.47 \mathrm{E}-05 \\
1.72 \mathrm{E}-05 \\
1.66 \mathrm{E}-05 \\
1.71 \mathrm{E}-05 \\
5.84 \mathrm{E}-05 \\
1.08 \mathrm{E}-04 \\
3.28 \mathrm{E}-05 \\
9.22 \mathrm{E}-06 \\
8.34 \mathrm{E}-06 \\
8.21 \mathrm{E}-05 \\
2.51 \mathrm{E}-05 \\
4.08 \mathrm{E}-06 \\
1.99 \mathrm{E}-05 \\
1.95 \mathrm{E}-05 \\
5.31 \mathrm{E}-05 \\
2.74 \mathrm{E}-05 \\
6.31 \mathrm{E}-06 \\
2.76 \mathrm{E}-05 \\
2.86 \mathrm{E}-05 \\
2.14 \mathrm{E}-05 \\
3.18 \mathrm{E}-05 \\
2.12 \mathrm{E}-05 \\
1.07 \mathrm{E}-05 \\
1.48 \mathrm{E}-06 \\
5.18 \mathrm{E}-06 \\
5.43 \mathrm{E}-06 \\
1.71 \mathrm{E}-05 \\
4.66 \mathrm{E}-06 \\
3.87 \mathrm{E}-05 \\
1.30 \mathrm{E}-06 \\
3.07 \mathrm{E}-07 \\
2.22 \mathrm{E}-05 \\
2.91 \mathrm{E}-06 \\
5.15 \mathrm{E}-05 \\
6.59 \mathrm{E}-06 \\
1.13 \mathrm{E}-05 \\
4.70 \mathrm{E}-05 \\
1.39 \mathrm{E}-05 \\
2.45 \mathrm{E}-05 \\
1.37 \mathrm{E}-06 \\
2.77 \mathrm{E}-05 \\
1.10 \mathrm{E}-05 \\
2.79 \mathrm{E}-06 \\
1.30 \mathrm{E}-05 \\
7.56 \mathrm{E}-06 \\
2.15 \mathrm{E}-05 \\
2.17 \mathrm{E}-05 \\
2.31 \mathrm{E}-05\end{array}$ & $\begin{array}{c}\text { TH229 } \\
3.53 \mathrm{E}-08 \\
4.45 \mathrm{E}-08 \\
2.60 \mathrm{E}-07 \\
4.93 \mathrm{E}-08 \\
1.16 \mathrm{E}-08 \\
3.67 \mathrm{E}-08 \\
1.55 \mathrm{E}-07 \\
3.19 \mathrm{E}-08 \\
1.90 \mathrm{E}-09 \\
6.26 \mathrm{E}-08 \\
5.93 \mathrm{E}-08 \\
2.31 \mathrm{E}-07 \\
6.00 \mathrm{E}-08 \\
1.16 \mathrm{E}-07 \\
1.02 \mathrm{E}-08 \\
1.91 \mathrm{E}-08 \\
2.74 \mathrm{E}-08 \\
5.11 \mathrm{E}-08 \\
3.12 \mathrm{E}-08 \\
2.65 \mathrm{E}-08 \\
2.52 \mathrm{E}-08 \\
8.29 \mathrm{E}-08 \\
1.69 \mathrm{E}-07 \\
4.48 \mathrm{E}-08 \\
6.49 \mathrm{E}-09 \\
8.60 \mathrm{E}-09 \\
6.91 \mathrm{E}-08 \\
1.97 \mathrm{E}-08\end{array}$ \\
\hline
\end{tabular}


HNF-SD-WM-TI-794, ReV. 0

ACTIVITY IN UNSEPARATEO FUEL (Curies decayed to $1 / 1 / 94$ )

\begin{tabular}{|c|c|c|c|c|c|c|c|c|c|}
\hline $\begin{array}{r}\text { isting } \# \text { ] } \\
976 \\
977 \\
978 \\
979 \\
980 \\
981 \\
982 \\
983 \\
984 \\
985 \\
986 \\
987 \\
988 \\
989 \\
990 \\
991 \\
992 \\
993 \\
994 \\
995 \\
996 \\
997 \\
998 \\
999 \\
1000 \\
1001 \\
1002 \\
1003 \\
1004 \\
1005 \\
1006 \\
1007 \\
1008 \\
1009 \\
1010 \\
1011 \\
1012 \\
1013 \\
1014 \\
1015 \\
1016 \\
1017 \\
1018 \\
1019 \\
1020 \\
1021 \\
1022 \\
1023 \\
1024 \\
1025 \\
1026 \\
1027 \\
1028 \\
1029 \\
1030 \\
1031 \\
1032 \\
1033 \\
1034 \\
1035 \\
1036 \\
1037 \\
1038 \\
1039 \\
1040\end{array}$ & $\begin{array}{l}\text { TH232 } \\
4.66 \mathrm{E}-10 \\
5.52 \mathrm{E}-10 \\
2.87 \mathrm{E}-09 \\
5.45 \mathrm{E}-10 \\
9.38 \mathrm{E}-11 \\
2.95 \mathrm{E}-10 \\
1.24 \mathrm{E}-09 \\
2.54 \mathrm{E}-10 \\
1.55 \mathrm{E}-11 \\
7.60 \mathrm{E}-10 \\
6.86 \mathrm{E}-10 \\
3.31 \mathrm{E}-09 \\
8.68 \mathrm{E}-10 \\
1.61 \mathrm{E}-09 \\
1.15 \mathrm{E}-10 \\
2.73 \mathrm{E}-10 \\
3.53 \mathrm{E}-10 \\
7.01 \mathrm{E}-10 \\
3.79 \mathrm{E}-10 \\
3.49 \mathrm{E}-10 \\
3.44 \mathrm{E}-10 \\
1.22 \mathrm{E}-09 \\
2.34 \mathrm{E}-09 \\
6.77 \mathrm{E}-10 \\
1.50 \mathrm{E}-10 \\
1.55 \mathrm{E}-10 \\
1.45 \mathrm{E}-09 \\
4.34 \mathrm{E}-10 \\
7.25 \mathrm{E}-11 \\
3.47 \mathrm{E}-10 \\
3.61 \mathrm{E}-10 \\
9.10 \mathrm{E}-10 \\
5.28 \mathrm{E}-10 \\
1.11 \mathrm{E}-10 \\
4.96 \mathrm{E}-10 \\
5.72 \mathrm{E}-10 \\
4.15 \mathrm{E}-10 \\
9.24 \mathrm{E}-10 \\
4.04 \mathrm{E}-10 \\
2.06 \mathrm{E}-10 \\
3.42 \mathrm{E}-11 \\
1.43 \mathrm{E}-10 \\
1.47 \mathrm{E}-10 \\
4.96 \mathrm{E}-10 \\
1.39 \mathrm{E}-10 \\
7.81 \mathrm{E}-10 \\
3.85 \mathrm{E}-11 \\
6.97 \mathrm{E}-12 \\
4.53 \mathrm{E}-10 \\
5.88 \mathrm{E}-11 \\
1.54 \mathrm{E}-09 \\
1.50 \mathrm{E}-10 \\
2.38 \mathrm{E}-10 \\
1.50 \mathrm{E}-09 \\
2.95 \mathrm{E}-10 \\
4.33 \mathrm{E}-10 \\
2.45 \mathrm{E}-11 \\
5.90 \mathrm{E}-10 \\
2.37 \mathrm{E}-10 \\
4.93 \mathrm{E}-11 \\
2.42 \mathrm{E}-10 \\
1.63 \mathrm{E}-10 \\
3.95 \mathrm{E}-10 \\
3.94 \mathrm{E}-10 \\
4.31 \mathrm{E}-10\end{array}$ & $\begin{array}{l}\text { PA231 } \\
7.28 \mathrm{E}-05 \\
8.25 \mathrm{E}-05 \\
3.93 \mathrm{E}-04 \\
7.46 \mathrm{E}-05 \\
2.15 \mathrm{E}-04 \\
6.50 \mathrm{E}-04 \\
2.62 \mathrm{E}-03 \\
3.95 \mathrm{E}-04 \\
5.45 \mathrm{E}-05 \\
1.11 \mathrm{E}-04 \\
9.58 \mathrm{E}-05 \\
5.38 \mathrm{E}-04 \\
1.45 \mathrm{E}-04 \\
2.62 \mathrm{E}-04 \\
1.55 \mathrm{E}-05 \\
4.55 \mathrm{E}-05 \\
5.44 \mathrm{E}-05 \\
1.14 \mathrm{E}-04 \\
5.55 \mathrm{E}-05 \\
5.47 \mathrm{E}-05 \\
5.58 \mathrm{E}-05 \\
2.01 \mathrm{E}-04 \\
3.71 \mathrm{E}-04 \\
1.14 \mathrm{E}-04 \\
3.24 \mathrm{E}-05 \\
2.91 \mathrm{E}-05 \\
2.95 \mathrm{E}-04 \\
9.08 \mathrm{E}-05 \\
1.48 \mathrm{E}-05 \\
7.26 \mathrm{E}-05 \\
7.11 \mathrm{E}-05 \\
1.93 \mathrm{E}-04 \\
1.07 \mathrm{E}-04 \\
2.29 \mathrm{E}-05 \\
1.01 \mathrm{E}-04 \\
1.17 \mathrm{E}-04 \\
8.46 \mathrm{E}-05 \\
1.83 \mathrm{E}-04 \\
8.47 \mathrm{E}-05 \\
4.19 \mathrm{E}-05 \\
8.71 \mathrm{E}-06 \\
3.01 \mathrm{E}-05 \\
3.18 \mathrm{E}-05 \\
9.84 \mathrm{E}-05 \\
2.70 \mathrm{E}-05 \\
1.59 \mathrm{E}-04 \\
7.50 \mathrm{E}-06 \\
1.83 \mathrm{E}-06 \\
9.24 \mathrm{E}-05 \\
1.25 \mathrm{E}-05 \\
3.00 \mathrm{E}-04 \\
3.94 \mathrm{E}-05 \\
4.99 \mathrm{E}-05 \\
3.17 \mathrm{E}-04 \\
6.08 \mathrm{E}-05 \\
9.05 \mathrm{E}-05 \\
5.13 \mathrm{E}-06 \\
1.19 \mathrm{E}-04 \\
4.68 \mathrm{E}-05 \\
1.04 \mathrm{E}-05 \\
4.93 \mathrm{E}-05 \\
3.41 \mathrm{E}-05 \\
8.12 \mathrm{E}-05-05 \\
8.72 \mathrm{E}-05\end{array}$ & $\begin{array}{l}\text { U232 } \\
1.75 E-03 \\
2.41 E-03 \\
1.59 E-02 \\
3.04 E-03 \\
2.04 E-04 \\
6.47 E-04 \\
2.77 E-03 \\
6.28 E-04 \\
3.03 E-05 \\
3.78 E-03 \\
3.81 E-03 \\
1.21 E-02 \\
3.30 E-03 \\
6.68 E-03 \\
7.54 E-04 \\
9.94 E-04 \\
1.54 E-03 \\
2.71 E-03 \\
1.89 E-03 \\
1.51 E-03 \\
1.32 E-03 \\
4.27 E-03 \\
9.34 E-03 \\
2.26 E-03 \\
2.08 E-04 \\
3.57 E-04 \\
2.59 E-03 \\
7.08 E-04 \\
1.32 E-04 \\
5.74 E-04 \\
7.59 E-04 \\
1.42 E-03 \\
1.11 E-03 \\
1.93 E-04 \\
9.13 E-04 \\
1.23 E-03 \\
8.57 E-04 \\
3.55 E-03 \\
7.63 E-04 \\
4.20 E-04 \\
4.20 E-05 \\
4.36 E-04 \\
4.02 E-04 \\
1.91 E-03 \\
5.73 E-04 \\
1.71 E-03 \\
1.59 E-04 \\
7.18 E-06 \\
1.01 E-03 \\
1.16 E-04 \\
6.43 E-03 \\
1.51 E-04 \\
5.16 E-04 \\
5.43 E-03 \\
6.68 E-04 \\
7.27 E-04 \\
4.21 E-05 \\
1.42 E-03 \\
6.22 E-04 \\
8.03 E-05 \\
4.70 E-04 \\
3.62 E-04 \\
7.05 E-04 \\
7.18 E-04 \\
8.59 E-04\end{array}$ & $\begin{array}{l}\text { U233 } \\
2.08 \mathrm{E}-05 \\
2.63 \mathrm{E}-05 \\
1.52 \mathrm{E}-04 \\
2.89 \mathrm{E}-05 \\
6.61 \mathrm{E}-06 \\
2.08 \mathrm{E}-05 \\
8.78 \mathrm{E}-05 \\
1.81 \mathrm{E}-05 \\
1.08 \mathrm{E}-06 \\
3.71 \mathrm{E}-05 \\
3.50 \mathrm{E}-05 \\
1.44 \mathrm{E}-04 \\
3.75 \mathrm{E}-05 \\
7.25 \mathrm{E}-05 \\
6.23 \mathrm{E}-06 \\
1.16 \mathrm{E}-05 \\
1.63 \mathrm{E}-05 \\
3.08 \mathrm{E}-05 \\
1.85 \mathrm{E}-05 \\
1.61 \mathrm{E}-05 \\
1.51 \mathrm{E}-05 \\
5.20 \mathrm{E}-05 \\
1.06 \mathrm{E}-04 \\
2.83 \mathrm{E}-05 \\
4.12 \mathrm{E}-06 \\
5.43 \mathrm{E}-06 \\
4.49 \mathrm{E}-05 \\
1.29 \mathrm{E}-05 \\
2.26 \mathrm{E}-06 \\
1.03 \mathrm{E}-05 \\
1.21 \mathrm{E}-05 \\
2.64 \mathrm{E}-05 \\
1.75 \mathrm{E}-05 \\
3.39 \mathrm{E}-06 \\
1.56 \mathrm{E}-05 \\
1.92 \mathrm{E}-05 \\
1.37 \mathrm{E}-05 \\
4.16 \mathrm{E}-05 \\
1.27 \mathrm{E}-05 \\
6.74 \mathrm{E}-06 \\
8.69 \mathrm{E}-07 \\
5.78 \mathrm{E}-06 \\
5.64 \mathrm{E}-06 \\
2.24 \mathrm{E}-05 \\
6.47 \mathrm{E}-06 \\
2.64 \mathrm{E}-05 \\
1.80 \mathrm{E}-06 \\
1.61 \mathrm{E}-07 \\
1.54 \mathrm{E}-05 \\
1.89 \mathrm{E}-06 \\
7.23 \mathrm{E}-05 \\
3.42 \mathrm{E}-06 \\
7.99 \mathrm{E}-06 \\
6.78 \mathrm{E}-05 \\
1.01 \mathrm{E}-05 \\
1.30 \mathrm{E}-05 \\
7.42 \mathrm{E}-07 \\
2.08 \mathrm{E}-05 \\
8.73 \mathrm{E}-06 \\
1.45 \mathrm{E}-06 \\
7.76 \mathrm{E}-06 \\
1.22 \mathrm{E}-06 \\
1.40 \mathrm{E}-05 \\
\end{array}$ & $\begin{array}{l}U 234 \\
3.98 E+00 \\
4.58 E+00 \\
2.79 E+01 \\
4.17 E+00 \\
9.88 E+00 \\
2.98 E+01 \\
1.21 E+02 \\
1.85 E+01 \\
2.48 E+00 \\
5.24 E+00 \\
4.55 E+00 \\
3.13 E+01 \\
7.16 E+00 \\
1.31 E+01 \\
7.84 E-01 \\
2.17 E+00 \\
2.55 E+00 \\
5.36 E+00 \\
2.62 E+00 \\
2.62 E+00 \\
2.61 E+00 \\
1.18 E+01 \\
2.20 E+01 \\
6.69 E+00 \\
1.82 E+00 \\
1.67 E+00 \\
1.72 E+01 \\
5.32 E+00 \\
8.74 E-01 \\
4.30 E+00 \\
4.28 E+00 \\
1.13 E+01 \\
6.93 E+00 \\
1.35 E+00 \\
6.04 E+00 \\
8.00 E+00 \\
5.58 E+00 \\
1.90 E+01 \\
5.62 E+00 \\
2.74 E+00 \\
8.74 E-01 \\
3.09 E+00 \\
3.29 E+00 \\
1.02 E+01 \\
2.82 E+00 \\
1.10 E+01 \\
7.84 E-01 \\
1.84 E-01 \\
6.49 E+00 \\
8.96 E-01 \\
3.16 E+01 \\
4.01 E+00 \\
3.74 E+00 \\
3.86 E+01 \\
4.51 E+00 \\
5.44 E+00 \\
3.14 E-01 \\
8.75 E+00 \\
3.40 E+00 \\
6.36 E-01 \\
3.09 E+00 \\
2.62 E+00 \\
5.23 E+00 \\
4.98 E+00 \\
5.43 E+00\end{array}$ & $\begin{array}{l}\text { U235 } \\
1.38 \mathrm{E}-01 \\
1.57 \mathrm{E}-01 \\
7.27 \mathrm{E}-01 \\
1.39 \mathrm{E}-01 \\
4.40 \mathrm{E}-01 \\
1.33 \mathrm{E}+00 \\
5.36 \mathrm{E}+00 \\
8.08 \mathrm{E}-01 \\
1.12 \mathrm{E}-01 \\
2.09 \mathrm{E}-01 \\
1.79 \mathrm{E}-01 \\
1.10 \mathrm{E}+00 \\
2.95 \mathrm{E}-01 \\
5.36 \mathrm{E}-01 \\
3.04 \mathrm{E}-02 \\
8.95 \mathrm{E}-02 \\
1.04 \mathrm{E}-01 \\
2.20 \mathrm{E}-01 \\
1.05 \mathrm{E}-01 \\
1.07 \mathrm{E}-01 \\
1.07 \mathrm{E}-01 \\
4.16 \mathrm{E}-01 \\
7.66 \mathrm{E}-01 \\
2.37 \mathrm{E}-01 \\
6.84 \mathrm{E}-02 \\
6.09 \mathrm{E}-02 \\
6.40 \mathrm{E}-01 \\
1.99 \mathrm{E}-01 \\
3.25 \mathrm{E}-02 \\
1.61 \mathrm{E}-01 \\
1.58 \mathrm{E}-01 \\
4.25 \mathrm{E}-01 \\
2.57 \mathrm{E}-01 \\
5.03 \mathrm{E}-02 \\
2.25 \mathrm{E}-01 \\
2.97 \mathrm{E}-01 \\
2.07 \mathrm{E}-01 \\
6.98 \mathrm{E}-01 \\
2.10 \mathrm{E}-01 \\
1.02 \mathrm{E}-01 \\
3.40 \mathrm{E}-02 \\
1.16 \mathrm{E}-01 \\
1.24 \mathrm{E}-01 \\
3.75 \mathrm{E}-01 \\
1.04 \mathrm{E}-01 \\
4.10 \mathrm{E}-01 \\
2.88 \mathrm{E}-02 \\
7.21 \mathrm{E}-03 \\
2.41 \mathrm{E}-01 \\
3.36 \mathrm{E}-02 \\
1.16 \mathrm{E}+00 \\
1.57 \mathrm{E}-01 \\
1.40 \mathrm{E}-01 \\
1.44 \mathrm{E}+00 \\
1.68 \mathrm{E}-01 \\
2.03 \mathrm{E}-01 \\
1.18 \mathrm{E}-02 \\
3.25 \mathrm{E}-01 \\
1.25 \mathrm{E}-01 \\
2.38 \mathrm{E}-02 \\
1.95 \mathrm{E}-01 \\
9.78 \mathrm{E}-02 \\
1.95 \mathrm{E}-01 \\
1.86 \mathrm{E}-01 \\
2.01 \mathrm{E}-01\end{array}$ & $\begin{array}{l}\text { U236 } \\
3.88 \mathrm{E}-01 \\
4.63 \mathrm{E}-01 \\
2.37 \mathrm{E}+00 \\
4.53 \mathrm{E}-01 \\
8.26 \mathrm{E}-02 \\
2.59 \mathrm{E}-01 \\
1.09 \mathrm{E}+00 \\
2.23 \mathrm{E}-01 \\
1.37 \mathrm{E}-02 \\
6.35 \mathrm{E}-01 \\
5.71 \mathrm{E}-01 \\
2.96 \mathrm{E}+00 \\
7.78 \mathrm{E}-01 \\
1.45 \mathrm{E}+00 \\
1.02 \mathrm{E}-01 \\
2.36 \mathrm{E}-01 \\
2.96 \mathrm{E}-01 \\
5.96 \mathrm{E}-01 \\
3.18 \mathrm{E}-01 \\
3.00 \mathrm{E}-01 \\
2.90 \mathrm{E}-01 \\
1.10 \mathrm{E}+00 \\
2.12 \mathrm{E}+00 \\
6.15 \mathrm{E}-01 \\
1.37 \mathrm{E}-01 \\
1.40 \mathrm{E}-01 \\
1.36 \mathrm{E}+00 \\
4.11 \mathrm{E}-01 \\
6.89 \mathrm{E}-02 \\
3.32 \mathrm{E}-01 \\
3.47 \mathrm{E}-01 \\
8.65 \mathrm{E}-01 \\
5.50 \mathrm{E}-01 \\
1.05 \mathrm{E}-01 \\
4.76 \mathrm{E}-01 \\
6.30 \mathrm{E}-01 \\
4.40 \mathrm{E}-01 \\
1.54 \mathrm{E}+00 \\
4.34 \mathrm{E}-01 \\
2.16 \mathrm{E}-01 \\
5.76 \mathrm{E}-02 \\
2.39 \mathrm{E}-01 \\
2.48 \mathrm{E}-01 \\
8.26 \mathrm{E}-01 \\
2.32 \mathrm{E}-01 \\
8.70 \mathrm{E}-01 \\
6.46 \mathrm{E}-02 \\
1.18 \mathrm{E}-02 \\
5.11 \mathrm{E}-01 \\
6.85 \mathrm{E}-02 \\
2.61 \mathrm{E}+00 \\
2.57 \mathrm{E}-01 \\
2.88 \mathrm{E}-01 \\
2.98 \mathrm{E}+00 \\
3.52 \mathrm{E}-01 \\
4.20 \mathrm{E}-01 \\
2.42 \mathrm{E}-02 \\
6.95 \mathrm{E}-01 \\
2.76 \mathrm{E}-01 \\
4.86 \mathrm{E}-02 \\
2.43 \mathrm{E}-01 \\
2.02 \mathrm{E}-01 \\
4.0 \mathrm{E}-01\end{array}$ & $\begin{array}{l}U 238 \\
2.78 E+00 \\
3.22 E+00 \\
1.55 E+01 \\
2.96 E+00 \\
1.00 E+01 \\
3.03 E+01 \\
1.23 E+02 \\
1.88 E+01 \\
2.51 E+00 \\
3.70 E+00 \\
3.22 E+00 \\
2.18 E+01 \\
4.99 E+00 \\
9.12 E+00 \\
5.57 E-01 \\
1.51 E+00 \\
1.79 E+00 \\
3.74 E+00 \\
1.85 E+00 \\
1.84 E+00 \\
1.82 E+00 \\
8.22 E+00 \\
1.54 E+01 \\
4.65 E+00 \\
1.24 E+00 \\
1.15 E+00 \\
1.18 E+01 \\
3.64 E+00 \\
5.98 E-01 \\
2.94 E+00 \\
2.94 E+00 \\
7.73 E+00 \\
4.75 E+00 \\
9.23 E-01 \\
4.14 E+00 \\
5.47 E+00 \\
3.82 E+00 \\
1.30 E+01 \\
3.84 E+00 \\
1.87 E+00 \\
5.90 E-01 \\
2.11 E+00 \\
2.24 E+00 \\
6.98 E+00 \\
1.94 E+00 \\
7.55 E+00 \\
5.39 E-01 \\
1.24 E-01 \\
4.44 E+00 \\
6.12 E-01 \\
2.18 E+01 \\
2.70 E+00 \\
2.55 E+00 \\
2.64 E+01 \\
3.08 E+00 \\
3.72 E+00 \\
2.15 E-01 \\
5.99 E+00 \\
2.34 E+00 \\
4.34 E-01 \\
2.11 E+00 \\
1.79 E+00 \\
3.57 E+00 \\
3.41 E+00 \\
3.72 E+00\end{array}$ & $\begin{array}{l}\text { NP237 } \\
1.17 \mathrm{E}-01 \\
1.51 \mathrm{E}-01 \\
8.84 \mathrm{E}-01 \\
1.69 \mathrm{E}-01 \\
2.58 \mathrm{E}-02 \\
8.10 \mathrm{E}-02 \\
3.43 \mathrm{E}-01 \\
7.22 \mathrm{E}-02 \\
4.14 \mathrm{E}-03 \\
2.19 \mathrm{E}-01 \\
2.08 \mathrm{E}-01 \\
8.35 \mathrm{E}-01 \\
2.22 \mathrm{E}-01 \\
4.33 \mathrm{E}-01 \\
3.88 \mathrm{E}-02 \\
6.73 \mathrm{E}-02 \\
9.51 \mathrm{E}-02 \\
1.78 \mathrm{E}-01 \\
1.09 \mathrm{E}-01 \\
9.47 \mathrm{E}-02 \\
8.68 \mathrm{E}-02 \\
3.02 \mathrm{E}-01 \\
6.23 \mathrm{E}-01 \\
1.64 \mathrm{E}-01 \\
2.25 \mathrm{E}-02 \\
3.06 \mathrm{E}-02 \\
2.52 \mathrm{E}-01 \\
7.21 \mathrm{E}-02 \\
1.28 \mathrm{E}-02 \\
5.83 \mathrm{E}-02 \\
6.92 \mathrm{E}-02 \\
1.48 \mathrm{E}-01 \\
1.04 \mathrm{E}-01 \\
1.91 \mathrm{E}-02 \\
8.84 \mathrm{E}-02 \\
1.17 \mathrm{E}-01 \\
8.15 \mathrm{E}-02 \\
3.04 \mathrm{E}-01 \\
7.59 \mathrm{E}-02 \\
4.00 \mathrm{E}-02 \\
5.97 \mathrm{E}-03 \\
4.16 \mathrm{E}-02 \\
4.05 \mathrm{E}-02 \\
1.64 \mathrm{E}-01 \\
4.77 \mathrm{E}-02 \\
1.61 \mathrm{E}-01 \\
1.33 \mathrm{E}-02 \\
1.10 \mathrm{E}-03 \\
9.47 \mathrm{E}-02 \\
1.17 \mathrm{E}-02 \\
5.35 \mathrm{E}-01 \\
2.35 \mathrm{E}-02 \\
5.04 \mathrm{E}-02 \\
5.19 \mathrm{E}-01 \\
6.36 \mathrm{E}-02 \\
7.36 \mathrm{E}-02\end{array}$ \\
\hline
\end{tabular}


HNF-SD-WM-TI-794, Rev. 0

ACTIVITY IN UNSEPARATED FUEL (Curies decayed to $1 / 1 / 94$ )

\begin{tabular}{|c|c|c|c|c|c|c|c|c|c|}
\hline $\begin{array}{r}\text { isting } \\
976 \\
977 \\
978 \\
979 \\
980 \\
981 \\
982 \\
983 \\
984 \\
985 \\
986 \\
987 \\
988 \\
989 \\
990 \\
991 \\
992 \\
993 \\
994 \\
995 \\
996 \\
997 \\
998 \\
999 \\
1000 \\
1001 \\
1002 \\
1003 \\
1004 \\
1005 \\
1006 \\
1007 \\
1008 \\
1009 \\
1010 \\
1011 \\
1012 \\
1013 \\
1014 \\
1015 \\
1016 \\
1017 \\
1018 \\
1019 \\
1020 \\
1021 \\
1022 \\
1023 \\
1024 \\
1025 \\
1026 \\
1027 \\
1028 \\
1029 \\
1030 \\
1031 \\
1032 \\
1033 \\
1034 \\
1035 \\
1036 \\
1037 \\
1038 \\
1039 \\
1040\end{array}$ & 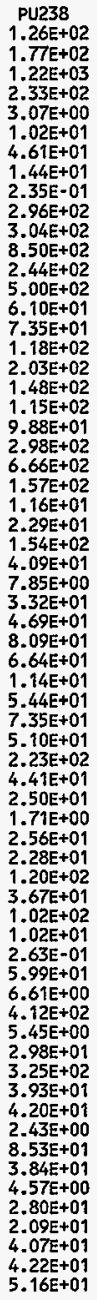 & $\begin{array}{l}P U 239 \\
7.34 E+02 \\
9.03 E+02 \\
4.88 E+03 \\
9.32 E+02 \\
5.52 E+02 \\
1.73 E+03 \\
7.30 E+03 \\
1.47 E+03 \\
9.22 E+01 \\
1.15 E+03 \\
1.05 E+03 \\
5.44 E+03 \\
1.32 E+03 \\
2.51 E+03 \\
1.89 E+02 \\
4.01 E+02 \\
5.23 E+02 \\
1.03 E+03 \\
5.74 E+02 \\
5.27 E+02 \\
5.01 E+02 \\
2.00 E+03 \\
3.97 E+03 \\
1.10 E+03 \\
1.82 E+02 \\
2.25 E+02 \\
1.96 E+03 \\
5.71 E+02 \\
9.96 E+01 \\
4.62 E+02 \\
5.26 E+02 \\
1.18 E+03 \\
8.05 E+02 \\
1.50 E+02 \\
6.89 E+02 \\
9.12 E+02 \\
6.36 E+02 \\
2.33 E+03 \\
6.03 E+02 \\
3.12 E+02 \\
5.32 E+01 \\
3.32 E+02 \\
3.29 E+02 \\
1.25 E+03 \\
3.61 E+02 \\
1.26 E+03 \\
1.00 E+02 \\
9.95 E+00 \\
7.40 E+02 \\
9.34 E+01 \\
4.05 E+03 \\
2.13 E+02 \\
4.01 E+02 \\
4.15 E+03 \\
5.01 E+02 \\
5.84 E+02 \\
3.37 E+01 \\
1.02 E+03 \\
4.19 E+02 \\
6.63 E+01 \\
3.52 E+02 \\
2.83 E+02 \\
5.61 E+02 \\
5.54 E+02 \\
6.30 E+02\end{array}$ & $\begin{array}{l}P U 240 \\
2.91 E+02 \\
3.88 \mathrm{E}+02 \\
2.37 \mathrm{E}+03 \\
4.53 \mathrm{E}+02 \\
3.94 \mathrm{E}+01 \\
1.28 \mathrm{E}+02 \\
5.59 \mathrm{E}+02 \\
1.48 \mathrm{E}+02 \\
4.61 \mathrm{E}+00 \\
5.70 \mathrm{E}+02 \\
5.51 \mathrm{E}+02 \\
2.02 \mathrm{E}+03 \\
5.41 \mathrm{E}+02 \\
1.08 \mathrm{E}+03 \\
1.04 \mathrm{E}+02 \\
1.64 \mathrm{E}+02 \\
2.43 \mathrm{E}+02 \\
4.42 \mathrm{E}+02 \\
2.85 \mathrm{E}+02 \\
2.41 \mathrm{E}+02 \\
2.15 \mathrm{E}+02 \\
7.20 \mathrm{E}+02 \\
1.53 \mathrm{E}+03 \\
3.85 \mathrm{E}+02 \\
4.33 \mathrm{E}+01 \\
6.57 \mathrm{E}+01 \\
5.08 \mathrm{E}+02 \\
1.42 \mathrm{E}+02 \\
2.58 \mathrm{E}+01 \\
1.15 \mathrm{E}+02 \\
1.44 \mathrm{E}+02 \\
2.88 \mathrm{E}+02 \\
2.11 \mathrm{E}+02 \\
3.82 \mathrm{E}+01 \\
1.78 \mathrm{0}+02 \\
2.36 \mathrm{E}+02 \\
1.65 \mathrm{E}+02 \\
6.37 \mathrm{E}+02 \\
1.50 \mathrm{E}+02 \\
8.08 \mathrm{E}+01 \\
9.98 \mathrm{E}+00 \\
8.26 \mathrm{E}+01 \\
7.83 \mathrm{E}+01 \\
3.42 \mathrm{E}+02 \\
1.02 \mathrm{E}+02 \\
3.26 \mathrm{E}+02 \\
2.82 \mathrm{E}+01 \\
1.79 \mathrm{E}+00 \\
1.91 \mathrm{E}+02 \\
2.28 \mathrm{E}+01 \\
1.14 \mathrm{E}+03 \\
3.80 \mathrm{E}+01 \\
9.98 \mathrm{E}+01 \\
1.03 \mathrm{E}+03 \\
1.28 \mathrm{E}+02 \\
1.45 \mathrm{E}+02 \\
8.39 \mathrm{E}+00 \\
2.67 \mathrm{E}+02 \\
1.14 \mathrm{E}+02 \\
1.62 \mathrm{E}+01 \\
9.11 \mathrm{E}+01 \\
6.98 \mathrm{E}+01 \\
1.40 \mathrm{E}+02 \\
1.41 \mathrm{E}+02 \\
1.65 \mathrm{E}+02\end{array}$ & $\begin{array}{l}\text { PU241 } \\
9.13 \mathrm{E}+03 \\
1.31 \mathrm{E}+04 \\
8.61 \mathrm{E}+04 \\
1.66 \mathrm{E}+04 \\
1.70 \mathrm{E}+02 \\
6.22 \mathrm{E}+02 \\
3.08 \mathrm{E}+03 \\
1.38 \mathrm{E}+03 \\
0.00 \mathrm{E}+00 \\
2.05 \mathrm{E}+04 \\
2.05 \mathrm{E}+04 \\
6.50 \mathrm{E}+04 \\
1.84 \mathrm{E}+04 \\
3.80 \mathrm{E}+04 \\
4.27 \mathrm{E}+03 \\
5.35 \mathrm{E}+03 \\
8.38 \mathrm{E}+03 \\
1.47 \mathrm{E}+04 \\
1.03 \mathrm{E}+04 \\
8.43 \mathrm{E}+03 \\
7.07 \mathrm{E}+03 \\
2.29 \mathrm{E}+04 \\
5.20 \mathrm{E}+04 \\
1.20 \mathrm{E}+04 \\
7.57 \mathrm{E}+02 \\
1.67 \mathrm{E}+03 \\
1.10 \mathrm{E}+04 \\
2.86 \mathrm{E}+03 \\
5.63 \mathrm{E}+02 \\
2.34 \mathrm{E}+03 \\
3.49 \mathrm{E}+03 \\
5.60 \mathrm{E}+03 \\
5.17 \mathrm{E}+03 \\
8.07 \mathrm{E}+02 \\
3.94 \mathrm{E}+03 \\
5.94 \mathrm{E}+03 \\
4.00 \mathrm{E}+03 \\
2.37 \mathrm{E}+04 \\
3.41 \mathrm{E}+03 \\
1.95 \mathrm{E}+03 \\
9.82 \mathrm{E}+01 \\
2.59 \mathrm{E}+03 \\
2.24 \mathrm{E}+03 \\
1.28 \mathrm{E}+04 \\
3.97 \mathrm{E}+03 \\
8.28 \mathrm{E}+03 \\
1.10 \mathrm{E}+03 \\
1.08 \mathrm{E}+01 \\
4.92 \mathrm{E}+03 \\
5.35 \mathrm{E}+02 \\
4.47 \mathrm{E}+04 \\
2.07 \mathrm{E}+02 \\
2.52 \mathrm{E}+03 \\
3.56 \mathrm{E}+04 \\
3.33 \mathrm{E}+03 \\
3.00 \mathrm{E}+03 \\
1.76 \mathrm{E}+02 \\
7.28 \mathrm{E}+03 \\
3.31 \mathrm{E}+03 \\
3.26 \mathrm{E}+02 \\
2.11 \mathrm{E}+03 \\
1.80 \mathrm{E}+03 \\
3.02 \mathrm{E}+03 \\
3.10 \mathrm{E}+03 \\
3.89 \mathrm{E}+03\end{array}$ & $\begin{array}{l}\text { PU242 } \\
6.17 \mathrm{E}-02 \\
9.06 \mathrm{E}-02 \\
7.21 \mathrm{E}-01 \\
1.38 \mathrm{E}-01 \\
0.00 \mathrm{E}+00 \\
0.00 \mathrm{E}+00 \\
9.87 \mathrm{E}-04 \\
2.15 \mathrm{E}-03 \\
0.00 \mathrm{E}+00 \\
1.73 \mathrm{E}-01 \\
1.89 \mathrm{E}-01 \\
3.90 \mathrm{E}-01 \\
1.18 \mathrm{E}-01 \\
2.49 \mathrm{E}-01 \\
3.92 \mathrm{E}-02 \\
3.58 \mathrm{E}-02 \\
6.32 \mathrm{E}-02 \\
1.02 \mathrm{E}-01 \\
8.65 \mathrm{E}-02 \\
5.99 \mathrm{E}-02 \\
4.97 \mathrm{E}-02 \\
1.33 \mathrm{E}-01 \\
3.15 \mathrm{E}-01 \\
6.80 \mathrm{E}-02 \\
1.46 \mathrm{E}-03 \\
7.69 \mathrm{E}-03 \\
3.53 \mathrm{E}-02 \\
7.52 \mathrm{E}-03 \\
1.79 \mathrm{E}-03 \\
6.08 \mathrm{E}-03 \\
1.31 \mathrm{E}-02 \\
1.30 \mathrm{E}-02 \\
1.58 \mathrm{E}-02 \\
2.38 \mathrm{E}-03 \\
1.24 \mathrm{E}-02 \\
1.64 \mathrm{E}-02 \\
1.14 \mathrm{E}-02 \\
5.79 \mathrm{E}-02 \\
7.93 \mathrm{E}-03 \\
5.61 \mathrm{E}-03 \\
0.00 \mathrm{E}-00 \\
4.36 \mathrm{E}-03 \\
2.64 \mathrm{E}-03 \\
3.11 \mathrm{E}-02 \\
1.03 \mathrm{E}-02 \\
2.26 \mathrm{E}-02 \\
2.87 \mathrm{E}-03 \\
0.00 \mathrm{E}+00 \\
1.33 \mathrm{E}-02 \\
1.03 \mathrm{E}-03 \\
1.16 \mathrm{E}-01 \\
0.00 \mathrm{E}+00 \\
5.28 \mathrm{E}-03 \\
5.46 \mathrm{E}-02 \\
7.96 \mathrm{E}-03 \\
7.68 \mathrm{E}-03 \\
4.43 \mathrm{E}-04 \\
1.99 \mathrm{E}-02 \\
1.04 \mathrm{E}-02 \\
7.28 \mathrm{E}-04 \\
6.33 \mathrm{E}-03 \\
3.69 \mathrm{E}-03 \\
7.38 \mathrm{~B}-03 \\
1.80 \mathrm{E}-03 \\
1.24 \mathrm{E}-02\end{array}$ & $\begin{array}{l}A M 241 \\
6.51 \mathrm{E}+02 \\
9.16 \mathrm{E}+02 \\
6.18 \mathrm{E}+03 \\
1.18 \mathrm{E}+03 \\
1.12 \mathrm{E}+01 \\
4.13 \mathrm{E}+01 \\
2.04 \mathrm{E}+02 \\
9.11 \mathrm{E}+01 \\
0.00 \mathrm{E}+00 \\
1.44 \mathrm{E}+03 \\
1.45 \mathrm{E}+03 \\
4.12 \mathrm{E}+03 \\
1.16 \mathrm{E}+03 \\
2.38 \mathrm{E}+03 \\
2.75 \mathrm{E}+02 \\
3.58 \mathrm{E}+02 \\
5.87 \mathrm{E}+02 \\
1.01 \mathrm{E}+03 \\
7.22 \mathrm{E}+02 \\
5.66 \mathrm{E}+02 \\
4.93 \mathrm{E}+02 \\
1.43 \mathrm{E}+03 \\
3.22 \mathrm{E}+03 \\
7.43 \mathrm{E}+02 \\
4.68 \mathrm{E}+01 \\
1.04 \mathrm{E}+02 \\
6.47 \mathrm{E}+02 \\
1.67 \mathrm{E}+02 \\
3.26 \mathrm{E}+01 \\
1.34 \mathrm{E}+02 \\
1.99 \mathrm{E}+02 \\
3.24 \mathrm{E}+02 \\
2.60 \mathrm{E}+02 \\
4.67 \mathrm{E}+01 \\
2.24 \mathrm{E}+02 \\
2.74 \mathrm{E}+02 \\
1.95 \mathrm{E}+02 \\
6.14 \mathrm{E}+02 \\
1.63 \mathrm{E}+02 \\
9.65 \mathrm{E}+01 \\
2.51 \mathrm{E}+00 \\
6.67 \mathrm{E}+01 \\
5.69 \mathrm{E}+01 \\
3.30 \mathrm{E}+02 \\
1.02 \mathrm{E}+02 \\
3.75 \mathrm{E}+02 \\
2.82 \mathrm{E}+01 \\
2.73 \mathrm{E}-01 \\
2.19 \mathrm{E}+02 \\
2.27 \mathrm{E}+01 \\
1.13 \mathrm{E}+03 \\
5.18 \mathrm{E}+00 \\
1.01 \mathrm{E}+02 \\
7.32 \mathrm{E}+02 \\
1.36 \mathrm{E}+02 \\
1.68 \mathrm{E}+02 \\
9.58 \mathrm{E}+00 \\
3.04 \mathrm{E}+02 \\
1.41 \mathrm{E}+02 \\
1.78 \mathrm{E}+01 \\
1.11 \mathrm{E}+02 \\
6.94 \mathrm{E}+01 \\
1.57 \mathrm{E}+02 \\
1.69 \mathrm{E}+02 \\
2.08 \mathrm{E}+02\end{array}$ & $\begin{array}{l}\text { AM243 } \\
7.28 \mathrm{E}-02 \\
1.10 \mathrm{E}-01 \\
1.10 \mathrm{E}+00 \\
2.10 \mathrm{E}-01 \\
0.00 \mathrm{E}+00 \\
0.00 \mathrm{E}+00 \\
2.57 \mathrm{E}-07 \\
6.39 \mathrm{E}-04 \\
0.00 \mathrm{E}+00 \\
2.54 \mathrm{E}-01 \\
3.03 \mathrm{E}-01 \\
4.48 \mathrm{E}-01 \\
1.36 \mathrm{E}-01 \\
2.91 \mathrm{E}-01 \\
6.69 \mathrm{E}-02 \\
4.13 \mathrm{E}-02 \\
8.04 \mathrm{E}-02 \\
1.20 \mathrm{E}-01 \\
1.27 \mathrm{E}-01 \\
7.28 \mathrm{E}-02 \\
5.82 \mathrm{E}-02 \\
1.51 \mathrm{E}-01 \\
3.68 \mathrm{E}-01 \\
7.59 \mathrm{E}-02 \\
3.46 \mathrm{E}-07 \\
7.07 \mathrm{E}-03 \\
1.85 \mathrm{E}-02 \\
1.65 \mathrm{E}-03 \\
9.41 \mathrm{E}-04 \\
1.33 \mathrm{E}-03 \\
9.88 \mathrm{E}-03 \\
3.25 \mathrm{E}-06 \\
9.39 \mathrm{E}-03 \\
9.90 \mathrm{E}-04 \\
6.50 \mathrm{E}-03 \\
8.61 \mathrm{E}-03 \\
6.00 \mathrm{E}-03 \\
4.38 \mathrm{E}-02 \\
1.74 \mathrm{E}-03 \\
2.95 \mathrm{E}-03 \\
0.00 \mathrm{E}+00 \\
9.57 \mathrm{E}-04 \\
6.26 \mathrm{E}-07 \\
2.35 \mathrm{E}-02 \\
8.63 \mathrm{E}-03 \\
1.19 \mathrm{E}-02 \\
2.40 \mathrm{E}-03 \\
0.00 \mathrm{E}+00 \\
6.98 \mathrm{E}-03 \\
2.57 \mathrm{E}-07 \\
9.68 \mathrm{E}-02 \\
0.00 \mathrm{E}+00 \\
1.16 \mathrm{E}-03 \\
1.20 \mathrm{E}-02 \\
3.31 \mathrm{E}-03 \\
1.68 \mathrm{E}-03 \\
9.72 \mathrm{E}-05 \\
1.19 \mathrm{E}-02 \\
7.86 \mathrm{E}-03 \\
1.82 \mathrm{E}-07 \\
3.32 \mathrm{E}-03 \\
8.10 \mathrm{E}-04 \\
1.50 \mathrm{n}\end{array}$ & 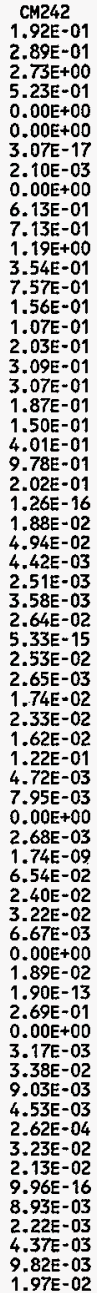 & 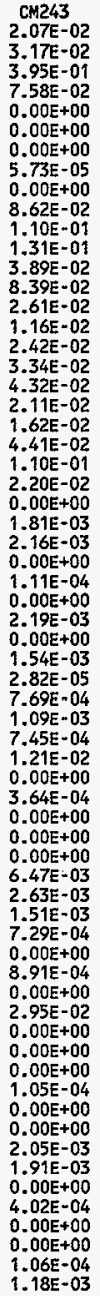 \\
\hline
\end{tabular}


HWF-SO-WM-TI-794, ReV. 0

ACTIVITY IN UNSEPARATED FUEL (Curies decayed to 1/1/94)

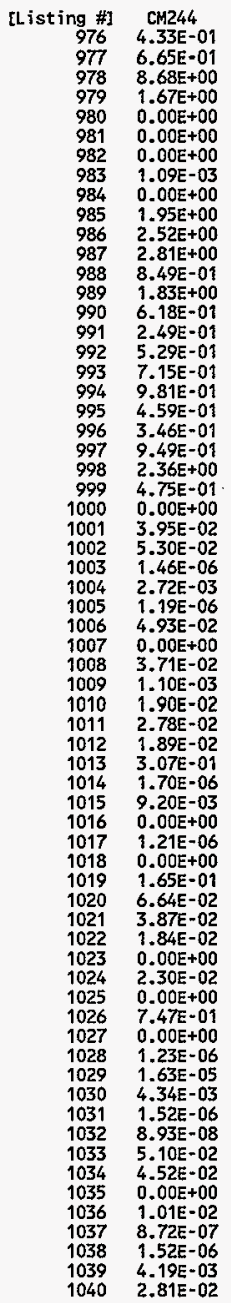


HNF-SD-WH-TI-794, ReV, O

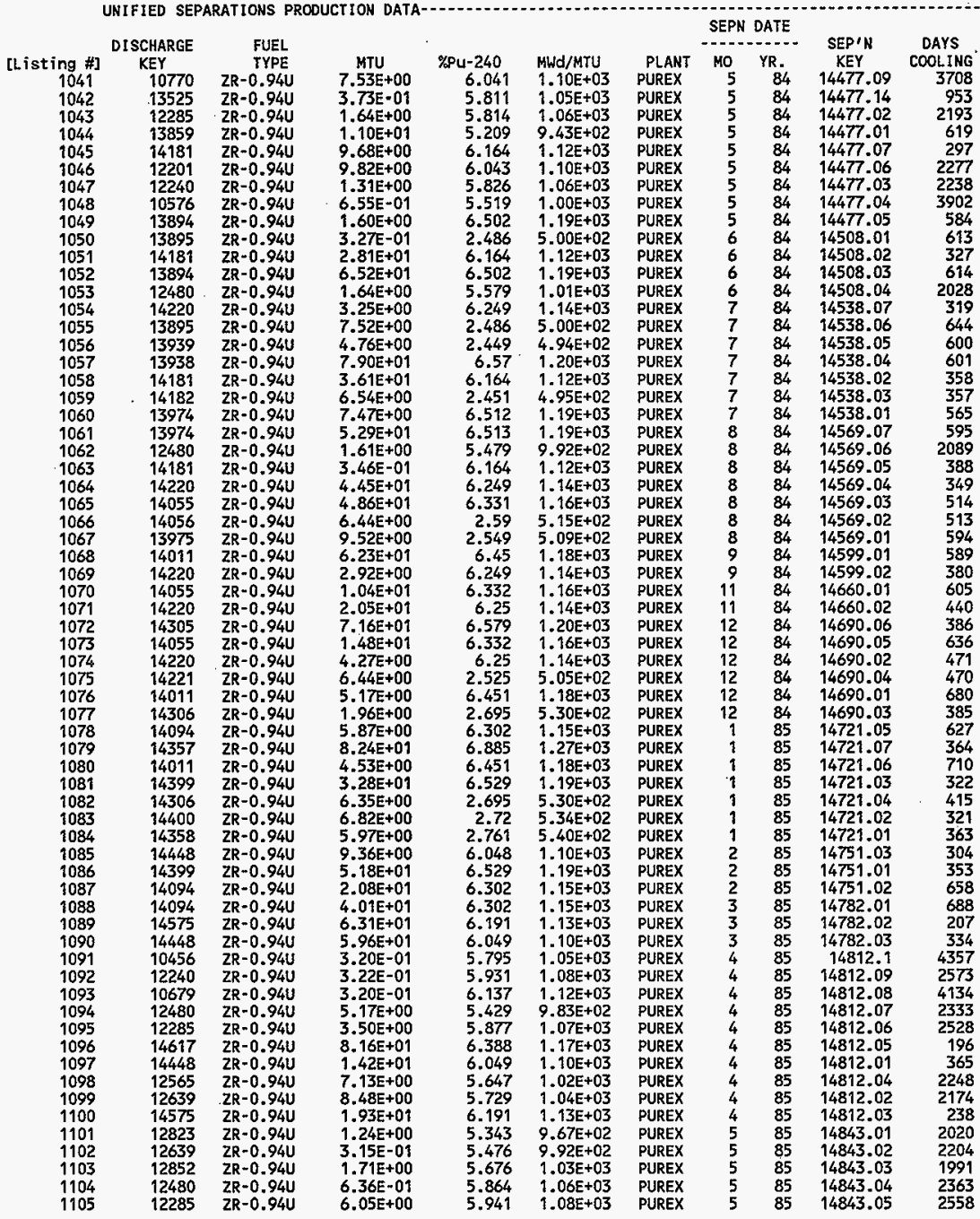


HNF-SD-HM-TI-794, ReV. 0

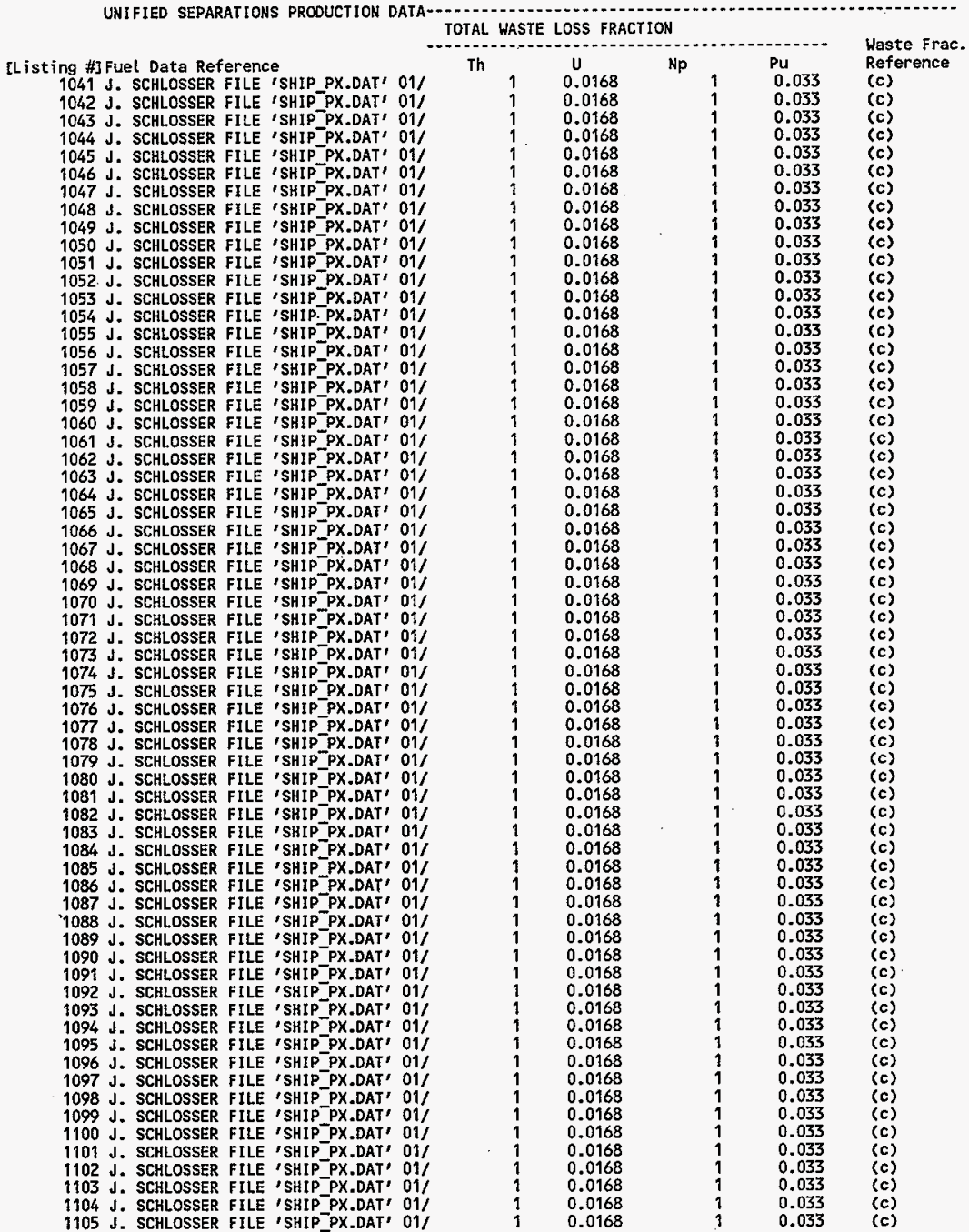


HWF-SD-WM-T1-794, Rev. 0

ACTIVITY IN UNSEPARATED FUEL (Curies decayed to 1/1/94)

\begin{tabular}{|c|c|c|c|c|c|c|c|c|c|}
\hline $\begin{array}{r}\text { [Listing \#] } \\
1041 \\
1042 \\
1043 \\
1044 \\
1045 \\
1046 \\
1047 \\
1048 \\
1049 \\
1050 \\
1051 \\
1052 \\
1053 \\
1054 \\
1055 \\
1056 \\
1057 \\
1058 \\
1059 \\
1060 \\
1061 \\
1062 \\
1063 \\
1064 \\
1065 \\
1066 \\
1067 \\
1068 \\
1069 \\
1070 \\
1071 \\
1072 \\
1073 \\
1074 \\
1075 \\
1076 \\
1077 \\
1078 \\
1079 \\
1080 \\
1081 \\
1082 \\
1083 \\
1084 \\
1085 \\
1086 \\
1087 \\
1088 \\
1089 \\
1090 \\
1091 \\
1092 \\
1093 \\
1094 \\
1095 \\
1096 \\
1097 \\
1098 \\
1099 \\
1100 \\
1101 \\
1102 \\
1103 \\
1104 \\
1105\end{array}$ & $\begin{array}{l}\text { H03 } \\
4.42 E+01 \\
3.21 E+00 \\
1.17 E+01 \\
8.89 E+01 \\
9.82 E+01 \\
7.19 E+01 \\
9.28 E+00 \\
3.39 E+00 \\
1.65 E+01 \\
1.39 E+00 \\
2.85 E+02 \\
6.72 E+02 \\
1.15 E+01 \\
3.36 E+01 \\
3.19 E+01 \\
2.01 E+01 \\
8.29 E+02 \\
3.67 E+02 \\
2.87 E+01 \\
7.81 E+01 \\
5.54 E+02 \\
1.11 E+01 \\
3.51 E+00 \\
4.61 E+02 \\
4.98 E+02 \\
2.89 E+01 \\
4.16 E+01 \\
6.48 E+02 \\
3.03 E+01 \\
1.06 E+02 \\
2.12 E+02 \\
7.96 E+02 \\
1.52 E+02 \\
4.42 E+01 \\
2.91 E+01 \\
5.38 E+01 \\
9.41 E+00 \\
6.02 E+01 \\
9.74 E+02 \\
4.71 E+01 \\
3.67 E+02 \\
3.05 E+01 \\
3.34 E+01 \\
2.94 E+01 \\
9.69 E+01 \\
5.80 E+02 \\
2.14 E+02 \\
4.11 E+02 \\
6.84 E+02 \\
6.17 E+02 \\
1.71 E+00 \\
2.32 E+00 \\
1.89 E+00 \\
3.52 E+01 \\
2.52 E+01 \\
9.22 E+02 \\
1.47 E+02 \\
5.13 E+01 \\
6.27 E+01 \\
2.09 E+02 \\
8.75 E+00 \\
2.22 E+00 \\
1.29 E+01 \\
4.70 E+00 \\
4.41 E+01\end{array}$ & $\begin{array}{l}\text { C14 } \\
8.91 \mathrm{E}-01 \\
4.24 \mathrm{E}-02 \\
1.86 \mathrm{E}-01 \\
1.12 \mathrm{E}+00 \\
1.17 \mathrm{E}+00 \\
1.16 \mathrm{E}+00 \\
1.49 \mathrm{E}-01 \\
7.04 \mathrm{E}-02 \\
2.05 \mathrm{E}-01 \\
1.74 \mathrm{E}-02 \\
3.40 \mathrm{E}+00 \\
8.37 \mathrm{E}+00 \\
1.78 \mathrm{E}-01 \\
3.99 \mathrm{E}-01 \\
4.01 \mathrm{E}-01 \\
2.51 \mathrm{E}-01 \\
1.03 \mathrm{E}+01 \\
4.38 \mathrm{E}+00 \\
3.45 \mathrm{E}-01 \\
9.62 \mathrm{E}-01 \\
6.81 \mathrm{E}+00 \\
1.72 \mathrm{E}-01 \\
4.19 \mathrm{E}-02 \\
5.47 \mathrm{E}+00 \\
6.06 \mathrm{E}+00 \\
3.54 \mathrm{E}-01 \\
5.17 \mathrm{E}-01 \\
7.93 \mathrm{E}+00 \\
3.59 \mathrm{E}-01 \\
1.29 \mathrm{E}+00 \\
2.51 \mathrm{E}+00 \\
9.31 \mathrm{E}+00 \\
1.85 \mathrm{E}+00 \\
5.24 \mathrm{E}-01 \\
3.47 \mathrm{E}-01 \\
6.58 \mathrm{E}-01 \\
1.11 \mathrm{E}-01 \\
7.28 \mathrm{E}-01 \\
1.13 \mathrm{E}+01 \\
5.76 \mathrm{E}-01 \\
4.23 \mathrm{E}+00 \\
3.59 \mathrm{E}-01 \\
3.88 \mathrm{E}-01 \\
3.44 \mathrm{E}-01 \\
1.11 \mathrm{E}+00 \\
6.69 \mathrm{E}+00 \\
2.58 \mathrm{E}+00 \\
4.98 \mathrm{E}+00 \\
7.68 \mathrm{E}+00 \\
7.07 \mathrm{E}+00 \\
3.62 \mathrm{E}-02 \\
3.74 \mathrm{E}-02 \\
3.85 \mathrm{E}-02 \\
5.46 \mathrm{E}-01 \\
4.02 \mathrm{E}-01 \\
1.03 \mathrm{E}+01 \\
1.69 \mathrm{E}+00 \\
7.85 \mathrm{E}-01 \\
9.49 \mathrm{E}-01 \\
2.35 \mathrm{E}+00 \\
1.29 \mathrm{E}-01 \\
3.36 \mathrm{E}-02 \\
1.89 \mathrm{E}-01 \\
7.29 \mathrm{E}-02 \\
7.04 \mathrm{E}-01\end{array}$ & $\begin{array}{l}\text { NIS9 } \\
5.32 \mathrm{E}-02 \\
2.53 \mathrm{E}-03 \\
1.11 \mathrm{E}-02 \\
6.67 \mathrm{E}-02 \\
6.98 \mathrm{E}-02 \\
6.94 \mathrm{E}-02 \\
8.90 \mathrm{E}-03 \\
4.21 \mathrm{E}-03 \\
1.22 \mathrm{E}-02 \\
1.05 \mathrm{E}-03 \\
2.03 \mathrm{E}-01 \\
4.98 \mathrm{E}-01 \\
1.06 \mathrm{E}-02 \\
2.38 \mathrm{E}-02 \\
2.40 \mathrm{E}-02 \\
1.51 \mathrm{E}-02 \\
6.11 \mathrm{E}-01 \\
2.61 \mathrm{E}-01 \\
2.07 \mathrm{E}-02 \\
5.73 \mathrm{E}-02 \\
4.06 \mathrm{E}-01 \\
1.03 \mathrm{E}-02 \\
2.50 \mathrm{E}-03 \\
3.26 \mathrm{E}-01 \\
3.61 \mathrm{E}-01 \\
2.12 \mathrm{E}-02 \\
3.10 \mathrm{E}-02 \\
4.72 \mathrm{E}-01 \\
2.14 \mathrm{E}-02 \\
7.70 \mathrm{E}-02 \\
1.50 \mathrm{E}-01 \\
5.55 \mathrm{E}-01 \\
1.10 \mathrm{E}-01 \\
3.12 \mathrm{E}-02 \\
2.08 \mathrm{E}-02 \\
3.92 \mathrm{E}-02 \\
6.65 \mathrm{E}-03 \\
4.34 \mathrm{E}-02 \\
6.72 \mathrm{E}-01 \\
3.43 \mathrm{E}-02 \\
2.52 \mathrm{E}-01 \\
2.16 \mathrm{E}-02 \\
2.33 \mathrm{E}-02 \\
2.07 \mathrm{E}-02 \\
6.62 \mathrm{E}-02 \\
3.98 \mathrm{E}-01 \\
1.54 \mathrm{E}-01 \\
2.96 \mathrm{E}-01 \\
4.58 \mathrm{E}-01 \\
4.22 \mathrm{E}-01 \\
2.16 \mathrm{E}-03 \\
2.23 \mathrm{E}-03 \\
2.30 \mathrm{E}-03 \\
3.26 \mathrm{E}-02 \\
2.40 \mathrm{E}-02 \\
6.12 \mathrm{E}-01 \\
1.00 \mathrm{E}-01 \\
4.69 \mathrm{E}-02 \\
5.66 \mathrm{E}-02 \\
1.40 \mathrm{E}-01 \\
7.69 \mathrm{E}-03 \\
2.01 \mathrm{E}-03 \\
4.35 \mathrm{E}-02 \\
4.20 \mathrm{E}-03\end{array}$ & $\begin{array}{l}\text { N163 } \\
5.78 \mathrm{E}+00 \\
2.91 \mathrm{E}-01 \\
1.24 \mathrm{E}+00 \\
7.71 \mathrm{E}+00 \\
8.14 \mathrm{E}+00 \\
7.76 \mathrm{E}+00 \\
9.96 \mathrm{E}-01 \\
4.55 \mathrm{E}-01 \\
1.42 \mathrm{E}+00 \\
1.21 \mathrm{E}-01 \\
2.36 \mathrm{E}+01 \\
5.78 \mathrm{E}+01 \\
1.20 \mathrm{E}+00 \\
2.77 \mathrm{E}+00 \\
2.77 \mathrm{E}+00 \\
1.74 \mathrm{E}+00 \\
7.09 \mathrm{E}+01 \\
3.04 \mathrm{E}+01 \\
2.40 \mathrm{E}+00 \\
6.65 \mathrm{E}+00 \\
4.71 \mathrm{E}+01 \\
1.16 \mathrm{E}+00 \\
2.91 \mathrm{E}-01 \\
3.80 \mathrm{E}+01 \\
4.20 \mathrm{E}+01 \\
2.46 \mathrm{E}+00 \\
3.58 \mathrm{E}+00 \\
5.49 \mathrm{E}+01 \\
2.50 \mathrm{E}+00 \\
8.96 \mathrm{E}+00 \\
1.75 \mathrm{E}+01 \\
6.48 \mathrm{E}+01 \\
1.28 \mathrm{E}+01 \\
3.65 \mathrm{E}+00 \\
2.42 \mathrm{E}+00 \\
4.56 \mathrm{E}+00 \\
7.74 \mathrm{E}-01 \\
5.05 \mathrm{E}+00 \\
7.86 \mathrm{E}+01 \\
3.99 \mathrm{E}+00 \\
2.95 \mathrm{E}+01 \\
2.51 \mathrm{E}+00 \\
2.72 \mathrm{E}+00 \\
2.41 \mathrm{E}+00 \\
7.76 \mathrm{E}+00 \\
4.66 \mathrm{E}+01 \\
1.79 \mathrm{E}+01 \\
3.45 \mathrm{E}+01 \\
5.38 \mathrm{E}+01 \\
4.94 \mathrm{E}+01 \\
2.33 \mathrm{E}-01 \\
2.50 \mathrm{E}-01 \\
2.49 \mathrm{E}-01 \\
3.67 \mathrm{E}+00 \\
2.69 \mathrm{E}+00 \\
7.21 \mathrm{E}+01 \\
1.18 \mathrm{E}+01 \\
5.28 \mathrm{E}+00 \\
6.39 \mathrm{E}+00 \\
1.64 \mathrm{E}+01 \\
8.71 \mathrm{E}-01 \\
2.26 \mathrm{E}-01 \\
1.28 \mathrm{E}+00 \\
4.89 \mathrm{E}-01 \\
4.70 \mathrm{E}+00\end{array}$ & $\begin{array}{l}\text { CO60 } \\
4.15 \mathrm{E}+00 \\
5.33 \mathrm{E}-01 \\
1.50 \mathrm{E}+00 \\
1.59 \mathrm{E}+01 \\
1.86 \mathrm{E}+01 \\
9.07 \mathrm{E}+00 \\
1.18 \mathrm{E}+00 \\
3.07 \mathrm{E}-01 \\
2.94 \mathrm{E}+00 \\
2.54 \mathrm{E}-01 \\
5.40 \mathrm{E}+01 \\
1.20 \mathrm{E}+02 \\
1.54 \mathrm{E}+00 \\
6.43 \mathrm{E}+00 \\
5.83 \mathrm{E}+00 \\
3.71 \mathrm{E}+00 \\
1.49 \mathrm{E}+02 \\
6.95 \mathrm{E}+01 \\
5.56 \mathrm{E}+00 \\
1.42 \mathrm{E}+01 \\
1.00 \mathrm{E}+02 \\
1.49 \mathrm{E}+00 \\
6.66 \mathrm{E}-01 \\
8.81 \mathrm{E}+01 \\
9.21 \mathrm{E}+01 \\
5.46 \mathrm{E}+00 \\
7.74 \mathrm{E}+00 \\
1.18 \mathrm{E}+02 \\
5.79 \mathrm{E}+00 \\
1.97 \mathrm{E}+01 \\
4.06 \mathrm{E}+01 \\
1.55 \mathrm{E}+02 \\
2.81 \mathrm{E}+01 \\
8.45 \mathrm{E}+00 \\
5.69 \mathrm{E}+00 \\
9.83 \mathrm{E}+00 \\
1.87 \mathrm{E}+00 \\
1.12 \mathrm{E}+01 \\
1.91 \mathrm{E}+02 \\
8.61 \mathrm{E}+00 \\
7.27 \mathrm{E}+01 \\
6.06 \mathrm{E}+00 \\
6.78 \mathrm{E}+00 \\
5.92 \mathrm{E}+00 \\
1.95 \mathrm{E}+01 \\
1.15 \mathrm{E}+02 \\
3.98 \mathrm{E}+01 \\
7.66 \mathrm{E}+01 \\
1.41 \mathrm{E}+02 \\
1.24 \mathrm{E}+02 \\
1.51 \mathrm{E}-01 \\
2.96 \mathrm{E}-01 \\
1.74 \mathrm{E}-01 \\
4.72 \mathrm{E}+00 \\
3.24 \mathrm{E}+00 \\
1.91 \mathrm{E}+02 \\
2.95 \mathrm{E}+01 \\
7.00 \mathrm{E}+00 \\
8.68 \mathrm{E}+00 \\
4.29 \mathrm{E}+01 \\
1.26 \mathrm{E}+00 \\
3.08 \mathrm{E}-01 \\
1.87 \mathrm{E}+00 \\
6.29 \mathrm{E}-01 \\
5.66 \mathrm{E}+00\end{array}$ & $\begin{array}{l}\text { SE79 } \\
1.10 \mathrm{E}-01 \\
5.24 \mathrm{E}-03 \\
2.30 \mathrm{E}-02 \\
1.39 \mathrm{E}-01 \\
1.45 \mathrm{E}-01 \\
1.44 \mathrm{E}-01 \\
1.85 \mathrm{E}-02 \\
8.74 \mathrm{E}-03 \\
2.53 \mathrm{E}-02 \\
2.19 \mathrm{E}-03 \\
4.20 \mathrm{E}-01 \\
1.03 \mathrm{E}+00 \\
2.21 \mathrm{E}-02 \\
4.93 \mathrm{E}-02 \\
5.04 \mathrm{E}-02 \\
3.16 \mathrm{E}-02 \\
1.27 \mathrm{E}+00 \\
5.40 \mathrm{E}-01 \\
4.34 \mathrm{E}-02 \\
1.19 \mathrm{E}-01 \\
8.40 \mathrm{E}-01 \\
2.14 \mathrm{E}-02 \\
5.17 \mathrm{E}-03 \\
6.75 \mathrm{E}-01 \\
7.49 \mathrm{E}-01 \\
4.45 \mathrm{E}-02 \\
6.51 \mathrm{E}-02 \\
9.78 \mathrm{E}-01 \\
4.44 \mathrm{E}-02 \\
1.60 \mathrm{E}-01 \\
3.11 \mathrm{E}-01 \\
1.15 \mathrm{E}+00 \\
2.29 \mathrm{E}-01 \\
6.48 \mathrm{E}-02 \\
4.37 \mathrm{E}-02 \\
8.12 \mathrm{E}-02 \\
1.40 \mathrm{E}-02 \\
8.99 \mathrm{E}-02 \\
1.39 \mathrm{E}+00 \\
7.11 \mathrm{E}-02 \\
5.22 \mathrm{E}-01 \\
4.52 \mathrm{E}-02 \\
4.89 \mathrm{E}-02 \\
4.33 \mathrm{E}-02 \\
1.37 \mathrm{E}-01 \\
8.24 \mathrm{E}-01 \\
3.19 \mathrm{E}-01 \\
6.14 \mathrm{E}-01 \\
9.48 \mathrm{E}-01 \\
8.74 \mathrm{E}-01 \\
4.49 \mathrm{E}-03 \\
4.63 \mathrm{E}-03 \\
4.77 \mathrm{E}-03 \\
6.78 \mathrm{E}-02 \\
4.99 \mathrm{E}-02 \\
1.27 \mathrm{E}+00 \\
2.08 \mathrm{E}-01 \\
9.74 \mathrm{E}-02 \\
1.18 \mathrm{E}-01 \\
2.90 \mathrm{E}-01 \\
1.60 \mathrm{E}-02 \\
4.17 \mathrm{E}-03 \\
2.35 \mathrm{E}-02 \\
9.03 \mathrm{E}-03 \\
8.71 \mathrm{E}-02\end{array}$ & $\begin{array}{l}\text { SR90 } \\
1.43 E+04 \\
8.17 E+02 \\
3.31 E+03 \\
2.22 E+04 \\
2.35 E+04 \\
2.05 E+04 \\
2.65 E+03 \\
1.13 E+03 \\
4.01 E+03 \\
3.59 E+02 \\
6.81 E+04 \\
1.64 E+05 \\
3.22 E+03 \\
8.01 E+03 \\
8.26 E+03 \\
5.19 E+03 \\
2.01 E+05 \\
8.76 E+04 \\
7.24 E+03 \\
1.89 E+04 \\
1.34 E+05 \\
3.12 E+03 \\
8.39 E+02 \\
1.10 E+05 \\
1.20 E+05 \\
7.36 E+03 \\
1.07 E+04 \\
1.56 E+05 \\
7.21 E+03 \\
2.57 E+04 \\
5.05 E+04 \\
1.87 E+05 \\
3.67 E+04 \\
1.05 E+04 \\
7.31 E+03 \\
1.30 E+04 \\
2.34 E+03 \\
1.45 E+04 \\
2.26 E+05 \\
1.14 E+04 \\
8.56 E+04 \\
7.59 E+03 \\
8.26 E+03 \\
7.30 E+03 \\
2.27 E+04 \\
1.35 E+05 \\
5.14 E+04 \\
9.90 E+04 \\
1.58 E+05 \\
1.44 E+05 \\
5.73 E+02 \\
6.63 E+02 \\
6.16 E+02 \\
9.91 E+03 \\
7.17 E+03 \\
2.11 E+05 \\
3.44 E+04 \\
1.43 E+04 \\
1.73 E+04 \\
4.81 E+04 \\
2.39 E+03 \\
6.16 E+02 \\
3.51 E+03 \\
1.32 E+03 \\
1.25 E+04\end{array}$ & $\begin{array}{l}Y 90 \\
1.43 \mathrm{E}+04 \\
8.17 \mathrm{E}+02 \\
3.31 \mathrm{E}+03 \\
2.22 \mathrm{E}+04 \\
2.35 \mathrm{E}+04 \\
2.05 \mathrm{E}+04 \\
2.65 \mathrm{E}+03 \\
1.13 \mathrm{E}+03 \\
4.01 \mathrm{E}+03 \\
3.59 \mathrm{E}+02 \\
6.81 \mathrm{E}+04 \\
1.64 \mathrm{E}+05 \\
3.22 \mathrm{E}+03 \\
8.01 \mathrm{E}+03 \\
8.26 \mathrm{E}+03 \\
5.19 \mathrm{E}+03 \\
2.02 \mathrm{E}+05 \\
8.76 \mathrm{E}+04 \\
7.25 \mathrm{E}+03 \\
1.89 \mathrm{E}+04 \\
1.34 \mathrm{E}+05 \\
3.12 \mathrm{E}+03 \\
8.39 \mathrm{E}+02 \\
1.10 \mathrm{E}+05 \\
1.20 \mathrm{E}+05 \\
7.36 \mathrm{E}+03 \\
1.07 \mathrm{E}+04 \\
1.56 \mathrm{E}+05 \\
7.21 \mathrm{E}+03 \\
2.57 \mathrm{E}+04 \\
5.05 \mathrm{E}+04 \\
1.87 \mathrm{E}+05 \\
3.67 \mathrm{E}+04 \\
1.05 \mathrm{E}+04 \\
7.31 \mathrm{E}+03 \\
1.30 \mathrm{E}+04 \\
2.34 \mathrm{E}+03 \\
1.45 \mathrm{E}+04 \\
2.26 \mathrm{E}+05 \\
1.14 \mathrm{E}+04 \\
8.56 \mathrm{E}+04 \\
7.59 \mathrm{E}+03 \\
8.26 \mathrm{E}+03 \\
7.30 \mathrm{E}+03 \\
2.27 \mathrm{E}+04 \\
1.35 \mathrm{E}+05 \\
5.14 \mathrm{E}+04 \\
9.90 \mathrm{E}+04 \\
1.58 \mathrm{E}+05 \\
1.45 \mathrm{E}+05 \\
5.73 \mathrm{E}+02 \\
6.63 \mathrm{E}+02 \\
6.16 \mathrm{E}+02 \\
9.91 \mathrm{E}+03 \\
7.17 \mathrm{E}+03 \\
2.11 \mathrm{E}+05 \\
3.44 \mathrm{E}+04 \\
1.43 \mathrm{E}+04 \\
1.73 \mathrm{E}+04 \\
4.82 \mathrm{E}+04 \\
2.39 \mathrm{E}+03 \\
6.16 \mathrm{E}+02 \\
3.51 \mathrm{E}+03 \\
1.32 \mathrm{E}+03 \\
1.25 \mathrm{E}+04\end{array}$ & $\begin{array}{l}2 R 93 \\
5.34 \mathrm{E}-01 \\
2.54 \mathrm{E}-02 \\
1.12 \mathrm{E}-01 \\
6.74 \mathrm{E}-01 \\
7.01 \mathrm{E}-01 \\
6.97 \mathrm{E}-01 \\
8.95 \mathrm{E}-02 \\
4.24 \mathrm{E}-02 \\
1.22 \mathrm{E}-01 \\
1.08 \mathrm{E}-02 \\
2.03 \mathrm{E}+00 \\
4.99 \mathrm{E}+00 \\
1.07 \mathrm{E}-01 \\
2.38 \mathrm{E}-01 \\
2.47 \mathrm{E}-01 \\
1.55 \mathrm{E}-01 \\
6.12 \mathrm{E}+00 \\
2.61 \mathrm{E}+00 \\
2.13 \mathrm{E}-01 \\
5.73 \mathrm{E}-01 \\
4.06 \mathrm{E}+00 \\
1.04 \mathrm{E}-01 \\
2.50 \mathrm{E}-02 \\
3.27 \mathrm{E}+00 \\
3.62 \mathrm{E}+00 \\
2.18 \mathrm{E}-01 \\
3.19 \mathrm{E}-01 \\
4.73 \mathrm{E}+00 \\
2.15 \mathrm{E}-01 \\
7.73 \mathrm{E}-01 \\
1.50 \mathrm{E}+00 \\
5.55 \mathrm{E}+00 \\
1.11 \mathrm{E}+00 \\
3.13 \mathrm{E}-01 \\
2.15 \mathrm{E}-01 \\
3.92 \mathrm{E}-01 \\
6.84 \mathrm{E}-02 \\
4.35 \mathrm{E}-01 \\
6.70 \mathrm{E}+00 \\
3.43 \mathrm{E}-01 \\
2.52 \mathrm{E}+00 \\
2.22 \mathrm{E}-01 \\
2.40 \mathrm{E}-01 \\
2.12 \mathrm{E}-01 \\
6.65 \mathrm{E}-01 \\
3.98 \mathrm{E}+00 \\
1.54 \mathrm{E}+00 \\
2.97 \mathrm{E}+00 \\
4.59 \mathrm{E}+00 \\
4.23 \mathrm{E}+00 \\
2.18 \mathrm{E}-02 \\
2.24 \mathrm{E}-02 \\
2.31 \mathrm{E}-02 \\
3.29 \mathrm{E}-01 \\
2.42 \mathrm{E}-01 \\
6.13 \mathrm{E}+00 \\
1.01 \mathrm{E}+00 \\
4.73 \mathrm{E}-01 \\
5.70 \mathrm{E}-01 \\
1.40 \mathrm{E}+00 \\
7.76 \mathrm{E}-02 \\
2.03 \mathrm{E}-02 \\
1.14 \mathrm{E}-01 \\
4.38 \mathrm{E}-02 \\
4.22 \mathrm{E}-01\end{array}$ \\
\hline
\end{tabular}


HNF-SD-WN-TI-794, ReV. 0

ACTIVITY IN UNSEPARATED FUEL (Curies decayed to $1 / 1 / 94$ )

\begin{tabular}{|c|c|c|c|c|c|c|c|c|c|}
\hline $\begin{array}{r}\text { [Listing \#] } \\
1041 \\
1042 \\
1043 \\
1044 \\
1045 \\
1046 \\
1047 \\
1048 \\
1049 \\
1050 \\
1051 \\
1052 \\
1053 \\
1054 \\
1055 \\
1056 \\
1057 \\
1058 \\
1059 \\
1060 \\
1061 \\
1062 \\
1063 \\
1064 \\
1065 \\
1066 \\
1067 \\
1068 \\
1069 \\
1070 \\
1071 \\
1072 \\
1073 \\
1074 \\
1075 \\
1076 \\
1077 \\
1078 \\
1079 \\
1080 \\
1081 \\
1082 \\
1083 \\
1084 \\
1085 \\
1086 \\
1087 \\
1088 \\
1089 \\
1090 \\
1091 \\
1092 \\
1093 \\
1094 \\
1095 \\
1096 \\
1097 \\
1098 \\
1099 \\
1100 \\
1101 \\
1102 \\
1103 \\
1104 \\
1105\end{array}$ & $\begin{array}{l}\text { NB93* } \\
3.24 \mathrm{E}-01 \\
1.13 \mathrm{E}-02 \\
5.87 \mathrm{E}-02 \\
2.84 \mathrm{E}-01 \\
2.79 \mathrm{E}-01 \\
3.70 \mathrm{E}-01 \\
4.73 \mathrm{E}-02 \\
2.61 \mathrm{E}-02 \\
5.13 \mathrm{E}-02 \\
4.49 \mathrm{E}-03 \\
8.08 \mathrm{E}-01 \\
2.09 \mathrm{E}+00 \\
5.51 \mathrm{E}-02 \\
9.41 \mathrm{E}-02 \\
1.03 \mathrm{E}-01 \\
6.41 \mathrm{E}-02 \\
2.55 \mathrm{E}+00 \\
1.04 \mathrm{E}+00 \\
8.41 \mathrm{E}-02 \\
2.37 \mathrm{E}-01 \\
1.68 \mathrm{E}+00 \\
5.33 \mathrm{E}-02 \\
9.96 \mathrm{E}-03 \\
1.29 \mathrm{E}+00 \\
1.48 \mathrm{E}+00 \\
8.83 \mathrm{E}-02 \\
1.31 \mathrm{E}-01 \\
1.94 \mathrm{E}+00 \\
8.47 \mathrm{E}-02 \\
3.15 \mathrm{E}-01 \\
5.93 \mathrm{E}-01 \\
2.15 \mathrm{E}+00 \\
4.50 \mathrm{E}-01 \\
1.24 \mathrm{E}-01 \\
8.40 \mathrm{E}-02 \\
1.61 \mathrm{E}-01 \\
2.64 \mathrm{E}-02 \\
1.76 \mathrm{E}-01 \\
2.58 \mathrm{E}+00 \\
1.41 \mathrm{E}-01 \\
9.60 \mathrm{E}-01 \\
8.53 \mathrm{E}-02 \\
9.05 \mathrm{E}-02 \\
8.09 \mathrm{E}-02 \\
2.50 \mathrm{E}-01 \\
1.52 \mathrm{E}+00 \\
6.24 \mathrm{E}-01 \\
1.20 \mathrm{E}+00 \\
1.68 \mathrm{E}+00 \\
1.59 \mathrm{E}+00 \\
1.35 \mathrm{E}-02 \\
1.19 \mathrm{E}-02 \\
1.41 \mathrm{E}-02 \\
1.69 \mathrm{E}-01 \\
1.27 \mathrm{E}-01 \\
2.23 \mathrm{E}+00 \\
3.80 \mathrm{E}-01 \\
2.40 \mathrm{E}-01 \\
2.88 \mathrm{E}-01 \\
5.13 \mathrm{E}-01 \\
3.82 \mathrm{E}-02 \\
1.02 \mathrm{E}-02 \\
5.58 \mathrm{E}-02 \\
2.25 \mathrm{E}-02 \\
2.22 \mathrm{E}-01\end{array}$ & $\begin{array}{l}T C 99 \\
3.68 E+00 \\
1.75 E+01 \\
7.69 E-01 \\
4.63 E+00 \\
4.83 E+00 \\
4.80 E+00 \\
6.17 E-01 \\
2.92 E-01 \\
8.44 E-01 \\
7.32 E-02 \\
1.40 E+01 \\
3.45 E+01 \\
7.37 E-01 \\
1.65 E+00 \\
1.68 E+00 \\
1.05 E+00 \\
4.23 E+01 \\
1.80 E+01 \\
1.45 E+00 \\
3.96 E+00 \\
2.80 E+01 \\
7.14 E-01 \\
1.73 E-01 \\
2.25 E+01 \\
2.50 E+01 \\
1.49 E+00 \\
2.17 E+00 \\
3.26 E+01 \\
1.48 E+00 \\
5.33 E+00 \\
1.04 E+01 \\
3.83 E+01 \\
7.63 E+00 \\
2.16 E+00 \\
1.46 E+00 \\
2.71 E+00 \\
4.65 E-01 \\
3.00 E+00 \\
4.64 E+01 \\
2.37 E+00 \\
1.74 E+01 \\
1.51 E+00 \\
1.63 E+00 \\
1.44 E+00 \\
4.58 E+00 \\
2.775 E+01 \\
1.07 E+01 \\
2.05 E+01 \\
3.17 E+01 \\
2.92 E+01 \\
1.50 E-01 \\
1.54 E-01 \\
1.59 E-01 \\
2.26 E+00 \\
1.66 E+00 \\
4.23 E+01 \\
6.95 E+00 \\
3.25 E+00 \\
3.92 E+00 \\
9.66 E+00 \\
5.34 E-01 \\
1.39 E-01 \\
7.83 E-01 \\
3.01 E-01 \\
2.91 E+00\end{array}$ & $\begin{array}{l}\text { RU106 } \\
1.47 E-01 \\
1.24 E+00 \\
5.28 E-01 \\
6.03 E+01 \\
1.19 E+02 \\
2.83 E+00 \\
3.89 E-01 \\
7.93 E-03 \\
1.23 E+01 \\
9.42 E-01 \\
3.46 E+02 \\
5.01 E+02 \\
7.25 E-01 \\
4.37 E+01 \\
2.16 E+01 \\
1.47 E+01 \\
6.66 E+02 \\
4.45 E+02 \\
3.19 E+01 \\
6.68 E+01 \\
4.74 E+02 \\
7.02 E-01 \\
4.27 E+00 \\
6.00 E+02 \\
4.88 E+02 \\
2.60 E+01 \\
3.25 E+01 \\
5.92 E+02 \\
3.94 E+01 \\
1.04 E+02 \\
2.77 E+02 \\
1.21 E+03 \\
1.49 E+02 \\
5.76 E+01 \\
3.47 E+01 \\
4.91 E+01 \\
1.31 E+01 \\
6.32 E+01 \\
1.64 E+03 \\
4.31 E+01 \\
6.57 E+02 \\
4.24 E+01 \\
5.47 E+01 \\
4.48 E+01 \\
1.87 E+02 \\
1.04 E+03 \\
2.24 E+02 \\
4.30 E+02 \\
1.64 E+03 \\
1.18 E+03 \\
3.28 E-03 \\
9.777 E-02 \\
5.33 E-03 \\
2.21 E+00 \\
1.14 E+00 \\
2.40 E+03 \\
2.82 E+02 \\
3.75 E+00 \\
5.24 E+00 \\
5.01 E+02 \\
9.95 E-01 \\
1.84 E-01 \\
1.55 E+00 \\
2.98 E-01 \\
2.00 E+00\end{array}$ & 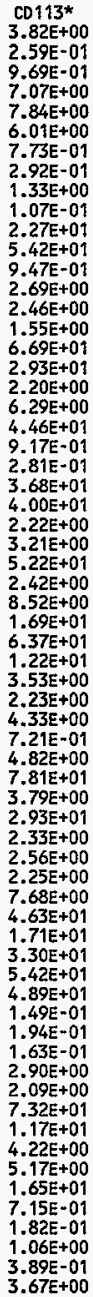 & 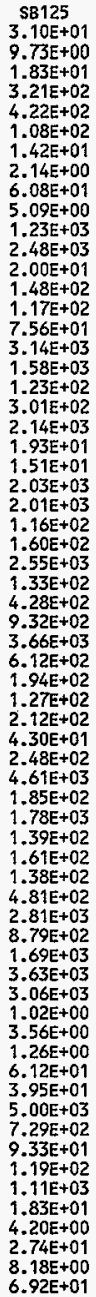 & $\begin{array}{l}\text { SN126 } \\
1.71 \mathrm{E}-01 \\
8.12 \mathrm{E}-03 \\
3.57 \mathrm{E}-02 \\
2.12 \mathrm{E}-01 \\
2.25 \mathrm{E}-01 \\
2.23 \mathrm{E}-01 \\
2.86 \mathrm{E}-02 \\
1.34 \mathrm{E}-02 \\
3.96 \mathrm{E}-02 \\
3.23 \mathrm{E}-03 \\
6.53 \mathrm{E}-01 \\
1.61 \mathrm{E}+00 \\
3.40 \mathrm{E}-02 \\
7.67 \mathrm{E}-02 \\
7.43 \mathrm{E}-02 \\
4.65 \mathrm{E}-02 \\
1.98 \mathrm{E}+00 \\
8.42 \mathrm{E}-01 \\
6.39 \mathrm{E}-02 \\
1.86 \mathrm{E}-01 \\
1.31 \mathrm{E}+00 \\
3.29 \mathrm{E}-02 \\
8.06 \mathrm{E}-03 \\
1.05 \mathrm{E}+00 \\
1.17 \mathrm{E}+00 \\
6.57 \mathrm{E}-02 \\
9.58 \mathrm{E}-02 \\
1.53 \mathrm{E}+00 \\
6.91 \mathrm{E}-02 \\
2.49 \mathrm{E}-01 \\
4.84 \mathrm{E}-01 \\
1.80 \mathrm{E}+00 \\
3.56 \mathrm{E}-01 \\
1.01 \mathrm{E}-01 \\
6.44 \mathrm{E}-02 \\
1.27 \mathrm{E}-01 \\
2.06 \mathrm{E}-02 \\
1.40 \mathrm{E}-01 \\
2.19 \mathrm{E}+00 \\
1.11 \mathrm{E}-01 \\
8.16 \mathrm{E}-01 \\
6.67 \mathrm{E}-02 \\
7.21 \mathrm{E}-02 \\
6.40 \mathrm{E}-02 \\
2.13 \mathrm{E}-01 \\
1.29 \mathrm{E}+00 \\
4.97 \mathrm{E}-01 \\
9.57 \mathrm{E}-01 \\
1.48 \mathrm{E}+00 \\
1.36 \mathrm{E}+00 \\
6.94 \mathrm{E}-03 \\
7.17 \mathrm{E}-03 \\
7.40 \mathrm{E}-03 \\
1.04 \mathrm{E}-01 \\
7.71 \mathrm{E}-02 \\
1.98 \mathrm{E}+00 \\
3.23 \mathrm{E}-01 \\
1.50 \mathrm{E}-01 \\
1.82 \mathrm{E}-01 \\
4.51 \mathrm{E}-01 \\
2.46 \mathrm{E}-02 \\
6.41 \mathrm{E}-03 \\
3.61 \mathrm{E}-02 \\
1.40 \mathrm{E}-02\end{array}$ & $\begin{array}{l}1129 \\
7.29 \mathrm{E}-03 \\
3.46 \mathrm{E}-04 \\
1.52 \mathrm{E}-03 \\
9.07 \mathrm{E}-03 \\
9.58 \mathrm{E}-03 \\
9.51 \mathrm{E}-03 \\
1.22 \mathrm{E}-03 \\
5.74 \mathrm{E}-04 \\
1.68 \mathrm{E}-03 \\
1.40 \mathrm{E}-04 \\
2.78 \mathrm{E}-02 \\
6.86 \mathrm{E}-02 \\
1.45 \mathrm{E}-03 \\
3.26 \mathrm{E}-03 \\
3.21 \mathrm{E}-03 \\
2.01 \mathrm{E}-03 \\
8.41 \mathrm{E}-02 \\
3.58 \mathrm{E}-02 \\
2.76 \mathrm{E}-03 \\
7.88 \mathrm{E}-03 \\
5.58 \mathrm{E}-02 \\
1.40 \mathrm{E}-03 \\
3.43 \mathrm{E}-04 \\
4.47 \mathrm{E}-02 \\
4.96 \mathrm{E}-02 \\
2.83 \mathrm{E}-03 \\
4.14 \mathrm{E}-03 \\
6.50 \mathrm{E}-02 \\
2.94 \mathrm{E}-03 \\
1.06 \mathrm{E}-02 \\
2.06 \mathrm{E}-02 \\
7.63 \mathrm{E}-02 \\
1.51 \mathrm{E}-02 \\
4.29 \mathrm{E}-03 \\
2.78 \mathrm{E}-03 \\
5.39 \mathrm{E}-03 \\
8.89 \mathrm{E}-04 \\
5.96 \mathrm{E}-03 \\
9.27 \mathrm{E}-02 \\
4.72 \mathrm{E}-03 \\
3.47 \mathrm{E}-02 \\
2.88 \mathrm{E}-03 \\
3.11 \mathrm{E}-03 \\
2.76 \mathrm{E}-03 \\
9.07 \mathrm{E}-03 \\
5.48 \mathrm{E}-02 \\
2.11 \mathrm{E}-02 \\
4.07 \mathrm{E}-02 \\
6.28 \mathrm{E}-02 \\
5.78 \mathrm{E}-02 \\
2.96 \mathrm{E}-04 \\
3.05 \mathrm{E}-04 \\
3.15 \mathrm{E}-04 \\
4.45 \mathrm{E}-03 \\
3.28 \mathrm{E}-03 \\
8.43 \mathrm{E}-02 \\
1.38 \mathrm{E}-02 \\
6.40 \mathrm{E}-03 \\
7.75 \mathrm{E}-03 \\
1.92 \mathrm{E}-02 \\
1.05 \mathrm{E}-03 \\
2.74 \mathrm{E}-04 \\
1.54 \mathrm{E}-03 \\
5.95 \mathrm{E}-03\end{array}$ & $\begin{array}{l}\text { CS134 } \\
5.22 \mathrm{E}+00 \\
3.04 \mathrm{E}+00 \\
4.26 \mathrm{E}+00 \\
9.55 \mathrm{E}+01 \\
1.62 \mathrm{E}+02 \\
2.55 \mathrm{E}+01 \\
3.28 \mathrm{E}+00 \\
3.10 \mathrm{E}-01 \\
2.34 \mathrm{E}+01 \\
7.54 \mathrm{E}-01 \\
4.70 \mathrm{E}+02 \\
9.47 \mathrm{E}+02 \\
4.62 \mathrm{E}+00 \\
5.82 \mathrm{E}+01 \\
1.73 \mathrm{E}+01 \\
1.10 \mathrm{E}+01 \\
1.21 \mathrm{E}+03 \\
6.15 \mathrm{E}+02 \\
1.89 \mathrm{E}+01 \\
1.17 \mathrm{E}+02 \\
8.30 \mathrm{E}+02 \\
4.47 \mathrm{E}+00 \\
5.90 \mathrm{E}+00 \\
7.98 \mathrm{E}+02 \\
7.60 \mathrm{E}+02 \\
1.84 \mathrm{E}+01 \\
2.41 \mathrm{E}+01 \\
9.99 \mathrm{E}+02 \\
5.24 \mathrm{E}+01 \\
1.62 \mathrm{E}+02 \\
3.67 \mathrm{E}+02 \\
1.54 \mathrm{E}+03 \\
2.32 \mathrm{E}+02 \\
7.65 \mathrm{E}+01 \\
2.03 \mathrm{E}+01 \\
8.30 \mathrm{E}+01 \\
7.51 \mathrm{E}+00 \\
9.47 \mathrm{E}+01 \\
2.09 \mathrm{E}+03 \\
7.27 \mathrm{E}+01 \\
7.63 \mathrm{E}+02 \\
2.44 \mathrm{E}+01 \\
2.87 \mathrm{E}+01 \\
2.45 \mathrm{E}+01 \\
1.93 \mathrm{E}+02 \\
1.21 \mathrm{E}+03 \\
3.36 \mathrm{E}+02 \\
6.46 \mathrm{E}+02 \\
1.55 \mathrm{E}+03 \\
1.22 \mathrm{E}+03 \\
1.52 \mathrm{E}-01 \\
8.31 \mathrm{E}-01 \\
2.08 \mathrm{E}-01 \\
1.37 \mathrm{E}+01 \\
9.08 \mathrm{E}+00 \\
2.26 \mathrm{E}+03 \\
2.92 \mathrm{E}+02 \\
2.20 \mathrm{E}+01 \\
2.97 \mathrm{E}+01 \\
4.74 \mathrm{E}+02 \\
4.44 \mathrm{E}+00 \\
9.78 \mathrm{E}-01 \\
6.90 \mathrm{E}+00 \\
1.97 \mathrm{E}+00\end{array}$ & 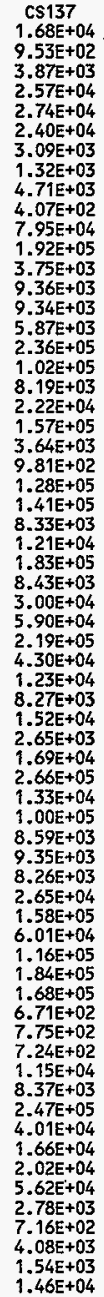 \\
\hline
\end{tabular}


HNF-SD-WH-TI-794, ReV. 0

ACTIVITY IN UNSEPARATED FUEL (Curies decayed to $1 / 1 / 94$ )

\begin{tabular}{|c|c|c|c|c|c|c|c|c|c|}
\hline $\begin{array}{c}\text { Listing } 4] \\
1041 \\
1042 \\
1043 \\
1044 \\
1045 \\
1046 \\
1047 \\
1048 \\
1049 \\
1050 \\
1051 \\
1052 \\
1053 \\
1054 \\
1055 \\
1056 \\
1057 \\
1058 \\
1059 \\
1060 \\
1061 \\
1062 \\
1063 \\
1064 \\
1065 \\
1066 \\
1067 \\
1068 \\
1069 \\
1070 \\
1071 \\
1072 \\
1073 \\
1074 \\
1075 \\
1076 \\
1077 \\
1078 \\
1079 \\
1080 \\
1081 \\
1082 \\
1083 \\
1084 \\
1085 \\
1086 \\
1087 \\
1088 \\
1089 \\
1090 \\
1091 \\
1092 \\
1093 \\
1094 \\
1095 \\
1096 \\
1097 \\
1098 \\
1099 \\
1100 \\
1901 \\
1102 \\
1103 \\
1104 \\
1105\end{array}$ & $\begin{array}{l}B A 137 * \\
1.59 E+04 \\
9.02 E+02 \\
3.66 E+03 \\
2.43 E+04 \\
2.59 E+04 \\
2.28 E+04 \\
2.93 E+03 \\
1.25 E+03 \\
4.46 E+03 \\
3.85 E+02 \\
7.52 E+04 \\
1.82 E+05 \\
3.55 E+03 \\
8.85 E+03 \\
8.84 E+03 \\
5.55 E+03 \\
2.24 E+05 \\
9.68 E+04 \\
7.75 E+03 \\
2.10 E+04 \\
1.49 E+05 \\
3.44 E+03 \\
9.28 E+02 \\
1.21 E+05 \\
1.33 E+05 \\
7.88 E+03 \\
1.15 E+04 \\
1.74 E+05 \\
7.97 E+03 \\
2.84 E+04 \\
5.58 E+04 \\
2.08 E+05 \\
4.06 E+04 \\
1.16 E+04 \\
7.82 E+03 \\
1.44 E+04 \\
2.51 E+03 \\
1.60 E+04 \\
2.52 E+05 \\
1.26 E+04 \\
9.49 E+04 \\
8.13 E+03 \\
8.84 E+03 \\
7.82 E+03 \\
2.50 E+04 \\
1.50 E+05 \\
5.69 E+04 \\
1.10 E+05 \\
1.74 E+05 \\
1.59 E+05 \\
6.35 E+02 \\
7.33 E+02 \\
6.85 E+02 \\
1.09 E+04 \\
7.92 E+03 \\
2.34 E+05 \\
1.58 E+04 \\
1.911 E+04 \\
5.32 E+04 \\
2.63 E+03 \\
6.78 E+02 \\
3.86 E+03 \\
1.45 E+03 \\
1.39 E+04\end{array}$ & $\begin{array}{l}54151 \\
3.88 E+02 \\
1.97 \mathrm{E}+01 \\
8.42 \mathrm{E}+01 \\
5.40 \mathrm{E}+02 \\
5.43 \mathrm{E}+02 \\
5.21 \mathrm{E}+02 \\
6.75 \mathrm{E}+01 \\
3.14 \mathrm{E}+01 \\
9.26 \mathrm{E}+01 \\
9.45 \mathrm{E}+00 \\
1.58 \mathrm{E}+03 \\
3.78 \mathrm{E}+03 \\
8.22 \mathrm{E}+01 \\
1.84 \mathrm{E}+02 \\
2.17 \mathrm{E}+02 \\
1.37 \mathrm{E}+02 \\
4.65 \mathrm{E}+03 \\
2.02 \mathrm{E}+03 \\
1.89 \mathrm{E}+02 \\
4.36 \mathrm{E}+02 \\
3.09 \mathrm{E}+03 \\
7.95 \mathrm{E}+01 \\
1.93 \mathrm{E}+01 \\
2.52 \mathrm{E}+03 \\
2.79 \mathrm{E}+03 \\
1.92 \mathrm{E}+02 \\
2.81 \mathrm{E}+02 \\
3.60 \mathrm{E}+03 \\
1.66 \mathrm{E}+02 \\
5.95 \mathrm{E}+02 \\
1.16 \mathrm{E}+03 \\
4.25 \mathrm{E}+03 \\
8.51 \mathrm{E}+02 \\
2.42 \mathrm{E}+02 \\
1.90 \mathrm{E}+02 \\
2.98 \mathrm{0}+02 \\
6.02 \mathrm{E}+01 \\
3.35 \mathrm{E}+02 \\
5.04 \mathrm{E}+03 \\
2.61 \mathrm{E}+02 \\
1.93 \mathrm{E}+03 \\
1.95 \mathrm{E}+02 \\
2.11 \mathrm{E}+02 \\
1.87 \mathrm{E}+02 \\
5.21 \mathrm{E}+02 \\
3.06 \mathrm{E}+03 \\
1.19 \mathrm{E}+03 \\
2.29 \mathrm{E}+03 \\
3.57 \mathrm{E}+03 \\
3.32 \mathrm{E}+03 \\
1.58 \mathrm{E}+01 \\
1.69 \mathrm{E}+01 \\
1.67 \mathrm{E}+01 \\
2.54 \mathrm{E}+02 \\
1.83 \mathrm{E}+02 \\
4.72 \mathrm{E}+03 \\
7.90 \mathrm{E}+02 \\
3.63 \mathrm{E}+02 \\
4.35 \mathrm{E}+02 \\
1.09 \mathrm{E}+03 \\
6.03 \mathrm{E}+01 \\
1.57 \mathrm{E}+01 \\
8.79 \mathrm{E}+01 \\
3.33 \mathrm{E}+01 \\
3.18 \mathrm{E}+02\end{array}$ & $\begin{array}{l}\text { EU152 } \\
3.60 \mathrm{E}-01 \\
2.41 \mathrm{E}-02 \\
8.91 \mathrm{E}-02 \\
5.53 \mathrm{E}-01 \\
7.85 \mathrm{E}-01 \\
5.75 \mathrm{E}-01 \\
7.10 \mathrm{E}-02 \\
2.39 \mathrm{E}-02 \\
1.45 \mathrm{E}-01 \\
2.27 \mathrm{E}-03 \\
2.28 \mathrm{E}+00 \\
5.86 \mathrm{E}+00 \\
8.10 \mathrm{E}-02 \\
2.75 \mathrm{E}-01 \\
5.21 \mathrm{E}-02 \\
3.07 \mathrm{E}-02 \\
7.22 \mathrm{E}+00 \\
3.00 \mathrm{E}+00 \\
4.36 \mathrm{E}-02 \\
6.80 \mathrm{E}-01 \\
4.82 \mathrm{E}+00 \\
7.84 \mathrm{E}-02 \\
2.87 \mathrm{E}-02 \\
3.77 \mathrm{E}+00 \\
4.08 \mathrm{E}+00 \\
5.17 \mathrm{E}-02 \\
6.80 \mathrm{E}-02 \\
5.64 \mathrm{E}+00 \\
2.48 \mathrm{E}-01 \\
8.71 \mathrm{E}-01 \\
1.73 \mathrm{E}+00 \\
6.90 \mathrm{E}+00 \\
1.25 \mathrm{E}+00 \\
3.62 \mathrm{E}-01 \\
4.73 \mathrm{E}-02 \\
4.68 \mathrm{E}-01 \\
1.83 \mathrm{E}-02 \\
4.93 \mathrm{E}-01 \\
9.18 \mathrm{E}+00 \\
4.10 \mathrm{E}-01 \\
3.18 \mathrm{E}+00 \\
5.93 \mathrm{E}-02 \\
6.49 \mathrm{E}-02 \\
5.72 \mathrm{E}-02 \\
7.51 \mathrm{E}-01 \\
5.02 \mathrm{E}+00 \\
1.75 \mathrm{E}+00 \\
3.37 \mathrm{E}+00 \\
5.56 \mathrm{E}+00 \\
4.78 \mathrm{E}+00 \\
1.32 \mathrm{E}-02 \\
1.81 \mathrm{E}-02 \\
1.54 \mathrm{E}-02 \\
2.38 \mathrm{E}-01 \\
1.89 \mathrm{E}-01 \\
7.96 \mathrm{E}+00 \\
1.14 \mathrm{E}+00 \\
3.61 \mathrm{E}-01 \\
4.68 \mathrm{E}-01 \\
1.70 \mathrm{E}+00 \\
5.89 \mathrm{E}-02 \\
1.50 \mathrm{E}-02 \\
9.06 \mathrm{E}-02 \\
3.51 \mathrm{E}-02 \\
3.42 \mathrm{E}-01\end{array}$ & $\begin{array}{l}E U 154 \\
4.11 E+01 \\
3.46 E+00 \\
1.16 E+01 \\
8.48 E+01 \\
1.17 E+02 \\
7.36 E+01 \\
9.19 E+00 \\
2.76 E+00 \\
2.08 E+01 \\
5.68 E-01 \\
3.41 E+02 \\
8.43 E+02 \\
1.09 E+01 \\
4.11 E+01 \\
1.31 E+01 \\
7.99 E+00 \\
1.04 E+03 \\
4.47 E+02 \\
1.16 E+01 \\
9.86 E+01 \\
6.98 E+02 \\
1.05 E+01 \\
4.28 E+00 \\
5.63 E+02 \\
6.02 E+02 \\
1.25 E+01 \\
1.71 E+01 \\
8.20 E+02 \\
3.70 E+01 \\
1.28 E+02 \\
2.59 E+02 \\
1.03 E+03 \\
1.84 E+02 \\
5.40 E+01 \\
1.22 E+01 \\
6.81 E+01 \\
4.33 E+00 \\
7.29 E+01 \\
1.35 E+03 \\
5.96 E+01 \\
4.77 E+02 \\
1.40 E+01 \\
1.55 E+01 \\
1.36 E+01 \\
1.15 E+02 \\
7.53 E+02 \\
2.59 E+02 \\
4.98 E+02 \\
8.55 E+02 \\
7.34 E+02 \\
1.48 E+00 \\
2.33 E+00 \\
1.74 E+00 \\
3.22 E+01 \\
2.46 E+01 \\
1.22 E+03 \\
1.75 E+02 \\
4.88 E+01 \\
6.28 E+01 \\
2.61 E+02 \\
8.19 E+00 \\
2.05 E+00 \\
1.25 E+01 \\
4.66 E+00 \\
4.43 E+01\end{array}$ & $\begin{array}{l}E U 155 \\
3.86 E+01 \\
5.31 E+00 \\
1.45 E+01 \\
1.64 E+02 \\
1.86 E+02 \\
8.70 E+01 \\
1.14 E+01 \\
2.91 E+00 \\
2.85 E+01 \\
2.90 E+00 \\
5.39 E+02 \\
1.17 E+03 \\
1.52 E+01 \\
6.39 E+01 \\
6.66 E+01 \\
4.26 E+01 \\
1.46 E+03 \\
6.90 E+02 \\
6.42 E+01 \\
1.38 E+02 \\
9.80 E+02 \\
1.47 E+01 \\
6.61 E+00 \\
8.76 E+02 \\
9.12 E+02 \\
6.24 E+01 \\
8.87 E+01 \\
1.16 E+03 \\
5.75 E+01 \\
1.95 E+02 \\
4.03 E+02 \\
1.52 E+03 \\
2.78 E+02 \\
8.40 E+01 \\
6.55 E+01 \\
9.60 E+01 \\
2.15 E+01 \\
1.11 E+02 \\
1.84 E+03 \\
8.41 E+01 \\
7.16 E+02 \\
6.95 E+01 \\
7.79 E+01 \\
6.79 E+01 \\
1.96 E+02 \\
1.13 E+03 \\
3.95 E+02 \\
7.59 E+02 \\
1.41 E+03 \\
1.25 E+03 \\
1.41 E+00 \\
2.85 E+00 \\
1.61 E+00 \\
4.69 E+01 \\
3.15 E+01 \\
1.89 E+03 \\
2.98 E+02 \\
6.91 E+01 \\
8.50 E+01 \\
4.30 E+02 \\
1.26 E+01 \\
3.07 E+00 \\
1.86 E+01 \\
6.14 E+00 \\
5.47 E+01\end{array}$ & $\begin{array}{l}\text { RA226 } \\
2.87 \mathrm{E}-06 \\
5.49 \mathrm{E}-08 \\
3.89 \mathrm{E}-07 \\
1.41 \mathrm{E}-06 \\
1.06 \mathrm{E}-06 \\
2.43 \mathrm{E}-06 \\
3.17 \mathrm{E}-07 \\
2.65 \mathrm{E}-07 \\
2.00 \mathrm{E}-07 \\
4.07 \mathrm{E}-08 \\
3.07 \mathrm{E}-06 \\
8.22 \mathrm{E}-06 \\
3.67 \mathrm{E}-07 \\
3.48 \mathrm{E}-07 \\
9.35 \mathrm{E}-07 \\
5.86 \mathrm{E}-07 \\
9.87 \mathrm{E}-06 \\
3.90 \mathrm{E}-06 \\
7.13 \mathrm{E}-07 \\
9.09 \mathrm{E}-07 \\
6.44 \mathrm{E}-06 \\
3.55 \mathrm{E}-07 \\
3.73 \mathrm{E}-08 \\
4.77 \mathrm{E}-06 \\
5.75 \mathrm{E}-06 \\
7.41 \mathrm{E}-07 \\
1.16 \mathrm{E}-06 \\
7.36 \mathrm{E}-06 \\
3.13 \mathrm{E}-07 \\
1.22 \mathrm{E}-06 \\
2.19 \mathrm{E}-06 \\
7.45 \mathrm{E}-06 \\
1.75 \mathrm{E}-06 \\
4.58 \mathrm{E}-07 \\
6.89 \mathrm{E}-07 \\
6.11 \mathrm{E}-07 \\
1.98 \mathrm{E}-07 \\
6.77 \mathrm{E}-07 \\
8.25 \mathrm{E}-06 \\
5.35 \mathrm{E}-07 \\
3.22 \mathrm{E}-06 \\
6.42 \mathrm{E}-07 \\
6.59 \mathrm{E}-07 \\
5.98 \mathrm{E}-07 \\
8.89 \mathrm{E}-07 \\
5.09 \mathrm{E}-06 \\
2.40 \mathrm{E}-06 \\
4.63 \mathrm{E}-06 \\
5.54 \mathrm{E}-06 \\
5.67 \mathrm{E}-06 \\
1.33 \mathrm{E}-07 \\
7.85 \mathrm{E}-08 \\
1.27 \mathrm{E}-07 \\
1.16 \mathrm{E}-06 \\
8.54 \mathrm{E}-07 \\
6.94 \mathrm{E}-06 \\
1.35 \mathrm{E}-06 \\
1.57 \mathrm{E}-06 \\
1.78 \mathrm{E}-06 \\
1.69 \mathrm{E}-06 \\
2.41 \mathrm{E}-07 \\
6.73 \mathrm{E}-08 \\
1.40 \mathrm{E}-07 \\
1.46 \mathrm{E}-07\end{array}$ & $\begin{array}{l}\text { RA228 } \\
1.65 \mathrm{E}-10 \\
3.79 \mathrm{E}-12 \\
2.46 \mathrm{E}-11 \\
9.60 \mathrm{E}-11 \\
7.81 \mathrm{E}-11 \\
1.54 \mathrm{E}-10 \\
1.99 \mathrm{E}-11 \\
1.46 \mathrm{E}-11 \\
1.47 \mathrm{E}-11 \\
2.41 \mathrm{E}-12 \\
2.26 \mathrm{E}-10 \\
6.03 \mathrm{E}-10 \\
2.31 \mathrm{E}-11 \\
2.59 \mathrm{E}-11 \\
5.53 \mathrm{E}-11 \\
3.46 \mathrm{E}-11 \\
7.27 \mathrm{E}-10 \\
2.88 \mathrm{E}-10 \\
4.29 \mathrm{E}-11 \\
6.71 \mathrm{E}-11 \\
4.75 \mathrm{E}-10 \\
2.24 \mathrm{E}-11 \\
2.76 \mathrm{E}-12 \\
3.54 \mathrm{E}-10 \\
4.21 \mathrm{E}-10 \\
4.46 \mathrm{E}-11 \\
6.91 \mathrm{E}-11 \\
5.45 \mathrm{E}-10 \\
2.33 \mathrm{E}-11 \\
8.98 \mathrm{E}-11 \\
1.63 \mathrm{E}-10 \\
5.64 \mathrm{E}-10 \\
1.29 \mathrm{E}-10 \\
3.40 \mathrm{E}-11 \\
4.18 \mathrm{E}-11 \\
4.53 \mathrm{E}-11 \\
1.22 \mathrm{E}-11 \\
4.98 \mathrm{E}-11 \\
6.40 \mathrm{E}-10 \\
3.96 \mathrm{E}-11 \\
2.46 \mathrm{E}-10 \\
3.96 \mathrm{E}-11 \\
4.10 \mathrm{E}-11 \\
3.71 \mathrm{E}-11 \\
6.65 \mathrm{E}-11 \\
3.88 \mathrm{E}-10 \\
1.77 \mathrm{E}-10 \\
3.40 \mathrm{E}-10 \\
4.22 \mathrm{E}-10 \\
4.24 \mathrm{E}-10 \\
7.37 \mathrm{E}-12 \\
4.96 \mathrm{E}-12 \\
7.26 \mathrm{E}-12 \\
7.24 \mathrm{E}-11 \\
5.37 \mathrm{E}-11 \\
1.38 \mathrm{E}-10 \\
1.15 \mathrm{E}-10 \\
1.29 \mathrm{E}-10 \\
1.54 \mathrm{E}-11 \\
4.25 \mathrm{E}-12 \\
2.20 \mathrm{E}-11 \\
9.21 \mathrm{E}-12 \\
9.23 \mathrm{E}-11\end{array}$ & $\begin{array}{l}\text { AC227 } \\
1.53 E-05 \\
3.17 E-07 \\
2.16 E-06 \\
8.24 E-06 \\
6.23 E-06 \\
1.34 E-05 \\
1.76 E-06 \\
1.41 E-06 \\
1.16 E-06 \\
2.46 E-07 \\
1.81 E-05 \\
4.777 E-05 \\
2.06 E-06 \\
2.05 E-06 \\
5.65 E-06 \\
3.55 E-06 \\
5.74 E-05 \\
2.29 E-05 \\
4.34 E-06 \\
5.29 E-06 \\
3.75 E-05 \\
1.99 E-06 \\
2.19 E-07 \\
2.81 E-05 \\
3.36 E-05 \\
4.49 E-06 \\
7.02 E-06 \\
4.29 E-05 \\
1.84 E-06 \\
7.17 E-06 \\
1.29 E-05 \\
4.39 E-05 \\
1.03 E-05 \\
2.69 E-06 \\
4.20 E-06 \\
3.56 E-06 \\
1.21 E-06 \\
3.96 E-06 \\
4.86 E-05 \\
3.12 E-06 \\
1.90 E-05 \\
3.91 E-06 \\
4.03 E-06 \\
3.65 E-06 \\
5.28 E-06 \\
3.000-05 \\
1.41 E-05 \\
2.71 E-05 \\
3.30 E-05 \\
3.36 E-05 \\
7.02 E-07 \\
4.36 E-07 \\
6.74 E-07 \\
6.51 E-06 \\
4.75 E-06 \\
4.13 E-05 \\
8.01 E-06 \\
8.83 E-06 \\
9.99 E-06 \\
1.01 E-05 \\
1.37 E-06 \\
3.80 E-07 \\
1.93 E-06 \\
8.08 E-07 \\
8.09 E-06\end{array}$ & $\begin{array}{l}\text { TH229 } \\
1.33 \mathrm{E}-08 \\
3.52 \mathrm{E}-10 \\
2.07 \mathrm{E}-09 \\
8.41 \mathrm{E}-09 \\
8.15 \mathrm{E}-09 \\
1.32 \mathrm{E}-08 \\
1.67 \mathrm{E}-09 \\
1.09 \mathrm{E}-09 \\
1.56 \mathrm{E}-09 \\
1.26 \mathrm{E}-10 \\
2.36 \mathrm{E}-08 \\
6.34 \mathrm{E}-08 \\
1.89 \mathrm{E}-09 \\
2.75 \mathrm{E}-09 \\
2.90 \mathrm{E}-09 \\
1.79 \mathrm{E}-09 \\
7.69 \mathrm{E}-08 \\
3.05 \mathrm{E}-08 \\
2.29 \mathrm{E}-09 \\
7.13 \mathrm{E}-09 \\
5.05 \mathrm{E}-08 \\
1.83 \mathrm{E}-09 \\
2.92 \mathrm{E}-10 \\
3.76 \mathrm{E}-08 \\
4.38 \mathrm{E}-08 \\
2.45 \mathrm{E}-09 \\
3.66 \mathrm{E}-09 \\
5.82 \mathrm{E}-08 \\
2.47 \mathrm{E}-09 \\
9.34 \mathrm{E}-09 \\
1.73 \mathrm{E}-08 \\
6.28 \mathrm{E}-08 \\
1.34 \mathrm{E}-08 \\
3.61 \mathrm{E}-09 \\
2.28 \mathrm{E}-09 \\
4.83 \mathrm{E}-09 \\
7.12 \mathrm{E}-10 \\
5.20 \mathrm{E}-09 \\
7.53 \mathrm{E}-08 \\
4.23 \mathrm{E}-09 \\
2.77 \mathrm{E}-08 \\
2.30 \mathrm{E}-09 \\
2.42 \mathrm{E}-09 \\
2.17 \mathrm{E}-09 \\
7.12 \mathrm{E}-09 \\
4.38 \mathrm{E}-08 \\
1.84 \mathrm{E}-08 \\
3.55 \mathrm{E}-08 \\
4.75 \mathrm{E}-08 \\
4.53 \mathrm{E}-08 \\
5.71 \mathrm{E}-10 \\
4.20 \mathrm{E}-10 \\
5.85 \mathrm{E}-10 \\
5.80 \mathrm{E}-09 \\
4.47 \mathrm{E}-09 \\
6.30 \mathrm{E}-08 \\
1.08 \mathrm{E}-08 \\
8.19 \mathrm{E}-09 \\
9.76 \mathrm{E}-09 \\
1.45 \mathrm{E}-08 \\
1.27 \mathrm{E}-09 \\
3.44 \mathrm{E}-10 \\
1.85 \mathrm{E}-09 \\
7.76 \mathrm{E}-10 \\
7.83 \mathrm{E}-09\end{array}$ \\
\hline
\end{tabular}


HNF-5D-WH-T1-794, ReV. D

ACTIVITY IN UNSEPARATED FUEL (Curies decayed to 1/1/94)

\begin{tabular}{|c|c|c|c|c|c|c|c|c|c|}
\hline $\begin{array}{r}\text { [Listing \#] } \\
1041 \\
1042 \\
1043 \\
1044 \\
1045 \\
1046 \\
1047 \\
1048 \\
1049 \\
1050 \\
1051 \\
1052 \\
1053 \\
1054 \\
1055 \\
1056 \\
1057 \\
1058 \\
1059 \\
1060 \\
1061 \\
1062 \\
1063 \\
1064 \\
1065 \\
1066 \\
1067 \\
1068 \\
1069 \\
1070 \\
1071 \\
1072 \\
1073 \\
1074 \\
1075 \\
1076 \\
1077 \\
1078 \\
1079 \\
1080 \\
1081 \\
1082 \\
1083 \\
1084 \\
1085 \\
1086 \\
1087 \\
1088 \\
1089 \\
1090 \\
1091 \\
1092 \\
1093 \\
1094 \\
1095 \\
1096 \\
1097 \\
1098 \\
1099 \\
1100 \\
1101 \\
1102 \\
1103 \\
1104 \\
1105\end{array}$ & $\begin{array}{l}\text { TH232 } \\
2.85 \mathrm{E}-10 \\
8.64 \mathrm{E}-12 \\
4.82 \mathrm{E}-11 \\
2.30 \mathrm{E}-10 \\
1.97 \mathrm{E}-10 \\
2.99 \mathrm{E}-10 \\
3.90 \mathrm{E}-11 \\
2.49 \mathrm{E}-11 \\
3.54 \mathrm{E}-11 \\
5.84 \mathrm{E}-12 \\
5.72 \mathrm{E}-10 \\
1.45 \mathrm{E}-09 \\
4.63 \mathrm{E}-11 \\
6.58 \mathrm{E}-11 \\
1.34 \mathrm{E}-10 \\
8.47 \mathrm{E}-11 \\
1.76 \mathrm{E}-09 \\
7.29 \mathrm{E}-10 \\
1.09 \mathrm{E}-10 \\
1.64 \mathrm{E}-10 \\
1.16 \mathrm{E}-09 \\
4.49 \mathrm{E}-11 \\
6.98 \mathrm{E}-12 \\
9.01 \mathrm{E}-10 \\
1.04 \mathrm{E}-09 \\
1.11 \mathrm{E}-10 \\
1.70 \mathrm{E}-10 \\
1.34 \mathrm{E}-09 \\
5.92 \mathrm{E}-11 \\
2.22 \mathrm{E}-10 \\
4.14 \mathrm{E}-10 \\
1.46 \mathrm{E}-09 \\
3.18 \mathrm{E}-10 \\
8.64 \mathrm{E}-11 \\
1.07 \mathrm{E}-10 \\
1.11 \mathrm{E}-10 \\
3.19 \mathrm{E}-11 \\
1.24 \mathrm{E}-10 \\
1.67 \mathrm{E}-09 \\
9.71 \mathrm{E}-11 \\
6.46 \mathrm{E}-10 \\
1.03 \mathrm{E}-10 \\
1.09 \mathrm{E}-10 \\
9.76 \mathrm{E}-11 \\
1.77 \mathrm{E}-10 \\
1.02 \mathrm{E}-09 \\
4.40 \mathrm{E}-10 \\
8.47 \mathrm{E}-10 \\
1.15 \mathrm{E}-09 \\
1.12 \mathrm{E}-09 \\
1.25 \mathrm{E}-11 \\
9.69 \mathrm{E}-12 \\
1.25 \mathrm{E}-11 \\
1.45 \mathrm{E}-10 \\
1.05 \mathrm{E}-10 \\
1.48 \mathrm{E}-09 \\
2.68 \mathrm{E}-10 \\
2.01 \mathrm{E}-10 \\
2.33 \mathrm{E}-10 \\
3.51 \mathrm{E}-10 \\
3.21 \mathrm{E}-11 \\
8.68 \mathrm{E}-12 \\
4.60 \mathrm{E}-11 \\
1.85 \mathrm{E}-11 \\
1.81 \mathrm{E}-10\end{array}$ & $\begin{array}{l}\text { PA231 } \\
5.82 \mathrm{E}-05 \\
1.79 \mathrm{E}-06 \\
9.97 \mathrm{E}-06 \\
5.00 \mathrm{E}-05 \\
4.02 \mathrm{E}-05 \\
6.11 \mathrm{E}-05 \\
8.05 \mathrm{E}-06 \\
5.28 \mathrm{E}-06 \\
7.02 \mathrm{E}-06 \\
1.52 \mathrm{E}-06 \\
1.16 \mathrm{E}-04 \\
2.89 \mathrm{E}-04 \\
9.77 \mathrm{E}-06 \\
1.33 \mathrm{E}-05 \\
3.50 \mathrm{E}-05 \\
2.22 \mathrm{E}-05 \\
3.50 \mathrm{E}-04 \\
1.48 \mathrm{E}-04 \\
2.87 \mathrm{E}-05 \\
3.25 \mathrm{E}-05 \\
2.30 \mathrm{E}-04 \\
9.46 \mathrm{E}-06 \\
1.41 \mathrm{E}-06 \\
1.82 \mathrm{E}-04 \\
2.11 \mathrm{E}-04 \\
2.88 \mathrm{E}-05 \\
4.43 \mathrm{E}-05 \\
2.66 \mathrm{E}-04 \\
1.20 \mathrm{E}-05 \\
4.50 \mathrm{E}-05 \\
8.39 \mathrm{E}-05 \\
2.90 \mathrm{E}-04 \\
6.44 \mathrm{E}-05 \\
1.75 \mathrm{E}-05 \\
2.80 \mathrm{E}-05 \\
2.21 \mathrm{E}-05 \\
8.21 \mathrm{E}-06 \\
2.51 \mathrm{E}-05 \\
3.24 \mathrm{E}-04 \\
1.93 \mathrm{E}-05 \\
1.29 \mathrm{E}-04 \\
2.66 \mathrm{E}-05 \\
2.80 \mathrm{E}-05 \\
2.51 \mathrm{E}-05 \\
3.63 \mathrm{E}-05 \\
2.03 \mathrm{E}-04 \\
8.90 \mathrm{E}-05 \\
1.71 \mathrm{E}-04 \\
2.33 \mathrm{E}-04 \\
2.31 \mathrm{E}-04 \\
2.59 \mathrm{E}-06 \\
1.99 \mathrm{E}-06 \\
2.54 \mathrm{E}-06 \\
3.09 \mathrm{E}-05 \\
2.19 \mathrm{E}-05 \\
2.95 \mathrm{E}-04 \\
5.51 \mathrm{E}-05 \\
4.25 \mathrm{E}-05 \\
4.85 \mathrm{E}-05 \\
7.12 \mathrm{E}-05 \\
6.85 \mathrm{E}-06 \\
1.85 \mathrm{E}-06 \\
9.69 \mathrm{E}-06 \\
3.83 \mathrm{E}-06 \\
3.73 \mathrm{E}-05\end{array}$ & $\begin{array}{c}\text { U23E } \\
5.58 \mathrm{E}-04 \\
2.71 \mathrm{E}-05 \\
1.17 \mathrm{E}-04 \\
6.51 \mathrm{E}-04 \\
7.79 \mathrm{E}-04 \\
7.51 \mathrm{E}-04 \\
9.41 \mathrm{E}-05 \\
4.06 \mathrm{E}-05 \\
1.44 \mathrm{E}-04 \\
6.54 \mathrm{E}-06 \\
2.26 \mathrm{E}-03 \\
5.84 \mathrm{E}-03 \\
1.08 \mathrm{E}-04 \\
2.69 \mathrm{E}-04 \\
1.50 \mathrm{E}-04 \\
9.29 \mathrm{E}-05 \\
7.16 \mathrm{E}-03 \\
2.95 \mathrm{E}-03 \\
1.28 \mathrm{E}-04 \\
6.70 \mathrm{E}-04 \\
4.75 \mathrm{E}-03 \\
1.05 \mathrm{E}-04 \\
2.82 \mathrm{E}-05 \\
3.68 \mathrm{E}-03 \\
4.09 \mathrm{E}-03 \\
1.35 \mathrm{E}-04 \\
1.94 \mathrm{E}-04 \\
5.53 \mathrm{E}-03 \\
2.42 \mathrm{E}-04 \\
8.71 \mathrm{E}-04 \\
1.69 \mathrm{E}-03 \\
6.48 \mathrm{E}-03 \\
1.25 \mathrm{E}-03 \\
3.53 \mathrm{E}-04 \\
1.31 \mathrm{E}=04 \\
4.59 \mathrm{E}-04 \\
4.33 \mathrm{E}-05 \\
4.90 \mathrm{E}-04 \\
8.24 \mathrm{E}-03 \\
4.02 \mathrm{E}-04 \\
2.94 \mathrm{E}-03 \\
1.40 \mathrm{E}-04 \\
1.52 \mathrm{E}-04 \\
1.34 \mathrm{E}-04 \\
7.25 \mathrm{E}-04 \\
4.64 \mathrm{E}-03 \\
1.74 \mathrm{E}-03 \\
3.35 \mathrm{E}-03 \\
5.14 \mathrm{E}-03 \\
4.62 \mathrm{E}-03 \\
2.18 \mathrm{E}-05 \\
2.38 \mathrm{E}-05 \\
2.41 \mathrm{E}-05 \\
3.25 \mathrm{E}-04 \\
2.51 \mathrm{E}-04 \\
7.11 \mathrm{E}-03 \\
1.10 \mathrm{E}-03 \\
4.78 \mathrm{E}-04 \\
5.96 \mathrm{E}-04 \\
1.57 \mathrm{E}-03 \\
7.70 \mathrm{E}-05 \\
2.00 \mathrm{E}-05 \\
1.16 \mathrm{E}-04 \\
4.56 \mathrm{E}-05 \\
4.48 \mathrm{E}-04\end{array}$ & 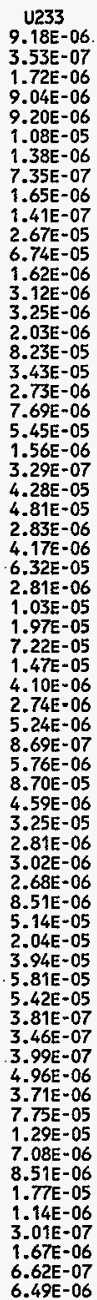 & $\begin{array}{l}\text { U234 } \\
3.66 \mathrm{E}+00 \\
1.79 \mathrm{E}=01 \\
7.87 \mathrm{E}-01 \\
5.37 \mathrm{E}+00 \\
4.70 \mathrm{E}+00 \\
4.77 \mathrm{E}+00 \\
6.31 \mathrm{E}-01 \\
3.21 \mathrm{E}-01 \\
7.68 \mathrm{E}-01 \\
1.61 \mathrm{E}-01 \\
1.36 \mathrm{E}+01 \\
3.16 \mathrm{E}+01 \\
7.96 \mathrm{E}-01 \\
1.57 \mathrm{E}+00 \\
3.71 \mathrm{E}+00 \\
2.37 \mathrm{E}+00 \\
3.87 \mathrm{E}+01 \\
1.73 \mathrm{E}+01 \\
3.26 \mathrm{E}+00 \\
3.63 \mathrm{E}+00 \\
2.57 \mathrm{E}+01 \\
7.71 \mathrm{E}-01 \\
1.65 \mathrm{E}-01 \\
2.16 \mathrm{E}+01 \\
2.39 \mathrm{E}+01 \\
3.18 \mathrm{E}+00 \\
4.78 \mathrm{E}+00 \\
2.99 \mathrm{E}+01 \\
1.42 \mathrm{E}+00 \\
5.10 \mathrm{E}+00 \\
9.92 \mathrm{E}+00 \\
3.51 \mathrm{E}+01 \\
7.30 \mathrm{E}+00 \\
2.07 \mathrm{E}+00 \\
3.21 \mathrm{E}+00 \\
2.48 \mathrm{E}+00 \\
9.66 \mathrm{E}-01 \\
2.87 \mathrm{E}+00 \\
3.98 \mathrm{E}+01 \\
2.17 \mathrm{E}+00 \\
1.60 \mathrm{E}+01 \\
3.13 \mathrm{E}+00 \\
3.38 \mathrm{E}+00 \\
3.00 \mathrm{E}+00 \\
4.55 \mathrm{E}+00 \\
2.52 \mathrm{E}+01 \\
1.02 \mathrm{E}+01 \\
1.96 \mathrm{E}+01 \\
3.03 \mathrm{E}+01 \\
2.90 \mathrm{E}+01 \\
1.56 \mathrm{E}-01 \\
1.57 \mathrm{E}-01 \\
1.58 \mathrm{E}-01 \\
2.52 \mathrm{E}+00 \\
1.73 \mathrm{E}+00 \\
3.88 \mathrm{E}+01 \\
6.90 \mathrm{E}+00 \\
3.51 \mathrm{E}+00 \\
4.08 \mathrm{E}+00 \\
9.25 \mathrm{E}+00 \\
5.93 \mathrm{E}-01 \\
1.55 \mathrm{E}-01 \\
8.46 \mathrm{E}-01 \\
3.13 \mathrm{E}-01 \\
2.95 \mathrm{E}+00\end{array}$ & $\begin{array}{l}\text { U235 } \\
1.36 \mathrm{E}-01 \\
6.68 \mathrm{E}-03 \\
2.93 \mathrm{E}-02 \\
2.02 \mathrm{E}-01 \\
1.74 \mathrm{E}-01 \\
1.77 \mathrm{E}-01 \\
2.35 \mathrm{E}-02 \\
1.21 \mathrm{E}-02 \\
2.83 \mathrm{E}-02 \\
6.31 \mathrm{E}-03 \\
5.06 \mathrm{E}-01 \\
1.16 \mathrm{E}+00 \\
2.98 \mathrm{E}-02 \\
5.83 \mathrm{E}-02 \\
1.45 \mathrm{E}-01 \\
9.30 \mathrm{E}-02 \\
1.43 \mathrm{E}+00 \\
6.39 \mathrm{E}-01 \\
1.28 \mathrm{E}-01 \\
1.34 \mathrm{E}-01 \\
9.47 \mathrm{E}-01 \\
2.89 \mathrm{E}-02 \\
6.12 \mathrm{E}-03 \\
7.99 \mathrm{E}-01 \\
8.86 \mathrm{E}-01 \\
1.24 \mathrm{E}-01 \\
1.87 \mathrm{E}-01 \\
1.10 \mathrm{E}+00 \\
5.25 \mathrm{E}-02 \\
1.89 \mathrm{E}-01 \\
3.67 \mathrm{E}-01 \\
1.30 \mathrm{E}+00 \\
2.70 \mathrm{E}-01 \\
7.66 \mathrm{E}-02 \\
1.26 \mathrm{E}-01 \\
9.16 \mathrm{E}-02 \\
3.77 \mathrm{E}-02 \\
1.06 \mathrm{E}-01 \\
1.46 \mathrm{E}+00 \\
8.01 \mathrm{E}-02 \\
5.89 \mathrm{E}-01 \\
1.22 \mathrm{E}-01 \\
1.32 \mathrm{E}-01 \\
1.17 \mathrm{E}-01 \\
1.69 \mathrm{E}-01 \\
9.30 \mathrm{E}-01 \\
3.78 \mathrm{E}-01 \\
7.27 \mathrm{E}-01 \\
1.12 \mathrm{E}+00 \\
1.08 \mathrm{E}+00 \\
5.81 \mathrm{E}-03 \\
5.82 \mathrm{E}-03 \\
5.87 \mathrm{E}-03 \\
9.44 \mathrm{E}-02 \\
6.45 \mathrm{E}-02 \\
1.43 \mathrm{E}+00 \\
2.56 \mathrm{E}-01 \\
1.32 \mathrm{E}-01 \\
1.52 \mathrm{E}-01 \\
3.43 \mathrm{E}-01 \\
2.23 \mathrm{E}-02 \\
5.81 \mathrm{E}-03 \\
3.17 \mathrm{E}-02 \\
1.17 \mathrm{E}-02 \\
1.10 \mathrm{E}-01\end{array}$ & $\begin{array}{l}\text { U236 } \\
2.89 \mathrm{E}-01 \\
1.40 \mathrm{E}-02 \\
6.15 \mathrm{E}-02 \\
4.04 \mathrm{E}-01 \\
3.73 \mathrm{E}-01 \\
3.76 \mathrm{E}-01 \\
4.93 \mathrm{E}-02 \\
2.46 \mathrm{E}-02 \\
6.23 \mathrm{E}-02 \\
1.04 \mathrm{E}-02 \\
1.08 \mathrm{E}+00 \\
2.55 \mathrm{E}+00 \\
6.12 \mathrm{E}-02 \\
1.26 \mathrm{E}-01 \\
2.39 \mathrm{E}-01 \\
1.53 \mathrm{E}-01 \\
3.13 \mathrm{E}+00 \\
1.38 \mathrm{E}+00 \\
2.10 \mathrm{E}-01 \\
2.94 \mathrm{E}-01 \\
2.08 \mathrm{E}+00 \\
5.92 \mathrm{E}-02 \\
1.32 \mathrm{E}-02 \\
1.72 \mathrm{E}+00 \\
1.91 \mathrm{E}+00 \\
2.06 \mathrm{E}-01 \\
3.09 \mathrm{E}-01 \\
2.42 \mathrm{E}+00 \\
1.13 \mathrm{E}-01 \\
4.07 \mathrm{E}-01 \\
7.92 \mathrm{E}-01 \\
2.84 \mathrm{E}+00 \\
5.83 \mathrm{E}-01 \\
1.65 \mathrm{E}-01 \\
2.07 \mathrm{E}-01 \\
2.01 \mathrm{E}-01 \\
6.31 \mathrm{E}-02 \\
2.29 \mathrm{E}-01 \\
3.29 \mathrm{E}+00 \\
1.76 \mathrm{E}-01 \\
1.29 \mathrm{E}+00 \\
2.04 \mathrm{E}-01 \\
2.21 \mathrm{E}-01 \\
1.96 \mathrm{E}-01 \\
3.59 \mathrm{E}-01 \\
2.04 \mathrm{E}+00 \\
8.13 \mathrm{E}-01 \\
1.57 \mathrm{E}+00 \\
2.42 \mathrm{E}+00 \\
2.28 \mathrm{E}+00 \\
1.21 \mathrm{E}-02 \\
1.23 \mathrm{E}-02 \\
1.25 \mathrm{E}-02 \\
1.92 \mathrm{E}-01 \\
1.34 \mathrm{E}-01 \\
3.14 \mathrm{E}+00 \\
5.44 \mathrm{E}-01 \\
2.70 \mathrm{E}-01 \\
3.17 \mathrm{E}-01 \\
7.38 \mathrm{E}-01 \\
4.52 \mathrm{E}-02 \\
1.18 \mathrm{E}-02 \\
6.50 \mathrm{E}-02 \\
2.44 \mathrm{E}-02 \\
2.31 \mathrm{E}-01\end{array}$ & $\begin{array}{c}\text { U238 } \\
2.53 \mathrm{E}+00 \\
1.23 \mathrm{E}-01 \\
5.39 \mathrm{E}-01 \\
3.66 \mathrm{E}+00 \\
3.22 \mathrm{E}+00 \\
3.26 \mathrm{E}+00 \\
4.32 \mathrm{E}-01 \\
2.19 \mathrm{E}-01 \\
5.27 \mathrm{E}-01 \\
1.09 \mathrm{E}-01 \\
9.34 \mathrm{E}+00 \\
2.17 \mathrm{E}+01 \\
5.44 \mathrm{E}-01 \\
1.08 \mathrm{E}+00 \\
2.50 \mathrm{E}+00 \\
1.60 \mathrm{E}+00 \\
2.66 \mathrm{E}+01 \\
1.18 \mathrm{E}+01 \\
2.20 \mathrm{E}+00 \\
2.49 \mathrm{E}+00 \\
1.76 \mathrm{E}+01 \\
5.27 \mathrm{E}-01 \\
1.13 \mathrm{E}-01 \\
1.48 \mathrm{E}+01 \\
1.64 \mathrm{E}+01 \\
2.14 \mathrm{E}+00 \\
3.23 \mathrm{E}+00 \\
2.05 \mathrm{E}+01 \\
9.71 \mathrm{E}-01 \\
3.50 \mathrm{E}+00 \\
6.80 \mathrm{E}+00 \\
2.41 \mathrm{E}+01 \\
5.00 \mathrm{E}+00 \\
1.42 \mathrm{E}+00 \\
2.17 \mathrm{E}+00 \\
1.70 \mathrm{E}+00 \\
6.52 \mathrm{E}-01 \\
1.97 \mathrm{E}+00 \\
2.74 \mathrm{E}+01 \\
1.49 \mathrm{E}+00 \\
1.10 \mathrm{E}+01 \\
2.11 \mathrm{E}+00 \\
2.28 \mathrm{E}+00 \\
2.02 \mathrm{E}+00 \\
3.11 \mathrm{E}+00 \\
1.73 \mathrm{E}+01 \\
6.99 \mathrm{E}+00 \\
1.35 \mathrm{E}+01 \\
2.08 \mathrm{E}+01 \\
1.98 \mathrm{E}+01 \\
1.06 \mathrm{E}-01 \\
1.07 \mathrm{E}-01 \\
1.08 \mathrm{E}-01 \\
1.72 \mathrm{E}+00 \\
1.18 \mathrm{E}+00 \\
2.66 \mathrm{E}+01 \\
4.72 \mathrm{E}+00 \\
2.40 \mathrm{E}+00 \\
2.79 \mathrm{E}+00 \\
6.34 \mathrm{E}+00 \\
4.05 \mathrm{E}-01 \\
1.06 \mathrm{E}-01 \\
5.78 \mathrm{E}-01 \\
2.14 \mathrm{E}-01 \\
2.02 \mathrm{E}+00\end{array}$ & $\begin{array}{l}\text { NP237 } \\
5.35 \mathrm{E}-02 \\
2.52 \mathrm{E}-03 \\
1.11 \mathrm{E}-02 \\
6.57 \mathrm{E}-02 \\
7.00 \mathrm{E}-02 \\
6.96 \mathrm{E}-02 \\
8.90 \mathrm{E}-03 \\
4.19 \mathrm{E}-03 \\
1.23 \mathrm{E}-02 \\
9.87 \mathrm{E}-04 \\
2.03 \mathrm{E}-01 \\
5.03 \mathrm{E}-01 \\
1.06 \mathrm{E}-02 \\
2.39 \mathrm{E}-02 \\
2.27 \mathrm{E}-02 \\
1.42 \mathrm{E}-02 \\
6.17 \mathrm{E}-01 \\
2.62 \mathrm{E}-01 \\
1.95 \mathrm{E}-02 \\
5.78 \mathrm{E}-02 \\
4.09 \mathrm{E}-01 \\
1.02 \mathrm{E}-02 \\
2.51 \mathrm{E}-03 \\
3.27 \mathrm{E}-01 \\
3.63 \mathrm{E}-01 \\
2.01 \mathrm{E}-02 \\
2.93 \mathrm{E}-02 \\
4.777 \mathrm{E}-01 \\
2.15 \mathrm{E}-02 \\
7.74 \mathrm{E}-02 \\
1.50 \mathrm{E}-01 \\
5.59 \mathrm{E}-01 \\
1.11 \mathrm{E}-01 \\
3.14 \mathrm{E}-02 \\
1.97 \mathrm{E}-02 \\
3.96 \mathrm{E}-02 \\
6.30 \mathrm{E}-03 \\
4.36 \mathrm{E}-02 \\
6.82 \mathrm{E}-01 \\
3.46 \mathrm{E}-02 \\
2.54 \mathrm{E}-01 \\
2.04 \mathrm{E}-02 \\
2.21 \mathrm{E}-02 \\
1.96 \mathrm{E}-02 \\
6.62 \mathrm{E}-02 \\
4.01 \mathrm{E}-01 \\
1.55 \mathrm{E}-01 \\
2.98 \mathrm{E}-01 \\
4.59 \mathrm{E}-01 \\
4.21 \mathrm{E}-01 \\
2.17 \mathrm{E}-03 \\
2.23 \mathrm{E}-03 \\
2.31 \mathrm{E}-03 \\
3.24 \mathrm{E}-02 \\
2.40 \mathrm{E}-02 \\
6.17 \mathrm{E}-01 \\
1.00 \mathrm{E}-01 \\
4.66 \mathrm{E}-02 \\
5.65 \mathrm{E}-02 \\
1.40 \mathrm{E}-01 \\
7.62 \mathrm{E}-03 \\
1.00 \mathrm{E}-03\end{array}$ \\
\hline
\end{tabular}


HNF-SD-WM-TI-794, Rev. 0

ACTIVITY IN UNSEPARATED FUEL (Curies decayed to 1/1/94)

\begin{tabular}{|c|c|c|c|c|c|c|c|c|c|}
\hline $\begin{array}{r}\text { isting } 10 \\
1041 \\
1042 \\
1043 \\
1044 \\
1045 \\
1046 \\
1047 \\
1048 \\
1049 \\
1050 \\
1051 \\
1052 \\
1053 \\
1054 \\
1055 \\
1056 \\
1057 \\
1058 \\
1059 \\
1060 \\
1061 \\
1062 \\
1063 \\
1064 \\
1065 \\
1066 \\
1067 \\
1068 \\
1069 \\
1070 \\
1071 \\
1072 \\
1073 \\
1074 \\
1075 \\
1076 \\
1077 \\
1078 \\
1079 \\
1080 \\
1081 \\
1082 \\
1083 \\
1084 \\
1085 \\
1086 \\
1087 \\
1088 \\
1089 \\
1090 \\
1091 \\
1092 \\
1093 \\
1094 \\
1095 \\
1096 \\
1097 \\
1098 \\
1099 \\
1100 \\
1101 \\
1102 \\
1103 \\
1104 \\
1105\end{array}$ & $\begin{array}{l}P U 238 \\
3.32 E+01 \\
1.62 E+00 \\
6.92 E+00 \\
3.69 E+01 \\
4.82 E+01 \\
4.48 E+01 \\
5.54 E+00 \\
2.31 E+00 \\
9.08 E+00 \\
2.45 E-01 \\
1.40 E+02 \\
3.68 E+02 \\
6.22 E+00 \\
1.68 E+01 \\
5.62 E+00 \\
3.41 E+00 \\
4.51 E+02 \\
1.84 E+02 \\
4.71 E+00 \\
4.23 E+01 \\
3.00 E+02 \\
6.03 E+00 \\
1.76 E+00 \\
2.30 E+02 \\
2.54 E+02 \\
5.22 E+00 \\
7.27 E+00 \\
3.49 E+02 \\
1.51 E+01 \\
5.41 E+01 \\
1.06 E+02 \\
4.13 E+02 \\
7.74 E+01 \\
2.20 E+01 \\
4.91 E+00 \\
2.90 E+01 \\
1.72 E+00 \\
3.05 E+01 \\
5.39 E+02 \\
2.54 E+01 \\
1.88 E+02 \\
5.57 E+00 \\
6.03 E+00 \\
5.34 E+00 \\
4.49 E+01 \\
2.97 E+02 \\
1.08 E+02 \\
2.08 E+02 \\
3.25 E+02 \\
2.86 E+02 \\
1.27 E+00 \\
1.41 E+00 \\
1.44 E+00 \\
1.85 E+01 \\
1.47 E+01 \\
4.59 E+02 \\
6.82 E+01 \\
2.75 E+01 \\
3.49 E+01 \\
9.92 E+01 \\
4.38 E+00 \\
1.14 E+00 \\
6.66 E+00 \\
2.67 E+00 \\
2.65 E+01\end{array}$ & $\begin{array}{l}\text { PU239 } \\
4.17 E+02 \\
1.99 E+01 \\
8.75 E+01 \\
5.35 E+02 \\
5.46 E+02 \\
5.44 E+02 \\
7.02 E+01 \\
3.35 E+01 \\
9.46 E+01 \\
8.90 E+00 \\
1.58 E+03 \\
3.86 E+03 \\
8.44 E+01 \\
1.86 E+02 \\
2.05 E+02 \\
1.28 E+02 \\
4.74 E+03 \\
2.03 E+03 \\
1.76 E+02 \\
4.44 E+02 \\
3.14 E+03 \\
8.18 E+01 \\
1.95 E+01 \\
2.54 E+03 \\
2.82 E+03 \\
1.80 E+02 \\
2.64 E+02 \\
3.66 E+03 \\
1.67 E+02 \\
6.01 E+02 \\
1.17 E+03 \\
4.30 E+03 \\
8.60 E+02 \\
2.44 E+02 \\
1.777 E+02 \\
3.04 E+02 \\
5.64 E+01 \\
3.39 E+02 \\
5.15 E+03 \\
2.66 E+02 \\
1.95 E+03 \\
1.83 E+02 \\
1.98 E+02 \\
1.75 E+02 \\
5.19 E+02 \\
3.09 E+03 \\
1.20 E+03 \\
2.31 E+03 \\
3.57 E+03 \\
3.31 E+03 \\
1.71 E+01 \\
1.76 E+01 \\
1.80 E+01 \\
2.60 E+02 \\
1.90 E+02 \\
4.75 E+03 \\
7.88 E+02 \\
3.72 E+02 \\
4.48 E+02 \\
1.09 E+03 \\
6.14 E+01 \\
1.60 E+01 \\
8.97 E+01 \\
3.44 E+01 \\
3.31 E+02\end{array}$ & 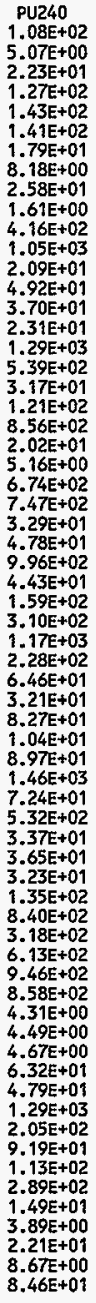 & $\begin{array}{l}P U 241 \\
2.51 E+03 \\
1.64 E+02 \\
6.12 E+02 \\
3.69 E+03 \\
5.35 E+03 \\
3.97 E+03 \\
4.88 E+02 \\
1.65 E+02 \\
9.95 E+02 \\
1.10 E+01 \\
1.55 E+04 \\
4.02 E+04 \\
5.52 E+02 \\
1.87 E+03 \\
2.54 E+02 \\
1.44 E+02 \\
4.96 E+04 \\
2.05 E+04 \\
2.04 E+02 \\
4.66 E+03 \\
3.31 E+04 \\
5.35 E+02 \\
1.96 E+02 \\
2.57 E+04 \\
2.79 E+04 \\
2.62 E+02 \\
3.31 E+02 \\
3.87 E+04 \\
1.69 E+03 \\
5.95 E+03 \\
1.18 E+04 \\
4.72 E+04 \\
8.51 E+03 \\
2.46 E+03 \\
2.30 E+02 \\
3.21 E+03 \\
9.61 E+01 \\
3.36 E+03 \\
6.31 E+04 \\
2.81 E+03 \\
2.17 E+04 \\
3.11 E+02 \\
3.41 E+02 \\
3.00 E+02 \\
5.09 E+03 \\
3.43 E+04 \\
1.19 E+04 \\
2.30 E+04 \\
3.78 E+04 \\
3.24 E+04 \\
9.16 E+01 \\
1.24 E+02 \\
1.08 E+02 \\
1.62 E+03 \\
1.29 E+03 \\
5.43 E+04 \\
7.74 E+03 \\
2.46 E+03 \\
3.20 E+03 \\
1.15 E+04 \\
3.99 E+02 \\
1.02 E+02 \\
6.16 E+02 \\
2.41 E+02 \\
2.35 E+03\end{array}$ & $\begin{array}{l}\text { PU242 } \\
7.51 E-03 \\
3.17 E-04 \\
1.39 E-03 \\
4.15 E-03 \\
1.07 E-02 \\
9.86 E-03 \\
1.12 E-03 \\
3.68 E-04 \\
2.35 E-03 \\
0.00 E+00 \\
3.11 E-02 \\
9.38 E-02 \\
1.03 E-03 \\
3.87 E-03 \\
0.00 E+00 \\
0.00 E+00 \\
1.15 E-01 \\
4.24 E-02 \\
0.00 E+00 \\
1.08 E-02 \\
7.64 E-02 \\
9.99 E-04 \\
4.06 E-04 \\
5.30 E-02 \\
5.87 E-02 \\
0.00 E+00 \\
0.00 E+00 \\
8.89 E-02 \\
3.48 E-03 \\
1.25 E-02 \\
2.44 E-02 \\
1.04 E-01 \\
1.79 E-02 \\
5.08 E-03 \\
0.00 E+00 \\
7.38 E-03 \\
0.00 E+00 \\
7.05 E-03 \\
1.53 E-01 \\
6.46 E-03 \\
4.74 E-02 \\
0.00 E+00 \\
0.00 E+00 \\
0.00 E+00 \\
9.41 E-03 \\
7.49 E-02 \\
2.50 E-02 \\
4.82 E-02 \\
7.44 E-02 \\
5.99 E-02 \\
2.54 E-04 \\
2.92 E-04 \\
3.27 E-04 \\
2.70 E-03 \\
2.82 E-03 \\
1.15 E-01 \\
1.43 E-02 \\
4.55 E-03 \\
6.66 E-03 \\
2.27 E-02 \\
6.37 E-04 \\
1.66 E-04 \\
1.10 E-03 \\
5.12 E-04 \\
5.50 E-03\end{array}$ & $\begin{array}{l}\text { AM241 } \\
1.32 \mathrm{E}+02 \\
4.39 \mathrm{E}+00 \\
2.28 \mathrm{E}+01 \\
8.91 \mathrm{E}+01 \\
1.17 \mathrm{E}+02 \\
1.51 \mathrm{E}+02 \\
1.84 \mathrm{E}+01 \\
9.00 \mathrm{E}+00 \\
2.39 \mathrm{E}+01 \\
2.62 \mathrm{E}-01 \\
3.39 \mathrm{E}+02 \\
9.63 \mathrm{E}+02 \\
1.96 \mathrm{E}+01 \\
4.05 \mathrm{E}+01 \\
6.02 \mathrm{E}+00 \\
3.37 \mathrm{E}+00 \\
1.17 \mathrm{E}+03 \\
4.47 \mathrm{E}+02 \\
4.42 \mathrm{E}+00 \\
1.09 \mathrm{E}+02 \\
7.73 \mathrm{E}+02 \\
1.89 \mathrm{E}+01 \\
4.28 \mathrm{E}+00 \\
5.54 \mathrm{E}+02 \\
6.35 \mathrm{E}+02 \\
5.92 \mathrm{E}+00 \\
7.66 \mathrm{E}+00 \\
8.94 \mathrm{E}+02 \\
3.64 \mathrm{E}+01 \\
1.35 \mathrm{E}+02 \\
2.55 \mathrm{E}+02 \\
9.91 \mathrm{E}+02 \\
1.94 \mathrm{E}+02 \\
5.32 \mathrm{E}+01 \\
4.91 \mathrm{E}+00 \\
7.42 \mathrm{E}+01 \\
2.00 \mathrm{E}+00 \\
7.57 \mathrm{E}+01 \\
1.30 \mathrm{E}+03 \\
6.49 \mathrm{E}+01 \\
4.42 \mathrm{E}+02 \\
6.46 \mathrm{E}+00 \\
6.85 \mathrm{E}+00 \\
6.13 \mathrm{E}+00 \\
1.02 \mathrm{E}+02 \\
6.97 \mathrm{E}+02 \\
2.69 \mathrm{E}+02 \\
5.17 \mathrm{E}+02 \\
7.23 \mathrm{E}+02 \\
6.47 \mathrm{E}+02 \\
5.13 \mathrm{E}+00 \\
4.67 \mathrm{E}+00 \\
5.76 \mathrm{E}+00 \\
5.73 \mathrm{E}+01 \\
4.82 \mathrm{E}+01 \\
1.02 \mathrm{E}+03 \\
1.55 \mathrm{E}+02 \\
8.54 \mathrm{E}+01 \\
1.09 \mathrm{E}+02 \\
2.21 \mathrm{E}+02 \\
1.29 \mathrm{E}+01 \\
3.45 \mathrm{E}+00 \\
1.98 \mathrm{E}+01 \\
8.53 \mathrm{E}+00 \\
8.76 \mathrm{E}+01\end{array}$ & 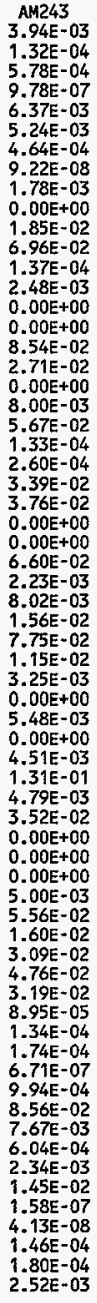 & $\begin{array}{l}\text { CN242 } \\
1.06 \mathrm{E}-02 \\
3.66 \mathrm{E}-04 \\
1.58 \mathrm{E}-03 \\
5.55 \mathrm{E}-09 \\
1.79 \mathrm{E}-02 \\
1.43 \mathrm{E}-02 \\
1.27 \mathrm{E}-03 \\
5.01 \mathrm{E}-16 \\
4.95 \mathrm{E}-03 \\
0.00 \mathrm{E}+00 \\
5.18 \mathrm{E}-02 \\
1.94 \mathrm{E}-01 \\
3.80 \mathrm{E}-04 \\
6.93 \mathrm{E}-03 \\
0.00 \mathrm{E}+00 \\
0.00 \mathrm{E}+00 \\
2.38 \mathrm{E}-01 \\
7.60 \mathrm{E}-02 \\
0.00 \mathrm{E}+00 \\
2.23 \mathrm{E}-02 \\
1.58 \mathrm{E}-01 \\
3.68 \mathrm{E}-04 \\
7.28 \mathrm{E}-04 \\
9.50 \mathrm{E}-02 \\
1.05 \mathrm{E}-01 \\
0.00 \mathrm{E}+00 \\
0.00 \mathrm{E}+00 \\
1.84 \mathrm{E}-01 \\
6.24 \mathrm{E}-03 \\
2.24 \mathrm{E}-02 \\
4.37 \mathrm{E}-02 \\
2.17 \mathrm{E}-01 \\
3.21 \mathrm{E}-02 \\
9.11 \mathrm{E}-03 \\
0.00 \mathrm{E}+00 \\
1.53 \mathrm{E}-02 \\
0.00 \mathrm{E}+00 \\
1.26 \mathrm{E}-02 \\
3.66 \mathrm{E}-01 \\
1.34 \mathrm{E}-02 \\
9.89 \mathrm{E}-02 \\
0.00 \mathrm{E}+00 \\
0.00 \mathrm{E}+00 \\
0.00 \mathrm{E}+00 \\
1.41 \mathrm{E}-02 \\
1.56 \mathrm{E}-01 \\
4.48 \mathrm{E}-02 \\
8.63 \mathrm{E}-02 \\
1.34 \mathrm{E}-01 \\
8.97 \mathrm{E}-02 \\
2.40 \mathrm{E}-04 \\
3.66 \mathrm{E}-04 \\
4.66 \mathrm{E}-04 \\
1.17 \mathrm{E}-11 \\
2.73 \mathrm{E}-03 \\
2.41 \mathrm{E}-01 \\
2.16 \mathrm{E}-02 \\
1.68 \mathrm{E}-03 \\
6.46 \mathrm{E}-03 \\
4.10 \mathrm{E}-02 \\
1.19 \mathrm{E}-11 \\
1.42 \mathrm{E}-12 \\
4.06 \mathrm{E}-04 \\
4.95 \mathrm{E}-04 \\
6.89 \mathrm{E}-03\end{array}$ & $\begin{array}{l}\text { CM243 } \\
4.78 \mathrm{E}-04 \\
4.64 \mathrm{E}-06 \\
1.88 \mathrm{E}-05 \\
0.00 \mathrm{E}+00 \\
1.29 \mathrm{E}-03 \\
7.23 \mathrm{E}-04 \\
1.50 \mathrm{E}-05 \\
0.00 \mathrm{E}+00 \\
4.97 \mathrm{E}-04 \\
0.00 \mathrm{E}+00 \\
3.75 \mathrm{E}-03 \\
1.91 \mathrm{E}-02 \\
0.00 \mathrm{E}+00 \\
5.71 \mathrm{E}-04 \\
0.00 \mathrm{E}+00 \\
0.00 \mathrm{E}+00 \\
2.35 \mathrm{E}-02 \\
6.24 \mathrm{E}-03 \\
0.00 \mathrm{E}+00 \\
2.20 \mathrm{E}-03 \\
1.56 \mathrm{E}-02 \\
0.00 \mathrm{E}+00 \\
5.98 \mathrm{E}-05 \\
7.82 \mathrm{E}-03 \\
8.58 \mathrm{E}-03 \\
0.00 \mathrm{E}+00 \\
0.00 \mathrm{E}+00 \\
1.82 \mathrm{E}-02 \\
5.14 \mathrm{E}-04 \\
1.83 \mathrm{E}-03 \\
3.60 \mathrm{E}-03 \\
2.18 \mathrm{E}-02 \\
2.62 \mathrm{E}-03 \\
7.50 \mathrm{E}-04 \\
0.00 \mathrm{E}+00 \\
1.54 \mathrm{E}-03 \\
0.00 \mathrm{E}+00 \\
1.03 \mathrm{E}-03 \\
4.23 \mathrm{E}-02 \\
1.33 \mathrm{E}-03 \\
9.99 \mathrm{E}-03 \\
0.00 \mathrm{E}+00 \\
0.00 \mathrm{E}+00 \\
0.00 \mathrm{E}+00 \\
8.01 \mathrm{E}-04 \\
1.58 \mathrm{E}-02 \\
3.67 \mathrm{E}-03 \\
7.06 \mathrm{E}-03 \\
1.13 \mathrm{E}-02 \\
5.10 \mathrm{E}-03 \\
0.00 \mathrm{E}+00 \\
1.03 \mathrm{E}-05 \\
2.16 \mathrm{E}-05 \\
0.00 \mathrm{E}+00 \\
0.00 \mathrm{E}+00 \\
1.25 \mathrm{E}-02 \\
0.00 \mathrm{E}+03 \\
0.00 \mathrm{E}+00 \\
7\end{array}$ \\
\hline
\end{tabular}


HNF-SD-WM-TI-794, ReV. 0

ACTIVITY IN UNSEPARATED FUEL (Curies decayed to 1/1/94)

\begin{tabular}{|c|c|}
\hline $\begin{array}{r}\text { Listing } 11 \\
1041 \\
1042 \\
1043 \\
1044 \\
1045 \\
1046 \\
1047 \\
1048 \\
1049 \\
1050 \\
1051 \\
1052 \\
1053 \\
1054 \\
1055 \\
1056 \\
1057 \\
1058 \\
1059 \\
1060 \\
1061 \\
1062 \\
1063 \\
1064 \\
1065 \\
1066 \\
1067 \\
1068 \\
1069 \\
1070 \\
1071 \\
1072 \\
1073 \\
1074 \\
1075 \\
1076 \\
1077 \\
1078 \\
1079 \\
1080 \\
1081 \\
1082 \\
1083 \\
1084 \\
1085 \\
1086 \\
1087 \\
1088 \\
1089 \\
1090 \\
1091 \\
1092 \\
1093 \\
1094 \\
1095 \\
1096 \\
1097 \\
1098 \\
1099 \\
1100 \\
1101 \\
1102 \\
1103 \\
1104 \\
1105\end{array}$ & $\begin{array}{l}\text { CM244 } \\
1.20 \mathrm{E}-02 \\
2.05 \mathrm{E}-04 \\
7.90 \mathrm{E}-04 \\
0.00 \mathrm{E}+00 \\
3.51 \mathrm{E}-02 \\
1.90 \mathrm{E}-02 \\
6.30 \mathrm{E}-04 \\
0.00 \mathrm{E}+00 \\
1.28 \mathrm{E}-02 \\
0.00 \mathrm{E}+00 \\
1.02 \mathrm{E}-01 \\
4.92 \mathrm{E}-01 \\
1.29 \mathrm{E}-07 \\
1.53 \mathrm{E}-02 \\
0.00 \mathrm{E}+00 \\
0.00 \mathrm{E}+00 \\
6.07 \mathrm{E}-01 \\
1.67 \mathrm{E}-01 \\
0.00 \mathrm{E}+00 \\
5.70 \mathrm{E}-02 \\
4.04 \mathrm{E}-01 \\
1.25 \mathrm{E}-07 \\
1.60 \mathrm{E}-03 \\
2.09 \mathrm{E}-01 \\
2.28 \mathrm{E}-01 \\
0.00 \mathrm{E}+00 \\
0.00 \mathrm{E}+00 \\
4.72 \mathrm{E}-01 \\
1.37 \mathrm{E}-02 \\
4.86 \mathrm{E}-02 \\
9.62 \mathrm{E}-02 \\
5.72 \mathrm{E}-01 \\
6.96 \mathrm{E}-02 \\
2.01 \mathrm{E}-02 \\
0.00 \mathrm{E}+00 \\
3.92 \mathrm{E}-02 \\
0.00 \mathrm{E}+00 \\
2.75 \mathrm{E}-02 \\
1.10 \mathrm{E}+00 \\
3.43 \mathrm{E}-02 \\
2.63 \mathrm{E}-01 \\
0.00 \mathrm{E}+00 \\
0.00 \mathrm{E}+00 \\
0.00 \mathrm{E}+00 \\
2.29 \mathrm{E}-02 \\
4.15 \mathrm{E}-01 \\
9.76 \mathrm{E}-02 \\
1.88 \mathrm{E}-01 \\
3.05 \mathrm{E}-01 \\
1.46 \mathrm{E}-01 \\
8.82 \mathrm{E}-08 \\
3.09 \mathrm{E}-04 \\
5.36 \mathrm{E}-04 \\
0.00 \mathrm{E}+00 \\
1.19 \mathrm{E}-06 \\
6.53 \mathrm{E}-01 \\
3.56 \mathrm{E}-02 \\
5.75 \mathrm{E}-07 \\
2.90 \mathrm{E}-00 \\
9.31 \mathrm{E}-02 \\
0.00 \mathrm{E}+00 \\
0.00 \mathrm{E}+00 \\
1.43 \mathrm{E}-07 \\
2.19 \mathrm{E}-07 \\
5.86 \mathrm{E}-03\end{array}$ \\
\hline
\end{tabular}


HNF-SD-WM-TI-794, ReV. 0

UNIFIED SEPARATIONS PRODUCTION DATA

\begin{tabular}{|c|c|c|c|c|c|c|c|c|c|c|}
\hline & & & & & & & & & & \\
\hline [Listing \#] & KEY & $\begin{array}{l}\text { FUEL } \\
\text { TYPE }\end{array}$ & NTU & \&Pu-240 & MWd/MTU & PLANT & MO & YR. & $\begin{array}{l}\text { SEP'N } \\
\text { KEY }\end{array}$ & $\begin{array}{l}\text { DAYS } \\
\text { COOLING }\end{array}$ \\
\hline 1106 & 14056 & $\mathrm{ZR}-1.1 \mathrm{U}$ & $3.82 E+00$ & 6.559 & $1.44 \mathrm{E}+03$ & PUREX & 5 & 85 & 14843.09 & 787 \\
\hline 1107 & 14618 & $2 R-1.1 U$ & $4.84 E+00$ & 6.427 & $1.41 \mathrm{E}+03$ & PUREX & 5 & 85 & 14843.1 & 225 \\
\hline 1108 & 13648 & $2 R-1.1 U$ & $4.70 E+00$ & 7.165 & $1.59 E+03$ & PUREX & 5 & 85 & 14843.06 & 1195 \\
\hline 1109 & 14576 & $\mathrm{ZR}-1.1 \mathrm{U}$ & $1.96 E+01$ & 6.484 & $1.43 \mathrm{E}+03$ & PUREX & 5 & 85 & 14843.08 & 267 \\
\hline 1110 & 14012 & $2 R-1.1 U$ & $1.59 \mathrm{E}+01$ & 6.571 & $1.45 E+03$ & PUREX & 5 & 85 & 14843.07 & 831 \\
\hline 1111 & 14056 & $\mathrm{ZR}-1 . \mathrm{U} \mathrm{U}$ & $7.88 \mathrm{E}+00$ & 6.559 & $1.44 E+03$ & PUREX & 6 & 85 & 14873.01 & 817 \\
\hline 1112 & 14221 & $2 R-1.1 U$ & $9.48 \mathrm{E}+00$ & 6.401 & $1.41 E+03$ & PUREX & 6 & 85 & 14873.02 & 652 \\
\hline 1113 & 14618 & $2 \mathrm{R}-1.1 \mathrm{U}$ & $1.52 E+01$ & 6.427 & $1.41 E+03$ & PUREX & 6 & 85 & 14873.03 & 255 \\
\hline 1114 & 14722 & $\mathrm{ZR}-0.94 \mathrm{U}$ & $3.68 E+00$ & 6.62 & $1.21 E+03$ & PUREX & 7 & 85 & 14903.01 & 182 \\
\hline 1115 & $\$ 4724$ & $2 R-0.94 U$ & $5.86 E+00$ & 7.658 & $1.43 \mathrm{E}+03$ & PUREX & 7 & 85 & 14903.02 & 180 \\
\hline 1116 & 14680 & $Z R=0.94 U$ & $5.05 E+01$ & 6.794 & $1.25 \mathrm{E}+03$ & PUREX & 7 & 85 & 14903.03 & 224 \\
\hline 1117 & 14221 & $Z R-1.1 U$ & $3.69 \mathrm{E}+00$ & 6.401 & $1.41 E+03$ & PUREX & 7 & 85 & 14903.07 & 683 \\
\hline 1118 & 14400 & $2 R-1.10$ & $1.81 E+01$ & 6.843 & $1.51 E+03$ & PUREX & 7 & 85 & 14903.06 & 504 \\
\hline 1119 & 14358 & $\mathrm{ZR}-1.1 \mathrm{U}$ & $1.59 E+01$ & 6.93 & $1.53 \mathrm{E}+03$ & PUREX & 7 & 85 & 14903.04 & 546 \\
\hline 1120 & 14306 & $2 R-1.10$ & 2.21E+01 & 6.788 & $1.50 E+03$ & PUREX & 7 & 85 & 14903.05 & 598 \\
\hline 1121 & 14680 & $2 \mathrm{R}-0.94 \mathrm{U}$ & $1.96 E+01$ & 6.794 & $1.25 E+03$ & PUREX & 8 & 85 & 14934.09 & 254 \\
\hline 1122 & 14756 & $Z R-0.94 U$ & $4.24 E+01$ & 6.232 & $1.14 E+03$ & PUREX & 8 & 85 & 14934.15 & 178 \\
\hline 1123 & 14757 & $2 R-0.94 U$ & $6.86 \mathrm{E}+00$ & 2.583 & $5.14 E+02$ & PUREX & 8 & 85 & 14934.14 & 177 \\
\hline 1124 & 14011 & $2 R-0.94 U$ & $2.57 E+00$ & 6.453 & $1.18 \mathrm{E}+03$ & PUREX & 8 & 85 & 14934.13 & 923 \\
\hline 1125 & 14094 & $2 R=0.94 \mathrm{U}$ & $7.04 E+00$ & 6.303 & $1.15 E+03$ & PUREX & 8 & 85 & 14934.12 & 840 \\
\hline 1126 & 14012 & $2 R-0.94 U$ & $.5 .98 E+00$ & 2.598 & $5.16 E+02$ & PUREX & 8 & 85 & 14934.11 & 922 \\
\hline 1127 & 14095 & $2 R-0.94 \mathrm{U}$ & $4.48 E+00$ & 2.439 & $4.93 E+02$ & PUREX & 8 & 85 & 14934.1 & 839 \\
\hline 1128 & 14446 & $Z R-0.94 U$ & $5.23 \mathrm{E}+00$ & 3.09 & $5.90 E+02$ & PUREX & 8 & 85 & 14934.08 & 488 \\
\hline 1129 & 14576 & $2 R-0.94 U$ & $7.38 \mathrm{E}+00$ & 2.559 & $5.10 E+02$ & PUREX & 8 & 85 & 14934.02 & 358 \\
\hline 1130 & 14449 & $Z R-0.94 U$ & $7.38 \mathrm{E}+00$ & 2.38 & $4.84 E+02$ & PUREX & 8 & 85 & 14934.01 & 485 \\
\hline 1131 & & $2 R-0.94 U$ & $1.03 \mathrm{E}+00$ & 1.178 & $3.21 E+02$ & PUREX & 8 & 85 & 14934.06 & 487 \\
\hline $\begin{array}{l}1132 \\
1133\end{array}$ & $\begin{array}{l}14723 \\
14722\end{array}$ & & & $\begin{array}{l}2.716 \\
6.621\end{array}$ & $\begin{array}{l}5.33 \mathrm{E}+02 \\
1.21 \mathrm{E}+03\end{array}$ & $\begin{array}{l}\text { PUREX } \\
\text { PUREX }\end{array}$ & $\begin{array}{l}8 \\
8\end{array}$ & $\begin{array}{l}85 \\
85\end{array}$ & & 211 \\
\hline $\begin{array}{l}1133 \\
1134\end{array}$ & $\begin{array}{l}14722 \\
14618\end{array}$ & $\begin{array}{l}2 R=0.94 U \\
2 R=0.94 U\end{array}$ & $\begin{array}{l}7.90 E+01 \\
7.22 E+00\end{array}$ & $\begin{array}{l}6.627 \\
2.538\end{array}$ & $\begin{array}{l}7.21 E+03 \\
5.07 E+02\end{array}$ & $\begin{array}{l}\text { PUREX } \\
\text { PUREX }\end{array}$ & $\begin{array}{l}8 \\
8\end{array}$ & $\begin{array}{l}85 \\
85\end{array}$ & $\begin{array}{l}14934.03 \\
14934.04\end{array}$ & $\begin{array}{l}212 \\
316\end{array}$ \\
\hline 1135 & 14681 & $2 R-0.94 U$ & $8.68 \mathrm{E}+00$ & 2.889 & $5.59 \mathrm{E}+02$ & PUREX & 8 & 85 & 14934.05 & $\begin{array}{l}310 \\
253\end{array}$ \\
\hline 1136 & 14757 & $Z R-0.94 U$ & $1.35 E+00$ & 2.583 & $5.14 E+02$ & PUREX & 9 & 8 & 14964.01 & 208 \\
\hline 1137 & 14577 & $2 R-0.94 U$ & $7.46 \mathrm{E}+00$ & 4.614 & $8.38 E+02$ & PUREX & 9 & 85 & 14964.02 & 388 \\
\hline 1138 & 14756 & $2 R-0.94 U$ & 2. $12 \mathrm{E}+01$ & 6.232 & $1.14 E+03$ & PUREX & 9 & 85 & 14964.03 & 209 \\
\hline 1139 & 11979 & $Z R-0.94 U$ & $1.48 \mathrm{E}+01$ & 13,486 & $2.95 E+03$ & PUREX & 10 & 85 & 14995.07 & 3016 \\
\hline 1140 & 10350 & $Z R=0.94 U$ & $6.33 \mathrm{E}-01$ & 12.45 & $2.64 E+03$ & PUREX & 10 & 85 & 14995.06 & 4645 \\
\hline 1141 & 10385 & $Z R=0.94 U$ & $9.79 \mathrm{E}-01$ & 12.504 & $2.65 E+03$ & PUREX & 10 & 85 & 14995.05 & 4610 \\
\hline 1142 & 12201 & $Z R=0.94 U$ & $1.88 E+01$ & 13.581 & $2.97 E+03$ & PUREX & 10 & 85 & 14995.03 & 2794 \\
\hline 1143 & 11372 & $\begin{array}{l}2 R=0.94 U \\
Z R-0.94 U\end{array}$ & $1.98 E+01$ & 13.663 & $3.00 E+03$ & PUREX & 10 & 85 & 5.02 & 3623 \\
\hline $\begin{array}{l}1144 \\
1145\end{array}$ & $\begin{array}{l}11709 \\
10982\end{array}$ & $\begin{array}{l}Z R-0.94 U \\
Z R-0.94 U\end{array}$ & $\begin{array}{l}1.10 \\
1.51\end{array}$ & $\begin{array}{l}13 . \\
13 .\end{array}$ & $\begin{array}{l}+03 \\
+03 \\
+03\end{array}$ & $\begin{array}{l}\text { PUREX } \\
\text { PUREX }\end{array}$ & 10 & $\begin{array}{l}85 \\
85\end{array}$ & 14995.01 & 3886 \\
\hline $\begin{array}{l}1145 \\
1146\end{array}$ & $\begin{array}{l}10982 \\
11540\end{array}$ & $2 R-0.94 U$ & 1.36E+01 & $\begin{array}{l}13.063 \\
13.402\end{array}$ & $\begin{array}{l}E+03 \\
E E+03\end{array}$ & $\begin{array}{l}\text { PUREX } \\
\text { PUREX }\end{array}$ & $\begin{array}{l}10 \\
11\end{array}$ & $\begin{array}{l}85 \\
85\end{array}$ & & $\begin{array}{l}4013 \\
3486\end{array}$ \\
\hline 1147 & 11449 & $Z R-0.94 U$ & $2.09 E+01$ & 13.563 & $2.97 E+03$ & PUREX & 11 & 85 & 15025.06 & $\begin{array}{l}3480 \\
3577\end{array}$ \\
\hline 1148 & 11897 & $Z R-0.94 U$ & $1.38 \mathrm{E}+01$ & 13.538 & $2.96 \mathrm{E}+03$ & PUREX & 11 & & 15025.02 & 3129 \\
\hline 1149 & 11733 & $2 R-0.94 U$ & $6.53 E-01$ & 13.353 & $2.90 E+03$ & PUREX & 11 & 85 & 5.04 & 3293 \\
\hline 1150 & 13685 & ZR-0.94U & $3.87 \mathrm{E}+01$ & 319 & $2.69 E+03$ & PUREX & 11 & & 5.03 & 1341 \\
\hline 1151 & $\begin{array}{l}11806 \\
12201\end{array}$ & $2 R-0.94 U$ & $\begin{array}{l}2.15 \mathrm{E}+01 \\
1\end{array}$ & 13.433 & $2.93 E+03$ & PUREX & 11 & & 15025.01 & $\begin{array}{l}3220 \\
3825\end{array}$ \\
\hline $\begin{array}{l}1152 \\
1153\end{array}$ & $\begin{array}{l}12201 \\
14832\end{array}$ & $\begin{array}{l}Z R-0.94 U \\
Z R-0.94 U\end{array}$ & $\begin{array}{l}1.28 E+00 \\
9.83 E+00\end{array}$ & $\begin{array}{r}13.433 \\
5.775\end{array}$ & $\begin{array}{l}2.93 E+03 \\
1.05 E+03\end{array}$ & $\begin{array}{l}\text { PUREX } \\
\text { PUREX }\end{array}$ & 11 & $\begin{array}{l}85 \\
85\end{array}$ & 15025.07 & $\begin{array}{r}2825 \\
224\end{array}$ \\
\hline 1154 & 13685 & $Z R-0.94 U$ & $1.98 \mathrm{E}+01$ & 12.621 & $2.69 E+03$ & $\begin{array}{l}\text { PUREX } \\
\text { PUREX }\end{array}$ & 12 & 8 & 15056.02 & 1371 \\
\hline 1155 & 14832 & $Z R=0.94 U$ & $5.91 \varepsilon+01$ & 5.784 & $E+03$ & PUREX & 1 & 8 & 6.01 & 255 \\
\hline 1156 & 14883 & $Z R-0.94 \mathrm{U}$ & $7 E+02$ & 5.918 & $E+03$ & PUREX & 2 & 8 & 15116.01 & 234 \\
\hline 1157 & 14925 & $Z R-0.94 U$ & $6 E+02$ & & $E+03$ & PUREX & 3 & & 15 & 222 \\
\hline 1758 & 13018 & ZR-0. & $0 E+01$ & 6.709 & $E+03$ & PUREX & 3 & 8 & 15 & 2129 \\
\hline $\begin{array}{l}1159 \\
1160\end{array}$ & $\begin{array}{l}14757 \\
13895\end{array}$ & ZR-1. IU & EE+01 & & $E+03$ & PUREX & 4 & & & $\begin{array}{r}421 \\
1383\end{array}$ \\
\hline $\begin{array}{l}1160 \\
1161\end{array}$ & $\begin{array}{l}13895 \\
14961\end{array}$ & $\begin{array}{l}Z R-1.1 U \\
Z R-0.94 U\end{array}$ & $\begin{array}{l}2.95 E+01 \\
3.08 E+01\end{array}$ & $\begin{array}{l}6.368 \\
5.765\end{array}$ & $\begin{array}{ll}O E+03 \\
5 E E+03\end{array}$ & PUREX & 4 & & & $\begin{array}{r}1283 \\
247\end{array}$ \\
\hline $\begin{array}{l}1161 \\
1162\end{array}$ & 14182 & $\begin{array}{l}2 R-0.94 U \\
Z R-1.1 U\end{array}$ & $\begin{array}{l}3.08 \mathrm{E}+01 \\
7.00 \mathrm{E}+01\end{array}$ & $\begin{array}{l}5.765 \\
6.287\end{array}$ & $\begin{array}{l}1.05 E+03 \\
1.38 E+03\end{array}$ & $\begin{array}{l}\text { PUREX } \\
\text { PUREX }\end{array}$ & $\begin{array}{l}5 \\
5\end{array}$ & $\begin{array}{l}86 \\
86\end{array}$ & $\begin{array}{l}15208.01 \\
15208.02\end{array}$ & $\begin{array}{r}247 \\
1026\end{array}$ \\
\hline 1163 & 14221 & $\mathrm{ZR}-1.1 \mathrm{U}$ & $3.83 E+00$ & 6.378 & $0 E+03$ & $\begin{array}{l}\text { PUREX } \\
\text { PUR }\end{array}$ & 5 & 8 & 15208.03 & 987 \\
\hline 1164 & 14961 & $\mathrm{ZR}-0.94 \mathrm{U}$ & $6.07 E+01$ & 5.665 & 1. $.03 E+03$ & PUREX & 6 & 86 & 15238.01 & 278 \\
\hline 1165 & 14963 & $2 R-0.94 U$ & $9 E-01$ & & $3 E+02$ & PUREX & 7 & 86 & 15269.02 & 306 \\
\hline 1166 & 34925 & $\mathrm{ZR}-0$. & 0E+01 & & $E+02$ & PUREX & 7 & & 15269 & 344 \\
\hline 1167 & 13724 & $\mathrm{ZR}-1$. & $3 E+00$ & & $=03$ & PUREX & 7 & 8 & 15269 & 1545 \\
\hline 1168 & 14833 & $2 R-1$ & $5 E+01$ & 5. & $\div 03$ & PUREX & 7 & 8 & 15269 & 43 \\
\hline 116 & 12240 & $Z R=0.9$ & $1 E-01$ & & 1.6 & PUREX & 8 & 8 & & 3060 \\
\hline & & ZR-0. & $1 E+00$ & & & PUREX & 8 & 86 & 15299.1 & 1205 \\
\hline
\end{tabular}


HNF-SD-WM-T1-794, ReV. 0

UNIFIED SEPARATIONS PRODUCTION DATA- - n.

TOTAL WASTE LOSS FRACTION

[Listing \#] Fuel Data Reference

$1106 \mathrm{~J}$. SCHLOSSER FILE 'SHIP_PX.DAT' 01/

1107 J. SCHLOSSER FILE 'SHIP_PX.DAT' 01/

$1108 \mathrm{~J}$. SCHLOSSER FILE 'SHIP-PX.DAT' O1'

$1109 \mathrm{~J}$. SCHLOSSER FILE 'SHIP'PX.DAT' O1/

$1110 \mathrm{~J}$. SCHLOSSER FILE 'SHIP_PX.DAY' 01/

$1111 \mathrm{~J}$. SCHLOSSER FILE 'SHIP'PX.DAT' 01/

1112 J. SCHLOSSER FILE 'SHIP'PX.DAT' O1/

1113 J. SCHLOSSER FILE 'SHIP_PX.DAT' O1/

$1114 \mathrm{~J}$. SCHLOSSER FILE 'SHIP-PX.DAT' 01/

1115 J. SCHLOSSER FILE 'SHIP-PX.DAT' OT/

$1116 \mathrm{~J}$. SCHLOSSER FILE 'SHIP-PX.DAT' 01/

$1117 \mathrm{~J}$. SCHLOSSER FILE 'SHIP'PX.DAT' 01 /

$1118 \mathrm{~J}$. SCHLOSSER FILE 'SHIP_PX.DAT' 01/

$1119 \mathrm{~J}$. SCHLOSSER FILE 'SHIP PX.DAT' 01/

1120 J. SCHLOSSER FILE 'SHIP'PX.DAT' O1/

$1121 \mathrm{~J}$. SCHLOSSER FILE 'SHIP_PX.DAT' 01/

$1122 \mathrm{~J}$. SCHLOSSER FILE 'SHIP'PX.DAT' O1/

$1123 \mathrm{~J}$. SCHLOSSER FILE 'SHIP.PX.DAT' 01/

$1124 \mathrm{~J}$. SCHLOSSER FILE 'SHIP.PX.DAT' O1/

1125 J. SCHLOSSER FILE 'SHIP PX.DAT' 01/

$1126 \mathrm{~J}$. SCHLOSSER FILE 'SHIP-PX.DAT' 01/

$1127 \mathrm{~J}$. SCHLOSSER FILE 'SHIP-PX.DAT' 01/

$1128 \mathrm{~J}$. SCHLOSSER FILE 'SHIP'PX.DAT' 01/

$1129 \mathrm{~J}$. SCHLOSSER FILE 'SHIP_PX.DAT' 01/

$1130 \mathrm{~J}$. SCHLOSSER FILE 'SHIP'PX.DAT' OI/

$1131 \mathrm{~J}$. SCHLOSSER FILE 'SHIP-PX.DAT' O1/

$1132 \mathrm{~J}$. SCHLOSSER FILE 'SHIP PX.DAT' 01/

1133 J. SCHLOSSER FILE 'SHIP'PX.DAT' 01/

1134 J. SCHLOSSER FILE 'SHIP'PX.DAT' 01/

1135 J. SCHLOSSER FILE 'SHIP'PX.DAT' 01/

$1136 \mathrm{~J}$. SCHLOSSER FILE 'SHIP.PX.DAT' 01/

$1137 \mathrm{~J}$. SCHLOSSER FILE 'SHIP PX.OAT' OY'

$1138 \mathrm{~J}$. SCHLOSSER FILE 'SHIP'PX.OAT' O1/

$1139 \mathrm{~J}$. SCHLOSSER FILE 'SHIP-PX.DAT' 01/

$1140 \mathrm{~J}$. SCHLOSSER FILE 'SHIP-PX.DAT' 01/

1141 3. SCHLOSSER FILE 'SHIP-PX.DAT' 01/

$1142 \mathrm{~J}$. SCHLOSSER FILE 'SHIP-PX.DAT' 01/

$1143 \mathrm{~J}$. SCHLOSSER FILE 'SHIP'PX.DAT' 01/

1144 J. SCHLOSSER FILE 'SHIP.PX.DAT' 01/

$1145 \mathrm{~J}$. SCHLOSSER FILE 'SHIP'PX.DAT' 01/

$1146 \mathrm{~J}$. SCHLOSSER FILE 'SHIP'PX.DAT' 01/

$1147 \mathrm{~J}$. SCHLOSSER FILE 'SHIP-PX.OAT' O1/

$1148 \mathrm{~J}$. SCHLOSSER FILE 'SHIP-PX.OAT' O1/

$1149 \mathrm{~J}$. SCHLOSSER FILE 'SHIP-PX.DAT' 01/

$1150 \mathrm{~J}$. SCHLOSSER FILE 'SHIP-PX.DAT' 01/

$1151 \mathrm{~J}$. SCHLOSSER FILE 'SHIP'PX DAT' O1/

1152 J. SCHLOSSER FILE 'SHIP_PX.DAT' 01/

$1153 \mathrm{~J}$. SCHLOSSER FILE 'SHIPIPX.DAT' 01/

$1154 \mathrm{~J}$. SCHLOSSER FILE 'SHIP'PX.DAT' 01/

$1155 \mathrm{~J}$. SCHLOSSER FILE 'SHIP-PX.DAT' 01/

$1156 \mathrm{~J}$. SCHLOSSER FILE 'SHIP'PX.DAT' 01/

1157 J. SCHLOSSER FILE 'SHIP-PX.DAT' 01/

$1158 \mathrm{~J}$. SCHLOSSER FILE 'SHIP_PX.DAT' OY'

$1159 \mathrm{~J}$. SCHLOSSER FILE 'SHIP'PX.DAT' OU,

$1160 \mathrm{~J}$. SCHLOSSER FILE 'SHIP-PX.DAT' O1/

1161 i. SCHLOSSER FILE 'SHIP-PX.DAT' 01/

1162 J. SCHLOSSER FILE 'SHIP'PX.DAT' 01/

$1163 \mathrm{~J}$. SCHLOSSER FILE 'SHIP PX.DAY' 01/

$1164 \mathrm{~J}$. SCHLOSSER FILE 'SHIP PX.DAT' 01/

$1165 \mathrm{~J}$. SCHLOSSER FILE 'SHIP.PX.DAT' 01/

$1166 \mathrm{~J}$. SCHLOSSER FILE 'SHIP-PX.DAT' 01/

$1167 \mathrm{~J}$. SCHLOSSER FILE 'SHIP-PX.DAT' O1'

$1168 \mathrm{~J}$. SCHLOSSER FILE 'SHIP PX.DAT' 01/

$1169 \mathrm{~J}$. SCHLOSSER FILE 'SHIP PX.DAT' 01/

$1170 \mathrm{~J}$. SCHLOSSER FILE 'SHIP_PX.DAT' O1/

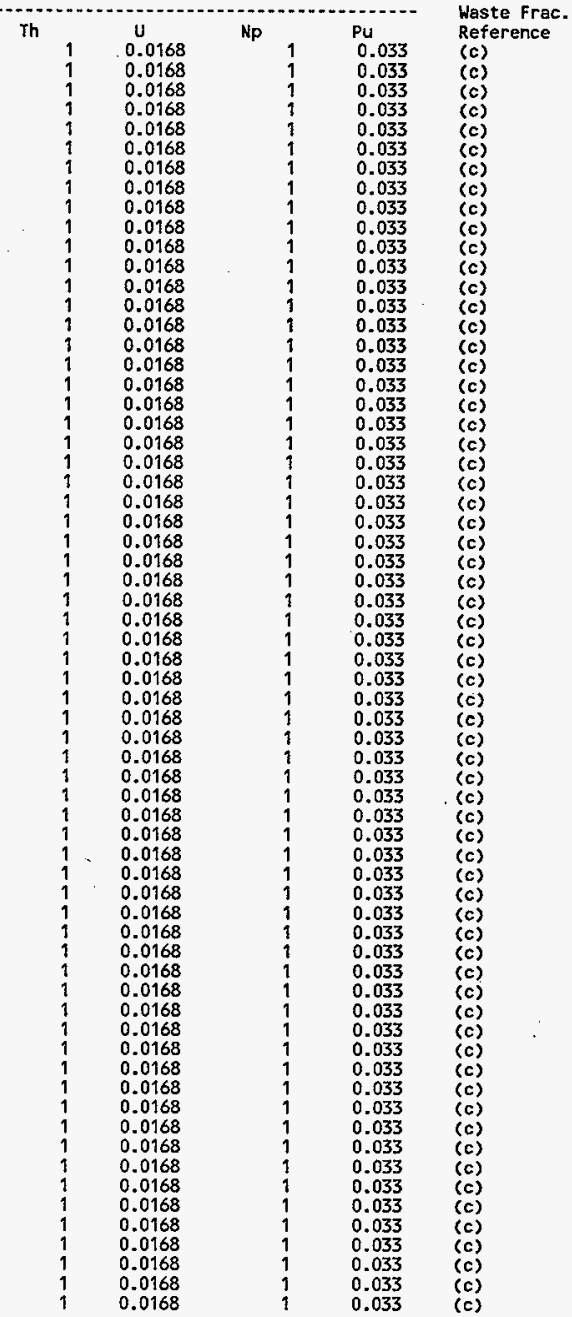


HNF-SD-WM-TI-794, Rev. 0

ACTIVITY IN UNSEPARATED FUEL (Curies decayed to 1/1/94)

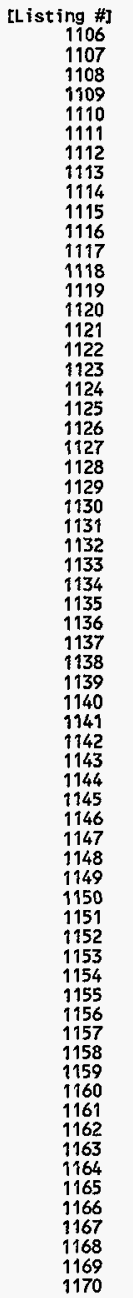

\begin{tabular}{|c|c|c|c|c|}
\hline 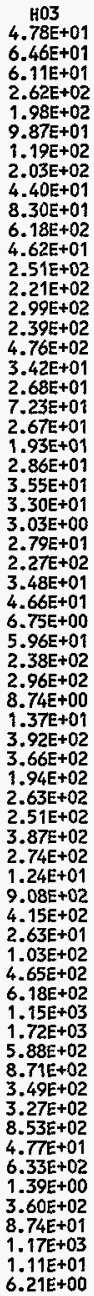 & 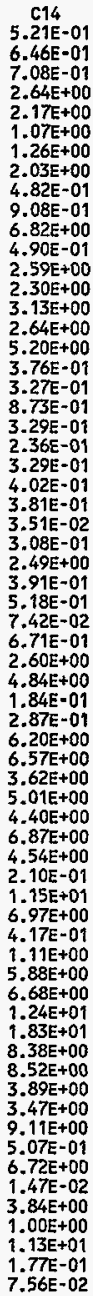 & 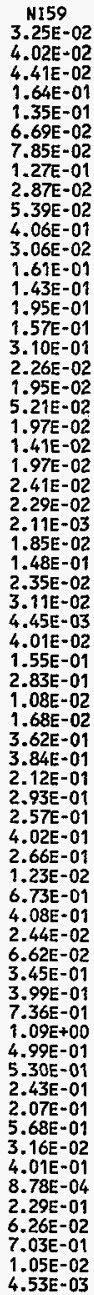 & 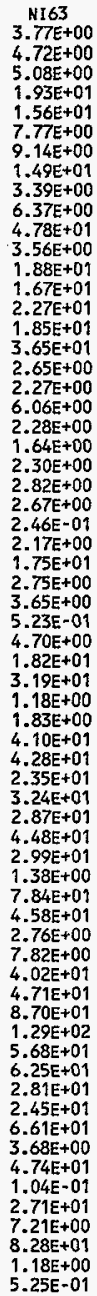 & 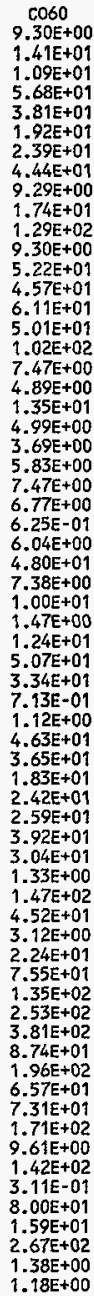 \\
\hline
\end{tabular}

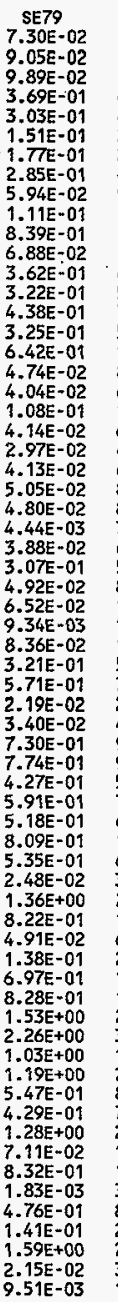

$9.51 \mathrm{E}-03$

\section{SR90 \\ $1.20 E+04$
$1.54 E+04$ \\ $1.57 \mathrm{E}+04$}

$6.28 \mathrm{E}+04$

4.97E+04

$2.47 E+04$

$2.93 \mathrm{E}+04$

$4.86 \mathrm{E}+04$

$9.94 E+03$

$1.84 \mathrm{E}+04$

$1.40 \mathrm{E}+05$

$1.14 E+04$

$6.06 E+04$

$5.38 \mathrm{E}+04$

$7.29 E+04$

$5.42 \mathrm{E}+04$

$1.08 E+05$

8. 19E+03

$6.46 \mathrm{E}+03$

1. $74 \mathrm{E}+04$

$6.83 \mathrm{E}+03$

$4.92 E+03$

$6.99 E+03$

$8.64 \mathrm{E}+03$

8.15E+03

$7.59 E+02$

$6.69 \mathrm{E}+03$

$5.13 E+04$

$8.44 E+03$

$1.12 \mathrm{E}+04$

$1.62 \mathrm{E}+03$

$1.41 \mathrm{E}+04$

5.39E+04

7. $41 \mathrm{E}+04$

2. $58 \mathrm{E}+03$

4.03E+03

$9.61 \mathrm{E}+04$

$9.66 \mathrm{E}+04$

$5.23 E+04$

$7.18 \mathrm{E}+04$

$6.54 E+04$

$1.01 \mathrm{E}+05$

6.91E+04

$3.16 \mathrm{E}+03$

2. $00 E+05$

1. $05 \mathrm{E}+05$

$6.47 \mathrm{E}+03$

$2.33 E+04$

$1.02 E+05$

$1.47 E+05$

2. $60 \mathrm{E}+05$

$3.84 \mathrm{E}+05$

$1.55 \mathrm{E}+05$

2.04E+05

$8.90 E+04$

$7.35 \mathrm{E}+04$

2.12E+05

$1.18 \mathrm{E}+04$

1. $43 \mathrm{E}+05$

$3.15 \mathrm{E}+02$

8.17E+04

$2.28 \mathrm{E}+04$

2. $75 E+05$

$3.00 E+03$

$1.57 \mathrm{E}+03$
Y90

1. $20 \mathrm{E}+04$

$1.54 E+04$

$1.57 \mathrm{E}+04$

$6.28 \mathrm{E}+04$

$4.97 \mathrm{E}+04$

$2.47 E+04$

$2.94 E+04$

$4.86 E+04$

$9.94 \mathrm{E}+03$

$1.84 E+04$

$1.40 E+05$

$1.14 \mathrm{E}+04$

$6.07 \mathrm{E}+04$

$5.38 E+04$

$7.29 E+04$

$5.42 \mathrm{E}+04$

$1.08 E+05$

$8.20 \mathrm{E}+03$

$6.46 \mathrm{E}+03$

$1.74 E+04$

$6.83 \mathrm{E}+03$

$4.92 \mathrm{E}+03$

$6.99 \mathrm{E}+03$

$8.65 \mathrm{E}+03$

$8.15 \mathrm{E}+03$

$7.59 \mathrm{E}+02$

$6.69 E+03$

$5.13 E+04$

8. $44 E+03$

$1.12 E+04$

1. $62 E+03$

$1.41 \mathrm{E}+04$

$5.40 \mathrm{E}+04$

$7.41 \mathrm{E}+04$

$2.58 \mathrm{E}+03$

$4.03 E+03$

$9.62 \mathrm{E}+04$

$9.66 \mathrm{E}+04$

$5.24 E+04$

$7.18 \mathrm{E}+04$

$6.54 E+04$

1.02E+05

$6.91 E+04$

3. $17 E+03$

2.00E+05

1.06E+05

$6.47 E+03$

$2.34 E+04$

1. $02 E+05$

$1.41 E+05$

2. $60 \mathrm{E}+05$

$3.85 E+05$

$1.55 E+05$

2.04E+05

8. $90 \mathrm{E}+04$

$7.35 \mathrm{E}+04$

2. $12 \mathrm{E}+05$

$1.18 E+04$

1. $43 \mathrm{E}+05$

3. $15 \mathrm{E}+02$

8.17E+04

2.28E+04

2. $75 \mathrm{E}+05$

$3.00 E+03$

$1.57 \mathrm{E}+03$
ZR93

$3.58 \mathrm{E}-01$

4.45E-01

$4.84 E-01$

$1.81 \mathrm{E}+00$

$1.49 \mathrm{E}+00$

$7.40 E-01$

8.68E-01

$1.40 \mathrm{E}+00$

2.87E-01

5.34E-01

4. $05 \mathrm{E}+00$

3.38E-01

$1.78 \mathrm{E}+00$

$1.58 E+00$

$2.15 \mathrm{E}+00$

$1.57 E+00$

$3.11 E+00$

2.32E-01

$1.95 \mathrm{E}-01$

5.22E-01

2.03E-01

$1.46 \mathrm{E}-01$

2. $02 E-01$

2.48E-01

2.35E-01

2.19E-02

$1.90 \mathrm{E}-01$

$1.48 E+00$

2.41E-01

3.19E-01

4.58E-02

4.07E-01

$1.55 \mathrm{E}+00$

$2.66 \mathrm{E}+00$

1.03E-01

$1.60 E-01$

$3.41 E+00$

$3.61 E+00$

$1.99 \mathrm{E}+00$

$2.76 E+00$

$2.42 E+00$

$3.78 \mathrm{E}+00$

$2.50 \mathrm{E}+00$

1.16E-01

$6.38 \mathrm{E}+00$

$3.83 \mathrm{E}+00$

29E-01

6.66E-01

$3.27 E+00$

$4.01 E+00$

$7.40 E+00$

$1.09 E+01$

$4.99 \mathrm{E}+00$

$5.84 E+00$

$2.69 E+00$

$2.08 \mathrm{E}+00$

$6.28 \mathrm{E}+00$

3. $49 \mathrm{E}-01$

$4.04 \mathrm{E}+00$

8.89E-03

$.31 \mathrm{E}+00$

$6.95 \mathrm{E}-01$

$7.20 \mathrm{E}+00$

$1.03 \mathrm{E}-01$

4.66E-02 
HNF-SD-WH-TI-794, Rev. 0

ACTIVITY IN UNSEPARATED FUEL (Curies decayed to $1 / 1 / 94$ )

\begin{tabular}{|c|c|c|c|c|c|c|c|c|c|}
\hline $\begin{array}{c}\text { isting } \\
1106 \\
1107 \\
1108 \\
1109 \\
1110 \\
1111 \\
1112 \\
1113 \\
1114 \\
1115 \\
1116 \\
1117 \\
1118 \\
1119 \\
1120 \\
1121 \\
1122 \\
1123 \\
1124 \\
1125 \\
1126 \\
1127 \\
1128 \\
1129 \\
1130 \\
1131 \\
1132 \\
1133 \\
1134 \\
1135 \\
1136 \\
1137 \\
1138 \\
1139 \\
1140 \\
1141 \\
1142 \\
1143 \\
1144 \\
1145 \\
1146 \\
1147 \\
1148 \\
1149 \\
1150 \\
1151 \\
1152 \\
1153 \\
1154 \\
1155 \\
1156 \\
1157 \\
1158 \\
1159 \\
1160 \\
1161 \\
1162 \\
1163 \\
1164 \\
1165 \\
1166 \\
1167 \\
1168 \\
1169 \\
1170\end{array}$ & $\begin{array}{l}\text { NB93* } \\
1.46 \mathrm{E}-01 \\
1.62 \mathrm{E}-01 \\
2.12 \mathrm{E}-01 \\
6.65 \mathrm{E}-01 \\
6.12 \mathrm{E}-01 \\
3.01 \mathrm{E}-01 \\
3.42 \mathrm{E}-01 \\
5.08 \mathrm{E}-01 \\
1.02 \mathrm{E}-01 \\
1.90 \mathrm{E}-01 \\
1.45 \mathrm{E}+00 \\
1.33 \mathrm{E}-01 \\
6.77 \mathrm{E}-01 \\
6.07 \mathrm{E}-01 \\
8.34 \mathrm{E}-01 \\
5.62 \mathrm{E}-01 \\
1.09 \mathrm{E}+00 \\
8.09 \mathrm{E}-02 \\
8.02 \mathrm{E}-02 \\
2.11 \mathrm{E}-01 \\
8.28 \mathrm{E}-02 \\
5.84 \mathrm{E}-02 \\
7.58 \mathrm{E}-02 \\
9.01 \mathrm{E}-02 \\
8.80 \mathrm{E}-02 \\
8.15 \mathrm{E}-03 \\
6.68 \mathrm{E}-02 \\
5.25 \mathrm{E}-01 \\
8.69 \mathrm{E}-02 \\
1.13 \mathrm{E}-01 \\
1.60 \mathrm{E}-02 \\
1.49 \mathrm{E}-01 \\
5.45 \mathrm{E}-01 \\
1.46 \mathrm{E}+00 \\
6.46 \mathrm{E}-02 \\
1.00 \mathrm{E}-01 \\
1.83 \mathrm{E}+00 \\
2.10 \mathrm{E}+00 \\
1.19 \mathrm{E}+00 \\
1.66 \mathrm{E}+00 \\
1.39 \mathrm{E}+00 \\
2.18 \mathrm{E}+00 \\
1.38 \mathrm{E}+00 \\
6.51 \mathrm{E}-02 \\
2.81 \mathrm{E}+00 \\
2.14 \mathrm{E}+00 \\
1.23 \mathrm{E}-01 \\
2.30 \mathrm{E}-01 \\
1.44 \mathrm{E}+00 \\
1.38 \mathrm{E}+00 \\
2.52 \mathrm{E}+00 \\
3.69 \mathrm{E}+00 \\
2.40 \mathrm{E}+00 \\
2.06 \mathrm{E}+00 \\
1.13 \mathrm{E}+00 \\
6.94 \mathrm{E}-01 \\
2.50 \mathrm{E}+00 \\
1.38 \mathrm{E}-01 \\
1.35 \mathrm{E}+00 \\
2.96 \mathrm{E}-03 \\
7.78 \mathrm{E}-01 \\
3.00 \mathrm{E}-01 \\
2.69 \mathrm{E}+00 \\
5.46 \mathrm{E}-02 \\
1.87 \mathrm{E}-02\end{array}$ & $\begin{array}{l}\text { TC99 } \\
2.45 \mathrm{E}+00 \\
3.03 \mathrm{E}+00 \\
3.31 \mathrm{E}+00 \\
1.24 \mathrm{E}+01 \\
1.02 \mathrm{E}+01 \\
5.05 \mathrm{E}+00 \\
5.92 \mathrm{E}+00 \\
9.54 \mathrm{E}+00 \\
1.98 \mathrm{E}+00 \\
3.71 \mathrm{E}+00 \\
2.80 \mathrm{E}+01 \\
2.30 \mathrm{E}+00 \\
1.21 \mathrm{E}+01 \\
1.08 \mathrm{E}+01 \\
1.47 \mathrm{E}+01 \\
1.09 \mathrm{E}+01 \\
2.14 \mathrm{E}+01 \\
1.58 \mathrm{E}+00 \\
1.35 \mathrm{E}+00 \\
3.60 \mathrm{E}+00 \\
1.38 \mathrm{E}+00 \\
9.89 \mathrm{E}-01 \\
1.38 \mathrm{E}+00 \\
1.69 \mathrm{E}+00 \\
1.60 \mathrm{E}+00 \\
1.48 \mathrm{E}-01 \\
1.29 \mathrm{E}+00 \\
1.02 \mathrm{E}+01 \\
1.64 \mathrm{E}+00 \\
2.17 \mathrm{E}+00 \\
3.12 \mathrm{E}-01 \\
2.79 \mathrm{E}+00 \\
1.07 \mathrm{E}+01 \\
1.91 \mathrm{E}+01 \\
7.32 \mathrm{E}-01 \\
1.14 \mathrm{E}+00 \\
2.44 \mathrm{E}+01 \\
2.59 \mathrm{E}+01 \\
1.43 \mathrm{E}+01 \\
1.98 \mathrm{E}+01 \\
1.74 \mathrm{E}+01 \\
2.71 \mathrm{E}+01 \\
1.79 \mathrm{E}+01 \\
8.29 \mathrm{E}-01 \\
4.55 \mathrm{E}+01 \\
2.75 \mathrm{E}+01 \\
1.64 \mathrm{E}+00 \\
4.59 \mathrm{E}+00 \\
2.33 \mathrm{E}+01 \\
2.76 \mathrm{E}+01 \\
5.10 \mathrm{E}+01 \\
7.55 \mathrm{E}+01 \\
3.45 \mathrm{E}+01 \\
3.99 \mathrm{E}+01 \\
1.83 \mathrm{E}+01 \\
1.43 \mathrm{E}+01 \\
4.28 \mathrm{E}+01 \\
2.38 \mathrm{E}+00 \\
2.78 \mathrm{E}+01 \\
6.10 \mathrm{E}-02 \\
1.59 \mathrm{E}+01 \\
4.73 \mathrm{E}+00 \\
5.31 \mathrm{E}+01 \\
7.19 \mathrm{E}-01 \\
3.17 \mathrm{E}-01\end{array}$ & $\begin{array}{l}\text { RU106 } \\
4.35 \mathrm{E}+01 \\
1.55 \mathrm{E}+02 \\
2.79 \mathrm{E}+01 \\
5.86 \mathrm{E}+02 \\
1.67 \mathrm{E}+02 \\
9.00 \mathrm{E}+01 \\
1.43 \mathrm{E}+02 \\
4.88 \mathrm{E}+02 \\
1.37 \mathrm{E}+02 \\
2.67 \mathrm{E}+02 \\
1.81 \mathrm{E}+03 \\
5.57 \mathrm{E}+01 \\
4.16 \mathrm{E}+02 \\
3.42 \mathrm{E}+02 \\
4.22 \mathrm{E}+02 \\
7.00 \mathrm{E}+02 \\
1.56 \mathrm{E}+03 \\
1.03 \mathrm{E}+02 \\
2.44 \mathrm{E}+01 \\
7.56 \mathrm{E}+01 \\
2.22 \mathrm{E}+01 \\
1.85 \mathrm{E}+01 \\
5.09 \mathrm{E}+01 \\
7.84 \mathrm{E}+01 \\
5.84 \mathrm{E}+01 \\
5.20 \mathrm{E}+00 \\
7.96 \mathrm{E}+01 \\
7.10 \mathrm{E}+02 \\
8.26 \mathrm{E}+01 \\
1.24 \mathrm{E}+02 \\
2.04 \mathrm{E}+01 \\
1.38 \mathrm{E}+02 \\
7.81 \mathrm{E}+02 \\
9.40 \mathrm{E}+00 \\
1.64 \mathrm{E}-02 \\
2.72 \mathrm{E}-02 \\
1.83 \mathrm{E}+01 \\
4.07 \mathrm{E}+00 \\
1.37 \mathrm{E}+00 \\
1.49 \mathrm{E}+00 \\
3.74 \mathrm{E}+00 \\
4.91 \mathrm{E}+00 \\
7.55 \mathrm{E}+00 \\
2.57 \mathrm{E}-01 \\
5.44 \mathrm{E}+02 \\
9.77 \mathrm{E}+00 \\
1.23 \mathrm{E}+00 \\
3.81 \mathrm{E}+02 \\
2.78 \mathrm{E}+02 \\
2.29 \mathrm{E}+03 \\
4.69 \mathrm{E}+03 \\
7.60 \mathrm{E}+03 \\
9.65 \mathrm{E}+01 \\
2.69 \mathrm{E}+03 \\
2.39 \mathrm{E}+02 \\
1.51 \mathrm{E}+03 \\
9.60 \mathrm{E}+02 \\
5.75 \mathrm{E}+01 \\
2.92 \mathrm{E}+03 \\
6.32 \mathrm{E}+00 \\
1.55 \mathrm{E}+03 \\
4.41 \mathrm{E}+01 \\
5.00 \mathrm{E}+03 \\
5.98 \mathrm{E}+01 \\
\end{array}$ & $\begin{array}{l}C D 113 * \\
3.53 E+00 \\
4.69 E+00 \\
4.59 \mathrm{E}+00 \\
1.91 \mathrm{E}+01 \\
1.46 \mathrm{E}+01 \\
7.27 \mathrm{E}+00 \\
8.69 \mathrm{E}+00 \\
1.48 \mathrm{E}+01 \\
3.48 \mathrm{E}+00 \\
6.67 \mathrm{E}+00 \\
4.92 \mathrm{E}+01 \\
3.38 \mathrm{E}+00 \\
1.84 \mathrm{E}+01 \\
1.63 \mathrm{E}+01 \\
2.20 \mathrm{E}+01 \\
1.90 \mathrm{E}+01 \\
3.75 \mathrm{E}+01 \\
2.59 \mathrm{E}+00 \\
2.15 \mathrm{E}+00 \\
5.79 \mathrm{E}+00 \\
2.05 \mathrm{E}+00 \\
1.48 \mathrm{E}+00 \\
2.19 \mathrm{E}+00 \\
2.70 \mathrm{E}+00 \\
2.51 \mathrm{E}+00 \\
2.28 \mathrm{E}-01 \\
2.11 \mathrm{E}+00 \\
1.80 \mathrm{E}+01 \\
2.64 \mathrm{E}+00 \\
3.54 \mathrm{E}+00 \\
5.11 \mathrm{E}-01 \\
4.63 \mathrm{E}+00 \\
1.87 \mathrm{E}+01 \\
2.74 \mathrm{E}+01 \\
8.30 \mathrm{E}-01 \\
1.30 \mathrm{E}+00 \\
3.61 \mathrm{E}+01 \\
3.44 \mathrm{E}+01 \\
1.83 \mathrm{E}+01 \\
2.49 \mathrm{E}+01 \\
2.35 \mathrm{E}+01 \\
3.63 \mathrm{E}+01 \\
2.54 \mathrm{E}+01 \\
1.15 \mathrm{E}+00 \\
7.97 \mathrm{E}+01 \\
3.86 \mathrm{E}+01 \\
2.43 \mathrm{E}+00 \\
8.04 \mathrm{E}+00 \\
4.08 \mathrm{E}+01 \\
4.84 \mathrm{E}+01 \\
9.02 \mathrm{E}+01 \\
1.36 \mathrm{E}+02 \\
4.85 \mathrm{E}+01 \\
6.34 \mathrm{E}+01 \\
2.58 \mathrm{E}+01 \\
2.55 \mathrm{E}+01 \\
6.25 \mathrm{E}+01 \\
3.49 \mathrm{E}+00 \\
4.94 \mathrm{E}+01 \\
1.07 \mathrm{E}-01 \\
2.79 \mathrm{E}+01 \\
6.44 \mathrm{E}+00 \\
8.36 \mathrm{E}+01 \\
9.57 \mathrm{E}-01 \\
4.78 \mathrm{E}-01\end{array}$ & $\begin{array}{c}\text { SB125 } \\
1.80 \mathrm{E}+02 \\
3.27 \mathrm{E}+02 \\
1.85 \mathrm{E}+02 \\
1.30 \mathrm{E}+03 \\
7.25 \mathrm{E}+02 \\
3.71 \mathrm{E}+02 \\
4.87 \mathrm{E}+02 \\
1.03 \mathrm{E}+03 \\
2.52 \mathrm{E}+02 \\
4.78 \mathrm{E}+02 \\
3.47 \mathrm{E}+03 \\
1.89 \mathrm{E}+02 \\
1.13 \mathrm{E}+03 \\
9.77 \mathrm{E}+02 \\
1.28 \mathrm{E}+03 \\
1.34 \mathrm{E}+03 \\
2.78 \mathrm{E}+03 \\
1.99 \mathrm{E}+02 \\
1.05 \mathrm{E}+02 \\
2.97 \mathrm{E}+02 \\
1.04 \mathrm{E}+02 \\
7.90 \mathrm{E}+01 \\
1.41 \mathrm{E}+02 \\
1.87 \mathrm{E}+02 \\
1.63 \mathrm{E}+02 \\
1.49 \mathrm{E}+01 \\
1.59 \mathrm{E}+02 \\
1.30 \mathrm{E}+03 \\
1.88 \mathrm{E}+02 \\
2.60 \mathrm{E}+02 \\
3.92 \mathrm{E}+01 \\
3.15 \mathrm{E}+02 \\
1.39 \mathrm{E}+03 \\
3.97 \mathrm{E}+02 \\
4.95 \mathrm{E}+00 \\
7.89 \mathrm{E}+00 \\
5.92 \mathrm{E}+02 \\
3.56 \mathrm{E}+02 \\
1.64 \mathrm{E}+02 \\
2.08 \mathrm{E}+02 \\
2.67 \mathrm{E}+02 \\
3.92 \mathrm{E}+02 \\
3.52 \mathrm{E}+02 \\
1.46 \mathrm{E}+01 \\
3.03 \mathrm{E}+03 \\
5.08 \mathrm{E}+02 \\
3.98 \mathrm{E}+01 \\
6.24 \mathrm{E}+02 \\
1.55 \mathrm{E}+03 \\
3.76 \mathrm{E}+03 \\
7.20 \mathrm{E}+03 \\
1.10 \mathrm{E}+04 \\
1.36 \mathrm{E}+03 \\
4.76 \mathrm{E}+03 \\
1.20 \mathrm{E}+03 \\
2.13 \mathrm{E}+03 \\
3.43 \mathrm{E}+03 \\
1.96 \mathrm{E}+02 \\
4.12 \mathrm{E}+03 \\
9.02 \mathrm{E}+00 \\
2.29 \mathrm{E}+03 \\
2.75 \mathrm{E}+02 \\
6.61 \mathrm{E}+03 \\
1.73 \mathrm{E}+01\end{array}$ & $\begin{array}{c}\text { SN126 } \\
1.07 \mathrm{E}-01 \\
1.32 \mathrm{E}-01 \\
1.46 \mathrm{E}-01 \\
5.40 \mathrm{E}-01 \\
4.43 \mathrm{E}-01 \\
2.20 \mathrm{E}-01 \\
2.57 \mathrm{E}-01 \\
4.15 \mathrm{E}-01 \\
9.31 \mathrm{E}-02 \\
1.77 \mathrm{E}-01 \\
1.32 \mathrm{E}+00 \\
1.00 \mathrm{E}-01 \\
5.31 \mathrm{E}-01 \\
4.72 \mathrm{E}-01 \\
6.42 \mathrm{E}-01 \\
5.11 \mathrm{E}-01 \\
9.99 \mathrm{E}-01 \\
6.98 \mathrm{E}-02 \\
6.31 \mathrm{E}-02 \\
1.68 \mathrm{E}-01 \\
6.10 \mathrm{E}-02 \\
4.37 \mathrm{E}-02 \\
6.14 \mathrm{E}-02 \\
7.45 \mathrm{E}-02 \\
7.06 \mathrm{E}-02 \\
6.43 \mathrm{E}-03 \\
5.73 \mathrm{E}-02 \\
4.81 \mathrm{E}-01 \\
7.25 \mathrm{E}-02 \\
9.64 \mathrm{E}-02 \\
1.38 \mathrm{E}-02 \\
1.27 \mathrm{E}-01 \\
4.99 \mathrm{E}-01 \\
1.02 \mathrm{E}+00 \\
3.82 \mathrm{E}-02 \\
5.95 \mathrm{E}-02 \\
1.30 \mathrm{E}+00 \\
1.38 \mathrm{E}+00 \\
7.60 \mathrm{E}-01 \\
1.05 \mathrm{E}+00 \\
9.22 \mathrm{E}-01 \\
1.44 \mathrm{E}+00 \\
9.52 \mathrm{E}-01 \\
4.41 \mathrm{E}-02 \\
2.38 \mathrm{E}+00 \\
1.46 \mathrm{E}+00 \\
8.74 \mathrm{E}-02 \\
2.12 \mathrm{E}-01 \\
1.22 \mathrm{E}+00 \\
1.28 \mathrm{E}+00 \\
2.37 \mathrm{E}+00 \\
3.53 \mathrm{E}+00 \\
1.62 \mathrm{E}+00 \\
1.75 \mathrm{E}+00 \\
7.96 \mathrm{E}-01 \\
6.63 \mathrm{E}-01 \\
1.86 \mathrm{E}+00 \\
1.04 \mathrm{E}-01 \\
1.28 \mathrm{E}+00 \\
2.79 \mathrm{E}-03 \\
7.31 \mathrm{E}-01 \\
2.04 \mathrm{E}-01 \\
2.29 \mathrm{E}+00 \\
3.50 \mathrm{E}-02 \\
1.41 \mathrm{E}-02\end{array}$ & $\begin{array}{c}1129 \\
4.73 \mathrm{E}-03 \\
5.86 \mathrm{E}-03 \\
6.45 \mathrm{E}-03 \\
2.39 \mathrm{E}-02 \\
1.97 \mathrm{E}-02 \\
9.76 \mathrm{E}-03 \\
1.14 \mathrm{E}-02 \\
1.84 \mathrm{E}-02 \\
3.95 \mathrm{E}-03 \\
7.50 \mathrm{E}-03 \\
5.60 \mathrm{E}-02 \\
4.45 \mathrm{E}-03 \\
2.35 \mathrm{E}-02 \\
2.09 \mathrm{E}-02 \\
2.84 \mathrm{E}-02 \\
2.17 \mathrm{E}-02 \\
4.25 \mathrm{E}-02 \\
3.01 \mathrm{E}-03 \\
2.68 \mathrm{E}-03 \\
7.15 \mathrm{E}-03 \\
2.63 \mathrm{E}-03 \\
1.88 \mathrm{E}-03 \\
2.64 \mathrm{E}-03 \\
3.21 \mathrm{E}-03 \\
3.05 \mathrm{E}-03 \\
2.79 \mathrm{E}-04 \\
2.47 \mathrm{E}-03 \\
2.04 \mathrm{E}-02 \\
3.13 \mathrm{E}-03 \\
4.16 \mathrm{E}-03 \\
5.94 \mathrm{E}-04 \\
5.43 \mathrm{E}-03 \\
2.12 \mathrm{E}-02 \\
4.18 \mathrm{E}-02 \\
1.58 \mathrm{E}-03 \\
2.46 \mathrm{E}-03 \\
5.35 \mathrm{E}-02 \\
5.67 \mathrm{E}-02 \\
3.13 \mathrm{E}-02 \\
4.33 \mathrm{E}-02 \\
3.80 \mathrm{E}-02 \\
5.93 \mathrm{E}-02 \\
3.92 \mathrm{E}-02 \\
1.81 \mathrm{E}-03 \\
9.83 \mathrm{E}-02 \\
6.02 \mathrm{E}-02 \\
3.60 \mathrm{E}-03 \\
9.05 \mathrm{E}-03\end{array}$ & 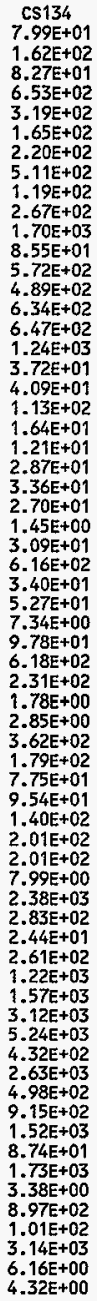 & $\begin{array}{c}\text { CS137 } \\
1.38 \mathrm{E}+04 \\
1.77 \mathrm{E}+04 \\
1.82 \mathrm{E}+04 \\
7.21 \mathrm{E}+04 \\
5.72 \mathrm{E}+04 \\
2.85 \mathrm{E}+04 \\
3.37 \mathrm{E}+04 \\
5.57 \mathrm{E}+04 \\
1.17 \mathrm{E}+04 \\
2.18 \mathrm{E}+04 \\
1.64 \mathrm{E}+05 \\
1.31 \mathrm{E}+04 \\
6.99 \mathrm{E}+04 \\
6.20 \mathrm{E}+04 \\
8.40 \mathrm{E}+04 \\
6.36 \mathrm{E}+04 \\
1.26 \mathrm{E}+05 \\
9.26 \mathrm{E}+03 \\
7.57 \mathrm{E}+03 \\
2.03 \mathrm{E}+04 \\
7.73 \mathrm{E}+03 \\
5.56 \mathrm{E}+03 \\
7.93 \mathrm{E}+03 \\
9.77 \mathrm{E}+03 \\
9.20 \mathrm{E}+03 \\
8.50 \mathrm{E}+02 \\
7.57 \mathrm{E}+03 \\
6.02 \mathrm{E}+04 \\
9.54 \mathrm{E}+03 \\
1.27 \mathrm{E}+04 \\
1.83 \mathrm{E}+03 \\
1.62 \mathrm{E}+04 \\
6.30 \mathrm{E}+04 \\
9.53 \mathrm{E}+04 \\
3.29 \mathrm{E}+03 \\
5.13 \mathrm{E}+03 \\
1.24 \mathrm{E}+05 \\
1.24 \mathrm{E}+05 \\
6.75 \mathrm{E}+04 \\
9.26 \mathrm{E}+04 \\
8.42 \mathrm{E}+04 \\
1.31 \mathrm{E}+05 \\
8.89 \mathrm{E}+04 \\
4.07 \mathrm{E}+03 \\
2.53 \mathrm{E}+05 \\
1.36 \mathrm{E}+05 \\
8.32 \mathrm{E}+03 \\
2.71 \mathrm{E}+04 \\
1.29 \mathrm{E}+05 \\
1.63 \mathrm{E}+05\end{array}$ \\
\hline
\end{tabular}


HNF-SD-WM-TI-794, ReV, 0

ACTIVITY IN UNSEPARATED FUEL (Curies decayed to $1 / 1 / 94$ )

\begin{tabular}{|c|c|c|c|c|c|c|c|c|c|}
\hline $\begin{array}{c}\text { [Listing } \#] \\
1106 \\
1107 \\
1108 \\
1109 \\
1110 \\
1111 \\
1112 \\
1113 \\
1114 \\
1115 \\
1116 \\
1117 \\
1118 \\
1119 \\
1120 \\
1121 \\
1122 \\
1123 \\
1124 \\
1125 \\
1126 \\
1127 \\
1128 \\
1129 \\
1130 \\
1131 \\
1132 \\
1133 \\
1134 \\
1135 \\
1136 \\
1137 \\
1138 \\
1139 \\
1140 \\
1141 \\
1142 \\
1143 \\
1144 \\
1145 \\
1146 \\
1147 \\
1148 \\
1149 \\
1150 \\
1151 \\
1152 \\
1153 \\
1154 \\
1155 \\
1156 \\
1157 \\
1158 \\
1159 \\
1160 \\
1161 \\
1162 \\
1163 \\
1164 \\
1165 \\
1166 \\
1167 \\
1168 \\
1169 \\
11170 \\
1169\end{array}$ & $\begin{array}{l}B A 137 \\
1.30 E+04 \\
1.68 E+04 \\
1.72 E+04 \\
6.82 E+04 \\
5.41 E+04 \\
2.69 E+04 \\
3.19 E+04 \\
5.27 E+04 \\
1.10 E+04 \\
2.07 E+04 \\
1.55 E+05 \\
1.24 E+04 \\
6.61 E+04 \\
5.86 E+04 \\
7.95 E+04 \\
6.02 E+04 \\
1.19 E+05 \\
8.76 E+03 \\
7.17 E+03 \\
1.92 E+04 \\
7.31 E+03 \\
5.26 E+03 \\
7.51 E+03 \\
9.25 E+03 \\
8.71 E+03 \\
8.04 E+02 \\
7.16 E+03 \\
5.69 E+04 \\
9.03 E+03 \\
1.20 E+04 \\
1.73 E+03 \\
1.53 E+04 \\
5.96 E+04 \\
9.02 E+04 \\
3.11 E+03 \\
4.85 E+03 \\
1.17 E+05 \\
1.18 E+05 \\
6.38 E+04 \\
8.76 E+04 \\
7.97 E+04 \\
1.24 E+05 \\
8.41 E+04 \\
3.85 E+03 \\
2.39 E+05 \\
1.28 E+05 \\
7.87 E+03 \\
2.57 E+04 \\
1.22 E+05 \\
1.55 E+05 \\
2.86 E+05 \\
4.25 E+05 \\
1.72 E+05 \\
2.23 E+05 \\
9.67 E+04 \\
8.08 E+04 \\
2.30 E+05 \\
1.28 E+04 \\
1.57 E+05 \\
3.44 E+02 \\
8.94 E+04 \\
2.47 E+04 \\
2.97 E+05 \\
3.43 E+03 \\
1.69 E+03\end{array}$ & 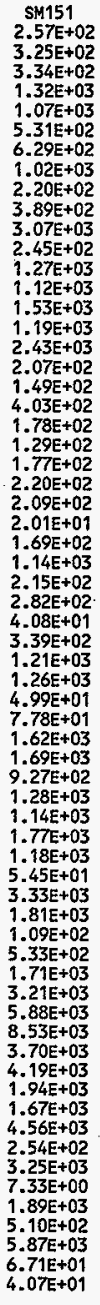 & $\begin{array}{l}\text { EU152 } \\
4.22 E-01 \\
5.48 \mathrm{E}-01 \\
6.16 \mathrm{E}-01 \\
2.30 \mathrm{E}+00 \\
1.74 \mathrm{E}+00 \\
8.70 \mathrm{E}-01 \\
1.01 \mathrm{E}+00 \\
1.73 \mathrm{E}+00 \\
3.89 \mathrm{E}-01 \\
9.11 \mathrm{E}-01 \\
5.80 \mathrm{E}+00 \\
3.94 \mathrm{E}-01 \\
2.34 \mathrm{E}+00 \\
2.07 \mathrm{E}+00 \\
2.79 \mathrm{E}+00 \\
2.20 \mathrm{E}+00 \\
3.84 \mathrm{E}+00 \\
6.02 \mathrm{E}-02 \\
2.30 \mathrm{E}-01 \\
5.89 \mathrm{E}-01 \\
4.75 \mathrm{E}-02 \\
3.01 \mathrm{E}-02 \\
7.30 \mathrm{E}-02 \\
6.27 \mathrm{E}-02 \\
5.12 \mathrm{E}-02 \\
0.00 \mathrm{E}+00 \\
5.48 \mathrm{E}-02 \\
2.01 \mathrm{E}+00 \\
6.14 \mathrm{E}-02 \\
1.04 \mathrm{E}-01 \\
1.19 \mathrm{E}-02 \\
3.03 \mathrm{E}-01 \\
1.92 \mathrm{E}+00 \\
6.89 \mathrm{E}+00 \\
1.94 \mathrm{E}-01 \\
3.03 \mathrm{E}-01 \\
9.10 \mathrm{E}+00 \\
8.59 \mathrm{E}+00 \\
4.57 \mathrm{E}+00 \\
6.21 \mathrm{E}+00 \\
5.89 \mathrm{E}+00 \\
9.08 \mathrm{E}+00 \\
6.39 \mathrm{E}+00 \\
2.89 \mathrm{E}-01 \\
1.92 \mathrm{E}+01 \\
9.69 \mathrm{E}+00 \\
6.12 \mathrm{E}-01 \\
7.39 \mathrm{E}-01 \\
9.84 \mathrm{E}+00 \\
4.45 \mathrm{E}+00 \\
8.59 \mathrm{E}+00 \\
1.44 \mathrm{E}+01 \\
5.33 \mathrm{E}+00 \\
8.14 \mathrm{E}+00 \\
2.95 \mathrm{E}+00 \\
2.35 \mathrm{E}+00 \\
7.19 \mathrm{E}+00 \\
4.02 \mathrm{E}-01 \\
4.42 \mathrm{E}+00 \\
8.15 \mathrm{E}-03 \\
2.29 \mathrm{E}+00 \\
6.66 \mathrm{E}-01 \\
8.74 \mathrm{E}+00 \\
1.51 \mathrm{E}-01 \\
1.29 \mathrm{E}-02\end{array}$ & $\begin{array}{l}E \cup 154 \\
6.16 E+01 \\
8.43 E+01 \\
8.56 E+01 \\
3.50 E+02 \\
2.54 E+02 \\
1.27 E+02 \\
1.51 E+02 \\
2.65 E+02 \\
5.96 E+01 \\
1.35 E+02 \\
8.77 E+02 \\
5.86 E+01 \\
3.48 E+02 \\
3.07 E+02 \\
4.12 E+02 \\
3.34 E+02 \\
6.00 E+02 \\
1.55 E+01 \\
3.35 E+01 \\
8.71 E+01 \\
1.15 E+01 \\
7.85 E+00 \\
1.55 E+01 \\
1.59 E+01 \\
1.37 E+01 \\
5.79 E-01 \\
1.33 E+01 \\
3.08 E+02 \\
1.56 E+01 \\
2.38 E+01 \\
3.05 E+00 \\
5.16 E+01 \\
3.00 E+02 \\
8.62 E+02 \\
2.03 E+01 \\
3.19 E+01 \\
1.16 E+03 \\
1.02 E+03 \\
5.32 E+02 \\
7.16 E+02 \\
7.11 E+02 \\
1.09 E+03 \\
7.94 E+02 \\
3.55 E+01 \\
2.64 E+03 \\
1.20 E+03 \\
7.79 E+01 \\
1.19 E+02 \\
1.35 E+03 \\
7.14 E+02 \\
1.38 E+03 \\
2.27 E+03 \\
7.11 E+02 \\
1.25 E+03 \\
4.29 E+02 \\
3.81 E+02 \\
1.07 E+03 \\
5.99 E+01 \\
7.21 E+02 \\
1.38 E+00 \\
3.79 E+02 \\
9.71 E+01 \\
1.39 E+03 \\
1.79 E+01 \\
2.91 E+00\end{array}$ & $\begin{array}{c}E U 155 \\
8.00 E+01 \\
1.24 E+02 \\
8.99 E+01 \\
4.94 E+02 \\
3.27 E+02 \\
1.65 E+02 \\
2.08 E+02 \\
3.90 E+02 \\
9.17 E+01 \\
1.62 E+02 \\
1.26 E+03 \\
8.08 E+01 \\
4.46 E+02 \\
3.90 E+02 \\
5.20 E+02 \\
4.90 E+02 \\
1.02 E+03 \\
8.68 E+01 \\
4.79 E+01 \\
1.34 E+02 \\
5.71 E+01 \\
4.24 E+01 \\
6.62 E+01 \\
8.64 E+01 \\
7.85 E+01 \\
7.51 E+00 \\
6.99 E+01 \\
4.74 E+02 \\
8.55 E+01 \\
1.15 E+02 \\
1.71 E+01 \\
1.33 E+02 \\
5.11 E+02 \\
2.14 E+02 \\
4.52 E+00 \\
7.12 E+00 \\
2.98 E+02 \\
2.30 E+02 \\
1.15 E+02 \\
1.51 E+02 \\
1.64 E+02 \\
2.48 E+02 \\
1.94 E+02 \\
8.45 E+00 \\
1.01 E+03 \\
2.88 E+02 \\
2.00 E+01 \\
2.30 E+02 \\
5.15 E+02 \\
1.39 E+03 \\
2.59 E+03 \\
3.82 E+03 \\
8.30 E+02 \\
1.68 E+03 \\
5.69 E+02 \\
7.55 E+02 \\
1.49 E+03 \\
8.38 E+01 \\
1.47 E+03 \\
3.32 E+00 \\
8.43 E+02 \\
1.41 E+02 \\
2.41 E+03 \\
1.14 E+01 \\
1.34 E+01\end{array}$ & 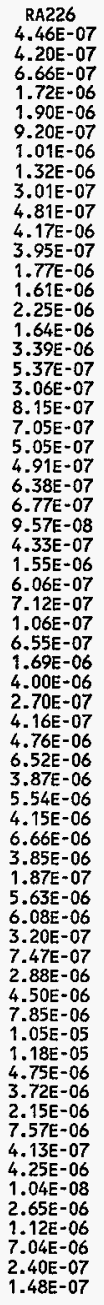 & $\begin{array}{l}\text { RA228 } \\
3.59 \mathrm{E}-11 \\
3.50 \mathrm{E}-11 \\
5.40 \mathrm{E}-11 \\
1.44 \mathrm{E}-10 \\
1.52 \mathrm{E}-10 \\
7.41 \mathrm{E}-11 \\
8.21 \mathrm{E}-11 \\
1.10 \mathrm{E}-10 \\
2.36 \mathrm{E}-11 \\
3.99 \mathrm{E}-11 \\
3.32 \mathrm{E}-10 \\
3.20 \mathrm{E}-11 \\
1.49 \mathrm{E}-10 \\
1.35 \mathrm{E}-10 \\
1.88 \mathrm{E}-10 \\
1.30 \mathrm{E}-10 \\
2.61 \mathrm{E}-10 \\
3.41 \mathrm{E}-11 \\
2.26 \mathrm{E}-11 \\
5.99 \mathrm{E}-11 \\
4.23 \mathrm{E}-11 \\
3.03 \mathrm{E}-11 \\
3.13 \mathrm{E}-11 \\
4.00 \mathrm{E}-11 \\
4.17 \mathrm{E}-11 \\
5.51 \mathrm{E}-12 \\
2.76 \mathrm{E}-11 \\
1.22 \mathrm{E}-10 \\
3.81 \mathrm{E}-11 \\
4.57 \mathrm{E}-11 \\
6.74 \mathrm{E}-12 \\
4.58 \mathrm{E}-11 \\
1.31 \mathrm{E}-10 \\
3.60 \mathrm{E}-10 \\
2.08 \mathrm{E}-11 \\
3.22 \mathrm{E}-11 \\
4.35 \mathrm{E}-10 \\
5.64 \mathrm{E}-10 \\
3.29 \mathrm{E}-10 \\
4.68 \mathrm{E}-10 \\
3.63 \mathrm{E}-10 \\
5.79 \mathrm{E}-10 \\
3.45 \mathrm{E}-10 \\
1.66 \mathrm{E}-11 \\
5.44 \mathrm{E}-10 \\
5.41 \mathrm{E}-10 \\
2.93 \mathrm{E}-11 \\
5.67 \mathrm{E}-11 \\
2.79 \mathrm{E}-10 \\
3.41 \mathrm{E}-10 \\
6.02 \mathrm{E}-10 \\
8.28 \mathrm{E}-10 \\
8.15 \mathrm{E}-10 \\
4.11 \mathrm{E}-10 \\
2.93 \mathrm{E}-10 \\
1.65 \mathrm{E}-10 \\
6.08 \mathrm{E}-10 \\
3.33 \mathrm{E}-11 \\
3.24 \mathrm{E}-10 \\
7.68 \mathrm{E}-13 \\
8.97 \mathrm{E}-10 \\
5.48 \mathrm{E}-11 \\
1.75 \mathrm{E}-10-11 \\
9.00 \mathrm{E}-12\end{array}$ & 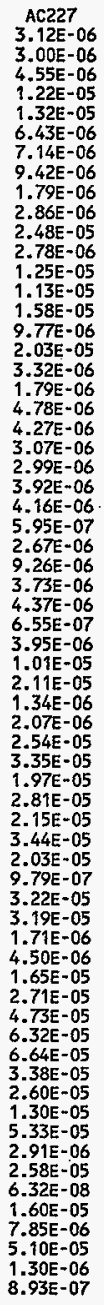 & $\begin{array}{l}\text { TH229 } \\
3.69 \mathrm{E}-09 \\
3.85 \mathrm{E}-09 \\
5.65 \mathrm{E}-09 \\
1.60 \mathrm{E}-08 \\
1.55 \mathrm{E}-08 \\
7.61 \mathrm{E}-09 \\
8.49 \mathrm{E}-09 \\
1.21 \mathrm{E}-08 \\
2.86 \mathrm{E}-09 \\
5.48 \mathrm{E}-09 \\
4.12 \mathrm{E}-08 \\
3.31 \mathrm{E}-09 \\
1.66 \mathrm{E}-08 \\
1.50 \mathrm{E}-08 \\
2.07 \mathrm{E}-08 \\
1.59 \mathrm{E}-08 \\
3.03 \mathrm{E}-08 \\
2.07 \mathrm{E}-09 \\
2.40 \mathrm{E}-09 \\
6.24 \mathrm{E}-09 \\
2.31 \mathrm{E}-09 \\
1.61 \mathrm{E}-09 \\
2.03 \mathrm{E}-09 \\
2.35 \mathrm{E}-09 \\
2.33 \mathrm{E}-09 \\
2.12 \mathrm{E}-10 \\
1.72 \mathrm{E}-09 \\
1.48 \mathrm{E}-08 \\
2.26 \mathrm{E}-09 \\
2.95 \mathrm{E}-09 \\
4.09 \mathrm{E}-10 \\
4.04 \mathrm{E}-09 \\
1.51 \mathrm{E}-08 \\
6.19 \mathrm{E}-08 \\
3.11 \mathrm{E}-09 \\
4.81 \mathrm{E}-09 \\
7.59 \mathrm{E}-08 \\
9.40 \mathrm{E}-08 \\
5.43 \mathrm{E}-08 \\
7.68 \mathrm{E}-08 \\
6.10 \mathrm{E}-08 \\
9.69 \mathrm{E}-08 \\
5.90 \mathrm{E}-08 \\
2.82 \mathrm{E}-09 \\
1.00 \mathrm{E}-07 \\
9.21 \mathrm{E}-08 \\
5.10 \mathrm{E}-09 \\
6.26 \mathrm{E}-09 \\
5.14 \mathrm{E}-08 \\
3.77 \mathrm{E}-08 \\
6.86 \mathrm{E}-08 \\
1.01 \mathrm{E}-07 \\
7.95 \mathrm{E}-08 \\
4.90 \mathrm{E}-08 \\
2.88 \mathrm{E}-08 \\
1.87 \mathrm{E}-08 \\
6.21 \mathrm{E}-08 \\
3.41 \mathrm{E}-09 \\
3.62 \mathrm{E}-08 \\
7.83 \mathrm{E}-11 \\
6.72 \mathrm{E}-08 \\
6.27 \mathrm{E}-09\end{array}$ \\
\hline
\end{tabular}


HNF-SD-WM-T1-794, ReV. 0

ACTIVITY IN UNSEPARATED FUEL (Curjes decayed to $1 / 1 / 94$ )

\begin{tabular}{|c|c|c|c|c|c|c|c|c|c|}
\hline $\begin{array}{c}\text { isting \#] } \\
1106 \\
1107 \\
1108 \\
1109 \\
1110 \\
1111 \\
1112 \\
1113 \\
1114 \\
1115 \\
1116 \\
1117 \\
1118 \\
1119 \\
1120 \\
1121 \\
1122 \\
1123 \\
1124 \\
1125 \\
1126 \\
1127 \\
1128 \\
1129 \\
1130 \\
1131 \\
1132 \\
1133 \\
1134 \\
1135 \\
1136 \\
1137 \\
1138 \\
1139 \\
1140 \\
1141 \\
1142 \\
1143 \\
1144 \\
1145 \\
1146 \\
1147 \\
1148 \\
1149 \\
1150 \\
1151 \\
1152 \\
1153 \\
1154 \\
1155 \\
1156 \\
1157 \\
1158 \\
1159 \\
1160 \\
1161 \\
1162 \\
1163 \\
1164 \\
1165 \\
1166 \\
1167 \\
1168 \\
1169 \\
1170\end{array}$ & $\begin{array}{l}\text { TH232 } \\
8.88 \mathrm{E}-11 \\
9.61 \mathrm{E}-11 \\
1.25 \mathrm{E}-10 \\
3.92 \mathrm{E}-10 \\
3.73 \mathrm{E}-10 \\
1.83 \mathrm{E}-10 \\
2.09 \mathrm{E}-10 \\
3.02 \mathrm{E}-10 \\
6.63 \mathrm{E}-11 \\
1.12 \mathrm{E}-10 \\
9.21 \mathrm{E}-10 \\
8.13 \mathrm{E}-11 \\
3.92 \mathrm{E}-10 \\
3.52 \mathrm{E}-10 \\
4.86 \mathrm{E}-10 \\
3.60 \mathrm{E}-10 \\
7.40 \mathrm{E}-10 \\
9.76 \mathrm{E}-11 \\
5.55 \mathrm{E}-11 \\
1.49 \mathrm{E}-10 \\
1.05 \mathrm{E}-10 \\
7.60 \mathrm{E}-11 \\
8.37 \mathrm{E}-11 \\
1.10 \mathrm{E}-10 \\
1.12 \mathrm{E}-10 \\
1.48 \mathrm{E}-11 \\
7.84 \mathrm{E}-11 \\
3.42 \mathrm{E}-10 \\
1.06 \mathrm{E}-10 \\
1.28 \mathrm{E}-10 \\
1.93 \mathrm{E}-11 \\
1.25 \mathrm{E}-10 \\
3.70 \mathrm{E}-10 \\
6.77 \mathrm{E}-10 \\
3.48 \mathrm{E}-11 \\
5.39 \mathrm{E}-11 \\
8.36 \mathrm{E}-10 \\
1.01 \mathrm{E}-09 \\
5.777 \mathrm{E}-10 \\
8.12 \mathrm{E}-10 \\
6.58 \mathrm{E}-10 \\
1.04 \mathrm{E}-09 \\
6.43 \mathrm{E}-10 \\
3.06 \mathrm{E}-11 \\
1.24 \mathrm{E}-09 \\
1.00 \mathrm{E}-09 \\
5.62 \mathrm{E}-11 \\
1.63 \mathrm{E}-10 \\
6.38 \mathrm{E}-10 \\
9.83 \mathrm{E}-10 \\
1.76 \mathrm{E}-09 \\
2.43 \mathrm{E}-09 \\
1.73 \mathrm{E}-09 \\
1.16 \mathrm{E}-09 \\
7.05 \mathrm{E}-10 \\
4.90 \mathrm{E}-10 \\
1.54 \mathrm{E}-09 \\
8.46 \mathrm{E}-11 \\
9.63 \mathrm{E}-10 \\
2.29 \mathrm{E}-12 \\
5.82 \mathrm{E}-10 \\
1.99 \mathrm{E}-10 \\
1.67 \mathrm{E}-09 \\
3.41 \mathrm{E}-11 \\
2.26 \mathrm{E}-11\end{array}$ & $\begin{array}{c}\text { PA231 } \\
1.95 \mathrm{E}-05 \\
2.14 \mathrm{E}-05 \\
2.61 \mathrm{E}-05 \\
8.64 \mathrm{E}-05 \\
8.21 \mathrm{E}-05 \\
4.03 \mathrm{E}-05 \\
4.65 \mathrm{E}-05 \\
6.74 \mathrm{E}-05 \\
1.31 \mathrm{E}-05 \\
2.07 \mathrm{E}-05 \\
1.79 \mathrm{E}-04 \\
1.81 \mathrm{E}-05 \\
8.43 \mathrm{E}-05 \\
7.58 \mathrm{E}-05 \\
1.05 \mathrm{E}-04 \\
7.06 \mathrm{E}-05 \\
1.50 \mathrm{E}-04 \\
2.53 \mathrm{E}-05 \\
1.11 \mathrm{E}-05 \\
3.02 \mathrm{E}-05 \\
2.71 \mathrm{E}-05 \\
1.99 \mathrm{E}-05 \\
2.10 \mathrm{E}-05 \\
2.85 \mathrm{E}-05 \\
2.93 \mathrm{E}-05 \\
4.21 \mathrm{E}-06 \\
2.01 \mathrm{E}-05 \\
6.78 \mathrm{E}-05 \\
2.74 \mathrm{E}-05 \\
3.26 \mathrm{E}-05 \\
4.99 \mathrm{E}-06 \\
2.84 \mathrm{E}-05 \\
7.51 \mathrm{E}-05 \\
8.69 \mathrm{E}-05 \\
4.67 \mathrm{E}-06 \\
7.23 \mathrm{E}-06 \\
1.07 \mathrm{E}-04 \\
1.28 \mathrm{E}-04 \\
7.33 \mathrm{E}-05 \\
1.03 \mathrm{E}-04 \\
8.40 \mathrm{E}-05 \\
1.33 \mathrm{E}-04 \\
8.24 \mathrm{E}-05 \\
3.91 \mathrm{E}-06 \\
1.73 \mathrm{E}-04 \\
1.28 \mathrm{E}-04 \\
7.23 \mathrm{E}-06 \\
3.42 \mathrm{E}-05 \\
8.88 \mathrm{E}-05 \\
2.06 \mathrm{E}-04 \\
3.65 \mathrm{E}-04 \\
4.90 \mathrm{E}-04 \\
3.41 \mathrm{E}-04 \\
2.50 \mathrm{E}-04 \\
1.58 \mathrm{E}-04 \\
1.03 \mathrm{E}-04 \\
3.44 \mathrm{E}-04 \\
1.89 \mathrm{E}-05 \\
2.03 \mathrm{E}-04 \\
5.02 \mathrm{E}-07 \\
1.26 \mathrm{E}-04 \\
4.61 \mathrm{E}-05 \\
3.88 \mathrm{E}-04 \\
5.84 \mathrm{E}-04\end{array}$ & $\begin{array}{l}\text { U232 } \\
3.47 \mathrm{E}-04 \\
4.19 \mathrm{E}-04 \\
5.12 \mathrm{E}-04 \\
1.75 \mathrm{E}-03 \\
1.44 \mathrm{E}-03 \\
7.16 \mathrm{E}-04 \\
8.22 \mathrm{E}-04 \\
1.32 \mathrm{E}-03 \\
3.38 \mathrm{E}-04 \\
7.23 \mathrm{E}-04 \\
4.95 \mathrm{E}-03 \\
3.20 \mathrm{E}-04 \\
1.78 \mathrm{E}-03 \\
1.59 \mathrm{E}-03 \\
2.16 \mathrm{E}-03 \\
1.89 \mathrm{E}-03 \\
3.46 \mathrm{E}-03 \\
1.44 \mathrm{E}-04 \\
2.27 \mathrm{E}-04 \\
5.87 \mathrm{E}-04 \\
1.26 \mathrm{E}-04 \\
8.77 \mathrm{E}-05 \\
1.38 \mathrm{E}-04 \\
1.54 \mathrm{E}-04 \\
1.42 \mathrm{E}-04 \\
1.03 \mathrm{E}-05 \\
1.21 \mathrm{E}-04 \\
1.74 \mathrm{E}-03 \\
1.50 \mathrm{E}-04 \\
2.09 \mathrm{E}-04 \\
2.84 \mathrm{E}-05 \\
3.57 \mathrm{E}-04 \\
1.73 \mathrm{E}-03 \\
7.28 \mathrm{E}-03 \\
2.41 \mathrm{E}-04 \\
3.76 \mathrm{E}-04 \\
9.34 \mathrm{E}-03 \\
9.77 \mathrm{E}-03 \\
5.36 \mathrm{E}-03 \\
7.39 \mathrm{E}-03 \\
6.56 \mathrm{E}-03 \\
1.02 \mathrm{E}-02 \\
6.81 \mathrm{E}-03 \\
3.15 \mathrm{E}-04 \\
1.57 \mathrm{E}-02 \\
1.05 \mathrm{E}-02 \\
6.28 \mathrm{E}-04 \\
6.94 \mathrm{E}-04 \\
8.05 \mathrm{E}-03 \\
4.18 \mathrm{E}-03 \\
7.85 \mathrm{E}-03 \\
1.24 \mathrm{E}-02 \\
5.91 \mathrm{E}-03 \\
5.85 \mathrm{E}-03 \\
2.53 \mathrm{E}-03 \\
2.16 \mathrm{E}-03 \\
5.91 \mathrm{E}-03 \\
3.28 \mathrm{E}-04 \\
4.12 \mathrm{E}-03 \\
8.31 \mathrm{E}-03 \\
2.25 \mathrm{E}-03 \\
6.11 \mathrm{E}-04 \\
6.78 \mathrm{E}-03 \\
1.60 \mathrm{E}-04 \\
2.99 \mathrm{E}-05\end{array}$ & $\begin{array}{l}\text { U233 } \\
4.05 E-06 \\
4.76 E-06 \\
5.71 E-06 \\
1.95 E-05 \\
1.69 E-05 \\
8.35 E-06 \\
9.64 E-06 \\
1.50 E-05 \\
3.59 E-06 \\
6.74 E-06 \\
5.10 E-05 \\
3.75 E-06 \\
1.95 E-05 \\
1.74 E-05 \\
2.38 E-05 \\
1.97 E-05 \\
3.87 E-05 \\
2.83 E-06 \\
2.61 E-06 \\
6.91 E-06 \\
2.64 E-06 \\
1.88 E-06 \\
2.55 E-06 \\
3.07 E-06 \\
2.95 E-06 \\
2.72 E-07 \\
2.33 E-06 \\
1.86 E-05 \\
2.98 E-06 \\
3.92 E-06 \\
5.59 E-07 \\
5.10 E-06 \\
1.93 E-05 \\
4.57 E-05 \\
1.99 E-06 \\
3.08 E-06 \\
5.74 E-05 \\
6.53 E-05 \\
3.688 E-05 \\
5.14 E-05 \\
4.31 E-05 \\
6.79 E-05 \\
4.32 E-05 \\
2.03 E-06 \\
9.27 E-05 \\
6.68 E-05 \\
3.86 E-06 \\
8.21 E-06 \\
4.75 E-05 \\
4.94 E-05 \\
9.08 E-05 \\
1.34 E-04 \\
7.27 E-05 \\
6.19 E-05 \\
3.07 E-05 \\
2.53 E-05 \\
7.00 E-05 \\
3.88 E-06 \\
4.91 E-05 \\
1.08 E-07 \\
2.82 E-05 \\
8.03 E-06 \\
8.15 E-05 \\
1.64 E-06 \\
6.03 E-00\end{array}$ & $\begin{array}{c}U 234 \\
1.85 E+00 \\
2.35 E+00 \\
2.27 E+00 \\
9.37 E+00 \\
7.69 E+00 \\
3.82 E+00 \\
4.58 E+00 \\
7.39 E+00 \\
1.78 E+00 \\
2.82 E+00 \\
2.41 E+01 \\
1.78 E+00 \\
8.75 E+00 \\
7.78 E+00 \\
1.06 E+01 \\
9.47 E+00 \\
2.06 E+01 \\
3.38 E+00 \\
1.25 E+00 \\
3.46 E+00 \\
2.96 E+00 \\
2.21 E+00 \\
2.57 E+00 \\
3.61 E+00 \\
3.58 E+00 \\
5.09 E-01 \\
2.67 E+00 \\
9.20 E+00 \\
3.52 E+00 \\
4.28 E+00 \\
6.68 E-01 \\
3.65 E+00 \\
1.03 E+01 \\
6.87 E+00 \\
2.96 E-01 \\
4.60 E-01 \\
8.78 E+00 \\
9.32 E+00 \\
5.34 E+00 \\
7.11 E+00 \\
6.24 E+00 \\
9.74 E+00 \\
6.44 E+00 \\
2.98 E-01 \\
1.84 E+01 \\
9.89 E+00 \\
5.91 E-01 \\
4.78 E+00 \\
9.40 E+00 \\
2.88 E+01 \\
5.17 E+01 \\
7.06 E+01 \\
3.10 E+01 \\
2.86 E+01 \\
1.43 E+01 \\
1.49 E+01 \\
3.35 E+01 \\
1.86 E+00 \\
2.95 E+01 \\
7.27 E=02 \\
1.80 E+01 \\
4.00 E+00 \\
4.49 E+01 \\
4.70 E-01 \\
6.44 E-01\end{array}$ & $\begin{array}{c}\text { U235 } \\
8.20 \mathrm{E}-02 \\
1.04 \mathrm{E}-01 \\
9.91 \mathrm{E}-02 \\
4.15 \mathrm{E}-01 \\
3.41 \mathrm{E}-01 \\
1.69 \mathrm{E}-01 \\
2.04 \mathrm{E}-01 \\
3.28 \mathrm{E}-01 \\
6.56 \mathrm{E}-02 \\
1.02 \mathrm{E}-01 \\
8.82 \mathrm{E}-01 \\
7.93 \mathrm{E}-02 \\
3.85 \mathrm{E}-01 \\
3.42 \mathrm{E}-01 \\
4.66 \mathrm{E}-01 \\
3.48 \mathrm{E}-01 \\
7.62 \mathrm{E}-01 \\
1.32 \mathrm{E}-01 \\
4.60 \mathrm{E}-02 \\
1.28 \mathrm{E}-01 \\
1.16 \mathrm{E}-01 \\
8.66 \mathrm{E}-02 \\
9.99 \mathrm{E}-02 \\
1.41 \mathrm{E}-01 \\
1.40 \mathrm{E}-01 \\
2.02 \mathrm{E}-02 \\
1.04 \mathrm{E}-01 \\
3.39 \mathrm{E}-01 \\
1.38 \mathrm{E}-01 \\
1.67 \mathrm{E}-01 \\
2.61 \mathrm{E}-02 \\
1.39 \mathrm{E}-01 \\
3.81 \mathrm{E}-01 \\
2.22 \mathrm{E}-01 \\
9.75 \mathrm{E}-03 \\
1.52 \mathrm{E}-02 \\
2.84 \mathrm{E}-01 \\
3.01 \mathrm{E}-01 \\
1.66 \mathrm{E}-01 \\
2.29 \mathrm{E}-01 \\
2.01 \mathrm{E}-01 \\
3.14 \mathrm{E}-01 \\
2.08 \mathrm{E}-01 \\
9.62 \mathrm{E}-03 \\
6.07 \mathrm{E}-01 \\
3.19 \mathrm{E}-01 \\
1.91 \mathrm{E}-02 \\
1.78 \mathrm{E}-01 \\
3.11 \mathrm{E}-01 \\
1.07 \mathrm{E}+00 \\
1.93 \mathrm{E}+00 \\
2.61 \mathrm{E}+00 \\
1.14 \mathrm{E}+00 \\
1.26 \mathrm{E}+00 \\
6.38 \mathrm{E}-01 \\
5.57 \mathrm{E}-01 \\
1.49 \mathrm{E}+00 \\
8.29 \mathrm{E}-02 \\
1.10 \mathrm{E}+00 \\
2.74 \mathrm{E}-03 \\
6.77 \mathrm{E}-01 \\
1.80 \mathrm{E}-01 \\
2.02 \mathrm{E}+00 \\
1.67 \mathrm{E}-02 \\
2.51 \mathrm{E}-02\end{array}$ & $\begin{array}{c}\text { U236 } \\
1.63 \mathrm{E}-01 \\
2.05 \mathrm{E}-01 \\
2.07 \mathrm{E}-01 \\
8.23 \mathrm{E}-01 \\
6.76 \mathrm{E}-01 \\
3.35 \mathrm{E}-01 \\
3.99 \mathrm{E}-01 \\
6.43 \mathrm{E}-01 \\
1.45 \mathrm{E}-01 \\
2.43 \mathrm{E}-01 \\
1.99 \mathrm{E}+00 \\
1.55 \mathrm{E}-01 \\
7.83 \mathrm{E}-01 \\
6.96 \mathrm{E}-01 \\
9.47 \mathrm{E}-01 \\
7.79 \mathrm{E}-01 \\
1.64 \mathrm{E}+00 \\
2.20 \mathrm{E}-01 \\
1.01 \mathrm{E}-01 \\
2.76 \mathrm{E}-01 \\
1.92 \mathrm{E}-01 \\
1.42 \mathrm{E}-01 \\
1.72 \mathrm{E}-01 \\
2.34 \mathrm{E}-01 \\
2.30 \mathrm{E}-01 \\
3.06 \mathrm{E}-02 \\
1.74 \mathrm{E}-01 \\
7.49 \mathrm{E}-01 \\
2.28 \mathrm{E}-01 \\
2.82 \mathrm{E}-01 \\
4.33 \mathrm{E}-02 \\
2.66 \mathrm{E}-01 \\
8.19 \mathrm{E}-01 \\
8.01 \mathrm{E}-01 \\
3.27 \mathrm{E}-02 \\
5.10 \mathrm{E}-02 \\
1.03 \mathrm{E}+00 \\
1.09 \mathrm{E}+00 \\
5.99 \mathrm{E}-01 \\
8.29 \mathrm{E}-01 \\
7.28 \mathrm{E}-01 \\
1.14 \mathrm{E}+00 \\
7.51 \mathrm{E}-01 \\
3.48 \mathrm{E}-02 \\
2.04 \mathrm{E}+00 \\
1.15 \mathrm{E}+00 \\
6.89 \mathrm{E}-02 \\
3.71 \mathrm{E}-01 \\
1.04 \mathrm{E}+00 \\
2.24 \mathrm{E}+00 \\
4.05 \mathrm{E}+00 \\
5.68 \mathrm{E}+00 \\
2.52 \mathrm{E}+00 \\
2.57 \mathrm{E}+00 \\
1.24 \mathrm{E}+00 \\
1.16 \mathrm{E}+00 \\
2.91 \mathrm{E}+00 \\
1.62 \mathrm{E}-01 \\
2.28 \mathrm{E}+00 \\
5.43 \mathrm{E}-03 \\
1.36 \mathrm{E}+00 \\
3.37 \mathrm{E}-01 \\
3.79 \mathrm{E}+00 \\
4.28 \mathrm{E}-02 \\
4.22 \mathrm{E}-02\end{array}$ & $\begin{array}{c}\text { U238 } \\
1.27 E+00 \\
1.61 E+00 \\
1.56 E+00 \\
6.41 E+00 \\
5.27 E+00 \\
2.61 E+00 \\
3.13 E+00 \\
5.05 E+00 \\
1.22 E+00 \\
1.95 E+00 \\
1.65 E+01 \\
1.22 E+00 \\
6.00 E+00 \\
5.33 E+00 \\
7.25 E+00 \\
6.51 E+00 \\
1.41 E+01 \\
2.28 E+00 \\
8.55 E-01 \\
2.37 E+00 \\
2.00 E+00 \\
1.49 E+00 \\
1.74 E+00 \\
2.44 E+00 \\
2.41 E+00 \\
3.42 E-01 \\
1.80 E+00 \\
6.32 E+00 \\
2.37 E+00 \\
2.89 E+00 \\
4.50 E-01 \\
2.48 E+00 \\
7.04 E+00 \\
4.92 E+00 \\
2.10 E-01 \\
3.27 E-01 \\
6.30 E+00 \\
6.68 E+00 \\
3.68 E+00 \\
5.09 E+00 \\
4.47 E+00 \\
6.98 E+00 \\
4.61 E+00 \\
2.14 E-01 \\
1.31 E+01 \\
7.08 E+00 \\
4.23 E-01 \\
3.27 E+00 \\
6.69 E+00 \\
1.97 E+01 \\
3.54 E+01 \\
4.84 E+01 \\
2.13 E+01 \\
1.96 E+01 \\
9.79 E+00 \\
1.02 E+01 \\
2.29 E+01 \\
1.27 E+00 \\
2.02 E+01 \\
4.95 E-00 \\
1.23 E+01 \\
2.73 E+00 \\
3.07 E+01 \\
3.26 E=01 \\
4.35 E=01\end{array}$ & $\begin{array}{l}\text { NP237 } \\
3.06 \mathrm{E}-02 \\
3.77 \mathrm{E}-02 \\
4.22 \mathrm{E}-02 \\
1.55 \mathrm{E}-01 \\
1.27 \mathrm{E}-01 \\
6.31 \mathrm{E}-02 \\
7.37 \mathrm{E}-02 \\
1.19 \mathrm{E}-01 \\
2.90 \mathrm{E}-02 \\
5.55 \mathrm{E}-02 \\
4.12 \mathrm{E}-01 \\
2.87 \mathrm{E}-02 \\
1.53 \mathrm{E}-01 \\
1.36 \mathrm{E}-01 \\
1.85 \mathrm{E}-01 \\
1.59 \mathrm{E}-01 \\
3.11 \mathrm{E}-01 \\
2.13 \mathrm{E}-02 \\
1.97 \mathrm{E}-02 \\
5.22 \mathrm{E}-02 \\
1.87 \mathrm{E}-02 \\
1.33 \mathrm{E}-02 \\
1.88 \mathrm{E}-02 \\
2.28 \mathrm{E}-02 \\
2.16 \mathrm{E}-02 \\
1.95 \mathrm{E}-03 \\
1.75 \mathrm{E}-02 \\
1.50 \mathrm{E}-01 \\
2.22 \mathrm{E}-02 \\
2.95 \mathrm{E}-02 \\
4.21 \mathrm{E}-03 \\
3.91 \mathrm{E}-02 \\
1.55 \mathrm{E}-01 \\
3.32 \mathrm{E}-01 \\
1.25 \mathrm{E}-02 \\
1.95 \mathrm{E}-02 \\
4.25 \mathrm{E}-01 \\
4.52 \mathrm{E}-01 \\
2.50 \mathrm{E}-01 \\
3.46 \mathrm{E}-01 \\
3.03 \mathrm{E}-01 \\
4.73 \mathrm{E}-01 \\
3.12 \mathrm{E}-01 \\
1.44 \mathrm{E}-02 \\
7.67 \mathrm{E}-01 \\
4.79 \mathrm{E}-01 \\
2.85 \mathrm{E}-02 \\
6.59 \mathrm{E}-02 \\
3.93 \mathrm{E}-01 \\
3.96 \mathrm{E}-01 \\
7.34 \mathrm{E}-01 \\
1.10 \mathrm{E}+00 \\
5.05 \mathrm{E}-01 \\
5.03 \mathrm{E}-01 \\
2.28 \mathrm{E}-01 \\
2.06 \mathrm{E}-01 \\
5.33 \mathrm{E}-01 \\
2.96 \mathrm{E}-02 \\
3.98 \mathrm{E}-01 \\
8.63 \mathrm{E}-04 \\
2.26 \mathrm{E}-01 \\
5.81 \mathrm{E}-02 \\
6.52 \mathrm{E}-01 \\
1.11 \mathrm{E}-02 \\
4.30 \mathrm{E}-03\end{array}$ \\
\hline
\end{tabular}


HNF-SD-WM-T1-794, Rev. 0

ACTIVITY IN UNSEPARATED FUEL (Curies decayed to $1 / 1 / 94$ )

\begin{tabular}{|c|c|c|c|c|c|c|c|c|c|}
\hline 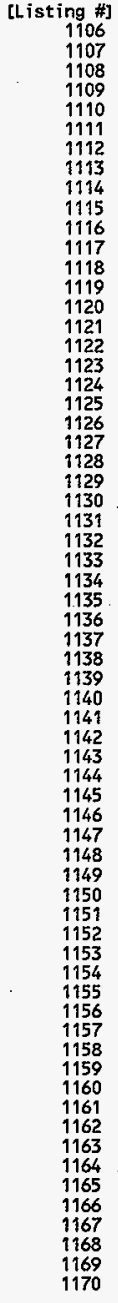 & $\begin{array}{l}\text { PU238 } \\
2.25 \mathrm{E}+01 \\
2.74 \mathrm{E}+01 \\
3.42 \mathrm{E}+01 \\
1.15 \mathrm{E}+02 \\
9.34 \mathrm{E}+01 \\
4.64 \mathrm{E}+01 \\
5.30 \mathrm{E}+01 \\
8.62 \mathrm{E}+01 \\
2.21 \mathrm{E}+01 \\
5.03 \mathrm{E}+01 \\
3.28 \mathrm{E}+02 \\
2.06 \mathrm{E}+01 \\
1.19 \mathrm{E}+02 \\
1.06 \mathrm{E}+02 \\
1.44 \mathrm{E}+02 \\
1.25 \mathrm{E}+02 \\
2.20 \mathrm{E}+02 \\
5.62 \mathrm{E}+00 \\
1.43 \mathrm{E}+01 \\
3.64 \mathrm{E}+01 \\
4.83 \mathrm{E}+00 \\
3.25 \mathrm{E}+00 \\
5.98 \mathrm{E}+00 \\
5.97 \mathrm{E}+00 \\
5.30 \mathrm{E}+00 \\
2.22 \mathrm{E}-01 \\
4.86 \mathrm{E}+00 \\
1.14 \mathrm{E}+02 \\
5.82 \mathrm{E}+00 \\
8.76 \mathrm{E}+00 \\
1.11 \mathrm{E}+00 \\
1.95 \mathrm{E}+01 \\
1.10 \mathrm{E}+02 \\
5.77 \mathrm{E}+02 \\
1.86 \mathrm{E}+01 \\
2.90 \mathrm{E}+01 \\
7.42 \mathrm{E}+02 \\
7.73 \mathrm{E}+02 \\
4.24 \mathrm{E}+02 \\
5.85 \mathrm{E}+02 \\
5.19 \mathrm{E}+02 \\
8.09 \mathrm{E}+02 \\
5.40 \mathrm{E}+02 \\
2.49 \mathrm{E}+01 \\
1.24 \mathrm{E}+03 \\
8.28 \mathrm{E}+02 \\
4.99 \mathrm{E}+01 \\
4.27 \mathrm{E}+01 \\
6.37 \mathrm{E}+02 \\
2.57 \mathrm{E}+02 \\
4.90 \mathrm{E}+02 \\
8.04 \mathrm{E}+02 \\
3.69 \mathrm{E}+02 \\
3.98 \mathrm{E}+02 \\
1.61 \mathrm{E}+02 \\
1.34 \mathrm{E}+02 \\
3.79 \mathrm{E}+02 \\
2.11 \mathrm{E}+01 \\
2.52 \mathrm{E}+02 \\
4.82 \mathrm{E}=01 \\
1.34 \mathrm{E}+02 \\
3.73 \mathrm{E}+01 \\
4.29 \mathrm{E}+02 \\
1.10 \mathrm{E}+01 \\
1.20 \mathrm{E}+00\end{array}$ & $\begin{array}{l}\text { PU239 } \\
2.28 E+02 \\
2.84 E+02 \\
3.04 E+02 \\
1.15 E+03 \\
9.47 E+02 \\
4.70 E+02 \\
5.53 E+02 \\
8.92 E+02 \\
2.22 E+02 \\
4.04 E+02 \\
3.11 E+03 \\
2.15 E+02 \\
1.12 E+03 \\
9.97 E+02 \\
1.36 E+03 \\
1.21 E+03 \\
2.42 E+03 \\
1.92 E+02 \\
1.51 E+02 \\
4.06 E+02 \\
1.68 E+02 \\
1.20 E+02 \\
1.66 E+02 \\
2.05 E+02 \\
1.95 E+02 \\
1.84 E+01 \\
1.57 E+02 \\
1.15 E+03 \\
1.99 E+02 \\
2.62 E+02 \\
3.78 E+01 \\
3.26 E+02 \\
1.21 E+03 \\
1.72 E+03 \\
6.82 E+01 \\
1.06 E+02 \\
2.20 E+03 \\
2.33 E+03 \\
1.29 E+03 \\
1.78 E+03 \\
1.56 E+03 \\
2.44 E+03 \\
1.61 E+03 \\
7.46 E+01 \\
4.24 E+03 \\
2.47 E+03 \\
1.48 E+02 \\
5.23 E+02 \\
2.17 E+03 \\
3.15 E+03 \\
5.80 E+03 \\
8.49 E+03 \\
3.85 E+03 \\
3.69 E+03 \\
1.71 E+03 \\
1.63 E+03 \\
4.01 E+03 \\
2.23 E+02 \\
3.18 E+03 \\
7.07 E+00 \\
1.83 E+03 \\
4.47 E+002 \\
5.02 E+03 \\
7.59 E+01 \\
3.84 E+01\end{array}$ & $\begin{array}{l}P U 240 \\
6.25 E+01 \\
7.66 E+01 \\
8.93 E+01 \\
3.17 E+02 \\
2.60 E+02 \\
1.29 E+02 \\
1.50 E+02 \\
2.41 E+02 \\
6.11 E+01 \\
1.24 E+02 \\
8.81 E+02 \\
5.82 E+01 \\
3.18 E+02 \\
2.83 E+02 \\
3.84 E+02 \\
3.39 E+02 \\
6.39 E+02 \\
3.50 E+01 \\
4.10 E+01 \\
1.07 E+02 \\
3.06 E+01 \\
2.17 E+01 \\
3.19 E+01 \\
3.74 E+01 \\
3.51 E+01 \\
2.93 E+00 \\
2.90 E+01 \\
3.15 E+02 \\
3.64 E+01 \\
4.94 E+01 \\
6.91 E+00 \\
7.30 E+01 \\
3.19 E+02 \\
9.39 E+02 \\
3.42 E+01 \\
5.33 E+01 \\
1.20 E+03 \\
1.27 E+03 \\
7.02 E+02 \\
9.71 E+02 \\
8.52 E+02 \\
1.33 E+03 \\
8.80 E+02 \\
4.08 E+01 \\
2.13 E+03 \\
1.35 E+03 \\
8.08 E+01 \\
1.32 E+02 \\
1.09 E+03 \\
7.94 E+02 \\
1.48 E+03 \\
2.28 E+03 \\
1.06 E+03 \\
1.05 E+03 \\
4.60 E+02 \\
4.12 E+02 \\
1.08 E+03 \\
5.99 E+01 \\
7.91 E+02 \\
1.66 E+00 \\
4.41 E+02 \\
1.14 E+02 \\
1.28 E+03 \\
2.61 E+03 \\
7.14 E+00\end{array}$ & $\begin{array}{c}P U 241 \\
2.43 E+03 \\
3.13 E+03 \\
3.63 E+03 \\
1.32 E+04 \\
1.00 E+04 \\
5.01 E+03 \\
5.80 E+03 \\
9.85 E+03 \\
2.65 E+03 \\
6.30 E+03 \\
3.98 E+04 \\
2.26 E+03 \\
1.36 E+04 \\
1.20 E+04 \\
1.62 E+04 \\
1.51 E+04 \\
2.61 E+04 \\
3.03 E+02 \\
1.58 E+03 \\
4.02 E+03 \\
2.40 E+02 \\
1.43 E+02 \\
4.19 E+02 \\
3.16 E+02 \\
2.42 E+02 \\
0.00 E+00 \\
2.89 E+02 \\
1.37 E+04 \\
3.09 E+02 \\
5.73 E+02 \\
5.98 E+01 \\
1.97 E+03 \\
1.30 E+04 \\
5.29 E+04 \\
1.47 E+03 \\
2.30 E+03 \\
6.97 E+04 \\
6.62 E+04 \\
3.53 E+04 \\
4.80 E+04 \\
4.53 E+04 \\
6.99 E+04 \\
4.90 E+04 \\
2.22 E+03 \\
1.42 E+05 \\
7.44 E+04 \\
4.68 E+03 \\
4.97 E+03 \\
7.27 E+04 \\
2.99 E+04 \\
5.79 E+04 \\
9.79 E+04 \\
3.69 E+04 \\
4.71 E+04 \\
1.69 E+04 \\
1.58 E+04 \\
4.11 E+04 \\
2.30 E+03 \\
2.96 E+04 \\
5.38 E+01 \\
1.52 E+04 \\
3.75 E+03 \\
4.88 E+04 \\
1.08 E+03 \\
6.91 E+01\end{array}$ & $\begin{array}{l}\text { PU242 } \\
5.75 E-03 \\
6.60 E=03 \\
1.05 E-02 \\
2.91 E-02 \\
2.39 E-02 \\
1.19 E-02 \\
1.29 E-02 \\
2.08 E-02 \\
5.74 E-03 \\
1.67 E-02 \\
9.23 E-02 \\
5.02 E-03 \\
3.31 E-02 \\
2.94 E-02 \\
4.00 E-02 \\
3.42 E-02 \\
4.97 E-02 \\
0.00 E+00 \\
3.57 E-03 \\
8.36 E-03 \\
0.00 E+00 \\
0.00 E+00 \\
0.00 E+00 \\
0.00 E+00 \\
0.00 E+00 \\
0.00 E+00 \\
0.00 E+00 \\
2.96 E-02 \\
0.00 E+00 \\
0.00 E+00 \\
0.00 E+00 \\
3.28 E-04 \\
2.48 E-02 \\
3.62 E-01 \\
1.12 E-02 \\
1.73 E-02 \\
4.63 E-01 \\
4.91 E-01 \\
2.71 E-01 \\
3.74 E-01 \\
3.29 E-01 \\
5.13 E-01 \\
3.39 E-01 \\
1.57 E-02 \\
6.93 E-01 \\
5.21 E-01 \\
3.111 E-02 \\
7.68 E-03 \\
3.55 E-01 \\
4.63 E-02 \\
9.56 E-02 \\
1.93 E-01 \\
9.97 E-02 \\
1.11 E E-01 \\
3.85 E-02 \\
2.40 E-02 \\
9.00 E-02 \\
5.00 E-03 \\
4.22 E-02 \\
4.35 E-05 \\
1.69 E-02 \\
7.23 E-03 \\
8.12 E-02 \\
4.47 E-03 \\
0.00 E+00\end{array}$ & $\begin{array}{c}A M 241 \\
5.54 E+01 \\
5.90 E+01 \\
9.39 E+01 \\
2.52 E+02 \\
2.32 E+02 \\
1.14 E+02 \\
1.25 E+02 \\
1.85 E+02 \\
4.82 E+01 \\
1.15 E+02 \\
7.33 E+02 \\
4.87 E+01 \\
2.76 E+02 \\
2.48 E+02 \\
3.40 E+02 \\
2.78 E+02 \\
4.66 E+02 \\
5.36 E+00 \\
3.64 E+01 \\
9.03 E+01 \\
5.50 E+00 \\
3.19 E+00 \\
8.29 E+00 \\
5.98 E+00 \\
4.80 E+00 \\
0.00 E+00 \\
5.17 E+00 \\
2.49 E+02 \\
5.76 E+00 \\
1.04 E+01 \\
1.06 E+00 \\
3.73 E+01 \\
2.33 E+02 \\
2.14 E+03 \\
8.49 E+01 \\
1.32 E+02 \\
2.68 E+03 \\
3.09 E+03 \\
1.74 E+03 \\
2.43 E+03 \\
2.03 E+03 \\
3.20 E+03 \\
2.03 E+03 \\
9.54 E+01 \\
3.67 E+03 \\
3.14 E+03 \\
1.80 E+02 \\
8.62 E+01 \\
1.88 E+03 \\
5.19 E+02 \\
9.85 E+02 \\
1.64 E+03 \\
1.14 E+03 \\
8.45 E+02 \\
4.05 E+02 \\
2.60 E+02 \\
8.99 E+02 \\
4.96 E+01 \\
4.88 E+02 \\
8.82 E=01 \\
2.54 E+02 \\
9.46 E+01 \\
8.47 E+02 \\
4.07 E+01 \\
1.54 E+00\end{array}$ & $\begin{array}{l}\text { AM243 } \\
4.37 E-03 \\
4.70 \mathrm{E}-03 \\
9.50 \mathrm{E}-03 \\
2.21 \mathrm{E}-02 \\
1.82 \mathrm{E}-02 \\
9.01 \mathrm{E}-03 \\
9.18 \mathrm{E}-03 \\
1.48 \mathrm{E}-02 \\
4.48 \mathrm{E}-03 \\
1.67 \mathrm{E}-02 \\
7.89 \mathrm{E}-02 \\
3.57 \mathrm{E}-03 \\
2.77 \mathrm{E}-02 \\
2.47 \mathrm{E}-02 \\
3.35 \mathrm{E}-02 \\
2.84 \mathrm{E}-02 \\
3.14 \mathrm{E}-02 \\
0.00 \mathrm{E}+00 \\
2.59 \mathrm{E}-03 \\
5.27 \mathrm{E}-03 \\
0.00 \mathrm{E}+00 \\
0.00 \mathrm{E}+00 \\
0.00 \mathrm{E}+00 \\
0.00 \mathrm{E}+00 \\
0.00 \mathrm{E}+00 \\
0.00 \mathrm{E}+00 \\
0.00 \mathrm{E}+00 \\
2.31 \mathrm{E}-02 \\
0.00 \mathrm{E}+00 \\
0.00 \mathrm{E}+00 \\
0.00 \mathrm{E}+00 \\
0.00 \mathrm{E}+00 \\
1.57 \mathrm{E}-02 \\
6.49 \mathrm{E}-01 \\
1.79 \mathrm{E}-02 \\
2.79 \mathrm{E}-02 \\
8.31 \mathrm{E}-01 \\
8.81 \mathrm{E}-01 \\
4.86 \mathrm{E}-01 \\
6.72 \mathrm{E}-01 \\
5.90 \mathrm{E}-01 \\
9.21 \mathrm{E}-01 \\
6.09 \mathrm{E}-01 \\
2.82 \mathrm{E}-02 \\
1.12 \mathrm{E}+00 \\
9.35 \mathrm{E}-01 \\
5.59 \mathrm{E}-02 \\
2.60 \mathrm{E}-03 \\
5.72 \mathrm{E}-01 \\
1.57 \mathrm{E}-02 \\
4.31 \mathrm{E}-02 \\
1.35 \mathrm{E}-01 \\
7.78 \mathrm{E}-02 \\
9.36 \mathrm{E}-02 \\
2.65 \mathrm{E}-02 \\
8.12 \mathrm{E}-03 \\
6.20 \mathrm{E}-02 \\
3.45 \mathrm{E}-03 \\
9.74 \mathrm{E}-03 \\
9.68 \mathrm{E}-09 \\
4.11 \mathrm{E}-06 \\
3.23 \mathrm{E}-03 \\
3.63 \mathrm{E}-02 \\
4.94 \mathrm{E}-03 \\
0.00 \mathrm{E}+00\end{array}$ & $\begin{array}{l}\text { CM242 } \\
1.21 E-02 \\
1.32 E-02 \\
2.61 E-02 \\
6.19 E-02 \\
5.04 E-02 \\
2.50 E-02 \\
2.56 E-02 \\
4.16 E-02 \\
1.27 E-02 \\
4.71 E-02 \\
2.22 E-01 \\
9.98 E-03 \\
7.71 E-02 \\
6.85 E-02 \\
9.30 E-02 \\
8.02 E-02 \\
8.87 E-02 \\
0.00 E+00 \\
7.22 E-03 \\
1.47 E-02 \\
0.00 E+00 \\
0.00 E+00 \\
0.00 E+00 \\
0.00 E+00 \\
0.00 E+00 \\
0.00 E+00 \\
0.00 E+00 \\
6.53 E-02 \\
0.00 E+00 \\
0.00 E+00 \\
0.00 E+00 \\
0.00 E+00 \\
4.43 E-02 \\
1.61 E+00 \\
4.46 E-02 \\
6.95 E-02 \\
2.07 E+00 \\
2.17 E+00 \\
1.19 E+00 \\
1.65 E+00 \\
1.46 E+00 \\
2.27 E+00 \\
1.51 E+00 \\
6.97 E-02 \\
2.89 E+00 \\
2.31 E+00 \\
1.39 E-01 \\
7.39 E-03 \\
1.48 E+00 \\
4.45 E-02 \\
1.22 E-01 \\
3.83 E-01 \\
2.15 E-01 \\
2.62 E-01 \\
7.39 E-02 \\
2.32 E-02 \\
1.73 E-01 \\
9.65 E-03 \\
2.79 E-02 \\
5.44 E-09 \\
2.26 E-06 \\
9.24 E-03 \\
1.06 E-01 \\
1.35 E-02 \\
0.00 E+00\end{array}$ & $\begin{array}{c}\text { CM243 } \\
1.23 \mathrm{E}-03 \\
1.28 \mathrm{E}-03 \\
3.02 \mathrm{E}-03 \\
6.43 \mathrm{E}-03 \\
5.09 \mathrm{E}-03 \\
2.53 \mathrm{E}-03 \\
2.44 \mathrm{E}-03 \\
4.04 \mathrm{E}-03 \\
1.37 \mathrm{E}-03 \\
6.20 \mathrm{E}-03 \\
2.61 \mathrm{E}-02 \\
9.49 \mathrm{E}-04 \\
8.72 \mathrm{E}-03 \\
7.73 \mathrm{E}-03 \\
1.05 \mathrm{E}-02 \\
9.18 \mathrm{E}-03 \\
7.32 \mathrm{E}-03 \\
0.00 \mathrm{E}+00 \\
6.95 \mathrm{E}-04 \\
1.18 \mathrm{E}-03 \\
0.00 \mathrm{E}+00 \\
0.00 \mathrm{E}+00 \\
0.00 \mathrm{E}+00 \\
0.00 \mathrm{E}+00 \\
0.00 \mathrm{E}+00 \\
0.00 \mathrm{E}+00 \\
0.00 \mathrm{E}+00 \\
7.06 \mathrm{E}-03 \\
0.00 \mathrm{E}+00 \\
0.00 \mathrm{E}+00 \\
0.00 \mathrm{E}+00 \\
0.00 \mathrm{E}+00 \\
3.65 \mathrm{E}-03 \\
3.12 \mathrm{E}-01 \\
7.21 \mathrm{E}-03 \\
1.13 \mathrm{E}-02 \\
4.06 \mathrm{E}-01 \\
4.07 \mathrm{E}-01 \\
2.21 \mathrm{E}-01 \\
3.03 \mathrm{E}-01 \\
2.76 \mathrm{E}-01 \\
4.28 \mathrm{E}-01 \\
2.91 \mathrm{E}-01 \\
1.34 \mathrm{E}-02 \\
5.60 \mathrm{E}-01 \\
4.45 \mathrm{E}-01 \\
2.73 \mathrm{E}-02 \\
0.00 \mathrm{E}+00 \\
2.87 \mathrm{E}-01 \\
0.00 \mathrm{E}+00 \\
3.58 \mathrm{E}-03 \\
3.68 \mathrm{E}-02 \\
2.12 \mathrm{E}-02 \\
3.04 \mathrm{E}-02 \\
6.64 \mathrm{E}-03 \\
0.00 \mathrm{E}+00 \\
1.58 \mathrm{E}-02 \\
8.82 \mathrm{E}-04 \\
0.00 \mathrm{E}+00 \\
0.00 \mathrm{E}+00 \\
0.00 \mathrm{E}+00 \\
2.78 \mathrm{E}-04 \\
3.35 \mathrm{E}-03\end{array}$ \\
\hline
\end{tabular}


ACTIVITY IN UNSEPARATED FUEL (Curies decayed to $1 / 1 / 94$ )

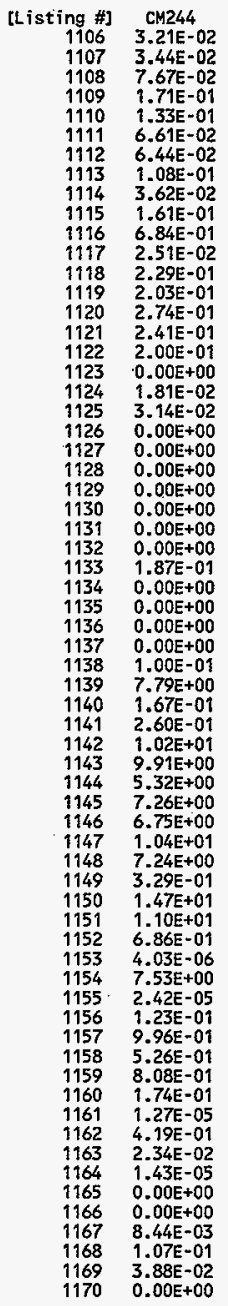


HNF-SD-WM-TI-794, Rev, 0

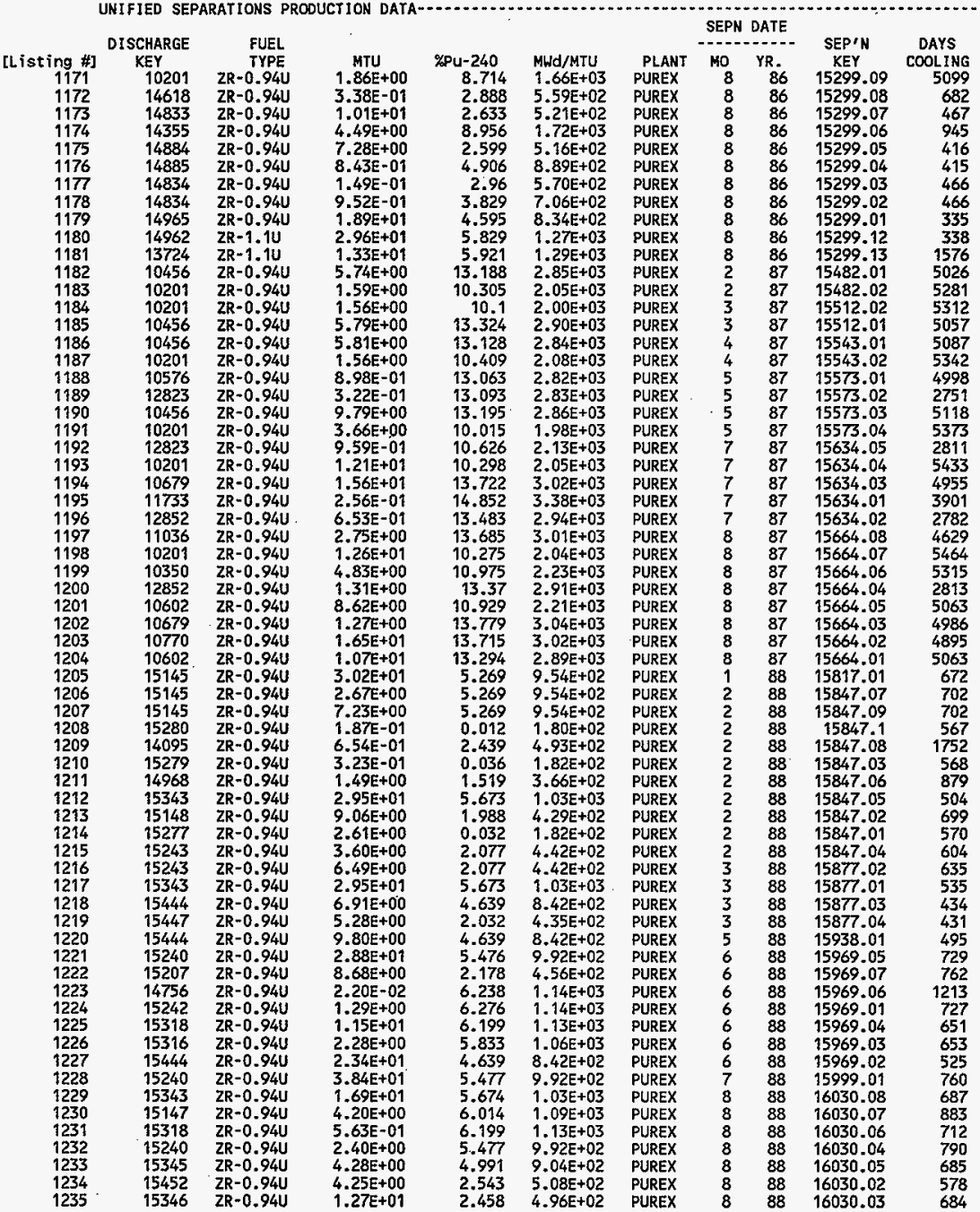


HNF-SD-WM-T1-794, ReV. 0

UNIFIED SEPARATIONS PRODUCTION DATA- TOTAL WASTE LOSS FRACTION
TON

[L.isting \#] Fuel Data Reference $1171 \mathrm{~J}$. SCHLOSSER FILE 'SHIP PX.DAT' 01/ 1172 J. SCHLOSSER FILE 'SHIP_PX.DAT' 01/ 1173 J. SCHLOSSER FILE 'SHIP_PX.DAT' O1/ $1174 \mathrm{~J}$. SCHLOSSER FILE 'SHIP-PX.DAT' 01/ 1175 J. SCHLOSSER FILE 'SHIP-PX.DAT' 01/ 1176 \$. SCHLOSSER FILE 'SHIP'PX.DAT' 01/ 1177 \&. SCHLOSSER FILE 'SHIP'PX.DAT' 01/ 1178 J. SCHLOSSER FILE 'SHIP_PX.DAT' 01/ $1179 \mathrm{~J}$. SCHLOSSER FILE 'SHIP'PX.DAT' 01/ $1180 \mathrm{~J}$. SCHLOSSER FILE 'SHIP_PX.DAT' 01/ $1181 \mathrm{~J}$. SCHLOSSER FILE 'SHIP-PX.DAT' 01/ $1182 \mathrm{~J}$. SCHLOSSER FILE 'SHIP-PX.DAT' 01/ $1183 \mathrm{~J}$. SCHLOSSER FILE 'SHIP-PX.DAT' 01/ $1184 \mathrm{~J}$. SCHLOSSER FILE 'SHIP-PX.OAT' O1/ $1185 \mathrm{~J}$. SCHLOSSER FILE 'SHIP_PX.DAT' O1/ $1186 \mathrm{~J}$. SCHLOSSER FILE 'SHIP_PX.DAT' OT/ $1187 \mathrm{~J}$. SCHLOSSER FILE 'SHIP-PX.DAT' 01/ $1188 \mathrm{~J}$. SCHLOSSER FILE 'SHIP.PX.DAT' 01/ $1189 \mathrm{~J}$. SCHLOSSER FILE 'SHIP.PX.DAT' 01/ $1190 \mathrm{~J}$. SCHLOSSER FILE 'SHIP'PX.DAT' 01/ 1191 J. SCHLOSSER FILE 'SHIP_PX.DAT' 01/ 1192 J. SCHLOSSER FILE 'SHIP'PX.DAT' 01/ 1193 J. SCHLOSSER FILE 'SHIP-PX.DAT' 01/ $1194 \mathrm{~J}$. SCHLOSSER FILE 'SHIPIPX.DAT' 01/ $1195 \mathrm{~J}$. SCHLOSSER FILE 'SHIP'PX.DAT' 01/ 1196 J. SCHLOSSER FILE 'SHIP_PX.DAT' 01/ $1197 \mathrm{~J}$. SCHLOSSER FILE 'SHIP-PX.DAT' 01/ $1198 \mathrm{~J}$. SCHLOSSER FILE 'SHIP-PX.DAT' 01/ 1199 J. SCHLOSSER FILE 'SHIP'PX.DAT' 01/ $1200 \mathrm{~J}$. SCHLOSSER FILE 'SHIP'PX.DAT' 01/ 1201 J. SCHLOSSER FILE 'SHIP_PX.DAT' 01/ 1202 J. SCHLOSSER FILE 'SHIP'PX.DAT' 01/ 1203 J. SCHLOSSER FILE 'SHIP'PX.DAT' 01/ 1204 J. SCHLOSSER FILE 'SHIP-PX.DAT' 01/ $1205 \mathrm{~J}$. SCHLOSSER FILE ISHIP-PX.DAT' O1/ $1206 \mathrm{~J}$. SCHLOSSER FILE 'SHIP_PX.DAT' 01/ $1207 \mathrm{j}$. SCHLOSSER FILE 'SHIPIPX.DAT' 01/ $1208 \mathrm{~J}$. SCHLOSSER FILE 'SHIP'PX.DAT' 01/ $1209 \mathrm{~J}$. SCHLOSSER FILE 'SHIP'PX.DAT' 01/ $1210 \mathrm{~J}$. SCHLOSSER FILE 'SHIP.PX.DAT' 01/ $1211 \mathrm{~J}$. SCHLOSSER FILE 'SHIP_PX.DAT' 01/ $1212 \mathrm{~J}$. SCHLOSSER FILE 'SHIP'PX.DAT' 01/ $1213 \mathrm{~J}$. SCHLOSSER FILE 'SHIP'PX.DAT' 01/ 1214 J. SCHLOSSER FILE 'SHIP_PX.DAT' O1/ $1215 \mathrm{~J}$. SCHLOSSER FILE 'SHIP.PX.OAT' O1/ $1216 \mathrm{~J}$. SCHLOSSER FILE 'SHIP_PX.DAT' O१/ $1217 \mathrm{~J}$. SCHLOSSER FILE 'SHIP_PX.DAT' 01/ $1218 \mathrm{~J}$. SCHLOSSER FILE 'SHIP-PX.DAT' O1/ 1219 J. SCHLOSSER FILE 'SHIP.PX.DAT' 01/ $1220 \mathrm{~J}$. SCHLOSSER FILE 'SHIP_PX.DAT' 01/ $1221 \mathrm{~J}$. SCHLOSSER FILE 'SHIP'PX.DAT' 01/ 1222 ง. SCHLOSSER FILE 'SHIP_PX.DAT' 01/ $1223 \mathrm{~J}$. SCHLOSSER FILE 'SHIP-PX.DAT' 01/ 1224 J. SCHLOSSER FILE 'SHIP-PX.DAT' 01/ $1225 \mathrm{~J}$. SCHLOSSER FILE 'SHIP-PX.DAT' O1/ $1226 \mathrm{~J}$. SCHLOSSER FILE 'SHIP'PX.DAT' 01/ 1227 J. SCHLOSSER FILE 'SHIP_PX.DAT' 01/ $1228 \mathrm{~d}$. SCHLOSSER FILE 'SHIP-PX.DAT' 01/ $1229 \mathrm{~J}$. SCHLOSSER FILE 'SHIP'PX.DAT' 01/ 1230 J. SCHLOSSER FILE 'SHIP_PX.DAT' 01/ 1231 3. SCHLOSSER FILE 'SHIP'PX.DAT' 01/ 1232 d. SCHLOSSER FILE 'SHIP PX.DAT' 01/ 1233 J. SCHLOSSER FILE 'SHIP-PX.DAT' 01/ $1234 \mathrm{~J}$. SCHLOSSER FILE 'SHIP PX.DAT' 01/ 1235 J. SCHLOSSER FILE 'SHIP_PX.DAT' 01/

Th

Th

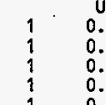

0.016

0.0168

0.0168

0.0168

0.0168

0.0168

0.0168

0.0168

0.0168

0.0168

0.0168

0.0168

0.0168

0.0168

0.0168

0.0168

0.0168

0.0168

0.0168

0.0168

0.0168

0.0168

0.0168

0.0168

0.0168

0.0168

0.0168

0.0168

0.0168

0.0168

0.0168

0.0168

0.0168

0.0168

0.0168

0.0168

0.0168

0.0168

0.0168

0.0168

0.0168

0.0168

0.0168

0.0168

0.0168

0.0168

0.0168

0.0168

0.0168

0.0168

0.0168

0.0168

0.0168

0.0168

0.0168

0.0168

0.0168

0.0168

0.0168

0.0168

0.0168

0.0168

0.0168
0.0168

Np

Pu

0.033

0.033

0.033

0.033

0.033

0.033

0.033

0.033

0.033

0.033

0.033

0.033

0.033

0.033

0.033

0.033

0.033

0.033

0.033

0.033

0.033

0.033

0.033

0.033

0.033

0.033

0.033

0.033

0.033

0.033

0.033

0.033

0.033

0.033

0.033

0.033

0.033

0.033

0.033

0.033

0.033

0.033

0.033

0.033

0.033

0.033

0.033

0.033

0.033

0.033

0.033

0.033

0.033

0.033

0.033

0.033

0.033

0.033

0.033

0.033

0.033

0.033

0.033

0.033

0.033
Waste Frac. Reference (c)

(c)

(c)

(c)

(c)

(c)

(c)

(c)

(c)

(c)

(c)

(c)

(c)

(c)

(c)

(c)

(c)

(c)

(c)

(c)

(c)

(c)

(c)

(c)

(c)

(c)

(c)

(c)

(c)

(c)

(c)

(c)

(c)

(c)

(c)

(c)

(c)

(c)

(c)

(c)

(c)

(c)

(c)

(c)

(c)

(c)

(c)

(c)

(c)

(c)

(c)

(c)

(c)

(c) 
HWF-SD-WM-TI-794, ReV. 0

ACTIVITY IN UNSEPARATED FUEL (Curies decayed to $1 / 1 / 94$ )

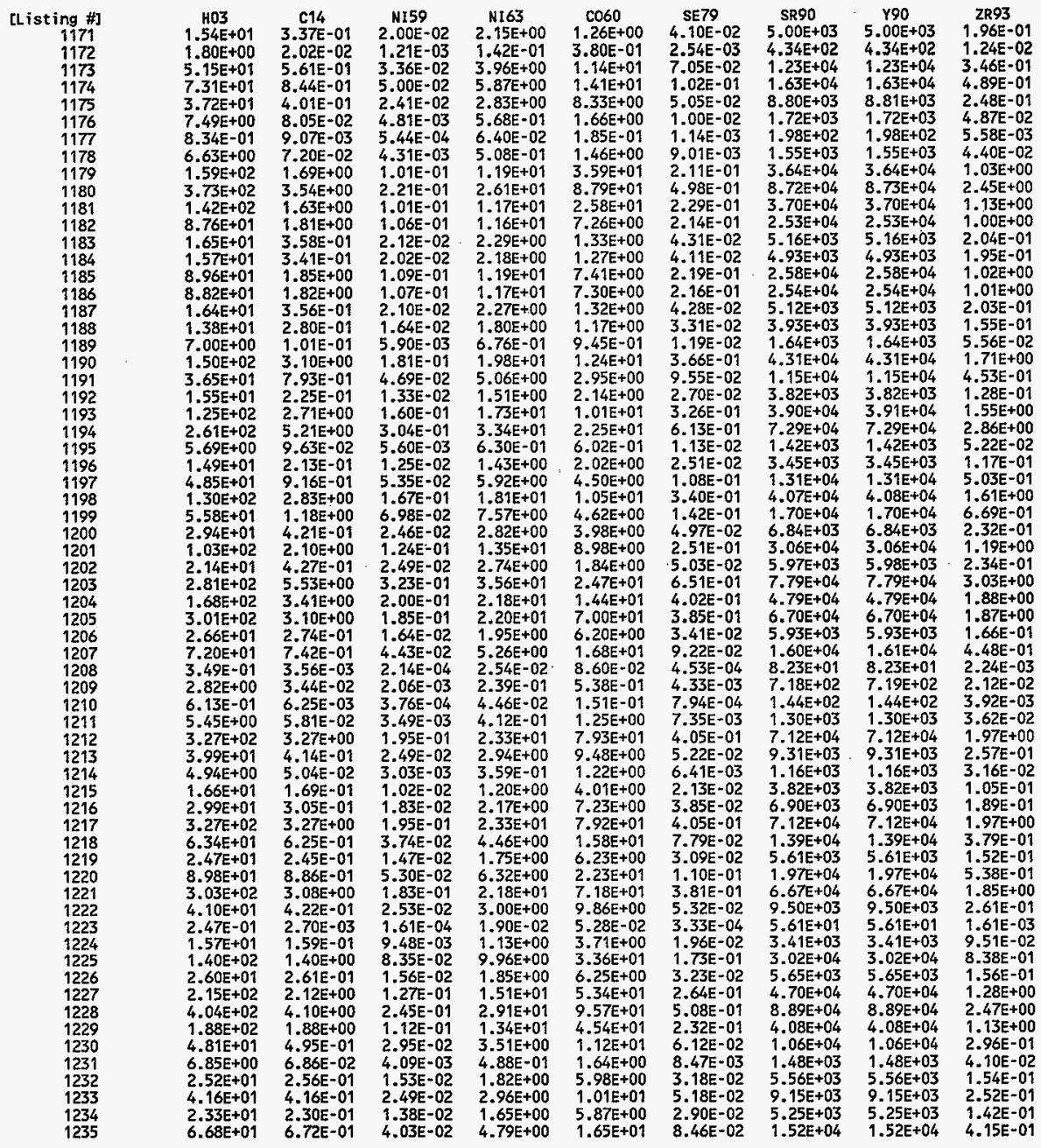


ACTIVITY IN UNSEPARATED FUEL (Curies decayed to 1/1/94)

\begin{tabular}{|c|c|c|c|c|c|c|c|c|c|}
\hline $\begin{array}{r}\text { [listing } \$ \text { ] } \\
1171 \\
1172 \\
1173 \\
1174 \\
1175 \\
1176 \\
1177 \\
1178 \\
1179 \\
1180 \\
1181 \\
1182 \\
1183 \\
1184 \\
1185 \\
1186 \\
1187 \\
1188 \\
1189 \\
1190 \\
1191 \\
1192 \\
1193 \\
1194 \\
1195 \\
1196 \\
1197 \\
1198 \\
1199 \\
1200 \\
1201 \\
1202 \\
1203 \\
1204 \\
1205 \\
1206 \\
1207 \\
1208 \\
1209 \\
1210 \\
1211 \\
1212 \\
1213 \\
1214 \\
1215 \\
1216 \\
1217 \\
1218 \\
1219 \\
1220 \\
1221 \\
1222 \\
1223 \\
1224 \\
1125 \\
1226 \\
1227 \\
1228 \\
1229 \\
1230 \\
1231 \\
1232 \\
1233 \\
1234 \\
1235\end{array}$ & $\begin{array}{l}\text { NB93* } \\
1.24 \mathrm{E}-01 \\
4.48 \mathrm{E}-03 \\
1.18 \mathrm{E}-01 \\
1.89 \mathrm{E}-01 \\
8.36 \mathrm{E}-02 \\
1.65 \mathrm{E}-02 \\
1.91 \mathrm{E}-03 \\
1.51 \mathrm{E}-02 \\
3.41 \mathrm{E}-01 \\
8.19 \mathrm{E}-01 \\
4.86 \mathrm{E}-01 \\
6.27 \mathrm{E}-01 \\
1.30 \mathrm{E}-01 \\
1.24 \mathrm{E}-01 \\
6.41 \mathrm{E}-01 \\
6.31 \mathrm{E}-01 \\
1.29 \mathrm{E}-01 \\
9.60 \mathrm{E}-02 \\
2.78 \mathrm{E}-02 \\
1.07 \mathrm{E}+00 \\
2.88 \mathrm{E}-01 \\
6.34 \mathrm{E}-02 \\
9.82 \mathrm{E}-01 \\
1.76 \mathrm{E}+00 \\
2.95 \mathrm{E}-02 \\
5.84 \mathrm{E}-02 \\
3.01 \mathrm{E}-01 \\
1.03 \mathrm{E}+00 \\
4.21 \mathrm{E}-01 \\
1.15 \mathrm{E}-01 \\
7.33 \mathrm{E}-01 \\
1.44 \mathrm{E}-01 \\
1.86 \mathrm{E}+00 \\
1.17 \mathrm{E}+00 \\
5.93 \mathrm{E}-01 \\
5.25 \mathrm{E}-02 \\
1.42 \mathrm{E}-01 \\
6.74 \mathrm{E}-04 \\
8.53 \mathrm{E}-03 \\
1.18 \mathrm{E}-03 \\
1.19 \mathrm{E}-02 \\
5.89 \mathrm{E}-01 \\
8.07 \mathrm{E}-02 \\
9.54 \mathrm{E}-03 \\
3.20 \mathrm{E}-02 \\
5.79 \mathrm{E}-02 \\
5.90 \mathrm{E}-01 \\
1.10 \mathrm{E}-01 \\
4.36 \mathrm{E}-02 \\
1.56 \mathrm{E}-01 \\
5.72 \mathrm{E}-01 \\
8.07 \mathrm{E}-02 \\
5.66 \mathrm{E}-04 \\
2.94 \mathrm{E}-02 \\
2.53 \mathrm{E}-01 \\
4.73 \mathrm{E}-02 \\
3.72 \mathrm{E}-01 \\
7.62 \mathrm{E}-01 \\
3.38 \mathrm{E}-01 \\
9.41 \mathrm{E}-02 \\
1.24 \mathrm{E}-02 \\
4.76 \mathrm{E}-02 \\
7.52 \mathrm{E}-02 \\
4.08 \mathrm{E}-02 \\
1.23 \mathrm{E}-01\end{array}$ & $\begin{array}{l}1 C 99 \\
1.37 \mathrm{E}+00 \\
8.46 \mathrm{E}=02 \\
2.35 \mathrm{E}+00 \\
3.42 \mathrm{E}+00 \\
1.68 \mathrm{E}+00 \\
3.34 \mathrm{E}-01 \\
3.80 \mathrm{E}-02 \\
3.00 \mathrm{E}-01 \\
7.03 \mathrm{E}+00 \\
1.67 \mathrm{E}+01 \\
7.67 \mathrm{E}+00 \\
7.17 \mathrm{E}+00 \\
1.44 \mathrm{E}+00 \\
1.37 \mathrm{E}+00 \\
7.33 \mathrm{E}+00 \\
7.21 \mathrm{E}+00 \\
1.43 \mathrm{E}+00 \\
1.11 \mathrm{E}+00 \\
3.98 \mathrm{E}-01 \\
1.22 \mathrm{E}+01 \\
3.19 \mathrm{E}+00 \\
9.01 \mathrm{E}-01 \\
1.09 \mathrm{E}+01 \\
2.05 \mathrm{E}+01 \\
3.77 \mathrm{E}-01 \\
8.41 \mathrm{E}-01 \\
3.61 \mathrm{E}+00 \\
1.14 \mathrm{E}+01 \\
4.74 \mathrm{E}+00 \\
1.66 \mathrm{E}+00 \\
8.40 \mathrm{E}+00 \\
1.68 \mathrm{E}+00 \\
2.18 \mathrm{E}+01 \\
1.35 \mathrm{E}+01 \\
1.28 \mathrm{E}+01 \\
1.14 \mathrm{E}+00 \\
3.08 \mathrm{E}+00 \\
1.51 \mathrm{E}-02 \\
1.44 \mathrm{E}-01 \\
2.65 \mathrm{E}-02 \\
2.45 \mathrm{E}-01 \\
1.35 \mathrm{E}+01 \\
1.74 \mathrm{E}+00 \\
2.14 \mathrm{E}-01 \\
7.11 \mathrm{E}-01 \\
1.28 \mathrm{E}+00 \\
1.35 \mathrm{E}+01 \\
2.60 \mathrm{E}+00 \\
1.03 \mathrm{E}+00 \\
3.68 \mathrm{E}+00 \\
1.27 \mathrm{E}+01 \\
1.77 \mathrm{E}+00 \\
1.11 \mathrm{E}-02 \\
6.56 \mathrm{E}-01 \\
5.78 \mathrm{E}+00 \\
1.08 \mathrm{E}+00 \\
8.80 \mathrm{E}+00 \\
1.70 \mathrm{E}+01 \\
7.76 \mathrm{E}+00 \\
2.04 \mathrm{E}+00 \\
2.83 \mathrm{E}-01 \\
1.06 \mathrm{E}+00 \\
1.73 \mathrm{E}+00 \\
9.67 \mathrm{E}=01 \\
2.82 \mathrm{E}+00\end{array}$ & 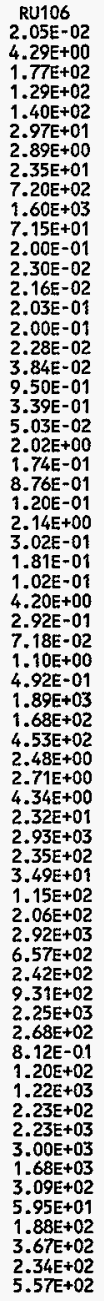 & $\begin{array}{l}C D 113 * \\
1.40 E+00 \\
1.37 E-01 \\
3.90 \mathrm{E}+00 \\
6.03 \mathrm{E}+00 \\
2.81 \mathrm{E}+00 \\
5.80 \mathrm{E}-01 \\
6.32 \mathrm{E}-02 \\
5.08 \mathrm{E}-01 \\
1.23 \mathrm{E}+01 \\
2.67 \mathrm{E}+01 \\
1.05 \mathrm{E}+01 \\
8.38 \mathrm{E}+00 \\
1.53 \mathrm{E}+00 \\
1.45 \mathrm{E}+00 \\
8.57 \mathrm{E}+00 \\
8.43 \mathrm{E}+00 \\
1.52 \mathrm{E}+00 \\
1.31 \mathrm{E}+00 \\
6.33 \mathrm{E}-01 \\
1.43 \mathrm{E}+01 \\
3.37 \mathrm{E}+00 \\
1.36 \mathrm{E}+00 \\
1.16 \mathrm{E}+01 \\
2.50 \mathrm{E}+01 \\
5.40 \mathrm{E}-01 \\
1.35 \mathrm{E}+00 \\
4.61 \mathrm{E}+00 \\
1.21 \mathrm{E}+01 \\
5.21 \mathrm{E}+00 \\
2.65 \mathrm{E}+00 \\
9.54 \mathrm{E}+00 \\
2.05 \mathrm{E}+00 \\
2.69 \mathrm{E}+01 \\
1.60 \mathrm{E}+01 \\
2.32 \mathrm{E}+01 \\
2.06 \mathrm{E}+00 \\
5.56 \mathrm{E}+00 \\
2.55 \mathrm{E}-02 \\
2.17 \mathrm{E}-01 \\
4.48 \mathrm{E}-02 \\
4.06 \mathrm{E}-01 \\
2.53 \mathrm{E}+01 \\
2.98 \mathrm{E}+00 \\
3.61 \mathrm{E}-01 \\
1.23 \mathrm{E}+00 \\
2.22 \mathrm{E}+00 \\
2.53 \mathrm{E}+01 \\
4.82 \mathrm{E}+00 \\
1.83 \mathrm{E}+00 \\
6.84 \mathrm{E}+00 \\
2.34 \mathrm{E}+01 \\
3.06 \mathrm{E}+00 \\
1.95 \mathrm{E}-02 \\
1.22 \mathrm{E}+00 \\
1.09 \mathrm{E}+01 \\
2.01 \mathrm{E}+00 \\
1.63 \mathrm{E}+01 \\
3.12 \mathrm{E}+01 \\
1.45 \mathrm{E}+01 \\
3.75 \mathrm{E}+00 \\
5.33 \mathrm{E}-01 \\
1.95 \mathrm{E}+00 \\
3.19 \mathrm{E}+00 \\
1.73 \mathrm{E}+00 \\
4.98 \mathrm{E}+00\end{array}$ & 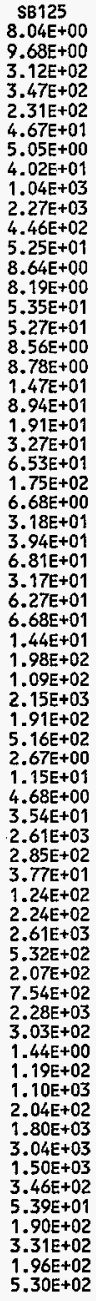 & $\begin{array}{l}\text { SH126 } \\
6.67 \mathrm{E}-02 \\
3.75 \mathrm{E}-03 \\
1.04 \mathrm{E}-01 \\
1.68 \mathrm{E}-01 \\
7.45 \mathrm{E}-02 \\
1.53 \mathrm{E}-02 \\
1.69 \mathrm{E}-03 \\
1.35 \mathrm{E}-02 \\
3.20 \mathrm{E}-01 \\
7.21 \mathrm{E}-01 \\
3.31 \mathrm{E}-01 \\
3.79 \mathrm{E}-01 \\
7.24 \mathrm{E}-02 \\
6.88 \mathrm{E}-02 \\
3.88 \mathrm{E}-01 \\
3.82 \mathrm{E}-01 \\
7.19 \mathrm{E}-02 \\
5.86 \mathrm{E}-02 \\
2.11 \mathrm{E}-02 \\
6.47 \mathrm{E}-01 \\
1.60 \mathrm{E}-01 \\
4.56 \mathrm{E}-02 \\
5.48 \mathrm{E}-01 \\
1.10 \mathrm{E}+00 \\
2.05 \mathrm{E}-02 \\
4.47 \mathrm{E}-02 \\
1.93 \mathrm{E}-01 \\
5.72 \mathrm{E}-01 \\
2.41 \mathrm{E}-01 \\
8.79 \mathrm{E}-02 \\
4.28 \mathrm{E}-01 \\
8.98 \mathrm{E}-02 \\
1.16 \mathrm{E}+00 \\
7.12 \mathrm{E}-01 \\
5.90 \mathrm{E}-01 \\
5.22 \mathrm{E}-02 \\
1.41 \mathrm{E}-01 \\
6.47 \mathrm{E}-04 \\
6.37 \mathrm{E}-03 \\
1.14 \mathrm{E}-03 \\
1.07 \mathrm{E}-02 \\
6.25 \mathrm{E}-01 \\
7.64 \mathrm{E}-02 \\
9.16 \mathrm{E}-03 \\
3.12 \mathrm{E}-02 \\
5.64 \mathrm{E}-02 \\
6.25 \mathrm{E}-01 \\
1.18 \mathrm{E}-01 \\
4.52 \mathrm{E}-02 \\
1.68 \mathrm{E}-01 \\
5.86 \mathrm{E}-01 \\
7.80 \mathrm{E}-02 \\
5.19 \mathrm{E}-04 \\
3.06 \mathrm{E}-02 \\
2.70 \mathrm{E}-01 \\
4.99 \mathrm{E}-02 \\
4.00 \mathrm{E}-01 \\
7.82 \mathrm{E}-01 \\
3.59 \mathrm{E}-01 \\
9.49 \mathrm{E}-02 \\
1.32 \mathrm{E}-02 \\
4.89 \mathrm{E}-02 \\
7.90 \mathrm{E}-02 \\
4.27 \mathrm{E}-02 \\
1.25 \mathrm{E}-01\end{array}$ & $\begin{array}{l}1129 \\
2.81 \mathrm{E}-03 \\
1.62 \mathrm{E}-04 \\
4.49 \mathrm{E}-03 \\
7.03 \mathrm{E}-03 \\
3.21 \mathrm{E}-03 \\
6.53 \mathrm{E}-04 \\
7.27 \mathrm{E}-05 \\
5.80 \mathrm{E}-04 \\
1.37 \mathrm{E}-02 \\
3.20 \mathrm{E}-02 \\
1.47 \mathrm{E}-02 \\
1.56 \mathrm{E}-02 \\
3.02 \mathrm{E}-03 \\
2.87 \mathrm{E}-03 \\
1.60 \mathrm{E}-02 \\
1.57 \mathrm{E}-02 \\
3.00 \mathrm{E}-03 \\
2.41 \mathrm{E}-03 \\
8.68 \mathrm{E}-04 \\
2.67 \mathrm{E}-02 \\
6.68 \mathrm{E}-03 \\
1.90 \mathrm{E}-03 \\
2.29 \mathrm{E}-02 \\
4.50 \mathrm{E}-02 \\
8.39 \mathrm{E}-04 \\
1.84 \mathrm{E}-03 \\
7.92 \mathrm{E}-03 \\
2.39 \mathrm{E}-02 \\
1.00 \mathrm{E}-02 \\
3.62 \mathrm{E}-03 \\
1.78 \mathrm{E}-02 \\
3.69 \mathrm{E}-03 \\
4.78 \mathrm{E}-02 \\
2.94 \mathrm{E}-02 \\
2.52 \mathrm{E}-02 \\
2.23 \mathrm{E}-03 \\
6.03 \mathrm{E}-03 \\
2.82 \mathrm{E}-05 \\
2.75 \mathrm{E}-04 \\
4.94 \mathrm{E}-05 \\
4.63 \mathrm{E}-04 \\
2.67 \mathrm{E}-02 \\
3.31 \mathrm{E}-03 \\
3.99 \mathrm{E}-04 \\
1.35 \mathrm{E}-03 \\
2.44 \mathrm{E}-03 \\
2.67 \mathrm{E}-02 \\
5.06 \mathrm{E}-03 \\
1.95 \mathrm{E}-03 \\
7.17 \mathrm{E}-03 \\
2.50 \mathrm{E}-02 \\
3.37 \mathrm{E}-03 \\
2.21 \mathrm{E}-05 \\
1.30 \mathrm{E}-03 \\
1.15 \mathrm{E}-02 \\
2.13 \mathrm{E}-03 \\
1.71 \mathrm{E}-02 \\
3.34 \mathrm{E}-02 \\
1.53 \mathrm{E}-02 \\
4.04 \mathrm{E}-03 \\
5.61 \mathrm{E}-04 \\
2.09 \mathrm{E}-03 \\
3.38 \mathrm{E}-03 \\
1.84 \mathrm{E}-03 \\
5.37 \mathrm{E}-03\end{array}$ & $\begin{array}{l}\text { CS134 } \\
1.80 \mathrm{E}+00 \\
1.94 \mathrm{E}+00 \\
6.04 \mathrm{E}+01 \\
2.14 \mathrm{E}+02 \\
4.53 \mathrm{E}+01 \\
1.66 \mathrm{E}+01 \\
1.06 \mathrm{E}+00 \\
1.10 \mathrm{E}+01 \\
3.50 \mathrm{E}+02 \\
1.11 \mathrm{E}+03 \\
1.64 \mathrm{E}+02 \\
2.07 \mathrm{E}+01 \\
2.38 \mathrm{E}+00 \\
2.20 \mathrm{E}+00 \\
2.11 \mathrm{E}+01 \\
2.08 \mathrm{E}+01 \\
2.36 \mathrm{E}+00 \\
3.56 \mathrm{E}+00 \\
1.01 \mathrm{E}+01 \\
3.53 \mathrm{E}+01 \\
5.11 \mathrm{E}+00 \\
1.73 \mathrm{E}+01 \\
1.80 \mathrm{E}+01 \\
7.67 \mathrm{E}+01 \\
4.14 \mathrm{E}+00 \\
2.27 \mathrm{E}+01 \\
1.87 \mathrm{E}+01 \\
1.87 \mathrm{E}+01 \\
9.74 \mathrm{E}+00 \\
4.35 \mathrm{E}+01 \\
2.18 \mathrm{E}+01 \\
6.28 \mathrm{E}+00 \\
8.85 \mathrm{E}+01 \\
4.44 \mathrm{E}+01 \\
8.77 \mathrm{E}+02 \\
7.77 \mathrm{E}+01 \\
2.10 \mathrm{E}+02 \\
1.29 \mathrm{E}-01 \\
1.76 \mathrm{E}+00 \\
2.27 \mathrm{E}-01 \\
4.60 \mathrm{E}+00 \\
1.21 \mathrm{E}+03 \\
4.73 \mathrm{E}+01 \\
1.83 \mathrm{E}+00 \\
2.18 \mathrm{E}+01 \\
3.93 \mathrm{E}+01 \\
1.20 \mathrm{E}+03 \\
2.03 \mathrm{E}+02 \\
3.67 \mathrm{E}+01 \\
2.88 \mathrm{E}+02 \\
9.90 \mathrm{E}+02 \\
5.46 \mathrm{E}+01 \\
6.43 \mathrm{E}-01 \\
5.93 \mathrm{E}+03 \\
5.60 \mathrm{E}+02 \\
9.66 \mathrm{E}+01 \\
6.89 \mathrm{E}+02 \\
1.32 \mathrm{E}+03 \\
6.90 \mathrm{E}+02 \\
1.62 \mathrm{E}+02 \\
2.74 \mathrm{E}+01 \\
8.25 \mathrm{E}+01 \\
1.34 \mathrm{E}+02 \\
4.26 \mathrm{E}+01 \\
1.09 \mathrm{E}+02\end{array}$ & 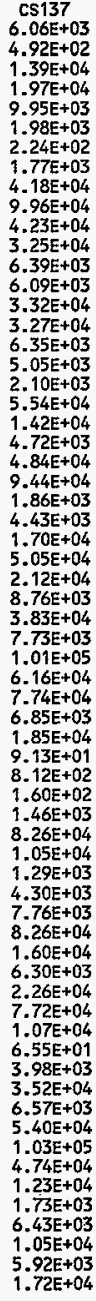 \\
\hline
\end{tabular}


HNF-SD-WH-TI-794, ReV. O

ACTIVITY IN UNSEPARATED FUEL. (Curies decayed to $1 / 1 / 94$ )

\begin{tabular}{|c|c|c|c|c|c|c|c|c|c|}
\hline $\begin{array}{r}\text { [Listing \#] } \\
1171 \\
1172 \\
1173 \\
1174 \\
1175 \\
1176 \\
1177 \\
1178 \\
1179 \\
1180 \\
1181 \\
1182 \\
1183 \\
1184 \\
1185 \\
1186 \\
1187 \\
1188 \\
1189 \\
1190 \\
1191 \\
1192 \\
1193 \\
1194 \\
1195 \\
1196 \\
1197 \\
1198 \\
1199 \\
1200 \\
1201 \\
1202 \\
1203 \\
1204 \\
1205 \\
1206 \\
1207 \\
1208 \\
1209 \\
1210 \\
1211 \\
1212 \\
1213 \\
1214 \\
1215 \\
1216 \\
1217 \\
1218 \\
1219 \\
1220 \\
1221 \\
1222 \\
1223 \\
1224 \\
1225 \\
1226 \\
1227 \\
1228 \\
1229 \\
1230 \\
1231 \\
1232 \\
1233 \\
1234 \\
1235\end{array}$ & $\begin{array}{l}B A 137 \\
5.73 \mathrm{E}+03 \\
4.65 \mathrm{E}+02 \\
1.31 \mathrm{E}+04 \\
1.86 \mathrm{E}+04 \\
9.42 \mathrm{E}+03 \\
1.87 \mathrm{E}+03 \\
2.12 \mathrm{E}+02 \\
1.68 \mathrm{E}+03 \\
3.96 \mathrm{E}+04 \\
9.42 \mathrm{E}+04 \\
4.00 \mathrm{E}+04 \\
3.07 \mathrm{E}+04 \\
6.05 \mathrm{E}+03 \\
5.76 \mathrm{E}+03 \\
3.14 \mathrm{E}+04 \\
3.09 \mathrm{E}+04 \\
6.00 \mathrm{E}+03 \\
4.78 \mathrm{E}+03 \\
1.98 \mathrm{E}+03 \\
5.24 \mathrm{E}+04 \\
1.34 \mathrm{E}+04 \\
4.47 \mathrm{E}+03 \\
4.58 \mathrm{E}+04 \\
8.93 \mathrm{E}+04 \\
1.76 \mathrm{E}+03 \\
4.19 \mathrm{E}+03 \\
1.61 \mathrm{E}+04 \\
4.77 \mathrm{E}+04 \\
2.01 \mathrm{E}+04 \\
8.28 \mathrm{E}+03 \\
3.62 \mathrm{E}+04 \\
7.32 \mathrm{E}+03 \\
9.53 \mathrm{E}+04 \\
5.82 \mathrm{E}+04 \\
7.32 \mathrm{E}+04 \\
6.48 \mathrm{E}+03 \\
1.75 \mathrm{E}+04 \\
8.64 \mathrm{E}+01 \\
7.68 \mathrm{E}+02 \\
1.52 \mathrm{E}+02 \\
1.38 \mathrm{E}+03 \\
7.81 \mathrm{E}+04 \\
9.90 \mathrm{E}+03 \\
1.22 \mathrm{E}+03 \\
4.07 \mathrm{E}+03 \\
7.34 \mathrm{E}+03 \\
7.81 \mathrm{E}+04 \\
1.51 \mathrm{E}+04 \\
5.96 \mathrm{E}+03 \\
2.14 \mathrm{E}+04 \\
7.30 \mathrm{E}+04 \\
1.01 \mathrm{E}+04 \\
6.19 \mathrm{E}+01 \\
3.77 \mathrm{E}+03 \\
3.33 \mathrm{E}+04 \\
6.21 \mathrm{E}+03 \\
5.11 \mathrm{E}+04 \\
9.73 \mathrm{E}+04 \\
4.48 \mathrm{E}+04 \\
1.17 \mathrm{E}+04 \\
1.63 \mathrm{E}+03 \\
6.08 \mathrm{E}+03 \\
9.97 \mathrm{E}+03 \\
5.60 \mathrm{E}+03 \\
1.62 \mathrm{E}+04\end{array}$ & $\begin{array}{l}\text { SM151 } \\
1.22 \mathrm{E}+02 \\
1.10 \mathrm{E}+01 \\
3.08 \mathrm{E}+02 \\
3.27 \mathrm{E}+02 \\
2.21 \mathrm{E}+02 \\
4.04 \mathrm{E}+01 \\
4.94 \mathrm{E}+00 \\
3.78 \mathrm{E}+01 \\
8.61 \mathrm{E}+02 \\
1.85 \mathrm{E}+03 \\
8.28 \mathrm{E}+02 \\
4.68 \mathrm{E}+02 \\
1.14 \mathrm{E}+02 \\
1.10 \mathrm{E}+02 \\
4.78 \mathrm{E}+02 \\
4.71 \mathrm{E}+02 \\
1.13 \mathrm{E}+02 \\
7.24 \mathrm{E}+01 \\
2.73 \mathrm{E}+01 \\
7.99 \mathrm{E}+02 \\
2.56 \mathrm{E}+02 \\
7.31 \mathrm{E}+01 \\
8.60 \mathrm{E}+02 \\
1.30 \mathrm{E}+03 \\
2.26 \mathrm{E}+01 \\
5.66 \mathrm{E}+01 \\
2.30 \mathrm{E}+02 \\
8.98 \mathrm{E}+02 \\
3.54 \mathrm{E}+02 \\
1.14 \mathrm{E}+02 \\
6.32 \mathrm{E}+02 \\
1.07 \mathrm{E}+02 \\
1.38 \mathrm{E}+03 \\
8.81 \mathrm{E}+02 \\
1.54 \mathrm{E}+03 \\
1.36 \mathrm{E}+02 \\
3.68 \mathrm{E}+02 \\
2.14 \mathrm{E}+00 \\
1.88 \mathrm{E}+01 \\
3.76 \mathrm{E}+00 \\
3.33 \mathrm{E}+01 \\
1.60 \mathrm{E}+03 \\
2.34 \mathrm{E}+02 \\
3.03 \mathrm{E}+01 \\
9.57 \mathrm{E}+01 \\
1.73 \mathrm{E}+02 \\
1.60 \mathrm{E}+03 \\
3.21 \mathrm{E}+02 \\
1.39 \mathrm{E}+02 \\
4.55 \mathrm{E}+02 \\
1.51 \mathrm{E}+03 \\
2.38 \mathrm{E}+02 \\
1.26 \mathrm{E}+00 \\
7.51 \mathrm{E}+01 \\
6.63 \mathrm{E}+02 \\
1.26 \mathrm{E}+02 \\
1.09 \mathrm{E}+03 \\
2.01 \mathrm{E}+03 \\
9.15 \mathrm{E}+02 \\
2.36 \mathrm{E}+02 \\
3.25 \mathrm{E}+01 \\
1.26 \mathrm{E}+02 \\
2.10 \mathrm{E}+02 \\
1.29 \mathrm{E}+02 \\
3.76 \mathrm{E}+02\end{array}$ & $\begin{array}{l}\text { EU152 } \\
2.17 \mathrm{E}-01 \\
4.01 \mathrm{E}-03 \\
9.46 \mathrm{E}-02 \\
1.02 \mathrm{E}+00 \\
6.82 \mathrm{E}-02 \\
4.19 \mathrm{E}-02 \\
1.86 \mathrm{E}-03 \\
2.48 \mathrm{E}-02 \\
8.01 \mathrm{E}-01 \\
2.80 \mathrm{E}+00 \\
1.08 \mathrm{E}+00 \\
2.05 \mathrm{E}+00 \\
2.98 \mathrm{E}-01 \\
2.75 \mathrm{E}-01 \\
2.09 \mathrm{E}+00 \\
2.06 \mathrm{E}+00 \\
2.96 \mathrm{E}-01 \\
3.21 \mathrm{E}-01 \\
1.58 \mathrm{E}-01 \\
3.49 \mathrm{E}+00 \\
6.40 \mathrm{E}-01 \\
2.82 \mathrm{E}-01 \\
2.26 \mathrm{E}+00 \\
6.29 \mathrm{E}+00 \\
1.45 \mathrm{E}-01 \\
3.43 \mathrm{E}-01 \\
1.16 \mathrm{E}+00 \\
2.36 \mathrm{E}+00 \\
1.10 \mathrm{E}+00 \\
6.62 \mathrm{E}-01 \\
2.02 \mathrm{E}+00 \\
5.15 \mathrm{E}-01 \\
6.76 \mathrm{E}+00 \\
3.92 \mathrm{E}+00 \\
1.87 \mathrm{E}+00 \\
1.66 \mathrm{E}-01 \\
4.48 \mathrm{E}-01 \\
0.00 \mathrm{E}+00 \\
4.40 \mathrm{E}-03 \\
0.00 \mathrm{E}+00 \\
1.19 \mathrm{E}-03 \\
2.27 \mathrm{E}+00 \\
3.50 \mathrm{E}-02 \\
0.00 \mathrm{E}+00 \\
1.66 \mathrm{E}-02 \\
3.00 \mathrm{E}-02 \\
2.27 \mathrm{E}+00 \\
3.22 \mathrm{E}-01 \\
2.16 \mathrm{E}-02 \\
4.56 \mathrm{E}-01 \\
2.00 \mathrm{E}+00 \\
4.72 \mathrm{E}-02 \\
2.00 \mathrm{E}-03 \\
1.26 \mathrm{E}-01 \\
1.12 \mathrm{E}+00 \\
1.89 \mathrm{E}-01 \\
1.09 \mathrm{E}+00 \\
2.66 \mathrm{E}+00 \\
1.30 \mathrm{E}+00 \\
3.66 \mathrm{E}-01 \\
5.49 \mathrm{E}-02 \\
1.66 \mathrm{E}-01 \\
2.37 \mathrm{E}-01 \\
3.92 \mathrm{E}-02 \\
1.04 \mathrm{E}-01\end{array}$ & $\begin{array}{l}\text { EU154 } \\
2.18 \mathrm{E}+01 \\
9.11 \mathrm{E}-01 \\
2.39 \mathrm{E}+01 \\
1.42 \mathrm{E}+02 \\
1.73 \mathrm{E}+01 \\
7.13 \mathrm{E}+00 \\
4.29 \mathrm{E}-01 \\
4.71 \mathrm{E}+00 \\
1.41 \mathrm{E}+02 \\
4.51 \mathrm{E}+02 \\
1.58 \mathrm{E}+02 \\
2.23 \mathrm{E}+02 \\
2.92 \mathrm{E}+01 \\
2.70 \mathrm{E}+01 \\
2.28 \mathrm{E}+02 \\
2.25 \mathrm{E}+02 \\
2.89 \mathrm{E}+01 \\
3.54 \mathrm{E}+01 \\
2.09 \mathrm{E}+01 \\
3.81 \mathrm{E}+02 \\
6.28 \mathrm{E}+01 \\
3.40 \mathrm{E}+01 \\
2.21 \mathrm{E}+02 \\
7.15 \mathrm{E}+02 \\
1.88 \mathrm{0}+01 \\
4.60 \mathrm{0}+01 \\
1.36 \mathrm{E}+02 \\
2.30 \mathrm{E}+02 \\
1.09 \mathrm{E}+02 \\
8.78 \mathrm{E}+01 \\
2.04 \mathrm{E}+02 \\
5.86 \mathrm{E}+01 \\
7.74 \mathrm{E}+02 \\
4.33 \mathrm{E}+02 \\
3.17 \mathrm{E}+02 \\
2.81 \mathrm{E}+01 \\
7.60 \mathrm{E}+01 \\
1.52 \mathrm{E}-03 \\
1.15 \mathrm{E}+00 \\
2.67 \mathrm{x}-03 \\
1.41 \mathrm{E}+00 \\
3.83 \mathrm{E}+02 \\
1.39 \mathrm{E}+01 \\
2.15 \mathrm{E}-02 \\
6.09 \mathrm{E}+00 \\
1.10 \mathrm{E}+01 \\
3.83 \mathrm{E}+02 \\
5.86 \mathrm{E}+01 \\
8.78 \mathrm{E}+00 \\
8.31 \mathrm{E}+01 \\
3.37 \mathrm{E}+02 \\
1.59 \mathrm{E}+01 \\
3.12 \mathrm{E}-01 \\
2.05 \mathrm{E}+01 \\
1.83 \mathrm{E}+02 \\
3.14 \mathrm{E}+01 \\
1.98 \mathrm{E}+02 \\
4.49 \mathrm{E}+02 \\
2.19 \mathrm{E}+02 \\
5.96 \mathrm{E}+01 \\
8.98 \mathrm{E}+00 \\
2.81 \mathrm{E}+01 \\
4.17 \mathrm{E}+01 \\
1.08 \mathrm{E}+01 \\
2.98 \mathrm{E}+01\end{array}$ & $\begin{array}{l}E U 155 \\
9.97 E+00 \\
4.36 E+00 \\
1.33 E+02 \\
1.20 E+02 \\
9.69 E+01 \\
1.78 E+01 \\
2.13 E+00 \\
1.63 E+01 \\
3.90 E+02 \\
7.97 E+02 \\
2.28 E+02 \\
4.52 E+01 \\
9.32 E+00 \\
9.02 E+00 \\
4.61 E+01 \\
4.54 E+01 \\
9.24 E+00 \\
7.30 E+00 \\
6.20 E+00 \\
7.70 E+01 \\
2.10 E+01 \\
1.55 E+01 \\
7.05 E+01 \\
1.39 E+02 \\
3.73 E+00 \\
1.31 E+01 \\
2.80 E+01 \\
7.35 E+01 \\
3.07 E+01 \\
2.62 E+01 \\
6.01 E+01 \\
1.14 E+01 \\
1.53 E+02 \\
8.96 E+01 \\
7.43 E+02 \\
6.58 E+01 \\
1.78 E+02 \\
1.08 E+00 \\
6.19 E+00 \\
1.90 E+00 \\
1.51 E+01 \\
8.30 E+02 \\
1.13 E+02 \\
1.53 E+01 \\
4.78 E+01 \\
8.62 E+01 \\
8.29 E+02 \\
1.73 E+02 \\
7.49 E+01 \\
2.45 E+02 \\
7.57 E+02 \\
1.17 E+02 \\
5.31 E-01 \\
3.77 E+01 \\
3.42 E+02 \\
6.49 E+01 \\
5.86 E+02 \\
1.01 E+03 \\
4.76 E+02 \\
1.14 E+02 \\
1.67 E+01 \\
6.31 E+01 \\
1.09 E+02 \\
6.94 E+01 \\
1.95 E+02\end{array}$ & $\begin{array}{l}\text { RA226 } \\
8.26 E-07 \\
2.88 E-08 \\
7.53 E-07 \\
4.57 E-07 \\
5.22 E-07 \\
6.17 E-08 \\
1.14 E-08 \\
7.15 E-08 \\
1.31 E-06 \\
2.04 E-06 \\
1.82 E-06 \\
2.38 E-06 \\
7.04 E-07 \\
6.88 E-07 \\
2.44 E-06 \\
2.40 E-06 \\
6.99 E-07 \\
3.57 E-07 \\
6.46 E-08 \\
4.07 E-06 \\
1.60 E-06 \\
1.92 E-07 \\
5.33 E-06 \\
6.10 E-06 \\
7.47 E-08 \\
1.31 E-07 \\
9.75 E-07 \\
5.56 E-06 \\
2.06 E-06 \\
2.66 E-07 \\
3.43 E-06 \\
5.00 E-07 \\
6.32 E-06 \\
4.31 E-06 \\
1.87 E-06 \\
1.65 E-07 \\
4.48 E-07 \\
1.02 E-08 \\
7.37 E-08 \\
1.79 E-08 \\
1.02 E-07 \\
1.60 E-06 \\
5.49 E-07 \\
1.45 E-07 \\
2.04 E-07 \\
3.68 E-07 \\
1.60 E-06 \\
3.44 E-07 \\
2.61 E-07 \\
4.88 E-07 \\
1.67 E-06 \\
5.05 E-07 \\
1.76 E-09 \\
7.57 E-08 \\
6.32 E-07 \\
1.26 E-07 \\
1.17 E-06 \\
2.23 E-06 \\
9.16 E-07 \\
2.61 E-07 \\
3.10 E-08 \\
1.39 E-07 \\
2.30 E-07 \\
2.07 E-07 \\
6.70 E-07\end{array}$ & $\begin{array}{l}\text { RA228 } \\
5.26 \mathrm{E}-11 \\
1.84 \mathrm{E}-12 \\
4.83 \mathrm{E}-11 \\
3.93 \mathrm{E}-11 \\
3.36 \mathrm{E}-11 \\
4.49 \mathrm{E}-12 \\
7.39 \mathrm{E}-13 \\
4.89 \mathrm{E}-12 \\
9.44 \mathrm{E}-11 \\
1.70 \mathrm{E}-10 \\
1.37 \mathrm{E}-10 \\
1.92 \mathrm{E}-10 \\
4.86 \mathrm{E}-11 \\
4.71 \mathrm{E}-11 \\
1.96 \mathrm{E}-10 \\
1.93 \mathrm{E}-10 \\
4.83 \mathrm{E}-11 \\
2.89 \mathrm{E}-11 \\
6.07 \mathrm{E}-12 \\
3.27 \mathrm{E}-10 \\
1.09 \mathrm{E}-10 \\
1.61 \mathrm{E}-11 \\
3.68 \mathrm{E}-10 \\
5.10 \mathrm{E}-10 \\
7.03 \mathrm{E}-12 \\
1.25 \mathrm{E}-11 \\
8.34 \mathrm{E}-11 \\
3.84 \mathrm{E}-10 \\
1.48 \mathrm{E}-10 \\
2.51 \mathrm{E}-11 \\
2.51 \mathrm{E}-10 \\
4.18 \mathrm{E}-11 \\
5.32 \mathrm{E}-10 \\
3.50 \mathrm{E}-10 \\
1.42 \mathrm{E}-10 \\
1.25 \mathrm{E}-11 \\
3.39 \mathrm{E}-11 \\
5.93 \mathrm{E}-13 \\
4.42 \mathrm{E}-12 \\
1.04 \mathrm{E}-12 \\
6.22 \mathrm{E}-12 \\
1.25 \mathrm{E}-10 \\
3.49 \mathrm{E}-11 \\
8.40 \mathrm{E}-12 \\
1.31 \mathrm{E}-11 \\
2.37 \mathrm{E}-11 \\
1.26 \mathrm{E}-10 \\
2.58 \mathrm{E}-11 \\
1.70 \mathrm{E}-11 \\
3.66 \mathrm{E}-11 \\
1.29 \mathrm{E}-10 \\
3.26 \mathrm{E}-11 \\
1.36 \mathrm{E}-13 \\
6.08 \mathrm{E}-12 \\
5.11 \mathrm{E}-11 \\
9.97 \mathrm{E}-12 \\
8.74 \mathrm{E}-11 \\
1.72 \mathrm{E}-10 \\
7.19 \mathrm{E}-11 \\
2.06 \mathrm{E}-11 \\
.2 .50 \mathrm{E}-12 \\
1.08 \mathrm{E}-11 \\
1.74 \mathrm{E}-11 \\
1.39 \mathrm{E}-11 \\
4.44 \mathrm{E}-11\end{array}$ & $\begin{array}{l}\text { AC227 } \\
4.20 \mathrm{E}-06 \\
1.76 \mathrm{E}-07 \\
4.66 \mathrm{E}-06 \\
2.67 \mathrm{E}-06 \\
3.24 \mathrm{E}-06 \\
3.75 \mathrm{E}-07 \\
7.01 \mathrm{E}-08 \\
4.38 \mathrm{E}-07 \\
8.00 \mathrm{E}-06 \\
1.49 \mathrm{E}-05 \\
1.27 \mathrm{E}-05 \\
1.18 \mathrm{E}-05 \\
3.53 \mathrm{E}-06 \\
3.45 \mathrm{E}-06 \\
1.21 \mathrm{E}-05 \\
1.19 \mathrm{E}-05 \\
3.50 \mathrm{E}-06 \\
1.78 \mathrm{E}-06 \\
3.54 \mathrm{E}-07 \\
2.02 \mathrm{E}-05 \\
8.03 \mathrm{E}-06 \\
1.05 \mathrm{E}-06 \\
2.67 \mathrm{E}-05 \\
3.05 \mathrm{E}-05 \\
3.92 \mathrm{E}-07 \\
7.21 \mathrm{E}-07 \\
4.95 \mathrm{E}-06 \\
2.78 \mathrm{E}-05 \\
1.03 \mathrm{E}-05 \\
1.46 \mathrm{E}-06 \\
1.73 \mathrm{E}-05 \\
2.50 \mathrm{E}-06 \\
3.17 \mathrm{E}-05 \\
2.15 \mathrm{E}-05 \\
1.14 \mathrm{E}-05 \\
1.01 \mathrm{E}-06 \\
2.74 \mathrm{E}-06 \\
6.58 \mathrm{E}-08 \\
4.48 \mathrm{E}-07 \\
1.15 \mathrm{E}-07 \\
6.40 \mathrm{E}-07 \\
9.80 \mathrm{E}-06 \\
3.45 \mathrm{E}-06 \\
9.33 \mathrm{E}-07 \\
1.28 \mathrm{E}-06 \\
2.32 \mathrm{E}-06 \\
9.82 \mathrm{E}-06 \\
2.13 \mathrm{E}-06 \\
1.66 \mathrm{E}-06 \\
3.03 \mathrm{E}-06 \\
1.02 \mathrm{E}-05 \\
3.18 \mathrm{E}-06 \\
1.05 \mathrm{E}-08 \\
4.62 \mathrm{E}-07 \\
3.87 \mathrm{E}-06 \\
7.73 \mathrm{E}-07 \\
7.22 \mathrm{E}-06 \\
1.37 \mathrm{E}-05 \\
5.62 \mathrm{E}-06 \\
1.59 \mathrm{E}-06 \\
1.89 \mathrm{E}-07 \\
8.54 \mathrm{E}-07 \\
1.42 \mathrm{E}-06 \\
1.31 \mathrm{E}-06 \\
4.22 \mathrm{E}-06\end{array}$ & $\begin{array}{l}\text { TH229 } \\
5.65 \mathrm{E}-09 \\
1.17 \mathrm{E}-10 \\
3.01 \mathrm{E}-09 \\
5.82 \mathrm{E}-09 \\
2.11 \mathrm{E}-09 \\
4.39 \mathrm{E}-10 \\
4.88 \mathrm{E}-11 \\
3.93 \mathrm{E}-10 \\
8.92 \mathrm{E}-09 \\
1.87 \mathrm{E}-08 \\
1.25 \mathrm{E}-08 \\
3.03 \mathrm{E}-08 \\
6.08 \mathrm{E}-09 \\
5.78 \mathrm{E}-09 \\
3.10 \mathrm{E}-08 \\
3.05 \mathrm{E}-08 \\
6.04 \mathrm{E}-09 \\
4.59 \mathrm{E}-09 \\
1.08 \mathrm{E}-09 \\
5.17 \mathrm{E}-08 \\
1.34 \mathrm{E}-08 \\
2.33 \mathrm{E}-09 \\
4.60 \mathrm{E}-08 \\
8.43 \mathrm{E}-08 \\
1.31 \mathrm{E}-09 \\
2.29 \mathrm{E}-09 \\
1.39 \mathrm{E}-08 \\
4.80 \mathrm{E}-08 \\
1.97 \mathrm{E}-08 \\
4.49 \mathrm{E}-09 \\
3.35 \mathrm{E}-08 \\
6.91 \mathrm{E}-09 \\
8.81 \mathrm{E}-08 \\
5.55 \mathrm{E}-08 \\
1.55 \mathrm{E}-08 \\
1.37 \mathrm{E}-09 \\
3.71 \mathrm{E}-09 \\
1.59 \mathrm{E}-11 \\
2.35 \mathrm{E}-10 \\
2.79 \mathrm{E}-11 \\
2.92 \mathrm{E}-10 \\
1.53 \mathrm{E}-08 \\
1.95 \mathrm{E}-09 \\
2.25 \mathrm{E}-10 \\
7.69 \mathrm{E}-10 \\
1.39 \mathrm{E}-09 \\
1.53 \mathrm{E}-08 \\
2.75 \mathrm{E}-09 \\
1.02 \mathrm{E}-09 \\
3.89 \mathrm{E}-09 \\
1.49 \mathrm{E}-08 \\
1.95 \mathrm{E}-09 \\
1.57 \mathrm{E}-11 \\
7.82 \mathrm{E}-10 \\
6.69 \mathrm{E}-09 \\
1.24 \mathrm{E}-09 \\
9.29 \mathrm{E}-09 \\
1.99 \mathrm{E}-08 \\
8.77 \mathrm{E}-09 \\
2.51 \mathrm{E}-09 \\
3.28 \mathrm{E}-10 \\
1.24 \mathrm{E}-09 \\
1.92 \mathrm{E}-09 \\
9.66 \mathrm{E}-10 \\
2.95 \mathrm{E}-09\end{array}$ \\
\hline
\end{tabular}


HNF-SD-WM-TI-794, ReV. 0

ACTIVITY IN UNSEPARATED FUEL (Curies decayed to 1/1/94)

\begin{tabular}{|c|c|c|c|c|c|c|c|c|c|}
\hline $\begin{array}{r}\text { [lsting } \\
1171 \\
1172 \\
1173 \\
1174 \\
1175 \\
1176 \\
1177 \\
1178 \\
1179 \\
1180 \\
1181 \\
1182 \\
1183 \\
1184 \\
1185 \\
1186 \\
1187 \\
1188 \\
1189 \\
1190 \\
1191 \\
1192 \\
1193 \\
1194 \\
1195 \\
1196 \\
1197 \\
1198 \\
1199 \\
1200 \\
1201 \\
1202 \\
1203 \\
1204 \\
1205 \\
1206 \\
1207 \\
1208 \\
1209 \\
1210 \\
1211 \\
1212 \\
1213 \\
1214 \\
1215 \\
1216 \\
1217 \\
1218 \\
1219 \\
1220 \\
1221 \\
1222 \\
1223 \\
1224 \\
1225 \\
1226 \\
1227 \\
1228 \\
1229 \\
1230 \\
1231 \\
1232 \\
1233 \\
1234 \\
1235\end{array}$ & $\begin{array}{l}\text { TH232 } \\
8.74 \mathrm{E}-11 \\
5.10 \mathrm{E}-12 \\
1.41 \mathrm{E}-10 \\
1.02 \mathrm{E}-10 \\
9.90 \mathrm{E}-11 \\
1.31 \mathrm{E}-11 \\
2.15 \mathrm{E}-12 \\
1.42 \mathrm{E}-11 \\
2.82 \mathrm{E}-10 \\
5.03 \mathrm{E}-10 \\
3.23 \mathrm{E}-10 \\
3.21 \mathrm{E}-10 \\
8.06 \mathrm{E}-11 \\
7.80 \mathrm{E}-11 \\
3.29 \mathrm{E}-10 \\
3.24 \mathrm{E}-10 \\
8.00 \mathrm{E}-111 \\
4.89 \mathrm{E}-11 \\
1.24 \mathrm{E}-11 \\
5.49 \mathrm{E}-10 \\
1.81 \mathrm{E}-10 \\
3.31 \mathrm{E}-11 \\
6.10 \mathrm{E}-10 \\
8.67 \mathrm{E}-10 \\
1.29 \mathrm{E}-11 \\
2.57 \mathrm{E}-11 \\
1.45 \mathrm{E}-10 \\
6.37 \mathrm{E}-10 \\
2.48 \mathrm{E}-10 \\
5.16 \mathrm{E}-11 \\
4.26 \mathrm{E}-10 \\
7.11 \mathrm{E}-11 \\
9.10 \mathrm{E}-10 \\
5.93 \mathrm{E}-10 \\
4.41 \mathrm{E}-10 \\
3.90 \mathrm{E}-11 \\
1.06 \mathrm{E}-10 \\
1.94 \mathrm{E}-12 \\
1.11 \mathrm{E}-11 \\
3.41 \mathrm{E}-12 \\
1.88 \mathrm{E}-11 \\
4.11 \mathrm{E}-10 \\
1.10 \mathrm{E}-10 \\
2.76 \mathrm{E}-11 \\
4.24 \mathrm{E}-11 \\
7.65 \mathrm{E}-11 \\
4.12 \mathrm{E}-10 \\
8.75 \mathrm{E}-11 \\
5.82 \mathrm{E}-11 \\
1.24 \mathrm{E}-10 \\
4.12 \mathrm{E}-10 \\
1.04 \mathrm{E}-10 \\
3.84 \mathrm{E}-13 \\
1.93 \mathrm{E}-11 \\
1.66 \mathrm{E}-10 \\
3.24 \mathrm{E}-11 \\
2.96 \mathrm{E}-10 \\
5.49 \mathrm{E}-10 \\
2.36 \mathrm{E}-10 \\
6.39 \mathrm{E}-11 \\
8.12 \mathrm{E}-12 \\
3.43 \mathrm{E}-11 \\
5.74 \mathrm{E}-11 \\
4.76 \mathrm{E}-11 \\
1.47 \mathrm{E}-10\end{array}$ & $\begin{array}{l}\text { PA231 } \\
1.49 \mathrm{E}-05 \\
1.30 \mathrm{E}-06 \\
3.63 \mathrm{E}-05 \\
1.74 \mathrm{E}-05 \\
2.56 \mathrm{E}-05 \\
2.92 \mathrm{E}-06 \\
5.45 \mathrm{E}-07 \\
3.38 \mathrm{E}-06 \\
6.39 \mathrm{E}-05 \\
1.17 \mathrm{E}-04 \\
7.48 \mathrm{E}-05 \\
4.13 \mathrm{E}-05 \\
1.24 \mathrm{E}-05 \\
1.21 \mathrm{E}-05 \\
4.23 \mathrm{E}-05 \\
4.16 \mathrm{E}-05 \\
1.23 \mathrm{E}-05 \\
6.29 \mathrm{E}-06 \\
1.64 \mathrm{E}-06 \\
7.06 \mathrm{E}-05 \\
2.82 \mathrm{E}-05 \\
5.05 \mathrm{E}-06 \\
9.35 \mathrm{E}-05 \\
1.08 \mathrm{E}-04 \\
1.53 \mathrm{E}-06 \\
3.33 \mathrm{E}-06 \\
1.82 \mathrm{E}-05 \\
9.76 \mathrm{E}-05 \\
3.65 \mathrm{E}-05 \\
6.80 \mathrm{E}-06 \\
6.27 \mathrm{E}-05 \\
8.88 \mathrm{E}-06 \\
1.14 \mathrm{E}-04 \\
7.63 \mathrm{E}-05 \\
9.57 \mathrm{E}-05 \\
8.47 \mathrm{E}-06 \\
2.29 \mathrm{E}-05 \\
5.95 \mathrm{E}-07 \\
2.90 \mathrm{E}-06 \\
1.04 \mathrm{E}-06 \\
5.22 \mathrm{E}-06 \\
8.71 \mathrm{E}-05 \\
2.96 \mathrm{E}-05 \\
8.43 \mathrm{E}-06 \\
1.14 \mathrm{E}-05 \\
2.05 \mathrm{E}-05 \\
8.72 \mathrm{E}-05 \\
1.98 \mathrm{E}-05 \\
1.57 \mathrm{E}-05 \\
2.81 \mathrm{E}-05 \\
8.82 \mathrm{E}-05 \\
2.78 \mathrm{E}-05 \\
7.80 \mathrm{E}-08 \\
3.95 \mathrm{E}-06 \\
3.39 \mathrm{E}-05 \\
6.79 \mathrm{E}-06 \\
6.71 \mathrm{E}-05 \\
1.18 \mathrm{E}-04 \\
1.00 \mathrm{E}-05 \\
1.32 \mathrm{E}-05 \\
7.36 \mathrm{E}-06 \\
1.27 \mathrm{E}-06 \\
1.24 \mathrm{E}-05 \\
3.85 \mathrm{E}-05 \\
\end{array}$ & $\begin{array}{l}\text { U232 } \\
2.91 E-04 \\
8.15 E-06 \\
2.17 E-04 \\
7.89 E-04 \\
1.55 E-04 \\
4.45 E-05 \\
3.66 E-06 \\
3.39 E-05 \\
8.91 E-04 \\
2.12 E-03 \\
9.90 E-04 \\
2.57 E-03 \\
3.72 E-04 \\
3.46 E-04 \\
2.63 E-03 \\
2.58 E-03 \\
3.69 E-04 \\
3.98 E-04 \\
1.49 E-04 \\
4.38 E-03 \\
8.05 E-04 \\
2.53 E-04 \\
2.81 E-03 \\
7.82 E-03 \\
1.64 E-04 \\
3.24 E-04 \\
1.39 E-03 \\
2.94 E-03 \\
1.32 E-03 \\
6.20 E-04 \\
2.36 E-03 \\
6.41 E-04 \\
8.32 E-03 \\
4.84 E-03 \\
1.79 E-03 \\
1.58 E-04 \\
4.29 E-04 \\
8.84 E-07 \\
1.28 E-05 \\
1.55 E-06 \\
1.83 E-05 \\
1.98 E-03 \\
1.42 E-04 \\
1.25 E-05 \\
5.91 E-05 \\
1.07 E-04 \\
1.98 E-03 \\
3.26 E-04 \\
8.39 E-05 \\
4.63 E-04 \\
1.82 E-03 \\
1.50 E-04 \\
1.80 E-06 \\
1.04 E-04 \\
9.14 E-04 \\
1.61 E-04 \\
1.11 E-03 \\
2.43 E-03 \\
1.13 E-03 \\
3.16 E-04 \\
4.48 E-05 \\
1.52 E-04 \\
2.299 E-04 \\
8.69 E-05 \\
2.50 E-04\end{array}$ & $\begin{array}{l}\text { U233 } \\
3.63 \mathrm{E}-06 \\
1.54 \mathrm{E}-07 \\
4.19 \mathrm{E}-06 \\
6.46 \mathrm{E}-06 \\
2.98 \mathrm{E}-06 \\
5.94 \mathrm{E}-07 \\
6.77 \mathrm{E}-08 \\
5.36 \mathrm{E}-07 \\
1.24 \mathrm{E}-05 \\
2.53 \mathrm{E}-05 \\
1.30 \mathrm{E}-05 \\
1.94 \mathrm{E}-05 \\
3.89 \mathrm{E}-06 \\
3.70 \mathrm{E}-06 \\
1.99 \mathrm{E}-05 \\
1.95 \mathrm{E}-05 \\
3.86 \mathrm{E}-06 \\
2.97 \mathrm{E}-06 \\
8.83 \mathrm{E}-07 \\
3.31 \mathrm{E}-05 \\
8.60 \mathrm{E}-06 \\
1.98 \mathrm{E}-06 \\
2.94 \mathrm{E}-05 \\
5.48 \mathrm{E}-05 \\
9.28 \mathrm{E}-07 \\
1.86 \mathrm{E}-06 \\
9.37 \mathrm{E}-06 \\
3.07 \mathrm{E}-05 \\
1.27 \mathrm{E}-05 \\
3.67 \mathrm{E}-06 \\
2.22 \mathrm{E}-05 \\
4.49 \mathrm{E}-06 \\
5.78 \mathrm{E}-05 \\
3.61 \mathrm{E}-05 \\
2.23 \mathrm{E}-05 \\
1.97 \mathrm{E}-06 \\
5.34 \mathrm{E}-06 \\
2.57 \mathrm{E}-08 \\
2.74 \mathrm{E}-07 \\
4.52 \mathrm{E}-08 \\
4.30 \mathrm{E}-07 \\
2.30 \mathrm{E}-05 \\
3.01 \mathrm{E}-06 \\
3.65 \mathrm{E}-07 \\
1.22 \mathrm{E}-06 \\
2.20 \mathrm{E}-06 \\
2.30 \mathrm{E}-05 \\
4.37 \mathrm{E}-06 \\
1.73 \mathrm{E}-06 \\
6.20 \mathrm{E}-06 \\
2.19 \mathrm{E}-05 \\
3.05 \mathrm{E}-06 \\
2.01 \mathrm{E}-08 \\
1.13 \mathrm{E}-06 \\
9.86 \mathrm{E}-06 \\
1.84 \mathrm{E}-06 \\
1.48 \mathrm{E}-05 \\
2.92 \mathrm{E}-05 \\
1.32 \mathrm{E}-05 \\
3.55 \mathrm{E}-06 \\
4.83 \mathrm{E}-07 \\
1.82 \mathrm{E}-06 \\
2.94 \mathrm{E}-06 \\
1.62 \mathrm{E}-06 \\
4.79 \mathrm{E}-06\end{array}$ & $\begin{array}{l}\text { U234 } \\
8.94 \mathrm{E}-01 \\
1.67 \mathrm{E}-01 \\
4.97 \mathrm{E}+00 \\
2.15 \mathrm{E}+00 \\
3.56 \mathrm{E}+00 \\
4.12 \mathrm{E}-01 \\
7.48 \mathrm{E}-02 \\
4.67 \mathrm{E}-01 \\
9.23 \mathrm{E}+00 \\
1.41 \mathrm{E}+01 \\
6.49 \mathrm{E}+00 \\
2.67 \mathrm{E}+00 \\
7.54 \mathrm{E}-01 \\
7.38 \mathrm{E}-01 \\
2.73 \mathrm{E}+00 \\
2.68 \mathrm{E}+00 \\
7.49 \mathrm{E}-01 \\
4.12 \mathrm{E}-01 \\
1.48 \mathrm{E}-01 \\
4.55 \mathrm{E}+00 \\
1.72 \mathrm{E}+00 \\
4.53 \mathrm{E}-01 \\
5.71 \mathrm{E}+00 \\
7.20 \mathrm{E}+00 \\
1.17 \mathrm{E}-01 \\
3.02 \mathrm{E}-01 \\
1.27 \mathrm{E}+00 \\
5.96 \mathrm{E}+00 \\
2.28 \mathrm{E}+00 \\
6.17 \mathrm{E}-01 \\
4.05 \mathrm{E}+00 \\
5.90 \mathrm{E}-01 \\
7.64 \mathrm{E}+00 \\
5.01 \mathrm{E}+00 \\
1.47 \mathrm{E}+01 \\
1.30 \mathrm{E}+00 \\
3.53 \mathrm{E}+00 \\
9.30 \mathrm{E}-02 \\
3.23 \mathrm{E}-01 \\
1.63 \mathrm{E}-01 \\
7.40 \mathrm{E}-01 \\
1.44 \mathrm{E}+01 \\
4.48 \mathrm{E}+00 \\
1.32 \mathrm{E}+00 \\
1.78 \mathrm{E}+00 \\
3.21 \mathrm{E}+00 \\
1.44 \mathrm{E}+01 \\
3.38 \mathrm{E}+00 \\
2.65 \mathrm{E}+00 \\
4.79 \mathrm{E}+00 \\
1.40 \mathrm{E}+01 \\
4.29 \mathrm{E}+00 \\
1.07 \mathrm{E}-02 \\
6.29 \mathrm{E}-01 \\
5.54 \mathrm{E}+00 \\
1.11 \mathrm{E}+00 \\
1.14 \mathrm{E}+01 \\
1.87 \mathrm{E}+01 \\
8.24 \mathrm{E}+00 \\
2.04 \mathrm{E}+00 \\
2.71 \mathrm{E}-01 \\
1.17 \mathrm{E}+00 \\
2.09 \mathrm{E}+00 \\
2.10 \mathrm{E}+00 \\
6.27 \mathrm{E}+00\end{array}$ & $\begin{array}{l}\text { U235 } \\
3.18 \mathrm{E}-02 \\
6.48 \mathrm{E}-03 \\
1.94 \mathrm{E}-01 \\
7.58 \mathrm{E}-02 \\
1.39 \mathrm{E}-01 \\
1.56 \mathrm{E}-02 \\
2.91 \mathrm{E}-03 \\
1.80 \mathrm{E}-02 \\
3.51 \mathrm{E}-01 \\
6.36 \mathrm{E}-01 \\
2.92 \mathrm{E}-01 \\
8.65 \mathrm{E}-02 \\
2.60 \mathrm{E}-02 \\
2.55 \mathrm{E}-02 \\
8.85 \mathrm{E}-02 \\
8.71 \mathrm{E}-02 \\
2.58 \mathrm{E}-02 \\
1.34 \mathrm{E}-02 \\
4.81 \mathrm{E}-03 \\
1.48 \mathrm{E}-01 \\
5.94 \mathrm{E}-02 \\
1.55 \mathrm{E}-02 \\
1.97 \mathrm{E}-01 \\
2.31 \mathrm{E}-01 \\
3.67 \mathrm{E}-03 \\
9.76 \mathrm{E}-03 \\
4.06 \mathrm{E}-02 \\
2.05 \mathrm{E}-01 \\
7.76 \mathrm{E}-02 \\
2.01 \mathrm{E}-02 \\
1.38 \mathrm{E}-01 \\
1.89 \mathrm{E}-02 \\
2.45 \mathrm{E}-01 \\
1.63 \mathrm{E}-01 \\
5.54 \mathrm{E}-01 \\
4.90 \mathrm{E}-02 \\
1.33 \mathrm{E}-01 \\
3.75 \mathrm{E}-03 \\
1.26 \mathrm{E}-02 \\
6.57 \mathrm{E}-03 \\
2.93 \mathrm{E}-02 \\
5.37 \mathrm{E}-01 \\
1.76 \mathrm{E}-01 \\
5.30 \mathrm{E}-02 \\
6.99 \mathrm{E}-02 \\
1.26 \mathrm{E}-01 \\
5.37 \mathrm{E}-01 \\
1.28 \mathrm{E}-01 \\
1.04 \mathrm{E}-01 \\
1.82 \mathrm{E}-01 \\
5.26 \mathrm{E}-01 \\
1.69 \mathrm{E}-01 \\
3.95 \mathrm{E}-04 \\
2.33 \mathrm{E}=02 \\
2.05 \mathrm{E}-01 \\
4.14 \mathrm{E}-02 \\
4.35 \mathrm{E}-01 \\
7.01 \mathrm{E}-01 \\
3.08 \mathrm{E}-01 \\
7.58 \mathrm{E}-02 \\
1.01 \mathrm{E}-02 \\
4.38 \mathrm{E}-02 \\
7.90 \mathrm{E}-02 \\
8.19 \mathrm{E}-02 \\
2.45 \mathrm{E}-01\end{array}$ & $\begin{array}{c}\text { U236 } \\
8.15 \mathrm{E}-02 \\
1.10 \mathrm{E}-02 \\
3.23 \mathrm{E}-01 \\
1.99 \mathrm{E}-01 \\
2.31 \mathrm{E}-01 \\
3.05 \mathrm{E}-02 \\
4.94 \mathrm{E}-03 \\
3.25 \mathrm{E}-02 \\
6.71 \mathrm{E}-01 \\
1.19 \mathrm{E}+00 \\
5.47 \mathrm{E}-01 \\
3.06 \mathrm{E}-01 \\
7.49 \mathrm{E}-02 \\
7.26 \mathrm{E}-02 \\
3.13 \mathrm{E}-01 \\
3.08 \mathrm{E}-01 \\
7.44 \mathrm{E}-02 \\
4.73 \mathrm{E}-02 \\
1.70 \mathrm{E}-02 \\
5.23 \mathrm{E}-01 \\
1.69 \mathrm{E}-01 \\
4.58 \mathrm{E}-02 \\
5.67 \mathrm{E}-01 \\
8.49 \mathrm{E}-01 \\
1.46 \mathrm{E}-02 \\
3.53 \mathrm{E}-02 \\
1.49 \mathrm{E}-01 \\
5.92 \mathrm{E}-01 \\
2.34 \mathrm{E}-01 \\
7.10 \mathrm{E}-02 \\
4.16 \mathrm{E}-01 \\
6.96 \mathrm{E}-02 \\
9.01 \mathrm{E}-01 \\
5.75 \mathrm{E}-01 \\
1.11 \mathrm{E}+00 \\
9.84 \mathrm{E}-02 \\
2.66 \mathrm{E}-01 \\
5.25 \mathrm{E}-03 \\
2.08 \mathrm{E}-02 \\
9.21 \mathrm{E}-03 \\
4.53 \mathrm{E}-02 \\
1.11 \mathrm{E}+00 \\
2.81 \mathrm{E}-01 \\
7.43 \mathrm{E}-02 \\
1.12 \mathrm{E}-01 \\
2.02 \mathrm{E}-01 \\
1.11 \mathrm{E}+00 \\
2.46 \mathrm{E}-01 \\
1.66 \mathrm{E}-01 \\
3.49 \mathrm{E}-01 \\
1.07 \mathrm{E}+00 \\
2.72 \mathrm{E}-01 \\
8.51 \mathrm{E}-04 \\
5.02 \mathrm{E}-02 \\
4.42 \mathrm{E}-01 \\
8.65 \mathrm{E}-02 \\
8.34 \mathrm{E}-01 \\
1.43 \mathrm{E}+00 \\
6.36 \mathrm{E}-01 \\
1.61 \mathrm{E}-01 \\
2.16 \mathrm{E}-02 \\
8.93 \mathrm{E}-02 \\
1.55 \mathrm{E}-01 \\
1.36 \mathrm{E}-01 \\
4.04 \mathrm{E}-01\end{array}$ & $\begin{array}{l}\text { U238 } \\
6.21 \mathrm{E}-01 \\
1.12 \mathrm{E}-01 \\
3.35 \mathrm{E}+00 \\
1.49 \mathrm{E}+00 \\
2.40 \mathrm{E}+00 \\
2.80 \mathrm{E}-01 \\
5.05 \mathrm{E}-02 \\
3.17 \mathrm{E}-01 \\
6.28 \mathrm{E}+00 \\
9.64 \mathrm{E}+00 \\
4.43 \mathrm{E}+00 \\
1.90 \mathrm{E}+00 \\
5.29 \mathrm{E}-01 \\
5.17 \mathrm{E}-01 \\
1.95 \mathrm{E}+00 \\
1.92 \mathrm{E}+00 \\
5.25 \mathrm{E}-01 \\
2.94 \mathrm{E}-01 \\
1.06 \mathrm{E}-01 \\
3.25 \mathrm{E}+00 \\
1.20 \mathrm{E}+00 \\
3.18 \mathrm{E}-01 \\
4.00 \mathrm{E}+00 \\
5.16 \mathrm{E}+00 \\
8.49 \mathrm{E}-02 \\
2.17 \mathrm{E}-01 \\
9.08 \mathrm{E}-01 \\
4.18 \mathrm{E}+00 \\
1.61 \mathrm{E}+00 \\
4.41 \mathrm{E}-01 \\
2.85 \mathrm{E}+00 \\
4.23 \mathrm{E}-01 \\
5.48 \mathrm{E}+00 \\
3.58 \mathrm{E}+00 \\
1.00 \mathrm{E}+01 \\
8.89 \mathrm{E}-01 \\
2.41 \mathrm{E}+00 \\
6.22 \mathrm{E}-02 \\
2.18 \mathrm{E}-01 \\
1.09 \mathrm{E}-01 \\
4.97 \mathrm{E}-01 \\
9.82 \mathrm{E}+00 \\
3.01 \mathrm{E}+00 \\
8.81 \mathrm{E}-01 \\
1.20 \mathrm{E}+00 \\
2.16 \mathrm{E}+00 \\
9.82 \mathrm{E}+00 \\
2.30 \mathrm{E}+00 \\
1.78 \mathrm{E}+00 \\
3.26 \mathrm{E}+00 \\
9.57 \mathrm{E}+00 \\
2.89 \mathrm{E}+00 \\
7.31 \mathrm{E}-03 \\
4.31 \mathrm{E}=01 \\
3.80 \mathrm{E}+00 \\
7.59 \mathrm{E}-01 \\
7.79 \mathrm{E}+00 \\
1.28 \mathrm{E}+01 \\
5.63 \mathrm{E}+00 \\
1.40 \mathrm{E}+00 \\
1.86 \mathrm{E}-01 \\
7.98 \mathrm{E}-01 \\
1.42 \mathrm{E}+00 \\
1.41 \mathrm{E}+00 \\
4.23 \mathrm{E}+00\end{array}$ & $\begin{array}{l}\text { NP237 } \\
2.13 \mathrm{E}-02 \\
1.15 \mathrm{E}-03 \\
3.18 \mathrm{E}-02 \\
5.28 \mathrm{E}-02 \\
2.28 \mathrm{E}-02 \\
4.71 \mathrm{E}-03 \\
5.16 \mathrm{E}-04 \\
4.15 \mathrm{E}-03 \\
9.85 \mathrm{E}-02 \\
2.05 \mathrm{E}-01 \\
9.42 \mathrm{E}-02 \\
1.25 \mathrm{E}-01 \\
2.34 \mathrm{E}-02 \\
2.22 \mathrm{E}-02 \\
1.28 \mathrm{E}-01 \\
1.26 \mathrm{E}-01 \\
2.33 \mathrm{E}-02 \\
1.93 \mathrm{E}-02 \\
6.85 \mathrm{E}-03 \\
2.13 \mathrm{E}-01 \\
5.16 \mathrm{E}-02 \\
1.46 \mathrm{E}-02 \\
1.77 \mathrm{E}-01 \\
3.62 \mathrm{E}-01 \\
6.77 \mathrm{E}-03 \\
1.46 \mathrm{E}-02 \\
6.35 \mathrm{E}-02 \\
1.85 \mathrm{E}-01 \\
7.84 \mathrm{E}-02 \\
2.86 \mathrm{E}-02 \\
1.39 \mathrm{E}-01 \\
2.97 \mathrm{E}-02 \\
3.84 \mathrm{E}-01 \\
2.35 \mathrm{E}-01 \\
1.82 \mathrm{E}-01 \\
1.61 \mathrm{E}-02 \\
4.37 \mathrm{E}-02 \\
1.96 \mathrm{E}-04 \\
1.94 \mathrm{E}-03 \\
3.44 \mathrm{E}-04 \\
3.25 \mathrm{E}-03 \\
1.94 \mathrm{E}-01 \\
2.33 \mathrm{E}-02 \\
2.77 \mathrm{E}-03 \\
9.52 \mathrm{E}-03 \\
1.72 \mathrm{E}-02 \\
1.94 \mathrm{E}-01 \\
3.64 \mathrm{E}-02 \\
1.38 \mathrm{E}-02 \\
5.16 \mathrm{E}-02 \\
1.81 \mathrm{E}-01 \\
2.38 \mathrm{E}-02 \\
1.61 \mathrm{E}-04 \\
9.50 \mathrm{E}-03 \\
8.37 \mathrm{E}-02 \\
1.55 \mathrm{E}-02 \\
1.23 \mathrm{E}-01 \\
2.42 \mathrm{E}-01 \\
1.11 \mathrm{E}-01 \\
2.95 \mathrm{E}-02 \\
4.10 \mathrm{E}-03 \\
1.51 \mathrm{E}-02 \\
2.44 \mathrm{E}-02 \\
1.30 \mathrm{E}-02 \\
3.80 \mathrm{E}-02\end{array}$ \\
\hline
\end{tabular}


ACTIVITY IN UNSEPARATEO FUEL (Curies decayed to 1/1/94)

\begin{tabular}{|c|c|c|c|c|c|c|c|c|c|}
\hline 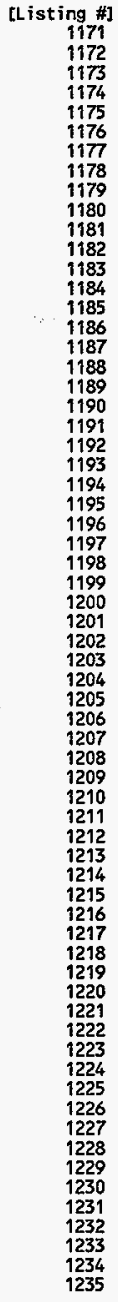 & $\begin{array}{l}P U 238 \\
2.00 \mathrm{E}+01 \\
3.40 \mathrm{E}-01 \\
8.56 \mathrm{E}+00 \\
5.72 \mathrm{E}+01 \\
6.13 \mathrm{E}+00 \\
2.53 \mathrm{E}+00 \\
1.54 \mathrm{E}-01 \\
1.69 \mathrm{E}+00 \\
4.92 \mathrm{E}+01 \\
1.35 \mathrm{E}+02 \\
6.05 \mathrm{E}+01 \\
2.02 \mathrm{E}+02 \\
2.70 \mathrm{E}+01 \\
2.50 \mathrm{E}+01 \\
2.06 \mathrm{E}+02 \\
2.03 \mathrm{E}+02 \\
2.68 \mathrm{E}+01 \\
3.12 \mathrm{E}+01 \\
1.18 \mathrm{E}+01 \\
3.44 \mathrm{E}+02 \\
5.80 \mathrm{E}+01 \\
1.87 \mathrm{E}+01 \\
2.04 \mathrm{E}+02 \\
6.21 \mathrm{E}+02 \\
1.34 \mathrm{E}+01 \\
2.59 \mathrm{E}+01 \\
1.10 \mathrm{E}+02 \\
2.13 \mathrm{E}+02 \\
9.77 \mathrm{E}+01 \\
4.92 \mathrm{E}+01 \\
1.74 \mathrm{E}+02 \\
5.09 \mathrm{E}+01 \\
6.61 \mathrm{E}+02 \\
3.80 \mathrm{E}+02 \\
1.07 \mathrm{E}+02 \\
9.46 \mathrm{E}+00 \\
2.56 \mathrm{E}+01 \\
5.73 \mathrm{E}-03 \\
4.75 \mathrm{E}-01 \\
1.00 \mathrm{E}-02 \\
4.88 \mathrm{E}-01 \\
1.24 \mathrm{E}+02 \\
4.67 \mathrm{E}+00 \\
8.10 \mathrm{E}-02 \\
2.01 \mathrm{E}+00 \\
3.62 \mathrm{E}+00 \\
1.24 \mathrm{E}+02 \\
1.86 \mathrm{E}+01 \\
2.78 \mathrm{E}+00 \\
2.64 \mathrm{E}+01 \\
1.11 \mathrm{E}+02 \\
5.26 \mathrm{E}+00 \\
1.14 \mathrm{E}-01 \\
6.81 \mathrm{E}+00 \\
6.01 \mathrm{E}+01 \\
1.03 \mathrm{E}+01 \\
6.30 \mathrm{E}+01 \\
1.49 \mathrm{E}+02 \\
7.12 \mathrm{E}+01 \\
2.02 \mathrm{E}+01 \\
2.94 \mathrm{E}+00 \\
9.28 \mathrm{E}+00 \\
1.35 \mathrm{E}+01 \\
3.43 \mathrm{E}+00 \\
9.61 \mathrm{E}+00\end{array}$ & $\begin{array}{l}P U 239 \\
1.45 E+02 \\
1.02 E+01 \\
2.85 E+02 \\
3.58 E+02 \\
2.04 E+02 \\
3.89 E+01 \\
4.59 E+00 \\
3.57 E+01 \\
8.22 E+02 \\
1.58 E+03 \\
7.25 E+02 \\
6.52 E+02 \\
1.44 E+02 \\
1.38 E+02 \\
6.66 E+02 \\
6.56 E+02 \\
1.43 E+02 \\
1.01 E+02 \\
3.62 E+01 \\
1.11 E+03 \\
3.22 E+02 \\
8.92 E+01 \\
1.09 E+03 \\
1.83 E+03 \\
3.23 E+01 \\
7.57 E+01 \\
3.22 E+02 \\
1.14 E+03 \\
4.62 E+02 \\
1.51 E+02 \\
8.20 E+02 \\
1.50 E+02 \\
1.94 E+03 \\
1.22 E+03 \\
1.48 E+03 \\
1.31 E+02 \\
3.55 E+02 \\
1.90 E+00 \\
1.76 E+01 \\
3.34 E+00 \\
3.02 E+01 \\
1.55 E+03 \\
2.14 E+02 \\
2.69 E+01 \\
8.71 E+01 \\
1.57 E+02 \\
1.55 E+03 \\
3.04 E+02 \\
1.26 E+02 \\
4.31 E+02 \\
1.46 E+03 \\
2.17 E+02 \\
1.26 E+00 \\
7.40 E+01 \\
6.52 E+02 \\
1.23 E+02 \\
1.03 E+03 \\
1.95 E+03 \\
8.87 E+02 \\
2.32 E+02 \\
3.19 E+01 \\
1.22 E+02 \\
2.00 E+02 \\
1.17 E+02 \\
3.43 E+02\end{array}$ & $\begin{array}{l}\text { PU240 } \\
4.97 \mathrm{E}+01 \\
1.92 \mathrm{E}+00 \\
5.24 \mathrm{E}+01 \\
1.27 \mathrm{E}+02 \\
3.75 \mathrm{E}+01 \\
8.95 \mathrm{E}+00 \\
8.64 \mathrm{E}-01 \\
7.37 \mathrm{E}+00 \\
1.84 \mathrm{E}+02 \\
4.03 \mathrm{E}+02 \\
1.85 \mathrm{E}+02 \\
3.47 \mathrm{E}+02 \\
5.95 \mathrm{E}+01 \\
5.58 \mathrm{E}+01 \\
3.55 \mathrm{E}+02 \\
3.49 \mathrm{E}+02 \\
5.90 \mathrm{E}+01 \\
5.36 \mathrm{E}+01 \\
1.93 \mathrm{E}+01 \\
5.93 \mathrm{E}+02 \\
1.30 \mathrm{E}+02 \\
3.82 \mathrm{E}+01 \\
4.50 \mathrm{E}+02 \\
1.02 \mathrm{E}+03 \\
1.97 \mathrm{E}+01 \\
4.13 \mathrm{E}+01 \\
1.79 \mathrm{E}+02 \\
4.69 \mathrm{E}+02 \\
2.06 \mathrm{E}+02 \\
8.05 \mathrm{E}+01 \\
3.65 \mathrm{E}+02 \\
8.35 \mathrm{E}+01 \\
1.08 \mathrm{E}+03 \\
6.52 \mathrm{E}+02 \\
3.54 \mathrm{E}+02 \\
3.14 \mathrm{E}+01 \\
8.49 \mathrm{E}+01 \\
2.73 \mathrm{E}-01 \\
3.16 \mathrm{E}+00 \\
4.79 \mathrm{E}-01 \\
4.99 \mathrm{E}+00 \\
3.86 \mathrm{E}+02 \\
3.68 \mathrm{E}+01 \\
3.87 \mathrm{E}+00 \\
1.52 \mathrm{E}+01 \\
2.73 \mathrm{E}+01 \\
3.86 \mathrm{E}+02 \\
6.81 \mathrm{E}+01 \\
2.18 \mathrm{E}+01 \\
9.66 \mathrm{E}+01 \\
3.57 \mathrm{E}+02 \\
3.81 \mathrm{E}+01 \\
3.32 \mathrm{E}-01 \\
1.96 \mathrm{E}+01 \\
1.73 \mathrm{E}+02 \\
3.11 \mathrm{E}+01 \\
2.31 \mathrm{E}+02 \\
4.76 \mathrm{E}+02 \\
2.21 \mathrm{E}+02 \\
5.99 \mathrm{E}+01 \\
8.45 \mathrm{E}+00 \\
2.98 \mathrm{E}+01 \\
4.66 \mathrm{E}+01 \\
2.14 \mathrm{E}+01 \\
6.19 \mathrm{E}+01\end{array}$ & $\begin{array}{c}\text { PU241 } \\
1.57 E+03 \\
2.21 E+01 \\
4.85 E+02 \\
7.12 E+03 \\
3.49 E+02 \\
2.75 E+02 \\
1.02 E+01 \\
1.54 E+02 \\
5.19 E+03 \\
1.56 E+04 \\
6.09 E+03 \\
1.58 E+04 \\
2.18 E+03 \\
2.01 E+03 \\
1.61 E+04 \\
1.59 E+04 \\
2.16 E+03 \\
2.47 E+03 \\
1.20 E+03 \\
2.69 E+04 \\
4.67 E+03 \\
2.02 E+03 \\
1.65 E+04 \\
4.90 E+04 \\
1.15 E+03 \\
2.61 E+03 \\
9.03 E+03 \\
1.72 E+04 \\
8.05 E+03 \\
5.01 E+03 \\
1.48 E+04 \\
4.01 E+03 \\
5.26 E+04 \\
3.02 E+04 \\
1.24 E+04 \\
1.10 E+03 \\
2.97 E+03 \\
0.00 E+00 \\
2.09 E+01 \\
0.00 E+00 \\
0.00 E+00 \\
1.52 E+04 \\
9.78 E+01 \\
0.00 E+00 \\
5.58 E+01 \\
1.01 E+02 \\
1.52 E+04 \\
2.08 E+03 \\
6.01 E+01 \\
2.95 E+03 \\
1.33 E+04 \\
1.82 E+02 \\
1.36 E+01 \\
8.52 E+02 \\
7.58 E+03 \\
1.27 E+03 \\
7.05 E+03 \\
1.77 E+04 \\
8.71 E+03 \\
2.47 E+03 \\
3.71 E+02 \\
1.11 E+03 \\
1.56 E+03 \\
1.93 E+02 \\
4.95 E+02\end{array}$ & $\begin{array}{l}\text { PU242 } \\
8.51 \mathrm{E}-03 \\
0.00 \mathrm{E}+00 \\
0.00 \mathrm{E}+00 \\
2.29 \mathrm{E}-02 \\
0.00 \mathrm{E}+00 \\
1.65 \mathrm{E}-04 \\
0.00 \mathrm{E}+00 \\
0.00 \mathrm{E}+00 \\
6.55 \mathrm{E}-04 \\
2.55 \mathrm{E}-02 \\
1.17 \mathrm{E}-02 \\
1.28 \mathrm{E}-01 \\
1.33 \mathrm{E}-02 \\
1.21 \mathrm{E}-02 \\
1.31 \mathrm{E}-01 \\
1.29 \mathrm{E}-01 \\
1.32 \mathrm{E}-02 \\
1.98 \mathrm{E}-02 \\
7.10 \mathrm{E}-03 \\
2.18 \mathrm{E}=01 \\
2.81 \mathrm{E}-02 \\
8.85 \mathrm{E}-03 \\
1.00 \mathrm{E}-01 \\
4.07 \mathrm{E}-01 \\
9.19 \mathrm{E}-03 \\
1.59 \mathrm{E}-02 \\
7.16 \mathrm{E}-02 \\
1.05 \mathrm{E}-01 \\
5.02 \mathrm{E}-02 \\
2.96 \mathrm{E}-02 \\
8.91 \mathrm{E}-02 \\
3.34 \mathrm{E}-02 \\
4.32 \mathrm{E}-01 \\
2.40 \mathrm{E}-01 \\
1.25 \mathrm{E}-02 \\
1.11 \mathrm{E}-03 \\
3.00 \mathrm{E}-03 \\
0.00 \mathrm{E}+00 \\
0.00 \mathrm{E}+00 \\
0.00 \mathrm{E}+00 \\
0.00 \mathrm{E}+00 \\
2.07 \mathrm{E}-02 \\
0.00 \mathrm{E}+00 \\
0.00 \mathrm{E}+00 \\
0.00 \mathrm{E}+00 \\
0.00 \mathrm{E}+00 \\
2.07 \mathrm{E}-02 \\
3.88 \mathrm{E}-04 \\
0.00 \mathrm{E}+00 \\
5.50 \mathrm{E}-04 \\
1.60 \mathrm{E}-02 \\
0.00 \mathrm{E}+00 \\
2.59 \mathrm{E}-05 \\
1.53 \mathrm{E}-03 \\
1.35 \mathrm{E}-02 \\
1.89 \mathrm{E}-03 \\
1.31 \mathrm{E}-03 \\
2.13 \mathrm{E}-02 \\
1.18 \mathrm{E}-02 \\
4.10 \mathrm{E}-03 \\
6.59 \mathrm{E}-04 \\
1.33 \mathrm{E}-03 \\
1.05 \mathrm{E}-03 \\
0.00 \mathrm{E}+00 \\
0.00 \mathrm{E}+00\end{array}$ & $\begin{array}{l}\text { AM241 } \\
9.29 \mathrm{E}+01 \\
4.12 \mathrm{E}-01 \\
8.33 \mathrm{E}+00 \\
1.48 \mathrm{E}+02 \\
5.89 \mathrm{E}+00 \\
4.64 \mathrm{E}+00 \\
1.76 \mathrm{E}-01 \\
2.65 \mathrm{E}+00 \\
8.48 \mathrm{E}+01 \\
2.58 \mathrm{E}+02 \\
1.53 \mathrm{E}+02 \\
8.92 \mathrm{E}+02 \\
1.29 \mathrm{E}+02 \\
1.39 \mathrm{E}+02 \\
9.12 \mathrm{E}+02 \\
8.97 \mathrm{E}+02 \\
1.28 \mathrm{E}+02 \\
1.37 \mathrm{E}+02 \\
3.93 \mathrm{E}+01 \\
1.52 \mathrm{E}+03 \\
2.77 \mathrm{E}+02 \\
6.61 \mathrm{E}+01 \\
9.77 \mathrm{E}+02 \\
2.65 \mathrm{E}+03 \\
4.94 \mathrm{E}+01 \\
8.52 \mathrm{E}+01 \\
4.53 \mathrm{E}+02 \\
1.02 \mathrm{E}+03 \\
4.64 \mathrm{E}+02 \\
1.64 \mathrm{E}+02 \\
8.08 \mathrm{E}+02 \\
2.17 \mathrm{E}+02 \\
2.79 \mathrm{E}+03 \\
1.66 \mathrm{E}+03 \\
1.89 \mathrm{E}+02 \\
1.67 \mathrm{E}+01 \\
4.53 \mathrm{E}+01 \\
0.00 \mathrm{E}+00 \\
4.65 \mathrm{E}-01 \\
0.00 \mathrm{E}+00 \\
0.00 \mathrm{E}+00 \\
2.14 \mathrm{E}+02 \\
1.48 \mathrm{E}+00 \\
0.00 \mathrm{E}+00 \\
8.12 \mathrm{E}-01 \\
1.47 \mathrm{E}+00 \\
2.14 \mathrm{E}+02 \\
2.77 \mathrm{E}+01 \\
8.00 \mathrm{E}-01 \\
3.93 \mathrm{E}+01 \\
1.95 \mathrm{E}+02 \\
2.69 \mathrm{E}+00 \\
2.42 \mathrm{E}-01 \\
1.26 \mathrm{E}+01 \\
1.08 \mathrm{E}+02 \\
1.80 \mathrm{E}+01 \\
9.39 \mathrm{E}+01 \\
2.60 \mathrm{E}+02 \\
1.23 \mathrm{E}+02 \\
3.78 \mathrm{E}+01 \\
5.29 \mathrm{E}+00 \\
1.63 \mathrm{E}+01 \\
2.17 \mathrm{E}+01 \\
2.57 \mathrm{E}+00 \\
6.89 \mathrm{E}+00\end{array}$ & $\begin{array}{l}\text { AM243 } \\
9.39 \mathrm{E}-03 \\
0.00 \mathrm{E}+00 \\
0.00 \mathrm{E}+00 \\
2.58 \mathrm{E}-02 \\
0.00 \mathrm{E}+00 \\
3.19 \mathrm{E}-08 \\
0.00 \mathrm{E}+00 \\
0.00 \mathrm{E}+00 \\
0.00 \mathrm{E}+00 \\
1.14 \mathrm{E}-02 \\
5.24 \mathrm{E}-03 \\
2.23 \mathrm{E}-01 \\
1.58 \mathrm{E}-02 \\
1.44 \mathrm{E}-02 \\
2.28 \mathrm{E}-01 \\
2.25 \mathrm{E}-01 \\
1.57 \mathrm{E}-02 \\
3.45 \mathrm{E}-02 \\
1.24 \mathrm{E}-02 \\
3.81 \mathrm{E}-01 \\
3.34 \mathrm{E}-02 \\
1.07 \mathrm{E}-02 \\
1.20 \mathrm{E}-01 \\
7.45 \mathrm{E}-01 \\
1.82 \mathrm{E}-02 \\
2.86 \mathrm{E}-02 \\
1.31 \mathrm{E}-01 \\
1.25 \mathrm{E}-01 \\
6.26 \mathrm{E}-02 \\
5.18 \mathrm{E}-02 \\
1.11 \mathrm{E}-01 \\
6.11 \mathrm{E}-02 \\
7.91 \mathrm{E}-01 \\
4.19 \mathrm{E}-01 \\
3.01 \mathrm{E}-06 \\
2.66 \mathrm{E}-07 \\
7.21 \mathrm{E}-07 \\
0.00 \mathrm{E}+00 \\
0.00 \mathrm{E}+00 \\
0.00 \mathrm{E}+00 \\
0.00 \mathrm{E}+00 \\
4.88 \mathrm{E}-03 \\
0.00 \mathrm{E}+00 \\
0.00 \mathrm{E}+00 \\
0.00 \mathrm{E}+00 \\
0.00 \mathrm{E}+00 \\
4.88 \mathrm{E}-03 \\
0.00 \mathrm{E}+00 \\
0.00 \mathrm{E}+00 \\
0.00 \mathrm{E}+00 \\
4.01 \mathrm{E}-06 \\
0.00 \mathrm{E}+00 \\
1.64 \mathrm{E}-05 \\
9.67 \mathrm{E}-04 \\
8.52 \mathrm{E}-03 \\
7.33 \mathrm{E}-04 \\
0.00 \mathrm{E}+00 \\
5.35 \mathrm{E}-06 \\
2.80 \mathrm{E}-03 \\
2.11 \mathrm{E}-03 \\
4.17 \mathrm{E}-04 \\
3.34 \mathrm{E}-07 \\
2.21 \mathrm{E}-07 \\
0.00 \mathrm{E}+00 \\
0.00 \mathrm{E}+00\end{array}$ & $\begin{array}{l}\text { CM242 } \\
2.50 \mathrm{E}-02 \\
0.00 \mathrm{E}+00 \\
0.00 \mathrm{E}+00 \\
7.23 \mathrm{E}-02 \\
0.00 \mathrm{E}+00 \\
9.57 \mathrm{E}-09 \\
0.00 \mathrm{E}+00 \\
0.00 \mathrm{E}+00 \\
0.00 \mathrm{E}+00 \\
3.34 \mathrm{E}-02 \\
1.50 \mathrm{E}-02 \\
5.46 \mathrm{E}-01 \\
4.22 \mathrm{E}-02 \\
3.82 \mathrm{E}-02 \\
5.59 \mathrm{E}-01 \\
5.50 \mathrm{E}-01 \\
4.19 \mathrm{E}-02 \\
8.45 \mathrm{E}-02 \\
3.13 \mathrm{E}-02 \\
9.33 \mathrm{E}-01 \\
8.88 \mathrm{E}-02 \\
2.94 \mathrm{E}-02 \\
3.19 \mathrm{E}-01 \\
1.81 \mathrm{E}+00 \\
4.41 \mathrm{E}-02 \\
7.16 \mathrm{E}-02 \\
3.20 \mathrm{E}-01 \\
3.33 \mathrm{E}-01 \\
1.66 \mathrm{E}-01 \\
1.31 \mathrm{E}-01 \\
2.95 \mathrm{E}-01 \\
1.48 \mathrm{E}-01 \\
1.93 \mathrm{E}+00 \\
1.03 \mathrm{E}+00 \\
4.14 \mathrm{E}-06 \\
3.68 \mathrm{E}-07 \\
9.97 \mathrm{E}-07 \\
0.00 \mathrm{E}+00 \\
0.00 \mathrm{E}+00 \\
0.00 \mathrm{E}+00 \\
0.00 \mathrm{E}+00 \\
1.44 \mathrm{E}-02 \\
0.00 \mathrm{E}+00 \\
0.00 \mathrm{E}+00 \\
0.00 \mathrm{E}+00 \\
0.00 \mathrm{E}+00 \\
1.44 \mathrm{E}-02 \\
0.00 \mathrm{E}+00 \\
0.00 \mathrm{E}+00 \\
0.00 \mathrm{E}+00 \\
8.77 \mathrm{E}-06 \\
0.00 \mathrm{E}+00 \\
4.64 \mathrm{E}-05 \\
2.81 \mathrm{E}-03 \\
2.50 \mathrm{E}-02 \\
2.15 \mathrm{E}-03 \\
0.00 \mathrm{E}+00 \\
1.17 \mathrm{E}-05 \\
8.26 \mathrm{E}-03 \\
6.06 \mathrm{E}-03 \\
1.22 \mathrm{E}-03 \\
7.31 \mathrm{E}-03 \\
5.71 \mathrm{E}-07 \\
0.00 \mathrm{E}+00 \\
0.00 \mathrm{E}+00\end{array}$ & $\begin{array}{l}\mathrm{CM} 243 \\
2.75 \mathrm{E}-03 \\
0.00 \mathrm{E}+00 \\
0.00 \mathrm{E}+00 \\
1.00 \mathrm{E}-02 \\
0.00 \mathrm{E}+00 \\
0.00 \mathrm{E}+00 \\
0.00 \mathrm{E}+00 \\
0.00 \mathrm{E}+00 \\
0.00 \mathrm{E}+00 \\
1.06 \mathrm{E}-03 \\
4.50 \mathrm{E}-04 \\
9.54 \mathrm{E}-02 \\
4.83 \mathrm{E}-03 \\
4.36 \mathrm{E}-03 \\
9.76 \mathrm{E}-02 \\
9.60 \mathrm{E}-02 \\
4.79 \mathrm{E}-03 \\
1.49 \mathrm{E}-02 \\
6.20 \mathrm{E}-03 \\
1.63 \mathrm{E}-01 \\
1.01 \mathrm{E}-02 \\
3.90 \mathrm{E}-03 \\
3.66 \mathrm{E}-02 \\
3.33 \mathrm{E}-01 \\
9.10 \mathrm{E}-03 \\
1.46 \mathrm{E}-02 \\
6.00 \mathrm{E}-02 \\
3.81 \mathrm{E}-02 \\
2.00 \mathrm{E}-02 \\
2.59 \mathrm{E}-02 \\
3.60 \mathrm{E}-02 \\
2.73 \mathrm{E}-02 \\
3.56 \mathrm{E}-01 \\
1.81 \mathrm{E}-01 \\
0.00 \mathrm{E}+00 \\
0.00 \mathrm{E}+00 \\
0.00 \mathrm{E}+00 \\
0.00 \mathrm{E}+00 \\
0.00 \mathrm{E}+00 \\
0.00 \mathrm{E}+00 \\
0.00 \mathrm{E}+00 \\
0.00 \mathrm{E}+00 \\
0.00 \mathrm{E}+00 \\
0.00 \mathrm{E}+00 \\
0.00 \mathrm{E}+00 \\
0.00 \mathrm{E}+00 \\
0.00 \mathrm{E}+00 \\
0.00 \mathrm{E}+00 \\
0.00 \mathrm{E}+00 \\
0.00 \mathrm{E}+00 \\
0.00 \mathrm{E}+00 \\
0.00 \mathrm{E}+00 \\
3.85 \mathrm{E}-06 \\
2.35 \mathrm{E}-04 \\
2.08 \mathrm{E}-03 \\
0.00 \mathrm{E}+00 \\
0.00 \mathrm{E}+00 \\
0.00 \mathrm{E}+00 \\
0.00 \mathrm{E}+00 \\
3.17 \mathrm{E}-04 \\
1.02 \mathrm{E}-04 \\
0.00 \mathrm{E}+00 \\
0.00 \mathrm{E} \\
0\end{array}$ \\
\hline
\end{tabular}


HNF-SD-WM-TI-794, ReV. 0

ACTIVITY IN UNSEPARATED FUEL (Curies decayed to 1/1/94)

\begin{tabular}{|c|c|}
\hline $\begin{array}{r}\text { issting } 1] \\
1171 \\
1172 \\
1173 \\
1174 \\
1175 \\
1176 \\
1177 \\
1178 \\
1179 \\
1180 \\
1181 \\
1182 \\
1183 \\
1184 \\
1185 \\
1186 \\
1187 \\
1188 \\
1189 \\
1190 \\
1191 \\
1192 \\
1193 \\
1194 \\
1195 \\
1196 \\
1197 \\
1198 \\
1199 \\
1200 \\
1201 \\
1202 \\
1203 \\
1204 \\
1205 \\
1206 \\
1207 \\
1208 \\
1209 \\
1210 \\
1211 \\
1212 \\
1213 \\
1214 \\
1215 \\
1216 \\
1217 \\
1218 \\
1219 \\
1220 \\
1221 \\
1222 \\
1223 \\
1224 \\
1225 \\
1226 \\
1227 \\
1228 \\
1229 \\
1230 \\
1231 \\
1232 \\
1233 \\
1234 \\
1235\end{array}$ & $\begin{array}{l}\text { CM244 } \\
5.97 \mathrm{E}-02 \\
0.00 \mathrm{E}+00 \\
0.00 \mathrm{E}+00 \\
2.56 \mathrm{E}-01 \\
0.00 \mathrm{E}+00 \\
0.00 \mathrm{E}+00 \\
0.00 \mathrm{E}+00 \\
0.00 \mathrm{E}+00 \\
0.00 \mathrm{E}+00 \\
3.39 \mathrm{E}-02 \\
1.37 \mathrm{E}-02 \\
2.24 \mathrm{E}+00 \\
1.05 \mathrm{E}-01 \\
9.44 \mathrm{E}-02 \\
2.29 \mathrm{E}+00 \\
2.25 \mathrm{E}+00 \\
1.04 \mathrm{E}-01 \\
3.50 \mathrm{E}-01 \\
1.59 \mathrm{E}-01 \\
3.82 \mathrm{E}+00 \\
2.20 \mathrm{E}-01 \\
9.33 \mathrm{E}-02 \\
7.92 \mathrm{E}-01 \\
7.91 \mathrm{E}+00 \\
2.27 \mathrm{E}-01 \\
3.75 \mathrm{E}-01 \\
1.44 \mathrm{E}+00 \\
8.26 \mathrm{E}-01 \\
4.38 \mathrm{E}-01 \\
6.66 \mathrm{E}-01 \\
7.98 \mathrm{E}-01 \\
6.48 \mathrm{E}-01 \\
8.48 \mathrm{E}+00 \\
4.26 \mathrm{E}+00 \\
0.00 \mathrm{E}+00 \\
0.00 \mathrm{E}+00 \\
0.00 \mathrm{E}+00 \\
0.00 \mathrm{E}+00 \\
0.00 \mathrm{E}+00 \\
0.00 \mathrm{E}+00 \\
0.00 \mathrm{E}+00 \\
7.49 \mathrm{E}-00 \\
0.00 \mathrm{E}+00 \\
0.00 \mathrm{E}+00 \\
0.00 \mathrm{E}+00 \\
0.00 \mathrm{E}+00 \\
7.49 \mathrm{E}-06 \\
0.00 \mathrm{E}+00 \\
0.00 \mathrm{E}+00 \\
0.00 \mathrm{E}+00 \\
0.00 \mathrm{E}+00 \\
0.00 \mathrm{E}+00 \\
1.05 \mathrm{E}-04 \\
6.54 \mathrm{E}-03 \\
5.80 \mathrm{E}-02 \\
5.90 \mathrm{E}-04 \\
0.00 \mathrm{E}+00 \\
0.00 \mathrm{E}+00 \\
4.29 \mathrm{E}-06 \\
9.51 \mathrm{E}-03 \\
2.84 \mathrm{E}-03 \\
0.00 \mathrm{E}+00 \\
0.00 \mathrm{E}+00 \\
0.00 \mathrm{E}+00 \\
0.00 \mathrm{E}+00\end{array}$ \\
\hline
\end{tabular}


HNF-SD-WM-TI-794, Rev. 0

UNIFIED SEPARATIONS PRODUCTION OATA

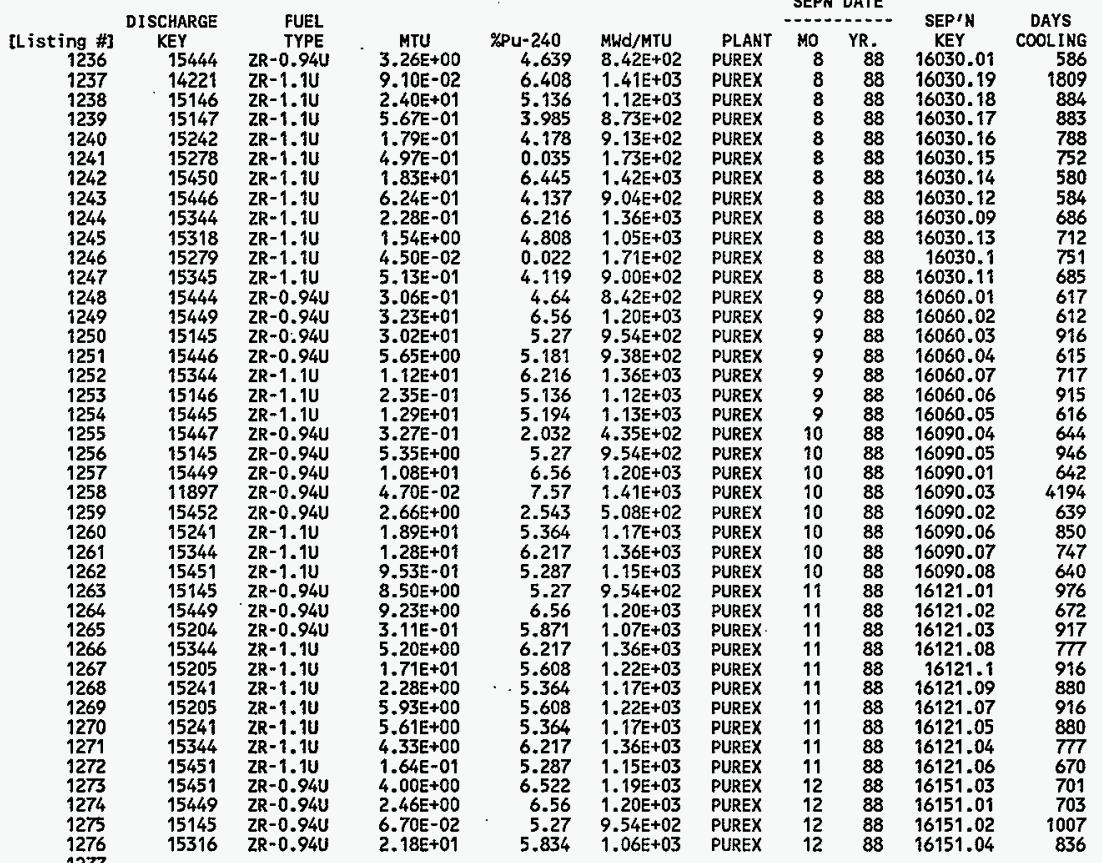


HNF-SD-WM-T1-794, Rev. 0

UNIFIED SEPARATIONS PRODUCTION DATA- TOTAL WASTE LOSS FRACTION

\section{[Listing \#] Fuel Data Reference}

$1236 \mathrm{~J}$. SCHLOSSER FILE 'SHIP_PX.DAT' 01/

$1237 \mathrm{~J}$. SCHLOSSER FILE 'SHIP'PX.DAT' 01/

$1238 \mathrm{~J}$. SCHLOSSER FILE 'SHIP-PX.DAT' 01/

$1239 \mathrm{~J}$. SCHLOSSER FILE 'SHIP'PX.DAT' 01/

$1240 \mathrm{~J}$. SCHLOSSER FILE 'SHIP'PX.DAT' 01/

$1241 \mathrm{~J}$. SCHLOSSER FILE 'SHIP-PX.DAT' 01/

$1242 \mathrm{~J}$. SCHLOSSER FILE 'SHIP'PX.DAT' 01/

$1243 \mathrm{~J}$. SCHLOSSER FILE 'SHIP_PX.DAT' 01/

$1244 \mathrm{~J}$. SCHLOSSER FILE 'SHIP'PX.DAT' O1'

$1245 \mathrm{~J}$. SCHLOSSER FILE 'SHIP_PX.DAT' O1/

$1246 \mathrm{~J}$. SCHLOSSER FILE 'SHIP_PX.DAT' O1/

$1247 \mathrm{~J}$. SCHLOSSER FILE 'SHIP'PX.DAT' 01/

$1248 \mathrm{~J}$. SCHLOSSER FILE 'SHIP_PX.DAT' 01/

$1249 \mathrm{j}$. SCHLOSSER FILE 'SHIP'PX.DAT' O1/

$1250 \mathrm{~J}$. SCHLOSSER FILE 'SHIP'PX.DAT' 01/

1251 \. SCHLOSSER FILE 'SHIP_PX.DAT' 01/

$1252 \mathrm{~J}$. SCHLOSSER FILE 'SHIP'PX.DAT' 01/

1253 J. SCHLOSSER FILE 'SHIP'PX.DAT' 01/

1254 j. SCHLOSSER FILE 'SHIP'PX.DAT' 01/

1255 J. SCHLOSSER FILE 'SHIP'PX.DAT' 01/

$1256 \mathrm{~J}$. SCHLOSSER FILE 'SHIP-PX.DAT' 01/

$1257 \mathrm{~s}$. SCHLOSSER FILE 'SHIP'PX.DAT' $01 /$

$1258 \mathrm{~J}$. SCHLOSSER FILE 'SHIP'PX.DAT' 01/

$1259 \mathrm{~J}$. SCHLOSSER FILE 'SHIP-PX.DAT' 01/

$1260 \mathrm{~d}$. SCHLOSSER FILE 'SHIP'PX.DAT' 01/

1261 3. SCHLOSSER FILE 'SHIP-PX.DAT' 01/

1262 i. SCHLOSSER FILE 'SHIP'PX.DAT' 01/

$1263 \mathrm{~J}$. SCHLOSSER FILE 'SHIP PX.DAT' 01/

1264 J. SCHLOSSER FILE 'SHIP_PX.DAT' 01/

$1265 \mathrm{~J}$. SCHLOSSER FILE 'SHIP PX.DAT' 01/

$1266 \mathrm{~J}$. SCHLOSSER FILE 'SHIP'PX.DAT' 01/

$1267 \mathrm{~J}$. SCHLOSSER FILE 'SHIPIPX.DAT' 01/

$1268 \mathrm{~J}$. SCHLOSSER FILE 'SHIP'PX.DAT' 01\%

$1269 \mathrm{~J}$. SCHLOSSER FILE 'SHIP_PX.DAT' 01/

$1270 \mathrm{~J}$. SCHLOSSER FILE 'SHIP PX.DAT' O1/

1271 J. SCHLOSSER FILE 'SHIP'PX.DAT' 01/

1272 J. SCHLOSSER FILE 'SHIP_PX.DAT' 01/

$1273 \mathrm{~J}$. SCHLOSSER FILE 'SHIP'PX.DAT' 01/

$1274 \mathrm{~J}$. SCHLOSSER FILE 'SHIP_PX.DAT' 01/

1275 J. SCHLOSSER FILE 'SHIP_PX.DAT' O1/

1276 J. SCHLOSSER FILE 'SHIP_PX.DAT' OI'

1277

1278

1279

1280

1281 (a) No data: Ave of T Plant 1953 thru 56.

1282 (b) Gydesen, HW-89085; Data from HW-63089-90 (Anderson Notebook.)

1283 (c) Generic factor for PUREX-Zr clad -- derived from core samples (TKs 101-, 102-AZ, 103-, 105 -AW)
Waste Frac.

Reference

(c)

(c)

(c)

(c)

(c)

(c)

(c)

(c)

(c)

(c)

(c)

(c)

(c)

(c)

(c)

(c)

(c)

(c)

(c)

(c)

(c)

(c)

(c)

(c)

(c)

(c)

(c)

(c)

(c)

(c)

1285

1286

1287

1288

1289

1290

1291

1292

1293

1294

1295

1296

1297

1298

1299

1300 
ACTIVITY IN UNSEPARATED FUEL (Curies decayed to $1 / 1 / 94$ )

\begin{tabular}{|c|c|c|c|c|c|c|c|c|c|}
\hline $\begin{array}{r}\text { [Listing } \mathbf{H}] \\
1236 \\
1237 \\
1238 \\
1239 \\
1240 \\
1241 \\
1242 \\
1243 \\
1244 \\
1245 \\
1246 \\
1247 \\
1248 \\
1249 \\
1250 \\
1251 \\
1252 \\
1253 \\
1254 \\
1255 \\
1256 \\
1257 \\
1258 \\
1259 \\
1260 \\
1261 \\
1262 \\
1263 \\
1264 \\
1265 \\
1266 \\
1267 \\
1268 \\
1269 \\
1270 \\
1271 \\
1272 \\
1273 \\
1274 \\
1275 \\
1276\end{array}$ & $\begin{array}{l}\text { H03 } \\
2.99 \mathrm{E}+01 \\
1.14 \mathrm{E}+00 \\
2.73 \mathrm{E}+02 \\
5.01 \mathrm{E}+00 \\
1.68 \mathrm{E}+00 \\
8.71 \mathrm{E}-01 \\
2.79 \mathrm{E}+02 \\
5.98 \mathrm{E}+00 \\
3.28 \mathrm{E}+00 \\
1.68 \mathrm{E}+01 \\
7.80 \mathrm{E}-02 \\
4.82 \mathrm{E}+00 \\
2.81 \mathrm{E}+00 \\
4.27 \mathrm{E}+02 \\
3.01 \mathrm{E}+02 \\
5.79 \mathrm{E}+01 \\
1.61 \mathrm{E}+02 \\
2.67 \mathrm{E}+00 \\
1.56 \mathrm{E}+02 \\
1.53 \mathrm{E}+00 \\
5.32 \mathrm{E}+01 \\
1.42 \mathrm{E}+02 \\
4.25 \mathrm{E}-01 \\
1.46 \mathrm{E}+01 \\
2.28 \mathrm{E}+02 \\
1.84 \mathrm{E}+02 \\
1.17 \mathrm{E}+01 \\
8.47 \mathrm{E}+01 \\
1.22 \mathrm{0}+02 \\
3.50 \mathrm{E}+00 \\
7.48 \mathrm{E}+01 \\
2.16 \mathrm{E}+02 \\
2.75 \mathrm{E}+01 \\
7.46 \mathrm{E}+01 \\
6.77 \mathrm{E}+01 \\
6.23 \mathrm{E}+01 \\
2.02 \mathrm{E}+00 \\
5.26 \mathrm{E}+01 \\
3.26 \mathrm{E}+01 \\
6.67 \mathrm{E}-01 \\
2.48 \mathrm{E}+02\end{array}$ & $\begin{array}{l}\text { C14 } \\
2.95 \mathrm{E}-01 \\
1.21 \mathrm{E}-02 \\
2.51 \mathrm{E}+00 \\
4.62 \mathrm{E}-02 \\
1.53 \mathrm{E}-02 \\
7.90 \mathrm{E}-03 \\
2.45 \mathrm{E}+00 \\
5.28 \mathrm{E}-02 \\
2.93 \mathrm{E}-02 \\
1.51 \mathrm{E}-01 \\
7.07 \mathrm{E}-04 \\
4.32 \mathrm{E}-02 \\
2.77 \mathrm{E}-02 \\
4.19 \mathrm{E}+00 \\
3.10 \mathrm{E}+00 \\
5.70 \mathrm{E}-01 \\
1.44 \mathrm{E}+00 \\
2.46 \mathrm{E}-02 \\
1.37 \mathrm{E}+00 \\
1.52 \mathrm{E}-02 \\
5.49 \mathrm{E}-01 \\
1.40 \mathrm{E}+00 \\
7.18 \mathrm{E}-03 \\
1.44 \mathrm{E}-01 \\
2.07 \mathrm{E}+00 \\
1.64 \mathrm{E}+00 \\
1.03 \mathrm{E}-01 \\
8.73 \mathrm{E}-01 \\
1.20 \mathrm{E}+00 \\
3.58 \mathrm{E}-02 \\
6.68 \mathrm{E}-01 \\
1.97 \mathrm{E}+00 \\
2.50 \mathrm{E}-01 \\
6.82 \mathrm{E}-01 \\
6.15 \mathrm{E}-01 \\
5.57 \mathrm{E}-01 \\
1.777 \mathrm{E}-02 \\
5.16 \mathrm{E}-01 \\
3.19 \mathrm{E}-01 \\
6.88 \mathrm{E}-03 \\
2.49 \mathrm{E}+00\end{array}$ & $\begin{array}{l}\text { NI59 } \\
1.76 \mathrm{E}-02 \\
7.54 \mathrm{E}-04 \\
1.57 \mathrm{E}-01 \\
2.90 \mathrm{E}-03 \\
9.56 \mathrm{E}-04 \\
4.98 \mathrm{E}-04 \\
1.53 \mathrm{E}-01 \\
3.30 \mathrm{E}-03 \\
1.83 \mathrm{E}-03 \\
9.42 \mathrm{E}-03 \\
4.46 \mathrm{E}-05 \\
2.70 \mathrm{E}-03 \\
1.65 \mathrm{E}-03 \\
2.50 \mathrm{E}-01 \\
1.85 \mathrm{E}-01 \\
3.40 \mathrm{E}-02 \\
8.95 \mathrm{E}-02 \\
1.54 \mathrm{E}-03 \\
8.56 \mathrm{E}-02 \\
9.10 \mathrm{E}-04 \\
3.28 \mathrm{E}-02 \\
8.32 \mathrm{E}-02 \\
4.27 \mathrm{E}-04 \\
8.65 \mathrm{E}-03 \\
1.29 \mathrm{E}-01 \\
1.02 \mathrm{E}-01 \\
6.43 \mathrm{E}-03 \\
5.21 \mathrm{E}-02 \\
7.13 \mathrm{E}-02 \\
2.13 \mathrm{E}-03 \\
4.17 \mathrm{E}-02 \\
1.23 \mathrm{E}-01 \\
1.56 \mathrm{E}-02 \\
4.26 \mathrm{E}-02 \\
3.84 \mathrm{E}-02 \\
3.47 \mathrm{E}-02 \\
1.11 \mathrm{E}-03 \\
3.07 \mathrm{E}-02 \\
1.90 \mathrm{E}-02 \\
4.11 \mathrm{E}-04 \\
1.49 \mathrm{E}-01\end{array}$ & $\begin{array}{l}\text { MI } 63 \\
2.10 E+00 \\
8.78 E-02 \\
1.86 E+01 \\
3.43 E-01 \\
1.13 E-01 \\
5.88 E-02 \\
1.83 E+01 \\
3.93 E-01 \\
2.18 E-01 \\
1.12 E+00 \\
5.27 E-03 \\
3.21 E-01 \\
1.97 E-01 \\
2.99 E+01 \\
2.20 E+01 \\
4.07 E+00 \\
1.07 E+01 \\
1.82 E-01 \\
1.02 E+01 \\
1.08 E-01 \\
3.89 E+00 \\
9.96 E+00 \\
4.76 E-02 \\
1.03 E+00 \\
1.54 E+01 \\
1.22 E+01 \\
7.67 E-01 \\
6.19 E+00 \\
8.53 E+00 \\
2.54 E-01 \\
4.96 E+00 \\
1.46 E+01 \\
1.85 E+00 \\
5.05 E+00 \\
4.56 E+00 \\
4.14 E+00 \\
1.32 E-01 \\
3.68 E+00 \\
2.28 E+00 \\
4.88 E-02 \\
1.77 E+01\end{array}$ & $\begin{array}{c}\text { CO60 } \\
7.43 E+00 \\
2.30 E-01 \\
6.69 E+01 \\
1.24 E+00 \\
4.23 E-01 \\
2.25 E-01 \\
7.24 E+01 \\
1.57 E+00 \\
8.34 E-01 \\
4.28 E+00 \\
2.01 E-02 \\
1.24 E+00 \\
6.98 E-01 \\
1.05 E+02 \\
7.00 E+01 \\
1.44 E+01 \\
4.09 E+01 \\
6.56 E-01 \\
4.06 E+01 \\
3.87 E-01 \\
1.24 E+01 \\
3.50 E+01 \\
4.99 E-02 \\
3.68 E+00 \\
5.70 E+01 \\
4.67 E+01 \\
3.06 E+00 \\
1.97 E+01 \\
3.00 E+01 \\
8.23 E-01 \\
1.90 E+01 \\
5.34 E+01 \\
6.87 E+00 \\
1.85 E+01 \\
1.69 E+01 \\
1.59 E+01 \\
5.26 E-01 \\
1.29 E+01 \\
8.00 E+00 \\
1.55 E-01 \\
5.975+01\end{array}$ & $\begin{array}{l}\text { SE79 } \\
3.67 \mathrm{E}-02 \\
1.70 \mathrm{E}-03 \\
3.55 \mathrm{E}-01 \\
6.58 \mathrm{E}-03 \\
2.17 \mathrm{E}-03 \\
1.15 \mathrm{E}-03 \\
3.44 \mathrm{E}-01 \\
7.50 \mathrm{E}-03 \\
4.11 \mathrm{E}-03 \\
2.14 \mathrm{E}-02 \\
1.03 \mathrm{E}-04 \\
6.14 \mathrm{E}-03 \\
3.45 \mathrm{E}-03 \\
5.17 \mathrm{E}-01 \\
3.85 \mathrm{E}-01 \\
7.08 \mathrm{E}-02 \\
2.02 \mathrm{E}-01 \\
3.48 \mathrm{E}-03 \\
1.94 \mathrm{E}-01 \\
1.91 \mathrm{E}-03 \\
6.81 \mathrm{E}-02 \\
1.72 \mathrm{E}-01 \\
8.80 \mathrm{E}-04 \\
1.82 \mathrm{E}-02 \\
2.93 \mathrm{E}-01 \\
2.31 \mathrm{E}-01 \\
1.45 \mathrm{E}-02 \\
1.08 \mathrm{E}-01 \\
1.48 \mathrm{E}-01 \\
4.42 \mathrm{E}-03 \\
9.38 \mathrm{E}-02 \\
2.78 \mathrm{E}-01 \\
3.52 \mathrm{E}-02 \\
9.61 \mathrm{E}-02 \\
8.68 \mathrm{E}-02 \\
7.82 \mathrm{E}-02 \\
2.50 \mathrm{E}-03 \\
6.36 \mathrm{E}-02 \\
3.94 \mathrm{E}-02 \\
8.54 \mathrm{E}-04 \\
3.08 \mathrm{E}-01\end{array}$ & $\begin{array}{c}\text { SR90 } \\
6.55 E+03 \\
2.82 E+02 \\
6.34 \mathrm{E}+04 \\
1.18 \mathrm{E}+03 \\
3.92 \mathrm{E}+02 \\
2.13 \mathrm{E}+02 \\
6.19 \mathrm{E}+04 \\
1.37 \mathrm{E}+03 \\
7.37 \mathrm{E}+02 \\
3.86 \mathrm{E}+03 \\
1.90 \mathrm{E}+01 \\
1.12 \mathrm{E}+03 \\
6.15 \mathrm{E}+02 \\
9.07 \mathrm{E}+04 \\
6.70 \mathrm{E}+04 \\
1.26 \mathrm{E}+04 \\
3.61 \mathrm{E}+04 \\
6.21 \mathrm{E}+02 \\
3.52 \mathrm{E}+04 \\
3.47 \mathrm{E}+02 \\
1.19 \mathrm{E}+04 \\
3.02 \mathrm{E}+04 \\
1.21 \mathrm{E}+02 \\
3.29 \mathrm{E}+03 \\
5.24 \mathrm{E}+04 \\
4.13 \mathrm{E}+04 \\
2.64 \mathrm{E}+03 \\
1.89 \mathrm{E}+04 \\
2.59 \mathrm{E}+04 \\
7.69 \mathrm{E}+02 \\
1.68 \mathrm{0}+04 \\
4.95 \mathrm{E}+04 \\
6.31 \mathrm{E}+03 \\
1.71 \mathrm{E}+04 \\
1.56 \mathrm{E}+04 \\
1.40 \mathrm{E}+04 \\
4.54 \mathrm{E}+02 \\
1.12 \mathrm{E}+04 \\
6.91 \mathrm{E}+03 \\
1.49 \mathrm{E}+02 \\
5.40 \mathrm{E}+04\end{array}$ & $\begin{array}{c}Y 90 \\
6.55 \mathrm{E}+03 \\
2.82 \mathrm{E}+02 \\
6.34 \mathrm{E}+04 \\
1.18 \mathrm{E}+03 \\
3.92 \mathrm{E}+02 \\
2.13 \mathrm{E}+02 \\
6.19 \mathrm{E}+04 \\
1.37 \mathrm{E}+03 \\
7.37 \mathrm{E}+02 \\
3.86 \mathrm{E}+03 \\
1.90 \mathrm{E}+01 \\
1.12 \mathrm{E}+03 \\
6.15 \mathrm{E}+02 \\
9.07 \mathrm{E}+04 \\
6.70 \mathrm{E}+04 \\
1.26 \mathrm{E}+04 \\
3.61 \mathrm{E}+04 \\
6.21 \mathrm{E}+02 \\
3.52 \mathrm{E}+04 \\
3.48 \mathrm{E}+02 \\
1.19 \mathrm{E}+04 \\
3.02 \mathrm{E}+04 \\
1.21 \mathrm{E}+02 \\
3.29 \mathrm{E}+03 \\
5.24 \mathrm{E}+04 \\
4.13 \mathrm{E}+04 \\
2.64 \mathrm{E}+03 \\
1.89 \mathrm{E}+04 \\
2.59 \mathrm{E}+04 \\
7.69 \mathrm{E}+02 \\
1.68 \mathrm{E}+04 \\
4.95 \mathrm{E}+04 \\
6.31 \mathrm{E}+03 \\
1.71 \mathrm{E}+04 \\
1.56 \mathrm{E}+04 \\
1.40 \mathrm{E}+04 \\
4.54 \mathrm{E}+02 \\
1.12 \mathrm{E}+04 \\
6.91 \mathrm{E}+03 \\
1.49 \mathrm{E}+02 \\
5.40 \mathrm{E}+04\end{array}$ & $\begin{array}{c}\text { ZR93 } \\
1.79 \mathrm{E}-01 \\
8.34 \mathrm{E}-03 \\
1.75 \mathrm{E}+00 \\
3.26 \mathrm{E}-02 \\
1.08 \mathrm{E}-02 \\
5.75 \mathrm{E}-03 \\
1.69 \mathrm{E}+00 \\
3.72 \mathrm{E}-02 \\
2.02 \mathrm{E}-02 \\
1.06 \mathrm{E}-01 \\
5.14 \mathrm{E}-04 \\
3.04 \mathrm{E}-02 \\
1.68 \mathrm{E}-02 \\
2.50 \mathrm{E}+00 \\
1.87 \mathrm{E}+00 \\
3.44 \mathrm{E}-01 \\
9.92 \mathrm{E}-01 \\
1.72 \mathrm{E}-02 \\
9.56 \mathrm{E}-01 \\
9.40 \mathrm{E}-03 \\
3.31 \mathrm{E}-01 \\
8.32 \mathrm{E}-01 \\
4.23 \mathrm{E}-03 \\
8.90 \mathrm{E}-02 \\
1.44 \mathrm{E}+00 \\
1.13 \mathrm{E}+00 \\
7.17 \mathrm{E}-02 \\
5.27 \mathrm{E}-01 \\
7.13 \mathrm{E}-01 \\
2.14 \mathrm{E}-02 \\
4.61 \mathrm{E}-01 \\
1.37 \mathrm{E}+00 \\
1.74 \mathrm{E}-01 \\
4.74 \mathrm{E}-01 \\
4.28 \mathrm{E}-01 \\
3.85 \mathrm{E}-01 \\
1.23 \mathrm{E}-02 \\
3.07 \mathrm{E}-01 \\
1.90 \mathrm{E}-01 \\
4.15 \mathrm{E}-03 \\
1.49 \mathrm{E}+00\end{array}$ \\
\hline
\end{tabular}


HNF-SD-WM-TI-794, ReV. O

ACTIVITY IN UNSEPARATED FUEL (Curies decayed to $1 / 9 / 94$ )

\begin{tabular}{|c|c|c|c|c|c|c|c|c|c|}
\hline $\begin{array}{c}\text { [Listing \#] } \\
1236 \\
1237 \\
1238 \\
1239 \\
1240 \\
1241 \\
1242 \\
1243 \\
1244 \\
1245 \\
1246 \\
1247 \\
1248 \\
1249 \\
1250 \\
1251 \\
1252 \\
1253 \\
1254 \\
1255 \\
1256 \\
1257 \\
1258 \\
1259 \\
1260 \\
1261 \\
1262 \\
1263 \\
1264 \\
1265 \\
1266 \\
1267 \\
1268 \\
1269 \\
1270 \\
1271 \\
1272 \\
1273 \\
1274 \\
1275 \\
1276\end{array}$ & $\begin{array}{l}\text { NB93* } \\
5.18 \mathrm{E}-02 \\
3.29 \mathrm{E}-03 \\
5.56 \mathrm{E}-01 \\
1.03 \mathrm{E}-02 \\
3.31 \mathrm{E}-03 \\
1.73 \mathrm{E}-03 \\
4.91 \mathrm{E}-01 \\
1.07 \mathrm{E}-02 \\
6.07 \mathrm{E}-03 \\
3.18 \mathrm{E}-02 \\
1.55 \mathrm{E}-04 \\
9.07 \mathrm{E}-03 \\
4.86 \mathrm{E}-03 \\
7.26 \mathrm{E}-01 \\
5.93 \mathrm{E}-01 \\
9.97 \mathrm{E}-02 \\
2.98 \mathrm{E}-01 \\
5.45 \mathrm{E}-03 \\
2.77 \mathrm{E}-01 \\
2.70 \mathrm{E}-03 \\
1.05 \mathrm{E}-01 \\
2.42 \mathrm{E}-01 \\
2.32 \mathrm{E}-03 \\
2.56 \mathrm{E}-02 \\
4.45 \mathrm{E}-01 \\
3.40 \mathrm{E}-01 \\
2.08 \mathrm{E}-02 \\
1.67 \mathrm{E}-01 \\
2.07 \mathrm{E}-01 \\
6.70 \mathrm{E}-03 \\
1.39 \mathrm{E}-01 \\
4.27 \mathrm{E}-01 \\
5.36 \mathrm{E}-02 \\
1.48 \mathrm{E}-01 \\
1.32 \mathrm{E}-01 \\
1.16 \mathrm{E}-01 \\
3.57 \mathrm{E}-03 \\
8.94 \mathrm{E}-02 \\
5.53 \mathrm{E}-02 \\
1.31 \mathrm{E}-03 \\
4.52 \mathrm{E}-01\end{array}$ & $\begin{array}{l}\text { TC99 } \\
1.23 \mathrm{E}+00 \\
5.69 \mathrm{E}-02 \\
1.19 \mathrm{E}+01 \\
2.21 \mathrm{E}-01 \\
7.28 \mathrm{E}-02 \\
3.84 \mathrm{E}-02 \\
1.15 \mathrm{E}+01 \\
2.51 \mathrm{E}-01 \\
1.38 \mathrm{E}-01 \\
7.16 \mathrm{E}-01 \\
3.44 \mathrm{E}-03 \\
2.06 \mathrm{E}-01 \\
1.15 \mathrm{E}-01 \\
1.72 \mathrm{E}+01 \\
1.28 \mathrm{E}+01 \\
2.36 \mathrm{E}+00 \\
6.76 \mathrm{E}+00 \\
1.17 \mathrm{E}-01 \\
6.49 \mathrm{E}+00 \\
6.38 \mathrm{E}-02 \\
2.27 \mathrm{E}+00 \\
5.75 \mathrm{E}+00 \\
2.94 \mathrm{E}-02 \\
6.05 \mathrm{E}-01 \\
9.80 \mathrm{E}+00 \\
7.73 \mathrm{E}+00 \\
4.87 \mathrm{E}-01 \\
3.62 \mathrm{E}+00 \\
4.93 \mathrm{E}+00 \\
1.48 \mathrm{E}-01 \\
3.14 \mathrm{E}+00 \\
9.30 \mathrm{E}+00 \\
1.18 \mathrm{E}+00 \\
3.22 \mathrm{E}+00 \\
2.91 \mathrm{E}+00 \\
2.62 \mathrm{E}+00 \\
8.38 \mathrm{E}-02 \\
2.12 \mathrm{E}+00 \\
1.31 \mathrm{E}+00 \\
2.85 \mathrm{E}-02 \\
1.03 \mathrm{E}+01\end{array}$ & $\begin{array}{l}\text { RU106 } \\
3.10 \mathrm{E}+02 \\
1.38 \mathrm{E}+00 \\
1.58 \mathrm{E}+03 \\
2.82 \mathrm{E}+01 \\
1.12 \mathrm{E}+01 \\
5.63 \mathrm{E}+00 \\
2.82 \mathrm{E}+03 \\
5.68 \mathrm{E}+01 \\
2.75 \mathrm{E}+01 \\
1.30 \mathrm{E}+02 \\
5.05 \mathrm{E}-01 \\
3.84 \mathrm{E}+01 \\
2.91 \mathrm{E}+01 \\
4.69 \mathrm{E}+03 \\
1.89 \mathrm{E}+03 \\
6.13 \mathrm{E}+02 \\
1.35 \mathrm{E}+03 \\
1.55 \mathrm{E}+01 \\
1.51 \mathrm{E}+03 \\
1.51 \mathrm{E}+01 \\
3.35 \mathrm{E}+02 \\
1.56 \mathrm{E}+03 \\
1.03 \mathrm{E}-02 \\
1.46 \mathrm{E}+02 \\
1.56 \mathrm{E}+03 \\
1.54 \mathrm{E}+03 \\
1.15 \mathrm{E}+02 \\
5.33 \mathrm{E}+02 \\
1.34 \mathrm{E}+03 \\
2.48 \mathrm{E}+01 \\
6.27 \mathrm{E}+02 \\
1.40 \mathrm{E}+03 \\
1.89 \mathrm{E}+02 \\
4.85 \mathrm{E}+02 \\
4.65 \mathrm{E}+02 \\
5.23 \mathrm{E}+02 \\
1.99 \mathrm{E}+01 \\
5.80 \mathrm{E}+02 \\
3.58 \mathrm{E}+02 \\
4.19 \mathrm{E}+00 \\
2.13 \mathrm{E}+03\end{array}$ & $\begin{array}{l}\text { CD113* } \\
2.28 \mathrm{E}+00 \\
8.35 \mathrm{E}-02 \\
1.92 \mathrm{E}+01 \\
3.49 \mathrm{E}-01 \\
1.17 \mathrm{E}-01 \\
5.80 \mathrm{E}-02 \\
1.99 \mathrm{E}+01 \\
4.15 \mathrm{E}-01 \\
2.33 \mathrm{E}-01 \\
1.17 \mathrm{E}+00 \\
5.19 \mathrm{E}-03 \\
3.35 \mathrm{E}-01 \\
2.14 \mathrm{E}-01 \\
3.33 \mathrm{E}+01 \\
2.32 \mathrm{E}+01 \\
4.44 \mathrm{E}+00 \\
1.14 \mathrm{E}+01 \\
1.89 \mathrm{E}-01 \\
1.09 \mathrm{E}+01 \\
1.13 \mathrm{E}-01 \\
4.11 \mathrm{E}+00 \\
1.11 \mathrm{E}+01 \\
3.65 \mathrm{E}-02 \\
1.09 \mathrm{E}+00 \\
1.61 \mathrm{E}+01 \\
1.31 \mathrm{E}+01 \\
8.22 \mathrm{E}-01 \\
6.54 \mathrm{E}+00 \\
9.50 \mathrm{E}+00 \\
2.72 \mathrm{E}-01 \\
5.32 \mathrm{E}+00 \\
1.53 \mathrm{E}+01 \\
1.94 \mathrm{E}+00 \\
5.29 \mathrm{E}+00 \\
4.78 \mathrm{E}+00 \\
4.44 \mathrm{E}+00 \\
1.42 \mathrm{E}-01 \\
4.10 \mathrm{E}+00 \\
2.53 \mathrm{E}+00 \\
5.15 \mathrm{E}-02 \\
1.92 \mathrm{E}+01\end{array}$ & $\begin{array}{c}\text { SB125 } \\
2.51 E+02 \\
4.68 E+00 \\
1.82 E+03 \\
3.35 E+01 \\
1.18 E+01 \\
6.19 E+00 \\
2.20 E+03 \\
4.69 E+01 \\
2.44 E+01 \\
1.23 E+02 \\
5.54 E-01 \\
3.58 E+01 \\
2.36 E+01 \\
3.61 E+03 \\
2.16 E+03 \\
4.87 E+02 \\
1.20 E+03 \\
1.79 E+01 \\
1.22 E+03 \\
1.28 E+01 \\
3.82 E+02 \\
1.20 E+03 \\
5.45 E-01 \\
1.23 E+02 \\
1.61 E+03 \\
1.37 E+03 \\
9.21 E+01 \\
6.07 E+02 \\
1.03 E+03 \\
2.60 E+01 \\
5.57 E+02 \\
1.49 E+03 \\
1.94 E+02 \\
5.16 E+02 \\
4.77 E+02 \\
4.65 E+02 \\
1.59 E+01 \\
4.45 E+02 \\
2.75 E+02 \\
4.78 E+00 \\
1.95 E+03\end{array}$ & $\begin{array}{c}\text { SN126 } \\
5.57 \mathrm{E}-02 \\
2.47 \mathrm{E}-03 \\
5.08 \mathrm{E}-01 \\
9.24 \mathrm{E}-03 \\
3.06 \mathrm{E}-03 \\
1.53 \mathrm{E}-03 \\
5.01 \mathrm{E}-01 \\
1.06 \mathrm{E}-02 \\
5.98 \mathrm{E}-03 \\
3.04 \mathrm{E}-02 \\
1.37 \mathrm{E}-04 \\
8.65 \mathrm{E}-03 \\
5.23 \mathrm{E}-03 \\
8.09 \mathrm{E}-01 \\
5.90 \mathrm{E}-01 \\
1.09 \mathrm{E}-01 \\
2.93 \mathrm{E}-01 \\
4.98 \mathrm{E}-03 \\
2.77 \mathrm{E}-01 \\
2.80 \mathrm{E}-03 \\
1.04 \mathrm{E}-01 \\
2.70 \mathrm{E}-01 \\
1.40 \mathrm{E}-03 \\
2.68 \mathrm{E}-02 \\
4.19 \mathrm{E}-01 \\
3.35 \mathrm{E}-01 \\
2.09 \mathrm{E}-02 \\
1.66 \mathrm{E}-01 \\
2.31 \mathrm{E}-01 \\
6.85 \mathrm{E}-03 \\
1.36 \mathrm{E}-01 \\
3.99 \mathrm{E}-01 \\
5.05 \mathrm{E}-02 \\
1.38 \mathrm{E}-01 \\
1.25 \mathrm{E}-01 \\
1.14 \mathrm{E}-01 \\
3.59 \mathrm{E}-03 \\
9.96 \mathrm{E}-02 \\
6.16 \mathrm{E}-02 \\
1.31 \mathrm{E}-03 \\
4.77 \mathrm{E}-01\end{array}$ & $\begin{array}{c}\text { I129 } \\
2.39 \mathrm{E}-03 \\
1.10 \mathrm{E}-04 \\
2.27 \mathrm{E}-02 \\
4.15 \mathrm{E}-04 \\
1.37 \mathrm{E}-04 \\
6.98 \mathrm{E}-05 \\
2.22 \mathrm{E}-02 \\
4.74 \mathrm{E}-04 \\
2.66 \mathrm{E}-04 \\
1.36 \mathrm{E}-03 \\
6.25 \mathrm{E}-06 \\
3.88 \mathrm{E}-04 \\
2.24 \mathrm{E}-04 \\
3.44 \mathrm{E}-02 \\
2.52 \mathrm{E}-02 \\
4.64 \mathrm{E}-03 \\
1.30 \mathrm{E}-02 \\
2.22 \mathrm{E}-04 \\
1.24 \mathrm{E}-02 \\
1.21 \mathrm{E}-04 \\
4.46 \mathrm{E}-03 \\
1.15 \mathrm{E}-02 \\
5.93 \mathrm{E}-05 \\
1.15 \mathrm{E}-03 \\
1.87 \mathrm{E}-02 \\
1.49 \mathrm{E}-02 \\
9.29 \mathrm{E}-04 \\
7.09 \mathrm{E}-03 \\
9.81 \mathrm{E}-03 \\
2.92 \mathrm{E}-04 \\
6.05 \mathrm{E}-03 \\
1.78 \mathrm{E}-02 \\
2.25 \mathrm{E}-03 \\
6.16 \mathrm{E}-03 \\
5.55 \mathrm{E}-03 \\
5.05 \mathrm{E}-03 \\
1.60 \mathrm{E}-04 \\
4.23 \mathrm{E}-03 \\
2.62 \mathrm{E}-03 \\
5.59 \mathrm{E}-05 \\
2.03 \mathrm{E}-02\end{array}$ & $\begin{array}{l}\text { CS134 } \\
9.59 \mathrm{E}+01 \\
2.11 \mathrm{E}+00 \\
7.99 \mathrm{E}+02 \\
1.12 \mathrm{E}+01 \\
4.24 \mathrm{E}+00 \\
1.72 \mathrm{E}-01 \\
1.32 \mathrm{E}+03 \\
1.77 \mathrm{E}+01 \\
1.38 \mathrm{E}+01 \\
5.22 \mathrm{E}+01 \\
1.54 \mathrm{E}-02 \\
1.32 \mathrm{E}+01 \\
9.01 \mathrm{E}+00 \\
2.00 \mathrm{E}+03 \\
8.77 \mathrm{E}+02 \\
2.13 \mathrm{E}+02 \\
6.78 \mathrm{E}+02 \\
7.83 \mathrm{E}+00 \\
5.73 \mathrm{E}+02 \\
2.32 \mathrm{E}+00 \\
1.55 \mathrm{E}+02 \\
6.67 \mathrm{E}+02 \\
1.54 \mathrm{E}-01 \\
2.67 \mathrm{E}+01 \\
7.54 \mathrm{E}+02 \\
7.75 \mathrm{E}+02 \\
4.55 \mathrm{E}+01 \\
2.47 \mathrm{E}+02 \\
5.73 \mathrm{E}+02 \\
1.20 \mathrm{E}+01 \\
3.16 \mathrm{E}+02 \\
7.29 \mathrm{E}+02 \\
9.09 \mathrm{E}+01 \\
2.52 \mathrm{E}+02 \\
2.24 \mathrm{E}+02 \\
2.64 \mathrm{E}+02 \\
7.83 \mathrm{E}+00 \\
2.47 \mathrm{E}+02 \\
1.53 \mathrm{E}+02 \\
1.95 \mathrm{E}+00 \\
9.29 \mathrm{E}+02\end{array}$ & $\begin{array}{c}\text { cS137 } \\
7.52 \mathrm{E}+03 \\
3.24 \mathrm{E}+02 \\
7.18 \mathrm{E}+04 \\
1.33 \mathrm{E}+03 \\
4.41 \mathrm{E}+02 \\
2.33 \mathrm{E}+02 \\
7.10 \mathrm{E}+04 \\
1.54 \mathrm{E}+03 \\
8.43 \mathrm{E}+02 \\
4.36 \mathrm{E}+03 \\
2.08 \mathrm{E}+04 \\
1.26 \mathrm{E}+03 \\
7.06 \mathrm{E}+02 \\
1.06 \mathrm{E}+05 \\
7.74 \mathrm{E}+04 \\
1.45 \mathrm{E}+04 \\
4.13 \mathrm{E}+04 \\
7.04 \mathrm{E}+02 \\
3.99 \mathrm{E}+04 \\
3.91 \mathrm{E}+02 \\
1.37 \mathrm{E}+04 \\
3.54 \mathrm{E}+04 \\
1.45 \mathrm{E}+02 \\
3.71 \mathrm{E}+03 \\
5.95 \mathrm{E}+04 \\
4.72 \mathrm{E}+04 \\
3.00 \mathrm{E}+03 \\
2.18 \mathrm{E}+04 \\
3.03 \mathrm{E}+04 \\
8.94 \mathrm{E}+02 \\
1.92 \mathrm{E}+04 \\
5.63 \mathrm{E}+04 \\
7.17 \mathrm{E}+03 \\
1.95 \mathrm{E}+04 \\
1.77 \mathrm{E}+04 \\
1.60 \mathrm{E}+04 \\
5.16 \mathrm{E}+02 \\
1.31 \mathrm{E}+04 \\
8.08 \mathrm{E}+03 \\
1.72 \mathrm{E}+02 \\
6.27 \mathrm{E}+04\end{array}$ \\
\hline
\end{tabular}


HNF-SD-WN-TI-794, ReV. 0

ACTIVITY IN UNSEPARATED FUEL (Curies decayed to $1 / 1 / 94$ )

\begin{tabular}{|c|c|c|c|c|c|c|c|c|c|}
\hline 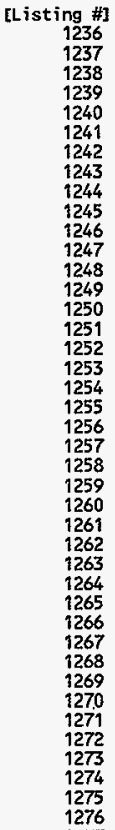 & $\begin{array}{l}B A 137 * \\
7.11 E+03 \\
3.06 E+02 \\
6.79 E+04 \\
1.26 E+03 \\
4.17 E+02 \\
2.20 E+02 \\
6.71 E+04 \\
1.46 E+03 \\
7.97 E+02 \\
4.13 E+03 \\
1.97 E+01 \\
1.19 E+03 \\
6.68 E+02 \\
1.00 E+05 \\
7.32 E+04 \\
1.37 E+04 \\
3.91 E+04 \\
6.66 E+02 \\
3.77 E+04 \\
3.70 E+02 \\
1.30 E+04 \\
3.35 E+04 \\
1.37 E+02 \\
3.51 E+03 \\
5.63 E+04 \\
4.47 E+04 \\
2.83 E+03 \\
2.06 E+04 \\
2.87 E+04 \\
8.46 E+02 \\
1.82 E+04 \\
5.33 E+04 \\
6.78 E+03 \\
1.85 E+04 \\
1.67 E+04 \\
1.52 E+04 \\
4.88 E+02 \\
1.24 E+04 \\
7.65 E+03 \\
1.63 E+02 \\
5.94 E+04\end{array}$ & $\begin{array}{c}\text { SM151 } \\
1.51 E+02 \\
6.05 E+00 \\
1.38 E+03 \\
2.68 E+01 \\
8.78 E+00 \\
5.32 E+00 \\
1.26 E+03 \\
3.04 E+01 \\
1.52 E+01 \\
8.42 E+01 \\
4.76 E-01 \\
2.49 E+01 \\
1.42 E+01 \\
1.95 E+03 \\
1.54 E+03 \\
2.84 E+02 \\
7.44 E+02 \\
1.35 E+01 \\
7.54 E+02 \\
8.63 E+00 \\
2.72 E+02 \\
6.51 E+02 \\
2.92 E+00 \\
8.06 E+01 \\
1.12 E+03 \\
8.50 E+02 \\
5.60 E+01 \\
4.32 E+02 \\
5.58 E+02 \\
1.72 E+01 \\
3.46 E+02 \\
1.05 E+03 \\
1.35 E+02 \\
3.64 E+02 \\
3.33 E+02 \\
2.88 E+02 \\
9.64 E+00 \\
2.40 E+02 \\
1.49 E+02 \\
3.41 E+00 \\
1.20 E+03\end{array}$ & $\begin{array}{c}\text { EU152 } \\
1.52 E-01 \\
9.69 \mathrm{E}-03 \\
1.64 \mathrm{E}+00 \\
2.01 \mathrm{E}-02 \\
7.29 \mathrm{E}-03 \\
0.00 \mathrm{E}+00 \\
2.33 \mathrm{E}+00 \\
2.59 \mathrm{E}-02 \\
2.62 \mathrm{E}-02 \\
9.10 \mathrm{E}-02 \\
0.00 \mathrm{E}+00 \\
2.09 \mathrm{E}-02 \\
1.42 \mathrm{E}-02 \\
3.69 \mathrm{E}+00 \\
1.87 \mathrm{E}+00 \\
3.59 \mathrm{E}-01 \\
1.29 \mathrm{E}+00 \\
1.61 \mathrm{E}-02 \\
9.33 \mathrm{E}-01 \\
1.43 \mathrm{E}-03 \\
3.31 \mathrm{E}-01 \\
1.23 \mathrm{E}+00 \\
4.77 \mathrm{E}-03 \\
2.46 \mathrm{E}-02 \\
1.47 \mathrm{E}+00 \\
1.47 \mathrm{E}+00 \\
7.50 \mathrm{E}-02 \\
5.27 \mathrm{E}-01 \\
1.05 \mathrm{E}+00 \\
2.57 \mathrm{E}-02 \\
5.98 \mathrm{E}-01 \\
1.48 \mathrm{E}+00 \\
1.77 \mathrm{E}-01 \\
5.13 \mathrm{E}-01 \\
4.35 \mathrm{E}-01 \\
4.99 \mathrm{E}-01 \\
1.29 \mathrm{E}-02 \\
4.54 \mathrm{E}-01 \\
2.81 \mathrm{E}-01 \\
4.15 \mathrm{E}-03 \\
1.82 \mathrm{E}+00\end{array}$ & $\begin{array}{l}E U 154 \\
2.76 E+01 \\
1.44 E+00 \\
2.79 E+02 \\
3.77 E+00 \\
1.35 E+00 \\
0.00 E+00 \\
3.83 E+02 \\
4.86 E+00 \\
4.31 E+00 \\
1.60 E+01 \\
0.00 E+00 \\
3.90 E+00 \\
2.60 E+00 \\
6.01 E+02 \\
3.17 E+02 \\
6.24 E+01 \\
2.11 E+02 \\
2.74 E+00 \\
1.63 E+02 \\
5.58 E-01 \\
5.62 E+01 \\
2.00 E+02 \\
5.65 E-01 \\
6.79 E+00 \\
2.48 E+02 \\
2.41 E+02 \\
1.29 E+01 \\
8.94 E+01 \\
1.72 E+02 \\
4.23 E+00 \\
9.82 E+01 \\
2.47 E+02 \\
2.99 E+01 \\
8.56 E+01 \\
7.36 E+01 \\
8.20 E+01 \\
2.22 E+00 \\
7.40 E+01 \\
4.58 E+01 \\
7.04 E-01 \\
3.02 E+02\end{array}$ & $\begin{array}{l}E U 155 \\
8.15 E+01 \\
2.00 E+00 \\
6.32 E+02 \\
1.23 E+01 \\
4.16 E+00 \\
2.54 E+00 \\
6.48 E+02 \\
1.56 E+01 \\
7.51 E+00 \\
4.11 E+01 \\
2.27 E-01 \\
1.23 E+01 \\
7.66 E+00 \\
1.06 E+03 \\
7.43 E+02 \\
1.53 E+02 \\
3.68 E+02 \\
6.19 E+00 \\
3.86 E+02 \\
4.64 E+00 \\
1.32 E+02 \\
3.52 E+02 \\
4.38 E-01 \\
4.35 E+01 \\
5.34 E+02 \\
4.21 E+02 \\
2.88 E+01 \\
2.09 E+02 \\
3.02 E+02 \\
8.51 E+00 \\
1.71 E+02 \\
4.94 E+02 \\
6.44 E+01 \\
1.71 E+02 \\
1.59 E+02 \\
1.43 E+02 \\
4.95 E+00 \\
1.30 E+02 \\
8.05 E+01 \\
1.65 E+00 \\
6.19 E+02\end{array}$ & $\begin{array}{c}\text { RA226 } \\
1.62 E-07 \\
9.77 \mathrm{E}-09 \\
1.48 \mathrm{E}-06 \\
3.48 \mathrm{E}-08 \\
1.03 \mathrm{E}-08 \\
2.72 \mathrm{E}-08 \\
9.29 \mathrm{E}-07 \\
3.06 \mathrm{E}-08 \\
1.24 \mathrm{E}-08 \\
8.42 \mathrm{E}-08 \\
2.43 \mathrm{E}-09 \\
2.70 \mathrm{E}-08 \\
1.52 \mathrm{E}-08 \\
1.63 \mathrm{E}-06 \\
1.87 \mathrm{E}-06 \\
2.77 \mathrm{E}-07 \\
6.08 \mathrm{E}-07 \\
1.45 \mathrm{E}-08 \\
6.52 \mathrm{E}-07 \\
1.60 \mathrm{E}-08 \\
3.31 \mathrm{E}-07 \\
5.43 \mathrm{E}-07 \\
1.29 \mathrm{E}-08 \\
1.30 \mathrm{E}-07 \\
1.10 \mathrm{E}-06 \\
6.94 \mathrm{E}-07 \\
4.68 \mathrm{E}-08 \\
5.26 \mathrm{E}-07 \\
4.65 \mathrm{E}-07 \\
1.86 \mathrm{E}-08 \\
2.82 \mathrm{E}-07 \\
1.02 \mathrm{E}-06 \\
1.32 \mathrm{E}-07 \\
3.53 \mathrm{E}-07 \\
3.25 \mathrm{E}-07 \\
2.35 \mathrm{E}-07 \\
8.05 \mathrm{E}-09 \\
2.00 \mathrm{E}-07 \\
1.24 \mathrm{E}-07 \\
4.15 \mathrm{E}-09 \\
1.20 \mathrm{E}-06\end{array}$ & $\begin{array}{l}\text { RA228 } \\
1.22 \mathrm{E}-11 \\
7.90 \mathrm{E}-13 \\
1.39 \mathrm{E}-10 \\
2.60 \mathrm{E}-12 \\
7.83 \mathrm{E}-13 \\
1.57 \mathrm{E}-12 \\
8.25 \mathrm{E}-11 \\
2.37 \mathrm{E}-12 \\
1.08 \mathrm{E}-12 \\
6.71 \mathrm{E}-12 \\
1.41 \mathrm{E}-13 \\
2.07 \mathrm{E}-12 \\
1.14 \mathrm{E}-12 \\
1.35 \mathrm{E}-10 \\
1.42 \mathrm{E}-10 \\
2.15 \mathrm{E}-11 \\
5.29 \mathrm{E}-11 \\
1.17 \mathrm{E}-12 \\
5.36 \mathrm{E}-11 \\
1.04 \mathrm{E}-12 \\
2.51 \mathrm{E}-11 \\
4.50 \mathrm{E}-11 \\
8.65 \mathrm{E}-13 \\
8.70 \mathrm{E}-12 \\
8.99 \mathrm{E}-11 \\
6.05 \mathrm{E}-11 \\
3.90 \mathrm{E}-12 \\
3.99 \mathrm{E}-11 \\
3.86 \mathrm{E}-11 \\
1.46 \mathrm{E}-12 \\
2.46 \mathrm{E}-11 \\
8.47 \mathrm{E}-11 \\
1.08 \mathrm{E}-11 \\
2.93 \mathrm{E}-11 \\
2.67 \mathrm{E}-11 \\
2.05 \mathrm{E}-11 \\
6.71 \mathrm{E}-13 \\
1.66 \mathrm{E}-11 \\
1.03 \mathrm{E}-11 \\
3.14 \mathrm{E}-13 \\
9.49 \mathrm{E}-11\end{array}$ & $\begin{array}{c}\text { AC227 } \\
1.01 E-06 \\
6.88 E-08 \\
1.10 E-05 \\
2.65 E-07 \\
7.82 E-08 \\
2.28 E-07 \\
6.83 E-06 \\
2.34 E-07 \\
9.10 E-08 \\
6.33 E-07 \\
2.04 E-08 \\
2.06 E-07 \\
9.44 E-08 \\
1.00 E-05 \\
1.14 E-05 \\
1.71 E-06 \\
4.46 E-06 \\
1.08 E-07 \\
4.90 E-06 \\
1.01 E-07 \\
2.02 E-06 \\
3.34 E-06 \\
6.95 E-08 \\
8.18 E-07 \\
8.14 E-06 \\
5.10 E-06 \\
3.50 E-07 \\
3.22 E-06 \\
2.86 E-06 \\
1.13 E-07 \\
2.07 E-06 \\
7.53 E-06 \\
9.80 E-07 \\
2.61 E-06 \\
2.42 E-06 \\
1.73 E-06 \\
6.02 E-08 \\
1.23 E-06 \\
7.63 E-07 \\
2.54 E-08 \\
7.34 E-06\end{array}$ & $\begin{array}{l}\text { TH229 } \\
1.29 \mathrm{E}-09 \\
8.15 \mathrm{E}-11 \\
1.22 \mathrm{E}-08 \\
2.20 \mathrm{E}-10 \\
7.04 \mathrm{E}-11 \\
3.46 \mathrm{E}-11 \\
1.09 \mathrm{E}-08 \\
2.24 \mathrm{E}-10 \\
1.35 \mathrm{E}-10 \\
6.82 \mathrm{E}-10 \\
3.10 \mathrm{E}-12 \\
1.91 \mathrm{E}-10 \\
1.21 \mathrm{E}-10 \\
1.92 \mathrm{E}-08 \\
1.55 \mathrm{E}-08 \\
2.54 \mathrm{E}-09 \\
6.63 \mathrm{E}-09 \\
1.20 \mathrm{E}-10 \\
5.93 \mathrm{E}-09 \\
6.32 \mathrm{E}-11 \\
2.74 \mathrm{E}-09 \\
6.39 \mathrm{E}-09 \\
8.77 \mathrm{E}-11 \\
6.06 \mathrm{E}-10 \\
9.77 \mathrm{E}-09 \\
7.57 \mathrm{E}-09 \\
4.47 \mathrm{E}-10 \\
4.36 \mathrm{E}-09 \\
5.47 \mathrm{E}-09 \\
1.77 \mathrm{E}-10 \\
3.08 \mathrm{E}-09 \\
9.45 \mathrm{E}-09 \\
1.18 \mathrm{E}-09 \\
3.27 \mathrm{E}-09 \\
2.90 \mathrm{E}-09 \\
2.57 \mathrm{E}-09 \\
7.69 \mathrm{E}-11 \\
2.36 \mathrm{E}-09 \\
1.46 \mathrm{E}-09 \\
3.44 \mathrm{E}-11 \\
1.18 \mathrm{E}-08\end{array}$ \\
\hline
\end{tabular}


HNF-SD-WM-TI-794, ReV. 0

ACIIVITY IN UNSEPARATED FUEL (Curies decayed to $1 / 1 / 94$ )

\begin{tabular}{|c|c|c|c|c|c|c|c|c|c|}
\hline $\begin{array}{c}\text { [Listing \#] } \\
1236 \\
1237 \\
1238 \\
1239 \\
1240 \\
1241 \\
1242 \\
1243 \\
1244 \\
1245 \\
1246 \\
1247 \\
1248 \\
1249 \\
1250 \\
1251 \\
1252 \\
1253 \\
1254 \\
1255 \\
1256 \\
1257 \\
1258 \\
1259 \\
1260 \\
1261 \\
1262 \\
1263 \\
1264 \\
1265 \\
1266 \\
1267 \\
1268 \\
1269 \\
1270 \\
1271 \\
1272 \\
1273 \\
1274 \\
1275 \\
1276\end{array}$ & $\begin{array}{l}\text { TH232 } \\
4.12 \mathrm{E}-11 \\
2.01 \mathrm{E}-12 \\
3.71 \mathrm{E}-10 \\
8.12 \mathrm{E}-12 \\
2.51 \mathrm{E}-12 \\
5.16 \mathrm{E}-12 \\
2.78 \mathrm{E}-10 \\
8.05 \mathrm{E}-12 \\
3.53 \mathrm{E}-12 \\
2.19 \mathrm{E}-11 \\
4.62 \mathrm{E}-13 \\
6.85 \mathrm{E}-12 \\
3.87 \mathrm{E}-12 \\
4.55 \mathrm{E}-10 \\
4.41 \mathrm{E}-10 \\
7.28 \mathrm{E}-11 \\
1.73 \mathrm{E}-10 \\
3.64 \mathrm{E}-12 \\
1.82 \mathrm{E}-10 \\
3.57 \mathrm{E}-12 \\
7.81 \mathrm{E}-11 \\
1.52 \mathrm{E}-10 \\
1.63 \mathrm{E}-12 \\
2.98 \mathrm{E}-11 \\
2.87 \mathrm{E}-10 \\
1.98 \mathrm{E}-10 \\
1.32 \mathrm{E}-11 \\
1.24 \mathrm{E}-10 \\
1.30 \mathrm{E}-10 \\
4.60 \mathrm{E}-12 \\
8.05 \mathrm{E}-11 \\
2.68 \mathrm{E}-10 \\
3.46 \mathrm{E}-11 \\
9.28 \mathrm{E}-11 \\
8.53 \mathrm{E}-11 \\
6.71 \mathrm{E}-11 \\
2.27 \mathrm{E}-12 \\
5.60 \mathrm{E}-11 \\
3.47 \mathrm{E}-11 \\
9.78 \mathrm{E}-13 \\
3.09 \mathrm{E}-10\end{array}$ & $\begin{array}{l}\text { PA231 } \\
9.34 \mathrm{E}-06 \\
4.48 \mathrm{E}-07 \\
9.26 \mathrm{E}-05 \\
2.25 \mathrm{E}-06 \\
6.83 \mathrm{E}-07 \\
2.07 \mathrm{E}-06 \\
6.25 \mathrm{E}-05 \\
2.19 \mathrm{E}-06 \\
8.05 \mathrm{E}-07 \\
5.64 \mathrm{E}-06 \\
1.85 \mathrm{E}-07 \\
1.86 \mathrm{E}-06 \\
8.77 \mathrm{E}-07 \\
9.11 \mathrm{E}-05 \\
9.57 \mathrm{E}-05 \\
1.58 \mathrm{E}-05 \\
3.95 \mathrm{E}-05 \\
9.08 \mathrm{E}-07 \\
4.54 \mathrm{E}-05 \\
9.59 \mathrm{E}-07 \\
1.69 \mathrm{E}-05 \\
3.04 \mathrm{E}-05 \\
3.01 \mathrm{E}-07 \\
7.75 \mathrm{E}-06 \\
7.03 \mathrm{E}-05 \\
4.51 \mathrm{E}-05 \\
3.24 \mathrm{E}-06 \\
2.69 \mathrm{E}-05 \\
2.60 \mathrm{E}-05 \\
9.61 \mathrm{E}-07 \\
1.84 \mathrm{E}-05 \\
6.42 \mathrm{E}-05 \\
8.47 \mathrm{E}-06 \\
2.22 \mathrm{E}-05 \\
2.09 \mathrm{E}-05 \\
1.53 \mathrm{E}-05 \\
5.58 \mathrm{E}-07 \\
1.12 \mathrm{E}-05 \\
6.94 \mathrm{E}-06 \\
2.12 \mathrm{E}-07 \\
6.44 \mathrm{E}-05\end{array}$ & $\begin{array}{l}\text { U232 } \\
1.54 E-04 \\
7.88 E-06 \\
1.34 E-03 \\
2.04 E-05 \\
6.94 E-06 \\
1.81 E-06 \\
1.54 E-03 \\
2.38 E-05 \\
1.80 E-05 \\
7.56 E-05 \\
1.62 E-07 \\
1.96 E-05 \\
1.45 E-05 \\
2.83 E-03 \\
1.79 E-03 \\
3.25 E-04 \\
8.84 E-04 \\
1.31 E-05 \\
7.19 E-04 \\
5.24 E-06 \\
3.17 E-04 \\
9.43 E-04 \\
5.63 E-06 \\
5.44 E-05 \\
1.13 E-03 \\
1.01 E-03 \\
5.59 E-05 \\
5.04 E-04 \\
8.08 E-04 \\
2.24 E-05 \\
4.11 E-04 \\
1.12 E-03 \\
1.37 E-04 \\
3.87 E-04 \\
3.37 E-04 \\
3.43 E-04 \\
9.61 E-06 \\
3.48 E-04 \\
2.16 E-04 \\
3.97 E-06 \\
1.55 E-03\end{array}$ & $\begin{array}{l}\text { U233 } \\
2.06 \mathrm{E}-06 \\
9.26 \mathrm{E}-08 \\
1.77 \mathrm{E}-05 \\
3.27 \mathrm{E}-07 \\
1.07 \mathrm{E}-07 \\
5.58 \mathrm{E}-08 \\
1.67 \mathrm{E}-05 \\
3.62 \mathrm{E}-07 \\
2.01 \mathrm{E}-07 \\
1.04 \mathrm{E}-06 \\
4.99 \mathrm{E}-09 \\
2.99 \mathrm{E}-07 \\
1.94 \mathrm{E}-07 \\
2.90 \mathrm{E}-05 \\
2.23 \mathrm{E}-05 \\
3.98 \mathrm{E}-06 \\
9.87 \mathrm{E}-06 \\
1.73 \mathrm{E}-07 \\
9.36 \mathrm{E}-06 \\
1.07 \mathrm{E}-07 \\
3.95 \mathrm{E}-06 \\
9.68 \mathrm{E}-06 \\
6.82 \mathrm{E}-08 \\
1.02 \mathrm{E}-06 \\
1.44 \mathrm{E}-05 \\
1.13 \mathrm{E}-05 \\
7.03 \mathrm{E}-07 \\
6.27 \mathrm{E}-06 \\
8.30 \mathrm{E}-06 \\
2.55 \mathrm{E}-07 \\
4.59 \mathrm{E}-06 \\
1.38 \mathrm{E}-05 \\
1.74 \mathrm{E}-06 \\
4.76 \mathrm{E}-06 \\
4.28 \mathrm{E}-06 \\
3.83 \mathrm{E}-06 \\
1.21 \mathrm{E}-07 \\
3.58 \mathrm{E}-06 \\
2.21 \mathrm{E}-06 \\
4.94 \mathrm{E}-08 \\
1.76 \mathrm{E}-05\end{array}$ & $\begin{array}{c}\text { U234 } \\
1.59 E+00 \\
4.41 E=02 \\
1.17 E+01 \\
2.78 E-01 \\
8.77 E-02 \\
2.48 E-01 \\
8.94 E+00 \\
3.03 E-01 \\
1.11 E-01 \\
7.52 E-01 \\
2.22 E-02 \\
2.48 E-01 \\
1.50 E-01 \\
1.56 E+01 \\
1.47 E+01 \\
2.71 E+00 \\
5.43 E+00 \\
1.15 E-01 \\
6.37 E+00 \\
1.62 E-01 \\
2.61 E+00 \\
5.22 E+00 \\
2.26 E-02 \\
1.31 E+00 \\
9.21 E+00 \\
6.20 E+00 \\
4.58 E-01 \\
4.15 E+00 \\
4.47 E+00 \\
1.51 E-01 \\
2.52 E+00 \\
8.34 E+00 \\
1.11 E+00 \\
2.89 E+00 \\
2.73 E+00 \\
2.10 E+00 \\
7.88 E-02 \\
1.93 E+00 \\
1.19 E+00 \\
3.27 E-02 \\
1.05 E+01\end{array}$ & $\begin{array}{l}\text { U235 } \\
6.05 \mathrm{E}-02 \\
1.96 \mathrm{E}-03 \\
5.38 \mathrm{E}-01 \\
1.32 \mathrm{E}-02 \\
4.14 \mathrm{E}-03 \\
1.30 \mathrm{E}-02 \\
3.98 \mathrm{E}-01 \\
1.43 \mathrm{E}-02 \\
4.95 \mathrm{E}-03 \\
3.49 \mathrm{E}-02 \\
1.17 \mathrm{E}-03 \\
1.17 \mathrm{E}-02 \\
5.68 \mathrm{E}-03 \\
5.77 \mathrm{E}-01 \\
5.54 \mathrm{E}-01 \\
1.02 \mathrm{E}-01 \\
2.43 \mathrm{E}-01 \\
5.28 \mathrm{E}-03 \\
2.93 \mathrm{E}-01 \\
6.36 \mathrm{E}-03 \\
9.81 \mathrm{E}-02 \\
1.92 \mathrm{E}-01 \\
8.20 \mathrm{E}-04 \\
5.13 \mathrm{E}-02 \\
4.21 \mathrm{E}-01 \\
2.77 \mathrm{E}-01 \\
2.09 \mathrm{E}-02 \\
1.56 \mathrm{E}-01 \\
1.65 \mathrm{E}-01 \\
5.63 \mathrm{E}-03 \\
1.13 \mathrm{E}-01 \\
3.79 \mathrm{E}-01 \\
5.07 \mathrm{E}-02 \\
1.31 \mathrm{E}-01 \\
1.25 \mathrm{E}-01 \\
9.40 \mathrm{E}-02 \\
3.60 \mathrm{E}-03 \\
7.10 \mathrm{E}-02 \\
4.39 \mathrm{E}-02 \\
1.23 \mathrm{E}-03 \\
3.93 \mathrm{E}-01\end{array}$ & $\begin{array}{c}\text { U236 } \\
1.16 \mathrm{E}-01 \\
3.84 \mathrm{E}-03 \\
9.37 \mathrm{E}-01 \\
2.06 \mathrm{E}-02 \\
6.59 \mathrm{E}-03 \\
1.39 \mathrm{E}-02 \\
7.78 \mathrm{E}-01 \\
2.28 \mathrm{E}-02 \\
9.51 \mathrm{E}-03 \\
5.89 \mathrm{E}-02 \\
1.25 \mathrm{E}-03 \\
1.86 \mathrm{E}-02 \\
1.09 \mathrm{E}-02 \\
1.27 \mathrm{E}+00 \\
1.11 \mathrm{E}+00 \\
2.05 \mathrm{E}-01 \\
4.66 \mathrm{E}-01 \\
9.18 \mathrm{E}-03 \\
5.10 \mathrm{E}-01 \\
1.02 \mathrm{E}-02 \\
1.97 \mathrm{E}-01 \\
4.23 \mathrm{E}-01 \\
1.94 \mathrm{E}-03 \\
8.50 \mathrm{E}-02 \\
7.49 \mathrm{E}-01 \\
5.33 \mathrm{E}-01 \\
3.72 \mathrm{E}-02 \\
3.13 \mathrm{E}-01 \\
3.63 \mathrm{E}-01 \\
1.18 \mathrm{E}-02 \\
2.17 \mathrm{E}-01 \\
6.89 \mathrm{E}-01 \\
9.02 \mathrm{E}-02 \\
2.39 \mathrm{E}-01 \\
2.22 \mathrm{E}-01 \\
1.81 \mathrm{E}-01 \\
6.40 \mathrm{E}-03 \\
1.56 \mathrm{E}-01 \\
9.68 \mathrm{E}-02 \\
2.47 \mathrm{E}-03 \\
8.23 \mathrm{E}-01\end{array}$ & $\begin{array}{c}\text { U238 } \\
1.08 \mathrm{E}+00 \\
3.02 \mathrm{E}-02 \\
7.95 \mathrm{E}+00 \\
1.88 \mathrm{E}-01 \\
5.94 \mathrm{E}-02 \\
1.65 \mathrm{E}-01 \\
6.12 \mathrm{E}+00 \\
2.05 \mathrm{E}-01 \\
7.56 \mathrm{E}-02 \\
5.11 \mathrm{E}-01 \\
1.48 \mathrm{E}-02 \\
1.68 \mathrm{E}-01 \\
1.02 \mathrm{E}-01 \\
1.07 \mathrm{E}+01 \\
1.00 \mathrm{E}+01 \\
1.85 \mathrm{E}+00 \\
3.71 \mathrm{E}+00 \\
7.80 \mathrm{E}-02 \\
4.33 \mathrm{E}+00 \\
1.09 \mathrm{E}-01 \\
1.78 \mathrm{E}+00 \\
3.58 \mathrm{E}+00 \\
1.56 \mathrm{E}-02 \\
8.85 \mathrm{E}-01 \\
6.27 \mathrm{E}+00 \\
4.24 \mathrm{E}+00 \\
3.12 \mathrm{E}-01 \\
2.83 \mathrm{E}+00 \\
3.07 \mathrm{E}+00 \\
1.03 \mathrm{E}-01 \\
1.73 \mathrm{E}+00 \\
5.68 \mathrm{E}+00 \\
7.55 \mathrm{E}-01 \\
1.97 \mathrm{E}+00 \\
1.86 \mathrm{E}+00 \\
1.44 \mathrm{E}+00 \\
5.36 \mathrm{E}-02 \\
1.32 \mathrm{E}+00 \\
8.18 \mathrm{E}-01 \\
2.23 \mathrm{E}-02 \\
7.21 \mathrm{E}+00\end{array}$ & $\begin{array}{c}\text { MP237 } \\
1.72 E-02 \\
7.07 \mathrm{E}-04 \\
1.43 \mathrm{E}-01 \\
2.57 \mathrm{E}-03 \\
8.53 \mathrm{E}-04 \\
4.11 \mathrm{E}-04 \\
1.43 \mathrm{E}-01 \\
2.95 \mathrm{E}-03 \\
1.70 \mathrm{E}-03 \\
8.52 \mathrm{E}-03 \\
3.67 \mathrm{E}-05 \\
2.41 \mathrm{E}-03 \\
1.61 \mathrm{E}-03 \\
2.52 \mathrm{E}-01 \\
1.82 \mathrm{E}-01 \\
3.35 \mathrm{E}-02 \\
8.35 \mathrm{E}-02 \\
1.40 \mathrm{E}-03 \\
7.79 \mathrm{E}-02 \\
8.53 \mathrm{E}-04 \\
3.23 \mathrm{E}-02 \\
8.38 \mathrm{E}-02 \\
4.42 \mathrm{E}-04 \\
8.17 \mathrm{E}-03 \\
1.18 \mathrm{E}-01 \\
9.55 \mathrm{E}-02 \\
5.88 \mathrm{E}-03 \\
5.14 \mathrm{E}-02 \\
7.18 \mathrm{E}-02 \\
2.12 \mathrm{E}-03 \\
3.89 \mathrm{E}-02 \\
1.13 \mathrm{E}-01 \\
1.43 \mathrm{E}-02 \\
3.92 \mathrm{E}-02 \\
3.52 \mathrm{E}-02 \\
3.24 \mathrm{E}-02 \\
1.01 \mathrm{E}-03 \\
3.10 \mathrm{E}-02 \\
1.92 \mathrm{E}-02 \\
4.05 \mathrm{E}-04 \\
1.48 \mathrm{E}-01\end{array}$ \\
\hline
\end{tabular}


HNF-SD-WM-TI-794, ReV. 0

ACIIVITY IN UNSEPARATED FUEL (Curies decayed to 1/1/94)

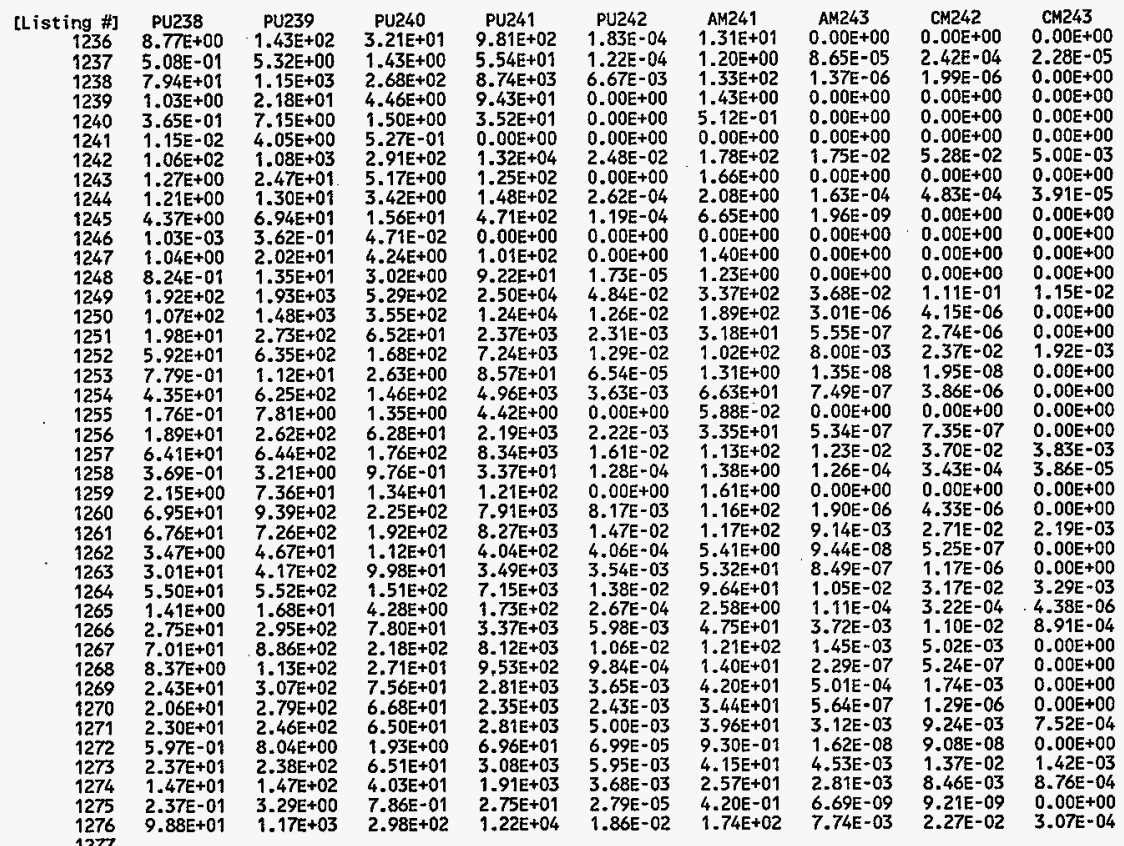


HNF-SD-WM-TI-794, ReV. 0

ACTIVITY IN UNSEPARATED FUEL (Curies decayed to $1 / 1 / 94$ )

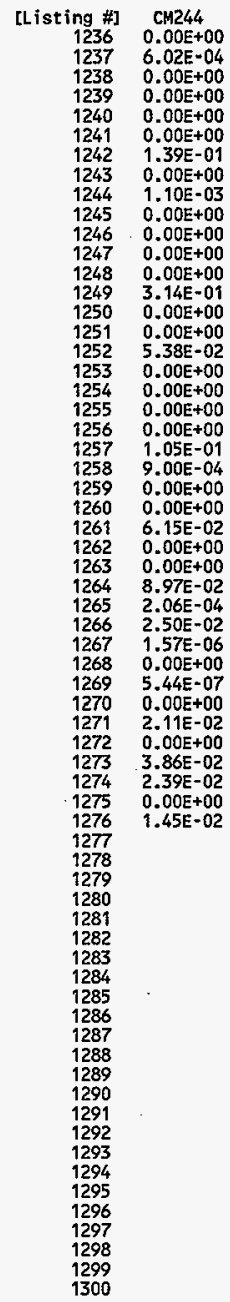

13.3367224

14.3859901

0.30491129

14.3274186

8.88500366

0.1770639

66.276112 
HNF-SD-WM-TI-794

Revision 0

APPENDIX B

DKPRO/ORIGEN2 ASSUMPTIONS AND FILES 
HNF-SD-WM-TI-794

Revision 0

This page intentionally left blank. 
HNF-SD-WM-TI-794

Revision 0

\section{APPENDIX B}

\section{DKPRO/ORIGEN2 ASSUMPTIONS AND FUES}

\section{B1.0 URANIUM FUEL IMPURITIES}

Table B-1 summarizes the isotopic composition of the fuel types used in the ORIGEN2 calculations of fuel inventories.

Table B-1. Uranium Fuel Isotopic Composition Used in ORIGEN2.

\begin{tabular}{|c|c|c|c|c|c|}
\hline Nuclide $(\mathrm{g})$ & $\begin{array}{c}\text { Single-pass } \\
\text { natural }\end{array}$ & $\begin{array}{c}\text { Single-pass } \\
\text { enriched }\end{array}$ & $\begin{array}{c}\text { N Reactor } \\
\text { MK-IV }\end{array}$ & \multicolumn{2}{|c|}{$\begin{array}{c}\text { N Reactor } \\
\text { MK-1A }\end{array}$} \\
\hline & AL-0.71U & AL-0.94U & ZR-0.94U & \multicolumn{2}{|c|}{ ZR-1.1U } \\
\hline${ }^{234} \mathrm{U}$ & $5.3100 \mathrm{E} 1$ & $8.0000 \mathrm{E} 1$ & $8.0000 \mathrm{E} 1$ & $8.0000 \mathrm{E} 1$ & $8.0000 \mathrm{E} 1$ \\
\hline${ }^{235} \mathrm{U}$ & $0.7110 \mathrm{E} 4$ & $0.9470 \mathrm{E} 4$ & $0.9470 \mathrm{E} 4$ & $0.9470 \mathrm{E} 4$ & $1.2500 \mathrm{E} 4$ \\
\hline${ }^{236} \mathrm{U}$ & $0.0000 \mathrm{E} 0$ & $4.0000 \mathrm{E} 2$ & $4.0000 \mathrm{E} 2$ & $4.0000 \mathrm{E} 2$ & $4.0000 \mathrm{E} 2$ \\
\hline${ }^{238} \mathrm{U}$ & $9.9284 \mathrm{E} 5$ & $9.9005 \mathrm{E} 5$ & $9.9005 \mathrm{E} 5$ & $9.9005 \mathrm{E} 5$ & $9.8692 \mathrm{E} 5$ \\
\hline Total U & $1.0000 \mathrm{E} 6$ & $1.0000 \mathrm{E} 6$ & $1.0000 \mathrm{E} 6$ & $1.0000 \mathrm{E} 6$ & $1.0000 \mathrm{E} 6$ \\
\hline
\end{tabular}

${ }^{*} \mathrm{ZR}-1.1 \mathrm{U}$ is a mixture of 0.94 percent ${ }^{235} \mathrm{U}$ inner fuel tube and 1.25 percent ${ }^{235} \mathrm{U}$ outer fuel tube, together averaging 1.1 percent ${ }^{235} \mathrm{U}$.

The MK-IV fuel contains two fuel elements, an inner tube and an outer tube. These elements start with the same 0.94 percent ${ }^{235} \mathrm{U}$ enrichment, but burn at different rates due to different neutron flux levels. The ORIGEN2 model created separate inventories for the inner tubes and outer tubes as a function of burnup, and then averaged the values together to create a composite inventory.

The MK-1A fuel actually contains two enrichments, 0.94 percent ${ }^{235} \mathrm{U}$ inner fuel tube and 1.25 percent ${ }^{235} \mathrm{U}$ outer fuel tube. The ORIGEN2 model created separate inventories for the inner tubes and outer tubes as a function of burnup, and then averaged the values together to create a composite that was labeled as 1.1 percent ${ }^{235} \mathrm{U}$.

Separate ORIGEN2 calculations were not made for the 2.1 percent ${ }^{235} \mathrm{U}$ "Co-Product" N Reactor fuel. For the calculations in this study, this fuel was treated like the 1.1 percent composite ${ }^{235} \mathrm{U} N$ Reactor fuel.

As mentioned in the assumptions (Section 4.0), a constant level of $400 \mathrm{p} / \mathrm{M}{ }^{236} \mathrm{U}$ was used for all of the enriched fuel. 


\section{HNF-SD-WM-TI-794 \\ Revision 0}

Table B-2 lists the impurities included in the single-pass reactor and N Reactor fuel compositions for the ORIGEN2 calculations of nuclide inventories.

Table B-2. Uranium Fuel Impurities.

\begin{tabular}{|c|c|c|}
\hline Nuclide & \multicolumn{2}{|c|}{ Fuel Impurities (g/MTU) } \\
\hline $\mathrm{H}$ & Single-Pass & N Reactor \\
\hline $\mathrm{Be}$ & - & 2. \\
\hline $\mathrm{B}$ & - & 10. \\
\hline $\mathrm{C}$ & - & 0.25 \\
\hline $\mathrm{N}$ & 50. & 550. \\
\hline $\mathrm{Mg}$ & 25. & 75. \\
\hline $\mathrm{Al}$ & - & 25. \\
\hline $\mathrm{Si}$ & 75. & 800 \\
\hline $\mathrm{Cr}$ & 65. & 124. \\
\hline $\mathrm{Mn}$ & 25. & 65. \\
\hline $\mathrm{Fe}$ & 150. & 25. \\
\hline $\mathrm{Ni}$ & 100. & 350. \\
\hline $\mathrm{Cu}$ & - & 100. \\
\hline $\mathrm{Zr}$ & - & 75. \\
\hline $\mathrm{Cd}$ & - & 65. \\
\hline
\end{tabular}


HNF-SD-WM-TI-794

Revision 0

\section{B2.0 CLADDING IMPURITIES}

Table B-3 lists the ratio of cladding to fuel for the different fuel types. Table B-4 lists the aluminum and zirconium cladding compositions used in the ORIGEN2 inventory calculations.

As described in Section 4.0 of the main report, several simplifying assumptions regarding the time variation in cladding impurities were made, such as the nitrogen level in both fuel and cladding leading to ${ }^{14} \mathrm{C}$, the nickel level in aluminum cladding leading to ${ }^{59} \mathrm{Ni}$ and ${ }^{63} \mathrm{Ni}$, and the cobalt level in both fuel and cladding leading to ${ }^{60} \mathrm{Co}$.

\section{B2.1 ALUMINUM CLADDING}

The amount of aluminum cladding is assumed to be $37.62 \mathrm{~kg} / \mathrm{MTU}$. The total aluminum cladding includes significant aluminum and minor elements in the aluminumsilicon layer. There is a big variation (factor of 100 difference) in the nickel content of two aluminum alloys used for cladding. A weighted average nickel concentration was used. The nickel impurity is a major source for activation products ${ }^{59} \mathrm{Ni}$ and ${ }^{63} \mathrm{Ni}$. Using the average nickel content overestimates the ${ }^{59} \mathrm{Ni}$ and ${ }^{63} \mathrm{Ni}$ production in the early years and underestimates their production after the nickel content was increased in the late 1950's.

The sum of the cladding related constituents is greater than one to account for the intermetallic layer and silicon from the aluminum-silicon layer. The intermetallic layer was accounted for by increasing the cladding values for copper by $1,900 \mathrm{~g} / \mathrm{MT}$ of cladding, tin by $4,000 \mathrm{~g} / \mathrm{MT}$, and lead by $60,000 \mathrm{~g} / \mathrm{MT}$. Silicon in the aluminum-silicon layer was accounted for by adding $35,300 \mathrm{~g} / \mathrm{MT}$ of cladding.

\section{B2.2 ZIRCONIUM CLADDING}

Zircalloy-2 alloy was used as cladding material for $\mathrm{N}$ Reactor fuel. Impurity estimates were based on the midrange or maximum impurity specification as available. The end caps of the N Reactor fuel are brazed to the rest of the fuel element with a 95 percent zirconium, 5 percent beryllium braze. The beryllium in the braze filler was not included in the impurities.

Table B-3. Uranium Fuel Cladding Fraction.

\begin{tabular}{|c|c|}
\hline Fuel Type & Cladđing Fraction $(\mathrm{kg} / \mathrm{MTU})$ \\
\hline Single-Pass & 37.62 \\
\hline MK-IV & 70.35 \\
\hline MK-1A & 85.65 \\
\hline
\end{tabular}


HNF-SD-WM-TI-794

Revision 0

Table B-4. Fuel Cladding Composition.

\begin{tabular}{|c|c|c|}
\hline \multirow[t]{2}{*}{ Element } & \multicolumn{2}{|c|}{ Cladding Composition (g/MT cladding) } \\
\hline & Aluminum & Zircaloy \\
\hline $\mathrm{H}$ & - & 25. \\
\hline $\mathrm{B}$ & 10. & 0.5 \\
\hline $\mathrm{C}$ & - & 275. \\
\hline $\mathrm{N}$ & 50. & 80. \\
\hline $\mathrm{Na}$ & - & 20. \\
\hline $\mathrm{Mg}$ & 100. & 20. \\
\hline $\mathrm{Al}$ & 982,600 . & 75. \\
\hline $\mathrm{Si}$ & 35,300 . & 100. \\
\hline $\mathrm{Ti}$ & 500. & 50. \\
\hline $\mathrm{V}$ & - & 50. \\
\hline $\mathrm{Cr}$ & 300. & 1000. \\
\hline $\mathrm{Mn}$ & 300. & 50. \\
\hline $\mathrm{Fe}$ & 5,000 & 1350. \\
\hline Co & 10. & 10. \\
\hline $\mathrm{Ni}$ & 7,800 & 550. \\
\hline $\mathrm{Cu}$ & 2,900 . & 50. \\
\hline $\mathrm{Zn}$ & 300. & - \\
\hline $\mathrm{Zr}$ & - & $981,391$. \\
\hline Mo & - & 50. \\
\hline $\mathrm{Cd}$ & 30. & 0.5 \\
\hline Sn & 4,100 & 14,500 . \\
\hline $\mathrm{Hf}$ & - & 200. \\
\hline W & - & 50. \\
\hline $\mathrm{Pb}$ & $60,100$. & 100. \\
\hline $\mathrm{Bi}$ & 100. & - \\
\hline U & - & 2.5 \\
\hline
\end{tabular}


HNF-SD-WM-TI-794

Revision 0

\section{B3.0 ORIGEN2 CROSS SECTIONS AND FILES}

ORIGEN2 cross section libraries include cross section sets generated specifically for $\mathrm{N}$ Reactor fuel. Four cross section sets are available in the ORIGEN2 library for MK-IV inner and outer fuel elements and MK-1A inner and outer fuel elements. These cross sections sets were used for the ORIGEN2 calculations for $\mathrm{N}$ Reactor fuel, with corrections for known problems. Burnup dependent cross sections for $\mathrm{N}$ Reactor fuel actinides are available as part of the ORIGEN2 cross section library, but these were not used. Table B-5 lists the cross section identifiers for the base set of fuel cross sections that were used.

Table B-5. ORIGEN2 N Reactor Cross Section Identifiers.

\begin{tabular}{|c|c|c|c|}
\hline Fuel Type & Activation & Fission Product & Actinide \\
\hline MK-IV inner & 411 & 413 & 412 \\
\hline MK-IV outer & 421 & 423 & 422 \\
\hline MK-1A inner & 431 & 433 & 432 \\
\hline MK-1A outer & 441 & 443 & 442 \\
\hline
\end{tabular}

These base sets of cross sections were used for both $\mathrm{N}$ Reactor and single-pass reactor calculations with various adjustments to the cross sections included as part of the ORIGEN2 input for each case. The ORIGEN2 cross section adjustments are summarized in Table B-6.

The ${ }^{243} \mathrm{Am}$ capture cross section was initially incorrectly set in the ORIGEN2 $\mathrm{N}$ Reactor cross sections. For capture cross sections, the ORIGEN2 code contains two capture cross sections, one for a capture that leads to the ground state of the daughter nuclide and one for a capture event that leads to the excited state of the daughter nuclide. When the ${ }^{243} \mathrm{Am}$ cross section was created the total cross section was used for the capture to the ground state of the daughter nuclide, and the capture to the excited state was set as the ratio of these two cross sections. This caused the ORIGEN2 capture cross section for ${ }^{243} \mathrm{Am}$ leading to an excited state of ${ }^{244} \mathrm{Am}$ to be a factor of about 18 times too high. The effect of this error was that ORIGEN2 overpredicted the ${ }^{244} \mathrm{Cm}$ (and nuclides beyond ${ }^{244} \mathrm{Cm}$ ) by about a factor of ten. An adjustment of the ${ }^{243} \mathrm{Am}$ capture cross section was made for all of the ORIGEN2 calculations used by DKPRO to correct this problem.

The N Reactor cross sections for ORIGEN2 were initially created by modifying selected actinide capture and fission cross sections in an existing base set of cross sections. The $(\mathrm{n}, 2 \mathrm{n})$ cross sections were not changed from the original set. For all of the ORIGEN2 calculations in this study, the ${ }^{238} \mathrm{U}$ cross section was adjusted to correct the $(n, 2 n)$ reaction that leads to ${ }^{237} \mathrm{~Np}$. 
Separate ORIGEN2 cross section sets do not exist for single-pass reactor fuel. For all of the single-pass reactor calculations, the $\mathrm{N}$ Reactor MK-IV inner element cross section set was used, with the ${ }^{235} U$ fission cross section adjusted to match the known plutonium conversion rates for the single-pass reactor fuel.

For the thorium calculations, the specific power level or burnup could not be treated the same as the uranium fuel. Therefore, the ${ }^{232} \mathrm{Th}$ capture cross section was adjusted to match the known ${ }^{233} \mathrm{U}$ production based on an average flux level from the driver fuel.

Table B-7 summarizes the ORIGEN2 file names associated with each fuel type. The inventory files were used as input to the DKPRO code, which interpolates between the values at the various burnup levels.

Table B-6. ORIGEN2 Cross Section Adjustment Input.

\begin{tabular}{|c|c|c|c|c|c|c|c|c|}
\hline & & $\begin{array}{l}\text { capture to } \\
\text { ground state }\end{array}$ & $\begin{array}{l}\mathrm{n}, 2 \mathrm{n} \text { to } \\
\text { ground state }\end{array}$ & $\begin{array}{l}n, 3 n \text { to } \\
\text { ground state }\end{array}$ & fission & $\begin{array}{l}\text { capture to } \\
\text { excited state }\end{array}$ & $\begin{array}{l}n, 2 n \text { to } \\
\text { excited state }\end{array}$ & \\
\hline \multicolumn{9}{|c|}{ N Reactor MKIV } \\
\hline 412 & 922380 & $6.755 \mathrm{E}-01$ & $2.500 \mathrm{E}-03$ & $4.597 \mathrm{E}-05$ & $6: 858 \mathrm{E}-02$ & .000 & .000 & -1.0 \\
\hline 412 & 952430 & $3.015 \mathrm{E}+00$ & $2.074 \mathrm{E}-04$ & $0.000 \mathrm{E}+00$ & $3.457 \mathrm{E}-01$ & $5.730 \mathrm{E}+01$ & $0.000 \mathrm{E}+00$ & -1.00 \\
\hline 422 & 922380 & $7.464 \mathrm{E}-01$ & $2.500 \mathrm{E}-03$ & 4.597E-05 & $5.978 \mathrm{E}-02$ & .000 & .000 & -1.0 \\
\hline 422 & 952430 & $3.144 \mathrm{E}+00$ & $2.074 \mathrm{E}-04$ & $0.000 \mathrm{E}+00$ & 3.193E-01 & $5.976 \mathrm{E}+01$ & $0.000 \mathrm{E}+00$ & -1.00 \\
\hline \multicolumn{9}{|c|}{ N Reactor MK1A } \\
\hline 432 & 922380 & 6.977E-01 & $2.500 \mathrm{E}-03$ & 4.597E-05 & $6.898 \mathrm{E}-02$ & .000 & .000 & -1.0 \\
\hline 432 & 952430 & $3.068 \mathrm{E}+00$ & $2.074 \mathrm{E}-04$ & $0.000 \mathrm{~B}+00$ & $3.448 \mathrm{E}-01$ & $5.830 \mathrm{E}+01$ & $0.000 \mathrm{E}+00$ & -1.00 \\
\hline 442 & 922380 & $7.601 \mathrm{E}-01$ & $2.500 \mathrm{E}-03$ & 4.597E-05 & $6.273 \mathrm{E}-02$ & .000 & .000 & -1.0 \\
\hline 442 & 952430 & $3.140 \mathrm{E}+00$ & $2.074 \mathrm{E}-04$ & $0.000 \mathrm{E}+00$ & $3.286 \mathrm{E}-01$ & $5.968 \mathrm{E}+01$ & $0.000 \mathrm{E}+00$ & -1.00 \\
\hline \multicolumn{9}{|c|}{ Single-Pass Reactors } \\
\hline 412 & 922350 & $1.317 \mathrm{E}+01$ & $2.696 \mathrm{E}-03$ & $1.160 \mathrm{E}-06$ & $0.900 \mathrm{E}+02$ & .000 & .000 & -1.0 \\
\hline 412 & 922380 & $6.755 \mathrm{E}-01$ & $2.500 \mathrm{E}-03$ & 4.597E-05 & $6.858 \mathrm{~B}-02$ & .000 & .000 & -1.0 \\
\hline 412 & 952430 & $3.015 \mathrm{E}+00$ & $2.074 \mathrm{E}-04$ & $0.000 \mathrm{E}+00$ & $3.457 \mathrm{E}-01$ & $5.730 \mathrm{E}+01$ & $0.000 \mathrm{~B}+00$ & -1.00 \\
\hline \multicolumn{9}{|c|}{ Thorium } \\
\hline 412 & 902320 & $1.500 \mathrm{E}+00$ & $5.089 \mathrm{E}-03$ & $2.660 \mathrm{~B}-05$ & $2.217 \mathrm{E}-02$ & .000 & .000 & -1.0 \\
\hline 412 & 922350 & $1.317 \mathrm{E}+01$ & $2.696 \mathrm{E}-03$ & $1.160 \mathrm{~B}-06$ & $1.000 \mathrm{E}+02$ & .000 & .000 & -1.0 \\
\hline 412 & 922380 & $6.755 \mathrm{E}-01$ & $2.500 \mathrm{~B}-03$ & $4.597 \mathrm{E}-05$ & $6.858 \mathrm{E}-02$ & .000 & .000 & -1.0 \\
\hline 412 & 952430 & $3.015 \mathrm{E}+00$ & $2.074 \mathrm{E}-04$ & $0.000 \mathrm{E}+00$ & $3.457 \mathrm{E}-01$ & $5.730 \mathrm{E}+01$ & $0.000 \mathrm{E}+00$ & -1.00 \\
\hline
\end{tabular}


HNF-SD-WM-TI-794

Revision 0

Table B-7. ORIGEN2 Input and Output Files.

\begin{tabular}{|c|c|c|c|c|}
\hline Fuel type & Input file & $\begin{array}{l}\text { Cross section } \\
\text { files }\end{array}$ & Output file & $\begin{array}{l}\text { Inventory files } \\
\text { (MWd/MTU) }\end{array}$ \\
\hline AL-0.71U & orig96sp.inp & $\begin{array}{l}\text { fort.9.mkiv } \\
\text { fort.3.sp }\end{array}$ & orig96sp.out & $\begin{array}{l}\text { SPNAT0.ORG (0) } \\
\text { SPNAT4.ORG }(400) \\
\text { SPNAT8.ORG }(800)\end{array}$ \\
\hline AL-0.94U & orig96sp.inp & $\begin{array}{l}\text { fort.9.mkiv } \\
\text { fort.3.sp }\end{array}$ & orig96sp.out & $\begin{array}{l}\text { SPENR0.ORG }(0) \\
\text { SPENR4.ORG }(400) \\
\text { SPENR8.ORG }(800)\end{array}$ \\
\hline ZR-0.94U & orig96mkiv.inp & $\begin{array}{l}\text { fort.9.mkiv } \\
\text { fort.3.mkiv }\end{array}$ & orig96mkiv.out & $\begin{array}{l}\text { MKIV00.ORG (0) } \\
\text { MKIV11.ORG }(1000) \\
\text { MKIV22.ORG }(2000) \\
\text { MKIV33.ORG }(3000)\end{array}$ \\
\hline ZR-1.1U & orig96mkla.inp & $\begin{array}{l}\text { fort.9.mkla } \\
\text { fort.3.mkla }\end{array}$ & orig96mk1a.out & $\begin{array}{l}\text { MK1A00.ORG }(0) \\
\text { MK1A11.ORG }(1000) \\
\text { MK1A22.ORG }(2000) \\
\text { MK1A33.ORG }(3000)\end{array}$ \\
\hline AL-OTH & orig96th.inp & $\begin{array}{l}\text { fort.9.mkiv } \\
\text { fort.3.th }\end{array}$ & orig96th.out & THORIUM.ORG \\
\hline
\end{tabular}


HNF-SD-WM-TI-794

Revision 0

This page intentionally left blank.

B-10 
HNF-SD-WM-TI-794

Revision 0

\section{B4.0 DKPRO FLLS}

The following are the DKPRO files used to generate the input to the HDW model. Listings of some of the files are provided.

Input Files:

UNIFIED6.PRN "Unified Separations Production File". (Data included in DKPRO output file - see Appendix A)

ORIGEN2.DK ORIGEN2/DKPRO decay library file

u6lanl.inp DKPRO input file

FILE UNIFIED6.PRN

FORMAT LANL

SPLIT

UNITS CURIES

OUTPUT

H3 $\quad$ C14 $\quad$ NI59 $\quad$ CO60 NI63

$\begin{array}{lllllll}\text { SE79 } & \text { SR90 } & \text { Y90 } & \text { ZR93 } & \text { NB93* } & \text { TC99 } & \text { RU106 }\end{array}$

CD113* SB125 SN126 $1129 \quad$ CS134

CS137 BA137* SM151 EU152 EU154 EU155

RA226 $\quad$ AC227 $\quad$ RA228 $\quad$ TH229 PA231

$\begin{array}{lllll}\text { TH232 } & \text { U232 } & \text { U233 } & \text { U234 } & \text { U235 }\end{array}$

$\begin{array}{llllll}\text { U236 } & \text { NP237 } & \text { PU238 } & \text { U238 } & \text { PU239 } & \text { PU240 }\end{array}$

AM241 PU241 PU242 CM242 AM243

CM243 CM244

ORIGDICT (DKPRO inventory files generated by ORIGEN2)

$\begin{array}{lll}\text { ZR-0.94U } & 0 & \text { MKIV00.ORG } \\ \text { ZR-0.94U } & 1,100 & \text { MKIV11.ORG } \\ \text { ZR-0.94U } & 2,200 & \text { MKIV22.ORG } \\ \text { ZR-0.94U } & 3,300 & \text { MKIV33.ORG } \\ \text { ZR-1.1U } & 0 & \text { MK1A00.ORG } \\ \text { ZR-1.1U } & 1,320 & \text { MK1A11.ORG } \\ \text { ZR-1.1U } & 2,640 & \text { MK1A22.ORG } \\ \text { ZR-1.1U } & 3,960 & \text { MK1A33.ORG } \\ \text { AL-0.71U } & 0 & \text { SPNAT0.ORG } \\ \text { AL-0.71U } & 400 & \text { SPNAT4.ORG } \\ \text { AL-0.71U } & 800 & \text { SPNAT8.ORG }\end{array}$




$\begin{array}{lll}\text { AL-0.94U } & 0 & \text { SPENR0.ORG } \\ \text { AL-0.94U } & 400 & \text { SPENR4.ORG } \\ \text { AL-0.94U } & 800 & \text { SPENR8.ORG } \\ \text { AL-0.0TH } & 1 & \text { THORIUM.ORG } \\ \text { ZR-2.1U } & 1,320 & \text { MK1A11.ORG } \\ \text { ZR-2.1U } & 2,640 & \text { MK1A22.ORG } \\ \text { ZR-2.1U } & 3,960 & \text { MK1A33.ORG }\end{array}$

u6prono.dir (DKPRO processing directives file)

FINAL 1/94 \# Decay to start of 1994 after other steps completed

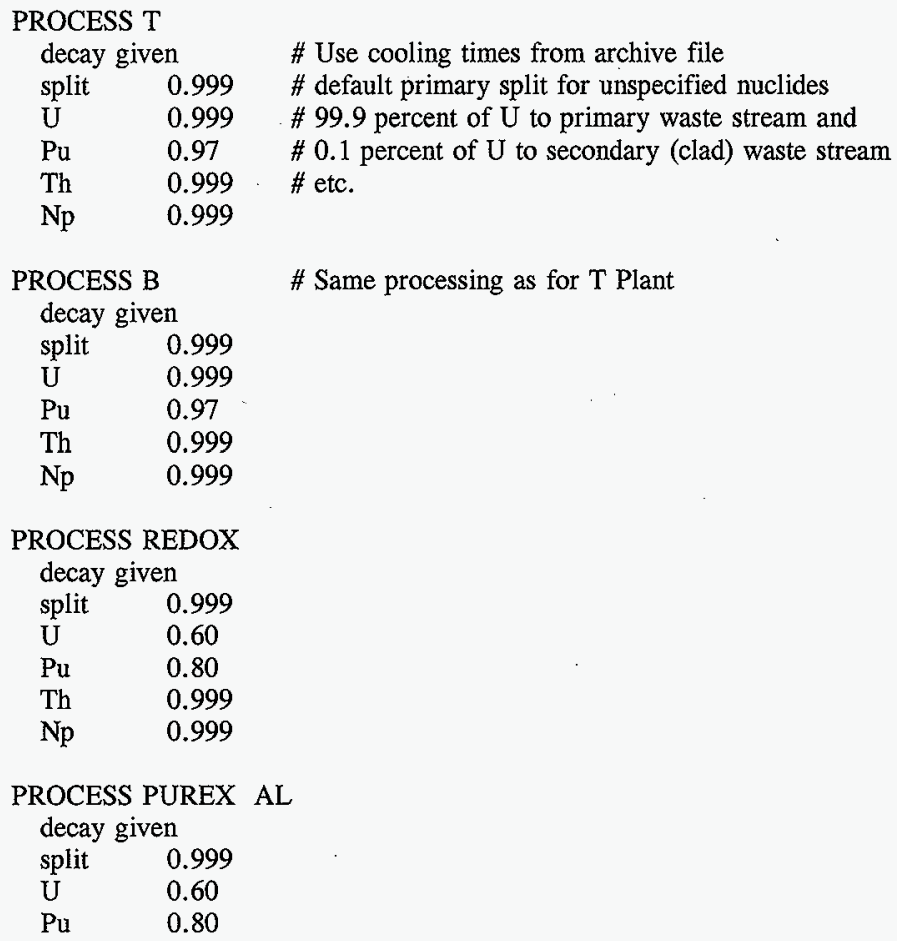


HNF-SD-WM-TI-794

Revision 0

SUMMARY.DIR (DKPRO summary output directives file)

SUMMARY T-plant

PLANT T

SUMMARY B-plant

PLANT B

SUMMARY Redox

PLANT REDOX

SUMMARY PureX

PLANT PUREX

Output Files:

u6summo.cur (DKPRO output summary file)

u6outno.cur (DKPRO output fuel activity record file) 
HNF-SD-WM-TI-794

Revision 0

This page intentionally left blank.

B-14 


\section{DISTRIBUTION SHEET}

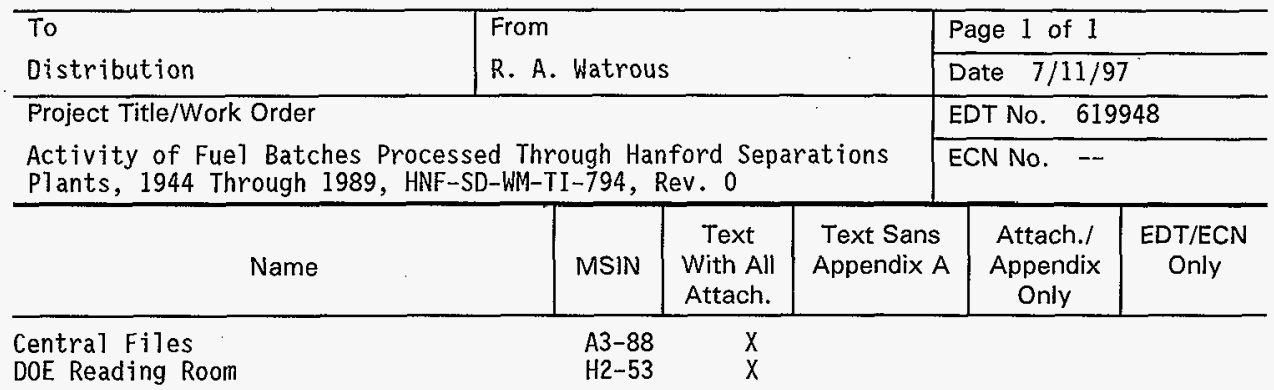

U.S. Department of Energy. Richland Operations office
D. D. Wodrich
S7-50
N. W. Will is
S7-54
$X$
$X$

Fluor Daniel Hanford Inc.

D. J. Washenfelder

$57-40$

$X$

Fluor Daniel Northwest

D. W. Wootan (5)

R. J. Puigh

H0-31

$\mathrm{HO}-31$

J. Greenborg

HO-35

$$
\begin{aligned}
& X \\
& X \\
& X
\end{aligned}
$$

Lockheed Martin Hanford Corporation

J. W. Cammann

K. M. Hodgson

R. A. Watrous (5)

TCSRC

File

$\begin{array}{llll}R 2-11 & & X \\ R 2-12 & & X \\ H 5-27 & & X \\ R 1-10 & X & \\ H 5-49 & X & \end{array}$

Science Applications International Corporation

M. D. LeClair H5-49

$X$

\section{OFFSITE}

Los ATamos National Laboratory

PO Box 1663

S. F. Agnew

CST-14, MS-J586

Los A1amos, NM 87545 